



\section{ADVANCES IN QUANTUM MECHANICS}

Edited by Paul Bracken 


\section{Advances in Quantum Mechanics}

http://dx.doi.org/10.5772/50232

Edited by Paul Bracken

\section{Contributors}

Tokuzo Shimada, Gabino Torres-Vega, Francisco Bulnes, Inge S. Helland, Rodolfo Esquivel, Nelson Flores-Gallegos, Stephen Fulling, Fernando Mera, Jan Jerzy Slawianowski, Vasyl Kovalchuk, Fujii, Argyris Nicolaidis, Rafael De Lima Rodrigues, Constancio Miguel Arizmendi, Omar Gustavo Zabaleta, Peter Enders, GianCarlo Ghirardi, Donald Jack Kouri, Cynthia Whitney, Francisco De Zela, Douglas Singleton, Seyed Mohammad Motevalli, Yasuteru Shigeta, Valeriy Sbitnev, Jonathan Bentwich, Miloš Vaclav Lokajíček, John Ralston, L. M. Arevalo Aguilar, Carlos Robledo Sanchez, Paulo Cesar Garcia Quijas, Balmakov, Maricel Agop, Bjorn Jensen, Sergio Curilef, Flavia Pennini, Paul Bracken

\section{(c) The Editor(s) and the Author(s) 2013}

The moral rights of the and the author(s) have been asserted. All rights to the book as a whole are reserved by INTECH. The book as a whole (compilation) cannot be reproduced, distributed or used for commercial or non-commercial purposes without INTECH's written permission. Enquiries concerning the use of the book should be directed to INTECH rights and permissions department (permissions@intechopen.com). Violations are liable to prosecution under the governing Copyright Law.

\section{(cc)BY}

Individual chapters of this publication are distributed under the terms of the Creative Commons Attribution 3.0 Unported License which permits commercial use, distribution and reproduction of the individual chapters, provided the original author(s) and source publication are appropriately acknowledged. If so indicated, certain images may not be included under the Creative Commons license. In such cases users will need to obtain permission from the license holder to reproduce the material. More details and guidelines concerning content reuse and adaptation can be foundat http://www.intechopen.com/copyright-policy.html.

\section{Notice}

Statements and opinions expressed in the chapters are these of the individual contributors and not necessarily those of the editors or publisher. No responsibility is accepted for the accuracy of information contained in the published chapters. The publisher assumes no responsibility for any damage or injury to persons or property arising out of the use of any materials, instructions, methods or ideas contained in the book.

First published in Croatia, 2013 by INTECH d.o.o.

eBook (PDF) Published by IN TECH d.o.o.

Place and year of publication of eBook (PDF): Rijeka, 2019.

IntechOpen is the global imprint of IN TECH d.o.o.

Printed in Croatia

Legal deposit, Croatia: National and University Library in Zagreb

Additional hard and PDF copies can be obtained from orders@intechopen.com

Advances in Quantum Mechanics

Edited by Paul Bracken

p. $\mathrm{cm}$.

ISBN 978-953-51-1089-7

eBook (PDF) ISBN 978-953-51-5036-7 


\section{We are IntechOpen, \\ the world's leading publisher of Open Access books}

\section{Built by scientists, for scientists}

\section{$4,200+$}

Open access books available

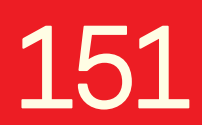

Countries delivered to

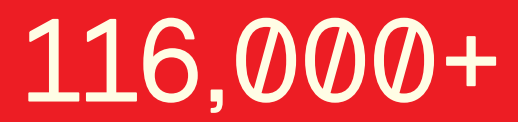

International authors and editors

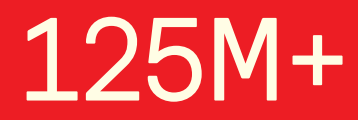

Downloads

Our authors are among the

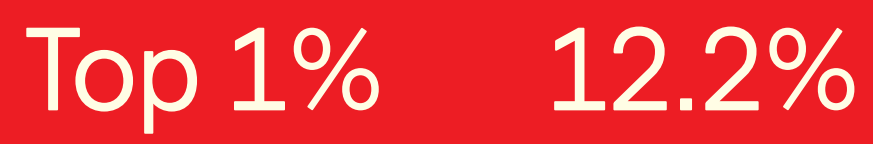

most cited scientists

Contributors from top 500 universities

\section{Interested in publishing with us? \\ Contact book.department@intechopen.com}

Numbers displayed above are based on latest data collected.

For more information visit www.intechopen.com 



\section{Meet the editor}

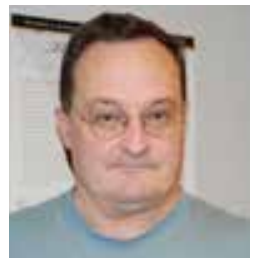

The editor Dr. Paul Bracken is a Professor currently a Professor in the Mathematics Department at the University of Texas in Edinburg, TX. He obtained his BSc degree from the University of Toronto and PhD degree from the University of Waterloo in Canada. His research interests include mathematical problems in quantum mechanics and quantum field theory, differential geometry and partial differential equations as well as gravity. He has published over one hundred papers in journals and books and as well presented about twenty talks at various meetings and conferences and have taught courses at all levels over the years. 



\section{Contents}

Preface XIII

Section 1 The Classical-Quantum Correspondence 1

Chapter 1 Classical and Quantum Conjugate Dynamics - The Interplay Between Conjugate Variables 3

Gabino Torres-Vega

Chapter 2 Classical and Quantum Correspondence in Anisotropic Kepler Problem 23

Keita Sumiya, Hisakazu Uchiyama, Kazuhiro Kubo and Tokuzo Shimada

Chapter 3 Charathéodory's "Royal Road" to the Calculus of Variations: A Possible Bridge Between Classical and Quantum Physics 41 Francisco De Zela

Chapter 4 The Improvement of the Heisenberg Uncertainty Principle 67 L. M. Arévalo Aguilar, C. P. García Quijas and Carlos RobledoSanchez

Section 2 The Schrödinger Equation 79

Chapter 5 Schrödinger Equation as a Hamiltonian System, Essential Nonlinearity, Dynamical Scalar Product and some Ideas of Decoherence 81 Jan J. Sławianowski and Vasyl Kovalchuk

Chapter 6 Schrödinger Equation and (Future) Quantum Physics 105 Miloš V. Lokajíček, Vojtěch Kundrát and Jiří Procházka

Chapter 7 Quantum Damped Harmonic Oscillator 133 Kazuyuki Fujii 
Section 3 Path Integrals 157

Chapter 8 The Schwinger Action Principle and Its Applications to Quantum Mechanics 159

Paul Bracken

Chapter 9 Generalized Path Integral Technique: Nanoparticles Incident on a Slit Grating, Matter Wave Interference 183

Valeriy I. Sbitnev

Chapter 10 Quantum Intentionality and Determination of Realities in the Space-Time Through Path Integrals and Their Integral Transforms 213

Francisco Bulnes

Section 4 Perturbation Theory 245

Chapter 11 Convergence of the Neumann Series for the Schrödinger Equation and General Volterra Equations in Banach Spaces 247

Fernando D. Mera and Stephen A. Fulling

Chapter 12 Quantum Perturbation Theory in Fluid Mixtures 269

S. M. Motevalli and M. Azimi

Chapter 13 Quantal Cumulant Mechanics as Extended Ehrenfest Theorem 293

Yasuteru Shigeta

Chapter 14 Unruh Radiation via WKB Method 317

Douglas A. Singleton

Section 5 Foundations of Quantum Mechanics 333

Chapter 15 A Basis for Statistical Theory and Quantum Theory 335 Inge S. Helland

Chapter 16 Relational Quantum Mechanics 361

A. Nicolaidis 
Chapter 17 On the Dual Concepts of 'Quantum State' and 'Quantum Process' 371

Cynthia Kolb Whitney

Chapter 18 The Computational Unified Field Theory (CUFT): A Candidate 'Theory of Everything' 395

Jonathan Bentwich

Chapter 19 Emergent un-Quantum Mechanics 437

John P. Ralston

Chapter 20 The Wigner-Heisenberg Algebra in Quantum Mechanics 477

Rafael de Lima Rodrigues

Chapter 21 New System-Specific Coherent States by Supersymmetric Quantum Mechanics for Bound State Calculations 499

Chia-Chun Chou, Mason T. Biamonte, Bernhard G. Bodmann and Donald J. Kouri

Section 6 Quantization and Entanglement 519

Chapter 22 Quantum Dating Market $\mathbf{5 2 1}$

C. M. Arizmendi and O. G. Zabaleta

Chapter 23 Quantization as Selection Rather than Eigenvalue Problem 543

Peter Enders

Chapter 24 Entanglement, Nonlocality, Superluminal Signaling and Cloning 565

GianCarlo Ghirardi

Chapter 25 The Husimi Distribution: Development and Applications 595 Sergio Curilef and Flavia Pennini

Section 7 Quantum Information and Related Topics 621

Chapter 26 The Quantum Mechanics Aspect of Structural Transformations in Nanosystems 623

M. D. Bal'makov 
Chapter 27 Decoding the Building Blocks of Life from the Perspective of Quantum Information 641

Rodolfo O. Esquivel, Moyocoyani Molina-Espíritu, Frank Salas, Catalina Soriano, Carolina Barrientos, Jesús S. Dehesa and José A. Dobado

Chapter 28 The Theoretical Ramifications of the Computational Unified Field Theory 671

Jonathan Bentwich

Chapter 29 Shannon Informational Entropies and Chemical

Reactivity 683

Nelson Flores-Gallegos

Chapter 30 A Novel Isospectral Deformation Chain in Supersymmetric Quantum Mechanics 707

Bjørn Jensen

Chapter 31 Quantum Effects Through a Fractal Theory of Motion 723

M. Agop, C.Gh. Buzea, S. Bacaita, A. Stroe and M. Popa 


\section{Preface}

It can be stated that one of the greatest creations of twentieth century physics has been quantum mechanics. This has brought with it a revolutionary view of the physical world in its wake initiated by the work of people like Bohr, Schrödinger, Heisenberg and Born, Pauli and Dirac and many others. The development of quantum mechanics has taken physics in a vastly new direction from that of classical physics from the very start. This is clear from the complicated mathematical formalism of quantum mechanics and the intrinsic statistical nature of measurement theory. In fact, there continue at present to be many developments in the subject of a very fundamental nature, such as implications for the foundations of physics, physics of entanglement, geometric phases, gravity and cosmology and elementary particles as well. Quantum mechanics has had a great impact on technology and in applications to other fields such as chemistry and biology. The intention of the papers in this volume is to give researchers in quantum mechanics, mathematical physics and mathematics an overview and introduction to some of the topics which are of current interest in this area.

Of the 29 chapters, the range of topics to be presented is limited to discussions on the foundations of quantum mechanics, the Schrödinger equation and quantum physics, the relationship of the classical-quantum correspondence, the impact of the path integral concept on quantum mechanics, perturbation theory, quantization and finally some informational-entropy aspects and application to biophysics. Many of the papers could be placed into more than one of these sections, so their breadth is quite substantial.

The book has been put together by a large international group of invited authors and it is necessary to thank them for their hard work and contributions to the book. I gratefully acknowledge with thanks to the assistance provided by Ms. Danijela Duric who was publishing manager during the publishing process, and Intech publishing group for the publication of the book.

Professor Paul Bracken

Department of Mathematics, University of Texas, Edinburg, TX 

Section 1

The Classical-Quantum Correspondence 

Chapter 1

\title{
Classical and Quantum Conjugate Dynamics - The Interplay Between Conjugate Variables
}

\author{
Gabino Torres-Vega \\ Additional information is available at the end of the chapter \\ http://dx.doi.org/10.5772/53598
}

\section{Introduction}

There are many proposals for writing Classical and Quantum Mechanics in the same language. Some approaches use complex functions for classical probability densities [1] and other define functions of two variables from single variable quantum wave functions [2,3]. Our approach is to use the same concepts in both types of dynamics but in their own realms, not using foreign unnatural objects. In this chapter, we derive many inter relationships between conjugate variables.

\subsection{Conjugate variables}

An important object in Quantum Mechanics is the eigenfunctions set $\{\mid n>\}_{n=0}^{\infty}$ of a Hermitian operator $\hat{F}$. These eigenfunctions belong to a Hilbert space and can have several representations, like the coordinate representation $\psi_{n}(q)=\langle q \mid n\rangle$. The basis vector used to provide the coordinate representation, $|q\rangle$, of the wave function are themselves eigenfunctions of the coordinate operator $Q$ We proceed to define the classical analogue of both objects, the eigenfunction and its support.

Classical motion takes place on the associated cotangent space $T^{*} Q$ with variable $z=(q, p)$, where $q$ and $p$ are $n$ dimensional vectors representing the coordinate and momentum of point particles. We can associate to a dynamical variable $F(z)$ its eigensurface, i.e. the level set

$$
\Sigma_{F}(f)=\left\{z \in T^{*} Q^{\mid} F(z)=f\right\}
$$


Where $f$ is a constant, one of the values that $F(z)$ can take. This is the set of points in phase space such that when we evaluate $F(z)$, we obtain the value $f$. Examples of these eigensurfaces are the constant coordinate surface, $q=X$, and the energy shell, $H(z)=E$, the surface on which the evolution of classical systems take place. These level sets are the classical analogues of the support of quantum eigenfunctions in coordinate or momentum representations.

Many dynamical variables come in pairs. These pairs of dynamical variables are related through the Poisson bracket. For a pair of conjugate variables, the Poisson bracket is equal to one. This is the case for coordinate and momentum variables, as well as for energy and time. In fact, according to Hamilton's equations of motion, and the chain rule, we have that

$$
\{t, H\}=\sum_{i}\left(\frac{\partial t}{\partial q^{i}} \frac{\partial H}{\partial p_{i}}-\frac{\partial H}{\partial q^{i}} \frac{\partial t}{\partial p_{i}}\right)=\sum_{i}\left(\frac{d t}{d q^{i}} \frac{d q^{i}}{d t}+\frac{d p_{i}}{d t} \frac{d t}{d p_{i}}\right)=\frac{d t}{d t}=1
$$

Now, a point in cotangent space can be specified as the intersection of $2 n$ hypersurfaces. A set of $2 n$ independent, intersecting, hypersurfaces can be seen as a coordinate system in cotangent space, as is the case for the hyper surfaces obtained by fixing values of coordinate and momentum, i.e. the phase space coordinate system with an intersection at $z=(q, p)$. We can think of alternative coordinate systems by considering another set of conjugate dynamical variables, as is the case of energy and time.

Thus, in general, the $T^{*} Q$ points can be represented as the intersection of the eigensurfaces of the pair of conjugate variables $F$ and $G$,

$$
\Sigma_{F G}(f, g)=\left\{z \in T^{*} Q^{\mid} F(z)=f, G(z)=g\right\}
$$

A point in this set will be denoted as an abstract bra $(f, g \mid$, such that $(f, g \mid u)$ means the function $u(f, g)$.

We can also have marginal representations of functions in phase space by using the eigensurfaces of only one of the functions,

$\Sigma_{F}(f)=\left\{z \in T^{*} Q \mid F(z)=f\right\}, \quad$ and $\quad \Sigma_{G}(g)=\left\{z \in T^{*} Q \mid G(z)=g\right\}$.

A point in the set $\Sigma_{F}(f)\left[\Sigma_{G}(g)\right]$ will be denoted by the bra $(f \mid[(g \mid)$ and an object like $(f \mid u)[(g \mid u)]$ will mean the $f[g]$ dependent function $u(f)[u(g)]$.

\subsection{Conjugate coordinate systems}

It is usual that the origin of one of the variables of a pair of conjugate variables is not well defined. This happens, for instance, with the pair of conjugate variables $q$ and $p$. Even though the momentum can be well defined, the origin of the coordinate is arbitrary on the trajectory of a point particle, and it can be different for each trajectory. A coordinate system fixes the origin of coordinates for all of the momentum eigensurfaces. 
A similar situation is found with the conjugate pair energy-time. Usually the energy is well defined in phase space but time is not. In a previous work, we have developed a method for defining a time coordinate in phase space [4]. The method takes the hypersurface $q^{1}=X$, where $X$ is fixed, as the zero time eigensurface and propagates it forward and backward in time generating that way a coordinate system for time in phase space.

Now, recall that any phase space function $G(z)$ generates a motion in phase space through a set of symplectic system of equations, a dynamical system,

$$
\frac{d z}{d f}=X_{G}, \quad X_{G}=\left(\frac{\partial G}{\partial p},-\frac{\partial G}{\partial q}\right),
$$

where $f$ is a variable with the same units as the conjugate variable $F(z)$. You can think of $G(z)$ as the Hamiltonian for a mechanical system and that $f$ is the time. For classical systems, we are considering conjugate pairs leading to conjugate motions associated to each variable with the conjugate variable serving as the evolution parameter (see below). This will be applied to the energy-time conjugate pair. Let us derive some properties in which the two conjugate variables participate.

\subsection{The interplay between conjugate variables}

Some relationships between a pair of conjugate variables are derived in this section. We will deal with general $F(z)$ and $G(z)$ conjugate variables, but the results can be applied to coordinate and momentum or energy and time or to any other conjugate pair.

The magnitude of the vector field $\left|X_{G}\right|$ is the change of length along the $f$ direction

$$
\left|X_{G}\right|=\sqrt{\frac{d q^{i}}{d f} \frac{d q^{i}}{d f}+\frac{d p_{i}}{d f} \frac{d p_{i}}{d f}}=\frac{d l_{F}}{d f},
$$

where $d l_{F}=\sqrt{\left(d q^{i}\right)^{2}+\left(d p^{i}\right)^{2}}$ is the length element.

A unit density with the eigensurface $\Sigma_{G}(g)$ as support

$$
(z \mid g)=\delta(z-v), \quad v \in \Sigma_{G}(g)
$$

is the classical analogue of the corresponding quantum eigenstate in coordinate $\langle q \mid g\rangle$ and momentum $\langle p \mid g\rangle$ representations. When $G(z)$ is evaluated at the points of the support of $(z \mid g)$, we get the value $g$. We use a bra-ket like notation to emphasise the similarity with the quantum concepts.

The overlap between a probability density with an eigenfunction of $\hat{F}$ or $\hat{G}$ provides marginal representations of a probability density, 


$$
\begin{array}{ll}
\varrho(f):=(f \mid \rho):=\int(f \mid z)(z \mid \rho) d z=\int \delta(z-f) \rho(z) d z, & f \in \sum_{F}(f) . \\
\varrho(g):=(g \mid \rho):=\int(g \mid z)(z \mid \rho) d z=\int \delta(z-g) \rho(z) d z, & g \in \sum_{G}(g) .
\end{array}
$$

But, a complete description of a function in $T^{*} Q$ is obtained by using the two dimensions unit density $(z \mid f, g)=\delta(z-(f, g))$, the eigenfunction of a location in phase space,

$$
\varrho(f, g):=(f, g \mid \rho):=\int(f, g \mid z)(z \mid \rho) d z=\int \delta(z-(f, g)) \rho(z) d z, \quad(f, g) \in \sum_{F G}(f, g) .
$$

In this way, we have the classical analogue of the quantum concepts of eigenfunctions of operators and the projection of vectors on them.

\subsection{Conjugate motions}

Two dynamical variables with a constant Poisson bracket between them induce two types of complementary motions in phase space. Let us consider two real functions $F(z)$ and $G(z)$ of points in cotangent space $z \in T^{*} Q$ of a mechanical system, and a unit Poisson bracket between them,

$$
\{F, G\}=\frac{\partial F}{\partial q^{i}} \frac{\partial G}{\partial p_{i}}-\frac{\partial G}{\partial q^{i}} \frac{\partial F}{\partial p_{i}}=1
$$

valid on some domain $\mathrm{D}=\mathrm{D}\left(\frac{\partial F}{\partial q^{i}}\right) \cap \mathrm{D}\left(\frac{\partial F}{\partial q^{i}}\right) \cap \mathrm{D}\left(\frac{\partial F}{\partial q^{i}}\right) \cap \mathrm{D}\left(\frac{\partial F}{\partial q^{i}}\right)$, according to the considered functions $F$ and $G$. The application of the chain rule to functions of $p$ and $q$, and Eq. (10), suggests two ways of defining dynamical systems for functions $F$ and $G$ that comply with the unit Poisson bracket. One of these dynamical systems is

$$
\frac{d p_{i}}{d F}=-\frac{\partial G}{\partial q^{i}}, \quad \frac{d q^{i}}{d F}=\frac{\partial G}{\partial p_{i}}
$$

With these replacements, the Poisson bracket becomes the derivative of a function with respect to itself

$$
\{F, G\}=\frac{\partial F}{\partial q^{i}} \frac{\partial q^{i}}{\partial F}+\frac{\partial p_{i}}{\partial F} \frac{\partial F}{\partial p_{i}}=\frac{\mathrm{d} F}{\mathrm{~d} F}=1
$$


Note that $F$ is at the same time a parameter in terms of which the motion of points in phase space is written, and also the conjugate variable to $G$.

We can also define other dynamical system as

$$
\frac{d p_{i}}{d G}=\frac{\partial F}{\partial q^{i}}, \quad \frac{d q^{i}}{d G}=-\frac{\partial F}{\partial p_{i}} .
$$

Now, $G$ is the shift parameter besides of being the conjugate variable to $F$. This also renders the Poisson bracket to the identity

$$
\{F, G\}=\frac{d p_{i}}{d G} \frac{\partial G}{\partial p_{i}}+\frac{d q^{i}}{d G} \frac{\partial G}{\partial q^{i}}=\frac{d G}{d G}=1
$$

The dynamical systems and vector fields for the motions just defined are

$$
\frac{d z}{d G}=X_{F}, \quad X_{F}=\left(-\frac{\partial F}{\partial p_{i}}, \frac{\partial F}{\partial q^{i}}\right), \quad \text { and } \quad \frac{d z}{d F}=X_{G}, \quad X_{G}=\left(\frac{\partial G}{\partial p_{i}},-\frac{\partial G}{\partial q^{i}}\right)
$$

Then, the motion along one of the $F$ or $G$ directions is determined by the corresponding conjugate variable. These vector fields in general are not orthogonal, nor parallel.

If the motion of phase space points is governed by the vector field (15), $F$ remains constant because

$$
\frac{d F}{d G}=\frac{\partial F}{\partial q^{i}} \frac{\partial q^{i}}{\partial G}+\frac{\partial F}{\partial p_{i}} \frac{\partial p_{i}}{\partial G}=\frac{\partial p_{i}}{\partial G} \frac{\partial q^{i}}{\partial G}-\frac{\partial q^{i}}{\partial G} \frac{\partial p_{i}}{\partial G}=0
$$

In contrast, when motion occurs in the $F$ direction, by means of Eq. (16), it is the $G$ variable the one that remains constant because

$$
\frac{d G}{d F}=\frac{\partial G}{\partial q^{i}} \frac{\partial q^{i}}{\partial F}+\frac{\partial G}{\partial p_{i}} \frac{\partial p_{i}}{\partial F}=-\frac{\partial p_{i}}{\partial F} \frac{\partial q^{i}}{\partial F}+\frac{\partial q^{i}}{\partial F} \frac{\partial p_{i}}{\partial F}=0
$$

Hence, motion originated by the conjugate variables $F(z)$ and $G(z)$ occurs on the shells of constant $F(z)$ or of constant $G(z)$, respectively.

The divergence of these vector fields is zero,

$$
\nabla \bullet X_{F}=-\frac{\partial}{\partial q^{i}} \frac{\partial F}{\partial p_{i}}+\frac{\partial}{\partial p_{i}} \frac{\partial F}{\partial q^{i}}=0, \quad \nabla \bullet X_{G}=\frac{\partial}{\partial q^{i}} \frac{\partial G}{\partial p_{i}}-\frac{\partial}{\partial p_{i}} \frac{\partial G}{\partial q^{i}}=0
$$


Thus, the motions associated to each of these conjugate variables preserve the phase space area.

A constant Poisson bracket is related to the constancy of a cross product because

$$
\begin{aligned}
& X_{G} \wedge X_{F}=\frac{d z}{d F} \wedge \frac{d z}{d G}=\left|\begin{array}{ccc}
\hat{q} & \hat{p} & \hat{n} \\
\frac{\partial G}{\partial p} & -\frac{\partial G}{\partial q} & 0
\end{array}\right|=\hat{n}\left(\frac{\partial G}{\partial p} \frac{\partial F}{\partial q}-\frac{\partial G}{\partial q} \frac{\partial F}{\partial p}\right)=\hat{n}\{F, G\} . \\
& -\frac{\partial F}{\partial p} \quad \frac{\partial F}{\partial q} \quad 0
\end{aligned}
$$

where $\hat{n}$ is the unit vector normal to the phase space plane. Then, the magnitudes of the vector fields and the angle between them changes in such a way that the cross product remains constant when the Poisson bracket is equal to one, i.e. the cross product between conjugate vector fields is a conserved quantity.

The Jacobian for transformations from phase space coordinates to $(f, g)$ variables is one for each type of motion:

$$
J=\left|\begin{array}{ll}
\frac{\partial q}{\partial f} & \frac{\partial p}{\partial f} \\
\frac{\partial q}{\partial g} & \frac{\partial p}{\partial g}
\end{array}\right|=\left|\begin{array}{cc}
\frac{\partial G}{\partial p} & -\frac{\partial G}{\partial q} \\
\frac{\partial q}{\partial g} & \frac{\partial p}{\partial g}
\end{array}\right|=\frac{\partial G}{\partial p} \frac{\partial p}{\partial g}+\frac{\partial G}{\partial q} \frac{\partial q}{\partial g}=\frac{d G}{d g}=1
$$

and

$$
J=\left|\begin{array}{ll}
\frac{\partial q}{\partial f} & \frac{\partial p}{\partial f} \\
\frac{\partial q}{\partial g} & \frac{\partial p}{\partial g}
\end{array}\right|=\left|\begin{array}{cc}
\frac{\partial q}{\partial f} & \frac{\partial p}{\partial f} \\
-\frac{\partial F}{\partial p} & \frac{\partial F}{\partial q}
\end{array}\right|=\frac{\partial F}{\partial q} \frac{\partial q}{\partial f}+\frac{\partial F}{\partial p} \frac{\partial p}{\partial f}=\frac{d F}{d f}=1
$$

We have seen some properties related to the motion of phase space points caused by conjugate variables.

\subsection{Poisson brackets and commutators}

We now consider the use of commutators in the classical realm.

The Poisson bracket can also be written in two ways involving a commutator. One form is

$$
\{F, G\}=\left(\frac{\partial G}{\partial p} \frac{\partial}{\partial q}-\frac{\partial G}{\partial q} \frac{\partial}{\partial p}\right) F=\left[L_{G}, F\right]=1,
$$


and the other is

$$
\{F, G\}=\left(\frac{\partial F}{\partial q} \frac{\partial}{\partial p}-\frac{\partial F}{\partial p} \frac{\partial}{\partial q}\right) G=\left[\mathrm{L}_{F}, G\right]=1
$$

With these, we have introduced the Liouville type operators

$$
\mathrm{L}_{F}=\frac{\partial F}{\partial q} \frac{\partial}{\partial p}-\frac{\partial F}{\partial p} \frac{\partial}{\partial q}=X_{F} \bullet \nabla, \quad \text { and } \mathrm{L}_{G}=\frac{\partial G}{\partial p} \frac{\partial}{\partial q}-\frac{\partial G}{\partial q} \frac{\partial}{\partial p}=X_{G} \bullet \nabla
$$

These are Lie derivatives in the directions of $X_{F}$ and $X_{G}$, respectively. These operators generate complementary motion of functions in phase space. Note that now, we also have operators and commutators as in Quantum Mechanics.

Conserved motion of phase space functions moving along the $f$ or $g$ directions can be achieved with the above Liouvillian operators as

$$
\frac{\partial}{\partial f}=-L_{G} \text {, and } \frac{\partial}{\partial g}=-L_{F} .
$$

Indeed, with the help these definitions and of the chain rule, we have that the total derivative of functions vanishes, i.e. the total amount of a function is conserved,

$$
\frac{d}{d f}=\frac{d q}{d f} \frac{\partial}{\partial q}+\frac{d p}{d f} \frac{\partial}{\partial p}+\frac{\partial}{\partial f}=\frac{d z}{d f} \bullet \nabla+\frac{\partial}{\partial f}=X_{G} \bullet \nabla+\frac{\partial}{\partial f}=L_{G}+\frac{\partial}{\partial f}=-\frac{\partial}{\partial f}+\frac{\partial}{\partial f}=0,
$$

and

$$
\frac{d}{d g}=\frac{d q}{d g} \frac{\partial}{\partial q}+\frac{d p}{d g} \frac{\partial}{\partial p}+\frac{\partial}{\partial g}=\frac{d z}{d g} \bullet \nabla+\frac{\partial}{\partial g}=X_{F} \bullet \nabla+\frac{\partial}{\partial g}=L_{F}+\frac{\partial}{\partial g}=-\frac{\partial}{\partial g}+\frac{\partial}{\partial g}=0 .
$$

Also, note that for any function $u(z)$ of a phase space point $z$, we have that

$$
\left[\mathrm{L}_{F}, u(z)\right]=\mathrm{L}_{F} u(z)=X_{F} \bullet \nabla u(z)=\frac{\mathrm{dz}}{\mathrm{dG}} \bullet \nabla \mathrm{u}(\mathrm{z})=-\frac{\partial}{\partial g} u(z)
$$

and

$$
\left[\mathrm{L}_{G}, u(z)\right]=\mathrm{L}_{G} u(z)=X_{G} \bullet \nabla u(z)=\frac{d z}{d F} \bullet \nabla u(z)=-\frac{\partial}{\partial f} u(z)
$$

which are the evolution equations for functions along the conjugate directions $f$ and $g$. These are the classical analogues of the quantum evolution equation $\frac{d}{d t}=\frac{1}{i \hbar}[, \hat{H}]$ for time dependent operators. The formal solutions to these equations are 


$$
u(z ; g)=e^{-g L_{F}} u(z), \quad \text { and } \quad u(z ; f)=e^{-f L_{G}} u(z) .
$$

With these equations, we can now move a function $u(z)$ on $T^{*} Q$ in such a way that the points of their support move according to the dynamical systems Eqs. (15) and the total amount of $u$ is conserved.

\subsection{The commutator as a derivation and its consequences}

As in quantum theory, we have found commutators and there are many properties based on them, taking advantage of the fact that a commutator is a derivation.

Since the commutator is a derivation, for conjugate variables $F(z)$ and $G(z)$ we have that, for integer $n$,

$$
\left[\mathbf{L}_{G}^{n}, F\right]=n \mathbf{L}_{G}^{n-1}, \quad\left[\mathrm{~L}_{G}, F^{n}\right]=n F^{n-1}, \quad\left[\mathbf{L}_{F}^{n}, G\right]=n \mathbf{L}_{F}^{n-1}, \quad\left[\mathrm{~L}_{F}, G^{n}\right]=n G^{n-1} .
$$

Based on the above equalities, we can get translation relationships for functions on $T^{*} Q$. We first note that, for a holomorphic function $u(x)=\sum_{n=0}^{\infty} u_{n} x^{n}$,

$$
\left[u\left(\mathrm{~L}_{G}\right), F\right]=\left[\sum_{n=0}^{\infty} u_{n} \mathrm{~L}_{G}^{n}, F\right]=\sum_{n=0}^{\infty} n u_{n} \mathrm{~L}_{G}^{n-1}=u^{\prime}\left(\mathrm{L}_{G}\right) .
$$

In particular, we have that

$$
\left[e^{f\left\llcorner_{G}\right.}, F\right]=f e^{f\left\llcorner_{G}\right.}
$$

Then, $e^{f \mathrm{~L}_{G}}$ is the eigenfunction of the commutator $[\bullet, F]$ with eigenvalue $f$.

From Eq. (32), we find that

$$
u\left(\mathrm{~L}_{G}\right) F-F u\left(\mathrm{~L}_{G}\right)=u^{\prime}\left(\mathrm{L}_{G}\right)
$$

But, if we multiply by $u^{-1}\left(\mathrm{~L}_{G}\right)$ from the right, we arrive to

$$
u\left(\mathrm{~L}_{G}\right) F u^{-1}\left(\mathrm{~L}_{G}\right)=F+u^{\prime}\left(\mathrm{L}_{G}\right) u^{-1}\left(\mathrm{~L}_{G}\right)
$$

This is a generalized version of a shift of $F$, and the classical analogue of a generalization of the quantum Weyl relationship. A simple form of the above equality, a familiar form, is obtained with the exponential function, i.e.

$$
e^{f\left\llcorner_{G}\right.} F e^{-f L_{G}}=F+f .
$$


This is a relationship that indicates how to translate the function $F(z)$ as an operator. When this equality is acting on the number one, we arrive at the translation property for $F$ as a function

$$
F(z ; f)=\left(e^{f \mathrm{~L}_{G}} F(z)\right)=F(z) e^{f \mathrm{~L}_{G}} 1+f e^{f \mathrm{~L}_{G}} 1=F(z)+f .
$$

This implies that

$$
\frac{d}{d f} F(z ; f)=1,
$$

i.e., up to an additive constant, $f$ is the value of $F(z)$ itself, one can be replaced by the other and actually they are the same object, with $f$ the classical analogue of the spectrum of a quantum operator.

Continuing in a similar way, we can obtain the relationships shown in the following diagram

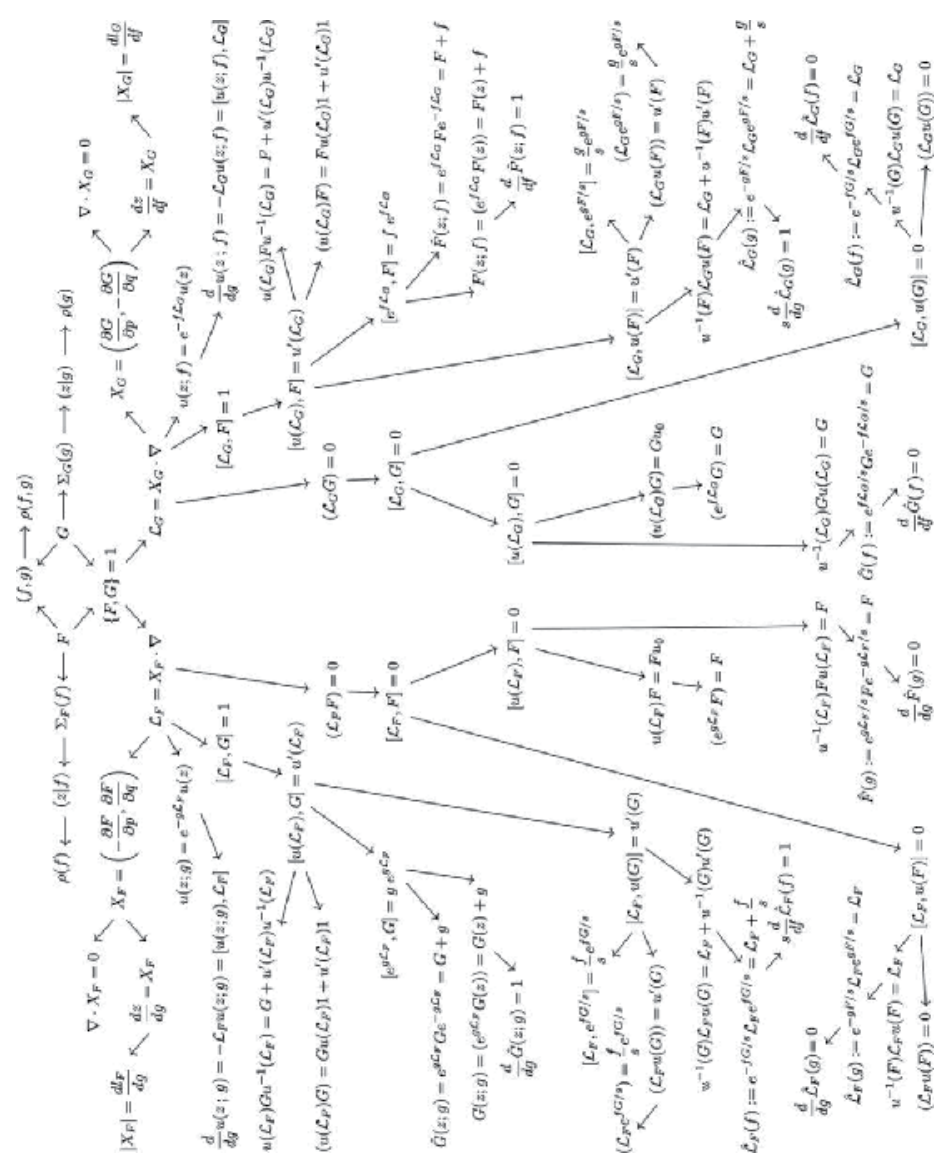

Diagram 1. 
where the constant $s$ has units of action, length times momentum, the same units as the quantum constant $\hbar$.

Some of the things to note are:

The operator $e^{g \mathrm{~L}_{F}}$ is the eigenoperator of the commutator $[\bullet, G]$ and can be used to generate translations of $G(z)$ as an operator or as a function. This operator is also the propagator for the evolution of functions along the $g$ direction. The variable $g$ is more than just a shift parameter; it actually labels the values that $G(z)$ takes, the classical analogue of the spectrum of a quantum operator.

The operators $L_{F}$ and $G(z)$ are also a pair of conjugate operators, as well as the pair $L_{G}$ and $F(z)$.

But $\mathrm{L}_{F}$ commutes with $F(z)$ and then it cannot be used to translate functions of $F(z), F(z)$ is a conserved quantity when motion occurs along the $G(z)$ direction.

The eigenfunction of $\left[\mathbf{L}_{F}, \bullet\right]$ and of $s \mathbf{L}_{F}$ is $e^{f G(z) / s}$ and this function can be used to shift $\mathbf{L}_{F}$ as an operator or as a function.

The variable $f$ is more than just a parameter in the shift of $s L_{F}$, it actually is the value that $s L_{F}$ can take, the classical analogue of the spectra of a quantum operator.

The steady state of $\mathrm{L}_{F}$ is a function of $F(z)$, but $e^{g F(z) / s}$ is an eigenfunction of $\mathrm{L}_{G}$ and of $\left[L_{G}, \bullet\right]$ and it can be used to translate $L_{G}$.

These comments involve the left hand side of the above diagram. There are similar conclusions that can be drawn by considering the right hand side of the diagram.

Remember that the above are results valid for classical systems. Below we derive the corresponding results for quantum systems.

\section{Quantum systems}

We now derive the quantum analogues of the relationships found in previous section. We start with a Hilbert space $\mathrm{H}$ of wave functions and two conjugate operators $\hat{F}$ and $G$ acting on vectors in $\mathrm{H}$, and with a constant commutator between them

$$
[\hat{F}, \hat{G}]=i \hbar
$$

together with the domain $D=D_{\lambda}(\hat{F} \hat{G}) \cap D(\hat{G} \hat{F})$ in which the commutator holds. Examples of these operators are coordinate $\hat{Q}$ and momentum $P$ operators, energy $H$ and time $T$ operators, creation $\hat{a}^{\dagger}$ and annihilation $\hat{a}$ operators. 
The eigenvectors of the position, momentum and energy operators have been used to provide a representation of wave functions and of operators. So, in general, the eigenvectors $|f\rangle$ and $|g\rangle$ of the conjugate operators $\hat{F}$ and $\hat{G}$ provide with a set of vectors for a representation of dynamical quantities like the wave functions $\langle f \mid \psi\rangle$ and $\langle g \mid \psi\rangle$.

With the help of the properties of commutators between operators, we can see that

$$
\left[\hat{F}^{n}, \hat{G}\right]=i \hbar \hat{F}^{n-1}, \quad\left[\hat{F}, \hat{G}^{n}\right]=i \hbar \hat{G}^{n-1}
$$

Hence, for a holomorphic function $u(z)=\sum_{n=0}^{\infty} u_{n} z^{n}$ we have that

$$
[\hat{u}(\hat{F}), \hat{G}]=i \hbar \hat{u}^{\prime}(\hat{F}), \quad[\hat{F}, \hat{u}(\hat{G})]=i \hbar \hat{u}^{\prime}(\hat{G})
$$

i.e., the commutators behave as derivations with respect to operators. In an abuse of notation, we have that

$$
\frac{1}{i \hbar}[\bullet, \hat{G}]=\frac{d \bullet}{d F}, \quad \frac{1}{i \hbar}[\hat{F}, \bullet]=\frac{d \bullet}{d G} .
$$

We can take advantage of this fact and derive the quantum versions of the equalities found in the classical realm.

A set of equalities is obtained from Eq. (43) by first writing them in expanded form as

$$
\hat{u}(\hat{F}) \hat{G}-\hat{G} \hat{u}(\hat{F})=i \hbar \hat{u}^{\prime}(\hat{F}), \quad \text { and } \quad \hat{F} \hat{u}(\hat{G})-\hat{u}(\hat{G}) \hat{F}=i \hbar \hat{u}^{\prime}(\hat{G})
$$

Next, we multiply these equalities by the inverse operator to the right or to the left in order to obtain

$$
\hat{u}(\hat{F}) \hat{G} \hat{u}^{-1}(\hat{F})=\hat{G}+i \hbar \hat{u}^{\prime}(\hat{F}) \hat{u}^{-1}(\hat{F}), \quad \text { and } \quad \hat{u}^{-1}(\hat{G}) \hat{F} \hat{u}(\hat{G})=\hat{F}+i \hbar \hat{u}^{-1}(\hat{G}) \hat{u}^{\prime}(\hat{G})
$$

These are a set of generalized shift relationships for the operators $\hat{G}$ and $\hat{F}$. The usual shift relationships are obtained when $u(x)$ is the exponential function, i.e.

$$
\hat{G}(g):=e^{-i g \hat{F} / \hbar} \hat{G} e^{i g \hat{F} / \hbar}=\hat{G}+g, \quad \text { and } \quad \hat{F}(f):=e^{i f \hat{G} / \hbar} \hat{F} e^{-i f \hat{G} / \hbar}=\hat{F}+f .
$$


Now, as in Classical Mechanics, the commutator between two operators can be seen as two different derivatives introducing quantum dynamical system as

$$
\begin{aligned}
& \frac{d \hat{P}(f)}{d f}=-\frac{\partial \hat{G}\left(\hat{Q_{N}} \hat{P}\right)}{\partial Q}=\frac{1}{i h}[\hat{P}(f), \hat{G}(\hat{Q}, \hat{P})], \quad \frac{d \hat{Q}(f)}{d f}=\frac{\partial \hat{G}\left(\hat{Q_{k}} \hat{P}\right)}{\partial P}=\frac{1}{i \hbar}[\hat{Q}(f), \hat{G}(\hat{Q}, \hat{P})], \\
& \frac{d \hat{P}(g)}{d g}=\frac{\partial \hat{F}\left(\hat{Q_{k}} \hat{P}\right)}{\partial Q}=\frac{1}{i \hbar}[\hat{F}(\hat{Q}, \hat{P}), \hat{P}(g)], \quad \text { and } \quad \frac{d \hat{Q}(g)}{d g}=-\frac{\partial \hat{F}\left(\hat{Q_{k}} \hat{P}\right)}{\partial P}=\frac{1}{i h}[\hat{F}(\hat{Q}, \hat{P}), \hat{Q}(g)]
\end{aligned}
$$

where

$$
\begin{aligned}
& \hat{P}(f)=e^{i f \hat{G} / \hbar} \hat{P} e^{-i f \hat{G} / \hbar}, \quad \hat{Q}(f)=e^{i f \hat{G} / \hbar} \hat{Q} e^{-i f \hat{G} / \hbar}, \\
& \hat{P}(g)=e^{-i g \hat{F} / \hbar} \hat{P} e^{i f \hat{F} / \hbar}, \quad \text { and } \quad \hat{Q}(g)=e^{-i g \hat{F} / \hbar} \hat{Q} e^{i g \hat{F} / \hbar}
\end{aligned}
$$

These equations can be written in the form of a set of quantum dynamical systems

$$
\frac{d \hat{z}}{d f}=\hat{X}_{G}, \quad \hat{X}_{G}=\left(\frac{\partial \hat{G}}{\partial P},-\frac{\partial \hat{G}}{\partial Q}\right), \quad \frac{d \hat{z}}{d g}=\hat{X}_{F}, \quad \hat{X}_{F}=\left(-\frac{\partial \hat{E}}{\partial P}, \frac{\partial \hat{E}}{\partial Q}\right),
$$

where $\hat{z}=(\hat{Q}, \hat{P})$

The inner product between the operator vector fields is

$$
\hat{X}_{F}^{\dagger} \bullet \hat{X}_{F}=\left(-\frac{\partial \hat{E}_{\hat{X}}}{\partial P}, \frac{\partial \hat{E}}{\partial Q}\right)^{+} \bullet\left(-\frac{\partial \hat{E}}{\partial P}, \frac{\partial \hat{E}}{\partial Q}\right)=\left(\frac{d \hat{Q}}{d g}\right)^{2}+\left(\frac{d \hat{P}}{d g}\right)^{2}:=\left(\frac{d \hat{l}_{F}}{d g}\right)^{2}
$$

where $\left(d \hat{l}_{F}\right)^{2}:=(d \hat{Q})^{2}+(d \hat{P})^{2}$, evaluated along the $g$ direction, is the quantum analogue of the square of the line element $\left(d l_{F}\right)^{2}=(d q)^{2}+(d p)^{2}$.

We can define many of the classical quantities but now in the quantum realm. Liouville type operators are

$$
\hat{\mathrm{L}}_{F}:=\frac{1}{i \hbar}[\hat{F}, \bullet], \quad \text { and } \quad \hat{\mathrm{L}}_{G}:=\frac{1}{i \hbar}[\bullet, \hat{G}]
$$

These operators will move functions of operators along the conjugate directions $\hat{G}$ or $\hat{F}$, respectively. This is the case when $G$ is the Hamiltonian $H$ of a physical system, a case in which we get the usual time evolution of operator. 
There are many equalities that can be obtained as in the classical case. The following diagram shows some of them:

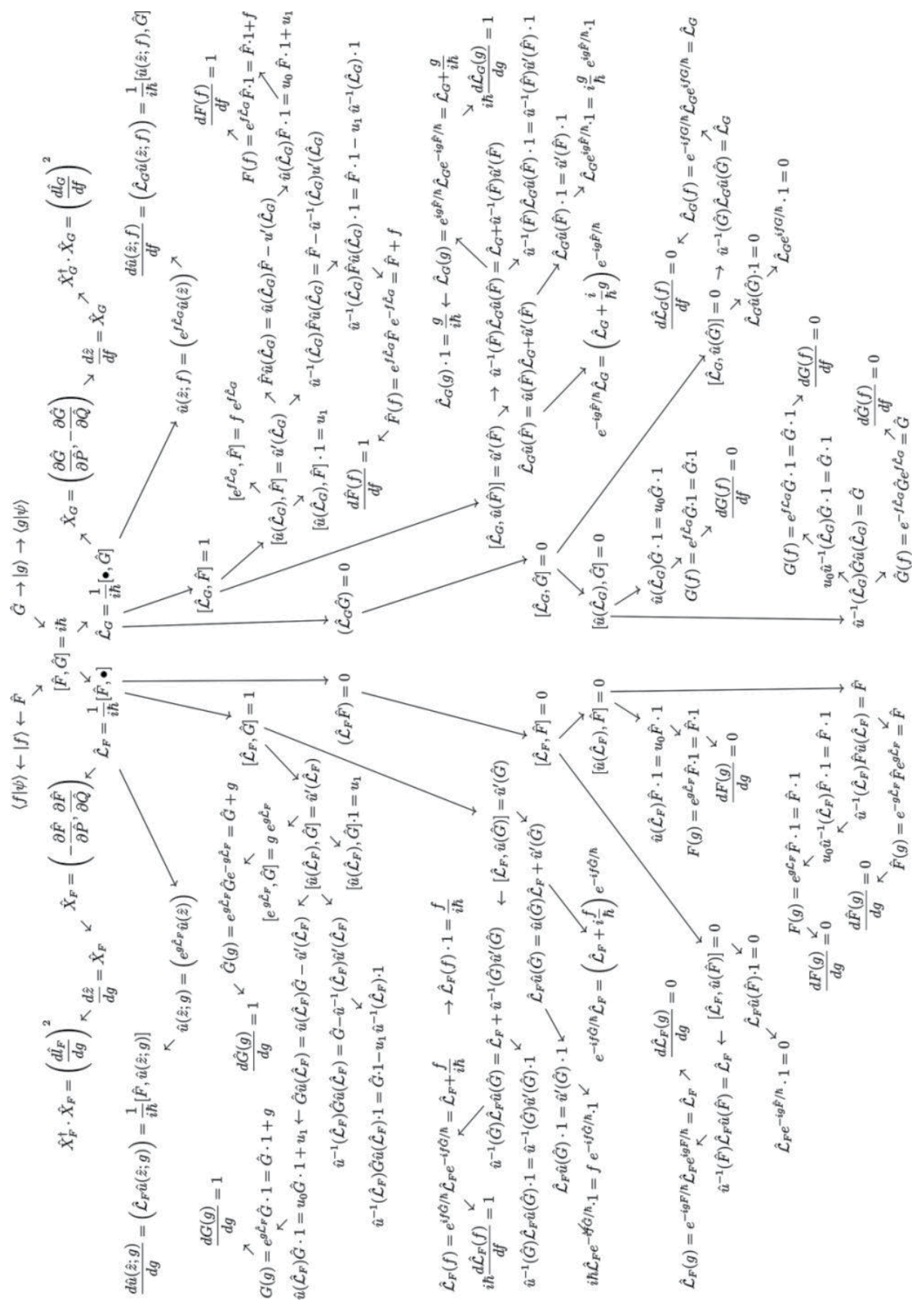

Diagram 2. 
Note that the conclusions mentioned at the end of the previous section for classical systems also hold in the quantum realm.

Next, we illustrate the use of these ideas with a simple system.

\section{Time evolution using energy and time eigenstates}

As a brief application of the abovee ideas, we show how to use the energy-time coordinates and eigenfunctions in the reversible evolution of probability densities.

Earlier, there was an interest on the classical and semi classical analysis of energy transfer in molecules. Those studies were based on the quantum procedure of expanding wave functions in terms of energy eigenstates, after the fact that the evolution of energy eigenstates is quite simple in Quantum Mechanics because the evolution equation for a wave function $i \hbar \frac{\partial}{\partial t}|\psi>=\hat{H}| \psi>$ is linear and contains the Hamiltonian operator. In those earlier calculations, an attempt to use the eigenfunctions of a complex classical Liouville operator was made [5-8]. The results in this chapter show that the eigenfunction of the Liouville operator $\mathrm{L}_{H}$ is $e^{g T(z)}$ and that it do not seems to be a good set of functions in terms of which any other function can be written, as is the case for the eigenfunctions of the Hamiltonian operator in Quantum Mechanics. In this section, we use the time eigenstates instead.

With energy-time eigenstates the propagation of classical densities is quite simple. In order to illustrate our procedure, we will apply it to the harmonic oscillator with Hamiltonian given by (we will use dimensionless units)

$$
H(z)=\frac{p^{2}}{2}+\frac{q^{2}}{2}
$$

Given and energy scaling parameter $E_{s}$ and the frequency $\omega$ of the harmonic oscillator, the remaining scaling parameters are

$$
p_{s}=\sqrt{m E_{s}}, \quad q_{s}=\sqrt{\frac{E_{s}}{m \omega^{2}}}, \quad t_{s}=\frac{1}{\omega} .
$$

We need to define time eigensurfaces for our calculations. The procedure to obtain them is to take the curve $q=0$ as the zero time curve. The forward and backward propagation of the zero time curve generates the time coordinate system in phase space. The trajectory generated with the harmonic oscillator Hamiltonian is

$$
q(t)=\sqrt{2 E} \cos \left(t+\frac{\pi}{2}\right), \quad p(t)=\sqrt{2 E} \sin \left(t+\frac{\pi}{2}\right) .
$$

With the choice of phase we have made, $q=0$ when $t=0$, which is the requirement for an initial time curve. Then, the equation for the time curve is 


$$
p=q \tan \left(t+\frac{\pi}{2}\right), \quad \text { or } q=p \cot \left(t+\frac{\pi}{2}\right) .
$$

These are just straight lines passing through the origin, equivalent to the polar coordinates. The value of time on these points is $t$, precisely. In Fig. 1, we show both coordinate systems, the phase space coordinates $(q, p)$, and the energy time coordinates $(E, t)$ on the plane. This is a periodic system, so we will only consider one period in time.

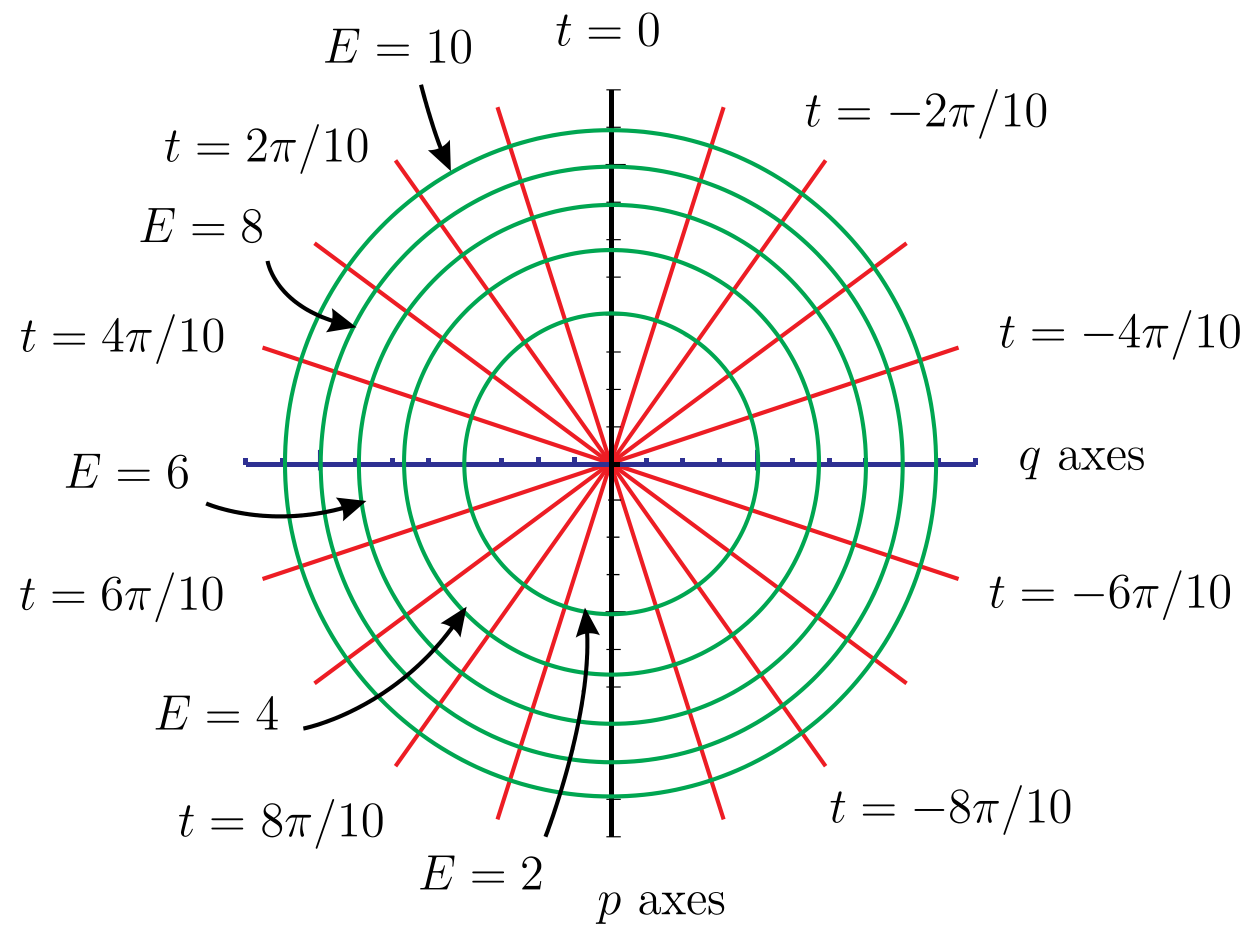

Figure 1. Two conjugate coordinate systems for the classical harmonic oscillator in dimensionless units. Blue and black lines correspond to the $(q, p)$ coordinates and the red and green curves to the $(E, t)$ coordinates.

At this point, there are two options for time curves. Both options will cover the plane and we can distinguish between the regions of phase space with negative or positive momentum. One is to use half lines and $t$ in the range from $-\pi$ to $\pi$, with the curve $t=0$ coinciding with the positive $p$ axes. The other option is to use the complete curve including positive 
and negative momentum values and with $t \in(-\pi / 2, \pi / 2)$. In the first option, the positive momentum part of a probability density will correspond to the range $t \in(-\pi / 2, \pi / 2)$, and the negative values will correspond to $t \in\left(-\pi,-\frac{\pi}{2}\right) \cup(\pi / 2, \pi)$. We take this option.

Now, based on the equalities derived in this chapter, we find the following relationship for a marginal density dependent only upon $H(z)$, assuming that the function $\rho(H)$ can be written as a power series of $H, \rho(H)=\sum_{i} \rho_{i} H^{i}$,

$$
e^{-\tau \mathbf{L}_{H}} \rho(H)=\sum_{n} \frac{(-\tau)^{n}}{n !} \mathbf{L}_{H}^{n} \sum_{i} \rho_{i} H^{i}=\sum_{i} \rho_{i} H^{i}=\rho(H)
$$

where we have made use of the equality $\mathrm{L}_{H} H=0$. Then, a function of $H$ does not evolve in time, it is a steady state. For a marginal function dependent upon $t$, we also have that

$$
e^{-\tau \mathrm{L}_{H}} \rho(t)=e^{\tau d / d t} \rho(t)=\rho(t+\tau)
$$

where we have made use of the result that $\frac{d}{d t}=-L_{H}$. Therefore, a function of $t$ is only shifted in time without changing its shape.

For a function of $H$ and $t$ we find that

$$
e^{-\tau \mathrm{L}_{H}} \rho(H, t)=e^{-\tau d / d t} \rho(H, t)=\rho(H, t+\tau)
$$

This means that evolution in energy-time space also is quite simple, it is only a shift of the function along the $t$ axes without a change of shape.

So, let us take a concrete probability density and let us evolve it in time. The probability density, in phase space, that we will consider is

$$
\rho(z)=H(z) e^{-\left(\left(q-q_{0}\right)^{2}+\left(p-p_{0}\right)^{2}\right) / 2 \sigma^{2}},
$$

with $\left(q_{0}, p_{0}\right)=(1,2)$ and $\sigma=1$. A contour plot of this density in phase-space is shown in (a) of Fig. 2. The energy-time components of this density are shown in (b) of the same figure. Time evolution by an amount $\tau$ correspond to a translation along the $t$ axes, from $t$ to $t+\tau$, without changing the energy values. This translation is illustrated in (d) of Fig. 2 in energy-time space and in (c) of the same figure in phase-space.

Recall that the whole function $\rho(z)$ is translated in time with the propagator $e^{-\tau L_{H}}$. Then, there are two times involved here, the variable $t$ as a coordinate and the shift in time $\tau$. The latter is the time variable that appears in the Liouville equation of motion $\frac{d \rho(z ; \tau)}{d \tau}=-\mathrm{L}_{H} \rho(z ; \tau)$. 

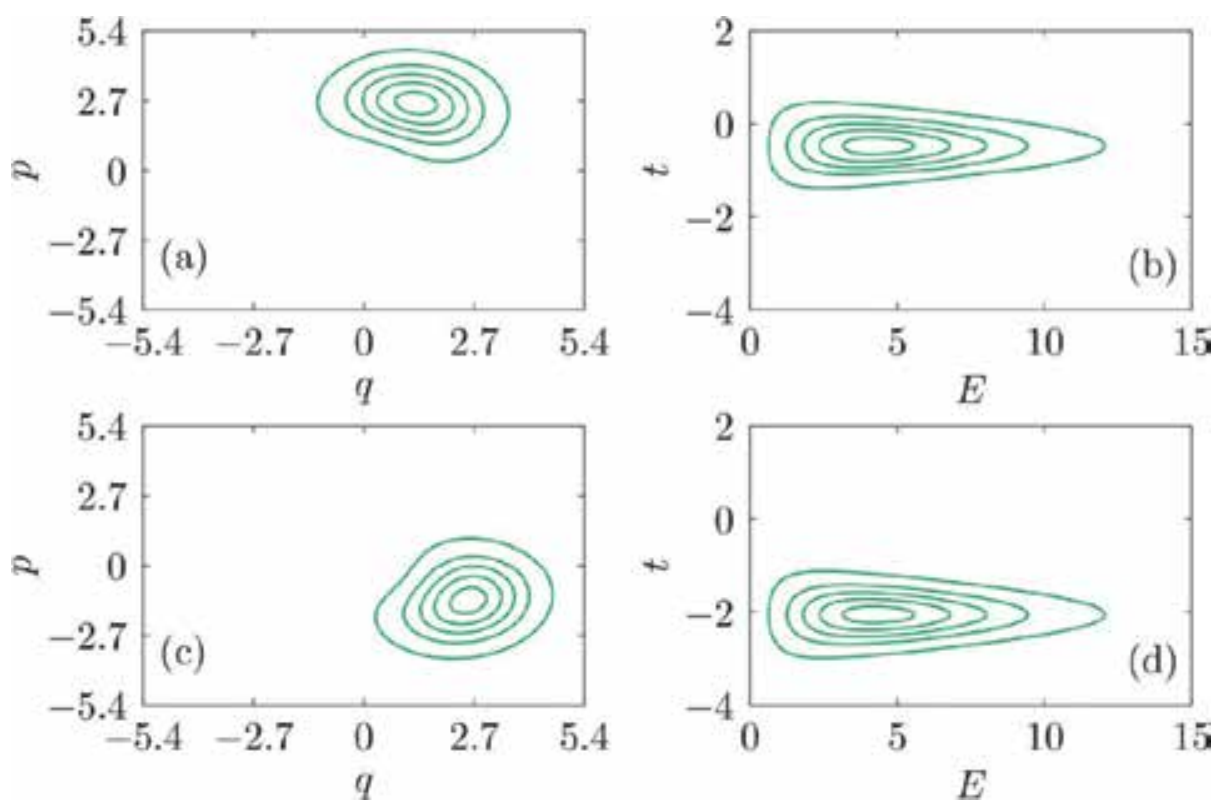

Figure 2. Contour plots of the time evolution of a probability density on phase-space and on energy-time space. Initial densities (a) in phase space, and (b) in energy-time space. (d) Evolution in energy-time space is accomplished by a shift along the taxes. (c) In phase space, the density is also translated to the corresponding time eigensurfaces.

This behaviour is also observed in quantum systems. Time eigenfunctions can be defined in a similar way as for classical systems. We start with a coordinate eigenfunction $|q\rangle$ for the eigenvalue $q=0$ and propagate it in time. This will be our time eigenstate

$$
\left.\right|_{t>=e^{\frac{i t H}{\hbar}}} \mid q=0>
$$

The projection of a wave function onto this vector is

$$
<t|\psi>=<q=0| e^{-\frac{i t \hat{H}}{\hbar}} \mid \psi>=\psi(\mathrm{q}=0 ; \mathrm{t}),
$$

Which is the time dependent wave function, in the coordinate representation, and evaluated at $q=0$. This function is the time component of the wave function.

The time component of a propagated wave function for a time $\tau$ is

$$
<t|\psi(\tau)>=<q=0| e^{-\frac{i t \hat{H}}{\hbar}} e^{-\frac{i \tau \hat{H}}{\hbar}}|\psi>=<t+\tau| \psi>
$$

Then, time evolution is the translation in time representation, without a change in shape. Note that the variable $\tau$ is the time variable that appears in the Schrödinger equation for the wave function. 
Now, assuming a discrete energy spectrum with energy eigenvalue $E_{n}$ and corresponding eigenfunction $|n\rangle$, in the energy representation we have that

$$
<n \mid \psi(\tau)>=\left\langle n\left|e^{-\frac{i \tau \hat{H}}{\hbar}}\right| \psi>=e^{-\frac{i \tau \mathrm{E}_{\mathrm{n}}}{\hbar}}\langle n| \psi>\right.
$$

i.e. the wave function in energy space only changes its phase after evolution for a time $\tau$.

\section{Concluding remarks}

Once that we have made use of the same concepts in both classical and quantum mechanics, it is more easy to understand quantum theory since many objects then are present in both theories.

Actually, there are many things in common for both classical and quantum systems, as is the case of the eigensurfaces and the eigenfunctions of conjugate variables, which can be used as coordinates for representing dynamical quantities.

Another benefit of knowing the influence of conjugate dynamical variables on themselves and of using the same language for both theories lies in that some puzzling things that are found in one of the theories can be analysed in the other and this helps in the understanding of the original puzzle. This is the case of the Pauli theorem [9-14] that prevents the existence of a hermitian time operator in Quantum Mechanics. The classical analogue of this puzzle is found in Reference [15].

These were some of the properties and their consequences in which both conjugate variables participate, influencing each other.

\section{Author details}

Gabino Torres-Vega

Physics Department, Cinvestav, México

\section{References}

[1] Woodhouse NMJ. Geometric Quantization. Oxford: Osford University Press; 1991.

[2] Wigner E. Phys Rev A 1932; 40749

[3] Husimi K. Proc Phys Math Soc Jpn 1940; 22264

[4] Torres-Vega G, Theoretical concepts of quantum mechanics. Rijeka: InTech; 2012. 
[5] Jaffé C. Classical Liouville mechanics and intramolecular relaxation dynamics. The Journal of Physical Chemistry 1984; 884829.

[6] Jaffé C and Brumer C. Classical-quantum correspondence in the distribution dynamics of integrable systems. Journal of Chemical Physics 1985; 822330.

[7] Jaffé C. Semiclassical quantization of the Liouville formulation of classical mechanics. Journal of Chemical Physics 1988; 887603.

[8] Jaffé C. Sheldon Kanfer and Paul Brumer, Classical analog of pure-state quantum dynamics. Physical Review Letters 1985; 548.

[9] Pauli W. Handbuch der Physics. Berlin: Springer-Verlag; 1926

[10] Galapon EA, Proc R Soc Lond A 2002; 458451

[11] Galapon EA, Proc R Soc Lond A 2002; 4582671

[12] Galapon EA, quant-ph/0303106

[13] Galindo A, Lett Math Phys 1984; 8495

[14] Garrison JC and Wong J, J Math Phys 1970; 112242

[15] Torres-Vega G, J Phys A: Math Theor 45, 215302 (2012) 



\title{
Classical and Quantum Correspondence in Anisotropic Kepler Problem
}

\author{
Keita Sumiya, Hisakazu Uchiyama, \\ Kazuhiro Kubo and Tokuzo Shimada \\ Additional information is available at the end of the chapter
}

http://dx.doi.org/10.5772/55208

\section{Introduction}

If the classical behavior of a given quantum system is chaotic, how is it reflected in the quantum properties of the system? To elucidate this correspondence is the main theme of the quantum chaos study. With the advent of nanophysics techniques, this has become also of experimental importance. With the advent of new technology, various quantum systems are now challenging us. These include nano-scale devices, laser trapping of atoms, the Bose-Einstein condensate, Rydberg atoms, and even web of chaos is observed in superlattices.

In this note we devote ourselves to the investigation of the quantum scars which occurs in the Anisotropic Kepler Problem (AKP) - the classical and quantum physics of an electron trapped around a proton in semiconductors. The merit of AKP is that its chaotic property can be controlled by changing the anisotropy from integrable Kepler limit down ergodic limit where the tori are completely collapsed and isolated unstable periodic orbits occupy the classical phase space. Thus in AKP we are able to investigate the classical quantum correspondence at varying chaoticity. Furthermore each unstable periodic orbit (PO) can be coded in a Bernoulli code which is a large merit in the formulation of quantum chaos in term of the periodic orbit theory (POT) $[1,2,6]$.

The AKP is an old home ground of the quantum chaos study. Its low energy levels were used as a test of the periodic orbit theory in the seminal work of Gutzwiller [3-7]. Then an efficient matrix diagonalization scheme was devised by Wintgen et al. (WMB method) [8]. With this method, the statistics of up to nearly 8000 AKP quantum levels were examined and it was found that the quantum level statistics of AKP change from Poisson to Wigner distribution with the increase of mass anisotropy [9]. Furthermore, an intriguing classical Poincaré surface of section (POS) was found at medium anisotropy $(\gamma \equiv m($ light $) / m$ (heavy) $=0.8)$, 
which indicates remnants of tori (cantori) in the classical phase space [9]. Thus, over two decades from the early 70th, AKP was a good testing ground of theories (along with billiards) as well as a constant source of important information to quantum chaos studies. However, there has not been much recent theory investigation on AKP. Especially, to our knowledge, the quantum scar of the classical periodic orbits in AKP has not been directly examined, even though intriguing phenomena was discovered by Heller [10] in 1984. On the other hand, for an analogous system - the hydrogen under a magnetic field (diamagnetic Kepler problem $(\mathrm{DKP})$ ), the scars of periodic orbits were extensively studied using highly efficient tool called as scar strength functions [11]. We note that AKP is by far simpler; for DKP it is necessary to code the POs by a sequence of symbols consisting of three letters.

Recently the level statistics of AKP was examined from the random matrix theory view [12]. It was considered that the AKP level statistic in the transitive region from Poisson to Wigner distribution correspond to the critical level statistics of an extended GOE random matrix theory and it was conjectured that the wave functions should exhibit characteristic multifractality. This aspect has been further developed in [13, 14]; it is considered that Anderson transition occurs in the quantum physics of a class of physical systems such as $\mathrm{AKP}$ and periodically driven kicked rotator in their critical parameter regions. Further very recently a well devised new solid state experiment has been conducted for AKP and ADKP $[15,16]$. We also refer [17] for a recent overview including this interesting conjecture.

Such is the case we have recently conducted AKP high accuracy matrix diagonalization based on the WMB method. This is not a perturbation calculation; the anisotropy term is not regarded as a perturbation and the full Hamiltonian matrix is diagonalized. Thus the approximation comes only from the size of the matrix. But, as a trade-off, a scaling parameter is unavoidably included; it is crucial to choose a correct parameter value at every anisotropy parameter. We have derived a simple rule of thumb to choose a suitable value [17]. After comparing with original WMB result in Sturmian basis, we have also worked with tensored-harmonic-wavefunction basis (THWFB) [11], which is more suitable for the Husimi function calculation to investigate the quantum scars. Our contribution here is the calculation of anisotropy term in the AKP Hamiltonian in THWFB [17], which is harder than the diamagnetic case. Comparing the results from two independent bases we have verified that both results agree completely thus the choices of scaling parameters (in both bases) are validated.

Aimed by these numerical data, we report in section 2 salient evidences of quantum scars in AKP for the first time. We compare the features of various known observables; thus this section will serve as a comparative test of methods and fulfills the gap in the literature pointed out above. Most interesting is the test using the scar strength function. We show that even in the ergodic regime $(\gamma=0.2)$, we can quantitatively observe that prominent periodic orbits systematically contribute to the quantum theory endowed with random energy spectrum.

In section 3 we investigate that how the scaring phenomena are affected by the variation of the anisotropy parameter. It is well known that the energy levels show successive avoiding crossings. On the other hand, in the periodic orbit formula, each term in the series for the density of states (DOS) consists of a contribution of an unstable PO with a pole (with an imaginary part given by the Lyapunov exponent of the PO) at the Bohr-Sommerfeld-type energy; thus each term smoothly varies with the anisotropy. We show that how these two 
seemingly contradicting features intriguingly compromise. The localization patterns in the wave functions or Husimi functions are swapped between two eigenstates of energy at every avoiding crossing. Repeating successively this swap process characteristic scarring patterns follow the POs responsible to them. In this sense the quantum scarring phenomena are robust. We conclude in section 4 .

\section{Manifestation of Scars in AKP}

We first explain how we have prepared the energy levels and wave functions. Then we introduce the indispensable ingredients to study the scars in AKP. After briefly explaining Husimi functions, we explain periodic orbit theory. The quantum scars will be observed along the classical unstable periodic orbits.

\subsection{Matrix diagonalization}

\subsubsection{AKP Hamiltonian}

The AKP Hamiltonian in the dimensionless form is given by

$$
H_{G}=\frac{1}{2 \mu} p_{x}^{2}+\frac{1}{2 v}\left(p_{y}^{2}+p_{z}^{2}\right)-\frac{1}{r}
$$

where $r=\sqrt{x^{2}+y^{2}+z^{2}}$ and $\mu>v[1,4]$ with which POT was formulated in the history, or equivalently it may be also written as $[9,11]$ (Harmonic basis)

$$
H_{W}^{88}=\frac{1}{2}\left(p_{x}^{2}+p_{y}^{2}\right)+\frac{\gamma}{2} p_{z}^{2}-\frac{1}{r}
$$

with $\gamma=v / \mu=1 / \mu^{2}$ or

$$
H_{W}^{87}=p_{x}^{2}+p_{y}^{2}+\gamma p_{z}^{2}-\frac{2}{r}
$$

as used in WMB (Sturmian basis) [8]. We recapitulate POT predictions in terms of (1), our formula for AKP eigenvalue calculation in tensored harmonic wave function basis in terms of (2), and we discuss quantum scars using energy values in (3) in order to facilitate comparison with literature.

\subsubsection{Matrix diagonalization in Sturmian basis}

We here summarize WMB method for efficient matrix diagonalization.

Firstly, in the Sturmian basis 


$$
\langle\vec{r} \mid n \ell m\rangle=\frac{1}{r} \sqrt{\frac{n !}{(2 \ell+n+1) !}} e^{-\frac{\lambda r}{2}}(\lambda r)^{\ell+1} L_{n}^{2 \ell+1}(\lambda r) Y_{\ell m}(\theta, \varphi)
$$

with a scaling parameter $\lambda$, the Schrödinger equation of the AKP becomes a matrix equation:

$$
\left[-\lambda \overleftrightarrow{\Delta^{(3)}}+(1-\gamma) \lambda \overleftrightarrow{\frac{\partial^{2}}{\partial z^{2}}}-2 \overleftrightarrow{\frac{1}{r}}\right] \Psi=\frac{E}{\lambda} \overleftrightarrow{\mathrm{Id}} \boldsymbol{\Psi}
$$

Dividing the whole equation by $\lambda$ and packing $E / \lambda^{2}$ into a parameter $\varepsilon$, one obtains

$$
\overleftrightarrow{M} \Psi \equiv\left[-\overleftrightarrow{\Delta^{(3)}}+(1-\gamma) \overleftrightarrow{\frac{\partial^{2}}{\partial z^{2}}}-\epsilon \overleftrightarrow{\mathrm{Id}}\right] \Psi=\frac{2}{\lambda} \Psi
$$

This $\epsilon$ is to be fixed at some constant value. In principle any value will do, but for finite size of Hamiltonian matrix, the best choice is given [17] approximately

$$
\varepsilon^{*} \simeq-\frac{1}{4} \gamma .
$$

With this choice, we can get the largest number of reliable energy levels at a given matrix size. The ratio of reliable levels to the matrix size can be estimated as

$$
R_{e f f} \simeq \sqrt{\gamma}
$$

After fixing $\varepsilon$, the diagonalization of (6) is performed for $2 / \lambda_{i}$ s and finally we obtain the energy eigenvalues by

$$
E_{i}=\varepsilon \lambda_{i}^{2}
$$

\subsubsection{Matrix diagonalization in Sturmian basis}

For the (tensored) harmonic wave function basis (THWFB) [11] we convert the Hamiltonian of AKP into the Hamiltonian of two of two-dimensional harmonic oscillators.

For this purpose semi-parabolic coordinates are introduced

$$
\mu \nu=\rho=\sqrt{x^{2}+y^{2}}, \frac{1}{2}\left(\mu^{2}-v^{2}\right)=z, \phi=\tan ^{-1}\left(\frac{y}{x}\right)
$$


and the AKP Schrödinger equation becomes

$$
\left[-\frac{1}{2\left(\mu^{2}+v^{2}\right)}\left(\Delta_{\mu}^{(2)}+\Delta_{v}^{(2)}\right)+\frac{1-\gamma}{2} \frac{\partial^{2}}{\partial z^{2}}-\frac{2}{\mu^{2}+v^{2}}\right]|\Psi\rangle=E|\Psi\rangle
$$

Multiplying by $\mu^{2}+v^{2}$ and swapping the Coulombic interaction term and the $E$ term one obtains

$$
\left[-\frac{1}{2}\left(\Delta_{\mu}^{(2)}+\Delta_{v}^{(2)}\right)+|E|\left(\mu^{2}+v^{2}\right)+\frac{1-\gamma}{2}\left(\mu^{2}+v^{2}\right) \frac{\partial^{2}}{\partial z^{2}}\right]|\mathbf{\Psi}\rangle=2|\mathbf{\Psi}\rangle .
$$

Thanks to the semi-parabolic coordinates, the Coulombic singularity has removed [19] for $\gamma=1$. Corresponding to the Sturmian basis with a scaling parameter $\lambda$ in (4), we introduce the harmonic wave function basis

$$
\langle\mu, v \mid i, j, \kappa\rangle=\frac{\kappa}{\pi} L_{i}\left(\kappa \mu^{2}\right) L_{j}\left(\kappa v^{2}\right) \exp \left(-\frac{\mu^{2}+v^{2}}{2}\right)
$$

with a scaling parameter $\kappa$ and, corresponding to $\varepsilon$ in (6), we introduce a parameter

$$
\tilde{\varepsilon}=2 \frac{|E|}{\kappa^{2}}
$$

and we solve (12) after transforming it into the matrix equation of WMB form with eigenvalues $\Lambda_{n}=2 / \kappa_{n}$. The matrix element calculation of the mass anisotropy term in (12) is somewhat involved and we refer to [17] for detail. Energy levels are then determined by

$$
E_{n}=-\frac{\kappa_{n}^{2}}{2} \tilde{\varepsilon}=-\frac{2}{\Lambda_{n}^{2}} \tilde{\varepsilon}
$$

The best value of $\tilde{\varepsilon}$ is given by

$$
\tilde{\varepsilon}^{*} \approx \gamma
$$

which is similar to (7).

We have found precise agreement between our calculations by the Sturmian basis and by the harmonic oscillator basis which in turn validates our choices of scaling parameter $\varepsilon$ and $\tilde{\varepsilon}$.

For the calculation of Husimi functions and scar strength function which uses Husimi functions, we use the THWFB since the projection of the basis functions to the Gaussian packets are easy to calculate [17]. 


\subsection{Husimi function}

Husimi function is defined via the scalar product of the wave function $|\psi\rangle$ with a coherent state(CHS) $\left|q_{0}, p_{0}\right\rangle$ of the system [11]:

$$
W_{\psi}^{H u s}\left(q_{0}, p_{0}\right)=\left|\left\langle\psi \mid q_{0}, p_{0}\right\rangle\right|^{2}
$$

A detailed account is given in [17].

\subsection{Periodic orbit theory}

\subsubsection{Periodic orbit theory and the density of state}

Let us recapitulate Gutzwiller's periodic orbit theory [4,20]. The starting point is Feynman's path integral formula for the propagator of a particle from $q^{\prime}$ to $q^{\prime \prime}$ during the time interval 0 to $T$;

$$
K\left(q^{\prime \prime}, q^{\prime}, T\right) \equiv\left\langle q^{\prime \prime}\left|\exp \left(-i \frac{H}{\hbar} T\right)\right| q^{\prime}\right\rangle=\int_{q^{\prime}}^{q^{\prime \prime}} D[q] e^{\frac{i}{\hbar} \int_{0}^{T} L(q, \dot{q}, t) d t}
$$

The Green function (response function) is given by the Fourier transformation of the propagator

$$
G\left(q^{\prime \prime}, q^{\prime}, E\right) \equiv-\frac{i}{\hbar} \int_{0}^{\infty} d t e^{\frac{i E t}{\hbar}} K\left(q^{\prime \prime}, q^{\prime}, T\right)
$$

where $E$ has infinitesimally small imaginary part for convergence. Thus we have

$$
G\left(q^{\prime \prime}, q^{\prime}, E\right)=\left\langle q^{\prime \prime}\left|\frac{1}{E+i \varepsilon-\hat{H}}\right| q^{\prime}\right\rangle=-\frac{i}{\hbar} \int_{0}^{\infty} d t e^{\frac{i E t}{\hbar}}\left[\int_{q^{\prime}}^{q^{\prime \prime}} D[q] e^{\left.\frac{i}{\hbar} \int_{0}^{T} L(q, \dot{q}, t) d t\right]}\right.
$$

By a stationary approximation we obtain a semiclassical formula for the Green function

$$
\tilde{G}\left(q^{\prime \prime}, q^{\prime}, E\right) \simeq \sum_{\Gamma} A_{\Gamma} \exp \left(\frac{i}{\hbar} S_{\Gamma}-i \frac{v_{\Gamma}}{2}\right)
$$

where $\Gamma$ denotes a classically arrowed orbit, $v_{\Gamma}$ is the number of conjugate points on the orbit, and the amplitude $A_{\Gamma}$ accounts for the Van Vleck determinant. Note that the principal function in (18) is changed into the action $S=\int_{q^{\prime}}^{q^{\prime \prime}} p d q$ and the phase $i \pi / 4$ from the stationary point approximation is shifted into $A_{\Gamma}$.

Now the density of states is given by 


$$
\rho(E) \equiv \sum_{n} \delta\left(E-E_{n}\right)=-\frac{1}{\pi} \operatorname{Im}\left(T_{r_{n}}\left(\frac{1}{E+i \varepsilon-\hat{H}}\right)\right)
$$

where in the second equality an identity $1 /(x+i \varepsilon)=P(1 / x)-i \pi \delta(x)$ is used and trace is taken over all energy eigenstates $\{|n\rangle\}$. Trading this tracing with the tracing over the eigenstates of coordinate operator $\{|q\rangle\}$, we obtain a semiclassical approximation for the DOS

$$
\rho(E) \approx-\left.\frac{1}{\pi} \operatorname{Im} \int d q^{\prime} \tilde{G}\left(q^{\prime \prime}, q^{\prime}, E\right)\right|_{q^{\prime \prime}=q^{\prime}}=-\frac{1}{\pi} \operatorname{Im} \int d q^{\prime} \sum_{\Gamma} A_{\Gamma} \exp \left(\frac{i}{\hbar} S_{\Gamma}-i \frac{v_{\Gamma}}{2}\right) .
$$

The integration over $q^{\prime}$ can be again approximated by a stationary phase approximation. Because

$$
p^{\prime \prime}=\frac{\partial S\left(q^{\prime \prime}, q^{\prime}\right)}{\partial q^{\prime \prime}}, \quad p^{\prime}=-\frac{\partial S\left(q^{\prime \prime}, q^{\prime}\right)}{\partial q^{\prime}}
$$

the stationary phase condition gives

$$
0=\frac{\partial S\left(q^{\prime}, q^{\prime}\right)}{\partial q^{\prime}}=p^{\prime \prime}-p^{\prime}
$$

which dictates the periodic orbits. We obtain finally the periodic orbit theory formula for the DOS

$$
\rho(E) \simeq \overline{\rho(E)}+\operatorname{Im} \sum_{r \in P O} \frac{T_{r}}{\pi \hbar} \sum_{n \neq 0} \frac{\exp \left\{i n\left[\frac{S_{r}}{\hbar}-\frac{\pi}{2} l_{r}\right]\right\}}{\left[\operatorname{det}\left(\left(M_{r}\right)^{n}-1\right)\right]^{\frac{1}{2}}}
$$

Here the first sum runs over all primitive POs and the $n$ sum counts the repetitions of each peridic orbit; $T_{r}, S_{r}$, and $l_{r}$ denote the period, action, and Maslov index of the primary PO, and the matrix $M$ stands for the monodromy matrix of the primary PO.

In AKP $m=0$ sector, the motion is restricted in a fixed plane which includes the heavy axis, and the problem essentially reduces to two dimensional one. (Later on the three dimensional feature is recovered only by the proper choice of the Maslov index [4]). As for AKP unstable periodic orbits, $M$ has two eigenvalues $e^{u}$ and $e^{-u}$ (hyperbolic case) and the determinant in (24) is given by

$$
\frac{1}{2}\left[\operatorname{det}\left(M^{n}-1\right)\right]^{\frac{1}{2}}=-i \sinh (n u / 2)
$$




\subsubsection{Naming of a periodic orbit}

In AKP every PO can be coded by the sign of the heavy axis coordinate when the heavy axis is crossed by it. Note that number of the crossings must be even $\left(2 n_{c}\right)$ for the orbit to close.

In this note we shall denote the PO according to Gutzwiller's identification number along with the Bernoulli sequence of POS. (See Table 1 in ref. [3] which gives a complete list ${ }^{1}$ of POs up to $n_{c}=5$ for the anisotropy $\gamma=0.2$. ) For instance, PO36 $(++-++-)$ is the identification number 6 among $n_{c}=3$ POs.

\subsubsection{The contribution of a periodic orbit to the density of state}

The contribution of a single periodic orbit $r$ to the DOS is estimated by a resummation of the sum over the repetition $\mathrm{j}$ (after the approximation $\sinh x \approx e^{x} / 2$ ),

$$
\left.\rho(E)\right|_{r} \approx T_{r} \sum_{m} \frac{u_{r} \hbar / 2}{\left(S_{r}-2 \pi \hbar(m+l / 4)\right)^{2}+\left(u_{r} \hbar / 2\right)^{2}} .
$$

This gives Lorentzian peak at

$$
S_{r}=2 \pi \hbar(m+l / 4)
$$

similar to the Bohr-Sommerfeld formula. In AKP the action $S$ is given as

$$
S_{r}(E)=\frac{T_{r}}{\sqrt{-2 E}} .
$$

Hence the peak position of the Lorentzian form in the energy is given by

$$
E_{r, m}=-\frac{1}{2}\left(\frac{T_{r}}{2 \pi \hbar(m+1)}\right)^{2}
$$

where Maslov index $l=4$ for three dimensions is taken.

We are aware that it is meant by (24) that the exact DOS with sharp delta function peaks on the energy axis corresponds to the sum of all PO contributions [20] (assuming convergence).

It is the collective addition of all POs that gives the dos. But, still, it is amusing to observe that the localization of wave functions occurs around the classical periodic orbits as we will see below.

\footnotetext{
${ }^{1}$ In $[3,5]$ an amazing approximation formula that gives a good estimate of the action of each periodic orbit from its symbolic code is presented. The trace formula has a difficulty coming from the proliferation of POs of long length. This approximation gives a nice way of estimating the sum. The table is created to fix the two parameters involved in the approximation. We thank Professor Gutzwiller for informing us of this formula a few years ago.
} 


\subsection{Scars as observed in the probability distributions and Husimi functions}

\subsubsection{The manifestation of the fundamental $\mathrm{FPO}(+-)$}

Let us start exploring the scars in AKP first by investigating the case of the fundamental periodic orbit FPO $(+-)$ which reduces the Kepler ellipse orbit in the limit $\gamma=1$.

In Figure 1, we show the wave function squared in the $\mu \nu$ plane and the Husimi distribution in the $\mu p_{\mu}$ plane. The FPO is shown by red line and compared with the probability distributions in the in the $\mu v$ plane. At high anisotropy the orbit is largely distorted. Still at chosen energy levels (upper row) we find clear localization around the FPO for both anisotropies. In the lower row we have displayed other energy eigenstates. For these energy levels we see also characteristic probability distribution patterns but not around FPO. Now let us look at the Husimi distributions. In case of energies in the upper diagrams we see very clearly that around the Poincare section of the FPO (the fixed points) the Husimi functions show clear scars, while in the lower we see anti-scars, the Husimi density is very low at the fixed points. It is clear that Husimi functions are superior observables. In this demonstration of scars we have scanned thousands of energy eigenstates and picked examples. Next task is to use the ability of POT predictions (24) to locate the scaring levels.

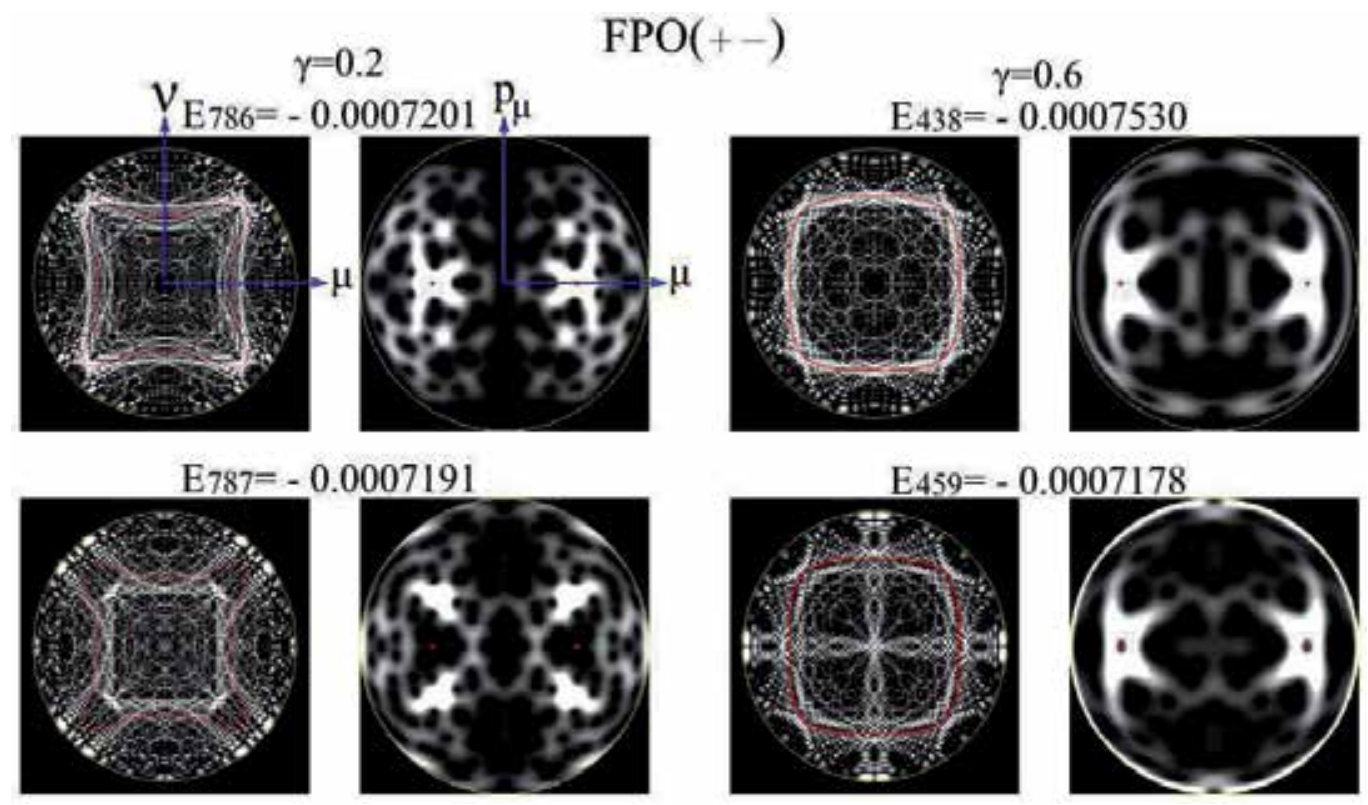

Figure 1. Scar and anti-scar phenomena with respect to the fundamental periodic orbit. The left set is for the anisotropy $\gamma=0.2$ and right for $\gamma=0.6$. In each set, the upper and lower row display prominent scar and anti-scar respectively, while the left and right columns exhibit the probability distribution on the $\mu \nu$ plane and Husimi distribution on the $\mu p_{\mu}$ plane $(v=0)$. The fundamental orbit is drawn by a red line on the $\mu \nu$ plane and its Poincaré section on the $\mu p_{\mu}$ plane by red points. The respective eigenvalues are $E_{786}, E_{787}, E_{438}, E_{459}$ in the $\mathrm{m}=0, \ell=$ even sector. Classical kinematical boundaries are shown by yellow circles. 


\subsubsection{PO prediction and AKP Scars}

As for the FPO the POT works quite well. Thus for this test we have selected more complicated PO PO22 $(+++-)$ and PO36 $(++-++-)$. These orbits wind around the heavy axis forth and back and presumably correspond to the bounce orbit in the billiard. ${ }^{2}$ The top row in Fig.2 shows the prediction from POT - the contribution of the single orbit to the DOS (26). We observe clearly the peak regions of POT prediction contains at least one energy eigenstate which shows the scar of the orbit. On the other hand we have checked that the relevant orbit pattern does not appear in the non-peak region of the POT prediction.

In this analysis the Husimi function again yields unmistakable information on the scaring.
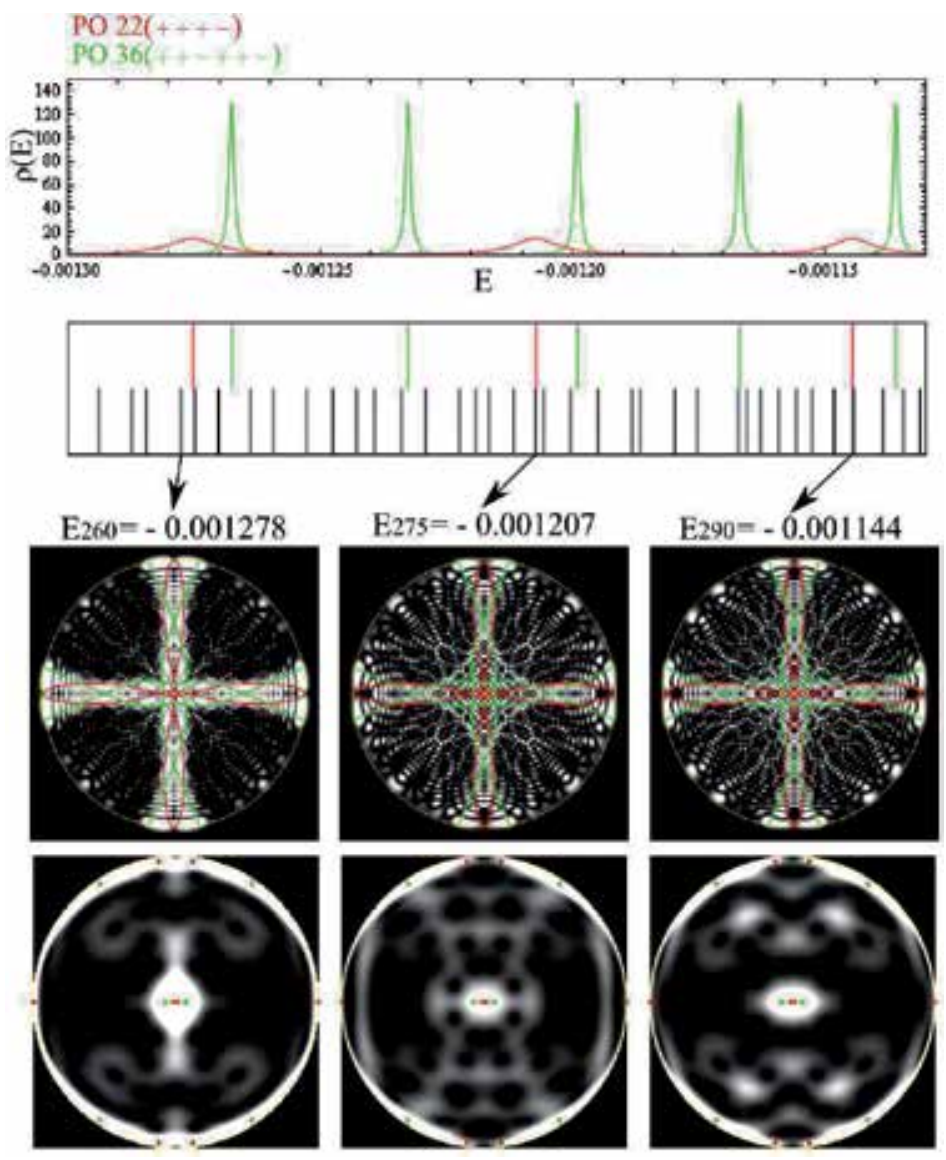

Figure 2. $\gamma=0.6, l=$ even, $m=0$. The upper diagrams: The red and green curves are contributions to the density of states $\rho(E)$ from bouncing-type periodic orbits PO22 $(+++-)$ and PO36 $(++-++-)$ respectively and the peak positions are compared with the $l$ even $m=0$ energy levels from matrix diagonalization (WMB with tensored harmonic oscillator basis). The lower: The quantum scars of these periodic orbits are exhibited on the probability distributions and the Husimi functions. (cf. Fig. 1).

\footnotetext{
${ }^{2}$ We thank Professor Toshiya Takami for explaining his articles [21,22] and pointing us this point.
} 


\subsection{Scars as analyzed by the scar strength function}

\subsubsection{Scar strength functions}

In an extensive analysis of scars in the diamagnetic hydrogen, a tool called as scar strength function (SSF) is presented [11]. It is defined as

$$
I_{n}^{P O}=\oint_{P O} d^{4} s W_{\psi_{n}}^{H u s}\left(\mu, v, p_{\mu}, p_{v}\right)\left(\oint_{P O} d^{4} s\right)^{-1}
$$

where the integral is to be performed along the $\mathrm{PO}$ with $d^{4} s=\sqrt{d \mu^{2}+d v^{2}+d p_{\mu}^{2}+d p_{v}^{2}}$. This quantity is exploiting to what extent a given PO is inducing localization along it in the Husimi function of a given energy eigenstate. Then spectral scar strength function is introduced as

$$
I^{P O}(E)=\sum_{n} I_{n}^{P O} \delta\left(E-E_{n}\right)
$$

This shows how the given $\mathrm{PO}$ affects each energy eigenstate in one function.

\subsubsection{The use of SSF $I^{P O}(E)$}

Let explore the region of high anisotropy $(\gamma=0.2)$ where the classical phase space is occupied by the unstable periodic orbits and chaoticity is rather high. We explore this region by the ability of $I^{P O}(E)$.

We start from FPO $(+-)$ in Fig. 3. The upper is the POT prediction curve, the middle is the SSF along with real eigenvalues and the bottom is as usual a direct comparison of FPO with wavefunctions squared as well as Husimi functions. The SSF is the quantum measure of scaring of a particular PO in consideration, while the FPO prediction is composed from purely classical information for the PO. When the curve of the contribution from a PO peaks, the SSF either peaks or reaches its minimum $\left(10^{-10}\right)$. The agreement in the energy values of the peaks (or dips) is quite remarkable. But we do not know why anti-scar occurs here. This anti-scar is interesting in that it produces a bright hallow just of the same size and position of the scar but the central core is missing.

\subsection{3. $I^{P O}(E)$ for various POs and their Fourier transform}

Let us now examine the case of several POs simultaneously in Fig. 4. The profiles, the SSF, and the Fourier transforms to the action space are listed in three columns. As Wintgen et al. write as 'the scars are the rules rather than exception' [11] we find that particular energy eigenstates give salient high scar function value while the other states give very low value of order even $10^{-10}$. Further more the Fourier transform $I^{P O}(S)$ of $I^{P O}(E)$ shows sequential peaks at equal $\Delta S$. We compare in Fig. $5 \Delta S_{r}^{Q M}$ and $S_{r}^{C l}$ (the measured spacing of the orbit and the action of the PO). They agree excellently; the POs live in quantum theory. 


\section{$\gamma=0.2 \mathrm{FPO}(+-) 210-302$ th}

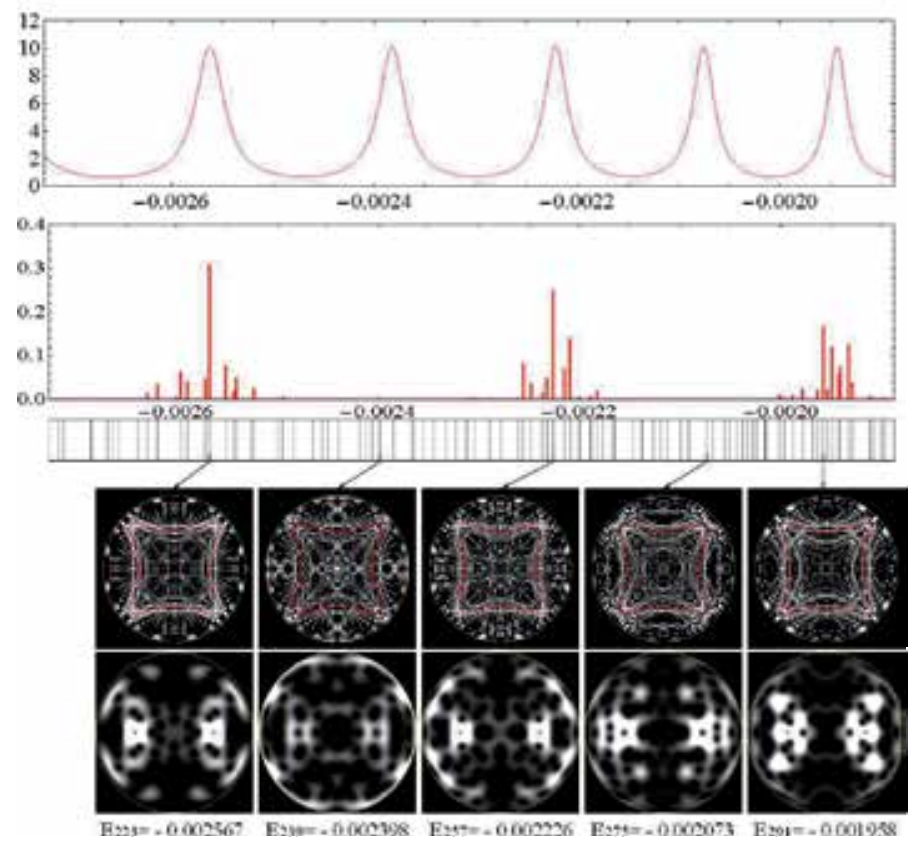

Figure 3. Contribution of FPO $(+-)$ to AKP. Upper two diagrams: The $(+-)$ contribution as a function of energy predicted by POT compared with the scar strength extraction from each of the energy eigenstates. Lower two diagrams: the scaring status of $(+-)$ at levels indicated by arrows are exhibited with respect to wave functions squared and Husimi functions. (cf. Fig. 1 and Fig. 2). Scar and anti-scar appear alternatively.

\subsection{Direct phase space observation of Scaring orbit}

The scar strength function is a useful tool which gives a list of numerical values which shows succinctly to which eigenstates the periodic orbit exerts its effect strongly. But we certainly want also visualized picture how the PO turns up in the 4 dimensional phase space. (Because $H=$ const., the actual independent variables are three, and we choose $\mu, v, p_{\mu}$.) The sample pictures are shown in Fig.6.

\section{Robustness of Scaring under the Variation of Anisotropy Parameter}

\subsection{Swap of the patterns under avoiding crossings}

It is well known that the patterns of wave functions (and of Husimi distributions) are swapped between the energy eigenstates via the avoiding level crossings, which is easy to demonstrate in terms a simple coupling model of two levels. Fig. 7 is a typical example of this phenomenon.

It is shown in [21,22] that with the aid of the diabatic transformation one can trace the localization on the transformed basis until very near to the minimum gap with an explicit evidence in the billiard scars. We have verified this issue in AKP. Furthermore it is conjectured that the long periodic orbits may interpolates two shorter orbits and they may be the cause of the avoiding crossings in this way. We are testing this conjecture in AKP. 


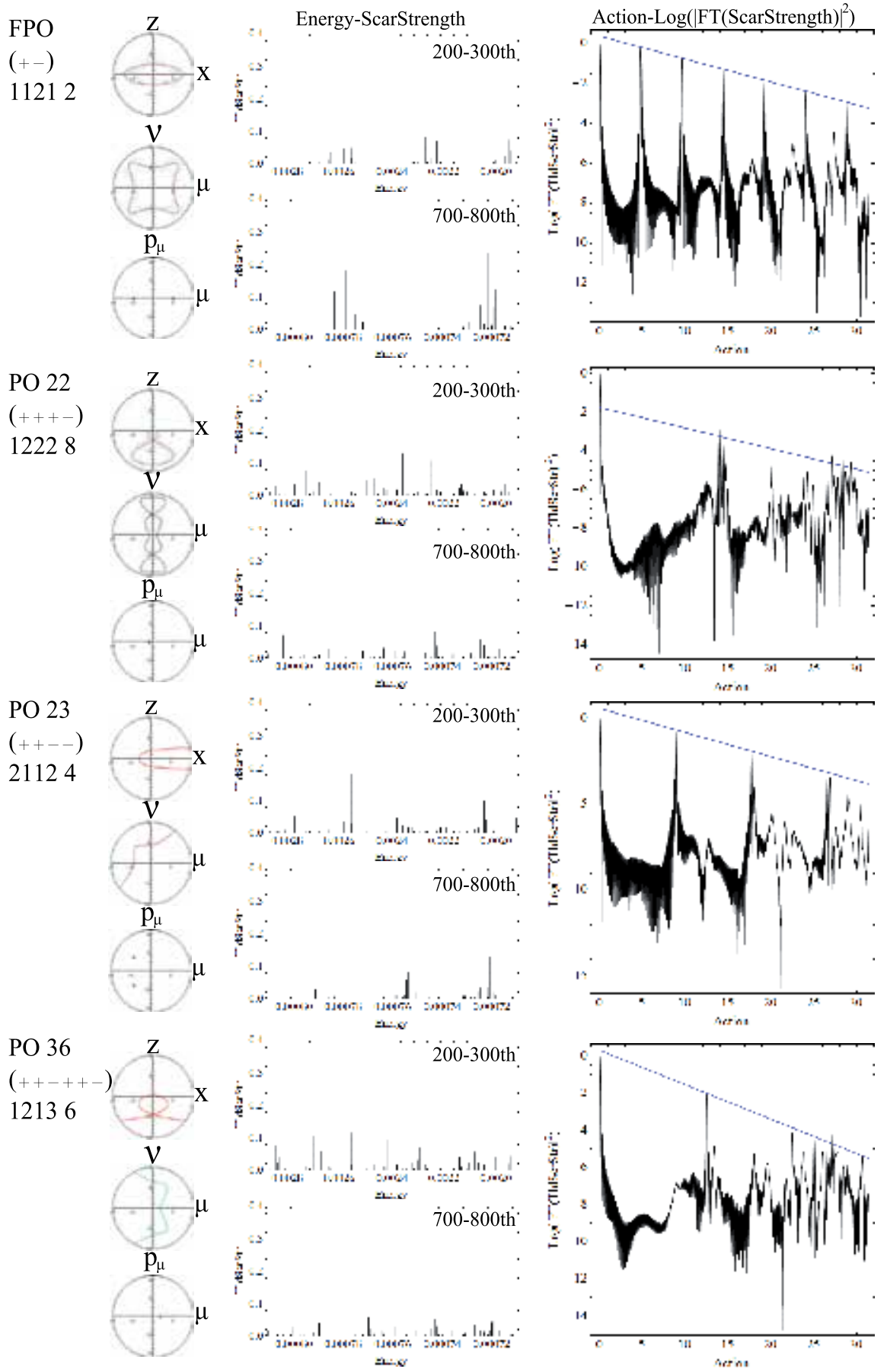

Figure 4. $\gamma=0.2$ Profiles of $\mathrm{PO}$, the scar strength function, and Fourier transformation of scar strength function to the action space. 


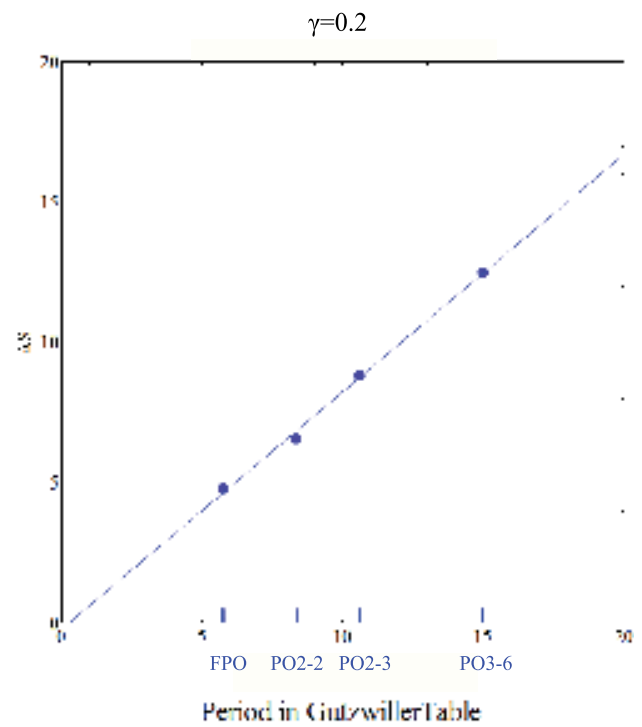

Figure 5. Plot of $\left(\Delta S_{r}^{Q M}, S_{r}^{C l}\right)$ for periodic orbits $r=\mathrm{FPO}, \mathrm{PO} 22, \mathrm{PO} 23, \mathrm{PO} 36$, where $\Delta S_{r}^{\mathrm{QM}}$ is measured from the third column of Fig. 4 and $S_{r}^{C l}$ is the action value of the classical orbit.
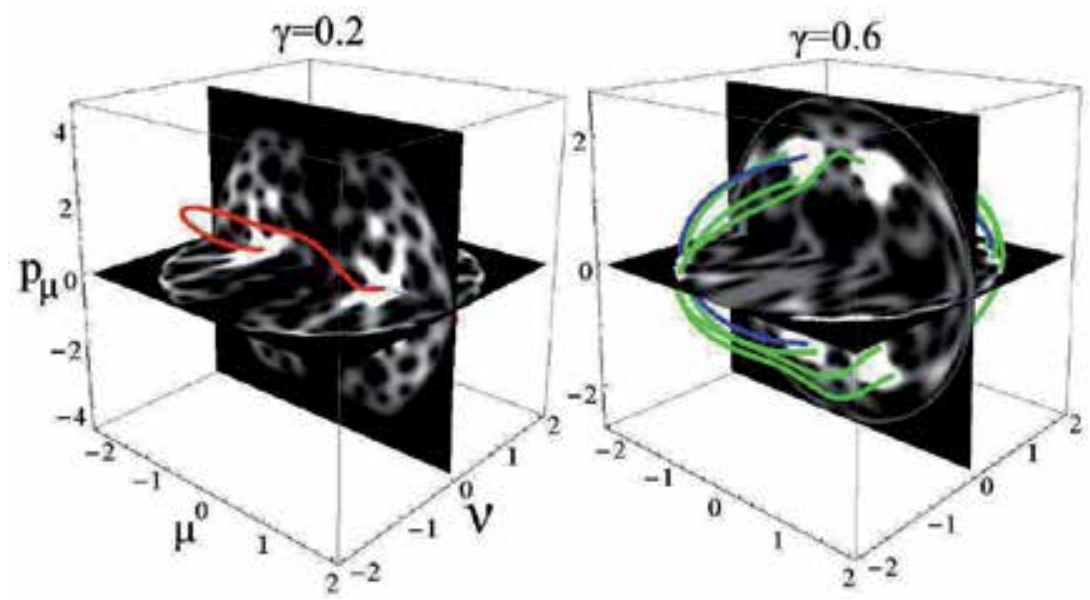

Figure 6. Two samples of density plot of Husimi functions in the 3 dimensional $\mu-v-p_{\mu}$ space. Left: $\gamma=0.2, W_{\text {Hus }}$ for $E_{786}=-0.0007201$. Red orbit is the FPO $(+-)$. Right: $\gamma=0.6, W_{\text {Hus }}$ for $E_{579}=-0.0005681$. Blue and green orbits are respectively $\mathrm{PO} 23(++--)$ and $\mathrm{PO} 37(++--+-)$.

\subsection{Robust association of localization with periodic orbits}

We have posed the following question in the introduction of this chapter.

1. Energy levels exhibit randomness at high anisotropy and change their values randomly repeating successive avoiding crossings when the anisotropy parameter is varied gradually. 


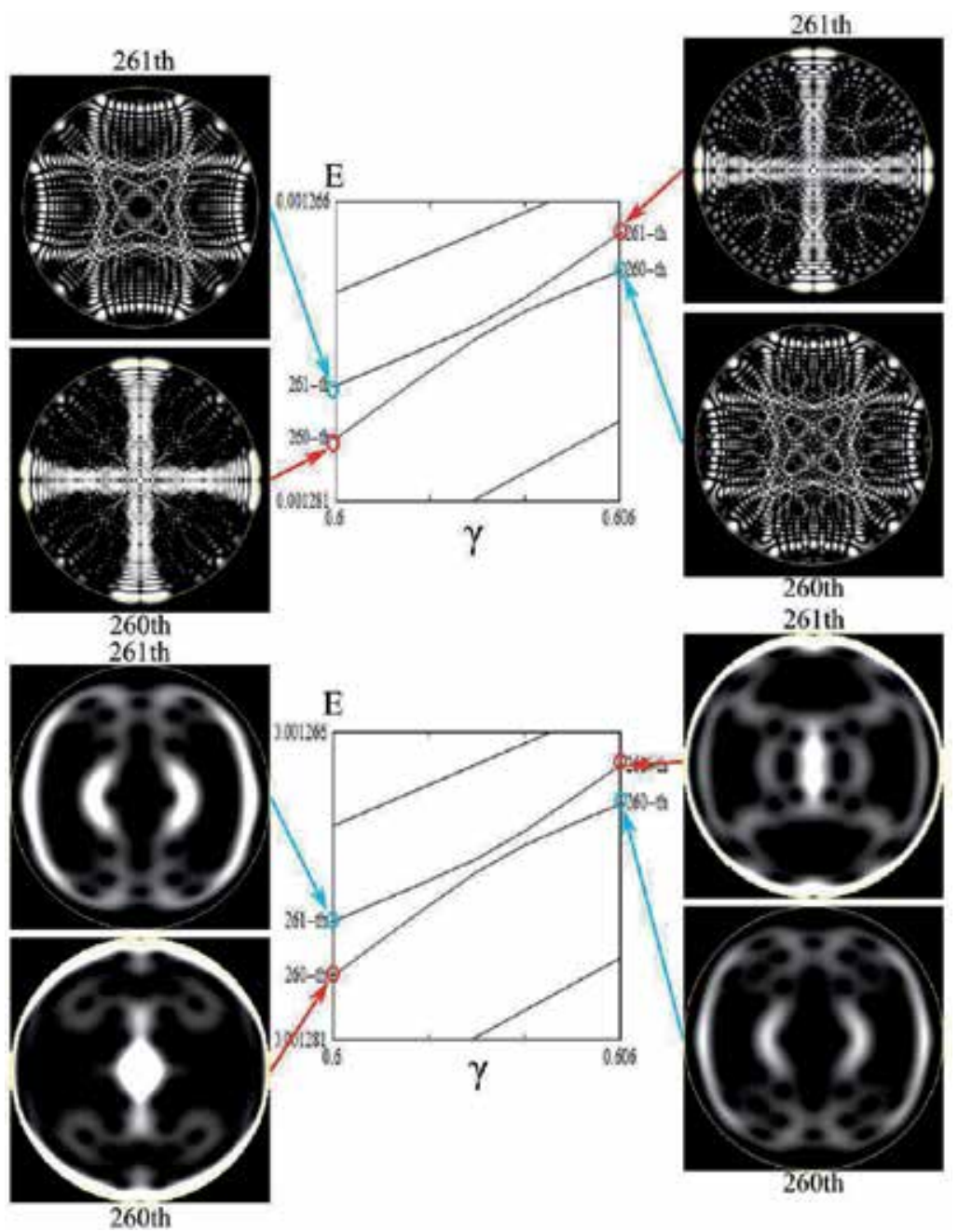

Figure 7. Avoiding level crossing between 260th and 261th energy eigen states under the variation of the anisotropy $\gamma \in$ $[0.6,0.606]$. Both the wave function squared and Husimi functions are swapped around the avoiding crossing.

2. On the other hand the peak locations (29) of the DOS as predicted by a single PO change smoothly with the anisotropy and the scar tends to be observed in the energy eigenstate around the peak position in the DOS as in Fig.2.

Aren't the two issues in contradiction? We have found that they can live together (within approximation of the fluctuation size). Most important point is that the swap of the localization patterns at avoiding crossing is in harmony with the transportation of them by the responsible PO orbits. Besides the POT prediction (29) does not imply the exact location of theappearance of the scar.

It has some allowance as recognized by the width of the modulation of SSF[11].

Let us explain this by Fig. 8. Here the anisotropy $\gamma$ is varied from 0.6 to 0.7 with inclement 0.001 . As for (1) we indeed observe both random fluctuation of energy levels as well as many 
avoided crossings. As for (2), we have picked the bouncing-type periodic orbit PO22 as an example. The predicted peak position (29) of its contribution to DOS varies with the change of $\gamma$ as shown by a red (almost straight) curve. This PO22 produces a salient cross-shaped scar at $E_{260}$ (and $E_{275}$ ) at $\gamma=0.6$. We have investigated how the cross-shaped scar travels in the spectrum space suffering many avoiding crossings. It reaches at $E_{276}$ (and $E_{291}$ ) at $\gamma=0.7$

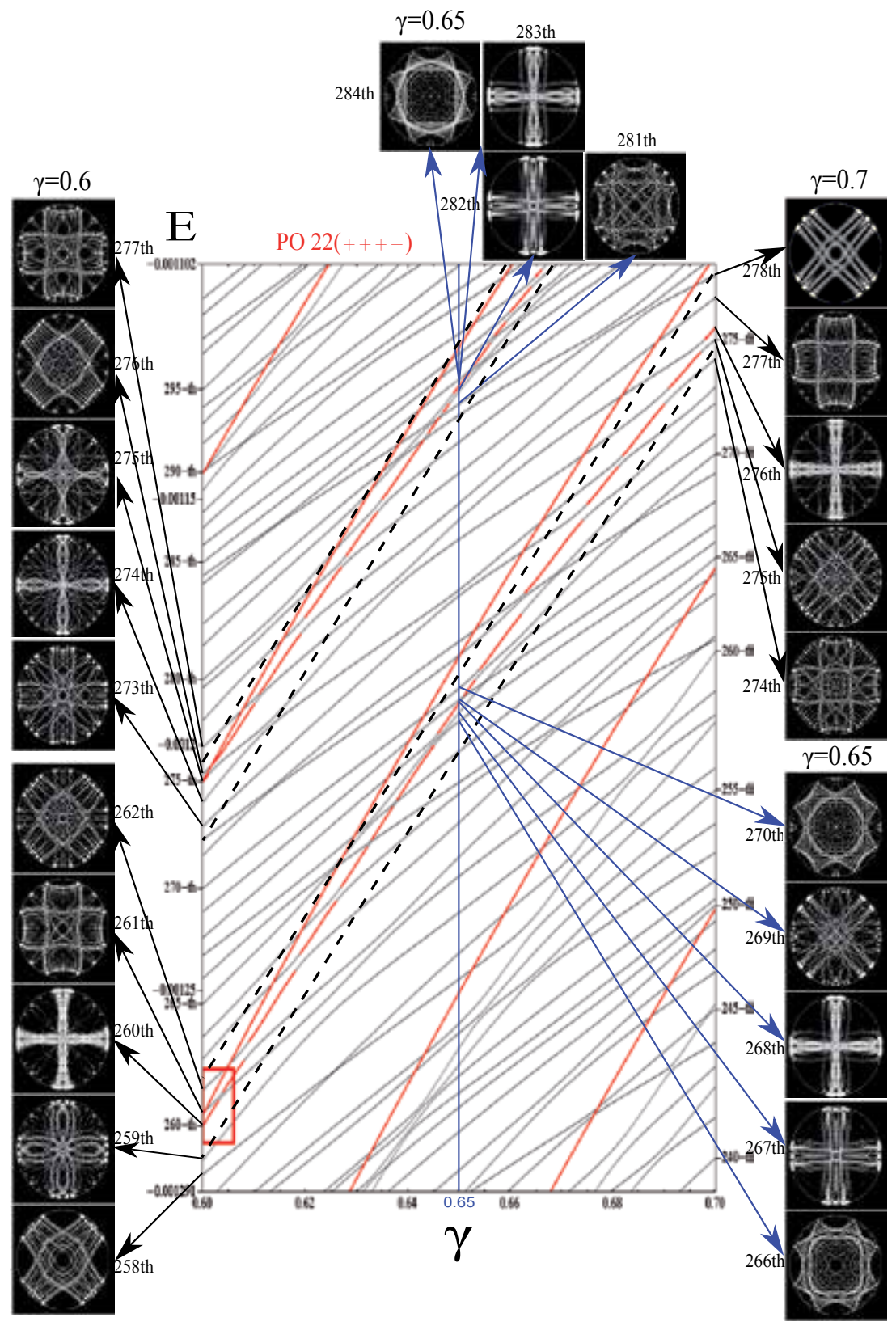

Figure 8. The spectrum lines in the wide interval $\gamma \in[0.6,0.7]$ investigated with increment 0.001 . The cross-shaped scar by PO22 travels within a belt bounded by two dashed lines. The POT prediction (24) is exhibited by a red curve. 
and the track in between is enclosed by a belt shown by two dashed lines. We clearly observe that the belt is closely associated by the POT prediction curve. In this sense the association is robust.

\section{Conclusion}

We have presented ample examples of scaring phenomena for the first time in AKP. Especially we have found how the fluctuation of energy levels and smooth POT prediction for the scaring levels are compromised by using the advantage of AKP endowed by a chaoticity changing parameter.

Although the theme is old, the scaring phenomenon is fascinating and we hope this contribution fulfills a gap in the literature.

\section{Acknowledgement}

Both KK and TS thank Professor Toshiya Takami for sharing his wisdom with us.

\section{Author details}

Keita Sumiya, Hisakazu Uchiyama, Kazuhiro Kubo and Tokuzo Shimada

Department of Physics, School of Science and Technology, Meiji University, Japan

\section{References}

[1] Gutzwiller, M. C. (1977). Bernoulli sequences and trajectories in the anisotropic Kepler problem, Journal of Mathematical Physics 18: 806-823.

[2] Devaney, R. L. (1979). Collision Orbits in the Anisotropic Kepler Problem, Inventions math. 45: 221-251.

[3] Gutzwiller, M. C. (1981). Periodic orbits in the anisotropic Kepler problem, in Devaney, R. L. \& Nitecki, Z. H. (ed.) Classical Mechanics and Dynamical systems, Marcel Dekker, New York, pp. 69-90.

[4] Gutzwiller, M. C. (1971). Periodic orbits and classical quantization conditions, Journal of Mathematical Physics 12: 343-358.

[5] Gutzwiller, M. C. (1980). Classical quantization of a hamiltonian with ergodic behavior, Physical Review Letters 45: 150-153.

[6] Gutzwiller, M. C. (1982). The quantization of a classically ergodic system, Physica D 5: 183-207.

[7] Gutzwiller, M. C. (1990). Chaos in Classical and Quantum Mechanics, Springer. 
[8] Wintgen, D., Marxer, H. \& Briggs, J. S. (1987). Efficient quantisation scheme for the anisotropic Kepler problem, Journal of Physics A 20: L965-L968.

[9] Wintgen, D. \& Marxer, H. (1988). Level statistics of a quantized cantori system, Physical Review Letters 60: 971-974.

[10] Heller, E. J. (1984). Bound-state eigenfunctions of classically chaotic hamiltonian systems: scars of periodic orbits, Physical Review Letters 53: 1515-1518.

[11] Müller, K. \& Wintgen, D. (1994). Scars in wavefunctions of the diamagnetic Kepler problem, Journal of Physics B: Atomic, Molecular and Optical Physics 27: 2693-2718.

[12] García-García, A. M. \& Verbaarschot, J. J. M. (2003). Critical statistics in quantum chaos and Calogero-Sutherland model at finite temperature, Physical Review E 67: 046104-1 $-046104-13$.

[13] García-García, A. M. (2007). Universality in quantum chaos and the one parameter scaling theory (a power point of a talk). http://www.tcm.phy.cam.ac.uk/ amg73/oslo2007.ppt.

[14] García-García, A. M. \& Wang, J. (2008). Universality in quantum chaos and the one-parameter scaling theory, Physical Review Letters 100: 070603-1 - 070603-4.

[15] Chen, Z. et al. (2009). Realization of Anisotropic Diamagnetic Kepler Problem in a Solid State Environment, Physical Review Letters 102: 244103.

[16] Zhou, W. (2010). Magnetic Field Control of the Quantum Chaotic Dynamics of Hydrogen Analogues in an Anisotropic Crystal Field, Physical Review Letters 105: 024101.

[17] Kubo, K. \& Shimada, T. (2011). Theoretical Concepts of Quantum Mechanics, InTech.

[18] Kustaanheimo, P. \& Stiefel, E. (1965). Perturbation theory of Kepler motion based on spinor regularization, Journal für die reine und angewandte Mathematik 218: 204-219.

[19] Husimi, K. (1940). Some formal properties of the density matrix, Proceedings of the Physico-Mathematical Society of Japan 22: 264-314.

[20] Wintgen, D. (1988). Semiclassical Path-Integral Quantization of Nonintegrable Hamiltonian Systems, Physical Review Letters 61: 1803-1806.

[21] Takami, T. (1992). Semiclassical Interpretation of Avoided Crossings for Classically Nonintegrable Systems, Physical Review Letters 68: 3371-3374.

[22] Takami, T. (1995). Semiclassical study of avoided crossings, Physical Review E 52: 2434. 


\section{Charathéodory's "Royal Road" to the Calculus of Variations: A Possible Bridge Between Classical and Quantum Physics}

Francisco De Zela

Additional information is available at the end of the chapter

http://dx.doi.org/10.5772/53843

\section{Introduction}

Constantin Carathéodory, a Greek-born, well-known German mathematician, is rarely mentioned in connection to physics. One of his most remarkable contributions to mathematics is his approach to the calculus of variations, the so-called Carathéodory's "royal road" [1]. Among physicists, Carathéodory's name is most frequently related to his contributions to the foundations of thermodynamics [2] and to topics of classical optics, though, as a pupil of Hermann Minkowski, he also worked on the development of especial relativity. In our opinion, however, Caratheodory's formulation of the variational problem deserves to be better known among physicists. For mathematicians, features like rigor and non-redundancy of basic postulates are of utmost importance. Among physicists, a more pragmatic attitude is usually behind efforts towards a theoretical construction, whose principal merit should be to offer an adequate description of Nature. Such a construction must provide us with predictive power. Rigor of the theoretical construction is necessary but not sufficient. Elegance - which is how non-redundancy and simplicity usually manifest themselves - can be sometimes just a welcome feature. Some other times, however, elegance has become a guiding principle when guessing at how Nature works. Nevertheless, once the basic principles of a theoretical construction have been identified, elegance may recede in favor of clarity, and redundancy might become acceptable. Such differences between the perspectives adopted by mathematicians and physicists have been presumably behind the different weight they have assigned to Caratheodory's achievements in the calculus of variations. To be sure, variational calculus does play a central role in physics, nowadays even more than ever before. It is by seeking for the appropriate Lagrangian that we hope to find out the most basic principles ruling physical behavior. Concepts like Feynman's path integral 
have become basic tools for the calculation of probability amplitudes of different processes, as well as for suggesting new developments in quantum field theory. Symmetry, such a basic concept underlying those aspects of Nature which appear to us in the form of interactions among fundamental particles, is best accounted for within the framework of a variational principle.

Within the domain of classical physics, only two fundamental interactions have been addressed: the gravitational and the electromagnetic interactions. The theoretical construction may correspondingly rest on two variational principles, one for gravitation and the other for electromagnetism. These principles lead to so-called "equations of motion": the Maxwell and the Lorentz equations for electromagnetism, and the Einstein and the geodesic equations for gravitation. All these differential equations can be derived as Euler-Lagrange equations from the appropriate Lagrangian or Lagrangian density.

The usual approach to variational calculus in physics starts by considering small variations of a curve which renders extremal the action integral $\int L d t$, with $L$ being the Lagrangian. This leads to the Euler-Lagrange equations of motion. By submitting $L$ to a Legendre transformation one obtains the corresponding Hamiltonian, in terms of which the Hamilton equations of motion can be established. By considering canonical transformations of these equations, one arrives at the Hamilton-Jacobi equation for a scalar function $S(t, x)$. It is last one that has been used to connect the classical approach with the quantum one, e.g., in Madelung's hydrodynamic model [3] or in Bohm's "hidden variables" approach [4]. This appears natural, because both the Hamilton-Jacobi and the Schrödinger equation rule the dynamics of quantities like $S(t, x)$ and $\psi(t, x)$, respectively, which are scalar fields. Their scalar nature is in fact irrelevant; they could be tensors and spinors. The relevant issue is that while the Euler-Lagrange and the Hamilton equations refer to a single path, quantum equations address a field. The quantum-classical connection thus requires making a field out of single paths, something which occurs by going to the Hamilton-Jacobi equation, or else by establishing a path-integral formulation, as Feynman did. The latter considers a family of trajectories and assigns a probability to each of them. Now, Carathéodory's approach has the advantage of addressing right from the start a field of extremals. In fact, as the calculus of variations shows, a solution of the extremal problem exists only when the sought-after extremal curve can be embedded in a field of similar extremals. Carathéodory exploited this fact by introducing the concepts of "equivalent variational problems" and the "complete figure". It is then possible to elegantly derive from a single statement the Euler-Lagrange and the Hamilton equations, as well as the Hamilton-Jacobi equation, all of them as field equations. The familiar Euler-Lagrange and Hamilton equations can be obtained afterwards by singling out a particular extremal of the field. But - as already stressed - it is not the inherent elegance of the formulation what drives our interest towards Carathéodory's approach. It is rather its potentiality as a bridge between classical and quantum formulations what should be brought to the fore. Indeed, Carathéodory's approach can provide new insights into the connection between classical and quantum formulations. These insights could go beyond those already known, which were obtained by extending the Hamilton-Jacobi equation with the inclusion of additional terms. By dealing with the other field equations that appear within Carathéodory's approach, one may hope to gain additional insight.

The present chapter, after discussing Carathéodory's approach, shows how one can classically explain two phenomena that have been understood as being exclusively quantum 
mechanical: superconductivity and the response of a sample of charged particles to an external magnetic field. The London equations of superconductivity were originally understood as an ad-hoc assumption, with quantum mechanics lying at its roots. On the other hand, according to classical mechanics there can be no diamagnetism and no paramagnetism at all. We will deal with these two issues, showing how it is possible to classically derive the London equations and the existence of magnetic moments. This is not to say that there is a classical explanation of these phenomena. What is meant is that, specifically, the London equations of superconductivity can be derived from a classical Lagrangian. It is worth noting that a previous attempt in this direction, due to W. F. Edwards [5], proved false [6-8]. The failure was due to an improper application of the principle of least action. The approach presented here is free from any shortcomings. It leads to the London equations both in the relativistic and in the nonrelativistic domains. It should be stressed that this does not explain the appearance of the superconducting phase. It only shows how the London equations follow from a purely classical approach. Also the expulsion of a magnetic field from the interior of a superconductor, i.e., the Meissner effect, follows. That is, perfect diamagnetism can be explained classically, as has been shown recently [9] but under restricted conditions. This is in contradiction with the Bohr-van Leeuwen theorem, according to which there can be no classical magnetism [10]. This point has been recently discussed (see, e.g., [11]) and it has been shown that the Bohr-van Leeuwen theorem does not hold when one uses the Darwin Hamiltonian, which was proposed back in 1920. The Darwin Hamiltonian contains additional terms with respect to the standard one that is used to describe a charged particle interacting with an electromagnetic field. Applying Carathéodory's approach it can be shown that it is unnecessary to go beyond the standard Hamiltonian or Lagrangian to conclude that a magnetic response may be explained classically. The main point is that the Bohr-van Leeuwen theorem did not consider a constant of the motion which in Carathéodory's approach naturally arises. By considering this constant of the motion, the possibility of magnetic response in a sample of charged particles automatically appears.

After dealing with the above two cases, the rest of the chapter will be devoted to show how gauge invariance can be considered within Carathéodory's framework. This may have some inspiring effect for future work aiming at exploring the quantum-classical correspondence.

\section{Carathéodory's royal road}

\subsection{Preliminaries}

Let us begin by recapitulating the approach usually employed in physics. For the sake of describing a particle's motion we use a variational principle based on a Lagrangian $L$. When describing the dynamics of a field we use instead a variational principle based on a Lagrangian density $\mathcal{L}$. The Euler-Lagrange equations are, respectively,

$$
\frac{d}{d t}\left(\frac{\partial L}{\partial \dot{x}^{i}}\right)-\frac{\partial L}{\partial x^{i}}=0,
$$

for a Lagrangian $L\left(t, x^{i}, \dot{x}^{i}\right)$, with $i=1, \ldots, n$, and

$$
\frac{\partial}{\partial x^{\mu}}\left(\frac{\partial \mathcal{L}}{\partial\left(\partial_{\mu} \psi^{i}\right)}\right)-\frac{\partial \mathcal{L}}{\partial \psi^{i}}=0
$$


for a Lagrangian density $\mathcal{L}\left(\psi^{i}, \partial_{\mu} \psi^{i}\right)$ that depends on $n$ fields $\psi^{i}$ and their derivatives $\partial_{\mu} \psi^{i}$ with respect to space-time coordinates $x^{\mu}$. The convention of summing over repeated indices has been used in Eq.(2), as we will do henceforth. The above equations are necessary conditions that are derivable from the action principle

$$
\delta I=0,
$$

with the action given by $I=\int L d t$ for the particle motion and $I=\int \mathcal{L} d^{4} x$ for the field dynamics. The variation $\delta$ means that we consider different paths joining some fixed initial and end points - hypersurfaces in the case of $\int \mathcal{L} d^{4} x$ - and seek for the path that affords $I$ an extremal value. Curves which are solutions of the Euler-Lagrange equations are called extremals.

Let us concentrate on the case $I=\int L d t$ in what follows and sketch how the standard derivation of Eq.(1) is usually obtained: one takes the variation $\delta \int L d t=$ $\int d t\left[\left(\partial L / \partial x^{i}\right) \delta x^{i}+\left(\partial L / \partial \dot{x}^{i}\right) \delta \dot{x}^{i}\right]$, and observing that $\delta \dot{x}^{i}=d\left(\delta x^{i}\right) / d t$, integration by parts gives $\delta I=\int d t\left(\partial L / \partial x^{i}-d\left(\partial L / \partial \dot{x}^{i}\right) / d t\right) \delta x^{i}=0$, where we have considered that $\delta x^{i}=0$ at the common endpoints of all the paths involved in the variation. The arbitrariness of $\delta x^{i}$ leads to Eq.(1) as a necessary condition for $\delta I$ to be zero.

The important case of a time-independent Lagrangian $(\partial L / \partial t=0)$ leads to the conservation of the quantity

$$
\frac{\partial L}{\partial \dot{x}^{i}} \dot{x}^{i}-L
$$

along an extremal, as can be seen by taking its time-derivative and using Eq.(1). By introducing the canonical momenta $p_{i}=\partial L(t, x, \dot{x}) / \partial \dot{x}^{i}$ and assuming that we can solve these equations for the $\dot{x}^{i}$ as functions of the new set of independent variables, $\dot{x}^{i}=\dot{x}^{i}\left(t, x^{i}, p_{i}\right)$, we can define a Hamiltonian $H(t, x, p)$ through the expression given by Eq.(4), written in terms of the new variables $\left(t, x^{i}, p_{i}\right)$ :

$$
H(t, x, p)=p_{i} \dot{x}^{i}(t, x, p)-L(t, x, \dot{x}(t, x, p)) .
$$

The Euler-Lagrange equations are then replaced by the Hamilton equations:

$$
\frac{d x^{i}}{d t}=\frac{\partial H}{\partial p_{i}}, \frac{d p_{i}}{d t}=-\frac{\partial H}{\partial x^{i}}
$$

Eq.(5) can be seen as a Legendre transformation leading from the set $\left(x^{i}, \dot{x}^{i}, t\right)$ to the set $\left(x^{i}, p_{i}, t\right)$ by means of the function $H(t, x, p)$. Taking the differential of $H(t, x, p)$ on the left-hand side of Eq.(5),

$$
d H=\frac{\partial H}{\partial t} d t+\frac{\partial H}{\partial x^{i}} d x^{i}+\frac{\partial H}{\partial p_{i}} d p_{i}
$$


and on the right-hand side,

$$
d H=p_{i} d \dot{x}^{i}+\dot{x}^{i} d p_{i}-\frac{\partial L}{\partial x^{i}} d x^{i}-\frac{\partial L}{\partial \dot{x}^{i}} d \dot{x}^{i}-\frac{\partial L}{\partial t} d t
$$

and replacing $\partial L / \partial \dot{x}^{i}$ by $p_{i}$, after equating both sides we see that Eqs.(6) must hold true, together with

$$
\frac{\partial H}{\partial t}=-\frac{\partial L}{\partial t}
$$

A third way to deal with the motion problem is given by the Hamilton-Jacobi equation. In order to introduce it, one usually starts by considering canonical transformations, i.e., those being of the form $\left\{x^{i}, p_{i}\right\} \rightarrow\left\{X^{i}(x, p, t), P_{i}(x, p, t)\right\}$ and leaving the action $I$ invariant. They lead to equations for $\left\{X^{i}, P_{i}\right\}$ that are similar to Eqs.(6) but with a new Hamiltonian, $K(t, X, P)$. From the set of canonical variables $\left\{x^{i}, p_{i}, X^{i}, P_{i}\right\}$ only $2 n$ of them are independent. One considers then four types of transformations, in accordance to the chosen set of independent variables: $\left\{x^{i}, X^{i}\right\},\left\{x^{i}, P_{i}\right\},\left\{p_{i}, X^{i}\right\}$, and $\left\{p_{i}, P_{i}\right\}$. The transformation from the old to the new canonical variables can be afforded by a so-called "generating function" $S$, which depends on the chosen set of independent variables and the time $t$. The old and new Hamiltonians are related by $K=H+\partial S / \partial t$. If we succeed in finding a transformation such that $K=0$, the Hamilton equations for $K$ can be trivially solved. One is thus led to seek for a transformation whose generating function is such that $K=0$. When the set of independent variables is $\left\{x^{i}, P_{i}\right\}$ the $p_{i}$ are given by $p_{i}=\partial S / \partial x^{i}$, while the new momenta have constant values $P_{i}=\alpha_{i}$ in virtue of $K=0$. This last equation reads, in terms of the original Hamiltonian,

$$
\partial S / \partial t+H\left(t, x^{i}, \partial S / \partial x^{i}\right)=0
$$

This is the Hamilton-Jacobi equation. It has played an important role beyond the context in which it originally arose, becoming a sort of bridge that links classical and quantum mechanics. As a first attempt to obtain a quantum-mechanical formalism it was Sommerfeld who, following Bohr, considered action-angle variables for the case of a conservative Hamiltonian, $\partial H / \partial t=0$. This Hamiltonian was furthermore assumed to allow the splitting of $S\left(t, x^{i}, \alpha_{i}\right)$ as $S=E t-\sum_{j} W_{j}\left(x^{i} ; \alpha_{i}\right)$. Restricting the treatment to cases where the relationship between the $p_{i}$ and the $x^{i}$, given through $p_{i}=\partial S / \partial x^{i}$, is such that the orbits $p_{i}=p_{i}\left(x^{j}, \alpha_{j}\right)$ are either closed (libration-like) or else periodic (rotation-like), action-angle variables can be introduced as new canonical variables [12]. By imposing that the action variables are integer-multiples of a fundamental action, i.e., Planck's $h$, it was possible to obtain a first formulation of quantum mechanics. This version is known as "old quantum mechanics". A second attempt went along Schrödinger's reinterpretation of the left-hand-side of Eq.(10) as a Lagrangian density of a new variational principle. Schrödinger considered first the case $\partial H / \partial t=0$, with $H=\sum_{i=1,3} p_{i}^{2} / 2 m+V$, and introduced $\psi$ through $S=k \ln \psi$, with $k$ a constant. From the left-hand side of Eq.(10), after multiplying it by $\psi^{2}$, Schrödinger obtained an expression that he took as a Lagrangian density: $\mathcal{L}=$ $\sum_{i=1,3} k^{2}\left(\partial \psi / \partial x^{i}\right)^{2} / 2 m+(V-E) \psi^{2}$. Inserting this $\mathcal{L}$ into the Euler-Lagrange equations (2) one readily obtains the (time-independent) Schrödinger equation. The constant $k$ could be 
identified with $\hbar$ by comparison with Bohr's energy levels in the case of the hydrogen atom $(V \sim 1 / r)$. We recall that parallel to this approach, another one, due to Heisenberg, Born, Jordan and Dirac, was constructed out of a reformulation of the action-angle formalism applied to multiple periodic motions. This reformulation led to a formalism in which the Poisson brackets were replaced by commutators, and the canonical variables by operators.

Coming back to the general action principle, we have so far followed the road usually employed by physicists. This road was build out of manifold contributions, made at different times and with different purposes. As a consequence, it lacks the unity and compactness that a mathematical theory usually has. At the beginning of the 20th century mathematicians were concerned with the construction and extension of a sound theory for the calculus of variations. It is in this context that Carathéodory made his contributions to the subject. They were thus naturally conceived from a mathematical viewpoint. Apparently, they added nothing new that could be of use for physicists, and so passed almost unnoticed to them. Our purpose here is to show how Carathéodory's formulation can provide physical insight and inspire new approaches. In the following, we give a short account of Carathéodory's approach. We will try to show the conceptual unity and potential usefulness that Carathéodory's formulation entails. Such a unity roots on the so-called complete figure that Carathéodory introduces as a central concept of his approach. It serves as the basis of a formulation in which the Euler-Lagrange, the Hamilton and the Hamilton-Jacobi equations appear as three alternative expressions of one and the same underlying concept.

\subsection{The non-homogeneous case}

Let us first consider the so-called non-homogeneous case, i.e., one in which the action principle - and with it the Euler-Lagrange equation - is not invariant under a change of the curve parameter. In physics, this parameter usually corresponds to time. By solving the equations of motion one obtains not only the geometrical path traced by the particle - or group of particles - being described, but also how, i.e., the rate at which this path is traveled. The non-homogeneous case applies to non-relativistic formulations.

The equation of motion follows from the variational principle $\delta \int L\left(t, x^{i}, \dot{x}^{i}\right) d t=0$. As physicists, we usually visualize the variational principle as expressing how Nature works: among all possible paths joining two given points, Nature chooses the one which affords $\int L d t$ an extremal value. In some sense, this presupposes a non-local behavior, as two distant points determine the extremal curve that should join them. This is reminiscent of the action-at-a-distance invoked by earlier formulations, in whose context the variational principle originally arose. The approach proposed by Carathéodory is more in accordance with our modern view of local interactions. He replaced the problem of finding an extremum for the action integral by one of finding a local extremal value for a function. Thus, the field concept is at the forefront, playing a major role.

Let us recall some important assumptions [1, 13-15] concerning the central problem of variational calculus:

a) To find an extremal curve $x^{i}=x^{i}(t)$ that satisfies $\delta \int L d t=0$ requires that we restrict ourselves to a simply-connected domain. Though apparently technical, this point might entail a profound physical significance. 
b) An extremal curve exists only in case that it can be embedded in a whole set of extremals, a so-called "Mayer field".

Now, having a field of curves is equivalent to defining a vector field $v^{i}\left(t, x^{j}\right)$ : at each point $x^{j}$ we just define $v^{i}\left(t, x^{j}\right)$ to be tangent to the unique curve which goes through $x^{j}$. In other words, the curves that constitute the field are integral curves of $v^{i}\left(t, x^{j}\right)$ :

$$
\frac{d x^{i}(t)}{d t}=v^{i}(t, x(t))
$$

Finding all the extremals $x^{i}(t)$ is thus equivalent to fixing $v^{i}(t, x)$. Once we have $v^{i}(t, x)$, the extremals can be obtained by integration of Eq.(11). The task of finding $v^{i}(t, x)$ can be approached locally. To this end, observe that the extremals we are seeking, for which $\delta \int L d t=0$, are also extremals of the modified, "equivalent variational problem", $\delta \int(L-d S / d t) d t=0$. This can be written as

$$
\delta \int\left(L-\partial_{t} S-\dot{x}^{i} \partial_{i} S\right) d t=0
$$

Now, assume that we are dealing with a particular Lagrangian $L^{*}(t, x, v)$, for which the following requirements are met: First, it is possible to find a vector field $v$ such that $L^{*}(t, x, v)=0$. Second, $L^{*}(t, x, w)>0$ for any other field $w \neq v$. It is then easy to show that the integral curves of $v$ are extremals of the variational problem $\delta \int L^{*} d t=0$. Of course, not every Lagrangian will satisfy the requirements we have put on $L^{*}$; but by making use of the freedom we have to change our original problem into an "equivalent variational problem", we let $L^{*}=L-d S / d t$ and seek for a vector field $v$ such that

$$
L(t, x, v)-\partial_{t} S-v^{i} \partial_{i} S=0
$$

identically, the value zero being an extremal one with respect to variations of $v$. This happens for a suitably chosen $S(t, x)$ that remains fixed in this context. The function $S(t, x)$ must be just the one for which the value of $\int\left(\partial_{t} S+\dot{x}^{i} \partial_{i} S\right) d t=\int L(t, x, \dot{x}) d t$, this last integral being calculated along an extremal curve. In other words, among all equivalent variational problems we seek the one for which the conditions put upon $L^{*}$ are fulfilled. Thus, for the extremal value being, e.g., a minimum, it must hold Eq.(13), while $L\left(t, x^{i}, w^{i}\right)-\partial_{t} S-w^{i} \partial_{i} S>$ 0 for any other field $w \neq v$. In this way our variational problem becomes a local one: $v$ has to be determined so as to afford an extremal value to the expression at the left-hand side of Eq.(13). Thus, taking the partial derivative of this expression with respect to $v$ and equating it to zero we obtain

$$
\frac{\partial S}{\partial x^{i}}=\frac{\partial L(t, x, v(t, x))}{\partial v^{i}} .
$$

Eqs.(13) and (14) are referred to as the fundamental equations in Carathéodory's approach. From these two equations we can derive all known results of the calculus of variations. We see, for instance, that defining $p_{i}=\partial L(t, x, v) / \partial v^{i}$, Eq.(14) gives $p_{i}=\partial S(t, x) / \partial x^{i}$. If 
we now introduce, by means of a Legendre transformation, the Hamiltonian $H(t, x, p)=$ $v^{i}(t, x, p) p_{i}-L(t, x, v(t, x, p))$, Eq.(13) reads

$$
\partial_{t} S+H\left(t, x^{i}, \partial_{i} S\right)=0
$$

which is the Hamilton-Jacobi equation. In this way we obtain an equation for $S$, the auxiliary function that was so far undetermined. It is also straightforward to deduce the Euler-Lagrange and the Hamilton equations within the present approach. For the sake of brevity, we will show how to derive the Euler-Lagrange equations in the homogeneous case only. The non-homogeneous case can be treated along similar lines.

\subsection{The homogeneous case}

Let us turn into the so-called homogeneous problem, the one appropriate for a relativistic formulation. In relativity, we consider a space-time continuum described by four variables $x^{\mu}$. Our variational principle is of the same form as before, i.e., $\delta \int L d \tau=0$; but we require it to be invariant under Lorentz transformations and under parameter changes. Indeed, all we need in order to fix the motion is the geometrical shape of the extremal curve $x^{\mu}(\tau)$ in space-time, so that the parameter $\tau$ has no physical meaning and the theory must be invariant under arbitrary changes of it. This is achieved when $L$ does not depend explicitly on $\tau$ and, furthermore, it is homogeneous of first degree in the generalized velocities $\dot{x}^{\mu}$ : $L\left(x^{\mu}, \alpha \dot{x}^{\mu}\right)=\alpha L\left(x^{\mu}, \dot{x}^{\mu}\right)$, for $\alpha \geq 0$. From this requirement, it follows the identity

$$
\dot{x}^{\mu} \frac{\partial L(x, \dot{x})}{\partial \dot{x}^{\mu}}=L,
$$

which holds true for homogeneous Lagrangians. This property, however, precludes us from introducing a Hamiltonian in a similar manner as we did in the non-homogeneous case. We come back to this point later on.

As before, we seek also now for a velocity field $v(x)$ and a function $S\left(x^{\mu}\right)$, such that

$$
L(x, v)-v^{\mu} \partial_{\mu} S=0
$$

the value zero being an extremal one with respect to $v$, for a suitably chosen $S(x)$ that remains fixed in this context. For a maximum, for example, it must hold $L\left(x^{\mu}, w^{\mu}\right)-w^{\mu} \partial_{\mu} S<0$ for any other field ${ }^{1} w \neq v$. Differentiating the left-hand side of Eq.(17) with respect to $v$ and equating the result to zero we get

$$
\frac{\partial S}{\partial x^{\mu}}=\frac{\partial L(x, v)}{\partial v^{\mu}} .
$$

\footnotetext{
${ }^{1}$ The considered fields $w^{\alpha}$ are essentially different from $v^{\alpha}$. A field $w^{\alpha}=\phi v^{\alpha}$, with $\phi$ a scalar function, is essentially the same as $v^{\alpha}$.
} 
From the fundamental equations, (17) and (18), we can derive all known results also in this case. In particular, we see that $S(x)$ must satisfy the integrability conditions

$$
\frac{\partial^{2} S}{\partial x^{\mu} \partial x^{\nu}}=\frac{\partial^{2} S}{\partial x^{v} \partial x^{\mu}}
$$

which are, as we will shortly see, at the very basis of the Euler-Lagrange equations. Indeed, from Eq.(17) we obtain, by taking the derivative with respect to $x^{\mu}$,

$$
\frac{\partial L}{\partial x^{\mu}}+\frac{\partial L}{\partial v^{\sigma}} \frac{\partial v^{\sigma}}{\partial x^{\mu}}=\frac{\partial v^{\sigma}}{\partial x^{\mu}} \frac{\partial S}{\partial x^{\sigma}}+v^{\sigma} \frac{\partial^{2} S}{\partial x^{\mu} \partial x^{\sigma}} .
$$

On using Eq.(18), Eq.(20) reduces to

$$
\frac{\partial L}{\partial x^{\mu}}=v^{\sigma} \frac{\partial^{2} S}{\partial x^{\mu} \partial x^{\sigma}}
$$

From Eqs.(18) and (19) we thus obtain

$$
\frac{\partial^{2} S}{\partial x^{\mu} \partial x^{\sigma}}=\frac{\partial^{2} S}{\partial x^{\sigma} \partial x^{\mu}}=\frac{\partial^{2} L}{\partial x^{\sigma} \partial v^{\mu}}+\frac{\partial^{2} L}{\partial v^{\tau} \partial v^{\mu}} \frac{\partial v^{\tau}}{\partial x^{\sigma}},
$$

so that

$$
\frac{\partial L}{\partial x^{\mu}}=v^{\sigma} \frac{\partial^{2} L}{\partial x^{\sigma} \partial v^{\mu}}+\frac{\partial^{2} L}{\partial v^{\tau} \partial v^{\mu}} \frac{\partial v^{\tau}}{\partial x^{\sigma}} v^{\sigma} .
$$

If we now evaluate this last relation along a single extremal, $d x^{\mu} / d \tau=v^{\mu}(x(\tau))$, we obtain, after recognizing the right hand side of Eq.(23) as $d\left(\partial L / \partial v^{\mu}\right) / d \tau$, the Euler-Lagrange equation:

$$
\frac{\partial L}{\partial x^{\mu}}=\frac{d}{d \tau}\left(\frac{\partial L}{\partial \dot{x}^{\mu}}\right)
$$

Eq.(23) is therefore more general than the Euler-Lagrange equation. The latter follows from Eq.(23); but not the other way around.

For the non-homogeneous case we obtain a similar result

$$
\frac{d}{d t}\left(\frac{\partial L}{\partial \dot{x}^{i}}\right)-\frac{\partial L}{\partial x^{i}}=0
$$

but with the important difference that now the curve-parameter $t$ is fixed: the solution of Eq.(25) provides us not only with the geometrical shape of the extremal curve, but also with the rate at which this curve is traced back. 


\subsection{The arbitrariness of the curve parameter}

Let us see how the arbitrariness of the curve parameter $\tau$ manifests itself when dealing with fields of extremals. It is usual to take advantage of such an arbitrariness in order to simplify the equations of motion. It is well known that in the cases of electromagnetism, for which $L(x, \dot{x})=m c\left(\eta_{\mu v} \dot{x}^{\mu} \dot{x}^{v}\right)^{1 / 2}+\frac{e}{c} A_{\mu}(x) \dot{x}^{\mu}$, and gravitation, for which $L(x, \dot{x})=\left(g_{\mu v}(x) \dot{x}^{\mu} \dot{x}^{\nu}\right)^{1 / 2}$, by choosing $\tau$ such that $\left(\eta_{\mu v} \dot{x}^{\mu} \dot{x}^{v}\right)^{1 / 2}=1$, and $\left(g_{\mu v}(x) \dot{x}^{\mu} \dot{x}^{v}\right)^{1 / 2}=1$, respectively, the equations of motion acquire a simple form. We are so led to ask whether the field $v$ satisfying the fundamental Eqs.(17) and (18) has a corresponding arbitrariness. That this is indeed the case can be seen as follows. We wish to prove that in case $v^{\mu}$ satisfies Eqs.(17) and (18), so does $w^{\mu}=\phi v^{\mu}$, with $\phi(x)>0$ an arbitrary, scalar function. From the homogeneity of the Lagrangian we have $L\left(x^{\mu}, \phi v^{\mu}\right)=\phi L\left(x^{\mu}, v^{\mu}\right)$, so that it is seen at once that $w^{\mu}$ satisfies Eq.(17) if $v^{\mu}$ does. Indeed, multiplying Eq.(17) by $\phi(x)>0$ leads to

$$
\phi(x)\left(L(x, v)-v^{\mu} \partial_{\mu} S\right)=L(x, \phi v)-\left(\phi v^{\mu}\right) \partial_{\mu} S=L(x, w)-w^{\mu} \partial_{\mu} S .
$$

The Lagrangian of the "equivalent variational problem" is $L^{*}=L-v^{\mu} \partial_{\mu} S$. Clearly, $L^{*}(x, \phi v)=\phi L^{*}(x, v)$, and hence it follows that

$$
\frac{\partial L^{*}(x, w)}{\partial v^{\mu}}=\frac{\partial L^{*}(x, w)}{\partial w^{v}} \frac{\partial w^{\nu}}{\partial v^{\mu}}=\frac{\partial L^{*}(x, w)}{\partial w^{\mu}} \phi
$$

On the other hand,

$$
\frac{\partial L^{*}(x, w)}{\partial v^{\mu}}=\frac{\partial}{\partial v^{\mu}}\left(\phi L^{*}(x, v)\right)=\phi \frac{\partial L^{*}(x, v)}{\partial v^{\mu}}=\phi\left(\frac{\partial L}{\partial v^{\mu}}-\frac{\partial S}{\partial x^{\mu}}\right)=0,
$$

on account of Eq.(17). In view of Eq.(27) we have then that $\partial L^{*}(x, w) / \partial w^{\mu}=0$. In summary, Eqs. $(17,18)$ hold with $v$ being replaced by $w$, so that both velocity fields solve our variational problem for the same $S(x)$. We have thus the freedom to choose $\phi$ according to our convenience. The integral curves of $v^{\mu}(x)$ and $w^{\mu}(x)$ coincide with each other, differing only in their parametrization.

\subsection{Hamiltonians}

The introduction of a Hamiltonian offers no problem in the non-homogeneous case, where it was defined as $H\left(x^{i}, p_{i}\right) \equiv \dot{x}^{i}(t, x, p) p_{i}-L(t, x, \dot{x}(t, x, p))$, with $p_{i} \equiv \partial L / \partial \dot{x}^{i}$; the condition for solving $\dot{x}^{i}$ in terms of $\left(x^{j}, p_{j}\right)$ being assumed to be fulfilled: $\operatorname{det}\left(\partial^{2} L / \partial \dot{x}^{i} \partial \dot{x}^{j}\right) \neq 0$. It is then straightforward $[1,13]$ to obtain

$$
\frac{\partial H}{\partial p_{i}}=\dot{x}^{i}=\frac{d x^{i}}{d t}
$$

which constitute half of the Hamilton equations. It is also easy to sow that $\partial H / \partial t=$ $-\partial L / \partial t$ and $\partial H / \partial x^{i}=-\partial L / \partial x^{i}$. Using this last result together with $p_{i}=\partial L / \partial \dot{x}^{i}$ in the 
Euler-Lagrange equation, Eq.(25), one gets

$$
\frac{d p_{i}}{d t}=-\frac{\partial H}{\partial x^{i}}
$$

the other half of the Hamilton equations.

In the homogeneous case, as already mentioned, the corresponding expression for $H$, i.e., $\dot{x}^{\mu} \partial L / \partial \dot{x}^{\mu}-L$, vanishes identically by virtue of Eq.(16) . It is nonetheless generally possible to introduce a Hamiltonian in a number of ways. Carathéodory's approach leads to an infinite set of Hamiltonians, from which we can choose the most suitable one for the problem at hand. We will not go into the details here, but refer the reader to the standard literature $[1,13]$ in which this material is discussed at length.

\section{Electromagnetism: The London equations and the Bohr-van Leeuwen theorem}

\subsection{The London equations of superconductivity}

As mentioned before, there are only two interactions relevant to classical physics: electromagnetism and gravitation. In electromagnetism, the Lagrangian is given by

$$
L(x, \dot{x})=m c\left(\eta_{\mu v} \dot{x}^{\mu} \dot{x}^{v}\right)^{1 / 2}+\frac{e}{c} A_{\mu}(x) \dot{x}^{\mu}
$$

Here, $\eta_{\mu v}=\operatorname{diag}(+1,-1,-1,-1)$ is the Minkowski metric tensor and summation over repeated indices from 0 to 3 is understood. The electromagnetic field is given by the four-potential $A_{\mu}$, whose components are $\phi\left(t, x^{i}\right)$ and $\mathbf{A}\left(t, x^{i}\right)$.

We are now in a position to show how the London equations follow as a logical consequence of the relations presented above, when we use Eq.(31). From Eqs.(18) and (19) we obtain, in general,

$$
\frac{\partial}{\partial x^{\mu}}\left(\frac{L(x, v(x))}{\partial v^{v}}\right)-\frac{\partial}{\partial x^{v}}\left(\frac{L(x, v(x))}{\partial v^{\mu}}\right)=0
$$

This equation can be used to obtain the relativistic version of the London equations: As stated before, because the Lagrangian is homogeneous of first order in $v$, this vector field can be chosen so as to satisfy $\left(v_{\mu} v^{\mu}\right)^{1 / 2}=1$ in the region of interest. From Eq.(32) and Eq.(31) we get

$$
\frac{\partial v_{v}}{\partial x^{\mu}}-\frac{\partial v_{\mu}}{\partial x^{v}}+\frac{e}{m c^{2}}\left(\frac{\partial A_{v}}{\partial x^{\mu}}-\frac{\partial A_{\mu}}{\partial x^{\nu}}\right)=0
$$

This condition leads to the London equations, if we go to the non-relativistic limit, $v^{2} / c^{2} \ll 1$. Indeed, after multiplication by $n e$, with $n$ meaning a uniform particle density, Eq.(33) can be 
brought into the form:

$$
\frac{\partial j_{v}}{\partial x^{\mu}}-\frac{\partial j_{\mu}}{\partial x^{v}}+\frac{n e^{2}}{m c^{2}}\left(\frac{\partial A_{v}}{\partial x^{\mu}}-\frac{\partial A_{\mu}}{\partial x^{v}}\right)=0
$$

where $j_{\mu} \equiv n e v_{\mu}$. In the non-relativistic limit Eq.(34) reduces, for $\mu, v=i, k=1,2,3$, to

$$
\frac{\partial j_{k}}{\partial x^{i}}-\frac{\partial j_{i}}{\partial x^{k}}=-\frac{n e^{2}}{m c}\left(\frac{\partial A_{k}}{\partial x^{i}}-\frac{\partial A_{i}}{\partial x^{k}}\right),
$$

where we have used $\dot{x}^{\mu}(\tau)=\gamma(1, \mathbf{v}(t) / c)$ with $\gamma \equiv\left(1-\mathbf{v}^{2} / c^{2}\right)^{-1 / 2} \approx 1$. In three-vector notation this equation reads

$$
\nabla \times \mathbf{j}=-\frac{n e^{2}}{m c} \nabla \times \mathbf{A}=-\frac{n e^{2}}{m c} \mathbf{B}
$$

which is the London equation [16]. Eq. (36), together with the steady-state Maxwell equation, $\nabla \times \mathbf{B}=(4 \pi / c) \mathbf{j}$, lead to $\nabla^{2} \mathbf{B}=\left(4 \pi n e^{2} / m c^{2}\right) \mathbf{B}$, from which the Meissner effect follows. By considering now the case $\mu=0, v=k=1,2,3$ in Eq.(34), we obtain

$$
\frac{\partial j_{k}}{\partial x^{0}}-\frac{\partial j_{0}}{\partial x^{k}}=\frac{n e^{2}}{m c^{2}}\left(\frac{\partial A_{0}}{\partial x^{k}}-\frac{\partial A_{k}}{\partial x^{0}}\right)
$$

Multiplying this equation by $-c^{2}$ and using three-vector notation it reads, with $j_{0}=c \rho$,

$$
\frac{\partial \mathbf{j}}{\partial t}+c^{2} \nabla \rho=\frac{n e^{2}}{m} \mathbf{E}
$$

This equation was also postulated by the London brothers as part of the phenomenological description of superconductors. It was guessed as a relativistic generalization of the equation that should hold for a perfect conductor. Without the $\rho$-term (which in our case vanishes due to the assumed uniformity of $n$ ) it is nothing but the Newton, or "acceleration" equation for charges moving under the force $e \mathbf{E}$. The $\rho$-term was originally conceived as a relativistic "time-like supplement" to the current $\mathbf{j}$ [16]. We see that the London equations are in fact the non-relativistic limit of an integrability condition, Eq.(33), which follows from the variational principle $\delta \int L d s=0$ alone. The physical content of this procedure appears when we interpret the integral curves of $v(x)$ as streamlines of an ideal fluid. By contracting Eq.(33) with $v^{\mu}$ and using $v^{\mu} \partial_{\nu} v_{\mu}=0$ (which follows from $v^{\mu} v_{\mu}=1$ ) we obtain

$$
v^{\mu} \frac{\partial v_{v}}{\partial x^{\mu}}=\frac{e}{m c^{2}} F_{v \mu} v^{\mu}
$$


with $F_{v \mu}=\partial_{v} A_{\mu}-\partial_{\mu} A_{v}$, which relates to $\mathbf{E}$ and $\mathbf{B}$ by $E_{i}=F_{0 i}$ and $B_{i}=-\epsilon_{i j k} F_{j k} / 2$, with $\epsilon_{i j k}$ the totally antisymmetric symbol and latin indices running from 1 to 3 . The nonrelativistic limit of Eq.(39) reads

$$
\frac{\partial \mathbf{v}}{\partial t}+(\mathbf{v} \cdot \nabla) \mathbf{v}=\frac{e}{m}\left(\mathbf{E}+\frac{\mathbf{v}}{c} \times \mathbf{B}\right)
$$

The left-hand side of this equation is the convective derivative, which reduces to $d \mathbf{v} / d t$ by restriction to a single extremal. Analogously, Eq.(39) becomes the well-known Lorentz equation when evaluated along a single extremal: $d x^{\mu} / d s=v^{\mu}(x(s))$. In this case, $v^{\mu}(x(s)) \partial v_{v}(x(s)) / \partial x^{\mu}=d v_{v}(s) / d s$. Thus, we see that the Lorentz equation for a single particle follows from the more general Eq.(39). For $\mu=0$ Eq.(39) gives an equation which can be derived from Eq.(40) by scalar multiplication with $\mathbf{v}$. This is the energy equation. It is worth mentioning that this last fact is a particular manifestation of a well-known result valid for homogeneous Lagrangians: only $n-1$ out of the $n$ Euler-Lagrange equations are independent from each other in this case, due to the identity $\dot{x}^{\mu} E_{\mu}=0$, with $E_{\mu} \equiv d\left(\partial L / \partial \dot{x}^{\mu}\right) / d \tau-\partial L / \partial x^{\mu}$ being the Euler vector[13]. Such a result follows from Eq.(16).

Some remarks are in place here. Our derivation of the London equations brings into evidence that they have a validity that goes beyond their original scope. They cannot be seen by themselves as characterizing the phenomenon of superconductivity. Instead, they describe a "dust" of charged particles moving along the extremals of the Lagrangian given by Eq.(31). The field $A^{\mu}$ under which these particles move could be produced by external sources, or else be the field resulting from the superposition of some external fields with those produced by the charges themselves. In this last case, the Maxwell and London equations constitute a self-consistent system. Only under special circumstances, the system of charges can be in a state of collective motion that may be described by the field of extremals obeying Eq.(39). This is the superconducting phase, for which quantum aspects are known to play a fundamental role[17]. However, once the phase transition from the normal to the superconducting state has occurred, it becomes possible to describe some aspects of the superconducting state by classical means. This is a case analogous to the one encountered in laser theory. Indeed, several features of a lasing system can be understood within a semi-classical laser theory, whereby the electromagnetic field is treated as a classical, non-quantized field. Perhaps some plasmas could reach the limit of perfect conductivity. However, in order to produce a Meissner-like effect some conditions should be met. It is necessary, for instance, that the available free-energy of the plasma is sufficient to overcome the magnetic field energy, so that the magnetic field can be driven out of the plasma [5-8]. The so-called helicity of the system should also play a role, attaining the value zero for the superconducting state to be reached [9].

In any case, we see that Carathéodory's approach can be a fruitful one in physics. In the case of superconductivity, from the sole assumption that the Lagrangian be given by Eq.(31) one can derive all the equations that were more or less guessed, in the course of almost twenty five years, since Kamerlingh Onnes discovered superconductivity in 1911, until the London model was proposed, in 1935. But beyond this, there are other aspects that can be illuminated by following Carathéodory's approach, as we shall see next. 


\subsection{Beyond the London equations}

Let us address the case when the charge density $\rho$ is not constant, as previously assumed. There is a close relationship between the norm of our velocity field, i.e., $\phi(x)=\left(v_{\mu} v^{\mu}\right)^{1 / 2}$, and $\rho(x)$. It can be shown that it is always possible to choose $v^{\mu}$ so that the continuity equation $\partial_{\mu} j^{\mu}=0$ holds. Here, $j^{\mu}:=\rho v^{\mu}$ and $\rho=n e c \phi^{-1}, n$ being a free parameter whose dimensions are 1 /volume. Indeed, in view of the aforementioned possibility of changing the field $v^{\mu}$ by $w^{\mu}=\tilde{\phi} v^{\mu}$, we can always satisfy the continuity equation. For, if $\partial_{\mu} j^{\mu}=-f$, we may choose $\tilde{j}^{\mu}=\tilde{\phi} j^{\mu}$ such that $\partial_{\mu} \tilde{j}^{\mu}=\tilde{\phi} \partial_{\mu} j^{\mu}+j^{\mu} \partial_{\mu} \tilde{\phi}=0$. Putting $\psi=\log \tilde{\phi}$, we need to solve $j^{\mu} \partial_{\mu} \psi=f$, which is always possible.

Coming back to our Lagrangian of Eq.(31), by replacing it in Eq.(18), we obtain

$$
v_{\mu}=\frac{\phi}{m c}\left(\partial_{\mu} S-\frac{e}{c} A_{\mu}\right)
$$

with $\phi:=\left(v_{\mu} v^{\mu}\right)^{1 / 2}$. Using the gauge freedom of $A_{\mu}$ we may replace this field by

$$
A_{\mu}^{\prime}=A_{\mu}-\frac{c}{e} \partial_{\mu} S
$$

in which case Eq.(41) reads

$$
v_{\mu}=-\phi\left(\frac{e}{m c^{2}}\right) A_{\mu}^{\prime}
$$

From this equation and $v_{\mu} v^{\mu}=\phi^{2}$ we get

$$
A_{\mu}^{\prime} A^{\prime \mu}=\left(\frac{m c^{2}}{e}\right)^{2} .
$$

Eq.(32) applied to the present case gives

$$
\frac{\partial}{\partial x^{\mu}}\left(\frac{v_{v}}{\phi}\right)-\frac{\partial}{\partial x^{v}}\left(\frac{v_{\mu}}{\phi}\right)+\frac{e}{m c^{2}}\left(\frac{\partial A_{v}}{\partial x^{\mu}}-\frac{\partial A_{\mu}}{\partial x^{\nu}}\right)=0
$$

It is clear that this equation holds for $A_{\mu}^{\prime}$ as well. Eq.(43) is a particular solution of this equation. By Fourier-transforming Eq.(45) we obtain, with $w^{\mu}:=v^{\mu} / \phi$,

$$
k^{\mu} w^{v}-k^{v} w^{\mu}=-\frac{e}{m c^{2}}\left(k^{\mu} A^{v}-k^{v} A^{\mu}\right)
$$

As for the Fourier-transformed version of Eq.(43), it is given by

$$
w_{\mu}(k)=-\frac{e}{m c^{2}} A_{\mu}^{\prime}(k)
$$


As we saw before, $v^{\mu}$ can be chosen so that $j_{\mu}=n e c w_{\mu}=(n e c / \phi) v_{\mu} \equiv \rho(x) v_{\mu}$ satisfies the continuity equation $\partial_{\mu} j^{\mu}=0$. The factor nec is included for dimensional purposes: $c / \phi$ has no dimensions and $n$ is a free parameter such that $n e$ has dimensions of charge per unit volume. While $n$ is a constant, $\rho(x)$ is a non-uniform charge density. Thus, the scalar field $\phi=\left(v_{\mu} v^{\mu}\right)^{1 / 2}$, the norm of the velocity field, is related to the density $\rho(x)$ by $\rho=n e c \phi^{-1}$.

Note that $\partial_{\mu} j^{\mu}=0$ implies a restriction on $\partial_{\mu} v^{\mu}$. To see this, observe that $\partial_{\mu} j^{\mu}=\rho \partial_{\mu} v^{\mu}+$ $v^{\mu} \partial_{\mu} \rho=0$. This can be rewritten as

$$
\frac{v^{\mu}}{\phi} \partial_{\mu} \phi=f
$$

with $\partial_{\mu} v^{\mu}=f$. On the other hand, from $v_{\mu} v^{\mu}=\phi^{2}$ it follows that $\phi \partial_{\mu} \phi=v^{v} \partial_{\mu} v_{v}$. Eq.(48) then implies that

$$
\frac{v^{\mu} v^{v}}{2 \phi^{2}}\left(\partial_{\mu} v_{v}+\partial_{\nu} v_{\mu}\right)=\partial_{\sigma} v^{\sigma} .
$$

It is also worth noting that instead of Eq.(39) we have now

$$
v^{\mu} \frac{\partial v_{v}}{\partial x^{\mu}}=\frac{e \phi}{m c^{2}} F_{v \mu} v^{\mu}+\left(v^{\mu} \frac{\partial}{\partial x^{\mu}} \ln \phi\right) v_{v}=\frac{e \phi}{m c^{2}} F_{\nu \mu} v^{\mu}+f v_{v} .
$$

We could argue that the second term on the right hand side is not physical, because we could choose $\phi=1$, as we did before, getting Eq.(39). However, such a choice is not available any longer when we invoke charge (or matter) conservation. In such a case, $\partial_{\mu} j^{\mu}=0$, and we must relate $j^{\mu}$ with $v^{\mu}$ by $j^{\mu}=\rho v^{\mu}$, so that the above considerations apply.

Coming back to Eq.(47), we see that it implies that $\partial_{\mu} A^{\prime \mu}=0$, i.e., $A^{\prime \mu}$ is in the Lorentz gauge. Because of $A_{\mu}^{\prime}=A_{\mu}-(c / e) \partial_{\mu} S$, the scalar function $S$ must satisfy

$$
\partial_{\mu} \partial^{\mu} S \equiv \square S=\frac{e}{c} \partial_{\mu} A^{\mu}
$$

Let us consider now Maxwell equations, $\partial_{\mu} F^{\mu v}=(4 \pi / c) j^{v}$. If we take $j^{v}$ to be the same as before, we are assuming that $F^{\mu v}$ is generated by the same currents upon which this field is acting. That is, we are considering a closed system of charges and fields. We have then, using $F^{\mu \nu}=\partial^{\mu} A^{\prime \nu}-\partial^{v} A^{\prime \mu}$ and $\partial_{\mu} A^{\prime \mu}=0$,

$$
\partial_{\mu} F^{\mu v}=\square A^{\prime v}=\frac{4 \pi}{c} j^{v},
$$

while from Eq. (47) we get

$$
A^{\prime v}=-\frac{m c}{n e^{2}} j^{v},
$$


so that we can write Eq. (52) as

$$
\square j^{v}=-\frac{4 \pi n e^{2}}{m c^{2}} j^{v} \equiv-\frac{1}{\lambda_{L}^{2}} j^{v},
$$

in which we have identified the London penetration length $\lambda_{L}$. This equation can be rewritten in the form of the Klein-Gordon equation:

$$
\left(\square+\lambda_{L}^{-2}\right) j^{v}=0
$$

with $\lambda_{L}$ replacing $\lambda_{C}=\hbar / m c$, the Compton wavelength that appears in the Klein-Gordon equation. For the steady-state $\left(\partial_{0} j^{v}=0\right)$, Eq.(54) reads

$$
\nabla^{2} j^{v}=+\frac{1}{\lambda_{L}^{2}} j^{v}
$$

Taking the usual configuration of a superconductor filling half the space $(z>0)$, the solution of this equation (satisfying appropriate boundary conditions: $\lim _{z \rightarrow \infty} j^{v}(z)=0$ ) is

$$
j^{v}(z)=\exp \left(-\frac{z}{\lambda_{L}}\right) j^{v}(0) .
$$

In general, however, Eq.(55) admits several other solutions that depend on the assumed boundary conditions. Note that Eq.(55) corresponds to a field-free case of the Klein-Gordon equation. This is because $j^{v} \sim A^{\prime v}$, so that electromagnetic fields and current share the same dynamics. This is a consequence of having assumed that the Euler-Lagrange equations (written as field equations) and Maxwell equations conform a closed system. Notably, $A^{\prime \prime}$ behaves like a source-free Proca field [18] whose mass (in units of inverse length) is fixed by $\lambda_{L}$.

\subsection{The Bohr-van Leeuwen theorem}

Dropping the prime, Eq.(53) gives $j^{v}=-\left(n e^{2} / m c\right) A^{v}$, which can be rewritten as $v^{v}=$ $-\left(e / m c^{2}\right) A^{v}$, with $v_{v} v^{v}=1$. We get thus $v_{v} A^{v}=-m c^{2} / e$, which in the nonrelativistic limit reads

$$
\mathbf{v} \cdot \mathbf{A}=\frac{m c^{3}}{e}
$$

This condition is important for the following reason. Our considerations have confirmed the possibility of classical diamagnetism, in contradiction with the Bohr-van Leeuwen theorem. Therefore, this theorem should be modified. Eq.(58) represents a constant of the motion that must be taken into account when constructing the phase density for a system of charged particles. The original version of the Bohr-van Leeuwen theorem did not consider condition (58). We will show next how this condition modifies the theorem. 
The Bohr-van Leeuwen theorem addresses a sample of charged particles subjected to a uniform magnetic field $\mathbf{B}$. The nonrelativistic Lagrangian of the system is $L=$ $\sum_{i=1}^{N}\left(m_{i} / 2\right) \mathbf{v}_{i}^{2}+\left(e_{i} / c\right) \mathbf{v}_{i} \cdot \mathbf{A}$. We can take $\mathbf{A}=\mathbf{B} \times \mathbf{r} / 2$. The partition function is given by

$$
Z=\int_{-\infty}^{+\infty} \ldots \int_{-\infty}^{+\infty} \exp \left[-\beta\left(\sum_{i} \dot{x}^{i} \frac{\partial L}{\partial \dot{x}^{i}}-L\right)\right]\left|\frac{\partial^{2} L}{\partial \dot{x}^{i} \partial \dot{x}^{j}}\right| d^{N} \tau,
$$

with $d^{N} \tau$ a properly normalized volume element in configuration space. We see that the terms in $L$ that depend on magnetic potentials are linear in the velocities, so that the integrand in $Z$ turns out to be independent of magnetic potentials. The Bohr-van Leeuwen theorem then follows: because $Z$ is independent of magnetic potentials, there is no effect on the system in response to $\mathbf{B}$. This prediction changes when we take into account the constant of motion, Eq.(58), or equivalently, $G:=(e \mathbf{B} / 2 m c) \cdot(\mathbf{r} \times \mathbf{v})=c^{2}$. For a sample of identical particles we define $G_{S}:=\sum_{i}(e \mathbf{B} / 2 m c) \cdot\left(\mathbf{r}_{i} \times \mathbf{v}_{i}\right) \equiv \sum_{i} \boldsymbol{\omega}_{L} \cdot\left(\mathbf{r}_{i} \times \mathbf{v}_{i}\right)$, with $\boldsymbol{\omega}_{L}$ the Larmor frequency. The phase density $D$ for the corresponding Hamiltonian $H=\sum_{i}(2 m)^{-1}\left(\mathbf{p}_{i}-\left(e_{i} / c\right) \mathbf{A}_{i}\right)^{2}$ is given by $D=Z^{-1} \exp \left(-\beta H-\lambda G_{s}\right)$, with $Z$ being the partition function that normalizes $D$ and $\beta=\left(k_{B} T\right)^{-1}$. Both $\lambda$ and $\beta$ are Lagrange multipliers, introduced to take account of the restrictions imposed by Eq. (58) and the fixed mean energy, respectively. Thus,

$$
D=\frac{1}{Z} \exp \left[\sum_{i}\left(\frac{-\beta\left(\mathbf{p}_{i}-(e / c) \mathbf{A}_{i}\right)^{2}}{2 m}+\lambda\left(\boldsymbol{\omega}_{L} \cdot \mathbf{r}_{i} \times \mathbf{v}_{i}\right)\right)\right]
$$

The single-particle velocity distribution that can be obtained from $D$ is proportional to

$$
\exp \left[-\frac{\beta m}{2}\left(\mathbf{v}-\frac{\lambda}{\beta m} \boldsymbol{\omega}_{L} \times \mathbf{r}\right)^{2}+\frac{\lambda^{2}}{2 \beta m}\left(\boldsymbol{\omega}_{L} \times \mathbf{r}\right)^{2}\right]
$$

This gives the mean velocity at $\mathbf{r}$. We have thus $\langle\mathbf{v}\rangle=(\lambda / \beta m) \boldsymbol{\omega}_{L} \times \mathbf{r}$, which determines the value of the Lagrange multiplier as $\lambda=\beta m$. The phase density can finally be written as

$$
D=\frac{1}{Z} \exp \left[-\beta\left(\sum_{i} \frac{m}{2} \mathbf{v}_{i}^{2}+\mathbf{B} \cdot \mathbf{M}\right)\right]
$$

with $\mathbf{M} \equiv \sum_{i}(e / 2 c) \mathbf{r}_{i} \times \mathbf{v}_{i}$ naturally arising as the magnetic moment of the system. A magnetic response shows up therefore also classically, contrary to what the original version of the Bohr-van Leeuwen theorem stated. It has been shown before that this theorem does not hold whenever the magnetic field produced by the moving charges is taken into account. Such a field is included in the Darwin Lagrangian [11], which is correct to order $(v / c)^{2}$. In our case, we do not need to modify the standard Lagrangian. 


\section{Hamilton-Jacobi equations without Hamiltonian}

We have already mentioned that for homogeneous Lagrangians the definition of a Hamiltonian is precluded by the vanishing of $\dot{x}^{\mu} \partial L / \partial \dot{x}^{\mu}-L$. It is nonetheless possible to introduce a Hamiltonian in a number of ways. Carathéodory's approach leads to an infinite set of Hamiltonians, from which we can choose the most suitable one for dealing with the problem at hand. Here, we focus on the two Lagrangians of interest to us, given by $L=m c\left(\eta_{\mu v} \dot{x}^{\mu} \dot{x}^{\nu}\right)^{1 / 2}+e A_{\mu}(x) \dot{x}^{\mu} / c$ for electromagnetism and

$$
L(x, \dot{x})=\left(g_{\mu v}(x) \dot{x}^{\mu} \dot{x}^{v}\right)^{1 / 2}
$$

for gravitation. We will prove that in these two particular cases it is possible to derive the equation which the function $S(x)$ has to satisfy, without having to introduce a Hamiltonian.

Let us start with gravitation. From Eq.(63) with $v^{\mu}$ replacing $\dot{x}^{\mu}$, it follows that

$$
\frac{\partial L}{\partial v^{\mu}}=\frac{1}{L} g_{\mu v} v^{v}
$$

Using $g_{\mu v} g^{v \sigma}=\delta_{\mu}^{\sigma}$ this equation leads to $v^{v}=L g^{\mu v} p_{\mu}$, with $p_{\mu} \equiv \partial L / \partial v^{\mu}$. Considering that $L^{2}=g_{\mu v} v^{\mu} v^{v}=L^{2} g^{\mu v} p_{\mu} p_{v}$, it follows $g^{\mu v} p_{\mu} p_{v}=1$. And because $\partial S / \partial x^{\mu}=\partial L / \partial v^{\mu}=p_{\mu}$, we obtain the Hamilton-Jacobi equation for $S$ :

$$
g^{\mu v}(x) \frac{\partial S}{\partial x^{\mu}} \frac{\partial S}{\partial x^{v}}=1
$$

In the electromagnetic case the corresponding Lagrangian leads, by the same token, to $v_{\mu}=$ $\left(\partial_{\mu} S-\frac{e}{c} A_{\mu}\right) \phi / m c$ with $\phi \equiv\left(\eta_{\mu v} v^{\mu} v^{v}\right)^{1 / 2}$, again as a consequence of $\partial S / \partial x^{\mu}=\partial L / \partial v^{\mu}$. By replacing the above expression of $v_{\mu}$ in $\phi^{2}=\eta^{\mu v} v_{\mu} v_{v}$, it follows the Hamilton-Jacobi equation

$$
\eta^{\mu \nu}\left(\frac{\partial S}{\partial x^{\mu}}-\frac{e}{c} A_{\mu}\right)\left(\frac{\partial S}{\partial x^{v}}-\frac{e}{c} A_{v}\right)=m^{2} c^{2} .
$$

We remark that there was no need to choose $v$ so as to satisfy either $\phi=$ const. in the electromagnetic case, or $L=$ const. in the gravitational case, as it is usually done for obtaining the respective Euler-Lagrange equations in their simplest forms. As a consequence, the constants appearing on the right-hand sides of Eqs.(65) and (66) are independent of the way by which we decide to fix the parameter $\tau$ of the extremal curves. Let us remark that it is not unusual to find in textbooks Eq.(65) written with $m^{2} c^{2}$ instead of the 1 on the right-hand side (see, e.g. [19]). This occurs because Eq.(65) is usually introduced as a generalization of Eq.(66), with $A_{\mu}=0$ (field-free case). Invoking the equivalence principle, one replaces $\eta^{\mu v}$ by $g^{\mu v}$ and so arrives at the equation which is supposed to describe a "free" particle moving in a curved space-time region. Now, the metric tensor $g^{\mu v}$ embodies all the information that determines how a test particle moves under gravity, irrespective of its inertial mass $m$. There 
is therefore no physical reason to put a term like $m^{2} c^{2}$ on the right-hand side of Eq.(65). To be sure, for all practical purposes it is irrelevant that we set any constant on the right-hand side of Eq.(65), as this constant will drop afterwards in the equations describing the motion. But, as a matter of principle, the mass of a test particle should not appear in an equation which describes how it moves under the sole action of gravity.

\section{Gauge invariance in electromagnetism and gravitation}

Gauge invariance is presently understood as a key principle that lies at the root of fundamental interactions. An equation like Schrödinger's (or Dirac's) for a free electron is invariant under the transformation $\psi \rightarrow \exp (i \alpha) \psi$, for constant $\alpha$. This is in accordance with the physical meaning of the wave-function and the way it enters in all expressions related to measurable quantities. However, one expects that Nature should respect such an invariance not only globally, i.e., with constant $\alpha$, but also locally, with $\alpha$ a function of time and position. It is, so to say, by recourse to the appropriate interaction that Nature manages to reach this goal. For achieving invariance under the $U(1)$ transformation $\psi \rightarrow \exp (i \alpha) \psi$, it is necessary to introduce a gauge field, in this case a field represented by $A_{\mu}(x)$, which couples to the particle. The equation for a free particle is correspondingly changed into one in which $A_{\mu}$ appears. In this context, gauge invariance means invariance under the simultaneous change $\psi \rightarrow \exp (i \alpha) \psi$ and an appropriate one for $A_{\mu}$. This last one must be so designed that the equation now containing $A_{\mu}$ remains invariant. The change of $A_{\mu}$ turns out to be $A_{\mu} \rightarrow A_{\mu}-(\hbar c / e) \partial_{\mu} \alpha$, which is the one corresponding to a gauge transformation of the electromagnetic field. Hence, one is led to interpret electromagnetic interactions as a consequence of local $U(1)$-invariance. Other fundamental interactions stem from similar gauge invariances: $S U(2) \times U(1)$ gives rise to electroweak interactions, $S U(3)$ to the strong interaction [20], and local Lorentz invariance to gravitation [21, 22].

In this Section we want to show how gauge invariance leads, within the classical context, to considerations paralleling those of quantum mechanics. Carathéodory's formulation will be particularly useful to this end. Let us start with the electromagnetic case. Replacing the Lagrangian of Eq.(31) in the fundamental Eq.(17), we get

$$
m c\left(v_{\mu} v^{\mu}\right)^{1 / 2}+\frac{e}{c} A_{\mu} v^{\mu}-v^{\mu} \partial_{\mu} S=0 .
$$

Now, the observable predictions we can make concern the integral curves of the velocity field $v^{\mu}$. This field remains invariant under the replacement

$$
A_{\mu}^{*}=A_{\mu}-\frac{c}{e} \partial_{\mu} W
$$

whenever a simultaneous change in $S$ is undertaken. This change is given by $S \rightarrow S^{*}=$ $S+W$. It leaves Eq.(67) unchanged, for a fixed $v^{\mu}(x)$. Eq.(66), to which the velocity field $v^{\mu}(x)$ belongs, is also fulfilled with $S^{*}$ and $A_{\mu}^{*}$. The quantum-mechanical counterpart of this result could have suggested such a conclusion, in view of the relationship $\psi \sim \exp (i S / \hbar)$. Indeed, a change $\psi \rightarrow \psi^{*}=\exp (i \alpha) \psi$ means that $\psi^{*} \sim \exp \left(i S^{*} / \hbar\right)$, with $S^{*}=S+W$, where $W=\hbar \alpha$. 
Now, we are naturally led to ask about a similar invariance in the gravitational case. Here, Eq.(17) reads

$$
\left(g_{\mu v} v^{\mu} v^{v}\right)^{1 / 2}-v^{\mu} \partial_{\mu} S=0,
$$

and we ask how a simultaneous change of $g_{\mu \nu}$ and $S$ might be, in order that $v^{\mu}$ remains fixed and with it the field of extremals. In the present case, it is better to start with Eq.(23) instead of Eq.(69). The reasons will become clear in what follows. Working out Eq.(23) for the present Lagrangian we obtain, after some manipulations,

$$
v^{\tau} \frac{\partial v^{v}}{\partial x^{\tau}}+g^{\mu v}\left(\partial_{\tau} g_{\mu \sigma}-\frac{1}{2} \partial_{\mu} g_{\sigma \tau}\right) v^{\sigma} v^{\tau}=\frac{\partial(\ln L(x, v(x)))}{\partial x^{\tau}} v^{v} v^{\tau}=\frac{\partial \Phi(x)}{\partial x^{\tau}} v^{v} v^{\tau},
$$

where $\Phi(x)=\ln L(x, v(x))$. The right-hand side of Eq.(70) can be written in the form $\frac{1}{2}\left(\delta_{\sigma}^{v} \partial_{\tau} \Phi+\delta_{\tau}^{v} \partial_{\sigma} \Phi\right) v^{\sigma} v^{\tau}$. This suggests us to symmetrize the coefficient of $v^{\sigma} v^{\tau}$ on the left-hand side, thereby obtaining

$$
g^{\mu v}\left(\partial_{\tau} g_{\mu \sigma}-\frac{1}{2} \partial_{\mu} g_{\sigma \tau}\right) v^{\sigma} v^{\tau}=\frac{1}{2} g^{\mu \nu}\left(\partial_{\tau} g_{\mu \sigma}+\partial_{\sigma} g_{\mu \tau}-\partial_{\mu} g_{\sigma \tau}\right) v^{\sigma} v^{\tau} \equiv \Gamma_{\sigma \tau}^{v} v^{\sigma} v^{\tau}
$$

with $\Gamma_{\sigma \tau}^{v}$ the Christoffel symbols. Eq.(70) then reads

$$
v^{\tau} \frac{\partial v^{v}}{\partial x^{\tau}}+\Gamma_{\sigma \tau}^{v} v^{\sigma} v^{\tau}=\frac{1}{2}\left(\delta_{\sigma}^{v} \partial_{\tau} \Phi+\delta_{\tau}^{v} \partial_{\sigma} \Phi\right) v^{\sigma} v^{\tau}
$$

Note that if we choose $v$ such that $L=$ const., then Eq.(72) becomes the usual geodesic equation, when it is calculated along an extremal curve, $d x^{\mu} / d \tau=v^{\mu}(x(\tau))$ :

$$
\frac{d \dot{x}^{\mu}}{d \tau}+\Gamma_{\sigma \rho}^{\mu} \frac{d x^{\sigma}}{d \tau} \frac{d x^{\rho}}{d \tau}=0
$$

If $L \neq$ const., we obtain a geodesic equation with a right-hand side of the form $(d f / d \tau) \dot{x}^{v}$. In both cases we obtain the same curves - geodesics - but with a different parametrization.

Now, assume that a change, $g_{\mu \sigma} \rightarrow g_{\mu \sigma}^{*}$ can be found, so that the corresponding $\Gamma_{\tau \sigma}^{* v}$ satisfy

$$
\Gamma_{\tau \sigma}^{* v}-\Gamma_{\tau \sigma}^{v}=\frac{1}{2}\left(\delta_{\sigma}^{v} \partial_{\tau} \Lambda(x)+\delta_{\tau}^{v} \partial_{\sigma} \Lambda(x)\right),
$$

with $\Lambda(x)$ being arbitrary. Such a change leads to an equation equivalent to Eq.(70), with $\Phi$ being replaced by $\Phi^{*}=\Phi+\Lambda$, and hence to the same extremals. In this way we recover an old result due to Weyl: if Christoffel symbols are related to each other by Eq.(74), then they have the same geodesics [23]. Given $g_{\mu v}$ and $\Lambda$, it is always possible to find a $g_{\mu \nu}^{*}$ satisfying Eq.(74). This is because this equation can be put in the form

$$
\partial_{\lambda} g^{* \mu \nu}=-g^{* \nu \sigma} \Omega_{\lambda \sigma}^{\mu}-g^{* \mu \sigma} \Omega_{\lambda \sigma^{\prime}}^{v}
$$


with $\Omega_{\lambda \sigma}^{\mu}:=\Gamma_{\lambda \sigma}^{\mu}+\frac{1}{2}\left(\delta_{\sigma}^{\mu} \partial_{\lambda} \Lambda+\delta_{\lambda}^{\mu} \partial_{\sigma} \Lambda\right)$, and it can be straightforwardly proved that the integrability conditions for the above equation are identically satisfied.

In fact, Weyl arrived at a relation like Eq.(74) but having the expression $w_{\tau} \delta_{\sigma}^{v}+w_{\sigma} \delta_{\tau}^{v}$ on the right hand side, with $w_{\sigma}$ taken to be a covariant vector. Now, it is easy to see that $w_{\sigma}$ must be a gradient. Indeed, after writing Eq.(74) in Weyl's form, with $w_{\mu}$ replacing $\frac{1}{2} \partial_{\mu} \Lambda$, we contract both sides of this equation with respect to $v$ and $\tau$, thereby obtaining $w_{\sigma}=\frac{1}{5}\left(\Gamma_{v \sigma}^{* v}-\Gamma_{v \sigma}^{v}\right)$. Using $\Gamma_{v \sigma}^{v}=\frac{1}{2} \partial_{\sigma} \ln g$, with $g=\left|\operatorname{det}\left(g_{\mu v}\right)\right|$, we get $w_{\sigma}=\partial_{\sigma}\left(\ln \left(g^{*} / g\right)\right) / 10$.

If we take geodesics as the only observable objects, then it is natural to seek transformations that leave them invariant. Such transformations are given by Eq.(74). However, a transformation of the metric tensor that fulfils Eq.(74) does not leave invariant Einstein's field equations:

$$
R_{\mu v}-\frac{1}{2} g_{\mu \nu}=\varkappa T_{\mu v}
$$

where, we recall, $R_{\mu \nu}=R_{\mu \nu \sigma}^{\sigma}$ is the Ricci tensor stemming from the Riemann tensor $R_{\mu \nu \sigma}^{\lambda}$ by contraction of $\lambda$ and $\sigma$, and $T_{\mu \nu}$ means the energy-momentum tensor.

If our transformations do not leave Eq.(76) invariant but we insist in viewing geodesic invariance as a fundamental requirement, then we are led to ask for alternative equations for the gravitational field. These equations should be invariant under Eq.(74). Weyl found a tensor that is invariant under Eq.(74), i.e., a candidate for replacing $R_{\mu v \sigma}^{\lambda}$ as the starting point of the sought-after equations. It is given by

$$
W_{\mu v \sigma}^{\lambda}=R_{\mu v \sigma}^{\lambda}-\frac{1}{4}\left(\delta_{\sigma}^{\lambda} R_{\mu v}-\delta_{v}^{\lambda} R_{\mu \sigma}\right)
$$

Unfortunately, any contraction of $W_{\mu \nu \sigma}^{\lambda}$ vanishes identically, thereby precluding an alternative setting of equations analogous to those of Einstein.

One could argue that it remains still open the possibility of changing our very starting point, so that we should look for a Lagrangian which does not depend on a metric tensor. A natural candidate for this would be an affine connection (the Christoffel symbols being a special case). However, we can show that, even if we start from very general assumptions, we will end up with a Lagrangian like that of Eq. (63). That is, if we take our variational principle in the general form $\delta \int L(x, v) d \tau=0$, and require that $L$ is invariant under local Lorentz transformations, then $L$ must be of the form $\left(g_{\mu v}(x) v^{\mu} v^{v}\right)^{1 / 2}$. The requirement of invariance under local Lorentz transformations follows from the principle of equivalence: at any given point we can choose our coordinate system so that a body subjected only to gravity appears to move freely in a small neighborhood of the given point. This requirement leads to the particular form of $L$ just given, as can be seen as follows [24]: From the homogeneity of $L$ with respect to $v$ it follows that we can write $L$ in the form $L=\left(g_{\mu v}(x, v) v^{\mu} v^{v}\right)^{1 / 2}$, with $g_{\mu v}(x, v):=\frac{1}{2} \partial^{2} L^{2}(x, v) / \partial v^{\mu} \partial v^{v}$. This puts our variational problem within the framework of Finsler spaces [25]. But local Lorentz invariance implies that $g_{\mu v}$ is independent of $v$, as we shall see, so that we end up within the framework of Riemann spaces, a special case of Finsler spaces. 
A transformation in the tangent space, $v \rightarrow w$, defined through $w^{\mu}=\tilde{\Lambda}_{v}^{\mu} v^{v}$, is a local Lorentz transformation if it satisfies $g_{\mu v}(x, v)=g_{\lambda \sigma}(x, v) \Lambda_{v}^{\lambda}(x) \Lambda_{v}^{\sigma}(x)$ at any fixed point $x$. Here, $\Lambda_{v}^{\mu}$ means the inverse of $\tilde{\Lambda}_{v}^{\mu}$. Invariance of $L$ under local Lorentz transformations means that $L\left(x^{\mu}, w^{\mu}\right)=L\left(x^{\mu}, \sim_{\nu}^{\mu} v^{v}\right)=L\left(x^{\mu}, v^{\mu}\right)$. From this equality, by taking partial derivatives with respect to $v$, we obtain the two following equations:

$$
\begin{gathered}
\frac{\partial L(x, w)}{\partial v^{\mu}} \Lambda_{v}^{\mu}=\frac{\partial L(x, v)}{\partial v^{v}} \\
\frac{\partial^{2} L(x, w)}{\partial v^{\mu} \partial v^{v}} \Lambda_{\sigma}^{\mu} \Lambda_{\tau}^{v}=\frac{\partial^{2} L(x, v)}{\partial v^{\sigma} \partial v^{\tau}}
\end{gathered}
$$

When these equations are substituted into the identity

$$
g_{\mu v}(x, v)=\frac{1}{2} \frac{\partial^{2} L^{2}}{\partial v^{\mu} \partial v^{v}}=\frac{\partial L}{\partial v^{\mu}} \frac{\partial L}{\partial v^{v}}+L \frac{\partial^{2} L}{\partial v^{\mu} \partial v^{v}}
$$

one obtains

$$
g_{\mu v}(x, w)=g_{\lambda \sigma}(x, v) \Lambda_{\mu}^{\lambda}(x) \Lambda_{v}^{\sigma}(x)
$$

We conclude therefore, in view of this last equation and the definition of the Lorentz transformation given above, that the equality $g_{\mu v}(x, w)=g_{\mu v}(x, v)$ holds true for any $w$ and $v$ that are connected to each other by a Lorentz transformation. Thus, setting $w=v+\delta v$, we obtain

$$
\frac{\partial g_{\mu v}(x, v)}{\partial v^{\lambda}}=\lim _{\delta v \rightarrow 0}\left(\frac{g_{\mu v}(x, v+\delta v)-g_{\mu v}(x, v)}{\delta v^{\lambda}}\right)=0 .
$$

Thus, $L$ must be of the form $\left(g_{\mu v}(x) v^{\mu} v^{v}\right)^{1 / 2}$. As we have seen, this result follows from the requirement of local Lorentz invariance. Such an assumption is the counterpart of the condition put by Helmholtz on a general metric space, in order to geometrically characterize Riemann spaces [23]. In this last case, local rotations played the role that is assigned to local Lorentz transformations in the physical case.

\section{Summary and conclusions}

Carathéodory's approach to the calculus of variations appears to be an appropriate tool for uncovering some aspects of the quantum-classical relationship. Because it describes a whole field of extremals rather than a single one, Carathéodory's approach is, by its very nature, more akin to the quantal formulation. It remains still open how to introduce in this framework the second basic element of the quantal formulation, namely probability. By blending field and probability issues, it is likely that the ensuing result shed some light on questions concerning the quantum-classical correspondence. Here, by way of 
illustration of the capabilities of Carathéodory's approach, we have dealt with the two fundamental interactions of classical physics: electromagnetism and gravitation. We have seen that the London equations of superconductivity can be formally derived from the standard Lagrangian of a particle interacting with a prescribed electromagnetic field. The London equations have therefore not a distinctive quantum-mechanical origin, as it is often assumed. This does not mean, however, that we can explain superconductivity by recourse to classical physics alone. The conditions under which a system of charged particles behaves as described by the standard, classical Lagrangian, might be explainable only through quantum mechanics.

In the gravitational case, we recovered Weyl's results about the invariance of geodesics under some special transformation of the Christoffel symbols. Carathéodory's fundamental equations led us to formulate Weyl's result without having to resort to the tools of differential geometry. Furthermore, we have seen that the Lagrangian $L=\left(g_{\mu v}(x) v^{\mu} v^{v}\right)^{1 / 2}$ is a direct consequence of the assumption of local Lorentz invariance. The underlying principle that led us to state the appropriate questions was the principle of gauge invariance, something usually tied to a quantal approach.

In summary, Carathéodory's approach to variational calculus represents an alternative way to introduce some of the most basic principles of classical physics. It unifies different aspects that otherwise appear to be independent from one another, and it can help us in our quest for delimiting the quantum-classical correspondence.

\section{Acknowledgements}

Partial financial support from DGI-PUCP and from the Science Department (PUCP) is gratefully acknowledged.

\section{Author details}

Francisco De Zela

Departamento de Ciencias, Sección Física, Pontificia Universidad Católica del Perú, Lima, Peru

\section{References}

[1] C. Carathéodory, Calculus of Variations and Partial Differential Equations of the First Order. Part II: Calculus of Variations, Holden-Day, San Francisco, 1967.

[2] O. Redlich, "Fundamental Thermodynamics since Carathéodory", Rev. Mod. Phys. vol. 40 pp. 556-563, 1968.

[3] P. R. Holland, The quantum theory of motion: an account of the de Broglie-Bohm causal interpretation of quantum mechanics, Cambridge University Press, Cambridge 1993.

[4] D. Bohm, "A suggested interpretation of the quantum theory in terms of 'hidden' variables", Phys. Rev. vol. 85, pp. 166-179, 1952. 
[5] W. Farrell Edwards, "Classical Derivation of the London Equations", Phys. Rev. Lett. vol. 47, pp. 1863-1866, 1981.

[6] F. S. Henyey, "Distinction between a Perfect Conductor and a Superconductor", Phys. Rev. Lett. vol. 49, pp. 416, 1982.

[7] B. Segall, L. L. Foldy, and R. W. Brown, "Comment on 'Classical Derivation of the London Equations"', Phys. Rev. Lett. vol. 49, p. 417, 1982.

[8] J. B. Taylor, "A classical derivation of the Meissner effect?", Nature vol. 299, pp. 681-682, 1982.

[9] S. M. Mahajan, "Classical Perfect Diamagnetism: Expulsion of Current from the Plasma Interior", Phys. Rev. Lett., vol. 100, pp. 075001-1-075001-4, 2008.

[10] R. Balian, From Microphysics to Macrophysics, Vol. I, Springer-Verlag, Berlin, Heidelberg, New York, 1991.

[11] H. Essén and M. C. N. Fiolhais, "Meissner effeect, diamagnetism, and classical physics - a review", Am. J. Phys., vol. 80, pp. 164-169, 2011.

[12] H. Goldstein, Classical Mechanics, 2nd. Ed., Addison-Wesley, Reading, Massachusetts, 1980.

[13] H. Rund, The Hamilton-Jacobi Theory in the Calculus of Variations, D.van Nostrand Comp., London, 1966.

[14] D. Lovelock and H. Rund, Tensors, Differential Forms, and Variational Principles Wiley, New York, 1975.

[15] G. A. Bliss, Lectures on the Calculus of Variations, University of Chicago Press, Chicago, 1946.

[16] F. London and H. London, "The Electromagnetic Equations of the Supraconductor", Proc. Roy. Soc.(London) vol. A149, pp. 71-88, 1935.

[17] J. Bardeen, L. N. Cooper, J. R. Schrieffer, "Theory of Superconductivity", Phys. Rev. vol. 108, pp. 1175-1204, 1957.

[18] J. D. Jackson, Classical Electrodynamics, 3rd edition, Wiley, New York, 1999.

[19] L. D. Landau, E. M. Lifshitz, The Classical Theory of Fields, Pergamon Press, Oxford, 1962.

[20] F. Halzen and A. D. Martin, Quarks and Leptons: An Introductory Course in Modern Particle Physics, Wiley, New York, 1984.

[21] R. Utiyama, "Invariant Theoretical Interpretation of Interaction", Phys. Rev. vol. 101, pp. 1597-1607, 1956.

[22] T. W. B. Kibble, "Lorentz Invariance and the Gravitational Field", J. Math. Phys. vol. 2, pp. 212-221, 1961. 
[23] D. Laugwitz, Differential and Riemannian Geometry , Academic Press, New York, 1965.

[24] F. De Zela, “Über mögliche Grenzen einer Gravitationstheorie”, Ann. der Physik, vol. 48, pp. 269-282, 1991.

[25] H. Rund, The differential geometry of Finsler spaces, Springer, Berlin, 1959. 



\title{
Chapter 4
}

\section{The Improvement of the Heisenberg Uncertainty Principle}

\author{
L. M. Arévalo Aguilar, C. P. García Quijas and \\ Carlos Robledo-Sanchez
}

Additional information is available at the end of the chapter

http://dx.doi.org/10.5772/54530

\section{Introduction}

One of the fundamental cornerstone of quantum mechanics is the Heisenberg uncertainty principle. This principle is so fundamental to quantum theory that it is believed that if a single phenomenon that could violate it is found then the whole building of quantum mechanics will fall apart. However, since the formulation of the uncertainty principle until today there is not clear and universal agreement in its formulation or interpretation. Even Heisenberg was not clear about the exact meaning of $p_{1}$ and $x_{1}$ in their first formulation of the uncertainty relations [1]:

$$
p_{1} q_{1} \sim h,
$$

nor in the interpretation of the uncertainty principle. According to Heisenberg, in Eq. (1) $q_{1}$ represents "the precision with which the value of $q$ is know ( $q_{1}$ is, say, the mean error of $q$ ), therefore here the wavelength of light. Let $p_{1}$ be the precision with which the value of $p$ is determinable; that is, here, the discontinuous change of $p$ in the Compton effect [1]". He also thought the uncertainty principle in terms of disturbance produced on an observable when it is measured its canonical counterpart.

The relevance of the uncertainty principle to Physics is that it introduced for the first time the indeterminacy in a physical theory, which mean the end of the era of certainty in Physics. That is to say, what uncertainty principle made evident was the peculiar characteristic of quantum theory of not being able to predict with certainty a property of a physical system; in words of Heisenberg: "...canonically conjugate quantities can be determined simultaneously only with a characteristic indeterminacy. This indeterminacy is the real basis for the occurrence of statistical relations in quantum mechanics [1]". 
Since now, you can perceive two different meanings of the Uncertainty Principle in the two quoted paragraphs above. In the first one, the uncertainty comes from a statistical property (according with Heisenberg, the mean error) of quantum theory; in the second meaning the uncertainty is a restriction to simultaneously measure two physical properties.

On the other hand, to elucidate the meaning of the time-energy uncertainty relation [1] $E_{1} t_{1} \sim h$ is quite difficult, for, contrary to the uncertainty relation given in Eq. (1), it is not possible to deduce it from the postulates of quantum mechanics, i. e. there is not an operator for time. In Heisenberg's paper the meaning of $t_{1}$ is the "time during which the atoms are under the influence of the deflecting field" and $E_{1}$ refers to the accuracy in the energy measurement. Heisenberg concludes that "a precise determination of energy can only be obtained at the cost of a corresponding uncertainty in the time [1]".

In this Chapter of the book, we will review the evolution of the Uncertainty Principle since its inception by Heisenberg until their application to measure entanglement. We will review some problems (usually untouched by quantum mechanic's textbooks) that the usual interpretation of the Uncertainty Principle have in terms of standard deviations and its dependence of the wave function. Also, we will review the efforts made to clarify the meaning of the Uncertainty Principle using uncertainty relations.

\section{The relation between the Heisenberg Uncertainty Principle and the Uncertainty Relations}

The uncertainty principle is one of the fundamental issues in which quantum theory differs from the classical theories, then since its formulation has attracted considerable attention, even from areas normally outside the scientific development. This has lead to create misunderstandings about the content of the principle. Thus, it is important to mention that when we say that there is a lower limit on irreducible uncertainty in the result of a measurement, what we mean is that the uncertainty is not due to experimental errors or to inaccuracies in the laboratory. Instead, the restriction attributed to the uncertainty principle is fundamental and inherent to the theory and is based on theoretical considerations in which it is assumed that all observations are ideal and perfectly accurate.

A reading of the original Heisenber's paper shows that he writes (i. e. believes) in some pharagraps that the indeterminacies comes from the observational procedures. For, in his original paper, Heisenberg stated [1] that the concepts of classical mechanics could be used analogously in quantum mechanics to describe a mechanical system, however, the use of such concepts are affected by an indeterminacy originated purely by the observational procedures used to determine simultaneously two canonically conjugate variables. This could be contrasted with the called Statistical Interpretation where it is tough that the wave function represents and ensemble of identical prepared system and, therefore, the indeterminacy comes form an intrinsic indeterminacy of the physical properties.

Usually, the uncertainty principle is stated in terms of uncertainty relations. One of the first way to obtain this indeterminacy relation is due to Robertson [2]. Here, instead, we use the textbooks approach to deduce the uncertainty relations from the quantum postulates $[3,4]$. This approach uses both the Schwarz inequality

$$
\langle\phi \mid \phi\rangle\langle\varphi \mid \varphi\rangle \geq|\langle\phi \mid \varphi\rangle|^{2},
$$


and the following quantum postulates:

- The state of a quantum system is represented by a wave function $\Psi(x, t)(|\Psi\rangle$, in Dirac notation).

- For every observable A there is a self-adjoint operator $\hat{A}$, its expectation value is given by $\langle\hat{A}\rangle=\int \Psi^{*}(x) \hat{A} \Psi(x) d x=\langle\Psi|\hat{A}| \Psi\rangle$.

Now, consider the following operators defined as ${ }^{1}$ :

$$
\begin{aligned}
\Delta \hat{A} & =\hat{A}-\langle\hat{A}\rangle \\
\Delta \hat{B} & =\hat{B}-\langle\hat{B}\rangle .
\end{aligned}
$$

Let them operate on an state $|\Psi\rangle$, given:

$$
\begin{gathered}
\Delta \hat{A}|\Psi\rangle=\left|\psi_{a}\right\rangle \\
\Delta \hat{B}|\Psi\rangle=\left|\psi_{b}\right\rangle .
\end{gathered}
$$

Therefore, using the Schwarz inequality given in the Eq. (2),

$$
\left\langle\psi_{a} \mid \psi_{a}\right\rangle\left\langle\psi_{b} \mid \psi_{b}\right\rangle \geq\left|\left\langle\psi_{a} \mid \psi_{b}\right\rangle\right|^{2}
$$

we arrive to:

$$
\left\langle\Delta \hat{A}^{2}\right\rangle\left\langle\Delta \hat{B}^{2}\right\rangle \geq|\langle\Delta \hat{A} \Delta \hat{B}\rangle|^{2},
$$

where $\left\langle\Delta \hat{A}^{2}\right\rangle=\left\langle\hat{A}^{2}\right\rangle-\langle\hat{A}\rangle^{2}=\delta A^{2}$ is the variance, the same for the operator $\hat{B}$. From the Eq. (6), it is not difficult to show that:

$$
\delta A \delta B \geq \sqrt{|\langle[\hat{A}, \hat{B}]\rangle|^{2}+|\langle\{\hat{A}, \hat{B}\}\rangle|^{2}}
$$

where $\{\hat{A}, \hat{B}\}=\hat{A} \hat{B}+\hat{B} \hat{A}$, and $\delta A$ and $\delta B$ are the standard deviation. It is worth to notice that the association of the standard deviation whit the uncertainty relations was not proposed by Heisenberg, it was Kennard and Robertson [2] who made this association. Although Heisenberg endorse it later. As it was stated above, Heisenberg associates $p_{1}$ and $q_{1}$ with the mean error, also in the same paper he associates these quantities with the widths of Gaussian functions representing the quantum states of the system.

Some problems arises with the textbooks uncertainty relations: $i$ ) They are given in terms of the standard deviation, ii) They depend on the state of the system. Additionally, iii) They

\footnotetext{
${ }^{1}$ There are others forms to obtain the uncertainty relations, this begin by defining an operator as $\hat{D}=\Delta \hat{A}+\lambda \Delta \hat{B}$ and, then, requiring that $\left\langle\hat{D}^{\dagger} \hat{D}\right\rangle \geq 0$.
} 
does not represent the meaning of the impossibilities of simultaneous measurement of two observables, iv) They does not quantify the role of the disturbance in the state after the measurement process. Finally, v) They does not address the concept of complementarity. There have been proposed some criteria to solve this problems, we are going to review this proposals in the next sections.

\section{Reformulations to the uncertainty principle}

In this section we will review some proposed solutions to the problems stated in the last paragraph of the previous section.

\subsection{The dependence on the standard deviation}

The principal criticism to the dependence of the uncertainty relation on the standard deviation comes from J. Hilgevoord and J. M. B. Uffink [5, 6]. Their argument is based on two reason, first, they argue that the standard deviation is an appropriate measure of the error of a measurement because errors usually follow a Gaussian distribution, and the standard deviation is an appropriate measurement of the spread of a Gaussian; however, this is not true for a general distribution. Secondly, they gave as a principal counter argument the fact that even for simple phenomenon as the single slit the standard deviation of momentum diverges. Their approach is inside the thinking that the uncertainty relations are the measure of the spread of the probability distribution, i. e. it is believed that $\Delta x$ and $\Delta p$ represents the probability distribution of the possibles properties of the system. In short, it represents the spread of values (of $\hat{x}$ or $\hat{p}$ ) that are intrinsic in the physical system that are available to appear after a measurement.

The principal counter argument with regard to the standard deviations comes from the single-slit experiment. In this case, it is supposed that the state of an income beam of particles is represented by plane waves. This plane wave represents a particle of precise momentum $p_{0}$. Then, the plane wave arrives at the single-slit and is diffracted by it. Therefore, the wave function at the screen, according to Hilgevoord and Uffink, is:

$$
\psi(x)= \begin{cases}(2 a)^{-1 / 2}, & \text { if }|x| \leq a \\ 0, & \text { if }|x|<a\end{cases}
$$

and

$$
\phi(p)=(a / \pi)^{1 / 2} \frac{\sin a p}{a p} .
$$

Now, the problem with the standard deviation, as defined in quantum mechanics, in this case is that it diverges:

$$
\Delta p=\left\langle\hat{p}^{2}\right\rangle-\langle\hat{p}\rangle^{2} \rightarrow \infty
$$

Therefore, these authors defined, instead of the standard deviation, the overall width $\left(W_{\psi}\right)$ and the mean peak width of $\psi$ as the smaller $W$ and $w$ that satisfies the following 
equations [7]:

$$
\begin{array}{r}
\int_{x_{0}-W / 2}^{x_{0}+W / 2}|\psi(x)|^{2} d x=N \\
\left|\int \psi^{*}\left(x^{\prime}\right) \psi\left(x^{\prime}-w\right) d x^{\prime}\right|^{2}=M^{2}
\end{array}
$$

These quantities, i. e. $W$ and $w$, provides a better characterization of the spread of the possible values of $\hat{x}$ and $\hat{p}$, in particular there is not any divergence in these numbers. Based in these definitions Hilgevoord and Uffink give the following uncertainty relations, that they propose as a substitute to the uncertainty relation given by Kennard $(\Delta x \Delta p \geq 1 / 2)$, [7]:

$$
\begin{aligned}
& w_{\phi} W_{\psi} \geq \arccos \left(\frac{M+1-N}{N}\right) \\
& w_{\psi} W_{\phi} \geq \arccos \left(\frac{M+1-N}{N}\right)
\end{aligned}
$$

these uncertainty relations works well for the single-slit and double-slit experiments.

\subsection{Entropic Uncertainty Relations}

In the quantum literature, there are many defined Entropic Uncertainty Relations. Mostly, they are based in terms of Shannon entropy $[8,9]$, although in last ten years there has been extension to other forms of entropy, like Renyi entropy [10]. In reference [11] there is a recent review of this research area.

One of the important result in this area was the one found by Deutsch [8]. What Deutsch pursuit was a quantitative expression of the Heisenberg uncertainty principle, he notice that the customary generalization has the drawback that the lower limit depends on the quantum state, that is:

$$
\Delta A \Delta B \geq \frac{1}{4}|\langle[\hat{A}, \hat{B}]\rangle|^{2} .
$$

Deutsch stress that the right hand side of the Equ. (12) does not has a lower bound but is a function of the state $|\psi\rangle$, even it vanishes for some choices of $|\psi\rangle$. So, in search of a quantity that could represent the uncertainty principle Deutsch propose some elementary properties, like for example that the lower limit must vanishes if the observables have an eigenstate in common. Based in this considerations he proposed the following entropic uncertainty relation:

$$
S_{\hat{A}}+S_{\hat{B}} \geq 2 \operatorname{Ln}\left(\frac{2}{1+\sup \{|\langle a \mid b\rangle|\}}\right),
$$

where $S_{\hat{A}}=-\sum_{a}|\langle a \mid \psi\rangle|^{2} \operatorname{Ln}|\langle a \mid \psi\rangle|^{2}$ and $S_{\hat{B}}=-\sum_{b}|\langle b \mid \psi\rangle|^{2} \operatorname{Ln}|\langle b \mid \psi\rangle|^{2}$ are the Shanon entropy, and $|a\rangle$ and $|b\rangle$ are, respectively, the eigenstates of $\hat{A}$ and $\hat{B}$. 
The next step in this line of research, was quite soon given by Hossein Partovi [12], he points out that the above uncertainty relation does not take into account the measurement process. Then, considering that the measuring device realizes a partitioning of the spectrum of the observable and the assignation of their corresponding probabilities, he proposes the following definition of entropy [12]:

$$
S_{A}=-\sum_{i} p_{i} \ln \left\{p_{i}\right\}
$$

where $p_{i}=\left\langle\psi\left|\hat{\pi}_{i}^{A}\right| \psi\right\rangle /\langle\psi \mid \psi\rangle$ and $\hat{\pi}_{i}^{A}$ is the projection onto the subspaces spanned by the states corresponding to the partition induced by the measuring apparatus [12]. In this case, $p_{i}$ gives the probability of obtaining the outcome of a measurement in a subset of the partition realized by the measuring apparatus. In this approach, the whole spectrum correspond to the observable $\hat{A}$ but its partitioning correspond to the measuring device. Using these considerations Hossein Patrovi proses the following lower bound for the uncertainty relation:

$$
S_{\hat{A}}+S_{\hat{B}} \geq 2 \operatorname{Ln}\left(\frac{2}{1+\sup _{i j}\left\{\left\|\hat{\pi}_{i}^{A}+\hat{\pi}_{j}^{B}\right\|\right\}}\right) .
$$

In the special case where the partition realized by the measuring device includes only one point of the spectrum of $\hat{A}$, i. e. $\hat{\pi}_{i}^{A}=\left|a_{i}\right\rangle\left\langle a_{i}\right|$ and $\hat{B}$, i. e. $\hat{\pi}_{j}^{B}=\left|b_{j}\right\rangle\left\langle b_{j}\right|$, then Equ. (15) reduces to Equ. (13). Finally, it is worth to mention that the Patrovi's formulation requires a formulation of the details of the measuring devices, specifically, the kind of partition that induces (or could be used) in the spectrum of the observable.

There were two additional improvement on the lower bound of the entropic uncertainty relations defined above. The first one was due to Bialynicki-Birula who presented, based in his earlier wok [9], a lower bound for the angle-angular momentum pair [13] $S^{\phi}+S^{L_{z}} \geq$ $-\ln (\Delta \phi / 2 \pi)$ and an improved lower bound for the position-momentum pair $S^{x}+S^{p} \geq$ $1-\ln (2)-\ln (\gamma)$, where $\gamma=\Delta x \Delta p / h$. The second one was proposed by Maasen and Uffink [14] who demonstrated, based on a previous work of Kraus [15], that

$$
S^{A}+S^{B} \geq-2 \ln (c)
$$

where $c=\max _{j k}\left|\left\langle a_{j} \mid b_{k}\right\rangle\right|$.

\subsection{Simultaneous measurement}

Whereas in the previous two subsection we treated the face of the Uncertainty Principle that is related with the probability distribution of observables of a given wave function, in this subsection we talk a bout a second version of The Uncertainty Principle. This version is related with the fact that it is not possible to determine simultaneously, with precision, two canonically conjugate observable and usually called joint measurement. This is stated, generally, as: "It is impossible to measure simultaneously two observables like, for example, position 
and momentum." So, this sub-research area is concerned with the simultaneous measurement of two observables.

One of the first work in this approach was that of Arthurs and Kelly [16], they analyze this problems as follows: First, they realize that as the problem is the measurement of two observables, then it is required two devices to perform the measurement. That is, the system is coupled to two devises. Then, they consider that as the two meter position commutes then it is possible to perform two simultaneous measurements of them. Therefore, the simultaneous measurement of the two meters constitutes a simultaneous measurement of two non-commuting observables of the system. As the two meters interacts with the quantum system, they consider the following Hamiltonian:

$$
\hat{H}_{\text {int }}=K\left(\hat{q} \hat{P}_{x}+\hat{p} \hat{P}_{y}\right)
$$

where $\hat{q}$ and $\hat{p}$ correspond to the position and momentum of the quantum system, respectively, and $\hat{P}_{x}$ and $\hat{P}_{y}$ are the momentum of the two independent meters. Using two Gaussian function as the initial wave function of the meters they arrive at the following uncertainty relation for the simultaneous measurement of two observables:

$$
\sigma_{x} \sigma_{p} \geq 1
$$

Therefore, the uncertainty relation of the simultaneous measurement of $\hat{q}$ and $\hat{p}$ is greater (by a factor of two) than the uncertainty relations based on the probability distribution of the two observables, the topic of the previous two sub-sections.

The next step in this approach was given by Arthurs and Goodman [17]. In this case, the approach is as follow: To perform a measurement, the system observables, $\hat{C}=\hat{C}_{1} \otimes \hat{I}_{2}$ and $\hat{D}=\hat{D}_{1} \otimes \hat{I}_{2}$, must be coupled to a measuring apparatus which is represented by the operators $\hat{R}=\hat{I}_{1} \otimes \hat{R}_{2}$ and $\hat{C}=\hat{C}_{1} \otimes \hat{I}_{2}$. Then, if we consider that there is access only to the meter operators then there must exist an uncertainty relations for these operators that puts a limit to the available information. Based in this consideration, they prove what they call a generalized uncertainty relation. To prove it they defined a noise operator by

$$
\begin{array}{r}
\hat{N}_{R}=\hat{R}-G_{R} \hat{C}(0), \\
\hat{N}_{S}=\hat{S}-G_{S} \hat{D}(0)
\end{array}
$$

where $\hat{C}(0)$ and $\hat{D}(0)$ are the system observables and $\hat{R}$ and $\hat{S}$ are the tracking apparatus observables, the latter obey the commutation rule $[\hat{R}, \hat{S}]=0$. Also, it is required that the correlation between the system observables and the meter has, on average, a perfect match, that is:

$$
\operatorname{Tr}\left(\hat{\rho} \hat{N}_{R, S}\right)=\langle\hat{R}\rangle-G_{R}\langle\hat{C}(0)\rangle=0 .
$$

Using the previous condition, i. e. Equ (20), it is possible to show that the noise operator is uncorrelated with all system operators like $\hat{C}$ and $\hat{D}$. Using all the previous properties of 
the system, meter and noise operators they arrive to the following generalized Heisenberg uncertainty relation:

$$
\sigma_{\xi} \sigma_{\eta} \geq|\operatorname{Tr}(\hat{\rho}[\hat{C}, \hat{D}])|,
$$

where $\hat{\rho}$ is the state of the system, $\sigma_{\xi}$ and $\sigma_{\eta}$ are, respectively the standard deviation of the normalized operators $\xi=\hat{R} / G_{R}$ and $\eta=\hat{R} / G_{R}$. This uncertainty relation is four times the corresponding uncertainty relation for $\hat{C}$ and $\hat{D}$. Notice that in the left hand side of the Eq. (21) there is information of the meter operator whereas in the right hand side there is information of the system operators and that we have access only to the meter system. In reference [18] there was published an experimental verification of this uncertainty relation.

\subsection{Disturbance due to measurement}

The disturbance produced on an observable due to the measurement of another observable is, perhaps, the face of the uncertainty principled most talked about but the least studied. This comes from the fact that in quantum mechanics any measurement introduces an unforeseeable disturbance in the measured quantum system. It was only recently that there have been some research and understanding of this effect.

Originally, the idea that the measuring process disturb observables comes from Heisenberg's analysis of the observation of an electron by means of a microscope. This kind of uncertainty principle is written down, to use recent terminology, as [19]:

$$
\epsilon(x) \eta(p) \geq \frac{1}{2}|\langle\psi|[\hat{x}, \hat{p}]| \psi\rangle|
$$

where $\epsilon(x)$ is the noise in the measurement in position and $\eta(p)$ is the disturbance caused by the apparatus [19]. Using a general description of measurement Ozawa demonstrated that the uncertainty relation for disturbance and noise given by the Eq. (22) does not accurately represent the disturbance process. He has show that this kind of uncertainty relation includes additional terms not present in Eq. (22). In the measurement process, the quantum system interacts with a measuring device. He considers that this devices measures observable $A$ precisely if its experimental probabilty distribution coincides with the theoretical probability distribution of the observable. In the measurement process, when the interaction have been turned off, the device is subject to a measurement of an observable $M$. Then, $\hat{A}^{\text {in }}=\hat{A} \otimes \hat{I}$ is the input observable, $\hat{A}^{\text {out }}=\hat{U}^{\dagger}(\hat{A} \otimes \hat{I}) \hat{U}$ is the observable after the mesaurement, $\hat{M}^{i n}=$ $\hat{I} \otimes \hat{M}$ is the device observable when the interaction begin, $\hat{M}^{\text {out }}=\hat{U}^{\dagger}(\hat{I} \otimes \hat{M}) \hat{U}$ and $\hat{U}$ is the unitary time evolution operator

To show that the original uncertainty relation need additional terms, he introduces the following noise $N(\hat{A})$ and disturbance $D(\hat{B})$ operators:

$$
\begin{array}{r}
N(\hat{A})=\hat{M}^{\text {out }}-\hat{A}^{\text {in }}, \\
D(\hat{B})=\hat{B}^{\text {out }}-\hat{B}^{\text {in }} .
\end{array}
$$


Using this operators, and considering that $\left[\hat{M}^{\text {out }}, \hat{B}^{\text {out }}\right]=0$, Ozawa was able to show the following uncertainty relation [19]:

$$
\epsilon(A) \eta(B)+\frac{1}{2}\left|\left\langle\left[N(\hat{A}), \hat{B}^{i n}\right]\right\rangle+\left\langle\left[\hat{A}^{\text {in }}, D(\hat{B})\right]\right\rangle\right| \geq \frac{1}{2}|\langle\psi|[\hat{A}, \hat{B}]| \psi\rangle|
$$

where the noise $\epsilon(A)$ was defined by Ozawa as the root-mean-square deviation of the experimental variable $\hat{M}^{\text {out }}$ from the theoretical variable $\hat{A}^{\text {in }}$ :

$$
\epsilon(A)=\left\langle\left(\hat{M}^{\text {out }}-\hat{A}^{\text {in }}\right)^{1 / 2}\right\rangle
$$

and the disturbance $\eta(B)$ on observable $\hat{B}$ is the change in the observable caused by the measurement process:

$$
\eta(B)=\left\langle\left(\hat{B}^{\text {out }}-\hat{B}^{\text {in }}\right)^{1 / 2}\right\rangle
$$

This uncertainty relation has been recently experimentally tested, see reference [20]

\section{Entanglement determination using entropic uncertainty relations}

Nowadays entanglement is considered as an useful resource to make non-clasical task. As a resource it is convenient to have adequate measures to quantify how much entanglement are in a given entangled state. However, until recently the most known proposed measures have the unwanted fact of being difficult to apply in experimental settings. Therefore, it was necessary to find out new ways of entanglement determination that enable that the amount of entanglement in a quantum state could be experimentally tested.

Recently there has been much research to proposed new entanglement determination based, mostly, in uncertainty relations. In this case, the entropic uncertainty relations helps to realize this task. Recently, Berta et. al. [21] have proposed a new uncertainty relation (based on that proposed in references $[8,14])$ to test the entanglement:

$$
S(R \mid B)+S(S \mid B) \log 2 c+S(A \mid B)
$$

to propose this equation Berta et. al. consider that the system, with observables $S$ and $R$, is entangled with a memori, with observable B, so in equation $S(R, B)$ is the von Neumann entropy and gives the uncertainty about the measurement of $R$ given information stored in a quantum memory, B. The term $\mathrm{S}(\mathrm{A} \mid \mathrm{B})$ quantifies the amount of entanglement between the particle and the memory. This relation was experimentally tested in reference [22]. 


\section{Conclusions}

In this chapter we review some of the most important improvements of the Heisenberg uncertainty relation. Although there are advances in their understanding and formulation, it remains yet as an open research area, specially in the quantification of entanglement.

\section{Acknowledgements}

We thanks Consejo Nacional de Ciencia y Tecnologia (CONACYT). L. M. Arévalo Aguilar acknowledge the support from Vicerrectoria de Investigación y Posgrado VIEP-BUAP under grand ARAL-2012-I. P. C. Garcia Quijas acknowledges CONACYT for a posdoctoral scholarship at the Universidad Autonoma de Guadalajara.

\section{Author details}

L. M. Arévalo Aguilar ${ }^{1, \star}$,

C. P. García Quijas ${ }^{2}$ and Carlos Robledo-Sanchez ${ }^{3}$

* Address all correspondence to: olareva@yahoo.com.mx

1 Facultad de Ciencias Físico Matemáticas, Benemérita Universidad Autónoma de Puebla, Puebla, México

2 Departamento de Fśica, Universidad de Guadalajara, Guadalajara, Jalisco, México

3 Facultad de Ciencias Fisico Matemáticas, Benemérita Universidad Autónoma de Puebla, Puebla, México

\section{References}

[1] W. Heisenberg, Zeitschrift fur Physik 43, 172 (1927). Translated in the book, Quantum Measurement, Weeler and Zurek editors.

[2] H. P. Robertson, Phys. Rev. 46, 794 (1934).

[3] D. Griffiths, Introduction to Quantum Mechanics, Addison-Wesley; 2nd edition (April 10, 2004).

[4] N. Zettili, Quantum Mechanics: Concepts and Applications, Wiley; 2 edition (March 4, 2009).

[5] J. Hilgevoord and J. M. M. Uffink, Eur. J. Phys. 6, 165 (1985).

[6] J. Hilgevoord and J. M. M. Uffink, The mathematical expression of the uncertainty principle, in the book Microphysical Reality and Quantum Formalism, 91-114, A. van der Merve et. al. Eds.

[7] J. M. M. Uffink and J. Hilgevoord, Found. Phys. 15, 925 (1985).

[8] D. Deutsch, Phys. Rev. Lett. 50, 631 (1983).

[9] I. Bialynicki-Birula and J. Mycielski, Commun. math. Phys. 44, 129 (1975). 
[10] I. Bialynicki-Birula, Phys. Rev. A 74, 052102 (2006).

[11] S. Wehner, New Jour. of Phys. 12, 025009 (2010).

[12] M. Hossein Partovi, Phys. Rev. Lett. 50, 1883 (1983).

[13] I. Bialynicki-Birula, Phys. Lett. A 103, 253 (1984).

[14] H. Maasen and J. B. Uffink, Phys. Rev. Lett. 60, 1103 (1988).

[15] Krauss, Phys. Rev. D 35, 3070 (1987).

[16] E. Arthurs and J. L. Kelly JR., Bell. Syst. Tech. J. 44, 725 (1965).

[17] E. Arthurs, M. S. Goodman, Phys. Rev. Lett. 60, 2447 (1988).

[18] A. Trifonov, G. Björk and J. Sỏderholm, Phys. Rev. Lett. 86, 4423 (2001).

[19] M. Ozawa, Phys. Rev. A67, 042105 (2003).

[20] J. Erhart, S. Sponar, G. Sulyok, G. Badurek, M. Ozawa and Y. Hasegawa, Nature Physics 8, 185Đ189 (2012).

[21] M. Berta, M. Christandl, R. Colbeck, J. M. Renes and R. Renner, Phys. Nature Physics 6, 659 (2010).

[22] Chuan-Feng Li, Jin-Shi Xu, Xiao-Ye Xu, Ke Li \& Guang-Can Guo, Nature Physics 7, 752 (2011). 



\section{Section 2}

\section{The Schrödinger Equation}



Chapter 5

\title{
Schrödinger Equation as a Hamiltonian System, Essential Nonlinearity, Dynamical Scalar Product and some Ideas of Decoherence
}

\author{
Jan J. Sławianowski and Vasyl Kovalchuk \\ Additional information is available at the end of the chapter
}

http://dx.doi.org/10.5772/53479

\section{Introduction}

Our main idea is to suggest some new model of nonlinearity in quantum mechanics. The nonlinearity we discuss is non-perturbative and geometrically motivated, in any case it is not an auxiliary correction to the linear background. It has a group-theoretic motivation based on the assumption of the "large" symmetry group. In a sense, it develops further our ideas suggested earlier in [1-4].

It is well known that quantum mechanics is still plagued by some paradoxes concerning decoherence, measurement process and the reduction of the state vector. In spite of certain optimistic opinions, the problem is still unsolved, although many interesting ideas have been formulated, like that about subsystems of a large (infinite) quantum system or stochastic quantum Markov processes with the spontaneous reduction of state vectors. There is still an opinion that the main problem is the linearity of the Schrödinger equation, which seems to be drastically incompatible with the mentioned problems $[5,6]$. But at the same time, that linearity works beautifully when describing the unobserved unitary quantum evolution, finding the energy levels and in all statistical predictions. It seems that either we are faced here with some completely new type of science, roughly speaking, based on some kind of solipsism with the irreducible role of human being in phenomena, or perhaps we deal with a very sophisticated and delicate nonlinearity which becomes active and remarkable just in the process of interaction between quantum systems and "large" classical objects.

The main idea is to analyse the Schrödinger equation and corresponding relativistic linear wave equations as usual self-adjoint equations of mathematical physics, thus ones derivable from variational principles. It is easy to construct their Lagrangians. Some problems appear when trying to formulate Hamiltonian formalism, because Lagrangians for the Schrödinger or Dirac equations are highly degenerate and the corresponding Legendre transformation is uninvertible and leads to constraints in the phase space. Nevertheless, using the Dirac formalism for such Lagrangians, one can find the corresponding Hamiltonian 
formalism. Incidentally, it turns out that introducing the second-order time derivatives to dynamical equations, even as small corrections, one can obtain the regular Legendre transformation. In non-relativistic quantum mechanics there are certain hints suggesting just such a modification in the nano-scale physics $[1,7,8]$. One can also show that in $\mathrm{SU}(2,2)$-invariant gauge models, i.e., roughly-speaking, in conformal theory, it is more natural to begin with the four-component Klein-Gordon amplitude and then to derive the Dirac behavior as an unexpected aspect of the Klein-Gordon theory [4, 9]. This leads us to certain interesting statements concerning the pairing of fundamental quarks and leptons in electroweak interactions.

We begin just like in [1] from the first- and second-order (in time) Schrödinger equations for a finite-level system, i.e., for the finite "configuration space". We construct the "direct nonlinearity" as a non-quadratic term in Lagrangian, but further on we concentrate on our main idea. It consists in that we follow the conceptual transition from the special to general relativity. Namely, just like in the passing to the theory of general relativity, the metric tensor loses its status of the absolute geometric object and becomes included into degrees of freedom (gravitational field), so in our treatment the Hilbert-space scalar product becomes a dynamical quantity which satisfies together with the state vector the system of differential equations. The main idea is that there is no fixed scalar product metric and the dynamical term of Lagrangian, describing the self-interaction of the metric, is invariant under the total group $\mathrm{GL}(n, \mathbb{C})$. But this invariance is possible only for models non-quadratic in the metric, just like in certain problems of the dynamics of "affinely-rigid" body [2,3]. There is a natural metric of this kind and it introduces to the theory a very strong nonlinearity which induces also the effective nonlinearity of the wave equation, even if there is no "direct nonlinearity" in it. The structure of Lagrangian and equations of motion is very beautiful, as usual in high-symmetry problems. Nevertheless, the very strong nonlinearity prevents us to find a rigorous solution. Nevertheless, there are some partial results, namely, if we fix the behaviour of wave function to some simple form and provide an academic discussion of the resulting behaviour of the scalar product, then it turns out that there are rigorous exponential solutions, including ones infinitely growing and ones exponentially decaying in future. This makes some hope for describing, e.g., some decay/reduction phenomena. Obviously, the full answer will be possible only when we will be able to find a rigorous solution for the total system. We are going to repeat the same discussion for the more realistic infinite-level system, when the wave function is defined somehow on the total configuration space like, e.g., the arithmetic space. As usual when passing from the finite to infinite dimensions, some essentially new features appear then, nevertheless, one can hope that the finite-dimensional results may be to some extent applicable. This will be done both in the usual non-relativistic Schrödinger wave equation and for the relativistic Klein-Gordon and Dirac equations. In any of those cases we are dealing with two kinds of degrees of freedom, i.e., dynamical variables: wave function and scalar product. They are mutually interacting.

All said above concerns the self-adjoint model of the Schrödinger equation, derivable from variational principle. However, one can also ask what would result if we admitted "dissipative" models, where the Schrödinger equation does possess some "friction-like" term. As yet we have not a ready answer, nevertheless, the question is well formulated and we will try to check what might appear in a consequence of such a generalization. Maybe some quantum model of dissipation, i.e., of the open system, but at a moment we are unable to answer the question. 


\section{Nonlinear Schrödinger equation as a self-adjoint equation of mathematical physics}

It is known, although not often noticed and declared, that the Schrödinger equation and other equations of quantum mechanics, including relativistic ones, are self-adjoint, i.e., derivable from variational principles. Therefore, they may be expressed in Hamiltonian terms, i.e., quantum mechanics becomes a kind of analytical mechanics, usually with an infinite number of degrees of freedom (excepting finite-level systems). So, as far as one deals with the unobserved quantum system, its evolution may be described within the classical mathematical framework of Hamiltonian mechanics and canonical transformations. Of course, this breaks down when quantum springs, jumps, occur, i.e., when one is faced with phenomena like macroscopic observation, measurements and decoherence. This happens when a small/quantum system interacts with a large/classical object showing some characteristic instability. There were various ways of explaining those catastrophic phenomena and their statistical rules. It is very interesting that those rules are based on the Hilbert space geometry, but in addition some unpredictable, statistical phenomena appear. There were many attempts of explanation, based either on the extension to larger, infinite systems or on the idea of spontaneous stochastic reduction. But one of the permanent motives is the hypothesis of nonlinearity, especially one which is "silent" in the evolution of the unobserved quantum system, but becomes essential in the process of interaction with the large and unstable classical system. This is also our line in this paper. Mathematical methods of nonlinear analytical mechanics just seem to suggest some attempts of solution.

To explain the main ideas we start from the simple finite-level system, i.e., one with a finite-dimensional unitary space of states. Let us denote this complex linear space by $W$ and put $\operatorname{dim}_{\mathbb{C}} W=n$. The dual, antidual and complex-conjugate spaces will be denoted respectively by $W^{*}, \bar{W}^{*}=\bar{W}^{*}$, and $\bar{W}$. As usual, $W^{*}$ is the space of $\mathbb{C}$-linear functions on $W$. Having the same finite dimension, the spaces $W, W^{*}$ are isomorphic, however in a non-canonical way until we introduce some unitary structure to $W$. But some comments are necessary concerning the complex conjugate spaces $\bar{W}^{*}=\bar{W}^{*}, \bar{W}$. It must be stressed that in general nothing like the complex structure is defined in $W$. It is a structure-less space and the half-linear (semi-linear) bar-operations are defined pointwisely. Therefore, for any $f \in W^{*}$ and for any $u \in W$ the corresponding $\bar{f} \in \bar{W}^{*}, \bar{u} \in \bar{W}$ are given by

$$
(\bar{f})(w)=\overline{f(w)}, \quad \bar{u}(g)=\overline{u(g)},
$$

where $w, g$ are arbitrary elements of $W$ and $W^{*}$. Therefore, under the bar-operation $\bar{W}$ is canonically anti-isomorphic with $W$ and $\bar{W}^{*}$ with $W^{*}$. Nevertheless, the bar-operation acts between different linear spaces and this is often essential. The spaces $W$ and $\bar{W}$, and similarly $W^{*}$ and $\bar{W}^{*}$ may be mutually identified only in important, nevertheless mathematically exceptional, situations when by the very definition $W$ is a linear subspace of the space of $\mathbb{C}$-valued functions on a given "configuration space" $Q$. Then we simply define pointwisely

$$
\bar{\psi}(q):=\overline{\psi(q)}
$$

and so $W$ becomes identical with $\bar{W}$. In general this is impossible. Let us mention of course that for the $n$-level system, $Q$ is an $n$-element set. 
Let us quote a few analytical formulas. We choose a pair of mutually dual bases $\left(e_{1}, \ldots, e_{n}\right)$, $\left(e^{1}, \ldots, e^{n}\right)$ in $W, W^{*}$ and induced pair of dual bases $\left(\bar{e}_{\overline{1}}, \ldots, \bar{e}_{\overline{1 n}}\right),\left(\bar{e}^{\overline{1}}, \ldots, \bar{e}^{\overline{1 n}}\right)$ in the complex-conjugate spaces $\bar{W}$ and $\bar{W}^{*} \simeq \bar{W}^{*}$. Then the complex conjugates of vectors

$$
u=u^{a} e_{a} \in W, \quad f=f_{a} e^{a} \in W^{*}
$$

are analytically expressed as

$$
\bar{u}=\bar{u}^{\bar{a}} \bar{e}_{\bar{a}}, \quad \bar{f}=\bar{f}_{\bar{a}} \bar{e}^{\bar{a}},
$$

where, obviously, $\bar{u}^{\bar{a}}, \bar{f}_{\bar{a}}$ are the usual complex conjugates of numbers $u^{a}, f_{a}$.

In quantum mechanics one uses often sesquilinear forms, usually Hermitian ones. Usually our sesquilinear forms are antilinear (half-linear) in the first argument and linear in the second, therefore,

$$
F(a u+b w, v)=\bar{a} F(u, v)+\bar{b} F(w, v)
$$

i.e., analytically

$$
F(u, w)=F_{\bar{a} b} \bar{u}^{\bar{a}} w^{b} .
$$

So, they are elements of $\bar{W}^{*} \otimes W^{*}$. For Hermitian forms we have

$$
F(u, w)=\overline{F(w, u)}, \quad F_{\bar{a} b}=\bar{F}_{b \bar{a}} .
$$

If $F$ is non-degenerate,

$$
\operatorname{det}\left[F_{\bar{a} b}\right] \neq 0,
$$

then the inverse form $F^{-1} \in W \otimes \bar{W}$ does exist with coefficients $F^{a \bar{b}}$ such that

$$
F^{a \bar{c}} F_{\bar{c} b}=\delta_{b}^{a}, \quad F_{\bar{a} c} F^{c \bar{b}}=\delta_{\bar{a}}{ }^{\bar{b}}
$$

For any quantum system there are two Hermitian forms: a) the scalar product $\Gamma \in \bar{W}^{*} \otimes W^{*}$ and $b$ ) the Hamiltonian form ${ }_{\Gamma} H$ obtained by the $\Gamma$-lowering of the first index of the Hamilton operator $H \in L(W) \simeq W \otimes W^{*}$. The Hamilton operator $H$ is $\Gamma$-Hermitian, i.e.,

$$
\Gamma(H \psi, \varphi)=\Gamma(\psi, H \varphi)
$$

Analytically the sesquilinear form ${ }_{\Gamma} H$,

$$
{ }_{\Gamma} H_{\bar{a} b}=\Gamma_{\bar{a} c} H_{b}^{c}
$$

is simply Hermitian without any relationship to $\Gamma$, and from the Langrangian point of view it is more fundamental than $H$ itself. The finite-level Schrödinger equation 


$$
i \hbar \frac{d \psi^{a}}{d t}=H^{a}{ }_{b} \psi^{b}
$$

is derivable from the Lagrangian

$$
L(1)=\frac{i \hbar}{2} \Gamma_{\bar{a} b}\left(\bar{\psi}^{\bar{a}} \dot{\psi}^{b}-\dot{\bar{\psi}}^{\bar{a}} \psi^{b}\right)-{ }_{\Gamma} H_{\bar{a} b} \bar{\psi}^{\bar{a}} \psi^{b} .
$$

Having in view some kind of "generality" it may be convenient to admit some general constant coefficients $\alpha, \gamma$ :

$$
L(1)=i \alpha \Gamma_{\bar{a} b}\left(\bar{\psi}^{\bar{a}} \dot{\psi}^{b}-\dot{\bar{\psi}}^{\bar{a}} \psi^{b}\right)-\gamma_{\Gamma} H_{\bar{a} b} \bar{\psi}^{\bar{a}} \psi^{b}
$$

It is seen that unlike in the Schrödinger equation, from the variational point of view ${ }_{\Gamma} H$ is more fundamental. It should be denoted rather as $\chi_{\bar{a} b}$, and $H^{a}{ }_{b}$ with the convention of the $\Gamma$-raised first index of $\chi$, as

$$
H^{a}{ }_{b}=\left({ }^{\Gamma} \chi\right)^{a}{ }_{b}=\Gamma^{a \bar{c}} \chi_{\bar{c} b} .
$$

One does not do so because of the prevailing role of Schrödinger equation over its variational interpretation. The Hermitian structure of $\Gamma$ and $\Gamma_{\Gamma} H$ imply that $L(1)$ is real. The descriptor (1) refers to the first-order polynomial dependence of $L(1)$ on the time derivatives of $\psi$. Obviously, the corresponding Legendre transformation leads to phase-space constraints and to the Dirac procedure in canonical formalism. It is interesting to admit some regularization by allowing $L$ to contain the terms quadratic in generalized velocities, just in the spirit of analytical mechanics. The corresponding Lagrangian will have the following form:

$$
L(1,2)=i \alpha \Gamma_{\bar{a} b}\left(\bar{\psi}^{\bar{a}} \dot{\psi}^{b}-\dot{\bar{\psi}} \overline{\bar{a}} \psi^{b}\right)+\beta \Gamma_{\bar{a} b} \dot{\bar{\psi}}^{\bar{a}} \dot{\psi}^{b}-\gamma_{\Gamma} H_{\bar{a} b} \bar{\psi}^{\bar{a}} \psi^{b}
$$

To be more precise, in the term quadratic in velocities one can admit some more general Hermitian form, not necessarily the one proportional to $\Gamma_{\bar{a} b}$. However, we do not do things like those in this paper. Let us stress that $\alpha, \beta, \gamma$ are real constants.

One circumstance must be stressed: we use as "independent" components $\psi^{a}, \bar{\psi}^{\bar{a}}$. The procedure is not new. The same is done in variational principles of field theory [10]. Lagrangians are real, based on Hermitian forms, therefore in variational procedure it is sufficient to subject, e.g., only $\bar{\psi}^{\bar{a}}$ to the modification $\bar{\psi}^{\bar{a}} \mapsto \bar{\psi}^{\bar{a}}+\delta \bar{\psi}^{\bar{a}}$. Then, e.g., for the action functional

one obtains

$$
I(1,2)=\int L(1,2) d t
$$

$$
\frac{\delta I(1,2)}{\delta \bar{\psi}^{\bar{a}}(t)}=2 i \alpha \Gamma_{\bar{a} b} \frac{d \psi^{b}}{d t}-\beta \Gamma_{\bar{a} b} \frac{d^{2} \psi^{b}}{d t^{2}}-\gamma_{\Gamma} H_{\bar{a} b} \psi^{b}
$$


and the resulting Schrödinger equation:

$$
2 i \alpha \frac{d \psi^{a}}{d t}-\beta \frac{d^{2} \psi^{a}}{d t^{2}}=\gamma H_{b}^{a} \psi^{b}
$$

And this is all, because the variation with respect to $\psi^{a}$ leads to the complex-conjugate equation. This is a convenient and commonly used procedure.

The language of analytical mechanics, in this case finite-dimensional one, opens some possibility of introducing nonlinearity to quantum-mechanical equations. The simplest way is to believe in Schrödinger equation but reinterpreting it in terms of Hamiltonian mechanics, to introduce some naturally looking nonlinear perturbations to it. The simplest way is to introduce to $L$ some non-quadratic potential term $\mathcal{V}(\psi, \bar{\psi})$ and the corresponding action term to $I$ :

$$
I(\mathcal{V})=\int \mathcal{V} d t
$$

For example, the simplest possibility is to use the term like

$$
\mathcal{V}(\psi, \bar{\psi})=f\left(\Gamma_{\bar{a} b} \bar{\psi}^{\bar{a}} \psi^{b}\right)
$$

with some model function $f: \mathbb{R} \rightarrow \mathbb{R}$. In various physical applications one uses often the quartic term:

$$
f(y)=\frac{\varkappa}{2}(y-b)^{2} .
$$

When using the $\mathcal{V}$-term, one obtains after the variational procedure the following nonlinear Schrödinger equation:

$$
2 i \alpha \frac{d \psi^{a}}{d t}-\beta \frac{d^{2} \psi^{a}}{d t^{2}}=\gamma H^{a}{ }_{b} \psi^{b}+f^{\prime} \psi^{a},
$$

where $f^{\prime}$ denotes the usual first-order derivative of $f$. This is the simplest model containing the superposition of first- and second-order time derivatives of $\psi$. It is very simple because of being a finite-level system and because of the direct introduction of nonlinearity as a perturbation of the primarily linear model. Nevertheless, it demonstrates some interesting features of nonlinearity and of the mixing of derivatives order.

The problem of the order of derivatives is strongly related to the structure of Hamiltonian mechanics of our systems. It occurs also in corresponding problems of field theory. Let us mention some elementary facts. As usual, it is convenient to use the doubled number of degrees of freedom $\psi^{a}, \bar{\psi}^{\bar{a}}$ and the corresponding canonical momenta $\pi_{a}, \bar{\pi}_{\bar{a}}$. The symplectic form is given by

$$
\omega=d \pi_{a} \wedge d \psi^{a}+d \bar{\pi}_{\bar{a}} \wedge d \bar{\psi}^{\bar{a}}
$$


and the resulting Poisson bracket is expressed as follows:

$$
\{F, G\}=\frac{\partial F}{\partial \psi^{a}} \frac{\partial G}{\partial \pi_{a}}+\frac{\partial F}{\partial \bar{\psi}^{\bar{a}}} \frac{\partial G}{\partial \bar{\pi}_{\bar{a}}}-\frac{\partial F}{\partial \pi_{a}} \frac{\partial G}{\partial \psi^{a}}-\frac{\partial F}{\partial \bar{\pi}_{\bar{a}}} \frac{\partial G}{\partial \bar{\psi}^{\bar{a}}} .
$$

The Hamiltonian vector field is given by

$$
X_{F}=\frac{\partial F}{\partial \pi_{a}} \frac{\partial}{\partial \psi^{a}}+\frac{\partial F}{\partial \bar{\pi}_{\bar{a}}} \frac{\partial}{\partial \bar{\psi}^{\bar{a}}}-\frac{\partial F}{\partial \psi^{a}} \frac{\partial}{\partial \pi_{a}}-\frac{\partial F}{\partial \bar{\psi}^{\bar{a}}} \frac{\partial}{\partial \bar{\pi}_{\bar{a}}} .
$$

It must be stressed that all dynamical quantities in this formalism are considered as independent on their complex conjugates:

$$
\left\langle d \psi^{a}, \frac{\partial}{\partial \psi^{b}}\right\rangle=\delta^{a}{ }_{b}, \quad\left\langle d \bar{\psi}^{\bar{a}}, \frac{\partial}{\partial \bar{\psi}^{\bar{b}}}\right\rangle=\delta^{\bar{a}} \bar{b}^{\prime}
$$

but

$$
\left\langle d \psi^{a}, \frac{\partial}{\partial \bar{\psi}^{\bar{b}}}\right\rangle=0, \quad\left\langle d \bar{\psi}^{\bar{a}}, \frac{\partial}{\partial \psi^{b}}\right\rangle=0,
$$

and similarly,

$$
\begin{gathered}
\left\langle d \pi_{a}, \frac{\partial}{\partial \pi_{b}}\right\rangle=\delta_{a}{ }^{b}, \quad\left\langle d \bar{\pi}_{\bar{a}}, \frac{\partial}{\partial \bar{\pi}_{\bar{b}}}\right\rangle=\delta_{\bar{a}}{ }^{b}, \\
\left\langle d \pi_{a}, \frac{\partial}{\partial \bar{\pi}_{\bar{b}}}\right\rangle=0, \quad\left\langle d \bar{\pi}_{\bar{a}}, \frac{\partial}{\partial \pi_{b}}\right\rangle=0 .
\end{gathered}
$$

All the remaining basic evaluations are vanishing, in particular those for $d \psi^{a}, d \bar{\psi}^{\bar{a}}$ with $\partial / \partial \pi_{b}$, $\partial / \partial \bar{\pi}_{\bar{b}}$, and similarly, for $d \pi_{a}, d \bar{\pi}_{\bar{a}}$ with $\partial / \partial \psi^{b}, \partial / \partial \bar{\psi}^{\bar{b}}$.

Let us write down the Hamilton equations of motion. Their form depends strongly on the occurrence of second time derivatives in the "Schrödinger equation". For simplicity let us begin with the assumption that $\beta \neq 0$ and our equation is second-order in time derivatives. Then the Legendre transformation is given by the formulas:

$$
\pi_{a}=i \alpha \bar{\psi}^{\bar{b}} \Gamma_{\bar{b} a}+\beta \dot{\bar{\psi}}^{\bar{b}} \Gamma_{\bar{b} a^{\prime}} \quad \bar{\pi}_{\bar{a}}=-i \alpha \Gamma_{\bar{a} b} \psi^{b}+\beta \Gamma_{\bar{a} b} \dot{\psi}^{b} .
$$

They are invertible and

$$
\dot{\psi}^{a}=\frac{1}{\beta} \Gamma^{a \bar{b}} \bar{\pi}_{\bar{b}}+\frac{i \alpha}{\beta} \psi^{a}, \quad \dot{\bar{\psi}}^{\bar{a}}=\frac{1}{\beta} \pi_{b} \Gamma^{b \bar{a}}-\frac{i \alpha}{\beta} \bar{\psi}^{\bar{a}}
$$


The Lagrangian "energy" function is given by

$$
\mathcal{E}=\beta \Gamma_{\bar{a} b} \dot{\bar{\psi}}^{\bar{a}} \dot{\psi}^{b}+\gamma_{\Gamma} H_{\bar{a} b} \bar{\psi}^{\bar{a}} \psi^{b}+\mathcal{V}(\psi, \bar{\psi})
$$

and substituting here the above inverse formula we obtain the "Hamilton function" in the sense of analytical mechanics:

$$
\mathcal{H}=\frac{1}{\beta}\left(\Gamma^{a \bar{b}} \pi_{a} \bar{\pi}_{\bar{b}}+i \alpha\left[\pi_{a} \psi^{a}-\bar{\pi}_{\bar{a}} \bar{\psi}^{\bar{a}}\right]\right)+\left(\frac{\alpha^{2}}{\beta} \Gamma_{\bar{a} b}+\gamma_{\Gamma} H_{\bar{a} b}\right) \bar{\psi}^{\bar{a}} \psi^{b}+\mathcal{V}(\psi, \bar{\psi}) .
$$

It is clear that the "energy" function is always globally defined in the tangent bundle, but the "Hamiltonian" $\mathcal{H}$ does exist as a function on the cotangent bundle only if $\beta$ does not vanish. The special case $\beta=0$ is essentially singular. Let us mention that it is a general rule that differential equations are catastrophically sensitive to the vanishing of coefficients at highest-order derivatives. In any case the Schrödinger equation modified by terms with second derivatives is essentially different than the usual, first-order equation. The problem has to do both with some doubts concerning the occurrence of second derivatives but also with certain hopes and new physical ideas. Obviously, if $\beta \neq 0$, the second-order Schrödinger equation is equivalent to the following canonical Hamilton equations:

$$
\frac{d \psi^{a}}{d t}=\left\{\psi^{a}, \mathcal{H}\right\}=\frac{\partial \mathcal{H}}{\partial \pi_{a}}, \quad \frac{d \pi_{a}}{d t}=\left\{\pi_{a}, \mathcal{H}\right\}=-\frac{\partial \mathcal{H}}{\partial \psi^{a}}
$$

Let us mention that there are various arguments for the second-order differential equations as fundamental ones for quantum theory. In a sense, in conformal SU(2,2)-ruled geometrodynamics, some kind of Dirac behaviour is a byproduct of the quadruplet of the gauge Klein-Gordon equation [4,9]. Besides, in nano-physics there are also some other arguments for the mixing of first- and second-order Schrödinger equations $[1,8]$. In the $\mathrm{SU}(2,2)$-gauge theory there are also some interesting consequences of this mixing within the framework of the standard model.

Nevertheless, it is also convenient to discuss separately the degenerate Schrödinger (Schrödinger-Dirac?) model based on the first derivatives. Our Legendre transformation becomes then

$$
\pi_{a}=i \alpha \bar{\psi}^{\bar{b}} \Gamma_{\bar{b} a^{\prime}} \quad \bar{\pi}_{\bar{a}}=-i \alpha \Gamma_{\bar{a} b} \psi^{b} .
$$

It does not depend on velocities at all. The same concerns the energy function:

$$
\mathcal{E}=\gamma_{\Gamma} H_{\bar{a} b} \bar{\psi}^{\bar{a}} \psi^{b}=\gamma \Gamma(\psi, \widehat{H} \psi)
$$

Strictly speaking, Hamiltonian is defined only on the manifold of "primary constraints" in the sense of Dirac, $M=\mathcal{L}(W \times \bar{W} \times W \times \bar{W}) \subset W \times \bar{W} \times W^{*} \times \bar{W}^{*}$, where $\mathcal{L}$ is just the 
above Legendre transformation. Some authors, including Dirac himself, define Hamiltonian $\mathcal{H}$ all over the phase space, however, it is then non-unique, and namely

$$
\mathcal{H}=\mathcal{H}_{0}+\lambda^{a}\left(\pi_{a}-i \alpha \bar{\psi}^{\bar{b}} \Gamma_{\bar{b} a}\right)+\bar{\lambda}^{\bar{a}}\left(\bar{\pi}_{\bar{a}}+i \alpha \Gamma_{\bar{a} b} \psi^{b}\right)
$$

where $\lambda^{a}, \bar{\lambda}^{\bar{a}}$ are Lagrange multipliers and

$$
\mathcal{H}_{0}=\gamma_{\Gamma} H_{\bar{a} b} \bar{\psi}^{\bar{a}} \psi^{b}+\mathcal{V}(\psi, \bar{\psi})
$$

One can easily show that the Dirac secondary constraints coincide with the primary ones, $M_{S}=M$, and the multipliers are given by

$$
\begin{aligned}
& \lambda^{a}=-\frac{i \gamma}{2 \alpha} H_{b}^{a} \psi^{b}-\frac{i}{2 \alpha} \Gamma^{a \bar{c}} \frac{\partial \mathcal{V}}{\partial \bar{\psi}^{\bar{c}}} \\
& \bar{\lambda}^{\bar{a}}=\frac{i \gamma}{2 \alpha} \bar{\psi}^{\bar{b}} H_{\bar{b}}{ }^{\bar{a}}+\frac{i}{2 \alpha} \frac{\partial \mathcal{V}}{\partial \psi^{c}} \Gamma^{c \bar{a}},
\end{aligned}
$$

where operations on indices of $H$ are meant in the sense of the metric tensor $\Gamma$.

It is clear that $M=M_{S}$ has the complex dimension $n$, but its real dimension is $2 n$, always even, as it should be with symplectic manifolds. The following quantities, $\pi$-s doubled in a consequence of this "complex-real",

$$
\Pi_{a}=2 i \alpha \bar{\psi}^{\bar{b}} \Gamma_{\bar{b} a^{\prime}} \quad \bar{\Pi}_{\bar{a}}=-2 i \alpha \Gamma_{\bar{a} b} \psi^{b},
$$

may be used to represent the canonical conjugate momenta. It follows in particular, that on the constraints submanifold $M$ we have the following Poisson brackets:

$$
\left\{\psi^{a}, \psi^{b}\right\}_{M}=0, \quad\left\{\bar{\psi}^{\bar{a}}, \bar{\psi}^{\bar{b}}\right\}_{M}=0, \quad\left\{\psi^{a}, \bar{\psi}^{\bar{b}}\right\}_{M}=\frac{1}{2 i \alpha} \Gamma^{a \bar{b}}
$$

Therefore, it is seen that up to normalization the complex conjugates $\bar{\psi}^{\bar{a}}$ coincide with canonical momenta conjugate to $\psi^{a}$. Using the standard properties of Poisson brackets we can write the resulting canonical equations in the bracket form:

$$
\frac{d \psi^{a}}{d t}=\left\{\psi^{a}, \mathcal{H}\right\}_{M}, \quad \frac{d \bar{\psi}^{\bar{a}}}{d t}=\left\{\bar{\psi}^{\bar{a}}, \mathcal{H}\right\}_{M}
$$

This implies, of course, the following well-known equation:

$$
i \hbar \frac{d \psi^{a}}{d t}=H^{a}{ }_{b} \psi^{b}+\frac{1}{2} \Gamma^{a \bar{b}} \frac{\partial \mathcal{V}}{\partial \bar{\psi}^{\bar{b}}}
$$


and, equivalently, its complex conjugate.

Let us stress that due to the $\mathcal{V}$-term, this is a nonlinear Schrödinger equation. The nonlinearity and its possible consequences for the decoherence and measurement problems depend on our invention in constructing the $\mathcal{V}$-model. Of course, the procedure is more promising for large, in particular infinite, systems, and the above finite-level framework is rather a toy model. This concerns both the first- and second-order Schrödinger equations. Nevertheless, in the above models nonlinearity was more or less introduced "by hand", as an additional perturbation term. Our main idea, we are going to describe it now, consists in introducing of nonlinearity in analogy to the passing from special to general relativity.

\section{Non-direct nonlinearity and the dynamical scalar product}

Let us remind some other, well-established nonlinearities of intrinsically geometric origin, appearing in physics. One of them is Einstein-Hilbert general relativity. It is well known that majority of well-established field theories is originally linear, and the nonlinearity appears in a consequence of their mutual interactions and symmetry principles. But there is one exceptional nonlinearity, namely that of gravitation theory. In special-relativistic physics the space-time arena is given by the flat Minkowski space. Its geometry is an absolute factor which restricts the symmetry to the Poincare group. But it is a strange and originally surprizing fact that physics does not like absolute objects. In general relativity the metric tensor becomes a dynamical quantity with the dynamics ruled by the Hilbert Lagrangian. It is so-to-speak an essentially nonlinear centre of physical reality. Its dynamics is essentially, non-perturbatively nonlinear and invariant under the infinite-dimensional group of the space-time diffeomorphisms (general covariance group). And automatically it becomes the group of symmetry of the whole physics. The dynamics is quasilinear, nevertheless by necessity nonlinear. The relationship between essential nonlinearity and large symmetry groups seems to be a general rule. Let us mention now two another, simpler examples from different branches of physics.

The first example belongs to mechanics of continua, first of all to plasticity theory, although elastic applications are also possible [11]. Let us consider a real linear space $V$ and the set $\operatorname{Sym}\left(V^{*} \otimes V^{*}\right)$ of symmetric metric tensors on $V$. It is obviously non-connected and consists of components characterized by the signature. Let us consider the manifold $\operatorname{Sym}^{+}\left(V^{*} \otimes V^{*}\right)$ of positively definite metrics. And now, assuming that the metrics elements of $\operatorname{Sym}^{+}\left(V^{*} \otimes V^{*}\right)$ describe some physical reality, let us ask for the metric structures, i.e., kinetic energy forms on $\operatorname{Sym}^{+}\left(V^{*} \otimes V^{*}\right)$. Of course, the simplest possibility is

$$
d s^{2}=G^{i j k l} d g_{i j} d g_{k l}, \quad \text { i.e., } \quad G=G^{i j k l} d g_{i j} \otimes d g_{k l},
$$

where $G^{i j k l}$ is constant and satisfies the natural nonsingularity and symmetry conditions:

$$
G^{i j k l}=G^{k l i j}, \quad G^{i j k l}=G^{j i k l}=G^{i j l k} .
$$

This metric on the manifold of metrics is flat. But this is rather strange and non-aesthetic. The natural question appears why not to use the following intrinsic metric: 


$$
d s^{2}=\lambda g^{j k} g^{l i} d g_{i j} d g_{k l}+\mu g^{j i} g^{l k} d g_{i j} d g_{k l},
$$

or, in more sophisticated terms:

$$
G=\lambda g^{j k} g^{l i} d g_{i j} \otimes d g_{k l}+\mu g^{j i} g^{l k} d g_{i j} \otimes d g_{k l}
$$

where $\lambda, \mu$ are constants and $\left[g^{i j}\right]$ is the contravariant inverse of $\left[g_{i j}\right]$, i.e., $g^{i k} g_{k j}=\delta^{i}{ }_{j}$. This metric structure is evidently non-Euclidean, Riemannian in $\operatorname{Sym}^{+}\left(V^{*} \otimes V^{*}\right)$, but it does not contain anything a priori fixed, but $\lambda, \mu$. To be more precise, it is only $\lambda$ that is essential up to normalization, because the $\mu$-term, being degenerate, is only an auxiliary correction. The corresponding kinetic energy of the $g$-process will be

$$
T=\frac{\lambda}{2} g^{j k} g^{l i} \frac{d g_{i j}}{d t} \frac{d g_{k l}}{d t}+\frac{\mu}{2} g^{j i} g^{l k} \frac{d g_{i j}}{d t} \frac{d g_{k l}}{d t}
$$

Expressions of this type are used, e.g., in incremental approaches to plasticity. They are also interesting in certain elastic problems and in defect theory.

Let us also mention about some other application. Consider the motion of material point with the mass $m$ and internal $g$-degrees of freedom. The corresponding kinetic energy will be given by

$$
T=\frac{m}{2} g_{i j} \frac{d x^{i}}{d t} \frac{d x^{j}}{d t}+\frac{\lambda}{2} g^{j k} g^{l i} \frac{d g_{i j}}{d t} \frac{d g_{k l}}{d t}+\frac{\mu}{2} g^{j i} g^{l k} \frac{d g_{i j}}{d t} \frac{d g_{k l}}{d t},
$$

obviously $x^{i}$ are here coordinates of the centre of mass. One can also introduce some potential term built of $x^{a}, g_{i j}$. In a sense, the structure of (50), (51) resembles that of generally-relativistic Lagrangians, obviously with the proviso that only the time derivatives occur, as we are dealing here with a system which does not possess any other continuous independent variables. Indeed, the main term of Hilbert Lagrangian begins from the expression proportional to

$$
g^{v \varkappa} g^{\mu \lambda} g^{\alpha \beta} g_{\mu v, \alpha} g_{\varkappa \lambda, \beta}
$$

where $g_{\mu v}$ is the space-time metric and $g^{\alpha \beta}$ is its contravariant inverse. Differentiation with respect to the space-time coordinates is meant here. The structural similarity to the prescription (50), (51) is obvious.

It is important that the both last expressions for $T$ are invariant under the total group $\mathrm{GL}(V)$, or rather under the semi-direct product $\mathrm{GL}(V) \times_{s} V$. Again the "large" symmetry group is responsible for the essential nonlinearity even of the geodetic models described by the expressions for $T$.

It is interesting to ask what changes appear when we assume $V$ to be a complex linear space and $g$ a sesquilinear Hermitian form. Obviously, instead of (50), (51) we will have then 


$$
\begin{aligned}
& T=\frac{\lambda}{2} g^{j \bar{k}} g^{l i} \frac{d g_{\overline{i j}}}{d t} \frac{d g_{\bar{k} l}}{d t}+\frac{\mu}{2} g^{j \bar{i}} g^{l \bar{k}} \frac{d g_{\overline{i j}}}{d t} \frac{d g_{\bar{k} l}}{d t}, \\
& T=\frac{m}{2} g_{\overline{i j}} \frac{d x^{\bar{i}}}{d t} \frac{d x^{j}}{d t}+\frac{\lambda}{2} g^{j \bar{k}} g^{l \bar{i}} \frac{d g_{\overline{i j}}}{d t} \frac{d g_{\bar{k} l}}{d t}+\frac{\mu}{2} g^{j \bar{i}} g^{l \bar{k}} \frac{d g_{\overline{i j}}}{d t} \frac{d g_{\bar{k} l}}{d t} .
\end{aligned}
$$

As usual, the matrix $\left[g^{i \bar{j}}\right]$ is reciprocal to $\left[g_{\bar{k} l}\right]$.

Let us observe that the models (50), (51), (53), (54) are structurally similar to our earlier affinely-invariant models of the affinely-rigid body [2, 3], i.e., roughly speaking to affinely-invariant geodetic models on the affine group. The idea there was that the material point was endowed with additional internal or collective degrees of freedom described by the attached linear basis $\left(\ldots, e_{A}, \ldots\right)$, or equivalently its dual $\left(\ldots, e^{A}, \ldots\right)$. The affinely-invariant kinetic energy was given by

$$
T=\frac{m}{2} C_{i j} \frac{d x^{i}}{d t} \frac{d x^{j}}{d t}+\frac{A}{2} \Omega^{i}{ }_{j} \Omega_{i}^{j}+\frac{B}{2} \Omega_{i}^{i} \Omega^{j}{ }_{j}
$$

where $C_{i j}=\eta_{A B} e^{A}{ }_{i} e^{B}{ }_{j}$ is the Cauchy deformation tensor, $\eta_{A B}$ is the fixed reference (material) metric, and $\Omega^{i}{ }_{j}$ is so-called affine velocity (affine generalization of angular velocity),

$$
\Omega_{j}^{i}=\frac{d e^{i} A}{d t} e^{A}
$$

This expression for $T$ is affinely invariant and in spite of its apparently strange structure it is dynamically applicable, due to its strongly non-quadratic prescription (nonlinearity of equations of motion).

Let us now go back to our quantum problem. First of all, let us notice that even in our finite-level system with the nonlinearity directly introduced to the Schrödinger equation, the procedure is in general non-trivial. There are two reasons for that: the possible time-dependence of the Hamiltonian $H^{a}{ }_{b}$, and the non-quadratic term $\mathcal{V}(\psi, \bar{\psi})$. Nevertheless, it is still a provisional solution.

Much more geometric is the following reasoning. To give up the fixed scalar product and to introduce instead the dynamical one, in analogy to general relativity and continuum mechanics. And then to define the kinetic energy for $\Gamma$ in analogy to (53):

$$
T=L[\Gamma]=\frac{A}{2} \Gamma^{b \bar{c}} \Gamma^{d \bar{a}} \dot{\Gamma}_{\bar{a} b} \dot{\Gamma}_{\bar{c} d}+\frac{B}{2} \Gamma^{b \bar{a}} \Gamma^{d \bar{c}} \dot{\Gamma}_{\bar{a} b} \dot{\Gamma}_{\bar{c} d}
$$

Therefore, the configuration space of our system consists of pairs $\left(\psi^{a}, \Gamma_{\bar{a} b}\right)$. The Lagrangian may be given by

$$
L[\psi, \Gamma]=L(1,2)[\psi, \Gamma]+\mathcal{V}[\psi, \Gamma]+L[\Gamma]
$$


where $L(1,2)[\psi, \Gamma], \mathcal{V}[\psi, \Gamma]$ are just the previously introduced models (16), (21), however with the dynamical, non-fixed $\Gamma$ subject to the variational procedure. Obviously, this complicates the Euler-Lagrange equations even in their parts following only from $L(1,2)+\mathcal{V}$.

The resulting theory is essentially nonlinear and invariant under the group $\mathrm{GL}(W)$, instead of the unitary group $\mathrm{U}(W, \Gamma)$ which preserves the traditional quantum mechanics. In a sense, the gap between quantum and classical mechanics diffuses. Not everything is quantum, not everything is classical. And the effective nonlinearity creates some hope for explaining the quantum paradoxes. Let us observe that the variation of $I[\Gamma]=\int L[\Gamma] d t$ leads to the following equations:

$$
-A \Gamma^{b \bar{n}}\left(\ddot{\Gamma}_{\bar{n} k}-\dot{\Gamma}_{\bar{n} l} \Gamma^{l \bar{c}} \dot{\Gamma}_{\bar{c} k}\right) \Gamma^{k \bar{a}}-B \Gamma^{l \bar{n}}\left(\ddot{\Gamma}_{\bar{n} l}-\dot{\Gamma}_{\bar{n} k} \Gamma^{k \bar{c}} \dot{\Gamma}_{\bar{c} l}\right) \Gamma^{b \bar{a}}=0 .
$$

As mentioned, the second term is merely a correction; the first term is essential. Let us notice that we did not perform variation in any other term of Lagrangian (58). It is so as if the $\psi^{a}$-degrees of freedom were non-excited. Of course, this is more than academic assumption, nevertheless convenient as a toy model. It is clear that the above equation (59) possesses solutions of the form:

$$
\Gamma_{\bar{r} s}=G_{\bar{r} z} \exp (E t)_{s}^{z}=\exp (F t)_{\bar{r}}^{\bar{z}} G_{\bar{z} s}
$$

where the initial condition for the scalar product $G=\Gamma(0)$ is a Hermitian sesquilinear form, $G \in \operatorname{Herm}\left(\bar{W}^{*} \otimes W^{*}\right)$. But this Hermitian property is to be preserved during the whole evolution. This will be the case when the linear mappings $E \in L(W), F \in L\left(\bar{W}^{*}\right)$ will be G-Hermitian, i.e., when the sesquilinear forms analytically given by

$$
{ }_{G} E_{\bar{r} s}=G_{\bar{r} z} E_{s}^{z}, \quad\left(F_{G}\right)_{\bar{r} s}=F_{\bar{r}}^{\bar{z}} G_{\bar{z} s}
$$

are Hermitian. Such solutions are analogous to our geodetic solutions in affinely-invariant models of the homogeneously deformable body [2,3]. Obviously, there is a deep geometric difference, because in mechanics of homogeneously deformable bodies one deals with real mixed tensors describing configurations, while here we are doing with complex sesquilinear forms. Nevertheless, the general philosophy is the same. Let us observe some interesting facts. Namely, the above evolution of $\Gamma$ may show all possible modes: it may be exponentially increasing, exponentially decaying, and even oscillatory. The point is how the initial data for $\Gamma(0)=G, E, F$ are fixed. Obviously, the academic model of the evolution of $\Gamma$ when $\psi$ is fixed is rather non-physical, nevertheless, there is a hope that the mentioned ways of behaviour may have something to do with decoherence and measurement paradoxes. Obviously, this hypothesis may be confirmed only a posteriori, by solving, at least approximately, the total system of equations derived from (58) and (63) below. In any case, it is almost sure that the supposed dynamics of $\Gamma$ should be based on (57) in (58). This follows from our demand of $\mathrm{GL}(W)$-invariance and from the analogy with general relativity and affine body dynamics. For example, we could try to use some fixed background metric $G$ and assume:

$$
L[G, \Gamma]=\frac{I}{2} G^{b \bar{c}} G^{d \bar{a}} \dot{\Gamma}_{\bar{a} b} \dot{\Gamma}_{\bar{c} d}+\frac{K}{2} G^{b \bar{a}} G^{d \bar{c}} \dot{\Gamma}_{\bar{a} b} \dot{\Gamma}_{\bar{c} d}
$$


When having $G$ at disposal, we can also define potential-like terms $\operatorname{Tr}\left({ }^{G} \Gamma^{p}\right)$, where ${ }^{G} \Gamma^{r}{ }_{s}:=$ $G^{r \bar{z}} \Gamma_{\bar{z} s}$. But of course, it seems aesthetically superfluous to fix some scalar products $G$ taken from nowhere, when the dynamical one is used. And it is only $L[\Gamma]$ (57) that seems to have a chance for solving the decoherence problem due to its strong, geometrically motivated nonlinearity.

In any case, the simplest $\mathrm{GL}(W)$-invariant Lagrangian seems to have the form:

$$
\begin{aligned}
L & =i \alpha_{1} \Gamma\left(\bar{\psi}^{\bar{a}} \dot{\psi}^{b}-\dot{\bar{\psi}}^{\bar{a}} \psi^{b}\right)+\alpha_{2} \Gamma_{\bar{a} b} \dot{\bar{\psi}}^{\bar{a}} \dot{\psi}^{b}+\left(\alpha_{3} \Gamma_{\bar{a} b}+\alpha_{4} H_{\bar{a} b}\right) \bar{\psi}^{\bar{a}} \psi^{b} \\
& +\alpha_{5} \Gamma^{d \bar{a}} \Gamma^{b \bar{c}} \dot{\Gamma}_{\bar{a} b} \dot{\Gamma}_{\bar{c} d}+\alpha_{6} \Gamma^{b \bar{a}} \Gamma^{d \bar{c}} \dot{\Gamma}_{\bar{a} b} \dot{\Gamma}_{\bar{c} d}-\mathcal{V}(\psi, \Gamma),
\end{aligned}
$$

where, e.g.,

$$
\mathcal{V}(\psi, \Gamma)=\frac{\varkappa}{2}\left(\Gamma_{\bar{a} b} \bar{\psi}^{\bar{a}} \psi^{b}-b\right)^{2}
$$

This expression (63) contains all the structural terms mentioned above and is $\mathrm{GL}(W)$-invariant. All quantities in it (except real constants) are dynamical variables and are subject to the variational procedure. We do not investigate in detail the resulting equations of motion. They are very complicated and describe the mutual interaction between $\psi^{a}, \Gamma \overline{a b}$. Nevertheless, their structure is interesting and instructive. Let us quote them for the above Lagrangian (63):

$$
\begin{aligned}
\frac{\partial L}{\partial \bar{\psi}^{\bar{a}}} & =\left(2 i \alpha_{1} \Gamma_{\bar{a} b}-\alpha_{2} \dot{\Gamma}_{\bar{a} b}\right) \dot{\psi}^{b}-\alpha_{2} \Gamma_{\bar{a} b} \ddot{\psi}^{b} \\
& +\left(i \alpha_{1} \dot{\Gamma}_{\bar{a} b}+\alpha_{3} \Gamma_{\bar{a} b}+\alpha_{4 \Gamma} H_{\bar{a} b}-\mathcal{V}^{\prime} \Gamma_{\bar{a} b}\right) \psi^{b}=0, \\
\frac{\partial L}{\partial \Gamma_{\bar{a} b}} & =-A \Gamma^{b \bar{n}}\left(\ddot{\Gamma}_{\bar{n} k}-\dot{\Gamma}_{\bar{n} l} \Gamma^{l \bar{c}} \dot{\Gamma}_{\bar{c} k}\right) \Gamma^{k \bar{a}}-B \Gamma^{l \bar{n}}\left(\ddot{\Gamma}_{\bar{n} l}-\dot{\Gamma}_{\bar{n} k} \Gamma^{k \bar{c}} \dot{\Gamma}_{\bar{c} l}\right) \Gamma^{b \bar{a}} \\
& +i \alpha_{1}\left(\bar{\psi}^{\bar{a}} \dot{\psi}^{b}-\dot{\bar{\psi}}^{\bar{a}} \psi^{b}\right)+\alpha_{2} \dot{\bar{\psi}}^{\bar{a}} \dot{\psi}^{b}+\left(\alpha_{3}-\mathcal{V}^{\prime}\right) \bar{\psi}^{\bar{a}} \psi^{b}=0 .
\end{aligned}
$$

In spite of their relatively complicated structure, these nonlinear equations are readable. For any case we have retained the direct nonlinearity term derived from $\mathcal{V}$. But the main idea of nonlinearity and large $\mathrm{GL}(W) \simeq \mathrm{GL}(n, \mathbb{C})$-symmetry is just the interaction between $\psi$ and $\Gamma$. And it is just the interaction of the generally-relativistic type. As seen, at the same time it is structurally similar to affinely-invariant geodetic models of elastic vibrations of the homogeneously deformable body $[2,3]$. Even independently on our quantum programme, this model is interesting in itself as an example of highly-symmetric dynamical system on a homogeneous space. Nonlinearity of the system is rational because the inverse matrix $\left[\Gamma^{a \bar{b}}\right]$ is a rational function of $\left[\Gamma_{\bar{c} d}\right]$. Therefore, there are some hopes for a solvability, perhaps at least qualitative or approximate, of the system (65), (66).

Let us notice that the $\alpha_{3}$-controlled term may be included into the $\alpha_{4}$-expression. We simply decided to write it separately to stress the special role of the Hamiltonian terms proportional to the identity operator. Let us stress that the Lagrangian (63) is not the only expression with 
the above enumerated properties. Rather, it is the simplest one. For example, one might replace $\Gamma^{a \bar{b}}$ by $\Gamma^{a \bar{b}}+\alpha_{7} \psi^{a} \bar{\psi}^{\bar{b}}$, etc. Perhaps this will modify somehow the resulting equations, but in a rather non-essential way. All important features are already predicted by equations following from (63). Let us remind that in (63) we have according to quantum mechanics

$$
\alpha_{1}=\frac{\hbar}{2}, \quad \alpha_{4}=-1
$$

The nonlinearity contained in (63) resembles in a sense the Thomas-Fermi approximation in quantum mechanics $[12,13]$. It may be considered as an alternative way of describing open systems.

When concentrating on the dynamical system model of Schrödinger equation, one is faced with the interesting question as to what might be described by admitting non-Hamiltonian forces. Perhaps nothing physical. There is nevertheless some possibility that such forces might be useful for describing quantum dissipative phenomena. However, at this stage we have no idea concerning this problem.

The $\mathrm{GL}(W) \simeq \mathrm{GL}(n, \mathbb{C})$-invariance of the Hamiltonian system implies the existence of $n^{2}$ complex constants of motion. We do not quote here their explicit form to avoid writing unnecessary and complicated formulas. Nevertheless, their existence is a remarkable property of the theory.

Let us stress that the strong and geometrically implied nonlinearity of equations following from (63) gives a chance for the macroscopic reinforcement and enhancement of quantum events.

It is very important to remember that in the model (63), (65), (66), based on the mutual interaction between $\psi$ and $\Gamma$, the "scalar product" $\Gamma$ is not a constant of motion. And there is an exchange of energy between $\psi$ - and $\Gamma$-degrees of freedom, especially in situations when the $\Gamma$-motion is remarkably excited. Therefore, if $\alpha_{5} \neq 0, \alpha_{6} \neq 0$, the quantity $\Gamma$, although fundamental for physical interpretation, in a sense loses its physical meaning of the scalar product. If $\alpha_{2}=0, \alpha_{5}=0, \alpha_{6}=0$, then the invariance of $\mathcal{L}$ under the $\mathrm{U}(1)$-group

$$
\psi \mapsto \exp \left(-\frac{i}{\hbar} e \chi\right) \psi
$$

implies, via Noether theorem, that

$$
e \frac{2 \alpha_{1}}{\hbar}\langle\psi \mid \psi\rangle=e \frac{2 \alpha_{1}}{\hbar} \Gamma_{\bar{a} b} \bar{\psi}^{\bar{a}} \psi^{b}
$$

is a constant of motion. And then the usual polarization formula for quadratic forms implies that $\langle\psi \mid \varphi\rangle=\Gamma_{\bar{a} b} \bar{\psi}^{\bar{a}} \varphi^{b}$ is preserved. If $\alpha_{2} \neq 0$, this procedure leads to the scalar product

$$
\Gamma_{\bar{a} b} \bar{\psi}^{\bar{a}} \varphi^{b}+\frac{i \alpha_{2}}{2 \alpha_{1}} \Gamma_{\bar{a} b}\left(\bar{\psi}^{\bar{a}} \dot{\varphi}^{b}-\dot{\bar{\psi}}^{\bar{a}} \varphi^{b}\right) .
$$


It fails to be positively definite, nevertheless, it seems to be physically interesting [1]. And finally, when $\alpha_{5} \neq 0, \alpha_{6} \neq 0$, Noether theorem tells us that the $\mathrm{U}(1)$-constant of motion differs form the above ones by the term proportional to $\Gamma^{a \bar{b}} \dot{\Gamma}_{\bar{b} a}$. But now $\Gamma$ is a dynamical variable and the last expression is not quadratic in $\left(\Gamma_{\bar{a} b}, \dot{\Gamma}_{\bar{a} b}\right)$. Because of this there is no polarization procedure and no well-defined Noether-based scalar product at all.

\section{Towards general systems}

The main ideas of our nonlinearity model in quantum mechanics were formulated and presented in the simplest case of finite-level systems. Let us mention now about a more general situation. We concentrate mainly on the non-relativistic Schrödinger mechanics. It is true that on the very fundamental level of theoretical physics one is interested mainly in relativistic theory. Nevertheless, the non-relativistic counterparts are also interesting at least from the methodological point of view; they just enable us to understand deeper the peculiarity of relativistic theory. And of course they may be also directly useful physically in situations where in condensed matter theory, relativistic effects are not relevant, e.g., in superconductivity and superfluidity.

Galilei group and Galilei space-time are structurally much more complicated than Poincare (inhomogeneous Lorentz) group and Minkowski space-time. Because of this, the construction of Lagrangian for the Schrödinger theory need some comments and a few steps of reasoning.

One is rather used to start with wave-mechanical ideas of Schrödinger equation, i.e.,

$$
i \hbar \frac{\partial \psi}{\partial t}=\mathbb{H} \psi
$$

rather than with some yet unspecified precisely field theory on the Galilei space-time. The standard scalar product of Schrödinger amplitudes on the three-dimensional Euclidean space will be denoted as usually by

$$
\left\langle\psi_{1} \mid \psi_{2}\right\rangle=\int \overline{\psi_{1}(x)} \psi_{2}(x) d_{3} x
$$

where, obviously, orthogonal Cartesian coordinates are meant. More generally, in curvilinear coordinates we would have

$$
\left\langle\psi_{1} \mid \psi_{2}\right\rangle=\int \overline{\psi_{1}(q)} \psi_{2}(q) \sqrt{|g(3)|} d_{3} q
$$

where $|g(3)|$ is an abbreviation for the determinant of the matrix of Euclidean metric tensor $g(3)$, and $q^{i}$ are generalized coordinates. The same formula is valid in the Riemann space, where all coordinates are "curvilinear". However, here we do not get into such details.

Hermitian conjugation of operators, $\mathbb{A} \mapsto \mathbb{A}^{+}$is meant in the usual sense of $L^{2}\left(\mathbb{R}^{3}\right)$ with the above scalar product,

$$
\left\langle\psi_{1} \mid \mathbb{A} \psi_{2}\right\rangle=\left\langle\mathbb{A}^{+} \psi_{1} \mid \psi_{2}\right\rangle,
$$


obviously with some additional remarks concerning domains. The Hamilton operator is self-adjoint, $\mathbb{H}^{+}=\mathbb{H}$, also with some care concerning the domains.

The Schrödinger equation is again variational with the Lagrangian

$$
\mathcal{L}=\mathcal{L}_{T}+\mathcal{L}_{H}
$$

where, $\mathcal{L}_{T}, \mathcal{L}_{H}$ denote respectively the terms depending linearly on first-order time derivatives and independent on them,

$$
\begin{aligned}
\mathcal{L}_{T} & =\frac{i \hbar}{2}\left(\bar{\psi} \frac{\partial \psi}{\partial t}-\frac{\partial \bar{\psi}}{\partial t} \psi\right)=-\operatorname{Im}\left(\hbar \bar{\psi} \frac{\partial \psi}{\partial t}\right)=\operatorname{Re}\left(i \hbar \bar{\psi} \frac{\partial \psi}{\partial t}\right) \\
\mathcal{L}_{H} & =-\bar{\psi}(\mathbb{H} \psi) .
\end{aligned}
$$

The total action is

$$
I=I_{T}+I_{H}=\int \mathcal{L}_{T} d t d_{3} x+\int \mathcal{L}_{H} d t d_{3} x,
$$

and the resulting variational derivative of $I$ equals

$$
\frac{\delta I}{\delta \bar{\psi}(t, x)}=-\mathbb{H} \psi+i \hbar \frac{\partial \psi}{\partial t}
$$

therefore, the stationary points are given indeed by the solutions of (71). In the very important special case of a material point moving in the potential field $V$,

$$
\mathbb{H}=-\frac{\hbar^{2}}{2 m} g^{i j} \frac{\partial^{2}}{\partial x^{i} \partial x^{j}}+V=-\frac{\hbar^{2}}{2 m} \Delta+V
$$

it is convenient and instructive to rewrite the Lagrangian term $\mathcal{L}_{H}$ in a variationally equivalent form structurally similar to the Klein-Gordon Lagrangian,

$$
\mathcal{L}_{H}^{\prime}=-\frac{\hbar^{2}}{2 m} g^{i j} \frac{\partial \bar{\psi}}{\partial x^{i}} \frac{\partial \psi}{\partial x^{j}}+V \bar{\psi} \psi,
$$

which differs from the original one by a total divergence term. In particular, there are no artificial second derivatives in $\mathcal{L}_{H}^{\prime}$. This resembles the situation one is faced with in General Relativity. For a free particle the total Lagrangian equals

$$
\mathcal{L}^{\prime}=\frac{i \hbar}{2}\left(\bar{\psi} \frac{\partial \psi}{\partial t}-\frac{\partial \bar{\psi}}{\partial t} \psi\right)-\frac{\hbar^{2}}{2 m} g^{i j} \frac{\partial \bar{\psi}}{\partial x^{i}} \frac{\partial \psi}{\partial x^{j}} .
$$

In this form, structurally as similar as possible to the Lagrangian for the Klein-Gordon field, the essential geometrical similarities and differences between Galilei and Poincare quantum symmetries are visible. 
The well-known expressions for the probability density and probability current are obtained in non-relativistic quantum mechanics on the basis of some rather rough and intuitive statistical concepts. However, when the Schrödinger equation is interpreted within the framework of field theory on the Galilean space-time, those concepts appear as direct consequences of Noether theorem just as said above in the finite number of levels part. The Schrödinger Lagrangian for a free particle is invariant under the group $\mathrm{U}(1)$ of global gauge transformations,

$$
\psi \mapsto \exp \left(-\frac{i}{\hbar} e \chi\right) \psi,
$$

where $e$ denotes the coupling constant (elementary charge) and $\chi$ is the gauge parameter. Within the Kaluza-Klein formulation of electrodynamics of point charges, the variable $\chi$ and electric charge $e$ are canonically conjugate quantities. The invariance under this gauge group implies the conservation law for the Galilean current $\left(j^{t}, \bar{j}\right)$, where

$$
j^{t}=\varrho=e \bar{\psi} \psi, \quad j^{a}=\frac{e \hbar}{2 i m}\left(\bar{\psi} \frac{\partial \psi}{\partial x^{a}}-\frac{\partial \bar{\psi}}{\partial x^{a}} \psi\right) .
$$

The resulting continuity equation has the usual form:

$$
\frac{\partial \varrho}{\partial t}+\frac{\partial j^{a}}{\partial x^{a}}=0, \quad \text { i.e., } \quad \frac{\partial \varrho}{\partial t}+\operatorname{div} \bar{j}=0 .
$$

Let us stress that all those formulas hold in rectilinear orthonormal coordinates. In general coordinates we would have to multiply the above expressions by $\sqrt{|g(3)|}$ so as to turn them respectively into scalar and contravariant vector densities. And so one does in a general Riemann space. Continuity equation is satisfied if $\psi$ is a solution of the Schrödinger equation.

The functional

$$
\psi \mapsto Q_{t}[\psi]=\int j^{t} d_{3} x=\int \varrho(t, x) d_{3} x
$$

describes the total charge at the time instant $t$. On the basis of field equations it is independent on time,

$$
\frac{d}{d t} Q_{t}[\psi]=0
$$

This is the global law of the charge conservation. Let us observe that $Q$ is a functional quadratic form of $\psi$. Its polarization reproduces the usual scalar product as a sesquilinear Hermitian form (up to a constant factor):

$$
4 e\langle\psi \mid \varphi\rangle=Q[\psi+\varphi]-Q[\psi-\varphi]-i Q[\psi+i \varphi]+i Q[\psi-i \varphi]
$$

In a consequence of charge conservation law $\langle\psi \mid \varphi\rangle$ is time-independent if $\psi, \varphi$ are solutions of the same Schrödinger equation. 
One can do the same for multiplets of scalar Schrödinger fields $\psi^{a}$, i.e., for the continuum of systems described in the previous section. Then we have

$$
\begin{aligned}
\mathcal{L}_{T} & =\frac{i \hbar}{2} \Gamma_{\bar{a} b}\left(\bar{\psi}^{\bar{a}} \frac{\partial \psi^{b}}{\partial t}-\frac{\partial \bar{\psi}^{\bar{a}}}{\partial t} \psi^{b}\right), \\
\langle\psi \mid \varphi\rangle & =\int \Gamma_{\bar{a} b} \bar{\psi}^{\bar{a}} \varphi^{b} \sqrt{|g(3)|} d_{3} q, \\
L_{T} & =\operatorname{Re}\left\langle\psi \mid i \hbar \frac{\partial \psi}{\partial t}\right\rangle=-\operatorname{Im}\left\langle\psi \mid \hbar \frac{\partial \psi}{\partial t}\right\rangle, \\
\mathcal{L}_{H} & =-\Gamma_{\bar{a} b} \bar{\psi}^{\bar{a}}(\mathbb{H} \psi)^{b}, \quad L_{H}=-\langle\psi \mid \mathbb{H} \psi\rangle .
\end{aligned}
$$

And again, for systems with scalar potentials, when

$$
(\mathbb{H} \psi)^{a}=-\frac{\hbar^{2}}{2 m} \Delta \psi^{a}+V_{b}^{a} \psi^{b}
$$

we can remove second derivatives as a divergence term and obtain the modified Lagrangian

$$
\mathcal{L}_{H}^{\prime}=-\frac{\hbar^{2}}{2 m} \Gamma_{\bar{a} b} \frac{\partial \bar{\psi}^{\bar{a}}}{\partial x^{i}} \frac{\partial \psi^{b}}{\partial x^{j}} g(3)^{i j}-V_{\bar{a} b} \bar{\psi}^{\bar{a}} \psi^{b},
$$

where

$$
V_{\bar{a} b}=\Gamma_{\bar{a} c} V_{b}^{c} .
$$

We have used here Cartesian coordinates for simplicity. Using the operator of linear momentum,

$$
\mathbb{P}_{a}=\frac{\hbar}{i} \frac{\partial}{\partial x^{a}}
$$

we can write

$$
L_{H}=-\frac{1}{2 m}\left\langle\mathbb{P}_{a} \psi \mid \mathbb{P}_{b} \psi\right\rangle g(3)^{a b}-\langle\psi \mid V \psi\rangle .
$$

In analogy to (16), (17), (21) we can write the direct-nonlinearity Lagrangian as follows:

$$
\begin{aligned}
\mathcal{L} & =i \alpha_{1} \Gamma_{\bar{a} b}\left(\bar{\psi}^{\bar{a}} \dot{\psi}^{b}-\overline{\bar{\psi}}^{\bar{a}} \psi^{b}\right)+\alpha_{2} \Gamma_{\bar{a} b} \dot{\bar{\psi}}^{\bar{a}} \dot{\psi}^{b} \\
& +\alpha_{3} \Gamma_{\bar{a} b} \frac{\partial \bar{\psi}^{\bar{a}}}{\partial x^{i}} \frac{\partial \psi^{b}}{\partial x^{j}} g(3)^{i j}+\alpha_{4} V_{\bar{a} b} \bar{\psi}^{\bar{a}} \psi^{b}-\mathcal{V}(\psi, \Gamma),
\end{aligned}
$$

where, e.g., the non-quadratic term $\mathcal{V}$ responsible for nonlinearity may be chosen in a quartic form (22). The combination of the $\alpha_{2^{-}}, \alpha_{3}$-terms may be purely academic, as explained above, but it may also describe something like the Klein-Gordon phenomena, when $\alpha_{2}$ and $\alpha_{3}$ are 
appropriately suited. Indeed, at an appropriate ratio $\alpha_{2}: \alpha_{3}$ the above expression for $\mathcal{L}$ becomes:

$$
\begin{aligned}
\mathcal{L} & =i \alpha_{1} \Gamma_{\bar{a} b}\left(\bar{\psi}^{\bar{a}} \dot{\psi}^{b}-\dot{\bar{\psi}} \overline{\bar{a}} \psi^{b}\right)+\alpha_{231} \Gamma_{\bar{a} b} \frac{\partial \bar{\psi}^{\bar{a}}}{\partial x^{\mu}} \frac{\partial \psi^{b}}{\partial x^{v}} g^{\mu v} \\
& -\alpha_{232} \Gamma_{\bar{a} b} \bar{\psi}^{\bar{a}} \psi^{b}+\alpha_{4} V_{\bar{a} b} \bar{\psi}^{\bar{a}} \psi^{b}-\mathcal{V}(\psi, \Gamma),
\end{aligned}
$$

where $g$ is the Minkowskian metric. For the notational simplicity we used Cartesian coordinates in this formula, to avoid the multiplying of $\mathcal{L}$ by $\sqrt{|g|}$. Of course, within relativistic framework it is rather artificial to superpose the $g$-Minkowskian terms with the non-relativistic $\alpha_{1}$-term. The consequent relativistic theory should rather combine the Klein-Gordon and Dirac terms. Therefore, $\mathcal{L}$ should be then postulated as:

$$
\begin{aligned}
\mathcal{L} & =i \alpha_{11} \Gamma^{\mu}{ }_{\bar{a} b}\left(\bar{\psi}^{\bar{a}} \frac{\partial \psi^{b}}{\partial x^{\mu}}-\frac{\partial \bar{\psi}^{\bar{a}}}{\partial x^{\mu}} \psi^{b}\right)+\alpha_{231} \Gamma_{\bar{a} b} \frac{\partial \bar{\psi}^{\bar{a}}}{\partial x^{\mu}} \frac{\partial \psi^{b}}{\partial x^{v}} g^{\mu \nu} \\
& -\alpha_{232} \Gamma_{\bar{a} b} \bar{\psi}^{\bar{a}} \psi^{b}+\alpha_{4} V_{\bar{a} b} \bar{\psi}^{\bar{a}} \psi^{b}-\mathcal{V}(\psi, \Gamma) .
\end{aligned}
$$

Here we do not fix entities and constants. As usual in the Dirac framework, $\Gamma$ in the $\alpha_{231^{-}}$, $\alpha_{232}$-terms is the sesquilinear Hermitian form of the neutral signature $(++--)$. And $\Gamma^{\mu}$ are sesquilinear Hermitian Dirac forms, i.e., raising their first index with the help of the reciprocal contravariant $\Gamma^{a \bar{b}}, \Gamma^{a \bar{c}} \Gamma_{\bar{c} b}=\delta^{a}{ }_{b}$, one obtains the Dirac matrices $\gamma^{\mu a}{ }_{b}=\Gamma^{a \bar{c}} \Gamma^{\mu}{ }_{\bar{c} b}$ satisfying the anticommutation rules:

$$
\gamma^{\mu} \gamma^{v}+\gamma^{v} \gamma^{\mu}=2 g^{\mu v} I_{4}
$$

Strictly speaking, the quantities $\Gamma_{\bar{a} b}$ at $\alpha_{231^{-}}, \alpha_{232}$-terms need not be the same. Nevertheless, in the fundamental quantum studies it is convenient to identify them. Let us mention, e.g., some ideas connected with the Dirac-Klein-Gordon equation appearing in certain problems of mathematical physics [2,3]. In particular, it turns out that the $\mathrm{SU}(2,2)$-ruled (conformally ruled) theory of spinorial geometrodynamics has a specially-relativistic limit based on Lagrangian similar to (100). In any case, the specially-relativistic theory based on the superposition of the Klein-Gordon and Dirac Lagrangians is interesting from the point of view of some peculiar kinship between pairs of fundamental particles. We mean pairs of fermions and pairs of quarks which occur in the standard model $[9,14,15]$.

Let us go back to our general model (98), not necessarily relativistic one. Its nonlinearity is contained only in the non-quadratic potential term $\mathcal{V}(\psi, \Gamma)$. Lagrangian $\mathcal{L}$ and the action are local in the $x$-space when the Hamilton operator is a sum of the position- and momentum-type operators. However, in general the corresponding contribution to action is given by

$$
I_{\chi}[\psi]=-\gamma \int \bar{\psi}^{\bar{a}}(t, x) \chi_{\bar{a} b}(x, y) \psi^{b}(t, y) d t d x d y
$$


therefore,

$$
\frac{\delta I_{\chi}}{\delta \bar{\psi}^{\bar{a}}(t, x)}=-\gamma \int \chi_{\bar{a} b}(x, y) \psi^{b}(t, y) d y .
$$

This integral expression implies that our equations of motion will be integro-differential ones. If we seriously assume that also the scalar product is given by the double spatial integral, so that, e.g.,

$$
I_{1}=i \alpha \int \Gamma_{\bar{a} b}(x, y)\left(\bar{\psi}^{\bar{a}}(t, x) \dot{\psi}^{b}(t, y)-\dot{\bar{\psi}}^{\bar{a}}(t, x) \psi^{b}(t, y)\right) d t d x d y,
$$

and in general

$$
\Gamma_{\bar{a} b}(x, y) \neq \Gamma_{\bar{a} b} \delta(x-y),
$$

then the variational derivative of $I_{1}$ also leads to the integro-differential equation, because

$$
\frac{\delta I_{1}}{\delta \bar{\psi}^{\bar{a}}(t, x)}=2 i \alpha \int \Gamma_{\bar{a} b}(x, y) \dot{\psi}^{b}(t, y) d y .
$$

The necessity of using integro-differential equations is embarrassing. However, the main difficulty appears when we wish to follow the finite-level systems dynamics in the general case. Namely, it was relatively easy to write formally something like the elements $\left[\Gamma^{a \bar{b}}\right]$ of the matrix reciprocal to $\left[\Gamma_{\bar{c} d}\right]$ for the finite-dimensional system. And one can try to follow this procedure for the dynamical scalar product in the general infinite-dimensional case. However, such a hybrid is not convincing. It would be then only "internal degrees of freedom" subject to the procedure of the dynamical scalar product. No doubt that this does not seem satisfactory. Let us write the dynamical scalar product of the Schrödinger-like quantum mechanics in the form:

$$
\langle\psi \mid \varphi\rangle=\int \Gamma_{\bar{a} b}(x, y) \bar{\psi}^{\bar{a}}(x) \varphi^{b}(y) d x d y
$$

where $\overline{\Gamma_{\bar{a} b}(x, y)}=\Gamma_{\bar{b} a}(y, x)$. Assuming in addition the translational invariance, we have $\Gamma(x, y)=\Gamma(x-y)$, i.e.,

$$
\langle\psi \mid \varphi\rangle=\int \Gamma_{\bar{a} b}(x-y) \bar{\psi}^{\bar{a}}(x) \varphi^{b}(y) d x d y .
$$

A simplifying assumption would be factorization

$$
\Gamma_{\bar{a} b}(x, y)=\Gamma_{\bar{a} b} \mathcal{K}(x, y)=\Gamma_{\bar{a} b} \mathcal{K}(x-y) .
$$

Let us remind that in the usual quantum mechanics without the dynamical scalar product, we have simply

$$
\Gamma_{\bar{a} b}(x, y)=\Delta_{\bar{a} b} \delta(x-y),
$$


where $\Delta$ is a positively definite algebraic scalar product for internal modes. In appropriately chosen basis we have simply $\Delta_{\bar{a} b}=\delta_{\bar{a} b}$. In the mentioned half-a-way approach, (110) is still valid but with the dynamical, time-dependent $\Delta_{\bar{a} b}$.

Much more reasonable, although incomparatively more difficult, would be a consequent approach based on the dynamical scalar product (107). Here we only mention some ideas. The main point is the construction of the full inverse of $\Gamma$ in (107), with the time-dependent $\Gamma$, i.e., analytically $\Gamma^{a \bar{b}}(x, y ; t)$ such that

$$
\int \Gamma^{a \bar{c}}(x, u ; t) \Gamma_{\bar{c} b}(u, y ; t) d u=\delta^{a}{ }_{b} \delta(x-y) .
$$

Obviously, in general it is a rather very difficult problem to find explicitly the formula for that inverse. One can try to discretize it by choosing some appropriate finite (or perhaps countable) family $\Omega$ of vectors $a_{p}$ such that

$$
\Gamma^{a \bar{b}}(x, y ; t)=\sum_{p \in \Omega} \Gamma^{a \bar{b}}(p ; t) \delta\left(x-y+a_{p}\right)
$$

This may be used as a basis for some discretization procedure like the finite-element method for finding $\Gamma^{a \bar{b}}(x, y ; t)$.

In any case, everything said in the former section about the dynamical scalar product and about the failure of the absolute scalar product (72), (73) remains true in wave mechanics on the differential configuration manifold. Although it is true that in this case everything becomes much more complicated.

\section{Acknowledgements}

This paper partially contains results obtained within the framework of the research project N N501 049540 financed from the Scientific Research Support Fund in 2011-2014 just as the previous contribution by Jan J. Sławianowski Order of Time Derivatives in Quantum-Mechanical Equations to the book Measurements in Quantum Mechanics [4]. The authors are greatly indebted to the Ministry of Science and Higher Education for this financial support.

\section{Author details}

Jan J. Sławianowski ${ }^{\star}$ and Vasyl Kovalchuk

* Address all correspondence to: jslawian@ippt.gov.pl

Institute of Fundamental Technological Research, Polish Academy of Sciences, Warsaw, Poland 


\section{References}

[1] Sławianowski JJ, Kovalchuk V. Schrödinger and Related Equations as Hamiltonian Systems, Manifolds of Second-Order Tensors and New Ideas of Nonlinearity in Quantum Mechanics. Reports on Mathematical Physics 2010;65(1) 29-76.

[2] Sławianowski JJ, Kovalchuk V, Sławianowska A, Gołubowska B, Martens A, Rożko EE, Zawistowski ZJ. Affine Symmetry in Mechanics of Collective and Internal Modes. Part I. Classical Models. Reports on Mathematical Physics 2004;54(3) 373-427.

[3] Sławianowski JJ, Kovalchuk V, Sławianowska A, Gołubowska B, Martens A, Rożko EE, Zawistowski ZJ. Affine Symmetry in Mechanics of Collective and Internal Modes. Part II. Quantum Models. Reports on Mathematical Physics 2005;55(1) 1-45.

[4] Sławianowski JJ. Order of Time Derivatives in Quantum-Mechanical Equations. In: Pahlavani MR. (ed.) Measurements in Quantum Mechanics. Rijeka: INTECH; 2012. p.57-74.

[5] Doebner HD, Goldin GA. Introducing Nonlinear Gauge Transformations in a Family of Nonlinear Schrödinger Equations. Phys. Rev. A 1996;54 3764-3771.

[6] Doebner HD, Goldin GA, Nattermann P. Gauge Transformations in Quantum Mechanics and the Unification of Nonlinear Schrödinger Equations. J. Math. Phys. 1999;40 49-63; quant-ph/9709036.

[7] Kozlowski M, Marciak-Kozlowska J. From Quarks to Bulk Matter. USA: Hadronic Press; 2001.

[8] Marciak-Kozlowska J, Kozlowski M. Schrödinger Equation for Nano-Science, cond-mat/0306699.

[9] Sławianowski JJ. New Approach to the U(2,2)-Symmetry in Spinor and Gravitation Theory. Fortschr. Phys./Progress of Physics 1996;44(2) 105-141.

[10] Bogoliubov NN, Shirkov DV. Quantum Fields. Reading, Mass.: Benjamin/Cummings Pub. Co.; 1982.

[11] Krawietz A. Natürliche Geometrie der Deformationsprozesse. ZAMM 1979;59 T199-T200.

[12] Landau LD, Lifshitz EM. Quantum Mechanics. London: Pergamon Press; 1958.

[13] Messiah A. Quantum Mechanics. Amsterdam: North-Holland Publishing Company; 1965.

[14] Dvoeglazov VV. The Barut Second-Order Equation, Dynamical Invariants and Interactions. J. Phys. Conf. Ser. 2005;24 236-240; math-ph/0503008.

[15] Kruglov SI. On the Generalized Dirac Equation for Fermions with Two Mass-States. An. Fond. Louis de Broglie 2004;29 1005-1-16; quant-ph0408056. 

Chapter 6

\title{
Schrödinger Equation and (Future) Quantum Physics
}

\author{
Miloš V. Lokajíček, Vojtěch Kundrát and \\ Jiř́ Procházka \\ Additional information is available at the end of the chapter
}

http://dx.doi.org/10.5772/53844

\section{Introduction}

The Schrödinger equation based on the Hamiltonian taken from the classical physics provides the solutions that may be correlated to the solutions of Hamilton equations or to their superpositions, if the additional assumptions introduced by Bohr have not been added; i.e., simple Hilbert space spanned on one set of Hamiltonian eigenfunctions and each vector representing pure state. It may describe, therefore, physical processes at microscopic as well as macroscopic levels; only the set of allowed states being partially limited. However, the given equation represents approximative phenomenological theory that is not able to explain the emergence of quantum states on the basis of Coulomb potential only; e.g., in the case of hydrogen atom such a state may arise evidently only if a repulsive short-ranged force (or some contact interaction) exists between electron and proton at low kinetic energy values. The dimensions and other properties of proton should play then important role in such a case. These characteristics may be derived from different experiments studying collisions between corresponding objects at different energies. They should be taken into account when the quantum physics is to pass from hitherto mere phenomenological description of physical evolution to causal realistic interpretation as it was common in classical physics. It concerns mainly the spontaneous transitions between different quantum states in atoms.

However, let us start with short summary of main points concerning the evolution of quantum mechanics theory during the 20th century. The Copenhagen quantum mechanics (based fundamentally on the Schrödinger equation [1] and proposed by Bohr [2] in 1927) has been taken as the only theory of microscopic physical processes till the end of the 20th century, even if it has involved a series of paradoxes corresponding in principle to internal contradictions contained in corresponding mathematical model. Already in 1933 Pauli [3] showed that the corresponding Hamiltonian was to exhibit continuous energy spectrum in the whole interval $E \in(-\infty,+\infty)$, which contradicted the existence of quantized energy 
states in closed systems. However, it has been possible to say that this fact did not raised any greater interest at that time.

More attention was devoted to the criticism of Einstein [4] in 1935, who showed with the help of a Gedankenexperiment that the given quantum theory required the existence of immediate interaction (or rather linkage) between two very distant matter objects, which was denoted by him as unacceptable on the basis of standard ontological experience. The given critique was refused by Bohr [5] having argued that this distant immediate interaction might exist between microscopic objects (at the difference to macroscopic phenomena). The world scientific community accepted fully Bohr's standpoint that was supported partially by the earlier argument of von Neumann [6] who refused the existence of any local (hidden) parameters in Copenhagen theory; the problem having been discussed several years ago. The argument of Grete Herrmann that the given conclusion of von Neumann was based on circular proof [7] was not taken into account, having remained practically unknown at that time.

A partial change occurred in 1952 when Bohm [8] showed that an additional (hidden) parameter existed already in the simplest solutions of Schrödinger equation. Two alternatives (Copenhagen mechanics and hidden-variable theory) were then considered in the microscopic region. The decision between them was expected to be done on the basis of experimental results when Bell [9] derived his inequalities in 1964 which were assumed to hold in the hidden-variable theory and not in the Copenhagen alternative. The corresponding experiments based in principle on the original proposal of Einstein were finished in 1982 and the inequalities of Bell were provably violated [10]. The Copenhagen quantum mechanics was then taken as the only physical theory valid for microscopic physical processes.

Only in the end of the last century it was shown for the first time that the given conclusion was based on the mistaking assumption and that the given inequalities did not hold in any quantum alternative based on Schrödinger equation [11]. In 2004 it was then shown by Rosinger [12] that Bell's inequalities contradicted also the inequalities derived by Boole (in 1854) for any probabilistic system $[13,14]$ :

$$
\begin{gathered}
\max \left\{p_{1}, p_{2}, \ldots ., p_{n}\right\} \leq P\left(A_{1} \cup A_{2} \cup \ldots \cup A_{n}\right) \leq \\
\min \left\{1, p_{1}+p_{2}+\ldots \ldots+p_{n}\right\} \\
\max \left\{0, p_{1}+p_{2}+\ldots .+p_{n}-n+1\right\} \leq \\
P\left(A_{1} \cap A_{2} \cap \ldots . \cap A_{n}\right) \leq \min \left\{1, p_{1}, p_{2}, \ldots . ., p_{n}\right\}
\end{gathered}
$$

where the first relation concerns the probabilities $p_{j}$ of possible alternative phenomena $A_{j}$ and the other one concerns those of contemporary phenomena.

More detailed analysis of the Bell inequalities problem may be found in [15]. It has been possible to conclude that these inequalities have been valid only in the classical physics, not in any kind of quantum theory. They have been mistakenly applied to experiment where photon polarizations have been measured while this possibility has been excluded by the assumption on the basis of which they were derived.

The consequences following from this fact have been summarized in [16]; more complete summary of all problems of contemporary quantum mechanics has been then presented in 
[17]. It has followed from these results that the Schrödinger equation itself may be denoted as common theory of all physical reality when the Hilbert space formed by its solutions has been adapted to given physical system (i.e., correspondingly extended) in contradiction to limiting and deforming conditions imposed by Bohr.

The given conclusion has followed practically from the fact that the Schrödinger equation may be derived for the set of statistical combinations of Hamilton equation solutions when the given set has been limited by a suitable condition (e.g., by Boltzmann statistics); see $[18,19]$. It means that any Schrödinger function $\psi(x, t)$ may represent always a classical state or a statistical combination of such states; see also [20] (or already quoted [17]).

However, the Schrödinger equation (including Coulomb potential only) represents approximative phenomenological theory only. It is not able to explain any emergence of quantum energy states. In such a case some additional properties of individual objects forming a quantized system must be taken into account. E.g., in the case of hydrogen atom any quantum state could not come into being only on the basis of Coulomb force if some further repulsive or contact interaction did not exist between electron and proton that would depend necessarily on dimensions and structures of main constituents. The study of corresponding structures of individual objects should represent, therefore, inseparable part of contemporary quantum physics.

These structures may be derived mainly from the data gained in experiments concerning the collisions between corresponding particles. However, it is not sufficient to look for a phenomenological description of some measured values. More detailed collision models must be made use of in such a case. They must be able to study the dependence on impact parameter value; e.g., at least the so called eikonal model should be made use of. Some results obtained on the given realistic basis will be introduced in the following; it will be discussed how they may be made use of in solving the problems of quantum physics in the next future.

In Sec. 2 we shall start with discussing the significance of the Schrödinger equation, based on the fact that it may be derived in principle from Hamilton equations. The necessity of repulsive force in the emergence of quantum states in closed systems will be then discussed in Sec. 3. The eikonal model of elastic collisions between hadrons and some results obtained for elastic proton-proton collisions in the impact parameter space will be presented in Sec. 4 . The problem of validity of optical theorem on which practically all contemporary elastic collision results have been based will be discussed in Sec. 5. In Sec. 6 new probabilistic ontological model enabling to study the existence of internal proton structures will be applied to experimental data and new results will be presented. In Sec. 7 some metaphysical consequences will be considered. Several open questions will be then mentioned in Sec. 8 .

\section{Schrödinger equation and Hilbert space structure}

The evolution of a physical system consisting of different matter objects may be described with the help of Hamilton equations

$$
\dot{q}_{j}=\frac{\partial H}{\partial p_{j}}, \quad \dot{p}_{j}=-\frac{\partial H}{\partial q_{j}}
$$


where $q_{j}$ and $p_{j}$ are space coordinates and momenta of all individual objects; the corresponding Hamiltonian given by

$$
H=\frac{p_{j}^{2}}{2 m}+V\left(q_{j}\right)
$$

represents the total energy of the given system.

Schrödinger equation may be then written as

$$
i \hbar \frac{\partial \psi(x, t)}{\partial t}=H \psi(x, t)
$$

where $x$ represents the set of coordinates and $q_{j}$ and $p_{j}$ in the Hamiltonian are substituted by operators $q_{o p}^{j}=x_{j}$ and $p_{o p}^{j}=-i \hbar \frac{\partial}{\partial x_{j}}$. Time-dependent physical quantities are then defined as expectation values of corresponding operators

$$
A(t)=\int \psi^{*}(x, t) A_{o p} \psi(x, t) d x
$$

where $A_{o p}$ is represented by corresponding combination of operators $q_{o p}^{j}$ and $p_{o p}^{j}$.

It has been introduced in the preceding section that any solution $\psi(x, t)$ of Schrödinger equation may be identified with a solution of Hamilton equations or with a superposition of these classical solutions. Any function $\psi(x, t)$ at a given $t$ may be then represented by a vector in the correspondingly constructed Hilbert space. This Hilbert space must consist of several mutually orthogonal Hilbert subspaces, each of them being spanned on the set of Hamiltonian eigenfunctions:

$$
H \psi_{E}\left(x_{j}\right)=E \psi_{E}\left(x_{j}\right) .
$$

Two identical $\psi(x, t)$ functions exhibiting opposite time derivatives must belong always to different subspaces (i.e., incoming and outgoing states of evolving physical system must be taken as fundamentally different). The arrangements of total Hilbert spaces differ somewhat for continuous and discrete parts of Hamiltonian energy spectrum; corresponding details being found in [21, 22].

In the system of two free particles in their center-of-mass system, e.g., the Hilbert space must consist of two mutually orthogonal Hilbert subspaces (see also [23, 24]) being formed by incoming and outgoing states:

$$
\mathcal{H} \equiv\left\{\Delta^{-} \oplus \Delta^{+}\right\} ;
$$

the given subspaces being mutually related with the help of evolution operator

$$
U(t)=e^{-i H t}
$$


The individual vectors of evolution trajectory correspond then to the different expectation values of time operator $T$ fulfilling the condition

$$
i[H, T]=1
$$

where the state corresponding to zero value of $T$ may be arbitrarily chosen; being usually attributed to the state when mutual particle distance (impact parameter) is minimal. Eq. (8) defines the time unit for a corresponding physical system; or for any subsystem that evolves independently of the whole greater system.

Any time evolution trajectory may be characterized by the Hamiltonian expectation value E that is conserved during the whole evolution. It is, of course, also the expectation value of the angular momentum operator

$$
M^{2}=M_{i} M_{i}, \quad M_{i}=\varepsilon_{i j k} q_{o p}^{j} p_{o p}^{k}
$$

commuting with the Hamiltonian

$$
\left[M^{2}, H\right]=0
$$

that is conserved. In principle it holds also $\left[M_{i}, H\right]=0$ and $\left[M_{i}, M^{2}\right]=0$; thus one of components $M_{i}$ should be also conserved. However, its value depends on the orientation of the coordinate system. It is always possible to choose it so that the expectation value of given $M_{i}$ is maximal. It means that any evolution trajectory of a given physical system is defined in addition to energy $E$ also by the value of $M^{2}$; and in principle also by the sign of corresponding $M_{i}$.

As to the closed physical systems they are standardly taken as corresponding to the discrete part of Hamiltonian spectrum. In such a case the Hilbert space should be at least doubled in comparison to Eq. (6) as two different kinds of incoming and outgoing states exist; for more details see [21, 22]. Some other problems concerning closed physical systems (e.g., their emergence) will be discussed in the next section.

Let us return, however, to the problem of free two-particle system. It is necessary to mention one additional possibility when two incoming particles having corresponding kinetic energy may form an unstable object that decays after a short time. In such a case the Hilbert space may be further extended:

$$
\mathcal{H} \equiv\left\{\Delta^{-} \oplus \Theta \oplus \Delta^{+}\right\}
$$

where $\Theta$ may represent the object (or physical system) arisen by merging of two incoming particles (belonging to $\Delta^{-}$subspace). This new object (or system) may be stable or (according to available free energy) unstable decaying into a state lying in $\Delta^{+}$or creating a further kind of physical systems or objects. The terms representing corresponding transition probabilities between different Hilbert subspaces must be added to earlier (classical) potential between two particles.

In this case it is, of course, necessary to respect always that the evolution goes in irreversible way. Evolution of the pair of colliding particles is described in the beginning in $\Delta^{-}$, particles 
going nearer one to the other (incoming). In dependence on impact parameter value they are scattered by mutual potential and continue in $\Delta^{+}$as outgoing pair. However, in the case of small impact parameter values (at corresponding energy values) they may form also one common object being represented by a vector in Hilbert subspace $\Theta$. The corresponding evolution may be described, of course, at the present on phenomenological level only as we do not know actual internal dynamics of arising object.

The object represented by the subspace $\Theta$ may be stable (e.g., creation of atom from nucleus and electron) or unstable (decaying in the same particle pair or in another one). At much higher energy values many other particles may be, of course, formed. In such a case the subspace $\Theta$ is to be substituted by a more complex system of products (or sums) of corresponding subspaces describing further evolution of separated physical systems.

While the simple subspaces (in which the interacting particles remain stable) may be in principle described with the help of corresponding Schrödinger equations the other processes require to be characterized by additional probabilities between concrete states in individual subspaces. There is not any interference between amplitudes from different orthogonal subspaces.

The individual (stable as well as unstable) objects represent closed physical systems that are characterized by some quantum physical values. Each object has some internal dynamics (eventually, exists in some different internal states - stable or unstable). And just these questions represent evidently one of the main problems of the future quantum physics.

\section{Closed systems and quantum states}

The idea of quantum states has been based on experimental data concerning the measured light spectra emitted by excited atoms, as it was formulated in two phenomenological postulates of N. Bohr [25]. These spectra have been correlated to transitions between different quantum energy levels. The existence of quantum states have been then derived with the help of Schrödinger equation containing Coulomb potential.

However, it is necessary to call attention to the fact that the Schrödinger equation provides an approximative phenomenological description of quantum phenomena only. It predicts and admits the existence of quantum states but it cannot explain at all how such a quantum state may arise when two corresponding objects (forming then the closed system) are mutually attracted and go always nearer one to the other. It is evident that the emergence of quantum state is not possible without the conjoint existence of short-ranged repulsive (or contact) force acting between these objects, too. One should expect that both the corresponding potentials (forces) are to be responsible for the final effect.

In the standard approach the corresponding quantum states are represented by special trajectories of electrons orbiting around a nucleus. It means that the quantum energy value should be correlated to a special value of angular momentum. It should be correlated also to dimensions of the physical system in the given state (and also to the dimensions of individual objects), which does not seem to have been analyzed sufficiently until now. It relates probably to the emergence of quantum states, which represents open question, too.

Let us start with this last problem in the case of hydrogen atom consisting of one electron and one proton. A stable hydrogen atom should arise always when a slowly moving electron 
appears in the neighborhood of a proton. The electron is attracted to a proton and it is evident that the given atom might be hardly formed if a repulsive (short-ranged) force between electron and proton did not exist. Some contact linkage of these two objects (e.g., some adhesive force between them) should be also taken into consideration.

At the present any force is being interpreted as the result of some potential. In such a case it might be expected that corresponding quantum states correspond to the distance when both these potentials compensate:

$$
V(x)=V_{\text {Coul }}(x)+V_{\text {rep }}(x) \cong 0 .
$$

The corresponding quantum states should then depend on the shapes of these two potentials. And we should ask how they might be influenced, e.g., by the proton or by its internal states that might be changeable as one must expect for the proton to exhibit some internal dynamics. The other question concerns then the problem how the energy of emitted photon is determined to correspond to the difference of corresponding quantum energy values and further which atom constituent emits the given photon.

It seems, therefore, that to expect for the quantum states to be mediated by some potential acting at the distance may be hardly convenient. The other possibility, i.e., the existence of weak adhesive force between electron and proton, might be more acceptable. In such a case the properties of hydrogen atom should follow from the properties of proton and its internal dynamics. The changes in the given adhesive linkage might be then responsible also for the energy of emitted photons.

In both the cases it is, of course, necessary to expect that the quantum states of hydrogen atom are to be fundamentally dependent on the dimensions and structure of proton. One must, therefore, assemble and analyze all corresponding characteristics that may be derived from available experimental data.

The characteristics of proton may be studied mainly with the help of experiments concerning the collisions between electrons and protons or between two protons. Unfortunately, in the corresponding analyzes only some mathematical models are usually used that describe some average phenomenological characteristics of given particles, which can hardly contribute to understanding the discussed problems of quantum physics. The models respecting the size and structures of individual particles must be made use of.

In the last time we have studied the elastic proton-proton collisions at higher values of collision energy using the eikonal model where the probability of different processes in the dependence on impact parameter values may be derived [26]; some conclusions differing from earlier ones having been obtained. First of all it has been demonstrated that there is not any reason for arguing that the elastic processes should be central, i.e., existing at very small (even zero) values of impact parameter. When any limiting condition (facilitating the calculations) has not been imposed the elastic collisions may be interpreted as peripheral in full agreement with ontological interpretation of microscopic objects; see more details in the next section. 


\section{Proton-proton collisions in impact parameter space}

In this section we shall present some results of elastic proton-proton collisions in the impact parameter space obtained earlier. The corresponding experimental data are represented by elastic differential cross section that is in the given case given by two different mutual interactions: Coulomb and strong ones. If the influence of proton spins is neglected one measures and establishes the dependence $\frac{\mathrm{d} \sigma^{C+N}(s, t)}{\mathrm{d} t}$ where $s$ is the square of center-of-mass energy and $t$ is the square of center-of-mass four-momentum transfer (it is zero or negative and it is a function of scattering angle).

It is not possible to measure the influence of individual interactions separately. In the standard theoretical framework any collision process is regarded as fully described provided its complex scattering amplitude is given. The measured differential cross section is written as

$$
\frac{\mathrm{d} \sigma^{C+N}(s, t)}{\mathrm{d} t}=\frac{\pi}{s p^{2}}\left|F^{C+N}(s, t)\right|^{2}
$$

where $p$ is the three-momentum of incident nucleon in the center-of-mass system $\left(s=4\left(p^{2}+\right.\right.$ $\left.m^{2}\right) ; m$ being mass of a proton); natural units have been used: $\hbar=c=1$. In the case of only hadronic (resp. Coulomb) elastic scattering it is, therefore, necessary to know amplitude $F^{N}(s, t)$ (resp. $F^{C}(s, t)$ ). And one must ask how to express complete amplitude $F^{C+N}(s, t)$ with the help of individual amplitudes $F^{N}(s, t)$ and $F^{C}(s, t)$.

Formulas still standardly used for complete elastic scattering amplitude $F^{C+N}(s, t)$ were derived under several very limiting assumptions. One of such commonly used formulas has been the simplified formula of West and Yennie (WY) published in 1968 [27] which was derived only for very low values of $|t|$ under assumption that the modulus of $F^{N}(s, t)$ was purely exponential in $t$ and the phase of $F^{N}(s, t)$ was $t$-independent; both these assumptions were supposed to be valid in the whole region of kinematically allowed values of $t$ at that time. Detailed discussion concerning both theoretical and experimental problems following from drastic limitation involved in this formula may be found, e.g., in [28].

The question has been raised whether it is possible to derive more general formula without any a priory limitation on $F^{C+N}(s, t)$, which could be used for more relevant analysis of experimental data. It has been possible to remove the most of earlier limitations if eikonal model has been applied to. According to [26] (in 1994) it has been possible to derive on the basis of the eikonal model more general formula for the complete elastic amplitude for any $s$ and $t$ with the accuracy up to terms linear in $\alpha$ and without any a priory restriction on hadronic amplitude $F^{N}(s, t)$.

If this hadronic amplitude $F^{N}(s, t)$ is given then some physically significant quantities may be calculated from it. For example, one may calculate distribution functions of total, elastic and inelastic hadronic collisions in the impact parameter space and if they are determined then one may evaluate mean-squares of impact parameter for corresponding processes. The mean-square of impact parameter in the case of elastic processes may be calculated from corresponding elastic distribution function $D_{e l}(s, b)$ as 


$$
\left\langle b^{2}(s)\right\rangle_{e l}=\frac{\int_{0}^{\infty} b \mathrm{~d} b b^{2} D_{e l}(s, b)}{\int_{0}^{\infty} b \mathrm{~d} b D_{e l}(s, b)} .
$$

and similarly we may define also $\left\langle b^{2}(s)\right\rangle_{\text {tot }}$ and $\left\langle b^{2}(s)\right\rangle_{\text {inel }}$ in the case of total and inelastic hadronic collisions. However, in eikonal model the mean-squares of all these processes may be derived directly from the $t$-dependent elastic hadronic amplitude $F^{N}(s, t)$ without trying to establish the whole distribution functions, which is mathematically much more complicated; detailed discussion how to actually determine all distribution functions on the basis of corresponding experimental data can be found in [29]. For a given hadronic amplitude $F^{N}(s, t)$ one may thus calculate relatively easily root-mean-squares $\sqrt{\left\langle b^{2}(s)\right\rangle_{e l}}$ and $\sqrt{\left\langle b^{2}(s)\right\rangle_{\text {inel }}}$ and compare both the values. If the value $\sqrt{\left\langle b^{2}(s)\right\rangle_{e l}}$ is lesser than $\sqrt{\left\langle b^{2}(s)\right\rangle_{\text {inel }}}$ then it would mean that elastic hadronic processes should be realized in average at lower impact parameter values than inelastic processes; the protons should be rather "transparent" which might be hardly acceptable from the ontological point of view. We may denote this situation as "central" behavior of elastic scattering. If the value $\sqrt{\left\langle b^{2}(s)\right\rangle_{e l}}$ is greater than $\sqrt{\left\langle b^{2}(s)\right\rangle_{\text {inel }}}$ then we denote situation as "peripheral" behavior of elastic scattering; the situation being in agreement with usual interpretation of two matter object collisions.

As already mentioned the corresponding proton-proton collision data have been interpreted with the help of rather simplified phenomenological mathematical models in the past. Some conclusions have been rather surprising. Especially, when it has been concluded that a rather great ratio of elastic processes has corresponded to purely central collisions (i.e., protons could scatter elastically even at impact parameter $b=0$ ). This kind of "transparency" of protons has been denoted already in 1979 in [30] as a "puzzle". In 1981 it has been then shown that the corresponding result has depended mainly on the $t$-dependence of the phase of elastic hadronic scattering amplitude, see [31]. The mentioned central behavior has been derived when the $t$-dependence of the phase has been strongly limited. And it has been shown that if the modulus is purely exponential and hadronic phase is practically $t$-independent in the whole region of kinematically allowed values of $t$ (at arbitrary collision energy $\sqrt{s}$ ) one obtains necessarily the mentioned central behavior of elastic hadronic collisions in the impact parameter space. These two (over)simplified assumptions have been commonly used in many analysis of corresponding experimental data (they are included also in the simplified WY formula).

Consequently, it has been very interesting to put the opposite question: How to modify the given mathematical model to obtain collision processes corresponding to usual ideas. The experimentally established elastic proton-proton differential cross section at energy of $\sqrt{s}=53 \mathrm{GeV}$ has been analyzed in the whole measured $t$-range with the help of more general eikonal formula used for complete scattering amplitude in [26]. It was possible to obtain acceptable fits for different $t$-dependencies of the phase (according to chosen parametrization). Two quite different dependencies of hadronic phase have been then shown in the quoted paper. The first phase was the so-called "standard" phase (used, e.g., in [32] for interpretation of experimental data) and the second phase corresponded to natural peripheral elastic collisions. Both the hadronic phases are plotted in Fig. 1. The root-mean-squares 


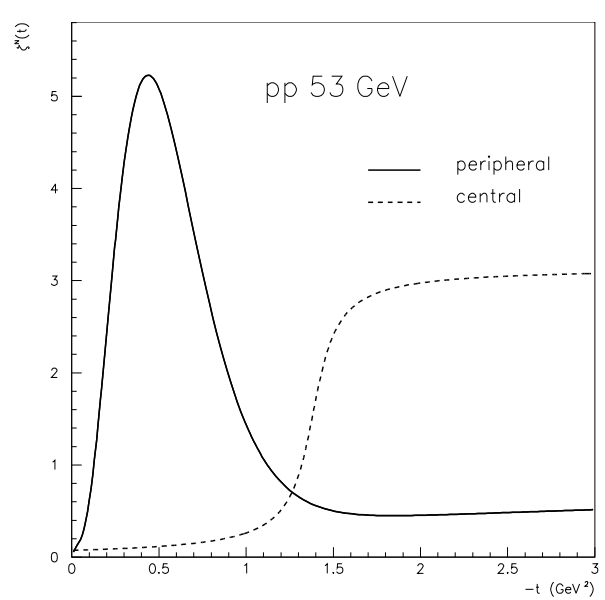

Figure 1. Two different hadronic phases $\zeta^{N}(s, t)$ defined by $F^{N}(s, t)=i\left|F^{N}(s, t)\right| e^{-i \zeta^{N}(s, t)}$ and fitted to corresponding experimental data at energy of $\sqrt{s}=53 \mathrm{GeV}$. Dashed line - standard hadronic phase admitting central elastic hadronic collisions; full line - peripheral phase.

corresponding to the two hadronic phases are then given in Table 1. The case with the standard hadronic phase leads to central elastic hadronic collisions. Similar result as in the case of proton-proton collisions was obtained also in the case of elastic antiproton-proton scattering at energy of $541 \mathrm{GeV}$ (see [26, 29]). The phase which leads to peripheral behavior of hadronic proton-proton collisions has very similar $t$-dependence as that obtained earlier at lower collision energy of $\sqrt{s}=23 \mathrm{GeV}$ in the already quoted older paper [31].

\begin{tabular}{lccccc}
\hline Data & Hadronic phase & Profile & $\sqrt{\left\langle b^{2}(s)\right\rangle_{\text {tot }}}$ & $\sqrt{\left\langle b^{2}(s)\right\rangle_{\text {el }}}$ & $\sqrt{\left\langle b^{2}(s)\right\rangle_{\text {inel }}}$ \\
\hline$p p 53 \mathrm{GeV}$ & peripheral & peripheral & 1.028 & 1.803 & 0.772 \\
$p p 53 \mathrm{GeV}$ & standard & central & 1.028 & 0.679 & 1.087 \\
$\bar{p} p 541 \mathrm{GeV}$ & peripheral & peripheral & 1.140 & 2.205 & 0.609 \\
$\bar{p} p 541 \mathrm{GeV}$ & standard & central & 1.140 & 0.756 & 1.220 \\
\hline
\end{tabular}

Table 1. Values of root-mean-squares (in femtometers) obtained from analysis of experimental data with standard and peripheral hadronic phase.

\begin{tabular}{lccc}
\hline Model & $\sqrt{\left\langle b^{2}(s)\right\rangle_{\text {tot }}}$ & $\sqrt{\left\langle b^{2}(s)\right\rangle_{e l}}$ & $\sqrt{\left\langle b^{2}(s)\right\rangle_{\text {inel }}}$ \\
\hline Bourelly et al. & 1.249 & 0.876 & 1.399 \\
Petrov et al. (2P) & 1.227 & 0.875 & 1.324 \\
Petrov et al. (3P) & 1.263 & 0.901 & 1.375 \\
Block et al. & 1.223 & 0.883 & 1.336 \\
Islam et al. & 1.552 & 1.048 & 1.659 \\
\hline
\end{tabular}

Table 2. Values of root-mean-squares (in femtometers) predicted by different models of proton-proton collisions at collision energy of $14 \mathrm{TeV}$. All the models predicts $\sqrt{\left\langle b^{2}(s)\right\rangle_{e l}}<\sqrt{\left\langle b^{2}(s)\right\rangle_{\text {inel }}}$, i.e., central behavior of elastic scattering.

The elastic and inelastic root-mean-squares have been calculated in [28] also for several relatively new phenomenological models proposed for elastic hadronic proton-proton scattering at energy of $14 \mathrm{TeV}$ (planned ultimate LHC collision energy). In all these cases it has been found that $\left\langle b^{2}(s)\right\rangle_{e l}<\left\langle b^{2}(s)\right\rangle_{\text {inel }}$, i.e., all these models correspond to central behavior of proton collisions, as shown in Table 2 . 
One of the results obtained in [26] was that the modulus of hadronic amplitude $\left|F^{N}(s, t)\right|$ was practically uniquely determined from corresponding measured elastic differential cross section while the hadronic phase remained quite undetermined, being only slightly limited due to Coulomb interaction. It should be determined on the basis of other physical requirements, not on the basis of some arbitrary assumptions. The results gained in elastic proton-proton collisions might be very helpful for nuclear and particle physics in general. However, practically in all current descriptions of proton-proton elastic scattering the validity of optical theorem which relates the imaginary part of hadronic amplitude to total hadronic cross section, has been taken as a basic assumption. The given problem will be discussed in the next section, as the given validity does not seem to be sufficiently reasoned.

As to the other problem mentioned in the end of preceding section (the use of mathematical models representing only average structure of colliding objects) it has been at least partially removed when the more general eikonal model has been applied to and the results in dependence on impact parameter values have been established. However, the main character of some average phenomenological characteristics obtained from hitherto phenomenological models remains, which can hardly contribute to understanding the results of contemporary quantum physics. A new more detailed model of proton-proton elastic collisions with realistic behavior of the collisions in the impact parameter space and respecting the sizes and structures of individual colliding particles will be formulated and applied in preliminary form to experimental data in Sec. 6 .

\section{Optical theorem and its validity}

Practically all hitherto mathematical models of proton collision processes (including the eikonal model described in Sec. 4) have been based on the assumption of optical theorem validity. This theorem has been taken from optical approaches where the total cross section was correlated to the imaginary part of complex index of refraction (see, e.g., the description of the given problem in [35]) and the behavior of light was studied on wave basis. The optical theorem has been applied then also to elastic particle collisions when the wave behavior has been attributed to all physical objects.

The approaches of deriving the validity of optical theorem in particle collisions have been summarized recently, e.g., in [36]. The goal of these approaches has consisted in deducing that it has held for the total cross section

$$
\sigma^{t o t}(s)=\sigma^{e l}(s)+\sigma^{i n e l}(s)=\frac{4 \pi}{p \sqrt{s}} \Im F(s, t=0)
$$

where the function $F(t)$ defines the elastic differential cross section

$$
\frac{d \sigma}{d t}=\frac{\pi}{s p^{2}}|F(s, t)|^{2}
$$

It holds then also

$$
F(s, t)=2 \pi \sqrt{s} \int_{0}^{\infty} b d b J_{0}(b \sqrt{-t}) \Gamma(b) .
$$


where $\Gamma(b)$ is the profile function in impact parameter space characterizing the probability of elastic processes in dependence on impact parameter values and on their initial distribution.

Until now there is not any reliable theory of elastic scattering and only different phenomenological models are practically available. The complex function

$$
F(s, t)=i|F(s, t)| e^{-i \zeta(s, t)}
$$

is being, therefore, derived usually from corresponding experimental data. However, from them only the modulus may be established while the phase $\zeta(t)$ remains quite undetermined. It is to be predicted on the basis of additional assumptions that may influence strongly the conclusions concerning the value of total cross section. Also the optical theorem has been practically always involved even if it has been derived on the basis of other additional assumptions that have not corresponded to actual characteristics of strong interactions as it was mentioned already earlier in [37].

In all attempts to derive the validity of optical theorem in particle collisions the standard approaches based on the Copenhagen quantum mechanics have been made use of; especially, the summation of amplitudes used commonly in S-matrix theory has been applied to. The states belonging to two mutually orthogonal Hilbert subspaces $\Delta^{-}$and $\Delta^{+}$(see Sec. (2)), i.e., the states at time $\tau=-\infty$ and at time $\tau=+\infty$, were taken as lying in one common Hilbert space and corresponding amplitudes were superposed, which cannot be done if the ontological approach is to be respected (i.e., time evolution described correctly with the help of time-dependent Schröedinger equation). It may be then denoted as surprising that the optical theorem has been applied to strong interaction only and never considered in the case of Coulomb interaction, even if the given derivation approach does not relate to a kind of interaction.

The final effect in collision processes of two protons is to depend on the conditions in individual events characterized mainly by corresponding impact parameter value. However, only the Coulomb elastic scattering comes into account for greater values of $b$ where short-ranged (or contact) strong interaction cannot exist while for lower values of $b$ elastic interaction (Coulomb or hadronic) may exist together with inelastic one. Consequently, as to the strong interaction the particles may continue in original motion practically without any disturbance; it means that such states represent a special set of states in the subspace $\Delta^{+}$which cannot be added if the total cross section corresponding to strong interaction is considered. Only the states scattered elastically by strong interaction may be involved.

It has been mentioned that for a given collision energy $\sqrt{s}$ only the modulus of hadronic amplitude $F(s, t)$ may be established from experimental data and the hadronic phase $\zeta(s, t)$ may be practically arbitrary. It means that very different characteristics may be attributed to elastic particle collisions; see the problem of centrality and peripherality of elastic hadronic processes discussed in preceding section. The choice of phase $t$-dependence has been then fundamentally influenced also by the application of the optical theorem, mainly in the region of very low values of $|t|$ where the measurement cannot be done. Even the separation of Coulomb and strong interactions might be fundamentally influenced when the parametrization of the strong part of scattering amplitude has allowed only the differential cross section decreasing monotony from the maximum value at $t=0$. In the case of 
short-ranged (contact) strong interactions it is necessary to admit that the maximum value may lie at non-zero value of $t$ as it will be demonstrated in the next section.

\section{Probabilistic ontological model of elastic proton-proton collisions}

It follows from the preceding that the Schrödinger equation leads to the same results as classical physics. However, in the classical physics based fully on ontological approach the individual matter objects represent always only the source of corresponding potentials mediating corresponding interactions. It may be applied fully to electromagnetic or gravitational forces. The strong (and also weak) interactions differ, however, rather strongly from these interactions. The short-ranged effect of the latter ones indicates that these forces should be denoted rather as contact ones; being zero at any greater distance. It means that in elastic proton-proton collisions (especially, at higher energy values) there is not practically any actual interference between Coulomb and strong interactions. In any event only one of these interactions is effective according to impact parameter value and the corresponding probabilities of individual contributions to elastic differential cross section may be added. However, in such a case it is necessary to take into account possible proton dimensions in collision instant. According to contemporary knowledge protons consist evidently of other objects that must be in mutual interaction. It is also necessary to expect that the particles having special value of spin must exhibit some internal dynamics. We are to accept that the proton may exist in different spontaneously changeable internal states that may have also different shapes and dimensions (as it has been proposed earlier in [38]). And we shall attempt to test whether these dimensional characteristics may become evident in elastic collision characteristics.

To make use of the given idea in the analysis of elastic proton-proton processes let us assume that a colliding proton may exist in $n$ different states, each being characterized by maximal possible dimension $d_{k}\left(d_{1} \geq \ldots \geq d_{k} \geq \ldots \geq d_{n}\right)$. The corresponding probabilities that proton is in such a state in the moment of interaction are then $p_{k}\left(\sum_{k} p_{k}=1\right)$.

If two such protons or other similar objects collide the different pairs of states may interact; the individual probabilities of such collision channels (or collision states) being equal to

$$
r_{k, l}= \begin{cases}p_{k} p_{l} & \text { if } k=l \text { or the colliding object are not identical } \\ 2 p_{k} p_{l} & \text { if } k \neq l \text { and the colliding object are identical, } k<l\end{cases}
$$

The factor 2 in the last relation follows from the fact that for $k \neq l$ and identical colliding objects the cases with interchanged collision types $k, l$ and $l, k$ are the same and the corresponding probabilities may be summed but we need to introduce convention $k<l$ to count each distinct collision type only once.

\subsection{Nuclear and Coulomb scattering}

As to the strong interactions (taken as contact ones) the maximum effective impact parameter for which two protons may still interact hadronically in corresponding collisions will be then equal to

$$
B_{k, l}=\left(d_{k}+d_{l}\right) / 2
$$


The two indexes $k$ and $l$ in $r_{k, l}$ and $B_{k, l}$ may be for convenience substituted by only one index $j$ using a one-to-one correspondence $(k, l) \leftrightarrow j$. In other words, if one object in a state $k$ collide with an another one in a state $l$ then this always implies unique collision type $j$ and vice versa. It is obvious that $\sum_{j} r_{j}=1$.

It is necessary to expect that for each $j$-th collision channel at any given impact parameter $b<B_{j}$ the value of scattering angle (or equivalently $|t|$ ) of two elastically (and hadronically only) scattered protons will belong to a limited interval of values beginning always with zero value. The corresponding frequency of individual values of scattering angle will go to a maximum and will diminish again to zero; the interval being reduced to one point at $b=B_{j}$. However, in the following very preliminary (and simplified) analysis of experimental data we have substituted the given interval by the corresponding mean value. Let us denote the corresponding function as $\bar{t}_{j}(b)$ which smoothly fall from zero to some lower negative values when $b$ will change from $B_{j}$ to lower values. The inverse function will be denoted as $\bar{b}_{j}(t)$.

We may write for partial elastic hadronic cross section corresponding to a $j$-th collision state following expression

$$
\frac{d \sigma_{j}^{N}(t)}{d t}=2 \pi P_{j}^{e l}\left(\bar{b}_{j}(t)\right) \bar{b}_{j}(t) \frac{d \bar{b}_{j}(t)}{d t}
$$

where the factor $P_{j}^{e l}(b)$ represents individual probability of elastic processes at corresponding impact parameter values $b$ if cylindrical symmetry in impact parameter dependence has been assumed. The elastic hadronic differential cross section is then given by the sum of individual contributions from all the collision states $j$ :

$$
\frac{d \sigma^{N}(t)}{d t}=\sum_{j} r_{j} \frac{d \sigma_{j}^{N}(t)}{d t} .
$$

Parameters $r_{j}$ in the last relation represent the weights (probabilities) of individual collision channels.

The behavior of the given pair of two colliding protons depends then on probabilities $p_{k}$ of individual states, their dimension values $d_{k}$ and on two series of functions $P_{j}^{e l}(b)$ and $\bar{b}_{j}(t)$ (or $\bar{t}_{j}(b)$ ). Their values or shapes are to be derived from corresponding experimental data. However, the function $P_{j}^{e l}(b)$ may be expressed as the product of two functions

$$
P_{j}^{e l}(b)=P_{j}^{\text {tot }}(b) P_{j}^{r a t}(b)
$$

where $P_{j}^{t o t}(b)$ is the probability of any mutual hadronic particle interaction (elastic as well as inelastic) at impact parameter $b$ corresponding to a $j$-th collision type while $P_{j}^{\text {rat }}(b)$ represents the corresponding ratio of elastic hadronic processes from all possible hadronic interactions. Both the functions $P_{j}^{t o t}(b)$ and $P_{j}^{r a t}(b)$ are evidently monotonous. The former one is to be non-increasing function of impact parameter $b$ while the latter one is non-decreasing in dependence on $b \in\left\langle 0, B_{j}\right\rangle$. The monotony of the functions brings very important 
simplification in the choice of parametrization of both the new functions; their values moving in the whole interval $\langle 0,1\rangle$. The probability of any inelastic process may be then defined as

$$
P_{j}^{\text {inel }}(b)=P_{j}^{\text {tot }}(b)-P_{j}^{e l}(b)
$$

It must hold always $P_{j}^{\text {tot }}(b)=P_{j}^{e l}(b)=P_{j}^{\text {inel }}(b)=0$ for any $b \geq B_{j}$. This will allow us to integrate in corresponding cases over finite interval of impact parameter $\left\langle 0, B_{j}\right\rangle$ instead of infinite interval $\langle 0, \infty\rangle$. We will use this fact in the following quite frequently; it also simplifies numerical calculations of corresponding expressions.

The (integrated) elastic hadronic cross section may be written with the help of Eq. (20) and Eq. (21) as

$$
\sigma^{e l, N}=\int_{t_{\min }}^{0} d t \frac{d \sigma^{N}(t)}{d t}=\sum_{j} r_{j} \sigma_{j}^{e l, N}
$$

where we have introduced elastic hadronic cross section just for $j$-th collision channel

$$
\sigma_{j}^{e l, N}=2 \pi \int_{0}^{B_{j}} d b P_{j}^{e l}(b) b
$$

In the given model it is possible to derive also total hadronic cross sections for individual collision types $\sigma_{j}^{t o t, N}$ from elastic experimental data without adding any further assumption; it holds

$$
\sigma_{j}^{t o t, N}=2 \pi \int_{0}^{B_{j}} d b P_{j}^{t o t}(b) b .
$$

The total hadronic cross section may be then written again as a sum of contributions from all the collision channels

$$
\sigma^{t o t, N}=\sum_{j} r_{j} \sigma_{j}^{t o t, N}
$$

And using the relation (23) the corresponding values for inelastic cross section may be established, too.

The mutual elastic collisions between two protons at smaller scattering angles (smaller momentum transfers $|t|$ ) are caused, however, not only by strong interactions that may be interpreted practically as contact but also by mutual Coulomb forces acting at distance. One can express then the experimentally measured elastic differential cross section as the sum of the two given interactions

$$
\frac{d \sigma^{C+N}(t)}{d t}=\sum_{j} r_{j} \frac{d \sigma_{j}^{N}(t)}{d t}+\frac{d \sigma^{C}(t)}{d t}
$$


where the Coulomb differential cross section will be established directly by fitting experimental data. It will not be determined with the help of formfactors from the standard Coulomb potential valid for pointlike particles as such approach does not correspond to reality. The infinite Coulomb elastic differential cross section for $t=0$ may be theoretically obtained at infinite distance while in real experiments the zero value of $t$ may exist inside narrow particle beam as combined effect from different surrounding scattering centers. The similar difference may concern, of course, also the frequencies for higher values of $|t|$ due to asymmetric positions of charged partons in individual protons.

In our model we have introduced some free parameters and some unknown functions which are to be determined from corresponding measured elastic differential cross section using formula (28). It is necessary to fit maximal dimensions $d_{k}$ of all the (considered) hadron states and their corresponding probabilities $p_{k}$ at the moment of collision. We also need to parametrize and then to fit three monotonic functions $P_{j}^{t o t}(b), P_{j}^{r a t}(b)$ and $\bar{b}_{j}(t)$ (or $\bar{t}_{j}(b)$ ) for each corresponding $j$-th hadronic collision channel. It is also the Coulomb interaction effect $\frac{d \sigma^{C}(t)}{d t}$ which needs to be determined from data. Several other very interesting and physically significant quantities may be then calculated from these parameters and functions as it has been shown in preceding.

\subsection{Analysis of experimental data}

We shall apply the given probabilistic model as we have already mentioned to the data obtained at ISR at CERN at the energy of $53 \mathrm{GeV}[33,34]$ (the same data as made use of in Sec. 4). We shall try to show that two proton states exhibiting the largest dimensions may be responsible for the part of differential elastic cross section data corresponding to $|t| \in(0 ., 1.25) \mathrm{GeV}^{2}$; see the corresponding part of experimental points shown in Fig. 2. As in the lower part of $|t|$ the density of measured points has been very great only one fifth of experimental points has been pictured in the interval $(0 ., 0.45) \mathrm{GeV}^{2}$.

If one proton in $k$-state $(k=1,2)$ collides with another proton in $l$-state $(l=1,2)$ we may define collision state $j$ using a following one-to-one correspondence $(k, l) \leftrightarrow j(k<l):(1,1) \leftrightarrow 1$, $(1,2) \leftrightarrow 2$ and $(2,2) \leftrightarrow 3$. We have thus three distinct collision types that will be responsible for the given part of elastic differential cross section.

It is, of course, necessary to parametrize suitably the corresponding functions used in the description of the given process. The following parameterizations of three monotonous functions $P_{j}^{t o t}(b), P_{j}^{r a t}(b)$ and $\bar{b}_{j}(t)$ have been used for individual collision channels

$$
P_{j}^{t o t}(b)= \begin{cases}1 & \text { if } 0 \leq b \leq \mu_{0, j} \\ \frac{e^{-\left(\mu_{1, j}\left(b-\mu_{0, j}\right)\right)^{\mu_{2, j}}}\left(1+\mu_{3, j}\right)}{1+\mu_{3, j} e^{-\left(\mu_{1, j}\left(b-\mu_{0, j}\right)\right)^{\mu_{2, j}}}} & \text { if } \mu_{0, j}<b<B_{j} \\ 0 & \text { if } B_{j} \leq b\end{cases}
$$




$$
\begin{gathered}
P_{j}^{r a t}(b)=\frac{e^{-\left(v_{0, j}\left(B_{j}-b\right)\right)^{v_{1, j}}}\left(1+v_{2, j}\right)}{1+v_{2, j} e^{-\left(v_{0, j}\left(B_{j}-b\right)\right)^{v_{1, j}}}} \\
\bar{b}_{j}(t)=B_{j}\left(\frac{2}{\pi} \arccos \left[\left(\frac{|t|}{\eta_{0, j}}\right)^{1 / \eta_{2, j}}\right]\right)^{1 / \eta_{1, j}}
\end{gathered}
$$

where $\mu_{0, j}, \mu_{1, j}, \mu_{2, j}, \mu_{3, j} ; v_{0, j}, v_{1, j}, v_{2, j} ; \eta_{0, j}, \eta_{1, j}, \eta_{2, j} \quad(j=1,2,3)$ are free parameters that are to be determined from experimental data together with parameters $p_{k}$ and $d_{k}(k=1,2)$.

As it has been already mentioned also the Coulomb interaction effect is to be determined from corresponding experimental data. To enable precise fit the following parametrization has been chosen

$$
\frac{d \sigma^{C}(t)}{d t}=\xi_{0}\left(1+\left(\xi_{1}|t|\right)^{\xi_{2}}\right) e^{-\xi_{3}|t|}+\xi_{4} e^{-\left(\xi_{5}|t|\right)^{\xi_{6}}} \frac{1+\xi_{7}}{1+\xi_{7} e^{-\left(\xi_{5}|t|\right)^{\xi_{6}}}}
$$

where $\xi_{i}(i=0, . ., 7)$ are additional free parameters that are to be derived from corresponding experimental data.

The number of free parameters may seem to be rather high. However, this is quite irrelevant at this stage of our research when quite new physical ideas are looked for and tested. The goal of our effort consists in principle in describing the characteristics of partial structures to a sufficient detail, to initiate further analysis with the help of other experiments.

The results of the corresponding fit are shown in Fig. 2. The probabilistic model can be fitted to experimental data practically in the whole considered interval of $|t| \in(0,1.25) \mathrm{GeV}^{2}$. Hadronic differential cross sections $\frac{d \sigma_{j}^{N}(t)}{d t}$ given by Eq. (20) for all the three collision types are plotted in Fig. 2, too. Only their parts given by $r_{j}$ parameters contribute to complete differential cross section $\frac{d \sigma^{C+N}(t)}{d t}$. The Coulomb differential cross section $\frac{d \sigma^{C}(t)}{d t}$ given by parametrization (32) is also shown in Fig. 2.

The following values of individual free parameters have been obtained on the basis of our analysis. The frequencies of two internal states considered in our fit to experimental data having the greatest dimensions are given by

$$
p_{1}=0.48, \quad p_{2}=0.39
$$

and the corresponding dimensions are

$$
d_{1}=2.50 \mathrm{fm}, \quad d_{2}=2.29 \mathrm{fm}
$$



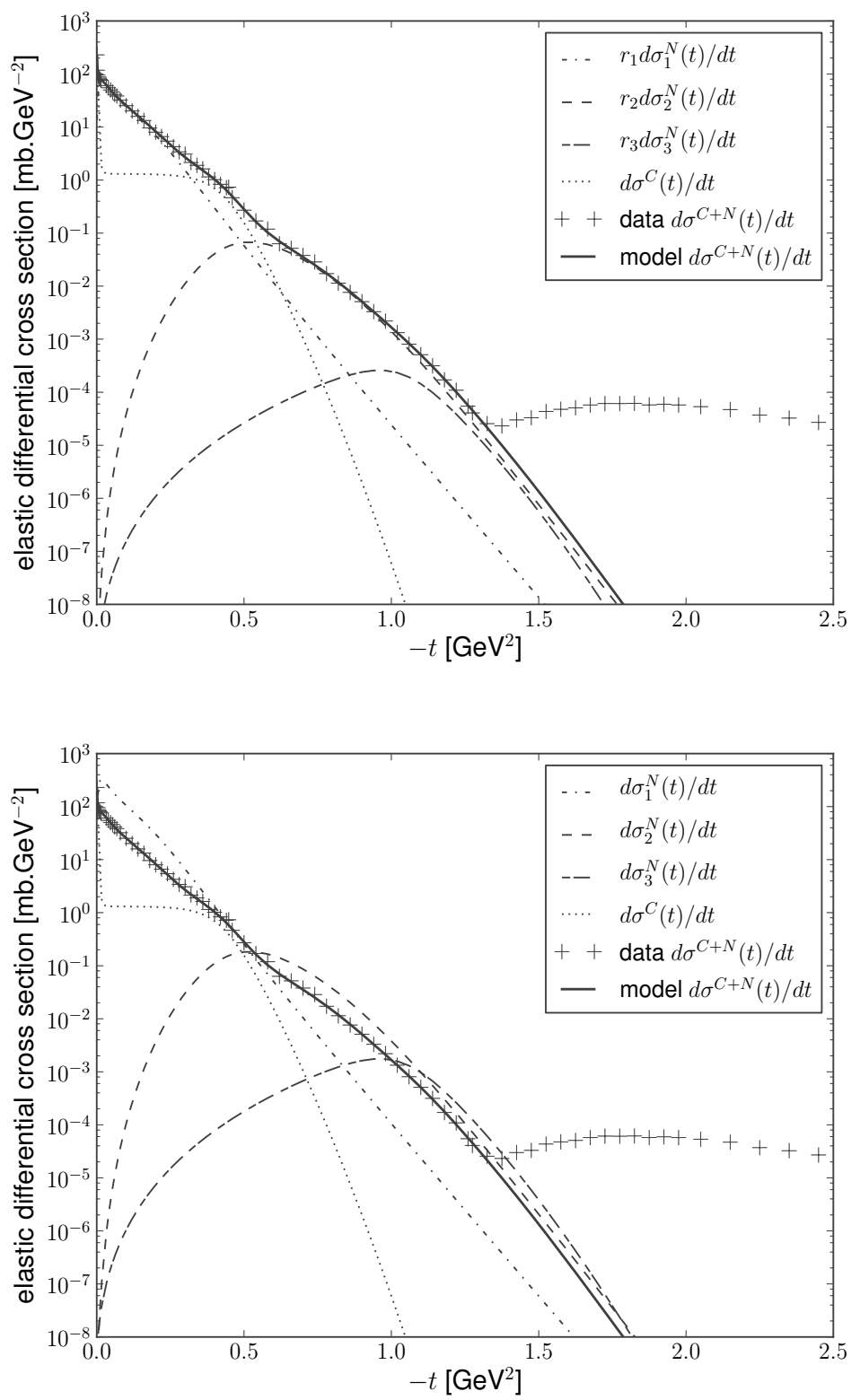

Figure 2. Differential elastic cross sections for proton-proton scattering at energy of $53 \mathrm{GeV}$; individual points - experimental data, full line - our probabilistic model fitted to experimental data, dashed line - Coulomb differential cross section $d \sigma^{C}(t) / d t$, three other lines - individual hadronic collision types (top - contributions $r_{j} d \sigma_{j}^{N}(t) / d t$ to complete differential cross $d \sigma^{C+N}(t) / d t$, bottom - individual differential cross sections $\left.d \sigma_{j}^{N}(t) / d t\right)$.

The functions $P_{j}^{\text {tot }}(b)$ and $P_{j}^{r a t}(b)$ (see Eqs. (29) and (30)) representing the $b$-dependences of probabilities of total and elastic collisions together with function $\bar{t}_{j}(b)$ (inverse function of 
$\bar{b}_{j}(t)$ given by Eq. (31)) are shown in Fig. 3. The values of free parameters in the functions describing the elastic scattering caused by strong interaction are shown in Table 3 for all the three considered collision states.

\begin{tabular}{cllccc}
\hline & & \multicolumn{1}{c}{$\mathbf{j}$} & $\mathbf{1}$ & $\mathbf{2}$ & $\mathbf{3}$ \\
\hline \multirow{4}{*}{$P_{j}^{\text {tot }}(b)$} & $\mu_{0, j}$ & {$[\mathrm{fm}]$} & 0.70 & 0.59 & 0.50 \\
& $\mu_{1, j}$ & {$\left[\mathrm{fm}^{-1}\right]$} & 0.95 & 2.35 & 4.34 \\
& $\mu_{2, j}$ & {$[1]$} & 5.90 & 2.55 & 1.69 \\
& $\mu_{3, j}$ & {$[1]$} & 193. & 995. & 15. \\
\hline \multirow{3}{*}{$P_{j}^{\text {rat }}(b)$} & $v_{0, j}$ & {$\left[\mathrm{fm}^{-1}\right]$} & 2.03 & 2.40 & 1.80 \\
& $v_{1, j}$ & {$[1]$} & 4.34 & 3.38 & 5.65 \\
& $v_{2, j}$ & {$[1]$} & 20.7 & 144. & 3742. \\
\hline \multirow{3}{*}{$\bar{b}_{j}(t)$} & $\eta_{0, j}$ & {$\left[\mathrm{GeV}^{2}\right]$} & 24.8 & 26.8 & 35.3 \\
& $\eta_{1, j}$ & {$[1]$} & 0.97 & 0.44 & 0.47 \\
& $\eta_{2, j}$ & {$[1]$} & 5.15 & 2.63 & 2.85 \\
\hline
\end{tabular}

Table 3. Values of free parameters of monotonous functions $P_{j}^{\text {tot }}(b), P_{j}^{r a t}(b)$ and $\bar{b}_{j}(t)$ for all the three collision types given by parametrizations (29), (30) and (31).

The additional effect caused by electric charges is then characterized by function (32) that contains some further free parameters. Their values obtained by fitting the given experimental data are

$$
\begin{gathered}
\xi_{0}=491 \mathrm{mb} \cdot \mathrm{GeV}^{-2}, \xi_{1}=265 \mathrm{GeV}^{-2}, \xi_{2}=3.70, \xi_{3}=742 \mathrm{GeV}^{-2}, \\
\xi_{4}=1.32 \mathrm{mb} \cdot \mathrm{GeV}^{-2}, \quad \xi_{5}=6.14 \mathrm{GeV}^{-2}, \xi_{6}=1.70, \quad \xi_{7}=106 .
\end{gathered}
$$

Once we have determined all the free parameters and unknown functions from the fit we may calculate several other physically significant quantities. Let us start with quantities which we can calculate for all the three individual collision states considered in our fit.

The parameters $r_{j}$ (see Eq. (18)) determining the contribution of individual channels (their probabilities) are in Table 4 . Corresponding maximal effective impact parameters $B_{j}$ for which protons might still interact via hadronic interaction calculated from Eq. (19) are shown, too; they are around $2.4 \mathrm{fm}$ slightly different for each collision type $j$. Further total, elastic and inelastic hadronic cross sections having been calculated for all three collision states $j$ are introduced in Table 4.

According to our very very rough model a colliding proton may be in one of two considered internal states with probability $p_{1}+p_{2}=0.86$. It means that in $14 \%$ of cases the proton is to be in different internal states with different maximal dimensions. The proton collisions go then in the given three channels with the probability $\sum_{j=1}^{3} r_{j}=p_{1}^{2}+2 p_{1} p_{2}+p_{2}^{2}=0.75$. The additional internal states may be responsible for measured differential cross section outside our considered $t$-range (for $|t|>1.25 \mathrm{GeV}^{2}$ ); partially also in combination with two already considered states, which is in agreement with preliminary tests done already by us, too. It means that the actual total and inelastic cross sections will be higher than the values 

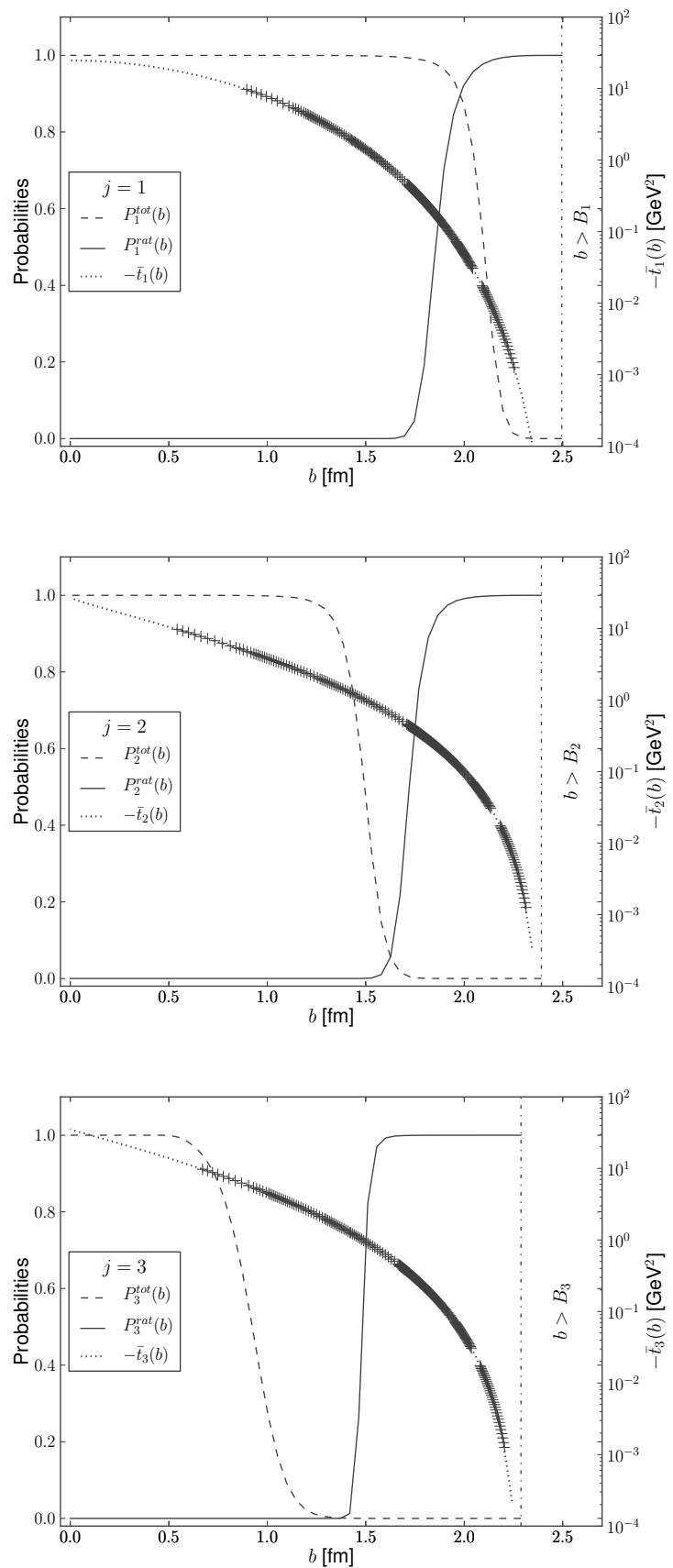

Figure 3. Functions $P_{j}^{\text {tot }}(b)$ and $P_{j}^{\text {rat }}(b)$ with opposite monotony (dashed and full lines) and functions $\left|\bar{t}_{j}(b)\right|$ (dotted line) for individual collision types (top $j=1$, middle $j=2$, bottom $j=3$ ). Individual points lying on $\left|\bar{t}_{j}(b)\right|$ curves correspond to values of impact parameters $b$ at experimentally established values of $t$ calculated using functions $\bar{b}_{j}(t)$. 


\begin{tabular}{cccccc}
\hline $\mathrm{j}$ & & $\mathbf{1}$ & $\mathbf{2}$ & $\mathbf{3}$ & $\sum_{j=1}^{3}$ \\
$\mathrm{k}, 1$ & & $\mathbf{1 , 1}$ & $\mathbf{1 , 2}$ & $\mathbf{2 , 2}$ & 0.75 \\
\hline$r_{j}$ & {$[1]$} & 0.23 & 0.37 & 0.15 & 0.75 \\
$B_{j}$ & {$[\mathrm{fm}]$} & 2.50 & 2.39 & 2.29 & - \\
\hline$\sigma_{j}^{\text {tot,N }}$ & {$[\mathrm{mb}]$} & 137. & 69.7 & 27.1 & - \\
$\sigma_{j}^{\text {el,N }}$ & {$[\mathrm{mb}]$} & 28.1 & $6.2 \times 10^{-2}$ & $7.2 \times 10^{-4}$ & - \\
$\sigma_{j}^{\text {inel }}$ & {$[\mathrm{mb}]$} & 109. & 69.7 & 27.1 & - \\
\hline$r_{j} \sigma_{j}^{\text {tot,N }}$ & {$[\mathrm{mb}]$} & 31.5 & 25.7 & 4.0 & 61.2 \\
$r_{j} \sigma_{j}^{\text {el,N }}$ & {$[\mathrm{mb}]$} & 6.5 & $2.3 \times 10^{-2}$ & $1.1 \times 10^{-4}$ & 6.5 \\
$r_{j} \sigma_{j}^{\text {inel }}$ & {$[\mathrm{mb}]$} & 25.0 & 25.7 & 4.0 & 54.7 \\
\hline
\end{tabular}

Table 4. Values of some physically significant quantities obtained as a result of the probabilistic ontological model fitted to corresponding experimental data at energy of $53 \mathrm{GeV}$.

introduced in Table 4: $\sigma^{t o t, N}>\sum_{j=1}^{3} r_{j} \sigma_{j}^{t o t, N}=61.2 \mathrm{mb} \quad$ (resp. $\sigma^{i n e l, N}>\sum_{j=1}^{3} r_{j} \sigma_{j}^{i n e l, N}=$ $54.7 \mathrm{mb}$ ), while the increase of elastic cross section may be neglected. The given values may be, of course, influenced by the very rough approximation neglecting the distribution of momentum transverses at any impact parameter value.

It is evident that according to our probabilistic model the Coulomb effect appears as significant till $|t| \cong 0.5 \mathrm{GeV}^{2}$, which might throw quite new light to the structure of charge distribution in individual protons and contribute fundamentally to our understanding of the internal structure of hadron objects.

The presented model has been based, of course, on one very simplifying and rough assumption $\left(\bar{t}_{j}(b)\right.$ - relating always only one momentum value to each impact parameter), which might significantly influence the corresponding numerical results. We are working already on the model that will respect the existence of momentum transfer spectrum for any impact parameter value, which should allow much more realistic fit of experimental data on the given basis. Also the fitting of experimental data at other energy values will be performed; the fits for higher values of $|t|$ will be done, too.

\section{Contemporary quantum physics and metaphysics}

We have mentioned already in the preceding that the new results require for the physicists to return to ontological basis the classical physics was based on. This basic approach changed fundamentally in the beginning of modern period when Descartes formulated his mathematically-philosophical concept in which any linkage between human knowledge and ontological approach to matter world was practically excluded. Any knowledge of matter existence started to be based fully on human thinking. Also the participation of human senses was excluded at that time, which was criticized by some philosophers. It led then to the positivism that influenced fully the thinking of European society in the course of the 19th century.

In the middle of the 19th century the given thinking dominated also scientific approaches and scientific knowledge. Boltzmann started it when he denoted one phenomenological characteristics of the system consisting of a great number of particles as natural law. It is possible to say that the given way was accomplished when Bohr formulated his Copenhagen quantum mechanics in 1927. He started from Schrödinger equation proposed in 1925 which 
itself was fully acceptable as the same results followed from it as from the classical concept of Galileo and Newton (only the set of corresponding states was smaller); see short paragraph (with corresponding quotations) in Sec. 1. However, Bohr deformed significantly physical conclusions (following from proper Schröedinger equation) by adding further very strong assumptions without any actual reason, which influenced fundamentally the evolution of quantum physics in the 20th century.

The corresponding (quantum) era of physical research started in principle at the break of the 19th and 20th centuries when black-body radiation was being intensively studied and at the same time new phenomena at the level of microscopic world were discovered (X and gamma radiations and electron (1895-1897)); and further, quantum energy transfer (1900) and photon existence (1904) were predicted. A broad space was opened for formulation of new hypotheses that could not be tested directly but only on the basis of indirect effects established with the help of macroscopic measuring devices. In such a situation the phenomenological models seemed to be very advantageous.

However, to understand the possibility of scientific knowledge it is necessary to realize what are the approaches of scientific research. As to the region of physical research it is possible to say that it is based on falsification approach. The basic step consists then in formulating some more general statements or unifying hypotheses with the help of our reason on the basis of observation and measurement; with the help of the approach making use of logical induction, or intuition. The goal of further approaches and analyses consists then in deriving all possible consequences that follow from a given hypothesis (or a set of hypotheses). As such hypotheses start always from a limited set of our pieces of knowledge it is clear in some cases immediately that they cannot sustain in further considerations. Generally, it is necessary to derive for any hypothesis all possible consequences with the help of logical deduction and to analyze, whether some logical contradictions between individual consequences do not exist, and further to compare these consequences to all possible observations of natural phenomena. If a contradiction is found the given hypothesis (or a set of hypotheses) must be refused, resp. modified, so as the given contradiction might be removed. If one does not come after sufficient falsifying effort to any contradiction the given set of hypotheses may be denoted as plausible; and it is possible to start from it in further considerations about the natural laws concerning the world and human being.

It follows from the preceding that the falsification approach represents important and practically basic knowledge method based on human reason, as K. Popper (1902-94) presented in the first half of the 20th century. One is never justified to denote our reason knowledge for a verified truth, as one can never grant that in following steps a logical contradiction or a contradiction to nature observation will not appear. On the other side one must accept any non-falsified hypothesis as plausible, even if it is in contradiction to another plausible hypothesis. All these statements or hypotheses must be fully tolerated. The decision concerning the preference of one of them must be left to other logical and experimental analyses. On the other side in contradiction to contemporary requirements of some human groups any falsified statement cannot be tolerated and must be decisively refused.

The preceding arguments have thrown new light also to the principle of falsifiability propagated in the region of physical research in the last century. The requirement of falsification tests to any statement has been interpreted as the possibility to prove the validity 
of the given statement, which contradicts the possibilities of our reason knowledge. At least some positive test should be necessary to admit the given statement as plausible; however, it can never represent any proof of its validity.

In the 20th century purely phenomenological view was taken as the knowledge basis and any ontological aspects have not been respected. Evidently mutually contradicting assumptions were being applied especially to elastic collision processes. It follows from these facts that the corresponding metaphysical view must be based on ontological view respecting fully our experience with matter nature.

\section{Some open questions}

One of the most important pieces of knowledge introduced in preceding sections is the fact that there is fundamental difference between Coulomb forces and strong (nuclear) interaction; the former acting at a distance and the latter representing a contact force. To interpret this force as the effect of some potential may be misleading. And it is the task of contemporary research to find some new approaches how to describe successfully the given phenomenon. The analyses of elastic collisions between the particles exhibiting corresponding interaction may be very helpful in this direction. It is even possible to say that the further progress of future quantum physics is strongly bound to the problem of particle structure. The existence and emergence of quantum states where not only the values of energy but also the values of angular momentum are quantized may be hardly understood without the progress in this region.

It follows from the preceding that it will be probably necessary to distinguish between two kinds of quantum states: one relating to the quantum states of atoms and the other relating to existence of individual matter objects characterized by special values of rest energy and angular momentum. The former relates to the mutual properties (interaction) of electrons and protons and the latter to mutual interactions of strongly interacting objects. It has been shown that the Coulomb interaction itself cannot explain the emergence of quantum states and that some additional interaction must exist between protons (nucleons) and electrons. And one should ask whether a kind of weak contact forces does not exist in addition to strong (nuclear) contact ones.

The existence of contact forces has opened a new question: How to describe the given situation with the help of corresponding mathematical models. It is evident that it will be necessary to limit the solutions of Schrödinger equation to subsystems exhibiting continuous evolution. This approach should be used in individual subsystems where no sudden changes (caused by contact forces) are occurring; and to describe the effect of contact forces probably as the passage to another subsystem being described again as developing continuously. The given situation may be represented in the Hilbert space consisting of individual mutually orthogonal subspaces defined with the help of corresponding Schrödinger equation describing respective continuous evolution.

The representation of physical processes in a suitable Hilbert space is surely very helpful. It means, of course, that we must represent the states corresponding to quantities conserving during the whole time evolution in mutually orthogonal subspaces. It concerns mainly two quantities: energy and angular momentum. The corresponding trajectories should always 
belong to mutually orthogonal subspaces. It may concern also the representation of states characterized by impact parameter values in the case of collision processes.

In any case it is possible to say that we have pass to the other ontological level that differs from that the classical physics has been based on and opens quite new questions. It is also the fact that these basic particles are to be characterized by special values of spins, testifying that these particles exhibit special internal dynamics which may lead to the existence of their different internal states. It has been demonstrated that these characteristics may be studied with the help of corresponding elastic collisions processes.

The given results might then help in revealing the internal dynamics running in corresponding objects. It is evident that practically all particles existing in the nature must be taken as complex quantum objects. The new results may contribute in looking for the characteristics of constituents of which the given particles consist and which are responsible for their structures. At the present the main attention is to be devoted to the protons and other hadrons; especially the reasons for their stability or instability should be analyzed.

From the presented results it is also possible to conclude that the contemporary quark theory having been proposed on the basis of phenomenological description of corresponding physical situation may hardly represent suitable basis to the given goal. It is, of course, necessary to expect that some basic objects (some "quasi-quarks") should exist that should exhibit some very strong (superstrong) contact interaction. This interaction kind might be responsible for forming some conglomerates equivalent to the so called partons corresponding in principle to experimentally observable hadrons. These partons might form changeable structures (being held mutually together by standard strong forces) of individual hadrons while they might be kicked out from the given object when sufficient amount of kinetic energy has been furnished to them in the corresponding collision event.

The preceding considerations have been based on the ontological approach respecting the basic matter properties when it has been shown that the past refusal of ontology led to key mistakes in the physics of the 20th century. One must be very careful in formulating and testing different hypotheses when some quite new properties of nanoscopic objects should be taken into account (e.g., the existence of contact forces). However, the ontological basis (including causal sequentiality) in the description of matter world should not be abandoned.

\section{Conclusion}

Even if the Schrödinger equation might represent in principle basic theory of the whole matter reality it corresponds to approximate phenomenological description only. Especially, it is not possible to explain at all how a quantum state may emerge. E.g., it is evident that the hydrogen atom arises always when slowly moving electron and proton meet and are attracted mutually by Coulomb force, which cannot be theoretically reasoned. It may occurred only if a kind of repulsive force between two given objects or a kind of impenetrability of proton having certain dimensions is to be involved. It means that the structure of protons (and other hadrons) represents indivisible part of the contemporary quantum physics.

Consequently, the concept of the hydrogen atom formed by proton and electron orbiting around should be taken hardly as acceptable. The adhesive merging of both the constituents must be regarded as much more probable concept. Here, of course, the existence of different internal proton structures should be responsible for divers quantum states of hydrogen 
atoms. It is necessary to look for the experiments how to get corresponding pieces of knowledge.

It has been demonstrated that some characteristics of these different proton structures might be derived from the data of elastic two-proton collisions. However, the earlier antiontological proton properties have been obtained on the basis of phenomenological models where some arbitrary additional assumptions have been added. The standard ontological characteristics have been obtained when the eikonal model enabling to test the dependence on impact parameter value has been made use of. When this ontological model has been further generalized (and corresponding probabilities have been derived directly from measured values) it has been shown that the momentum transfer dependence of differential cross section may be reconstructed as the consequence of colliding protons exhibiting a series of structures of different external dimensions. It opens new way how the existence of quantum atom states might be interpreted on much more realistic basis.

It represents also important argument that the purely phenomenological approach to physical reality should be abandoned as quite insufficient. It is necessary to return to ontological approach on which all successes of the classical physics were based and from which practically all world civilization emerged. Some interesting orientation results have been already obtained with the help of the mentioned very rough model. Now the attention is to be devoted to its generalization to correspond fully to all ontological requirements and not to involve any unphysical limitation.

The given results should contribute mainly to understand better the existence of atom quantum states; and how they may be influenced by proton structure. It is necessary to analyze corresponding data from experiments that might help in this direction; mainly elastic collision experiments may be very helpful. However, it is not more possible to look for a phenomenological description of measured values only, but for the interpretation of corresponding processes on ontological basis.

However, it may be also helpful to answer the question how it was possible that the Copenhagen alternative was influencing scientific thinking in greater part of the past century. It followed from the fact that two different kinds of quantum physics have existed; one based on the Copenhagen quantum mechanics and looking for the support of quantum paradoxes and the other one solving in principle successfully different physical and technological problems on the basis of standard Schrödinger approach (no additional assumption having been added - without mentioning it explicitly). It is possible to say that it followed from the fact that the scientific thinking in the modern period was fundamentally influenced by mathematical philosophy of Descartes refusing ontological approach. It was also the reason why also Einstein's criticism based on ontological argument has been refused by scientific community. Our main contemporary task consists in devoting more attention to the ontological properties of physical objects.

\section{Author details}

Miloš V. Lokajíček*, Vojtěch Kundrát and Jiří Procházka

* Address all correspondence to: lokaj@fzu.cz; kundrat@fzu.cz; prochazka@fzu.cz

Institute of Physics of the AS CR, Prague, Czech Republic 


\section{References}

[1] E. Schrödinger: Quantisierung als Eigenwertproblem; Ann. Phys. 79, 361-76; 489-527; 80, 437-90; 81, 109-39 (1926).

[2] N. Bohr: The quantum postulate and the development of atomic theory; Nature 121, 580-90 (1928).

[3] W. Pauli: Die allgemeinen Prinzipien der Wellenmechanik; Handbuch der Physik XXIV, Springer, Berlin, p. 140 (1933).

[4] A. Einstein, B. Podolsky, N. Rosen: Can quantum-mechanical description of physical reality be considered complete?; Phys. Rev. 47, 777-80 (1935).

[5] N. Bohr: Can quantum-mechanical description of physical reality be considered complete?; Phys. Rev. 48, 696-702 (1935).

[6] J. von Neumann: Mathematische Grundlagen der Quantenmechanik; Springer (1932).

[7] Grete Herrmann: Die Naturphilosophischen Grundlagen der Quantenmechanik; Abhandlungen der Fries'schen Schule 6, 75-152 (1935).

[8] D. Bohm: A suggested interpretation of the quantum theory in terms of "hidden variables"; Phys. Rev. 85, 180-93 (1952).

[9] J. S. Bell: On the Einstein Podolsky Rosen paradox; Physics 1, 195-200 (1964).

[10] A. Aspect, P. Grangier, G. Roger: Experimental realization of Einstein-Podolsky-Rosen-Bohm Gedankenexperiment: A new violation of Bell's inequalities; Phys. Rev. Lett. 49, 91-4 (1982).

[11] M. V. Lokajíček: Locality problem, Bell's inequalities and EPR experiments; /arXiv:quant-ph/9808005 (1998).

[12] E. E. Rosinger: George Boole and the Bell inequalities; /arXiv:quant-ph/0406004.

[13] G. Boole: On the Theory of Probabilities; Philos. Trans.; R. Soc., London 152, 225-52 (1862).

[14] N. Vorob'ev: Theor. Probab. Appl. 7, 147 (1952).

[15] M. V. Lokajíček: The assumption in Bell's inequalities and entanglement problem; J. Comp. Theor. Nanosci. (accepted for publication), /arXiv:1108.0922.

[16] M. V. Lokajíček: Einstein-Bohr controversy and theory of hidden variables; NeuroQuantology (section: Basics of Quantum Physics) 8 (2010), issue 4, 638-45; see also /arXiv:1004.3005[quant-ph].

[17] M. V. Lokajíček: Einstein-Bohr controversy after 75 years, its actual solution and consequences; Some Applications of Quantum Mechanics (ed. M. R. Pahlavani), InTech Publisher (February 2012), 409-24. 
[18] U. Hoyer: Synthetische Quantentheorie; Georg Olms Verlag, Hildesheim (2002).

[19] H. Ioannidou: A new derivation of Schrödinger equation; Lett. al Nuovo Cim. 34, 453-8 (1982).

[20] M. V. Lokajíček: Schrödinger equation, classical physics and Copenhagen quantum mechanics; New Advances in Physics 1, No. 1, 69-77 (2007); see also /arxiv/quant-ph/0611176.

[21] P. Kundrát, M. Lokajíček: Three-dimensional harmonic oscillator and time evolution in quantum mechanics; Phys. Rev. A 67, art. 012104 (2003).

[22] P. Kundrát, M. Lokajíček: Irreversible time flow and Hilbert space structure; New Research in Quantum Physics (eds. Vl. Krasnoholovets, F. Columbus), Nova Science Publishers, Inc., pp. 17-41 (2004).

[23] P.D.Lax, R.S.Phillips: Scattering theory; Academic Press (1967).

[24] P.D.Lax, R.S.Phillips: Scattering theory for automorphic functions; Princeton (1976).

[25] N. Bohr: On the constitution of atoms and molecules; Philosophical Magazine 26, 1-24 (1913).

[26] V. Kundrát, M. V. Lokajíček: High-energy elastic scattering amplitude of unpolarized and charged hadrons; Z. Phys. C 63, 619-29 (1994).

[27] G. B. West and D. R. Yennie: Coulomb Interference in High-Energy Scattering; Phys. Rev. 172, 1413 (1968).

[28] J. Kašpar et al.: Phenomenological models of elastic nucleon scattering and predictions for LHC; Nucl. Phys. B 843, 84 (2011).

[29] V. Kundrát, M. Lokajíček and D. Krupa: Impact parameter structure derived from elastic collisions; Physics Letters B 544, 132 (2002).

[30] G. Giacomelli, M. Jacob: Physics at the CERN-ISR; Phys. Rep. 55, 1 (1979).

[31] V. Kundrát, M. Lokajíček Jr., M. V. Lokajíček: Are elastic collisions central or peripheral?; Czech. J. Phys. B 31, 1334 (1981).

[32] J. L. Bailly et al. (EHS-RCBC Collaboration): An impact parameter analysis of proton-proton elastic and inelastic interactions at $360 \mathrm{GeV} / \mathrm{c}$; Z. Phys. C 37, 7 (1987).

[33] J. Bystricky et al., in Nucleon-nucleon and kaon-nucleon scattering edited by H. Schopper(Landolt-Börnstein Series, Vol. 1), (Springer, Berlin, 1980).

[34] M. K. Carter, P. D. B. Collins and M. R. Whalley, Compilation of Nucleon-Nucleon and Nucleon-Antinucleon Elastic Scattering Data, RAL-86-002, preprint.

[35] R. G. Newton: Optical theorem and beyond; Am. J. Phys. 44, 639-42 (1976). 
[36] V. Barone, E. Predazzi: High-energy particle diffraction; Springer-Verlag (2002).

[37] M. V. Lokajíček, V. Kundrát: Optical theorem and elastic nucleon scattering; /arXiv:0906.3961 (see also Proc. of 13th Int. Conf., Blois Workshop; /arXiv:1002.3527 [hep-ph]).

[38] M. V. Lokajíček, V. Kundrát: Elastic pp scattering and the internal structure of colliding protons; (2009) /arXiv:0909.3199[hep-ph]. 
Chapter 7

\title{
Quantum Damped Harmonic Oscillator
}

\author{
Kazuyuki Fujii \\ Additional information is available at the end of the chapter
}

http://dx.doi.org/10.5772/52671

\section{Introduction}

In this chapter we introduce a toy model of Quantum Mechanics with Dissipation. Quantum Mechanics with Dissipation plays a crucial role to understand real world. However, it is not easy to master the theory for undergraduates. The target of this chapter is eager undergraduates in the world. Therefore, a good toy model to understand it deeply is required.

The quantum damped harmonic oscillator is just such a one because undergraduates must use (master) many fundamental techniques in Quantum Mechanics and Mathematics. That is, harmonic oscillator, density operator, Lindblad form, coherent state, squeezed state, tensor product, Lie algebra, representation theory, Baker-Campbell-Hausdorff formula, etc.

They are "jewels" in Quantum Mechanics and Mathematics. If undergraduates master this model, they will get a powerful weapon for Quantum Physics. I expect some of them will attack many hard problems of Quantum Mechanics with Dissipation.

The contents of this chapter are based on our two papers [3] and [6]. I will give a clear and fruitful explanation to them as much as I can.

\section{Some preliminaries}

In this section let us make some reviews from Physics and Mathematics within our necessity.

\subsection{From physics}

First we review the solution of classical damped harmonic oscillator, which is important to understand the text. For this topic see any textbook of Mathematical Physics.

The differential equation is given by

$$
\ddot{x}+\omega^{2} x=-\gamma \dot{x} \Longleftrightarrow \ddot{x}+\gamma \dot{x}+\omega^{2} x=0 \quad(\gamma>0)
$$


where $x=x(t), \dot{x}=d x / d t$ and the mass $m$ is set to 1 for simplicity. In the following we treat only the case $\omega>\gamma / 2$ (the case $\omega=\gamma / 2$ may be interesting).

Solutions (with complex form) are well-known to be

$$
x_{ \pm}(t)=e^{-\left(\frac{\gamma}{2} \pm i \sqrt{\omega^{2}-\left(\frac{\gamma}{2}\right)^{2}}\right) t},
$$

so the general solution is given by

$$
\begin{aligned}
x(t)=\alpha x_{+}(t)+\bar{\alpha} x_{-}(t) & =\alpha e^{-\left(\frac{\gamma}{2}+i \sqrt{\omega^{2}-\left(\frac{\gamma}{2}\right)^{2}}\right) t}+\bar{\alpha} e^{-\left(\frac{\gamma}{2}-i \sqrt{\omega^{2}-\left(\frac{\gamma}{2}\right)^{2}}\right) t} \\
& =\alpha e^{-\left(\frac{\gamma}{2}+i \omega \sqrt{1-\left(\frac{\gamma}{2 \omega}\right)^{2}}\right) t}+\bar{\alpha} e^{-\left(\frac{\gamma}{2}-i \omega \sqrt{1-\left(\frac{\gamma}{2 \omega}\right)^{2}}\right) t}
\end{aligned}
$$

where $\alpha$ is a complex number. If $\gamma / 2 \omega$ is small enough we have an approximate solution

$$
x(t) \approx \alpha e^{-\left(\frac{\gamma}{2}+i \omega\right) t}+\bar{\alpha} e^{-\left(\frac{\gamma}{2}-i \omega\right) t} .
$$

Next, we consider the quantum harmonic oscillator. This is well-known in textbooks of Quantum Mechanics. As standard textbooks of Quantum Mechanics see [2] and [11] ([2] is particularly interesting).

For the Hamiltonian

$$
H=H(q, p)=\frac{1}{2}\left(p^{2}+\omega^{2} q^{2}\right)
$$

where $q=q(t), p=p(t)$, the canonical equation of motion reads

$$
\dot{q} \equiv \frac{\partial H}{\partial p}=p, \quad \dot{p} \equiv-\frac{\partial H}{\partial q}=-\omega^{2} q .
$$

From these we recover the equation

$$
\ddot{q}=-\omega^{2} q \Longleftrightarrow \ddot{q}+\omega^{2} q=0
$$

See (2.1) with $q=x$ and $\lambda=0$.

Next, we introduce the Poisson bracket. For $A=A(q, p), B=B(q, p)$ it is defined as

$$
\{A, B\}_{c} \equiv \frac{\partial A}{\partial q} \frac{\partial B}{\partial p}-\frac{\partial A}{\partial p} \frac{\partial B}{\partial q}
$$

where $\{,\}_{\mathcal{C}}$ means classical. Then it is easy to see

$$
\{q, q\}_{c}=0, \quad\{p, p\}_{c}=0, \quad\{q, p\}_{c}=1
$$


Now, we are in a position to give a quantization condition due to Dirac. Before that we prepare some notation from algebra.

Square matrices $A$ and $B$ don't commute in general, so we need the commutator

$$
[A, B]=A B-B A \text {. }
$$

Then Dirac gives an abstract correspondence $q \longrightarrow \hat{q}, \quad p \longrightarrow \hat{p} \quad$ which satisfies the condition

$$
[\hat{q}, \hat{q}]=0, \quad[\hat{p}, \hat{p}]=0, \quad[\hat{q}, \hat{p}]=i \hbar \mathbf{1}
$$

corresponding to (2.6). Here $\hbar$ is the Plank constant, and $\hat{q}$ and $\hat{p}$ are both Hermite operators on some Fock space (a kind of Hilbert space) given in the latter and $\mathbf{1}$ is the identity on it. Therefore, our quantum Hamiltonian should be

$$
H=H(\hat{q}, \hat{p})=\frac{1}{2}\left(\hat{p}^{2}+\omega^{2} \hat{q}^{2}\right)
$$

from (2.4). Note that a notation $H$ instead of $\hat{H}$ is used for simplicity. From now on we consider a complex version. From (2.4) and (2.8) we rewrite like

$$
H(q, p)=\frac{1}{2}\left(p^{2}+\omega^{2} q^{2}\right)=\frac{\omega^{2}}{2}\left(q^{2}+\frac{1}{\omega^{2}} p^{2}\right)=\frac{\omega^{2}}{2}\left(q-\frac{i}{\omega} p\right)\left(q+\frac{i}{\omega} p\right)
$$

and

$$
\begin{aligned}
H(\hat{q}, \hat{p}) & =\frac{\omega^{2}}{2}\left(\hat{q}^{2}+\frac{1}{\omega^{2}} \hat{p}^{2}\right)=\frac{\omega^{2}}{2}\left\{\left(\hat{q}-\frac{i}{\omega} \hat{p}\right)\left(\hat{q}+\frac{i}{\omega} \hat{p}\right)-\frac{i}{\omega}[\hat{q}, \hat{p}]\right\} \\
& =\frac{\omega^{2}}{2}\left\{\left(\hat{q}-\frac{i}{\omega} \hat{p}\right)\left(\hat{q}+\frac{i}{\omega} \hat{p}\right)+\frac{\hbar}{\omega}\right\}=\omega \hbar\left\{\frac{\omega}{2 \hbar}\left(\hat{q}-\frac{i}{\omega} \hat{p}\right)\left(\hat{q}+\frac{i}{\omega} \hat{p}\right)+\frac{1}{2}\right\}
\end{aligned}
$$

by use of (2.7), and if we set

$$
a^{\dagger}=\sqrt{\frac{\omega}{2 \hbar}}\left(\hat{q}-\frac{i}{\omega} \hat{p}\right), \quad a=\sqrt{\frac{\omega}{2 \hbar}}\left(\hat{q}+\frac{i}{\omega} \hat{p}\right)
$$

we have easily

$$
\left[a, a^{\dagger}\right]=\frac{\omega}{2 \hbar}\left[\hat{q}+\frac{i}{\omega} \hat{p}, \hat{q}-\frac{i}{\omega} \hat{p}\right]=\frac{\omega}{2 \hbar}\left\{-\frac{2 i}{\omega}[\hat{q}, \hat{p}]\right\}=\frac{\omega}{2 \hbar}\left\{-\frac{2 i}{\omega} \times i \hbar\right\}=\mathbf{1}
$$

by use of (2.7). As a result we obtain a well-known form

$$
H=\omega \hbar\left(a^{\dagger} a+\frac{1}{2}\right), \quad\left[a, a^{\dagger}\right]=\mathbf{1}
$$

Here we used an abbreviation $1 / 2$ in place of $(1 / 2) 1$ for simplicity. 
If we define an operator $N=a^{\dagger} a$ (which is called the number operator) then it is easy to see the relations

$$
\left[N, a^{\dagger}\right]=a^{\dagger}, \quad[N, a]=-a, \quad\left[a, a^{\dagger}\right]=\mathbf{1} .
$$

For the proof a well-known formula $[A B, C]=[A, C] B+A[B, C]$ is used. Note that $a a^{\dagger}=$ $a^{\dagger} a+\left[a, a^{\dagger}\right]=N+1$. The set $\left\{a^{\dagger}, a, N\right\}$ is just a generator of Heisenberg algebra and we can construct a Fock space based on this. Let us note that $a, a^{\dagger}$ and $N$ are called the annihilation operator, creation one and number one respectively.

First of all let us define a vacuum $|0\rangle$. This is defined by the equation $a|0\rangle=0$. Based on this vacuum we construct the $n$ state $|n\rangle$ like

$$
|n\rangle=\frac{\left(a^{\dagger}\right)^{n}}{\sqrt{n !}}|0\rangle \quad(0 \leq n)
$$

Then we can easily prove

$$
a^{\dagger}|n\rangle=\sqrt{n+1}|n+1\rangle, \quad a|n\rangle=\sqrt{n}|n-1\rangle, \quad N|n\rangle=n|n\rangle
$$

and moreover can prove both the orthogonality condition and the resolution of unity

$$
\langle m \mid n\rangle=\delta_{m n}, \quad \sum_{n=0}^{\infty}|n\rangle\langle n|=\mathbf{1} .
$$

For the proof one can use for example

$$
a^{2}\left(a^{\dagger}\right)^{2}=a\left(a a^{\dagger}\right) a^{\dagger}=a(N+1) a^{\dagger}=(N+2) a a^{\dagger}=(N+2)(N+1)
$$

by (2.11), therefore we have

$$
\left\langle 0\left|a^{2}\left(a^{\dagger}\right)^{2}\right| 0\right\rangle=\langle 0|(N+2)(N+1)| 0\rangle=2 ! \Longrightarrow\langle 2 \mid 2\rangle=1 .
$$

The proof of the resolution of unity may be not easy for readers (we omit it here).

As a result we can define a Fock space generated by the generator $\left\{a^{\dagger}, a, N\right\}$

$$
\mathcal{F}=\left\{\left.\sum_{n=0}^{\infty} c_{n}|n\rangle \in \mathbf{C}^{\infty}\left|\sum_{n=0}^{\infty}\right| c_{n}\right|^{2}<\infty\right\}
$$

This is just a kind of Hilbert space. On this space the operators (= infinite dimensional matrices) $a^{\dagger}, a$ and $N$ are represented as 


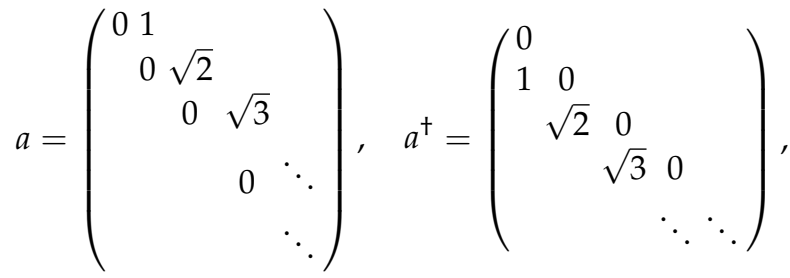

$$
\begin{aligned}
& N=a^{\dagger} a=\left(\begin{array}{cccc}
0 & & & \\
& 1 & & \\
& 2 & & \\
& & 3 & \\
& & & \ddots
\end{array}\right)
\end{aligned}
$$

by use of (2.12).

Note We can add a phase to $\left\{a, a^{\dagger}\right\}$ like

$$
b=e^{i \theta} a, \quad b^{\dagger}=e^{-i \theta} a^{\dagger}, \quad N=b^{\dagger} b=a^{\dagger} a
$$

where $\theta$ is constant. Then we have another Heisenberg algebra

$$
\left[N, b^{\dagger}\right]=b^{\dagger}, \quad[N, b]=-b, \quad\left[b, b^{\dagger}\right]=\mathbf{1} .
$$

Next, we introduce a coherent state which plays a central role in Quantum Optics or Quantum Computation. For $z \in \mathbf{C}$ the coherent state $|z\rangle \in \mathcal{F}$ is defined by the equation

$$
a|z\rangle=z|z\rangle \text { and } \quad\langle z \mid z\rangle=1
$$

The annihilation operator $a$ is not hermitian, so this equation is never trivial. For this state the following three equations are equivalent :

$$
\left\{\begin{array}{l}
\text { (1) } \quad a|z\rangle=z|z\rangle \text { and }\langle z \mid z\rangle=1, \\
\text { (2) }|z\rangle=e^{z a^{+}-\bar{z} a}|0\rangle, \\
\text { (3) }|z\rangle=e^{-\frac{|z|^{2}}{2}} \sum_{n=0}^{\infty} \frac{z^{n}}{\sqrt{n !}}|n\rangle .
\end{array}\right.
$$

The proof is as follows. From (1) to (2) we use a popular formula

$$
e^{A} B e^{-A}=B+[A, B]+\frac{1}{2 !}[A,[A, B]]+\cdots
$$


( $A, B:$ operators) to prove

$$
e^{-\left(z a^{\dagger}-\bar{z} a\right)} a e^{z a^{\dagger}-\bar{z} a}=a+z .
$$

From (2) to (3) we use the Baker-Campbell-Hausdorff formula (see for example [17])

$$
e^{A} e^{B}=e^{A+B+\frac{1}{2}[A, B]+\frac{1}{6}[A,[A, B]]+\frac{1}{6}[B,[A, B]]+\cdots} .
$$

If $[A,[A, B]]=0=[B,[A, B]]$ (namely, $[A, B]$ commutes with both $A$ and $B$ ) then we have

$$
e^{A} e^{B}=e^{A+B+\frac{1}{2}[A, B]}=e^{\frac{1}{2}[A, B]} e^{A+B} \Longrightarrow e^{A+B}=e^{-\frac{1}{2}[A, B]} e^{A} e^{B}
$$

In our case the condition is satisfied because of $\left[a, a^{\dagger}\right]=1$. Therefore we obtain a (famous) decomposition

$$
e^{z a^{\dagger}-\bar{z} a}=e^{-\frac{|z|^{2}}{2}} e^{z a^{\dagger}} e^{-\bar{z} a} .
$$

The remaining part of the proof is left to readers.

From the equation (3) in (2.16) we obtain the resolution of unity for coherent states

$$
\iint \frac{d x d y}{\pi}|z\rangle\left\langle z\left|=\sum_{n=0}^{\infty}\right| n\right\rangle\langle n|=\mathbf{1} \quad(z=x+i y) .
$$

The proof is reduced to the following formula

$$
\iint \frac{d x d y}{\pi} e^{-|z|^{2}} \bar{z}^{m} z^{n}=n ! \delta_{m n} \quad(z=x+i y)
$$

The proof is left to readers. See [14] for more general knowledge of coherent states.

\subsection{From mathematics}

We consider a simple matrix equation

$$
\frac{d}{d t} X=A X B
$$

where

$$
X=X(t)=\left(\begin{array}{ll}
x_{11}(t) & x_{12}(t) \\
x_{21}(t) & x_{22}(t)
\end{array}\right), \quad A=\left(\begin{array}{ll}
a_{11} & a_{12} \\
a_{21} & a_{22}
\end{array}\right), \quad B=\left(\begin{array}{ll}
b_{11} & b_{12} \\
b_{21} & b_{22}
\end{array}\right)
$$


A standard form of linear differential equation which we usually treat is

$$
\frac{d}{d t} \mathbf{x}=C \mathbf{x}
$$

where $\mathbf{x}=\mathbf{x}(t)$ is a vector and $C$ is a matrix associated to the vector. Therefore, we want to rewrite (2.20) into a standard form.

For the purpose we introduce the Kronecker product of matrices. For example, it is defined as

$$
\begin{aligned}
A \otimes B & =\left(\begin{array}{ll}
a_{11} & a_{12} \\
a_{21} & a_{22}
\end{array}\right) \otimes B \equiv\left(\begin{array}{lll}
a_{11} B & a_{12} B \\
a_{21} B & a_{22} B
\end{array}\right) \\
& =\left(\begin{array}{llll}
a_{11} b_{11} & a_{11} b_{12} & a_{12} b_{11} & a_{12} b_{12} \\
a_{11} b_{21} & a_{11} b_{22} & a_{12} b_{21} & a_{12} b_{22} \\
a_{21} b_{11} & a_{21} b_{12} & a_{22} b_{11} & a_{22} b_{12} \\
a_{21} b_{21} & a_{21} b_{22} & a_{22} b_{21} & a_{22} b_{22}
\end{array}\right)
\end{aligned}
$$

for $A$ and $B$ above. Note that recently we use the tensor product instead of the Kronecker product, so we use it in the following. Here, let us list some useful properties of the tensor product

$$
\begin{array}{ll}
\text { (1) } & \left(A_{1} \otimes B_{1}\right)\left(A_{2} \otimes B_{2}\right)=A_{1} A_{2} \otimes B_{1} B_{2}, \\
\text { (2) } & (A \otimes E)(E \otimes B)=A \otimes B=(E \otimes B)(A \otimes E), \\
\text { (3) } e^{A \otimes E+E \otimes B}=e^{A \otimes E} e^{E \otimes B}=\left(e^{A} \otimes E\right)\left(E \otimes e^{B}\right)=e^{A} \otimes e^{B}, \\
\text { (4) } & (A \otimes B)^{\dagger}=A^{\dagger} \otimes B^{\dagger}
\end{array}
$$

where $E$ is the unit matrix. The proof is left to readers. [9] is recommended as a general introduction.

Then the equation (2.20) can be written in terms of components as

$$
\left\{\begin{array}{l}
\frac{d x_{11}}{d t}=a_{11} b_{11} x_{11}+a_{11} b_{21} x_{12}+a_{12} b_{11} x_{21}+a_{12} b_{21} x_{22} \\
\frac{d x_{12}}{d t}=a_{11} b_{12} x_{11}+a_{11} b_{22} x_{12}+a_{12} b_{12} x_{21}+a_{12} b_{22} x_{22} \\
\frac{d x_{21}}{d t}=a_{21} b_{11} x_{11}+a_{21} b_{21} x_{12}+a_{22} b_{11} x_{21}+a_{22} b_{21} x_{22} \\
\frac{d x_{22}}{d t}=a_{21} b_{12} x_{11}+a_{21} b_{22} x_{12}+a_{22} b_{12} x_{21}+a_{22} b_{22} x_{22}
\end{array}\right.
$$

or in a matrix form

$$
\frac{d}{d t}\left(\begin{array}{l}
x_{11} \\
x_{12} \\
x_{21} \\
x_{22}
\end{array}\right)=\left(\begin{array}{llll}
a_{11} b_{11} & a_{11} b_{21} & a_{12} b_{11} & a_{12} b_{21} \\
a_{11} b_{12} & a_{11} b_{22} & a_{12} b_{12} & a_{12} b_{22} \\
a_{21} b_{11} & a_{21} b_{21} & a_{22} b_{11} & a_{22} b_{21} \\
a_{21} b_{12} & a_{21} b_{22} & a_{22} b_{12} & a_{22} b_{22}
\end{array}\right)\left(\begin{array}{l}
x_{11} \\
x_{12} \\
x_{21} \\
x_{22}
\end{array}\right)
$$


If we set

$$
X=\left(\begin{array}{ll}
x_{11} & x_{12} \\
x_{21} & x_{22}
\end{array}\right) \Longrightarrow \widehat{X}=\left(x_{11}, x_{12}, x_{21}, x_{22}\right)^{T}
$$

where $T$ is the transpose, then we obtain a standard form

$$
\frac{d}{d t} X=A X B \Longrightarrow \frac{d}{d t} \widehat{X}=\left(A \otimes B^{T}\right) \widehat{X}
$$

from (2.21).

Similarly we have a standard form

$$
\frac{d}{d t} X=A X+X B \Longrightarrow \frac{d}{d t} \widehat{X}=\left(A \otimes E+E \otimes B^{T}\right) \widehat{X}
$$

where $E^{T}=E$ for the unit matrix $E$.

From these lessons there is no problem to generalize (2.23) and (2.24) based on $2 \times 2$ matrices to ones based on any (square) matrices or operators on $\mathcal{F}$. Namely, we have

$$
\left\{\begin{array}{l}
\frac{d}{d t} X=A X B \Longrightarrow \frac{d}{d t} \widehat{X}=\left(A \otimes B^{T}\right) \widehat{X} \\
\frac{d}{d t} X=A X+X B \Longrightarrow \frac{d}{d t} \widehat{X}=\left(A \otimes I+I \otimes B^{T}\right) \widehat{X} .
\end{array}\right.
$$

where $I$ is the identity $E$ (matrices) or $\mathbf{1}$ (operators).

\section{Quantum damped harmonic oscillator}

In this section we treat the quantum damped harmonic oscillator. As a general introduction to this topic see [1] or [16].

\subsection{Model}

Before that we introduce the quantum harmonic oscillator. The Schrödinger equation is given by

$$
i \hbar \frac{\partial}{\partial t}|\Psi(t)\rangle=H|\Psi(t)\rangle=\left(\omega \hbar\left(N+\frac{1}{2}\right)\right)|\Psi(t)\rangle
$$

by (2.10) (note $N=a^{\dagger} a$ ). In the following we use $\frac{\partial}{\partial t}$ instead of $\frac{d}{d t}$.

Now we change from a wave-function to a density operator because we want to treat a mixed state, which is a well-known technique in Quantum Mechanics or Quantum Optics.

If we set $\rho(t)=|\Psi(t)\rangle\langle\Psi(t)|$, then a little algebra gives 


$$
i \hbar \frac{\partial}{\partial t} \rho=[H, \rho]=[\omega \hbar N, \rho] \Longrightarrow \frac{\partial}{\partial t} \rho=-i[\omega N, \rho] .
$$

This is called the quantum Liouville equation. With this form we can treat a mixed state like

$$
\rho=\rho(t)=\sum_{j=1}^{N} u_{j}\left|\Psi_{j}(t)\right\rangle\left\langle\Psi_{j}(t)\right|
$$

where $u_{j} \geq 0$ and $\sum_{j=1}^{N} u_{j}=1$. Note that the general solution of (3.1) is given by

$$
\rho(t)=e^{-i \omega N t} \rho(0) e^{i \omega N t}
$$

We are in a position to state the equation of quantum damped harmonic oscillator by use of (3.1).

Definition The equation is given by

$$
\frac{\partial}{\partial t} \rho=-i\left[\omega a^{\dagger} a, \rho\right]-\frac{\mu}{2}\left(a^{\dagger} a \rho+\rho a^{\dagger} a-2 a \rho a^{\dagger}\right)-\frac{v}{2}\left(a a^{\dagger} \rho+\rho a a^{\dagger}-2 a^{\dagger} \rho a\right)
$$

where $\mu, v(\mu>v \geq 0)$ are some real constants depending on the system (for example, a damping rate of the cavity mode $)^{1}$.

Note that the extra term

$$
-\frac{\mu}{2}\left(a^{\dagger} a \rho+\rho a^{\dagger} a-2 a \rho a^{\dagger}\right)-\frac{v}{2}\left(a a^{\dagger} \rho+\rho a a^{\dagger}-2 a^{\dagger} \rho a\right)
$$

is called the Lindblad form (term). Such a term plays an essential role in Decoherence.

\subsection{Method of solution}

First we solve the Lindblad equation :

$$
\frac{\partial}{\partial t} \rho=-\frac{\mu}{2}\left(a^{\dagger} a \rho+\rho a^{\dagger} a-2 a \rho a^{\dagger}\right)-\frac{v}{2}\left(a a^{\dagger} \rho+\rho a a^{\dagger}-2 a^{\dagger} \rho a\right) .
$$

Interesting enough, we can solve this equation completely.

\footnotetext{
${ }^{1}$ The aim of this chapter is not to drive this equation. In fact, its derivation is not easy for non-experts, so see for example the original papers [15] and [10], or [12] as a short review paper
} 
Let us rewrite (3.3) more conveniently using the number operator $N \equiv a^{\dagger} a$

$$
\frac{\partial}{\partial t} \rho=\mu a \rho a^{\dagger}+v a^{\dagger} \rho a-\frac{\mu+v}{2}(N \rho+\rho N+\rho)+\frac{\mu-v}{2} \rho
$$

where we have used $a a^{\dagger}=N+\mathbf{1}$.

From here we use the method developed in Section 2.2. For a matrix $X=\left(x_{i j}\right) \in M(\mathcal{F})$ over $\mathcal{F}$

$$
X=\left(\begin{array}{cccc}
x_{00} & x_{01} & x_{02} & \cdots \\
x_{10} & x_{11} & x_{12} & \cdots \\
x_{20} & x_{21} & x_{22} & \cdots \\
\vdots & \vdots & \vdots & \ddots
\end{array}\right)
$$

we correspond to the vector $\widehat{X} \in \mathcal{F}^{\operatorname{dim}_{\mathrm{C}} \mathcal{F}}$ as

$$
X=\left(x_{i j}\right) \longrightarrow \widehat{X}=\left(x_{00}, x_{01}, x_{02}, \cdots ; x_{10}, x_{11}, x_{12}, \cdots ; x_{20}, x_{21}, x_{22}, \cdots ; \cdots \cdots\right)^{T}
$$

where $T$ means the transpose. The following formulas

$$
\widehat{A X B}=\left(A \otimes B^{T}\right) \widehat{X}, \quad(A \widehat{X+X} B)=\left(A \otimes \mathbf{1}+\mathbf{1} \otimes B^{T}\right) \widehat{X}
$$

hold for $A, B, X \in M(\mathcal{F})$, see (2.25).

Then (3.4) becomes

$$
\begin{aligned}
\frac{\partial}{\partial t} \widehat{\rho} & =\left\{\mu a \otimes\left(a^{\dagger}\right)^{T}+v a^{\dagger} \otimes a^{T}-\frac{\mu+v}{2}(N \otimes \mathbf{1}+\mathbf{1} \otimes N+\mathbf{1} \otimes \mathbf{1})+\frac{\mu-v}{2} \mathbf{1} \otimes \mathbf{1}\right\} \widehat{\rho} \\
& =\left\{\frac{\mu-v}{2} \mathbf{1} \otimes \mathbf{1}+v a^{\dagger} \otimes a^{\dagger}+\mu a \otimes a-\frac{\mu+v}{2}(N \otimes \mathbf{1}+\mathbf{1} \otimes N+\mathbf{1} \otimes \mathbf{1})\right\} \widehat{\rho}
\end{aligned}
$$

where we have used $a^{T}=a^{\dagger}$ from the form (2.15), so that the solution is formally given by

$$
\widehat{\rho}(t)=e^{\frac{\mu-v}{2}} t e^{t\left\{v a^{+} \otimes a^{+}+\mu a \otimes a-\frac{\mu+v}{2}(N \otimes \mathbf{1}+\mathbf{1} \otimes N+\mathbf{1} \otimes \mathbf{1})\right\}} \widehat{\rho}(0) .
$$

In order to use some techniques from Lie algebra we set

$$
K_{3}=\frac{1}{2}(N \otimes \mathbf{1}+\mathbf{1} \otimes N+\mathbf{1} \otimes \mathbf{1}), \quad K_{+}=a^{\dagger} \otimes a^{\dagger}, \quad K_{-}=a \otimes a \quad\left(K_{-}=K_{+}^{\dagger}\right)
$$

then we can show the relations

$$
\left[K_{3}, K_{+}\right]=K_{+}, \quad\left[K_{3}, K_{-}\right]=-K_{-}, \quad\left[K_{+}, K_{-}\right]=-2 K_{3} .
$$


This is just the $s u(1,1)$ algebra. The proof is very easy and is left to readers.

The equation (3.8) can be written simply as

$$
\widehat{\rho}(t)=e^{\frac{\mu-v}{2} t} e^{t\left\{v K_{+}+\mu K_{-}-(\mu+v) K_{3}\right\}} \widehat{\rho}(0),
$$

so we have only to calculate the term

$$
e^{t\left\{v K_{+}+\mu K_{-}-(\mu+v) K_{3}\right\}},
$$

which is of course not simple. Now the disentangling formula in [4] is helpful in calculating (3.11).

If we set $\left\{k_{+}, k_{-}, k_{3}\right\}$ as

$$
k_{+}=\left(\begin{array}{ll}
0 & 1 \\
0 & 0
\end{array}\right), \quad k_{-}=\left(\begin{array}{cc}
0 & 0 \\
-1 & 0
\end{array}\right), \quad k_{3}=\frac{1}{2}\left(\begin{array}{cc}
1 & 0 \\
0 & -1
\end{array}\right) \quad\left(k_{-} \neq k_{+}^{\dagger}\right)
$$

then it is very easy to check the relations

$$
\left[k_{3}, k_{+}\right]=k_{+}, \quad\left[k_{3}, k_{-}\right]=-k_{-}, \quad\left[k_{+}, k_{-}\right]=-2 k_{3} .
$$

That is, $\left\{k_{+}, k_{-}, k_{3}\right\}$ are generators of the Lie algebra $s u(1,1)$. Let us show by $\operatorname{SU}(1,1)$ the corresponding Lie group, which is a typical noncompact group.

Since $S U(1,1)$ is contained in the special linear group $S L(2 ; \mathbf{C})$, we assume that there exists an infinite dimensional unitary representation $\rho: S L(2 ; \mathbf{C}) \longrightarrow U(\mathcal{F} \otimes \mathcal{F})$ (group homomorphism) satisfying

$$
d \rho\left(k_{+}\right)=K_{+}, \quad d \rho\left(k_{-}\right)=K_{-}, \quad d \rho\left(k_{3}\right)=K_{3} .
$$

From (3.11) some algebra gives

$$
\begin{aligned}
e^{t\left\{v K_{+}+\mu K_{-}-(\mu+v) K_{3}\right\}} & =e^{t\left\{v d \rho\left(k_{+}\right)+\mu d \rho\left(k_{-}\right)-(\mu+v) d \rho\left(k_{3}\right)\right\}} \\
& =e^{d \rho\left(t\left(v k_{+}+\mu k_{-}-(\mu+v) k_{3}\right)\right)} \\
& =\rho\left(e^{t\left(v k_{+}+\mu k_{-}-(\mu+v) k_{3}\right)}\right) \quad(\Downarrow \text { by definition }) \\
& \equiv \rho\left(e^{t A}\right)
\end{aligned}
$$


and we have

$$
\begin{aligned}
e^{t A} & =e^{t\left\{v k_{+}+\mu k_{-}-(\mu+v) k_{3}\right\}} \\
& =\exp \left\{t\left(\begin{array}{rr}
-\frac{\mu+v}{2} & v \\
-\mu & \frac{\mu+v}{2}
\end{array}\right)\right\} \\
& =\left(\begin{array}{cc}
\cosh \left(\frac{\mu-v}{2} t\right)-\frac{\mu+v}{\mu-v} \sinh \left(\frac{\mu-v}{2} t\right) & \frac{2 v}{\mu-v} \sinh \left(\frac{\mu-v}{2} t\right) \\
-\frac{2 \mu}{\mu-v} \sinh \left(\frac{\mu-v}{2} t\right) & \cosh \left(\frac{\mu-v}{2} t\right)+\frac{\mu+v}{\mu-v} \sinh \left(\frac{\mu-v}{2} t\right)
\end{array}\right) .
\end{aligned}
$$

The proof is based on the following two facts.

$$
(t A)^{2}=t^{2}\left(\begin{array}{cc}
-\frac{\mu+v}{2} & v \\
-\mu & \frac{\mu+v}{2}
\end{array}\right)^{2}=t^{2}\left(\begin{array}{cc}
\left(\frac{\mu+v}{2}\right)^{2}-\mu v & 0 \\
0 & \left(\frac{\mu+v}{2}\right)^{2}-\mu v
\end{array}\right)=\left(\frac{\mu-v}{2} t\right)^{2}\left(\begin{array}{ll}
1 & 0 \\
0 & 1
\end{array}\right)
$$

and

$$
e^{X}=\sum_{n=0}^{\infty} \frac{1}{n !} X^{n}=\sum_{n=0}^{\infty} \frac{1}{(2 n) !} X^{2 n}+\sum_{n=0}^{\infty} \frac{1}{(2 n+1) !} X^{2 n+1} \quad(X=t A) .
$$

Note that

$$
\cosh (x)=\frac{e^{x}+e^{-x}}{2}=\sum_{n=0}^{\infty} \frac{x^{2 n}}{(2 n) !} \quad \text { and } \quad \sinh (x)=\frac{e^{x}-e^{-x}}{2}=\sum_{n=0}^{\infty} \frac{x^{2 n+1}}{(2 n+1) !} .
$$

The remainder is left to readers.

The Gauss decomposition formula (in $S L(2 ; \mathbf{C})$ )

$$
\left(\begin{array}{ll}
a & b \\
c & d
\end{array}\right)=\left(\begin{array}{ll}
1 & \frac{b}{d} \\
0 & 1
\end{array}\right)\left(\begin{array}{ll}
\frac{1}{d} & 0 \\
0 & d
\end{array}\right)\left(\begin{array}{ll}
1 & 0 \\
c & 1
\end{array}\right) \quad(a d-b c=1)
$$

gives the decomposition 


$$
\begin{aligned}
& e^{t A}=\left(\begin{array}{l}
1 \frac{\frac{2 v}{\mu-v} \sinh \left(\frac{\mu-v}{2} t\right)}{\cosh \left(\frac{\mu-v}{2} t\right)+\frac{\mu+v}{\mu-\nu} \sinh \left(\frac{\mu-v}{2} t\right)} \\
0
\end{array}\right) \times \\
& \left(\begin{array}{cc}
\frac{1}{\cosh \left(\frac{\mu-v}{2} t\right)+\frac{\mu+v}{\mu-v} \sinh \left(\frac{\mu-v}{2} t\right)} & 0 \\
0 & \cosh \left(\frac{\mu-v}{2} t\right)+\frac{\mu+v}{\mu-v} \sinh \left(\frac{\mu-v}{2} t\right)
\end{array}\right) \times \\
& \left(\begin{array}{cc}
1 & 0 \\
-\frac{2 \mu}{\mu-v} \sinh \left(\frac{\mu-v}{2} t\right) & 1 \\
\cosh \left(\frac{\mu-v}{2} t\right)+\frac{\mu+v}{\mu-\nu} \sinh \left(\frac{\mu-v}{2} t\right) & 1
\end{array}\right)
\end{aligned}
$$

and moreover we have

$$
\begin{aligned}
& e^{t A}=\exp \left(\begin{array}{l}
0 \frac{\frac{2 v}{\mu-v} \sinh \left(\frac{\mu-v}{2} t\right)}{\cosh \left(\frac{\mu-v}{2} t\right)+\frac{\mu+v}{\mu-v} \sinh \left(\frac{\mu-v}{2} t\right)} \\
0
\end{array}\right) \times \\
& \exp \left(\begin{array}{cc}
-\log \left(\cosh \left(\frac{\mu-v}{2} t\right)+\frac{\mu+v}{\mu-v} \sinh \left(\frac{\mu-v}{2} t\right)\right) & 0 \\
0 & \log \left(\cosh \left(\frac{\mu-v}{2} t\right)+\frac{\mu+v}{\mu-\nu} \sinh \left(\frac{\mu-v}{2} t\right)\right)
\end{array}\right) \times \\
& \exp \left(\begin{array}{cc}
0 & 0 \\
-\frac{2 \mu}{\mu-v} \sinh \left(\frac{\mu-v}{2} t\right) & 0
\end{array}\right) \\
& =\exp \left(\frac{\frac{2 v}{\mu-v} \sinh \left(\frac{\mu-v}{2} t\right)}{\cosh \left(\frac{\mu-v}{2} t\right)+\frac{\mu+v}{\mu-v} \sinh \left(\frac{\mu-v}{2} t\right)} k_{+}\right) \times \\
& \exp \left(-2 \log \left(\cosh \left(\frac{\mu-v}{2} t\right)+\frac{\mu+v}{\mu-v} \sinh \left(\frac{\mu-v}{2} t\right)\right) k_{3}\right) \times \\
& \exp \left(\frac{\frac{2 \mu}{\mu-v} \sinh \left(\frac{\mu-v}{2} t\right)}{\cosh \left(\frac{\mu-v}{2} t\right)+\frac{\mu+v}{\mu-v} \sinh \left(\frac{\mu-v}{2} t\right)} k_{-}\right) \text {. }
\end{aligned}
$$

Since $\rho$ is a group homomorphism $(\rho(X Y Z)=\rho(X) \rho(Y) \rho(Z))$ and the formula $\rho\left(e^{L k}\right)=$ $e^{L d \rho(k)}\left(k=k_{+}, k_{3}, k_{-}\right)$holds we obtain 


$$
\begin{aligned}
\rho\left(e^{t A}\right) & \exp \left(\frac{\frac{2 v}{\mu-v} \sinh \left(\frac{\mu-v}{2} t\right)}{\cosh \left(\frac{\mu-v}{2} t\right)+\frac{\mu+v}{\mu-v} \sinh \left(\frac{\mu-v}{2} t\right)} d \rho\left(k_{+}\right)\right) \times \\
& \exp \left(-2 \log \left(\cosh \left(\frac{\mu-v}{2} t\right)+\frac{\mu+v}{\mu-v} \sinh \left(\frac{\mu-v}{2} t\right)\right) d \rho\left(k_{3}\right)\right) \times \\
& \exp \left(\frac{\frac{2 \mu}{\mu-v} \sinh \left(\frac{\mu-v}{2} t\right)}{\cosh \left(\frac{\mu-v}{2} t\right)+\frac{\mu+v}{\mu-v} \sinh \left(\frac{\mu-v}{2} t\right)} d \rho\left(k_{-}\right)\right)
\end{aligned}
$$

As a result we have the disentangling formula

$$
\begin{aligned}
e^{t\left\{v K_{+}+\mu K_{-}-(\mu+v) K_{3}\right\}=} & \exp \left(\frac{\frac{2 v}{\mu-v} \sinh \left(\frac{\mu-v}{2} t\right)}{\cosh \left(\frac{\mu-v}{2} t\right)+\frac{\mu+v}{\mu-v} \sinh \left(\frac{\mu-v}{2} t\right)} K_{+}\right) \times \\
& \exp \left(-2 \log \left(\cosh \left(\frac{\mu-v}{2} t\right)+\frac{\mu+v}{\mu-v} \sinh \left(\frac{\mu-v}{2} t\right)\right) K_{3}\right) \times \\
& \exp \left(\frac{\frac{2 \mu}{\mu-v} \sinh \left(\frac{\mu-v}{2} t\right)}{\cosh \left(\frac{\mu-v}{2} t\right)+\frac{\mu+v}{\mu-v} \sinh \left(\frac{\mu-v}{2} t\right)} K_{-}\right)
\end{aligned}
$$

by (3.13).

In the following we set for simplicity

$$
\begin{aligned}
E(t) & =\frac{\frac{2 \mu}{\mu-v} \sinh \left(\frac{\mu-v}{2} t\right)}{\cosh \left(\frac{\mu-v}{2} t\right)+\frac{\mu+v}{\mu-v} \sinh \left(\frac{\mu-v}{2} t\right)}, \\
F(t) & =\cosh \left(\frac{\mu-v}{2} t\right)+\frac{\mu+v}{\mu-v} \sinh \left(\frac{\mu-v}{2} t\right), \\
G(t) & =\frac{\frac{2 v}{\mu-v} \sinh \left(\frac{\mu-v}{2} t\right)}{\cosh \left(\frac{\mu-v}{2} t\right)+\frac{\mu+v}{\mu-v} \sinh \left(\frac{\mu-v}{2} t\right)} .
\end{aligned}
$$

Readers should be careful of this "proof", which is a heuristic method. In fact, it is incomplete because we have assumed a group homomorphism. In order to complete it we want to show a disentangling formula like

$$
e^{t\left\{v K_{+}+\mu K_{-}-(\mu+v) K_{3}\right\}}=e^{f(t) K_{+}} e^{g(t) K_{3}} e^{h(t) K_{-}}
$$


with unknowns $f(t), g(t), h(t)$ satisfying $f(0)=g(0)=h(0)=0$. For the purpose we set

$$
A(t)=e^{t\left\{v K_{+}+\mu K_{-}-(\mu+v) K_{3}\right\}}, \quad B(t)=e^{f(t) K_{+}} e^{g(t) K_{3}} e^{h(t) K_{-}} .
$$

For $t=0$ we have $A(0)=B(0)=$ identity and

$$
\dot{A}(t)=\left\{v K_{+}+\mu K_{-}-(\mu+v) K_{3}\right\} A(t) .
$$

Next, let us calculate $\dot{B}(t)$. By use of the Leibniz rule

$$
\begin{aligned}
\dot{B}(t) & =\left(\dot{f} K_{+}\right) e^{f(t) K_{+}} e^{g(t) K_{3}} e^{h(t) K_{-}}+e^{f(t) K_{+}}\left(\dot{g} K_{3}\right) e^{g(t) K_{3}} e^{h(t) K_{-}}+e^{f(t) K_{+}} e^{g(t) K_{3}}\left(\dot{h} K_{-}\right) e^{h(t) K_{-}} \\
& =\left\{\dot{f} K_{+}+\dot{g} e^{f K_{+}} K_{3} e^{-f K_{+}}+\dot{h} e^{f K_{+}} e^{g K_{3}} K_{-} e^{-g K_{3}} e^{-f K_{+}}\right\} e^{f(t) K_{+}} e^{g(t) K_{3}} e^{h(t) K_{-}} \\
& =\left\{\dot{f} K_{+}+\dot{g}\left(K_{3}-f K_{+}\right)+\dot{h} e^{-g}\left(K_{-}-2 f K_{3}+f^{2} K_{+}\right)\right\} B(t) \\
& =\left\{\left(\dot{f}-\dot{g} f+\dot{h} e^{-g} f^{2}\right) K_{+}+\left(\dot{g}-2 \dot{h} e^{-g} f\right) K_{3}+\dot{h} e^{-g} K_{-}\right\} B(t)
\end{aligned}
$$

where we have used relations

$$
\begin{aligned}
& e^{f K_{+}} K_{3} e^{-f K_{+}}=K_{3}-f K_{+}, \\
& e^{g K_{3}} K_{-} e^{-g K_{3}}=e^{-g} K_{-} \quad \text { and } e^{f K_{+}} K_{-} e^{-f K_{+}}=K_{-}-2 f K_{3}+f^{2} K_{+} .
\end{aligned}
$$

The proof is easy. By comparing coefficients of $\dot{A}(t)$ and $\dot{B}(t)$ we have

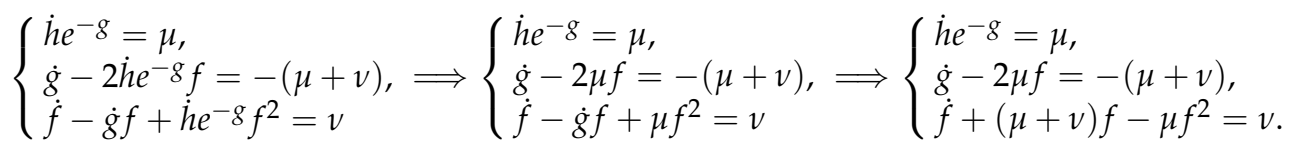

Note that the equation

$$
\dot{f}+(\mu+v) f-\mu f^{2}=v
$$

is a (famous) Riccati equation. If we can solve the equation then we obtain solutions like

$$
f \Longrightarrow g \Longrightarrow h
$$

Unfortunately, it is not easy. However there is an ansatz for the solution, $G, F$ and $E$. That is,

$$
f(t)=G(t), \quad g(t)=-2 \log (F(t)), \quad h(t)=E(t)
$$

in (3.15). To check these equations is left to readers (as a good exercise). From this

$$
A(0)=B(0), \quad \dot{A}(0)=\dot{B}(0) \Longrightarrow A(t)=B(t) \text { for all } t
$$


and we finally obtain the disentangling formula

$$
e^{t\left\{v K_{+}+\mu K_{-}-(\mu+v) K_{3}\right\}}=e^{G(t) K_{+}} e^{-2 \log (F(t)) K_{3}} e^{E(t) K_{-}}
$$

with (3.15).

Therefore (3.8) becomes

$\widehat{\rho}(t)=e^{\frac{\mu-v}{2} t} \exp \left(G(t) a^{\dagger} \otimes a^{\dagger}\right) \exp (-\log (F(t))(N \otimes \mathbf{1}+\mathbf{1} \otimes N+\mathbf{1} \otimes \mathbf{1})) \exp (E(t) a \otimes a) \widehat{\rho}(0)$ with (3.15). Some calculation by use of (2.22) gives

$$
\begin{aligned}
& \widehat{\rho}(t) \frac{e^{\frac{\mu-v}{2} t}}{\bar{F}(t)} \exp \left(G(t) a^{\dagger} \otimes a^{T}\right)\left\{\exp (-\log (F(t)) N) \otimes \exp (-\log (F(t)) N)^{T}\right\} \times \\
& \exp \left(E(t) a \otimes\left(a^{\dagger}\right)^{T}\right) \hat{\rho}(0)
\end{aligned}
$$

where we have used $N^{T}=N$ and $a^{\dagger}=a^{T}$. By coming back to matrix form by use of (3.6) like

$$
\begin{aligned}
\exp \left(E(t) a \otimes\left(a^{\dagger}\right)^{T}\right) \widehat{\rho}(0) & =\sum_{m=0}^{\infty} \frac{E(t)^{m}}{m !}\left(a \otimes\left(a^{\dagger}\right)^{T}\right)^{m} \widehat{\rho}(0) \\
& =\sum_{m=0}^{\infty} \frac{E(t)^{m}}{m !}\left(a^{m} \otimes\left(\left(a^{\dagger}\right)^{m}\right)^{T}\right) \widehat{\rho}(0) \longrightarrow \sum_{m=0}^{\infty} \frac{E(t)^{m}}{m !} a^{m} \rho(0)\left(a^{\dagger}\right)^{m}
\end{aligned}
$$

we finally obtain

$$
\begin{aligned}
& \rho(t)=\frac{e^{\frac{\mu-v}{2}} t}{F(t)} \times \\
& \sum_{n=0}^{\infty} \frac{G(t)^{n}}{n !}\left(a^{\dagger}\right)^{n}\left[\exp (-\log (F(t)) N)\left\{\sum_{m=0}^{\infty} \frac{E(t)^{m}}{m !} a^{m} \rho(0)\left(a^{\dagger}\right)^{m}\right\} \exp (-\log (F(t)) N)\right] a^{n} .
\end{aligned}
$$

This form is very beautiful but complicated!

\subsection{General solution}

Last, we treat the full equation (3.2)

$$
\frac{\partial}{\partial t} \rho=-i \omega\left(a^{\dagger} a \rho-\rho a^{\dagger} a\right)-\frac{\mu}{2}\left(a^{\dagger} a \rho+\rho a^{\dagger} a-2 a \rho a^{\dagger}\right)-\frac{v}{2}\left(a a^{\dagger} \rho+\rho a a^{\dagger}-2 a^{\dagger} \rho a\right) .
$$


From the lesson in the preceding subsection it is easy to rewrite this as

$$
\frac{\partial}{\partial t} \widehat{\rho}=\left\{-i \omega K_{0}+v K_{+}+\mu K_{-}-(\mu+v) K_{3}+\frac{\mu-v}{2} \mathbf{1} \otimes \mathbf{1}\right\} \widehat{\rho}
$$

in terms of $K_{0}=N \otimes \mathbf{1}-\mathbf{1} \otimes N$ (note that $N^{T}=N$ ). Then it is easy to see

$$
\left[K_{0}, K_{+}\right]=\left[K_{0}, K_{3}\right]=\left[K_{0}, K_{-}\right]=0
$$

from (3.9), which is left to readers. That is, $K_{0}$ commutes with all $\left\{K_{+}, K_{3}, K_{-}\right\}$. Therefore

$$
\begin{aligned}
\widehat{\rho}(t) & =e^{-i \omega t K_{0}} e^{t\left\{v K_{+}+\mu K_{-}-(\mu+v) K_{3}+\frac{\mu-v}{2} \mathbf{1} \otimes \mathbf{1}\right\}} \widehat{\rho}(0) \\
& =e^{\frac{\mu-v}{2} t} \exp \left(-i \omega t K_{0}\right) \exp \left(G(t) K_{+}\right) \exp \left(-2 \log (F(t)) K_{3}\right) \exp \left(E(t) K_{-}\right) \widehat{\rho}(0) \\
& =e^{\frac{\mu-v}{2} t} \exp \left(G(t) K_{+}\right) \exp \left(\left\{-i \omega t K_{0}-2 \log (F(t)) K_{3}\right\}\right) \exp \left(E(t) K_{-}\right) \widehat{\rho}(0),
\end{aligned}
$$

so that the general solution that we are looking for is just given by

$$
\begin{aligned}
\rho(t)=\frac{e^{\frac{\mu-v}{2}} t}{F(t)} \sum_{n=0}^{\infty} & \frac{G(t)^{n}}{n !}\left(a^{\dagger}\right)^{n}[\exp (\{-i \omega t-\log (F(t))\} N) \times \\
& \left.\left\{\sum_{m=0}^{\infty} \frac{E(t)^{m}}{m !} a^{m} \rho(0)\left(a^{\dagger}\right)^{m}\right\} \exp (\{i \omega t-\log (F(t))\} N)\right] a^{n}
\end{aligned}
$$

by use of (3.17) and (3.18).

Particularly, if $v=0$ then

$$
\begin{aligned}
& E(t)=\frac{2 \sinh \left(\frac{\mu}{2} t\right)}{\cosh \left(\frac{\mu}{2} t\right)+\sinh \left(\frac{\mu}{2} t\right)}=1-e^{-\mu t}, \\
& F(t)=\cosh \left(\frac{\mu}{2} t\right)+\sinh \left(\frac{\mu}{2} t\right)=e^{\frac{\mu}{2} t} \\
& G(t)=0
\end{aligned}
$$

from (3.15), so that we have

$$
\rho(t)=e^{-\left(\frac{\mu}{2}+i \omega\right) t N}\left\{\sum_{m=0}^{\infty} \frac{\left(1-e^{-\mu t}\right)^{m}}{m !} a^{m} \rho(0)\left(a^{\dagger}\right)^{m}\right\} e^{-\left(\frac{\mu}{2}-i \omega\right) t N}
$$

from (3.21).

\section{Quantum counterpart}

In this section we explicitly calculate $\rho(t)$ for the initial value $\rho(0)$ given in the following. 
4.1. Case of $\rho(0)=|0\rangle\langle 0|$

Noting $a|0\rangle=0\left(\Leftrightarrow 0=\langle 0| a^{\dagger}\right)$, this case is very easy and we have

$$
\rho(t)=\frac{e^{\frac{\mu-v}{2}} t}{F(t)} \sum_{n=0}^{\infty} \frac{G(t)^{n}}{n !}\left(a^{\dagger}\right)^{n}|0\rangle\left\langle 0\left|a^{n}=\frac{e^{\frac{\mu-v}{2}} t}{F(t)} \sum_{n=0}^{\infty} G(t)^{n}\right| n\right\rangle\langle n|=\frac{e^{\frac{\mu-v}{2} t}}{F(t)} e^{\log G(t) N}
$$

because the number operator $N\left(=a^{\dagger} a\right)$ is written as

$$
N=\sum_{n=0}^{\infty} n|n\rangle\langle n|\Longrightarrow N| n\rangle=n|n\rangle
$$

, see for example (2.15). To check the last equality of (4.1) is left to readers. Moreover, $\rho(t)$ can be written as

$$
\rho(t)=(1-G(t)) e^{\log G(t) N}=e^{\log (1-G(t))} e^{\log G(t) N}
$$

see the next subsection.

\subsection{Case of $\rho(0)=|\alpha\rangle\langle\alpha|(\alpha \in \mathbf{C})$}

Remind that $|\alpha\rangle$ is a coherent state given by (2.16) $\left(a|\alpha\rangle=\alpha|\alpha\rangle \Leftrightarrow\langle\alpha| a^{\dagger}=\langle\alpha| \bar{\alpha}\right)$. First of all let us write down the result :

$$
\rho(t)=e^{|\alpha|^{2} e^{-(\mu-v) t} \log G(t)+\log (1-G(t))} \exp \left\{-\log G(t)\left(\alpha e^{-\left(\frac{\mu-v}{2}+i \omega\right) t} a^{\dagger}+\bar{\alpha} e^{-\left(\frac{\mu-v}{2}-i \omega\right) t} a-N\right)\right\}
$$

with $G(t)$ in (3.15). Here we again meet a term like (2.3)

$$
\alpha e^{-\left(\frac{\mu-v}{2}+i \omega\right) t} a^{\dagger}+\bar{\alpha} e^{-\left(\frac{\mu-v}{2}-i \omega\right) t} a
$$

with $\lambda=\frac{\mu-v}{2}$.

Therefore, (4.3) is just our quantum counterpart of the classical damped harmonic oscillator. The proof is divided into four parts.

[First Step] From (3.21) it is easy to see

$$
\sum_{m=0}^{\infty} \frac{E(t)^{m}}{m !} a^{m}|\alpha\rangle\left\langle\alpha\left|\left(a^{\dagger}\right)^{m}=\sum_{m=0}^{\infty} \frac{E(t)^{m}}{m !} \alpha^{m}\right| \alpha\right\rangle\left\langle\alpha\left|\bar{\alpha}^{m}=\sum_{m=0}^{\infty} \frac{\left(E(t)|\alpha|^{2}\right)^{m}}{m !}\right| \alpha\right\rangle\left\langle\alpha\left|=e^{E(t)|\alpha|^{2}}\right| \alpha\right\rangle\langle\alpha| .
$$


[Second Step] From (3.21) we must calculate the term

$$
e^{\gamma N}|\alpha\rangle\left\langle\alpha\left|e^{\bar{\gamma} N}=e^{\gamma N} e^{\alpha a^{+}-\bar{\alpha} a}\right| 0\right\rangle\langle 0| e^{-\left(\alpha a^{+}-\bar{\alpha} a\right)} e^{\bar{\gamma} N}
$$

where $\gamma=-i \omega t-\log (F(t))$ (note $\bar{\gamma} \neq-\gamma$ ). It is easy to see

$$
e^{\gamma N} e^{\alpha a^{+}-\bar{\alpha} a}|0\rangle=e^{\gamma N} e^{\alpha a^{+}-\bar{\alpha} a} e^{-\gamma N} e^{\gamma N}|0\rangle=e^{e^{\gamma N}\left(\alpha a^{+}-\bar{\alpha} a\right) e^{-\gamma N}}|0\rangle=e^{\alpha e^{\gamma} a^{+}-\bar{\alpha} e^{-\gamma} a}|0\rangle
$$

where we have used

$$
e^{\gamma N} a^{\dagger} e^{-\gamma N}=e^{\gamma} a^{\dagger} \text { and } e^{\gamma N} a e^{-\gamma N}=e^{-\gamma} a
$$

The proof is easy and left to readers. Therefore, by use of Baker-Campbell-Hausdorff formula (2.17) two times

$$
\begin{aligned}
e^{\alpha e^{\gamma} a^{+}-\bar{\alpha} e^{-\gamma} a}|0\rangle & =e^{-\frac{|\alpha|^{2}}{2}} e^{\alpha e^{\gamma} a^{\dagger}} e^{-\bar{\alpha} e^{-\gamma} a}|0\rangle=e^{-\frac{|\alpha|^{2}}{2}} e^{\alpha e^{\gamma} a^{\dagger}}|0\rangle \\
& =e^{-\frac{|\alpha|^{2}}{2}} e^{\frac{|\alpha|^{2}}{2} e^{\gamma+\bar{\gamma}}} e^{\alpha e^{\gamma} a^{\dagger}-\bar{\alpha} e^{\bar{\gamma}} a}|0\rangle=e^{-\frac{|\alpha|^{2}}{2}\left(1-e^{\gamma+\bar{\gamma}}\right)}\left|\alpha e^{\gamma}\right\rangle
\end{aligned}
$$

and we obtain

$$
e^{\gamma N}|\alpha\rangle\left\langle\alpha\left|e^{\bar{\gamma} N}=e^{-|\alpha|^{2}\left(1-e^{\gamma+\bar{\gamma}}\right)}\right| \alpha e^{\gamma}\right\rangle\left\langle\alpha e^{\gamma}\right|
$$

with $\gamma=-i \omega t-\log (F(t))$.

[Third Step] Under two steps above the equation (3.21) becomes

$$
\rho(t)=\frac{e^{\frac{\mu-v}{2} t} e^{|\alpha|^{2}\left(E(t)-1+e^{\gamma+\gamma}\right)}}{F(t)} \sum_{n=0}^{\infty} \frac{G(t)^{n}}{n !}\left(a^{\dagger}\right)^{n}\left|\alpha e^{\gamma}\right\rangle\left\langle\alpha e^{\gamma}\right| a^{n} .
$$

For simplicity we set $z=\alpha e^{\gamma}$ and calculate the term

$$
(\sharp)=\sum_{n=0}^{\infty} \frac{G(t)^{n}}{n !}\left(a^{\dagger}\right)^{n}|z\rangle\langle z| a^{n} .
$$

Since $|z\rangle=e^{-|z|^{2} / 2} e^{z a^{\dagger}}|0\rangle$ we have 


$$
\begin{aligned}
(\sharp) & =e^{-|z|^{2}} \sum_{n=0}^{\infty} \frac{G(t)^{n}}{n !}\left(a^{\dagger}\right)^{n} e^{z a^{+}}|0\rangle\langle 0| e^{\bar{z} a} a^{n} \\
& =e^{-|z|^{2}} e^{z a^{+}}\left\{\sum_{n=0}^{\infty} \frac{G(t)^{n}}{n !}\left(a^{\dagger}\right)^{n}|0\rangle\langle 0| a^{n}\right\} e^{\bar{z} a} \\
& =e^{-|z|^{2}} e^{z a^{+}}\left\{\sum_{n=0}^{\infty} G(t)^{n}|n\rangle\langle n|\right\} e^{\bar{z} a} \\
& =e^{-|z|^{2}} e^{z a^{+}} e^{\log G(t) N} e^{\bar{z} a}
\end{aligned}
$$

by (4.1). Namely, this form is a kind of disentangling formula, so we want to restore an entangling formula.

For the purpose we use the disentangling formula

$$
e^{\alpha a^{+}+\beta a+\gamma N}=e^{\alpha \beta \frac{e^{\gamma}-(1+\gamma)}{\gamma^{2}}} e^{\alpha \frac{e^{\gamma}-1}{\gamma} a^{+}} e^{\gamma N} e^{\beta \frac{e^{\gamma}-1}{\gamma} a}
$$

where $\alpha, \beta, \gamma$ are usual numbers. The proof is given in the fourth step. From this it is easy to see

$$
e^{u a^{\dagger}} e^{v N} e^{w a}=e^{-\frac{u w\left(e^{v}-(1+v)\right)}{\left(e^{v}-1\right)^{2}}} e^{\frac{u v}{e^{v}-1} a^{\dagger}+\frac{v w}{e^{v}-1} a+v N}
$$

Therefore $(u \rightarrow z, v \rightarrow \log G(t), w \rightarrow \bar{z})$

$$
(\sharp)=e^{-|z|^{2}} e^{\frac{|z|^{2}(1+\log G(t)-G(t))}{(1-G(t))^{2}}} e^{\frac{\log G(t)}{G(t)-1} z a^{\dagger}+\frac{\log G(t)}{G(t)-1} \bar{z} a+\log G(t) N},
$$

so by noting

$$
z=\alpha e^{\gamma}=\alpha \frac{e^{-i \omega t}}{F(t)} \quad \text { and } \quad|z|^{2}=|\alpha|^{2} e^{\gamma+\bar{\gamma}}=|\alpha|^{2} \frac{1}{F(t)^{2}}
$$

we have

$$
\begin{aligned}
\rho(t) & =\frac{e^{\frac{\mu-v}{2} t}}{F(t)} e^{|\alpha|^{2}(E(t)-1)} e^{|\alpha|^{2} \frac{1+\log G(t)-G(t)}{F(t)^{2}(1-G(t))^{2}}} e^{\frac{\log G(t)}{F(t)(G(t)-1)} \alpha e^{-i \omega t} a^{\dagger}+\frac{\log G(t)}{F(t)(G(t)-1)} \bar{\alpha} e^{i \omega t} a+\log G(t) N} \\
& =\frac{e^{\frac{\mu-v}{2} t}}{F(t)} e^{|\alpha|^{2}\left\{E(t)-1+\frac{1+\log G(t)-G(t)}{F(t)^{2}(1-G(t))^{2}}\right\}} e^{\frac{\log G(t)}{F(t)(G(t)-1)} \alpha e^{-i \omega t} a^{\dagger}+\frac{\log G(t)}{F(t)(G(t)-1)} \bar{\alpha} e^{i \omega t} a+\log G(t) N} .
\end{aligned}
$$

By the way, from (3.15) 


$$
G(t)-1=-\frac{e^{\frac{\mu-v}{2} t}}{F(t)}, \quad \frac{1}{F(t)(G(t)-1)}=-e^{-\frac{\mu-v}{2} t}, \quad E(t)-1=-\frac{e^{-\frac{\mu-v}{2} t}}{F(t)}
$$

and

$$
\begin{aligned}
\frac{1-G(t)+\log G(t)}{F(t)^{2}(G(t)-1)^{2}} & =e^{-(\mu-v) t}\left\{\frac{e^{\frac{\mu-v}{2} t}}{F(t)}+\log G(t)\right\} \\
& =\frac{e^{-\frac{\mu-v}{2} t}}{F(t)}+e^{-(\mu-v) t} \log G(t) \\
& =-(E(t)-1)+e^{-(\mu-v) t} \log G(t)
\end{aligned}
$$

we finally obtain

$$
\begin{aligned}
\rho(t) & =(1-G(t)) e^{|\alpha|^{2} e^{-(\mu-v) t} \log G(t)} e^{-\log G(t)\left\{\alpha e^{-i \omega t} e^{-\frac{\mu-v}{2} t} a^{\dagger}+\bar{\alpha} e^{i \omega t} e^{-\frac{\mu-v}{2} t} a-N\right\}} \\
& =e^{|\alpha|^{2} e^{-(\mu-v) t} \log G(t)+\log (1-G(t))} e^{-\log G(t)\left\{\alpha e^{-\left(\frac{\mu-v}{2}+i \omega\right) t} a^{\dagger}+\bar{\alpha} e^{-\left(\frac{\mu-v}{2}-i \omega\right) t} a-N\right\} .} .
\end{aligned}
$$

[Fourth Step] In last, let us give the proof to the disentangling formula (4.4) because it is not so popular as far as we know. From (4.4)

$$
\begin{aligned}
\alpha a^{\dagger}+\beta a+\gamma N & =\gamma a^{\dagger} a+\alpha a^{\dagger}+\beta a \\
& =\gamma\left\{\left(a^{\dagger}+\frac{\beta}{\gamma}\right)\left(a+\frac{\alpha}{\gamma}\right)-\frac{\alpha \beta}{\gamma^{2}}\right\} \\
& =\gamma\left(a^{\dagger}+\frac{\beta}{\gamma}\right)\left(a+\frac{\alpha}{\gamma}\right)-\frac{\alpha \beta}{\gamma}
\end{aligned}
$$

we have

$$
\begin{aligned}
e^{\alpha a^{+}+\beta a+\gamma N} & =e^{-\frac{\alpha \beta}{\gamma}} e^{\gamma\left(a^{+}+\frac{\beta}{\gamma}\right)\left(a+\frac{\alpha}{\gamma}\right)} \\
& =e^{-\frac{\alpha \beta}{\gamma}} e^{\frac{\beta}{\gamma} a} e^{\gamma a^{+}\left(a+\frac{\alpha}{\gamma}\right)} e^{-\frac{\beta}{\gamma} a} \\
& =e^{-\frac{\alpha \beta}{\gamma}} e^{\frac{\beta}{\gamma} a} e^{-\frac{\alpha}{\gamma} a^{+}} e^{\gamma a^{+} a} e^{\frac{\alpha}{\gamma} a^{+}} e^{-\frac{\beta}{\gamma} a} .
\end{aligned}
$$


Then, careful calculation gives the disentangling formula (4.4) $\left(N=a^{\dagger} a\right)$

$$
\begin{aligned}
e^{-\frac{\alpha \beta}{\gamma}} e^{\frac{\beta}{\gamma} a} e^{-\frac{\alpha}{\gamma} a^{+}} e^{\gamma N} e^{\frac{\alpha}{\gamma} a^{+}} e^{-\frac{\beta}{\gamma} a} & =e^{-\frac{\alpha \beta}{\gamma}} e^{-\frac{\alpha \beta}{\gamma^{2}}} e^{-\frac{\alpha}{\gamma} a^{+}} e^{\frac{\beta}{\gamma} a} e^{\gamma N} e^{\frac{\alpha}{\gamma} a^{+}} e^{-\frac{\beta}{\gamma} a} \\
& =e^{-\left(\frac{\alpha \beta}{\gamma}+\frac{\alpha \beta}{\gamma^{2}}\right)} e^{-\frac{\alpha}{\gamma} a^{+}} e^{\frac{\beta}{\gamma} a} e^{\gamma N} e^{\frac{\alpha}{\gamma} a^{+}} e^{-\frac{\beta}{\gamma} a} \\
& =e^{-\left(\frac{\alpha \beta}{\gamma}+\frac{\alpha \beta}{\gamma^{2}}\right)} e^{-\frac{\alpha}{\gamma} a^{+}} e^{\gamma N} e^{\frac{\beta}{\gamma} e^{\gamma} a} e^{\frac{\alpha}{\gamma} a^{+}} e^{-\frac{\beta}{\gamma} a} \\
& =e^{-\left(\frac{\alpha \beta}{\gamma}+\frac{\alpha \beta}{\gamma^{2}}\right)+\frac{\alpha \beta}{\gamma^{2}} e^{\gamma}} e^{-\frac{\alpha}{\gamma} a^{+}} e^{\gamma N} e^{\frac{\alpha}{\gamma} a^{+}} e^{\frac{\beta}{\gamma} e^{\gamma} a} e^{-\frac{\beta}{\gamma} a} \\
& =e^{\alpha \beta \frac{e^{\gamma}-1-\gamma}{\gamma^{2}}} e^{-\frac{\alpha}{\gamma} a^{+}} e^{\frac{\alpha}{\gamma}} e^{\gamma} a^{+} e^{\gamma N} e^{\beta \frac{\beta \gamma-1}{\gamma} a} \\
& =e^{\alpha \beta \frac{e^{\gamma}-1-\gamma}{\gamma^{2}}} e^{\alpha \frac{e^{\gamma}-1}{\gamma} a^{+}} e^{\gamma N} e^{\beta \frac{e^{\gamma}-1}{\gamma} a}
\end{aligned}
$$

by use of some commutation relations

$$
e^{s a} e^{t a^{\dagger}}=e^{s t} e^{t a^{\dagger}} e^{s a}, \quad e^{s a} e^{t N}=e^{t N} e^{s e^{t} a}, \quad e^{t N} e^{s a^{+}}=e^{s e^{t} a^{\dagger}} e^{t N}
$$

The proof is simple. For example,

$$
e^{s a} e^{t a^{+}}=e^{s a} e^{t a^{\dagger}} e^{-s a} e^{s a}=e^{t e^{s a} a^{\dagger} e^{-s a}} e^{s a}=e^{t\left(a^{\dagger}+s\right)} e^{s a}=e^{s t} e^{t a^{\dagger}} e^{s a} .
$$

The remainder is left to readers.

We finished the proof. The formula (4.3) is both compact and clear-cut and has not been known as far as we know. See [1] and [16] for some applications.

In last, let us present a challenging problem. A squeezed state $|\beta\rangle(\beta \in \mathbf{C})$ is defined as

$$
|\beta\rangle=e^{\frac{1}{2}\left(\beta\left(a^{+}\right)^{2}-\bar{\beta} a^{2}\right)}|0\rangle
$$

See for example [4]. For the initial value $\rho(0)=|\beta\rangle\langle\beta|$ we want to calculate $\rho(t)$ in (3.21) like in the text. However, we cannot sum up it in a compact form like (4.3) at the present time, so we propose the problem,

Problem sum up $\rho(t)$ in a compact form.

\section{Concluding remarks}

In this chapter we treated the quantum damped harmonic oscillator, and studied mathematical structure of the model, and constructed general solution with any initial condition, and gave a quantum counterpart in the case of taking coherent state as an initial condition. It is in my opinion perfect. 
However, readers should pay attention to the fact that this is not a goal but a starting point. Our real target is to construct general theory of Quantum Mechanics with Dissipation.

In the papers [7] and [8] (see also [13]) we studied a more realistic model entitled "Jaynes-Cummings Model with Dissipation" and constructed some approximate solutions under any initial condition. In the paper [5] we studied "Superluminal Group Velocity of Neutrinos" from the point of view of Quantum Mechanics with Dissipation.

Unfortunately, there is no space to introduce them. It is a good challenge for readers to read them carefully and attack the problems.

\section{Acknowledgments}

I would like to thank Ryu Sasaki and Tatsuo Suzuki for their helpful comments and suggestions.

\section{Author details}

Kazuyuki Fujii

* Address all correspondence to: fujii@yokohama-cu.ac.jp

International College of Arts and Sciences, Yokohama City University, Yokohama, Japan

\section{References}

[1] H. -P. Breuer and F. Petruccione : The theory of open quantum systems, Oxford University Press, New York, 2002.

[2] P. Dirac: The Principles of Quantum Mechanics, Fourth Edition, Oxford University Press, 1958.

This is a bible of Quantum Mechanics.

[3] R. Endo, K. Fujii and T. Suzuki : General Solution of the Quantum Damped Harmonic Oscillator, Int. J. Geom. Methods. Mod. Phys, 5 (2008), 653, arXiv : 0710.2724 [quant-ph].

[4] K. Fujii : Introduction to Coherent States and Quantum Information Theory, quant-ph/0112090.

This is a kind of lecture note based on my (several) talks.

[5] K. Fujii : Superluminal Group Velocity of Neutrinos : Review, Development and Problems, Int. J. Geom. Methods Mod. Phys, 10 (2013), 1250083, arXiv:1203.6425 [physics].

[6] K. Fujii and T. Suzuki : General Solution of the Quantum Damped Harmonic Oscillator II : Some Examples, Int. J. Geom. Methods Mod. Phys, 6 (2009), 225, arXiv : 0806.2169 [quant-ph].

[7] K. Fujii and T. Suzuki : An Approximate Solution of the Jaynes-Cummings Model with Dissipation, Int. J. Geom. Methods Mod. Phys, 8 (2011), 1799, arXiv : 1103.0329 [math-ph]. 
[8] K. Fujii and T. Suzuki : An Approximate Solution of the Jaynes-Cummings Model with Dissipation II : Another Approach, Int. J. Geom. Methods Mod. Phys, 9 (2012), 1250036, arXiv : 1108.2322 [math-ph].

[9] K. Fujii and et al ; Treasure Box of Mathematical Sciences (in Japanese), Yuseisha, Tokyo, 2010.

I expect that the book will be translated into English.

[10] V. Gorini, A. Kossakowski and E. C. G. Sudarshan ; Completely positive dynamical semigroups of N-level systems, J. Math. Phys, 17 (1976), 821.

[11] H. S. Green : Matrix Mechanics, P. Noordhoff Ltd, Groningen, 1965.

This is my favorite textbook of elementary Quantum Mechanics.

[12] K. Hornberger : Introduction to Decoherence Theory, in "Theoretical Foundations of Quantum Information", Lecture Notes in Physics, 768 (2009), 221-276, Springer, Berlin, quant-ph/061211.

[13] E. T. Jaynes and F. W. Cummings : Comparison of Quantum and Semiclassical Radiation Theories with Applications to the Beam Maser, Proc. IEEE, 51 (1963), 89.

[14] J. R. Kauder and Bo-S. Skagerstam : Coherent States-Applications in Physics and Mathematical Physics, World Scientific, Singapore, 1985.

This is a kind of dictionary of coherent states.

[15] G. Lindblad; On the generator of quantum dynamical semigroups, Commun. Math. Phys, 48 (1976), 119.

[16] W. P. Schleich : Quantum Optics in Phase Space, WILEY-VCH, Berlin, 2001.

This book is strongly recommended although it is thick.

[17] C. Zachos : Crib Notes on Campbell-Baker-Hausdorff expansions, unpublished, 1999, see http://www.hep.anl.gov/czachos/index.html.

I expect that this (crib) note will be published in some journal. 
Section 3

\section{Path Integrals}



Chapter 8

\title{
The Schwinger Action Principle and Its Applications to Quantum Mechanics
}

\author{
Paul Bracken \\ Additional information is available at the end of the chapter
}

http://dx.doi.org/10.5772/53472

\section{Introduction}

In physics it is generally of interest to understand the dynamics of a system. The way the dynamics is to be specified and studied invariably depends on the scale of the system, that is whether it is macroscopic or microscopic. The formal machinery with which the world is explained and understood depends at which of these two levels an experiment is conducted [1]. At the classical level, the dynamics of the system can be understood in terms of such things as trajectories in space or space-time.

In fact, classical mechanics can be formulated in terms of a principle of stationary action to obtain the Euler-Lagrange equations. To carry this out, an action functional has to be defined. It is written as $S$ and given by

$$
S[\mathbf{q}(t)]=\int_{t_{1}}^{t_{2}} L\left(q^{i}(t), \dot{q}^{i}(t), t\right) d t .
$$

The action depends on the Lagrangian, written $L$ in (1). It is to be emphasized that the action is a functional, which can be thought of as a function defined on a space of functions. For any given trajectory or path in space, the action works out to be a number, so $S$ maps paths to real numbers.

One way to obtain equations of motion is by means of Hamilton's principle. Hamilton's principle states that the actual motion of a particle with Lagrangian $L$ is such that the action functional is stationary. This means the action functional achieves a minimum or maximum value. To apply and use this principle, Stationary action must result in the Euler-Lagrange equations of motion. Conversely, if the Euler-Lagrange equations are imposed, the action functional should be stationary. As is well known, the Euler-Lagrange equations provide a system of second-order differential equations for the path. This in turn leads to other approaches to the same end. 
As an illustration, the momentum canonically conjugate to the coordinate $q^{i}$ is defined by

$$
p_{i}=\frac{\partial L}{\partial \dot{q}^{i}}
$$

The dependence on the velocity components $\dot{q}^{i}$ can be eliminated in favor of the canonical momentum. This means that (2) must be solved for the $\dot{q}^{i}$ in terms of the $q^{i}$ and $p_{i}$, and the inverse function theorem states this is possible if and only if $\left(\partial p_{i} / \partial \dot{q}^{j}\right) \neq 0$. Given a non-singular system all dependence on $\dot{q}^{i}$ can be eliminated by means of a Legendre transformation

$$
H(\mathbf{q}, \mathbf{p}, t)=p_{i} \dot{q}^{i}-L(\mathbf{q}, \dot{\mathbf{q}}, t) .
$$

The Hamiltonian equations are obtained by considering the derivatives of the Hamiltonian with respect to $q^{i}$ and $p_{i}$. The action in terms of $H$ is written

$$
S[\mathbf{q}, \mathbf{p}]=\int_{t_{1}}^{t_{2}} d t\left[p_{i} \dot{q}^{i}-H(\mathbf{q}, \mathbf{p}, t)\right]
$$

such that the action now depends on both $\mathbf{q}$ and $\mathbf{p}$. Using this the principle of stationary action can then be modified so that Hamilton's equations result.

In passing to the quantum domain, the concept of path or trajectory is of less importance, largely because it has no meaning. In quantum physics it can no longer be assumed that the interaction between system and measuring device can be made arbitrarily small and that there are no restrictions on what measurements can be made on the system either in terms of type or in accuracy. Both these assumptions tend to break down at the scales of interest here, and one is much more interested in states and observables, which replace the classical idea of a trajectory with well defined properties [2,3].

Now let us follow Dirac and consider possible measurements on a system as observables. Suppose $A_{i}$ denotes any observable with $a_{i}$ as a possible outcome of any measurement of this observable. As much information as possible can be extracted with regard to a quantum mechanical system by measuring some set of observables $\left\{A_{i}\right\}_{i=1}^{n}$ without restriction. Thus, the observables should be mutually compatible because the measurement of any observable in the set does not affect the measurement of any of the other observables. The most information that can be assembled about a system is the collection of numbers $\left\{a_{i}\right\}_{i=1}^{n}$, which are possible values for the set of mutually compatible observables, and this set specifies the state of the system.

\section{Schwinger's action principle}

In Dirac's view of quantum mechanics, the state of a system is associated with a vector in a complex vector space $V$. The knowledge of the values for a complete set of mutually compatible observables gives the most information about a state. It can then be assumed that $\{|a\rangle\}$, where $|a\rangle=\left|a_{1}, \cdots, a_{n}\right\rangle$, the set of all possible states, forms a basis for $V$. Associated 
with any vector space $V$ is the dual space $V^{*}$ whose elements are referred to as bras in Dirac's terminology. A basis for $V^{*}$ is denoted by $\{\langle a|\}$ and is dual to $\{|a\rangle\}$. The quantities satisfy

$$
\left\langle a^{\prime} \mid a^{\prime \prime}\right\rangle=\delta\left(a^{\prime}, a^{\prime \prime}\right)
$$

where $\delta\left(a^{\prime}, a^{\prime \prime}\right)$ is the Kronecker delta if $a^{\prime}$ is a discrete set, and the Dirac delta if it is continuous. The choice of a complete set of mutually compatible observables is not unique. Suppose $\{|b\rangle\}$ also provides a basis for $V$ relevant to another set of mutually compatible observables $B_{1}, B_{2}, \cdots$. Since $\{|a\rangle\}$ and $\{|b\rangle\}$ are both bases for $V$, this means that one set of basis vectors can be expressed in terms of the other set

$$
|b\rangle=\sum_{a}|a\rangle\langle a \mid b\rangle
$$

and the $\langle a \mid b\rangle$ coefficients are some set of complex numbers, so that $\langle b \mid a\rangle^{*}=\langle a \mid b\rangle$. If there is a third basis for $V$ provided by $\{|c\rangle\}$, then these complex numbers are related by means of

$$
\langle a \mid c\rangle=\sum_{b}\langle a \mid b\rangle\langle b \mid c\rangle
$$

Schwinger's action principle is based on the types of transformation properties of the transformation functions which can be constructed from this basis set [4].

Suppose the transformation function is subjected to, as Schwinger asserted, any conceivable infinitesimal variation. Then, by performing an arbitrary variation of (7), it follows that

$$
\delta\langle a \mid c\rangle=\sum_{b}[(\delta\langle a \mid b\rangle)\langle b \mid c\rangle+\langle a \mid b\rangle(\delta\langle b \mid c\rangle)]
$$

and moreover,

$$
\delta\langle a \mid b\rangle=\delta\langle b \mid a\rangle^{*} .
$$

Now a new operator can be defined which evaluates this actual variation when it is placed between the relevant state vectors. Define this operator to be $\delta W_{a b}$, so that it has the following action between states

$$
\delta\langle a \mid b\rangle=\frac{i}{\hbar}\left\langle a\left|\delta W_{a b}\right| b\right\rangle .
$$

Including the factor of $\hbar$ gives the operator units of action. Using (10) in (8) produces,

$$
\left\langle a\left|\delta W_{a c}\right| c\right\rangle=\sum_{b}\left[\left\langle a\left|\delta W_{a b}\right| b\right\rangle\langle b \mid c\rangle+\langle a \mid b\rangle\left\langle b\left|\delta W_{b c}\right| c\right\rangle\right]=\left\langle a\left|\delta W_{a b}+\delta W_{b c}\right| c\right\rangle,
$$

using the completeness relation (7). Now it follows from (11) that

$$
\delta W_{a c}=\delta W_{a b}+\delta W_{b c}
$$


In the case in which the $a$ and $b$ descriptions are identified then using $\delta\left\langle a \mid a^{\prime}\right\rangle=0$, there results

$$
\delta W_{a a}=0 .
$$

Identifying the $a$ and $c$ pictures in (11) gives,

$$
\delta W_{b a}=-\delta W_{a b}
$$

The complex conjugate of (10) with $a$ and $b$ descriptions reversed implies

$$
-\frac{i}{\hbar}\left\langle b\left|\delta W_{b a}\right| a\right\rangle^{*}=\frac{i}{\hbar}\left\langle a\left|\delta W_{a b}\right| b\right\rangle .
$$

This has the equivalent form

$$
-\left\langle a\left|\delta W_{b a}^{+}\right| b\right\rangle=\left\langle a\left|\delta W_{a b}\right| b\right\rangle
$$

Using (14), this yields the property

$$
\delta W_{b a}^{\dagger}=\delta W_{a b}
$$

The basic properties of the transformation function and the definition (10) have produced all of these additional properties [5,6].

In the Heisenberg picture, the basis kets become time dependent. The transformation function relates states which are eigenstates of different complete sets of commuting observables at different times. Instead of using different letters, different subscripts 1, 2 can be used to denote different complete sets of commuting observables. In this event, (10) takes the form

$$
\delta\left\langle a_{2}^{\prime}, t_{2} \mid a_{1}^{\prime}, t_{1}\right\rangle=\frac{i}{\hbar}\left\langle a_{2}^{\prime}, t_{2}\left|\delta W_{21}\right| a_{1}^{\prime}, t_{1}\right\rangle .
$$

The assumption at the heart of this approach is that the operator $\delta W_{21}$ in (18) is obtained from the variation of a single operator $W_{21}$. This is referred to as the action operator.

To adapt the results of the previous notation to the case with subscripts, we should have

$$
W_{31}=W_{32}+W_{21}, \quad W_{11}=0, \quad W_{21}=-W_{12}=W_{21}^{\dagger} .
$$

At this point, a correspondence between the Schwinger action principle and the classical principle of stationary action can be made. Suppose the members of a complete set of commuting observables $A_{1}$ which have eigenvectors $\left|a_{1}, t\right\rangle$ in the Heisenberg picture are deformed in some fashion at time $t_{1}$. For example, take the alteration in the observables to correspond to a unitary transformation $A \rightarrow U^{\dagger} A U$ such that $U^{\dagger}=U^{-1}$. To remain 
eigenstates of the transformed operator, it must be that states transform as $|a\rangle \rightarrow U^{\dagger}|a\rangle$. Thinking of the transformation as being infinitesimal in nature, the operator $U$ can be written $U=I+\frac{i}{\hbar} G$, where $G$ is Hermitean. It is then possible to define a variation

$$
\delta\left|a_{1}, t_{1}\right\rangle=-\frac{i}{\hbar} G_{1}\left|a_{1}, t_{1}\right\rangle .
$$

Here operator $G$ is a Hermitian operator and depends only on the observables $A_{1}$ at the time $t_{1}$. Similarly, if observables $A_{2}$ are altered at $t_{2}$, it is the case that

$$
\delta\left\langle a_{2}, t_{2}\right|=\frac{i}{\hbar}\left\langle a_{2}, t_{2}\right| G_{2}
$$

and the operator $G_{2}$ depends only on observables $A_{2}$ at time $t_{2}$. If both sets $A_{1}, A_{2}$ are altered infinitesimally, then the change in the transformation function is given by

$$
\delta\left\langle a_{2}, t_{2} \mid a_{1}, t_{1}\right\rangle=\frac{i}{\hbar}\left\langle a_{2}, t_{2}\left|G_{2}-G_{1}\right| a_{1}, t_{1}\right\rangle
$$

Comparing this with (18), it is concluded that

$$
\delta W_{21}=G_{2}-G_{1}
$$

If the time evolution from state $\left|a_{1}, t_{1}\right\rangle$ to $\left|a_{2}, t_{2}\right\rangle$ can be thought of as occurring continuously in time, then $W_{21}$ can be expressed as

$$
W_{21}=\int_{t_{1}}^{t_{2}} L(t) d t
$$

where $L(t)$ is called the Lagrange operator. As a consequence of (23), it follows that if the dynamical variables which enter $L(t)$ are altered during an arbitrary infinitesimal change between $t_{1}$ and $t_{2}$, then it must be that

$$
\delta W_{21}=0
$$

The operator equations of motion are implied in this result. The usual form for the Lagrange operator is

$$
L(t)=\frac{1}{2}\left(p_{i} \dot{x}^{i}+\dot{x}^{i} p_{i}\right)-H(\mathbf{x}, \mathbf{p}, t) .
$$

The first term has been symmetrized to give a Hermitian $L(t)$, as the operators $\dot{x}^{i}$ and $p_{i}$ do not as usual commute. It is assumed the Hamiltonian $H$ is a Hermitian operator. The action operator (24) is used to calculate the variation $\delta W_{21}$. In order to vary the endpoints $t_{1}, t_{2}$, 
we follow Schwinger exactly and change the variable of integration from $t$ to $\tau$ such that $t=t(\tau)$. This allows for the variation of the functional dependence of $t$ to depend on $\tau$ with the variable of integration $\tau$ held fixed. Then $W_{21}$ takes the form,

$$
W_{21}=\int_{\tau_{1}}^{\tau_{2}} d \tau\left[\frac{1}{2}\left(P_{i} \frac{d y^{i}}{d \tau}+\frac{d y^{i}}{d \tau} P_{i}\right)-\tilde{H}(\mathbf{y}, \mathbf{P}, \tau) \frac{d t}{d \tau}\right]
$$

where in (27),

$$
\tilde{H}(\mathbf{y}, \mathbf{P}, \tau)=H(\mathbf{x}, \mathbf{p}, t)
$$

Thus $y^{i}(\tau)=x^{i}(t)$ and $P_{i}(\tau)=p_{i}(t)$ when the transformation $t=t(\tau)$ is implemented. Evaluating the infinitesimal variation of (27), it is found that

$$
\delta W_{21}=\int_{\tau_{1}}^{\tau_{2}} d \tau\left[\frac{1}{2} \delta P_{i} \frac{d y^{i}}{d \tau}+\frac{1}{2} P_{i} \delta\left(\frac{d y^{i}}{d \tau}\right)+\frac{1}{2} \delta\left(\frac{d y^{i}}{d \tau}\right) P_{i}+\frac{1}{2} \frac{d y^{i}}{d \tau} \delta P_{i}-\delta \tilde{H} \frac{d t}{d \tau}-\tilde{H} \delta\left(\frac{d t}{d \tau}\right)\right]
$$

Moving the operator $\delta$ through the derivative, this becomes

$$
\begin{gathered}
\delta W_{21}=\int_{t_{1}}^{t_{2}} d \tau\left\{\frac{1}{2}\left(\delta P_{i} \frac{d y^{i}}{d \tau}+\frac{d y^{i}}{d \tau} \delta P_{i}-\frac{d P_{i}}{d \tau} \delta y^{i}-\delta y^{i} \frac{d P_{i}}{d \tau}\right)-\delta \tilde{H} \frac{d t}{d \tau}+\frac{d \tilde{H}}{d \tau} \delta t\right. \\
\left.+\frac{d}{d \tau}\left[\frac{1}{2}\left(P_{i} \delta x^{i}+\delta x^{i} P_{i}\right)-\tilde{H} \delta t\right]\right\} .
\end{gathered}
$$

No assumptions with regard to the commutation properties of the variations with the dynamical variables have been made yet. It may be assumed that the variations are multiples of the identity operator, which commutes with everything. After returning to the variable $t$ in the integral in $\delta W_{21}$, the result is

$$
\delta W_{21}=\int_{t_{1}}^{t_{2}} d t\left(\delta p_{i} \dot{x}^{i}-\dot{p}_{i} \delta x^{i}+\frac{d H}{d t} \delta t-\delta H\right)+G_{2}-G_{1} .
$$

Here $G_{1}$ and $G_{2}$ denote the quantity

$$
G=p_{i} \delta x^{i}-H \delta t
$$

when it is evaluated at the two endpoints $t=t_{1}$ and $t=t_{2}$. If we define,

$$
\delta H=\delta x^{i} \frac{\partial H}{\partial x^{i}}+\delta p_{i} \frac{\partial H}{\partial p_{i}}+\delta t \frac{\partial H}{\partial t}
$$


then $\delta W_{21}$ can be expressed in the form,

$$
\delta W_{21}=\int_{t_{1}}^{t_{2}} d t\left\{\delta p_{i}\left(\dot{x}^{i}-\frac{\partial H}{\partial p_{i}}\right)-\delta x^{i}\left(\dot{p}_{i}+\frac{\partial H}{\partial x^{i}}\right)+\left(\frac{d H}{d t}-\frac{\partial H}{\partial t}\right) \delta t\right\}+G_{2}-G_{1} .
$$

Taking the variations with endpoints fixed, it follows that $G_{1}=G_{2}=0$. Consequently, the operator equations of motion which follow from equating $\delta W_{21}$ in (34) to zero are then

$$
\dot{x}^{i}=\frac{\partial H}{\partial p_{i}}, \quad \dot{p}_{i}=-\frac{\partial H}{\partial x^{i}}, \quad \frac{d H}{d t}=\frac{\partial H}{\partial t} .
$$

The results produced in this way are exactly of the form of the classical Hamilton equations of motion, and the derivatives in the first two equations of (35) are with respect to operators.

\section{Commutation relations}

Let $B$ represent any observable and consider the matrix element $\left\langle a|B| a^{\prime}\right\rangle$. If the variables $A$ are subjected to a unitary transformation $A \rightarrow \bar{A}=U A U^{\dagger}$, where $U$ is a unitary operator, then the eigenstates $|a\rangle$ are transformed into $|\bar{a}\rangle=U|a\rangle$ having the eigenvalue $a$. Define the operator $\bar{B}$ to be

$$
\bar{B}=U B U^{\dagger}
$$

These operators have the property that

$$
\left\langle\bar{a}|\bar{B}| \bar{a}^{\prime}\right\rangle=\left\langle a|B| a^{\prime}\right\rangle
$$

Let $U$ now be an infinitesimal unitary transformation which can be expressed in the form

$$
U=I-\frac{i}{\hbar} G_{a}
$$

In (38), $G_{a}$ is a Hermitian quantity and can depend on observables $A$. Consequently, if $\delta_{G_{a}}$ is the change produced by the canonical transformation whose generator is $G_{a}$, then

$$
\delta_{G_{a}}|a\rangle=|\bar{a}\rangle-|a\rangle=-\frac{i}{\hbar} G_{a}|a\rangle,
$$

and $\bar{B}$ is given by

$$
\bar{B}=\left(I-\frac{i}{\hbar} G_{a}\right) B\left(I+\frac{i}{\hbar} G_{a}\right)=B-\frac{i}{\hbar}\left[G_{a}, B\right]
$$


The change in the matrix element $\left\langle a|B| a^{\prime}\right\rangle$ can then be considered to be entirely due to the change in the state vector, with $B$ held fixed. To first order in the operator $G_{a}$, there results

$$
\delta_{G_{a}}\left\langle a|B| a^{\prime}\right\rangle=\left\langle\bar{a}|B| \bar{a}^{\prime}\right\rangle-\left\langle a|B| a^{\prime}\right\rangle=\left\langle a\left|\frac{i}{\hbar}\left[G_{a}, B\right]\right| a^{\prime}\right\rangle
$$

Alternatively, this can be approached by taking the change in $\left\langle a|B| a^{\prime}\right\rangle$ to be due to a change in the operator $B$, but with the states held fixed. Define then the change in $B$ as $\delta_{G_{a}} B$ such that

$$
\delta_{G_{a}}\left\langle a|B| a^{\prime}\right\rangle=\left\langle a\left|\delta_{G_{a}} B\right| a^{\prime}\right\rangle .
$$

If the result obtained for the change in $\left\langle a|B| a^{\prime}\right\rangle$ is not to depend on which of these approaches is taken, by comparing (41) and (42) it is concluded that

$$
\delta_{G_{a}} B=\frac{i}{\hbar}\left[G_{a}, B\right]
$$

As an example, let the change in the operators $A$ at times $t_{1}$ and $t_{2}$ be due to a change in time $t \rightarrow t+\delta t$ with the $x^{i}$ fixed at times $t_{1}$ and $t_{2}$ so that the generator for this tranformation is given by $G(t)=-H(t) \delta t$ and (43) in this case is

$$
\delta_{G_{a}} B=\frac{i}{\hbar}[B, H] \delta t
$$

where $B$ and $H$ are evaluated at the same time. It is only the change in $B$ resulting from a change in operators which is considered now. Specifically, if $B=B[A(t), t]$, then $\bar{B}=$ $B[A(t+\delta t), t]$ and so

$$
\delta B=-\delta t\left(\frac{d B}{d t}-\frac{\delta B}{\delta t}\right) .
$$

Using (45) in (44), a result in agreement with the Heisenberg picture equation of motion results,

$$
\frac{d B}{d t}=\frac{\partial B}{\partial t}+\frac{i}{\hbar}[H, B]
$$

The Heisenberg equation of motion has resulted by this process. If $B$ is taken to be the operator $B=x^{i}$, then (46) becomes,

$$
\dot{x}^{i}=\frac{i}{\hbar}\left[H, x^{i}\right]
$$

If $B=p_{i}$ is used in (46), there results

$$
\dot{p}_{i}=\frac{i}{\hbar}\left[H, p_{i}\right]
$$


The general equations of motion have been produced [7].

The canonical commutation relations are also a consequence of the action principle. To see this, first fix $\delta t$ at the times $t_{1}$ and $t_{2}$ but permit $\delta x^{i}$ to vary. Then (32) gives the generator of this transformation

$$
G=p_{i} \delta x^{i}
$$

Then for any operator $B$, equation (43) gives

$$
\delta B=\frac{i}{\hbar} \delta x^{i}\left[p_{i}, B\right]
$$

Putting $B=x^{i}$ and $B=p_{j}$ respectively in (50) leads to the following pair of commutation relations

$$
\left[x^{i}, p_{j}\right]=i \hbar \delta_{j}^{i}, \quad\left[p_{i}, p_{j}\right]=0
$$

If $B$ has a dependence on $x$, then $\delta B=B[x(t)]-B[x(t)-\delta x(t)]$ implies

$$
\left[p_{i}, B\right]=-\frac{i}{\hbar} \frac{\partial}{\partial x^{i}} B
$$

In order to obtain the commutator $\left[x^{i}, x^{j}\right]$, the freedom of altering the Lagrangian operator by the addition of a total time derivative can be used. Suppose that

$$
F=\frac{1}{2}\left(p_{i} x^{i}+x^{i} p_{i}\right)
$$

which satisfies $F^{\dagger}=F$, and consequently

$$
\delta F=\frac{1}{2}\left(\delta p_{i} x^{i}+p_{i} \delta x^{i}+\delta x^{i} p_{i}+x^{i} \delta p_{i}\right)=\delta p_{i} x^{i}+p_{i} \delta x^{i}
$$

Then $\bar{G}$ is calculated to be

$$
\bar{G}=G-\delta F=-\delta p_{i} x^{i}-H \delta t .
$$

Taking $\delta t=0$ so that $G_{p}$ can be defined as $G_{p}=-\delta p_{i} x^{i}$, it follows that

$$
\delta B=-\frac{i}{\hbar} \delta p_{i}\left[x^{i}, B\right]
$$

for any operator $B$. Taking $B=p_{j}$, the bracket $\left[x^{i}, p_{j}\right]=i \hbar \delta_{j}^{i}$ is obtained from (56), and when $B=x^{j}$, using the independence of $\delta x^{i}$ and $\delta p_{i}$ there results the bracket 


$$
\left[x^{i}, x^{j}\right]=0 .
$$

It has been shown that the set of canonical commutation relations can be obtained from this action principle.

Consider a matrix element $\langle a|F(A, B)| b\rangle$ where $A$ and $B$ each represent a complete set of mutually compatible observables and $F(A, B)$ is some function, and it is not assumed the observables from the two different sets commute with each other. If the commutator $[B, A]$ is known, it is always possible to order the operators in $F(A, B)$ so all $A$ terms are to the left of $B$, which allows the matrix element to be evaluated. Let

$$
\mathcal{F}(A, B)=F(A, B)
$$

denote the operator where the commutation relation for $[A, B]$ has been used to move all occurrences of $A$ in $F(A, B)$ to the left of all $B$, and (58) is said to be well-ordered

$$
\langle a|F(A, B)| b\rangle=\mathcal{F}(a, b)\langle a \mid b\rangle .
$$

The matrix element of $F(A, B)$ is directly related to the transformation function $\langle a \mid b\rangle$.

The idea of well-ordering operators can be used in the action principle. Define

$$
\delta \mathcal{W}_{21}=\delta W_{21}
$$

be the well-ordered form of $\delta W_{21}$. Then

$$
\delta\left\langle a_{2}, t_{2} \mid a_{1}, t_{1}\right\rangle=\frac{i}{\hbar} \delta \mathcal{W}_{21}\left\langle a_{2}, t_{2} \mid a_{1}, t_{1}\right\rangle
$$

where $\delta \mathcal{W}_{21}$ denotes the replacement of all operators with their eigenvalues. Equation (61) can be integrated to yield

$$
\left\langle a_{2}, t_{2} \mid a_{1}, t_{1}\right\rangle=\exp \left(\frac{i}{\hbar} \mathcal{W}_{21}\right)
$$

Using (23) and (32) in the action principle,

$$
\begin{gathered}
\delta\left\langle a_{2}, t_{2} \mid a_{1}, t_{1}\right\rangle=\frac{i}{\hbar} \delta x^{i}\left(t_{2}\right)\left\langle a_{2}, t_{2}\left|p\left(t_{2}\right)\right| a_{1}, t_{1}\right\rangle-\frac{i}{\hbar} \delta t_{2}\left\langle a_{2}, t_{2}\left|H\left(t_{2}\right)\right| a_{1}, t_{1}\right\rangle \\
-\frac{i}{\hbar} \delta x^{i}\left(t_{1}\right)\left\langle a_{2}, t_{2}\left|p_{i}\left(t_{1}\right)\right| a_{1}, t_{1}\right\rangle+\frac{i}{\hbar} \delta t_{1}\left\langle a_{2}, t_{2}\left|H\left(t_{1}\right)\right| a_{1}, t_{1}\right\rangle .
\end{gathered}
$$


Since $\delta \mathcal{W}_{21}$ is well-ordered, it is possible to write

$$
\delta\left\langle a_{2}, t_{2} \mid a_{1}, t_{1}\right\rangle=\frac{i}{\hbar}\left\langle a_{2}, t_{2}\left|\delta \mathcal{W}_{21}\right| a_{1}, t_{1}\right\rangle
$$

Comparing this with (63), it must be that

$$
\frac{\partial \mathcal{W}_{21}}{\partial x^{i}\left(t_{2}\right)}=p_{i}\left(t_{2}\right), \quad \frac{\partial \mathcal{W}_{21}}{\partial t_{2}}=-H\left(t_{2}\right), \quad \frac{\partial \mathcal{W}_{21}}{\partial x^{i}\left(t_{1}\right)}=-p_{i}\left(t_{1}\right), \quad \frac{\partial \mathcal{W}_{21}}{\partial t_{1}}=H\left(t_{1}\right)
$$

If we consider a matrix element $\left\langle x_{2}, t_{2} \mid x_{1}, t_{1}\right\rangle$, which could be regarded as the propagator, we then have a form of (62),

$$
\left\langle\mathbf{x}_{2}, t_{2} \mid \mathbf{x}_{1}, t_{1}\right\rangle=\exp \left(\frac{i}{\hbar} \mathcal{W}_{21}\right)
$$

The arbitrary integration constant is determined by requiring that $\left\langle\mathbf{x}_{2}, t_{2} \mid \mathbf{x}_{1}, t_{1}\right\rangle=\delta\left(\mathbf{x}_{2}, \mathbf{x}_{1}\right)$. Any other transformation function can be recovered from the propagator equation

$$
\left\langle a_{2}, t_{2} \mid a_{1}, t_{1}\right\rangle=\int d^{n} x_{2} \int d^{n} x_{1}\left\langle a_{2}, t_{2} \mid \mathbf{x}_{2}, t_{2}\right\rangle\left\langle\mathbf{x}_{2}, t_{2} \mid \mathbf{x}_{1}, t_{1}\right\rangle\left\langle\mathbf{x}_{1} t_{1} \mid a_{1}, t_{1}\right\rangle,
$$

which follows from the completeness relation. Another way of formulating transition amplitudes will be seen when the path integral approach is formulated.

\section{Action principle adapted to case of quantum fields}

The Schwinger action principle, much like the Feynman path integral, concentrates on the transition amplitude between two quantum states. The action principle will be formulated here for a local field theory. Classically, a local field is a function which depends only on a single spacetime point, rather than on an extended region of spacetime. The theory can be quantized by replacing the classical fields $\phi^{i}(x)$ with field operators $\varphi^{i}(x)$.

Let $\Sigma$ denote a spacelike hypersurface. This means that any two points on $\Sigma$ have a spacelike separation and consequently must be causally disconnected. As a consequence of this, the values of the field at different points of the surface $\Sigma$ must be independent. If $x_{1}$ and $x_{2}$ are two spacetime points of $\Sigma$, then

$$
\left[\varphi^{i}\left(x_{1}\right), \varphi^{j}\left(x_{2}\right)\right]=0 .
$$

This follows since it must be the case that a measurement at $x_{1}$ must not influence one at $x_{2}$. A fundamental assumption of local field theory is that a complete set of commuting observables can be constructed based on the fields and their derivatives on the surface $\Sigma$. Let $\tau$ denote such a complete set of commuting observables on $\Sigma$ such that $\tau^{\prime}$ represents the eigenvalues of the observables. A quantum state is then denoted by

$$
\left|\tau^{\prime}, \Sigma\right\rangle
$$


Since causality properties are important in field theory, the surface $\Sigma$ is written explicitly in the ket. The Heisenberg picture has been adopted here. The states are then time-independent with the time dependence located in the operators. This is necessary for manifest covariance, since the time and space arguments of the field are not treated differently.

Suppose that $\Sigma_{1}$ and $\Sigma_{2}$ are two spacelike hypersurfaces such that all points of $\Sigma_{2}$ are to the future of $\Sigma_{1}$. Let $\tau_{1}$ be a complete set of commuting observables defined on $\Sigma_{1}$, and $\tau_{2}$ a complete set of observables defined on $\Sigma_{2}$, such that these observables have the same eigenvalue spectrum. Then $\tau_{1}$ and $\tau_{2}$ should be related by a unitary transformation

$$
\tau_{2}=U_{12} \tau_{1} U_{12}^{-1}
$$

The eigenstates are related by

$$
\left|\tau_{2}^{\prime}, \Sigma_{2}\right\rangle=U_{12}\left|\tau_{1}^{\prime}, \Sigma_{1}\right\rangle
$$

In (71), $U_{12}$ is a unitary operator giving the evolution of the state in the spacetime between the two spacelike hypersurfaces. The transition amplitude is defined by

$$
\left\langle\tau_{2}^{\prime}, \Sigma_{2} \mid \tau_{1}^{\prime}, \Sigma_{1}\right\rangle=\left\langle\tau_{1}^{\prime}, \Sigma_{1}\left|U_{12}^{-1}\right| \tau_{1}^{\prime}, \Sigma_{1}\right\rangle
$$

The unitary operator $U_{12}$ depends on a number of details of the quantum system, namely, the choice made for the commuting observables $\tau$, and the spacelike hypersurfaces $\Sigma_{1}$ and $\Sigma_{2}$. A change in any of these quantities will induce a change in the transformation function according to

$$
\delta\left\langle\tau_{2}^{\prime}, \Sigma_{2} \mid \tau_{1}^{\prime}, \Sigma_{1}\right\rangle=\left\langle\tau_{1}^{\prime}, \Sigma_{1}\left|\delta U_{12}^{-1}\right| \tau_{1}^{\prime}, \Sigma_{1}\right\rangle .
$$

The unitary operator $U_{12}$ can be expressed in the form

$$
U_{12}=\exp \left(-\frac{i}{\hbar} S_{12}\right)
$$

in which $S_{12}^{+}=S_{12}$ is a Hermitian operator. Moreover, beginning with $U_{12} U_{12}^{-1}=I$ and using (74), it is found that

$$
\delta U_{12}^{-1}=-U_{12}^{-1} \delta U_{12} U_{12}^{-1}=\frac{i}{\hbar} U_{12}^{-1} \delta S_{12} .
$$

The change in the transformation function can be written in terms of the operator in (74) as

$$
\delta\left\langle\tau_{2}^{\prime}, \Sigma_{2} \mid \tau_{1}^{\prime}, \Sigma_{1}\right\rangle=\frac{i}{\hbar}\left\langle\tau_{2}^{\prime}, \Sigma_{2}\left|\delta S_{12}\right| \tau_{1}^{\prime}, \Sigma_{1}\right\rangle
$$

Equation (76) can be regarded as a definition of $\delta S_{12}$. In order that $\delta S_{12}$ be consistent with the basic requirement that

$$
\left\langle\tau_{2}^{\prime}, \Sigma_{2} \mid \tau_{1}^{\prime}, \Sigma_{1}\right\rangle^{*}=\left\langle\tau_{1}^{\prime}, \Sigma_{1} \mid \tau_{2}^{\prime}, \Sigma_{2}\right\rangle
$$


it must be that $\delta S_{12}$ is Hermitean. If $\Sigma_{3}$ is a spacelike hypersurface, all of whose points lie to the future of those on $\Sigma_{2}$, the basic law for composition of probability amplitudes is

$$
\left\langle\tau_{3}^{\prime}, \Sigma_{3} \mid \tau_{1}^{\prime}, \Sigma_{1}\right\rangle=\sum_{\tau_{2}^{\prime}}\left\langle\tau_{3}^{\prime}, \Sigma_{3} \mid \tau_{2}^{\prime}, \Sigma_{2}\right\rangle\left\langle\tau_{2}^{\prime}, \Sigma_{2} \mid \tau_{1}^{\prime}, \Sigma_{1}\right\rangle
$$

Varying both sides of the expression in (77),

$$
\begin{gathered}
\left\langle\tau_{3}^{\prime}, \Sigma_{3}\left|\delta S_{13}\right| \tau_{1}^{\prime}, \Sigma_{1}\right\rangle=\sum_{\tau_{2}^{\prime}}\left\{\left\langle\tau_{3}^{\prime}, \Sigma_{3}\left|\delta S_{23}\right| \tau_{2}^{\prime}, \Sigma_{2}\right\rangle\left\langle\tau_{2}^{\prime}, \Sigma_{2} \mid \tau_{1}^{\prime}, \Sigma_{1}\right\rangle+\left\langle\tau_{3}^{\prime}, \Sigma_{3} \mid \tau_{2}^{\prime}, \Sigma_{2}\right\rangle\left\langle\tau_{2}^{\prime}, \Sigma_{2}\left|\delta S_{12}\right| \tau_{1}^{\prime}, \Sigma_{1}\right\rangle\right. \\
=\left\langle\tau_{3}^{\prime}, \Sigma_{3}\left|\delta S_{23}+\delta S_{12}\right| \tau_{1}^{\prime}, \Sigma_{1}\right\rangle
\end{gathered}
$$

Comparing both ends of the result in (78), it follows that

$$
\delta S_{13}=\delta S_{23}+\delta S_{12}
$$

In the limit $\Sigma_{2} \rightarrow \Sigma_{1}$, it must be that

$$
\delta S_{12}=0 .
$$

In the limit in which $\Sigma_{3} \rightarrow \Sigma_{1}$, it follows that

$$
\delta S_{21}=-\delta S_{12}
$$

If the operators in $\tau_{1}$ and $\tau_{2}$ undergo infinitesimal, unitary transformations on the hypersurfaces $\Sigma_{1}$ and $\Sigma_{2}$, respectively, and only on these two hypersurfaces, then the change in the transformation function has the form

$$
\delta\left\langle\tau_{2}^{\prime}, \Sigma_{2} \mid \tau_{1}^{\prime}, \Sigma_{1}\right\rangle=\frac{i}{\hbar}\left\langle\tau_{2}^{\prime}, \Sigma_{2}\left|F_{2}-F_{1}\right| \tau_{1}^{\prime}, \Sigma_{1}\right\rangle
$$

Here $F_{1}$ and $F_{2}$ are Hermitean operators constructed from a knowledge of the fields and their derivatives on $\Sigma_{1}$ and $\Sigma_{2}$. The result (80) is of the form (76) provided that

$$
\delta S_{12}=F_{2}-F_{1}
$$

A generator $F$ of this type on a spacelike hypersurface $\Sigma$ should be expressible in the form,

$$
F=\int_{\Sigma} d \sigma_{x} n^{\mu} F_{\mu}(x)
$$

and $d \sigma_{x}$ is the area element on $\Sigma, n^{\mu}$ is the outward unit normal to $\Sigma$, and $F_{\mu}(x)$ may be put together based on a knowledge of the fields on the surface $\Sigma$. 
The points of $\Sigma$ are all spacelike separated, hence independent and so the result follows by adding up all of these independent contributions. Applying (82) to $\Sigma_{1}$ and $\Sigma_{2}$ assuming that $F_{\mu}(x)$ is defined throughout the spacetime region bounded by these two surfaces, $\delta S_{12}$ can be expressed as

$$
\delta_{12} S=\int_{\Sigma_{2}} d \sigma_{x} n^{\mu} F_{\mu}(x)-\int_{\Sigma_{1}} d \sigma_{x} n^{\mu} F_{\mu}(x)=\int_{\Omega_{12}} d v_{x} \nabla^{\mu} F_{\mu}(x)
$$

In (83), $\Omega_{12}$ is the spacetime region bounded by $\Sigma_{1}$ and $\Sigma_{2}$, and $d v_{x}$ is the invariant volume. This assumes that the operators are changes only on $\Sigma_{1}$ and $\Sigma_{2}$. However, suppose the operators are changed in the spacetime region between $\Sigma_{1}$ and $\Sigma_{2}$. Assume once more that $\delta S_{12}$ can be expressed as a volume integral as,

$$
\delta S_{12}=\int_{\Omega_{12}} d v_{x} \delta \mathcal{L}(x)
$$

for some $\delta \mathcal{L}(x)$. Combining these two types of variation yields

$$
\delta S_{12}=F_{2}-F_{1}+\int_{\Omega_{12}} d v_{x} \delta \mathcal{L}(x)=\int_{\Omega_{12}} d v_{x}\left[\delta \mathcal{L}(x)+\nabla^{\mu} F_{\mu}(x)\right]
$$

It is an important result of $(85)$ that altering $\delta \mathcal{L}$ by the addition of the divergence of a vector field will result in a unitary transformation of the states on $\Sigma_{1}$ and $\Sigma_{2}$.

To summarize, the fundamental assumption of the Schwinger action principle is that $\delta S_{12}$ may be obtained from a variation of

$$
S_{12}=\int_{\Omega_{12}} d v_{x} \mathcal{L}(x)
$$

where $\mathcal{L}(x)$ is a Lagrangian density. The density depends on the fields and their derivatives at a single spacetime point. Since $\delta S_{12}$ is required to be Hermitian, $S_{12}$ must be Hermitian and similarly, so must the Lagrangian density.

\section{Correspondence with Feynman path integrals}

Suppose a classical theory described by the action $S[\varphi]$ is altered by coupling the field to an external source $J_{i}=J_{I}(x)$. By external it is meant that it has no dependence on the field $\varphi^{i}$, and $i$ stands for $(I, x)$. For example, $F_{i}[\varphi] \sigma^{i}$ is an abbreviation for

$$
F_{, i}[\varphi] \sigma^{i}=\int d^{n} x^{\prime} \frac{\delta F[\varphi(x)]}{\delta \varphi^{I}\left(x^{\prime}\right)} \sigma^{i}\left(x^{\prime}\right)
$$

The idea of introducing external sources originates with Schwinger. As he states, causality and space-time uniformity are the creative principles of source theory. Uniformity in 
space-time also has a complementary momentum-energy implication, illustrated by the source idea [5,8]. Not only for the special balance of energy and momentum involved in the emission or absorption of a single particle is the source defined and meaningful. Given a sufficient excess of energy over momentum, or an excess of mass, several particles can be emitted or absorbed. For example, consider the emission of a pair of charged particles by an extended photon source. This process is represented as the conversion of a virtual photon into a pair of real particles. In ordinary scattering, particle-particle scattering, the particles persist while exchanging a space-like virtual photon. Another is an annihilation of the particle-antiparticle pair, producing a time-like virtual photon, which quickly decays back into particles.

Let us choose a simple scalar theory

$$
S_{J}[\varphi]=S[\varphi]+\int J_{I}(x) \varphi^{I}(x) d^{n} x,
$$

and try to establish a connection between the action principle introduced here and a path integral picture [9]. The transition amplitude $\left\langle\tau_{2}^{\prime}, \Sigma_{2} \mid \tau_{1}^{\prime}, \Sigma_{1}\right\rangle$ discussed in the previous section may be regarded as a functional of the source term $J_{I}(x)$. This will be denoted explicitly by writing $\left\langle\tau_{2}^{\prime}, \Sigma_{2} \mid \tau_{1}^{\prime}, \Sigma_{1}\right\rangle_{J}$. If the Schwinger action principle is applied to this modified theory

$$
\delta\left\langle\tau_{2}^{\prime}, \Sigma_{2} \mid \tau_{1}^{\prime}, \Sigma_{1}\right\rangle_{J}=\frac{i}{\hbar}\left\langle\tau_{2}^{\prime}, \Sigma_{2}\left|\delta S_{J}\right| \tau_{1}^{\prime}, \Sigma_{1}\right\rangle_{J}
$$

The classical field $\varphi^{I}(x)$ has been replaced by the operator $\phi^{I}(x)$. Moreover, in addition to its previous meaning, $\delta$ now includes a possible change in the source. By considering the variation to be with respect to the dynamical variables which are held fixed on $\Sigma_{1}$ and $\Sigma_{2}$, the operator field equations are obtained as

$$
\frac{\delta S[\phi]}{\delta \phi^{I}(x)}+J_{I}(x)=0
$$

Assume that the variation in (88) is one in which the dynamical variables are held fixed and only the source is altered. Since the source enters in the simple way given in (87),

$$
\delta\left\langle\tau_{2}^{\prime}, \Sigma_{2} \mid \tau_{1}^{\prime}, \Sigma_{1}\right\rangle=\frac{i}{\hbar} \int \delta J_{I}(x)\left\langle\tau_{2}^{\prime}, \Sigma_{2}\left|\phi^{I}(x)\right| \tau_{1}^{\prime}, \Sigma_{1}\right\rangle_{J} d^{n} x
$$

The result in (90) may be rewritten in the equivariant form,

$$
\frac{\delta\left\langle\tau_{2}^{\prime}, \Sigma_{2} \mid \tau_{1}^{\prime}, \Sigma_{1}\right\rangle}{\delta J_{i}(x)}=\frac{i}{\hbar}\left\langle\tau_{2}^{\prime}, \Sigma_{2}\left|\phi^{i}(x)\right| \tau_{1}^{\prime}, \Sigma_{1}\right\rangle[J] .
$$

To simplify this, it can be written in the alternate form,

$$
\frac{\delta\langle 2 \mid 1\rangle}{\delta J_{i}}=\frac{i}{\hbar}\left\langle 2\left|\phi^{i}\right| 1\right\rangle[J]
$$


An abbreviated notation for the initial and final state has been introduced. This result can be varied with respect to the source which gives

$$
\delta \frac{\delta\langle 2 \mid 1\rangle[J]}{\delta J_{i}}=\frac{i}{\hbar} \delta\left\langle 2\left|\phi^{i}\right| 1\right\rangle[J]
$$

To evaluate (93), a spatial hypersurface $\Sigma^{\prime}$ is introduced which resides to the future of $\Sigma_{1}$ and the past of $\Sigma_{2}$ and contains the spacetime point on which the $\phi^{i}(x)$ depend. Any source variation can be represented as the sum of a variation which vanishes to the future of $\Sigma^{\prime}$, but is non-zero to the past, and one which vanishes to the past of $\Sigma^{\prime}$ but is nonzero to the future. Consider the case where $\delta J_{i}$ vanishes to the future. In this event, any amplitude of the form $\left\langle 2\left|\phi^{i}\right| \tau^{\prime}\right\rangle[J]$, where $\left|\tau^{\prime}\right\rangle$ represents a state on $\Sigma^{\prime}$, can not be affected by the variation of the source since $\delta J_{i}$ will vanish to the future of $\Sigma^{\prime}$. By using the completeness relation

$$
\left\langle 2\left|\phi^{i}\right| 1\right\rangle[J]=\sum_{\tau^{\prime}}\left\langle 2\left|\phi^{i}\right| \tau^{\prime}\right\rangle\left\langle\tau^{\prime} \mid 1\right\rangle[J]
$$

it follows that the right-hand side of (93) may be reexpressed with the use of

$$
\delta\left\langle 2\left|\phi^{i}\right| 1\right\rangle[J]=\sum_{\tau^{\prime}}\left\langle 2\left|\phi^{i}\right| \tau^{\prime}\right\rangle \delta\left\langle\tau^{\prime} \mid 1\right\rangle[J]
$$

The Schwinger action principle then implies that

$$
\delta\left\langle\tau^{\prime} \mid 1\right\rangle[J]=\frac{i}{\hbar} \delta J_{k}\left\langle\tau^{\prime}\left|\phi^{k}\right| 1\right\rangle[J]
$$

Substituting (95) into (94) leads to the conclusion that

$$
\delta\left\langle 2\left|\phi^{i}\right| 1\right\rangle[J]=\frac{i}{\hbar} \delta J_{j} \sum_{\tau^{\prime}}\left\langle 2\left|\phi^{i}\right| \tau^{\prime}\right\rangle\left\langle\tau^{\prime}\left|\phi^{j}\right| 1\right\rangle[J]=\frac{i}{\hbar} \delta J_{j}\left\langle 2\left|\phi^{i} \phi^{j}\right| 1\right\rangle[J] .
$$

Since it can be said that $\delta J_{j}$ vanishes to the future of $\Sigma^{\prime}$, which contains the spacetime point of $\phi^{i}$, the spacetime point of $\phi^{j}$ must lie to the past of the former.

Consider the case in which $\delta J_{j}$ vanishes to the past of $\Sigma^{\prime}$. A similar argument yields the same conclusion as (96), but with $\phi^{j}$ to the left of $\phi^{i}$. Combining this set of results produces the following conclusion

$$
\frac{\delta\left\langle 2\left|\phi^{i}\right| 1\right\rangle}{\delta J_{j}}=\frac{i}{\hbar}\left\langle 2\left|T\left(\phi^{i} \phi^{j}\right)\right| 1\right\rangle[J] .
$$

In (97), $T$ is the chronological, or time, ordering operator, which orders any product of fields in the sequence of increasing time, with those furthest to the past to the very right. 
Differentiating (91) and using the result (97), we get

$$
\frac{\delta^{2}\langle 2 \mid 1\rangle[J]}{\delta J_{i} \delta J_{j}}=\left(\frac{i}{\hbar}\right)\left\langle 2\left|T\left(\phi^{i} \phi^{j}\right)\right| 1\right\rangle[J] .
$$

This can be generalized, and omitting details,

$$
\frac{\delta^{n}\langle 2 \mid 1\rangle[J]}{\delta J_{i_{1}} \cdots \delta J_{i_{n}}}=\left(\frac{i}{\hbar}\right)^{n}\left\langle 2\left|T\left(\phi^{i_{1}} \cdots \phi^{i_{n}}\right)\right| 1\right\rangle[J] .
$$

The amplitude $\langle 2 \mid 1\rangle[J]$ may be defined by a Taylor expansion about $J_{i}=0$, so using the previous result

$$
\langle 2 \mid 1\rangle[J]=\sum_{n=0}^{\infty} \frac{1}{n !}\left(\frac{i}{\hbar}\right)^{n} J_{i_{1}} \cdots J_{i_{n}}\left\langle 2\left|T\left(\phi^{i_{1}} \cdots \phi^{i_{n}}\right)\right| 1\right\rangle[J=0] .
$$

The series may be formally summed to yield

$$
\langle 2 \mid 1\rangle[J]=\left\langle 2\left|T\left(\exp \left(\frac{i}{\hbar} J_{i} \phi^{i}\right)\right)\right| 1\right\rangle[J=0]
$$

and $J_{i}$ is set to zero everywhere on the right-hand side except in the exponential.

The action $S[\phi]$ can be expanded in a Taylor series about $\phi^{i}=0$, indicating differentiation with a comma,

$$
S[\phi]=\sum_{n=0}^{\infty} \frac{1}{n !} S_{, i_{1} \cdots i_{n}}[\phi=0] \phi^{i_{1}} \cdots \phi^{i_{n}} .
$$

Similarly, the derivative of $S$ with respect to the field has the expansion [7],

$$
S_{, i}[\phi]=\sum_{n=0}^{\infty} \frac{1}{n !} S_{, i i_{1} \cdots i_{n}}[\phi=0] \phi^{i_{1}} \cdots \phi^{i_{n}} .
$$

If $\phi^{i}$ is replaced by $\frac{\hbar}{i} \frac{\delta}{\delta J_{i}}$ in this expression and then operate on $\langle 2 \mid 1\rangle[J]$ with $S_{, i}\left[\frac{\hbar}{i} \frac{\delta}{\delta J_{i}}\right]$, and use (102), the following differential equation arises

$$
S_{, i}\left[\frac{\hbar}{i} \frac{\delta}{\delta J_{i}}\right]\langle 2 \mid 1\rangle[J]=\left\langle 2\left|T\left(S_{, i}[\phi] \exp \left(\frac{i}{\hbar} J_{i} \phi^{i}\right)\right)\right| 1\right\rangle[J=0] .
$$

The operator equation of motion (89) implies

$$
S_{, i}\left[\frac{\hbar}{i} \frac{\delta}{\delta J_{i}}\right]\langle 2 \mid 1\rangle[J]=-J_{i}\langle 2 \mid 1\rangle[J]
$$


This results in a differential equation for the transition amplitude. In order to solve equation (105), the functional analogue of a Fourier transform may be used

$$
\langle 2 \mid 1\rangle=\int\left(\prod_{I} d \varphi^{I}(x)\right) F[\varphi] \exp \left(\frac{i}{\hbar} \int J_{I}\left(x^{\prime}\right) \varphi^{I}\left(x^{\prime}\right) d^{n} x^{\prime}\right) .
$$

The integration in (106) extends over all fields which correspond to the choice of states described by $|1\rangle$ and $|2\rangle$. The functional $F[\varphi]$ which is to be thought of as the Fourier transform of the transformation function, is to be determined by requiring (106) satisfy (105)

$$
\begin{gathered}
0=\int\left(\prod_{I, x} d \varphi^{I}(x)\right)\left\{S_{, i}[\varphi]+J_{i}\right\} F[\varphi] \exp \left(\frac{i}{\hbar} \int J_{I}\left(x^{\prime}\right) \varphi^{I}\left(x^{\prime}\right) d^{n} x^{\prime}\right) \\
=\int\left(\prod_{I} d \varphi^{I}(x)\right)\left\{S_{, i}[\varphi] F[\varphi]+\frac{\hbar}{i} F[\varphi] \frac{\delta}{\delta \varphi^{i}}\right\} \exp \left(\frac{i}{\hbar} \int J_{I}\left(x^{\prime}\right) \varphi^{I}\left(x^{\prime}\right) d^{n} x^{\prime}\right) .
\end{gathered}
$$

Upon carrying out an integration by parts on the second term here

$$
\begin{gathered}
0=\int\left(\prod_{i} d \varphi^{i}\right)\left\{S_{, i}[\varphi] F[\varphi]-\frac{\hbar}{i} F_{, i}[\varphi]\right\} \exp \left(\frac{i}{\hbar} \int J_{I}\left(x^{\prime}\right) \varphi^{I}\left(x^{\prime}\right) d^{n} x^{\prime}\right) \\
+\left.\frac{\hbar}{i} F[\varphi] \exp \left(\frac{i}{\hbar} \int J_{I}\left(x^{\prime}\right) \varphi^{I}\left(x^{\prime}\right) d^{n} x^{\prime}\right)\right|_{\varphi_{1}} ^{\varphi_{2}} .
\end{gathered}
$$

Assuming the surface term at the end vanishes, it follows from (107) that

$$
F[\varphi]=\mathcal{N} \exp \left(\frac{i}{\hbar} S[\varphi]\right)
$$

where $\mathcal{N}$ is any field-independent constant. The condition for the surface term to vanish is that the action $S[\varphi]$ be the same on both surfaces $\Sigma_{1}$ and $\Sigma_{2}$. This condition is usually fulfilled in field theory by assuming that the fields are in the vacuum state on the initial and final hypersurface.

The transformation function can then be summarized as

$$
\langle 2 \mid 1\rangle[J]=\mathcal{N} \int\left(\prod_{i} d \varphi^{i}\right) \exp \left(\frac{i}{\hbar}\left\{S[\varphi]+J_{i} \varphi^{i}\right\}\right) .
$$

This is one form of the Feynman path integral, or functional integral, which represents the transformation function. This technique turns out to be very effective with further modifications applied to the quantization of gauge theories. These theories have been particularly successful in understanding the weak and strong interactions [10]. 


\section{QED - A physical example and summary}

A given elementary interaction implies a system of coupled field equations. Thus for the photon and the charged spin $1 / 2$ particle as in quantum electrodynamics [5],

$$
\begin{gathered}
{[\gamma(-i \partial-e q A(x))+m] \psi(x)=\eta^{A}(x),} \\
-\partial^{2} A^{\mu}(x)+\partial^{\mu} \partial A(x)=J^{\mu}(x)+\frac{1}{2} \psi(x) \gamma^{0} \gamma^{\mu} e q \psi(x)-\int d x^{\prime} f^{\mu}\left(x-x^{\prime}\right) \psi\left(x^{\prime}\right) \gamma^{0} i e q \eta^{A}\left(x^{\prime}\right) .
\end{gathered}
$$

Since this is a nonlinear system, the construction of the fields in terms of the sources will be given by doubly infinite power series. The succesive terms of this series $W_{n v}$ with $n$ paricles and $v$ photon sources represent increasingly complicated physical processes. One of the simplest terms in the interaction skeleton will be discussed below to the point of obtaining experimental consequences.

There are two asymmetrical ways to eliminate the fields. First, introduce the formal solution of the particle field equation

$$
\psi^{A}(x)=\int d x^{\prime} G_{+}^{A}\left(x, x^{\prime}\right) \eta^{A}(x)
$$

and $G_{+}^{A}\left(x, x^{\prime}\right)$ is the Green function

$$
[\gamma(-i \partial-e q A(x))+m] G_{+}^{A}\left(x, x^{\prime}\right)=\delta\left(x-x^{\prime}\right) .
$$

This gives the partial action

$$
W=\int d x\left[J^{\mu} A_{\mu}-\frac{1}{4} F^{\mu v} F_{\mu v}\right]+\frac{1}{2} \int d x d x^{\prime} \eta^{A}(x) \gamma^{0} G_{+}^{A}\left(x, x^{\prime}\right) \eta^{A}\left(x^{\prime}\right) .
$$

The stationarity requirement on variations of $A_{\mu}$ recovers the Maxwell equation above. If we eliminate the vector potential

$$
\begin{gathered}
A_{\mu}^{f}(x)=\int d x^{\prime} D_{+}\left(x-x^{\prime}\right)\left[J^{\mu}(x)+j_{\text {cons }}^{\mu}\right]+\partial_{\mu} \lambda(x) . \\
j_{\text {cons }}^{\mu}(x)=j^{\mu}(x)-\int d x^{\prime} f^{\mu}\left(x-x^{\prime}\right) \partial_{v}^{\prime}\left(x^{\prime}\right),
\end{gathered}
$$

and the gauge condition determines $\lambda(x)$.

Finally, the first few successive $W_{2 v}$ are written out, noting each particle source is multiplied by a propagation function $G_{+}\left(x, x^{\prime}\right)$ to form the field $\psi$,

$$
W_{21}=\frac{1}{2} \int d^{4} x \psi(x) \gamma^{0} e q \gamma A(x) \psi(2)
$$




$$
W_{22}=\frac{1}{2} \int d x d x^{\prime} \psi(x) \gamma^{0} e q \gamma A(x) G_{+}\left(x-x^{\prime}\right) \operatorname{eq\gamma } A\left(x^{\prime}\right) \psi\left(x^{\prime}\right)
$$

As a brief introduction to how this formalism can lead to important physical results, let us look at a specific term like $W_{21}$, the interaction energy of an electron with a static electromagnetic field $A_{\mu}^{\text {ext }}$

$$
E=\int d^{3} x j_{\mu} A_{e x t}^{\mu}=e \int d^{3} x \bar{\psi}_{p^{\prime}}\left(\gamma_{\mu}+\Gamma_{\mu}^{R}\left(p^{\prime}, p\right)+\frac{i}{4 \pi} \Pi_{\mu v}^{R} i D^{v \sigma} \gamma_{\sigma}\right) \psi_{p} A_{e x t}^{\mu}
$$

These terms include the bare electron-photon term, the electron-photon correction terms and then the photon vacuum-polarization correction term, $R$ means a renormalized quantity and $\gamma_{\mu}$ denote Dirac matrices. The self-energy correction is left out, because for free particles, it contributes only to charge and mass renormalization. The polarization tensor $\Pi_{\mu \nu}\left(q^{2}\right)$ is given by

$$
\Pi_{\mu v}\left(q^{2}\right)=\left(q^{2} g_{\mu \nu}-q_{\mu} q_{v}\right) \Pi\left(q^{2}\right),
$$

where $\Pi\left(q^{2}\right)$ is the polarization function. A simple result is obtained in the limit of low momentum transfer, $q^{2} \rightarrow 0$, which is also of special physical significance and the case of interest here. The renormalized polarization function is

$$
\Pi^{R}\left(q^{2}\right)=-\frac{e^{2}}{\pi} \frac{q^{2}}{m^{2}}\left(\frac{1}{15}+\frac{1}{140} \frac{q^{2}}{m^{2}}+\cdots\right)
$$

The regularized vertex function is

$$
\Gamma_{\mu}^{R}\left(p^{\prime}, p\right)=\gamma_{\mu} F_{1}\left(q^{2}\right)+\frac{i}{2 m} \sigma_{\mu v} q^{v} F_{2}\left(q^{2}\right)
$$

The functions $F_{1}\left(q^{2}\right)$ and $F_{2}\left(q^{2}\right)$ are called form factors. The electron gets an apparent internal structure by the interaction with the virtual radiation field which alters it from a pure Dirac particle. In the limit, $q^{2} \rightarrow 0$, these functions can be calculated to be

$$
F_{1}\left(q^{2}\right)=\frac{\alpha}{3 \pi} \frac{q^{2}}{m^{2}}\left(\ln \left(\frac{m}{\mu}\right)-\frac{3}{8}\right), \quad F_{2}\left(q^{2}\right)=\frac{\alpha}{2 \pi}
$$

Substituting all of these factors and $D_{F}^{\mu v}\left(q^{2}\right)=-4 \pi g^{\mu v} / q^{2}$ into (111) yields for small values of $q^{2}$,

$$
E=e \int d^{3} x \bar{\psi}_{p^{\prime}}\left\{\gamma_{\mu}\left[1+\frac{\alpha}{3 \pi} \frac{q^{2}}{m^{2}}\left(\ln \left(\frac{m}{\mu}\right)-\frac{3}{8}-\frac{1}{5}\right)+\frac{\alpha}{2 \pi} \frac{i}{2 m} \sigma_{\mu \nu} q^{v}\right\} \psi_{p} A_{\text {ext }}^{\mu} .\right.
$$

Note $\mu$ appears in (115) as an elementary attempt to regularize a photon propagator in one of the terms and does not interfere further with the application at this level and $\alpha$ is the fine 
structure constant. The Gordon decomposition allows this to be written

$$
E=e \int d^{3} x \bar{\psi}_{p^{\prime}}\left\{\frac{1}{2 m}\left(p+p^{\prime}\right)_{\mu}\left[1+\frac{\alpha}{3 \pi} \frac{q^{2}}{m^{2}}\left(\ln \left(\frac{m}{\mu}\right)-\frac{3}{8}-\frac{1}{5}\right)\right]+\left(1+\frac{\alpha}{2 \pi}\right) \frac{i}{2 m} \sigma_{\mu \nu} q^{v}\right\} \psi_{p} A_{\text {ext }}^{\mu} .
$$

The momentum factors can be transformed into gradients in configuration space, thus $q_{\mu} \rightarrow$ $i \partial_{\mu}$ acts on the photon field and $p_{\mu}^{\prime}=-i \overleftarrow{\partial}_{\mu} p_{\mu}=i \partial_{\mu}$ act on the spinor field to the left and right respectively. Then (117) becomes

$$
\begin{gathered}
E=e \int d^{3} x\left\{\frac{i}{2 m} \bar{\psi}_{p^{\prime}}(x)\left(\partial_{\mu}-\overleftarrow{\partial}\right) \psi_{p}\left[1-\frac{\alpha}{3 \pi} \frac{1}{m^{2}}\left(\ln \left(\frac{m}{\mu}\right)-\frac{3}{8}-\frac{1}{5}\right)\right] A_{\text {ext }}^{\mu}\right. \\
\left.-\left(1+\frac{\alpha}{2 \pi}\right) \frac{1}{2 m} \bar{\psi}_{p}(x) \sigma_{\mu \nu} \psi_{p}(x) \partial^{v} A_{\text {ext }}^{\mu}\right\} .
\end{gathered}
$$

The first term contains the convection current of the electron which interacts with the potential. In the special case of a purely magnetic field the second part can be identified as the dipole energy. By introducing the electromagnetic field strength tensor $F^{\mu v}=\partial^{\mu} A^{v}-\partial^{v} A^{\mu}$ and using the antisymmetry of $\sigma_{\mu \nu}=\frac{i}{2}\left[\gamma_{\mu}, \gamma_{\nu}\right]$, the second part is

$$
W_{m a g}=e\left(1+\frac{\alpha}{2 \pi}\right) \frac{1}{4 m} \int d^{3} x \bar{\psi}(x) \sigma_{\mu \nu} \psi(x) F^{\mu v}
$$

When $F^{\mu v}$ represents a pure magnetic field, $F^{12}=-B^{3}, \sigma_{12}=\Sigma_{3}$ with cyclic permutations and the interaction energy becomes

$$
W_{\text {mag }}=-\frac{e}{4 m}\left(1+\frac{\alpha}{2 \pi}\right) 2 \int d^{3} x \bar{\psi}(x) \vec{\Sigma} \psi(x) \cdot \vec{B}=-\langle\vec{\mu}\rangle \cdot \vec{B} .
$$

The magnetic moment is given by

$$
\langle\vec{\mu}\rangle=\frac{e \hbar}{2 m c}\left(1+\frac{\alpha}{2 \pi}\right) 2\langle\vec{S}\rangle=g \mu_{B}\langle\vec{S}\rangle .
$$

The magnetic moment is thus proportional to the spin expectation value of the electron. The proportionality factor is the so called $g$-factor

$$
g=2\left(1+\frac{\alpha}{2 \pi}\right)=2(1+0.00116141)
$$

The first point to note is that the value of the $g$-factor obtained including quantum mechanics differs from the classical value of 2 . The result in (122) was first calculated by Schwinger and it has been measured to remarkable accuracy many times. A modern experimental value for the $g$-factor is

$$
g_{\exp }=2(1+0.001159652193),
$$


and only the last digit is uncertain.

Of course Schwinger's calculation has been carried out to much further accuracy and is the subject of continuing work. At around order $\alpha^{4}$, further corrections must be included, such as such effects as virtual hadron creation. The pure-QED contributions are represented by coefficients $C_{i}$ as a power series in powers of $\alpha / \pi$, which acts as a natural expansion parameter for the calculation

$$
g_{\text {theo }}=2\left[1+C_{1}\left(\frac{\alpha}{\pi}\right)+C_{2}\left(\frac{\alpha}{\pi}\right)^{2}+C_{3}\left(\frac{\alpha}{\pi}\right)^{3}+\cdots\right]
$$

Not all of the assumptions made in classical physics apply in quantum physics. In particular, the assumption that it is possible, at least in principle to perform a measurement on a given system in a way in which the interaction between the measured and measuring device can be made as small as desired. In the absence of concepts which follow from observation, principles such as the action principle discussed here are extremely important in providing a direction in which to proceed to formulate a picture of reality which is valid at the microscopic level, given that many assumptions at the normal level of perception no longer apply. These ideas such as the action principle touched on here have led to wide ranging conclusions about the quantum world and resulted in a way to produce useful tools for calculation and results such as transition amplitudes, interaction energies and the result concerning the $g$ factor given here.

\section{Author details}

Paul Bracken

^ Address all correspondence to: bracken@panam.edu

Department of Mathematics, University of Texas, Edinburg, TX, USA

\section{References}

[1] A. L. Fetter and J. D. Walecka, Theoretical Mechanics of particles and Continua, Dover, Mineola, NY, 2003.

[2] J. Schwinger, Quantum Mechanics, Springer Verlag, Berlin Heidelberg, 2001.

[3] J. Schwinger, Selected Papers on Quantum Electrodynamics, Dover, NY, 1958.

[4] J. Schwinger, The theory of quantized fields. I, Phys. Rev. 82, 914-927 (1951).

[5] J. Schwinger, Particles Sources and Fields, Vols I-III, Addison-Wesley, USA, 1981.

[6] D. Toms, The Schwinger Action Principle and Effective Action, Cambridge University Press, Cambridge, 2007.

[7] S. S. Schweber, An Introduction to Relativistic Quantum Field Theory, Row, Peterson and Co, Evanston, 1961. 
[8] W. K. Burton, Equivalence of the Lagrangian formulations of quantum field theory due to Feynman and Schwinger, Nuovo Cimento, 1, 355-357 (1955).

[9] P. Bracken, Quantum Mechanics in Terms of an Action Principle, Can. J. Phys. 75, 261-271 (1991).

[10] K. Fujikawa, Path integral measure for gauge theories with fermions, Phys. Rev. D 21, 2848-2858 (1980). 

Chapter 9

\title{
Generalized Path Integral Technique: Nanoparticles Incident on a Slit Grating, Matter Wave Interference
}

\author{
Valeriy I. Sbitnev \\ Additional information is available at the end of the chapter \\ http://dx.doi.org/10.5772/53471
}

\section{Introduction}

One of the crises of contemporary mathematics belongs in part to the subject of the infinite and infinitesimals [1]. It originates from the barest necessity to develop a rigorous language for description of observable physical phenomena. It was a time when foundations of integral and differential calculi were developed. A theoretical foundation for facilitation of understanding of classical mechanics is provided by the concepts of absolute time and space originally formulated by Sir Isaac Newton [2]. Space is distinct from body. And time passes uniformly without regard to whether anything happens in the world. For this reason Newton spoke of absolute space and absolute time as of a "container" for all possible objects and events. The space-time container is absolutely empty until prescribed metric and a reference frame are introduced. Infinitesimals are main tools of differential calculus [3, 4] within chosen reference frames.

Infinitesimal increment being a cornerstone of theoretical physics has one receptee default belief, that increment $\delta V$ tending to zero contains a lot of events to be under consideration. Probability of detection of a particle within this infinitesimal volume $\rho(\vec{r}) \delta V$ is adopted as a smooth differentiable function with respect to its argument. From experience we know that for reproducing the probability one needs to accumulate enormous amount of events occurring within this volume. On the other hand we know, that as $\delta V$ tends to zero we lose information about amount of the events. What is more, the information becomes uncertain. It means the infinitesimal increment being applied in physics faces with a conflict of depth of understanding physical processes on such minuscule scales. This trouble is avoided in quantum mechanics by proclamation that infinitesimal increments are operators, whereas observables are averaged on an ensemble. 
In light of classical views Newton maintained the theory that light was made up of tiny particles, corpuscles. They spread through space in accordance with law of classical mechanics. Christian Huygens (a contemporary of Newton), believed that light was made up of waves vibrating up and down perpendicularly to direction of the light propagation. It comes into contradiction with Newtonian idea about corpuscular nature of light. Huygens was a proclaimer of wave mechanics as opposite to classical mechanics [5].

We abstain from allusion to physical vacuum but expand Huygensian idea to its logical completion. Let us imagine that all Newtonian absolute space is not empty but is populated everywhere densely by Huygensian vibrators. The vibrators are silent at absence of wave propagating through. But as soon as a wave front reaches some surface all vibrators on this surface begin to radiate at a frequency resonant with that of incident wave. From here it follows, that in each point of the space there are vibrators with different frequencies ranging from zero frequency up to infinite. All are silent in absence of an external wave perturbance. Thus, the infinitesimal volume $\delta V$ is populated by infinite amount of the vibrators with frequencies ranging from zero to infinity. They are virtual vibrators facilitating propagation of waves through space.

Let us return to our days. One believes that besides matter and physical fields there is nothing more in the universe. Elementary particles are only a building material of "eternal and indestructible" substance of the cosmos. However we should avow that all observed matter and physical fields, are not the basis of the world, but they are only a small part of the total quantum reality. Physical vacuum in this picture constitutes a basic part of this reality. In particular, modern conception of the physical vacuum covers Huygens's idea perfectly. All space is fully populated by virtual particle-antiparticle pairs situated on a ground level. Such a particle-antiparticle pair has zero mass, zero charge, and zero magnetic moment. Famous Dirac' sea (Dirac postulated that the entire universe is entirely filled by particles with negative energy) is boundless space of electron-positron pairs populated everywhere densely - each quantum state possessing a positive energy is accompanied by a corresponding state with negative energy. Electron has positive mass and positron has the same mass but negative; electron has negative charge and positron has the same positive charge; when electron and positron dance in pair theirs magnetic moments have opposite orientations, so magnetic moment of the pair is zero.

Let electron and positron of a virtual pair rotate about mass center of this pair. Rotation of the pairs happens on a Bohr orbit. Energy level of the first Bohr orbit, for example, $m v^{2} / 2$, is about $14 \mathrm{eV}$. Here $m$ is electron mass and $v$ is its velocity (on the first Bohr orbit the velocity is about $2.18810^{6} \mathrm{~m} / \mathrm{s}$ ). Energy of the pair remains zero since positron has the same energy but with negative sign. Quantum fluctuations around this zero energy are as zero oscillations of electromagnetic field. Observe that, energy releasing of electron and positron from vacuum occurs at $m c^{2}=1.022 \mathrm{MeV}$. So we see that there are about $7.510^{4} \mathrm{Bohr}$ orbits lying below this energy. That is, there is a vast scope for occupation of different Bohr orbits by the virtual electron-positron pairs.

A short outline given above is a basis for understanding of interference effects to be described below. 
Ones suppose that random fluctuations of electron-positron pairs take place always. What is more, these fluctuations are induced by other pairs and by particles traveling through this random conglomerate. Edward Nelson has described mathematical models [6, 7] representing the above random fluctuations as Brownian motions of particles that are subjected by random impacts from particles populating aether (Nelson's title of a lower environment). The model is viewed as a Markov process

$$
d x(t)=b(x(t), t) d t+d w(t)
$$

loaded by a Wiener term $w$ with diffusion coefficient equal to $\hbar / 2 m$, where $m$ is a mass of the particle and $\hbar$ is the reduced Planck constant. In this perspective Nelson has considered two Markov processes complementary to each other. One is described by forward-difference operator in the time, here $b(x(t), t)$ is a velocity calculated forward. And other equation is described by backward-difference operator in the time with the velocity $b^{\dagger}(x(t), t)$ calculated backward. In general $b(x(t), t) \neq b^{\dagger}(x(t), t)[6,7]$. The two complementary processes, by means of transition to two new variables, real and imaginary, finally lead to emergence of the Schrödinger equation.

Nelson' vision that aether fluctuations look as Brownian movements of subparticles with $\hbar / 2 m$ being the diffusion coefficient of the movements, correlates with Feynman's ideas about quantum fluctuations of virtual particles in vacuum [8]. The Feynman path integral is akin to the Einstein-Smoluchowski integral equation [9]. The latter computes transition probability density. We shall deal with the modified Feynman path integral loaded by a temperature multiplied by the Boltzmann constant. At that, probability amplitude stays as a fundamental mathematical object at all stages of computations. As an example we shall consider emergence of interference patterns at scattering heavy particles on gratings. The particles are heavy in the sense that they adjoin to both realms, quantum and classical. They are nanoparticles. Such nanoparticles have masses about $100 \mathrm{amu}$ and more, as, for example, fullerene molecules [10] shown in Fig. 1. It is remarkable that there are many experiments with such molecules showing interference patterns in the near field [11-18]. On the other hand these molecules are so large, that they behave themselves as classical particles at ordinary conditions.

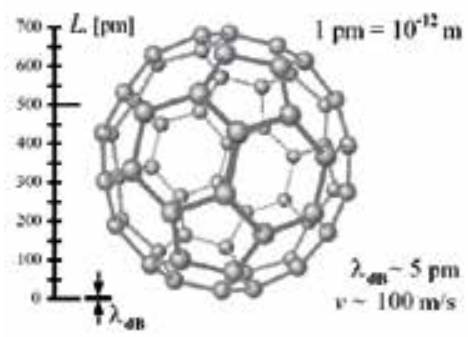

Figure 1. The fullerene molecule $C_{60}$ consists of 60 carbon atoms. Its radius is about $700 \mathrm{pm}$. De Broglie wavelength of the molecule, $\lambda_{\mathrm{dB}}$, is about $5 \mathrm{pm}$ at a flight velocity $v=100 \mathrm{~m} / \mathrm{s}$ [18]. The molecules are prepared in a thermal emission gun which has temperature about $1000 \mathrm{~K}$. It means that carbon atoms accomplish thermal fluctuations. 
The article consists of five sections. Sec. 2 introduces a general conception of the path integral that describes transitions along paths both of classical and quantum particles. Here we fulfill expansions in the Taylor series of terms presented in the path integral. Depending on type of presented parameters we disclose either the Schrödinger equation or diffusion-drift equation containing extra term, osmotic diffusion. In the end of the section we compute passing nanoparticles through $N$-slit grating. Sec. 3 deals with interference patterns from the $N$-slit grating. Specifically, we study blurring of the Talbot carpet (an interference pattern emergent under special conditions imposed on the grating $[19,20])$ arising under decoherence of incident on the grating nanoparticles. In Sec. 4 we find equations for computing the Bohmian trajectories. Also we compute variance of momenta along the trajectories. These computations lead to emergence of the uncertainty conditions. In concluding Sec. 5 we summarize results. For confirmation of existence of the Bohmian trajectories here we mention interference experiments with single silicon oil droplet [21].

\section{Generalized path integral}

Let many classical particles occupy a volume $V$ and they move with different velocities in different directions. Let us imagine that there is a predominant orientation along which ensemble of the particles drifts. As a rule, one chooses a small volume $\delta V$ in order to evaluate such a drift, Fig. 2. Learning of statistical mechanics begins with assumption that the volume should contain many particles.

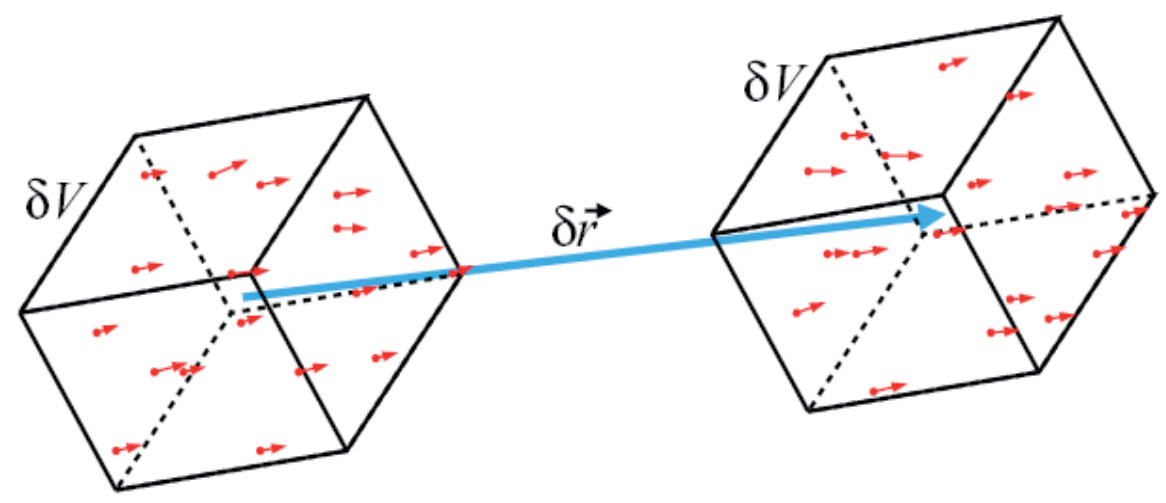

Figure 2. Infinitesimal volume $\delta V$ contains many particles moving with different velocities having predominant orientation along blue arrow. The infinitesimal volume $\delta V$, as a mental image, is shifted along the same orientation.

The problem is to find transition probabilities that describe transition of the particle ensemble from one statistical state to another. These transient probabilities can be found through solution of the integral Einstein-Smoluchowski equation [9]. This equation in mathematical physics is known as Chapman-Kolmogorov [22-24] equation.. This equation looks as follows 


$$
p\left(\vec{r}_{2}, \vec{r}_{1} ; t+\delta \tau\right)=\int_{R} p\left(\vec{r}_{2}, \vec{r} ; t+\delta \tau, t\right) p\left(\vec{r}, \vec{r}_{1} ; t\right) d V
$$

This equation describes a Markovian process without memory. That is, only previous state bears information for the next state. Integration here is fulfilled over all a working scene $\mathrm{R}$, encompassing finite space volume. Infinitesimal volume $\delta V$ in the integral tends to zero. It should be noted, however, that this volume should contain as many particles as possible for getting a satisfactory statistical pattern. One can see that this claim enters in conflict with the assumption $\delta V \rightarrow 0$.

Next we shall slightly modify approach to this problem. Essential difference from the classical probability theory is that instead of the probabilities we shall deal with probability amplitudes. The transition amplitudes can contain also imaginary terms. They bear information about phase shifts accumulated along paths. In that way, a transition from an initial state $\vec{q}_{0}$ to a final state $\vec{q}_{1}$ through all intermediate positions $\vec{q}_{x}$ given on a manifold $\mathrm{R}^{3}$ (see Fig. 2 ) is represented by the following path integral

$$
\psi\left(\vec{q}_{1}, \vec{q}_{0} ; t+\delta t\right)=\int_{R} K\left(\vec{q}_{1}, \vec{q}_{x} ; t+\delta t, t\right) \psi\left(\vec{q}_{x}, \vec{q}_{0} ; t\right) D^{3} q_{x}
$$

Here function $\psi(\ldots)$ is a probability amplitude. Probability density $p(\ldots)$, in turn, is represented by square of modulo of the probability amplitude, namely, $p(\ldots)=|\psi(\ldots)|^{2}$. Integral kernel $K\left(\vec{q}_{1}, \vec{q}_{x} ; t+\delta t, t\right)$ represents the transition amplitude from an intermediate state $\vec{q}_{x}$ to a final state $\vec{q}_{1}$. It is called propagator $[8,25,26]$. We suppose that the propagator has the following standard form

$$
K\left(\vec{q}_{1}, \vec{q}_{x} ; t+\delta t, t\right)=\frac{1}{A} \exp \left\{-\frac{L\left(\vec{q}_{x}, \dot{\vec{q}}_{x}\right) \delta t}{\Gamma}\right\},
$$

where denominator $\Gamma$ under exponent is a complex-valued quantity, i.e., $\Gamma=\beta+\mathbf{i} \hbar$. The both parameters, $\beta$ and the reduced Planck constant $\hbar$, have dimensionality of energy multiplied by time. From here it follows that $\beta=2 k_{\mathrm{B}} T \delta t$. Here $k_{\mathrm{B}}$ is Boltzmann constant and $T$ is temperature. So, we can write

$$
\Gamma=2 k_{\mathrm{B}} T \delta t+\mathrm{i} \hbar
$$

Factor 2 at the first term is conditioned by the fact that the kernel $K$ relates to transitions of the probability amplitude $\psi$, not the probability $p$. Observe that a fullerene molecular beam 
for interference experiment prepared in a Knudsen cell at T=1070 K [27] spreads further in a vacuum. That is, fullerene molecules keep this temperature. From here it follows, that thermal fluctuations of carbon atoms from equilibrium occur at that temperature as the fullerene molecules propagate further within the vacuum chamber. One can see that at $T=1000 \mathrm{~K}$ the term $2 k_{\mathrm{B}} T \delta t$ may be about $\hbar$ if $\delta t$ is about $10^{-14} \mathrm{~s}$.

Next let us imagine that particles pass through a path length one by one. That is, they do not collide with each other along the path length. The particles are complex objects, however. They are nanoparticles. Fullerene molecule, for example, contains 60 carbon atoms, Fig. 1. Conditionally we can think that the atoms are connected with each other by springs simulating elastic vibrations. In this view the Lagrangian $L\left(\vec{q}_{x}, \vec{q}_{x}\right)$ can be written in the following form

$$
L\left(\vec{q}_{x}, \dot{\vec{q}}_{x}\right)=\sum_{k=1}^{N}\left[\frac{m}{2} \frac{\left.\left(\vec{q}_{1, k}-\vec{q}_{x, k}\right)\right)^{2}}{\delta t^{2}}-U\left(\vec{q}_{x, k}\right)\right]
$$

Here $N$ is amount of atoms, constituent complex molecule, and $m$ is mass of a single atom. By supposing that there are no quantum permutations between atoms we may expand the Lagrangian further

$$
\begin{aligned}
L\left(\vec{q}_{x}, \dot{\vec{q}}_{x}\right) & =\frac{m}{2} \sum_{k=1}^{N} \frac{\left(\vec{q}_{1, k}-\vec{q}_{1}+\vec{q}_{1}-\vec{q}_{x, k}+\vec{q}_{x}-\vec{q}_{x}\right)^{2}}{\delta t^{2}}-\sum_{k=1}^{N} U\left(\vec{q}_{x, k}\right) \\
& =\frac{m_{N}}{2} \frac{\left(\vec{q}_{1}-\vec{q}_{x}\right)^{2}}{\delta t^{2}}-m_{N} \frac{\left(\vec{q}_{1}-\vec{q}_{x}\right)}{\delta t} \frac{1}{N} \sum_{k=1}^{N} \frac{\left(\vec{\delta}_{1, k}-\vec{\delta}_{x, k}\right)}{\delta t}+\frac{m}{2} \sum_{k=1}^{N} \frac{\left(\vec{\delta}_{1, k}-\vec{\delta}_{x, k}\right)^{2}}{\delta t^{2}}-\sum_{k=1}^{N} U\left(\vec{q}_{x, k}\right) .
\end{aligned}
$$

Here we admit that $\vec{q}_{x}$ and $\vec{q}_{1}$ are coordinates of center of mass of that complex molecule in intermediate and final positions. And $m_{N}=N m$ is a mass of the molecule referred to its center of mass. Small deviations $\vec{\delta}_{1, k}=\left(\vec{q}_{1}-\vec{q}_{1, k}\right)$ and $\vec{\delta}_{x, k}=\left(\vec{q}_{x}-\vec{q}_{x, k}\right)$ are due to oscillations of the $k$ th atoms with respect to the center of mass.

Let us consider the second row in Eq. (7). First of all we note that the term $\left(\vec{q}_{1}-\vec{q}_{x}\right) / \delta t$ represents a velocity of movement of the center of mass. In such a case the first term is a kinetic energy of the center of mass. The second term represents product of the center mass velocity on an averaged velocity of partial oscillations of atoms constituting this molecule. The averaged velocity we believe vanishes because of conservation of total momentum. The third term represents a thermal kinetic energy of partial oscillating atoms constituting this molecular object. This energy is small enough. But it is sufficient to exhibit itself in the Casimir effect. The last term is a total potential energy $U\left(\vec{q}_{x}\right)$ in the point $\vec{q}_{x}$. 
The path integral (3) contains functions depending only on coordinates $\vec{q}_{0}, \vec{q}_{1}, \vec{q}_{x}$ relating to positions of the center of mass. Whereas the Lagrangian (6) gives description for behavior of each atom constituent the complex molecule. Here we shall suppose that oscillations of all atoms are noncoherent. And consequently they do not give contribution to interference effect on output. We believe that these oscillations provide a thermal noise. And next we shall replace this oscillating background by a corresponding thermal term. For this reason, we believe that along with the reduced Planck constant $\hbar$ the parameter $2 k_{\mathrm{B}} T \delta t$ in Eq. (5) can be different from zero as well.

\subsection{Expansion of the path integral}

The next step is to expand terms, ingoing into the integral (3), into Taylor series. The wave function written on the left is expanded up to the first term

$$
\psi\left(\vec{q}_{1}, \vec{q}_{0} ; t+\delta t\right) \approx \psi\left(\vec{q}_{1}, \vec{q}_{0} ; t\right)+\frac{\partial \psi}{\partial t} \delta t .
$$

As for the terms under the integral, here we preliminarily make some transformations. We define a small increment

$$
\vec{\xi}=\vec{q}_{1}-\vec{q}_{x} \Rightarrow D^{3} q_{x}=-D^{3} \xi
$$

The Lagrangian (7) is rewritten, in such a case, in the following view

$$
L\left(\vec{q}_{x}, \dot{\vec{q}}_{x}\right)=\frac{m_{N}}{2} \frac{\xi^{2}}{\delta t^{2}}-m_{N} \frac{\xi}{\delta t} \frac{1}{N} \sum_{k=1}^{N} \frac{\left(\vec{\delta}_{1, k}-\vec{\delta}_{x, k}\right)}{\delta t}+\frac{m}{2} \sum_{k=1}^{N} \frac{\left(\vec{\delta}_{1, k}-\vec{\delta}_{x, k}\right)^{2}}{\delta t^{2}}-U\left(\vec{q}_{x}\right)
$$

Here $U\left(\vec{q}_{x}\right)$ is sum of all potentials $U\left(\vec{q}_{x, k}\right)$ given in the center of mass. Further we shall deal with the path integral (3) where the kernel $K$ contains the Lagrangian given from Eq. (10). The under integral function $\psi\left(\vec{q}_{x}, \vec{q}_{0} ; t\right)=\psi\left(\vec{q}_{1}-\vec{\xi}, \vec{q}_{0} ; t\right)$ is subjected to expansion into the Taylor series up to the second terms of the expansion

$$
\psi\left(\vec{q}_{1}-\vec{\xi}, \vec{q}_{0} ; t\right) \approx \psi\left(\vec{q}_{1}, \vec{q}_{0} ; t\right)-(\nabla \psi, \vec{\xi})+\nabla^{2} \psi \cdot \xi^{2} / 2
$$

The potential energy $U\left(\vec{q}_{x}\right)=U\left(\vec{q}_{1}-\vec{\xi}\right)$ is subjected to expansion into the Taylor series by the small parameter $\vec{\xi}$ also. Here we restrict themselves by the first two terms of the expansion, $U\left(\vec{q}_{1}\right)-\left(\nabla U\left(\vec{q}_{1}\right), \vec{\xi}\right)$.

Taking into account the expressions (8)-(11) and substituting theirs into Eq. (3) we get 


$$
\begin{aligned}
\psi\left(\vec{q}_{1}, \vec{q}_{0} ; t\right)+\frac{\partial \psi}{\partial t} \delta t & =-\frac{1}{A} \int_{R^{3}} \exp \left\{-\frac{1}{\Gamma}(\frac{m_{N}}{2} \frac{\xi^{2}}{\delta t}-\underbrace{\xi \frac{m_{N}}{N} \sum_{k=1}^{N} \frac{\left(\vec{\delta}_{1, k}-\vec{\delta}_{x, k}\right)}{\delta t}}_{(a)}+\underbrace{\left[\frac{m}{2} \sum_{k=1}^{N} \frac{\left(\vec{\delta}_{1, k}-\vec{\delta}_{x, k}\right)^{2}}{\delta t^{2}}\right] \delta t}_{(b)}\right. \\
& \left.\left.-\left(U\left(\vec{q}_{1}\right)-\left(\nabla U\left(\vec{q}_{1}\right), \vec{\xi}\right)\right) \delta t\right)\right\}\left(\psi\left(\vec{q}_{1}, \vec{q}_{0} ; t\right)-(\nabla \psi, \vec{\xi})+\nabla^{2} \psi \cdot \xi^{2} / 2\right) D^{3} \xi .
\end{aligned}
$$

First, we consider terms enveloped by braces (a) and (b): (a) here displacement $\left(\vec{\delta}_{1, k}-\vec{\delta}_{x, k}\right)$ divided by $\delta t$ is a velocity $\vec{v}_{k}$ of $\mathrm{kth}$ atom at its deviation from a steady position relative to the center of mass. Summation through all deviations of atoms divided by $N$ gives averaged velocity, $\vec{v}$, of all atoms with respect to the center of mass. This averaged velocity, as we mentioned above, vanishes. The velocity can be nonzero only in a case when external forces push coherently all atoms. This case we do not consider here. (b) this term is a thermal kinetic energy, $T_{N}$, of the atoms oscillating about the center of mass. Observe that energy of thermal fluctuations, $T_{N}$, is proportional to $k_{\mathrm{B}} T$. Because of its presence in the propagator intensity of an interference pattern diminishes in general. Further we shall add this term into the potential energy as some constant component.

In the light of the above observation we rewrite Eq. (12) as follows

$$
\begin{aligned}
\psi\left(\vec{q}_{1}, \vec{q}_{0} ; t\right)+\frac{\partial \psi}{\partial t} \delta t & =-\frac{1}{A} \int_{R^{3}} \exp \left\{-\frac{m_{N}}{2 \Gamma} \frac{\xi^{2}}{\delta t}\right\}(1+\underbrace{m_{N} \vec{v}}_{(a)} \frac{\vec{\xi}}{\Gamma}+\underbrace{T_{N}}_{(b)} \frac{\delta t}{\Gamma}+\left(U\left(\vec{q}_{1}\right)-\left(\nabla U\left(\vec{q}_{1}\right), \vec{\xi}\right)\right) \frac{\delta t}{\Gamma}) \\
& \times\left(\psi\left(\vec{q}_{1}, \vec{q}_{0} ; t\right)-(\nabla \psi, \vec{\xi})+\nabla^{2} \psi \cdot \xi^{2} / 2\right) D^{3} \xi .
\end{aligned}
$$

Here we have expanded preliminarily exponents to the Taylor series up to the first term of the expansion. Exception relates to the term $\exp \left\{-m_{N} \xi^{2} / 2 \Gamma \delta t\right\}$ which remains in its original form. This exponent integrated over all space $\mathrm{R}^{3}$ results in

$$
-\frac{1}{A} \int_{R^{3}} \exp \left\{-\frac{m_{N}}{2 \Gamma} \frac{\xi^{2}}{\delta t}\right\} D^{3} \xi=-\frac{1}{A}\left(\frac{2 \pi \Gamma \delta t}{m_{N}}\right)^{3 / 2}=1 \quad \Rightarrow \quad A=-\left(\frac{2 \pi \Gamma \delta t}{m_{N}}\right)^{3 / 2} .
$$

To derive outcomes of integration of terms containing factors $(\nabla \psi, \vec{\xi})$ and $\nabla^{2} \psi \cdot \xi^{2} / 2$ we mention the following integrals [8]

$$
\frac{1}{A} \int_{R^{3}} \exp \left\{-\frac{m_{N}}{2 \Gamma} \frac{\xi^{2}}{\delta t}\right\} \xi D^{3} \xi=0 \quad \text { and } \quad \frac{1}{A} \int_{R^{3}} \exp \left\{-\frac{m_{N}}{2 \Gamma} \frac{\xi^{2}}{\delta t}\right\} \xi^{2} D^{3} \xi=\frac{\Gamma}{m_{N}} \delta t
$$


In the light of this observation let us now solve integral (13) accurate to terms containing $\delta t$ not higher the first order:

$$
\psi\left(\vec{q}_{1}, \vec{q}_{0} ; t\right)+\frac{\partial \psi}{\partial t} \delta t=\psi\left(\vec{q}_{1}, \vec{q}_{0} ; t\right)+\frac{\Gamma}{2 m_{N}} \nabla^{2} \psi \delta t+\frac{1}{\Gamma}\left(U\left(\vec{q}_{1}\right)-T_{N}\right) \psi \delta t .
$$

We note that the term $(\vec{v}, \nabla \psi)$ here is absent since we consider $\vec{v}=0$, as was mentioned above. By reducing from the both sides the function $\psi\left(\vec{q}_{1}, \vec{q}_{0} ; t\right)$ we come to the following differential equation

$$
\frac{\partial \psi\left(\vec{q}_{1}, \vec{q}_{0} ; t\right)}{\partial t}=\frac{\Gamma}{2 m_{N}} \nabla^{2} \psi\left(\vec{q}_{1}, \vec{q}_{0} ; t\right)+\frac{1}{\Gamma}\left(U\left(\vec{q}_{1}\right)-T_{N}\right) \psi\left(\vec{q}_{1}, \vec{q}_{0} ; t\right) .
$$

The parameter

$$
\frac{\Gamma}{2 m_{N}}=\frac{2 k_{\mathrm{B}} T \delta t}{2 m_{N}}+\mathbf{i} \frac{\hbar}{2 m_{N}}
$$

is seen to be as a complex-valued diffusion coefficient consisting of real and imaginary parts.

\subsubsection{Temperature is zero}

Let $k_{\mathrm{B}} T \delta t=0$. It means that $\Gamma=\mathrm{i} \hbar$. Also $T_{N}=0$. One can see that Eq. (17) is reduced to

$$
\mathbf{i} \hbar \frac{\partial \psi\left(\vec{q}_{1}, \vec{q}_{0} ; t\right)}{\partial t}=-\frac{\hbar^{2}}{2 m_{N}} \Delta \psi\left(\vec{q}_{1}, \vec{q}_{0} ; t\right)+U\left(\vec{q}_{1}\right) \psi\left(\vec{q}_{1}, \vec{q}_{0} ; t\right) .
$$

It is the Schrödinger equation.

\subsubsection{Temperature is not zero}

Let $k_{\mathrm{B}} T \delta t>\hbar$. We can suppose that $\Gamma=k_{\mathrm{B}} T \delta t$. Eq. (17) takes a form

$$
\frac{\partial \psi\left(\vec{q}_{1}, \vec{q}_{0} ; t\right)}{\partial t}=D \Delta \psi\left(\vec{q}_{1}, \vec{q}_{0} ; t\right)+\frac{\left(U\left(\vec{q}_{1}\right)-T_{N}\right)}{2 m_{N} D} \psi\left(\vec{q}_{1}, \vec{q}_{0} ; t\right) .
$$

Here 


$$
D=\frac{k_{\mathrm{B}} T \delta t}{m_{N}}
$$

is the diffusion coefficient. The coefficient has dimensionality of [length ${ }^{2}$ time $\left.^{-1}\right]$. It is a factor of proportionality representing amount of substance diffusing across a unit area in a unit time - concentration gradient in unit time.

We can see that Eq. (20) deals with the amplitude function $\psi$, not a concentration. However, a measurable function is $\rho=|\psi|^{2}$ - concentration having dimensionality of [(amount of substance) length ${ }^{-3}$. Let us multiply Eq. (20) from the left by $2 \psi$. First we note that the combination $\quad 2 \psi \Delta \psi=2 \psi \nabla \psi^{-1} \psi \nabla \psi=2\left(\psi \nabla \psi^{-1}\right)(\psi \nabla \psi)+2 \nabla(\psi \nabla \psi) \quad$ results in $-(1 / 2)(\nabla \ln (\rho) \cdot \nabla \rho)+\Delta \rho$. As a result we come to a diffusion-drift equation describing diffusion in a space loaded by a potential field $\left(U\left(\vec{q}_{1}\right)-T_{N}\right)$ :

$$
\frac{\partial \rho}{\partial t}+\frac{D}{2}(\nabla \ln (\rho), \nabla \rho)=D \Delta \rho+\frac{\left(U\left(\vec{q}_{1}\right)-T_{N}\right)}{2 m_{N} D} \rho
$$

Extra term $(D / 2) \nabla \ln (\rho)$ in this diffusion-drift equation is a velocity of outflow of the particles from volume populated by much more number of particles than in adjacent volume. The term $-\ln (\rho)$ is entropy of a particle ensemble. From here it follows that $\nabla \ln (\rho)$ describes inflow of the particles to a region where the entropy is small. Observe that the velocity

$$
\vec{u}=D \frac{\nabla \rho}{\rho}=D \nabla \ln (\rho)
$$

is an osmotic velocity required of the particle to counteract osmotic effects [6]. Namely, imagine a suspension of many Brownian particles within a physical volume acted on by an external, virtual in general, force. This force is balanced by an osmotic pressure force of the suspension [6]:

$$
\vec{K}=k_{\mathrm{B}} T \frac{\nabla \rho}{\rho} .
$$

From here it is seen, that the osmotic pressure force arises always when density difference exists and especially when the density tends to zero. And vice-versa, the force disappears in extra-dense media with spatially homogeneous distribution of particles. As states the second law of thermodynamics spontaneous processes happen with increasing entropy. The osmosis evolves spontaneously because it leads to increase of disorder, i.e., with increase of entropy. When the entropy gradient becomes zero the system achieves equilibrium, osmotic forces vanish. 
Due to appearance of the term $(D / 2) \nabla \ln (\rho)$ in Eq. (22) the diffusion equation becomes nonlinear. It is interesting to note, running ahead, that this osmotic term reveals many common with the quantum potential, which is show further. Observe that the both expressions contain the term $\nabla \rho / \rho=\nabla \ln (\rho)$ relating to gradient of the quantum entropy $S_{Q}=-\ln (\rho) / 2$ [28].

Reduction to PDEs, Eq. (19) and Eq. (22), was done with aim to show that the both quantum and classical realms adjoin with each other much more closely, than it could seem with the first glance. Further we shall return to the integral path paradigm [8] and calculate patterns arising after passing particles through gratings. We shall combine quantum and classical realms by introducing the complex-valued parameter $\Gamma=2 k_{\mathrm{B}} T \delta t+\mathrm{i} \hbar$.

\subsection{Paths through $N$-slit grating}

Computation of a passing particle through a grating is based on the path integral technique [8]. We begin with writing the path integral that describes passing the particle through a slit made in an opaque screen that is situated perpendicularly to axis $z$, Fig. 3. For this reason we need to describe a movement of the particle from a source to the screen and its possible deflection at passing through the slit, see Fig. 4. At that we need to evaluate all possible deflections.

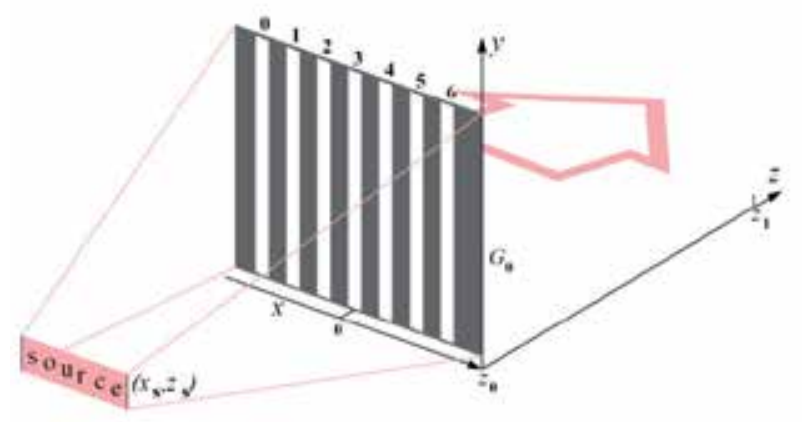

Figure 3. Interferometry from one grating $G_{0}$ situated transversely to a particle beam emitted from a distributed source.

We believe, that before the screen and after it, the particle (fullerene molecule, for example) moves as a free particle. Its Lagrangian, rewritten from Eq. (17), describes its deflection from a straight path in the following form

$$
L=m_{N} \frac{\dot{x}^{2}}{2}-m_{N}(\dot{x}, \vec{v})+T_{N}
$$

The first term relates to movement of the center of mass of the molecule. So that $m_{N}$ is mass of the molecule and $\dot{x}$ is its transversal velocity, i.e., the velocity lies in transversal direction to the axis $z$. The second term is conditioned by collective fluctuations of atoms constituent 
this molecule. This term is nonzero when atoms have predominant fluctuations along axis $x$. For the sake of simplicity we admit that $\vec{v}$ is constant. The third term is a constant and comes from Eq. (17). It can be introduced into the normalization factor. For that reason we shall ignore this term in the following computations. A longitudinal momentum, $p_{z}$ is much greater than its transverse component $[16,17,29]$ and we believe it is constant also. By translating a particle's position on a small distance $\delta x=\left(x_{\mathrm{b}}-x_{\mathrm{a}}\right)<<1$ for a small time $\delta t=\left(t_{\mathrm{b}}-t_{\mathrm{a}}\right)<<1$ we find that a weight factor of such a translation has the following form

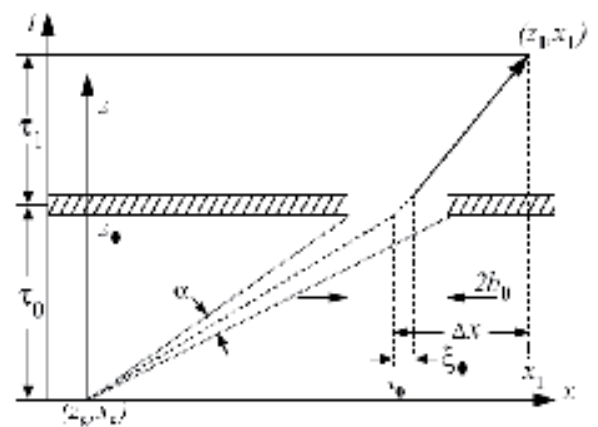

Figure 4. Passage of a particle along path $\left(z_{s}, x_{5}\right) \rightarrow\left(z_{0}, x_{0}\right) \rightarrow\left(z_{1}, x_{1}\right)$ through a screen containing one slit with a width equal to $2_{\mathrm{b} 0}$. Divergence angle of particles incident on the slit, $a$, tends to zero as the source is removed to infinity.

$$
\exp \left\{-\frac{L \delta t}{\Gamma}\right\}=\exp \left\{-\frac{m_{N}}{2 \Gamma} \frac{\left(x_{b}-x_{a}\right)^{2}}{\left(t_{b}-t_{a}\right)}+\frac{m_{N} v}{\Gamma}\left(x_{b}-x_{a}\right)\right\}
$$

The particles flying to the grating slit along a ray $\alpha$, Fig. 4 , pass through the slit within a range from $x_{0}-b_{0}$ to $x_{0}+b_{0}$ with high probability. The path integral in that case reads

$$
\psi\left(x_{1}, x_{0}, x_{s}\right)=\int_{-b_{0}}^{b_{0}} K\left(x_{1}, \tau_{0}+\tau_{1} ; x_{0}+\xi_{0}, \tau_{0}\right) K\left(x_{0}+\xi_{0}, \tau_{0} ; x_{s}, 0\right) d \xi_{0} .
$$

Integral kernel (propagator) for the particle freely flying is as follows [8, 26]

$$
K\left(x_{b}, t_{b} ; x_{a}, t_{a}\right)=\left[\frac{m_{N}\left(1-2 v / v_{(a, b)}\right)}{2 \pi \Gamma\left(t_{b}-t_{a}\right)}\right]^{1 / 2} \exp \left\{-\frac{m_{N}\left(x_{b}-x_{a}\right)^{2}}{2 \Gamma\left(t_{b}-t_{a}\right)}\left(1-2 \frac{v}{v_{(a, b)}}\right)\right\}
$$

Here $v_{(a, b)}=\left(x_{b}-x_{a}\right) /\left(t_{b}-t_{b}\right)$ is a velocity of the molecule on a segment from $x_{a}$ to $x_{b}$. And $v$ is an average velocity of collective deflection of atoms constituent the molecule. It was defined 
in Eq. (12). We shall believe that the ratio $v / v_{(a, b)}$ is small enough. We can define a new renormalized mass $\{m\}_{N}=m_{N}\left(1-v / v_{(a, b)}\right)$ and further we shall deal with this mass.

\subsubsection{Passing of a particle through slit}

By substituting the kernel (28) into the integral (27) we obtain the following detailed form

$$
\psi\left(x_{1}, x_{0}, x_{s}\right)=\int_{-b_{0}}^{b_{0}}\left(\frac{\{m\}_{N}}{2 \pi \Gamma \tau_{1}}\right)^{1 / 2} \exp \left\{-\frac{\{m\}_{N}\left(x_{1}-\left(x_{0}+\xi_{0}\right)\right)^{2}}{2 \Gamma \tau_{1}}\right\}\left(\frac{\{m\}_{N}}{2 \pi \Gamma \tau_{0}}\right)^{1 / 2} \exp \left\{-\frac{\{m\}_{N}\left(\left(x_{0}+\xi_{0}\right)-x_{s}\right)^{2}}{2 \Gamma \tau_{0}}\right\} d \xi_{0} .
$$

The integral is computed within a finite interval $\left[-b_{0},+b_{0}\right]$. Observe, that the integrating can be broadened from $-\infty$ to $+\infty$. But in this case we need to load the integral by the step function equal to unit within the finite interval $\left[-b_{0},+b_{0}\right]$ and it vanishes outside of the interval. The step function, that simulate a single slit, can be approximated by the following a set of the Gaussian functions [30]

$$
G(\xi, b, \eta, K)=\frac{1}{\eta} \sqrt{\frac{2}{\pi}} \sum_{k=1}^{K} \exp \left\{-\frac{(K \xi-b(K-(2 k-1)))^{2}}{2(b \eta)^{2}}\right\}
$$

Here parameter $b$ is a half-width of the slit, real $\eta>0$ is a tuning parameter, and $K$ takes integer values. At $K=1$ this form-factor degenerates to a single Gaussian function. And at $K \rightarrow \infty$ this function tends to an infinite collection of the Kronecker deltas which fill everywhere densely the step function. We rewrite Eq. (29) with inserting this form-factor

$$
\psi\left(x_{1}, x_{0}, x_{s}\right)=\int_{-\infty}^{\infty} G\left(\xi_{0}, b_{0}\right) \frac{\{m\}_{N}}{2 \pi \Gamma \sqrt{\tau_{1} \tau_{0}}} \exp \left\{-\frac{\{m\}_{N}}{2 \Gamma}\left(\frac{\left(x_{1}-\left(x_{0}+\xi_{0}\right)\right)^{2}}{\tau_{1}}+\frac{\left(\left(x_{0}+\xi_{0}\right)-x_{s}\right)^{2}}{\tau_{0}}\right)\right\} d \xi_{0} .
$$

We do not write parameters $\eta$ and $K$ in the Gaussian form-factor and for the sake of simplicity further we shall consider they equal to 1 . That is, for simulating the slit we select a single Gaussian function.

\subsubsection{Definition of new working parameters}

First we replace the flight times $\tau_{0}$ and $\tau_{1}$ by flight distances $\left(z_{0}-z_{\mathrm{s}}\right)$ and $\left(z_{1}-z_{0}\right)$, see Fig. 4 . This replacement reads

$$
\left\{\begin{array}{l}
\tau_{0}=\left(z_{0}-z_{s}\right) / v_{z} \\
\tau_{1}=\left(z_{1}-z_{0}\right) / v_{z}
\end{array}\right.
$$


where $v_{z}$ is a particle velocity along the axis $z$.

There is, however, one more parameter of time that is represented in definition of the coefficient $\Gamma=2 k_{\mathrm{B}} T \delta t+\mathbf{i} \hbar$. It is a small time increment $\delta t$. The parameter $\delta t$ first appears in the path integral (3) as the time increment along a path. In accordance with the uncertainty principle $\delta t$ should be more or equal to the ratio of $h$, Planck constant, to energy of occurring events. In a case of a flying particle through vacuum it can be minimal energy of vacuum fluctuations (it is about energy of the first Bohr orbit of electron-positron pair that is about $14 \mathrm{eV}$ ). Evaluation gives $\delta t \sim 2.810^{-16} \mathrm{~s}$. From here it follows, that $2 k_{\mathrm{B}} T \delta t$ is less than $\hbar$ on about one order at $T=1000 \mathrm{~K}$ (almost temperature of fullerene evaporation from the Knudsen cell [27]).

Emergence of the term $2 k_{\mathrm{B}} T \delta t$ can be induced by existence of quantum drag [31] owing to different conditions for quantum fluctuations both inside of the fullerene molecule and outside what can induce weak Casimir forces. Because of the weak Casimir force the quantum drag does not lead to decoherence at least in the near zone. However further we shall see that a weak washing out of the Talbot interference pattern is due to existence of this term.

Let us divide the parameter $\Gamma$ by $\{m\}_{N} v_{z}$

$$
\frac{\Gamma}{\{m\}_{N} v_{z}}=\frac{2 k_{\mathrm{B}} T}{\{m\}_{N} v_{z}} \delta t+\mathbf{i} \frac{\hbar}{\{m\}_{N} v_{z}} .
$$

Here $p_{z}=\{m\}_{N} v_{z}$ is a particle momentum along axis $z$. We can define the de Broglie wavelength $\lambda_{\mathrm{dB}}=h / p_{z}$ where $h=2 \pi \hbar$ is the Planck constant. Let us also define a length $\delta_{T}=$ $4 \pi k_{\mathrm{B}} T \delta t /\left(\{m\}_{N} v_{z}\right)$. In this view we can rewrite Eq. (33) as follows

$$
\frac{\Gamma}{\{m\}_{N} v_{z}}=\frac{\delta_{T}}{2 \pi}+\mathbf{i} \frac{\lambda_{\mathrm{dB}}}{2 \pi}=\frac{1}{2 \pi} \Lambda .
$$

The length $\delta_{T}$ tends to zero as $T \rightarrow 0$. At $T=1000 \mathrm{~K}$ and at adopted $\delta t=2.810^{-16} \mathrm{~s}$ we have $\delta_{T}$ $\approx 0.4 \mathrm{pm}$. On the other hand, the de Broglie wavelength, $\lambda_{\mathrm{dB}}$, evaluated for the fullerene molecule moving with the velocity $v_{z}=100 \mathrm{~m} / \mathrm{s}$ is about $5 \mathrm{pm}$ [18]. So, we can see that the length $\delta_{T}$ is less of the de Broglie wavelength on about one order and smaller. A signification of the length $\delta_{T}$ is that it determines decoherence of a particle beam. Decoherence of flying particles occurs the quickly, the larger $\delta_{T}$. Observe that the length $\delta_{T}$ has a close relation with the coherence width - a main parameter in the generalized Gaussian Schell-model [32, 33].

\section{Wave function behind the grating}

Wave function from one slit after integration over $\xi_{0}$ from $-\infty$ to $+\infty$ has the following view [28] 


$$
\psi\left(x, z, x_{0}, x_{s}\right)=\sqrt{\frac{2}{\pi \Lambda \Sigma_{0}\left(z_{0}-z_{s}\right)}} \exp \left\{-\frac{\pi}{\Lambda}\left[\frac{\left(x-x_{0}\right)^{2}}{\left(z-z_{0}\right)}\left(1-\frac{\Xi_{0}^{2}}{\Sigma_{0}}\right)+\frac{\left(x_{0}-x_{s}\right)^{2}}{\left(z_{0}-z_{s}\right)}\right]\right\}
$$

Here argument of $\psi$-function contains apart $x$ also $z$ in order to emphasize that we carry out observation in the point $(z, x)$, see Fig. 4 . The factor $(2 / \pi)^{1 / 2}$ comes from (30). Parameters $\Xi_{0}$ and $\Sigma_{0}$ read

$$
\Xi_{0}=1-\frac{\left(x_{0}-x_{s}\right)\left(z-z_{0}\right)}{\left(z_{0}-z_{s}\right)\left(x-x_{0}\right)} \quad \text { and } \quad \Sigma_{0}=\frac{\left(z-z_{s}\right)}{\left(z_{0}-z_{s}\right)}+\frac{\Lambda\left(z-z_{0}\right)}{2 \pi b_{0}^{2}}
$$

In order to simplify records here we omit subscript 1 at $x$ and $z$ - an observation point that is situated after the slit.

Let us consider that an opaque screen contains $N_{0}$ slits spaced through equal distance, $d$, from each other. Numeration of the slits is given as it is shown in Fig. $3, n_{0}=0,1,2, \ldots, N_{0}-1$. Sum of all wave functions (35), each of which calculates outcome from an individual slit, gives a total effect in the point $(z, x)$ where a detector is placed:

$$
\left|\Psi_{0}\left(x, z, d, x_{s}, \Lambda\right)\right\rangle=\sum_{n_{0}=0}^{N_{0}-1} \psi\left(x, z,\left(n_{0}-\frac{N_{0}-1}{2}\right) d, x_{x}\right)
$$

Probability density in the vicinity of the observation point $(x, z)$ reads

$$
p(x, z)=\left\langle\Psi_{0}\left(x, z, d, x_{s}, \Lambda\right) \mid \Psi_{0}\left(x, z, d, x_{s}, \Lambda\right)\right\rangle
$$

Calculation of the wave function (37) is fulfilled for the grating containing $N_{0}=32$ slits. Distance between slits is $d=10^{5} \lambda_{\mathrm{dB}}$. So at $\lambda_{\mathrm{dB}}=5 \mathrm{pm}$ the distance is equal to $500 \mathrm{~nm}$. Requirement $\lambda_{\mathrm{dB}} \ll d$ and $N_{0}$ tending to infinity together with a condition that the particle beam is paraxial, that is, $x_{\mathrm{s}}=0$ and $z_{\mathrm{s}} \rightarrow-\infty$, provides emergence in the near-field of an interference pattern, named Talbot carpet $[19,20]$. Here a spacing along interference patterns is measured in the Talbot length

$$
z_{\mathrm{T}}=2 \frac{d^{2}}{\lambda_{\mathrm{dB}}}
$$

which is a convenient natural length at representation of interference patterns. Since we restrict themselves by finite $N_{0}$ we have a defective carpet, which progressively collapses as a 
spacing from the slit increases. Fig. 5 shows the Talbot carpet, being perfect in the vicinity of the grating slit; it is destroyed progressively with increasing $z_{\mathrm{T}}$. As for the Talbot carpet we have the following observation. We see that in a cross-section $z=z_{\mathrm{T}} / 2$ image reproduces radiation of the slits but phase-shifted by half period between them. At $z=z_{\mathrm{T}}$ radiation of the slits is reproduced again on the same positions where the slits are placed. And so forth.

Evaluation of sizes of the interference pattern is given by ratio of the Talbot length to a length of the slit grating. In our case the Talbot length is $z_{\mathrm{T}}=0.1 \mathrm{~m}$. And length of the slit grating is about $N_{0} d=1.610^{-5} \mathrm{~m}$. From here we find that the ratio is 6250 . It means that the interference pattern shown in Fig. 5 represents itself a very narrow strip.

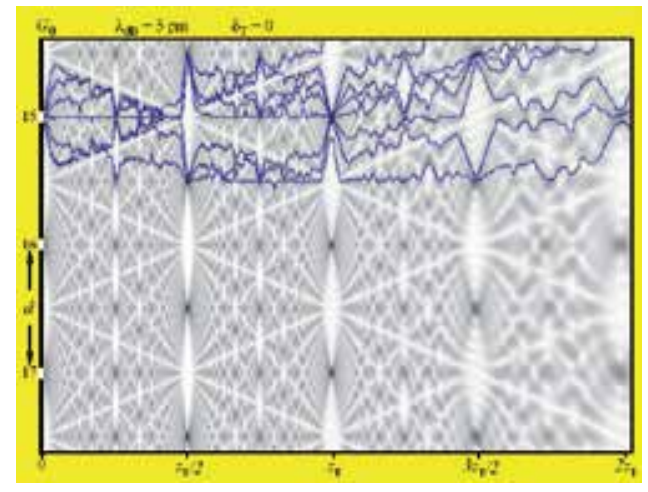

Figure 5. Interference pattern in the near field. It is shown only the central part of the grating containing $N_{0}=32$ slits, $\lambda_{\mathrm{dB}}=5 \mathrm{pm}, d=500 \mathrm{~nm}$, and $\delta_{\mathrm{T}}=0$. In the upper part of the figure a set of the Bohmian trajectories, looking like on zigzag curves, drawn in dark blue color is shown.

Zigzag curves, drawn in the upper part of Fig. 5 by dark blue color, show Bohmian trajectories that start from the slit No. 15. One can see that particles prefer to move between nodes having positive interference effect and avoid empty lacunas. However the above we noted, that the ratio of the Talbot length to the length of the grating is about $6250>1$. It means that really the Bohmian trajectories look almost as straight lines slightly divergent apart. Zigzaglike behavior of the trajectories is almost invisible. Such an almost feebly marked zigzag-like behavior may be induced by fluctuations of virtual particles escorting the real particle.

As soon as we add the term $k_{\mathrm{B}} T \delta t$ different from zero $(T>0 \mathrm{~K})$ we observe blurring the interference pattern. The blurring is the stronger, the larger $k_{\mathrm{B}} T \delta t$. For comparison see Figs. 6 and 7. Here instead of $k_{\mathrm{B}} T \delta t$ we write a more convenient parameter, the coherence length $\delta_{\mathrm{T}}$. This length characterizes a dispersed divergence from initially tuned the de Broglie wavelength. Such a disperse medium can be due to quantum drag on the vacuum fluctuations. Here the Bohmian trajectories are not shown, since because of the thermal term $k_{\mathrm{B}} T \delta t>0$ a Brownian like scattering of the trajectories arises. This scattering we shall discuss later on. 


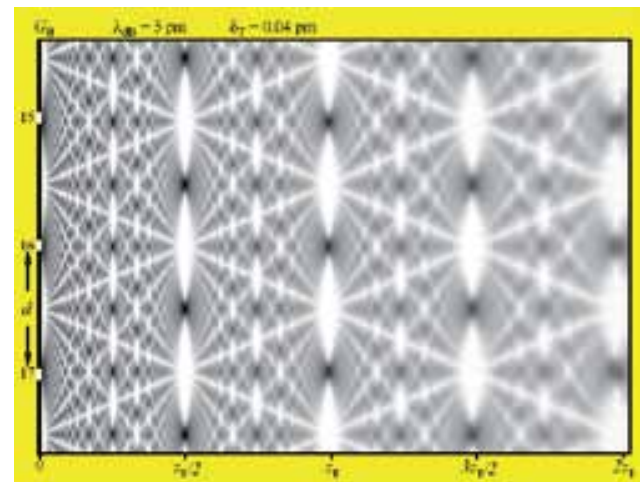

Figure 6. Blurred interference pattern in the near field. It is shown only the central part of the grating containing $N_{0}=$ 32 slits; $d=500 \mathrm{~nm}, \delta_{T}=0.04 \mathrm{pm} \ll<\lambda_{\mathrm{dB}}=5 \mathrm{pm}$.

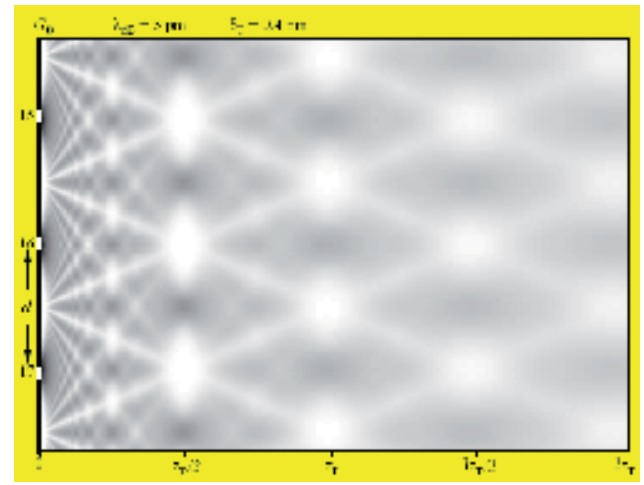

Figure 7. Blurred interference pattern in the near field. It is shown only the central part of the grating containing $N_{0}=$ 32 slits; $d=500 \mathrm{~nm}, \delta_{\mathrm{T}}=0.4 \mathrm{pm}<\lambda_{\mathrm{dB}}=5 \mathrm{pm}$.

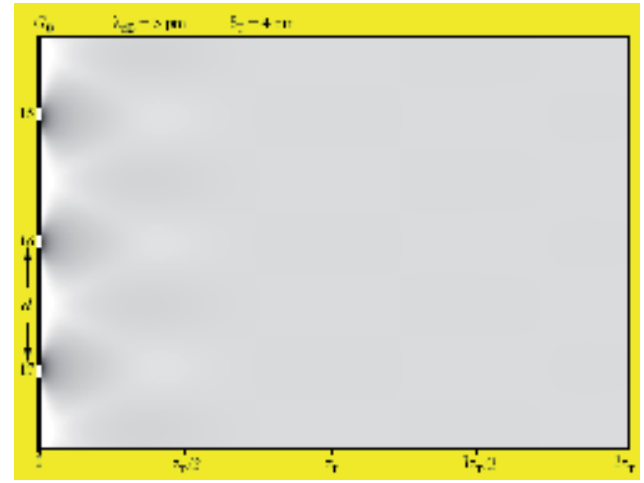

Figure 8. Destroyed interference pattern because of large $\delta_{\mathrm{T}}=4 \mathrm{pm} \sim \lambda_{\mathrm{dB}}=5 \mathrm{pm} . N_{0}=32$ slits, $d=500 \mathrm{~nm}$. 
We may think that the technical vacuum can be not perfect. It causes additional scattering of particles on residual gases. Because of this additional scattering the interference pattern can be destroyed entirely, as shown in Fig. 8 .

Now let us draw dependence of the probability density $p(x, z)$ as a function of $x$ at fixed $z$. In other words, we calculate interference fringes in a cross-section of the interference patterns at $z=z_{\mathrm{T}} / 2$ for different values of the length $\delta_{\mathrm{T}}$. Such a cross-section is chosen because a self image of the slit grating appears phase-shifted by half period of the grating. For that reason we should see the interference fringes spaced between the slit sources of radiation.

Fig. 9 shows three characteristic patterns of the interference fringes. In Fig. 9(a) almost ideal interference fringes are shown obtained at $\delta_{\mathrm{T}}<\lambda_{\mathrm{dB}}$. Fig. 9(b) shows interference fringes obtained at $\delta_{\mathrm{T}} \sim \lambda_{\mathrm{dB}}$. It is instructive to compare these interference fringes with those that have been measured in experiments [14,34]. And Fig. 9(c) shows disappearance of interference fringes because of strong scattering of the particles on residual gases in vacuum, $\delta_{\mathrm{T}}>\lambda_{\mathrm{dB}}$.

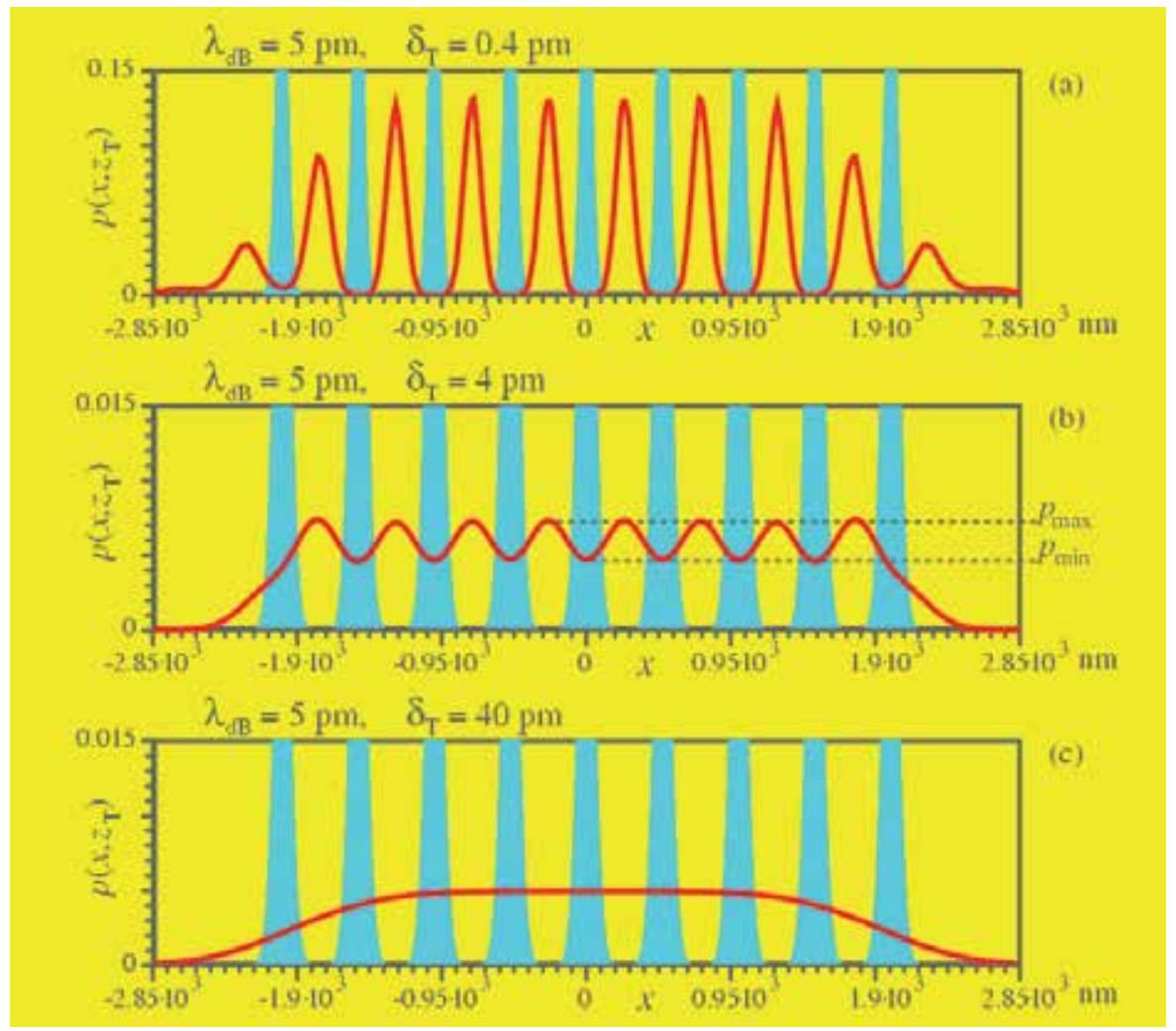

Figure 9. Interference fringes in cross-section of the density distribution pattern by the Talbot half-length, $z=z_{\top} / 2$, (the fringes are drawn in red): (a) $\delta_{T}=0.4 \mathrm{pm}$, almost coherent beam; (b) $\delta_{\top}=4 \mathrm{pm}$, weak coherence; (c) $\delta_{T}=40 \mathrm{pm}$, entirely noncoherent beam. Cyan strips show luminosity of slits. The grating consists of $N_{0}=9$ slits. Collapse of the interference pattern on edges of the grating is due to its finite length. Therefore visibility of the interference fringes is evaluated only for 5 central slits. 
Disappearance of interference fringes is numerically evaluated by calculating a characteristic called visibility [14, 34]. The fringe visibility [27] is represented as a ratio of difference between maximal and minimal intensities of the fringes to their sum:

$$
V=\frac{P_{\max }-P_{\min }}{P_{\max }+P_{\min }} .
$$

Evaluation of $P_{\max }$ and $P_{\min }$ is shown in Fig. 9(b). As follows from the figure, the evaluations are fulfilled in a central region of the grating. That is, edges of the grating have to be left far off from the measured zone. The visibility $V$ as a function of the parameter $\delta_{\mathrm{T}}$ is shown in Fig. 10. One can see that crossover from almost perfect interference fringes, $V=1$, up to their absence, $V=0$, begins near $\delta_{\mathrm{T}} \sim \lambda_{\mathrm{dB}}$. Transition from almost coherent particle beam to incoherent is a cause of such a crossover [30].

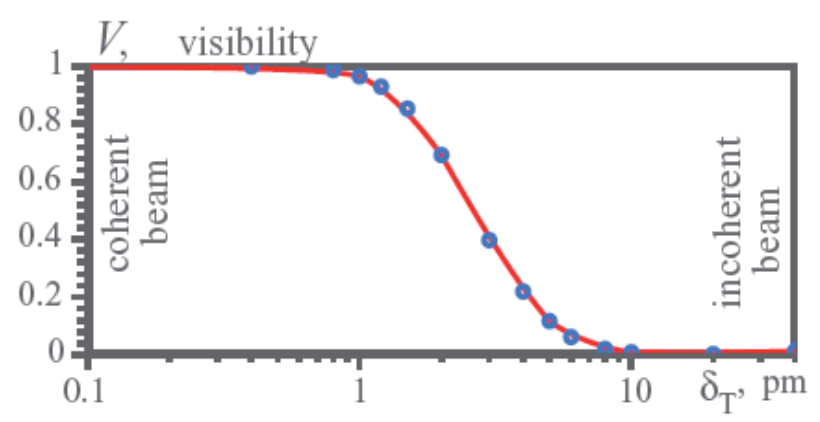

Figure 10. Visibility of interference fringes as a function of the parameter $\delta_{T}$ ranging from 0.1 to $40 \mathrm{pm}$. Wavelength of a matter wave is $\lambda_{\mathrm{dB}}=5 \mathrm{pm}$.

\section{Bohmian trajectories and variance of momenta and positions along paths}

Here we repeat computations of David Bohm [35] which lead to the Hamiltoton-Jacobi equation loaded by the quantum potential and, as consequence, to finding Bohmian trajectories. But instead of the Schrödinger equation we choose Eq. (17) that contains complex-valued parameter $\Gamma=\beta+\mathrm{i} \hbar$ :

$$
(\beta-\mathbf{i} \hbar) \frac{\partial \psi}{\partial t}=\frac{\left(\beta^{2}+\hbar^{2}\right)}{2 m_{N}} \nabla^{2} \psi+\frac{(\beta-\mathbf{i} \hbar)}{(\beta+\mathbf{i} \hbar)} V \psi .
$$

Here $\beta=2 k_{\mathrm{B}} T \delta t$ (in particular, the diffusion coefficient reads $\left.D=\beta / 2 m_{N}\right)$ and $V=U\left(\vec{q}_{1}\right)-T_{N}$. 
Further we apply polar representation of the wave function, $\psi=R \exp \{\mathrm{iS} / \hbar\}$. It leads to obtaining two equations for real and imaginary parts that deal with real-valued functions $R$ and $S$. The function $R$ is the amplitude of the wave function and $S / \hbar$ is its phase. After series of computations, aim of which is to put together real and imaginary terms, we obtain the following equations

$$
\begin{gathered}
\frac{\partial S}{\partial t}+\beta \frac{1}{R} \frac{\partial R}{\partial t}=-\underbrace{\frac{\left(\beta^{2}+\hbar^{2}\right)}{2 m_{N} \hbar^{2}}(\nabla S)^{2}}_{(\mathrm{a})}+\underbrace{\frac{\left(\beta^{2}+\hbar^{2}\right)}{2 m_{N}} \frac{\nabla^{2} R}{R}}_{(\mathrm{b})}+\frac{\left(\beta^{2}-\hbar^{2}\right)}{\left(\beta^{2}+\hbar^{2}\right)} V, \\
\frac{\beta}{\hbar^{2}} \frac{\partial S}{\partial t}-\frac{1}{R} \frac{\partial R}{\partial t}=\frac{\left(\beta^{2}+\hbar^{2}\right)}{2 m_{N} \hbar^{2}} \underbrace{\left(\nabla^{2} S+2 \frac{1}{R} \nabla R \nabla S\right)}_{(\mathrm{c})}-\frac{2 \beta}{\left(\beta^{2}+\hbar^{2}\right)} V .
\end{gathered}
$$

Firstly, we can see that at $\beta=0$ Eq. (42) reduces to the modified Hamilton-Jacobi equation due to loaded the quantum potential that is enveloped here by brace (b). And Eq. (43) reduces to the continuity equation. These equations read

$$
\begin{gathered}
\frac{\partial S}{\partial t}=-\underbrace{\frac{1}{2 m_{N}}(\nabla S)^{2}}_{(\mathrm{a})}+\underbrace{\frac{\hbar^{2}}{2 m_{N}} \frac{\nabla^{2} R}{R}}_{(\mathrm{b})}+V, \\
-\frac{1}{R} \frac{\partial R}{\partial t}=\frac{1}{2 m_{N}} \underbrace{\left(\nabla^{2} S+2 \frac{1}{R} \nabla R \nabla S\right.}_{\text {(c) }}) .
\end{gathered}
$$

Terms enveloped by braces (a), (b), and (c) are the kinetic energy of the particle, the quantum potential, and the right part is a kernel of the continuity equation (45), respectively. In particular, the term $2 \nabla R / R=\nabla \ln \left(R^{2}\right)$ relates to the osmotic velocity, see Eq. (23). Eqs. (44) and (45) are the same equations obtained by Bohm [35]. From historical viewpoint it should be noted that the same equations were published by Madelung ${ }^{1}$ in 1926 [36].

Momentum of the particle reads

$$
\vec{p}=m_{N} \vec{v}=\nabla S
$$

where $\vec{v}$ is its current velocity. The de Broglie equation relates the momentum $p$ to the wavelength $\lambda_{\mathrm{dB}}=h / p$, where $h=2 \pi \hbar$ is the Planck constant. Now, as soon as we found the current velocity 


$$
\vec{v}=\frac{1}{m_{N}} \nabla S=\frac{\hbar}{m_{N}} \operatorname{Im}\left(|\psi\rangle^{-1} \nabla|\psi\rangle\right)
$$

positions of the particle in each current time beginning from the grating' slits up to a detector is calculated by the following formula

$$
\vec{r}(t+\delta t)=\vec{r}(t)+\vec{v}(t) \delta t
$$

Here $t$ is a current time that starts from $t=0$ on a slit source and $\delta t$ is an arbitrarily small increment of time. Some calculated trajectories of particles, the Bohmian trajectories, are shown in upper part of Fig. 5. It should be noted that the Bohmian trajectories follow from exact solutions of Eqs. (44)-(45). These equations give a rule for finding geodesic trajectories and secants of equal phases, $S / \hbar$, at given boundary conditions. The geodesic trajectories point to tendency of the particle migration along paths. And the secant surfaces describe a coherence of all the passing particles created on a single source.

In case of $\beta \gg \hbar$ we have the following two equations

$$
\begin{gathered}
\frac{\partial R}{\partial t}=-\underbrace{\frac{\beta}{2 m_{N} \hbar^{2}} R(\nabla S)^{2}}_{(\mathrm{a})}+\underbrace{\frac{\beta}{2 m_{N}} \nabla^{2} R}_{(\mathrm{b})}, \\
\frac{\partial S}{\partial t}=\frac{\beta}{2 m_{N}} \underbrace{\left(\nabla^{2} S+2 \frac{1}{R} \nabla R \nabla S\right)},
\end{gathered}
$$

Here we take into consideration that in the first equation we may replace $(\nabla S / \hbar)^{2}=4 \pi^{2} / \lambda_{\mathrm{dB}}^{2}$. In the second equation we may replace the term $\nabla S$ by $m_{N} \vec{v}$ as follows from Eq. (46). We notice also, that $2 R^{-1} \nabla R=2 \nabla \ln (R)=\nabla \ln (\rho)$. And $\beta / 2 m_{N}=D$ is the diffusion coefficient. Now we may rewrite Eqs. (49)-(50) as follows

$$
\begin{gathered}
\frac{\partial R}{\partial t}=\underbrace{D \nabla^{2} R}_{(\mathrm{b})}-\underbrace{\frac{4 \pi^{2}}{\lambda_{\mathrm{dB}}^{2}} D R}_{(\mathrm{a})}, \\
\frac{\partial S}{\partial t}=D \underbrace{\left.D \nabla^{2} S+m_{N}(\nabla \ln (\rho), \vec{v})\right)}_{(\mathrm{c})}=D \nabla^{2} S+m_{N}(\vec{u}, \vec{v}) .
\end{gathered}
$$


Here $\vec{u}=D \nabla \ln (\rho)$ is the osmotic velocity defined in Eq. (23). We got the two diffusion equations coupled with each other through sources. Namely, this coupling is provided due to the de Broglie wavelength and the osmotic velocity which can change with time. These diffusion equations cardinally differ from Eqs. (44)-(45). Because of diffusive nature of these supplementary parts blurring of interference patterns occurs. It leads to degeneration of the Bohmian trajectories to Brownian ones.

\subsection{Dispersion of trajectories and the uncertainty principle}

As for the Bohmian trajectories there is a problem concerning their possible existence. As follows from Eqs. (46) and (48) in each moment of time there are definite values of the momentum and the coordinate of a particle moving along the Bohmian trajectory. This statement enters in conflict with the uncertainty principle.

Here we try to retrace emergence of the uncertainty principle stemming from standard probability-theoretical computations of expectation value and variance of a particle momentum. We adopt a wave function in the polar representation

$$
|\Psi\rangle=\operatorname{Rexp}\{\mathbf{i} S / \hbar\}
$$

where $R=(\rho)^{1 / 2}$ is the amplitude of the wave function $\left(\rho=R^{2}=\langle\Psi \mid \Psi\rangle\right.$ is the probability density) and $S / \hbar$ is its phase. Momentum operator $\hat{p}=-i \hbar \nabla$ and corresponding velocity operator $v=-\boldsymbol{i}(\hbar / m) \nabla$ are kinetic operators in quantum mechanics. Here $m$ is mass of the particle. Expectation value of the velocity operator reads

$$
\vec{V}_{g}=\frac{1}{\langle\Psi \mid \Psi\rangle}\left\langle\Psi\left|-\mathbf{i} \frac{\hbar}{m} \nabla\right| \Psi\right\rangle=\frac{1}{m}\left(\nabla S+\mathbf{i} \hbar \nabla S_{Q}\right) .
$$

The velocity $\mathrm{V}_{\mathrm{g}}$ is seen to be complex-valued. Here $S_{Q}=-\ln (R)=-\ln (\rho) / 2$ is the quantum entropy [28] and $(\hbar / 2 m)$ is the quantum diffusion coefficient $[6,7]$. Therefore its imaginary part is a quantum osmotic velocity

$$
\vec{u}_{Q}=-(\hbar / m) \nabla S_{Q}=(\hbar / 2 m) \nabla \ln (\rho) .
$$

It is instructive to compare this velocity with the classical osmotic velocity given in Eq. (23). As can see the osmotic velocity stems from gradient of entropy that evaluates degree of order and disorder on a quantum level, likely of vacuum fluctuations.

Real part of Eq. (54) gives the current velocity $\vec{v}$ defined by Eq. (47). It should be noted that because of existence of imaginary unit in definition of the momentum operator, real part of Eq. (54) is taken as the current velocity. Whereas imaginary unit is absent in Eq. (47). Therefore at computing the current velocity by Eq. (47) we take imaginary part. 
Let us now calculate variance of the velocity $\mathrm{V}_{\mathrm{g}}$. This computation reads

$$
\operatorname{Var}\left(V_{g}\right)=\frac{1}{\langle\Psi \mid \Psi\rangle}\left\langle\Psi\left|\left(-\mathbf{i} \frac{\hbar}{m} \nabla-\vec{V}_{g}\right)^{2}\right| \Psi\right\rangle=\frac{1}{\langle\Psi \mid \Psi\rangle}\left\langle\Psi|\mathbf{i} \frac{\hbar}{m} \nabla \vec{V}_{g}+\underbrace{\frac{\hbar}{m} \vec{V}_{g} \nabla+V_{g}^{2}}_{(\mathrm{d})}-\frac{\hbar^{2}}{m^{2}} \Delta| \Psi\right\rangle
$$

Terms over bracket (d) kill each other as follows from Eq. (54). It is reasonable in the perspective to multiply $\operatorname{Var}\left(\mathrm{V}_{\mathrm{g}}\right)$ by $m / 2$

$$
\frac{m}{2} \operatorname{Var}\left(V_{g}\right)=-\frac{1}{\langle\Psi \mid \Psi\rangle}\left\langle\Psi\left|\frac{\hbar^{2}}{2 m} \Delta\right| \Psi\right\rangle+\mathbf{i} \frac{\hbar}{2} \nabla \vec{V}_{g}
$$

So, this expression has a dimensionality of energy. The first term to be computed represents the following result

$$
-\frac{1}{\langle\Psi \mid \Psi\rangle}\left\langle\Psi\left|\frac{\hbar^{2}}{2 m} \Delta\right| \Psi\right\rangle=\underbrace{\frac{1}{2 m}(\nabla S)^{2}}_{\text {(a) }}-\underbrace{\frac{\hbar^{2}}{2 m} \frac{\nabla^{2} R}{R}}_{(\mathrm{b})}-\mathbf{i} \frac{\hbar}{2 m} \underbrace{\left(\Delta S+2 \frac{(\nabla S, \nabla R)}{R}\right)}_{\text {(c) }} .
$$

Here the term enveloped by bracket (a) is a kinetic energy of the particle, the term enveloped by bracket (b) with negative sign added is the quantum potential $Q$, and the term enveloped by bracket (c) comes from the continuity equation. See for comparison Eqs. (44) and (45). We rewrite the quantum potential as follows

$$
Q=-\frac{\hbar^{2}}{2 m} \frac{\nabla^{2} R}{R}=-\frac{\hbar^{2}}{2 m}\left(\nabla S_{Q}\right)^{2}+\frac{\hbar^{2}}{2 m} \nabla^{2} S_{Q}
$$

As for the second term in Eq. (57) we have $i(\hbar / 2) \nabla \vec{V}_{g}=i(\hbar / 2 m) \Delta S-\left(\hbar^{2} / 2 m\right) \Delta S_{Q}$. It is follows from computation by Eq. (54). As a result, the expression (57) takes the following view

$$
\frac{m}{2} \operatorname{Var}\left(V_{g}\right)=\frac{1}{2 m}(\nabla S)^{2}-\frac{\hbar^{2}}{2 m}\left(\nabla S_{Q}\right)^{2}-\mathbf{i} \frac{\hbar}{m} \frac{(\nabla S, \nabla R)}{R}
$$

One can see that the variance consists of real and imaginary parts. Observe that the right side is represented through square of gradient of the complexified action [28], namely $\left(\nabla\left(S+i \hbar S_{Q}\right)\right)^{2} / 2 m$. We shall not consider here the imaginary part. Instead we shall consider the real part of this expression. It reads 


$$
\frac{m}{2} \operatorname{Re}\left(\operatorname{Var}\left(V_{g}\right)\right)=\frac{1}{2 m}(\nabla S)^{2}-\hbar\left(\frac{\hbar}{2 m}\left(\nabla S_{Q}\right)^{2}\right)
$$

The first term in this expression represents kinetic energy, $E$, of the particle. The second term, stemming from the quantum potential, contains under braces a term having dimensionality of inverse time, that is, of frequency

$$
\omega_{Q}=\frac{\hbar}{2 m}\left(\nabla S_{Q}\right)^{2}
$$

This frequency multiplied by $\hbar$ represents an energy binding a particle with vacuum fluctuations. This energy, as follows from Eq. (61), is equal to the particle mass multiplied by squared the osmotic velocity (55) and divided by 2 . It is an osmotic kinetic energy. In the light of the above said we rewrite Eq. (61) in the following way

$$
\frac{m}{2} \operatorname{Re}\left(\operatorname{Var}\left(V_{g}\right)\right)=E-\hbar \omega_{Q} \geq 0 .
$$

Let we have two Bohmian trajectories. Along one trajectory we have $E_{1}-\hbar \omega_{\mathrm{Q}, 1}$, and along other trajectory we have a perturbed value $E_{2}-\hbar \omega_{\mathrm{Q}, 2}$. Subtracting one from other we have

$$
\delta E-\hbar \delta \omega_{Q} \geq 0
$$

One can suppose that emergence of the second trajectory was conditioned by a perturbation of the particle moving along the first trajectory. If it is so, then emergence of the second trajectory stems from an operation of measurement of some parameters of the particle. One can think that duration of the measurement is about $\delta t=1 / \delta \omega_{\mathrm{Q}}$. From here we find

$$
\delta E \delta t \geq \hbar
$$

Now let us return to Eq. (48) and rewrite it in the following view

$$
\delta \vec{r}(t)=\vec{v}_{1}(t) \delta t \geq \vec{v}_{1}(t) \hbar / \delta E .
$$

The initial Bohmian trajectory is marked here by subscript 1 . Observe that $\delta E=m\left(v_{2}^{2}-v_{1}^{2}\right) / 2 \approx m \vec{v}_{1} \delta \vec{v}$. Here we have calculated $v_{2}^{2}=\left(\vec{v}_{1}+\delta \vec{v}\right)^{2} \approx v_{1}^{2}+2 \vec{v}{ }_{1} \delta \vec{v}$. Substituting computations of $\delta E$ into Eq. (66) we obtain finally 


$$
\delta \vec{p} \delta \vec{r} \geq \hbar
$$

Here we take into account $\delta \vec{p}=m \delta \vec{v}$.

\section{Concluding remarks}

Each nanoparticle incident on a slit grating passes only through a single slit. Its path runs along a Bohmian trajectory which is represented as an optimal path for the nanoparticle migrating from a source to a detector. Unfortunately, the Bohmian trajectory can not be observable since a serious obstacle for the observation comes from the uncertainty principle. In other words, an attempt to measure any attribute of the nanoparticle, be it position or orientation, i.e., the particle momentum, leads to destroying information relating to future history of the nanoparticle. What is more, any collision of the nanoparticle with a foreign particle destroys the Bohmian trajectory which could give a real contribution to the interference pattern. It relates closely with quality of vacuum. In the case of a bad vacuum such collisions will occur frequently. They lead to destruction of the Bohmian trajectories. Actually, they degenerate to Brownian trajectories.

Excellent article [21] of Couder \& Fort with droplets gives, however, a clear hint of what happens when the nanoparticle passes through a single grating slit. In the light of this hint we may admit that the particle "bouncing at moving through vacuum" generates a wave at each bounce. So, a holistic quantum mechanical object is the particle + wave. Here the wave to be generated by the particle plays a role of the pilot-wave first formulated by Lui de Broglie and later developed by Bohm [37]. It is interesting to note in this context, that the pilotwaves have many common with Huygens waves [5].

A particle passing through vacuum generates waves with wavelength that is inversely proportional to its momentum (it follows from the de Broglie formula, $\lambda=h / p$, where $h$ is Planck's constant). One can guess that a role of the vacuum in the experiment of Couder \& Fort takes upon itself a silicon oil surface with subcritical Faraday ripples activated on it [21]. Observe that pattern of the ripples is changed in the vicinity of extraneous bodies immersed in the oil which simulate grating slits. Interference of the ripples with waves generated by the bouncing droplets provides optimal paths for the droplets traveling through the slits and further. As a result we may observe an interference pattern emergent depending on amount of slits in the grating and distance between them.

Now we may suppose that the subcritical Faraday ripples on the silicon oil surface simulate vacuum fluctuations. Consequently, the vacuum fluctuations change their own pattern near the slit grating depending on amount of slits and distance between them. We may imagine that the particle passing through vacuum (bouncing through, Fig. 11) initiates waves which interfere with the vacuum fluctuations. As a result of such an interference the particle moves along an optimal path - along the Bohmian trajectory. Mathematically the bounce is imitated by an exponential term $\exp \{\mathrm{i} S / \hbar\}$, where the angle $S / \hbar$ parametrizes the group of rotation 
given on a circle of unit radius. So, the path along which the particle moves is scaled by this unitary group, $\mathrm{U}(1)$, due to the exponential mapping of the phase $S / \hbar$ on the circle.

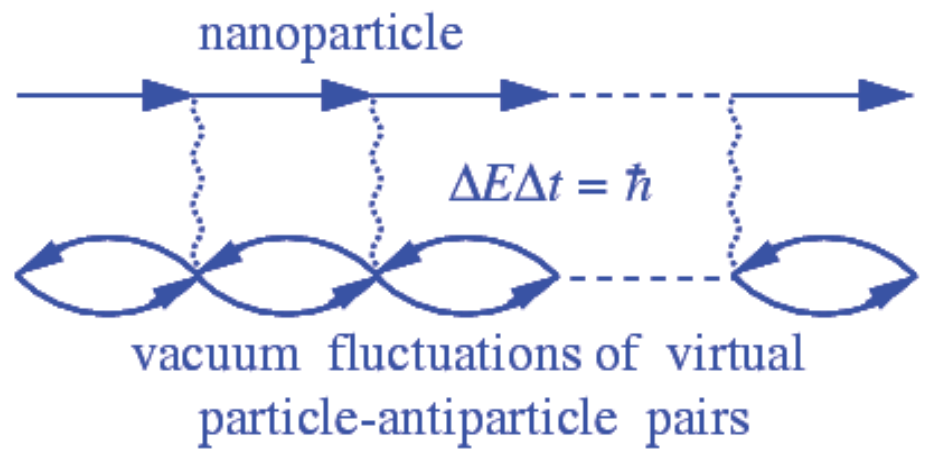

Figure 11. Bouncing a nanoparticle at moving through vacuum. Vertical dotted sinusoidal curves depict exchange by energy $\Delta E$ with vacuum virtual particle-antiparticle pairs over period of about $\Delta t=\hbar / \Delta E$.

In conclusion it would be like to remember remarkable reflection of Paul Dirac. In 1933 Paul Dirac drew attention to a special role of the action $S$ in quantum mechanics [38] - it can exhibit itself in expressions through $\exp \{\mathrm{iS} / \hbar\}$. In 1945 he emphasized once again, that the classical and quantum mechanics have many general points of crossing [39]. In particular, he had written in this article: "We can use the formal probability to set up a quantum picture rather close to the classical picture in which the coordinates $\mathrm{q}$ of a dynamical system have definite values at any time. We take a number of times $t_{1}, t_{2}, t_{3}, \ldots$ following closely one after another and set up the formal probability for the q's at each of these times lying within specified small ranges, this being permissible since the q's at any time all commutate. We then get a formal probability for the trajectory of the system in quantum mechanics lying within certain limits. This enables us to speak of some trajectories being improbable and others being likely."

\section{Acknowledgement}

Author thanks Miss Pipa (administrator of Quantum Portal) for preparing programs that permitted to calculate and prepare Figures 5 to 8. Author thanks also O. A. Bykovsky for taking my attention to a single-particle interference observed for macroscopic objects by Couder and Fort and V. Lozovskiy for some remarks relating to the article.

\section{Author details}

Valeriy I. Sbitnev 
St.-Petersburg Nuclear Physics Institute, NRC Kurchatov Institute, Gatchina, Russia

\section{References}

[1] Dauben JW. Abraham Robinson: The creation of nonstandard analysis. A personal and mathematical odyssey. Princeton, NJ: Princeton University Press; 1995.

[2] Stanford Encyclopeia of Philosophy: Newton's Views on Space, Time, and Motion. http://plato.stanford.edu/entries/newton-stm/ (accessed 12 August 2004).

[3] Henson CW. Foundations of nonstandard analysis. In: Arkeryd L.O., Cutland N. J., \& Henson C. W., (eds.) Nonstandard Analysis, Theory and application. The Netherlands: Kluwer Acad. Publ.; 1997,p. 1-51.

[4] Nelson E. Radically Elementary Probability Theory. Princeton, New Jersey: Princeton Univ. Press; 1987.

[5] Huygens C. Treatise on Light. Gutenberg eBook, No. 14725. http://www.gutenberg.org/files/14725/14725-h/14725-h.h (accessed 18 January 2005)

[6] Nelson E. Dynamical theories of Brownian motion. Princeton, New Jersey: Princeton Univ. Press; 2001.

[7] Nelson E. Quantum fluctuations. Princeton Series in Physics. Princeon, New Jersey: Princeton Univ. Press; 2001.

[8] Feynman RP., Hibbs A. Quantum mechanics and path integrals. N. Y.: McGraw Hill; 1965.

[9] Einstein A., von Smoluchowski M. Brownian motion. Moscow-Leningrad: ONTI (in Russian); 1936.

[10] Sidorov LN., Troyanov SI. At the dawn of a new chemistry of fullerenes. PRIRODA 2011; 1153(9) 22-30.

[11] Arndt M., Hackermüller L., Reiger E. Interferometry with large molecules: Exploration of coherence, decoherence and novel beam methods. Brazilian Journal of Physics 2005; 35(2A) 216-223.

[12] Brezger B., Arndt M., Zeilinger A. Concepts for near-field interferometers with large molecules. J. Opt. B: Quantum Semiclass. Opt. 2003; 5(2) S82-S89.

[13] Brezger B., Hackermüller L., Uttenthaler S., Petschinka J., Arndt M., Zeilinger A. Matter-Wave Interferometer for Large Molecules. Phys. Rev. Lett. 2002; 88100404.

[14] Gerlich S., Eibenberger S., Tomandl M., Nimmrichter S., Hornberger K., Fagan P. J., Tuxen J., Mayor M., Arndt, M. Quantum interference of large organic molecules. Nature Communications 2011; (2) 263. http://www.nature.com/ncomms/journal/v2/n4/ full/ncomms1263.html (accessed 5 April 2011) 
[15] Hornberger K., Hackermüller L., Arndt M. Inuence of molecular temperature on the coherence of fullerenes in a near-field interferometer. Phys. Rev. A. 2005; 71, 023601.

[16] Hornberger K., Sipe JP., Arndt M. Theory of decoherence in a matter wave TalbotLau interferometer. Phys. Rev. A. 2004; 70, 053608.

[17] Nimmrichter S., Hornberger K. Theory of near-fieldmatter wave interference beyond the eikonal approximation. Phys. Rev. A. 2008; 78, 023612.

[18] Juffmann,T., Truppe S., Geyer P., Major AG., Deachapunya S., Ulbricht H., Arndt, M. Wave and particle in molecular interference lithography. Phys. Rev. Lett. 2009; 103, 263601.

[19] Berry M., Klein S. Integer, fractional and fractal Talbot effects, Journal of Modern Optics, 1996; 43(10) 2139-2164.

[20] Berry M., Marzoli L., Schleich W. Quantum carpets, carpets of light. Physics World, 2001; (6), 39-44.

[21] Couder Y., Fort E. Single-Particle Diffraction and Interference at a Macroscopic Scale. Phys. Rev. Lett. 2006; 97 (6), 154101(4).

[22] Stratonovich RL. Topics in the theory of random noise. N.Y.: Gordon and Breach; 1963.

[23] Gnedenko BV. Theory of Probability. N.Y.: Gordon \& Breach; 1997.

[24] Ventzel AD. The course of stochastic processes theory. Moskow: Nauka (in Russian); 1975.

[25] Grosche C. An introduction into the Feynman path integral. http://arXiv.org/abs/hepth/9302097 (accessed 20 February 1993).

[26] MacKenzie R. Path integral methods and applications. http://arXiv.org/abs/quant-ph/ 0004090 (accessed 20 April 2000).

[27] Juffmann T., Milic A., Müllneritsch M., Asenbaum P., Tsukernik A., Tüxen J., Mayor M., Cheshnovsky O., Arndt, M. Real-time single-molecule imaging of quantum interference. Nature Nanotechnology - Letter. 2012; 7, 297-300. http://www.nature.com/ nnano/journal/v7/n5/full/nnano.2012.34.html?WT.ec_id=NNANO-201205 (accessed 25 March 2012)

[28] Sbitnev VI. Bohmian trajectories and the path integral paradigm - complexified Lagrangian mechanics. In: Pahlavani MR. (ed.) Theoretical Concepts of Quantum Mechanics. Rijeka: InTech; 2011 p313-340. http://www.intechopen.com/books/ theoretical-concepts-of-quantum-mechanics/the-path-integral-paradigm-and-bohmian-trajectories-from-the-lagrangian-mechanics-to-complexified-on (accessed 2004-2012)

[29] Nairz O., Arndt M., Zeilinger A. Quantum interference experiments with large molecules. Am. J. Phys. 2003; 71 (4), 319-325. 
[30] Sbitnev VI. Matter waves in the Talbot-Lau interferometry, http://arxiv.org/abs/ 1005.0890 (accessed 17 September 2010).

[31] Volokitin AI. Quantum drag and graphene. PRIRODA, 2011; 1153(9), 13-21.

[32] Mandel L., Wolf E. Optical coherence and quantum optics. Cambridge: Cambridge University Press; 1995.

[33] McMorran B.,Cronin AD. Model for partial coherence and wavefront curvature in grating interferometers. Phys. Rev. A. 2008; 78 (1), 013601(10).

[34] Gerlich S., Hackermüller L., Hornberger K., Stibor A., Ulbricht H., Gring M., Goldfarb F., Savas T., Müri M., Mayor M., Arndt, M. A Kapitza-Dirac-Talbot-Lau interferometer for highly polarizable molecules. Nature Physics. 2007; 3, 711-715.

[35] Bohm D. A suggested interpretation of the quantum theory in terms of "hidden variables", I \& II. Physical Review, 1952; 85, 166-179 \& 180-193.

[36] Madelung E. Quantentheorie in Hydrodynamischer form. Zts. f. Phys. 1926; 40, 322-326.

[37] Stanford Encyclopeia of Philosophy: Bohmian Mechanics. http://plato.stanford.edu/ entries/qm-bohm/ (accessed 26 October 2001).

[38] Dirac PAM. The Lagrangian in Quantum Mechanics. Physikalische Zeitschrift der Sowjetunion. 1933; 3, 64-72.

[39] Dirac PAM. On the analogy between classical and quantum mechanics. Rev. Mod. Phys. 1945; 17 (2 \& 3), 195-199. 



\section{Quantum Intentionality and Determination of Realities in the Space-Time Through Path Integrals and Their Integral Transforms}

Francisco Bulnes

Additional information is available at the end of the chapter

http://dx.doi.org/10.5772/53439

\section{Introduction}

In the universe three fundamental realities exist inside our perception, which share messages and quantum processes: the physical, energy and mental reality. These realities happen at all times and they are around us like part of our existence spending one to other one across organised transformations which realise a linking field - energy-matter across the concept of conscience of a field on the interpretation of the matter and space to create a reality non-temporal that only depends on the nature of the field, for example, the gravitational field is a reality in the space - time that generates a curved space for the presence of masses. At macroscopic level and according to the Einsteinian models the time is a flexible band that acts in form parallel to the space. Nevertheless, studying the field at microscopic level dominated by particles that produce gravity, the time is an intrinsic part of the space (there is no distinction between one and other), since the particles contain a rotation concept (called spin) that is intrinsic to the same particles that produce gravity from quantum level [1].Then the gravitational field between such particles is an always present reality and therefore non-temporal. The time at quantum level is the distance between cause and effect, but the effect (gravitational spin) is contained in the proper particle that is their cause on having been interrelated with other particles and vice versa the effect contains the cause since the particle changed their direction [1].

Then the action of any field that is wished transforms their surrounding reality which must spill through the component particles of the space - time, their nature and to transmit it in organised form, which is legal, because the field is invariant under movements of the proper space, and in every particle there sublies a part of the field through their spinor. 
Three fundamental realities perceived by our anthropometric development of the universe; field - energy- matter between three different but indistinguishable realities are realised at macroscopic level: one is the material reality which is determined by their atomic linkage between material particles (atoms constituted by protons, neutrons and electrons), an energy reality, called also quantum reality, since the information in this reality area exchanges the matter happen through sub-particles (bosons, fermions, gluons, etc) and finally a virtual reality that sublies like fundamental field and that is an origin of the changes of spin of the subparticles and their support doing that they transform these into others and that they transform everything around him (Higgs field). The integration of these three realities will be called by us a hyper-reality by us. The hyper-reality contains to the quantum reality and to the reality perceived by our senses (material reality).

Consider $\mathbb{R}^{\mathrm{d}} \times \mathrm{I}_{t}$, like the space - time where happens the transitions of energy states into space - time, and let $u, v$, elements of this space, the integral of all the continuous possible paths to particle $x(s)$, that transit from energy state in $u$, to an energy state in $v$, in $\mathbb{R}^{\mathrm{d}} \times \mathrm{I}_{t}$, is

$$
\mathrm{I}(L, x(t))=\int_{\mathcal{C}^{\mu, j}[0, t]} \exp \left\{\frac{i}{h} \mathfrak{J}(x)\right\} d x,
$$

where $h$, is the constant of Max Planck, and the action $\mathfrak{I}$, is the one realised by their Lagrangian $L$.

Since we have mentioned, the action of a field is realised being a cause and effect, for which it must be a cause and effect in each of the component particles, "waking up" the particle conscience to particle being transmitted this way without any exception. This action must infiltrate to the field itself that it sublies in the space and that it is shaped by the proper particles that compose it creating a certain co-action that is major than their algebraic sum [2].The configuration space $C_{n, m}=\left\{\gamma_{t} \in \Omega(\Gamma) \mid \gamma t \rightarrow \Gamma \rightarrow \Gamma / \gamma\right\}$ [3], is the model created by the due action to each corresponding trajectory to the different splits it. Is clear here we must have in mind all the paths in the space-time $M$, that contribute to interference amplitude in this space, remaining the path of major statistical weight. The intention takes implicitly a space $C_{n}, m$. Any transformation that wants to realise of a space, has as constant the same energy that comes from the permanent field of the matter and which is determined by the quantum field of the particles $x(s)$, constituents of the space and matter. If we want to define a conscience in the above mentioned field, that is to say, an action that involves an intention is necessary to establish it inside the argument of the action. Likewise, if $x(s) \in \Omega$, and $\Im(x(s))$, there is their action due to a field of particles $X$, and there is spilled an intention defined by (1) the length and breadth of the space $M$, such that satisfies the property of synergy [2], for all the possible trajectories that they fill $\Omega$, we have that

$$
\mathfrak{I}_{\text {TOTAL }} \geq \int_{\mathrm{E}^{-}}^{\mathrm{E}^{+}}\left\{\sum_{j} \int_{\gamma_{t}} \mathfrak{J}_{j}(x(s)) d(x(s))\right\} d \mu,
$$


where the entire action (2) is an intentional action (for the whole infinity of paths $\gamma_{t}$, that defines $\Gamma$, and that are trajectories of the space - time $\left.\Omega \subset \mathbb{R}^{3} \times \mathrm{I}_{t}\right)$, if and only if $\mathrm{O}_{\mathrm{c}}(x, \dot{x}(s))=L(\phi(x)$, $\partial \phi(x)) d \phi$, where then

$$
\mathfrak{I}_{\text {TOTAL }}=\int_{\Gamma}\left\{\mathfrak{I}\left[\int_{\Omega(\Gamma)} O_{\mathrm{c}}(\phi(x)) d(\phi(x))\right] \mathrm{\mu}_{x}=\left(E^{+}-E^{-}\right) \int_{-\infty}^{+\infty}\left\{\int_{\Omega(\Gamma)} \mathfrak{I}(x(s)) \mathrm{d} x(s)\right\} \mu_{s^{\prime}}\right.
$$

where the energy factor $E^{+}-E^{-}$, represents the energy needed by the always present force to realise the action and $O_{c}$, is the conscience operator which defines the value or record of the field $X$ (direction), on every particle of the space $\Omega(\Gamma)$, which along their set of trajectories $\Gamma$, realizes the action of permanent field $\Im_{O c}$, it being fulfilled that

$$
\mathfrak{I}_{O_{\mathrm{c}}}(\phi(x))=\int_{X(\mathrm{M})} O_{\mathrm{c}}(\phi(x)) d \phi(x)
$$

where the operator $\mathfrak{J} \circ$, invests an energy quasi-infinite, encapsulated in a microscopic region of the space (quantum space $\mathcal{M}$ ), and with applications and influence in an unlimited space of the sub-particles (boson space). Likewise a photon of certain class $\phi(x)$, will be generated by the quantum field (if it manages to change its field spin) and will be moved for the intention on a trajectory $\Gamma$, by the path integral

$$
\mathrm{I}\left(\mathfrak{J}_{O_{\mathrm{c}}}(\phi(x))\right)=\int_{\Gamma}\left[\int_{X(\Omega(\Gamma))} O_{\mathrm{c}}(\phi(x)) d(\phi(x))\right] \mathrm{\mu}_{\Gamma^{\prime}}
$$

Interesting applications of the formula (3) to nano-sciences will happen at the end of the present chapter. Also it will be demonstrated that (3) is a quantum integral transform of bundles or distortions of energy in the space - time if it involves a special kernel. The bundle stops existing if there is applied certain intention (path integral transform). The operator $\mathrm{Oc}_{\mathrm{c}}$ involves a connection of the tangent bundle of the space of trajectories $\Omega(\Gamma)$.

The operator $O_{c}$, include a connection of the tangent bundle of the space of trajectories $\Omega(\Gamma)$. The integral (5) will determine on certain hypotheses the interdependence between the material, quantum and virtual realities in $M$.

Def. 2. 1 (intentional action of the $X)$. Let $X$, be a field acting on the particles $x_{1}(s), x_{2}(s), \ldots, \in \mathbb{M}$, and let $\mathfrak{I}$, be their action on the above mentioned particles under an operator who recognizes the "target" in $\mathbb{M}$, (conscience operator). We say that $\mathfrak{I}$, is an conscientious intentional action (or simply intention) of the field $X$, if and only if:

a. $\quad \mathfrak{I}$, is the determination of the field $X$, to realise or execute, (their force $\mathrm{F}(x)$ ),

b. $\quad \mathfrak{I}$, recognizes well their target, it is known what the field $X$ wants to do (their direction $\Leftrightarrow$ she follows a configuration patron) 
Consider a particle system $p_{1}, p_{2}, \ldots$ in a space - time $\mathbb{M} \cong \mathbb{R}^{4}$. Let $x(t) \in \Omega(\Gamma) \subset \mathbb{R}^{3} \times I_{t}$, be a trajectory which predetermines a position $x \in \mathbb{R}^{3}$, for all time $t \in \mathrm{I}$. A field $X$, that infiltrates its action to the whole space of points predetermined by all the trajectories $x_{1}(t), x_{2}(t), x_{3}(t)$, $\ldots, \in \Omega(\Gamma)$, is the field that predetermines the points $\phi_{i}\left(x_{i}(t)\right)$, which are fields whose determination is given by the action of the field $X$, and evaluated in the position of every particle. Every point have a defined force by the action $\mathfrak{I}$, of $X$, along the geodesic $\gamma$ t, and determined direction by their tangent bundle given for $T *^{1}(\Omega(\Gamma))$, that is the cotangent space $\mathrm{T}^{*}(\Omega(\Gamma))[4]$, which give the images of the states under Lagrangian, that is to say, the field provides of direction to every point $\phi_{i}$, because their tangent bundle has a subjacent spinor bundle $s$ [5], where the field $X$, comes given as $X=\left.\sum_{i} \phi^{i} \frac{\partial}{\partial \phi^{i}}\right|_{\left(x^{i}, \phi^{i}\right)}, \forall \phi_{1}, \phi_{2}, \phi_{3}, \ldots \in *^{1}$, on every particle $p_{\mathrm{i}}=x_{\mathrm{i}}(t)(\mathrm{i}=1,2, \ldots)$. Then to direct an intention is the map or connection:

$$
O_{c}: T \Omega(\Gamma) \rightarrow T *^{1}(\Omega(\Gamma))\left(\cong T^{*}(\Omega(\Gamma)),\right.
$$

with rule of correspondence

$$
\left(x^{i}, \partial_{t} x^{i}\right) I \rightarrow\left(\phi^{i}, \partial_{m} \phi^{i}\right)
$$

which produces one to us ith- state of field energy $\phi \dot{b}[6]$, where the action $\mathfrak{I}$, of the field $X$, infiltrates and transmits from particle to particle in the whole space $\Omega(\Gamma)$, using a configuration given by their Lagrangian $L$ (conscience operator), along all the trajectories of $\Omega(\Gamma)$. Then of a sum of trajectories $\int D_{\mathrm{F}}(x(t))$, one has the sum $\int d(\phi(x))$, on all the possible field configurations $C_{n, m}$. Extending these intentions to whole space $\Omega(\Gamma) \subset \mathbb{M}$, on all the elections of possible paths whose statistical weight corresponds to the determined one by the intention of the field, and realising the integration in paths for an infinity of particles - fields in $\mathrm{T} \Omega(\Gamma)$, it is had that

$$
\mathrm{I}\left(\phi^{i}(x)\right)=\int_{T \Omega(\Gamma)} \omega(\phi(x))=\lim _{\substack{\mathrm{N} \rightarrow \infty \\ \delta s \rightarrow 0}} \frac{1}{B} \int_{-\infty}^{+\infty} \frac{d \phi^{1}}{B} \cdots \int_{-\infty}^{+\infty} \frac{d \phi^{\mathrm{n}}}{B} \cdots=\prod_{i=1}^{\infty} \int_{-\infty}^{+\infty} \mathrm{e}^{\mathrm{i} \Im\left[\phi^{i}, \partial_{\mu} \phi^{i}\right]} d \phi^{i}(x(s)),
$$

where $B=\left[\frac{m}{2 \pi \hbar i \delta s}\right]^{1 / 2}$, is the amplitude of their propagator and in the second integral of (8), we have expressed the Feynman integral using the form of volume $\omega(\phi(x))$, of the space of all the paths that add in $\mathrm{T} \Omega(\Gamma)$, to obtain the real path of the particle (where we have chosen quantized trajectories, that is to say, $\left.\int d(\phi(x))\right)$. Remember that the sum of all these paths is the interference amplitude between paths that is established under an action whose Lagrangian is $\omega(\phi(x))=\mathfrak{I}_{\xi}(x) \mathrm{d} \phi(x)$, where, if $\Omega(\mathbb{M})$, is a complex with $\mathbb{M}$, the space-time, and $C(\mathbb{M})$, is a complex or configuration space on $\mathbb{M}$, (interfered paths in the experiment given by multiple split [7]), endowed with a pairing

$$
\int: C(\mathbb{M}) \times \Omega^{*}(\mathbb{M}) \rightarrow \mathbb{R}
$$


where $\Omega^{*}(M)$, is some dual complex ("forms on configuration spaces"), i.e. such that "Stokes theorem" holds:

$$
\int_{\Omega \times C} \omega=\langle\mathfrak{I}, \mathrm{d} \omega\rangle
$$

then the integrals given by (8) can be written (to $m$-border points and $n$-inner points (see figure 1a))) as:

$$
\begin{aligned}
& \int_{\mathrm{T} \Omega(\Gamma)} \Im(\phi(x)) d \phi=\int_{\Omega\left(\Gamma_{t^{1}}\right) \times \ldots \times \Omega\left(\Gamma_{t^{\mathrm{m}}}\right) \times \ldots} \mathfrak{J}_{\mathrm{q}} \mathrm{d} \phi_{1}^{m_{1}} \ldots \mathrm{d} \phi_{n}^{m_{n}} \ldots \\
& =\int_{\Omega\left(\Gamma_{t^{1}}\right)}\left(\int_{\Omega\left(\Gamma_{t^{2}}\right) \ldots}\left(\int_{\Omega\left(\Gamma_{t^{\mathrm{m}}}\right)} \mathfrak{I d} \phi_{1}^{m_{n}}\right) \ldots \mathrm{d} \phi_{n}^{m_{1}}\right) \ldots,
\end{aligned}
$$

This is an infiltration in the space-time by the direct action $\mathfrak{I}[2,3]$, that happens in the space $\Omega \times C$, to each component of the space $\Omega(\Gamma)$, through the expressed Lagrangian in this case by $\omega$, de (10). In (11), the integration of the space realises with the infiltration of the time, integrating only energy state elements of the field.

The design of some possible spintronic devices that show the functioning of this process of transformation in the space $\mathrm{M}$, will be included in this chapter.

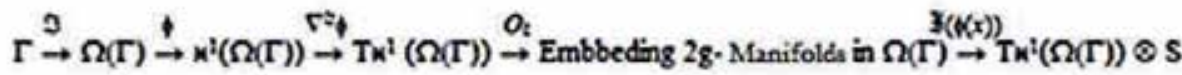

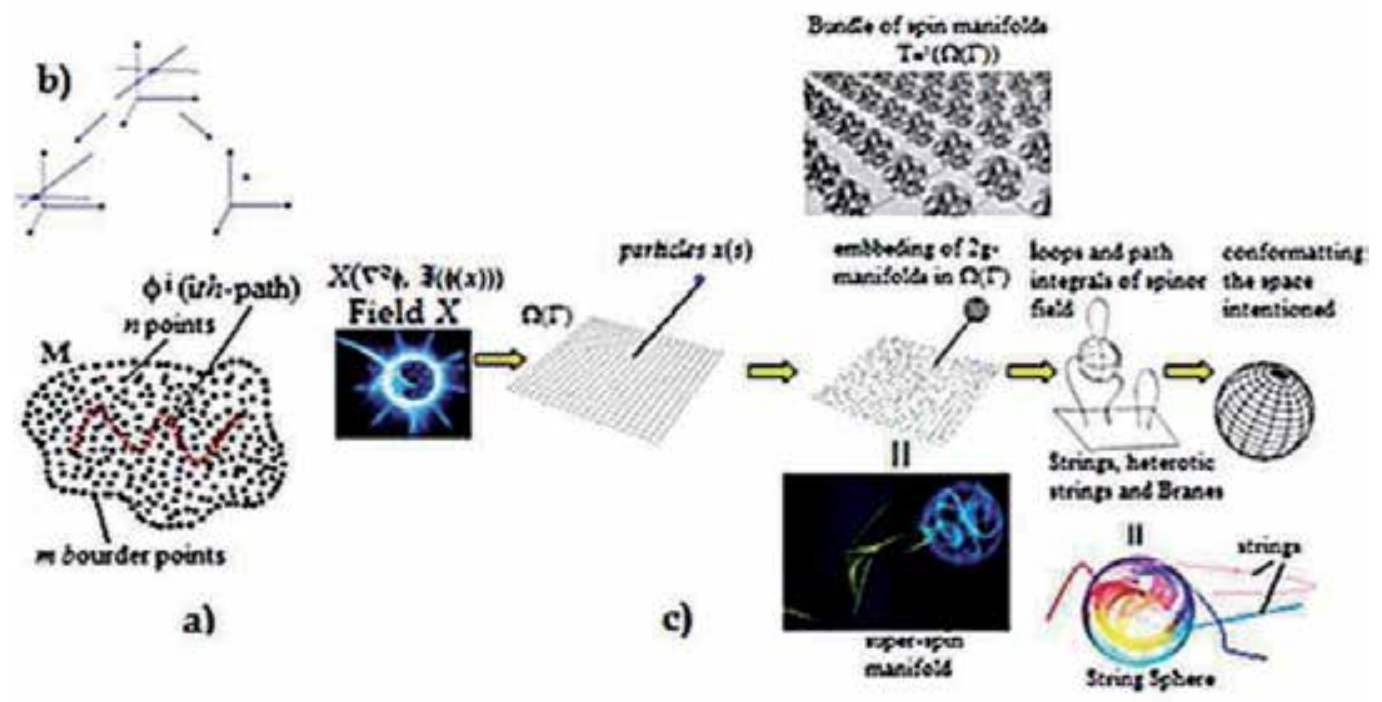

Figure 1. In a) The configuration space $C_{n, m}$, is the model created by the due action to each corresponding trajectory to the different splits. b) Example of a doble fibration to explain the relation between two realities of a space of particles: the bundle of lines $L$, and the ordinary space $\mathbb{R}^{3}$. c) Way in as a quantum field $X$, which acts on a space - time to change its reality, that is to say, to spill their intention. 


\section{Conscience operators and configuration spaces}

We consider $\mathbb{M} \cong \mathbb{R}^{3} \times \mathrm{I}_{t}$, the space-time of certain particles $x(s)$, in movement, and let $L$, be an operator that explains certain law of movement that governs the movement of the set of particles in $\mathbb{M}$, in such a way that the energy conservation law is applied for the total action of each one of their particles. The movement of all the particles of the space M, is given geometrically for their tangent vector bundle TM. Then the action due to $L$, on $M$, is defined like [8]:

$$
\mathfrak{I}_{L}: T_{M} \rightarrow \mathrm{R},
$$

with rule of correspondence

$$
\Im(x(s))=\operatorname{FluxL}(x(s)) x(s)
$$

and whose energy due to the movement is

$$
E=\mathfrak{I}-L,
$$

But this energy is due from their Lagrangian $L \in \mathrm{C}^{\infty}(T M, \mathbb{R})$, defined like [9]

$$
L(x(s), \dot{x}(s), s)=T(x(s), \dot{x}(s), s)-V(x(s), \dot{x}(s), s),
$$

If we want to calculate the action defined in (7) and (8), along a given path $\Gamma=x(s)$, we have that the action is

$$
\mathfrak{I}_{\Gamma}=\int_{\Gamma} L(x(s), x(s), s) d s
$$

If this action involves an intention (that is to say, it is an intentional action) then the action is translated in all the possible field configurations, considering all the variations of the action along the fiber derivative defined by the Lagrangian $L$. Of this way, the conscience operator is the map

$$
O_{c}: \mathrm{TM} \rightarrow \mathrm{T}^{*} \mathrm{M},
$$

with corresponding rule

$$
O_{c}(v) w=\left.\frac{d}{d t} L(v+t w)\right|_{t=0}
$$

That is, $O_{\mathrm{c}}(\boldsymbol{v}) w$, is the derivative of $L$, along the fiber in direction $w$. In the case of $v=x^{\prime}(s)$, and $q=$ $x(s), \forall \boldsymbol{q} \in \mathrm{M}, L(\boldsymbol{q}, \boldsymbol{v})=E-V=1 / 2<\boldsymbol{v}, \boldsymbol{v}>-\mathrm{V}(\boldsymbol{q})$, we see that $O_{c}(\boldsymbol{v}) \boldsymbol{w}=<\mathrm{v}, \mathrm{w}>$, so we recover the usual map s $\mathbf{s}^{\mathrm{b}}: \mathrm{TM} \rightarrow \mathrm{T}^{*} \mathrm{M}$, (with ${ }^{\mathrm{b}}$ Euclidean in $\mathbb{R}^{3}$ ) associated with the bilinear form $<,>$. Is here where the spin structure subjacent appears in the momentum of the particle $x(s)$. 
As we can see, $\mathrm{T}^{*} \mathrm{M}$, carries a canonical symplectic form, which we call $\omega$. Using $O_{c}$, we obtain a closed two-form $\omega L$, on TM, by setting

$$
\omega_{L}=\left(O_{c}\right) * \omega,
$$

Considering the local coordinates $\left(\phi^{i}, \partial_{\mu} \phi^{i}\right)$, to $\omega L$, modeling the space-time $\mathbb{M}$, through $\mathcal{H}$ spaces, we have that (19) is

$$
\omega_{L}=\frac{\partial^{2} L}{\partial \phi^{i} \partial \partial_{\mu} \phi^{j}} \mathrm{~d} \phi^{i} \wedge \mathrm{d} \phi^{j}+\frac{\partial^{2} \mathrm{~L}}{\partial \phi^{i} \partial \partial_{\mu} \phi^{j}} \mathrm{~d} \phi^{i} \wedge \mathrm{d} \partial_{\mu} \phi^{j},
$$

Likewise, the variation of the action from the operator $O_{c}=d \Im(\phi)=L\left(\phi, \partial_{\mu} \phi\right) d \phi$, is translated in the differential

$$
\mathrm{d} \Im(\phi) h=\int_{\Gamma}\left(\frac{\partial L}{\partial \phi}-\frac{d}{d t} \frac{\partial L}{\partial \dot{\phi}}\right)(\phi(s), \dot{\phi}(s)) h(s) d s,
$$

where $h(s): \Gamma \rightarrow T M$, and is such that $\tau_{\mathrm{M}} \mathrm{O} h=\Gamma$ and $h\left(x_{1}\right)=h\left(x_{2}\right)=0$, to extreme points of $\Gamma, x\left(s_{1}\right)=p$ y $x\left(s_{2}\right)=q$. The total differential (21) is the symplectic form $\omega L$, that constructs the application of the field intention expanding $2 n$-coordinates in $(20)$. The space $*^{1}(\Omega(\Gamma))$, is the space of differentiable vector fields on $\Omega(\Gamma)$, and $\Omega(\Gamma)$, is the manifold of trajectories (spacetime of curves) that satisfies the variation principle given by the Lagrange equation that expresses the force $F\left(x(s)^{j}\right),(j=1,2, \ldots, n)$ generated by a field that generates one "conscience" of order given by their Lagrangian (to see the figure 2).

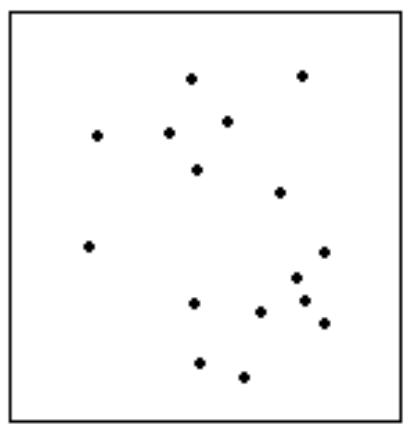

a)

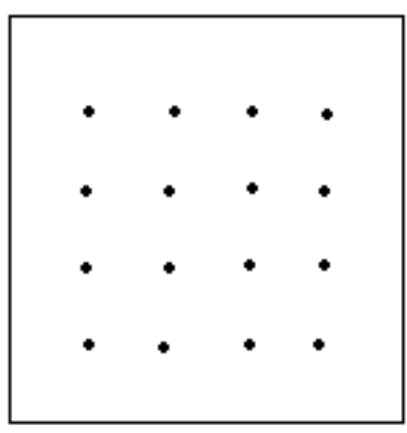

b)

Figure 2. a) The particles act in free form in the space - time without the action of a quantum field that spills a force that generates an order conscience. b) A force $\mathrm{F}\left(x(s)^{\prime}\right)$, is spilled, generated by a field that generates a "conscience" of order given by their Lagrangian. For it, there is not to forget the principle of conservation energy re-interpreted in the Lagrange equations and given for this force like $\frac{d}{d t}\left(\frac{\partial T}{\partial \dot{x}^{j}}\right)-\frac{\partial T}{\partial x^{j}}=F^{j}(x(s))$, (also acquaintances as "living forces") transmitting their momentum in every ith-particle of the space $E$, creating a infiltred region by path integrals of trajectories $\Omega(\Gamma)$, where the actions have effect. Here $T$, is their kinetic energy. 
How does it influence the above mentioned intention in the space - time? what is the handling of the force $\mathrm{Fj}(x(s))$ ? What is the quantum mechanism that makes possible the transformation of a body or space dictated by this intention?

It is necessary to have two aspects clear: the influence grade on the space, and a property that the field itself "wakes up" in the space or body to be transformed though the quantum information $\phi(\mathrm{x})$, their particles. Consider the integral (8) and their Green function for $n$, states $\phi\left(x_{j}\right)(j=1,2, \ldots n)$ :

$$
\mathrm{G}^{(n)}\left(x_{1}, x_{2}, \ldots, x_{n}\right)=\frac{\prod_{i=1}^{n} \int_{-\infty}^{+\infty} \mathrm{e}^{\mathrm{i} \Im\left[\phi^{i}, \partial_{\mu} \phi^{i}\right]} d \phi^{i}(x(s))}{\int_{-\infty}^{+\infty} \mathrm{e}^{\mathrm{i} \Im\left[\phi^{i}, \partial_{\mu} \phi^{i}\right]} d \phi^{\mathrm{i}}(x(s))}
$$

These Green's functions can most straightforwardly be evaluated by use of generating functional where we are using an external force $\mathrm{F}^{j}(x(s))$, given by the intention

$$
\mathrm{W}\left[\mathrm{F}^{j}(x(s))\right]=\mathrm{N} \int_{-\infty}^{+\infty} \exp \left\{\mathrm{i} \Im\left(\phi^{j}, \partial_{\mu} \phi^{j}\right)+\mathrm{i} \int_{\Omega(\Gamma)} \mathrm{F}^{j}(x(s)) \phi^{\mathrm{j}}(x)\right\} d(x(s)),
$$

This operator is the operator of execution $\exp _{\mathfrak{F}(\phi)}$, which establishes in general form (5) that has been studied and applied in other developed research (see $[2,10,11]$ as an example).

Then the influence realised on the space $\Omega(\Gamma) \subset M$, that there bears the functional one (23) that involves the force of the intention given by the field (observe that the second addend of the argument of exp, is the action which is realised from the exterior on the space $\Omega(\Gamma)$ ) can go according to the functional derivative:

$$
\mathrm{G}^{(n)}\left(x_{1}, x_{2}, \ldots, x_{n}\right)=\left.(-\mathrm{i})^{n} \frac{1}{\mathrm{~W}[0]} \frac{\delta^{n}}{\delta \mathrm{F}^{j}\left(x_{1}\right) \cdots \delta \mathrm{F}^{j}\left(x_{n}\right)} \mathrm{W}\left[\mathrm{F}^{j}(x(s))\right]\right|_{\mathrm{F}^{j}=0},
$$

where $\frac{\delta^{n}}{\delta F^{j}\left(x_{1}\right) \cdots \delta F^{j}\left(x_{n}\right)}$, describe the functional differentiation of nth-order, defined by the formula [6]

$$
\begin{aligned}
& \frac{\delta^{n}\left(\mathrm{~F}^{j}(x)\right)}{\delta \mathrm{F}^{j}\left(x_{1}\right) \cdots \delta \mathrm{F}^{j}\left(x_{n}\right)}=\frac{\delta^{n}}{\delta \mathrm{F}^{j}\left(x_{1}\right) \cdots \delta \mathrm{F}^{j}\left(x_{n}\right)} \int_{\Omega(\Gamma) \subset \mathrm{E}^{n}} \delta\left(x-x_{1}\right) \mathrm{F}^{j}\left(x_{1}\right) \delta\left(x-x_{2}\right) \mathrm{F}^{j}\left(x_{2}\right) \cdots \\
& \cdots \delta\left(x-x_{n}\right) \mathrm{F}^{j}\left(x_{n}\right) \mathrm{d} x_{1} \cdots \mathrm{d} x_{n}=\delta\left(x-x_{1}\right) \cdots \delta\left(x-x_{n}\right),
\end{aligned}
$$

where these derivatives express impulses (force) of every particle placed in the positions $x_{1}$, $\ldots, x_{n}$. In case of receiving the influence of the field $X$, these impulses will be directed by the 
derivative of their Lagrangian density $\mathcal{L}^{(0)}$, that is a consequence of the differential (21), (whereas, by the application of their conscience operator $O_{c}$ ) know

$$
\partial_{\mu} \frac{\delta L^{(0)}}{\delta \partial_{\mu} \phi(x)}-\frac{\delta L^{(0)}}{\delta \phi(x)}=0,
$$

But the equation (26) is the quantum wave equation (bearer of the information (configuration and momentum of the intention)) due to $O_{c}$, to the time $s$. Then the generating functional takes the form (23), considering the property of the operator $O_{c}$, given through the operator $\mathcal{O}\left(x-x_{j}\right)^{1}$ :

$$
\begin{aligned}
& W\left[\mathrm{~F}^{j}(x(s))\right]=\mathrm{N} \int_{-\infty}^{+\infty} \exp \left\{-\frac{\mathrm{i}}{2} \int_{\Omega(\Gamma)} \phi(x) \mathrm{O}\left(x-x_{j}\right) \phi\left(x_{j}\right)-\mathrm{i} \int_{\Omega(\mathrm{\Gamma})} \mathrm{F}^{j}(x(s)) \mathrm{o}^{-1}\left(x-x_{j}\right) \mathrm{F}^{j}\left(x_{j}(s)\right)\right\} d(\phi(x)) \\
& \left.=\mathrm{N} \int_{-\infty}^{+\infty} \exp \left\{-\frac{\mathrm{i}}{2} \int_{\Omega(\Gamma)} \phi^{\prime}(x) \mathrm{o}\left(x-x_{j}\right) \phi^{\prime}\left(x_{j}\right)-\mathrm{i} \int_{\Omega(\Gamma)} \mathrm{F}^{j}(x(s)) \mathrm{O}^{-1}\left(x-x_{j}\right) \mathrm{F}^{j}\left(x_{j}(s)\right)\right\} d\left(\phi^{\prime} x\right)\right),
\end{aligned}
$$

Where we have used $[d \phi(x)]=\left[d \phi^{\prime}(x)\right]$.

The intention infiltrated by the conscience given for $O_{c}$, establishes that the differential of the action $d \mathfrak{I}(\phi) h$, given by (21) (using the energy (amplitude) that their propagator contributes $\left.D_{F}\right)$ can be visualised inside the configuration space through their boarder points ("targets" of the intention of the field $X$, and that happen in $\partial M$ [12]),being also the interior points of the space $\mathbb{M}$, int $\mathbb{M}$, are the proper sources of the field (particles of the space $\mathbb{M}$, that generate the field $X$ ). Then the intention of the field $X$, is the total action

$$
\mathfrak{I}_{T}=\mathfrak{I}_{\partial \mathrm{M}}\left(\mathfrak{I}_{\text {int } \mathrm{M}}\right),
$$

where this is a composition of the actions $\mathfrak{I}_{i n t_{M},}$ and $\mathfrak{J}_{\partial \mathrm{M}}$. These actions have codimensions strata $k$, and $n-k$, respectively, when we want to form the space M, using path integrals [].

To extract the intrinsic properties of integrals over configurating spaces, we will follow the proof of the formality theorem [13], and record the relevant facts in our homologicalphysical interpretation: admissible graphs are "cobordisms" $\omega(\gamma) \rightarrow[m]$, when Un is thought as a state-sum model [14]. The graphs are also interpreted as "extensions" $\gamma \rightarrow \Gamma \rightarrow \gamma^{\prime}$, when considering the associated Hopf algebra structure. The implementations of these tools were done in the [3]. Remember that using the Stokes theorem (10) a Lagrangian on the class $G$, of Feynman graphs is a $k$-linear map $\omega: H \rightarrow \Omega^{\bullet}(\mathrm{M})$, associating to any Feynman graph $\Gamma$, a

\footnotetext{
${ }^{1}$ The operator $\mathcal{O}\left(x-x_{j}\right)=\left(\square_{x}+m^{2}-i \varepsilon\right) \delta^{n}\left(x-x_{j}\right)$, and such that to their inverse $\mathcal{O}^{-1}\left(x-x_{j}\right)$, the functional property is had:

$\int_{\Omega(\Gamma)} \mathrm{O}^{-1}\left(x-x_{j}\right) \mathrm{O}\left(x_{j}-y\right) d^{n} x_{j}=\delta^{n}(x-y)$,
} 
closed volume form on $\mathfrak{I}(\Gamma)$, vanishing on the boundaries, i.e. for any subgraph $\gamma \rightarrow \Gamma$ (viewed as a sub-object) meeting the boundary of $\Gamma:[s] \rightarrow[t]$ (viewed as a cobordism), $\omega(\gamma)$ $=0$. Then an action given by $\mathfrak{I}_{i n t_{\mathrm{M}},}$ is defined through their interior as:

Def. 2. 1. An action on $G\left(\right.$ "T int $\left.^{\prime \prime}\right)$, is a character $W: H \rightarrow \mathbb{R}$, which is a cocycle in the associated $\mathrm{D} G$-coalgebra $\left(\mathrm{T}\left(H^{*}\right), D\right)$, where $\mathcal{G}$, is a class of Feynman graph.

Let $C_{n, m}$, be the configuration space of $n$, interior points and $m$, boundary points in the manifold $M$, with boundary $\partial M$ I (that is to say. [13], upper half-plane $H$ ). Its elements will be thought as (geometric) "representations of cobordisms" (enabling degrees of freedom with constraints). Then the action in (28) takes the form

$$
\{\{\omega(\gamma)=0\} \stackrel{[n]}{\longrightarrow}[m]\} \stackrel{\mathrm{x}(\mathrm{s})}{\longrightarrow}\{\{\omega(\Gamma)=0\} \stackrel{[m]}{\longrightarrow} \partial \mathrm{M}\},
$$

Let $H$, be the Hopf algebra (associative algebra used to the quantised action in the space-time), of a class of Feynman graphs $G$ [12]. If $\Gamma$, is such a graph, then configurations are attached to their vertices, while momentum are attached to edges in the two dual representations (Feynman rules in position and momentum spaces). This duality is represented by a pairing between a "configuration functor" (typically $C_{\Gamma}$, (configuration space of subgraphs and strings [15], and a "Lagrangian" (e.g. $\omega$, determined by its value on an edge, i.e. by a propagator $D_{\mathrm{F}}$ ). Together with the pairing (typically integration) representing the action, they are thought as part of the Feynman model of the state space of a quantum system. The differential (21) considering the DG-structure [12], in the class of Feynman graphs $\mathcal{G}$, can be defined as one graph homology differential:

$$
d \Gamma=\sum_{\mathbf{e} \in E_{\Gamma}} \pm \Gamma / \gamma_{\mathrm{e}^{\prime}}
$$

where the sum is over the edges of $\Gamma, \gamma_{e}$ is the one-edge graph, and $\Gamma / \gamma_{e}$, is the quotient (forget about the signs for now).

We can give a major generalisation of this graphical homological version of the differential establishing the graduated derivation that comes from considering $H=\mathrm{T}(\mathfrak{g})$, the tensor algebra with reduced co-product

$$
\Delta \Gamma=\sum_{\gamma \rightarrow \Gamma \rightarrow \gamma^{\prime}} \gamma \otimes \gamma^{\prime},
$$

Consider the following basic properties of the operators $O_{\text {c. Let }} \mathcal{O}\left(x-x^{\prime}\right)$, defined in the footnote 1 , and $\phi(x) \in \mathcal{H}$, where the space is the set of points $\mathcal{H}=\left\{\phi(x) \in[\mathrm{m}] \mid[\mathrm{m}] \subset \mathrm{T}^{*} \mathrm{M}\right\}^{2}[8]$.

\footnotetext{
2 The corresponding cotangent space to vector fields is: $\mathrm{T}^{*} *^{1}(\mathrm{M})=\left\{\left(\phi, \partial_{\mu} \phi\right) \in \mathcal{H} \times \mathrm{T} *^{1}(\mathrm{M}) \mid \partial_{\mu} \phi=\nabla_{\mu} \xi, \forall \xi \in *^{1}(\mathbb{M})\right\}$.

Here $[\mathrm{m}]=\mathrm{T}^{*} \mathrm{C}_{n, m}$.
} 
Points of phase space are called states of the particle system acting in the cotangent space of M. Thus, to give the state of a system, one must specify their configuration and momentum.

a)

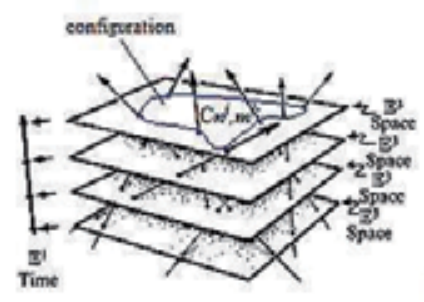

b)

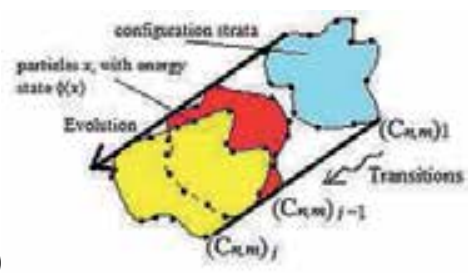

c)

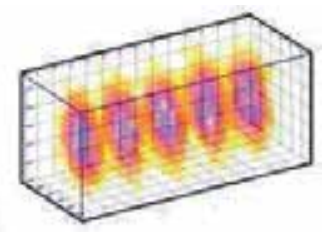

Figure 3. a) In every plane there is a particle configuration for a given time. b) The evolution of the particles along everything $t$, happens for a succession of configurations through which the particles system spends different strata codimension one. The causal structure of the space - time is invariant for every particle along the transformation process. d) Strata Evolution strata of the configuration space $C_{n, m}$ in the space-time $\mathbb{E}^{4}$. The translation obeys to evolution of Lagrangian system given by $L\left(\phi_{1}, \ldots, \phi_{n}\right)$.

Example 1. Let $\pi: \mathrm{T}^{*} \mathrm{M} \rightarrow \mathbb{M}$, be and $\gamma: \mathbb{R} \rightarrow \mathrm{TC}_{n, m}$, then $\pi \circ \gamma: \mathbb{R} \rightarrow \mathbb{M}$, describes the curve in the configuration space which describes the sequence of configurations through which the particles system passes to different strata of co-dimension one (see figure 2). Every strata correspond to a phase space of $m$, particles that are moved by curve $\gamma$ and directed from their energy states $d \phi(x)$, by $\pi$, to $n$, particles $\phi(x)$.

This defines our intentional conscience. Then are true the following properties:

i. $\mathrm{O}\left(\mathrm{x}-\mathrm{x}^{\prime}\right) \phi(\mathrm{x})=\delta\left(\mathrm{x}-\mathrm{x}^{\prime}\right) \phi(\mathrm{x}), \forall \mathrm{x}, \mathrm{x}^{\prime} \in \mathrm{M}$,

ii. $\operatorname{Oc}(\mathrm{x}(\mathrm{s})) \phi\left(\mathrm{x}^{\prime}(\mathrm{s})\right)=\mathrm{O}\left(\mathrm{x}-\mathrm{x}^{\prime}\right), \forall \mathrm{x}, \mathrm{x}^{\prime} \in \mathrm{M}$, and $\mathrm{s} \leq \mathrm{t}$,

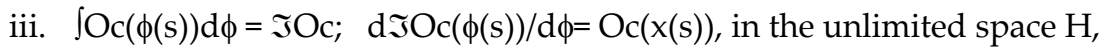

iv. Oc $=\delta\left(s-s^{\prime}\right)$, if and only if $\delta x(s) / \delta x\left(s^{\prime}\right)=\delta\left(s-s^{\prime}\right), \forall s \leq t$, then $F(x(s))=x(s)$,

v. $\mathrm{O}^{-1}\left(\mathrm{x}-\mathrm{x}^{\prime}\right) \mathrm{Oc}(\mathrm{x}(\mathrm{s}))=-\Delta \mathrm{F}\left(\mathrm{x}-\mathrm{x}^{\prime}\right) \delta\left(\mathrm{x}-\mathrm{x}^{\prime}\right), \forall \mathrm{x}, \mathrm{x}^{\prime} \in \mathrm{M}$, and $\mathrm{s} \leq \mathrm{t}$,

vi. $\int \mathrm{H} O c(\phi(s)) d \phi=\int \Omega O\left(x-x^{\prime}\right) x(s) d(x(s))$.

On the one hand, $\int_{\mathcal{H}} O_{c}(\phi(s)) \phi(x) d x=\int_{\mathcal{H}} O_{c}(\phi(s)) d \phi=\Sigma \int_{\Gamma} \mathcal{O}\left(x-x^{\prime}\right) x(s) d s$. Also

$$
\int_{\Omega(\Gamma)} O_{\mathrm{c}}(\phi(s)) x(s) d s=\int_{\mathrm{H}} \mathrm{O}\left(x-x^{\prime}\right) \phi(x) d x
$$

then $i$, is satisfied. To the property $i i$. is necessary consider $\mathcal{O}\left(x-x^{\prime}\right)=\left(\square_{x}+m^{2}-i \varepsilon\right) \phi\left(x-x^{\prime}\right)$ [6]. But the operator $\mathrm{O}_{c}$, is the defined as

$$
O_{\mathrm{c}}(x(s)) \phi\left(x^{\prime}\right)=\left(\frac{d \mathfrak{I}_{O_{\mathrm{c}}}}{d \phi}\right) \phi\left(x^{\prime}\right),
$$

Considering the operator $\mathcal{O}\left(x-x^{\prime}\right)$, we have

$$
\mathrm{O}\left(x-x^{\prime}\right) d \phi=d \Im_{O_{c}} \phi\left(x^{\prime}\right)
$$


Integrating both members on unlimited space $\mathcal{H} \times \Omega(\Gamma)$, (applying the principle of Stokes integration given by (10)) we have that integral identity is valid for whole space. Then is verifying ii. The property iii., is directly consequence of (19), (20) and (21), considering the Stokes theorem given in (10), therefore $O_{c}(x(s))$, is such that $\omega_{L}=\left(O_{c}\right)^{*} \omega$, considering $\omega=d \phi$. Then

$$
O_{\mathrm{c}}=\int_{\Omega(\Gamma) \times \mathrm{H}} \omega_{L}=\int_{\Omega(\Gamma)}\left(O_{\mathrm{c}}\right)^{*} \omega,
$$

which is a integral of type (10). Indeed,

$$
\mathfrak{J}_{O_{c}}=\int_{H \times \Omega(\Gamma)} \omega_{L}=\int_{\mathrm{H}} O_{\mathrm{c}}(x(s)) * \omega=\int_{\mathrm{H}} O_{\mathrm{c}}(x(s)) d \phi=\int_{\mathrm{H}} d \Im_{O_{\mathrm{c}}}(\phi),
$$

The derivative in the last integral from (33) is the total differential given by (21) from where we have the derivative formula in the context of the unlimited space $\mathcal{H}$.

The property iv., require demonstrate two implication where both implications are reciprocates. If $O_{c}\left(x\left(s^{\prime}\right)\right)=\delta\left(s-s^{\prime}\right)$, then all intention on trajectory defined $\Gamma$, its had that

$$
\int_{\Gamma} O_{c}(x(s)) x(s) \mathrm{d} s=\int_{\Gamma} \delta\left(s-s^{\prime}\right) x(s) d s,
$$

But for the differential (21) and the second member of the integral (34) we have

$$
\delta \mathfrak{I}_{O_{c}}\left(x\left(s^{\prime}\right)\right)=\delta\left(\int_{\Gamma} \delta\left(s-s^{\prime}\right) x\left(s^{\prime}\right) d s^{\prime}\right),
$$

since $\frac{\delta}{\delta x\left(s^{\prime}\right)}\left(\int_{\Gamma} \delta\left(s-s^{\prime}\right) x\left(s^{\prime}\right) d s^{\prime}\right)=\delta\left(s-s^{\prime}\right)$, then $\delta\left(\int_{\Gamma} \delta\left(s-s^{\prime}\right) x\left(s^{\prime}\right) d s^{\prime}=\delta x(s)\right.$, and for other side

$$
\delta\left(\int_{\Gamma} \delta\left(s-s^{\prime}\right) x\left(s^{\prime}\right) d s^{\prime}\right)=\delta\left(s-s^{\prime}\right) \delta x\left(s^{\prime}\right)
$$

from where $\frac{\delta x(s)}{\delta x\left(s^{\prime}\right)}=\delta\left(s-s^{\prime}\right), s \leq t^{3}$. But this implies directly $\mathrm{F}(x(s))=x(s)$. This property tell us that we can have influence on the space $\mathrm{M}$, considering only a curve any of the space where the influence of the field exists like the force $\delta\left(s-s^{\prime}\right)$, since the space is infiltrated by

${ }^{3}$ In the general sense the functional derivative $\frac{\delta \phi_{a}(y)}{\delta \phi_{b}(x)}=\delta_{b a} \delta^{n}(y-x)$, implies

$\delta \phi_{b}(y)=\sum_{a} \int \delta^{n}(y-x) \delta \phi_{a}(x) \delta_{b a} d x$

but does not imply

$\delta \phi_{b}(y)=\delta_{b a} \delta^{n}(y-x) \delta \phi_{a}(x)$. 
the force $\mathrm{F}$, and this produces the permanent state of energy generated by every component of the space. On the other side, if $\frac{\delta x(s)}{\delta x\left(s^{\prime}\right)}=\delta\left(s-s^{\prime}\right), s \leq t$ which is equivalent to $\frac{\delta}{\delta x\left(s^{\prime}\right)}\left(\int_{\Gamma} \delta\left(s-s^{\prime}\right) x\left(s^{\prime}\right) d s^{\prime}\right)=\delta\left(s-s^{\prime}\right)$, . But integrating (21) we have

$$
\int_{\Gamma} \delta \Im_{O_{c}}\left(x\left(s^{\prime}\right)\right)=\int_{\Gamma} \delta\left(s-s^{\prime}\right) x\left(s^{\prime}\right) d s^{\prime},
$$

which for before implication is $\delta\left(s-s^{\prime}\right) \delta x\left(s^{\prime}\right)$. But

$$
\int_{\Gamma}\left\{O_{c}\left(x\left(s^{\prime}\right)\right) x\left(s^{\prime}\right)+\delta\left(s-s^{\prime}\right) x\left(s^{\prime}\right)\right\} d s^{\prime}=0,
$$

This integral is valid $\forall \Gamma \subset M$ M. Thus $O_{\mathrm{c}}\left(x\left(s^{\prime}\right)\right)=\delta\left(s-s^{\prime}\right)$. With this, the demostration of $i v$., is completed. The identity in $v$, happens because $\mathcal{O}^{-1}\left(x-x^{\prime}\right)=-\Delta_{F}\left(x-x^{\prime}\right)$, considering the before property (simple conaequence of the property $i v$ ) [6].

The identity in vi., happens in the phase space created by the cotangent space due to the image of the differential (21). Therefore, both members of integral identity will have to coincide in the intention given by $\mathfrak{J}$ oc. Indeed, consider the integral

$$
\int_{\mathrm{H}} O_{\mathrm{c}}(\phi(x)) d \phi=\int_{\Omega(\Gamma)}\left(O_{\mathrm{c}}\right) * \omega=\int_{\mathrm{H}} d \mathfrak{I}_{O \mathrm{c}}=\mathfrak{I}_{O \mathrm{C}}
$$

On the other side, inside the quantum wave equation:

$$
\begin{aligned}
& \int_{\Omega(\Gamma)} O\left(x-x^{\prime}\right) \mathrm{d} x(s)=\int_{\Omega(\Gamma)}\left(\square_{x}+m^{2}-\mathrm{i} \varepsilon\right) \omega=\int_{\Omega}\left(\square_{x}+m^{2}-\mathrm{i} \varepsilon\right) \phi(x) d x \\
& =\int_{\mathrm{H}}\left(\square_{x}+m^{2}-\mathrm{i} \varepsilon\right) d \phi=\int_{\mathrm{H}} d \Im_{O \mathrm{C}}(\phi),
\end{aligned}
$$

Joining (35) with (36) we have vi.

\section{Quantum intentionality and organized transformations}

Considering the quantizations of our Lagrangian system describe in (11), (18) and (19) on $\mathbb{R}^{n}$, $n \geq 2$, coordinated by $\left\{x^{j}\right\}$, we describe terms of a graded commutative $\mathrm{C}^{\infty}(\mathrm{M})$-algebra $H$, with generating elements

$$
\left\{\partial_{m} x^{a}, \partial_{m} x_{l}^{a}, \partial_{m} x^{a}{ }_{l 1 l 2}, \ldots, \partial_{m} x^{a}{ }_{l 1} \cdots l k, \ldots\right\},
$$

and the bi-graded differential algebra $H^{*}$, of differential forms (the Chevalley-Eilenberg differential calculus) over $H^{0}$, as an $\mathbb{R}$-algebra [1-3]. One can think of generating elements (37) of $H$, as being sui generis coordinates of even and odd fields and their partial derivatives. 
The graded commutative $\mathbb{R}$-algebra $H^{0}$, is provided with the even graded derivations (called total derivatives)

$$
d_{\lambda}=\partial_{\lambda}+\sum_{0 \leq \Lambda \Lambda \mid} \partial_{\lambda+\Lambda}^{a} \partial_{a}^{\Lambda}, \mathrm{d}_{\Lambda}=\mathrm{d}_{\lambda_{1}} \cdots \mathrm{d}_{\lambda_{k}}
$$

where $\Lambda=\left(\lambda_{1} \ldots \lambda_{k}\right),|\Lambda|=k$, and $\lambda+\Lambda=\left(\lambda, \lambda_{1}, \ldots, \lambda_{k}\right)$ are symmetric multi-indices. One can think of even elements

$$
L=\left(x^{l}, \partial_{\mu} x^{a}\right) d^{n} x, \quad \delta L=d \partial_{\mu} x^{a} \wedge E_{a} d^{n} x=\sum_{0 \leq|\Lambda|}(-1)^{|\Lambda|} d \partial_{\mu} x^{a} \wedge d_{\Lambda}\left(\partial_{a}{ }^{\Lambda} L\right) d^{n} x
$$

where we observe that $\delta L$, is the 2-form given by $\omega L$, in the formula (20) with $n=2$, and $\Lambda=\lambda_{1} \lambda_{2}$.

Now we consider the dual part of the space $(H, \Omega(\Gamma))$, that is to say, the space $\left(H^{*}, L\right)$, be

We consider quantize this Lagrangian system in the framework of perturbative Euclidean QFT. We suppose that $\mathrm{L}$, is a Lagrangian of Euclidean fields on $\Omega(\Gamma) \subseteq \mathbb{R}^{n}$. The key point is that the algebra of Euclidean quantum fields $B_{\Phi}$, as like as $H^{0}$, is graded commutative. It is generated by elements $\phi_{a}^{\lambda \Lambda}, x \in \Omega(\Gamma)$. For any $x \in \Omega(\Gamma)$, there is a homomorphism belonging to space $H \rightarrow \operatorname{Hom}(\mathrm{T}(H), D$ ) (with homomorphisms $\operatorname{Hom}(\mathrm{T}(H), D)$ given for $D G$-algebra of cycles)

$$
\gamma_{\mathrm{x}}: \mathrm{I}_{a_{1} \cdots a_{r}}^{\Lambda_{1} \cdots \Lambda_{r}} \partial x_{\Lambda_{1}}^{a_{1}} \cdots \partial x_{\Lambda_{r}}^{a_{r}} \mapsto \mathrm{I}_{a_{1} \cdots a_{r}}^{\Lambda_{1} \cdots \Lambda_{r}}(x) \phi_{x \Lambda_{1}}^{a_{1}} \cdots \phi_{x \Lambda_{r}}^{a_{r}}, \quad \mathrm{I}_{a_{1} \cdots a_{r}}^{\Lambda_{1} \cdots \Lambda_{r}} \in \mathrm{C}^{\infty}(\Omega(\Gamma))
$$

Of the algebra $H^{0}$, of classical fields to the algebra $\mathrm{B}_{\Phi}$, which sends the basic elements

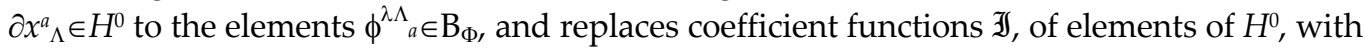
their values $\mathfrak{I}(x)$ (executions) at a point $x$. Then a state $<_{,}>$of $\mathrm{B}_{\Phi}$, is given by symbolic functional integrals

$$
<\phi_{x_{1}}^{a_{1}} \cdots \phi_{x_{k}}^{a_{k}}>\frac{1}{\mathrm{~N}} \int_{\mathrm{H}} \phi_{x_{1}}^{a_{1}} \cdots \phi_{x_{k}}^{a_{k}} \exp \left\{-\int_{\Omega(\Gamma)} O_{\mathrm{c}}\left(\phi_{x \Lambda}^{a}\right) d^{n} x\right\} \prod_{x}\left[d \phi_{x}^{a}\right]
$$

where this is an integral of type $\int_{H \times \Omega(\Gamma)} O_{c}(x(s)) \mathrm{d} \phi$, as give by the properties. When the intention expands to the whole space, infiltrating their information on the tangent spaces images of the cotangent bundle $\mathrm{T}^{*} \mathrm{M}$, (given by the imagen of $\phi(x)$, under $d \mathfrak{J}^{\circ} \mathrm{c}$ ). Then their intentionality will be the property of the field to spill or infiltrate their intention from a nano level of strings inside the quantum particles. Then from the energy states of the particles, and considering the intention spilled in them given by $O_{c}(\phi)$, we have the homomorphism (40) that establishes the action from $\mathbb{M}(\cong \Omega(\Gamma))$, to $\partial \mathbb{M}$, for their transformation through the 
action $\mathfrak{I}_{\mathrm{T}}$, defined in (28) to any derivation given through their conscience operator (fiber (18)), like the graded derivation $\tilde{\partial}$, (considering the derivatives $\left.O_{c}(x)=\left(O_{c}\right)^{*} \omega, D_{\mathrm{F}}=d \phi\right)$ :

$$
\tilde{\partial}: \phi_{x \Lambda}^{a} \mapsto\left(x, \partial_{\mu}^{a} x\right) \mapsto\left(O_{c}(x), \omega\right) \mapsto\left(O_{c}^{*}(x), \omega_{L}\right)=O_{c}^{*}\left(\phi_{x \Lambda}^{a}\right) \omega,
$$

of the algebra of quantum fields $B_{\Phi}$. With an odd parameter $\alpha$, let us consider the automorphism

$$
\hat{\mathrm{U}}=\exp \{\alpha \tilde{\partial}\}=\mathrm{Id}+\alpha \tilde{\partial},
$$

Of the algebra $\mathrm{B}_{\Phi}$. This automorphism yields a new state $<,>$, of $\mathrm{B}_{\Phi}$, given by the equality

$$
<\phi_{x_{1}}^{a_{1}} \cdots \phi_{x_{k}}^{a_{k}}>\frac{1}{\mathrm{~N}^{\prime}} \int_{\mathrm{H}} \hat{U}\left(\phi_{x_{1}}^{a_{1}}\right) \cdots \hat{U}\left(\phi_{x_{k}}^{a_{k}}\right) \exp
$$

where the energy state has survived, since $d \hat{U}\left(\phi_{x}^{a}\right)=d \varphi_{x}^{a}$. That because the intention is the same. The intention has not changed.

What happens towards the interior of every particle? what is the field intention mechanism inside every particle?

To answer these questions we have to internalise the actions of field $X$, on the particles of the space $\mathbb{M}$, and consider their spin. But for it, it is necessary to do the immersion of the Lagrangian $\omega$, defined as the map

$$
w: \mathrm{L} \rightarrow \mathrm{M}^{2 n}, 4
$$

with rule of correspondence

$$
Z_{i} \mapsto w\left(Z_{i}\right),
$$

where the image of the 1 -form $\omega$, that the Lagrangian defines, $\omega\left(Z_{i}\right)$, is a symplectic form [8], and the variable $Z_{i}$, is constructed through the algebraic equations $\mathrm{W}^{a}\left(Z_{i}\right)=0$ [16].They describe the $k$-dimensional hypersurfaces denoted by $S$, such that $S \subset \mathcal{H}$, where $\mathcal{H}$, is the phase space defined in the section 2. The index $a=1, \ldots, q$ runs over the number of polynomials $\mathrm{W}^{a}\left(Z_{i}\right)$, in the variables $\mathrm{Zi}$ and $\mathrm{i}$ runs over the dimension of the ambient manifold which is assumed to be $\mathbb{C}^{\mathrm{N}}$. If the space is a complete intersection, the constraints

\footnotetext{
${ }^{4}$ Having chosen $\mathbb{M}^{2 n}$, is to consider the two components of any point in the space $\mathrm{C}^{\mathrm{N}}$, (that we are considering isomorfo to the ambient space of any quantum particle $x(s)$, in the space-time) to have the two components that characterise any quantum particle $x(\mathrm{~s})$, that is their spin (direction) and their energy state (density of energy or "living force of the particle"). $\mathbb{L}$, is the corresponding Lagrangian submanifold of the symplectic structure given by $(\mathbb{M} 2 \mathrm{2m}, \omega)$.
} 
$\mathrm{W}^{a}\left(Z_{i}\right)$ (there is exact solution to $\mathrm{W}^{a}\left(Z_{i}\right)=0$ ), are linearly independent and the differential form

$$
\Theta^{(n-k)}=\epsilon_{a_{1} \cdots a_{N-k}} d W^{a_{1}} \wedge \cdots \wedge d W^{a_{N-K}},
$$

is not vanishing. In this case, $\mathrm{q}=\mathrm{N}-k$ and the dimension of the surface is easily determined. For example, if the hypersurface is described by a single algebraic equation $W(Z)=0$, the form (44) is given by $\Theta^{(1)}=d \mathrm{~W}$. On the other hand, if the hypersurface is not a complete intersection, then there exists a differential form

$$
\Theta^{(n-k)}=\mathrm{T}_{A\left[a_{1} \cdots a_{\mathrm{N}-k}\right]} d W^{a_{1}} \wedge \cdots \wedge d W^{a_{N-K}} \wedge \eta^{A,(\mathrm{~N}-k-q)},
$$

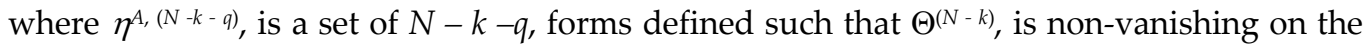
constraints $\mathrm{W}^{a}\left(Z_{i}\right)=0$, and $\left.T_{\mathrm{A},[a 1} \ldots a N-k\right]$, is a numerical tensor which is antisymmetric in the indices $a_{1} \ldots a_{q}$. The construction of $\eta^{A,(N-k-q)}$, depends upon the precise form of the algebraic manifold (variety of the equations $\mathrm{W}^{a}\left(Z_{i}\right)=0$ ). In some cases a general form can be given, but in general it is not easy to find it and we did not find a general procedure for that computation.

To construct a global form on the space $S$, one can use a modification of the Griffiths residue method [16], by observing that given the global holomorphic form on the ambient space $\Omega^{(N)}=\epsilon^{i_{1} \cdots i_{N}} d Z_{i_{1}} \wedge \cdots \wedge d Z_{i_{N}}$, we can decompose the $\left\{Z Z_{i}\right\}^{\prime}$, into a set of coordinates $Y^{a}=$ $\mathrm{W}^{a}(Z)$, and the rest. By using the contraction with respect to $q$, vectors $\left\{\underline{Z}^{a_{i}}\right\}$, the top form for $S$, can be written as

$$
\Omega^{(k)}=\frac{\underline{\mathrm{Z}}_{1}^{a} \cdots \mathrm{l}_{\underline{Z}_{\theta}^{a}} \Omega^{(\mathrm{N})}}{\underline{\mathrm{Z}}_{1}^{a} \cdots \mathrm{l}_{\mathrm{Z}_{\theta}^{a}} \Theta^{(\mathrm{N}-k)}},
$$

which is independent from $\left\{\underline{Z}^{a_{i}}\right\}$, as can be easily proved by using the constraints $\mathrm{W}^{a}\left(Z_{i}\right)=0$. Notice that this form is nowhere-vanishing and non singular only the case of CY-space (Calabi-Yau manifold). The calabi-Yau manifols is a spin manifold and their existence in the our space $\mathbb{M}^{2 n}$, like product of this construction is the first evidence that a spin manifold is the spin of our space-time due to their holomorphicity [17]. The vectors $\left\{\underline{Z}^{a}\right\}$, play the role of gauge fixing parameters needed to choose a polarisation of the space $S$, into the ambient space.

For example, in the case of pure spinor we have: the ambient form $\Omega^{(16)}=\epsilon_{\alpha_{1} \cdots \alpha} d \lambda_{i_{1}} \wedge \cdots \wedge d \lambda_{i_{6}}$, and $\Theta^{(5)}=\lambda \gamma^{m} d \lambda \lambda \gamma^{m} d \lambda \lambda \gamma^{m} d \lambda d \lambda \gamma_{m n p} d \lambda$. From these data, we can get the holomorphic top form $\Omega^{(11)}$, by introducing 5 , independent parameters $\underline{\lambda}$, and by using the formula (46). 
The latter is independent from the choice of parameters $\underline{\lambda}$, (however, some care has to be devoted to the choice of the contour of integration and of the integrand: in the minimal formalism, the presence of delta function $\delta(\lambda)$, might introduce some singularities which prevent from proving the independence from $\underline{\lambda}$, as was pointed out in [18], [19]). Using $\Omega^{(k)}$ $\wedge \Omega^{(k)}$, one can compute the correlation functions by integrating globally defined functions. When the space is Calabi-Yau, it also exists a globally-defined nowhere vanishing holomorphic form $\left.\Omega_{h o l}{ }^{(k}\right|_{0)}$, such that $\left.\Omega_{h o l}{ }^{(k)}\right|_{0)} \wedge \Omega_{h o l} \underline{k}^{\left(k_{0}\right)}$, is proportional to $\Omega^{(k)} \wedge \Omega^{(\underline{k})}$. The ratio of the two top forms is a globally defined function on the CY-space. In the case of the holomorphic measure $\Omega_{h o l}\left(k_{0}\right)_{0}$, the integration of holomorphic functions is related to the definition of a contour $\gamma \in S$, in the complex space

$$
<\prod_{\mathrm{A}} \mathrm{o}\left(\mathrm{Z}_{i}, p_{A}\right)>\int_{\gamma \in \mathrm{S}} \Omega^{(k, 0)} \prod_{A} \mathrm{o}_{0}\left(\mathrm{Z}_{i}, p_{A}\right)
$$

where $\mathcal{O}\left(Z_{i}, p_{A}\right)$, are the vertex operators of the theory localized at the points $p_{A}$, of the Riemann surface and $\mathcal{O}_{0}\left(Z_{i}, p_{A}\right)$, is the zero-mode component of the vertex operators. Newly our conscience operator come given by the form $\Omega^{(k, 0)}$.

Example 2. All Calabi-Yau manifolds are spin. In hypothetical quantum process (from point of view QFT), to obtain a Calabi-Yau manifold is necessary add (or sum) strings in all directions. In the inverse imaginary process, all these strings define a direction or spin. The strings themselves are Lagrangian submanifolds whose Lagrangian action is a path integral.

In mathematics, an isotropic manifold is a manifold in which the geometry doesn't depend on directions. A simple example is the surface of a sphere. This directional independence grants us freedom to generate a quantum dimension process, since it does not import what direction falls ill through a string, the space is the same way affected and it presents the same aspect in any direction that is observed creating this way their isotropy.

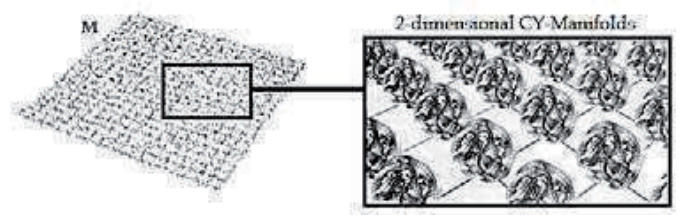

(a)

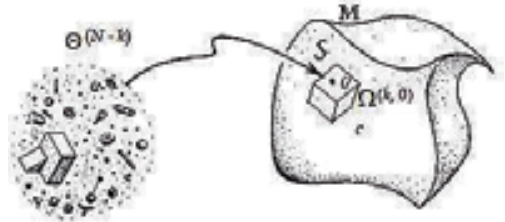

(b)

Figure 4. a) For instance, let us consider the hypersurface $S: \Sigma_{i} Z_{i}^{2}=0$, in $\mathbb{C}^{\mathrm{N}}$. This equation can be put in the form using generalized coordinates $S\left(u_{i}\right)=w z$, where $i$, runs over $i=1,2, \ldots, N-2$, coordinates and $w, z$, are two combinations of the $Z^{\prime} s$. $S\left(u_{i}\right)$, is a polynomial of the coordinates $u_{i}$. For a given $N$, they are local CY-manifolds (spin manifolds) and there exist a globally-defined a organized transformation inside space $\mathbf{M}[$ [20]. b) Intention inside particle Q.

The importance of this isotropy property in our spin manifold, helps us to establish that the transformations applied to the space that are directed to use (awakening) their nanostructure do it through an organized transformation that introduces the time as isotropic 
variable, creating a momentary timelessness in the space where the above mentioned transformation is created. Then the intentionality like a organized transformation is a coaction compose by field that act to realise the transformation of space and the field of the proper space that is transformed. Then the symplectic structure subjacent in $\mathbb{M}$, receives sense.

A transformation T, it is said organized if in whole stage of execution of the transformation, isotropic images are obtained of the original manifold (object space of the transformation), under a finite number of endomorphisms of the underlying group to the manifold that coacts with this transformation.

Likewise, if $\mathcal{T}$, is a transformation on the space $M$, whose subjacent group $G$, have endomorphisms $\sigma_{1}, \ldots, \sigma_{\mathrm{n}}$, such that $\sigma_{1} \mathcal{T}(\mathrm{M}), \ldots, \sigma_{\mathrm{n}} \mathcal{T}(\mathrm{M})$, are isotropic then the infinite tensor product of isotropic submanifolds is a isotropic manifold, and is a organized transform equivalent to tensor product of spin representations $\sigma_{1} \mathcal{T}(\mathrm{M}) \otimes \ldots \otimes \sigma_{\mathrm{n}} \mathcal{T}(\mathrm{M}) \otimes \ldots[2,5]$.

\section{Quantum integral transforms: Elimination of distortions and quantum singularities}

One of the quantum phenomena that can form or provoke the conditions of formation of singularities at this level is the propensity of a quantum system to develop scattering phenomena for the appearance of the anomalous states of energy, as antimatter energy or energy of particles of matter in the free state [21], which crowds (a big number of particles overlaps) due to the accumulation of the states of energy of the past or future (to see table 1) [21, 22], which on have different existence time and to having met their corresponding pairs of particles (particle/anti-particle pair) provoke bundles of energy that there form in the space time $M \times \mathrm{I}_{t}$, singularities of certain weight (for mechanisms that can be explained inside the actions in $S U(3)$ and $S U(2)[11,21])$ due to its energy charge [21].

Studies in astrophysics and experiments in the CERN (Organisation Européenne pour la Recherche Nucléaire) establish that a similar mechanism although with substantial differences (known also like Schwinger mechanism) can explain the formation of a singularity such as the fundamental singularity (big-bang). This one establishes that the gravitational field turns into virtual pairs of particle- antiparticle of an environment of quantum gap in authentic pair's particle-antiparticle. If the black hole (singularity of the Universe) is done of matter (antimatter), it might repel violently to thousands of million antiparticles (particles), expelling them to the space in a second fraction, creating an event of ejection very similar to a Big-Bang. Nevertheless, in case of a singularity in the region of space - time of the particles in a quantum ambience is different in that aspect, since the small mass of a singularity *(s), might perform the order of the Planck mass, which is approximately $2 \times 10^{-8} \mathrm{~kg}$ or $1,1 \times 10^{19} \mathrm{GeV}$. To this scale, the formulation of the thermodynamic theory of singularities of the space macroscopic time predicts that the 


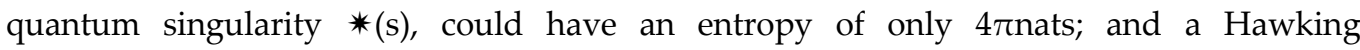
temperature of needing quantum thermal energy comparable approximately to the mass of the finished singularity; and a Compton wavelength equivalent within a radius of Schwarzschild of the singularity in the Universe (this distance being equivalent to the Planck length). This is the point where the classic gravitational description of the object is not valid, being probably very important the quantum effects of the gravity. But there exists another mechanism or thermodynamic limit that is fundamental in the theory of the quantum dispersion and of the formation of quantum singularities.

The past and future in the quantum scattering phenomena

\begin{tabular}{|c|c|c|}
\hline $\mathrm{Z}^{+} / \mathrm{Z}^{-}$ & $\begin{array}{c}\text { Particle } \\
\phi(1)\end{array}$ & $\begin{array}{c}\text { Anti-particle } \\
\phi(-1)\end{array}$ \\
\hline Input & $\begin{array}{c}\bullet \longrightarrow \phi(1) \\
\text { Positive Future }\end{array}$ & $\begin{array}{c}\bullet \longleftarrow-\phi(1) \\
\text { Negative Future }\end{array}$ \\
\hline Output & $\begin{array}{l}\circ \longrightarrow \bullet(1) \\
\text { Positive Past }\end{array}$ & $\begin{array}{l}\circ \longleftarrow-\phi(1) \\
\text { Negative Past }\end{array}$ \\
\hline
\end{tabular}

Table 1.

Proposition 1. The energy of the singularity is born of the proper state of altered energy of the quantum space-time, although without a clear distinction of a suitable path of the particles (without a normal sequence of superposition of (past and future) particles (a path does not exist)). Then in this absence of paths, the singularity arises.

Proof. It is necessary to demonstrate that the past and present particles overlap without a normal sequential order in the causality, passing to the unconscious one $\left(O_{c}=0\right.$, since ${ }^{L} \Im_{0}=0$ or $\omega L=0$ ) for fluctuations of energy that have a property of adherence to the transition of energy states forming energy bundles ([23]) that alter the normal behavior of the particles in the atoms. In effect, this happens when the quantum energy fragments and the photons exchange at electromagnetic level do not take in finished form (there is no exchange in the virtual field of the $S O(3)$ ). For which there is no path that the execution operator $\mathfrak{I}$, (see the section 2) could resolve through of a path integral between two photons (virtual particles). In this case the path integral does not exist. For the adherent effect, the virtual particles that do not manage to be exchanged accumulate forming the altered energy states (an excess very nearby of particles of the certain class (inclusive anti-particles of certain class) they add to themselves to the adherent space of photon (in this case the adherent photon is an excited photon $\operatorname{int} \mathcal{E} \cup \delta(\mathcal{E})$, (where $\mathcal{E}$, is the influence of the singularity) with the infinitesimal 
nearby of points given by $\delta(\mathcal{E})$ [24])) which are the bundles of energy that defines the singularities.

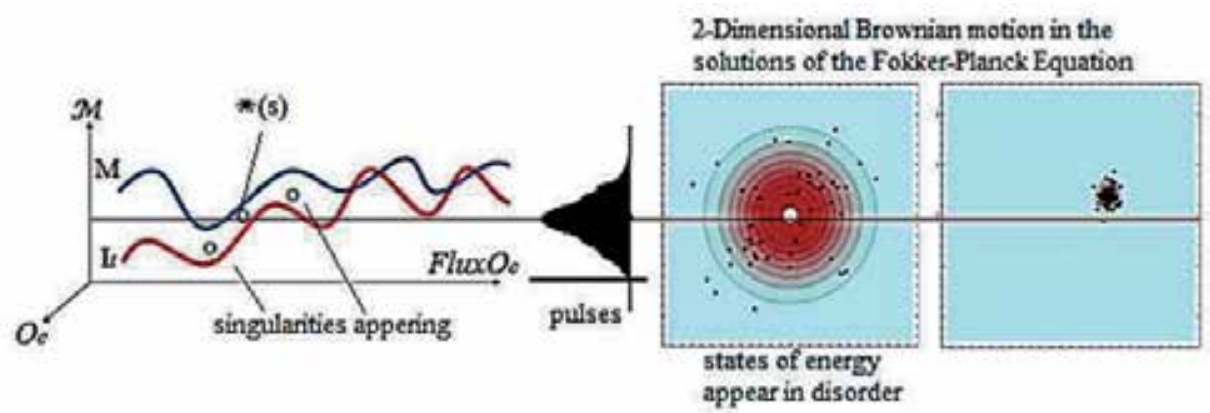

Figure 5. In a). is the elastic band of the space-time $M$, and b), result the pulse solutions of the Fokker-Planck Equation. In c), the singularity is being perturbed by states of different particles interacting and creating big scattering (the red circles are perturbations created by the states of different particles creating scattering with a big level of particles pair annihilating. This produce defecting evolution in every sub-particles and increase of inflation in quantum level of the space-time). In d), the singularity is formed.

Let $\hat{U}_{0}(t, s)$, the operator of evolution [6], of a particle $x(t)$, in the space of transition of the levels of conscience operator $O_{c}$, to all time $t \geq s$. Whose operator limits of $s$ (that is to say, coming to the process of understanding of a concept, (border conditions of $\left.\hat{U}_{0}(t, s)\right)$ ), satisfy

$$
\lim _{t \rightarrow s^{+}} \hat{U}_{0}(t, s)=1,[31]
$$

But there are waves of certain level (Table 1), that act like moderators of wave length to the operator of evolution defined by $\psi(s)$, satisfying that

$$
\hat{U}_{0}(t, s) \mid \psi(s)>=\left\{\begin{array}{cc}
\mid \psi(s)>, & t \geq s \\
0, & t<0
\end{array},\right.
$$

having then that the singularity *(s), that is object of quantum transformation to along of the time, is that product obtained by the integral transform

$$
*(s)=\int_{X(C)} O_{C}(x(t)) w(s, t) d t=\left(\mathrm{E}^{+}-\mathrm{E}^{-}\right) \sqrt{\frac{m}{2 \pi \mathrm{i} n}} \int_{-\infty}^{\infty}\left\{\int_{\mathrm{C}} \mathrm{e}^{\frac{\mathrm{i} m\left(x-x^{\prime}\right)^{2}}{2 \pi}} \hat{U}_{0}(s, t) d x\right\} d t,
$$

where the singularity changes the time $t \geq s$, for the evolution operator $\hat{U}_{0}(t, s),[11]$, due to the evolution of the quantum system in the space-time. But by evolution operator $[6,21]$, this evolution comes given by the anomalous energy $\left(E^{-}-E^{+}\right)$[6] (see figure 3), which establishes the energy load functions $w^{+}(s, t)-w^{-}(s, t)$, that define the energy load function $w(s, t)$, at time $t \geq s$, where $w(s, t)=\left(\mathrm{E}^{-}-\mathrm{E}^{+}\right) U_{0}(s, t) \mathrm{E}^{-}$and $\mathrm{E}^{+}$, are amplitudes of the curves $\mathrm{M}$ $=\mathrm{I} t x(s), \mathrm{I} t=\mathrm{M} x^{-1}(s), \forall$ and $\mathrm{M}, \mathrm{I} t$, functions of evolution curves of space-time see figure 3$)$. 
Then a corrective action is the inverse transform that transform the energy load function in energy useful to the process of re-establishment on the quantum space (remember that it is necessary to release the bundle of energy captive). How this inverse transformation realise?

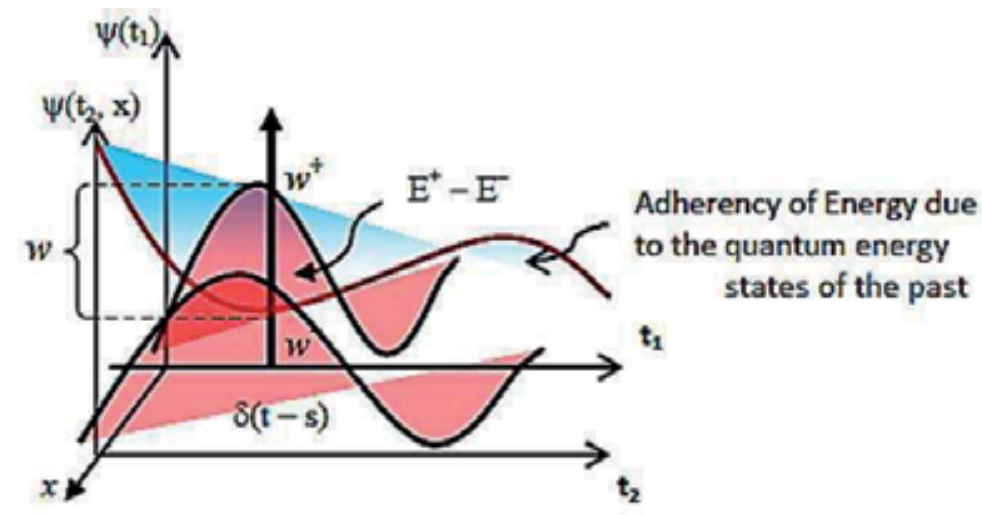

Figure 6. a). This is the graph that shows the formation of singularities of quantum type by the energy load (positives and negatives), rational amplitudes (extremes and defects), and bundle energy created and defined by $\mathrm{E}^{-}-\mathrm{E}^{+}$. The load is pre-determined by the impulse $\delta(t-s)$, which focalize the field action. The adherence zones are propitious to the formation of singularities with all conditions described. The wave function $\psi$, stays under constant regime in space evolution, which is $\psi=\psi(x, 0)$. In the second wave, the corresponding wave function is for other transition time (other evolution space), under the same conditions.

Lemma 1. Let $\mathcal{M}=M \times I_{t}$, the unlimited space of the quantum space (Fock space [24]). A particle $x(t)$, that is focalized by a bad evolution given for the energy load function $w(t, s)$, comes given for

$$
x(s)=\phi x(t) d x(t)=\int_{-\infty}^{+\infty} \delta(t-s) x(t) d t,
$$

Then to time $t=s$, begin the singularity.

Proof. We consider the function $w(t, s)$, like a Green function on the interval $t \geq s$. Given that this function is focalised for the emotional interpretation which is fed by the proper energy of the deep quantum energy (since it produce an auto-disipant effect that deviates the evolution of every particles [23]), then $\mathrm{O}_{c}\left(x(t), x^{\prime}(t)\right) x(t)=\nabla^{2} w(t, s)=\delta(t-s)$ [25]. By the nature of Green function of the weight function $w(t, s)$, we have

$$
\begin{aligned}
& \int_{M} \phi\left(x^{\prime}\right) x(t) d x(t)=\int_{I_{t}}\left[\int_{M} \phi(x) \delta\left(x-x^{\prime}\right) d x(t)\right] x(t) \mu_{t}=\int_{-\infty}^{+\infty} O_{c}(x(t)) x(t) \mu_{t} \\
& =\int_{-\infty}^{+\infty} \nabla^{2} w(s, t) x(t) \mu_{t}=\int_{-\infty}^{+\infty} \delta(t-s) x(t) d t
\end{aligned}
$$

Then all particle $x(t)$, in the space-time $M \times I_{t}$, affected by this regime to time $t=s$, and after, take the form (that is to say, to past and future particles) 


$$
\int_{-\infty}^{s} O_{c}(x(t)) x(t) d t+\int_{s}^{+\infty} O_{c}(x(t)) x(t) d t=0+\int_{s}^{+\infty} \delta(t-s) x(t) d t,
$$

where the first integral is equal to cero, because there is no singularity before $s$, (the evolution happens after the time $t \geq s$ (see (49)). But this evolution is anomalous, since to all $t$ $<s$, includes a captive energy not assimilated to $t=s$ (this because it does not have a conscience operator at this moment (part of (28) defined by $\left.\mathrm{O}^{0} \mathrm{c}\right)$ ). Then

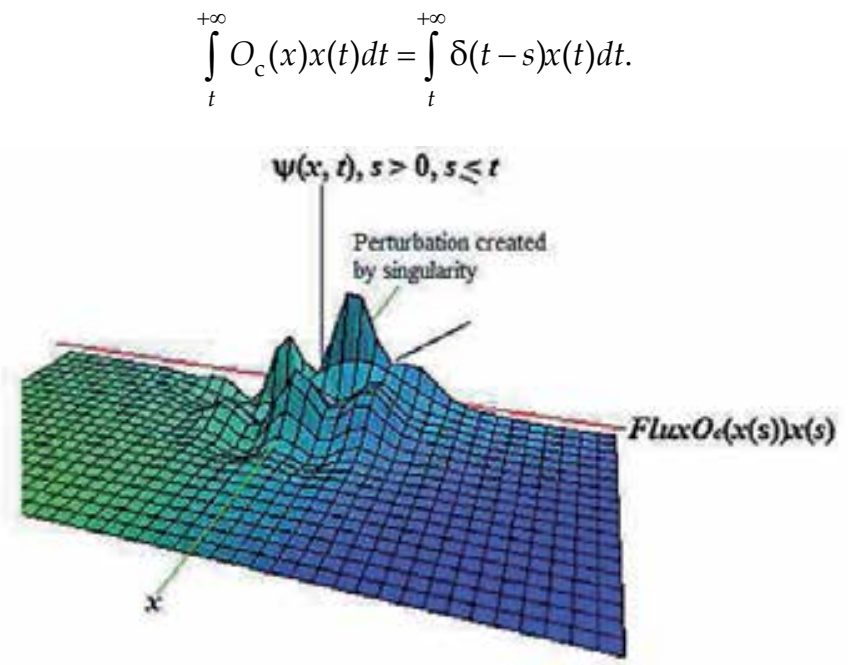

Figure 7. The surface represents the perturbation given by the existence of a singularity in the space-time of the quantum zone. The computational model obeys the solution of Planck-Fokker partial differential equation. The waves of perturbed state begins to $s \leq t$, such as it was predicted in the lemma 1. The surface include the waves established in figure 3, given by $\left.z=\operatorname{Plot} 3 D\left(\exp \left(-1 / 2\left(x^{\wedge} 2+y^{\wedge} 2\right)\right)(\cos (4 x)+\sin (2 x)+3 \sin (1 / 3 y)+\cos (5 y)) \ln (2 x)\right)\right)$, through the space-time 4.0 program. Observe that is present a kernel of transformation for normal distribution given by $\exp \left(-1 / 2\left(x^{2}+y^{2}\right)\right)$ that will appear in the transform that defines the singularity when a particle is not appropriately assimilate. The normal distribution kernel is the statistical weight that establishes the appearance of an abnormal evolution created by the existence of the singularity. That is to say, the singularity is detected by the anomalous effects that are glimpsed in the flux of the operator $O_{c}$, and that are observed in the surface bundle.

Theorem. 1. (F. Bulnes). Let consider a conscience operator with singularity $O_{c}($ * $)$, for the presence of an energy load $w(s, t)$. Then the elimination of the singularity $*(s)$, comes given for

$$
x(t)=\text { correction + restoring }=\int_{X(C)} O_{c}(*(s)) w(t, s) d t=\operatorname{dim} \Lambda \int_{C}\left\{\frac{1}{A} \prod_{j=1}^{\infty}\left\{\int_{-\infty}^{+\infty} \phi\left(n_{j}\right) F\left(n_{j}\right)\right\} d x(t)\right\},
$$

where $\operatorname{dim} \Lambda(\alpha)$, is the Neumann dimension corresponding to the Weyl camera of the roots $\alpha_{\mathrm{j}}$, [26, 27], used in the rotation process to eliminate the deviation [11], created by the singularity.

Proof. Consider an arbitrary irreducible diagram with nodes with $w$-parts (parts of diagrams with nodes of weight $w(t, s))$. Suppose that this points "nodes" with weight $w$, determines the singularity given by (50). In fact, by the theory of Van Hove on the singularities in the 
thermodynamic limit [23], each transition matrix of energy states has a correspondence with the product of Hermitian matrices of the corresponding evolution operators that to this case on a node en $t=s$, are given by $v_{\alpha}\left(\mathrm{s}^{\prime}\right)=e^{i s^{\prime} h 0} v_{\alpha} e^{-i s^{\prime} h}$, where we have used the lemma 1 , to the arising of the quantum impurity in the space-time $\mathbb{M}$ (quantum singularity) in the image space of the conscience operator $\mathrm{TM}^{*}$, located in $\mathbb{R}^{3} \times \mathrm{I}_{t}$, in the point or node $(\cong \mathcal{G}[12,23](w$ diagram)) $t=s$, corresponding of root space $\Phi_{\alpha}$. Then considering a irreducible diagram containing a $w(t, s)$-part, their contribution will be contained in $\mathbf{r}-\mathbf{R}$-space (which is the $\mathbf{E}^{+}$ - $\mathrm{E}^{-}$-space (see figure 2)) [23] by the function $\delta(t-s)$ :

$$
x(t)=\int_{C}\left\{\prod_{j=1}^{\infty} \delta^{(3)}\left(\mathbf{r}^{j}\right) e^{-\mathrm{i} H_{0} t} \hat{U}(t, s)\right\} d^{3} r^{j} d^{3} R^{j} e^{\mathrm{i} H_{0} s} n_{\mathbf{r}}\left(\mathbf{R}, s^{j}\right),
$$

to jth-particle in the interaction of a jth-thought to time $s$. The diagrams drawn here (figure 3) may be interpreted to represent the evolution of particles contributing the position density matrix $n_{\mathbf{r}}(\mathbf{R}, t)$ at $t=t$, in the corresponding path integral of correction. The true correction comes given by the evolution created in the quantum process of transformation of the functional $O_{c}(* *(s))$, where the information $*(s)$, must be changed when $\lim _{t \rightarrow s} \pm \mathrm{i} w(t, s)=n(s)$ (is to say, when $\left.M=I_{t}\right)$. If we call $t_{j}=s$, (j-th-time of evolution in the thought $\stackrel{t \rightarrow s}{\text { process }}$ ) then the integral (53), takes the form

$$
\left.e^{-\mathrm{i}\left(t-t_{\mathrm{j}}\right) H_{0}}\left\{1+\sum_{1}^{\infty}(-\mathrm{i}) \lambda \int_{0}^{j t_{1}} d t_{1} \int_{0}^{t_{2}} d t_{2} \cdots \int_{0}^{t_{\mathrm{j}-1}} d t_{j} \mathrm{v}\left(t_{1}\right) \mathrm{v}\left(t_{2}\right) \cdots \mathrm{v}\left(t_{j}\right)\right\}=\prod_{j=1}^{\infty}\left\{\int_{-\infty}^{+\infty} \phi\left(n_{j}\right) F\left(n_{j}\right)\right\} d x(t)\right\},
$$

where the states $\phi$, are established in the density matrix $\underline{n}(0)$, that to a vertex of $\mathcal{G}, \quad(w$ diagram), arrange, those perturbations $B$ (see figure 3 ), that they gave origin to the singularity, with the corresponding arrange of those positive perturbations $A$, that will realises the corrective action to transform the singularity signal $*(s)$, of an adequate thought given by $x(t)$. Due to that, the information given by the product $A \underline{n}(0) B$, must be changed when $\lim _{t \rightarrow s} \pm \mathrm{i} w(t, s)=\overline{n(s)}$, then a $w$-diagram must be change for $\Phi \alpha$-diagram [27]. But this live in the quantum field of the space-time $\mathrm{TM}^{*}$, that is to say, in the corresponding zone of the executive operator $\mathfrak{I}$. Then in the material space-time (Einstein universe), the displacement of energy needs inside this transformation the application of an invariant given in the quantum space that guarantee that the new particle (boson) obtained let that correct. This is given by number $\operatorname{dim} \Lambda(\alpha)$, since it depends on the roots system to the representation of the corresponding action group [27], that recover the recognition action. By the integral (4), the transformation due to the new conscience operator created in $\mathrm{TM}^{*}$ zone obtained on whole the space-time is,

$$
\int_{X(C)} O_{c}\left(\frac{x(t)}{\operatorname{dim} \Lambda}\right) d x(t)=\int_{X(C)}\left\{\frac{1}{A} \prod_{j=1}^{\infty}\left\{\int_{-\infty}^{+\infty} \phi\left(n_{j}\right) F\left(n_{j}\right)\right\} d x(t)\right\},
$$

that is the result wanted. 


\section{Re-composition and determination of the realities}

We consider the space-time $\mathbb{M}$, like space where $\mathbb{R}^{\mathrm{d}} \times \mathrm{I}_{t}$, is the macroscopic component of the space-time and we called $\mathbb{F}$, the microscopic component of the space-time of ratio $10^{-33} \mathrm{~cm}$ (length of a string [21]). For previously described the quantum zone of the space-time $\mathcal{M}$, is connected with $\mathcal{N}$, which will called virtual zone of the space-time (zone of the space-time where the process and transformation of the virtual particles happen) are connected by possibilities causal space generated by certain class of photons and by the material particles interacting in the material space time, with permanent energy and the material particles recombining their states they become in waves on having moved in $\mathbb{R}^{\mathrm{d}} \times \mathrm{I}_{t}$, on any path of Feynman. Likewise we can define the space of this double fibration of quantum processing as:

$$
\mathcal{L}=\left\{O_{c}\left(\phi, \partial_{\mu} \phi, x(t), t\right) \in C^{2}\left(\mathrm{R}^{d} \times I_{t}\right) \mid \frac{\partial^{2}}{\partial t^{2}}-\nabla^{2}\left(O_{c}\left(\phi, \partial_{\mu} \phi, x(t), t\right)\right)=0\right\},
$$

with the states $\phi$, of quantum field are in the quantum zone $\mathcal{M}$. Let $\mathcal{N}$, the ambi-space (set of connection and field) defined as:

$$
\mathcal{N}=\left\{(X, \nabla) \in M \times L \mid \nabla_{X^{\prime} Y^{\prime}}^{X Y} \Psi+\Phi(X)=0\right\}
$$

where $\nabla$, is the connection of virtual field $X$, with the quantum field $Y$, and $\Psi$, is the field whose action is always present to create perceptions in the quantum zone connected with $\Phi$ (2-form)[28]. Then we can create the correspondence given by the double fibration [29]:

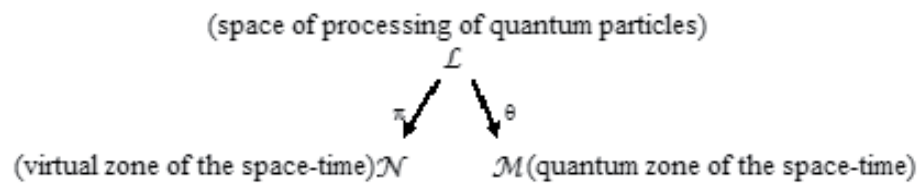

This double fibration conformed the interrelation between $\mathcal{M}$, and $\mathcal{N}$. $\forall x(t) \in \mathcal{M}$, give beginning to a complex submanifold (that represents the spaces where are the quantum hologram) that includes all these quantum images given by quantum holograms, why? Because this complex submanifolds, considering the causal structure given in the space-time by the light cones (see figure 6 a)) [26], of all trajectories that follow a particle in the spacetime [29], they can write using (57) as:

$$
\Theta_{x}=\theta\left[\pi^{-1}(x)\right]
$$

of $\mathcal{N}$, such that $\Theta_{\mathrm{x}} \cong \mathbb{P}^{1} \times \mathbb{P}^{1}$, which by space-time properties to quantum level represent the space of all light rays that transit through $x$, conforming a hypersurface (projective surface) that is a light surface. This surface is called the sky $x$ [30]. A sky in this context represents the set of light rays through $x$ (bosons) that it comes of the virtual field. 
If $\mathcal{M} \cong \mathbb{C}^{4}$, then $\mathbb{M}=\mathcal{M} \times \mathrm{Q}_{x}$, is the complete universe (include the cosmogonist perception by the super-symmetry specialist [31]). But, what is there of our quantum universe with regard to our real universe (included the material part given by the atoms)?

The answer is the same, we have an universe of ten dimensions and $M=\mathcal{N} \times \mathcal{M}$, where the quantum representation of the object $x(s)$, is the quantum space-time $\mathcal{M}=\mathbb{R}^{3} \times I$, (which is the Einstein cosmogonist perception) then the cosmo-vision of the virtual particles is $\mathbb{C}^{2} \times \mathrm{Q}_{x}$, [21], then the execution operator $\mathfrak{I}$, that proceeds to connect virtual particles through the paths which have path integrals on double fibration, establishing the material-quantumvirtual connection required to a total reality:
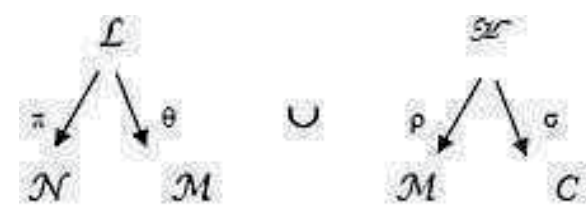

where $C$, is the material part connected with the quantum zone of the space-time (space taken by atoms) $\mathcal{M}$. The corresponding path integral that connects virtual particles in the whole fibration is the integral of line type (5) defining feedback connection:

$$
\mathrm{I}\left(\mathfrak{I}_{\mathrm{Q}_{x}}(x(s))\right)=\oint_{\Gamma} O_{\mathrm{c}}\left(\theta\left(\pi^{-1}\left(\sigma\left(\rho^{-1}(x)\right)\right)\right) \mu_{s^{\prime}}\right.
$$

always with the space $\left\{x(s) \in \mathcal{M} \mid \Theta_{x} \subset \mathcal{N}\right\}$, to the permanent field actions. Then the reality state is the obtained through the integral of perception (60), considering the fibre of the corresponding reality in the argument of the operator $O_{c}$, of the integrating from (60).

\section{Applications to the nanosciences}

\subsection{Nanomedicine}

The integral medicine into of the class of alternative medicine, fundament their methods of cure in to health and reactive the vital field $X$, of the human body $B$, the regeneration of the centers of energy of $B$, and the corrections and restoration of the flux of energy Flux, in and in each organ $\mathbf{B}$, of the human body $B$, taking constant of gradient of their electromagnetic current, voltage and resistance, obtaining of this manner, the balance of each organ in sunstone with the other organs to characterize to $B$, like complete synergic system in equilibrium and harmony [10].

Now, the cure that is realized to nano-metric scale must be executed with a synergic action of constant field [33], equal to effect in each atom of our body to unison of real conscience of cure (duality mind-body [11]). Of this way, the conscience of $B$, is the obtained synergy by the atoms in this sense and that will come reflected in the reconstitution of the vital field $X$. 
Then under this reinterpretation, the sickness is only an effect of the fragmentation of this real conscience of cure of $B$, that is deduced by disconnections and disparity of atoms [11]. The integral medicine helps to recover the continuity of this conscience through of the electronic memory of health of the proper body $[11,22]$ (see the figure $6 \mathrm{c}$ )).

\subsection{Quantology and neurosciences}

Let $\mathfrak{A l l}$, the mind space and their organic component (material component) the brain space c. Also we consider the quantum component of the mind given by the space-time $\mathcal{M}$. Studies realised in statistical mechanics have revealed that the Bose-Einstein statistics stretches to accentuate the low energy levels. This reflects his closeness to the emission of a virtual field, where the virtual particles are not detected in a virtual energy sea. This allows to surmise that the radiation that takes place from the virtual field to the quantum field of $\mathfrak{A l}$, is composed by photons type bosons (that is to say it obeys this Bose-Einstein statistics), since the quantum field interacts with the material particles that contains the material field of the mind which is anchored in the brain c, like material organ.

Theorem (F. Bulnes). [21] The total Lagrangian of mental field comes given by the superior action whose total conscience is

$$
\mathrm{O}_{\text {total }}=\mathrm{O}_{\mathrm{QCD}}\left(\mathrm{O}_{\mathrm{EM}}\right)
$$

to one total action defined by the groups $S U(3)$, (quantum and virtual field) and $S U(2)$, (material field).

Proof. The Lagrangian of the theory is an invariant of Lorentz and invariant under local transformations of phase of the group SU(3), (for the charge of color) and has the following form [31]:

$$
\mathrm{L}_{\text {TOTAL }}=\left\{\bar{q} \mathrm{i} \gamma^{\mu} \partial_{\mu} q-\bar{q} m q-b \bar{q} \gamma^{\mu} \mathrm{T}_{a} q \mathrm{~b}_{\mu \nu}^{a}-\frac{1}{4} \mathrm{~b}_{\mu \nu}^{a} \mathrm{~b}_{a}^{\mu \nu}\right\},
$$

This corresponds to the space of the mind $\mathfrak{A l}=\mathcal{M}+\mathrm{C}$, where $O_{E M}$, put in C, (neurological studies have proved that the process of thought in the level at least visible is of electromagnetic type) signals through charges in the synapses and neurons of c. Nevertheless these charges produce in one level deeper. The tensor $\mathfrak{b}^{\mathfrak{a}}{ }_{\mu v}$ is anti-symmetric and represent a bosonic field created by the interaction of quarks $q, p=\bar{q}$, and $[p, q]=\bar{q} \mathrm{q}-\mathrm{q} \bar{q}$. Whose bosonic field has all the particles of spin 0 , and the trace of tensor $\mathfrak{b}_{\mu \nu}{ }_{\mu \nu}$, has electromagnetic components conformed by the photons that are stable on the limit of the transformation $\mathrm{q}(x(s)) \mathrm{I} \rightarrow$

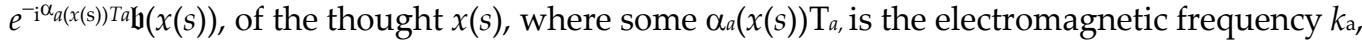
of the term $i k_{\mathrm{a} v}[7,1026]$, where in these cases the last term in the second member of (62), is $1 / 4 F_{\mu \nu} F^{\mu \nu}$. Then the $S U(2)$-actions are included in the $S U(3)$, actions and their points are electromagnetic particles transformed by these rules like photons (= thoughts in $\mathfrak{A t l}$ ) [28]. 


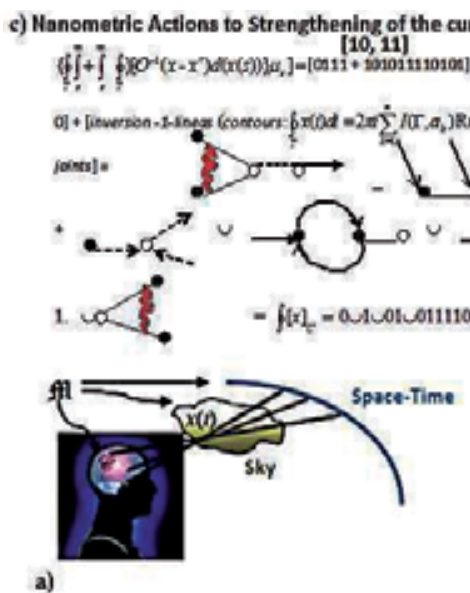

a)

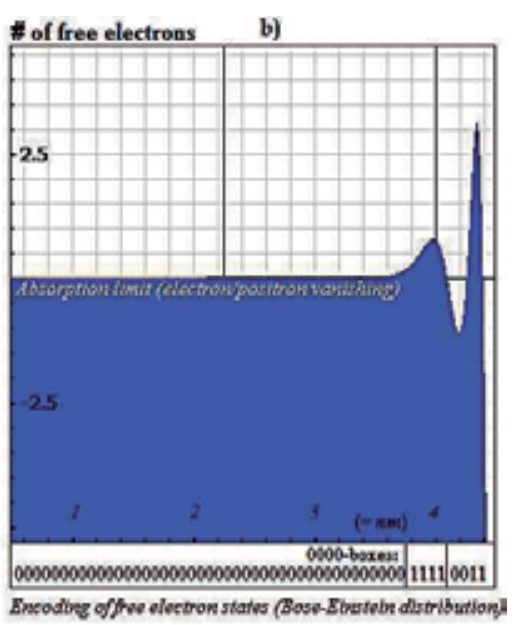

Figure 8. a). Concept of the topological space $(s k y[21,30])$ and the cosmogonist perceptions of the mind. The conscience operator of the different realities is given by (60). b) Modelling from boson-shape distribution in localizing of symptoms in the mind by cerebral signals with low interchange electrons [24, 25, 32, 33]. Bose-Einstein distribution was published in [22]. c) Total Action due Gelsem including the action de Bervul and Ignamara. The corresponding quantum intelligence code of this total action is given by 01111010110110110110111110 , [7]. The corresponding code given in black include de codes of Gelsem and Bervul. The code in orange is the code of Gelsemium equal to code of Gelsem. This quantum code was used to cure a patient with a digestive illness [11, 21, 22].

\subsection{Electromagnetic vehicle with levitation magnetic conscience}

Considering some applications of quantum electrodynamics in the design of flaying vehicles self-supported and their magnetic levitation, we find the magnetic conscience operator is defined for the transmitting of the diamagnetic property every particle of the ship structure.

This vehicle is controlled by one microchip that is programmed by conscience operators algebra of electromagnetic type that leads to the flow of Eddy currents, the iso-rotations and suspension of the special geometrical characteristics vehicle, generating also on the vehicle structure certain "magnetic conscience" that provokes all movements like succeeding the sidereal objects in the universe [26,34]. This magnetic conscience is generating by the proper particles of the ship structure transmitted for the interaction of superconductor inside the reactor with the magnetic field generated by the rotating rings under the ship. By so doing, the Eddy's currents in the "skin effect" around the structure of the ship are given by the actions [35]

$$
\mathfrak{I}_{\text {ship }}=\mathfrak{I}_{M}+\mathfrak{I}_{\text {rot }}
$$

and using the quantum $\mathbb{E} \otimes$ 每-fields to create (magnetic conscious operator given by the integrals [36])

$$
H\left(A, \mathfrak{J}_{M}\right)=\int_{\text {ship }}\left\{L_{M}-\mathrm{H}^{2} / 8 \pi\right\} d \mathrm{~V},
$$




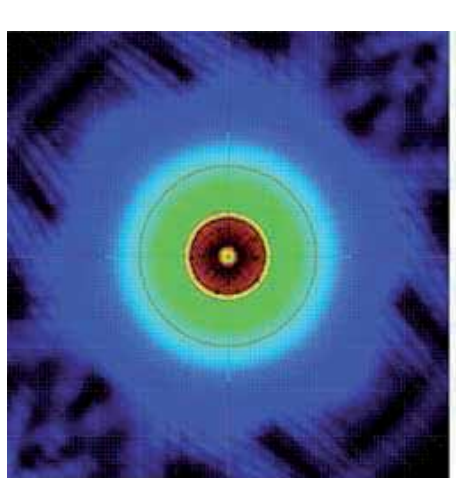

(a)

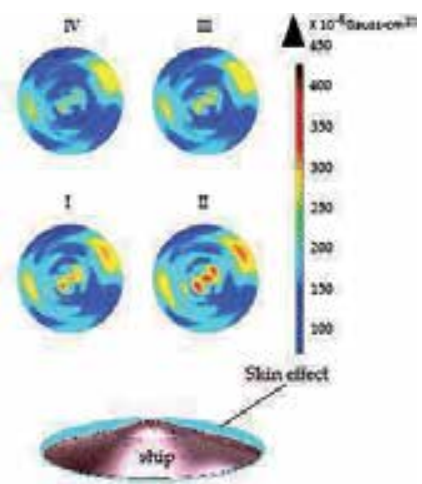

(b)

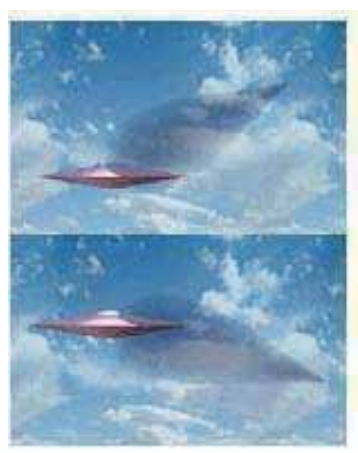

(c)

Figure 9. a). The green color represents the state of quantum particles in transition to obtain the anti-gravity states through the interaction of the $\mathbb{E} \otimes$ 䄸-fields used on the structure of the vehicle. The blue flux represents Eddy's currents that interact with the lines of magnetic field to produce an diamagnetic effect in the top part of the vehicle. The red central ring is the magnetic field that generates the twistor surface (this computational simulation is published in $[35,36])$. b) Top part of the flying plate showing the generation of the effect skin obtained in the interaction superconductor - magnetic field. The regions in light and obscure blue represent the flow of Eddy's currents, in this top part of the vehicle. The Skin effect is derived by the quantum interaction by the actions of $\mathfrak{E} \otimes \mathbb{I _ { 1 }}$ $[26,37]$. c) The microscopic effects of the electromagnetic fields (quantum densities of field) created inside the algebra $\mathbb{E} \otimes \mathbb{Z}$, create an effect of macro-particle of the vehicle [36] (the vehicle and their electromagnetic revetment behaves like a particle) where their displacement is realized in instantaneous form and their direction it is a macro-spin projected from the magnetic conscious operator (from $\mathfrak{\Im}_{M}$, from (63)) of the ship which defines their angular moment $[35,36]$.

\section{Apendix}

\section{Technical notation}

$O_{c}$ - Is an operator that involves the Lagrangian but directing this Lagrangian in one specific fiber (direction) prefixing tha Lagrangian action in one direction. This is defined as the map: $O_{c}$ $:$ TM $\rightarrow \mathrm{TM}^{*}$, with rule of correspondence $w \mapsto O_{\mathrm{c}}(v) w$, where $w=L(v)$, with $L$, the classic Lagrangian. This defines the quantum conscience. If we locally restrict to $O_{c}$, that is to say, on the tangent space $\mathrm{T}_{x} \mathbb{M} \times \mathrm{T}_{x} \mathbb{M}, \forall x \in \mathbb{M}(\cong \Omega(\Gamma))$, we have that

$$
O_{c}: \mathrm{T}_{x} \mathrm{Mr} \times \mathrm{T}_{x} \mathrm{MI}(\stackrel{\cong}{\text { locally }} \text { TMI }) \longrightarrow \mathrm{TM}^{*},
$$

with rule of correspondence

$$
(v, w) \longrightarrow O_{c}(v) w,
$$

$O_{\mathrm{c}}(v) w$, generalise the means of $O_{\mathrm{c}}(v) v=L(v), \forall v \in \mathrm{T}_{x} \mathbb{M}, \forall \mathbf{x} \in \Omega(\Gamma)$. Likewise, if $\mathfrak{I}: \mathrm{TM} \rightarrow \mathbb{R}$, with rule of correspondence $L(v) \mapsto \Im(L(v))=O_{C}(v) v$, then the total action along the trajectory $\Gamma$, will be 


$$
\mathfrak{I}_{\Gamma}=\int_{\Omega(\Gamma)} O_{\mathrm{c}}(v) v=\int_{\Omega(\Gamma)} L(v),
$$

In the forms language, the conscience operator comes given by the map $\omega_{L}: \mathrm{TM} \rightarrow \mathrm{TM}^{*}$, with rule of correspondence given by (19). The quantum conscience shape a continuous flux of energy with an intention, involving a smooth map $\pi$ (defined in the example 1). Then the conscience operator is related with the action $\mathfrak{I}$, and the trajectories $\gamma_{t}$, through of the following diagram:

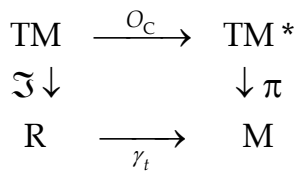

$O_{c}(*)$ - Conscience Operator in the singularity *. This is a kernel of the quantum inverse transfom of path integrals to eliminate singularities. Their direct transform use the kernel $O_{c}(x(t))$.

$\mathcal{O}_{0}\left(Z_{i}, p_{A}\right)$ - Vertex operator of the theory given by the equations $\mathrm{W}^{a}\left(Z_{i}\right)=0$, localized at the points $p_{A}$, of the Riemann surface $S$.

$\mathcal{O}\left(x-x^{\prime}\right)$ - Is the functional operator $\mathcal{O}\left(x-x_{j}\right)=\left(\square x+m^{2}-i \varepsilon\right) \delta^{n}\left(x-x_{j}\right)$. This operator involves to electronic propagator in a pulse impulse.

OQCD - Quantum chromodynamics conscience operator. Their Lagrangian density using the quantum chromodynamics is $\mathcal{L}_{E M}=\sum_{n}\left(i h c \Psi_{n} \nabla \psi_{n}-m_{n} c^{2} \Psi_{n} \psi_{n}\right)-1 / 4 G_{\mu \nu}^{\alpha} G_{\alpha}{ }^{\mu \nu}$, Where $\theta$, is the QCD gauge covariant derivative (in Feynman notation $\left.\xi^{\sigma} D \sigma\right), n=1,2, \ldots 6$ counts the quark types, and is the gluon field strength tensor.

$O_{E M}$ - Conscience operator defined through of the Lagrangian to quantum electromagnetic field (these like gauge fields). Their Lagrangian density is $\mathcal{L}_{E M}=$ $i h c \Psi \nabla \psi-m c^{2} \Psi \psi-(1 / 4 \mu 0) F_{\mu v} F^{\mu v}$. where $F^{\mu v}$, is the electromagnetic tensor, $D$, is the gauge covariant derivative, and $\theta$, is Feynman notation for $\xi^{\sigma} D \sigma$.

$O_{\text {total }}$ - Total quantum conscience operator. This is the composition of operators $O E M$ followed OQCD.

$\Im_{\mathrm{Oc}}-$ Action that involves a conscience operator $\mathrm{Oc}_{\mathrm{c}}$

$\Omega^{(k, 0)}$ - Differential form to complex hypersurfaces of dimension $\mathrm{k}$. This form is analogous to the form $\omega L$, and involves the conscience operator $\left(O_{c}\right)^{*}$.

$\Theta_{\mathrm{x}}$ - Fibers of the topological space $\mathrm{Q}_{x}$, called sky conformed by the light rays through $x$ (bosons) that it comes of the virtual field. This is a conscience operator when realises the reality transformation by the double fibration. 
$\mathfrak{J}_{\partial \mathrm{M}^{-}}$Action that have codimension strata $n-k$. This action is due to the differential $d \mathfrak{I}(\phi) h$.

$\mathfrak{J}_{\text {int }_{\mathrm{M}}}-$ Action that have codimension strata $k$. This action is due by $\mathfrak{I}(\phi)$.

\section{Author details}

Francisco Bulnes

Department of Research in Mathematics and Engineering, TESCHA, Mexico

\section{Acknowledgements}

I am grateful with Carlos Sotero, Eng., for the help offered for the digital process of the images that were included in this chapter.

\section{References}

[1] Sobreiro R., editor. Quantum Gravity. Rijeka: InTech; 2012.

http://www.intechopen.com/books/quantum-gravity- (accessed 20 January 2012).

[2] Bulnes F. Analysis of prospective and development of effective technologies through integral synergic operators of the mechanics. In: ISPJAE, Superior Education Ministry of Cuba (eds.) $14^{\text {th }}$ Scientific Convention of Engineering and Arquitecture: proceedings of the 5th Cuban Congress of Mechanical Engineering, December 2-5, 2008, CCIA2008, 2-5 December, 2008, Havana, Cuba.

[3] Bulnes F. Theoretical Concepts of Quantum Mechanics. In: Mohammad Reza Pahlavani (ed.) Correction, Alignment, Restoration and Re-Composition of Quantum Mechanical Fields of Particles by Path Integrals and Their Applications. Rijeka: InTech; 2012. p Available from http://www.intechopen.com/books/theoretical-concepts-of-quantummechanics/correction-alignment-restoration-and-re-composition-of-fields-of-particlesby-path-integrals-and-the

[4] Marsden, JE.; Abraham, R. Manifolds, tensor analysis and applications. Massachusetts: Addison-Wesley; 1993.

[5] Lawson, HB.; Michelsohn, ML. Spin Geometry, Princeton University Press; 1989.

[6] Holstein, BR. Topics in Advanced Quantum Mechanics. CA, USA: Addison-Wesley Publishing Company; 1992.

[7] Feynman, RP; Leighton, RB; Sands, M. Electromagnetism and matter (Vol. II). USA: Addison-Wesley; 1964.

[8] Warner, FW. Foundations of Differential Manifolds and Lie Groups, New York: Springer, 1983. 
[9] Sokolnikoff, IS. Tensor Analysis: Theory and Applications. New York: Wiley and Sons; 1951.

[10] Bulnes F; Bulnes H. F; Hernandez E; Maya J. Integral Medicine: New Methods of Organ-Regeneration by Cellular Encoding through Path Integrals applied to the Quantum Medicine. Journal of Nanotechnology in Engineering and Medicine ASME 2010; 030019(1) 7.

[11] Bulnes F; Bulnes H. F; Hernandez E; Maya J. Diagnosis and Spectral Encoding in Integral Medicine through Electronic Devices Designed and Developed by Path Integrals. Journal of Nanotechnology in Engineering and Medicine ASME 2011; 021009(2)10.

[12] Ionescu LM. Considerations on some algebraic properties of Feynman integrals. Surveys in Mathematics and Its Applications. Vol. 3 (2008). p79-110.

[13] Kontsevich M, Deformation quantization of Poisson manifolds I. In: Joseph A, Mignot F, Murat F, Prum B, Rentschler R.(eds.) Proceedings of the First European Congress of Mathematics, Vol. II, 6-10 July 1992, Paris, France. Progr. Math., 120, Birkhäuser, Basel, 1994.

[14] Kontsevich M. Feynman diagrams and low-dimensional topology. In: Joseph A, Mignot F, Murat F, Prum B, Rentschler R.(eds.) Proceedings of the first European congress of mathematics (ECM), Vol. II, 6-10 July 1992, Paris, France, Progr. Math., 120, Birkhäuser, Basel, 1994.

[15] Bulnes F. Cohomology of Cycles and Integral Topology. In: Bulnes, F. (ed.) Meeting of 27- 29 Autumn 2008, Mexico City, Appliedmath 4, IM-UNAM, Mexico, 2008. www.Appliedmath4.ipn.mx

[16] Griffiths P; Harris J. Principles of Algebraic Geometry. USA: Wiley-Interscience; 1994.

[17] Gross M; Huybrechts D; Joyce D. Calabi-Yau Manifolds and Related Geometries. Norway: Springer, 2001.

[18] Hoogeveen J; Skenderis K. Decoupling of unphysical states in the minimal pure spinor formalism I. JHEP 1001 (2010) 041. [arXiv:0906.3368].

[19] Berkovits N; Hoogeveen J; Skenderis K. Decoupling of unphysical states in the minimal pure spinor formalism II. JHEP 0909 (2009) 035. [arXiv:0906.3371]

[20] Kibbe TWB. Geometrization of Quantum Mechanics. Springer Online Journal Archives 1860-2000 1979; 65(2) 189-201.

[21] Bulnes F; Bulnes HF; Cote D. Symptom Quantum Theory: Loops and Nodes in Psychology and Nanometric Actions by Quantum Medicine on the Mind Mechanisms Programming Path Integrals. Journal of Smart Nanosystems in Engineering and Medicine 2012; 1(1) 97-121.

[22] Bulnes F; Bulnes HF. Quantum Medicine Actions: Programming Path Integrals on Integral Mono-Pharmacists for Strengthening and Arranging of the Mind on Body. Journal of Frontiers of Public Health 2012; accepted. 
[23] Fujita S. Introduction to non-equilibrium quantum statistical mechanics. Malabar, Fla: W. Krieger Pub. Co. 1983.

[24] Simon B; Reed M. Mathematical methods for physics, Vol. I (functional analysis). New York: Academic Press, 1972.

[25] Eleftherios N; Economou E; Economou N. Green's Functions in Quantum Physics. Berlin Germany: Springer, 2006.

[26] Bulnes F, Doctoral course of mathematical electrodynamics. In: National Polytechnique Institute (ed.) Appliedmath3: Advanced Courses: Proceedings of the Applied Mathematics International Congress, Appliedmath3, 25-29 October SEPI-IPN, México, 2006.

[27] Bulnes F, Conferences of Mathematics: Seminar of Representation Theory of Reductive Lie Groups. Mexico: Compilation of Institute of Mathematics (ed.), UNAM Publications, 2000.

[28] Aharonov Y; Bohm D. Significance of electromagnetic potentials in quantum theory. Phys. Rev. 115 (1959) 485-491.

[29] Bulnes F; Shapiro M. On general theory of integral operators to analysis and geometry (Monograph in Mathematics). Mexico: SEPI-IPN, IMUMAM, 2007.

[30] LeBrun ER, Twistors, Ambitwistors and Conformal Gravity. In: Bailey TN, Baston RJ (ed.) Twistors in Mathematics and Physics, UK: Cambridge University; 1990. p71-86.

[31] Hughston LP; Shaw WT. Classical Strings in Ten Dimensions. Proceedings of the Royal Society of London. Series A, Mathematical and Physical Sciences, Vol 414. No. 1847 (December 8). UK, 1987.

[32] Dossey L. Space, Time and Medicine. Boston: Shambhala, USA, 1982.

[33] Truesdell C; Topin RA. The classical fields theories (in encyclopedia of physics, Vol. III/1). Berlin: Springer-Verlag. 1960.

[34] Bulnes F. Special dissertations of Maxwell equations. Mexico: unpublished. Only registered SEP, 1996.

[35] Bulnes F; Hernández E; Maya J. Design and Development of an Impeller Synergic System of Electromagnetic Type for Levitation, Suspension and Movement of Symmetrical Body. Imece2010: Fluid Flow, Heat Transfer and Thermal Systems Part A and B: Proceedings of $11^{\text {th }}$ Symposium on Advances in Materials Processing Science and Manufacturing, 12-18 November2010, British Columbia, Canada, 2010.

[36] Bulnes F; Maya J; Martínez I. Design and Development of Impeller Synergic Systems of Electromagnetic Type to Levitation/Suspension Flight of Symmetrical Bodies. Journal of Electromagnetic Analysis and Applications 2012 1(4) 42-52. See in: http://www.scirp.org/journal/PaperInformation.aspx?paperID=17151

[37] Bulnes F. Foundations on possible technological applications of the mathematical electrodynamics. Masterful Conference in Section of Postgraduate Studies and Research (SEPI), National Polytechnic Institute, Federal District, Mexico 2007. 


\section{Section 4}

\section{Perturbation Theory}





\title{
Convergence of the Neumann Series for the Schrödinger Equation and General Volterra Equations in Banach Spaces
}

\author{
Fernando D. Mera and Stephen A. Fulling \\ Additional information is available at the end of the chapter
}

http://dx.doi.org/10.5772/52489

\section{Introduction}

The time-dependent Schrödinger equation, like many other time-evolution equations, can be converted along with its initial data into a linear integral equation of Volterra type (defined below). Such an equation can be solved formally by iteration (the Picard algorithm), which produces a Neumann series whose $j$ th term involves the $j$ th power of an integral operator. The Volterra structure of the integral operator ensures that the time integration in this term is over a $j$-simplex, so that its size is of the order of $1 / j$ !. One would therefore expect to be able to prove that the series converges, being bounded by an exponential series. The difficulty in implementing this idea is that the integrand usually is itself an operator in an infinite-dimensional vector space (for example, representing integration over the spatial variables of a wave function). If one can prove that this operator is bounded, uniformly in its time variables, with respect to some Banach-space norm, then one obtains a convergence theorem for the Neumann series. This strategy is indeed implemented for the heat equation in the books of the Rubinsteins [1] and Kress [2]. The objective of the thesis [3] was to treat the Schrödinger equation as much as possible in parallel with this standard treatment of the heat equation. This article reports from the thesis a summary of the rigorous framework of the problem, the main theorem, and the most elementary applications of the theorem.

We stress that the situation for time-evolution equations is different (in this respect, nicer) than for the Laplace and Poisson equations, which are the problems studied in most detail in most graduate textbooks on partial differential equations, such as [4]. In that harmonic potential theory the problem is similarly reduced to an integral equation, but the integral equation is not of Volterra type and therefore the Neumann series does not converge 
automatically. The terms are bounded by a geometric series but not an exponential one, so to prove convergence it is not enough to show that the Banach-space operator has finite norm; the norm would need to be less than unity, whereas in the PDE application it turns out to be exactly unity. Therefore, in the theory of elliptic PDEs the Neumann series is not used to prove existence of a solution; instead, the Fredholm theory is used to prove existence more abstractly. In time-evolution problems the concrete convergence of the series gives rigorous meaning to formal constructions used by physicists, such as path integrals and perturbation series.

The similarities between the Schrödinger equation and the heat equation were used in [3] to create a theoretical framework for representing and studying the solutions to the Schrödinger problem, which is summarized here. As much as possible, we use the books [1,2] as guides to treat the quantum problem like a heat problem. However, the parallel between the heat equation and the Schrödinger equation is a limited one, because the exponential decay of the heat equation's fundamental solution is not available here. Therefore, different formulations and proofs needed to be constructed for the basic representation theorems in section 2, as well as for the main theorem in section 4 . For example, the Poisson integral formula (14) with the Schrödinger kernel (11) is shown to hold in the "Abel summable" sense [5, Sec. 1.5][6, Sec. 6.2].

Section 2 is devoted to the basic integral representation of a solution of the Schrödinger equation in terms of prescribed data and the fundamental solution (11). Here, unlike [3], we do not consider boundary-value problems, so the representation consists of two terms, a Poisson integral incorporating the initial data and a source integral. (In a boundary-value problem there is a third term incorporating boundary data.) For the free Schrödinger equation (6) with a known nonhomogeneous term $F(x, t)$, the source integral (10) simply gives the contribution of $F$ to the solution. In the more interesting case of a homogeneous equation including a potential, $F$ involves the unknown function (multiplied by the potential), so the representation theorem yields an integral equation that must be solved. The crucial feature of the integral operator in (10) is that the upper limit of the time integration is $t$, the time variable of the solution, rather than $+\infty$ or some constant. This is the Volterra structure that causes the iterative solution of the equation to converge exponentially. Thus the initial-value problem for the Schrödinger PDE has been expressed as a Volterra integral equation of the second kind with respect to time. Our main task is to use the Picard-Neumann method of successive approximation to construct the unique solution of this integral equation. The abstract theory of such iterative solutions for linear operators in arbitrary Banach spaces is outlined in section 3 .

The main theorem is proved in section 4 . It treats a Volterra integral equation for a function of $t$ taking values at each $t$ in some Banach space, $\mathcal{B}$, such as $L^{2}\left(\mathbb{R}^{3}\right)$. More precisely, one has bounded operators $A(t, \tau): \mathcal{B} \rightarrow \mathcal{B}$, with the bound independent of the time variables, that satisfy the Volterra property that $A(t, \tau)=0$ unless $\tau<t$. It can then be proved inductively that the $j$ th term of the Neumann series has norm proportional to $t^{j} / j !$. The conclusion is that the series converges in the topology of $L^{\infty}((0, T) ; \mathcal{B})$ for $t<T$. A variant with $L^{\infty}$ replaced by $L^{p}$ is also given.

In section 5 the main theorem is applied to some simple and familiar cases. First, we consider classical integral equations, such as one with a kernel that is Hilbert-Schmidt in space and Volterra in time. Then we return to the Schrödinger problem set up in section 2, with a 
bounded potential function. In that case the unitarity of the free Schrödinger evolution operator between fixed times is the key to proving boundedness of the integral operator, and the resulting Neumann series is a standard form of time-dependent perturbation theory.

\section{The Poisson integral and source integral theorems}

The wavefunction $\Psi(x, t)$ of a nonrelativistic particle in $\mathbb{R}^{n}$ is a solution to the Schrödinger equation,

$$
H \Psi(x, t)=i \hbar \partial_{t} \Psi(x, t),
$$

where $H$ is the Hamiltonian, given by

$$
H=H_{0}+V \equiv \frac{1}{2 m} p^{2}+V(x, t) \equiv-\frac{\hbar^{2}}{2 m} \Delta_{x}+V(x, t)
$$

In the "free" case, $V(x, t)=0$, the equation becomes

$$
i \hbar \partial_{t} \Psi(x, t)=-a^{2} \Delta_{x} \Psi(x, t), \quad \forall(x, t) \in \mathbb{R}^{n} \times \mathbb{R},
$$

where

$$
a^{2}=\frac{\hbar^{2}}{2 m}
$$

For the differential operator appearing in (3) we introduce the notation

$$
L=a^{2} \Delta_{x}+i \hbar \partial_{t}
$$

Unlike the corresponding operator for the heat equation, $L$ is formally self-adjoint with respect to the usual $L^{2}$ inner product.

We now consider the more general equation

$$
L u(x, t) \equiv a^{2} \Delta_{x} u(x, t)+i \hbar \partial_{t} u(x, t)=F(x, t)
$$

again in all of space-time. If the source term $F(x, t)$ is prescribed, (6) is a nonhomogeneous version of the free Schrödinger equation. In order to get an integral equation for the homogeneous problem with a potential $V(x, t)$, however, we will later take $F(x, t)$ to be $V(x, t) u(x, t)$. In any case, one imposes the initial condition

$$
u(x, 0)=f(x), \quad \forall(x, t)=(x, 0) \in \mathbb{R}^{n} \times\{t=0\}
$$

and usually concentrates attention tacitly on $t>0$. 
The initial-value problem for (6) with the nonhomogeneous initial condition (7) can be reduced to the analogous problem with homogeneous initial condition by decomposing the solution $u$ into two integral representations:

$$
u(x, t)=\Phi(x, t)+\Pi(x, t)
$$

where $\Phi(x, t)$, called the source term, contains the effects of $F$ and has null initial data, while $\Pi(x, t)$, the Poisson integral term, solves the homogeneous equation (3) with the data (7). We shall show (Theorem 2) that

$$
\Pi(x, t)=e^{-i t H_{0} / \hbar} f(x)=\int_{\mathbb{R}^{n}} K_{\mathrm{f}}(x, y, t) f(y) d y
$$

and

$$
\Phi(x, t)=\int_{0}^{t} e^{-i t H_{0} / \hbar} e^{i \tau H_{0} / \hbar} F(\cdot, \tau) d \tau=-\frac{i}{\hbar} \int_{0}^{t} \int_{\mathbb{R}^{n}} K_{\mathrm{f}}(x, y, t-\tau) F(y, \tau) d y d \tau
$$

Here $K_{\mathrm{f}}(x, y, t)$ is the fundamental solution (free propagator) to the Schrödinger equation (3) in $\mathbb{R}^{n}$, which is given by

$$
K_{\mathrm{f}}(x, y, t) \equiv K_{\mathrm{f}}(x-y, t)=\left(\frac{m}{2 \pi \hbar i t}\right)^{n / 2} e^{i m|x-y|^{2} / 2 \hbar t}, \quad \forall x, y \in \mathbb{R}^{n}, t \neq 0
$$

The formula (9) is equivalent to the statement that $K_{\mathrm{f}}(x, y, t)$ as a function of $(x, t)$ satisfies the homogeneous free Schrödinger equation and the initial condition

$$
K_{\mathrm{f}}(x, y, 0)=\lim _{t \downarrow \tau} K_{\mathrm{f}}(x, y, t-\tau)=\delta(x-y)
$$

Thus $K_{\mathrm{f}}(x, y, t)$ vanishes as a distribution as $t \rightarrow 0$ in the region $x \neq y$, even though as a function it does not approach pointwise limits there. The formula (10) is equivalent to the alternative characterization that $K_{\mathrm{f}}$ is the causal solution of the nonhomogeneous equation

$$
L K_{\mathrm{f}}(x, y, t-\tau)=\delta(x-y) \delta(t-\tau)
$$

where $L$ acts on the $(x, t)$ variables.

The following theorem introduces the Poisson integral, which gives the solution of the initial-value problem for the free Schrödinger equation. Our discussion of the Poisson integral is somewhat more detailed than that of Evans [7], especially concerning the role of Abel summability. 
Theorem 1. Let $f(x)$ be a function on $\mathbb{R}^{n}$ such that $\left(1+|y|^{2}\right) f(y) \in L^{1}\left(\mathbb{R}^{n}\right)$. Then the Poisson integral

$$
u(x, t)=K_{\mathrm{f}} * f=\int_{\mathbb{R}^{n}} K_{\mathrm{f}}(x-y, t) f(y) d y
$$

exists and is a solution of the equation

$$
L u(x, t)=a^{2} \Delta_{x} u(x, t)+i \hbar \partial_{t} u(x, t)=0, \quad \forall(x, t) \in \mathbb{R}^{n} \times \mathbb{R},
$$

and it satisfies the initial condition (7) in the sense of Abel summability. The Poisson integral defines a solution of the free Schrödinger equation in $\mathbb{R}^{n} \times\{t \neq 0\}$ (including negative $t$ ). This solution is extended into $\mathbb{R}^{n} \times \mathbb{R}$ by the initial condition $u(x, 0)=f(x)$ at all points $x$ at which $f$ is continuous.

Proof. If $|y|^{2} f(y) \in L^{1}\left(\mathbb{R}^{n}\right)$, then the order of differentiation and integration in (15), (14) can be interchanged to verify that the Poisson integral solves the Schrödinger equation. This hypothesis is obtained from [7, Chapter IV].

The harder part is verifying the initial value. Assuming $t>0$, let $y=x+\gamma z$, where $\gamma^{2}=$ $2 \hbar t / m$; then we can rewrite the Poisson integral as

$$
u(x, t)=\left(\frac{1}{\pi i}\right)^{n / 2} \int_{\mathbb{R}^{n}} e^{i|z|^{2}} f(x+\gamma z) d z
$$

where $|z|=|x-y| / \gamma$. Let $\epsilon$ be any positive number. Then

$$
(\pi i)^{n / 2} u(x, t)=\int_{\mathbb{R}^{n}} e^{i|z|^{2}} f(x+\gamma z) d z=I_{1}+I_{2}+I_{3}
$$

where

$$
\begin{gathered}
I_{1}=\int_{|z| \leq \epsilon} e^{i|z|^{2}}\{f(x+\gamma z)-f(x)\} d z, \\
I_{2}=\int_{|z| \geq \epsilon} e^{i|z|^{2}} f(x+\gamma z) d z, \\
I_{3}=\int_{|z| \leq \epsilon} e^{i|z|^{2}} f(x) d z .
\end{gathered}
$$

To dispose of $I_{1}$, let $x$ be a point in $\mathbb{R}^{n}$ where $f$ is continuous: $\forall \eta>0 \exists \delta>0$ such that $\forall y \in \mathbb{R}^{n}$ with $|y-x|<\delta$ one has $|f(y)-f(x)|<\eta$. Given $\epsilon$ (however large) and $\eta$ (however small), choose $t$ (hence $\gamma$ ) so small that $\gamma \epsilon<\delta$; then $|f(x+\gamma z)-f(x)|<\eta$ for all $z$ such that $|z| \leq \epsilon$. Therefore, 


$$
\left|I_{1}\right| \leq \eta \int_{|z| \leq \epsilon} d z
$$

which can be made arbitrarily small in the limit $t \rightarrow 0$.

On the other hand, since $f \in L^{1}\left(\mathbb{R}^{n}\right)$,

$$
\left|I_{2}\right| \leq \int_{|z| \geq \epsilon}|f(x+\gamma z)| d z \rightarrow 0
$$

(not necessarily uniformly in $x$ ) as $\epsilon \rightarrow \infty$. Thus the initial value $u\left(x, 0^{+}\right)$comes entirely from $I_{3}$.

To evaluate $I_{3}$ we use the Fresnel integral formula

$$
\int_{\mathbb{R}^{n}} e^{i|z|^{2}} d z=(\pi i)^{n / 2}
$$

A proof of (23) with $n=1$, which converges classically, appears in [8, pp. 82-83]. The one-dimensional formula appears to imply the product version by

$$
\int_{\mathbb{R}^{n}} e^{i|z|^{2}} d z=\int_{\mathbb{R}^{n}} \exp \left(i \sum_{k=1}^{n} z_{k}^{2}\right) d z=\prod_{k=1}^{n} \int_{-\infty}^{\infty} e^{i z_{k}^{2}} d z_{k}=\prod_{k=1}^{n}(\pi i)^{1 / 2}=(\pi i)^{n / 2}
$$

Therefore, we have

$$
\lim _{\epsilon \rightarrow \infty} I_{3}=(\pi i)^{n / 2} f(x)
$$

which is what we want to prove.

However, the integral on the left side of (23) is rather questionable when $n>1$, so we reconsider it in polar coordinates:

$$
\int_{\mathbb{R}^{n}} e^{i|z|^{2}} d z=\int_{0}^{\infty} \int_{S^{n-1}} e^{i \rho^{2}} \rho^{n-1} d \rho d \Omega \equiv \omega_{n} \int_{0}^{\infty} \rho^{n-1} e^{i \rho^{2}} d \rho .
$$

The surface area of the unit $n$-sphere is

$$
\omega_{n}=2 \pi^{n / 2} / \Gamma\left(\frac{n}{2}\right)
$$

With the substitutions $t=\rho^{2}, m=(n-2) / 2$, we obtain

$$
\int_{\mathbb{R}^{n}} e^{i|z|^{2}} d z=\frac{\omega_{n}}{2} \int_{0}^{\infty} t^{m} e^{i t} d t
$$


which technically is not convergent. Therefore. we insert the Abel factor $e^{-\alpha t}(\alpha>0)$ into (26) to get

$$
A(\alpha) \equiv \frac{\omega_{n}}{2} \int_{0}^{\infty} e^{-\alpha t} t^{m} e^{i t} d t
$$

This integral is convergent, and it can be transformed as

$$
\frac{2 A(\alpha)}{\omega_{n}}=\lim _{r \rightarrow \infty} \int_{0}^{r} e^{-\alpha t} t^{m} e^{i t} d t=\lim _{r \rightarrow \infty} i \int_{0}^{i r} e^{-i \alpha z}(i z)^{m} e^{-z} d z
$$

The path of integration can be moved back to the positive real axis, because the integral over the arc of radius $r$ tends to 0 . Thus

$$
\frac{2 A(\alpha)}{\omega_{n}}=i^{n / 2} \int_{0}^{\infty} e^{-i \alpha z} z^{(n / 2)-1} e^{-z} d z
$$

and in the limit

$$
A(0)=\frac{1}{2} \omega_{n} i^{n / 2} \Gamma\left(\frac{n}{2}\right)=(\pi i)^{n / 2} .
$$

This analysis confirms (23) in an alternative way and gives it a rigorous meaning.

This completes the proof that the Poisson integral has the initial value $u(x, 0)=f(x)$ at all points $x$ where $f$ is continuous.

Theorem 2 establishes formula (10) rigorously. Our proof is partly based on [9], which considers the nonhomogeneous Schrödinger equation (6) in the more abstract form

$$
i \hbar \frac{\partial u(t)}{\partial t}=H_{0} u(t)+F(t)
$$

Here and later, $I$ will denote the time interval $(0, T)$, where $T$ is a positive constant. In Theorem 2 we deal with the space $L^{\infty}(I ; \mathcal{B})$ of functions $u(t)$ taking values in the Banach space $\mathcal{B}$, equipped with the norm (cf. Definition 5)

$$
\|u\|_{L^{\infty}(I ; \mathcal{B})}=\inf \left\{M \geq 0:\|u(t)\|_{\mathcal{B}} \leq M \quad \text { for almost all } t \in[0, T]\right\}
$$

Theorem 2. Let $f(x)$ belong to some Banach space $\mathcal{B}$ of functions on $\mathbb{R}^{n}$ that includes those for which $\left(1+|y|^{2}\right) f(y) \in L^{1}\left(\mathbb{R}^{n}\right)$. Furthermore, suppose that the source term $F(x, t)$ is continuous in $t$ and satisfies the condition

$$
\|F(\cdot, t)\|_{L^{1}\left(\mathbb{R}^{n}\right)} \leq \xi(t), \quad\|\xi\|_{L^{\infty}(I)} \leq M
$$


for some positive constant $M$. The solution of the initial-value problem for the nonhomogeneous Schrödinger equation (6) can be represented in the form $u=\Pi+\Phi$ of (8), where the initial term is

$$
\Pi(x, t)=\int_{\mathbb{R}^{n}} K_{\mathrm{f}}(x, y, t) f(y) d y
$$

and the source term is

$$
\Phi(x, t)=-\frac{i}{\hbar} \int_{0}^{t} \int_{\mathbb{R}^{n}} K_{\mathrm{f}}(x, y, t-\tau) F(y, \tau) d y d \tau
$$

Here $K_{\mathrm{f}}(x, y, t)$ is the fundamental solution (11) and $u(x, 0)=f(x)$. The solution $u$ belongs to the Banach space $L^{\infty}(I ; \mathcal{B})$.

Proof. Theorem 1 shows that the Poisson integral $\Pi$ solves the initial-value problem for the homogeneous Schrödinger equation. We claim that the solution of the full problem has the Volterra integral representation

$$
\begin{gathered}
u(x, t)=\int_{\mathbb{R}^{n}} K_{\mathrm{f}}(x, y, t) f(y) d y-\frac{i}{\hbar} \int_{0}^{t} \int_{\mathbb{R}^{n}} K_{\mathrm{f}}(x, y, t-\tau) F(y, \tau) d y d \tau \\
\equiv \Pi(x, t)+\Phi(x, t) .
\end{gathered}
$$

By applying the Schrödinger operator (5) to $u(t)$, we have

$$
\begin{aligned}
L u= & L \Pi+L \Phi=a^{2} \Delta_{x} \Phi+i \hbar \frac{\partial \Phi}{\partial t} \\
= & a^{2}\left(-\frac{i}{\hbar}\right) \Delta_{x} \int_{\mathbb{R}^{n}} K_{\mathrm{f}}(x, y, t-\tau) F(y, \tau) d y d \tau \\
& +i \hbar \frac{\partial}{\partial t}\left(-\frac{i}{\hbar} \int_{0}^{t} \int_{\mathbb{R}^{n}} K_{\mathrm{f}}(x, y, t-\tau) F(y, \tau) d y d \tau\right) \\
= & \int_{0}^{t} \int_{\mathbb{R}^{n}} L K_{\mathrm{f}}(x, y, t-\tau) F(y, \tau) d y d \tau \\
& +\lim _{t \downarrow \tau} \int_{\mathbb{R}^{n}} K_{\mathrm{f}}(x, y, t-\tau) F(y, \tau) d y .
\end{aligned}
$$

But $L K_{\mathrm{f}}(x, y, t-\tau)=0$ for all $t>\tau$, and Theorem 1 shows that $K_{\mathrm{f}}(x, y, t-\tau) \rightarrow \delta(x-y)$. Therefore, we have

$$
L \Phi=F(x, t) .
$$

Furthermore, it is clear that $F(x, 0)=0$. Therfore, by linearity the sum $u=\Pi+\Phi$ solves the problem.

Another way to express (34) is via unitary operators: 


$$
u(t)=e^{-i t H_{0} / \hbar} f(x)-\frac{i}{\hbar} \int_{0}^{t} e^{-i t H_{0} / \hbar} e^{i \tau H_{0} / \hbar} F(\tau) d \tau .
$$

Consider the integral

$$
\begin{aligned}
-i \hbar^{-1} \int_{0}^{t} e^{i \tau H_{0} / \hbar} L u(\tau) d \tau & =-i \hbar^{-1} \int_{0}^{t} e^{i \tau H_{+} 0 / \hbar}\left(-a \Delta u(\tau)+i \hbar \frac{\partial u(\tau)}{\partial \tau}\right) d \tau \\
& =-i \hbar^{-1} \int_{0}^{t} e^{i \tau H_{0} / \hbar}\left(-H_{0} u(\tau)+i \hbar \frac{\partial u}{\partial \tau}\right) d \tau \\
& =\int_{0}^{t} \frac{\partial}{\partial \tau}\left(e^{i \tau H_{0} / \hbar} u(\tau)\right) d \tau \\
& =e^{i t H_{0} / \hbar} u(t)-u(0) .
\end{aligned}
$$

This calculation implies that

$$
u(t)=e^{-i t H_{0} / \hbar} u(0)-i \hbar^{-1} e^{-i t H_{0} / \hbar} \int_{0}^{t} e^{i \tau H_{0} / \hbar} L u(\tau) d \tau,
$$

which is equivalent to (37) and to the Volterra integral formula (34). The expression $u(t)-$ $e^{-i t H_{0} / \hbar} u(0)$ is simply the source term $\Phi(x, t)$. Taking its Banach space norm and using the unitarity of the evolution operator $e^{-i t H_{0} / \hbar}$ and the fundamental theorem of calculus, we have

$$
\begin{aligned}
\|\Phi(t)\| & =\left\|i \hbar^{-1} e^{-i t H_{0} / \hbar} \int_{0}^{t} e^{i \tau H_{0} / \hbar} L u(\tau) d \tau\right\| \\
& \leq \frac{1}{h} \int_{0}^{t}\|L u\| d \tau \leq \frac{1}{\hbar} \int_{0}^{t} \xi(\tau) d \tau \leq \frac{M t}{\hbar},
\end{aligned}
$$

because of (31). Therefore, $\Phi \rightarrow 0$ when $t \rightarrow 0$. Since $e^{-i t H_{0} / \hbar} u(0)$ is another way of writing $\Pi(x, t)$, we have again established that $(8)$ is the desired solution.

Remark 3. The $L^{1}$ condition of Theorem 1 has not been used in the second, more abstract proof of Theorem 2 , because the limits $(t \downarrow 0)$ are being taken in the topology of the quantum Hilbert space $L^{2}\left(\mathbb{R}^{n}\right)$, not pointwise.

Corollary 4. The homogeneous Schrödinger initial-value problem,

$$
i \hbar \partial_{t} \Psi(x, t)=-a^{2} \Delta \Psi(x . t)+V(x, t) \Psi(x, t), \quad \Psi(x, 0)=f(x),
$$

is equivalent to a nonhomogeneous Volterra integral equation of the second kind,

$$
\Psi(x, t)=\int_{\mathbb{R}^{n}} K_{\mathrm{f}}(x, y, t) f(y) d y-\frac{i}{\hbar} \int_{0}^{t} \int_{\mathbb{R}^{n}} K_{\mathrm{f}}(x, y, t-\tau) V(y, \tau) \Psi(y, \tau) d y d \tau .
$$

Proof. (42) is (34) with the source $F$ in (6) identified with $V \Psi$. 


\section{Integral equations and Neumann series}

In this section we introduce integral operators in arbitrary Banach spaces in order to set up a framework for constructing solutions to the Schrödinger equation. This section is a preliminary to the general Volterra theorems that are proved in section 4 . It uses as a foundation Kress's treatment of linear integral equations [2].

In operator notation, an integral equation of the second kind has the structure

$$
\phi-\hat{Q} \phi=f
$$

where $\hat{Q}$ is a bounded linear operator from a Banach space $\mathcal{W}$ to itself, and $\phi$ and $f$ are in $\mathcal{W}$. A solution $\phi$ exists and is unique for each $f$ if and only if the inverse operator $(1-\hat{Q})^{-1}$ exists (where 1 indicates the identity operator). For Volterra operators, the focus of our attention, the existence of the inverse operator will become clear below. Equivalently, the theorems of the next section will prove that the spectral radius of a Volterra operator is zero. For these purposes we need to work in Lebesgue spaces $\mathcal{W}=L^{p}(I ; \mathcal{B})$ (including, especially, $p=\infty$ ) of functions of $t$ to obtain useful estimates.

Definition 5. Let $(\Omega, \Sigma, \mu)$ be a measure space and $\mathcal{B}$ be a Banach space. The collection of all essentially bounded measurable functions on $\Omega$ taking values in $\mathcal{B}$ is denoted $L^{\infty}(\Omega, \mu ; \mathcal{B})$, the reference to $\mu$ being omitted when there is no danger of confusion. The essential supremum of a function $\varphi: \Omega \rightarrow \mathcal{B}$ is given by

$$
\|\varphi\|_{L^{\infty}(\Omega ; \mathcal{B})}=\inf \left\{M \geq 0:\|\varphi(x)\|_{\mathcal{B}} \leq M \text { for almost all } x\right\}
$$

Definition 6. Let $\mathcal{B}_{1}$ and $\mathcal{B}_{2}$ be Banach spaces and $\Omega$ be some measurable space. For each $(x, y) \in$ $\Omega \times \Omega \equiv \Omega^{2}$ let $A(x, y): \mathcal{B}_{1} \rightarrow \mathcal{B}_{2}$ be a bounded linear operator, and suppose that the function $A(\cdot, \cdot)$ is measurable. At each $(x, y)$ define its norm

$$
\|A(x, y)\|_{\mathcal{B}_{1} \rightarrow \mathcal{B}_{2}}=\inf \left\{M \geq 0:\|A(x, y) \phi\| \leq M\|\phi\|, \quad \forall \phi \in \mathcal{B}_{1}\right\}
$$

If $\mathcal{B}_{1}=\mathcal{B}_{2}=\mathcal{B}$, then one abbreviates $\|A(x, y)\|_{\mathcal{B}_{1} \rightarrow \mathcal{B}_{2}}$ as $\|A(x, y)\|_{\mathcal{B}}$ or even $\|A(x, y)\|$. Now define the uniform norm

$$
\begin{gathered}
\|A\|_{L^{\infty}\left(\Omega^{2} ; \mathcal{B}_{1} \rightarrow \mathcal{B}_{2}\right)} \equiv \inf \left\{M \geq 0:\|A(x, y)\| \leq M \text { for almost all }(x, y) \in \Omega^{2}\right\} \\
\equiv \operatorname{ess} \sup _{(x, y) \in \Omega^{2}}\|A(x, y)\|_{\mathcal{B}_{1} \rightarrow \mathcal{B}_{2}}
\end{gathered}
$$

and call $A(\cdot, \cdot)$ a uniformly bounded operator kernel if $\|A\|_{L^{\infty}\left(\Omega^{2} ; \mathcal{B}_{1} \rightarrow \mathcal{B}_{2}\right)}$ is finite.

Definition 7. In Definition 6 let $\Omega=I=(0, T)$. If $A$ is a uniformly bounded operator kernel, the operator $\hat{Q}$ defined by

$$
\hat{Q} f(t)=\int_{0}^{t} A(t, \tau) f(\tau) d \tau
$$

is called a bounded Volterra operator on $L^{\infty}(I ; \mathcal{B})$ with kernel A. 
Remark 8. In (47) one may write the integration as $\int_{0}^{T} \cdots d \tau$ if one has defined $A(t, \tau)$ to be 0 whenever $\tau>t$. In that case $A$ is called a Volterra kernel.

In Corollary 4 we have reformulated the Schrödinger equation as an integral equation of the second kind. The existence and uniqueness of its solution can be found by analysis of the Neumann series. The successive approximations (Picard's algorithm)

$$
\phi_{N}=\hat{Q} \phi_{N-1}+f=\sum_{j=0}^{N} \hat{Q}^{j} f
$$

converge to the exact solution of the integral equation (43), if some technical conditions are satisfied. In the terminology of an arbitrary Banach space, one must establish that

1. the function $\phi_{0} \equiv f$ belongs to a Banach space $\mathcal{B}$,

2. the integral operator $\hat{Q}$ is a bounded Volterra operator on $L^{\infty}(I ; \mathcal{B})$, and

3. the infinite (Neumann) series $\phi=\sum_{j=0}^{\infty} \hat{Q}^{j} f$ is a convergent series with respect to the topology of $L^{\infty}(I ; \mathcal{B})$.

If these three conditions are satisfied, then the Neumann series provides the exact solution to the integral equation (43). In the Schrödinger case, therefore, it solves the original initial-value problem for the Schrödinger equation. This program will be implemented in detail in the next two sections.

\section{Volterra kernels and successive approximations}

In this section we implement the method of successive approximations set forth in section 3 . The Volterra operator has a nice property, known as the simplex structure, which makes its infinite Neumann series converge. This claim is made precise in our main theorems.

It follows from the convergence of the Neumann series that the spectral radius of the Volterra integral operator of the second kind is zero. In Kress's treatment of the heat equation [2] the logic runs in the other direction - convergence follows from a theorem on spectral radius. For the Schrödinger equation we find it more convenient to prove convergence directly.

\section{Hypotheses}

- $\mathcal{B}$ is a Banach space, and $I=(0, T)$ is an interval, with closure $\bar{I}$.

- For all $(t, \tau) \in \bar{I}^{2}, A(t, \tau)$ is a linear operator from $\mathcal{B}$ to $\mathcal{B}$.

- the operator kernel $A(t, \tau)$ is measurable and uniformly bounded, in the sense of Definition 6, with bound $\|A\|_{L^{\infty}\left(I^{2} ; \mathcal{B} \rightarrow \mathcal{B}\right)}=D$.

- $A(t, \tau)$ satisfes the Volterra condition, $A(t, \tau)=0$ if $\tau>t$. 
Our primary theorem, like the definitions in section 3 , deals with the space $L^{\infty}(I ; \mathcal{B})$. We also provide variants of the theorem and the key lemma for other Lebesgue spaces, $L^{1}(I ; \mathcal{B})$ and $L^{p}(I ; \mathcal{B})$. In each case, the space $\mathcal{B}$ is likely, in applications, to be itself a Lebesgue space of functions of a spatial variable, $L^{m}\left(\mathbb{R}^{n}\right)$, with no connection between $m$ and $p$.

The first step of the proof is a fundamental lemma establishing a bound on the Volterra operator that fully exploits its simplex structure. This argument inductively establishes the norm of each term in the Neumann series, from which the convergence quickly follows. In the lemmas, $j$ (the future summation index) is understood to be an arbitrary nonnegative integer (or even a real positive number).

Lemma 9. Let the Volterra integral operator, $\hat{Q}: L^{\infty}(I ; \mathcal{B}) \rightarrow L^{\infty}(I ; \mathcal{B})$, be defined by

$$
\hat{Q} \phi(t)=\int_{0}^{T} A(t, \tau) \phi(\tau) d \tau=\int_{0}^{t} A(t, \tau) \phi(\tau) d \tau
$$

Let $\phi \in L^{\infty}(I ; \mathcal{B})$ and assume that $\exists C>0$ such that for each subinterval $J_{t}$ of the form $(0, t)$, we

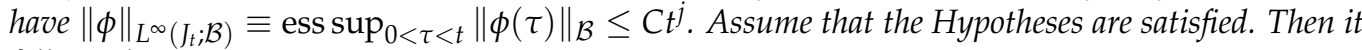
follows that

$$
\|\hat{Q} \phi\|_{L^{\infty}\left(J_{t}, \mathcal{B}\right)} \leq \frac{D C}{j+1} t^{j+1}
$$

Proof. Recall that $D$ is defined so that $\|A(t, \tau)\| \leq D<\infty$ for all $(t, \tau) \in \bar{I}^{2}$. The $L^{\infty}\left(J_{t} ; \mathcal{B}\right)$ norm of the function $\hat{Q} \phi(\cdot)$ is

$$
\begin{aligned}
\|\hat{Q} \phi\|_{L^{\infty}\left(J_{t} ; \mathcal{B}\right)} & =\sup _{t_{1} \leq t}\left\|\int_{0}^{t_{1}} A(t, \tau) \phi(\tau) d \tau\right\| \leq \sup _{t_{1} \leq t} \int_{0}^{t_{1}}\|A(t, \tau) \phi(\tau)\| d \tau \\
& \leq \sup _{t_{1} \leq t} \int_{0}^{t_{1}}\|A(t, \tau)\|\|\phi(\tau)\| d \tau \leq \sup _{t_{1} \leq t} \int_{0}^{t_{1}} D C \tau^{j} d \tau \\
& =\sup _{t_{1} \leq t} D C \frac{t_{1}^{j+1}}{j+1}=\frac{D C t^{j+1}}{j+1} \cdot \square
\end{aligned}
$$

Lemma 10. Let the Volterra integral operator, $\hat{Q}: L^{1}(I ; \mathcal{B}) \rightarrow L^{1}(I ; \mathcal{B})$, be defined by (49). Let $\phi \in L^{1}(I ; \mathcal{B})$, and assume that $\exists C>0$ such that for each subinterval $J_{t}=(0, t)$, we have

$$
\|\phi\|_{L^{1}\left(J_{t} ; \mathcal{B}\right)} \equiv \int_{0}^{t}\|\phi(\tau)\| d \tau \leq C t^{j}
$$

Assume that the Hypotheses are satisfied. Then it follows that

$$
\|\hat{Q} \phi\|_{L^{1}\left(J_{t}, \mathcal{B}\right)} \leq \frac{D C}{j+1} t^{j+1}
$$


Proof. The argument is the same as before, except that the $L^{1}\left(J_{t} ; \mathcal{B}\right)$ norm of $\hat{Q} \phi(\cdot)$ is

$$
\begin{aligned}
\|\hat{Q} \phi\|_{L^{1}\left(J_{t} ; \mathcal{B}\right)} & =\int_{0}^{t}\left\|\int_{0}^{t_{1}} A(t, \tau) \phi(\tau) d \tau\right\| d t_{1} \leq \int_{0}^{t} \int_{0}^{t_{1}}\|A(t, \tau)\|\|\phi(\tau)\| d \tau \\
& \leq \int_{0}^{t} D \int_{0}^{t_{1}}\|\phi(\tau)\| d \tau d t_{1} \leq \int_{0}^{t} D\|\phi\|_{L^{1}\left(J_{t_{1}} ; \mathcal{B}\right)} d t_{1} \\
& \leq \int_{0}^{t} D C t_{1}^{j} d t_{1}=\frac{D C t^{j+1}}{j+1} \cdot
\end{aligned}
$$

Corollary 11. Let the Volterra integral operator, $\hat{Q}: L^{p}(I ; \mathcal{B}) \rightarrow L^{p}(I ; \mathcal{B})$, where $1<p<\infty$, be defined by (49). Let $\phi \in L^{p}(I ; \mathcal{B})$ and assume that $\exists C>0$ such that for each subinterval $J_{t}=(0, t)$, we have

$$
\|\phi\|_{L^{p}\left(J_{t} ; \mathcal{B}\right)} \equiv\left(\int_{0}^{t}\|\phi(\tau)\|_{\mathcal{B}}^{p} d \tau\right)^{1 / p} \leq C t^{n} .
$$

Assume that the Hypotheses are satisfied. Then it follows that

$$
\|\hat{Q} \phi\|_{L^{p}\left(J_{t}, \mathcal{B}\right)} \leq \frac{D C}{j+1} t^{j+1}
$$

Proof. This follows from Lemmas 9 and 10 by the Riesz-Thorin theorem [10, pp. 27-28].

It may be of some interest to see how the $L^{p}$ theorem can be proved directly. The proof of the needed lemma uses Folland's proof of Young's inequality [4] as a model.

Lemma 12. Let the Volterra integral operator, $\hat{Q}: L^{p}(I ; \mathcal{B}) \rightarrow L^{p}(I ; \mathcal{B})$, where $1<p<\infty$, be defined by (49). Let $\phi \in L^{p}(I ; \mathcal{B})$ and assume that $\exists C>0$ such that for each subinterval $J_{t}=(0, t)$, we have

$$
\|\phi\|_{L^{p}\left(J_{t} ; \mathcal{B}\right)} \equiv\left(\int_{0}^{t}\|\phi(\tau)\|_{\mathcal{B}}^{p} d \tau\right)^{1 / p} \leq C t^{n} .
$$

Assume that the Hypotheses are satisfied. Then it follows that

$$
\|\hat{Q} \phi\|_{L^{p}\left(J_{t}, \mathcal{B}\right)} \leq \frac{D C t^{j+1}}{[p(j+1)]^{1 / p}} .
$$

Proof. Let $q$ be the conjugate exponent $\left(p^{-1}+q^{-1}=1\right)$. The Banach-space norm of $\hat{Q} \phi(t)$ satisfies 


$$
\begin{aligned}
\left\|\hat{Q} \phi\left(t_{1}\right)\right\|_{\mathcal{B}} & \left.\leq\left(\int_{0}^{t_{1}}\left\|A\left(t_{1}, \tau\right)\right\|\right) d \tau\right)^{1 / q}\left(\int_{0}^{t_{1}}\left\|A\left(t_{1}, \tau\right)\right\|\|\phi(\tau)\|^{p} d \tau\right)^{1 / p} \\
& \leq D^{1 / q}\left(\int_{0}^{t_{1}} d \tau\right)^{1 / q}\left(\int_{0}^{t_{1}} D\|\phi(\tau)\|^{p} d \tau\right)^{1 / p} \\
& \leq D^{1 / q} D^{1 / p} t_{1}^{1 / q}\left(\int_{0}^{t_{1}}\|\phi(\tau)\|^{p} d \tau\right)^{1 / p} \\
& \leq D t_{1}^{1 / q}\left(\int_{0}^{t_{1}}\|\phi(\tau)\|^{p} d \tau\right)^{1 / p} .
\end{aligned}
$$

Then we must raise both sides to the $p$ th power and integrate, seeing by Fubini's theorem that

$$
\begin{aligned}
\int_{0}^{t_{1}}\left\|\hat{Q} \phi\left(t_{1}\right)\right\|^{p} d t_{1} & \leq \int_{0}^{t} D^{p} t_{1}^{p / q} \int_{0}^{t_{1}}\|\phi(\tau)\|^{p} d \tau d t_{1} \leq \int_{0}^{t} D^{p} \int_{0}^{t} t_{1}^{p / q}\|\phi\|_{L^{p}\left(J_{t_{1}} ; \mathcal{B}\right)}^{p} d t_{1} \\
& \leq D^{p} \int_{0}^{t} C^{p} t_{1}^{n p+p / q} d t_{1} \leq D^{p} C^{p} \frac{t^{n p+p / q+1}}{n p+\frac{p}{q}+1}=D^{p} C^{p} \frac{t^{(j+1) p}}{j p+p}
\end{aligned}
$$

since $1+\frac{p}{q}=p$. Now take the $p$ th root, getting

$$
\|\hat{Q} \phi\|_{L^{p}\left(J_{t} ; \mathcal{B}\right)} \leq D C \frac{t^{j+1}}{[p(j+1)]^{1 / p}} .
$$

The following theorem is the main theorem of [3]; its proof is corrected here.

Theorem 13. ( $L^{\infty}$ Volterra Theorem) Let the Hypotheses be satisfied, and let $\hat{Q}$ be defined by (49). Let $f$ belong to $L^{\infty}(I ; \mathcal{B})$. Then the Volterra integral equation

$$
\phi=\hat{Q} \phi+f
$$

can be solved by successive approximations. That is, the Neumann series for $\phi$,

$$
\phi=\sum_{j=0}^{\infty} \hat{Q}^{j} f
$$

converges in the topology of $L^{\infty}(I ; \mathcal{B})$.

Proof. Let $\|f\|_{L^{\infty}(I ; \mathcal{B})}=C_{0}$. Of course, $\|f\|_{L^{\infty}\left(J_{t} ; \mathcal{B}\right)} \leq C_{0}$ on a smaller interval, $J_{t}=(0, t)$, so by Lemma 9 with $j=0$, 


$$
\left\|\hat{Q}^{1} f\right\|_{L^{\infty}\left(J_{t} ; \mathcal{B}\right)} \leq D C_{0} t .
$$

Then by inductively applying Lemma 9 with $C=D^{j-1} C_{0} /(j-1)$ ! , we see that the $j$ th term of the Neumann series, $\hat{Q}^{j} f$, has, because of its simplex structure, the bound

$$
\left\|\hat{Q}^{j} f\right\|_{L^{\infty}\left(J_{t} ; \mathcal{B}\right)} \leq D^{j} C_{0} \frac{t^{j}}{j !}
$$

Therefore, the series (63) is majorized by

$$
C_{0} \sum_{n=0}^{\infty} \frac{D^{j} t^{j}}{j !}=\|f\|_{L^{\infty}(I ; \mathcal{B})} e^{D t}
$$

for all $t \in(0, T]$. Therefore, the Neumann series converges in the topology of $L^{\infty}(I ; \mathcal{B})$.

The $L^{\infty}$ norm on the time behavior is the most natural and likely one to apply to solutions of a time-evolution equation (especially for the Schrödinger equation with $\mathcal{B}$ a Hilbert space, because of the unitary of the evolution). However, the other $L^{p}$ norms may prove to be useful, and it is easy to generalize the theorem to them. Note that the appropriate condition on $A(t, \tau)$ is still the uniform boundedness of Definition 6 .

Theorem 14. ( $L^{1}$ Volterra Theorem) Let the Hypotheses be satisfied, and let $\hat{Q}$ be defined by (49). Let $f$ belong to $L^{1}(I ; \mathcal{B})$. Then the Volterra integral equation $\phi=\hat{Q} \phi+f$ can be solved by successive approximations. That is, the Neumann series for $\phi,(63)$, converges in the topology of $L^{1}(I ; \mathcal{B})$.

Proof. Let $\|f\|_{L^{1}(I ; \mathcal{B})}=C_{0}$ and argue as before, except that Lemma 10 is used to bound all the terms $\left\|\hat{Q}^{j} f\right\|_{L^{1}\left(J_{t} ; \mathcal{B}\right)}$.

Theorem 15. ( $L^{p}$ Volterra Theorem) Let the Hypotheses be satisfied, and let $\hat{Q}$ be defined by (49). Let $f$ belong to $L^{p}(I ; \mathcal{B})$. Then the Volterra integral equation $\phi=\hat{Q} \phi+f$ can be solved by successive approximations. That is, the Neumann series converges in the topology of $L^{p}(I ; \mathcal{B})$.

Proof. The proof based on the Riesz-Thorin theorem goes exactly like the previous two, using Corollary 11. To prove the theorem directly, let $\|f\|_{L^{p}(I ; \mathcal{B})}=C_{0}$ and use Lemma 12 inductively to show

$$
\left\|\hat{Q}^{j} f\right\|_{L^{p}\left(J_{t} ; \mathcal{B}\right)} \leq \frac{D^{j}}{p^{j / p}}\|f\|_{L^{p}(I ; \mathcal{B})} \frac{t^{j}}{(j !)^{1 / p}} .
$$

To see whether the series

$$
\sum_{j=0}^{\infty}\left\|\hat{Q}^{j} f\right\|_{L^{p}(I ; \mathcal{B})}=\|f\|_{L^{p}(I ; \mathcal{B})} \sum_{j=0}^{\infty} \frac{D^{j}}{p^{j / p}} \frac{t^{j}}{(j !)^{1 / p}}
$$


is convergent, we use the ratio test. Let

$$
L=\lim _{j \rightarrow \infty}\left|\frac{a_{j+1}}{a_{j}}\right|, \quad a_{j}=\left\|\hat{Q}^{j} f\right\|_{L^{p}(I ; \mathcal{B})} .
$$

Let $M(p)=D p^{-1 / p}$. Then

$$
\begin{aligned}
L & =\lim _{n \rightarrow \infty} \frac{\|f\|_{L^{p}(I ; \mathcal{B})} M(p)^{j+1} t^{j+1}}{[(j+1) !]^{1 / p}} \cdot \frac{(j !)^{1 / p}}{\|f\|_{L^{p}(I ; \mathcal{B})} M(p)^{j} t^{j}} \\
& =M(p) t \lim _{j \rightarrow \infty}(j+1)^{-1 / p}=0,
\end{aligned}
$$

Thus $L<1$, and by the ratio-test and series-majorization theorems, the Neumann series converges absolutely in the topology of $L^{p}(I ; \mathcal{B})$.

\section{Applications of the Volterra theorem}

In this section we present some quick applications of the general Volterra theorem of section 4 . The conclusions are already well known, or are obvious generalizations of those that are, so these examples just show how they fit into the general framework. More serious applications are delayed to later papers. The first set of examples comprises some of the standard elementary types of Volterra integral equations [11-13], generalized to vector-valued functions and functions of additional variables. The second application is to the Schrödinger problem set up in section 2 ; the result is essentially what is known in textbooks of quantum mechanics as "time-dependent perturbation theory".

Although we use the $L^{\infty}$ version of the theorem, Theorem 13, one could easily apply Theorems 14 and 15 as well. Thus a general setting for many examples is the generic double Lebesgue space defined as follows. As usual, let $I=(0, T)$ be the maximal time interval considered. In the role of $\mathcal{B}$, consider the Lebesgue space $L^{m}\left(\mathbb{R}^{n}\right)$ of functions of an $n$-dimensional spatial variable. Then $L^{p, m}\left(I ; \mathbb{R}^{n}\right)$ is the Banach space of functions on $I$ taking values in $L^{m}\left(\mathbb{R}^{n}\right)$ and subjected to the $L^{p}$ norm as functions of $t$. Thus

$$
L^{p, m}\left(I ; \mathbb{R}^{n}\right)=\left\{\phi:\left(\int_{I}\left[\int_{\mathbb{R}^{n}}|\phi(y, \tau)|^{m} d y\right]^{p / m} d \tau\right)^{1 / p} \equiv\|\phi\|_{L^{p, m}\left(I ; \mathbb{R}^{n}\right)}<\infty\right\} .
$$

When either $p$ or $m$ is $\infty$, the Lebesgue norm is replaced by the essential supremum in the obvious way.

\subsection{Classical integral equations}

\subsubsection{Spatial variables}

For $x$ and $y$ in $\mathbb{R}^{n}$, let $K(x, t ; y, \tau)$ be a uniformly bounded complex-valued function, satisfying the Volterra condition in $(t, \tau)$. The Volterra operator kernel $A(t, \tau): L^{m}\left(\mathbb{R}^{n}\right) \rightarrow$ 
$L^{m}\left(\mathbb{R}^{n}\right)$ is defined by

$$
A(t, \tau) \phi(x)=\int_{\mathbb{R}^{n}} K(x, t ; y, \tau) \phi(y) d y,
$$

for $\phi \in L^{m}\left(\mathbb{R}^{n}\right)$. (We shall be using this equation for functions $\phi$ that depend on $\tau$ as well as $y$, so that $A(t, \tau) \phi$ is a function of $(x, t, \tau)$.) To assure that $A(t, \tau)$ is a bounded Banach-space operator, we need to impose an additional technical condition on the kernel function $K$. The simplest possibility is to exploit the generalized Young inequality [4, Theorem (0.10)].

Suppose that we wish to treat functions $\phi(y, \tau) \in L^{\infty, \infty}\left(I ; \mathbb{R}^{n}\right)$. Then (72) leads to

$$
\begin{aligned}
|[A(t, \tau) \phi](x, t, \tau)| & \leq \int_{\mathbb{R}^{n}}|K(x, t ; y, \tau)||\phi(y, \tau)| d y \\
& \leq\|\phi\|_{L^{\infty, \infty}\left(I ; \mathbb{R}^{n}\right)} \int_{\mathbb{R}^{n}}|K(x, t ; y, \tau)| d y
\end{aligned}
$$

Therefore, if

$$
\int_{\mathbb{R}^{n}}|K(x, t ; y, \tau)| d y \leq D
$$

uniformly in $(x, t, \tau)$, then

$$
\|A(t, \tau) \phi\|_{L^{\infty, \infty}\left(I ; \mathbb{R}^{n}\right)} \leq D\|\phi\|_{L^{\infty, \infty}\left(I ; \mathbb{R}^{n}\right)}
$$

That is,

$$
\|A\|_{L^{\infty}\left(I^{2} ; \mathcal{B} \rightarrow \mathcal{B}\right)} \leq D,
$$

and Lemma 9 applies. Theorem 13 therefore proves that the Volterra integral equation $\phi=$ $\hat{Q} \phi+f$ can be solved by iteration within $L^{\infty, \infty}\left(I ; \mathbb{R}^{n}\right)$.

Now suppose instead that we want to work in $L^{\infty, 1}\left(I ; \mathbb{R}^{n}\right)$. In place of (73) we have

$$
\begin{aligned}
|A(t, \tau) \phi(x, t, \tau)| & \leq \int_{\mathbb{R}^{n}}|K(x, t ; y, \tau)||\phi(y, \tau)| d y \\
& \leq\|\phi\|_{L^{\infty, 1}\left(I ; \mathbb{R}^{n}\right)} \sup _{y}|K(x, t ; y, \tau)| .
\end{aligned}
$$

This time we need the condition that

$$
\int_{\mathbb{R}^{n}}|K(x, t ; y, \tau)| d x \leq D
$$

uniformly in $(y, t, \tau)$; then

$$
\|A(t, \tau) \phi\|_{L^{\infty, 1}\left(I ; \mathbb{R}^{n}\right)} \leq D\|\phi\|_{L^{\infty, 1}\left(I ; \mathbb{R}^{n}\right)}
$$


in place of (75). The argument concludes as before, using Lemma 10 and Theorem 14.

For $L^{\infty, p}\left(I ; \mathbb{R}^{n}\right)$, the generalized Young inequality [4] assumes both (74) and (78) and assures that

$$
\|A(t, \tau) \phi\|_{L^{\infty, p}\left(I ; \mathbb{R}^{n}\right)} \leq D\|\phi\|_{L^{\infty, p}\left(I ; \mathbb{R}^{n}\right)} .
$$

The argument concludes as before, using Lemma 12 and Theorem 15, proving convergence of the Neumann series within $L^{\infty, p}\left(I ; \mathbb{R}^{n}\right)$.

\subsubsection{Vector-valued functions}

A similar but simpler situation is where $\mathcal{B}$ is finite-dimensional, say $\mathbb{C}^{n}$. Then $A(t, \tau)$ is an $n \times n$ matrix. Boundedness of $A$ as an operator is automatic, but uniformity in the time variables is still a nontrivial condition. The theorem then gives a vectorial generalization of the usual Neumann series for a scalar Volterra equation.

\subsubsection{Hilbert-Schmidt operators}

Although the generalized Young approach yields a theorem for $\mathcal{B}=L^{2}\left(\mathbb{R}^{n}\right)$, the boundedness of operators on that space is often proved from a stronger condition on their kernels. In our context a Hilbert-Schmidt kernel is a function $K: \mathbb{R}^{n} \times \mathbb{R} \times \mathbb{R}^{n} \times \mathbb{R} \rightarrow \mathbb{C}$ for which

$$
\left(\int_{\mathbb{R}^{n} \times \mathbb{R}^{n}}|K(x, t ; y, \tau)|^{2} d x d y\right)^{1 / 2} \equiv\|K(t, \tau)\|_{L^{2}\left(\mathbb{R}^{2 n}\right)} \leq D<\infty,
$$

and, of course, we also want it to be Volterra in $(t, \tau)$. In other words, $K(x, t ; y, \tau)$ belongs to $L^{\infty, 2}\left(I^{2} ; \mathbb{R}^{2 n}\right)$ and vanishes (or is ignored in the integrals) when $\tau>t$. Then $A(t, \tau): L^{2}\left(\mathbb{R}^{n}\right) \rightarrow L^{2}\left(\mathbb{R}^{n}\right)$ defined by (72) is a Hilbert-Schmidt operator, which under our assumptions is uniformly bounded with norm at most $D$.

In parallel with (73) or (77) one has

$$
\begin{aligned}
|A(t, \tau) \phi(x, t, \tau)| & \leq \int_{\mathbb{R}^{n}}|K(x, t ; y, \tau)||\phi(y, \tau)| d y \\
& \leq\left(\int_{\mathbb{R}^{n}}|K(x, t ; y, \tau)|^{2} d y\right)^{1 / 2}\left(\int_{\mathbb{R}^{n}}|\phi(y, \tau)|^{2} d y\right)^{1 / 2}
\end{aligned}
$$

and hence

$$
\|A(t, \tau) \phi(\cdot)\|_{L^{2}\left(\mathbb{R}^{n}\right)} \leq\|K(t, \tau)\|_{L^{2}\left(\mathbb{R}^{2 n}\right)}\|\phi(\tau)\|_{L^{2}\left(\mathbb{R}^{n}\right)} \leq\|K\|_{L^{\infty, 2}\left(I^{2} ; \mathbb{R}^{2 n}\right)}\|\phi(\tau)\|_{L^{2}\left(\mathbb{R}^{n}\right)} .
$$

Therefore, 


$$
\|A\|_{L^{\infty}\left(I^{2} ; \mathcal{B} \rightarrow \mathcal{B}\right)} \leq D
$$

and hence Lemma 9 and Theorem 13 apply as usual, establishing convergence of the Picard solution of $\phi=\hat{Q} \phi+f$ in the topology of $L^{\infty, 2}\left(I, \mathbb{R}^{n}\right)$.

\subsection{Perturbation theory for the Schrödinger equation}

In Corollary 4 we converted the time-dependent Schrödinger problem to a Volterra integral equation, (42), wherein $K_{\mathrm{f}}(x, y, t)=(4 \pi i t)^{-n / 2} e^{i|x-y|^{2} / 4 t}$. The solution of that equation by iteration (successive approximations, Picard algorithm, Neumann series) is effectively a power series in the potential $V$, so it is the same thing as a perturbation calculation with respect to a coupling constant multiplying $V$.

In this problem the Banach space $\mathcal{B}$ is the Hilbert space $L^{2}\left(\mathbb{R}^{n}\right)$. (To assure pointwise convergence to the initial data, according to Theorem 2 and Remark 3, we should also take the intersection with $L^{1}\left(\mathbb{R}^{n} ;\left(1+|x|^{2}\right)^{-1} d x\right)$.) In order for our method to work simply, we must assume that $V(x, t)$ is a bounded potential. It may be time-dependent, but in that case its bound should be independent of $t$. That is, we assume

$$
V \in L^{\infty}\left(\mathbb{R}^{n} \times I\right) ; \quad|V(y, t)| \leq D \text { (almost everywhere). }
$$

Note that the role of $f$ in the abstract Volterra equation (62) is played by the entire first integral term in (42), $\int K_{\mathrm{f}}(x, y, t) f(y) d y$.

From the other term of (42) we extract the kernel function

$$
K(x, t ; y, \tau)=-\frac{i}{\hbar} K_{\mathrm{f}}(x, y, t-\tau) V(y, \tau)
$$

It satisfies neither the Hilbert-Schmidt condition (81) nor the generalized Young conditions (73) and (77). However, the resulting operator kernel can be factored as

$$
A(t, \tau)=-\frac{i}{\hbar} U_{\mathrm{f}}(t-\tau) \hat{V}(\tau)
$$

where $U_{\mathrm{f}}$ is the free time evolution (9) implemented by the kernel $K_{\mathrm{f}}$, and $\hat{V}$ is the operator of pointwise multiplication by the potential $V(y, \tau)$. It is well known [7, Chapter 4] that $U_{\mathrm{f}}(t)=e^{-i t H_{0} / \hbar}$ is unitary, and hence its norm as an operator on $L^{2}\left(\mathbb{R}^{n}\right)$ is 1 . On the other hand,

$$
\begin{aligned}
\|V(\tau) f(\tau)\|_{L^{2}\left(\mathbb{R}^{n}\right)}^{2} & =\int_{\mathbb{R}^{n}}|V(y, \tau) f(y, \tau)|^{2} d y \\
& \leq D^{2} \int_{\mathbb{R}^{n}}|f(y, \tau)|^{2} d y=D^{2}\|f(\tau)\|_{L^{2}\left(\mathbb{R}^{n}\right)}^{2}
\end{aligned}
$$


so the operator norm of $\hat{V}(\tau)$ is

$$
\|\hat{V}(\tau)\|=\|V(\cdot, \tau)\|_{L^{\infty}\left(\mathbb{R}^{n}\right)} \leq\|V\|_{L^{\infty}\left(I \times \mathbb{R}^{n}\right)} \leq D
$$

Therefore, the norm of the product operator is

$$
\|A(t, \tau)\|=\left\|(i \hbar)^{-1} U_{\mathrm{f}}(t-\tau) \hat{V}(\tau)\right\| \leq D / \hbar
$$

Therefore, Lemma 9 and Theorem 13 apply to the integral equation (42), and we reach the desired conclusion:

Theorem 16. If the potential $V(x, t)$ is uniformly bounded, then the time-dependent Schrödinger problem described in Corollary 4 can be solved by iteration. That is, the perturbation (Neumann) series converges in the topology of $L^{\infty}\left((0, T), L^{2}\left(\mathbb{R}^{n}\right)\right)$ for any finite, positive $T$.

\section{Concluding remarks}

Most mathematical physics literature on the Schrödinger equation (for example, [14]) works in an abstract Hilbert-space framework and concentrates on proving that particular second-order elliptic Hamiltonian operators are self-adjoint, then describing their spectra and other properties. Here we have investigated a different aspect of the subject; we regard the time-dependent Schrödinger equation as a classical partial differential equation analogous to the heat or wave equation and study it by classical analysis.

The similarities between the Schrödinger and heat equations were exploited to create the theoretical framework, and then their technical differences were addressed. In section 2 the structure of solutions in terms of the free propagator $K_{\mathrm{f}}$ was worked out, and thereby the initial-value problem was recast as an integral equation.

The key feature of that equation is its Volterra character: It involves integration only up to the time in question. In this respect it is like the heat equation and unlike, for instance, the Poisson equation. The consequence of the Volterra property is that when the equation is solved by iteration, the $j$ th iterate involves integration over a $j$-dimensional simplex (not a hypercube). The resulting volume factor of $(j !)^{-1}$ suggests that the series should converge.

The implementation of that idea in any particular case requires some technical work to prove that the operators $A(t, \tau)$ connecting any two times are bounded, and uniformly so. In section 4 we showed, in the setting of any Banach space, that that hypothesis is sufficient to establish the convergence of the Neumann series. In section 5 we verified the hypothesis in several simple examples, including the Schrödinger problem with a bounded potential.

In future work we hope to apply the Volterra theorem in contexts more complicated than the simple examples presented here. Preliminary work on those applications appears in Chapters 8 and 9 of [3]. Chapter 9 and [15] (see also [16]) implement an idea due to Balian and Bloch [17] to use a semiclassical Green function to construct a perturbation expansion for a smooth potential $V(x, t)$. The solution of the Schrödinger equation is approximated in terms of classical paths, and the resulting semiclassical propagator $K_{\mathrm{scl}}=A e^{i S / \hbar}$ is used as the building block for the exact propagator. The result is a series in $\hbar$, rather than in a 
coupling constant as in Theorem 16. The domain of validity of the construction in its simplest form is limited because the caustic structure of $K_{\mathrm{scl}}$ can spoil the uniform boundedness; improvements are an open field of research.

Chapter 8 dealt with the application of the Volterra method to boundary-value problems for the Schrödinger equation. Following the heat-equation theory $[1,2,18]$, the solutions were formally represented as single-layer and double-layer potentials, giving rise to Volterra integral equations on the boundary. Unfortunately, the proof in [3] of the existence and boundedness of the resulting operators is defective. The problem remains under investigation, and we hope that generalizing the Volterra theorem to a less obvious space (similarly to Theorems 14 and 15) will provide the answer.

\section{Acknowledgements}

We are grateful to Ricardo Estrada, Arne Jensen, Peter Kuchment, and Tetsuo Tsuchida for various pieces of helpful advice. The paper [18] originally led us to the mathematical literature [1] on the series solution of the Schrödinger equation. This research was supported by National Science Foundation Grants PHY-0554849 and PHY-0968269.

\section{Author details}

Fernando D. Mera and Stephen A. Fulling*

* Address all correspondence to: fulling@math.tamu.edu

Departments of Mathematics and Physics, Texas A\&M University, College Station, TX, USA

\section{References}

[1] I. Rubinstein and L. Rubinstein. Partial Differential Equations in Classical Mathematical Physics. Cambridge University Press, New York, 1998.

[2] R. Kress. Linear Integral Equations. Springer-Verlag, New York, second edition, 1999.

[3] F. D. Mera. The Schrödinger equation as a Volterra problem. Master's thesis, Texas A\&M University, College Station, TX, May 2011.

[4] G. B. Folland. Introduction to Partial Differential Equations. Princeton University Press, Princeton, New Jersey, second edition, 1995.

[5] G. H. Hardy. Divergent Series. Chelsea Publishing Co., New York, second edition, 1991.

[6] R. R. Estrada and R. P. Kanwal. A Distributional Approach to Asymptotics: Theory and Applications. Birkhäuser, Boston, second edition, 2002.

[7] L.C. Evans. Partial Differential Equations, Graduate Studies in Mathematics, volume 19. American Mathematical Society, Providence, RI, second edition, 2010.

[8] G. F. Carrier, M. Krook, and C. E. Pearson. Functions of a Complex Variable: Theory and Technique. Society for Industrial and Applied Mathematics, New York, 1966. 
[9] G. A. Hagedorn and A. Joye. Semiclassical dynamics with exponentially small error estimates. Commun. Math. Phys., 207:439-465, 1999.

[10] M. Reed and B. Simon. Methods of Modern Mathematical Physics II: Fourier Analysis, Self-Adjointness. Academic Press, New York, 1975.

[11] W. V. Lovitt. Linear Integral Equation. Dover Publications, New York, 1950.

[12] F.G. Tricomi. Integral Equations. Dover Publications, New York, 1985.

[13] B. L. Moiseiwitsch. Integral Equations. Dover Publications, New York, 2005.

[14] H. L. Cycon, R. G. Froese, W. Kirsch, and B. Simon. Schrödinger Operators with Application to Quantum Mechanics and Global Geometry. Springer, Berlin, 1987.

[15] F. D. Mera, S. A. Fulling, J. D. Bouas, and K. Thapa. WKB approximation to the power wall. in preparation.

[16] J. D. Bouas, S. A. Fulling, F. D. Mera, K. Thapa, C. S. Trendafilova, and J. Wagner. Investigating the spectral geometry of a soft wall. In A. Barnett, C. Gordon, P. Perry, and A. Uribe, editors, Spectral Geometry, Proceedings of Symposia in Pure Mathematics, Vol. 84, 2012, pp. 139-154

[17] R. Balian and C. Bloch. Solution of the Schrödinger equation in terms of classical paths. Ann. Phys., 85:514-545, 1974.

[18] I. Pirozhenko, V. V. Nesterenko, and M. Bordag. Integral equations for heat kernel in compound media. J. Math. Phys, 46:042305, 2005. 
Chapter 12

\title{
Quantum Perturbation Theory in Fluid Mixtures
}

\author{
S. M. Motevalli and M. Azimi \\ Additional information is available at the end of the chapter \\ http://dx.doi.org/10.5772/54056
}

\section{Introduction}

Experimental assessment of macroscopic thermo-dynamical parameters under extreme conditions is almost impossible and very expensive. Therefore, theoretical EOS for further experiments or evaluation is inevitable. In spite of other efficient methods of calculation such as integral equations and computer simulations, we have used perturbation theory because of its extensive qualities. Moreover, other methods are more time consuming than perturbation theories. When one wants to deal with realistic intermolecular interactions, the problem of deriving the thermodynamic and structural properties of the system becomes rather formidable. Thus, perturbation theories of liquid have been devised since the mid-20th century. Thermodynamic perturbation theory offers a molecular, as opposed to continuum approach to the prediction of fluid thermodynamic properties. Although, perturbation predictions are not expected to rival those of advanced integral-equations or large scale computer simulations methods, they are far more numerically efficient than the latter approaches and often produced comparably accurate results.

Dealing with light species such as $\mathrm{He}$ and $\mathrm{H}_{2}$ at low temperature and high densities makes it necessary taking into account quantum mechanical effects. Quantum rules and shapes related with the electronic orbital change completely the macroscopic properties.

Furthermore, for this fluid mixture, the quantum effect has been exerted in terms of first order quantum mechanical correction term in the Wigner-Kirkwood expansion. This term by generalizing the Wigner-Kirkwood correction for one component fluid to binary mixture produce acceptable results in comparison with simulation and other experimental data. Since utilizing Wigner-Kirkwood expansion in temperatures below $50 \mathrm{~K}$ bears diverges, we preferred to restrict our investigations in ranges above those temperatures from 50 to 4000 degrees. In these regions our calculations provide more acceptable results in comparison with other studies. 
This term make a negligible contribution under high temperatures conditions. Taking into account various contributions, we have utilized an improved version of the equation of state to study the Helmholtz free energy $F$, to investigate the effects of $P$ and $T$ on thermodynamic properties of helium and hydrogen isotopes mixtures over a wide range of densities. We also have studied effects of concentrations of each component on macroscopic parameters. In addition, comparisons among various perturbation and ideal parts have been presented in logarithmic diagrams for different densities and concentrations for evaluation of perturbation terms validity in respect to variables ranges.

The first section is dedicated to a brief description of Wigner expansion which leads to derivation of first quantum correction term in free energy. With the intention of describing effects of quantum correction term we have explained theoretical method of our calculations in the frame work of statistical perturbation theory of free energy in section two. In section three we have depicted diagrams resulted from our theoretical evaluations and gave a brief explanation for them. In section four we have focused on the description of our calculations and its usages in different areas. Finally, some applications of this study have been introduced in the last section.

\section{Quantum correction term}

Considering quantum system of $N$ identical particles of mass $m$ confined to the region of $\Lambda$ with the interacting potential of $U$. This structure is considered in $v$-dimension space $\left(R^{v}\right)$. In the absence of external fields the Hamiltonian of particles is given as

$$
H=\frac{1}{2 m}(-i \hbar \vec{\nabla})^{2}+U(\vec{r})
$$

Where, $\hbar$ is the Plank constant. The equilibrium statistical mechanics of the particle system is studied in the canonical ensemble at the temperature $T$ (or, alternatively, the inverse temperature $\beta=1 / k_{B} T$ with $k_{B}$ being Boltzmann's constant). Quantum effects will be considered via de Broglie wavelength $\lambda=\hbar \sqrt{\beta / m}$. For a typical microscopic length of particles $l$, for sufficiently small dimensionless parameter $\lambda / l$ semi-classical regime is dominant. In such system Boltzmann density in configuration space $\vec{r}$ can be expanded in powers of $\lambda^{2}$ within the wellknown Wigner-Kirkwood expansion [1, 2]. In the case of an inverse-power-law repulsive potential $V(r)=V_{0}(a / r)^{n}$ from the range $1<n<\infty$, the Wigner-Kirkwood expansion turns out to be analytic in $\lambda^{2}$ [3]. In the hard-core limit $n \rightarrow \infty$, this expansion is not further correct and one has the non-analyticity of type $\left(\lambda^{2}\right)^{1 / 2}$, as was shown in numerous analytic studies [4-7]. In contrast to the bulk case, the resulting Boltzmann density involves also position dependent terms which are non-analytic in $\lambda$. Under some condition about the classical density profile, the analyticity in $\lambda$ is restored by integrating the Boltzmann density over configuration space. 


\subsection{Wigner-Kirkwood expansion}

To have an analytical equation for quantum effects in fluid we must derive partition function of it. In approximating partition function we need to evaluate Boltzmann density. Consequently having an expansion of quantum correction terms it is necessary to expand Boltzmann density. Considering system of $N$ particles in the infinite space in standard Wigner-Kirkwood expansion [1, 2] fermions or boson exchange effects between quantum particles have been neglected. In the "bulk" regime, equilibrium quantities of this system in the nearly classical regime can be expanded in powers of $h^{2}$. In this section, we review briefly the derivation of this expansion for utilizing it in statistical perturbation framework. The Boltzmann density $B_{\beta}$ in configuration space $\vec{r}$ can be formally written in the basis of plane waves as a $v N$-dimensional integral defined in an infinite domain $R^{v}$ :

$$
B_{\beta}=\left\langle\vec{r}\left|e^{-\beta H}\right| \vec{r}\right\rangle=\int \frac{d \vec{P}}{(2 \pi \hbar)^{\nu N}} e^{-(i / \hbar) \vec{p} \cdot \vec{r}} e^{-\beta H} e^{(i / \hbar) \vec{p} \cdot \vec{r}}
$$

Where $\vec{p}=\left(p_{1}, p_{2}, p_{3}, \ldots\right)$ is the $v N$-dimensional momentum vector. Instead of considering we take the Laplace transform of this operator with respect to the inverse temperature $\beta$,

$$
\int_{0}^{\beta} d \beta e^{-\beta H} e^{-\beta z}=\frac{1}{H+Z}
$$

Via integrating equation 2 in respect to $\beta$ we have

$$
\int_{0}^{\beta} d \beta\left\langle\vec{r}\left|e^{-\beta H}\right| \vec{r}\right\rangle e^{-\beta z}=\int \frac{d \vec{P}}{(2 \pi \hbar)^{\nu N}} e^{-(i / \hbar) \vec{p} \cdot \vec{r}} \frac{1}{H+Z} e^{(i / \hbar) \vec{p} \cdot \vec{r}}
$$

Let us introduce following definition

$$
H+z=D+Q
$$

That $Q$ and $D$ respectively represent

$$
\begin{gathered}
Q=\frac{1}{2 m}(-i \hbar \vec{\nabla})^{2}-\frac{1}{2 m} \vec{P}^{2} \\
D=\frac{1}{2 m} \vec{P}^{2}+U(\vec{r})+z
\end{gathered}
$$


One can expand

$$
\frac{1}{H+Z}=\frac{1}{D}-\frac{1}{D} Q \frac{1}{D}+\frac{1}{D} Q \frac{1}{D} Q \frac{1}{D}-\ldots
$$

$Q$, operates in the following manner

$$
Q\left[f(\vec{r}) e^{(i / \hbar) \vec{p} \cdot \vec{r}}\right]=e^{-(i / \hbar) \vec{p} \cdot \vec{r}}\left[\frac{i \hbar}{m} \vec{p} \cdot \vec{\nabla}+\frac{\hbar^{2}}{2 m} \vec{\nabla}^{2}\right] f(\vec{r})
$$

And then we can find that

$$
\begin{aligned}
& e^{-(i / \hbar) \vec{p} \cdot \vec{r}} \frac{1}{H+z} e^{(i / \hbar) \vec{p} \cdot \vec{r}}=\frac{1}{D} \sum_{n=0}^{\infty}\left\{\left[\frac{i \hbar}{m} \vec{p} \cdot \vec{\nabla}+\frac{\hbar^{2}}{2 m} \vec{\nabla}^{2}\right] \frac{1}{D}\right\}^{n} \\
& \int \frac{d \vec{P}}{(2 \pi \hbar)^{v N}} e^{-(i / \hbar) \vec{p} \cdot \vec{r}} \frac{1}{H+z} e^{(i / \hbar) \vec{p} \cdot \vec{r}}=\int \frac{d \vec{P}}{(2 \pi \hbar)^{v N}} \frac{1}{D} \sum_{n=0}^{\infty}\left\{\left[\frac{i \hbar}{m} \vec{p} \cdot \vec{\nabla}+\frac{\hbar^{2}}{2 m} \vec{\nabla}^{2}\right] \frac{1}{D}\right\}^{n}= \\
& \int_{0}^{\beta} d \beta\left\langle\vec{r}\left|e^{-\beta H}\right| \vec{r}\right\rangle e^{-\beta z}=\sum_{n=0}^{\infty} \int \frac{d \vec{P}}{(2 \pi \hbar)^{v N}} \frac{1}{D}\left\{\left[\frac{i \hbar}{m} \vec{p} \cdot \vec{\nabla}+\frac{\hbar^{2}}{2 m} \vec{\nabla}^{2}\right] \frac{1}{D}\right\}^{n}
\end{aligned}
$$

So we have expanded series in $\hbar^{2 n}$ which enable us power series of $\hbar^{n}$. It remains to define $1 / D^{j}$

$$
\frac{1}{D^{j}}=\int_{0}^{\infty} d \beta \frac{1}{(j-1) !} \beta^{j-1} e^{-\beta D}=\int_{0}^{\infty} d \beta e^{-\beta z} \frac{1}{(j-1) !} \beta^{j-1} e^{-\beta\left[\vec{p}^{2} / 2 m+U(\vec{r})\right]}
$$

and finally integrating on the momentum variables $\vec{p}$, the Boltzmann density in configuration space is obtained as the series

$$
\left\langle\vec{r}\left|e^{-\beta H}\right| \vec{r}\right\rangle=\sum_{n=0}^{\infty} B_{\beta}^{(n)}(\vec{r}),
$$

where 


$$
\begin{aligned}
& B_{\beta}^{(0)}(\vec{r})=\frac{1}{(\sqrt{2 \pi} \lambda)^{v N}} e^{-\beta U} \\
& B_{\beta}^{(1)}(\vec{r})=\frac{1}{(\sqrt{2 \pi} \lambda)^{v N}} e^{-\beta U} \lambda^{2}\left[\frac{-\beta}{4} \vec{\nabla}^{2} U+\frac{\beta^{2}}{6}(\vec{\nabla} U)^{2}\right], \\
& B_{\beta}^{(2)}(\vec{r})=\frac{1}{(\sqrt{2 \pi} \lambda)^{v N}} e^{-\beta U}\left\{\lambda^{2}\left[\frac{\beta}{6} \vec{\nabla}^{2} U-\frac{\beta^{2}}{8}(\vec{\nabla} U)^{2}\right]+O \lambda^{4}\right\}
\end{aligned}
$$

We conclude that the quantum Boltzmann density in configuration space is given, to order $\lambda^{2}$, by

$$
\begin{aligned}
& \left\langle\vec{r}\left|e^{-\beta H}\right| \vec{r}\right\rangle=\frac{1}{(\sqrt{2 \pi} \lambda)^{v N}} e^{-\beta U}\left\{1+\lambda^{2}\left[\frac{-\beta}{12} \vec{\nabla}^{2} U+\frac{\beta^{2}}{24}(\vec{\nabla} U)^{2}\right]+O \lambda^{4}\right\}= \\
& \frac{1}{(\sqrt{2 \pi} \lambda)^{v N}}\left\{e^{-\beta U}\left[1-\frac{\lambda^{2} \beta}{24} \vec{\nabla}^{2} U\right]+\frac{\lambda^{2}}{24} \vec{\nabla}^{2} e^{-\beta U}+O \lambda^{4}\right\}
\end{aligned}
$$

Integrating Boltzmann density ignoring exchange effects over configuration space will result in partition function of fluids mixture.

$$
Z_{q u}=\frac{1}{N !} \int_{V} d \vec{r}\left\langle\vec{r}\left|e^{-\beta H}\right| \vec{r}\right\rangle
$$

Substituting the $\lambda$-expansion of the Boltzmann density (12A) into formula (13), the quantum partition function takes the expansion form

$$
Z_{q u}=\frac{1}{N !} \int_{\Lambda} d \vec{r} \frac{1}{(\sqrt{2 \pi} \lambda)^{v N}}\left\{e^{-\beta U}\left[1-\frac{\lambda^{2} \beta}{24} \vec{\nabla}^{2} U\right] 1+\frac{\lambda^{2}}{24} \vec{\nabla}^{2} e^{-\beta U}+O \lambda^{4}\right\}
$$

For expressing macroscopic physical quantities, one defines the quantum average of a function $f(\vec{r})$ as follows

$$
\langle f\rangle_{q u}=\frac{1}{Z_{q u} N !} \int_{\Lambda} d \vec{r}\left\langle\vec{r}\left|e^{-\beta H}\right| \vec{r}\right\rangle f(\vec{r})
$$

At the one-particle level, one introduces the particle density 


$$
n_{q u}(r)=\left\langle\sum_{j=1}^{N} \delta\left(r-r_{j}\right)\right\rangle_{q u}
$$

At the two-particle level, the two-body density is given by

$$
n_{q u}^{(2)}\left(r, r^{\prime}\right)=\left\langle\sum_{\substack{j, k=1 \\ j \neq k}}^{N} \delta\left(r-r_{j}\right) \delta\left(r^{\prime}-r_{j}\right)\right\rangle_{q u}
$$

And the pair distribution function

$$
g_{q u}\left(r, r^{\prime}\right)=\frac{n_{q u}^{(2)}\left(r, r^{\prime}\right)}{n_{q u}(r) n_{q u}\left(r^{\prime}\right)}
$$

The classical partition function and the classical average of a function $f(\vec{r})$ are defined as follows

$$
\begin{gathered}
Z=\frac{1}{N !} \int_{\Lambda} \frac{\overrightarrow{d r}}{(\sqrt{2 \pi} \lambda)^{v N}} e^{-\beta U(\vec{r})} \\
\langle f\rangle=\frac{1}{Z N !} \int_{\Lambda} \frac{d \vec{r}}{(\sqrt{2 \pi} \lambda)^{v N}} e^{-\beta U(\vec{r})} f(\vec{r})
\end{gathered}
$$

Consequently with the definition of equation 19 one can derive below equation for $Z_{q u}$

$$
\begin{gathered}
Z_{q u}=Z\left\{1-\lambda^{2} \frac{\beta}{24}\left\langle\vec{\nabla}^{2} U\right\rangle+O \lambda^{4}\right\} \\
\beta F_{q u}=-\ln \left(Z_{q u}\right) \\
\ln \left(Z_{q u}\right)=\ln (Z)+\ln \left(1-\lambda^{2} \frac{\beta}{24}\left\langle\vec{\nabla}^{2} U\right\rangle+O \lambda^{4}+\ldots\right)
\end{gathered}
$$


Since we have $\ln (1-x)=-x-x^{2} / 2-\ldots$ we can expand the second term in the right side. By means of equation 18 in deriving $\left\langle\vec{\nabla}^{2} U\right\rangle$ we can have explicit formula for the second term of which indicates the first term of Wigner-Kirkwood correction part that is consist of the second derivative of potential function that leads to below equation for quantum correction term with the number density of $n$ we have

$$
F_{q u}^{(1)}=\frac{h^{2} N_{A} n \beta}{96 \pi^{2} m} \int_{\sigma^{0}}^{\infty} \nabla^{2} U(r) g(r) d r
$$

$g(r)$ represents radial distribution function, which is a measure of the spatial structure of the particles in reference system, is the expected number of particles at a distance $r . N_{A}$ is Avogadro constant and $\sigma^{0}$ is the distance in which potential function effectively tend to zero.

\subsection{Free energy}

Generalizing to multi-component system we have [8]

$$
F_{q u}^{(1)}=\frac{h^{2} N_{A} n \beta}{96 \pi^{2}} \sum_{i, j} \frac{c_{i} c_{j}}{m_{i j}} \int_{\sigma_{i j}^{0}}^{\infty} \nabla^{2} u_{i j}(r) g_{i j}(r) 4 \pi r^{2} \overline{V_{i j}} d r
$$

$m_{11}=m_{1}, \quad m_{22}=m_{2}, \quad m_{12}=c_{1} m_{1}+c_{2} m_{2}$

$m_{i}$ is the ith particle's mass. $\overline{V_{i j}}$ is the average molecular volume. Distribution function defines probability of finding particle at particular point $r$. In many literatures that have studied distribution function found it more versatile to use Laplace transform of this function $G(s)$.

$$
G_{i j}(s)=\int_{0}^{\infty} r g_{i j}(r) e^{-s r} d r
$$

In this chapter the two formula which use RDF, we will encounter below integral equation that need expansion.

$$
I=\int_{\sigma_{0}}^{\infty} r \phi(r) r g(r) d r=\sigma_{0}^{3} \int_{1}^{\infty} x \phi(x) x g(x) d x=\sigma_{0}^{3}\left\{\int_{0}^{\infty} x^{2} \phi(x) g(x) d x-\int_{0}^{1} x^{2} \phi(x) g(x) d x\right\}
$$

On the right side of above equation from the right in the first equation we approximate distribution function with its values at contact points. This choice has been resulted from the behavior of molecules of which their repulsive interactions dominate their attractive potential. 
However, for the second term $\left(I^{\prime}\right)$ we will use change in integrals to employ Laplace transform of RDF instead of RDF directly.

$$
x g(x)=\frac{1}{2 \pi i} \int_{\gamma-i \infty}^{\gamma-i \infty} G(s) e^{-s x} d s
$$

Substituting above equation in $I^{\prime}$ we have

$$
I^{\prime}=\int_{0}^{\infty} \varphi(s) G(s) d s
$$

Where $\varphi(s)$ represents

$$
\varphi(s)=\frac{1}{2 \pi i} \int_{\gamma-i \infty}^{\gamma-i \infty} x \phi(x) e^{-s x} d x
$$

That indicates inverse Laplace of $x \phi(x)$. So it suffices to just define inverse Laplace of potential function multiplied by $x$.

Therefore, Using Laplace transform of RDF G(s) [9] quantum correction term for DY potential turn out to be

$$
F^{Q}=\frac{h^{2} N_{A} n \beta}{24 \pi} \sum_{i, j} \frac{c_{i} c_{j} \varepsilon_{i j} A_{i j} \overline{V_{i j}}}{m_{i j} \sigma_{i j}^{0}}\left(\lambda_{i j}^{2} e^{\lambda_{i j}} G\left(\frac{\lambda_{i j}}{\sigma_{i j}^{0}}\right)-v_{i j}^{2} e^{v_{i j}} G\left(\frac{v_{i j}}{\sigma_{i j}^{0}}\right)\right)
$$

$c_{i}$ is the $i$ particle's concentration and $n$ represents number density. $A_{i j}, \lambda_{i j}$ and $v_{i j}$ are controlling parameters of double Yukawa(DY). $\varepsilon_{i j}$ is the attractive well depth of mutual interacting potential.

\section{Framework}

The derivation of the thermodynamic and structural properties of a fluid system becomes a rather difficult problem when one wants to deal with realistic intermolecular interactions. For that reason, since the mid-20th century, simplifying attempts to (approximately) solve this problem have been devised, among which the perturbation theories of liquids have played a prominent role [10]. In this instance, the key idea is to express the actual potential in terms of a reference potential (that in terms of Ross perturbation theory Helmholtz free energy is 
expressed as of the "unperturbed" system) plus a correction term. This in turn implies that the thermodynamic and structural properties of the real system may be expressed in terms of those of the reference system which, of course, should be known. In the case of two component fluids, a natural choice for the reference system is the hard-sphere fluid, even for this simple system the thermodynamic and structural properties are known only approximately. Let us now consider a system defined by a pair interaction potential $u(r)$. The usual perturbation expansion for the Helmholtz free energy, $F$, to first order in $\beta=1 / k_{\beta} T$, with $T$ being the absolute temperature and $k_{B}$ being the Boltzmann constant, leads to $F$. Common starting point of many thermodynamic perturbation theories is an expansion of the Helmholtz free energy, the resulting first-order prediction for a fluid composed of particles helium and hydrogen is given via the following equation.

$$
F=F^{t}+F^{Q}+F^{H B}+F^{i d}
$$

The terms respectively are perturbation, Quantum, hard convex body and ideal terms. Perturbation term due to long range attraction of potential is given by [10]

$$
F^{t}=2 \pi n \sum_{i, j} c_{i} c_{j} \int_{\sigma_{i j}^{0}}^{\infty} u_{i j}(r) g_{i j}^{H S}\left(r, \rho, \sigma_{i j}\right) 4 \pi r^{2} \overline{V_{i j}} d r
$$

Via Laplace transform of RDF $\left(r g_{i j}^{H S}\right)$ in calculation of first order perturbation contribution due to long-ranged attraction for DY potential we can employ below equation:

$$
F^{t}=k T \sum_{i, j} c_{i} c_{j} \varepsilon_{i j} \sigma_{i j}^{0} A_{i j} \bar{V}_{i j}\left(e^{\lambda_{i j}} G\left(\frac{\lambda_{i j}}{\sigma_{i j}^{0}}\right)-e^{v_{i j}} G\left(\frac{v_{i j}}{\sigma_{i j}^{0}}\right)\right)-\delta F^{t}
$$

$\overline{V_{i j}}$ the average molecular volume defined as:

$$
\overline{V_{i j}}=1+\frac{\left(n_{i}^{\prime}-1\right)}{\sigma_{i i}{ }^{3}}\left[\frac{3}{2}\left({\sigma_{i i}}^{2}+{\sigma_{i j}}^{2}\right) l_{i}-\frac{1}{2}\left(l_{i}\right)^{3}-\frac{3}{2}\left[\left(\sigma_{i i}+\sigma_{j j}\right)^{2}-l_{i}^{2}\right]^{\frac{1}{2}} \sin ^{-1}\left[\frac{l_{i}}{\sigma_{i i}+\sigma_{i j}}\right]\left(\sigma_{i j}{ }^{2}\right)\right]
$$

Where $n^{\prime}{ }_{i}$ define the number of element in a molecule, $l_{i}$ is distance of centre to centre for each molecule. $\delta F^{t}$ corresponds to the interval of $\left[\sigma_{i j}, \sigma_{i j}^{0}\right]$ which long range attractive range is not further applicable. Consequently, we prefer to use the contact value of hard sphere RDF at $r=\sigma_{i j}$. By this approach we can express this term as: 


$$
\delta F^{t} \approx \frac{n}{2 k T} \sum_{i, j} c_{i} c_{j} \int_{\sigma_{i j}}^{\sigma_{i j}^{0}} u_{i j}(r) g_{i j}^{H S}\left(\sigma_{i j}\right) 4 \pi r^{2} \overline{V_{i j}} d r
$$

$g_{i j}^{H S}\left(\sigma_{i j}\right)$ is the contact value of radial distribution function. $\sigma_{i j}$ stands for separation distance at contact between the centers of two interacting fluid particles, with species $i$ and $j$. Although via minimization of Helmholtz free energy we can achieve value for hard sphere diameter, we preferred to use its analytical form due to its practical approach [17]. Hard sphere diameter will be calculated by means of Barker-Henderson equation as a function of interacting potential and temperature. Using Gauss-Legendre qudrature integration method we are able to evaluate its values numerically.

$$
\sigma_{i j}=\int_{0}^{\sigma_{i j}^{0}}\left(1-\exp \left(-\beta u_{i j}^{D Y}(r)\right)\right) d r
$$

$F^{H B}$, Helmholtz free energy of hard convex body is given by following equation:

$$
F^{H B}=a_{\text {mix }}\left(F^{H S}+F^{n d}\right)
$$

Non-sphericity parameter $a_{\text {mix }}$ for the scaling theory [11] is defined as

$$
a_{\text {mix }}=\frac{1}{3 \pi} \frac{\sum_{i, j} c_{i} c_{j} V_{i j}^{e f}\left(V_{i j}^{\text {eff }}\right)^{\prime}\left(V_{i j}^{\text {eff }}\right)^{\prime \prime}}{\sum_{i, j} c_{i} c_{j} V_{i j}^{e f f}}, \quad V_{i j}^{e f f}=\frac{\pi}{6} \sigma_{i j}^{3} \overline{V_{i j}}
$$

$\left(V_{i j}^{e f f}\right)^{\prime}$ and $\left(V_{i j}^{e f f}\right)^{\prime \prime}$ are the first and second partial derivatives of $V_{i j}^{\text {eff }}$ with respect to $\sigma_{i i}$ and $\sigma_{j j}$. From Boublik, Mansoori, Carnahan, Starling, Leland (BMCSL) $[12,8]$ with correction term of Barrio [13] on EOS, the Helmholtz free energy, $F^{H S}$ for hard sphere term becomes:

$$
\begin{aligned}
& \frac{F^{H S}}{K T}=\frac{\eta_{3}\left[\xi_{1}+\left(2-\eta_{3}\right) \xi_{2}\right]}{1-\eta_{3}}+\frac{\eta_{3} \xi_{3}}{\left(1-\eta_{3}\right)^{2}}+\left(\xi_{3}+2 \xi_{2}-1\right) \ln \left(1-\eta_{3}\right), \\
& \xi_{1}=\frac{3 \eta_{1} \eta_{2}}{\eta_{0} \eta_{3}}, \quad \xi_{2}=\frac{\eta_{1} \eta_{2}}{\eta_{3}{ }^{2}}\left(\eta_{4} z_{1}+\eta_{0} z_{2}\right), \quad \xi_{3}=\frac{\eta_{2}{ }^{3}}{\eta_{0} \eta_{3}{ }^{3}}
\end{aligned}
$$




$$
\begin{aligned}
& z_{1}=2 c_{1} c_{2} \sigma_{11} \sigma_{22}\left(\frac{\sigma_{11}-\sigma_{22}}{\sigma_{11}+\sigma_{22}}\right) \\
& z_{2}=c_{1} c_{2} \sigma_{11} \sigma_{22}{ }^{3}\left({\sigma_{11}}^{2}-\sigma_{22}{ }^{2}\right)
\end{aligned}
$$

The correction term due to nonadditivity of the hard sphere diameter is the first order perturbation correction [14]

$$
F^{n d}=-k T \pi n c_{1} c_{2}\left(\sigma_{11}+\sigma_{22}\right)\left(\sigma_{11}+\sigma_{22}-2 \sigma_{12}\right) g_{12}^{H S}\left(\sigma_{12}\right)
$$

In Eq. (41), $g_{12}^{H S}\left(\sigma_{12}\right)$ refer to as hard sphere radial distribution function at $r=\sigma_{12}$ contact point by inclusion of Barrio and Solana correction on equation of state of BMCSL. Undoubtedly, the availability of the analytical HS RDF obtained from the solution to the corresponding PercusYevick (PY) equation represented a major step toward the successful application of the perturbation theory of liquids to more realistic inter-particle potentials. However, the lack of thermodynamic consistency between the virial and compressibility routes to the equation of state present in the PY approximation (as well as in other integral equation theories) is a drawback that may question the results derived from its use within a perturbation treatment. Fortunately, for our purposes, another analytical approximation for the RDF of the HS fluid, which avoids the thermodynamic consistency problem, has been more recently derived [15, 16]. We used improved RDF that yields exact asymptotic expression for the thermodynamic properties. However, we have used improved version of RDF that yields exact asymptotic expression for the thermodynamic properties. This have been derived by inclusion of Barrio and Solana correction on EOS of BMCSL at $r=\sigma_{12}[9]$

$$
\begin{gathered}
g_{i j}^{H S}\left(\sigma_{i j}\right)=g_{i j}^{B M C S L}\left(\sigma_{i j}\right)+g_{i j}^{B S}\left(\sigma_{i j}\right) \\
g_{12}^{B M C S L}\left(\sigma_{12}\right)=\frac{1}{1-\eta_{3}}+\frac{3 \eta_{2}}{\left(1-\eta_{3}\right)^{2}} \frac{\sigma_{i i} \sigma_{j j}}{\sigma_{i i}+\sigma_{i j}}+\frac{2 \eta_{2}{ }^{2}}{\left(1-\eta_{3}\right)^{3}}\left(\frac{\sigma_{i i} \sigma_{j j}}{\sigma_{i i}+\sigma_{j j}}\right)^{2}, \\
g_{12}^{B S}\left(\sigma_{12}\right)=\frac{1-\delta_{i j} c_{i}}{2} \frac{\eta_{1} \eta_{2}}{\left(1-\eta_{3}\right)^{2}}\left(\frac{\sigma_{i i} \sigma_{i j}}{\sigma_{i i}+\sigma_{i j}}\right)^{2}\left(\sigma_{11}-\sigma_{22}\right)\left(\delta_{i j}+\left(1-\delta_{i j}\right) \frac{\sigma_{22}}{\sigma_{11}}\right)
\end{gathered}
$$

$\eta_{i}=\frac{\pi}{6} n \sum_{j} c_{j} \sigma_{j j}{ }^{i}$

$\delta_{i j}$ is the Kronecker delta function. For additive mixtures $\sigma_{i j}$ is arithmetic mean of hard-core diameters of each species. Otherwise, the system is said to be non-additive. 
The ideal free energy with $N$ particle for the atomic and molecular components of fluid mixture are given by,

$$
F^{i d}(n, T)=\frac{3}{2} \ln \left(\frac{h^{2}}{2 \pi k T m_{1}^{c_{1}} m_{2}^{c_{2}}}\right)+\ln n+\sum_{i} c_{i} \ln c_{i}-1
$$

Compressibility factor of ideal term is one and $Z^{H B}$ would be estimated with the following derivation of related Helmholtz free energy

$$
Z^{H B}=n \frac{\partial}{\partial n} \frac{F^{H B}}{k T}
$$

For the perturbation term due to long rage attraction of potential tail employing (44) we will have

$$
\begin{aligned}
& Z^{t}=\frac{2 \pi n}{k T} \sum_{i, j} c_{i} c_{j} \varepsilon_{i j} \sigma_{i j}^{0} A_{i j} \bar{V}_{i j}\left(e^{\lambda_{i j}}\left(G\left(\frac{\lambda_{i j}}{\sigma_{i j}^{0}}\right)-n \frac{\partial}{\partial n} G\left(\frac{\lambda_{i j}}{\sigma_{i j}^{0}}\right)\right)-e^{v_{i j}}\left(G\left(\frac{v_{i j}}{\sigma_{i j}^{0}}\right)-n \frac{\partial}{\partial n} G\left(\frac{v_{i j}}{\sigma_{i j}^{0}}\right)\right)-\delta Z^{t}\right. \\
& \delta Z^{t} \approx \frac{n}{2 k T} \sum_{i, j} c_{i} c_{j}\left(g_{i j}^{H S}\left(\sigma_{i j}\right)+n \frac{\partial}{\partial n} g_{i j}^{H S}\left(\sigma_{i j}\right)\right) \int_{\sigma_{i j}}^{\sigma_{i j}^{0}} u_{i j}^{D Y}(r) 4 \pi r^{2} \overline{V_{i j}} d r
\end{aligned}
$$

Numerical integration has been used for calculation of $\delta Z^{t}$ in the range of $\left[\sigma_{i j}\right.$, $\left.\sigma_{i j}^{0}\right]$. Expressions for first order perturbation and quantum correction term of compressibility factor are achievable via applying (44) for the free energy part of the quantum correction term.

$$
Z^{Q}=\frac{h^{2} N_{A} n \beta^{2}}{24 \pi} \sum_{i, j} \frac{c_{i} c_{j} \varepsilon_{i j} A_{i j} \overline{j_{i j}}}{m_{i j} \sigma_{i j}^{0}}\left(\lambda_{i j}^{2} e^{\lambda_{i j}}\left(G\left(\frac{\lambda_{i j}}{\sigma_{i j}^{0}}\right)-n \frac{\partial}{\partial n} G\left(\frac{\lambda_{i j}}{\sigma_{i j}^{0}}\right)\right)-v_{i j}^{2} e^{v_{i j}}\left(G\left(\frac{v_{i j}}{\sigma_{i j}^{0}}\right)-n \frac{\partial}{\partial n} G\left(\frac{v_{i j}}{\sigma_{i j}^{0}}\right)\right)\right)
$$

Summation over compressibility factors gives the total pressure of mixture

$$
P=n k T\left(1+Z^{H B}+Z^{t}+Z^{Q}\right)
$$

Defining Gibbs free energy provides information at critical points of phase stability diagram. Concavity and convexity of Gibbs diagram indicates if mixture is in one phase or not, 


$$
G=F+\frac{N}{n} P
$$

Furthermore, Gibbs excess free energy is an appropriate measure in the definition of phase stability. Negative values for this energy describe stable state. This is expressed as

$$
G_{x s}=G-\sum_{i} c_{i} G_{i}^{0}-N k T \sum_{i} c_{i} \ln c_{i}
$$

That $G_{i}^{0}$ represents the Gibbs free energy of pure fluid of species i. Concentration-concentration structure factor is defined as

$$
S_{c c}(0)=N k T\left(\frac{\partial^{2}}{\partial c^{2}} G\right)_{T, P, N}^{-1}
$$

Compairing this equation with $S_{c c}^{i d}$ enable us to define degree of hetero-coordination. In a given composition if $S_{c c}(0)<<S_{c c}^{i d}$ then unlike atoms tend to pair as nearest neighbors (heterocoordination) and when $S_{c c}(0)>>S_{c c}^{i d}$ then like atoms are preferred as a neighbor.

\subsection{Potentials}

It is convenient to consider interacting potential with short-range sharply repulsive and longer-range attractive tail and treat them within a combined potential. The most practical method for the repulsive term of potential is the hard-sphere model with the benefit of preventing particles overlap. Furthermore, attractive or repulsive tails may be included using a perturbation theory. It is incontrovertible to generalize this potential to multi-component mixtures. This behavior is conveyed in double Yukawa (DY) potential which provides accurate thermodynamic properties of fluid in low temperatures and high density [18, 19]. At first we define DY potential as its effects on pressure of $\mathrm{He}-\mathrm{H}_{2}$ mixture has been studied in this work, written as:

$$
u_{i j}^{D Y}(r)=\varepsilon_{i j} A_{i j} \frac{\sigma_{i j}^{0}}{r}\left[e^{\lambda_{i j}\left(1-r / \sigma_{i j}^{0}\right)}-e^{v_{i j}\left(1-r / \sigma_{i j}^{0}\right)}\right]
$$

$A_{i j}, \lambda_{i j}, v_{i j}$ are controlling parameters. These parameters for $\mathrm{He}$ and $\mathrm{H}_{2}$ are listed in table 1 with their reference [20]. 


\begin{tabular}{cccc}
\hline & $\mathrm{He}-\mathrm{He}$ & $\boldsymbol{H e}-\boldsymbol{H}_{2}$ & $\boldsymbol{H}_{\mathbf{2}}-\boldsymbol{H}_{\mathbf{2}}$ \\
\hline$\sigma^{0}$ & 2.634 & 2.970 & 2.978 \\
\hline$A$ & 2.548 & 2.801 & 3.179 \\
\hline$\varepsilon / k_{B}$ & 10.57 & 15.50 & 36.40 \\
\hline$U$ & 3.336 & 3.386 & 3.211 \\
\hline$\lambda$ & 12.204 & 10.954 & 9.083 \\
\hline
\end{tabular}

Table 1. Potential parameters for $\mathrm{He}, \mathrm{H}_{2}$ interactions for DY potential [20].

For the atomic and molecular fluids studies in this mixture, these particles interact via a exponential six (exp-6) or Double Yukawa (DY) potential energy function [20]. The fluids considered in this work are binary mixtures that their constituents are spherical particles of two species, $i$ and $j$, interacting via pair potential $u_{i j}(r)$.

$$
u_{i j}^{\exp -6}(r)= \begin{cases}\infty & r<\sigma_{\infty} \\ \varepsilon_{i, j} \frac{\alpha_{i j}}{\alpha_{i j}-6}\left(6 \frac{6}{\alpha_{i j}} \exp \left(\alpha_{i j}\left(1-\frac{r}{\sigma_{\min , i j}}\right)\right)-\left(\frac{\sigma_{\min , i j}}{r}\right)^{6}\right) & r>\sigma_{\infty}\end{cases}
$$

So we consider two-component fluid interacting via Buckingham potential $u_{i j}(r)$ between molecules of types $i$ and $j$. This potential is more realistic than square-well or Yukawa type potential for hydrogen isotope's mixture [21] at high temperatures. Because of same atomic structure of hydrogen and its isotopes, the three constant of potential are same for hydrogen isotopes. These constants obtained experimentally from molecular scattering [22].

$\sigma_{\min }$ indicate the range of interaction and the parameter $\alpha$ regulates the stiffness of repulsion. For hydrogen and helium type atoms these parameters have been organized in Table 2. It is well known that the long range attractive part of exp-6 potential is similar to Lenard-Jones potential.

In view of the energy equation (32), one can readily obtain equation for total pressure and different contributions to pressure from standard derivation of respective Helmholtz free energy. By the exp-6 potential, we have computed the Helmholtz free energy. The ten-point Gausses quadrature has been used to calculate integrals in quantum correction and perturbation contribution. The calculated pressure for $D_{2}+T_{2}$ fluid mixture with equal mole fraction and at temperature of $T=100^{\circ} \mathrm{K}$ is showed logarithmically in figure 1 . As it is clear from this figure, the effect of hard sphere term of pressure in given rang of temperature is significant and the range of pressure variation is wider than ideal part. As it is mentioned earlier the difference between isotopes is simply related to the neutron number in each nucleus and correction due to difference in mass which involves in non-additive correction term doesn't 
affect sensibly. In addition, this figure shows the predicted equimolar surface of the deuterium and tritium mixture for quantum correction term. This part is the most significant contribution at low temperature and varying smoothly in higher temperature. At very high densities, perturbation term contribution increases sharply with reducing density. Also, terms, $P^{t}, P^{Q}$, $P^{H S}$ and $P^{i d}$, tend to infinity as $\rho \rightarrow \infty$.

\begin{tabular}{cccc}
\hline & $\mathrm{He}-\mathrm{He}$ & $\boldsymbol{H e}-\boldsymbol{H}_{2}$ & $\boldsymbol{H}_{2}-\boldsymbol{H}_{2}$ \\
\hline$a$ & 13.10 & 12.7 & 11.1 \\
\hline$\varepsilon / k_{B}$ & 10.80 & 15.50 & 36.40 \\
\hline$\sigma_{\min }$ & 0.29673 & 0.337 & 0.343 \\
\hline
\end{tabular}

Table 2. Potential parameters for $\mathrm{He}, \mathrm{H}_{2}$ interactions for exp-6 potential [20]

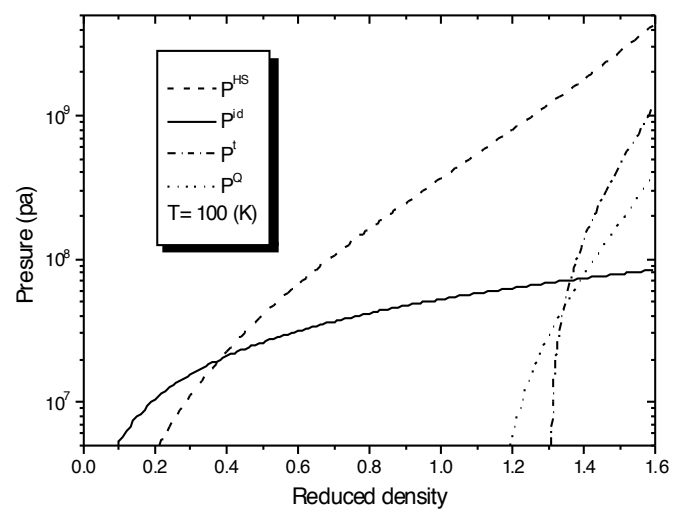

Figure 1. Different contributions of pressure as a function of reduced density for $T=100^{\circ} \mathrm{K}$ for fluid mixture of deuterium and tritium

\section{Results}

For helium-hydrogen mixtures different parts of pressure due to correction terms and ideal parts have been showed in figure 2 at $T=100^{\circ} \mathrm{K}$. Ideal pressure at reduced density of approximately zero, to about 0.25 rises drastically. However, afterward it soars gently up to $100 \mathrm{M}$ (pa). Pressure due to hard sphere is the most significant contribution except that it is less than perturbation part at value of 1.5 for reduced density. Effects of perturbation and Quantum correction are important in high densities. In low densities, these contributions are insignificant and may possibly be ignored. Non-additive part has been caused by dissimilarity of particles which surges steadily from the beginning. 


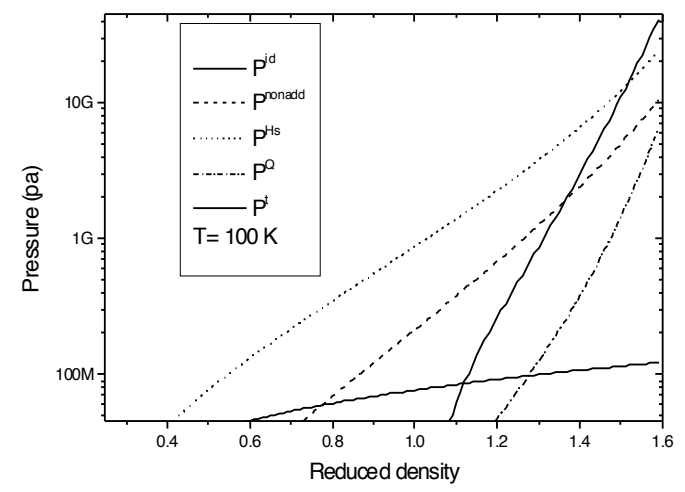

Figure 2. Different contribution of correction terms on pressure of helium-hydrogen mixture at $T=100$, che $=0.5$ vs. reduce density

Gibbs excess free energy which is a measure for indicating phase stability of matters has been depicted in figure 3. Stability is limited to the areas that Gibbs excess free energy tends to negative values. This figure explains that stability rages for helium-hydrogen mixture at room temperature is confide in the boundaries in which helium concentration is less than 0.1 .

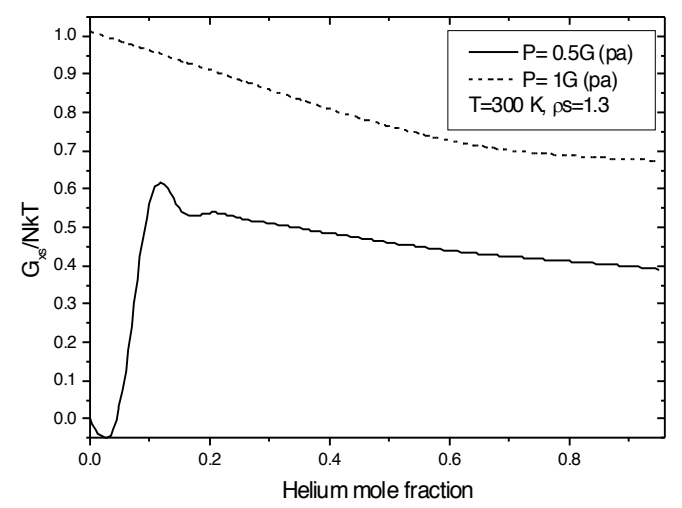

Figure 3. Gibbs excess free energy for helium-hydrogen mixture

Table 3 presents a comparison between results of pressure from this work using DY potential in place of exp-6, Monte-Carlo simulations and additionally study of reference [23] Obviously, there are appreciable adaption among our investigation results and $\mathrm{MC}$ which proves validity of our calculations. As Table 3, exhibits in low temperatures DY potential have more consistent results in comparison with exp-6. However, values of pressure extracted using DY potential cannot adjust with simulation resembling exp-6. Moreover, at higher temperatures after $T=1000^{\circ} \mathrm{K}$, DY potential is not good choice for evaluating EOS of hydrogen and helium mixture. We clarify our deduction presenting comparison between effects of these two potentials over wide ranges of temperatures. 


\begin{tabular}{rrrrrrrr}
\hline \multicolumn{1}{c}{$\boldsymbol{T}(\boldsymbol{K})$} & $\boldsymbol{C}_{\mathrm{He}}$ & \multicolumn{1}{c}{$\boldsymbol{\eta}$} & $\boldsymbol{P}_{\boldsymbol{M C}}$ & $\boldsymbol{P}[\mathbf{1 9 ]}$ & $\boldsymbol{P}[\mathbf{2 3}]$ & $\boldsymbol{P}_{D Y}[\mathbf{1 9 ]}$ \\
\hline 300 & 0.25 & 1.101 & 0.433 & 2.3090 & 2.7039 & 1.9664 & 2.8678 \\
\hline 300 & 0.5 & 1.101 & 0.400 & 1.8560 & 1.7001 & 1.5729 & 1.8402 \\
\hline 300 & 0.75 & 1.101 & 0.367 & 1.4240 & 1.2816 & 1.3160 & 1.3887 \\
\hline 1000 & 0.5 & 1.223 & 0.335 & 4.5100 & 4.4205 & 4.1094 & 4.9406 \\
\hline 1000 & 0.75 & 1.223 & 0.307 & 3.7150 & 3.5190 & 3.5904 & 3.9328 \\
\hline 4000 & 0.5 & 1.376 & 0.247 & 12.4300 & 12.0832 & 12.1014 & 14.154 \\
\hline 4000 & 0.5 & 1.572 & 0.282 & 16.3300 & 16.4485 & 16.4720 & 19.859 \\
\hline
\end{tabular}

Table 3. Comparison of values of pressure results from our study [19], Monte-Carlo simulation [24] and Isam Ali's study [23].

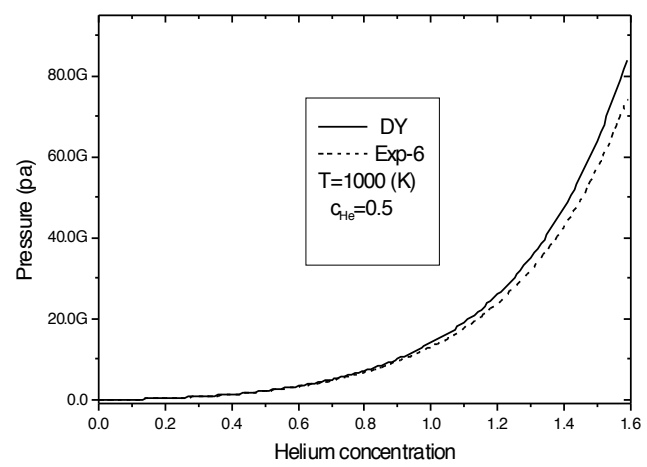

Figure 4. Comparision of efect of DY and EXP- 6 potential on pressure of mixture in che $=0.5, T=300 \mathrm{vs}$. Reduced density

Providing evidence of gradual divergence of DY and exp-6 potentials, a comparative figure has been made in figure 4 for helium-hydrogen mixtures. This figure shows more steepening effects of DY on total pressure of this mixture. Both potentials engender increase in pressure, except that, Buckingham affects moderately on pressure increase. The exp-6's more steady behavior makes it adjustable with previous studies and MC simulation.

In figures 5, 6, 7, 8 we tried to give information about effects of quantum correction term on total pressure of helium-hydrogen and deuterium-tritium mixtures at the high reduced density of 1.3. This correction term has been plotted in 3-dimensional diagram in figure 5 . This term is approximately zero for temperatures higher than $200(\mathrm{~K})$. Figure 6 represents that for hydrogen rich mixture at low temperature due to quantum effects pressure rise is significant. For effectual discussion on the effects of this term we have described $P Q / P$ in figure 7 for helium-hydrogen and in figure 8 for deuterium tritium mixture. For the third picture increase in pressure is similar to what have been elaborated for figures 5 and 6 . For figure 8 this manner remains analogous to helium-hydrogen mixture and temperatures next to $100(\mathrm{~K})$. However, for temperature lower than this it would behave inversely. For this range any increase in tritium concentration bears decrease in pressure. 


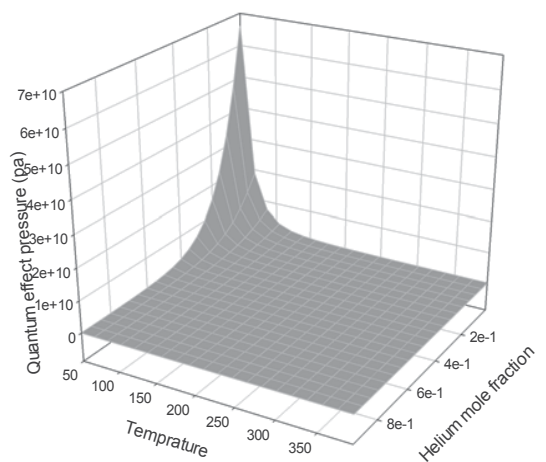

Figure 5. Pressure of quantum correction term at $\rho s=1.3$ for helium-hydrogen mixture.

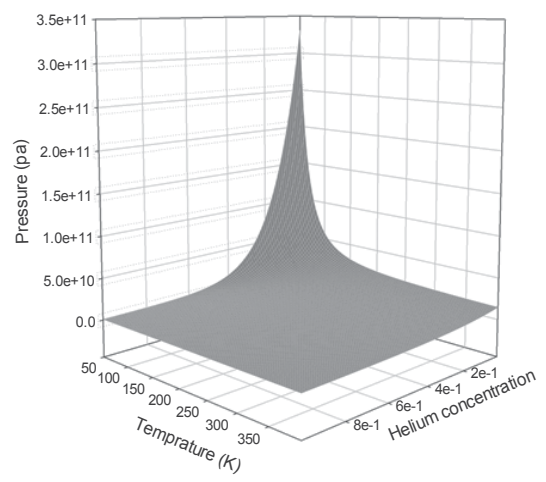

Figure 6. Total pressure from $50 \mathrm{~K}$ at $\rho_{S}=1.3$ for helium-hydrogen mixture.

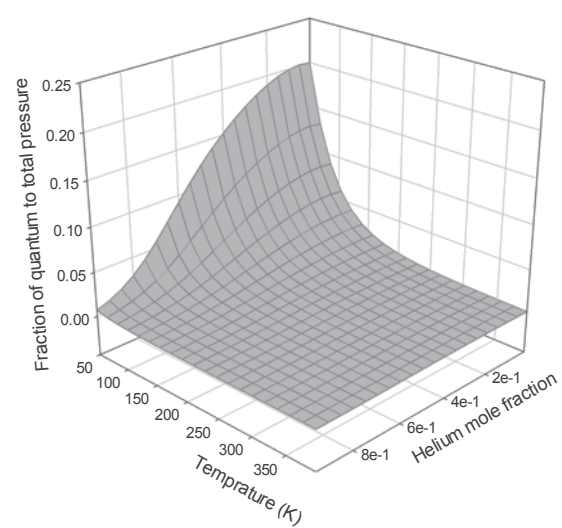

Figure 7. Fraction of quantum perturbation term to total pressure for helium-hydrogen mixture. 


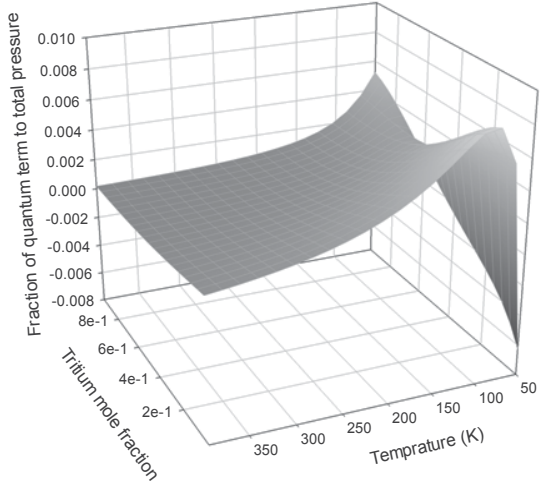

Figure 8. Fraction of quantum perturbation term to total pressure for Deuterium-Tritium mixture.

\section{Conclusion}

An Equation of state of hydrogen-helium mixture has been studied up to 90G (pa) pressure and temperature equal to $4000 \circ \mathrm{K}$. We have used perturbation theory as an adequate theory for describing EOS of fluid mixtures. As well, by using this theory we can add extra distributive terms as perturb part which makes it more applicable than other theories. Considering this advantage, we can spread it out with additional terms for investigation on other states of matter like plasma in the direction of compares with experimental data. Otherwise, using simulation methods, for evaluating our theoretical results. Such as ab initio simulations with the code VASP,[25] which combines classical molecular dynamics simulation for the ions with electrons, behave in quantum mechanical system by means of finite temperature density functional theory [26]. In this chapter, two potentials have been presented, which we have used them for hydrogen isotopes and helium, and their mixtures. By means of comparison with Monte Carlo simulation and results of refrence [14] in Table 3 we could prove that exp-6 potential is more beneficial than DY in wider ranges of variables, since its application in this theory shows more convergent results in comparison with MC simulation [24]. Also exp-6 potential is a good choice of potential since it allows us to elevate temperature and density [28]. But as hydrogen molecules dissociation occurs [28] for pressures more than $100 \mathrm{G}$ (pa), this effect must be accounted. Therefore, we have restricted ourselves to pressures below 100G (pa).

Furthermore, we have used Wertheim RDF which enables us to use this EOS for extended values of temperature. As well, we have compared different contributions of pressure to represent which one is more effective in different density and temperature regimes. By finding the most effective parts of pressure contributions in each ranges of independent variables (Temperature, reduced density, mole fraction), we can omit the less significant parts which are considered ignorable in value, to decrease unnecessary efforts. Likewise, we can speculate 
from Fig. 1 that in low temperature and high densities, long range perturbation term has the most significant effect in comparison with other parts. On the other hand, hard sphere part can be assumed as the most noticeable part in high temperature ranges. Moreover, comparison of DY and exp-6 potentials effects, on pressure of this mixture has been studied to express benefits of using exp-6 potential for higher temperatures and densities. Additionally, as it is obvious in high temperature and density difference between effects of two potentials are considerable for this equimolar mixture. This discriminating property makes exp-6 potential preferable.

Furthermore, this approach has been used to evaluate EOS of $D_{2}+T_{2}$ mixture. Also, we have used this method to compare different contribution parts of pressure. These comparisons indicate that in low temperature quantum effects are more important, however in high temperatures, hard sphere part is the most effective. The last two three dimensional diagrams reveals the importance of quantum term in comparison with total pressure. However, for temperatures below $100(\mathrm{k})$ for deuterium-tritium mixture negative pressure express that in low tritium concentration, deuterium rich fluid tend to consolidate.

\section{Applications}

One of the topics which can count on a great deal of interest from both theoretical and experimental physics is research in fluid mixture properties. These interests, not only comprise in the wide abundance of mixtures in our everyday life and in our universe but also the surprising new phenomena which were detected in the laboratories responsible for this increased attention. Mixtures, in general, have a much richer phase diagram than their pure constituents and various effects can be observed only in multi-component systems.

These kinds of studies have allowed a more complete modeling of mixture and consequently a better prediction and a more accurate calculation of thermodynamic quantities of mixture, such as activity coefficient, partial molar volume, phase behavior, local composition in general and have promoted a deeper understanding of the microscopic structure of mixtures.

Furthermore, for astronomical applications it is known that most of giant gas planets are like Jupiter is consisting primarily of hydrogen and helium. Modeling the interior of such planets requires an accurate equation of state for hydrogen-helium mixtures at high pressure and temperature conditions similar to those in planetary interiors [29]. Thus, the characterization of such system by statistical perturbation calculations will help us to answer questions concerning the inner structure of planets, their origin and evolution [29, 30].

In addition, in perturbation consideration of plasma via chemical picture, perturbation corrections will be included by means of additional free energy correction terms. Therefore, in considering transition behavior of molecular fluid to fully ionized plasma these terms are suitable in studying the neutral interaction parts. Consequently this will help us in studying inertial confinement fusion [31] and considering plasma as a fluid mixture in tokomak [32]. 


\section{Author details}

S. M. Motevalli and M. Azimi

Department of Physics, Faculty of Science, University of Mazandaran, Babolsar, Iran

\section{References}

[1] Wigner E. P. On the Quantum Correction for Thermodynamic Equilibrium. Physical Review 40, 749 (1932).

[2] Kirkwood J. G. Quantum Statistics of Almost Classical Assemblies. Physical Review 45,116 (1934).

[3] DeWitt H. E. Analytic Properties of the Quantum Corrections to the Second Virial Coefficient. Journal of Mathematical Physics 3, 1003 (1962).

[4] Hill R. N. Quantum Corrections to the Second Virial Coefficient at High Temperatures. Journal of Mathematical Physics 9, 1534 (1968).

[5] Jancovici B. Quantum-Mechanical Equation of State of a Hard-Sphere Gas at High Temperature. Physical Review 178, 295 (1969).

[6] Pisani C. and McKellar B. H. J, Semiclassical propagators and Wigner-Kirkwood expansions for hard-core potentials. Physical Review A 44, 1061 (1991).

[7] Mason E. A Siregar J. and Huang Y. Simplified calculation of quantum corrections to the virial coefficients of hard convex bodies. Molecular Physics 73, 1171 (1991).

[8] Mansoori G. A., Carnahan N. F., Starling K. E., and T. W. Leland. Equilibrium Thermodynamic Properties of the Mixture of Hard Spheres. Journal of Chemical Physics 54, 1523 (1971).

[9] Tang Y. and Lu B. C.Y. Improved expressions for the radial distribution function of hard spheres. Journal of Chemical Physics 103, 7463 (1995).

[10] Barker J. A. and Henderson D., Perturbation Theory and Equation of State for Fluids: The Square-Well Potential. Journal of Chemical Physics 47, 2856 (1967).

[11] Largo L. and Solana J. R., Equation of state for fluid mixtures of hard spheres and linear homo-nuclear fused hard spheres Physical Review E 58, 2251 (1998).

[12] Boublik T. Hard-Sphere Equation of State. Journal of Chemical Physics 54, 471 (1970).

[13] Barrio C. and Solana J. Consistency conditions and equation of state for additive hard-sphere fluid mixtures. Journal of Chemical Physics 113, 10180 (2000). 
[14] Leonard P. J. Henderson D. and Barker J., Molecular Physic 21, 107 (1971).

[15] Yuste S. B. and Santos A., Radial distribution function for hard spheres. Physical Review A 43, 5418 (1991).

[16] Yuste S. B. Lopez de Haro M. and Santos A., Structure of hard-sphere meta-stable fluids. Physical Review E 53, 4820 (1996).

[17] Tang Y., Jianzhong W. Journal of Chemical Physics 119, 7388 (2003).

[18] Garcia A. and Gonzalez D. J. Physical Chemistry Liquid 18, 91 (1988).

[19] Motevalli S. M., Pahlavani and M. R. Azimi M. Theoretical Investigations of Properties of Hydrogen and Helium Mixture Based on Perturbation Theory. , International Journal of Modern Physic B 26, 1250103 (2012).

[20] Ree F. H. Mol. Phys. 96, 87 (1983).

[21] Paricaud P. A general perturbation approach for equation of state development. Journal of Chemical Physics. 124, 154505 (2006).

[22] Kuijper A. D. et al., Fluid-Fluid Phase Separation in a Repulsive $\alpha$-exp-6 Mixture . Europhys. Lett. 13, 679 (1990).

[23] Ali I. et al., Thermodynamic properties of $\mathrm{He}-\mathrm{H} 2$ fluid mixtures over a wide range of temperatures and pressures. Physical Review E 69, 056104 (2004).

[24] Ree F. H. Simple mixing rule for mixtures with exp-6 interactions. Journal of Chemical Physics 78, 409 (1983).

[25] Kresse G. and Hafner J. Ab initio molecular dynamics for liquid metals. Physical Review B 47, 558 (1993).

[26] Lorenzen W. Halts B. and Redmer R. Metallization in hydrogen-helium mixtures Physical Review B 84, 235109 (2011).

[27] Ross M. Ree F. H. and D. A. Young, The equation of state of molecular hydrogen at very high density. Journal of Chemical Physics 79, 1487 (1983).

[28] Chen Q. F. and Cai L. C. Equation of State of Helium-Hydrogen and Helium-Deuterium Fluid Mixture at High Pressures and Tempratures. International of Journal of Thermodynamics 2, 27 (2006).

[29] Guillot T., D. J. Stevenson, W. B. Hubbard, and D. Saumon, in Jupiter, edited by Bagenal F., Chapter three. University of Arizona Press, Tucson; 2003. p35-57.

[30] Saumon D. and Guillot T. Shock Compression of Deuterium and the Interiors of Jupiter and Saturn. The Astrophysical Journal 609. 1170 (2004). 
[31] Collins G. W., Da Silva L. B., Celliers P., Gold D. M., Foord M. E., Wallace R. J., Ng A., Weber S. V., Budil K. S., Cauble R. Measurements of the Equation of State of Deuterium at the Fluid Insulator-Metal Transition. Science 281, 1178 (1998).

[32] Hakel P. and Kilcrease D. P. A New Chemical-Picture-Based Model for Plasma Equation-of-State Calculations, 14th APS Topical Conference on Atomic Processes in Plasma, (2004). 

Chapter 13

\title{
Quantal Cumulant Mechanics as Extended Ehrenfest Theorem
}

\author{
Yasuteru Shigeta \\ Additional information is available at the end of the chapter \\ http://dx.doi.org/10.5772/53703
}

\section{Introduction}

Since Schrödinger proposed wave mechanics for quantum phenomena in 1926 [1-4], referred as Schrödinger equation named after his name, this equation has been applied to atom-molecules, condensed matter, particle, and elementary particle physics and succeeded to reproduce various experiments. Although the Schrödinger equation is in principle the differential equation and difficult to solve, by introducing trial wave functions it is reduced to matrix equations on the basis of the variational principle. The accuracy of the approximate Schrödinger equation depends strongly on the quality of the trial wave function. He also derived the time-dependent Schrödinger equation by imposing the time-energy correspondence. This extension opened to describe time-dependent phenomena within quantum mechanics. However there exist a few exactly solvable systems so that the methodology to solve Schrödinger equation approximately is extensively explored, yet.

In contrast to the time-dependent wave mechanics, Heisenberg developed the equations of motion (EOM) derived for time-dependent operator rather than wave function [5]. This equation is now referred as the Heisenberg' EOM. This equation is exactly equivalent to the time-dependent Schrödinger equation so that the trials to solve the Heisenberg' EOM rather than Schrödinger one were also done for long time. For example, the Dyson equation, which is the basic equation in the Green's function theory, is also derived from the Heisenberg' EOM. Various approximate methods were deviced to solve the Dyson equation for nuclear and electronic structures.

In this chapter, we propose a new approximate methodology to solve dynamical properties of given systems on the basis of quantum mechanics starting from the Heisenberg' EOM. First, theoretical background of the method is given for one-dimensional systems and an extension to multi-dimensional cases is derived. Then, we show three applications in molecu- 
lar physics, i.e. the molecular vibration, the proton transfer reaction, and the quantum structural transition, respectively. Finally, we give conclusion at the last part.

\section{Theoretical background}

\subsection{Heisenberg' equation of motion and Ehrenfest theorem}

When the Hamiltonian does not explicitly depend on time, by defining time-dependent of an arbitrary operator $A$ in the Heisenberg representation as

$$
\hat{A}(t)=e^{-\frac{i}{\hbar} \hat{H} t} \hat{A}(0) e^{\frac{i}{\hbar} \hat{H} t}
$$

The Heisenberg' equation of motion (EOM) is given as

$$
\frac{\partial \hat{A}(t)}{\partial t}=\frac{1}{i \hbar}[\hat{A}(t), \hat{H}]
$$

where $\hat{H}$ is the Hamiltonian operator and $h=2 \pi \hbar$ is the Planck's constant. As an expectation value of $A$ with respect to $\psi$ is expressed as $\langle A\rangle \equiv\langle\psi|A| \psi\rangle$, the Heisenberg' EOM is rewritten as

$$
\frac{\partial\langle\hat{A}(t)\rangle}{\partial t}=\frac{1}{i \hbar}\langle[\hat{A}(t), \hat{H}]\rangle \equiv \frac{1}{i \hbar}\left\langle e^{-\frac{i}{\hbar} \hat{H} t}[\hat{A}, \hat{H}] e^{\frac{i}{\hbar} \hat{H} t}\right\rangle .
$$

For one-dimensional case, the Hamiltonian operator is expressed as a sum of the kinetic and the potential operator as

$$
\hat{H}=\frac{\hat{p}^{2}}{2 m}+V(\hat{q}) .
$$

The Heisenberg' EOMs for both a coordinate and a momentum are derived as

$$
\left\{\begin{array} { l } 
{ \frac { \partial \langle \hat { q } ( t ) \rangle } { \partial t } = \frac { \langle \hat { p } ( t ) \rangle } { m } } \\
{ \frac { \partial \langle \hat { p } ( t ) \rangle } { \partial t } = - \langle V ^ { ( 1 ) } ( \hat { q } ( t ) ) \rangle }
\end{array} \rightarrow \left\{\begin{array}{l}
\dot{q}(t)=\frac{p(t)}{m} \\
\dot{p}(t)=-\left\langle V^{(1)}(\hat{q}(t))\right\rangle
\end{array} .\right.\right.
$$


These equations resemble corresponding Newton' EOMs as

$$
\begin{aligned}
& \dot{q}(t)=\frac{p(t)}{m} \\
& \dot{p}(t)=-V^{(1)}(q(t)) .
\end{aligned}
$$

This relationship is so-called Ehrenfest's theorem [6]. A definite difference between Heisenberg' and Nowton' EOMs is that the expectation value of the potential operator appears in the former. If one approximates the expectation value as

$$
\langle V(\hat{q}(t))\rangle \approx V(\langle\hat{q}(t)\rangle)
$$

the same structure of the EOM is immediately derived. However, there is no guarantee that this approximation always holds for general cases. Including this approximation is also referred as the Ehrenfest's theorem.

In general, Taylor expansion of the potential energy term,

$$
\langle V(\hat{q}(t))\rangle=V(0)+V^{(1)}(0)\langle\hat{q}(t)\rangle+\frac{1}{2 !} V^{(2)}(0)\left\langle\hat{q}^{2}(t)\right\rangle+\frac{1}{3 !} V^{(3)}(0)\left\langle\hat{q}^{3}(t)\right\rangle+\cdots
$$

gives a infinite series of higher-order derivatives, $V^{(m)}(0)$, times expectation values of higher-powers of coordinate moment operators, $\left\langle\hat{q}^{m}(t)\right\rangle \quad(m=1,2, \cdots)$. Introducing a fluctuation operator of $A$ as $\delta A \equiv A-\langle A\rangle$ and the expectation values of the higher-order central moment $\left\langle\delta \hat{q}^{m}(t)\right\rangle \equiv\left\langle(\hat{q}(t)-\langle\hat{q}(t)\rangle)^{m}\right\rangle \quad(m=2,3, \cdots)$, the Taylor series is rewritten as

$$
\langle V(\hat{q}(t))\rangle=V(\langle\hat{q}(t)\rangle)+\frac{1}{2 !} V^{(2)}(\langle\hat{q}(t)\rangle)\left\langle\delta \hat{q}^{2}(t)\right\rangle+\frac{1}{3 !} V^{(3)}(\langle\hat{q}(t)\rangle)\left\langle\delta \hat{q}^{3}(t)\right\rangle+\cdots,
$$

The first term appears in Eq. (2-7) and the other terms are neglected by the approximation made before. This relation indicates that the difference between classical mechanics and quantum mechanics is existence of higher-order moment.

\subsection{Quantized Hamilton dynamics and quantal cumulant dynamics}

Ehrenfest' theorem fulfills for the arbitrary wave function. In previous studies, effects of the higher-order moments on dynamics were explored. The most of studies treat second-order 
term with the potential being a series of $q$. For example, Prezhdo and co-workers derived EOMs for three additional moments of $\left\langle\hat{q}^{2}(t)\right\rangle,\left\langle\hat{p}^{2}(t)\right\rangle$, and $\left\langle\langle\hat{q}(t) \hat{p}(t))_{\mathrm{s}}\right\rangle$ and solved the EOMs by truncating the potential term up to fourth-order power series. The subscript s represent a symmetric sum of the operator product defined as $\left\langle\langle\hat{q}(t) \hat{p}(t))_{\mathrm{s}}\right\rangle=\frac{1}{2}\langle(\hat{q}(t) \hat{p}(t)+\hat{p}(t) \hat{q}(t))\rangle$. Judging from previous works, this formalism is essentially the same as Gaussian wave packet method. Prezhdo also proposed a correction to the higher-order moments [8]. Nevertheless their formalism could not be applied general potential without any approximation such as the truncation.

Recently Shigeta and co-workers derived a general expression for the expectation value of an arbitrary operator by means of cumulants rather than moments [9-19]. For one-dimensional case, the expectation value of a differential arbitrary operator, $A_{\mathrm{s}}(\hat{q}, \hat{p})$, that consists of the symmetric sum of power series of $q$ and $p$ is derived as

$$
\left\langle A_{\mathrm{s}}(\hat{q}(t), \hat{p}(t))\right\rangle=\exp \left(\sum_{m} \sum_{0 \leq l \leq m} \frac{\lambda_{l, m-l}(t)}{l !(m-l) !} \frac{\partial^{m}}{\partial q^{l} \partial p^{m-l}}\right) A(q, p),
$$

where we introduced the general expression for the cumulant $\lambda_{m, n}(t) \equiv\left\langle\left(\delta \hat{q}^{m}(t) \delta \hat{p}^{n}(t)\right)_{s}\right\rangle$, in which the subscripts mean $m$-th order and $n$-th order with respect to the coordinate and momentum, respectively [20-22]. Using the expression, the expectation value of the potential is

$$
\langle V(\hat{q}(t))\rangle=\exp \left(\sum_{m=2} \frac{\lambda_{m, 0}(t)}{m !} \frac{\partial^{m}}{\partial q^{m}}\right) V(q)
$$

Thus, when the anharmonicity of the potential is remarkable, it is expected that the higherorder cumulants play important role in their dynamics. Indeed, for the harmonic oscillator case, only the second-order cumulant appears as

$$
\langle V(\hat{q}(t))\rangle=\frac{m \omega^{2}}{2}\left(q(t)^{2}+\lambda_{2,0}(t)\right)
$$

and the other higher-order terms do not.

Up to the second-order, Heisenberg' EOMs for cumulants are given by 


$$
\left\{\begin{array}{rl}
\dot{q}(t) & =\frac{p(t)}{m} \\
\dot{p}(t) & =-\tilde{V}^{(1,0)}\left(q(t), \lambda_{2,0}(t)\right) \\
\dot{\lambda}_{2,0}(t) & =\frac{2 \lambda_{1,1}(t)}{m} \\
\dot{\lambda}_{1,1}(t) & =\frac{\lambda_{0,2}(t)}{m}-\lambda_{2,0}(t) \tilde{V}^{(2,0)}\left(q(t), \lambda_{2,0}(t)\right) \\
\dot{\lambda}_{0,2}(t) & =-2 \lambda_{1,1}(t) \tilde{V}^{(2,0)}\left(q(t), \lambda_{2,0}(t)\right)
\end{array},\right.
$$

where $\widetilde{V}$ is second-order "quantal" potential defined as

$$
\tilde{V}\left(q(t), \lambda_{2,0}(t)\right) \equiv\langle V(\hat{q}(t))\rangle_{2}=\left.\exp \left(\frac{\lambda_{2,0}}{2} \frac{\partial^{2}}{\partial q^{2}}\right) V(q)\right|_{q=\langle\hat{q}(t)\rangle} .
$$

$\widetilde{V}^{(n, 0)}$ is the $n$-th derivative of $\widetilde{V}$ with respect to $q$. It is easily seen that the quantal potential is a finite series with respect to the cumulant by expanding as a Taylor series as

$$
\langle V(\hat{q}(t))\rangle_{2}=V(\langle\hat{q}(t)\rangle)+\frac{\lambda_{2,0}}{2} V^{(2)}(\langle\hat{q}(t)\rangle)+\frac{\lambda_{2,0}^{2}}{8} V^{(4)}(\langle\hat{q}(t)\rangle)+\cdots,
$$

It is noteworthy that the first and second terms of above equation corresponds to the first and second terms of Eq. (2-9), on the other hand, the other term are different each other.

Now we here give an expression to the quantal potential that has complicated form like as in Eq. (2-14). By using the famous formula for the Gaussian integral

$$
\int_{-\infty}^{\infty} \exp \left[-\left(a r^{2}+b r\right)\right] d r=\int_{-\infty}^{\infty} \exp \left[-a\left(r+\frac{b}{2 a}\right)^{2}+\frac{b^{2}}{4 a}\right] d r=\sqrt{\frac{\pi}{a}} \exp \left(\frac{b^{2}}{4 a}\right)
$$

the exponential operator appearing in Eq. (2-14) is rewritten as,

$$
\exp \left(\frac{\lambda_{2,0}}{2} \frac{\partial^{2}}{\partial q^{2}}\right)=\frac{1}{\sqrt{2 \pi \lambda_{2,0}}} \int_{-\infty}^{\infty} \exp \left[-\left(\frac{r^{2}}{2 \lambda_{2,0}}+r \frac{\partial}{\partial q}\right)\right] d r
$$


The first derivative operator term in right hand side of the above equation can act to the potential with the relationship of $\exp \left[r \frac{\partial}{\partial q}\right] f(q)=f(q+r)$ as

$$
\begin{aligned}
\exp \left(\frac{\lambda_{2,0}}{2} \frac{\partial^{2}}{\partial q^{2}}\right) V(q) & =\int_{-\infty}^{\infty} \frac{d r}{\sqrt{2 \pi \lambda_{2,0}}} \exp \left[-\frac{r^{2}}{2 \lambda_{2,0}}\right] V(q+r) \\
& =\int_{-\infty}^{\infty} \frac{d r}{\sqrt{2 \pi \lambda_{2,0}}} \exp \left[-\frac{(q-r)^{2}}{2 \lambda_{2,0}}\right] V(r) .
\end{aligned}
$$

Therefore it is possible to estimate potential energy term without the truncation of the potential. However the analytic integration is not always has the closed form and the numerical integration does not converge depending on the kind of the potential. For the quantal potential including third and higher-order culumant, it is convenient to use the Fourier integral instead of Gaussian integral. Nevertheless this scheme also has problems concerning about the integrability and its convergence.

\subsection{Energy conservation law and least uncertainty state}

For the EOMs of Eq. (2-13), there exists first integral that always hold for. Now defining a function,

$$
\gamma(t)=\lambda_{2,0}(t) \lambda_{0,2}(t)-\lambda_{1,1}^{2}(t)
$$

and differentiating it result in

$$
\begin{aligned}
\dot{\gamma}(t) & =\dot{\lambda}_{2,0}(t) \lambda_{0,2}(t)+\lambda_{2,0}(t) \dot{\lambda}_{0,2}(t)-2 \lambda_{1,1}(t) \dot{\lambda}_{1,1}(t) \\
& =\frac{2 \lambda_{1,1}(t)}{m} \lambda_{0,2}(t)-2 \lambda_{2,0} \lambda_{1,1}(t) V^{(2,0)}\left(q(t), \lambda_{2,0}(t)\right) \\
& -2 \lambda_{1,1}(t)\left\{\frac{\lambda_{0,2}(t)}{m}-\lambda_{0,2}(t) V^{(2,0)}\left(q(t), \lambda_{2,0}(t)\right)\right\} \\
& =0 .
\end{aligned}
$$

Thus, this function is a time-independent constant. It is well-known that the least uncertainty state fulfills $\gamma=\frac{\hbar^{2}}{4}$. By setting the adequate parameter, one can incorporate the Heisenberg' uncertainty principle and thus least uncertainty relation into EOMs. Using this value, one can delete one cumulant from EOMs, for example 


$$
\lambda_{0,2}(t)=\frac{\lambda_{1,1}^{2}(t)}{\lambda_{2,0}(t)}+\frac{\hbar^{2}}{4 \lambda_{2,0}(t)}
$$

Now by considering the dimension we define new coordinate and momentum as

$$
\begin{aligned}
& p_{\lambda}(t)=\frac{\lambda_{1,1}(t)}{\sqrt{\lambda_{2,0}(t)}} \\
& q_{\lambda}(t)=\sqrt{\lambda_{2,0}(t)} .
\end{aligned}
$$

The second-order momentum cumulant $\lambda_{0,2}(t)$ is rewritten using them as

$$
\lambda_{0,2}(t)=p_{\lambda}^{2}(t)+\frac{\hbar^{2}}{4 q_{\lambda}^{2}(t)}
$$

Total energy are expressed using the cumulant variables as

$$
E_{2}(t)=\langle H\rangle_{2}=\frac{p^{2}(t)+\lambda_{0,2}(t)}{2 m}+\int \frac{d r}{\sqrt{2 \pi \lambda_{2,0}(t)}} \exp \left(-\frac{(r-q(t))^{2}}{2 \lambda_{2,0}(t)}\right) V(r) .
$$

Above expression indicates that the energy does not depend on $\lambda_{1,1}(t)$. Differentiating the energy with respect to time gives the energy conservation law. The proof of the energy conservation law is give below.

$$
\begin{aligned}
\dot{E}_{2}(t) & =\frac{2 \dot{p}(t) p(t)+\dot{\lambda}_{0,2}(t)}{2 m}-\dot{q}(t) \int \frac{d r}{\sqrt{2 \pi \lambda_{2,0}(t)}} \frac{(r-q(t))}{\lambda_{2,0}(t)} \exp \left(-\frac{(r-q(t))^{2}}{2 \lambda_{2,0}(t)}\right) V(r) . \\
& +\int \frac{d r}{\sqrt{2 \pi \lambda_{2,0}^{3}(t)}} \dot{\lambda}_{2,0}(t)\left(1+\frac{(r-q(t))^{2}}{2 \lambda_{2,0}(t)}\right) \exp \left(-\frac{(r-q(t))^{2}}{2 \lambda_{2,0}(t)}\right) V(r) \\
& =0
\end{aligned}
$$


By means of the new coordinate and momentum, the total energy is rewritten as

$$
E_{2}=\frac{p^{2}(t)+p_{\lambda}^{2}(t)}{2 m}+\frac{\hbar^{2}}{8 m q_{\lambda}^{2}(t)}+\frac{1}{q_{\lambda}(t)} \int \frac{d r}{\sqrt{2 \pi}} \exp \left(-\frac{(r-q(t))^{2}}{2 q_{\lambda}^{2}(t)}\right) V(r) .
$$

This equation tells us that the effective potential derived from the kinetic energy term affect the dynamics of $q(t)$ via dynamics of $q_{\lambda}(t)$. A variational principle of $E_{2}$,

$$
\frac{\partial E_{2}}{\partial p}=\frac{\partial E_{2}}{\partial p_{\lambda}}=\frac{\partial E_{2}}{\partial q}=\frac{\partial E_{2}}{\partial q_{\lambda}}=0
$$

gives stationary state that fulfills the least uncertainty condition as

$$
\begin{aligned}
& \frac{\partial E_{2}}{\partial p}=\frac{p}{m}=0 \\
& \frac{\partial E_{2}}{\partial p_{\lambda}}=\frac{p_{\lambda}}{m}=0 \\
& \frac{\partial E_{2}}{\partial q}=V_{2}^{(1,0)}\left(q, q_{\lambda}\right)=0 \\
& \frac{\partial E_{2}}{\partial q_{\lambda}}=-\frac{\hbar^{2}}{4 m q_{\lambda}^{3}}+q_{\lambda} V_{2}^{(2,0)}\left(q, q_{\lambda}\right)=0
\end{aligned}
$$

For both momenta, the solutions of the above variational principle are zero. On the other hand, the solutions for the coordinates strongly depend on the shape of the give potential. As an exactly soluble case, we here consider the harmonic oscillator. The variational condition gives a set of solutions as $\left(p, p_{\lambda}, q, q_{\lambda}\right)=(0,0,0, \sqrt{\hbar / 2 m \omega})$. The corresponding energy $E_{2}=\frac{\hbar \omega}{2}$ is the same as the exact ground state energy. The cumulant variables estimated from the solutions result in $\left(\lambda_{2,0}, \lambda_{1,1}, \lambda_{0,2}\right)=\left(\frac{\hbar}{2 m \omega}, 0, \frac{m \hbar \omega}{2}\right)$ being the exact expectation values for the ground state. Thus the present scheme with the least uncertainty relation is reasonable at least for the ground state.

\subsection{Distribution function and joint distribution}

In order to visualize the trajectory in this theory, we here introduce distribution function as a function of coordinate and second-order cumulant variables. Now the density finding a 
particle at $r$ is the expectation value of the density operator, $\delta(\hat{q}-r)$, with a useful expression as

$$
\rho(r)=\langle\delta(\hat{q}-r)\rangle=\lim _{\beta \rightarrow \infty} \sqrt{\frac{\beta}{\pi}}\left\langle\exp \left(-\beta(\hat{q}-r)^{2}\right)\right\rangle .
$$

Thus the second-order expression for the density is evaluated as

$$
\rho_{2}(r)=\frac{1}{\sqrt{2 \pi \lambda_{2,0}}} \exp \left(-\frac{1}{2 \lambda_{2,0}}(r-q)^{2}\right)
$$

This density shows that the distribution is a Gaussian centered at $q$ with a width depending on the cumulant $\lambda_{2,0}$. Thus the physical meaning of the second-order cumulant $\lambda_{2,0}$ results in the width of the distribution. As the integration of this density for the whole space becomes unity, the density is normalized. Therefore the density has the physical meaning of probability. Comparison with Eq. (2-18), the potential energy is rewritten by means of the density as

$$
\langle V(\hat{q})\rangle_{2}=\int \frac{d r}{\sqrt{2 \pi \lambda_{2,0}}} \exp \left(-\frac{(r-q)^{2}}{2 \lambda_{2,0}}\right) V(r) \equiv \int d r \rho_{2}(r) V(r) .
$$

This expression indicates that the expectation value of the potential is related to the mean average of the potential with weight $\rho_{2}(r)$. The same relationship holds for the momentum distribution.

In principle, one cannot determine the position and momentum at the same time within the quantum mechanics. In other words, resolution of phase space is no more than the Planck' constant, $h$. In contrast to the quantum mechanics, we can define the joint distribution function on the basis of the present theory as

$$
\rho_{\text {joint }}(r, s)=\left\langle(\delta(\hat{q}-r) \delta(\hat{p}-s))_{s}\right\rangle
$$

The second-order expression is given by

$$
\rho_{\text {joint }}(r, s)=\frac{1}{2 \pi \sqrt{\gamma}} \exp \left[-\frac{\lambda_{0,2}(r-q)^{2}-2 \lambda_{1,1}(r-q)(s-p)+\lambda_{2,0}(s-p)^{2}}{2 \gamma}\right] .
$$


In contrast to the energy, the joint distribution depends on all the cumulant variables. In the phase space, this joint distribution has the elliptic shape rotated toward $r$-s axes. This joint distribution corresponds not to a simple coherent state, but to a squeezed-coherent state.

Using the joint distribution, the expectation value of the arbitrary operator is evaluated via

$$
\left\langle A_{\mathrm{s}}(\hat{q}, \hat{p})\right\rangle=\iint A(r, s) \rho_{\text {joint }}(r, s) d r d s
$$

In this sense, this theory is one of variants of the quantum distribution function theory. This joint distribution fulfills the following relations as

$$
\begin{aligned}
\int \rho_{\text {joint }}(r, s) d s & =\rho(r) \\
\int \rho_{\text {joint }}(r, s) d r & =\rho_{\text {momentum }}(s) \\
\iint \rho_{\text {joint }}(r, s) d r d s & =1 .
\end{aligned}
$$

Moreover the coordinate, momentum, and cumulants are derived by means of the joint distribution as

$$
\begin{aligned}
q & =\iint r \rho_{\text {joint }}(r, s) d r d s \\
p & =\iint s \rho_{\text {joint }}(r, s) d r d s \\
\lambda_{2,0} & =\iint(r-q)^{2} \rho_{\text {joint }}(r, s) d r d s \\
\lambda_{1,1} & =\iint(r-q)(s-p) \rho_{\text {joint }}(r, s) d r d s \\
\lambda_{0,2} & =\iint(s-p)^{2} \rho_{\text {joint }}(r, s) d r d s .
\end{aligned}
$$

\subsection{Extension to multi-dimensional systems}

The Hamiltonian of an $n$-dimensional $N$ particle system including a two-body interaction is written by

$$
\hat{H}=\sum_{I=1}^{N} \frac{\hat{\mathbf{P}}_{I}^{2}}{2 m_{I}}+\sum_{I>J}^{N} V\left(\left|\hat{\mathbf{Q}}_{I}-\hat{\mathbf{Q}}_{J}\right|\right),
$$

where $\hat{\boldsymbol{Q}}_{I}=\left(\hat{q}_{I 1}, \hat{q}_{I 2}, \cdots, \hat{q}_{I n}\right)$ and $\hat{\boldsymbol{P}}_{I}=\left(\hat{p}_{I 1}, \hat{p}_{I 2}, \cdots, \hat{p}_{I n}\right)$, and $m_{I}$ represent a vector of $I$-th position operator, that of momentum operator, and mass, respectively. We here assume that the potential $V(r)$ is a function of the inter-nuclear distance $r$. Using the definitions of the second-order single-particle cumulants given by 


$$
\begin{aligned}
& \xi_{I, k l}=\left\langle\left(\delta \hat{q}_{I k} \delta \hat{q}_{I l}\right)_{\mathrm{s}}\right\rangle \\
& \eta_{I, k l}=\left\langle\left(\delta \hat{p}_{I k} \delta \hat{p}_{I l}\right)_{\mathrm{s}}\right\rangle, \\
& \zeta_{I, k l}=\left\langle\left(\delta \hat{q}_{I k} \delta \hat{p}_{I l}\right)_{\mathrm{s}}\right\rangle
\end{aligned}
$$

the total energy is derived as an extension of Eq. (2-26) by

$$
E_{2}=\sum_{I=1}^{N} \frac{\mathbf{P}_{I}^{2}+\eta_{I} \cdot \mathbf{1}_{n}}{2 m_{I}}+\sum_{I>J}^{N} \tilde{V}_{2}\left(\mathbf{Q}_{I}-\mathbf{Q}_{J}, \boldsymbol{\xi}_{I}+\boldsymbol{\xi}_{J}\right)
$$

where $\boldsymbol{P}_{I}$ and $\boldsymbol{Q}_{I}$ are $n$-dimensional momentum and coordinae and $\mathbf{1}_{n}=\left(\begin{array}{llll}1 & 1 & \cdots & 1\end{array}\right)$ is $n$-dimensional identity vector. $V(Q, \xi)$ is the second-order quantal potential given as

$$
\tilde{V}_{2}(\mathbf{Q}, \xi)=\int \frac{d \mathbf{r}}{\sqrt{(2 \pi)^{n} \operatorname{det}|\xi|}} \exp \left(-\frac{1}{2}(\mathbf{Q}-\mathbf{r})^{T} \xi^{-1}(\mathbf{Q}-\mathbf{r})\right) V(|\mathbf{r}|),
$$

where $\xi$ is an $n$ by $n$ matrix composed of the position cumulant variables. From Heisenberg uncertainty relation and the least uncertainty, the total energy of Eq. (2-39) is rewritten as

$$
E_{2}^{\mathrm{LQ}}=\sum_{I=1}^{N} \frac{\mathbf{P}_{I}^{2}}{2 m_{I}}+\sum_{i} \frac{\hbar^{2}}{8 m_{I}} \operatorname{Tr}\left(\boldsymbol{\xi}_{I}^{-1}\right)+\sum_{I>J}^{N} \tilde{V}_{2}\left(\mathbf{Q}_{I}-\mathbf{Q}_{J}, \boldsymbol{\xi}_{I}+\boldsymbol{\xi}_{J}\right) .
$$

From Heisenberg EOM, EOMs up to the second-order cumulants are given by

$$
\begin{aligned}
\dot{q}_{I k} & =\frac{p_{I k}}{m_{I}} \\
\dot{p}_{I k} & =-\tilde{W}_{2}^{\left(\mathbf{1}_{I k}\right)}\left(\left\{\mathbf{q}_{I}-\mathbf{q}_{J}\right\},\left\{\boldsymbol{\xi}_{I}+\boldsymbol{\xi}_{J}\right\}\right) \\
\dot{\xi}_{I, k l} & =\frac{\zeta_{I, k l}+\zeta_{I, l k}}{m_{I}} \\
\dot{\eta}_{I, k l} & =-\sum_{m}\left[\zeta_{I, m l} \tilde{W}_{2}^{\left(\mathbf{2}_{I m, I k}\right)}\left(\left\{\mathbf{q}_{I}-\mathbf{q}_{J}\right\},\left\{\boldsymbol{\xi}_{I}+\boldsymbol{\xi}_{J}\right\}\right)+\zeta_{I, m k} \tilde{W}_{2}^{\left(\mathbf{2}_{I m, l l}\right)}\left(\left\{\mathbf{q}_{I}-\mathbf{q}_{J}\right\},\left\{\boldsymbol{\xi}_{I}+\boldsymbol{\xi}_{J}\right\}\right)\right] \\
\zeta_{I, k l} & =\frac{\eta_{I, k l}}{m_{I}}-\sum_{m} \xi_{i, k m} \tilde{W}_{2}^{\left(\mathbf{2}_{I m, l l}\right)}\left(\left\{\mathbf{q}_{I}-\mathbf{q}_{J}\right\},\left\{\boldsymbol{\xi}_{I}+\boldsymbol{\xi}_{J}\right\}\right),
\end{aligned}
$$


where $\widetilde{W}_{2}^{\left(\mathbf{1}_{I k}\right)}\left(\left\{\boldsymbol{Q}_{I}-\boldsymbol{Q}_{J}\right\},\left\{\xi_{I}+\xi_{J}\right\}\right)$ and $\widetilde{W}_{2}^{\left(\mathbf{2}_{I k, I m}\right)}\left(\left\{\boldsymbol{Q}_{I}-\boldsymbol{Q}_{J}\right\},\left\{\xi_{I}+\xi_{J}\right\}\right)$ are the $1^{\text {st }}$ and $2^{\text {nd }}$ derivatives of the sum of the quantal potentials with respect to the position $q_{i k}$ and to $q_{i k}$ and $q_{i m}$ defined as

$$
\begin{gathered}
\tilde{W}_{2}^{\left(\mathbf{1}_{I k}\right)}\left(\left\{\mathbf{Q}_{I}-\mathbf{Q}_{J}\right\},\left\{\boldsymbol{\xi}_{I}+\boldsymbol{\xi}_{J}\right\}\right)=\sum_{J} \frac{\partial \tilde{V}_{2}\left(\mathbf{Q}_{I}-\mathbf{Q}_{J}, \boldsymbol{\xi}_{I}+\boldsymbol{\xi}_{J}\right)}{\partial q_{I k}} \\
\tilde{W}_{2}^{\left(\mathbf{2}_{I k, I m}\right)}\left(\left\{\mathbf{Q}_{I}-\mathbf{Q}_{J}\right\},\left\{\boldsymbol{\xi}_{I}+\boldsymbol{\xi}_{J}\right\}\right)=\sum_{J} \frac{\partial^{2} \tilde{V}_{2}\left(\mathbf{Q}_{I}-\mathbf{Q}_{J}, \boldsymbol{\xi}_{I}+\boldsymbol{\xi}_{J}\right)}{\partial q_{I k} \partial q_{I m}} .
\end{gathered}
$$

In contrast to the one-dimensional problems, second-order cumulants are represented as matrices. Thus, the total degrees of freedom are $24 \mathrm{~N}$ for 3-dimensional cases. For the latter convenience, we here propose two different approximations. The one is the diagonal approximation, where the all off-diagonal elements are neglected, and the spherical approximation, where the all diagonal cumulants are the same in addition to the diagonal approximation. In the following, we apply the present methods for several multi-dimensional problems. We hereafter refer our method as QCD2.

\section{Applications}

\subsection{Application to molecular vibration}

Here we evaluate the vibrational modes from the results obtained from molecular dynamics (MD) simulations. Since the force field based model potentials, which are often used in molecular dynamics simulations, are empirical so that they sometimes leads to poor results for molecular vibrations. For quantitative results in any MD study, the accuracy of the PES is the other important requirement as well as the treatment of the nuclear motion. Here we use an efficient representation of the PES derived from $a b$ initio electronic structure methods, which is suitable for both molecular vibration and the QCD scheme in principle. In order to include anharmonic effects, multi-dimensional quartic force field (QFF) approximation [23] is applied as

$$
V_{\mathrm{QFF}}\left(\left\{\hat{Q}_{i}\right\}\right)=V_{0}+\sum_{i} \frac{h_{i i}}{2} \hat{Q}_{i}^{2}+\sum_{i j k} \frac{t_{i j k}}{6} \hat{Q}_{i} \hat{Q}_{j} \hat{Q}_{k}+\sum_{i j k l} \frac{u_{i j k l}}{24} \hat{Q}_{i} \hat{Q}_{j} \hat{Q}_{k} \hat{Q}_{l}
$$

where $V_{0}, h_{i i}, t_{i j k}$, and $u_{i j k l}$ denote the potential energy and its second-, third- and fourth-order derivatives with respect to a set of normal coordinates $\left\{\hat{Q}_{i}\right\}$, at the equilibrium geometry, respectively. To further reduce the computational cost for multi-dimensional cases, an $n$ - 
mode coupling representation of QFF ( $n \mathrm{MR}-\mathrm{QFF}$ ) was applied [23], which includes mode couplings up to $n$ modes.

By taking each normal mode as the degree of freedom in the dynamics simulation, the Hamiltonian for QCD2 with $n \mathrm{MR}-\mathrm{QFF}$ as the potential energy is

$$
\hat{H}\left(\left\{\hat{P}_{i}\right\},\left\{\hat{Q}_{i}\right\}\right)=\sum_{i} \frac{\hat{P}_{i}^{2}}{2}+V_{\mathrm{QFF}}^{n-\operatorname{mode}}\left(\left\{\hat{Q}_{i}\right\}\right)
$$

where $V_{Q F F}^{n-\text { mode }}$ denotes $n \mathrm{MR}$-QFF. In this Hamiltonian we neglected the Watson term, which represents the vibrational-rotational coupling. Mass does not appear in the equations since the QFF normal coordinate is mass weighted. Therefore, the time evolution of variables of QCD2 with 1MR-QFF (general expressions are not shown for simplicity) is derived as

$$
\begin{aligned}
\dot{Q}_{i} & =P_{i} \\
\dot{P}_{i} & =-h_{i i} Q_{i}+\frac{t_{i i i}}{2}\left(Q_{i}^{2}+\lambda_{2 i, 0 i}\right)+\frac{u_{i i i i}}{6} Q_{i}\left(Q_{i}^{2}+2 \lambda_{2 i, 0 i}\right) \\
\dot{\lambda}_{2 i, 0 i} & =2 \lambda_{1 i, 1 i} \\
\dot{\lambda}_{1 i, 1 i} & =\lambda_{0 i, 2 i}-\lambda_{2 i, 0 i}\left[h_{i i}+t_{i i i} Q_{i}+\frac{u_{i i i i}}{2}\left(Q_{i}^{2}+\lambda_{2 i, 0 i}\right)\right] \\
\dot{\lambda}_{0 i, 2 i} & =-2 \lambda_{1 i, 1 i}\left[h_{i i}+t_{i i i} Q_{i}+\frac{u_{i i i i}}{2}\left(Q_{i}^{2}+\lambda_{2 i, 0 i}\right)\right] .
\end{aligned}
$$

For molecules with more than 1 degree of freedom, we applied 3MR-QFF, because it has been shown by various examples that the 3MR-QFF is sufficient to describe fundamental modes as well as more complex overtone modes. The QCD2 and classical simulations were performed numerically with a fourth-order Runge-Kutta integrator. For formaldehyde $\left(\mathrm{CH}_{2} \mathrm{O}\right)$ and formic acid $(\mathrm{HCOOH}), 3 \mathrm{MR}-\mathrm{QFF}$ PES was generated at the level of MP2/aug-ccpVTZ [24, 25] using GAMESS [26] and Gaussian03 [27] program packages. In this work, the results obtained by our method are compared with those by vibrational self-consistent field method (VSCF) with full second-order perturbation correction (VPT2), which is based on the quantum mechanics and accurate enough to treat molecular vibrations.

We here present results of the spectral analysis of trajectories obtained from the simulation that can be compared with other theoretical calculations and experimental results. The Fourier transform of any dynamical variables obtained from the trajectories of MD simulations is related to spectral densities. In particular, Fourier transform of velocity autocorrelation function gives the density of vibrational states. In addition, the power spectrum of the time series or autocorrelation function of each normal coordinate shows the contribution to frequency peaks of the spectrum obtained from velocity autocorrelation. Here we adopted the latter procedure. The time interval used was $0.1 \mathrm{fs}$ and total time is $1 \mathrm{ps}$ for all MD and 
QCD simulations. The resolution in the frequency domain is less than $1 \mathrm{~cm}^{-1}$, which is enough accuracy for the analysis of the molecular vibrations of interest. If a longer time trajectory is obtained, the resolution of the Fourier spectrum becomes fine.

Since each normal mode is taken as the degree of freedom explicitly in the present dynamics simulation, the interpretation and analysis of the results can directly be related with each normal mode. The results are shown in Table 1 . The table indicates that the harmonic and QFF approximation of the PES results in a large deviation between each other. Therefore, anharmonicity of the potential must be considered to perform reliable simulations. The table shows that for the analysis of fundamental frequencies, the QCD2 has higher accuracy than the classical results, which can be compared with the VPT2 results in all cases. In spite of the high accuracy, the computational cost of the QCD2 remains low even when applied to larger systems. For $\mathrm{HCOOH}$ molecule, the QFF is so anharmonic that the classical simulation does not give clear vibrational frequencies due to the chaotic behavior of the power spectrum. The QCD may suppress the chaotic motion as seen in the full quantum mechanics.

\begin{tabular}{|c|c|c|c|c|c|c|}
\hline & Mode & NMA & MD & QCD & VPT2 & Exp. \\
\hline \multirow{6}{*}{$\mathrm{H}_{2} \mathrm{CO}$} & $v_{1}$ & 3040 & 2901 & 2843 & 2866 & 2843 \\
\hline & $v_{2}$ & 2997 & 2868 & 2838 & 2849 & 2782 \\
\hline & $v_{3}$ & 1766 & 1764 & 1723 & 1734 & 1746 \\
\hline & $v_{4}$ & 1548 & 1504 & 1509 & 1515 & 1500 \\
\hline & $v_{5}$ & 1268 & 1247 & 1250 & 1251 & 1250 \\
\hline & $v_{6}$ & 1202 & 1166 & N/A & 1189 & 1167 \\
\hline \multirow{9}{*}{$\mathrm{HCOOH}$} & $v_{1}$ & 3739 & $N / A$ & 3527 & 3554 & 3570 \\
\hline & $v_{2}$ & 3126 & $\mathrm{~N} / \mathrm{A}$ & 2980 & 2989 & 2943 \\
\hline & $v_{3}$ & 1794 & $\mathrm{~N} / \mathrm{A}$ & 1761 & 1761 & 1770 \\
\hline & $v_{4}$ & 1409 & N/A & 1377 & 1385 & 1387 \\
\hline & $v_{5}$ & 1302 & N/A & 1270 & 1231 & 1229 \\
\hline & $v_{6}$ & 1130 & N/A & 1120 & 1097 & 1105 \\
\hline & $v_{7}$ & 626 & N/A & 631 & 620 & 625 \\
\hline & $v_{8}$ & 1058 & \multirow{2}{*}{ N/A } & \multirow{2}{*}{ N/A } & 1036 & 1033 \\
\hline & $/ v_{9}$ & 1676 & & & 1642 & 1638 \\
\hline
\end{tabular}

Table 1. 


\subsection{Proton transfer reaction in guanine-cytosine base pair}

DNA base pairs have two and three inter-base hydrogen bonds for Adenine-Thymine and Guanine-Cytosine pairs, respectively. Proton transfer reactions among based were theoretically investigated by quantum chemical methods and further quantum mechanical analyses for decases [28-33]. In order to investigate dynamical stability of proton-transferred structures of the model system consisting DNA bases, we here perform QCD2 simulations of a model Guanine-Cytosine base pair. The model potential is given by

$$
V^{\mathrm{GC}}(x, y, z)=\sum_{i, j, k} h_{i, j, k}^{\prime} x^{i} y^{j} z^{k}
$$

where parameters in the model potential are given by Villani's paper [30, 31], which is fifthorder polynomials with respect to the coordinates for GC pairs and determined by the first principle calculations (B3LYP/cc-pVDZ). The reaction coordinates $x, y$, and $z$ are shown in the figure. The corresponding quantal potentials are explicitly given by

$$
\tilde{V}_{2}^{\mathrm{GC}}(x, y, z, \xi, \eta, \zeta)=\sum_{l, m, n=0}^{2} \sum_{i=0}^{5-2 l} \sum_{j=0}^{5-2 m} \sum_{k=0}^{5-2 n} H_{i, j, k}^{l, m, n} x^{i} y^{j} z^{k}\left(\frac{\xi}{2}\right)^{l}\left(\frac{\eta}{2}\right)^{m}\left(\frac{\zeta}{2}\right)^{n}
$$

with

$$
H_{i, j, k}^{l, m, n}=\frac{(i+2 l) !}{i ! l !} \frac{(j+2 m) !}{j ! m !} \frac{(k+2 n) !}{k ! n !} h_{i+2 l, j+2 m, k+2 n}^{\prime}
$$

where Greek characters denote the cumulant variables. In order to avoid the particles escaping from the bottoms, we have added the well-like potential is defined as

$$
V_{\text {well }}\left(\left\{q_{i}\right\}\right)=V_{0}\left[1+\prod_{i=x, y, z}\left(\theta_{b}\left(q_{i}-q_{i \max }\right)-\theta_{b}\left(q_{i}-q_{i \min }\right)\right)\right]
$$

where $q_{i \max }$ and $q_{i \min }$ are maximum and minimum range of potential and $V_{0}$ is height of the well-like potential. An approximate Heaviside function is given by

$$
\theta_{b}(x)=\frac{\operatorname{erf}(\sqrt{b} x)+1}{2}
$$

where $b$ is an effective width of the approximate Heaviside function and guarantees smoothness of the potential. Using this approximate Heaviside function, the quantal potential for 
the well-like potential is analytically derived. When $V_{0}$ is appropriately large, the particles stay around minima during dynamics simulations. We set $b=100, q_{i \max }=2.0(\AA), q_{i \min }=0.4(\AA)$, and $V_{0}=0.05$ (a.u.). Both $q_{i \max }$ and $q_{i \min }$ are in a reasonable range for the coordinate of the proton, because the distances between heavy elements $(\mathrm{O}$ and $\mathrm{N})$ of the DNA bases are approximately 2.7 3.0 ( $\mathrm{\AA})$ and roughly speaking the bond length of $\mathrm{OH}$ and $\mathrm{NH}$ are almost 1.0 $\sim 1.1(\AA)$. The ordinary PES analysis gives both global and metastable structures for the GC pair. The former structure is the original Watson-Crick type and the latter is double protontransferred one as easily found in (a) and (c). No other proton-transferred structure is found on the PES.

In the actual calculations, the time interval used was $0.1 \mathrm{fs}$, total time is $2 \mathrm{ps}$. The initial conditions of the variables can be determined by the least quantal energy principle. In figures 2 we have depicted phase space $(x / p x, y / p y, z / p z)$ structures of a trajectory obtained by the QCD simulations. For cases (a) and (b), the dynamical feature of the closed orbits is the same except for its amplitudes. The phase space of the $x / p x$ is compact, on the other hand, that of $z / p z$ is loose in comparison with that of $y / p y$. The explicit isotope effects on the phase space structure are found in the cases of (c) and (d). In Fig. 2-(c), the nuclei initially located at the metastable structure go out from the basin and strongly vibrate around the global minimum due to tunneling. On the other hand, the deuterated isotopomer remains around the metastable structure. It is concluded that the metastable structure of the protonated isotopomer is quantum mechanically unstable, though it is classically stable based on the PES analysis. Therefore, it is important to take the quantum effects into isotope effects on the metastable structure with a small energy gap.

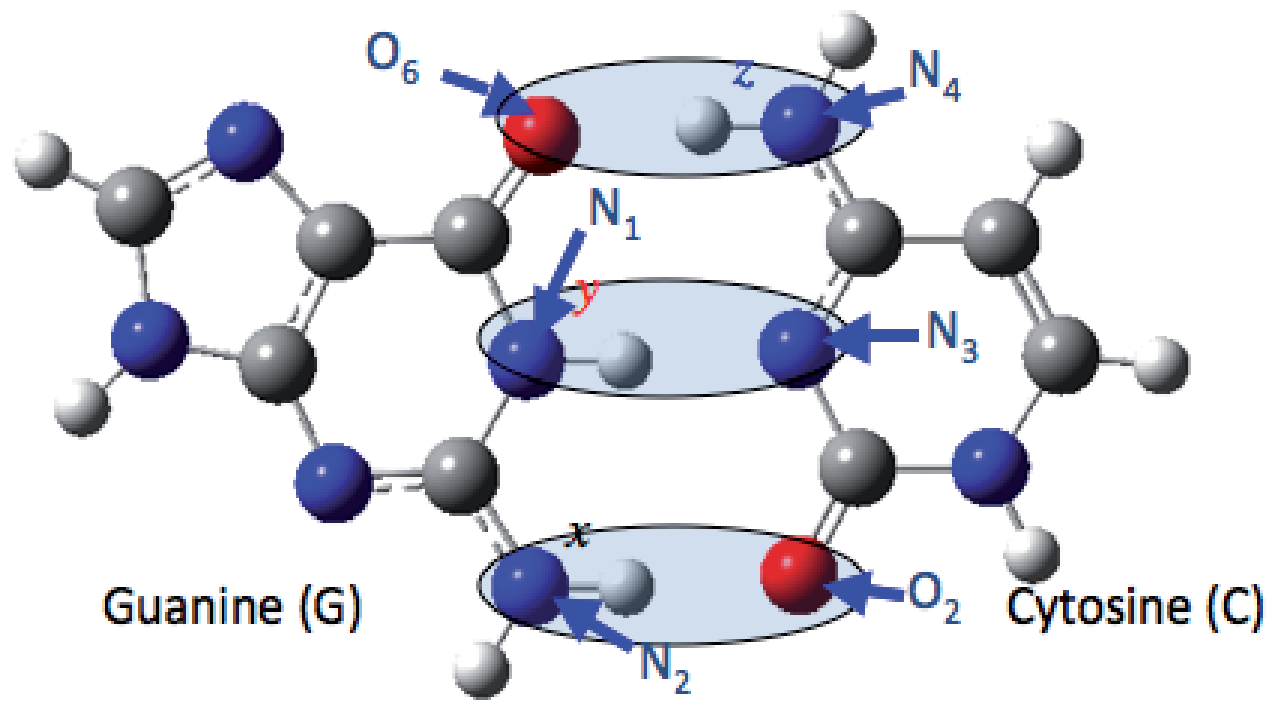

Figure 1. A Model for multiple proton transfer reactions in GC pairs. $x, y$, and $z$ are reaction coordinates of the proton transfer reactions 


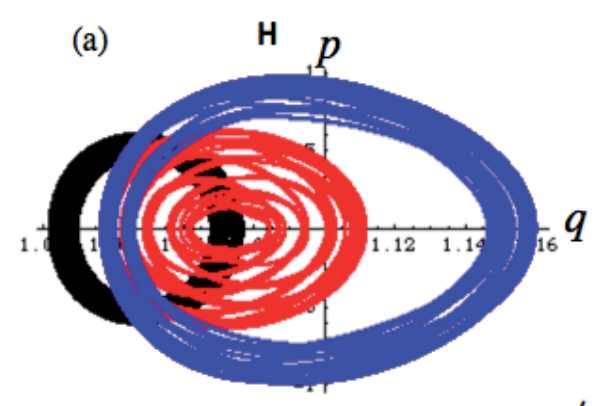

$x-p x / y-p y / z-p z$

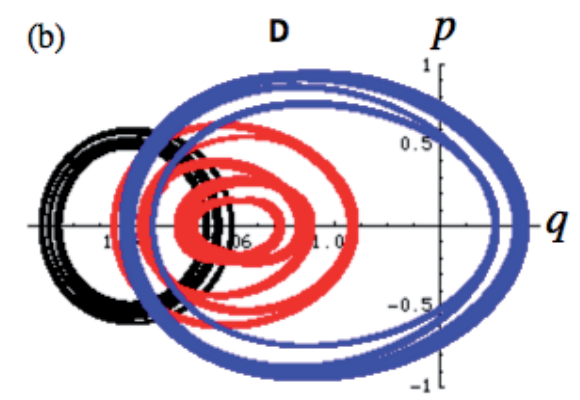

(c)

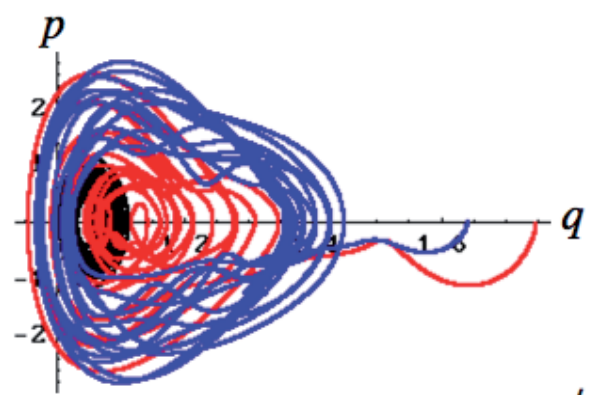

(d)

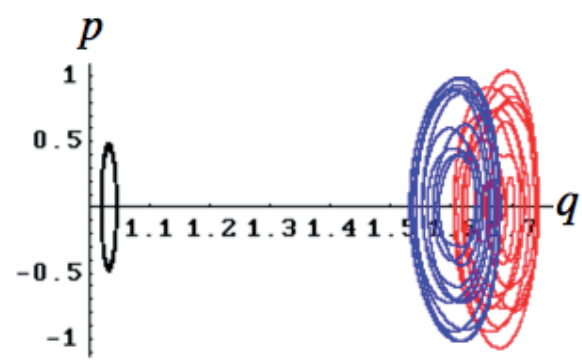

$x-p x / y-p y / z-p z$

Figure 2. QCD phase space structures of the GC pair, where $q=x, y$, or $z$ and $p=p_{x}, p_{y}$, or $p_{z}$, respectively. (a) and (b) are initially located around the global minimum for the protonated and deuterated cases. (c) and (d) are initially located around the metastable structure for the protonated and deuterated cases.

\subsection{Quantal structural transition of finite clusters}

Melting behavior of the finite quantum clusters were extensively investigated by many researchers using different kind of methodologies [34-39]. We here investigate the melting behavior of $n$ particle Morse clusters (abbreviated as $M_{n}$ ) by means of the QCD2 method. The Morse potential has following form:

$$
V_{M}(r)=D_{\mathrm{e}}\left[\exp \left(-2 \rho\left(r-R_{\mathrm{e}}\right)\right)-2 \exp \left(-\rho\left(r-R_{\mathrm{e}}\right)\right)\right]
$$

where $D_{\mathrm{e}}, R_{\mathrm{e}}$ and $\rho$ are parameters for a depth, a position of minimum, and a curvature of potential. In order to evaluate the quantal potential for the Morse potential, we adopt a Gaussian fit for the potential as

$$
V_{G}(r)=\sum_{i=1}^{N_{G}} c_{i} e^{-\alpha_{i} r^{2}},
$$


which has an analytic form of the quantal potential and coefficients $\left\{c_{i}\right\}$ are obtained by a least square fit for a set of even-tempered exponent with upper and lower bounds $\left(\alpha_{\text {upper }}=10^{6}\right.$ and $\alpha_{\text {lower }}=10^{-3}$ ). By choosing the number of Gaussians, $N_{G}$, the set of the coefficients is explicitly determined and we here set $N_{G}=41$.

We here evaluate optimized structures of $\mathrm{M}_{\mathrm{n}}$ clusters ( $\left.\mathrm{n}=3-7\right)$ for $D_{e}=1, R_{\mathrm{e}}=1$, and $\alpha=1$. The classical global minimum structures of $\mathrm{M}_{3}, \mathrm{M}_{4}, \mathrm{M}_{5}, \mathrm{M}_{6}$, and $\mathrm{M}_{7}$ structure have $\mathrm{C}_{3 \mathrm{v}}, \mathrm{T}_{\mathrm{d}}, \mathrm{D}_{3 \mathrm{~h}}$, $\mathrm{O}_{\mathrm{h}}$, and $\mathrm{D}_{5 \mathrm{~h}}$ symmetry respectively. Table 2 lists energy for each method. We found that the diagonal approximation causes the artificial symmetry breaking and the spherical approximation gives less accurate results. Original approximation gives the most accurate and correctly symmetric global minimum structures. The diagonal approximation gives the same results by the original one for $\mathrm{M}_{6}$ due to the same reason denoted before. The error of both the diagonal and spherical approximation decreases with the increase of the number of the particles. It is expected that both approximations work well for many particle systems instead of the original one. In particular the error of the diagonal approximation is $0.006 \%$ for the $\mathrm{M}_{7}$ cluster. This fact tells us that the diagonal approximation is reliable for the $\mathrm{M}_{7}$ cluster at least the stable structure.

For the analyses on quantum melting behavior, the parameters of the Morse potential are chosen as $D_{e}=1, R_{\mathrm{e}}=3$, and $\alpha=1$. Table 2 also lists nearest and next nearest distances obtained by the original and classical ones. As found in this Table, all the distances elongate with respect to classical ones. For example, the distances of $\mathrm{M}_{3}$ and $\mathrm{M}_{4}$ elongate by $7.2 \%$ and $7.8 \%$, respectively. It is notable that these distances does not equally elongate. The ratio between the original and classical ones is different for the nearest and next nearest distances, i.e. $8.16 \%$ and $6.20 \%$ for $\mathrm{M}_{5}, 7.36 \%$ and $3.91 \%$ for $\mathrm{M}_{6}$, and $8.70 \%$ and $6.79 \%$ for $\mathrm{M}_{7}$, respectively. In future works, we investigate influence of these behaviors on the structural transition (deformation) of the quantum Morse clusters in detail.

In order to measure the melting behavior of the finite clusters, we here use the Lindemann index defined as

$$
\sigma=\frac{2}{N(N-1)} \sum_{i j} \frac{\sqrt{\left\langle r_{i j}^{2}\right\rangle-\left\langle r_{i j}\right\rangle^{2}}}{\left\langle r_{i j}\right\rangle}
$$

where $\left\langle r_{i j}\right\rangle$ is a long time-averaged distance between $i$ and $j$-th particles. In the present approach, there exist two possible choices of the average. One is the quantum mechanical average within the second-order QCD approach, $\left\langle\hat{r}_{i j}\right\rangle_{\mathrm{QCD} 2}=\left\langle\left|\hat{\boldsymbol{q}}_{i}-\boldsymbol{\boldsymbol { q }}_{j}\right|\right\rangle_{\mathrm{QCD} 2}$, which include information of both the classical position and the second-order position cumulant simultaneously, and the other is the average evaluated by means of the classical positions appearing in the QCD approach, $\left\langle r_{i j}\right\rangle=\left\langle\left|\boldsymbol{q}_{i}-\boldsymbol{q}_{j}\right|\right\rangle$. We perform real-time dynamics simulation to obtain the Lindemann index for both systems, where we adopt $m=100$. 
The Lindemann indexes obtained by CD and QCD are illustrated in Fig. 1. In the figure, there exist three different regions. Until a freezing point, the Lindemann index gradually increases as the increase of the additional kinetic energy. In this region, the structural transition does not actually occur and the cluster remains stiff. This phase is called "solid-like phase". On the other hand, above a melting point, the structural transition often occurs and the cluster is soft. This phase is called "liquid-like phase". Between two phases, the Lindemann index rapidly increases. This phase is referred as "coexistence phase", which is not allowed for the bulk systems and peculiar to the finite systems. For both the solid- and liquidlike phases, the Lindeman index does not deviate too much. However that of the coexistence phase fluctuates due to a choice of the initial condition. In comparison with $C D$ and QCD results, the transition temperatures of $\mathrm{QCD}$ are lower than those of the $\mathrm{CD}$ reflecting the quantum effects. The freezing and melting temperatures are about 0.35 and 0.42 for QCD and about 0.41 and 0.60 for CD, respectively. Since the zero-point vibrational energy is included in QCD, the energy barrier between the basin and transition state become lower so that the less temperature is needed to overcome the barrier. This is so-called quantum softening as indicated by Doll and coworker for the Neon case by means of the path-integral approach. Our real-time dynamics well reproduce their tendency for this static property. On the other hand, behavior of Lidemann indexes from $\left\langle\hat{r}_{i j}\right\rangle_{\mathrm{QCD} 2}=\left\langle\left|\hat{\boldsymbol{q}}_{i}-\hat{\boldsymbol{q}}_{j}\right|\right\rangle_{\mathrm{QCD} 2}$ and $\left\langle r_{i j}\right\rangle=\left\langle\left|\boldsymbol{q}_{i}-\boldsymbol{q}_{j}\right|\right\rangle$ is slight different, whereas the transition temperature is the same. In the solid-like phase the Lindemann index obtained by the classical dynamics is equivalent to that of the classical contribution from QCD approach. On the other hand, in the liquid-like phase the Lindemann index is equivalent to that by the classical dynamics. This fact originates from the fact that the high temperature limit of the quantum results coincides with that of the classical one. It is stressed here that the cumulant variables, which contributes to not only to the quantum delocalization but also to the thermal fluctuation.

\begin{tabular}{lllllll}
\hline & \multicolumn{3}{c}{ Energy } & & Distance \\
\cline { 2 - 6 } & Spherical & Diagonal & Original & Classical & Original & Classical \\
\hline $\mathbf{M}_{\mathbf{3}}$ & -2.52135 & -2.55978 & -2.56510 & -3.00000 & 1.07198 & 1 \\
\hline $\mathbf{M}_{\mathbf{4}}$ & -5.20344 & -5.22683 & -5.24237 & -6.0000 & 1.07811 & 1 \\
\hline $\mathbf{M}_{\mathbf{5}}$ & -8.71392 & -8.74464 & -8.76049 & -9.85233 & 0.99212 & 0.91725 \\
\hline $\mathbf{M}_{\mathbf{6}}$ & -13.1836 & -13.2141 & -13.2141 & -14.7182 & 1.15085 & 1.08364 \\
\hline $\mathbf{M}_{\mathbf{7}}$ & -18.4016 & -18.4404 & -18.4415 & -20.3282 & 1.37518 & 1.32343 \\
\hline
\end{tabular}

Table 2. Nearest and next nearest distances and energy for global minimum obtained by cumulant and classical dynamics for 3-dimensional $\mathrm{M}_{n}$ clusters ( $\left.n=3-7\right)$. 


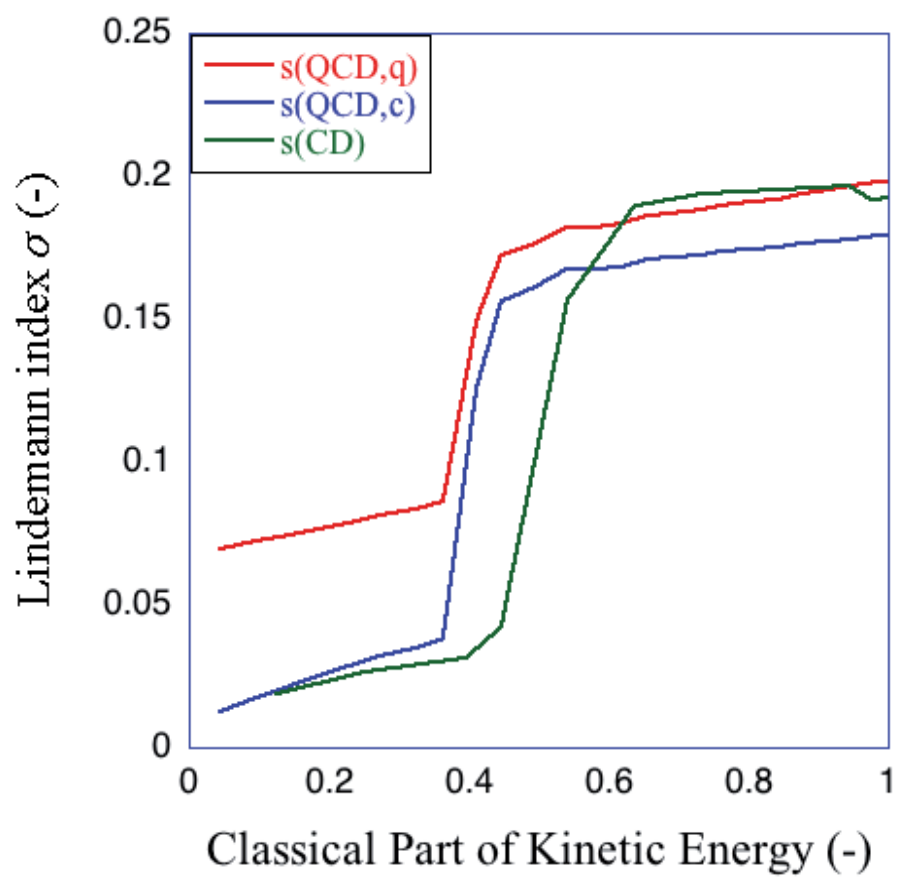

Figure 3. Static Lindemann indexes evaluated from QCD quantum distance (red), QCD classical distance (blue), and CD classical distance (green).

\section{Summary}

As an extension to the mechanics concerning about Ehrenfest theorem, we formulated a quantal cumulant mechanics (QCM) and corresponding dynamic method (QCD). The key point is the use of a position shift operator acting on the potential operator and introducing the cumulant variables to evaluate it, so that one need not truncate the potential, and it does not require separating into quantum and classical parts. In particular, we derived the coupled equation of motion (EOM) for the position, momentum, and second-order cumulants of the product of the momentum and position fluctuation operators. The EOM consists of variables and a quantal potential and its derivatives, where the quantal potential is expressed as an exponential function of the differential operator acting on the given potential. We defined density and joint density evaluated from the cumulant expansion scheme. It is clearly found that the present second-order approach gives a Gaussian density distribution spanned both on position and momentum space. Since the density is normalized, the joint density is considered exactly as probability distribution. We also indicated the relation between the joint density and cumulant variables as expectation values calculated from the distribution. We extended the QCD for the one-dimensional system in to treat the multi-dimensional systems. We derived the EOMs with the $24 \mathrm{~N}$ dimensional phase space. 
As numerical examples, we performed four applications to the simple systems. The first is the application to molecular vibrations. At first we showed that the normal mode analysis is extended to the effective potential appeared in the QCD. We illustrated that the anharmonic contribution is taken into account through mixing between the ordinary and the extended coordinates. The QCD simulations for the ab initio derived quartic force field are performed. The vibrational frequencies obtained from its power spectrum are good agreement with those obtained by accurate methods such as VPT2 and VCI.

The second is the proton transfer reactions in model DNA base pairs. We numerically showed the geometric isotope effects on the stability of the proton-transferred structures of the DNA base pairs as a function of the mass. We performed QCD simulations in order to investigate dynamical stability of the proton-transferred GC pair. The results showed that the proton-transferred structure of the protonated isotopomer is dynamically unstable and that of deuterated isotopomer remains stable. In former case, dynamically induced transition from the metastable to global minimum occurs. It is relevant to include dynamical effects to treat quantum isotope effects on the proton transfer reactions.

The last application is structural transition of finite quantum Morse clusters. We first compared the energy of the stable structures of the classical $\mathrm{M}_{n}$ cluster with those of quantum counterpart and found that the quantum effects due to zero point vibration is remarkable for small system and suppressed for larger. Then we performed the real-time dynamics to evaluate the Lindemann index to characterize the dynamical effects on the melting for $M_{7}$ cluster. In between solid-like and liquid-like phases (so-called coexistence phase), structural changes of the cluster occur intermittently.

\section{Acknowledgements}

This study is supported by a Grant-in-Aid for Young Scientists (A) (No. 22685003) from Japan Society for the Promotion of the Science (JSPS) and also by a CREST program from Japan Science and Technology Agency (JST).

\section{Author details}

\section{Yasuteru Shigeta ${ }^{1,2}$}

1 Department of Materials Engineering Science, Graduate School of Engineering Sciences, Osaka University, Machikaneyama-cho, Toyonaka, Osaka, Japan

2 Japan Science and Technology Agency, Kawaguchi Center Building, Honcho, Kawaguchishi, Saitama, Japan 


\section{References}

[1] Schrödinger, E. (1926). Quantisierung als Eigenwertproblem (Erste Mitteilung). Annalen der Physik, Vol. 79, No. 4, (April, 1926), pp. 361-376. ISSN: 0003-3804

[2] Schrödinger, E. (1926). Quantisierung als Eigenwertproblem (Zweite Mitteilung). Annalen der Physik, Vol. 79, No. 6, (May, 1926), pp. 489-527. ISSN: 0003-3804

[3] Schrödinger, E. (1926). Quantisierung als Eigenwertproblem (Dritte Mitteilung: Storungstheorie, mit Anwendung auf den Starkeffekt der Balmerlinien). Annalen der Physik, Vol. 80, No. 13, (September, 1926), pp. 437-490. ISSN: 0003-3804.

[4] Schrödinger, E. (1927). Quantisierung als Eigenwertproblem (Vierte Mitteilung). Annalen der Physik, Vol 81, No. 18, (September, 1927), pp. 109-139. 0003-3804.

[5] Heisenberg, W. (1943). The observable quantities in the theory of elementary particles. III. Zeitschrift für physik, Vol 123, No. 1-2, (March, 1943) pp. 93-112. ISSN: 0044-3328.

[6] Ehrenfest, P. (1927). Bemerkung über die angenäherte Gültigkeit der klassischen Mechanik innerhalb der Quantenmechanik. Zeitschrift für physik, Vol 45, No. 7-8, (July, 1927), pp. 455-472. ISSN: 0044-3328.

[7] Prezhdo, O.V. \& Pereverzev, Y.V. (2000). Quantized Hamilton dynamics. Journal of Chemical Physics, Vol. 113, No. 16, (October 22, 2000), pp. 6557-6565. ISSN: 0021-9606.

[8] Prezhdo, O.V. (2006). Quantized Hamilton Dynamics. Theoretical Chemistry Accounts, Vol. 116, No. 1-3, (August 2006), pp. 206-218. ISSN: 1432-881X and references cited therein.

[9] Miyachi, H.; Shigeta, Y.; Hirao K. (2006). Real time mixed quantum-classical dynamics with ab initio quartic force field: Application to molecular vibrational frequency analysis. Chemical Physics Letters, Vol. 432, No. 4-6, (December 11, 2006) 585-590. ISSN: 0009-2614

[10] Shigeta, Y.; Miyachi, H.; Hirao, K. (2006). Quantal cumulant dynamics: General theory. Journal of Chemical Physics, Vol. 125: 244102. ISSN: 0021-9606.

[11] Shigeta, Y.; Miyachi, H.; Hirao, K. (2007). Quantal cumulant dynamics II: An efficient time-reversible integrator. Chemical Physics Letters, Vol. 443, No. (AUG 6 2007), 414-419. ISSN: 0009-2614

[12] Shigeta Y. (2007). Quantal Cumulant Dynamics for Dissipative Systems. AIP proceedings Vol. 963, (2007), 1317.

[13] Shigeta Y. (2008). Quantal cumulant dynamics III: A quantum confinement under a magnetic field. Chemical Physics Letters, Vol. 461, No. 4-6, (August 20, 2008), 310-315. ISSN: 0009-2614 
[14] Shigeta Y. (2008). Distribution function in quantal cumulant dynamics. Journal of Chemical Physics, Vol. 128, No. 16, (April 28, 2008) 161103. ISSN: 0021-9606.

[15] Shigeta, Y.; Miyachi, H.; Matsui, T.; Hirao, K. (2008) Dynamical quantum isotope effects on multiple proton transfer reactions. Bulletin of the Chemical Society Japan. Vo. 81, No. 10, (October 15, 2008), 1230 -1240. ISSN: 0009-2673.

[16] Pereverzev, Y.V.; Pereverzev, A.; Shigeta, Y.; Prezhdo, O.V. (2008) Correlation functions in quantized Hamilton dynamics and quantal cumulant dynamics. Journal of Chemical Physics, Vol. 129, No. 14, (October 14, 2008), 144104. ISSN: 0021-9606.

[17] Shigeta, Y. Molecular Theory Including Quantum Effects and Thermal Fluctuations, the Bulletin of Chemical Society Japan, Vo. 82, No. 11, (November 15, 2009), 1323-1340. ISSN: 0009-2673.

[18] Shigeta, Y.; Miyachi, H.; Matsui, T.; Yokoyama, N.; Hirao, K. "Quantum Theory in Terms of Cumulant Variables", Progress in Theoretical Chemistry and Physics, Vol. 20, "Advances in the Theory of Atomic and Molecular Systems - Dynamics, Spectroscopy, Clusters, and Nanostructures", edited by Piecuch, P.; Maruani, J.; DelgadoBarrio, G.; Wilson, S., pp. 3-34, Springer, 2009, 3-34.

[19] Shigeta, Y.; Inui, T.; Baba, T.; Okuno, K.; Kuwabara, H.; Kishi, R.; Nakano, M. (2012) International Journal of Quantum Chemistry. in press, (2012). ISSN: 0020-7608.

[20] Mayer, J. E. (1937). The Statistical Mechanics of Condensing Systems I. Journal of Chemical Physics, Vol. 5, (January, 1937), 67-73. ISSN: 0021-9606.

[21] Kubo, R. (1962). Generalized Cumulant Expansion Method. Journal of Physical Society of Japan, Vol. 17, No. 7, (July, 1962) 1100-1120. ISSN: 0031-9015.

[22] Mandal. S.H.; Sanyal, G.; Mukherjee, D. (1998). A Thermal Cluster-Cumulant Theory. Lecture Notes in Physics, Vol. 510, (1998), 93-117. ISSN: 0075-8450.

[23] Yagi, K.; Hirao, K.; Taketsugu, T.; Schmidt, M.W.; Gordon, M.S. (2004). Ab initio vibrational state calculations with a quartic force field: Applications to $\mathrm{H}_{2} \mathrm{CO}, \mathrm{C}_{2} \mathrm{H}_{4}$, $\mathrm{CH}_{3} \mathrm{OH}, \mathrm{CH}_{3} \mathrm{CCH}$, and $\mathrm{C}_{6} \mathrm{H}_{6}$. Journal of Chemical Physics, Vol. 121, No. 3 (July 15, 2004) 1383-1389. ISSN: 0021-9606.

[24] Möller, C; Plesset, M.S. (1934). Note on an approximation treatment for many-electron systems. Physical Review, Vol. 46, No. 7, (October, 1934), 618-622. ISSN: 0031-899X.

[25] Lendall, R.A.; Dunning Jr, T.H.; Harrison, R.J. (1992). Electron affinities of the firstrow atoms revisited. Systematic basis sets and wave functions. Journal of Chemical Physics, Vol. 96, No. 9, (May 1, 1992), 6796-6806. ISSN: 0021-9606.

[26] M.W. Schmidt et al (1993) General Atomic and Molecular Electronic-structure System. Journal Computational Chemistry, Vol. 14, No. 11, (November, 1993), 1347-1363. ISSN: 0192-8651.

[27] M.J. Frisch et al (2004) Gaussian 03 (Revision C.02). Gaussian Inc Wallingford CT 
[28] Florián, J.; Hrouda, V.; Hobza, P. (1994). Proton Transfer in the Adenine-Thymine Base Pair. Journal of the American Chemical Society, Vol. 116, No. 4, (February 23, 1994), 1457-1460. ISSN: 0002-7863.

[29] Floriaán J, Leszczyn'sky J (1996) Spontaneous DNA Mutation Induced by Proton Transfer in the Guanine-Cytosine Base Pair: An Energetic Perspective. Journal of the American Chemical Society, Vol. 118, No. 12, (March 27, 1996), 3010-3017. ISSN: 0002-7863.

[30] Villani G (2005) Theoretical investigation of hydrogen transfer mechanism in adenine-thymine base pair. Chemical Physics, Vol. 316 No. 1-3, (September 19, 2005), 1-8. ISSN: 0301-0104.

[31] Villani G (2006) Theoretical investigation of hydrogen transfer mechanism in the guanine-cytosine base pair Chemical Physics, Vol. 324, No. 2-3, (MAY 31 2006), 438 -446. ISSN: 0301-0104.

[32] Matsui T, Shigeta Y, Hirao K (2006) Influence of Pt complex binding on the guanine cytosine pair: A theoretical study. Chemical Physics Letters, Vol. 423, No 4-6, (June 1, 2006), 331-334. ISSN: 0009-2614.

[33] Matsui, T.; Shigeta, Y.; Hirao, K. (2007). Multiple proton-transfer reactions in DNA base pairs by coordination of Pt complex. Journal of Chemical Physics B, Vol. 111, No. 5, (February 8, 2007), 1176-1181. ISSN: 1520-6106.

[34] Ceperley, D.M. (1995). Path-integrals in the theory of condensed Helium. Review Modern Physics, Vol. 67, No. 2, (April 1995), 279-355. ISSN: 0034-6861.

[35] Chakravarty, C. (1995). Structure of quantum binary clusters. Physical Review Letters, Vol. 75, No. 9, (August 28, 1995), 1727-1730. ISSN: 0031-9007.

[36] Chakravarty, C. (1996). Cluster analogs of binary isotopic mixtures: Path integral Monte Carlo simulations. Journal of Chemical Physics, Vol. 104, No. 18, (May 8, 1996), 7223-7232. ISSN: 0021-9606.

[37] Predescu, C.; Frantsuzov, P.A.; Mandelshtam, V.A. (2005). Journal of Chemical Physics, Vol. 122, No. 15, (April 15, 2005), 154305. ISSN: 0021-9606.

[38] Frantsuzov, P.A.; Meluzzi, D.; Mandelshtam, V.A. (2006). Structural transformations and melting in neon clusters: Quantum versus classical mechanics. Physical Review Letters, Vol. 96, No. 11, (March 24, 2006), 113401. ISSN: 0031-9007

[39] Heller, E. J. (1975). Time-dependent approach to semiclassical dynamics. Journal of Chemical Physics, Vol. 62, No. 4, (1975), 1544-1555. ISSN: 0021-9606. 


\title{
Unruh Radiation via WKB Method
}

\author{
Douglas A. Singleton
}

Additional information is available at the end of the chapter

http://dx.doi.org/10.5772/53898

\section{Introduction}

Quantum mechanics has many features which are distinct from classical physics. Perhaps none more so than tunneling - the ability of a quantum particle to pass through some potential barrier even when, classically, it would not have enough energy to do so. The examples of tunneling phenomenon range from the nuclear (e.g. alpha decay of nuclei) to the molecular(oscillations of the ammonia molecule). Every text book on quantum mechanics devotes a good fraction of of page space to tunneling (usually introduced via the tunneling through a one dimensional step potential) and its applications.

In general, tunneling problems can not be solved, easily or at all, in closed, analytic form and so one must resort to various approximation techniques. One of the first and mostly useful approximations techniques is the WKB method [1] names after its co-discovers Wentzel, Krammers and Brillouin. For a particle with an energy $E$ and rest mass $m$ moving in a one-dimensional potential $V(x)$ (where $E<V(x)$ for some range of $x$, say $a \leq x \leq b$, which is the region through which the particle tunnels) the tunneling amplitude is given by

$$
\exp \left[-\frac{1}{\hbar} \int_{a}^{b}[2 m(V(x)-E)]^{1 / 2} d x\right]=\exp \left[-\frac{1}{\hbar} \int_{a}^{b} p(x) d x\right]
$$

where $p(x)$ is the canonical momentum of the particle. Taking the square of (1) gives the probability for the particle to tunnel through the barrier.

In this chapter we show how the essentially quantum field theory phenomenon of Unruh radiation [4] can be seen as a tunneling phenomenon and how one can calculate some details of Unruh radiation using the WKB method. Unruh radiation is the radiation seen by an observer who accelerates through Minkowski space-time. Via the equivalence principle (i.e. the local equivalence between observations in a gravitational field versus in an accelerating 
frame) Unruh radiation is closely related to Hawking radiation [2] - the radiation seen by an observer in the space-time background of a Schwarzschild black hole.

In the WKB derivation of Unruh radiation presented here we do not recover all the details of the radiation that the full quantum field theory calculation of the Unurh effect yields. The most obvious gap is that from the quantum field theory calculations it is known that Unruh radiation as well as Hawking radiation have a thermal/Planckian spectrum. In the simple treatment given here we do not obtain the thermal character of the spectrum of Unruh radiation but rather one must assume the spectrum is thermal (however as shown in [5] one can use the density matrix formalism to obtain the thermal nature of Unruh radiation as well as Hawking radiation in the WKB tunneling approach). The advantage of the present approach (in contrast to the full quantum field theory calculation) is that it easy to apply to a wide range of observer and space-times. For example, an observer in de Sitter space-time (the space-time with a positive cosmological constant) will see Hawking-Gibbons radiation [3]; an observer in the Friedmann-Robertson-Walker metric of standard Big Bang cosmology will see Hawking-like radiation [6]. One can easily calculate the basic thermal features of many space-times (e.g. Reissner-Nordstrom [9], de Sitter [14], Kerr and Kerr-Newmann [15, 16], Unruh [17]) using the WKB tunneling method. Additionally, one can easily incorporate the Hawking radiation of particles with different spins [18] and one can begin to take into account back reaction effects on the metric $[9,10,19]$ i.e. the effect that due to the emission of Hawking radiation the space-time will change which in turn will modify the nature of subsequent Hawking radiation.

The WKB tunneling method of calculating the Unruh and Hawking effects also corresponds the heuristic picture of Hawking radiation given in the original work by Hawking (see pg. 202 of [2]). In this paper Hawking describes the effect as a tunneling outward of positive energy modes from behind the black hole event horizon and a tunneling inward of negative energy modes. However only after a span of about twenty five years where mathematical details given to this heuristic tunneling picture with the works [7-10]. These works showed that the action for a particle which crosses the horizon of some space-time picked up an imaginary contribution on crossing the horizon. This imaginary contribution was then interpreted as the tunneling probability.

One additional advantage of the WKB tunneling method for calculating some of the features of Hawking and Unruh radiation is that this method does not rely on quantum field theory techniques. Thus this approach should make some aspects of Unruh radiation accessible to beginning graduate students or even advanced undergraduate students.

Because of the strong equivalence principle (i.e., locally, a constant acceleration and a gravitational field are observationally equivalent), the Unruh radiation from Rindler space-time is the prototype of this type of effect. Also, of all these effects - Hawking radiation, Hawking-Gibbons radiation - Unruh radiation has the best prospects for being observed experimentally [20-23]. This WKB approach to Unruh radiation draws together many different areas of study: (i) classical mechanics via the Hamilton-Jacobi equations; (ii) relativity via the use of the Rindler metric; (iii) relativistic field theory through the Klein-Gordon equation in curved backgrounds; (iv) quantum mechanics via the use of the WKB-like method applied to gravitational backgrounds; (v) thermodynamics via the use of the Boltzmann distribution to extract the temperature of the radiation; (vi) mathematical methods in physics via the use of contour integrations to evaluate the imaginary part of 
the action of the particle that crosses the horizon. Thus this single problem serves to show students how the different areas of physics are interconnected.

Finally, through this discussion of Unruh radiation we will highlight some subtle features of the Rindler space-time and the WKB method which are usually overlooked. In particular, we show that the gravitational WKB amplitude has a contribution coming from a change of the time coordinate from crossing the horizon [14]. This temporal contribution is never encountered in ordinary quantum mechanics, where time acts as a parameter rather than a coordinate. Additionally we show that the invariance under canonical transformations of the tunneling amplitude for Unurh radiation is crucially important to obtaining the correct results in the case of tunneling in space-time with a horizon.

\section{Some details of Rindler space-time}

We now introduce and discuss some relevant features of Rindler space-time. This is the space-time seen by an observer moving with constant proper acceleration through Minkowski space-time. Thus in some sense this is distinct from the case of a gravitational field since here we are dealing with flat, Minkowski space-time but now seen by an accelerating observer. However, because of the equivalence principle this discussion is connected to situations where one does have gravitational fields such as Hawking radiation in the vicinity of a black hole.

The Rindler metric can be obtained by starting with the Minkowski metric, i.e., $d s^{2}=$ $-d t^{2}+d x^{2}+d y^{2}+d z^{2}$, where we have set $c=1$, and transforming to the coordinates of the accelerating observer. We take the acceleration to be along the $x$-direction, thus we only need to consider a 1+1 dimensional Minkowski space-time

$$
d s^{2}=-d t^{2}+d x^{2}
$$

Using the Lorentz transformations (LT) of special relativity, the worldlines of an accelerated observer moving along the $x$-axis in empty spacetime can be related to Minkowski coordinates $t, x$ according to the following transformations

$$
\begin{aligned}
& t=\left(a^{-1}+x_{R}\right) \sinh \left(a t_{R}\right) \\
& x=\left(a^{-1}+x_{R}\right) \cosh \left(a t_{R}\right),
\end{aligned}
$$

where $a$ is the constant, proper acceleration of the Rindler observer measured in his instantaneous rest frame. One can show that the acceleration associated with the trajectory of (3) is constant since $a_{\mu} a^{\mu}=\left(d^{2} x_{\mu} / d t_{R}^{2}\right)^{2}=a^{2}$ with $x_{R}=0$. The trajectory of (3) can be obtained using the definitions of four-velocity and four-acceleration of the accelerated observer in his instantaneous inertial rest frame [24]. Another derivation of (3) uses a LT to relate the proper acceleration of the non-inertial observer to the acceleration of the inertial observer [25]. The text by Taylor and Wheeler [26] also provides a discussion of the Rindler observer.

The coordinates $x_{R}$ and $t_{R}$, when parametrized and plotted in a spacetime diagram whose axes are the Minkowski coordinates $x$ and $t$, result in the familiar hyperbolic trajectories (i.e., $x^{2}-t^{2}=a^{-2}$ ) that represent the worldlines of the Rindler observer. 
Differentiating each coordinate in (3) and substituting the result into (2) yields the standard Rindler metric

$$
d s^{2}=-\left(1+a x_{R}\right)^{2} d t_{R}^{2}+d x_{R}^{2} .
$$

When $x_{R}=-\frac{1}{a}$, the determinant of the metric given by (4), $\operatorname{det}\left(g_{a b}\right) \equiv g=-\left(1+a x_{R}\right)^{2}$, vanishes. This indicates the presence of a coordinate singularity at $x_{R}=-\frac{1}{a}$, which can not be a real singularity since (4) is the result of a global coordinate transformation from Minkowski spacetime. The horizon of the Rindler space-time is given by $x_{R}=-\frac{1}{a}$.

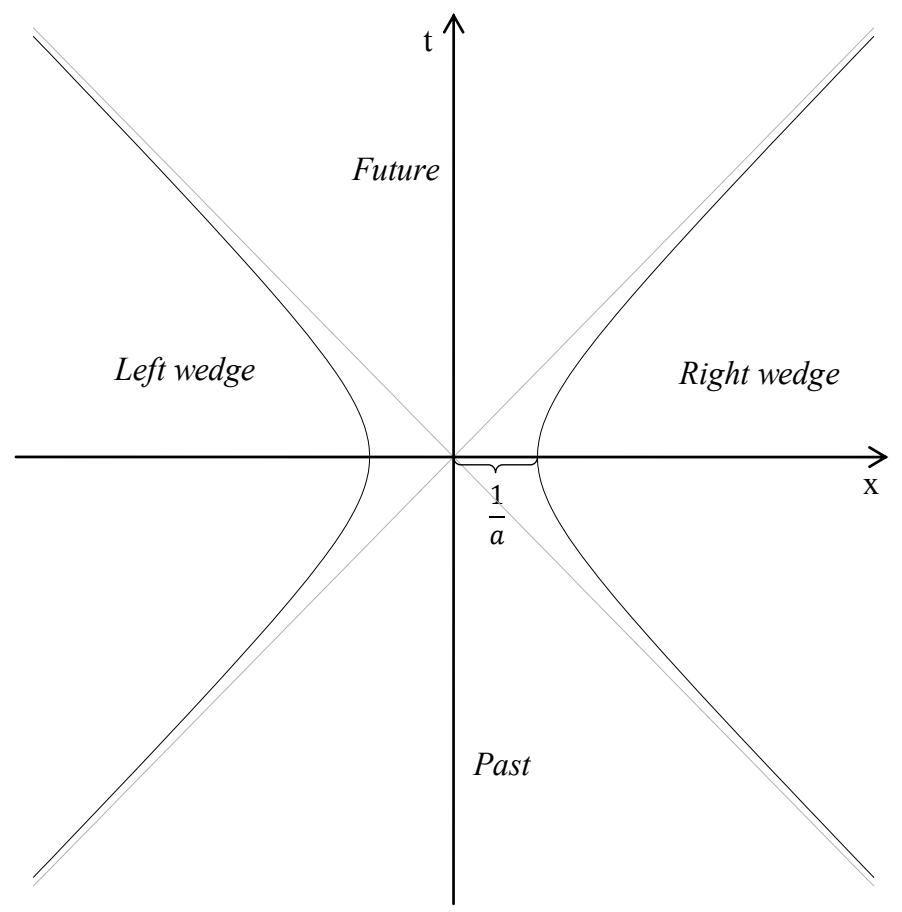

Figure 1. Trajectory of the Rindler observer as seen by the observer at rest.

In the spacetime diagram shown above, the horizon for this metric is represented by the null asymptotes, $x= \pm t$, that the hyperbola given by (3) approaches as $x$ and $t$ tend to infinity [27]. Note that this horizon is a particle horizon, since the Rindler observer is not influenced by the whole space-time, and the horizon's location is observer dependent [28].

One can also see that the transformations (3) that lead to the Rindler metric in (4) only cover a quarter of the full Minkowski space-time, given by $x-t>0$ and $x+t>0$. This portion of Minkowski is usually labeled Right wedge. To recover the Left wedge, one can modify the second equation of (3) with a minus sign in front of the transformation of the $x$ coordinate, thus recovering the trajectory of an observer moving with a negative acceleration. In fact, we will show below that the coordinates $x_{R}$ and $t_{R}$ double cover the region in front of the horizon, $x_{R}=-\frac{1}{a}$. In this sense, the metric in (4) is similar to the Schwarzschild metric written in isotropic coordinates. For further details, see reference [28]. 
There is an alternative form of the Rindler metric that can be obtained from (4) by the following transformation:

$$
\left(1+a x_{R}\right)=\sqrt{\left|1+2 a x_{R^{\prime}}\right|} .
$$

Using the coordinate transformation given by (5) in (4), we get the following Schwarzschild-like form of the Rindler metric

$$
d s^{2}=-\left(1+2 a x_{R^{\prime}}\right) d t_{R^{\prime}}^{2}+\left(1+2 a x_{R^{\prime}}\right)^{-1} d x_{R^{\prime}}^{2} .
$$

If one makes the substitution $a \rightarrow G M / x_{R^{\prime}}^{2}$ one can see the similarity to the usual Schwarzschild metric. The horizon is now at $x_{R^{\prime}}=-1 / 2 a$ and the time coordinate, $t_{R^{\prime}}$, does change sign as one crosses $x_{R^{\prime}}=-1 / 2 a$. In addition, from (5) one can see explicitly that as $x_{R^{\prime}}$ ranges from $+\infty$ to $-\infty$ the standard Rindler coordinate will go from $+\infty$ down to $x_{R}=-1 / a$ and then back out to $+\infty$.

The Schwarzschild-like form of the Rindler metric given by (6) can also be obtained directly from the 2-dimensional Minkowski metric (2) via the transformations

$$
\begin{aligned}
& t=\frac{\sqrt{1+2 a x_{R^{\prime}}}}{a} \sinh \left(a t_{R^{\prime}}\right) \\
& x=\frac{\sqrt{1+2 a x_{R^{\prime}}}}{a} \cosh \left(a t_{R^{\prime}}\right)
\end{aligned}
$$

for $x_{R^{\prime}} \geq-\frac{1}{2 a}$, and

$$
\begin{aligned}
& t=\frac{\sqrt{\left|1+2 a x_{R^{\prime}}\right|}}{a} \cosh \left(a t_{R^{\prime}}\right) \\
& x=\frac{\sqrt{\left|1+2 a x_{R^{\prime}}\right|}}{a} \sinh \left(a t_{R^{\prime}}\right)
\end{aligned}
$$

for $x_{R^{\prime}} \leq-\frac{1}{2 a}$. Note that imposing the above conditions on the coordinate $x_{R^{\prime}}$ fixes the signature of the metric, since for $x_{R^{\prime}} \leq-\frac{1}{2 a}$ or $1+2 a x_{R^{\prime}} \leq 0$ the metric signature changes to $(+,-)$, while for $1+2 a x_{R^{\prime}} \geq 0$ the metric has signature $(-,+)$. Thus one sees that the crossing of the horizon is achieved by the crossing of the coordinate singularity, which is precisely the tunneling barrier that causes the radiation in this formalism. As a final comment, we note that the determinant of the metric for (4) is zero at the horizon $x_{R}=-1 / a$, while the determinant of the metric given by (6) is 1 everywhere.

\section{The WKB/Tunneling method applied to Rindler space-time}

In this section we study a scalar field placed in a background metric. Physically, these fields come from the quantum fields, i.e., vacuum fluctuations, that permeate the space-time given by the metric. By applying the WKB method to this scalar field, we find that the phase of the scalar field develops imaginary contributions upon crossing the horizon. The exponential of 
these imaginary contributions is interpreted as a tunneling amplitude through the horizon. By assuming a Boltzmann distribution and associating it with the tunneling amplitude, we obtain the temperature of the radiation.

To begin we derive the Hamilton-Jacobi equations for a scalar field, $\phi$, in a given background metric. In using a scalar field, we are following the original works [2, 4]. The derivation with spinor or vector particles/fields would only add the complication of having to carry around spinor or Lorentz indices without adding to the basic understanding of the phenomenon. Using the WKB approach presented here it is straightforward to do the calculation using spinor[18] or vector particles. The scalar field in some background metic, $g^{\mu v}$ is taken to satisfy the Klein-Gordon (KG) equation

$$
\left(\frac{1}{\sqrt{-g}} \partial_{\mu}\left(\sqrt{-g} g^{\mu \nu} \partial_{v}\right)-\frac{m^{2} c^{2}}{\hbar^{2}}\right) \phi=0,
$$

where $c$ is the speed of light, $\hbar$ is Planck's constant, $m$ is the mass of the scalar field and $g_{\mu v}$ is the background metric. For Minkowski space-time, the (9) reduces to the free Klein-Gordon equation, i.e., $\left(\square-m^{2} c^{2} / \hbar^{2}\right) \phi=\left(-\partial^{2} / c^{2} \partial t^{2}+\nabla^{2}-m^{2} c^{2} / \hbar^{2}\right) \phi=0$. This equation is nothing other than the fundamental relativistic equation $E^{2}-p^{2} c^{2}=m^{2} c^{4}$ with $E \rightarrow i \hbar \partial_{t}$ and $p \rightarrow-i \hbar \nabla$.

Setting the speed of light $c=1$, multiplying (9) by $-\hbar$ and using the product rule, (9) becomes

$$
\begin{aligned}
& \frac{-\hbar^{2}}{\sqrt{-g}}\left[\left(\partial_{\mu} \sqrt{-g}\right) g^{\mu v} \partial_{\nu} \phi+\sqrt{-g}\left(\partial_{\mu} g^{\mu v}\right) \partial_{\nu} \phi+\right. \\
& \left.\sqrt{-g} g^{\mu v} \partial_{\mu} \partial_{\nu} \phi\right]+m^{2} \phi=0 .
\end{aligned}
$$

The above equation can be simplified using the fact that the covariant derivative of any metric $g$ vanishes

$$
\nabla_{\alpha} g^{\mu v}=\partial_{\alpha} g^{\mu v}+\Gamma_{\alpha \beta}^{\mu} g^{\beta v}+\Gamma_{\alpha \beta}^{v} g^{\mu \beta}=0,
$$

where $\Gamma_{\alpha \beta}^{\mu}$ is the Christoffel connection. All the metrics that we consider here are diagonal so $\Gamma_{\alpha \beta}^{\mu}=0$, for $\mu \neq \alpha \neq \beta$. It can also be shown that

$$
\Gamma_{\mu \gamma}^{\mu}=\partial_{\gamma}(\ln \sqrt{-g})=\frac{\partial_{\gamma} \sqrt{-g}}{\sqrt{-g}}
$$

Using (11) and (12), the term $\partial_{\mu} g^{\mu v}$ in (10) can be rewritten as

$$
\partial_{\mu} g^{\mu \nu}=-\Gamma_{\mu \gamma}^{\mu} g^{\gamma v}-\Gamma_{\mu \rho}^{v} g^{\mu \rho}=-\frac{\partial_{\gamma} \sqrt{-g}}{\sqrt{-g}} g^{\gamma v}
$$


since the harmonic condition is imposed on the metric $g^{\mu v}$, i.e., $\Gamma_{\mu \rho}^{v} g^{\mu \rho}=0$. Thus (10) becomes

$$
-\hbar^{2} g^{\mu v} \partial_{\mu} \partial_{\nu} \phi+m^{2} \phi=0 .
$$

We now express the scalar field $\phi$ in terms of its action $S=S(t, \vec{x})$

$$
\phi=\phi_{0} e^{\frac{i}{\hbar} S(t, \vec{x})},
$$

where $\phi_{0}$ is an amplitude [29] not relevant for calculating the tunneling rate. Plugging this expression for $\phi$ into (14), we get

$$
-\hbar g^{\mu v}\left(\partial_{\mu}\left(\partial_{v}(i S)\right)\right)+g^{\mu v} \partial_{\nu}(S) \partial_{\mu}(S)+m^{2}=0 .
$$

Taking the classical limit, i.e., letting $\hbar \rightarrow 0$, we obtain the Hamilton-Jacobi equations for the action $S$ of the field $\phi$ in the gravitational background given by the metric $g_{\mu v}$,

$$
g^{\mu v} \partial_{\nu}(S) \partial_{\mu}(S)+m^{2}=0
$$

For stationary space-times (technically space-times for which one can define a time-like Killing vector that yields a conserved energy, $E)$ the action $S$ can be split into a time and space part, i.e., $S(t, \vec{x})=E t+S_{0}(\vec{x})$.

If $S_{0}$ has an imaginary part, this then gives the tunneling rate, $\Gamma_{Q M}$, via the standard WKB formula. The WKB approximation tells us how to find the transmission probability in terms of the incident wave and transmitted wave amplitudes. The transition probability is in turn given by the exponentially decaying part of the wave function over the non-classical (tunneling) region [30]

$$
\Gamma_{Q M} \propto e^{-\operatorname{Im} \frac{1}{\hbar} \oint p_{x} d x} .
$$

The tunneling rate given by (18) is just the lowest order, quasi-classical approximation to the full non-perturbative Schwinger [31] rate. ${ }^{1}$

In most cases (with an important exception of Painlevé-Gulstrand form of the Schwarzschild metric which we discuss below), $p^{\text {out }}$ and $p^{\text {in }}$ have the same magnitude but opposite signs. Thus $\Gamma_{Q M}$ will receive equal contributions from the ingoing and outgoing particles, since the sign difference between $p^{\text {out }}$ and $p^{\text {in }}$ will be compensated for by the minus sign that is picked up in the $p^{\text {in }}$ integration due to the fact that the path is being traversed in the

\footnotetext{
${ }^{1}$ The Schwinger rate is found by taking the Trace-Log of the operator $\left(\square_{g}-m^{2} c^{2} / \hbar^{2}\right)$, where $\square_{g}$ is the d'Alembertian in the background metric $g_{\mu v}$, i.e., the first term in (9). As a side comment, the Schwinger rate was initially calculated for the case of a uniform electric field. In this case, the Schwinger rate corresponded to the probability of creating particle-antiparticle pairs from the vacuum field at the expense of the electric field's energy. This electric field must have a critical strength in order for the Schwinger effect to occur. A good discussion of the calculation of the Schwinger rate for the usual case of a uniform electric field and the connection of the Schwinger effect to Unruh and Hawking radiation can be found in reference [32].
} 
backward $x$-direction. In all quantum mechanical tunneling problems that we are aware of this is the case: the tunneling rate across a barrier is the same for particles going right to left or left to right. For this reason, the tunneling rate (18) is usually written as [30]

$$
\Gamma_{Q M} \propto e^{\mp 2 \operatorname{Im} \frac{1}{\hbar} \int p_{x}^{\text {out in }} d x}
$$

In (19) the - sign goes with $p_{x}^{\text {out }}$ and the + sign with $p_{x}^{\text {in }}$.

There is a technical reason to prefer (18) over (19). As was remarked in references [33-35], equation (18) is invariant under canonical transformations, whereas the form given by (19) is not. Thus the form given by (19) is not a proper observable.

Moreover, we now show that the two formulas, (18) and (19), are not even numerically equivalent when one applies the WKB method to the Schwarzschild space-time in Painlevé-Gulstrand coordinates. The Painlevé-Gulstrand form of the Schwarzschild space-time is obtained by transforming the Schwarzschild time $t$ to the Painlevé-Gulstrand time $t^{\prime}$ using the transformation

$$
d t=d t^{\prime}-\frac{\sqrt{\frac{2 M}{r}} d r}{1-\frac{2 M}{r}} .
$$

Applying the above transformation to the Schwarzshild metric gives us the Painlevé-Gulstrand form of the Schwarzschild space-time

$$
d s^{2}=-\left(1-\frac{2 M}{r}\right) d t^{\prime 2}+2 \sqrt{\frac{2 M}{r}} d r d t^{\prime}+d r^{2}
$$

The time is transformed, but all the other coordinates $(r, \theta, \phi)$ are the same as the Schwarzschild coordinates. If we use the metric in (21) to calculate the spatial part of the action as in (35) and (29), we obtain

$$
\begin{aligned}
S_{0} & =-\int_{-\infty}^{\infty} \frac{d r}{1-\frac{2 M}{r}} \sqrt{\frac{2 M}{r}} E \\
& \pm \int_{-\infty}^{\infty} \frac{d r}{1-\frac{2 M}{r}} \sqrt{E^{2}-m^{2}\left(1-\frac{2 M}{r}\right)} .
\end{aligned}
$$

Each of these two integrals has an imaginary contribution of equal magnitude, as can be seen by performing a contour integration. Thus one finds that for the ingoing particle (the + sign in the second integral) one has a zero net imaginary contribution, while from for the outgoing particle (the - sign in the second integral) there is a non-zero net imaginary contribution. Also as anticipated above the ingoing momentum (i.e, the integrand in (22) with the + sign in the second term) is not equal to the outgoing momentum (i.e, the integrand in (22) with the - sign in the second term) In these coordinates there is a difference by a 
factor of two between using (18) and (19) which comes exactly because the tunneling rates from the spatial contributions in this case do depend upon the direction in which the barrier (i.e., the horizon) is crossed. The Schwarzcshild metric has a similar temporal contribution as for the Rindler metric [36]. The Painlevé-Gulstrand form of the Schwarzschild metric actually has two temporal contributions: (i) one coming from the jump in the Schwarzschild time coordinate similar to what occurs with the Rindler metric in (7) and (8); (ii) the second temporal contribution coming from the transformation between the Schwarzschild and Painlevé-Gulstrand time coordinates in (20). If one integrates equation (20), one can see that there is a pole coming from the second term. One needs to take into account both of these time contributions in addition to the spatial contribution, to recover the Hawking temperature. Only by adding the temporal contribution to the spatial part from (18), does one recover the Hawking temperature [36] $T=\frac{\hbar}{8 \pi M}$. Thus for both reasons - canonical invariance and to recover the temperature - it is (18) which should be used over (19), when calculating $\Gamma_{Q M}$. In ordinary quantum mechanics, there is never a case - as far as we know - where it makes a difference whether one uses (18) or (19). This feature - dependence of the tunneling rate on the direction in which the barrier is traverse - appears to be a unique feature of the gravitational WKB problem. So in terms of the WKB method as applied to the gravitational field, we have found that there are situations (e.g. Schwarzschild space-time in Painlevé-Gulstrand coordinates) where the tunneling rate depends on the direction in which barrier is traversed so that (18) over (19) are not equivalent and will thus yield different tunneling rates, $\Gamma$.

For the case of the gravitational WKB problem, equation (19) only gives the imaginary contribution to the total action coming from the spatial part of the action. In addition, there is a temporal piece, $E \Delta t$, that must be added to the total imaginary part of the action to obtain the tunneling rate. This temporal piece originates from an imaginary change of the time coordinate as the horizon is crossed. We will explicitly show how to account for this temporal piece in the next section, where we apply the WKB method to the Rindler space-time. This imaginary part of the total action coming from the time piece is a unique feature of the gravitational WKB problem. Therefore, for the case of the gravitational WKB problem, the tunneling rate is given by

$$
\Gamma \propto e^{-\frac{1}{\hbar}\left[\operatorname{Im}\left(\oint p_{x} d x\right)-E \operatorname{Im}(\Delta t)\right]}
$$

In order to obtain the temperature of the radiation, we assume a Boltzmann distribution for the emitted particles

$$
\Gamma \propto e^{-\frac{E}{T}},
$$

where $E$ is the energy of the emitted particle, $T$ is the temperature associated with the radiation, and we have set Boltzmann's constant, $k_{B}$, equal to 1 . Equation (25) gives the probability that a system at temperature $T$ occupies a quantum state with energy $E$. One weak point of this derivation is that we had to assume a Boltzmann distribution for the radiation while the original derivations $[2,4]$ obtain the thermal spectrum without any assumptions. Recently, this shortcoming of the tunneling method has been addressed in reference [5], where the thermal spectrum was obtained within the tunneling method using density matrix techniques of quantum mechanics. 
By equating (25) and (24), we obtain the following formula for the temperature $T$

$$
T=\frac{E \hbar}{\operatorname{Im}\left(\oint p_{x} d x\right)-E \operatorname{Im}(\Delta t)} .
$$

\section{Unruh radiation via $\mathrm{WKB} /$ tunneling}

We now apply the above method to the alternative Rindler metric previously introduced. For the $1+1$ Rindler space-times, the Hamilton-Jacobi equations (H-J) reduce to

$$
g^{t t} \partial_{t} S \partial_{t} S+g^{x x} \partial_{x} S \partial_{x} S+m^{2}=0
$$

For the Schwarzschild-like form of Rindler given in (6) the H-J equations are

$$
-\frac{1}{\left(1+2 a x_{R^{\prime}}\right)}\left(\partial_{t} S\right)^{2}+\left(1+2 a x_{R^{\prime}}\right)\left(\partial_{x} S\right)^{2}+m^{2}=0
$$

Now splitting up the action $S$ as $S(t, \vec{x})=E t+S_{0}(\vec{x})$ in (27) gives

$$
-\frac{E}{\left(1+2 a x_{R^{\prime}}\right)^{2}}+\left(\partial_{x} S_{0}\left(x_{R^{\prime}}\right)\right)^{2}+\frac{m^{2}}{1+2 a x_{R^{\prime}}}=0
$$

From (28), $S_{0}$ is found to be

$$
S_{0}^{ \pm}= \pm \int_{-\infty}^{\infty} \frac{\sqrt{E^{2}-m^{2}\left(1+2 a x_{R^{\prime}}\right)}}{1+2 a x_{R^{\prime}}} d x_{R^{\prime}}
$$

In (29), the + sign corresponds to the ingoing particles (i.e., particles that move from right to left) and the - sign to the outgoing particles (i.e., particles that move left to right). Note also that (29) is of the form $S_{0}=\int p_{x} d x$, where $p_{x}$ is the canonical momentum of the field in the Rindler background. The Minkowski space-time expression for the momentum is easily recovered by setting $a=0$, in which case one sees that $p_{x}=\sqrt{E^{2}-m^{2}}$.

From (29), one can see that this integral has a pole along the path of integration at $x_{R^{\prime}}=-\frac{1}{2 a}$. Using a contour integration gives an imaginary contribution to the action. We will give explicit details of the contour integration since this will be important when we try to apply this method to the standard form of the Rindler metric (4) (see Appendix I for the details of this calculation). We go around the pole at $x_{R^{\prime}}=-\frac{1}{2 a}$ using a semi-circular contour which we parameterize as $x_{R^{\prime}}=-\frac{1}{2 a}+\epsilon e^{i \theta}$, where $\epsilon \ll 1$ and $\theta$ goes from 0 to $\pi$ for the ingoing path and $\pi$ to 0 for the outgoing path. These contours are illustrated in the figure below. With this parameterization of the path, and taking the limit $\epsilon \rightarrow 0$, we find that the imaginary part of (29) for ingoing $(+)$ particles is 


$$
S_{0}^{+}=\int_{0}^{\pi} \frac{\sqrt{E^{2}-m^{2} \epsilon e^{i \theta}}}{2 a \epsilon e^{i \theta}} i \epsilon e^{i \theta} d \theta=\frac{i \pi E}{2 a},
$$

and for outgoing (-) particles, we get

$$
S_{0}^{-}=-\int_{\pi}^{0} \frac{\sqrt{E^{2}-m^{2} \epsilon e^{i \theta}}}{2 a \epsilon e^{i \theta}} i \epsilon e^{i \theta} d \theta=\frac{i \pi E}{2 a} .
$$

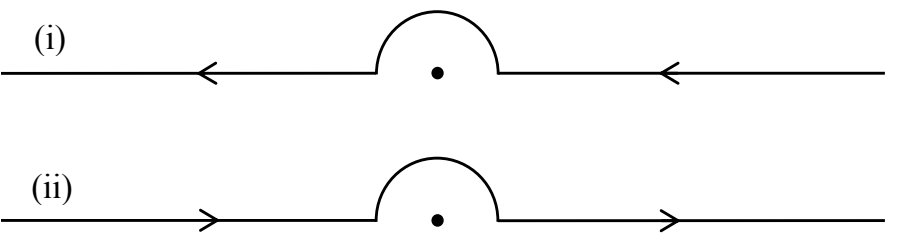

Figure 2. Contours of integration for (i) the ingoing and (ii) the outgoing particles.

In order to recover the Unruh temperature, we need to take into account the contribution from the time piece of the total action $S(t, \vec{x})=E t+S_{0}(\vec{x})$, as indicated by the formula of the temperature, (26), found in the previous section. The transformation of (7) into (8) indicates that the time coordinate has a discrete imaginary jump as one crosses the horizon at $x_{R^{\prime}}=-\frac{1}{2 a}$, since the two time coordinate transformations are connected across the horizon by the change $t_{R^{\prime}} \rightarrow t_{R^{\prime}}-\frac{i \pi}{2 a}$, that is,

$$
\sinh \left(a t_{R^{\prime}}\right) \rightarrow \sinh \left(a t_{R^{\prime}}-\frac{i \pi}{2}\right)=-i \cosh \left(a t_{R^{\prime}}\right)
$$

Note that as the horizon is crossed, a factor of $i$ comes from the term in front of the hyperbolic function in (7), i.e.,

$$
\sqrt{1+2 a x_{R^{\prime}}} \rightarrow i \sqrt{\left|1+2 a x_{R^{\prime}}\right|}
$$

so that (8) is recovered.

Therefore every time the horizon is crossed, the total action $S(t, \vec{x})=S_{0}(\vec{x})+E t$ picks up a factor of $E \Delta t=-\frac{i \pi E}{2 a}$. For the temporal contribution, the direction in which the horizon is crossed does not affect the sign. This is different from the situation for the spatial contribution. When the horizon is crossed once, the total action $S(t, \vec{x})$ gets a contribution of $E \Delta t=-\frac{i E \pi}{2 a}$, and for a round trip, as implied by the spatial part $\oint p_{x} d x$, the total contribution is $E \Delta t_{\text {total }}=-\frac{i E \pi}{a}$. So using the equation for the temperature (26) developed in the previous section, we obtain 


$$
T_{\text {Unruh }}=\frac{E \hbar}{\frac{\pi E}{a}+\frac{\pi E}{a}}=\frac{\hbar a}{2 \pi},
$$

which is the Unruh temperature. The interesting feature of this result is that the gravitational WKB problem has contributions from both spatial and temporal parts of the wave function, whereas the ordinary quantum mechanical WKB problem has only a spatial contribution. This is natural since time in quantum mechanics is treated as a distinct parameter, separate in character from the spatial coordinates. However, in relativity time is on equal footing with the spatial coordinates.

\section{Conclusions and summary}

We have given a derivation of Unruh radiation in terms of the original heuristic explanation as tunneling of virtual particles tunneling through the horizon [2]. This tunneling method can easily be applied to different space-times and to different types of virtual particles. We chose the Rindler metric and Unruh radiation since, because of the local equivalence of acceleration and gravitational fields, it represents the prototype of all similar effects (e.g. Hawking radiation, Hawking-Gibbons radiation).

Since this derivation touches on many different areas - classical mechanics (through the $\mathrm{H}-\mathrm{J}$ equations), relativity (via the Rindler metric), relativistic field theory (through the Klein-Gordon equation in curved backgrounds), quantum mechanics (via the WKB method for gravitational fields), thermodynamics (via the Boltzmann distribution to extract the temperature), and mathematical methods (via the contour integration to obtain the imaginary part of the action) - this single problem serves as a reminder of the connections between the different areas of physics.

This derivation also highlights several subtle points regarding the Rindler metric and the WKB tunneling method. In terms of the Rindler metric, we found that the different forms of the metric (4) and (6) do not cover the same parts of the full spacetime diagram. Also, as one crosses the horizon, there is an imaginary jump of the Rindler time coordinate as given by comparing (7) and (8).

In addition, for the gravitational WKB problem, $\Gamma$ has contributions from both the spatial and temporal parts of the action. Both these features are not found in the ordinary quantum mechanical WKB problem.

As a final comment, note that one can define an absorption probability (i.e., $P_{a b s} \propto\left|\phi_{\text {in }}\right|^{2}$ ) and an emission probability (i.e., $P_{\text {emit }} \propto\left|\phi_{\text {out }}\right|^{2}$ ). These probabilities can also be used to obtain the temperature of the radiation via the "detailed balance method" [8]

$$
\frac{P_{\text {emit }}}{P_{\text {abs }}}=e^{-E / T} .
$$

Using the expression of the field $\phi=\phi_{0} e^{\frac{i}{\hbar} S(t, \vec{x})}$, the Schwarzschild-like form of the Rindler metric given in (6), and taking into account the spatial and temporal contributions gives an 
an absorption probability of

$$
P_{a b s} \propto e^{\frac{\pi E}{a}-\frac{\pi E}{a}}=1
$$

and an emission probability of

$$
P_{\text {emit }} \propto e^{-\frac{\pi E}{a}-\frac{\pi E}{a}}=e^{-\frac{2 \pi E}{a}} .
$$

The first term in the exponents of the above probabilities corresponds to the spatial contribution of the action $S$, while the second term is the time piece. When using this method, we are not dealing with a directed line integral as in (18), so the spatial parts of the absorption and emission probability have opposite signs. In addition, the absorption probability is 1 , which physically makes sense - particles should be able to fall into the horizon with unit probability. If the time part were not included in $P_{a b s}$, then for some given $E$ and $a$ one would have $P_{a b s} \propto e^{\frac{\pi E}{a}}>1$, i.e., the probability of absorption would exceed 1 for some energy. Thus for the detailed balance method the temporal piece is crucial to ensure that one has a physically reasonable absorption probability.

\section{Appendix I: Unruh radiation from the standard Rindler metric}

For the standard form of the Rindler metric given by (4), the Hamilton-Jacobi equations become

$$
-\frac{1}{\left(1+a x_{R}\right)^{2}}\left(\partial_{t} S\right)^{2}+\left(\partial_{x} S\right)^{2}+m^{2}=0 .
$$

After splitting up the action as $S(t, \vec{x})=E t+S_{0}(\vec{x})$, we get

$$
-\frac{E}{\left(1+a x_{R}\right)^{2}}+\left(\partial_{x} S_{0}\left(x_{R}\right)\right)^{2}+m^{2}=0
$$

The above yields the following solution for $S_{0}$

$$
S_{0}^{ \pm}= \pm \int_{-\infty}^{\infty} \frac{\sqrt{E^{2}-m^{2}\left(1+a x_{R}\right)^{2}}}{1+a x_{R}} d x_{R}
$$

where the $+(-)$ sign corresponds to the ingoing (outgoing) particles.

Looking at (35), we see that the pole is now at $x_{R}=-1 / a$ and a naive application of contour integration appears to give the results $\pm \frac{i \pi E}{a}$. However, this cannot be justified since the two forms of the Rindler metric - (4) and (6) - are related by the simple coordinate transformation (5), and one should not change the value of an integral by a change of variables. The resolution to this puzzle is that one needs to transform not only the integrand but the path of integration, so applying the transformation (5) to the semi-circular contour $x_{R^{\prime}}=-\frac{1}{2 a}+\epsilon e^{i \theta}$ gives $x_{R}=-\frac{1}{a}+\frac{\sqrt{\epsilon}}{a} e^{i \theta / 2}$. Because $e^{i \theta}$ is replaced by $e^{i \theta / 2}$ due to the square root in the transformation (5), the semi-circular contour of (30) is replaced by a quarter-circle, which then leads to a contour integral of $i \frac{\pi}{2} \times$ Residue instead of $i \pi \times$ Residue. Thus both forms of Rindler yield the same spatial contribution to the total imaginary part of the action. 


\section{Acknowledgements}

This work supported in part by a DAAD research grant.

\section{Author details}

Douglas A. Singleton

California State University, Fresno, Department of Physics, Fresno, CA, USA

Institut für Mathematik, Universität Potsdam, Potsdam, Germany

\section{References}

[1] L. Brillouin, "La mécanique ondulatoire de Schrödinger: une méthode générale de resolution par approximations successives", Comptes Rendus de l'Academie des Sciences 183, 24 Ú26 (1926) H. A. Kramers, "Wellenmechanik und halbzählige Quantisierung", Zeit. f. Phys. 39, $828 U$ U840 (1926); G. Wentzel, "Eine Verallgemeinerung der Quantenbedingungen für die Zwecke der Wellenmechanik", Zeit. f. Phys. 38, 518 Ü529 (1926)

[2] S.W. Hawking, "Particle creation by black holes," Comm. Math. Phys. 43, 199-220 (1975).

[3] G.W. Gibbons and S.W Hawking, "Cosmological event horizons, thermodynamics, and particle creation," Phys. Rev. D 15, 2738-2751 (1977).

[4] W.G. Unruh, “Notes on black hole evaporation," Phys. Rev. D 14, 870-892 (1976).

[5] R. Banerjee and B.R. Majhi, "Hawking black body spectrum from tunneling mechanism," Phys. Lett. B 675, 243-245 (2009)

[6] T. Zhu, , Ji-Rong Ren, and D, Singleton, "Hawking-like radiation as tunneling from the apparent horizon in a FRW Universe", Int. J. Mod. Phys. D 19 159-169 (2010)

[7] P. Kraus and F. Wilczek, "Effect of self-interaction on charged black hole radiance," Nucl. Phys. B 437 231-242 (1995); E. Keski-Vakkuri and P. Kraus, "Microcanonical D-branes and back reaction," Nucl. Phys. B 491 249-262 (1997).

[8] K. Srinivasan and T. Padmanabhan, "Particle production and complex path analysis," Phys. Rev. D 60024007 (1999); S. Shankaranarayanan, T. Padmanabhan and K. Srinivasan, "Hawking radiation in different coordinate settings: complex paths approach," Class. Quant. Grav. 19, 2671-2688 (2002).

[9] M.K. Parikh and F. Wilczek, "Hawking radiation as tunneling," Phys. Rev. Lett. 85, 5042-5045 (2000).

[10] M.K. Parikh, “A secret tunnel through the horizon," Int. J. Mod. Phys. D 13, 2351-2354 (2004).

[11] Y. Sekiwa, "Decay of the cosmological constant by Hawking radiation as quantum tunneling", arXiv:0802.3266 
[12] G.E. Volovik, “On de Sitter radiation via quantum tunneling," arXiv:0803.3367 [gr-qc]

[13] A.J.M. Medved, “A Brief Editorial on de Sitter Radiation via Tunneling," arXiv:0802.3796 [hep-th]

[14] V. Akhmedova, T. Pilling, A. de Gill and D. Singleton, "Temporal contribution to gravitational WKB-like calculations," Phys. Lett. B 666 269-271 (2008). arXiv:0804.2289 [hep-th]

[15] Qing-Quan Jiang, Shuang-Qing $\mathrm{Wu}$, and $\mathrm{Xu}$ Cai, "Hawking radiation as tunneling from the Kerr and Kerr-Newman black holes," Phys. Rev. D 73, 064003 (2006) [hep-th/0512351]

[16] Jingyi Zhang, and Zheng Zhao, "Charged particles tunneling from the Kerr-Newman black hole," Phys. Lett. B 638, 110-113 (2006) [gr-qc/0512153]

[17] V. Akhmedova, T. Pilling, A. de Gill, and D. Singleton, "Comments on anomaly versus WKB/tunneling methods for calculating Unruh radiation," Phys. Lett. B 673, 227-231 (2009). arXiv:0808.3413 [hep-th]

[18] R. Kerner and R. B. Mann, "Fermions tunnelling from black holes," Class. Quant. Grav. 25, 095014 (2008). arXiv:0710.0612

[19] A.J.M. Medved and E. C. Vagenas, "On Hawking radiation as tunneling with back-reaction" Mod. Phys. Lett. A 20, 2449-2454 (2005) [gr-qc/0504113]; A.J.M. Medved and E. C. Vagenas, "On Hawking radiation as tunneling with logarithmic corrections", Mod. Phys. Lett. A 20, 1723-1728 (2005) [gr-qc/0505015]

[20] J.D. Jackson "On understanding spin-flip synchrotron radiation and the transverse polarization of electrons in storage rings" Rev. Mod. Phys. 48, 417-433 (1976)

[21] J.S. Bell and J.M. Leinaas, "Electrons As Accelerated Thermometers" Nucl. Phys. B 212, 131-150 (1983); J.S. Bell and J.M. Leinaas, "The Unruh Effect And Quantum Fluctuations Of Electrons In Storage Rings" Nucl. Phys. B 284, 488-508 (1987)

[22] E. T. Akhmedov and D. Singleton, “On the relation between Unruh and Sokolov-Ternov effects", Int. J. Mod. Phys. A 22, 4797-4823 (2007). hep-ph/0610391; E. T. Akhmedov and D. Singleton, "On the physical meaning of the Unruh effect", JETP Phys. Letts. 86, 615-619 (2007). arXiv:0705.2525 [hep-th]

[23] A. Retzker, J.I. Cirac, M.B. Plenio, and B. Reznik, Phys. Rev. Lett. 101, 110402 (2008).

[24] C. W. Misner, K. S. Thorne and J. A. Wheeler, Gravitation, W. H. Freeman and Company, San Francisco, 1973

[25] P.M. Alsing and P.W. Milonni, "Simplified derivation of the Hawking-Unruh temperature for an accelerated observer in vacuum," Am. J. Phys. 72, 1524-1529 (2004). quant-ph/0401170

[26] E. F. Taylor and J. A. Wheeler, Spacetime Physics, Freeman, San Francisco, 1966. 
[27] N. D. Birrell and P. C. W. Davies, Quantum Fields in Curved Space, Cambridge University Press, New York, 1982.

[28] M. Visser, Lorentzian Wormholes: from Einstein to Hawking, AIP Press, New York, 1995.

[29] L. D. Landau and E. M. Lifshitz, Quantum Mechanics: Non-Relativistic Theory, (Vol. 3 of Course of Theoretical Physics), 3rd ed., Pergamon Press, New York, 1977.

[30] D. J. Griffiths, Introduction to Quantum Mechanics, 2nd ed., Pearson Prentice Hall, Upper Saddle River, NJ, 2005.

[31] J. Schwinger, “On Gauge Invariance and Vacuum Polarization", Phys. Rev. 82, 664-679 (1951).

[32] B.R. Holstein, "Strong field pair production", Am. J. Phys. 67, 499-507 (1999).

[33] B. D. Chowdhury, "Problems with tunneling of thin shells from black holes," Pramana 70, 593-612 (2008). hep-th/0605197

[34] E.T. Akhmedov, V. Akhmedova, and D. Singleton, "Hawking temperature in the tunneling picture," Phys. Lett. B 642, 124-128 (2006). hep-th/0608098

[35] E.T. Akhmedov, V. Akhmedova, T. Pilling, and D. Singleton, "Thermal radiation of various gravitational backgrounds," Int. J. Mod. Phys. A 22, 1705-1715 (2007). hep-th/0605137

[36] E.T. Akhmedov, T. Pilling, D. Singleton, "Subtleties in the quasi-classical calculation of Hawking radiation," Int. J. Mod. Phys. D, 17 2453-2458 (2008). GRF "Honorable mention" essay, arXiv:0805.2653 [gr-qc] 


\section{Section 5}

Foundations of Quantum Mechanics 

Chapter 15

\title{
A Basis for Statistical Theory and Quantum Theory
}

\author{
Inge S. Helland
}

Additional information is available at the end of the chapter

http://dx.doi.org/10.5772/53702

\section{Introduction}

Compaired to other physical theories, the foundation of quantum mechanics is very formal and abstract. The pure state of a system is defined as a complex vector (or ray) in some abstract vector space, the observables as Hermitian operators on this space. Even a modern textbook like Ballentine [1] starts by introducing two abstract postulates:

1. To each dynamical variable there corresponds a linear operator, and the possible values of the dynamical variable are the eigenvalues of the operator.

2. To each state there corresponds a unique operator $\rho$. The average value of a dynamical variable $\mathbf{R}$, represented by the operator $R$, in the state given by the operator $\rho$ is given by

$$
\langle\mathbf{R}\rangle=\frac{\operatorname{Tr}(\rho R)}{\operatorname{Tr}(\rho)} .
$$

Here $\mathrm{Tr}$ is the trace operator. The discussion in [1] goes on by arguing that $R$ must be Hermitian (have real eigenvalues) and that $\rho$ ought to be positive with trace 1 . An important special case is when $\rho$ is one-dimensional: $\rho=|\psi\rangle\langle\psi|$ for a vector $|\psi\rangle$. Then the state is pure, and is equivalently specified by the vector $|\psi\rangle$. In general the formula (1) is a consequence of Born's formula: The probability of observing a pure state $|\phi\rangle$ when the system is prepared in a pure state $|\psi\rangle$ is given by $|\langle\phi \mid \psi\rangle|^{2}$.

From these two postulates a very rich theory is deduced, a theory which has proved to be in agreement with observations in each case where it has been tested. Still, the abstract nature of the basic postulates leaves one a little uneasy: Is it possible to find another basis which is more directly connected to what one observes in nature? The purpose of this chapter is to show that to a large extent one can give a positive answer to this question. 
Another problem is that there are many interpretations of quantum mechanics. In this chapter I will choose an epistemic interpretation: Quantum mechanics is related to the knowledge we get about nature, not directly to how nature 'is'. The latter aspect - the ontological aspect of nature - is something we can talk about when all observers agree on the same information. Any knowledge about nature is found through an epistemic process - an experiment or an observational study. Typically we ask a question: What is $\theta$ ? And after the epistemic process is completed, nature gives an answer, in the simplest case: $\theta=u_{k}$, where $u_{k}$ is one of several possible values. Here $\theta$ is what we will call an epistemic conceptual variable or e-variable, a variable defined by an observer or by a group of observers and defining the epistemic process.

In all empirical sciences, epistemic questions like this are posed to nature. It is well known that the answers are not always that simple. Typically we end up with a confidence interval (a frequentist concept) or a credibility interval (a Bayesian concept) for $\theta$. This leads us into statistical science. In statistics, $\theta$ is most often called a parameter, and is often connected to a population of experimental units. But there are instances also in statistics where we want to predict a value for a single unit. The corresponding intervals are then called prediction intervals. In this chapter we will also use $\theta$ for an unknown variable for a single unit, which is a situation very often met in physics. This is the generalization we think about when we in general call $\theta$ an e-variable, not a parameter. Also, the notion of a parameter may have a different meaning in physics, so by this we will avoid confusion.

A more detailed discussion than what can be covered here, can be found in Helland [2].

\section{A basis for statistics}

Every experiment or observational study is made in a context. Part of the context may be physical, another part may be historical, including earlier experiments. Also, the status of the observer(s) may be seen as a part of the context, and another part of the context may be conceptual, including a goal for the study. In all our discussion, we assume that we have conditioned upon the context $\tau$. We can imagine the context formulated as a set of propositions. But propositional calculus corresponds to set theory, as both are Boolean algebras. Therefore we can here in principle use the familiar concept of conditioning as developed in Kolmogorov's theory of probability, where it is defined as a Radon-Nikodym derivative. Readers unfamiliar to this mathematics may think of a more intuitive conditioning concept.

In addition, for every experiment, we have an e-variable of interest $\theta$ and we have data $z$. A basis for all statistical theory is the statistical model, the distribution of $z$ as a function of $\theta$. Conceptual variables which are not of interest, may be taken as part of the context $\tau$. The density of the statistical model, seen as a function of $\theta$, is called the likelihood. We will assume throughout:

1) The distribution of $z$, given $\tau$, depends on an unknown e-variable $\theta$.

2) If $\tau$ or part of $\tau$ has a distribution, this is independent of $\theta$. The part of $\tau$ which does not have a distribution is functionally independent of $\theta$.

A function of the data is called a statistic $t(z)$. Often it is of interest to reduce the data to a sufficient statistic, a concept due to R. A. Fisher. 


\section{Definition 1.}

We say that $t=t(z)$ is a $\tau$-sufficient for $\theta$ if the conditional distribution of $z$, given $t, \tau$ and $\theta$ is independent of $\theta$.

The intuitive notion here is that if the distribution of $z$, given $t$ is independent of $\theta$, the distribution of the whole data set might as well be generated by the distribution of $t$, given $\theta$ together with some random mechanism which is totally independent of the conceptual variable of interest. This is the basis for

The sufficiency principle.

Consider an experiment in a context $\tau$, let $z$ be the data of this experiment, and let $\theta$ be the e-variable of interest. Let $t=t(z)$ be a $\tau$-sufficient statistic for $\theta$. Then, if $t\left(z_{1}\right)=t\left(z_{2}\right)$, the data $z_{1}$ and $z_{2}$ contain the same experimental evidence about $\theta$ in the context $\tau$.

Here 'experimental evidence' is left undefined. The principle is regarded as intuitively obvious by most statisticians.

Another principle which is concidered intuitively obvious by most statisticians, is

The conditionality principle 1.

Suppose that there are two experiments $E_{1}$ and $E_{2}$ with common conceptual variable of interest $\theta$ and with equivalent contexts $\tau$. Consider a mixed experiment $E^{*}$, whereby $u=1$ or $u=2$ is observed, each having probability $1 / 2$ (independent of $\theta$, the data of the experiments and the contexts), and the experiment $E_{u}$ is then performed. Then the evidence about $\theta$ from $E^{*}$ is just the evidence from the experiment actually performed.

Two contexts $\tau$ and $\tau^{\prime}$ are defined to be equivalent if there is a one-to-one correspondence between them: $\tau^{\prime}=f(\tau)$; $\tau=f^{-1}\left(\tau^{\prime}\right)$. The principle can be motivated by simple examples. From these examples one can also deduce

The conditionality principle 2.

In the situation of conditionality principle 1 one should in any statistical analysis condition upon the outcome of the coin toss.

It caused much discussion among statisticians when Birnbaum [3] proved that the sufficiency principle and the conditionality principle 1 together imply

The likelihood principle.

Consider two experiments with equivalent contexts $\tau$, and assume that $\theta$ is the same full e-variable in both experiments. Suppose that the two observations $z_{1}^{*}$ and $z_{2}^{*}$ have proportional likelihoods in the two experiments. Then these two observations produce the same evidence on $\theta$ in this context.

It is crucial for the present chapter that these principles may be generalized from experiments to any epistemic processes involving data such that 1) and 2) are satisfied.

An important special case of the likelihood principle is when $E_{1}$ and $E_{2}$ are the same experiment and $z_{1}^{*}$ and $z_{2}^{*}$ have equal likelihoods. Then the likelihood principle says that any experimental evidence on $\theta$ must only depend on the likelihood (given the context). Without taking the context into account this is really controversial. It seems like common statistical methods like confidence intervals and test of hypotheses are excluded. But this is 
saved when we can take confidence levels, alternative hypotheses, test levels etc. as part of the context.

A discussion of these common statistical methods will not be included here; the reader is referred to [2] for this. Also, a discussion of the important topic of model reduction in statistics will be omitted here. Sometimes a statistical model contains more structure than what has been assumed here; for instance group actions may be defined on the space of e-variables. Then any model reduction should be to an orbit or to a set of orbits for the group; for examples, see [2].

\section{Inaccessible conceptual variables and quantum theory}

An e-variable as it is used here is related to the question posed in an epistemic process: What is the value of $\theta$ ? Sometimes we can obtain an accurate answer to such a question, sometimes not. We call $\theta$ accessible if we in principle can devise an experiment such that $\theta$ can be assessed with arbitrary accuracy. If this in principle is impossible, we say that $\theta$ is inaccessible.

Consider a single medical patient which at time $t=0$ can be given one out of two mutually exclusive treatments $\mathrm{A}$ or $\mathrm{B}$. The time $\theta^{A}$ until recovery given treatment $\mathrm{A}$ can be measured accurately by giving this treatment and waiting a sufficiently long time, likewise the time $\theta^{B}$ until recovery given treatment $\mathrm{B}$. But consider the vector $\phi=\left(\theta^{A}, \theta^{B}\right)$. This vector can not be assessed with arbitrary accuracy by any person neither before, during nor after treatment. The vector $\phi$ is inaccessible. A similar phenomenon occurs in all counterfactual situations.

Many more situations with inaccessible conceptual variables can be devised. Consider a fragile apparatus which is destroyed after a single measurement of some quantity $\theta_{1}$, and let $\theta_{2}$ be another quantity which can only be measured by dismantling the apparatus. Then $\phi=\left(\theta_{1}, \theta_{2}\right)$ is inaccessible. Or consider two sensitive questions to be posed to a single person at some moment of time, where we expect that the order in which the questions are posed may be relevant for the answers. Let $\left(\theta_{1}, \theta_{2}\right)$ be the answers when the questions are posed in one order, and let $\left(\theta_{3}, \theta_{4}\right)$ be the answers when the questions are posed in the opposite order. Then the vector $\phi=\left(\theta_{1}, \theta_{2}, \theta_{3}, \theta_{4}\right)$ is inaccessible.

I will approach quantum mechanics by looking upon it as an epistemic science and pointing out the different inaccessible conceptual variables. First, by Heisenberg's uncertainty principle, the vector $(\xi, \pi)$ is inaccessible, where $\xi$ is the theoretical position and $\pi$ is the theoretical momentum of a particle. This implies that $\left(\xi\left(t_{1}\right), \xi\left(t_{2}\right)\right)$, the positions at two different times, is an inaccessible vector. Hence the trajectory of the particle is inaccessible. In the two-slit experiment $(\alpha, \theta)$ is inaccessible, where $\alpha$ denotes the slit that the particle goes through, and $\theta$ is the phase of the particle's wave as it hits the screen.

In this chapter I will pay particular attention to a particle's spin/ angular momentum. The spin or angular momentum vector is inaccessible, but its component $\lambda^{a}$ in any chosen direction $a$ will be accessible.

It will be crucial for my discussion that even though a vector is inaccessible, it can be seen upon as an abstract quantity taking values in some space and one can often act on it by group actions. Thus in the medical example which started this section, a change of time units will affect the whole vector $\phi$, and a spin vector can be acted upon by rotations. 


\section{The maximal symmetrical epistemic setting}

A general setting will be descibed, and then I will show that spin and angular momentum are special cases of this setting. This is called the maximal symmetrical epistemic setting.

Consider an inaccessible conceptual variable $\phi$, and let there be accessible e-variables $\lambda^{a}(\phi)$ $(a \in \mathcal{A})$ indexed by some set $\mathcal{A}$. Thus for each $a$, one can ask the question: What is the value of $\lambda^{a}$ ? and get some information from experiment. To begin with, assume that these are maximally accessible, more precisely maximal in the ordering where $\alpha<\beta$ when $\alpha=f(\beta)$ for some $f$. This can be assumed by Zorn's lemma, but it will later be relaxed. For $a \neq b$ let there be an invertible transformation $g_{a b}$ such that $\lambda^{b}(\phi)=\lambda^{a}\left(g_{a b}(\phi)\right)$.

In general, let a group $H$ act on a conceptual variable $\phi$. A function $\eta(\phi)$ is said to be permissible with respect to $H$ if $\eta\left(\phi_{1}\right)=\eta\left(\phi_{2}\right)$ implies $\eta\left(h \phi_{1}\right)=\eta\left(h \phi_{2}\right)$ for all $h \in H$. Then one can define a corresponding group $\tilde{H}$ acting upon $\eta$. For a given function $\eta(\phi)$ there is a maximal group with respect to which it is permissible.

Now fix $0 \in \mathcal{A}$ and let $G^{0}$ be the maximal group under which $\lambda^{0}(\phi)$ is permissible. Take $G^{a}=g_{a 0} G^{0} g_{0 a}$, and let $G$ be the smallest group containing $G^{0}$ and all the transformations $g_{a 0}$. It is then easy to see that $G^{a}$ is the maximal group under which $\lambda^{a}(\phi)$ is permissible, and that $G$ is the group generated by $G^{a} ; a \in \mathcal{A}$ and the transformations $g_{a b}$. Make the following assumptions about $G$ :

a) It is a locally compact topological group satisfying weak conditions such that an invariant measure $\rho$ exists on the space $\Phi$ of $\phi^{\prime}$ s.

b) $\lambda^{a}(\phi)$ varies over an orbit or a set of orbits of the smaller group $G^{a}$. More precisely: $\lambda^{a}$ varies over an orbit or a set of orbits of the corresponding group $\tilde{G}^{a}$ on its range.

c) $G$ is generated by the product of elements of $G^{a}, G^{b}, \ldots ; a, b, \ldots \in \mathcal{A}$.

As an important example, let $\phi$ be the spin vector or the angular momentum vector for a particle or a system of particles. Let $G$ be the group of rotations of the vector $\phi$, that is, the group which fixes the norm $\|\phi\|$. Next, choose a direction $a$ i space, and focus upon the spin component in this direction: $\zeta^{a}=\|\phi\| \cos (\phi, a)$. The largest subgroup $G^{a}$ with respect to which $\zeta^{a}(\phi)$ is permissible, is given by rotations around $a$ together with a reflection in a plane pependicular to $a$. However, the action of the corresponding group $\tilde{G}^{a}$ on $\zeta^{a}$ is just a reflection together with the identity.

Finally introduce model reduction. As mentioned at the end of the previous section, such a model reduction for $\zeta^{a}$ should be to an orbit or to a set of orbits for the group $\tilde{G}^{a}$ as acting on $\zeta^{a}$. These orbits are given as two-point sets $\pm c$ together with the single point 0 . To conform to the ordinary theory of spin/angular momentum, I will choose the set of orbits indexed by an integer or half-integer $j$ and let the reduced set of orbits be $-j,-j+1, \ldots, j-1, j$. Letting $\lambda^{a}$ be the e-variable $\zeta^{a}$ reduced to this set of orbits of $\tilde{G}^{a}$, and assuming it to be a maximally accessible e-variable, we can prove the general assumptions of the maximal symmetrical epistemic setting (except for the case $j=0$, where we must redefine $G$ to be the trivial group). For instance, here is an indication of the proof leading to assumption c) above: given $a$ and $b$, a transformation $g_{a b}$ sending $\lambda_{a}(\phi)$ onto $\lambda_{b}(\phi)$ can be obtained by a reflection in a plane orthogonal to the two vectors $a$ and $b$, a plane containing the midline between $a$ and $b$.

The case with one orbit and $c=j=1 / 2$ corresponds to electrons and other spin $1 / 2$ particles. 
In general, assumption b) in the maximal symmetrical epistemic setting may be motivated in a similar manner: First, a conceptual variable $\zeta^{a}$ is introduced for each $a$ through a chosen focusing, then define $G^{a}$ as the maximal group under which $\zeta^{a}(\phi)$ is permissible, with $\tilde{G}^{a}$ being the corresponding group acting on $\zeta^{a}$. Finally define $\lambda^{a}$ as the reduction of $\zeta^{a}$ to a set of orbits of $\tilde{G}^{a}$. The content of assumption $b$ ) is that it is this $\lambda^{a}$ which is maximally accessible. This may be regarded as the quantum hypothesis.

\section{Hilbert space, pure states and operators}

Consider the maximal symmetrical epistemic setting. The crucial step towards the formalism of quantum mechanics is to define a Hilbert space, that is, a complete inner product space which can serve as a state space for the system.

By assumption a) there exists an invariant measure $\rho$ for the group's action: $\rho(g A)=\rho(A)$ for all $g \in G$ and all Borel-measurable subsets $A$ of the space $\Phi$ of inaccessible conceptual variables. If $G$ is transitive on $\Phi$, then $\rho$ is unique up to a multiplicative constant. For compact groups $\rho$ can be normalized, i.e., taken as a probability measure. For each $a$ define

$$
H^{a}=\left\{f \in L^{2}(\Phi, \rho): f(\phi)=r\left(\lambda^{a}(\phi)\right) \text { for some function } r .\right\}
$$

Thus $H^{a}$ is the set of $L^{2}$-functions that are functions of $\lambda^{a}(\phi)$. Since $H^{a}$ is a closed subspace of the Hilbert space $L^{2}(\Phi, \rho)$, it is itself a Hilbert space. To define our state space $H$, we now fix an arbitrary index $a=0 \in \mathcal{A}$, and take

$$
H=H^{0} .
$$

First look at the case where the accessible e-variables take a finite, discrete set of values. Let $\left\{u_{k}\right\}$ be the set of possible values of $\lambda^{a}$. Since $\lambda^{a}(\cdot)$ is maximal, $\left\{u_{k}\right\}$ can be taken to be independent of $a$, see [2]. Now go back to the definition of an epistemic process: We start by choosing $a$, that is, ask an epistemic question: What is the value of $\lambda^{a}$ ? After the process we get some information; I will here look upon the simple case where we get full knowledge: $\lambda^{a}=u_{k}$. I define this as a pure state of the system; it can be characterized by the indicator function $\mathbf{1}\left(\lambda^{a}(\phi)=u_{k}\right)$. This is a function in $H^{a}$, but I will show below that one can find an invertible operator $V^{a}$ such that

$$
f_{k}^{a}(\phi)=V^{a} \mathbf{1}\left(\lambda^{a}(\phi)=u_{k}\right)
$$

is a unique function in $H=H^{0}$. Since $H$ in this case is a $K$-dimensional vector space, where $K$ is the number of values $u_{k}$, we can regard $f_{k}^{a}$ as a $K$-dimensional vector. To conform to the ordinary quantum mechanical notation, I write this as a ket-vector $|a ; k\rangle=f_{k}^{a}$. It is easy to see that $\{|0 ; k\rangle ; k=1, \ldots, K\}$ is an orthonormal basis of $H$ when $\rho$ is normalized to be 1 for the whole space $\Phi$. I will show below that $\{|a ; k\rangle ; k=1, \ldots, K\}$ has the same property. My main point is that $|a ; k\rangle$ is characterized by and characterizes a question: What is $\lambda^{a}$ ? together with an answer: $\lambda^{a}=u_{k}$. This is a pure state for the maximal symmetrical epistemic setting. 
I will also introduce operators by

$$
A^{a}=\sum_{k=1}^{K} u_{k}|a ; k\rangle\langle a ; k|,
$$

where $\langle a ; k|$ is the bra vector corresponding to $|a ; k\rangle$. This is by definition the observator corresponding to the e-variable $\lambda^{a}$. Since $\lambda^{a}$ is maximal, $A^{a}$ will have non-degenerate eigenvalues $u_{k}$. Knowing $A^{a}$, we will have information of all possible values of $\lambda^{a}$ together with information about all possible states connected to this variable.

The rest of this section will be devoted to proving (2) and showing the properties of the state vectors $|a ; k\rangle$. To allow for future generalizations I now allow the accessible e-variables $\lambda^{a}$ to take any set of values, continuous or discrete. The discussion will by necessity be a bit technical. First I define the (left) regular representation $U$ for a group $G$. For given $f \in L^{2}(\Phi, \rho)$ and given $g \in G$ we define a new function $U(g) f$ by

$$
U(g) f(\phi)=f\left(g^{-1} \phi\right)
$$

Without proof I mention 5 properties of the set of operators $U(g)$ :

- $U(g)$ is linear: $U(g)\left(a_{1} f_{1}+a_{2} f_{2}\right)=a_{1} U(g) f_{1}+a_{2} U(g) f_{2}$.

- $U(g)$ is unitary: $\left\langle U(g) f_{1}, f_{2}\right\rangle=\left\langle f_{1}, U(g)^{-1} f_{2}\right\rangle$ in $L^{2}(\Phi, \rho)$.

- $U(g)$ is bounded: $\sup _{f:\|f\|=1}\|U(g) f\|=1<\infty$.

- $U(\cdot)$ is continuous: If $\lim g_{n}=g_{0}$ in the group topology, then $\lim U\left(g_{n}\right)=U\left(g_{0}\right)$ (in the matrix norm in the finite-dimensional case, which is what I will focus on here, in general in the topology of bounded linear operators).

- $U(\cdot)$ is a homomorphism: $U\left(g_{1} g_{2}\right)=U\left(g_{1}\right) U\left(g_{2}\right)$ for all $g_{1}, g_{2}$ and $U(e)=I$ for the unit element.

The concept of homomorphism will be crucial in this section. In general, a homomorphism is a mapping $k \rightarrow k^{\prime}$ between groups $K$ and $K^{\prime}$ such that $k_{1} \rightarrow k_{1}^{\prime}$ and $k_{2} \rightarrow k_{2}^{\prime}$ implies $k_{1} k_{2} \rightarrow k_{1}^{\prime} k_{2}^{\prime}$ and such that $e \rightarrow e^{\prime}$ for the identities. Then also $k^{-1} \rightarrow\left(k^{\prime}\right)^{-1}$ when $k \rightarrow k^{\prime}$.

A representation of a group $K$ is a continuous homomorphism from $K$ into a group of invertible operators on some vector space. If the vector space is finite dimensional, the linear operators can be taken as matrices. There is a large and useful mathematical theory about operator (matrix) representations of groups; some of it is sketched in Appendix 3 of [2]. Equation (3) gives one such representation of the basic group $G$ on the vector space $L^{2}(\Phi, \rho)$.

Proposition 1.

Let $U_{a}=U\left(g_{0 a}\right)$ with $g_{a b}$ defined in the beginning of Section 4. Then

$$
H^{a}=U_{a}^{-1} H \text { through } r\left(\lambda^{a}(\phi)\right)=U_{a}^{-1} r\left(\lambda^{0}(\phi)\right) .
$$


Proof. If $f \in H^{a}$, then $f(\phi)=r\left(\lambda^{a}(\phi)\right)=r\left(\lambda^{0}\left(g_{0 a} \phi\right)\right)=U\left(g_{0 a}\right)^{-1} r\left(\lambda^{0}(\phi)\right)=U_{a}^{-1} f_{0}(\phi)$, where $f_{0} \in H=H^{0}$.

Since $a=0$ is a fixed but arbitrary index, this gives in principle a unitary connection between the different choices of $H$, different representations of the 'Hilbert space apparatus'. However this connection cannot be used directly in (2), since if $f_{k}^{a}=\mathbf{1}\left(\lambda^{a}=u_{k}\right)$ is the state function representing the question: What is $\lambda^{a}$ ? together with the answer $\lambda^{a}=u_{k}$, then we have

$$
U_{a} f_{k}^{a}=U\left(g_{0 a}\right) \mathbf{1}\left(\lambda^{0}\left(g_{0 a} \phi\right)=u_{k}\right)=U\left(g_{0 a}\right) U\left(g_{0 a}\right)^{-1} \mathbf{1}\left(\lambda^{0}(\phi)=u_{k}\right)=f_{k}^{0}
$$

Thus by this simple transformation the indicator functions in $H$ are not able to distinguish between the different questions asked.

Another reason why the simple solution is not satisfactory is that the regular representation $U$ will not typically be a representation of the whole group $G$ on the Hilbert space $H$. This can however be amended by the following theorem. Its proof and the resulting discussion below are where the Assumption c) of the maximal symmetrical epistemic setting is used. Recall that throughout, upper indices $\left(G^{a}, g^{a}\right)$ are for the subgroups of $G$ connected to the accessible variables $\lambda^{a}$, similarly $\left(\tilde{G}^{a}, \tilde{g}^{a}\right)$ for the group (elements) acting upon $\lambda^{a}$. Lower indices (e.g., $\left.U_{a}=U\left(g_{0 a}\right)\right)$ are related to the transformations between these variables.

\section{Theorem 1.}

(i) A representation (possibly multivalued) $V$ of the whole group $G$ on $H$ can always be found.

(ii) For $g^{a} \in G^{a}$ we have $V\left(g^{a}\right)=U_{a} U\left(g^{a}\right) U_{a}^{+}$.

Proof. (i) For each $a$ and for $g^{a} \in G^{a}$ define $V\left(g^{a}\right)=U\left(g_{0 a}\right) U\left(g^{a}\right) U\left(g_{a 0}\right)$. Then $V\left(g^{a}\right)$ is an operator on $H=H^{0}$, since it is equal to $U\left(g_{0 a} g^{a} g_{a 0}\right)$, and $g_{0 a} g^{a} g_{a 0} \in G^{0}$ by the construction of $G^{a}$ from $G^{0}$. For a product $g^{a} g^{b} g^{c}$ with $g^{a} \in G^{a}, g^{b} \in G^{b}$ and $g^{c} \in G^{c}$ we define $V\left(g^{a} g^{b} g^{c}\right)=$ $V\left(g^{a}\right) V\left(g^{b}\right) V\left(g^{c}\right)$, and similarly for all elements of $G$ that can be written as a finite product of elements from different subgroups.

Let now $g$ and $h$ be any two elements in $G$ such that $g$ can be written as a product of elements from $G^{a}, G^{b}$ and $G^{c}$, and similarly $h$ (the proof is similar for other cases.) It follows that $V(g h)=V(g) V(h)$ on these elements, since the last factor of $g$ and the first factor of $h$ either must belong to the same subgroup or to different subgroups; in both cases the product can be reduced by the definition of the previous paragraph. In this way we see that $V$ is a representation on the set of finite products, and since these generate $G$ by Assumption c), and since $U$, hence by definition $V$, is continuous, it is a representation of $G$.

Since different representations of $g$ as a product may give different solutions, we have to include the possibility that $V$ may be multivalued.

(ii) Directly from the proof of (i).

What is meant by a multivalued representation? As an example, consider the group SU(2) of unitary $2 \times 2$ matrices. Many books in group theory will state that there is a homomorphism from $S U(2)$ to the group $S O(3)$ of real 3-dimensional rotations, where the kernel of the homomorphism is $\pm I$. This latter statement means that both $+I$ and $-I$ are mapped into the identity rotation by the homomorphism. 
In this case there is no unique inverse $S O(3) \rightarrow S U(2)$, but nevertheless we may say informally that there is a multivalued homomorphism from $S O(3)$ to $S U(2)$. Here is a way to make this precise:

Extend $S U(2)$ to a new group with elements $(g, k)$, where $g \in S U(2)$ and $k$ is an element of the group $K=\{ \pm 1\}$ with the natural multiplication. The multiplication in this extended group is defined by $\left(g_{1}, k_{1}\right) \cdot\left(g_{2}, k_{2}\right)=\left(g_{1} g_{2}, k_{1} k_{2}\right)$, and the inverse by $(g, k)^{-1}=\left(g^{-1}, k^{-1}\right)$. Then there is an invertible homomorphism between this extended group and $S O(3)$.

A similar construction can be made with the representation $V$ of Theorem 1 .

\section{Theorem 2.}

(i) There is an extended group $G^{\prime}$ such that $V$ is a univariate representation of $G^{\prime}$ on $H$.

(ii) There is a unique mapping $G^{\prime} \rightarrow G$, denoted by $g^{\prime} \rightarrow g$, such that $V\left(g^{\prime}\right)=V(g)$. This mapping is a homomorphism.

Proof. (i) Assume as in Theorem 1 that we have a multivalued representation $V$ of $G$. Define a larger group $G^{\prime}$ as follows: If $g^{a} g^{b} g^{c}=g^{d} g^{e} g^{f}$, say, with $g^{k} \in G^{k}$ for all $k$, we define $g_{1}^{\prime}=g^{a} g^{b} g^{c}$ and $g_{2}^{\prime}=g^{d} g^{e} g^{f}$. A similar definition of new group elements is done if we have equality of a limit of such products. Let $G^{\prime}$ be the collection of all such new elements that can be written as a formal product of elements $g^{k} \in G^{k}$ or as limits of such symbols. The product is defined in the natural way, and the inverse by for example $\left(g^{a} g^{b} g^{c}\right)^{-1}=$ $\left(g^{c}\right)^{-1}\left(g^{b}\right)^{-1}\left(g^{a}\right)^{-1}$. By Assumption 2c), the group $G^{\prime}$ generated by this construction must be at least as large as $G$. It is clear from the proof of Theorem 1 that $V$ also is a representation of the larger group $G^{\prime}$ on $H$, now a one-valued representation.

(ii) Again, if $g^{a} g^{b} g^{c}=g^{d} g^{e} g f=g$, say, with $g^{k} \in G^{k}$ for all $k$, we define $g_{1}^{\prime}=g^{a} g^{b} g^{c}$ and $g_{2}^{\prime}=g^{d} g^{e} g^{f}$. There is a natural map $g_{1}^{\prime} \rightarrow g$ and $g_{2}^{\prime} \rightarrow g$, and the situation is similar for other products and limits of products. It is easily shown that this mapping is a homomorphism.

Note that while $G$ is a group of transformations on $\Phi$, the extended group $G^{\prime}$ must be considered as an abstract group.

Theorem 3.

(i) For $g^{\prime} \in G^{\prime}$ there is a unique $g^{0} \in G^{0}$ such that $V\left(g^{\prime}\right)=U\left(g^{0}\right)$. The mapping $g^{\prime} \rightarrow g^{0}$ is a homomorphism.

(ii) If $g^{\prime} \rightarrow g^{0}$ by the homomorphism of $(i)$, and $g^{\prime} \neq e^{\prime}$ in $G^{\prime}$, then $g^{0} \neq e$ in $G^{0}$.

Proof. (i) Consider the case where $g^{\prime}=g^{a} g^{b} g^{c}$ with $g^{k} \in G^{k}$. Then by the proof of Theorem 1:

$$
\begin{gathered}
V\left(g^{\prime}\right)=U_{a} U\left(g^{a}\right) U_{a}^{\dagger} U_{b} U\left(g^{b}\right) U_{b}^{\dagger} U_{c} U\left(g^{c}\right) U_{c}^{\dagger}=U\left(g_{0 a} g^{a} g_{a 0} g_{0 b} g^{b} g_{b 0} g_{0 c} g^{c} g_{c 0}\right) \\
=U\left(g^{0}\right)
\end{gathered}
$$

where $g^{0} \in G^{0}$. The group element $g^{0}$ is unique since the decomposition $g^{\prime}=g^{a} g^{b} g^{c}$ is unique for $g^{\prime} \in G^{\prime}$. The proof is similar for other decompositions and limits of these. By the construction, the mapping $g^{\prime} \rightarrow g^{0}$ is a homomorphism. 
(ii) Assume that $g^{0}=e$ and $g^{\prime} \neq e^{\prime}$. Since $U\left(g^{0}\right) \tilde{f}\left(\lambda^{0}(\phi)\right)=\tilde{f}\left(\lambda^{0}\left(\left(g^{0}\right)^{-1}(\phi)\right)\right)$, it follows from $g^{0}=e$ that $U\left(g^{0}\right)=I$ on $H$. But then from (i), $V\left(g^{\prime}\right)=I$, and since $V$ is a univariate representation, it follows that $g^{\prime}=e^{\prime}$, contrary to the assumption.

The theorems 1-3 are valid in any maximal symmetrical epistemic setting. I will now again specialize to the case where the accessible e-variables $\lambda$ have a finite discrete range. This is often done in elementary quantum theory texts, in fact also in recent quantum foundation papers, and in our situation it has several advantages:

- It is easy to interprete the principle that $\lambda$ can be estimated with any fixed accuracy.

- In particular, confidence regions and credibility regions for an accessible e-variable can be taken as single points if observations are accurate enough.

- The operators involved will be much simpler and are defined everywhere.

- The operators $A^{a}$ can be understood directly from the epistemic setting; see above.

So look at the statement $\lambda^{a}(\phi)=u_{k}$. This means two things: 1) One has sought information about the value of the maximally accessible e-variable $\lambda^{a}$, that is, asked the question: What is the value of $\lambda^{a}$ ? 2) One has obtained the answer $\lambda^{a}=u_{k}$. This information can be thought of as a perfect measurement, and it can be represented by the indicator function $\mathbf{1}\left(\lambda^{a}(\phi)=u_{k}\right)$, which is a function in $H^{a}$. From Proposition 1, this function can by a unitary transformation be represented in $H$, which now is a vector space with a discrete basis, a finite-dimensional vector space: $U_{a} f_{k}^{a}$. However, we have seen that this tentative state definition $U_{a} \mathbf{1}\left(\lambda^{a}(\phi)=\right.$ $\left.u_{k}\right)=U\left(g_{0 a}\right) \mathbf{1}\left(\lambda^{0}\left(g_{0 a} \phi\right)=u_{k}\right)$ led to ambiguities. These ambiguities can be removed by replacing the two $g_{0 a}$ 's here in effect by different elements $g_{0 a i}^{\prime}$ of the extended group $G^{\prime}$. Let $g_{0 a 1}^{\prime}$ and $g_{0 a 2}^{\prime}$ be two different such elements where both $g_{0 a 1}^{\prime} \rightarrow g_{0 a}$ and $g_{0 a 2}^{\prime} \rightarrow g_{0 a}$ according to Theorem 2 (ii). I will prove in a moment that this is in fact always possible when $g_{0 a} \neq e$. Let $g_{a}^{\prime}=\left(g_{0 a 1}^{\prime}\right)^{-1} g_{0 a 2}^{\prime}$, and define

$$
f_{k}^{a}(\phi)=V\left(g_{a}^{\prime}\right) U_{a} \mathbf{1}\left(\lambda^{a}(\phi)=u_{k}\right)=V\left(g_{a}^{\prime}\right) f_{k}^{0}(\phi)
$$

This gives the relation (2).

In order that the interpretation of $f_{k}^{a}$ as a state $|a ; k\rangle$ shall make sense, I need the following result. I assume that $\tilde{G}^{0}$ is non-trivial.

\section{Theorem 4.}

a) Assume that two vectors in $H$ satisfy $|a ; i\rangle=|b ; j\rangle$, where $|a ; i\rangle$ corresponds to $\lambda^{a}=u_{i}$ for one perfect measurement and $|b ; j\rangle$ corresponds to $\lambda^{b}=u_{j}$ for another perfect measurement. Then there is a one-to-one function $F$ such that $\lambda^{b}=F\left(\lambda^{a}\right)$ and $u_{j}=F\left(u_{i}\right)$. On the other hand, if $\lambda^{b}=F\left(\lambda^{a}\right)$ and $u_{j}=F\left(u_{i}\right)$ for such a function $F$, then $|a ; i\rangle=|b ; j\rangle$.

b) Each $|a ; k\rangle$ corresponds to only one $\left\{\lambda^{a}, u_{k}\right\}$ pair except possibly for a simultaneous one-to-one transformation of this pair.

Proof. a) I prove the first statement; the second follows from the proof of the first statement. Without loss of generality consider a system where each e-variable $\lambda$ takes only two values, say 0 and 1 . Otherwise we can reduce to a degerate system with just these two values: The 
statement $|a ; i\rangle=|b ; j\rangle$ involves, in addition to $\lambda^{a}$ and $\lambda^{b}$, only the two values $u_{i}$ and $u_{j}$. By considering a function of the maximally accessible e-variable (compare the next section), we can take one specific value equal to 1 , and the others collected in 0 . By doing this, we also arrange that both $u_{i}$ and $u_{j}$ are 1 , so we are comparing the state given by $\lambda^{a}=1$ with the state given by $\lambda^{b}=1$.

By the definition, $|a ; 1\rangle=|b ; 1\rangle$ can be written

$$
V\left(g_{a}^{\prime}\right) U_{a} \mathbf{1}\left(\lambda^{a}(\phi)=1\right)=V\left(g_{b}^{\prime}\right) U_{b} \mathbf{1}\left(\lambda^{b}(\phi)=1\right)
$$

for group elements $g_{a}^{\prime}$ and $g_{b}^{\prime}$ in $G^{\prime}$.

Use Theorem 3(i) and find $g_{a}^{0}$ and $g_{b}^{0}$ in $G^{0}$ such that $V\left(g_{a}^{\prime}\right)=U\left(g_{a}^{0}\right)$ and $V\left(g_{b}^{\prime}\right)=U\left(g_{b}^{0}\right)$. Therefore

$$
\begin{gathered}
U\left(g_{a}^{0}\right) U\left(g_{0 a}\right) \mathbf{1}\left(\lambda^{a}(\phi)=1\right)=U\left(g_{b}^{0}\right) U\left(g_{0 b}\right) \mathbf{1}\left(\lambda^{b}(\phi)=1\right) ; \\
\mathbf{1}\left(\lambda^{a}(\phi)=1\right)=U\left(g^{0}\right) \mathbf{1}\left(\lambda^{b}(\phi)=1\right)=\mathbf{1}\left(\lambda^{b}\left(\left(g^{0}\right)^{-1} \phi\right)=1\right),
\end{gathered}
$$

for $g^{0}=\left(g_{0 a}\right)^{-1}\left(g_{a}^{0}\right)^{-1} g_{b}^{0} g_{0 b}$.

Both $\lambda^{a}$ and $\lambda^{b}$ take only the values 0 and 1 . Since the set where $\lambda^{b}(\phi)=1$ can be transformed into the set where $\lambda^{a}(\phi)=1$, we must have $\lambda^{a}=F\left(\lambda^{b}\right)$ for some transformation $F$.

b) follows trivially from a).

\section{Corollary.}

The group $G$ is properly contained in $G^{\prime}$, so the representation $V$ of Theorem 1 is really multivalued.

Proof. If we had $G^{\prime}=G$, then $|a ; k\rangle$ and $|b ; k\rangle$ both reduce to $U_{a} \mathbf{1}\left(\lambda^{a}(\phi)=u_{k}\right)=U_{b} \mathbf{1}\left(\lambda^{b}(\phi)=\right.$

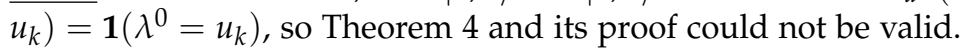

Theorem 4 and its corollary are also valid in the situation where we are interested in just two accessible variables $\lambda^{a}$ and $\lambda^{b}$, which might as well be called $\lambda^{0}$ and $\lambda^{a}$. We can then provisionally let the group $G$ be generated by $g_{0 a}, g_{a 0}=g_{0 a}^{-1}$ and all elements $g^{0}$ and $g^{a}$. The earlier statement that it is always possible to find two different elements $g_{0 a 1}^{\prime}$ and $g_{0 a 2}^{\prime}$ in $G^{\prime}$ which are mapped onto $g_{0 a}$ follows.

Finally we have

\section{Theorem 5.}

For each $a \in \mathcal{A}$, the vectors $\{|a ; k\rangle ; k=1,2, \ldots\}$ form an orthonormal basis for $H$.

Proof. Taking the invariant measure $\rho$ on $H$ as normalized to 1 , the indicator functions $|0 ; k\rangle=$ $\left.\overline{\mathbf{1}\left(\lambda^{0}(\phi)\right.}=u_{k}\right)$ form an orthonormal basis for $H$. Since the mapping $|0 ; k\rangle \rightarrow|a ; k\rangle$ is unitary, the Theorem follows.

So if $b \neq a$ and $k$ is fixed, there are complex constants $c_{k i}$ such that $|b ; k\rangle=\sum_{i} c_{k i}|a ; i\rangle$. This opens for the interference effects that one sees discussed in quantum mechanical texts. In 
particular $|a ; k\rangle=\sum_{i} d_{k i}|0 ; i\rangle$ for some constants $d_{k i}$. This is the first instance of something that we also will meet later in different situations: New states in $H$ are found by taking linear combinations of a basic set of state vectors.

\section{The general symmetrical epistemic setting}

Go back to the definition of the maximal symmetrical epistemic setting. Let again $\phi$ be the inaccessible conceptual variable and let $\lambda^{a}$ for $a \in \mathcal{A}$ be the maximal accessible conceptual variables, functions of $\phi$. Let the corresponding induced groups $G^{a}$ and $G$ satisfy the assumptions a)-c). Finally, let $t^{a}$ for each $a$ be an arbitrary function on the range of $\lambda^{a}$, and assume that we observe $\theta^{a}=t^{a}\left(\lambda^{a}\right) ; a \in \mathcal{A}$. We will call this the symmetrical epistemic setting; it is no longer necessarily maximal with respect to the observations $\theta^{a}$.

Consider first the quantum states $|a ; k\rangle$. We are no longer interested in the full information on $\lambda^{a}$, but keep the Hilbert space as in Section 5, and now let $h_{k}^{a}(\phi)=\mathbf{1}\left(t^{a}\left(\lambda^{a}\right)=t^{a}\left(u_{k}\right)\right)=$ $\mathbf{1}\left(\theta^{a}=u_{k}^{a}\right)$, where $u_{k}^{a}=t^{a}\left(u_{k}\right)$. We let again $g_{0 a 1}^{\prime}$ and $g_{0 a 2}^{\prime}$ be two distinct elements of $G^{\prime}$ such that $g_{0 a i}^{\prime} \rightarrow g_{0 a}$, define $g_{a}^{\prime}=\left(g_{0 a 1}^{\prime}\right)^{-1} g_{0 a 2}^{\prime}$ and then

$$
|a ; k\rangle=V\left(g_{a}^{\prime}\right) U_{a} h_{k}^{a}=V\left(g_{a}^{\prime}\right)|0 ; k\rangle
$$

where $|0 ; k\rangle=h_{k}^{0}$.

Interpretation of the state vector $|a ; k\rangle$ :

1) The question: 'What is the value of $\theta^{a}$ ?' has been posed. 2) We have obtained the answer $\theta^{a}=u_{k}^{a}$. Both the question and the answer are contained in the state vector.

From this we may define the operator connected to the e-variable $\theta^{a}$ :

$$
A^{a}=\sum_{k} u_{k}^{a}|a ; k\rangle\left\langle a ; k\left|=\sum_{k} t^{a}\left(u_{k}\right)\right| a ; k\right\rangle\langle a ; k|
$$

Then $A^{a}$ is no longer necessarily an operator with distinct eigenvalues, but $A^{a}$ is still Hermitian: $A^{a \dagger}=A^{a}$.

Interpretation of the operator $A^{a}$ :

This gives all possible states and all possible values corresponding to the accessible e-variable $\theta^{a}$.

The projectors $|a ; k\rangle\langle a ; k|$ and hence the ket vectors $|a ; k\rangle$ are no longer uniquely determined by $A^{a}$ : They can be transformed arbitrarily by unitary transformations in each space corresponding to one eigenvalue. In general I will redefine $|a ; k\rangle$ by allowing it to be subject to such transformations. These transformed eigenvectors all still correspond to the same eigenvalue, that is, the same observed value of $\theta^{a}$ and they give the same operators $A^{a}$. In particular, in the maximal symmetric epistemic setting I will allow an arbitrary constant phase factor in the definition of the $|a ; k\rangle$ 's. 
As an example of the general construction, assume that $\lambda^{a}$ is a vector: $\lambda^{a}=\left(\theta^{a_{1}}, \ldots, \theta^{a_{m}}\right)$. Then one can write a state vector corresponding to $\lambda^{a}$ as

$$
|a ; k\rangle=\left|a_{1} ; k_{1}\right\rangle \otimes \ldots \otimes\left|a_{m} ; k_{m}\right\rangle
$$

in an obvious notation, where $a=\left(a_{1}, \ldots, a_{m}\right)$ and $k=\left(k_{1}, \ldots, k_{m}\right)$. The different $\theta^{\prime}$ s may be connected to different subsystems.

So far I have kept the same groups $G^{a}$ and $G$ when going from $\lambda^{a}$ to $\theta^{a}=t^{a}\left(\lambda^{a}\right)$, that is from the maximal symmetrical epistemic setting to the general symmetrical epistemic setting. This implies that the (large) Hilbert space will be the same. A special case occurs if $t^{a}$ is a reduction to an orbit of $G^{a}$. This is the kind of model reduction mentioned at the end of Section 2. Then the construction of the previous sections can also be carried with a smaller group action acting just upon an orbit, resulting then in a smaller Hilbert space. In the example of the previous paragraph it may be relevant to consider one Hilbert space for each subsystem. The large Hilbert space is however the correct space to use when the whole system is considered.

Connected to a general physical system, one may have many e-variables $\theta$ and corresponding operators $A$. In the ordinary quantum formalism, there is well-known theorem saying that, in my formulation, $\theta^{1}, \ldots, \theta^{n}$ are compatible, that is, there exists an e-variable $\lambda$ such that $\theta^{i}=t^{i}(\lambda)$ for some functions $t^{i}$ if and only if the corresponding operators commute:

$$
\left[A^{i}, A^{j}\right] \equiv A^{i} A^{j}-A^{j} A^{i}=0 \text { for all } i, j
$$

(See Holevo [4].) Compatible e-variables may in principle be estimated simultaneously with arbitrary accuracy.

The way I have defined pure state, the only state vectors that are allowed, are those which are eigenvectors of some physically meaningful operator. This is hardly a limitation in the spin/angular momentum case where operators corresponding to all directions are included. Nevertheless it is an open question to find general conditions under which all unit vectors in $H$ correspond to states $|a ; k\rangle$ the way I have defined them. It is shown in [5] that this holds under no further conditions for the spin $1 / 2$ case.

\section{Link to statistical inference}

Assume now the symmetrical epistemic setting. We can think of a spin component in a fixed direction to be assessed. To assume a state $|a ; k\rangle$ is to assume perfect knowledge of the e-variable $\theta^{a}: \theta^{a}=u_{k}^{a}$. Such perfect knowledge is rarely available. In practice we have data $z^{a}$ about the system, and use these data to obtain knowledge about $\theta^{a}$. Let us start with Bayesian inference. This assumes prior probabilities $\pi_{k}^{a}$ on the values $u_{k^{\prime}}^{a}$ and after the inference we have posterior probabilities $\pi_{k}^{a}\left(z^{a}\right)$. In either case we summarize this information in the density operator:

$$
\sigma^{a}=\sum_{k} \pi_{k}^{a}|a ; k\rangle\langle a ; k| .
$$


Interpretation of the density operator $\sigma^{a}$ :

1) We have posed the question 'What is the value of $\theta^{a} ?^{\prime}$ 2) We have specified a prior or posterior probability distribution $\pi_{k}^{a}$ over the possible answers. The probability for all possible answers to the question, formulated in terms of state vectors, can be recovered from the density operator.

A third possibility for the probability specifications is a relatively new, but important concept of a confidence distribution ([6], [7]). This is a frequentist alternative to the distribution connected to a parameter (here: e-variable). The idea is that one looks at a one-sided confidence interval for any value of the confidence coefficient $\gamma$. Let the data be $z$, and let $(-\infty, \beta(\gamma, z)]$ be such an interval. Then $\beta(\gamma)=\beta(\gamma, z)$ is an increasing function. We define $H(\cdot)=\beta^{-1}(\cdot)$ as the confidence distribution for $\theta$. This $H$ is a cumulative distribution function, and in the continuous case it is characterized with the property that $H(\beta(\gamma, z))$ has a uniform distribution over $[0,1]$ under the model. For discrete $\theta^{a}$ the confidence distribution function $H^{a}$ is connected to a discrete distribution, which gives the probabilities $\pi_{k}^{a}$. Extending the argument in [7] to this situation, this should not be looked upon as a distribution of $\theta^{a}$, but a distribution for $\theta^{a}$, to be used in the epistemic process.

Since the sum of the probabilities is 1 , the trace (sum of eigenvalues) of any density operator is 1 . In the quantum mechanical literature, a density operator is any positive operator with trace 1 .

Note that specification of the accessible e-variables $\theta^{a}$ is equivalent to specifying $t\left(\theta^{a}\right)$ for any one-to-one function $t$. The operator $t\left(A^{a}\right)$ has then distinct eigenvalues if and only if the operator $A^{a}$ has distinct eigenvalues. Hence it is enough in order to specify the question 1) to give the set of orthonormal vectors $|a ; k\rangle$.

Given the question $a$, the e-variable $\theta^{a}$ plays the role similar to a parameter in statistical inference, even though it may be connected to a single unit. Inference can be done by preparing many independent units in the same state. Inference is then from data $z^{a}$, a part of the total data $z$ that nature can provide us with. All inference theory that one finds in standard texts like [8] applies. In particular, the concepts of unbiasedness, equivariance, minimaxity and admissibility apply. None of these concepts are much discussed in the physical literature, first because measurements there are often considered as perfect, at least in elementary texts, secondly because, when measurements are considered in the physical literature, they are discussed in terms of the more abstract concept of an operator-valued measure; see below.

Whatever kind of inference we make on $\theta^{a}$, we can take as a point of departure the statistical model and the likelihood principle of Section 2 . Hence after an experiment is done, and given some context $\tau$, all evidence on $\theta^{a}$ is contained in the likelihood $p\left(z^{a} \mid \tau, \theta^{a}\right)$, where $z^{a}$ is the portion of the data relevant for inference on $\theta^{a}$, also assumed discrete. This is summarized in the likelihood effect:

$$
E\left(z^{a}, \tau\right)=\sum_{k} p\left(z^{a} \mid \tau, \theta^{a}=u_{k}^{a}\right)|a ; k\rangle\langle a ; k|
$$

Interpretation of the likelihood effect $E\left(z^{a}, \tau\right)$ : 
1) We have posed some inference question on the accessible e-variable $\theta^{a}$. 2) We have specified the relevant likelihood for the data. The likelihood for all possible answers of the question, formulated in terms of state vectors, can be recovered from the likelihood effect.

Since the focused question assumes discrete data, each likelihood is in the range $0 \leq p \leq 1$. In the quantum mechanical literature, an effect is any operator with eigenvalues in the range $[0,1]$.

Return now to the likelihood principle of Section 2. The following principle follows.

The focused likelihood principle (FLP)

Consider two potential experiments in the symmetrical epistemic setting with equivalent contexts $\tau$, and assume that the inaccessible conceptual variable $\phi$ is the same in both experiments. Suppose that the observations $z_{1}^{*}$ and $z_{2}^{*}$ have proportional likelihood effects in the two experiments, with a constant of proportionality independent of the conceptual variable. Then the questions posed in the two experiments are equivalent, that is, there is an e-variable $\theta^{a}$ which can be considered to be the same in the two experiments, and the two observations produce the same evidence on $\theta^{a}$ in this context.

In many examples the two observations will have equal, not only proportional, likelihood effects. Then the FLP says simply that the experimental evidence is a function of the likelihood effect.

In the FLP we have the freedom to redefine the e-variable in the case of coinciding eigenvalues in the likelihood effect, that is, if $p\left(z^{a} \mid \tau, \theta^{a}=u_{k}\right)=p\left(z^{a} \mid \tau, \theta^{a}=u_{l}\right)$ for some $k$, l. An extreme case is the likelihood effect $E\left(z^{a}, \tau\right)=I$, where all the likelihoods are 1 , that is, the probability of $z$ is 1 under any considered model. Then any accessible e-variable $\theta^{a}$ will serve our purpose.

We are now ready to define the operator-valued measure in this discrete case:

$$
M^{a}(B \mid \tau)=\sum_{z^{a} \in B} E\left(z^{a}, \tau\right)
$$

for any Borel set in the sample space for experiment $a$. Its usefulness will be seen after we have discussed Born's formula. Then we will also have background for reading much of [9], a survey over quantum statistical inference.

\section{Rationality and experimental evidence}

Throughout this section I will consider a fixed context $\tau$ and a fixed epistemic setting in this context. The inaccessible e-variable is $\phi$, and I assume that the accessible e-variables $\theta^{a}$ take a discrete set of values. Let the data behind the potential experiment be $z^{a}$, also assumed to take a discrete set of values.

Let first a single experimentalist $A$ be in this situation, and let all conceptual variables be attached to $A$, although he also has the possibility to receiving information from others through part of the context $\tau$. He has the choice of doing different experiments $a$, and he also has the choice of choosing different models for his experiment through his likelihood $p_{A}\left(z^{a} \mid \tau, \theta^{a}\right)$. The experiment and the model, hence the likelihood, should be chosen before the data are obtained. All these choices are summarized in the likelihood effect $E$, a function 
of the at present unknown data $z^{a}$. For use after the experiment, he should also choose a good estimator/predictor $\widehat{\theta^{a}}$, and he may also have to choose some loss function, but the principles behind these latter choices will be considered as part of the context $\tau$. If he chooses to do a Bayesian analysis, the estimator should be based on a prior $\pi\left(\theta^{a} \mid \tau\right)$. We assume that $A$ is trying to be as rational as possible in all his choices, and that this rationality is connected to his loss function or to other criteria.

What should be meant by experimental evidence, and how should it be measured? As a natural choice, let the experimental evidence that we are seeking, be the marginal probability of the obtained data for a fixed experiment and for a given likelihood function. From the experimentalist $A$ 's point of view this is given by:

$$
p_{A}^{a}\left(z^{a} \mid \tau\right)=\sum_{k} p_{A}\left(z^{a} \mid \tau, \theta^{a}=u_{k}\right) \pi_{A}\left(\theta^{a}=u_{k} \mid \tau\right)
$$

assuming the likelihood chosen by $A$ and $A^{\prime}$ s prior $\pi_{A}$ for $\theta^{a}$. In a non-Bayesian analysis, we can let $p_{A}^{a}\left(z^{a} \mid \tau\right)$ be the probability given the true value $u_{k}^{0}$ of the e-variable: $p_{A}^{a}\left(z^{a} \mid \tau\right)=$ $p_{A}\left(z^{a} \mid \tau, \theta^{a}=u_{k}^{0}\right)$. In general, take $p_{A}^{a}\left(z^{a} \mid \tau\right)$ as the probability of the part of the data $z^{a}$ which $A$ assesses in connection to his inference on $\theta^{a}$. By the FLP - specialized to the case of one experiment and equal likelihoods - this experimental evidence must be a function of the likelihood effect: $p_{A}^{a}\left(z^{a} \mid \tau\right)=q_{A}\left(E\left(z^{a}\right) \mid \tau\right)$.

We have to make precise in some way what is meant by the rationality of the experimentalist $A$. He has to make many difficult choices on the basis of uncertain knowledge. His actions can partly be based on intuition, partly on experience from similar situations, partly on a common scientific culture and partly on advices from other persons. These other persons will in turn have their intuition, their experience and their scientific education. Often $A$ will have certain explicitly formulated principles on which to base his decisions, but sometimes he has to dispense with the principles. In the latter case, he has to rely on some 'inner voice', a conviction which tells him what to do.

We will formalize all this by introducing a perfectly rational superior actor $D$, to which all these principles, experiences and convictions can be related. We also assume that $D$ can observe everything that is going on, in particular $A$, and that he on this background can have some influence on $A^{\prime}$ s decisions. The real experimental evidence will then be defined as the probability of the data $z^{a}$ from $D^{\prime}$ 's point of view, which we assume also to give the real objective probabilities. By the FLP this must again be a function of the likelihood effect $E$, where the likelihood now may be seen as the objectively correct model.

$$
p^{a}\left(z^{a} \mid \tau\right)=q\left(E\left(z^{a}\right) \mid \tau\right)
$$

As said, we assume that $D$ is perfectly rational. This can be formalized mathematically by considering a hypothetical betting situation for $D$ against a bookie, nature $N$. A similar discussion was recently done in [10] using a more abstract language. Note the difference to the ordinary Bayesian assumption, where $A$ himself is assumed to be perfectly rational. This difference is crucial to me. I do not see any human scientist, including myself, as being 
perfectly rational. We can try to be as rational as possible, but we have to rely on some underlying rational principles that partly determine our actions.

So let the hypothetical odds of a given bet for $D$ be $(1-q) / q$ to 1 , where $q$ is the probability as defined by (4). This odds specification is a way to make precise that, given the context $\tau$ and given the question $a$, the bettor's probability that the experimental result takes some value is given by $q$ : For a given utility measured by $x$, the bettor $D$ pays in an amount $q x$ the stake - to the bookie. After the experiment the bookie pays out an amount $x$ - the payoff - to the bettor if the result of the experiment takes the value $z^{a}$, otherwise nothing is payed.

The rationality of $D$ is formulated in terms of

The Dutch book principle.

No choice of payoffs in a series of bets shall lead to a sure loss for the bettor.

For a related use of the same principle, see [11].

Assumption D.

Consider in some context $\tau$ a maximal symmetrical epistemic setting where the FLP is satisfied, and the whole situation is observed and acted upon by a superior actor $D$ as described above. Assume that $D$ 's probabilities q given by (4) are taken as the experimental evidence, and that $D$ acts rationally in agreement with the Dutch book principle.

A situation where all the Assumption D holds together with the assumptions of a symmetric epistemic setting will be called a rational epistemic setting.

Theorem 6.

Assume a rational epistemic setting. Let $E_{1}$ and $E_{2}$ be two likelihood effects in this setting, and assume that $E_{1}+E_{2}$ also is a likelihood effect. Then the experimental evidences, taken as the probabilities of the corresponding data, satisfy

$$
q\left(E_{1}+E_{2} \mid \tau\right)=q\left(E_{1} \mid \tau\right)+q\left(E_{2} \mid \tau\right)
$$

Proof. The result of the theorem is obvious, without making Assumption D, if $E_{1}$ and $E_{2}$ are likelihood effects connected to experiments on the same e-variable $\theta^{a}$. We will prove it in general. Consider then any finite number of potential experiments including the two with likelihood effects $E_{1}$ and $E_{2}$. Let $q_{1}=q\left(E_{1} \mid \tau\right)$ be equal to (4) for the first experiment, and let $q_{2}=q\left(E_{2} \mid \tau\right)$ be equal to the same quantity for the second experiment. Consider in addition the following randomized experiment: Throw an unbiased coin. If head, choose the experiment with likelihood effect $E_{1}$; if tail, choose the experiment with likelihood effect $E_{2}$. This is a valid experiment. The likelihood effect when the coin shows head is $\frac{1}{2} E_{1}$, when it shows tail $\frac{1}{2} E_{2}$, so that the likelihood effect of this experiment is $E_{0}=\frac{1}{2}\left(E_{1}+E_{2}\right)$. Define $q_{0}=q\left(E_{0}\right)$. Let the bettor bet on the results of all these 3 experiments: Payoff $x_{1}$ for experiment 1, payoff $x_{2}$ for experiment 2 and payoff $x_{0}$ for experiment 0 .

I will divide into 3 possible outcomes: Either the likelihood effect from the data $z$ is $E_{1}$ or it is $E_{2}$ or it is none of these. The randomization in the choice of $E_{0}$ is considered separately from the result of the bet. (Technically this can be done by repeating the whole series of 
experiments many times with the same randomization. This is also consistent with the conditionality principle.) Thus if $E_{1}$ occurs, the payoff for experiment 0 is replaced by the expected payoff $x_{0} / 2$, similarly if $E_{2}$ occurs. The net expected amount the bettor receives is then

$$
\begin{gathered}
x_{1}+\frac{1}{2} x_{0}-q_{1} x_{1}-q_{2} x_{2}-q_{0} x_{0}=\left(1-q_{1}\right) x_{1}-q_{2} x_{2}-\left(1-2 q_{0}\right) \frac{1}{2} x_{0} \text { if } E_{1} \\
x_{2}+\frac{1}{2} x_{0}-q_{1} x_{1}-q_{2} x_{2}-q_{0} x_{0}=-q_{1} x_{1}-\left(1-q_{2}\right) x_{2}-\left(1-2 q_{0}\right) \frac{1}{2} x_{0} \text { if } E_{2} \\
-q_{1} x_{1}-q_{2} x_{2}-2 q_{0} \cdot \frac{1}{2} x_{0} \text { otherwise. }
\end{gathered}
$$

The payoffs $\left(x_{1}, x_{2}, x_{0}\right)$ can be chosen by nature $N$ in such a way that it leads to sure loss for the bettor $D$ if not the determinant of this system is zero:

$$
0=\left|\begin{array}{ccc}
1-q_{1} & -q_{2} & 1-2 q_{0} \\
-q_{1} & 1-q_{2} & 1-2 q_{0} \\
-q_{1} & -q_{2} & -2 q_{0}
\end{array}\right|=q_{1}+q_{2}-2 q_{0} .
$$

Thus we must have

$$
q\left(\frac{1}{2}\left(E_{1}+E_{2}\right) \mid \tau\right)=\frac{1}{2}\left(q\left(E_{1} \mid \tau\right)+q\left(E_{2} \mid \tau\right)\right) .
$$

If $E_{1}+E_{2}$ is an effect, the common factor $\frac{1}{2}$ can be removed by changing the likelihoods, and the result follows.

Corollary.

Assume a rational epistemic setting. Let $E_{1}, E_{2}, \ldots$ be likelihood effects in this setting, and assume that $E_{1}+E_{2}+\ldots$ also is a likelihood effect. Then

$$
q\left(E_{1}+E_{2}+\ldots \mid \tau\right)=q\left(E_{1} \mid \tau\right)+q\left(E_{2} \mid \tau\right)+\ldots
$$

Proof. The finite case follows immediately from Theorem 6 . Then the infinite case follows from monotone convergence.

The result of this section is quite general. In particular the loss function and any other criterion for the success of the experiments are arbitrary. So far I have assumed that the choice of experiment $a$ is fixed, which implies that it is the same for $A$ and for $D$. However, the result also applies to the following more general situation: Let $A$ have some definite purpose of his experiment, and to achieve that purpose, he has to choose the question $a$ in a clever manner, as rationally as he can. Assume that this rationality is formalized through the actor $D$, who has the ideal likelihood effect $E$ and the experimental evidence $p(z \mid \tau)=q(E \mid \tau)$. If two such questions shall be chosen, the result of Theorem 6 holds, with essentially the same proof. 


\section{The Born formula}

\subsection{The basic formula}

Born's formula is the basis for all probability calculations in quantum mechanics. In textbooks it is usually stated as a separate axiom, but it has also been argued for by using various sets of assumptions; see [12] for some references. Here I will base the discussion upon the result of Section 8 .

I begin with a recent result by Busch [13], giving a new version of a classical mathematical theorem by Gleason. Busch's version has the advantage that it is valid for a Hilbert space of dimension 2, which Gleason's original theorem is not, and it also has a simpler proof. For a proof for the finite-dimensional case, see Appendix 5 of [2].

Let in general $H$ be any Hilbert space. Recall that an effect $E$ is any operator on the Hilbert space with eigenvalues in the range $[0,1]$. A generalized probability measure $\mu$ is a function on the effects with the properties

(1) $0 \leq \mu(E) \leq 1$ for all $E$,

(2) $\mu(I)=1$,

(3) $\mu\left(E_{1}+E_{2}+\ldots\right)=\mu\left(E_{1}\right)+\mu\left(E_{2}\right)+\ldots$ whenever $E_{1}+E_{2}+\ldots \leq I$.

Theorem 7. (Busch, 2003).

Any generalized probability measure $\mu$ is of the form $\mu(E)=\operatorname{Tr}(\sigma E)$ for some density operator $\sigma$.

It is now easy to see that $q(E \mid \tau)=p(z \mid \tau)$ on the ideal likelihood effects of Section 8 is a generalized probability measure if Assumption D holds: (1) follows since $q$ is a probability; (2) since $E=I$ implies that the likelihood is 1 for all values of the e-variable, hence $p(z)=1$; finally (3) is a concequence of the corollary of Theorem 6. Hence there is a density operator $\sigma=\sigma(\tau)$ such that $p(z \mid \tau)=\operatorname{Tr}(\sigma(\tau) E)$ for all ideal likelihood effects $E=E(z)$.

Define now a perfect experiment as one where the measurement uncertainty can be disregarded. The quantum mechanical literature operates very much with perfect experiments which give well-defined states $|k\rangle$. From the point of view of statistics, if, say the $99 \%$ confidence or credibility region of $\theta^{b}$ is the single point $u_{k}^{b}$, we can infer approximately that a perfect experiment has given the result $\theta^{b}=u_{k}^{b}$.

In our symmetric epistemic setting then: We have asked the question: 'What is the value of the accessible e-variable $\theta^{b}$ ?', and are interested in finding the probability of the answer $\theta^{b}=u_{j}^{b}$ though a perfect experiment. This is the probability of the state $|b ; j\rangle$. Assume now that this probability is sought in a context $\tau=\tau^{a, k}$ defined as follows: We have previous knowledge of the answer $\theta^{a}=u_{k}^{a}$ of another accessible question: What is the value of $\theta^{a}$ ? That is, we know the state $|a ; k\rangle$. If $\theta^{a}$ is maximally accessible, this is the maximal knowledge about the system that $\tau$ may contain; in general we assume that the context $\tau$ does not contain more information about this system. It can contain irrelevant information, however. 
Theorem 8. (Born's formula)

Assume a rational epistemic setting. In the above situation we have:

$$
P\left(\theta^{b}=u_{j}^{b} \mid \theta^{a}=u_{k}^{a}\right)=|\langle a ; k \mid b ; j\rangle|^{2} .
$$

Proof. Fix $j$ and $k$, let $|v\rangle$ be either $|a ; k\rangle$ or $|b ; j\rangle$, and consider likelihood effects of the form $E=|v\rangle\langle v|$. This corresponds in both cases to a perfect measurement of a maximally accessible parameter with a definite result. By Theorem 7 there exists a density operator $\sigma^{a, k}=\sum_{i} \pi_{i}\left(\tau^{a, k}\right)|i\rangle\langle i|$ such that $q\left(E \mid \tau^{a, k}\right)=\left\langle v\left|\sigma^{a, k}\right| v\right\rangle$, where $\pi_{i}\left(\tau^{a, k}\right)$ are non-negative constants adding to 1 . Consider first $|v\rangle=|a ; k\rangle$. For this case one must have $\sum_{i} \pi_{i}\left(\tau^{a, k}\right)|\langle i \mid a ; k\rangle|^{2}=1$ and thus $\sum_{i} \pi_{i}\left(\tau^{a, k}\right)\left(1-|\langle i \mid a ; k\rangle|^{2}\right)=0$. This implies for each $i$ that either $\pi_{i}\left(\tau^{a, k}\right)=0$ or $|\langle i \mid a ; k\rangle|=1$. Since the last condition implies $|i\rangle=|a ; k\rangle$ (modulus an irrelevant phase factor), and this is a condition which can only be true for one $i$, it follows that $\pi_{i}\left(\tau^{a, k}\right)=0$ for all other $i$ than this one, and that $\pi_{i}\left(\tau^{a, k}\right)=1$ for this particular $i$. Summarizing this, we get $\sigma^{a, k}=|a ; k\rangle\langle a ; k|$, and setting $|v\rangle=|b ; j\rangle$, Born's formula follows, since $q\left(E \mid \tau^{a, k}\right)$ in this case is equal to the probability of the perfect result $\theta^{b}=u_{j}^{b}$.

\subsection{Consequences}

Here are three easy consequences of Born's formula:

(1) If the context of the system is given by the state $|a ; k\rangle$, and $A^{b}$ is the operator corresponding to the e-variable $\theta^{b}$, then the expected value of a perfect measurement of $\theta^{b}$ is $\left\langle a ; k\left|A^{b}\right| a ; k\right\rangle$.

(2) If the context is given by a density operator $\sigma$, and $A$ is the operator corresponding to the e-variable $\theta$, then the expected value of a perfect measurement of $\theta$ is $\operatorname{Tr}(\sigma A)$.

(3) In the same situation the expected value of a perfect measurement of $f(\theta)$ is $\operatorname{Tr}(\sigma f(A))$.

Proof of (1):

$$
\begin{aligned}
& \mathrm{E}\left(\theta^{b} \mid \theta^{a}=u_{k}^{a}\right)=\sum_{i} u_{i}^{b} P\left(\theta^{b}=u_{i}^{b} \mid \theta^{a}=u_{k}^{a}\right) \\
& =\sum_{i} u_{i}^{b}\langle a ; k \mid b ; i\rangle\langle b ; i \mid a ; k\rangle=\left\langle a ; k\left|A^{b}\right| a ; k\right\rangle .
\end{aligned}
$$

These results give an extended interpretation of the operator $A$ compared to what I gave in Section 5: There is a simple formula for all expectations in terms of the operator. On the other hand, the set of such expectations determine the state of the system. Also on the other hand: If $A$ is specialized to an indicator function, we get back Born's formula, so the consequences are equivalent to this formula.

As an application of Born's formula, we give the transition probabilities for electron spin. I will, for a given direction $a$, define the e-variable $\theta^{a}$ as +1 if the measured spin component by a perfect measurement for the electron is $+\hbar / 2$ in this direction, $\theta^{a}=-1$ if the component is $-\hbar / 2$. Assume that $a$ and $b$ are two directions in which the spin component can be measured. 
Proposition 2.

For electron spin we have

$$
P\left(\theta^{b}= \pm 1 \mid \theta^{a}=+1\right)=\frac{1}{2}(1 \pm \cos (a \cdot b)) .
$$

This is proved in several textbooks, for instance [4], from Born's formula. A similar proof using the Pauli spin matrices is also given in [5].

Finally, using Born's formula, we deduce the basic formula for quantum measurement. Let the state of a system be given by a density matrix $\rho=\rho(\eta)$, where $\eta$ is an unknown statistical parameter, and let the measurements be determined by an operator-valued measure $M(\cdot)$ as defined in Section 7. Then the probability distribution of the observations is given by

$$
P(B ; \eta)=\operatorname{Tr}(\rho(\eta) M(B))
$$

This, together with an assumption on the state after measurement, is the basis for [9].

\section{Entanglement, EPR and the Bell theorem}

The total spin components in different directions for a system of two spin 1/2 particles satisfy the assumptions of a maximal symmetric epistemic setting. Assume that we have such a system where $j=0$, that is, the state is such that the total spin is zero. By ordinary quantum mechanical calculations, this state can be explicitly written as

$$
|0\rangle=\frac{1}{\sqrt{2}}(|1,+\rangle \otimes|2,-\rangle-|1,-\rangle \otimes|2,+\rangle),
$$

where $|1,+\rangle \otimes|2,-\rangle$ is a state where particle 1 has a spin component $+\hbar / 2$ and particle 2 has a spin component $-\hbar / 2$ along the $z$-axis, and vice versa for $|1,-\rangle \otimes|2,+\rangle$. This is what is called an entangled state, that is, a state which is not a direct product of the component state vectors. I will follow my own programme, however, and stick to the e-variable description.

Assume further that the two particles separate, the spin component of particle 1 is measured in some direction by an observer Alice, and the spin component of particle 2 is measured by an observer Bob. Before the experiment, the two observers agree both either to measure spin in some fixed direction $a$ or in another fixed direction $b$, orthogonal to $a$, both measurements assumed for simplicity to be perfect. As a final assumption, let the positions of the two observers at the time of measurement be spacelike, that is, the distance between them is so large that no signal can reach from one to the other at this time, taking into account that signals cannot go faster that the speed of light by the theory of relativity.

This is Bohm's version of the situation behind the argument against the completeness of quantum mechanics as posed by Einstein et al. [14] and countered by Bohr [15], [16]. This discussion is still sometimes taken up today, although most physicists now support Bohr. So will I, but I will go a step further. The main thesis in [14] was as follows: If, without in any 
way disturbing a system, we can predict the value of a physical quantity, then there exists an element of physical reality corresponding to this physical reality. Bohr answered by introducing a strict interpretation of this criterion: To ascribe reality to $P$, the measurement of an observable whose outcome allows for the prediction of $P$, must actually be performed, or one must give a description of how it can be performed. Several authors have argued that Einstein's criterion of reality lead an assumption of non-locality: Signals between observers with a spacelike separation must travel faster than light. Recently, it has been shown in [17] that the possibility is open to interpret the non-locality theorems in the physical literature as arguments supporting the strict criterion of reality, rather than a violation of locality. I agree with this last interpretation.

I will be very brief on this discussion here. Let $\lambda$ be the spin component in units of $\hbar / 2$ as measured by Alice, and let $\eta$ be the spin component in the same units as measured by Bob. Alice has a free choice between measuring in the the directions $a$ and in the direction $b$. In both cases, her probability is $1 / 2$ for each of $\lambda= \pm 1$. If she measures $\lambda^{a}=+1$, say, she will predict $\eta^{a}=-1$ for the corresponding component measured by Bob. According to Einstein et al. [14] there should then be an element of reality corresponding to this prediction, but if we adapt the strict interpretation of Bohr here, there is no way in which Alice can predict Bob's actual real measurement at this point of time. Bob on his side has also a free choice of measurement direction $a$ or $b$, and in both cases he has the probability $1 / 2$ for each of $\eta= \pm 1$. The variables $\lambda$ and $\eta$ are conceptual, the first one connected to Alice and the second one connected to Bob. As long as the two are not able to communicate, there is no sense in which we can make statements like $\eta=-\lambda$ meaningful.

The situation changes. however, if Alice and Bob meet at some time after the measurement. If Alice then says 'I chose to make a measurement in the direction $a$ and got the result $u^{\prime}$ and Bob happens to say 'I also chose to make a measurement in the direction $a$, and then I got the result $v^{\prime}$, then these two statements must be consistent: $v=-u$. This seems to be a necessary requirement for the consistency of the theory. There is a subtle distinction here. The clue is that the choices of measurement direction both for Alice and for Bob are free and independent. The directions are either equal or different. If they should happen to be different, there is no consistency requirement after the measurement, due to the assumed orthogonality of $a$ and $b$. Note again that we have an epistemic interpretation of quantum mechanics. At the time of measurement, nothing exists except the observations by the two observers.

Let us then look at the more complicated situation where $a$ and $b$ are not necessarily orthogonal, where Alice tosses a coin and measures in the direction $a$ if head and $b$ if tail, while Bob tosses an independent coin and measures in some direction $c$ if head and in another direction $d$ if tail. Then there is an algebraic inequality

$$
\lambda^{a} \eta^{c}+\lambda^{b} \eta^{c}+\lambda^{b} \eta^{d}-\lambda^{a} \eta^{d} \leq 2
$$

Since all the conceptual variables take values \pm 1 , this inequality follows from

$$
\left(\lambda^{a}+\lambda^{b}\right) \eta^{c}+\left(\lambda^{b}-\lambda^{a}\right) \eta^{d}= \pm 2 \leq 2
$$

Now replace the conceptual variables here with actual measurements. Taking then formal expectations from (6), assumes that the products here have meaning as random variables; 
in the physical literature this is stated as an assumption of realism and locality. This leads formally to

$$
E\left(\widehat{\lambda^{a}} \widehat{\eta^{c}}\right)+E\left(\widehat{\lambda^{b}} \widehat{\eta^{c}}\right)+E\left(\widehat{\lambda^{b} \eta^{d}}\right)-E\left(\widehat{\lambda^{a}} \widehat{\eta^{d}}\right) \leq 2
$$

This is one of Bell's inequalities, called the CHSH inequality.

On the other hand, using quantum-mechanical calculations, that is Born's formula, from the basic state (5), shows that $a, b, c$ and $d$ can be chosen such that Bell's inequality (7) is violated. This is also confirmed by numerous experiments with electrons and photons.

From our point of view the transition from (6) to (7) is not valid. One can not take the expectation term by term in equation (6). The $\lambda^{\prime}$ s and $\eta^{\prime}$ s are conceptual variables belonging to different observers. Any valid statistical expectation must take one of these observers as a point of departure. Look at (6) from Alice's point of view, for instance. She starts by tossing a coin. The outcome of this toss leads to some e-variable $\lambda$ being measured in one of the directions $a$ or $b$. This measurement is an epistemic process, and any prediction based upon this measurement is a new epistemic process. During these processes she must obey Conditionality principle 2 of Section 2. By this conditionality principle she should condition upon the outcome of the coin toss. So in any prediction she should condition upon the choice $a$ or $b$. It is crucial for this argument that the prediction of an e-variable is an epistemic process, not a process where ordinary probability calculations can be immediately used.

By doing predictions from her measurement result, she can use Born's formula. Suppose that she measures $\lambda^{a}$ and finds $\lambda^{a}=+1$, for instance. Then she can predict the value of $\lambda^{c}$ and hence $\eta^{c}=-\lambda^{c}$. Thus she can (given the outcome $a$ of the coin toss) compute the expectation of the first term (6). similarly, she can compute the expectation of the last term in (6). But there is no way in which she simultaneously can predict $\lambda^{b}$ and $\eta^{d}$. Hence the expectation of the second term (and also, similarly the third term) in (6) is for her meaningless. A similar conclusion is reached if the outcome of the coin toss gives $b$. And of course a similar conclusion is valid if we take Bob's point of view. Therefore the transition from (6) to (7) is not valid, not by non-locality, but by a simple use of the conditionality principle. This can also in some sense be called lack of realism: In this situation is it not meaningful to take expectation from the point of view of an impartial observer. By necessity one must see the situation from the point of view of one of the observers Alice or Bob.

Entanglement is very important in modern applications of quantum mechanics, not least in quantum information theory, including quantum computation. It is also an important ingredient in the theory of decoherence [18], which explains why ordinary quantum effects are not usually visible on a larger scale. Decoherence theory shows the importance of the entanglement of each system with its environment. In particular, it leads in effect to the conclusion that all observers share common observations after decoherence between the system and its environment, and this can then be identified with the 'objective' aspects of the world; which is also what the superior actor $D$ of Section 8 would find.

\section{Position as an e-variable and the Schrödinger equation}

So far I have looked at e-variables taking a finite discrete set of values, but the concept of an e-variable carries over to the continuous case. Consider the motion of a non-relativistic 
one-dimensional particle. Its position $\xi$ at some time $t$ can in principle be determined by arbitrary accuracy, resulting in an arbitrarily short confidence interval. But momentum and hence velocity cannot be determined simultaneously with arbitrary accuracy, hence the vector $(\xi(s), \xi(t))$ for positions at two different time points is inaccessible. Now fix some time $t$. An observer $i$ may predict $\xi(t)$ by conditioning on some $\sigma$-algebra $\mathcal{P}_{i}$ of information from the past. This may be information from some time point $s_{i}<t$, but it can also take other forms. We must think of different observers as hypothetical; only one of them can be realized. Nevertheless one can imagine that all this information, subject to the choice of observer later, is collected in an inaccessible $\sigma$-algebra $\mathcal{P}_{t}$, the past of $\xi(t)$. The distribution of $\xi(t)$, given the past $\mathcal{P}_{t}$, for each $t$, can then be represented as a stochastic process.

In the simplest case one can then imagine $\{\xi(s) ; s \geq 0\}$ as an inaccessible Markov process: The future is independent of the past, given the present. Under suitable regularity conditions, a continuous Markov process will be a diffusion process, i.e., a solution of a stochastic differential equation of the type

$$
d \xi(t)=b(\xi(t), t) d t+\sigma(\xi(t), t) d w(t)
$$

Here $b(\cdot, \cdot)$ and $\sigma(\cdot, \cdot)$ are continuous functions, also assumed differentiable, and $\{w(t) ; t \geq$ $0\}$ is a Wiener process. The Wiener process is a stochastic process with continuous paths, independent increments $w(t)-w(s), w(0)=0$ and $E\left((w(t)-w(s))^{2}\right)=t-s$. Many properties of the Wiener process have been studied, including the fact that its paths are nowhere differentiable. The stochastic differential equation (8) must therefore be defined in a particular way; for an introduction to Itô calculus or Stochastic calculus; see for instance [19].

So far we have considered observers making predictions of the present value $\xi(t)$, given the past $\mathcal{P}_{t}$. There is another type of epistemic processes which can be described as follows: Imagine an actor $\mathrm{A}$ which considers some future event for the particle, lying in a $\sigma$-algebra $\mathcal{F}_{j}$. He asks himself in which position he should place the particle at time $t$ as well as possible in order to have this event fulfilled. In other words, he can adjust $\xi(t)$ for this purpose. Again one can collect the $\sigma$-algebras for the different potential actors in one big inaccessible $\sigma$-algebra $\mathcal{F}_{t}$, the future after $t$. The conditioning of the present, given the future, defines $\{\xi(t) ; t \geq 0\}$ as a new inaccessible stochastic process, with now $t$ running backwards in time. In the simplest case this is a Markov process, and can be described by a stochastic differential equation

$$
d \xi(t)=b_{*}(\xi(t), t) d t+\sigma_{*}(\xi(t), t) d w_{*}(t),
$$

where again $w_{*}(t)$ is a Wiener process.

Without having much previous knowledge about modern stochastic analysis and without knowing anything about epistemic processes, Nelson [20] formulated his stochastic mechanics, which serves our purpose perfectly. Nelson considered the multidimensional case, but for simplicity, I will here only discuss a one-dimensional particle. Everything can be generalized.

Nelson discussed what corresponds to the stochastic differential equations (8) and (9) with $\sigma$ and $\sigma_{*}$ constant in space and time. Since heavy particles fluctuate less than light particles, 
he assumed that these quantities vary inversely with mass $m$, that is, $\sigma^{2}=\sigma_{*}^{2}=\hbar / m$. The constant $\hbar$ has dimension action, and turns out to be equal to Planck's constant divided by $2 \pi$. This assumes that $\sigma^{2}=\sigma_{*}^{2}$, a fact that Nelson actually proved in addition to proving that

$$
b_{*}=b-\sigma^{2}(\ln \rho)_{x},
$$

where $\rho=\rho(x, t)$ is the probability density of $\xi(t)$.

Introduce $u=\left(b-b_{*}\right) / 2$ and $v=\left(b+b_{*}\right) / 2$. Then $R=\frac{1}{2} \ln \rho(x, t)$ satisfies $R_{x}=m u / \hbar$. Let $S$ be defined up to an additive constant by $S_{x}=m v / \hbar$ and define the wave function of the particle by $f=\exp (R+i S)$. Then $|f(x, t)|^{2}=\rho(x, t)$ as it should. By defining the acceleration of the particle in a proper way and using Newton's second law, a set of partial differential equations for $u$ and $v$ can be found, and by choosing the additive constant in $S$ properly, one deduces from these equations

$$
i \hbar \frac{\partial}{\partial t} f(x, t)=\left[\frac{1}{2 m}\left(-i \hbar \frac{\partial}{\partial x}\right)^{2}+V(x)\right] f(x, t),
$$

where $V(x)$ is the potential energy. The details of these derivations can be found in [2] with more details in [20]. Identifying $-i \hbar \frac{\partial}{\partial x}$ as the operator for momentum, we see that (10) is the Schrödinger equation for the particle.

\section{Conclusion}

Even though the mathematics here is more involved, the approach of the present chapter (expressed in more detail in [2]) should serve to take some of the mystery off the ordinary formal introduction to quantum theory. A challenge for the future will be to develop the corresponding relativistic theory, by using representations of the Poincaré group together with an argument like that in Section 11. Also, one should seek a link to elementary particle physics using the relevant Lie group theory. Group theory is an important part of physics, and it should come as no surprise that this also is relevant to the foundation of quantum mechanics.

\section{Author details}

Inge S. Helland

* Address all correspondence to: ingeh@math.uio.no

Department of Mathematics, University of Oslo, Blindern, Oslo, Norway

\section{References}

[1] Ballentine, L. E. (1998). Quantum Mechanics. A Modern Development. Singapore: World Scientific.

[2] Helland, I. S. (2012). A Unified Scientific Basis for Inference. Submitted as a Springer Brief; arXiv:1206.5075. 
[3] Birnbaum, A. (1962). On the foundation of statistical inference. Journal of the American Statistical Association 57, 269-326.

[4] Holevo A.S. (2001). Statistical Structure of Quantum Theory. Berlin: Springer-Verlag.

[5] Helland, I.S. (2010). Steps Towards a Unified Basis for Scientific Models and Methods. Singapore: World Scientific.

[6] Schweder, T. and Hjort, N.L. (2002). Confidence and likelihood. Scandinavian Journal of Statistics 29, 309-332.

[7] Xie, M. and Singh, K. (2012). Confidence distributions, the frequentist distribution estimator of a parameter - a review. To appear in International Statistical Review

[8] Lehmann, E.L. and Casella, G. (1998). Theory of Point Estimation. New York: Springer.

[9] Barndorff-Nielsen, O.E., Gill, R.D. and Jupp, P.E. (2003). On quantum statistical inference. Journal of the Royal Statistical Society B 65, 775-816.

[10] Hammond, P.J. (2011). Laboratory games and quantum behaviour. The normal form with a separable state space. Working paper. University of Warwick, Dept of Economics.

[11] Caves, C.M., Fuchs, C.A. and Schack, R. (2002). Quantum probabilities as Bayesian probabilities. Physical Review A65, 022305.

[12] Helland, I.S. (2008). Quantum mechanics from focusing and symmetry. Foundations of Physics 38, 818-842.

[13] Busch, P. (2003). Quantum states and generalized observables: A simple proof of Gleason's Theorem. Physical Review Letters 91 (12), 120403.

[14] Einstein, A., Podolsky, B. and Rosen, N. (1935). Can quantum mechanical description of physical reality be considered complete? Physical Review 47, 777-780.

[15] Bohr, N. (1935a). Quantum mechanics and physical reality. Nature 136, 65.

[16] Bohr, N. (1935b). Can quantum mechanical description of physical reality be considered complete? Physical Review 48, 696-702.

[17] Nisticó, G. and Sestito, A. (2011). Quantum mechanics, can it be consistent with reality? Foundatons of Physics 41, 1263-1278.

[18] Schlosshauer, M. (2007). Decoherence and the Quantum-to Classical Transition. Berlin: Springer-Verlag.

[19] Klebaner, F.C. (1998). Introduction to Stochastic Calculus with Applications. London: Imperial College Press.

[20] Nelson, E. (1967). Dynamic Theories of Brownian Motion. Princeton: Princeton University Press. 


\title{
Relational Quantum Mechanics
}

\author{
A. Nicolaidis \\ Additional information is available at the end of the chapter
}

http://dx.doi.org/10.5772/54892

\section{Introduction}

Quantum mechanics (QM) stands out as the theory of the 20th century, shaping the most diverse phenomena, from subatomic physics to cosmology. All quantum predictions have been crowned with full success and utmost accuracy. Yet, the admiration we feel towards QM is mixed with surprise and uneasiness. QM defies common sense and common logic. Various paradoxes, including Schrodinger's cat and EPR paradox, exemplify the lurking conflict. The reality of the problem is confirmed by the Bell's inequalities and the GHZ equalities. We are thus led to revisit a number of old interlocked oppositions: operator operand, discrete - continuous, finite -infinite, hardware - software, local - global, particular - universal, syntax - semantics, ontological - epistemological.

The logic of a physical theory reflects the structure of the propositions describing the physical system under study. The propositional logic of classical mechanics is Boolean logic, which is based on set theory. A set theory is deprived of any structure, being a plurality of structure-less individuals, qualified only by membership (or non-membership). Accordingly a set-theoretic enterprise is analytic, atomistic, arithmetic. It was noticed as early as 1936 by Neumann and Birkhoff that the quantum real needs a non-Boolean logical structure. On numerous cases the need for a novel system of logical syntax is evident. Quantum measurement bypasses the old disjunctions subject-object, observer-observed. The observer affects the system under observation and the borderline between ontological and epistemological is blurred. Correlations are not anymore local and a quantum system embodies multiple entanglements. The particular-universal dichotomy is also under revision. While a single quantum event is particular, a plethora of quantum events leads to universal patterns. Viewing the quantum system as a system encoding information, we understand that the usual distinction between hardware and software is not relevant. Most importantly, if we consider the opposing terms being-becoming, we realize that the emphasis is sifted to the becoming, the movement, the process. The underlying dynamics is governed by relational 
principles and we have suggested [1] that the relational logic of C. S. Peirce may serve as the conceptual foundation of QM.

Peirce, the founder of American pragmatism, made important contributions in science, philosophy, semeiotics and notably in logic. Many scholars (Clifford, Schröder, Whitehead, Lukasiewicz) rank Peirce with Leibniz and Aristotle in the history of thought. Logic, in its most general sense, is the formal science of representation, coextensive with semeiotics. Algebraic logic attempts to express the laws of thought in the form of mathematical equations, and Peirce incorporated a theory of relations into algebraic logic $[2,3]$. Relation is the primary irreducible datum and everything is expressed in terms of relations. A relational formulation is bound to be synthetic, holistic, geometric. Peirce invented also a notation for quantifiers and developed quantification theory, thus he is regarded as one of the principal founders of modern logic.

In the next section we present the structures of the relational logic and a representation of relation which will lead us to the probability rule of QM. In the third section we analyze a discrete system and demonstrate the non-commutation of conjugate operators. In the last section we present the conclusions and indicate directions for future work.

\section{The logic of relations and the quantum rules}

The starting point is the binary relation $S_{i} R S_{j}$ between the two 'individual terms' (subjects) $S_{j}$ and $S_{i}$. In a short hand notation we represent this relation by $R_{i j}$. Relations may be composed: whenever we have relations of the form $R_{i j}, R_{j l}$, a third transitive relation $R_{i l}$ emerges following the rule $[2,3]$

$$
R_{i j} R_{k l}=\delta_{j k} R_{i l}
$$

In ordinary logic the individual subject is the starting point and it is defined as a member of a set. Peirce, in an original move, considered the individual as the aggregate of all its relations

$$
S_{i}=\sum_{j} R_{i j}
$$

It is easy to verify that the individual $S_{i}$ thus defined is an eigenstate of the $R_{i i}$ relation

$$
R_{i i} S_{i}=S_{i} .
$$

The relations $R_{i i}$ are idempotent

$$
R_{i i}^{2}=R_{i i}
$$

and they span the identity

$$
\sum_{i} R_{i i}=\mathbf{1}
$$

The Peircean logical structure bears great resemblance to category theory, a remarkably rich branch of mathematics developed by Eilenberg and Maclane in 1945 [4]. In categories 
the concept of transformation (transition, map, morphism or arrow) enjoys an autonomous, primary and irreducible role. A category [5] consists of objects A, B, C,... and arrows (morphisms) $\mathrm{f}, \mathrm{g}, \mathrm{h}, \ldots$. Each arrow $\mathrm{f}$ is assigned an object $A$ as domain and an object $B$ as codomain, indicated by writing $f: A \rightarrow B$. If $g$ is an arrow $g: B \rightarrow C$ with domain $B$, the codomain of $\mathrm{f}$, then $\mathrm{f}$ and $\mathrm{g}$ can be "composed" to give an arrow gof $: A \rightarrow C$. The composition obeys the associative law $h o(g \circ f)=(h \circ g)$ of. For each object $A$ there is an arrow $1_{A}: A \rightarrow A$ called the identity arrow of $A$. The analogy with the relational logic of Peirce is evident, $R_{i j}$ stands as an arrow, the composition rule is manifested in eq. (1) and the identity arrow for $A \equiv S_{i}$ is $R_{i i}$. There is an important literature on possible ways the category notions can be applied to physics; specifically to quantising space-time [6], attaching a formal language to a physical system [7], studying topological quantum field theories $[8,9]$, exploring quantum issues and quantum information theory [10].

A relation $R_{i j}$ may receive multiple interpretations: as the proof of the logical proposition $i$ starting from the logical premise $j$, as a transition from the $j$ state to the $i$ state, as a measurement process that rejects all impinging systems except those in the state $j$ and permits only systems in the state $i$ to emerge from the apparatus. We proceed to a representation of $R_{i j}$

$$
R_{i j}=\left|r_{i}\right\rangle\left\langle r_{j}\right|
$$

where state $\left\langle r_{i}\right|$ is the dual of the state $\left|r_{i}\right\rangle$ and they obey the orthonormal condition

$$
\left\langle r_{i} \mid r_{j}\right\rangle=\delta_{i j}
$$

It is immediately seen that our representation satisfies the composition rule eq. (1). The completeness, eq.(5), takes the form

$$
\sum_{i}\left|r_{i}\right\rangle\left\langle r_{i}\right|=\mathbf{1}
$$

All relations remain satisfied if we replace the state $\left|r_{i}\right\rangle$ by $\left|\varrho_{i}\right\rangle$, where

$$
\left|\varrho_{i}\right\rangle=\frac{1}{\sqrt{N}} \sum_{n}\left|r_{i}\right\rangle\left\langle r_{n}\right|
$$

with $N$ the number of states. Thus we verify Peirce's suggestion, eq. (2), and the state $\left|r_{i}\right\rangle$ is derived as the sum of all its interactions with the other states. $R_{i j}$ acts as a projection, transferring from one $r$ state to another $r$ state

$$
R_{i j}\left|r_{k}\right\rangle=\delta_{j k}\left|r_{i}\right\rangle \text {. }
$$

We may think also of another property characterizing our states and define a corresponding operator

$$
Q_{i j}=\left|q_{i}\right\rangle\left\langle q_{j}\right|
$$


with

$$
Q_{i j}\left|q_{k}\right\rangle=\delta_{j k}\left|q_{i}\right\rangle
$$

and

$$
\sum_{i}\left|q_{i}\right\rangle\left\langle q_{i}\right|=\mathbf{1}
$$

Successive measurements of the $q$-ness and $r$-ness of the states is provided by the operator

$$
R_{i j} Q_{k l}=\left|r_{i}\right\rangle\left\langle r_{j} \mid q_{k}\right\rangle\left\langle q_{l}\right|=\left\langle r_{j} \mid q_{k}\right\rangle S_{i l}
$$

with

$$
S_{i l}=\left|r_{i}\right\rangle\left\langle q_{l}\right|
$$

Considering the matrix elements of an operator $A$ as $A_{n m}=\left\langle r_{n}|A| r_{m}\right\rangle$ we find for the trace

$$
\operatorname{Tr}\left(S_{i l}\right)=\sum_{n}\left\langle r_{n}\left|S_{i l}\right| r_{n}\right\rangle=\left\langle q_{l} \mid r_{i}\right\rangle
$$

From the above relation we deduce

$$
\operatorname{Tr}\left(R_{i j}\right)=\delta_{i j}
$$

Any operator can be expressed as a linear superposition of the $R_{i j}$

$$
A=\sum_{i, j} A_{i j} R_{i j}
$$

with

$$
A_{i j}=\operatorname{Tr}\left(A R_{j i}\right)
$$

The individual states can be redefined

$$
\begin{aligned}
& \left|r_{i}\right\rangle \rightarrow e^{i \varphi_{i}}\left|r_{i}\right\rangle \\
& \left|q_{i}\right\rangle \rightarrow e^{i \theta_{i}}\left|q_{i}\right\rangle
\end{aligned}
$$

without affecting the corresponding composition laws. However the overlap number $\left\langle r_{i} \mid q_{j}\right\rangle$ changes and therefore we need an invariant formulation for the transition $\left|r_{i}\right\rangle \rightarrow\left|q_{j}\right\rangle$. This is provided by the trace of the closed operation $R_{i i} Q_{j j} R_{i i}$

$$
\operatorname{Tr}\left(R_{i i} Q_{j j} R_{i i}\right) \equiv p\left(q_{j}, r_{i}\right)=\left|\left\langle r_{i} \mid q_{j}\right\rangle\right|^{2}
$$


The completeness relation, eq. (13), guarantees that $p\left(q_{j}, r_{i}\right)$ may assume the role of a probability since

$$
\sum_{j} p\left(q_{j}, r_{i}\right)=1
$$

We discover that starting from the relational logic of Peirce we obtain the essential law of Quantum Mechanics. Our derivation underlines the outmost relational nature of Quantum Mechanics and goes in parallel with the analysis of the quantum algebra of microscopic measurement presented by Schwinger [11].

\section{The emergence of Planck's constant}

Consider a chain of $N$ discrete states $\left|a_{k}\right\rangle$, with $k=1,2, \ldots, N$. A relation $R$ acts like a shift operator

$$
\begin{aligned}
R\left|a_{k}\right\rangle & =\left|a_{k+1}\right\rangle \\
R\left|a_{N}\right\rangle & =\left|a_{1}\right\rangle
\end{aligned}
$$

$N$ is the period of $R$

$$
R^{N}=\mathbf{1}
$$

The numbers which satisfy $a^{N}=1$ are given by

$$
a_{k}=\exp \left(2 \pi i \frac{k}{N}\right) \quad k=1,2, \ldots, N
$$

Then we have

$$
R^{N}-1=\left(\frac{R}{a_{k}}\right)^{N}-1=\left[\left(\frac{R}{a_{k}}\right)-1\right] \sum_{j=0}^{N-1}\left(\frac{R}{a_{k}}\right)^{j}=0
$$

$R$ has a set of eigenfunctions

$$
R\left|b_{i}\right\rangle=b_{i}\left|b_{i}\right\rangle
$$

with $b_{i}$ the $N$-th root of unity $\left(b_{i}=a_{i}\right)$. It is decomposed like

$$
R=\sum_{j} b_{j}\left|b_{j}\right\rangle\left\langle b_{j}\right|
$$

Notice that we may write

$$
\left|b_{j}\right\rangle\left\langle b_{j}\right|=\frac{1}{N} \sum_{k=1}^{N}\left(\frac{R}{b_{j}}\right)^{k}
$$


The above projection operator acting upon $\left|a_{N}\right\rangle$ will give

$$
\left|b_{j}\right\rangle\left\langle b_{j} \mid a_{N}\right\rangle=\frac{1}{N} \sum_{k=1}^{N}\left(\frac{1}{b_{j}}\right)^{k}\left|a_{k}\right\rangle
$$

Matching from the right with $\left\langle a_{N}\right|$ we obtain

$$
\left\langle a_{N} \mid b_{j}\right\rangle\left\langle b_{j} \mid a_{N}\right\rangle=\frac{1}{N}
$$

We adopt the positive root

$$
\left\langle b_{j} \mid a_{N}\right\rangle=\frac{1}{\sqrt{N}}
$$

and equ. (32) becomes

$$
\left|b_{j}\right\rangle=\frac{1}{\sqrt{N}} \sum_{k=1}^{N} \exp \left[-2 \pi i \frac{j k}{N}\right]\left|a_{k}\right\rangle
$$

Inversely we have the decomposition

$$
\left|a_{m}\right\rangle=\frac{1}{\sqrt{N}} \sum_{n=1}^{N} \exp \left[2 \pi i \frac{m n}{N}\right]\left|a_{n}\right\rangle .
$$

We introduce another relation $Q$ acting like shift operator

$$
\begin{aligned}
\left\langle b_{k}\right| Q & =\left\langle b_{k+1}\right| \\
\left\langle b_{N}\right| Q & =\left\langle b_{1}\right|
\end{aligned}
$$

The relation $Q$ receives the decomposition

$$
Q=\sum_{j} a_{j}\left|a_{j}\right\rangle\left\langle a_{j}\right|
$$

Consider now

$$
\begin{gathered}
\left\langle b_{k}\right| Q R=\left\langle b_{k+1}\right| R=\exp \left[2 \pi i \frac{(k+1)}{N}\right]\left\langle b_{k+1}\right| \\
\left\langle b_{k}\right| R Q=\exp \left[2 \pi i \frac{k}{N}\right]\left\langle b_{k}\right| Q=\exp \left[2 \pi i \frac{k}{N}\right]\left\langle b_{k+1}\right|
\end{gathered}
$$


We conclude that the conjugate operators $R$ and $Q$ do not commute

$$
Q R=\exp \left[2 \pi i \frac{1}{N}\right] R Q
$$

Similarly

$$
Q^{n} R^{m}=\exp \left[2 \pi i \frac{n m}{N}\right] R^{m} Q^{n}
$$

In our discrete model the non-commutativity is determined by $N$. As $N \rightarrow \infty$ the relation-operators $Q$ and $R$ commute. However it would be hasty to conclude that as $N \rightarrow \infty$ we reach the continuum. The transition from the discrete to the continuum is a subtle affair and many options are available. Let us define

$$
L=N a \quad p=\frac{2 \pi}{L}
$$

Then

$$
\exp \left[2 \pi i \frac{1}{N}\right]=\exp [i p a]
$$

What counts is the size of the available phase space and we may use Planck's constant $h$ as a unit measuring the number of phase space cells. Using rather $\exp \left[\frac{i}{h} p a\right]$, equ.(42) becomes

$$
Q R=\exp \left[\frac{i}{\hbar} p a\right] R Q
$$

Approaching the continuum we may replace the discrete operators by exponential forms

$$
\begin{aligned}
& R=\exp \left[\frac{i}{\hbar} p X\right] \\
& Q=\exp \left[\frac{i}{\hbar} a P\right] .
\end{aligned}
$$

With $R$ and $Q$ unitary operators, $X$ and $P$ are hermitian operators. From equs. (46), (47), (48), we deduce

$$
[X, P]=i \hbar
$$

The foundational non-commutative law of Quantum Mechanics testifies that there is a limit size $\hbar \sim p a$ in dividing the phase space. With $p \sim m v \simeq m c$ we understand that $a$ represents the Compton wavelength. 


\section{Conclusions}

We are used first to wonder about particles or states and then about their interactions. First to ask about "what is it" and afterwards "how is it". On the other hand, quantum mechanics displays a highly relational nature. We are led to reorient our thinking and consider that things have no meaning in themselves, and that only the correlations between them are "real" [12]. We adopted the Peircean relational logic as a consistent framework to prime correlations and gain new insights into these theories. The logic of relations leads us naturally to the fundamental quantum rule, the probability as the square of an amplitude. The study of a simple discrete model, once extended to the continuum, reveals that only finite degrees of freedom can live in a given phase space. The "granularity" of phase space (how many cells reside within a given phase space) is determined by Planck's constant $h$.

Discerning the foundations of a theory is not simply a curiosity. It is a quest for the internal architecture of the theory, offering a better comprehension of the entire theoretical construction and favoring the study of more complex issues. We have indicated elsewhere [13] that a relation may be represented by a spinor. The Cartan - Penrose argument $[14,15]$, connecting spinor to geometry, allowed us to study geometries using spinors. Furthermore we have shown that space-time may emerge as the outcome of quantum entanglement [16].

It isn't inappropriate to connect category theory and relational logic, the conceptual foundations of quantum mechanics, to broader philosophical interrogations. Relational and categorical principles have been presented by Aristotle, Leibniz, Kant, Peirce, among others. Relational ontology is one of the cornerstones of Christian theology, advocated consistently by the Fathers (notably by Saint Gregory Palamas). We should view then science as a "laboratory philosophy" and always link the meaning of concepts to their operational or practical consequences.

\section{Acknowledgment}

This work has been supported by the Templeton Foundation.

\section{Author details}

A. Nicolaidis

* Address all correspondence to: nicolaid@auth.gr

Theoretical Physics Department, Aristotle University of Thessaloniki, Thessaloniki, Greece

\section{References}

[1] A. Nicolaidis, Categorical Foundation of Quantum Mechanics and String Theory, Int. J. Mod. Phys. A24: 1175 - 1183, 2009

[2] C. S. Peirce, Description of a notation for the logic of relatives, resulting from an amplification of the conceptions of Boole's calculus of logic, Memoirs of the American Academy of Sciences 9, pp 317-378 (1870) 
[3] C. S. Peirce, On the algebra of logic, American Journal of Mathematics 3, pp 15-57 (1880)

[4] S. Eilenberg and S. Maclane, General theory of natural equivalences, Transactions of the American Mathematical Society, 58, pp 239-294 (1945)

[5] F. Lawvere and S. Schanuel, Conceptual Mathematics: A first indroduction to categories, Cambridge University Press (1997)

[6] C. Isham, A New Approach to Quantising Space-Time: I. Quantising on a General Category, Adv. Theor. Math. Phys. 7, 331-367 (2003)

[7] A. Doring and C. Isham, A topos foundation for Theoretical Physics: I. Formal languages for physics, J. Math. Phys. 49: 053515, 2008

[8] J. Baez and J. Dolan, Higher-dimensional algebra and topological quantum field theory, J. Math. Phys. 36, 6073 (1995)

[9] J. Baez, Quantum quandaries: A category-theoretic perspective, quant-ph/0404040 preprint, to appear in Structural Foundations of Quantum Gravity, Oxford University Press

[10] S. Abramsky and B. Coecke, A categorical sematics of quantum protocols, Proceedings of the 19th IEEE conference on Logic in Computer Science (LiCS'04). IEEE Computer Science Press (2004)

[11] J. Schwinger, Proc. N.A. S. 45, 1542 (1959)

[12] Vlatko Vedral, Quantum physics: Entanglement hits the big time, Nature 425, 28-29 (4 September 2003)

[13] A. Nicolaidis and V. Kiosses, Spinor Geometry, Int. J. of Mod. Phys. A27, Issue 22, id. 1250126, arXiv:1201.6231

[14] E. Cartan, Les groupes projectifs qui ne laissent invariante aucune multiplicité plane, Bull. Soc. Math. France 41, 53-96 (1913); E. Cartan, Leçons sur la théorie des spineurs, vols 1 and 2, Exposés de Geométrie, Hermann, Paris (1938)

[15] R. Penrose, A spinor approach to General Relativity, Annals of Physics, 10, 171 201 (1960); R. Penrose and W. Rindler, Spinors and space - time, Vol. 1, Cambridge University Press (1984)

[16] A. Nicolaidis and V. Kiosses, Quantum Entanglement on Cosmological Scale, to appear 



\title{
On the Dual Concepts of 'Quantum State' and 'Quantum Process'
}

\author{
Cynthia Kolb Whitney \\ Additional information is available at the end of the chapter
}

http://dx.doi.org/10.5772/53326

\section{Introduction}

Many of us who made a living in the $20^{\text {th }}$ century did so by functioning as some kind of engineer. Though schooled mostly in Physics, this author often functioned in those days as an engineer. It was a good continuing education. One aspect of it was the big tool kit in use. For example, some subject systems were best viewed in the frequency domain: a system functioning as a filter would suppress some frequencies and enhance others. But other systems were better viewed in the time domain: a system functioning as a controller would take a time series of input signals and produce a time series of output commands. Neither approach was considered more right, or more fundamental, than the other. They were complementary.

But in the $20^{\text {th }}$ century, things felt less eclectic in Physics. Especially in the literature of Quantum Mechanics (QM), there often seemed to be a lot of passion about what viewpoint was allowed, and what viewpoint was not allowed. We were taught that it just was not correct to think of an atom as a nucleus with electrons in orbits around it. There could not be orbits; there had to be only 'orbitals', a new word coined to refer to complex wave functions that extended over all space, and provided only spatial densities of probability, in the form of squared amplitude. Except for its phase factor, there was no sense of time-line to an orbital. It was a stable state.

So in QM, the emphasis was all on the stable states. Between the stable states, there could occur transitions, resulting in emission or absorption of a photon, but the state transitions themselves were essentially instantaneous, and not open to study. This emphasis on the stable states, and the avoidance of the transitions between them, implied that questions about the details of state transitions should be regarded as illegitimate.

Back at the turn of the $20^{\text {th }}$ century, there was a good reason for the avoidance of details about process in QM: we did not understand how any atom could resist one totally destructive 
process that was expected within the context of classical electrodynamics. Even the simplest atom, the Hydrogen atom, was expected to continuously radiate away its orbit energy, and so quickly collapse. So ever paying attention to any details of process looked fraught with peril.

A way of escaping the issue of process in QM came along with the discovery of the photon. Quantization of light was implied in the spectrum of blackbody radiation, and demonstrated in the photoelectric effect. Those developments gave us Planck's constant. Planck's constant provided a constraint for defining the ground state of the Hydrogen atom. So Schrödinger wrote it into his equation for the wave function of the electron in the Hydrogen atom. The Schrödinger equation produced a set of solutions representing a whole family of stationary states for the Hydrogen atom. We could forget process, and focus on those stationary states.

But today, one of the big application areas for QM is in development of computational approaches fitting the name Quantum Chemistry (QC). Chemistry is largely about reactions, and certainly every reaction is a complex process. So the chemical reactions are like the quantum state transitions: they have, not only the stationary state before, and the stationary state after, but also something of interest in between. So the historical injunction against inquiry into the specifics of quantum state transitions tends to inhibit the full application of QM to the process-related problems that QC presents. So consideration of process is no longer avoidable for QM.

Fortunately, history is never the final story; it exists mainly to be updated from time to time. A Chapter in an earlier Book in this series (Whitney, 2012) argued that our understanding of Maxwell's Electromagnetic Theory (EMT) at the turn of the $20^{\text {th }}$ century was incomplete. When we develop a description for the photon based on EMT, we learn some facts that have bearing on the communication between the electron and the proton in the Hydrogen atom, and how that communication in turn supports the continuing existence of the atom as a system. So consideration of a quantum process becomes less perilous for QM.

But the practical difficulties are numerous. In many cases, molecules involve numbers of atoms that are too small for any kind of statistical ideas to be applicable, but too large for traditional QM calculations to be practical; hence, they are altogether awkward to address. Furthermore, Chemistry is all about reactions, which can involve many molecules. And sometimes there are multiple reaction steps, or even multiple paths, each one with multiple steps, each one with a time line worthy of detailed numerical study.

The practical difficulties of QC arise largely because the most common way of thinking about $\mathrm{QM}$ is still in terms of wave functions. Their amplitudes are squared to make probability density functions, multiplied by functions or differential operators representing variables of interest, and integrated over argument variables. It can add up to way too much computation.

So how can QM better meet the needs of QC? A potentially helpful concept comes out of QM: the concept of duality. Abad and Huichalaf (2012) describe it in terms of seeming contradiction, and seeming is certainly the right characterization, for duality is not really a contradiction at all. Consider, for example, the traditional wave vs. particle duality of light. The earlier Chapter (Whitney, 2012) presented a model for the photon based on Maxwell's four coupled field equations, together with boundary conditions representing the source and the receiver of the 
photon. The Maxwell photon model is pulse-like at emission, and evolves into an extended wave-like condition, and then collects back into a more confined pulse-like condition for absorption. In QM, all observable objects are like the Maxwell photon model, in that they present both particle-like and wave-like aspects. Which aspect is seen just depends on when and how one observes the object.

To meet the needs of QC, we clearly need to develop and exploit another duality analogous to the traditional wave $v$ s. particle duality of observed objects. We need a duality of observer descriptions: state descriptions vs. process descriptions. Where the traditional QM approach starts with the idea of quantum state, which is something stationary, the dual approach must start with the idea of quantum process, which naturally has a time line to it. The temporal evolution of the Maxwell photon model is fundamentally a process, and it can be followed in detail, with no untoward disasters, so it is a promising point of departure for this work.

One key thing about photons is that they have finite energy. In this respect, photons are completely different from infinite plane waves, which have infinite energy. The Maxwell photon model has the finite total energy as needed. That energy is always trapped in the space between the source and the receiver. So another key role in the photon model is played by mathematical boundary conditions. Section 2 picks up where the earlier work left off, discussing in more detail what the boundary conditions are, what they do, and how they do it.

Photons were not known in Maxwell's day, so the implication of their finite energy was not then appreciated. Coupled with their finite propagation speed, their finite energy causes the definite Arrow of Time that has long been considered such a mystery in Physics. Many people suppose that the Arrow of Time has to do with Thermodynamics, because that subject deals with entropy and irreversibility. Searching for a mechanism, many people would think of friction in Newtonian mechanics. When told that Electrodynamics displays the Arrow of Time, many people think first of the friction-like effect of radiation reaction acting on accelerating charges. But actually, the Arrow of Time is present quite apart from anything that happens to material particles. It appears in the photon itself.

There is a reason why this fact was not emphasized a long time ago. Section 3 recalls how Maxwell's coupled field equations were immediately inserted, one into another, in order to reduce the set of four coupled field equations into a set of two un-coupled wave equations. The two un-coupled wave equations clearly display the finite propagation speed, but they totally hide the effect of finite energy.

The problem is this: the two un-coupled wave equations are less restrictive than the four coupled field equations are, so they have a larger set of solution functions, some of which do not also solve the four coupled field equations. One example of such a solution is a finite-energy pair of orthogonal E and B pulses that travels without distortion and faithfully delivers information.

In the early $20^{\text {th }}$ century, Einstein used this kind of solution for the role of 'signal' in his Special Relativity Theory (SRT). His goal was to capture the spirit of Maxwell's EMT into SRT. But his signal model is inadequate for that job. The Maxwell photon model better captures the spirit of Maxwell, and only slightly modifies the SRT results, and better ties those results into QM. 
What about the QM of material particles? Section 4 revisits the Schrödinger equation. Viewed as the analog to a statement from Newtonian mechanics, it has no essential irreversibility to it. That is one reason why its solutions are stable states. That is why something else is needed for the study of transitions between the quantum states of atoms, and for the numerous chemical processes that QC should address.

Section 5 points out that the main thing for Chemistry is not revealed in the Hydrogen atom, due to the fact that the Hydrogen atom has only one electron. The main thing for Chemistry is the variety of relationships among multiple electrons. Chemistry data suggest that electrons can sometimes actually attract, rather than repel, each other. That propensity can be important in driving chemical reactions. The calculation approach suitable for QC was introduced in the earlier Chapter (Whitney 2012). It is called Algebraic Chemistry (AC). It is a big subject, detailed further in a full Book (Whitney 2013).

Section 6 concludes this Chapter. It draws a lesson from all the problems treated here. The lesson is that we sometimes actually make problems very much worse than they need to be by oversimplifying them. Many seeming mysteries in physical science are nothing but our own creations.

\section{More about photons}

The earlier Chapter (Whitney, 2012) revisited the quantum of light, the photon, and its relationship to Classical Electrodynamics. That Chapter argued that a simple mental picture of a photon as a pair of electric and magnetic field pulses that travel together, but do not change their pulse shapes, does not comport with Maxwell's four coupled field equations. Instead, there has to be a temporal evolution, first from pulses emitted by a source, into a waveform shape extended in the propagation direction, then back to a more compact shape, concluding with absorption by a receiver; in short, a whole time-line process.

This Section gives further mathematical detail about the temporal evolution of the photon waveform. In summary, the evolution begins with emission of Gaussian field pulses at the photon source. After emission, the fields develop according to Maxwell's coupled field equations. The development is constrained and guided by boundary conditions that represent the initial source of the photon and the ultimate receiver of the photon. In the end, all the energy accumulates near the receiver, and can finally be swallowed by it.

In more detail, Maxwell's coupled field equations cause the Gaussians in E and B to beget firstorder Hermite polynomials in B and E, and then those beget second-order Hermite polynomials in $\mathrm{E}$ and $\mathrm{B}$, and so on, indefinitely as time goes on. The roots of each newly generated Hermite polynomial interleave with the roots of the previously generated Hermite polynomial, with one more root being added at each step of the process. This process is illustrated with Figure 1 in (Whitney, 2012). It shows spreading of the waveform in its propagation direction.

Next, there have to be boundary conditions to represent the source and the receiver. The boundary conditions can be like those representing the mirrors in a laser cavity: they can enforce a zero in the $\mathrm{E}$ field at the boundary locations. As a result of the zero-E condition at 
the source, the spreading never causes any backflow of field energy into the space behind the source. And as a result of the zero-E condition at the receiver, the waveform spreading never causes any field energy to propagate into the space beyond the receiver. So eventually all the energy just 'piles up' before its final absorption into the receiver.

A mental picture of the photon in terms of electric and magnetic fields is quite complicated, even without the boundary conditions. There have to be two field vectors $E$ and $B$ in orthogonal directions to create a cross product, the Poynting vector of energy propagation. And there have to be two such pairs of fields, one a quarter cycle out of phase with the other in both space and time, to create circular polarization. That makes a quartet of field vectors to think about. Then to create the boundary conditions, there have to be two more such quartets of field vectors, arranged to propagate in the opposite direction, and placed to provide the E-field cancellations at the boundaries. But each of these field vector quartets slightly spoils the boundary condition fixed by the other one. So then an infinite regression of more and more field quartets is demanded.

A mental picture of the photon can be formulated much more simply in terms of its overall profile of energy density, $\left(E^{2}+B^{2}\right) / 2$. Before the boundary conditions are imposed, the energy density profile is always a simple Gaussian, the height of which decreases over time, and the width of which increases over time. Then to impose the boundary conditions, the infinite Gaussian tails get folded, and refolded infinitely many times over, back into the space between the source and the receiver. That means the energy density profile is a slightly deformed from Gaussian in the vicinity of the source, and the vicinity of the receiver: cut off sharply at those points, and slightly more than doubled in height near them, because zero $E$ means double $B$ and double energy density. Of course, the total energy, the integral of the energy density profile between the source and the receiver, never changes.

Figure 1 illustrates this mental picture of the photon as a changing energy density profile with constant total energy. The three data series plotted correspond to the energy density profiles near the beginning, in the middle, and near the end, of the photon propagation scenario. Take note of the phenomenon of waveform spreading. It is not reversible. It shows the Arrow of Time.

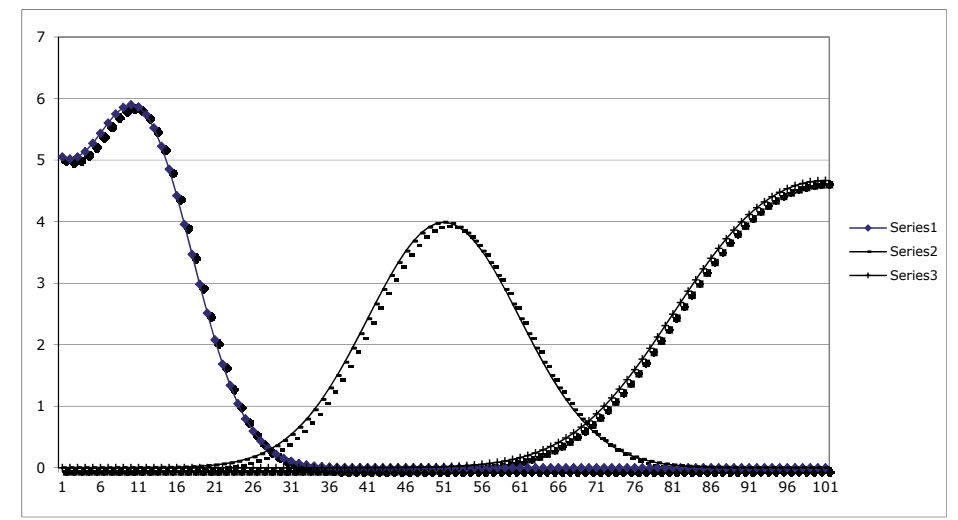

Figure 1. Photon energy density profile at three stages. 


\section{On the arrow of time}

There is an interesting history to the Arrow of Time, and it begins with the beginning of Mathematical Physics itself. Newton developed his laws of mechanics at a time of great interest in celestial events. In that sphere, the absence of contact invites the assumption of no friction, and in the absence of friction, Newtonian mechanics is invariant under time reversal. In this case, there is in principle no Arrow of Time. However, in practice there is a related problem about chaos, which develops in the mathematics when three or more bodies interact. It was certainly impossible to solve for three trajectories in closed form, i.e. as simple functions of time. Even today, it is not entirely clear exactly what is possible to do.

Maxwell developed his electromagnetic theory at a time of developing industrial technology. In that domain, the first thing encountered is limitation. With real-world machines, there is always an Arrow of Time, and it is best described with the science of Thermodynamics. In Maxwell's electrodynamics, there are two important features. First, there is not instantaneousaction-at-a-distance; there is a finite propagation speed. It shows up clearly with propagating waves. Second, there can be infinite plane waves in the mathematics, but not in physical reality. Reality is always about finite energy.

The finite propagation speed was discovered first. People quickly transformed Maxwell's four coupled field equations into something more familiar: two un-coupled wave equations. They then often fixated on the infinite plane wave solutions of those equations. The implications of finite energy were then left for investigation later. The job was partially addressed in the study of Optics: finite apertures create diffraction patterns transverse to the propagation direction. But the implications of pulsing in the propagation direction itself would become interesting only much later; for example, with the invention of pulsed lasers.

But much work was left undone at that time. So let us pursue the investigation a little further now. In modern notation and Gaussian units, Maxwell's four coupled field equations go

$\nabla \boldsymbol{B}=0, \nabla \quad \boldsymbol{D}=4 \pi \rho, \nabla \times \boldsymbol{E}+\frac{1}{c} \mathrm{\partial} \boldsymbol{B} / \mathrm{\partial} t=0, \nabla \times \boldsymbol{H}-\frac{1}{c} \partial \boldsymbol{D} / \partial t=\frac{4 \pi}{c} J$.

Here $\boldsymbol{B}$ is the magnetic field vector and $\boldsymbol{E}$ is the electric field vector. In free space $\boldsymbol{D}=\varepsilon_{0} \boldsymbol{E}$, $\boldsymbol{H}=\boldsymbol{B} / \mu_{0}, 1 / c=\sqrt{\varepsilon_{0} \mu_{0}}$, and charge density $\rho$ and current density vector $\boldsymbol{J}$ are zero.

The two uncoupled wave equations go

$\nabla^{2} \boldsymbol{E}-\frac{1}{c^{2}} \partial^{2} \boldsymbol{E} / \partial t^{2}=0, \nabla^{2} \boldsymbol{B}-\frac{1}{c^{2}} \partial^{2} \boldsymbol{B} / \partial t^{2}=0$.

where $1 / c^{2}=\varepsilon_{0} \mu_{0}$.

Because the time derivatives in the two un-coupled wave equations are second order, the sign attributed to time cancels out. So the two un-coupled wave equations are invariant under time reversal. This fact means the two un-coupled wave equations are not equivalent to the four coupled field equations. The two un-coupled wave equations have a larger set of solutions than do the four coupled field equations. So while all of the solutions to the four coupled field 
equations will satisfy the two un-coupled wave equations, only some of the solutions to the two un-coupled wave equations will satisfy the four coupled field equations.

So some solutions to the two un-coupled wave equations do not satisfy the four coupled field equations. The infinite plane wave is one of those. It has the parameter $c$, which was interpreted as the speed of propagation for light, not just in the context of the infinite plane wave, but also in every other context; in particular, in the context of the concept of signal that Einstein used in the development of Special Relativity Theory (SRT).

But the signal for SRT cannot be just an infinite plane wave. A signal has to have some discernable feature in order to carry any information. Bracken (2012) discusses the mathematical concept of information, and its relation to entropy. The infinite plane wave has a deltafunction spectrum, meaning just one wavelength, zero entropy, and hence zero information content.

Instead of an infinite plane wave, a signal needs finite $E$ and B pulses. These can be constructed from a spectrum of infinite plane waves with infinitesimal amplitudes. But as we have seen, such pulses have to evolve over time into spread-out waveforms. Pulses without such evolution can satisfy the two uncoupled wave equations, but they cannot satisfy Maxwell's four coupled field equations.

The purpose of the SRT signal concept was to capture the essence of Maxwell's EMT into SRT. So in retrospect, it does not seem very appropriate to have used a model for the signal that does not satisfy Maxwell's four coupled field equations. It seems more appropriate to use the Maxwell photon model instead. Einstein did develop SRT at nearly the same time as he did his Nobel-prize winning work about the photoelectric effect - and the photon. But mysteriously, the idea that the SRT signal is similar to the photon did not come up at that time.

Better late than never, we can tackle that problem now. Clearly, at the beginning of the propagation scenario, the boundary condition at the source dominates the energy profile, and at the end of the propagation scenario, the boundary condition at the receiver dominates the energy profile. From this assessment, it is easy to imagine a generalization of the propagation scenario. Like the mirrors in a tunable laser, the source and the receiver in the propagation scenario can be imagined to move relative to each other. This possibility then suggests the deep question:

\section{What is the frame of reference for light speed $c$ ?}

This question lies at the heart of SRT. There, the reference for light speed $c$ is any and all observers. That is Einstein's Second Postulate. To be fair, it was also the hidden Assumption of all prior works in Electrodynamics. So Einstein is to be credited for making it so explicit.

But a Postulate is a rather formalized way of stating an Assumption, and, as such, does not invite as much scrutiny, or convey as much reward when scrutiny is given, as an Assumption would do. Linguistically, the Second Postulate may seem quite incomprehensible, but its status of Postulate, rather than Assumption, invites a level of deference. This appears to be a partial answer for the 'Why?' articulated above. 
However, use of the Maxwell photon model instead of the Einstein signal does allow a more linguistically normal kind of statement. Observe that during times soon after the emission, the boundary condition that is numerically more significant is the one demanding zero $\mathrm{E}$ at the source. But during times just before the reception, the boundary condition that is numerically more significant is the one demanding zero $\mathrm{E}$ at the receiver. The consequence is that the photon energy starts out traveling at speed $c$ relative to the source, but then finishes up traveling at speed $c$ relative to the receiver. In between, the reference for $c$ has to transition gradually from one frame to the other, along with the numerical dominance of the corresponding boundary conditions.

This analysis leaves us with a conundrum: If the Maxwell photon model can be adopted as the signal for developing SRT, then the Einstein's Second Postulate is true at the moment of reception, but not generally true at any moment before that event. So then SRT cannot be universally valid. But if the Maxwell photon model cannot be adopted as the signal for developing SRT, then SRT should not be considered as founded in Maxwell's EMT. So then SRT is not founded in any prior science. This conundrum does not disqualify SRT as a useful theory, but it does mean that prudence demands investigation of other theories too.

Use of the Maxwell photon model instead of Einstein's Second Postulate does produce results somewhat different from those of Einstein's SRT. Fortunately, it is not very difficult to work out these slightly different results. The different results that are important for connecting better with QM are given in Whitney (2012). In summary, they are the following: 1) Within the Hydrogen atom, there exists not just the one electromagnetic process, the radiation from the accelerating electron; there exist three electromagnetic processes. The other two are: 2) torquing internal to the atomic system, and 3) circular motion of its center of mass. The torquing produces an energy gain mechanism that more than compensates the energy loss due to radiation. But the center-of-mass motion amplifies the radiation loss, bringing the atomic system into its final balance. The combination of these three electromagnetic processes, instead of just one, makes it possible to model a stable Hydrogen atom electromagnetically.

Here is how finite signal propagation speed causes the second and third effects. Each particle is attracted to a former position of the other. The forces are not central, and are not even balanced. The non-centrality centrality causes the torquing. The force imbalance causes the center of mass circulation. That in turn causes Thomas rotation, which amplifies the radiation. It is interesting that Thomas rotation was first discovered in the context of sequential Lorentz transformations, and is generally believed to be a consequence of SRT. See, for example, De Zela (2012). But Thomas rotation actually does not depend on Lorentz transformations; it emerges just as well from sequential Galilean transformations. It is not a relativistic effect.

\section{More about the hydrogen atom}

The treatment of the Hydrogen atom given in Whitney 2012 was the first-order approximation, in which the cosine and the sine of an angle traversed around the circular orbit were repre- 
sented to first order, by unity and by the angle itself. Let us now go further. The total radiation power changes from

$P_{\text {total radiated }} \approx 2^{4}\left(2 e^{2} / 3 c^{3}\right) a_{\mathrm{e}}^{2}=\left(2^{5} e^{6} / m_{\mathrm{e}}^{2}\right) / 3 c^{3}\left(r_{\mathrm{e}}+r_{\mathrm{p}}\right)^{4}$

to

$P_{\text {total radiated }} \approx\left[\left(2^{5} e^{6} / m_{\mathrm{e}}^{2}\right) / 3 c^{3}\left(r_{\mathrm{e}}+r_{\mathrm{p}}\right)^{4}\right] \times \cos ^{2}\left(v_{\mathrm{e}} / 2 c\right)$.

The total torquing power changes from

$P_{\text {torquing }} \approx \frac{m_{\mathrm{e}}}{m_{\mathrm{p}}} \frac{r_{\mathrm{e}} \Omega_{\mathrm{e}}^{2}}{c} \frac{e^{2}}{\left(r_{\mathrm{e}}+r_{\mathrm{p}}\right)}=\left(e^{4} / m_{\mathrm{p}}\right) / c\left(r_{\mathrm{e}}+r_{\mathrm{p}}\right)^{3}$

to

$P_{\text {torquing }}=\left[\left(e^{4} / m_{\mathrm{p}}\right) / c\left(r_{\mathrm{e}}+r_{\mathrm{p}}\right)^{3}\right] \times \frac{1}{2}\left[\frac{\sin \left(v_{\mathrm{e}} / 2 c\right)}{\left(v_{\mathrm{e}} / 2 c\right)}+\frac{\sin \left(v_{\mathrm{p}} / 2 c\right)}{\left(v_{\mathrm{p}} / 2 c\right)}\right]$.

Observe that both of these expressions are oscillatory. But where $P_{\text {total radiated }}$ is confined to non-negative values, $P_{\text {torquing }}$ is not. So while $P_{\text {total radiated }}$ always represents a mechanism for energy loss, $P_{\text {torquing }}$ does not always represent a mechanism for energy gain. For electron orbit radius $r_{\mathrm{e}}$ below the Hydrogen ground state orbit, $P_{\text {torquing }}$ oscillates between giving energy gain and giving energy loss. Therefore, there exist many values of $r_{\mathrm{e}}$ where $P_{\text {total radiated }}=P_{\text {torquing' }}$ and the Hydrogen atom can exist, and even persist. These represent 'substates' of the Hydrogen atom. Such states are sometimes discussed in the literature of experimental physics, but never in the literature of theoretical physics. For one thing, they seem to involve orbit speeds beyond the speed of light, and so violate SRT. For another thing, they are not within the universe of discourse of the Schrödinger approach to the Hydrogen atom.

\section{The Schrödinger approach to the hydrogen atom}

Schrödinger's famous equation representing a particle, such as an electron, reads:

$i \hbar \frac{\partial}{\partial t} \Psi(r, t)=\left[-\frac{\hbar^{2}}{2 m} \nabla^{2}+V(r, t)\right] \Psi(r, t)$,

where $\Psi(r, t)$ is the wave function, $r$ is position in three-dimensional space, and $t$ is time. The $\hbar$ is the reduced Planck's constant $h / 2 \pi, m$ is the particle mass, $\nabla^{2}$ is the usual three-space second-derivative operator, and $V(r, t)$ is the potential energy, created for example by a nucleus. For an atom that is not moving, and is not perturbed by some measuring device, $V(r, t)$ is time-invariant, reducing to $V(r)$.

Schrödinger's equation is about a wave function, and not about a particle. So in the beginning, it seemed to have no clear foundation in the science prior to its own time. It was taken as a gift from heaven. But actually, Schrödinger's equation does have a foundation - just not entirely within Physics, but also partly within Engineering Science. The following analysis shows that Schrödinger's equation reduces to a classical equation based on Newton's laws. The reduction uses Fourier transforms, a tool very commonly used in Engineering Science. 
Consider first the wave function $\Psi(r, t)$. By definition, it satisfies the normalization condition $\int_{-\infty}^{\infty} \Psi(r, t) \Psi^{*}(r, t) d^{3} r=\int_{-\infty}^{\infty}|\Psi(\boldsymbol{r}, t)|^{2} d^{3} \boldsymbol{r}=1$.

The function $\Psi(r, t)$ has four-dimensional Fourier transform $\Psi(p, E)$, where $p$ is momentum and $E$ is total energy. This Fourier transform is defined by:

$\Psi(p, E)=\frac{1}{(2 \pi)^{2}} \int_{-\infty}^{\infty} \Psi(\boldsymbol{r}, t) \exp [i(p \quad r-E \cdot t) / \hbar] d^{3} \boldsymbol{r} d t$

This function too satisfies a normalization condition

$\int_{-\infty}^{\infty} \Psi(p, E) \Psi^{*}(p, E) d^{3} p=\int_{-\infty}^{\infty}|\Psi(p, E)|^{2} d^{3} p=1$.

The corresponding inverse Fourier transform is defined by

$\Psi(r, t)=\frac{1}{(2 \pi)^{2}} \int_{-\infty}^{\infty} \Psi(p, E) \exp [-i(p \quad r-E \cdot t) / \hbar] d^{3} p d E$.

(An aside: definitions of Fourier transforms for other applications sometimes deploy the factors of $2 \pi$ differently, although always such that the round trip from one space to the other and back again has $1 / 2 \pi$ for each dimension.)

Observe first of all that if $\Psi(r, t)$ is very sharply peaked over a small range $\Delta \boldsymbol{r}$, say centered at $\boldsymbol{r}=\mathbf{0}$, then $\Psi(\boldsymbol{p}, E)$ will be very spread out over a large range $\Delta \boldsymbol{p}$, centered at $\boldsymbol{p}=\mathbf{0}$. And vice versa: large $\Delta r$ makes for small $\Delta p$. That means the Fourier pair of functions $\Psi(r, t)$ and $\Psi(p, E)$ automatically generates a relationship that looks like the Heisenberg uncertainty relationship.

The product $\Delta r \quad \Delta p$ has its minimum possible value when $\Psi(r, t)$ is a Gaussian function, in which case $\Psi(p, E)$ is also a Gaussian function. For the Physics application, the product $\left(\begin{array}{ll}\Delta & \Delta p\end{array}\right)_{\text {Gaussian }}$ is Planck's constant $h$.

There is no way around the law $\Delta r \quad \Delta p \geq(\Delta r \quad \Delta p)_{\text {Gaussian. }}$. It is a property of Mathematics in general, and not of QM in particular. But physicists do worry about its meaning for QM. For example, Cini (2012) seems ready to remove the deBroglie and Schrödinger classical probability wave approaches from the main narrative of QM, and begin it instead with quantum field theory. One problem with this strategy is the risk of putting too much trust in SRT, which appears possibly flawed in its founding Postulate.

In contrast to physicists, engineers just accept the law $\Delta r \quad \Delta p>(\Delta r \quad \Delta p)_{\text {Gaussian }}$ because in their world there never exists a measurement without a spread, and they regard any proposed perfectly precise physical quantity as just a metaphysical idea, and not a real physical thing.

The present analysis proceeds in that spirit. The next step is to rewrite Schrödinger's equation in the form:

$\left[-\frac{\hbar^{2}}{2 m} \nabla^{2} \Psi(r, t)\right] \Psi^{*}(r, t)+[V(r, t) \Psi(r, t)] \Psi^{*}(r, t)=\left[i \hbar \frac{\partial}{\partial t} \Psi(r, t)\right] \Psi^{*}(r, t)$. 
With its seemingly superfluous $\Psi^{*}(r, t)$ factors, this form of Schrödinger's equation looks more complicated than the original form. But this form ultimately leads to tremendous simplification and explanatory power. And, as in Berkdemir (2012), the objective here is mainly pedagogical.

The first term on the left side of the rewritten Schrödinger's equation is:

$$
\begin{gathered}
{\left[-\frac{\hbar^{2}}{2 m} \nabla^{2} \Psi(r, t)\right] \Psi^{*}(r, t)=} \\
\frac{1}{(2 \pi)^{2}} \int_{-\infty}^{\infty} \frac{p^{2}}{2 m} \Psi(p, E) \exp [-i(p \quad r-E \cdot t) / \hbar] d^{3} p d E \frac{1}{(2 \pi)^{2}} \int_{-\infty}^{\infty} \Psi^{*}\left(p^{\prime}, E^{\prime}\right) \exp \left[+i\left(p^{\prime} \quad r-E^{\prime} \cdot t\right) / \hbar\right] d^{3} p^{\prime} d E^{\prime} \\
=\int_{-\infty}^{\infty} \frac{p^{2}}{2 m} \Psi(p, E) \Psi^{*}(p, E) d^{3} p d E=\left\langle\frac{p^{2}}{2 m}\right\rangle
\end{gathered}
$$

where $\langle>$ indicates statistically average value.

The second term on the left side is just:

$$
\int_{-\infty}^{\infty} V(r, t) \Psi(r, t) \Psi^{*}(r, t) d^{3} r d t=\langle V(r, t)\rangle
$$

The one term on the right side is:

$$
\begin{gathered}
{\left[i \hbar \frac{\partial}{\partial t} \Psi(r, t)\right] \Psi^{*}(r, t)=} \\
\frac{1}{(2 \pi)^{2}} \int_{-\infty}^{\infty} E \Psi(p, E) \exp [-i(p \quad r-E \cdot t) / \hbar] d^{3} p d E \frac{1}{(2 \pi)^{2}} \int_{-\infty}^{\infty} \Psi^{*}\left(p^{\prime}, E^{\prime}\right) \exp \left[+i\left(p^{\prime} \quad r-E \cdot t\right) / \hbar\right] d^{3} p^{\prime} d E^{\prime} \\
=\int_{-\infty}^{\infty} E \Psi(p, E) \Psi^{*}(p, E) d^{3} p d E=\langle E\rangle
\end{gathered}
$$

So viewed in this way, Schrödinger's equation reads:

$\left\langle\frac{p^{2}}{2 m}\right\rangle+\langle V(\boldsymbol{r}, t)\rangle=\langle E\rangle$.

This presentation of Schrödinger's equation just says that the classical kinetic energy plus the potential energy makes the total energy. This is basically a statement from classical mechanics, ultimately derivable from Newton's laws.

Observe that if $V(r, t)$ is time invariant, and so reduces to just $V(r)$, then $|\Psi(\boldsymbol{r}, t)|$ is also time invariant, and reduces to just $|\Psi(r)|$. That is, time then enters into the wave function $\Psi(r, t)$ only through its un-observable phase factor. That is why $\Psi(r, t)$ represents a stationary state for the Hydrogen atom.

Observe too that, however one writes Schrödinger's equation, there is no parameter $c$, or any other trace of Maxwell theory in it. That is why Schrödinger's equation cannot give any clue about sub-states of the Hydrogen atom.

Observe next that, in giving only stationary $\Psi(r, t)$ solutions, Schrödinger's equation does not reveal the irreversibility that we know exists in our macroscopic world. Lunin (2012) has identified this absence of irreversibility in Schrödinger's equation as an unsolved problem. Skála \& Kapsa (2012) have noted that the measuring apparatus is not described in QM, and 
they have worked out an approach to deal with that deficit. Streklas (2012) has done the same with regard to the surrounding environment in which the system sits.

(An aside: the background could include a gravitational field, and Streklas notes how classical General Relativity Theory (GRT) breaks down at the Planck scale. As a cause for this failure, I suspect the SRT background from which GRT developed.)

In the present exposition, the time variation of $V(r, t)$ can represent the intrusion of a measurement process, or an environmental factor, or some other disturbance, and then Schrödinger's equation can capture the phenomenon of irreversibility.

Can the foundation for Schrödinger's equation be further explicated by using the proposed Maxwell photon model? Recall that in its emission / propagation / reception scenario, the Maxwell photon model naturally displays particle-like localization at the two ends, and wavelike periodicity in the middle. The middle part of the photon scenario establishes a precedent for he use of the wave function as the subject of the Schrödinger equation.

The Maxwell photon model also helps explain why Schrödinger's equation seemingly demands complex numbers, while Physics before that time used them for convenience, but not out of necessity. Recall that that the Maxwell photon model has a second $\boldsymbol{E}, \quad \boldsymbol{B}$ vector pair a quarter cycle out of phase with the first $E, \quad B$ vector pair to make the circular polarization. That sort of phase issue naturally brings complex numbers.

Also, recall that the important output from the Maxwell photon model was its energy density, defined in terms of squared electric and magnetic fields. If we represented the fields a quarter cycle out of phase as imaginary numbers, then we would need fields, not just squared, but multiplied by complex conjugate fields. That operation would resemble the familiar $|\Psi|^{2}=\Psi \Psi^{*}$ operation for probability density.

Finally, the Maxwell photon model can help clarify the issue of 'duality'. The word has been taken to suggest a mysteriously simultaneous wave-particle character. But the general Schrödinger equation, with a time-dependent $V(r, t)$ to represent some sort of measurement process, could certainly display the same less mysterious, more pedestrian, kind of duality that the Maxwell photon displays: sequential particle-like and wave-like behaviors.

Schrödinger's equation gives a lot more than just the ground state of Hydrogen. Like Maxwell's equations, it admits an infinite set of solutions. They are currently understood as representing an infinite set of excited states of the Hydrogen atom.

What exactly are excited states of the Hydrogen atom? The usual understanding is that they refer to something like spherical neighborhoods around the nucleus at larger radii, and that the electron can live in any one of these neighborhoods, and if it tumbles into a lower neighborhood, then a photon will be released.

I want to encourage readers to consider also any and all alternative interpretations that may be offered for the meaning of the term 'excited state'. My own working idea (Whitney 2012) is that 'excited state' does not refer to an attribute that a single Hydrogen atom can possess. The Hydrogen atom is too simple; it has too few degrees of freedom. My mental image of 'excited state' is a system involving, not one, but several, Hydrogen atoms. 
The basis for such a candidate interpretation is that a balance between radiation and torquing works out, not only for two charges of opposite sign, but also for two, or more, charges of the same sign - if superluminal orbit speeds are allowed. And what is there to disallow them? The only factors are Einstein's Second Postulate and his resulting SRT, which together embed a rash denial of the well-known Arrow of Time. So be prudent; don't a priori disallow superluminal orbit speeds.

The idea of the excited state of an atom as being actually a system of several atoms answers a need that was identified in Gevorkyan (2012). He pointed to spontaneous transition between quantum levels of a system as a hard-to-explain phenomenon. Indeed it is hard to explain in terms of excited states of a single atom. But it is easy to explain in terms of a system involving several atoms: the system can simply disintegrate back into several isolated single atoms.

\section{Quantum chemistry}

The main thing for Chemistry is not revealed in the Hydrogen atom, due to the fact that the Hydrogen atom has only one electron. The main thing for Chemistry is the variety of relationships among multiple electrons. Chemistry data suggest that electrons can sometimes actually attract, rather than repel, each other. That propensity can be important in driving chemical reactions. A basis for understanding that process is needed, and it cannot be found in the Schrödinger equation, or in any extension of Quantum Mechanics that injects Special Relativity Theory.

Buzea, Agop, \& Nejneru (2012) investigate the Bohm/Vigier approach and the Madelung approach for the kinds of problems that Chemistry presents. The former approach relies at its outset on SRT, which seems risky to those of us who doubt SRT. The latter approach invokes a 'quantum potential' for interaction with a 'subquantic medium'. That sounds like 'aether', and seems risky to those of us who doubt the existence of 'aether'.

So perhaps additional approaches are still to be welcomed. One such approach was introduced in Whitney (2012), is expanded in Whitney (2013, in press), and is discussed further below.

The approach is called Algebraic Chemistry (AC). The name reflects the fact AC is carried out entirely with algebra, and not numerical integration. In fact, the math is hardly even algebra, since only the occasional square root goes beyond simple Arithmetic. Such simple math suffices because the AC approach is based on scaling laws. The model for the Hydrogen atom is the prototype for similar models of the atoms of all the other elements. The input information for all atoms is consists ionization potentials. The raw data set looks quite daunting, but as reported in Whitney (2012), the data fall into neat patterns when scaled by $M / Z$ where $M$ is the nuclear mass number and $Z$ is the nuclear charge.

This $M / Z$ scaling produces a variable $I P_{1, Z}$ that we call 'population generic' because information about any element can be inferred from information about other elements. The AC Hydrogen-based model invites the division of each ionization potential $I P_{1, Z}$ into two parts, 
one part being $I P_{1,1}$ for the Hydrogen-like interaction of the electron population as a whole with the nucleus, and the other part being the increment $\triangle I P_{1, Z}=I P_{1, Z}-I P_{1,1}$ for the electronelectron interactions.

\section{Modeling the energy requirements for making ions}

The $I P_{1, Z}$ and $\Delta I P_{1, Z}$ for 118 elements are given in Whitney (2012). The utility of that data lies in the larger universe of inferences it supports. It can be used to estimate the actual energy involved in creating any ionization state of any element. To refer to the data $I P_{1, Z}$ and $\triangle I P_{1, Z}$ used, the term 'population generic' applies. To refer to the inferences made, the term 'element-specific' applies.

The formulae used are essentially the same for every element, so let us use the symbol ' ${ }_{Z} E^{\prime}$ for an arbitrary element, so we can write the formulae in a symbolic way. First consider the transition ${ }_{Z} \mathrm{E} \rightarrow{ }_{Z} \mathrm{E}^{+}$. It definitely takes an energy investment of $I P_{1, Z} \times Z / M_{Z}$, where the factors of $Z$ and $1 / M_{Z}$ restore the population-generic information $I P_{1, Z}$ to element-specific information. This energy investment corresponds to a potential 'wall' to be gotten over. The wall has two parts, $I P_{1,1} \times Z / M_{Z}$ and $\Delta I P_{1, Z} \times Z / M_{Z}$. The transition ${ }_{Z} \mathrm{E} \rightarrow{ }_{Z} \mathrm{E}^{+}$may also consume some heat, or generate some heat, as the remaining $Z-1$ electrons form new relationships, not necessarily instantaneously. This process constitutes adjustment to the rock pile, or the ditch, on the other side of the potential wall. It is represented by a term $-\Delta I P_{1, Z-1} \times(Z-1) / M_{Z}$, where the factors of $(Z-1)$ and $1 / M_{Z}$ restore the population-generic information $-\triangle I P_{1, Z-1}$ to element-specific information tailored for ${ }_{Z} E$. Thus altogether, ${ }_{Z} E \rightarrow{ }_{Z} E^{+}$takes:

$I P_{1,1} \times Z / M_{Z}+\Delta I P_{1, Z} \times Z / M_{Z}-\Delta I P_{1, Z-1} \times(Z-1) / M_{Z}$

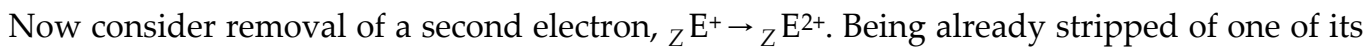
electrons, the ${ }_{Z} \mathrm{E}^{+}$system has less internal Coulomb attraction than neutral ${ }_{Z} E$ has. So the factor of $Z$ multiplying $I P_{1,1}$ for ${ }_{Z} \mathrm{E} \rightarrow{ }_{Z} \mathrm{E}^{+}$has to change to something smaller. Since Coulomb attraction generally reflects the product of the number of positive charges (here $Z$ ) and the number of negative charges (here $Z-1$ ), the reduced factor is $\sqrt{Z \times(Z-1)}$. Given this factor, ${ }_{Z} \mathrm{E}^{+} \rightarrow{ }_{\mathrm{Z}} \mathrm{E}^{2+\text { takes: }}$

$I P_{1,1} \times \sqrt{Z \times(Z-1)} / M_{Z}+\Delta I P_{1, Z-1} \times(Z-1) / M_{Z}-\Delta I P_{1, Z-2} \times(Z-2) / M_{Z}$

Observe that putting the steps ${ }_{Z} \mathrm{E} \rightarrow{ }_{Z} \mathrm{E}^{+}$and ${ }_{Z} \mathrm{E}^{+} \rightarrow{ }_{Z} \mathrm{E}^{2+}$ together, the terms involving $\triangle I P_{1, Z-1}$ cancel, leaving that altogether, ${ }_{Z} \mathrm{E} \rightarrow{ }_{Z} \mathrm{E}^{2+}$ takes:

$I P_{1,1} \times Z / M_{Z}+I P_{1,1} \sqrt{Z \times(Z-1)} / M_{Z}+\Delta I P_{1, Z} \times Z / M_{Z}-\Delta I P_{1, Z-2} \times(Z-2) / M_{Z}$.

This reduction to just two terms involving $\Delta I P^{\prime}$ s is typical of all sequential ionizations, of however many steps. Observe too that in the cumulative, we have two terms in $I P_{1,1}$ $I P_{1,1} \times Z / M_{Z}$ and $I P_{1,1} \sqrt{Z \times(Z-1)} / M_{Z}$. This makes sense since two electrons are removed. 
From the above, it should be clear how to proceed with stripping however many more electrons you may be interested in removing. Now consider adding an electron to ${ }_{Z} E$. The problem is similar to removing an electron from ${ }_{Z+1} E$, but in reverse. So, ${ }_{Z} E \rightarrow{ }_{Z} E^{-}$takes:

$-I P_{1,1} \times \sqrt{Z \times(Z+1)} / M_{Z}-\Delta I P_{1, Z+1}(Z+1) / M_{Z}+\Delta I P_{1, Z} Z / M_{Z}$

Going one step further, the problem of adding another electron to ${ }_{Z} \mathrm{E}^{-}$is similar to removing an electron from ${ }_{Z+2} E$, but in reverse. So, $\mathrm{E}^{-} \rightarrow{ }_{Z} \mathrm{E}^{2-}$ takes:

$-I P_{1,1} \times \sqrt{Z \times(Z+2)} / M_{Z}-\Delta I P_{1, Z+2} \times(Z+2) / M_{Z}+\Delta I P_{1, Z+1} \times(Z+1) / M_{Z}$

Observe that putting the steps ${ }_{Z} \mathrm{E} \rightarrow{ }_{Z} \mathrm{E}^{-}$and ${ }_{Z} \mathrm{E}^{-} \rightarrow{ }_{Z} \mathrm{E}^{2-}$ together, the terms involving $\triangle I P_{1, Z+1}$ cancel, leaving that altogether, ${ }_{Z} \mathrm{E} \rightarrow{ }_{Z} \mathrm{E}^{2-}$ takes:

$-I P_{1,1} \times \sqrt{Z \times(Z+1)} / M_{Z}-I P_{1,1} \times \sqrt{Z \times(Z+2)} / M_{Z}+\Delta I P_{1, Z} \times Z / M_{Z}-\Delta I P_{1, Z+2} \times(Z+2) / M_{Z}$

From the above, it should be clear how to proceed with adding however many more electrons you may be interested in adding.

To illustrate the development and use of information, consider a few example elements: Hydrogen, Carbon and Oxygen. The steps to develop essential information for Hydrogen are:

Write Formulae:

${ }_{1} \mathrm{H} \rightarrow{ }_{1} \mathrm{H}^{+}: I P_{1,1} \times 1 / M_{1}+\Delta I P_{1,1} \times 1 / M_{1}-\Delta I P_{1,0} \times 0 / M_{1}$ (Note: $\Delta I P_{1,1} \equiv 0, \quad$ and $\Delta I P_{1,0}$ does not exist.)

${ }_{1} \mathrm{H} \rightarrow{ }_{1} \mathrm{H}^{-}:-I P_{1,1} \times \sqrt{1 \times 2} / M_{1}-\Delta I P_{1,2} \times 2 / M_{1}+\Delta I P_{1,1} \times 1 / M_{1}$

Insert Data:

${ }_{1} \mathrm{H} \rightarrow{ }_{1} \mathrm{H}^{+}: 14.250 \times 1 / 1.008+0-0$

${ }_{1} \mathrm{H} \rightarrow{ }_{1} \mathrm{H}^{-}:-14.250 \times 1.4142 / 1.008-35.625 \times 2 / 1.008+0 \times 1 / 1.008$

Evaluate Formulae:

${ }_{1} \mathrm{H} \rightarrow{ }_{1} \mathrm{H}^{+}: 14.1369+0-0=14.1369 \mathrm{eV}$

${ }_{1} \mathrm{H} \rightarrow{ }_{1} \mathrm{H}^{-}:-19.9924-70.6845+0=-90.6769 \mathrm{eV}$

The steps to develop the information needed for Carbon are far more numerous because it routinely gives or takes so many electrons. The steps are:

Write Formulae:

${ }_{6} \mathrm{C} \rightarrow{ }_{6} \mathrm{C}^{+}: I P_{1,1} \times 6 / M_{6}+\Delta I P_{1,6} \times 6 / M_{6}-\Delta I P_{1,5} \times 5 / M_{6}$

${ }_{6} \mathrm{C}^{+} \rightarrow{ }_{6} \mathrm{C}^{2+}: I P_{1,1} \times \sqrt{6 \times 5} / M_{6}+\Delta I P_{1,5} \times 5 / M_{6}-\Delta I P_{1,4} \times 4 / M_{6}$

${ }_{6} \mathrm{C}^{2+} \rightarrow{ }_{6} \mathrm{C}^{3+}: I P_{1,1} \times \sqrt{6 \times 4} / M_{6}+\Delta I P_{1,4} \times 4 / M_{6}-\Delta I P_{1,3} \times 3 / M_{6}$ 


$$
\begin{aligned}
& { }_{6} \mathrm{C}^{3+} \rightarrow{ }_{6} \mathrm{C}^{4+}: I P_{1,1} \times \sqrt{6 \times 3} / M_{6}+\Delta I P_{1,3} \times 3 / M_{6}-\Delta I P_{1,2} \times 2 / M_{16} \\
& { }_{6} \mathrm{C} \rightarrow{ }_{6} \mathrm{C}^{-}:-I P_{1,1} \times \sqrt{6 \times 7} / M_{6}-\Delta I P_{1,7} \times 7 / M_{6}+\Delta I P_{1,6} \times 6 / M_{6} \\
& { }_{6} \mathrm{C}^{-} \rightarrow{ }_{6} \mathrm{C}^{2-}:-I P_{1,1} \times \sqrt{6 \times 8} / M_{6}-\Delta I P_{1,8} \times 8 / M_{6}+\Delta I P_{1,7} \times 7 / M_{6} \\
& { }_{6} \mathrm{C}^{2-} \rightarrow{ }_{6} \mathrm{C}^{3-}:-I P_{1,1} \times \sqrt{6 \times 9} / M_{6}-\Delta I P_{1,9} \times 9 / M_{6}+\Delta I P_{1,8} \times 8 / M_{6} \\
& { }_{6} \mathrm{C}^{3-} \rightarrow{ }_{6} \mathrm{C}^{4-}:-I P_{1,1} \times \sqrt{6 \times 10} / M_{6}-\Delta I P_{1,10} \times 10 / M_{6}+\Delta I P_{1,9} \times 9 / M_{6}
\end{aligned}
$$

Insert Data:

$$
\begin{aligned}
& { }_{6} \mathrm{C} \rightarrow{ }_{6} \mathrm{C}^{+}: 14.250 \times 6 / 12.011+7.320 \times 6 / 12.011-2.805 \times 5 / 12.011 \\
& { }_{6} \mathrm{C}^{+} \rightarrow{ }_{6} \mathrm{C}^{2+}: 14.250 \times 5.4772 / 12.011+2.805 \times 5 / 12.011-9.077 \times 4 / 12.011 \\
& { }_{6} \mathrm{C}^{2+} \rightarrow{ }_{6} \mathrm{C}^{3+}: 14.250 \times 4.8990 / 12.011+9.077 \times 4 / 12.011-(-1.781) \times 3 / 12.011 \\
& { }_{6} \mathrm{C}^{3+} \rightarrow{ }_{6} \mathrm{C}^{4+}: 14.250 \times 4.2426 / 12.011+(-1.781) \times 3 / 12.011-35.625 \times 2 / 12.011 \\
& { }_{6} \mathrm{C} \rightarrow{ }_{6} \mathrm{C}^{-}:-14.250 \times 6.4807 / 12.011-13.031 \times 7 / 12.011+7.320 \times 6 / 12.011 \\
& { }_{6} \mathrm{C}^{-} \rightarrow{ }_{6} \mathrm{C}^{2-}:-14.250 \times 6.9282 / 12.011-13.031 \times 8 / 12.011+13.031 \times 7 / 12.011 \\
& { }_{6} \mathrm{C}^{2-} \rightarrow{ }_{6} \mathrm{C}^{3-}:-14.250 \times 7.3485 / 12.011-20.254 \times 9 / 12.011+13.031 \times 8 / 12.011 \\
& { }_{6} \mathrm{C}^{3-} \rightarrow{ }_{6} \mathrm{C}^{4-}:-14.250 \times 7.7460 / 12.011-29.391 \times 10 / 12.011+20.254 \times 9 / 12.011
\end{aligned}
$$

Evaluate Formulae:

$$
\begin{aligned}
& { }_{6} \mathrm{C} \rightarrow{ }_{6} \mathrm{C}^{+}: 7.1185+3.6566-1.1677=9.6074 \mathrm{eV} \\
& { }_{6} \mathrm{C}^{+} \rightarrow{ }_{6} \mathrm{C}^{2+}: 6.4982+1.1678-3.0229=4.6431 \mathrm{eV} \\
& { }_{6} \mathrm{C}^{2+} \rightarrow{ }_{6} \mathrm{C}^{3+}: 5.8122+3.0229+0.4448=9.2799 \mathrm{eV} \\
& { }_{6} \mathrm{C}^{3+} \rightarrow{ }_{6} \mathrm{C}^{4+}: 5.0335-0.4448-5.9321=-1.3434 \mathrm{eV} \\
& { }_{6} \mathrm{C} \rightarrow{ }_{6} \mathrm{C}^{-}:-7.6888-7.5945+3.6566=-11.6267 \mathrm{eV} \\
& { }_{6} \mathrm{C}^{-} \rightarrow{ }_{6} \mathrm{C}^{2-}:-8.2197-8.6794+7.5945=-9.3046 \mathrm{eV} \\
& { }_{6} \mathrm{C}^{2-} \rightarrow{ }_{6} \mathrm{C}^{3-}:-8.7184-15.1766+8.6794=-15.2156 \mathrm{eV} \\
& { }_{6} \mathrm{C}^{3-} \rightarrow{ }_{6} \mathrm{C}^{4-}:-9.1900-24.4701+15.1766=-18.4835 \mathrm{eV}
\end{aligned}
$$

Evaluate sums:

$$
\begin{aligned}
& { }_{6} \mathrm{C} \rightarrow{ }_{6} \mathrm{C}^{2+}: 9.6074+4.6431=14.2505 \mathrm{eV} \\
& { }_{6} \mathrm{C} \rightarrow{ }_{6} \mathrm{C}^{3+}: 14.2505+9.2799=23.5304 \mathrm{eV}
\end{aligned}
$$




$$
\begin{aligned}
& { }_{6} \mathrm{C} \rightarrow{ }_{6} \mathrm{C}^{4+}: 23.5304-1.3434=22.187 \mathrm{eV} \\
& { }_{6} \mathrm{C} \rightarrow{ }_{6} \mathrm{C}^{2-}:-11.6267-9.3046=-20.9313 \mathrm{eV} \\
& { }_{6} \mathrm{C} \rightarrow{ }_{6} \mathrm{C}^{3-}:-20.9313-15.2156=-36.1469 \mathrm{eV} \\
& { }_{6} \mathrm{C} \rightarrow{ }_{6} \mathrm{C}^{4-}:-36.1469-18.4835=-54.6304 \mathrm{eV}
\end{aligned}
$$

The steps to develop information for Oxygen are:

Write Formulae:

$$
\begin{aligned}
& { }_{8} \mathrm{O} \rightarrow{ }_{8} \mathrm{O}^{+}: I P_{1,1} \times 8 / M_{8}+\Delta I P_{1,8} \times 8 / M_{8}-\Delta I P_{1,7} \times 7 / M_{8} \\
& { }_{8} \mathrm{O}^{+} \rightarrow{ }_{8} \mathrm{O}^{2+}: I P_{1,1} \times \sqrt{8 \times 7} / M_{8}+\Delta I P_{1,7} \times 7 / M_{8}-\Delta I P_{1,6} \times 6 / M_{8} \\
& { }_{8} \mathrm{O} \rightarrow{ }_{8} \mathrm{O}^{-}:-I P_{1,1} \times \sqrt{8 \times 9} / M_{8}-\Delta I P_{1,9} \times 9 / M_{8}+\Delta I P_{1,8} \times 8 / M_{8} \\
& { }_{8} \mathrm{O}^{-} \rightarrow{ }_{8} \mathrm{O}^{2-}:-I P_{1,1} \times \sqrt{8 \times 10} / M_{8}-\Delta I P_{1,10} \times 10 / M_{8}+\Delta I P_{1,9} \times 9 / M_{8}
\end{aligned}
$$

Insert Data:

$$
\begin{aligned}
& { }_{8} \mathrm{O} \rightarrow{ }_{8} \mathrm{O}^{+}: 14.250 \times 8 / 15.999+13.031 \times 8 / 15.999-13.031 \times 7 / 15.999 \\
& { }_{8} \mathrm{O}^{+} \rightarrow{ }_{8} \mathrm{O}^{2+}: 14.250 \times 7.4833 / 15.999+13.031 \times 7 / 15.999-7.320 \times 6 / 15.999 \\
& { }_{8} \mathrm{O} \rightarrow{ }_{8} \mathrm{O}^{-}:-14.250 \times 8.4853 / 15.999-20.254 \times 9 / 15.999+13.031 \times 8 / 15.999 \\
& { }_{8} \mathrm{O}^{-} \rightarrow{ }_{8} \mathrm{O}^{2-}:-14.250 \times 8.9443 / 15.999-29.391 \times 10 / 15.999+20.254 \times 9 / 15.999
\end{aligned}
$$

Evaluate Formulae:

$$
\begin{aligned}
& { }_{8} \mathrm{O} \rightarrow{ }_{8} \mathrm{O}^{+}: 7.1254+6.5159-5.7014=7.9399 \mathrm{eV} \\
& { }_{8} \mathrm{O}^{+} \rightarrow{ }_{8} \mathrm{O}^{2+}: 6.6652+5.7014-2.7452=9.6214 \mathrm{eV} \\
& { }_{8} \mathrm{O} \rightarrow{ }_{8} \mathrm{O}^{-}:-7.5577-11.3936+6.5159=-12.4354 \mathrm{eV} \\
& { }_{8} \mathrm{O}^{-} \rightarrow{ }_{8} \mathrm{O}^{2-}:-7.9665-18.3705+11.3936=-14.9434 \mathrm{eV}
\end{aligned}
$$

Evaluate sums:

$$
\begin{aligned}
& { }_{8} \mathrm{O} \rightarrow{ }_{8} \mathrm{O}^{2+}: 7.9399+9.6214=17.5613 \mathrm{eV} \\
& { }_{8} \mathrm{O} \rightarrow{ }_{8} \mathrm{O}^{2-}:-12.4354-14.9434=-27.3788 \mathrm{eV}
\end{aligned}
$$

What can we tell from all this information? Consider a few of the molecules that these elements can make. The simplest one is the Hydrogen molecule $\mathrm{H}_{2}$. Forming it takes $14.1369-90.6769=-76.54 \mathrm{eV}$. That number is very negative, which means the Hydrogen molecule forms quickly, even explosively. Isolated neutral Hydrogen atoms are rare in Nature. Even at very low density, in deep space, Hydrogen atoms would rather form molecules, or 
form plasma, than remain as neutral atoms. How ironic it is that the prototypical atom for the development of QM was something that is hardly to be found in Nature!

Another simple molecule is $\mathrm{O}_{2}$. This oxygen molecule illustrates the interesting possibility of more than one ionic configuration, a situation that turns out to be the case for many molecules. $\mathrm{O}_{2}$ can be ${ }_{8} \mathrm{O}^{+}+{ }_{8} \mathrm{O}^{-}$or ${ }_{8} \mathrm{O}^{2+}+{ }_{8} \mathrm{O}^{2-}$. Forming ${ }_{8} \mathrm{O}^{+}+{ }_{8} \mathrm{O}^{-}$takes $7.9399-12.4354=-4.4955 \mathrm{eV}$, and forming ${ }_{8} \mathrm{O}^{2+}+{ }_{8} \mathrm{O}^{2-}$ takes $17.5613-27.3788=-9.8175 \mathrm{eV}$. Although the second ionic configuration is better in terms of energy, the two are close. In situations like this, both ionic configurations exist, in proportions determined by thermodynamic entropy maximization, which depends on temperature.

The fact that the two ionic configurations of $\mathrm{O}_{2}$ are so close in energy means that transitions between them are easy. This can make $\mathrm{O}_{2}$ an absorber and emitter of low energy photons; i.e. infrared photons, i.e. heat. This can in turn make Oxygen act as a so-called 'greenhouse gas'; i.e., a contributor to atmospheric warming. But as $\mathrm{O}_{2}$-consuming animals, we just never speak of $\mathrm{O}_{2}$ in such a derogatory way.

Consider $\mathrm{H}_{2} \mathrm{O}$. Water again illustrates the possibility of more than one ionic configuration for a given molecule. Water is known to dissociate into the naked proton $\mathrm{H}^{+}$and the hydroxyl radical $\mathrm{OH}^{-}$. In turn, the hydroxyl radical has to be the combination of ions $\mathrm{O}^{2-}+\mathrm{H}^{+}$; there is not an alternative form using $\mathrm{H}^{-}$, because then $\mathrm{O}$ would have to be neutral. So we might well imagine that $\mathrm{H}_{2} \mathrm{O}$ had ionic configuration $2 \mathrm{H}^{+}+\mathrm{O}^{2-}$. But the formation of that ionic configuration takes $2 \times 14.1369-27.3788=+0.8950 \mathrm{eV}$, a slightly positive energy. That can't be right for the common water molecule covering our planet. So in fact, common water must not live in the ionic configuration to which it dissociates, and thereby dies. Therefore, consider the alternative ionic configuration $2 \mathrm{H}^{-}+\mathrm{O}^{2+}$. This one requires $2 \times(-90.6769)+17.5613=-163.7925$ $\mathrm{eV}$. This is a decidedly negative energy, and so is believable for a decidedly stable molecule.

Water in the normal $2 \mathrm{H}^{-}+\mathrm{O}^{2+}$ ionic configuration has to form a tetrahedron, with two $\mathrm{H}^{+}$naked protons on two vertices and two $2 \mathrm{e}^{-}$electron pairs on and the other two vertices. That is why the water molecule we know has a bend to it. Viewing the Hydrogen nuclei as lying on arms originating from the Oxygen nucleus, the angle of the bend is the angle characteristic of arms from the center to two corners of a regular tetrahedron - on the order of $109.5^{\circ}$.

The other ionic configuration for water, $2 \mathrm{H}^{+}+\mathrm{O}^{2-}$, is the charge mirror image of the commonly known one. But it has to be a completely different shape: not tetrahedral, but instead linear. It just looks like $\mathrm{H} \quad \mathrm{O} \quad \mathrm{H}$, where the dots mean 'chemical bond'. This form of water apparently does exist, but only in a very un-natural circumstance. There exists an electrochemically created substance known as 'Brown's gas' that has occasioned some impossibly wild claims about energy generation, but has also been investigated quite legitimately for applications in welding. A linear isomer of water is thought to be the active ingredient in Brown's gas.

The story of water tells us that even the most familiar of compounds can have some very interesting isomers. The conclusion to be drawn is that any molecule with three or more atoms 
can have isomers that differ, certainly in ionic configuration, but probably also in molecular shape, and in resultant chemical properties.

The very negative $-163.7925 \mathrm{eV}$ result for forming the $2 \mathrm{H}^{-}+\mathrm{O}^{2+}$ ionic configuration of normal water is what makes the burning of all hydrocarbons so worthwhile as energy sources. It is the main thing, but it generally attracts no mention. Other reaction products get all the attention.

Consider $\mathrm{CO}_{2}$. Carbon dioxide is a normal atmospheric constituent, and a product of hydrocarbon combustion, a possible contributor to global warming, and sometimes a target for government regulation.

$\mathrm{CO}_{2}$ again illustrates the possibility of several ionic configurations for a molecule. $\mathrm{CO}_{2}$ has at least four plausible ionization configurations: $\mathrm{C}^{4+}+2 \mathrm{O}^{2-}, \mathrm{C}^{4-}+2 \mathrm{O}^{2+}, \mathrm{C}^{2+}+2 \mathrm{O}^{-}$, and $\mathrm{C}^{2-}+\mathrm{O}^{+}$. The energy requirements to make them from the neutral atoms are:

$\mathrm{C}^{4+}+2 \mathrm{O}^{2-}: 22.187+2 \times(-27.3788)=22.187-54.7576=-32.5706 \mathrm{eV}$

$\mathrm{C}^{4-}+2 \mathrm{O}^{2+}:-54.6304+2 \times 17.5613=-54.6304+35.1226=-19.5078 \mathrm{eV}$

$\mathrm{C}^{2+}+2 \mathrm{O}^{-}: 14.2505+2 \times(-12.4354)=14.2505-24.8708=-10.6203 \mathrm{eV}$

$\mathrm{C}^{2-}+2 \mathrm{O}^{+}:-20.9313+2 \times 7.9399=-20.9313+15.8798=-5.0515 \mathrm{eV}$

The first ionic configuration listed is the one favored electrically, but the others must also occur, all in thermodynamically determined proportion. The fact that there are so many possibilities for just this one little tri-atomic molecule means that QC can benefit from using AC to identify all the possibilities in a situation, and select rationally among them, and spend computation power wisely.

\section{Single-electron state filling over the periodic table}

Nobody is yet satisfied that we completely understand the Periodic Table. QM informs us of single electron states and their quantum numbers, and we can tell from spectroscopic data what single electron states are filled for each element, and we can see what the governing rule is, but we do not understand why that is the rule, and we do not understand why there are exceptions to the rule.

The normal order of state filling can be described in terms of the quantum numbers $n=1,2,3, \ldots$ for radial level, $l=0,1, \ldots n-1$ for orbital angular momentum, and $s=-1 / 2,+1 / 2$ for spin. The normal order of state filling goes with increasing $n+l$, with all of $s=-1 / 2$ first, and then all of $s=-1 / 2$. So that makes the normal order:

In Period $1: n=1, l=0, s=-1 / 2$ and $n=1, \quad l=0, s=+1 / 2$;

Then in Period 2:

$n=2, l=0, s=-1 / 2, \quad n=2, l=0, s=+1 / 2 \quad$ and $n=2, l=1, s=-1 / 2 \quad$ three times, $n=2, \quad l=1, \quad s=+1 / 2$ three times; 
Then in Period 3:

$n=3, l=0, s=-1 / 2, \quad n=3, l=0, s=+1 / 2 \quad$ and $n=3, l=1, s=-1 / 2 \quad$ three times, $n=3, l=1, \quad s=+1 / 2$ three times;

Then in Period 4:

$n=4, l=0, s= \pm 1 / 2, n=3, l=2, s=-1 / 2$ five times, $n=3, l=2, s=+1 / 2$ five times,

$n=4, l=1, \quad s=-1 / 2$ three times, $n=4, l=1, s=+1 / 2$ three times;

Then in Period 5:

$n=5, l=0, s= \pm 1 / 2, n=4, l=2, s=-1 / 2$ five times, $n=4, l=2, s=+1 / 2$ five times,

$n=5, l=1, \quad s=-1 / 2$ three times, $n=5, l=1, s=+1 / 2$ three times;

Then in Period 6:

$n=6, l=0, \quad s= \pm 1 / 2, n=5, l=3, \quad s=-1 / 2$ seven times, $n=5, l=3, s=+1 / 2$ seven times,

$n=5, l=3, s=-1 / 2$ five times, $n=5, l=3, s=+1 / 2$ five times,

$n=6, l=1, s=-1 / 2$ three times, $n=6, l=1, s=+1 / 2$ three times;

Then in Period 7:

$n=7, l=0, \quad s= \pm 1 / 2, n=6, \quad l=3, \quad s=-1 / 2$ seven times, $n=6, l=3, \quad s=+1 / 2$ seven times,

$n=6 l=3, s=-1 / 2$ five times, $n=6 l=3, s=+1 / 2$ five times,

$n=7, l=1, \quad s=-1 / 2$ three times, $n=7, l=1, s=+1 / 2$ three times.

It is perhaps possible to do enough QM calculations to develop a numerical explanation for this pattern. But we do not have from QM any higher-level, conceptual explanation for this pattern.

And on top of that, there are 19 exceptions to the pattern. They are:

In Period 4: Chromium ${ }_{24} \mathrm{Cr}$, Copper ${ }_{29} \mathrm{Cu}$;

In Period 5: Niobium ${ }_{41} \mathrm{Nb}$, Molybdenum ${ }_{42} \mathrm{Mo}$, Rubidium ${ }_{44} \mathrm{Ru}$, Rhodium ${ }_{45} \mathrm{Rh}$, Palladium ${ }_{46} \mathrm{Pd}$, Silver ${ }_{47} \mathrm{Ag}$;

In Period 6: Lanthanum ${ }_{57} \mathrm{La}$, Cerium ${ }_{58} \mathrm{Ce}$, Gadolinium ${ }_{64} \mathrm{Gd}$, Platinum ${ }_{78} \mathrm{Pt}$, Gold ${ }_{79} \mathrm{Au}$;

In Period 7: Actinium ${ }_{89} \mathrm{Ac}$, Thallium ${ }_{90} \mathrm{Th}$, Protactinium ${ }_{91} \mathrm{~Pa}$, Uranium ${ }_{92} \mathrm{U}$, Neptunium ${ }_{93} \mathrm{~Np}$, Cerium ${ }_{96} \mathrm{Cm}$.

So it is a good project for AC to try to improve this situation, both in regard to explaining the pattern, and in regard to explaining the exceptions.

The Hydrogen-based model used for AC makes the electron population a rather localized subsystem, orbiting the nucleus, rather than enclosing it. The electron subsystem is composed 
of electron rings spinning at superluminal speeds, stacked together like little magnets. This model creates a hierarchy of magnetic confinement levels. Two rings with two electrons each create a 'magnetic bottle', and it can contain up to two geometrically smaller rings with three electrons each. Two such three-electron rings create a stronger 'magnetic thermos jug'. That can contain up to two geometrically smaller rings with five electrons each. Two such fiveelectron rings create an even stronger 'magnetic Dewar flask'. It is capable of containing up to two geometrically smaller rings with seven electrons each.

The electron state filling sequence is determined by a rather 'fractal' looking algorithm: Always build and store an electron ring for the largest number of electrons possible, where 'possible' means having a suitable magnetic confinement volume available to fit into, and where 'suitable' means created by two electron rings with smaller electron count, and not yet filled with two electron rings of larger electron count. Sometimes only a new two-electron ring is possible, and that is what starts a new period in the Periodic Table.

So there follows the expected order for the filling of single electron states across the Periodic Table.

AC can also identify factors that account for individual exceptions. The worst exception is Palladium, because it has not just one, but two, violations of the nominal pattern. Here is the explanation for Palladium. According to the nominal electron filling pattern, ${ }_{46} \mathrm{Pd}$ would have a not yet used space for two three-electron rings, and hence it would have an un-used twoelectron ring. Total consumption of that un-used two-electron ring into an unfilled fiveelectron ring allows an extremely symmetric stack of filled electron rings. It goes: 2, 3, 5,5, 3, $2,3,3,2,3,5,5,3,2$. The opportunity for such symmetry is what trumps the nominal pattern.

Here is a list of brief comments about all of the exceptions:

Chromium ${ }_{24} \mathrm{Cr}$ robs one electron from a two-electron ring to complete a five-electron ring.

Copper ${ }_{29} \mathrm{Cu}$ robs one electron from a two-electron ring to complete a five-electron ring.

Niobium ${ }_{41} \mathrm{Nb}$ robs one electron from a two-electron ring to complete a five-electron ring.

Molybdenum ${ }_{42}$ Mo robs one electron from a two-electron ring to complete a five-electron ring.

Rubidium ${ }_{44} \mathrm{Ru}$ robs one electron from a two-electron ring to complete a five-electron ring.

Rhodium ${ }_{45} \mathrm{Rh}$ robs one electron from a two-electron ring to complete a five-electron ring.

Palladium ${ }_{46} \mathrm{Pd}$ completely consumes a two-electron ring to complete a five-electron ring.

Silver ${ }_{47} \mathrm{Ag}$ robs one electron from a two-electron ring to complete a five-electron ring.

Lanthanum ${ }_{57} \mathrm{La}$ puts an electron in a five-electron place instead of a seven-electron place.

Cerium ${ }_{58} \mathrm{Ce}$ puts an electron in a five-electron place instead of a seven-electron place.

Gadolinium ${ }_{64} \mathrm{Gd}$ puts an electron in a five-electron place instead of a seven-electron place. 
Platinum ${ }_{78} \mathrm{Pt}$ robs one electron from a two-electron ring to complete a five-electron ring. Gold ${ }_{79} \mathrm{Au}$ robs one electron from a two-electron ring to complete a five-electron ring. Actinium ${ }_{89}$ Ac puts an electron in a five-electron place instead of a seven-electron place.

Thallium ${ }_{90}$ Th puts an electron in a five-electron place instead of a seven-electron place.

Protactinium ${ }_{91} \mathrm{~Pa}$ puts an electron in a five-electron place instead of a seven-electron place.

Uranium ${ }_{92} \mathrm{U}$ puts an electron in a five-electron place instead of a seven-electron place.

Neptunium ${ }_{93} \mathrm{~Np}$ puts an electron in a five-electron place instead of a seven-electron place.

Cerium ${ }_{96} \mathrm{Cm}$ puts an electron in a five-electron place instead of a seven-electron place.

\section{Conclusion}

It is this author's opinion is that a more fully developed quantum mechanics, giving equal attention to both stable states, and the transitions between them, would fulfill a property that the subject matter of quantum mechanics has always demanded: some kind of duality. All of the objects of study in quantum mechanics exhibit a wave-particle duality, and the theory itself needs a corresponding kind of duality: attention both to the definition of stable states, and to the study of details of state transitions.

This paper attempts to make some progress in that direction. It gives several examples of old problems treated with a new approach. The first of these concerns the nature of the photon. There is a perception that the discovery of the photon marks a departure from Maxwell. This author disagrees with that perception. The second problem concerns Schrödinger's equation. There is a perception that Schrödinger's equation marks a departure from Newton, and the classical physics of particles. This author disagrees with that perception too. The third problem has to do with the application of QM in Chemistry. There is a perception that such an application of QM demands extensive computer calculation. This author believes in an alternative approach based on scaling laws: Algebraic Chemistry.

All of these problems illustrate a reasoned concern about the current practice of Physics. I think Physics sometimes goes a step too far in the direction of reductionism. Einstein's signal for SRT, with only a speed parameter, is a step too far. The photon concept without Maxwell's equations is a step too far. An atom with a stationary nucleus is a step too far. Schrödinger's equation without the $\Psi^{*}$ factor is a step too far. A single atom with multiple excited states is a step too far. And so on.

Consider some history. At the turn of the $20^{\text {th }}$ century, there was a lot of work concerning a isolated electron. Why does the electron not explode due to internal Coulomb forces? Why does the equation of motion for the electron allow run-away solutions that do not occur in Nature? There are many such puzzles. Chapter 17 in the standard textbook by Jackson (1975) 
discusses radiation damping, self-fields of a particle, scattering and absorption of radiation by a bound system, all from the classical viewpoint and from the viewpoint of SRT. It is a status report, not a final resolution. These matters are still not fully resolved. I suspect too much reductionism as their cause.

Always remember: Sometimes, backing off from reductionism, and analyzing a slightly more complicated problem, actually leads to simpler results.

\section{Author details}

Cynthia Kolb Whitney

Galilean Electrodynamics, USA

\section{References}

[1] Abad, L.V., \& S. C. Huichalaf (2012) Complementarity in Quantum Mechanics and Classical Statistical Mechanics, Chapter 1 in Theoretical Concepts of Quantum Mechanics, Ed. M.R. Pahlavani, InTech.

[2] Berkdemir, C. (2012) Application of the Nikiforov-Uvarov Method in Quantum Mechanics, Chapter 11 in Theoretical Concepts of Quantum Mechanics, Ed. M.R. Pahlavani, InTech.

[3] Bracken, P. (2012) Quantum Mechanics Entropy and a Quantum Version of the H theorem, Chapter 20 in Theoretical Concepts of Quantum Mechanics, Ed. M.R. Pahlavani, InTech.

[4] Buzea, Calin Gh., M. Agop, \& C. Nejneru (2012) Correspondences of Scale Relativity Theory with Quantum Mechanics, Chapter 18 in Theoretical Concepts of Quantum Mechanics, Ed. M.R. Pahlavani, InTech.

[5] Cini, M. (2012) The Physical Nature of Wave/Particle Duality, Chapter 2 in Theoretical Concepts of Quantum Mechanics, Ed. M.R. Pahlavani, InTech.

[6] De Zela, F. (2012) The Pancharatnam-Berry Phase: Theoretical and Eperimental Aspects, Chapter 14 in Theoretical Concepts of Quantum Mechanics, Ed. M.R. Pahlavani, InTech.

[7] Gevorkyan, A.S. (2012) Nonrelativistic Quantum Mechanics with Fundamental Environment, Chapter 8 in Theoretical Concepts of Quantum Mechanics, Ed. M.R. Pahlavani, InTech.

[8] Jackson, J.D. (1975) Classical Electrodynamics, John Wiley \& Sons, New York, etc. 
[9] Lunin, N.V. (2012) The Group Theory and Non-Euclidean Superposition Principle in Quantum Mechanics, Chapter 13 in Theoretical Concepts of Quantum Mechanics, Ed. M.R. Pahlavani, InTech.

[10] Skála, L., \& V. Kapsa (2012) Quantum Mechanics and Statistical Description of Results of Measurement, Chapter 10 in Theoretical Concepts of Quantum Mechanics, Ed. M.R. Pahlavani, InTech.

[11] Streklas, A. (2012) Non Commutative Quantum Mechanics in Time-Dependent Backgrounds, Chapter 9 in Theoretical Concepts of Quantum Mechanics, Ed. M.R. Pahlavani, InTech.

[12] Torres-Vega, G. (2012), Correspondence, Time, Energy, Uncertainty, Tunnelling, and Collapse of Probability Densities, Chapter 4 in Theoretical Concepts of Quantum Mechanics, Ed. M.R. Pahlavani, InTech.

[13] Whitney, C. K. (2012) Better Unification for Physics in General Through Quantum Mechanics in Particular, Chapter 7 in Theoretical Concepts of Quantum Mechanics, Ed. M.R. Pahlavani, InTech.

[14] Whitney, C.K. (2013) Algebraic Chemistry: Applications and Origins, Nova Science Publishers. 
Chapter 18

\title{
The Computational Unified Field Theory (CUFT): A Candidate 'Theory of Everything'
}

\author{
Jonathan Bentwich \\ Additional information is available at the end of the chapter \\ http://dx.doi.org/10.5772/53804
}

Einstein: "Our experience hitherto justifies us in believing that nature is the realization of the simplest conceivable
mathematical ideas... In a certain sense, therefore, I hold it true that pure thought can grasp reality, as the ancients dreamed" (1933)

\section{Introduction}

Two previous articles (Bentwich, 2012: a \& b) have postulated the existence of a new (hypothetical) Computational Unified Field Theory (CUFT) which appears to be capable of bridging the gap between Quantum Mechanics and Relativity Theory within a conceptually higher-ordered ('D2') Universal Computational Principle (' ' '), thereby representing a potential candidate for a 'Theory of Everything' (TOE) (Brumfiel, 2006; Ellis, 1986; Greene, 2003). The CUFT is based on five basic theoretical postulates which include: a) the discovery of a new computational 'Duality Principle' (Bentwich, 2003: a, b, c, 2004, 2006), e.g., which proves that it is not possible to determine the "existence" or "non-existence" of any particular 'y' element based on any direct or indirect interaction/s with any exhaustive hypothetical series of ' $x$ ' factor/s (termed: a 'SelfReferential Ontological Computational System' (SROCS), but only based on a conceptually higher-ordered 'D2' computational framework which can compute the "simultaneous cooccurrences" of any exhaustive hypothetical 'x-y' series. The validity of the Duality Principlehas been demonstrated for a series of key scientific (computational SROCS) paradigms, including: Darwin's 'Natural Selection Principle' (and associated 'Genetic Encoding' hypothesis, Neuroscience'sPsychophysicalProblem(PPP) of human Consciousnessas well as toallother (inductive or deductive) 'Gödel-like' SROCS computational paradigms (for which there is a knowable 
empirical capacity to determine the values of the ' $x$ ' and 'y' elements) (Bentwich, 2012: $a$ \& b); the existence of such a higher-ordered 'D2' 'Universal Computational Principle' (termed:'',' denoted by the Hebrew letter "yud") which carries out an extremely rapid computation (e.g., 'c $\left.{ }^{2} / h^{\prime}\right)$ of a series of 'Universal Simultaneous Computational Frames' (USCF's) that comprise the entire corpus of spatial pixels in the universe (e.g., computed simultaneously at any minimal Planck's 'h' time interval); c) The existence of three Computational Dimensions: 'Framework' (e.g., 'frame' vs. 'object'), 'Consistency' (e.g., 'consistent' vs. 'inconsistent') and 'Locus' (e.g., 'local', vs. global') whose various combinations gives rise to the four basic 'physical' properties of 'space', 'time', 'energy' and 'mass' (i.e., through the four possible combinations of Framework and Consistency), and to all relativistic effects (e.g., through these four secondary computational 'physical' properties' combinations with Locus' two abovementioned levels); d) The 'Computational Invariance Principle' (e.g., based on 'Ockham's razor'), which proves that since only the 'Universal Computational Principle' (' '') exists invariantly - i.e., both as producing and underlying each of the USCF's secondary computational four 'physical' properties (of 'space', 'time', 'energy' and 'mass) and as existing solely (and independently) "in-between" any two subsequent USCF's frames, whereas these four secondary computational 'physical' properties represent computationally variant features (e.g., since they are computed based on different computational combinations and only exist "during" the USCF's frames but not "in-between" any two such USCF's frames), then we may only regard the computationally invariant Universal Computational Principle(' ' as "real" whereas the computationally variant four secondary computational 'physical' properties (of 'space', 'time', 'energy' and 'mass') must be viewed as "phenomenal" or "unreal" (i.e., relative to their solely underlying computationally invariant Universal Computational Principle); and e) The 'Universal Consciousness Principle' which proves that since (based on the previous 'Computational Invariance Principle') only the Universal Computational Principle solely exists both "in-between" any two subsequent USCF's and as producing all USCF's four secondary computational 'physical' properties, then it necessarily follows that this (solely existing) Universal Computational Principle must also possess an equivalent 'Universal Consciousness Principle' capacity to produce-sustain or evolve allexhaustive(hypothetical)spatial pixels in the universe (i.e., across any two subsequent USCF's)...

The discovery of this new hypothetical CUFT has been accompanied by the identification of specific (empirical) 'critical predictions' for which the CUFT significantly differs from both quantum and relativistic models of the physical reality, e.g., including: 1) embedding of the (known) relativistic "E=Mc" equation and Quantum 'Uncertainty Principle's complimentary pairs (e.g., of 'space and energy' or of 'time and mass') within a broader (novel) 'Universal Computational Formula':

$$
\frac{c^{2} x^{\prime}}{h}=\frac{s x e}{t}
$$

2) the CUFT's differential critical prediction regarding the greater number of (consistent) presentations of a more "massive" element (e.g., across a series of USCF's), relative to the number of presentations of a less massive element; and 3) A hypothetical capacity to "reverse the flow of time" based on the measurement of any given object's sequence of spatial electromagnetic values (across a given series of USCF's) and the application of the appropriate electromagnetic modulation values (applied to any of its identified spatial-electromagnetic 
pixels across the measured USCF's series) that is necessary in order to attain the reversed spatial-temporal electromagnetic sequence (e.g., comprising that object's reversed spatialtemporal sequence). (Such methodology could also hypothetically lead to the "de-materialization" or "materialization" of any given object or event).

Due to the fact that the current Scientific framework is anchored and based (entirely) upon a Cartesian 'materialistic-reductionistic' assumption wherein it is assumed that any given (or even hypothetical) phenomenon- element- or natural law (represented as: 'y') is reducible to- or can be explained- solely based on a certain number of physical interactions between this 'y' element and any exhaustive hypothetical ' $x$ ' factor/s, element/s, phenomenon/a or events etc. - e.g., giving rise to the Duality Principle's (abovementioned) SROCS computational structure; and due to the transcendence of such 'computationally invalid' SROCS structure (Bentwich, 2012:a \&b) by the Duality Principleand itsembedding within theCUFT'shigher-ordered 'D2':Universal Computational/Universal Consciousness Principle theoretical framework; it was previously suggested that to the extent that the CUFT may be validated experimentally (e.g., such as for instance through an empirical validation of one (or more) of its (abovementioned) differential critical predictions - then this may lead to a 'paradigmatic shift' from the current Cartesian 'materialistic-reductionsitic' theoretical framework towards a conceptually higher-ordered singular Universal Computational/Universal Consciousness Principle which explains the physical universe in terms of its apparent production by a singular non-material, a-causal D2 computation which gives rise to all apparent (secondary computational) properties of 'space', 'time', 'energy' and 'mass'.

However, to the extent that the CUFT is corroborated empirically (e.g., especially in terms of the validation of its previously outlined critical empirical predictions), then the possible theoretical ramifications of its signified (potential) scientific paradigmatic shift must be further explored: Hence, the primary aim of the current chapter is to investigate the various potential theoretical ramifications of the CUFT as a candidate 'Theory of Everything' which points at the existence of the singular "reality" of the Universal Computational/Consciousness Principle as underlying all four basic physical properties (of 'space', 'time', 'energy' and 'mass') as well as all other inductive or deductive ' $x-y$ ' relationships; and finally also the possible relationship between this Universal Consciousness Principle and our individual human consciousness (e.g., which in fact may lead to a further modification of the CUFT's Universal Computational Formula based on the recognition of the potential gradations of individual human consciousness as embedded within the full expansiveness of the Universal Consciousness)...

\section{The universal computational principle's D2 A-causal computation}

We therefore (first) aim at fully integrating between the CUFT's Duality Principle proof for the conceptual computational inability to determine the "existence" or "non-existence" of any particular ' $y$ ' element based on any exhaustive hypothetical series of interactions with any ' $x$ ' factor/s (e.g., constituting a SROCS computational paradigm) - but only based on a conceptually higher-ordered (D2) Universal Computational Principle which computes the "simulta- 
neous co-occurrences" of any (empirically computable) exhaustive hypothetical series of 'x-y' pairs; and between the CUFT's Universal Consciousness Principle assertion that that higher-ordered (singular) Universal Computational Principle is (in fact) the only "real" element that truly exists - both as solely producing all USCF's secondary computational physical properties (of 'space', 'time', 'energy' and 'mass') and as existing solely (and independently of any such USCF's secondary computational properties) "in-between" any two subsequent USCF's: The starting point (to attain this first aim) is the CUFT's Duality Principle proof that all (hypothetical inductive or deductive) scientific SROCS paradigms must be constrained by a conceptually higher-ordered (D2) Universal Computational Principle which alone is capable of computing the "simultaneous co-occurrences" of any exhaustive hypothetical 'x-y' pairs series: Thus, both in the case of the CUFT's unification between quantum and relativistic models of physical reality as well as in the case of all other (hypothetical) inductive or deductive SROCS computational paradigms (e.g., for which there is an empirically known capacity to determine the values of any exhaustive ' $y$ ' and ' $x$ ' pairs series) the CUFT's Duality Principle has shown that the sole means for computing all of these (exhaustive hypothetical) quantum, relativistic, inductive or deductive ' $x$-y' relationships is based on the operation of the (conceptually higher-ordered) 'Universal Computational Principle' ('") which computes the "simultaneous co-occurrences" of all of these 'x-y' pairs series (e.g., which comprise a series of USCF's frames). Hence, we realize that all physical quantum or relativistic ' $x-y$ ' relationships as well as all (hypothetical) inductive (logical or mathematical) or deductive (e.g., including all natural sciences) ' $x-y$ ' relationships - are underline by the singular Universal Computational Principle's (') computation of the "simultaneous co-occurrences" of each of these 'x-y' pairs series... But, since according to the CUFT's 'Computational Invariance' and 'Universal Consciousness Principle' theoretical postulates the sole (and singular) computationally invariant "reality" that exists both as producing any USCF's series and which also (solely) exists "in-between" any two subsequent USCF's is that Universal Computational Principle which is equated with a Universal Consciousness Principle (e.g., capable of "producing", "retaining" and "evolving" any of the multifarious spatial pixels across subsequent USCF's frames) - whereas all physical properties - quantum or relativistic, or any inductive or deductive ' $x$ - $y$ ' relationships may only be as "phenomenal" or "unreal" due to their basic computationally variant properties). We are therefore forced to conclude that the sole production- sustenance- or any evolution- in any physical (quantum or relativistic), inductive or deductive (exhaustive hypothetical) ' $x-y$ ' pairs' series is based on the operation of the singular Universal Consciousness Principle which underlies the production of all USCF's frames and also exists independently "in-between" any two such (subsequent) USCF's. Thus, we accomplish our first aim of fully integrating between the CUFT Duality Principle's constraint of all scientific (inductive or deductive) scientific SROCS paradigms, e.g., based on the operation of the singular (conceptually higher-ordered) Universal Computational Principle ('") and the CUFT's (Computational Invariance and Universal Consciousness theoretical postulates') assertion regarding the sole "reality" of this Universal Computational/Consciousness Principle (e.g., both as producing all USCF's derived secondary computational physical properties or inductive or deductive 'x-y' relationships): This implies that instead of the existence of any "real" "material-causal" relationship between any quantum or relativistic (or any other exhaustive hypothetical inductive or deductive) ' $x$ ' 
and 'y' factor/s, elements, events etc. - the sole "reality" is of the existence of the singular 'Universal Consciousness Principle' which produces- sustains- and can evolve- all exhaustive hypothetical physical (quantum or relativistic) or inductive or deductive 'x-y pairs' series, e.g., based on its sole production of the USCF's frames and its independent existence in between any two such USCF's frames...

\section{The exhaustiveness of the universal consciousness principle for all natural phenomena}

Based on the CUFT's postulation of this singular Universal Computational/Consciousness Principle as comprising the only "real" (computationally invariant) principle which producesall USCF's (secondary computational) 'physical' properties (e.g., of 'space', time', 'energy' and 'mass'), and which also solely exists "in-between" any two (subsequent) USCF's, it is worthwhile to consider the broader applicability of this Universal Computation/Consciousness Principle ('") as underlying- and constraining- all (empirically knowable) hypothetical inductive or deductive ' $x-y$ ' relationship/s; Perhaps the best starting point for the such a comprehensive endeavor is to reexamine the computational SROCS structure underlying Gödel's Incompleteness Theorem (GIT) equivalent computational paradigms: This is because It is hereby hypothesized that an application of the Duality Principle to generalized deductive or inductive computational SROCS paradigms may bear equivalence to a certain aspect of Gödel's Incompleteness Theorem (GIT) - while allowing Science to advance beyond the mathematical constraint imposed by GIT; The basic hypothesis (advanced here) is that the Duality Principle sets a conceptual computational constraint upon all logical, mathematical or (indeed) scientific SROCS exhaustive relationship/s between any two given ' $x$ ' and ' $y$ ' elements - for which there exists an empirically known or determinable result (e.g., an empirically known capacity of the specific logical, mathematical, or other scientific computational system's to determine whether a particular given 'y' value or entity etc. "exists" or "doesn't exist");

In the general case of all (hypothetical) inductive ' $x$ ' and 'y' relationships, the Duality Principle constrains all SROCS paradigms of the form:

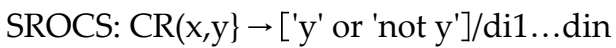

In the (generalized) case of all hypothetical deductive SROCS paradigms the Duality Principle constraint may be apply to a specific formalization of any computational system that attempts to determine the 'truth-value' (e.g., true: 't' or false: 'not t') of any hypothetical (exhaustive) Mathematical System ('Sm') based on an (exhaustive hypothetical) series of direct or indirect conceptual relationship/s between that given System and its definition of the 'true' (' $t$ ') value of that System, thus:

$\mathrm{CR}\left\{\mathrm{S}^{\prime} m^{\prime}, \mathrm{t}\right\} \rightarrow[$ 't' or 'not 't $] /$ di1 ...din

In fact, it is suggested that GIT proof may be equivalent to the Duality Principle's constraint of the (abovementioned) SRONCS special 'negative' computational outcome case: 
SRONCS: CR $\{$ 'Sm', t $\} \rightarrow$ 'not t' /di1...din

which was proven (by the Duality Principle) to inevitably lead to both 'logical inconsistency' and 'computational indeterminacy', e.g., stemming from the SRONCS logically contradictory assertion wherein the particular 'y' ('t') element both "exists" AND "doesn't exist" at the same computational level ('di1...din') - which necessarily also leads to such SRONCS structure's conceptual inability to determine whether that particular ' $y$ ' (' $t$ ') value "exists" or "doesn't exist"... But, for all of those logical, mathematical or inductive computational systems for which there exists an empirical capacity to determine whether any such particular System is "true" $(' t$ ') or 'false (' $f$ ') - since this empirical capacity contradicts the (abovementioned) SRONCS's inevitably ensuing 'logical inconsistency' and 'computational indeterminacy', then the Duality Principle asserts that there must exist a conceptually higher-ordered D2 computational framework capable of computing the simultaneous co-occurrences of any exhaustive hypothetical series of $\left\{\right.$ 'Sm' $\left.^{\prime} \mathrm{t}[1 \ldots \mathrm{n}]\right\} \ldots$ It is suggested that GIT's logical-mathematical proof may replicate the Duality Principle assertion regarding the inevitable 'logical inconsistency' and 'computational indeterminacy' that ensue from a SRONCS computational structure.

The principle difference between the Duality Principle's conceptual computational proof and GIT's logical mathematical proof is that whereas GIT focuses on the inevitable 'logical inconsistency' and (subsequent) 'computational indeterminacy' that arise from any SRONCS computational structure - whereas the Duality Principle goes further to investigate the empirical-computational ramifications of those specific computational systems for which there is a proven empirical capacity to determine whether or not any such (particular) ' $y$ ' value "exists" or "doesn't exist"; which therefore points at the inevitable existence of a conceptually higherordered D2 computational framework which can determine the "co-occurrences" of any (hypothetical) ['S' and 't'/'not t'] pairs' series... Thus, the Duality Principle's focus only on those logical, mathematical or scientific Systems for which there exists an empirical evidence for their capacity to determine the "truth" or "false" value of any given proposition or entity etc. - for which the Duality Principle proves that there exists a conceptually higher-ordered 'D2' computational framework that is capable of determining the "co-existence" of any (exhaustive hypothetical) pairs of 'Sm' and ' $t$ ' values.

In fact, as has been shown previously (Bentwich, 2011c) since there can only exist one (singular) such conceptually higher-ordered 'D2' computational framework (e.g., as underlying any and all SROCS computational systems) and based on its identification as no other than the Computational Unified Field Theory's singular D2 rapid series of USCF's), then we are led to the inevitable conclusion that all logical, mathematical or scientific (e.g., empirically knowable SROCS) paradigms must be embedded within the CUFT singular D2 rapid series of USCF's....

In order to formally present the exhaustiveness of the Duality Principle (e.g., as embedded within the CUFT's D2 framework) it may be helpful to formalize the conceptual computational constraint imposed by the Duality Principle on all (exhaustive hypothetical) inductive or deductive relationships in this manner:

SROCS $[1 \ldots z]: R\{x, y[1 \ldots n]\} \rightarrow[' y$ ' or 'not y']/di1...din 
wherein any (exhaustive hypothetical) logical, mathematical, computational or scientific SROCS paradigm/s is one which attempts to determine the "existence" or "non-existence" of any given ' $y$ ' element (or particular ' $y$ ' value) based on its direct or indirect physical or conceptual relationship/s with an exhaustive series of (hypothetical) 'x' series (e.g., at any single or multiple: 'di1...din' computational levels) - and for which there is a known (or knowable) empirical capacity to determine whether the particular ' $y$ ' entity or value "exists" or "doesn't exist".

Interestingly, once the Duality Principle narrows down the computational definition of all those (apparent) logical, mathematical, computational or (any other hypothetical) scientific SROCS paradigms - to only those computational systems for which there exists an empirical capacity to determine whether any particular ' $y$ ' entity "exists" or "doesn't exist", then according to the computational Duality Principle it (in effect) compliments (and may transcend) Gödel's Incompleteness Theorem, i.e., through the recognition of a (singular) conceptually higherordered 'D2' computational framework which is no other than the Universal Computational Principle's (extremely rapid) computation of a series of Universal Simultaneous Computational Frames (USCF's) - which also determines the simultaneous "co-occurrences" of any (exhaustive hypothetical) series of any ' $x$ ' and ' $y$ ' factors underlying all of (inductive or deductive) relationships, laws, phenomena (e.g., that can be known)... This is because based on the Duality Principle's narrowed down computational definition of any (exhaustive hypothetical) SROCS (inductive or deductive) scientific paradigm that possesses the general format:

$\operatorname{PR}\{x, y\} \rightarrow\left[y^{\prime}\right.$ or 'not y']/di1...din

and for which there exists an empirically known (or knowable) outcome (e.g., 'y' or 'not y'), the Duality Principle's computational proof (shown previously: Bentwich, 2011c \& d) indicated that such scientific SROCS paradigms can only be computed by the conceptually higherordered 'D2' computational framework (e.g., which was also shown to be equivalent to the abovementioned rapid series of USCF's).

In other words, the generalized format of the Duality Principle - when narrowed down to only those (apparently) computational SROCS paradigms for which there exist an empirical proof for the capacity of any such (inductive or deductive) computational system/s to determine whether any particular 'y' or 'not y' outcome exists (e.g., at any given spatial-temporal point/s) - also necessarily provides us with the Duality Principle's asserted conceptual computational proof for the existence of a singular higher-ordered 'D2' USCF's based computation of the co-occurrences of any exhaustive hypothetical series of (particular) ' $x$ ' and ' $y$ ' pairs. Indeed, it is suggested that in the particular case of 'Gödel's Incompleteness Theorem' (GIT) which constitutes the state of the art known conceptual mathematical constraint imposed on our capacity to construct any (hypothetical deductive) logical or mathematical System - i.e., as necessarily containing certain mathematical statement/s which either lead to 'logical inconsistency' (e.g., such as in the basic case of the "liar's paradox" - as embedded within GIT), or which cannot be determined from within any such (exhaustive hypothetical) Mathematical System - the generalized form of the Duality Principle provides us with a clear indication that even though the consistency of certain mathematical (SRONCS) statements cannot be deter- 
mined from within any (exhaustive hypothetical) Mathematical System, their consistency can be determined by the conceptually higher-ordered 'D2' (USCF based) computational framework...

As such, the computational Duality Principle offers us a potentially significant alternative to 'Gödel's Incompleteness Theorem's (GIT) 'negative' constraint set upon the capacity to construct any consistent logical, mathematical (e.g., or indeed scientific - as shown later on) computational System based on the realization that any such (exhaustive hypothetical) logical or mathematical (empirically validated) System can be formulated based on a conceptually higher-ordered 'D2 a-causal' computational framework (Bentwich, 2012: a \& b) that is capable of computing the simultaneous "co-occurrences" of any such (exhaustive hypothetical) pairs series of logical or mathematical System and corresponding ' $t$ ' (truth-value) definition/s; This is due to the fact that based on the (abovementioned) Duality Principle's strict definition of only those mathematical or logical systems for which there is a known (empirical) capacity to compute the "truth" or "false" value of any System (or statement within a given System) - its proof for the conceptual computational inability of any (such) SROCS/SRONCS system to carry out such computation at the same computational level as any direct or indirect (conceptual) interaction between the System and the 'truth value' definition but only at a conceptually higher-ordered 'D2 a-causal' computational framework (Bentwich, 2012a) points at the capacity of any such hypothetical logical or mathematical System to determine the simultaneous "co-occurrences" of any series of 'System' and 'truth-value' definition/s (e.g., at the 'D2 a-causal' computational framework)... In other words, once the (generalized) Duality Principle narrows down the computational definition of any possible logical or mathematical SROCS paradigm (e.g., of the form: $C R\{S, t\} \rightarrow[$ 't' or 'not t']) to only those Systems for which there is a known empirical capacity to determine whether any given System or any given statement/s within a given System), then we necessarily obtain the Duality Principle's conceptual computational proof for the capacity to produce a particular series of logical or mathematical systems that are capable of determining whether they are 'true' or 'false' - based on a conceptually higher-ordered 'D2 a-causal' computational framework. In that sense, this generalized Duality Principle format (e.g., for empirically computable logical or mathematical systems) seems to transcend Gödel's Incompleteness Theorem's strict constraints set on the construction of any logical or mathematical consistent systems, e.g., as devoid of any 'logical inconsistency' or 'mathematical indeterminacy' (as also defined previously: Bentwich, 2012a); this is because such generalized Duality Principle format in fact asserts the capacity to construct specific logical or mathematical systems for which we can determine their truth-value based on a conceptually higher-ordered 'D2 a-causal' computational framework which can compute the simultaneous "co-occurrences" of any exhaustive hypothetical pairs series of mathematical system and truth-value (e.g., 't' or 'not t'). Indeed, based on the Duality Principle's previously proven singularity of such conceptually higher-ordered 'D2 a-causal' computational framework (e.g., which has been furthermore shown to be synonymous with the Computational Unified Field Theory's rapid series of Universal Simultaneous Computational Frames) - the generalized Duality Principle's proof indicates that for all empirically determinable logical or mathematical systems there necessarily exists only one singular higher-ordered D2 (USCF's) computational framework that is capable of determining any exhaustive hypothetical pairs 
(series) of logical or mathematical system and corresponding 'truth-value' (e.g., 't' or 'not t') outcome.

Finally, it is suggested that this generalized format of the Duality Principle offers a constructive computational alternative to GIT's failing of Hilbert's famous 'Mathematical Program' - to base all of Mathematics on the basis of Logic (e.g., and moreover attempt to base the whole of Science upon the foundations of such a logical-mathematical structure); This is because GIT essentially proved that any exhaustive hypothetical (SRONCS) Mathematical System (e.g., of the form: $\mathrm{CR}\{\mathrm{S}, \mathrm{t}\} \rightarrow$ 'not $\mathrm{t}$ ') necessarily leads to both 'logical inconsistency' and 'mathematical inconsistency' - i.e., as also indicated by the Duality Principle's analysis of any such SRONCS computational structure); As such, GIT evinces - as does the Duality Principle, that such SRONCS computational structure cannot be computed, e.g., from within the confinements of any such SRONCS Mathematical System... However, since GIT does not go further to investigate whether any such specific (apparent SRONCS) computational system can determine empirically whether any given statement (found within that System) is 'true' or 'false', then the theoretical assertion made by GIT is that for any given (exhaustive hypothetical) mathematical system there exist certain SRONCS statement/s which cannot be proven from within such system... Hence, the theoretical ramification of GIT was taken to indicate that the whole of Logic and Mathematics cannot be based on any exhaustive hypothetical logical or mathematical system (i.e., regardless of its potential complexity etc.) - which essentially failed Hilbert's 'Mathematical Program' to base the whole of Mathematics (e.g., and by extension potentially also the whole of deductive and inductive Science) upon the foundations of any given logical (or mathematical system)... However, if we are to accept the Duality Principle's (generalized format) proof for the specifically defined logical or mathematical systems - i.e., of the form: $\mathrm{CR}\{S, t\} \rightarrow[$ 't' or 'not $\mathrm{t}$ '] for which there exist an empirical capacity to determine whether any given statement found within such system/s is 'true' or 'false', then the Duality Principle in fact proves that for such (empirically determinable) logical or mathematical systems there exists a conceptually higher-ordered computational framework (e.g., 'D2') which can determine the simultaneous "co-occurrences" of any exhaustive hypothetical series of 'statement' and 'true' or 'false' pairs! Thus, the Duality Principle in effect offers a higher-ordered 'hierarchicaldualistic' ('D2') alternative (i.e., for those specific logical or mathematical systems for which there is a known capacity to determine the "true" or "false" value of any given statement) - to GIT's asserted conceptual computational inability to determine the consistency or computability of any mathematical system that can contain a SRONCS statement!

Therefore, it may be said that GIT proved the inevitable 'logical inconsistency' and 'computational indeterminacy' of any given SRONCS statement - and subsequently 'extrapolated' from the existence of such 'logically inconsistent' and 'computationally indeterminable' SRONCS statements that the whole of Logic or Mathematics is "flawed" in that there is no possibility to construct any exhaustive hypothetical logical or mathematical system that will be free of any such logical inconsistencies or computational indeterminacy... In contrast, the (generalized) Duality Principle views the existence of any such SRONCS statement/s - as embedded within the general SROCS/SRONCS scientific computational structure and asserts that for any such scientific (e.g., inductive or deductive) SROCS/SRONCS structure for which there is a capacity 
to determine whether any given statement possesses a "true" or "false" value there must exist a conceptually higher-ordered (singular) 'D2' computational framework that is capable of computing the simultaneous "co-occurrences" of any series of pairs of 'statement' and 'true' or 'false'; Hence, the (generalized) Duality Principle evinces the existence of a whole series of (deductive or inductive) scientific SROCS/SRONCS paradigms for which there is an empirically proven capacity to determine the truth value (e.g., "true" or "false") of any given statement/s - which point at the (inevitable) existence of a conceptually higher-ordered (singular) 'D2 a-causal' computational framework that computes any exhaustive hypothetical series of statement and truth value pairs... Therefore, the Duality Principle in fact replaces GIT's strict assertion wherein it is not possible to construct any logically consistent and computationally determinable logical or mathematical system - instead pointing at the existence of a whole series of inductive or deductive (apparently SROCS/SRONCS) computational systems for which there exists an empirically proven capacity to determine whether any given statement is 'true' or 'false' and which is necessarily computed by that singular conceptually higherordered 'D2 a-causal' computational framework that can compute the simultaneous "cooccurrences" of any 'statement' and 'true'/'false' value. Hence, the (generalized) Duality Principle points at the existence of a singular conceptually higher-ordered 'D2' computational framework - upon which all of the empirically 'known' (or 'knowable') inductive or deductive relationships has to be based; It is therefore suggested that a new hierarchical-dualistic formalization of (deductive and inductive) Science has to be anchored in- and based upon- this singular conceptually higher-ordered 'D2 a-causal' computational framework, which has been previously shown to be no different than the Computational Unified Field Theory's (CUFT) D2 Universal Computational Principle ('") based rapid series of Universal Simultaneous Computational Frames (USCF's)...

Therefore, the Duality Principle's (generalized) resolution of GIT consists of the precise definition of only those deductive systems or statements possessing the (apparent) SROCS form:

$\mathrm{CR}\left\{\{x, y\} \rightarrow\left[y^{\prime}\right.\right.$ or 'not y']/di1...din; or

$\mathrm{CR}\{\mathrm{S}, \mathrm{t}\} \rightarrow\left[\mathrm{t}^{\prime}\right.$ or 'not t']/di1...din

which are known empirically to be capable of determining whether any given 'y' element (or value) "exists" or "doesn't exist" or whether a given System or statement possesses a 'true' or 'false' value (e.g., as defined by that System or statement);

Indeed, based on this (narrower) definition of only those deductive (apparent) SROCS paradigms for which there is a capacity to determine the "existence" or "non-existence" of a particular 'y' value or 'truth value' (i.e., 'true' [t] or 'false' [f]) the (generalized) Duality Principle proves that there must exist a conceptually higher-ordered (singular) 'a-causal D2' computational framework which can compute the simultaneous "co-occurrences" of any exhaustive hypothetical pairs of the deductive system's (or statement's) abovementioned 'x' and 'y' factors or of any exhaustive hypothetical pairs of 'S' and ' $t$ ' values, thus:

D2: $([\{S\{1 \ldots n\}, t\} i \ldots\{S\{1 \ldots n\}, t\} z]$, or $[\{x\{1 \ldots n\} i, y i\} \ldots\{x\{1 \ldots n\} z, y z\}])$ 
Note that this particular definition of the (generalized) Duality Principle asserts that for all of those deductive systems (or statements) for which there exists an empirical proof for their capacity to determine the "existence" or "non-existence" of any particular 'y' element/s (or factor/s) or any particular 't' value (e.g., 'true' or 'false') - the Duality Principle proves that there must exist a conceptually higher-ordered 'D2 a-causal' computational framework which can compute the simultaneous "co-occurrences" of any exhaustive hypothetical series of ' $\mathrm{S}$ ' and ' $\mathrm{t}$ ' pairs or of ' $x$ ' and ' $y$ ' pairs; This implies that through the narrower definition of only those empirical deductive computational systems (or statements) for which there is a capacity to determine their (particular) 'y' or 'truth value' - the (generalized) Duality Principle is able to go beyond GIT's negative SRONCS' assertion wherein for all logical or mathematical systems there exist specific statements that cannot be determined from (within that system) or which lead to logical inconsistency (of that system), thereby opening the door for a novel 'hierarchicaldualistic' definition of those deductive systems that can be known and which do not lead to any logical inconsistencies...

Finally, it is suggested that based on the equivalence of GIT (deductive) SROCS computational structure to all (previously: Bentwich, 2011d) analyzed (inductive) SROCS scientific paradigms:

$\operatorname{PR}\{x, y\} \rightarrow[$ 'y' or 'not y']/di1...din; or

$\mathrm{CR}\left\{\{x, y\} \rightarrow\left[y^{\prime}\right.\right.$ or 'not y']/di1...din;

Then according to the Duality Principle's computational-empirical proof (Bentwich, 2011c \& d), i.e. indicating that it is not possible (in principle) to compute the "existence" or "nonexistence" of any such particular 'y' entity (or value) based on its direct (or indirect) physical or conceptual relationship/s with another ' $x$ ' entity (e.g., at any 'di1...din' computational level/ s) - but rather the empirically proven capacity of specific computational systems to compute the simultaneous "co-occurrences" of any (exhaustive hypothetical) series of pairs of ' $x$ ' and ' $y$ ' (occurring at any known given spatial-temporal point/s or at any known computational level/ s or instance/s). Indeed, based on the (abovementioned) Duality Principle's (generalized) particular definition of any such inductive or deductive SROCS scientific paradigm as possessing both the (above outlined) inductive or deductive SROCS computational structure and the empirical capacity to determine whether any given 'y' element "exists" or "doesn't exist", as well as the Duality Principle's proof for the existence of a conceptually higher-ordered (singular) 'D2 a-causal' (USCF's based) computational framework - then this evinces the fact that all empirically determinable scientific (inductive or deductive) SROCS paradigms must all be computed by this singular conceptually higher-ordered D2 USCF's series computational framework...

Therefore, the next (logical) step may be to consider all of the previously demonstrated scientific SROCS paradigms constrained by the Duality Principle (including: Darwin's Natural Selection Principle, the Genetic Encoding Hypothesis, Neuroscience's (materialistic-reductionistic) Psycho-Physical Problem of human consciousness, as well as Gödel's Incompleteness Theorem's replacement by the generalized Duality Principle's proof for the capacity to determine the simultaneous "co-occurrences" of any ' $x$ ' and ' $y$ ' (deductive or inductive) 
exhaustive hypothetical pairs (e.g., for all those empirical computational systems for which there is a known empirical capacity to determine the "existence" or "non-existence" of any given 'y' element or value/s); Specifically, the next section attempts to fully integrate between all known (any determinable) inductive or deductive scientific SROCS paradigms (e.g., delineated previously: Bentwich, 2012: a \& b, and above) as necessarily comprising- and being embedded within- the singular conceptually higher-ordered 'D2 a-causal' computational framework which was already shown to be no other than the CUFT's rapid series of USCF's computed solely by the Universal Computational Principle, '').

Previously (Bentwich, 2012b) it was shown that each of a series of key scientific SROCS paradigms (including: Darwin's Natural Selection Principle, the Genetic Encoding Hypothesis and Neuroscience's Psycho-Physical Problem) are necessarily constrained by the Duality Principle - indicating that their empirically proven capacity to determine the "existence" or "non-existence" of their (particular) 'y' element may not be based on any direct or indirect physical interaction between that given ' $y$ ' element and any (exhaustive hypothetical) $x$-series of the form:

SROCS: PR $\{x, y\} \rightarrow[$ 'y' or 'not 'y' $\backslash /$ di1 ...din

Instead, the Duality Principle pointed at the (inevitable) existence of a conceptually higherordered 'D2 a-causal' computational framework which is capable of computing the simultaneous "co-occurrences" of any (exhaustive hypothetical) ' $x$ ' and 'y' pairs series - as embedded within the Computational Unified Field Theory's singular D2 rapid series of USCF's (which are computed by the Universal Computational Principle, '"'); Thus, for instance, both Darwin's Natural Selection Principle's SROCS and (associated) Genetic Encoding SROCS computational structures were shown to constrained by the Duality Principle - pointing at an (inevitable) singular conceptually higher-ordered 'D2 a-causal' USCF's (rapid) series which is computed by the Universal Computational Principle and which computes the simultaneous "co-occurrences" of any exhaustive hypothetical series of 'organism' ('o') and 'Environmental Factors' $(\mathrm{E}(1 \ldots \mathrm{n}))$ or of 'Genetic Factors' and 'Phenotype property', or of Genetic Encoding and Protein Synthesis' etc. Likewise, it was hypothesized that the four computational SROCS levels constituting Neuroscience's (materialistic-reductionistic) Psycho-Physical Problem are also necessarily constrained by the Duality Principle - also pointing at the same singular conceptually higher-ordered 'D2 a-causal' computational framework constituting the rapid USCF's series that is computed by the Universal Computational Principle ('"), which also embeds within each of those rapid series of USCF's the simultaneous "co-occurrences" of any (exhaustive hypothetical) 'Psychophysical Stimulus' and (corresponding) 'Neural Activation' pairs; or any 'Neural Activation' and 'Functional Activation' pairs, or any 'Functional Activation' and 'Pheneomenological Experience' pairs; or any 'Phenomenological Experience' and 'SelfConsciousness' pairs... In much the same manner, the abovementioned analysis offered by the generalized Duality Principle for Gödel's Incompleteness Theorem (GIT) and subsequent (narrower) definition of all those scientific (inductive or deductive) SROCS paradigms (which can be determined empirically) also pointed at the existence of a singular conceptually higherordered 'D2 a-causal' USCF's based computational framework that can compute any (exhaus- 
tive hypothetical) ' $x$ ' and 'y' pairs series as "co-occurring" simultaneously (e.g., as embedded within any single or multiple USCF's frames).

Thus, an application of one of the key theoretical postulates of the 'Computational Unified Field Theory' (CUFT), namely: the computational 'Duality Principle' to a series of central (inductive or deductive) scientific SROCS paradigms (including: Darwin's Natural Selection Principle, the Genetic Encoding hypothesis, Neuroscience's Psycho-Physical Problem and 'Gödel's Incompleteness Theorem' and broader hierarchical-dualistic reformalization of all determinable deductive apparently SROCS paradigms) pointed at the need to reformulate all such scientific SROCS paradigms based on a singular conceptually higher-ordered 'D2 acausal' computational framework which was previously shown (Bentwich, 2012: a \& b) to be no other than the 'Universal Computational Principle' computed rapid series of USCF's (as delineated by the Computational Unified Field Theory); There are several potentially far reaching theoretical ramifications for this new hypothetical assertion made by the Computational Unified Field Theory (CUFT) and embedded computational 'Duality Principle': First, to the extent that the CUFT and Duality Principle's (abovementioned) applied scientific SROCS paradigms (e.g., including: Darwin's Natural Selection Principle and Genetic Encoding hypothesis, Neuroscience's Psycho-Physical Problem and Gödel's Incompleteness Theorem etc.) may be corroborated, then we must accept that all of these inductive and deductive scientific paradigms must be reformulated based on the recognition of a singular conceptually higher-ordered CUFT's based 'D2 a-causal' USCF's computational framework;

Specifically, the acceptance of the Duality Principle - e.g., as one of the key postulates of the CUFT as well as a basic constraint for each of these major scientific paradigms forces us to relinquish the current 'material-causal' working assumption underlying each of these scientific SROCS paradigms (i.e., of the general form: $\operatorname{PR}\{x, y\} \rightarrow\left[y^{\prime}\right.$ or 'not y'] or CR $\{x . y\} \rightarrow\left[y^{\prime}\right.$ ' or 'not y'] or $\operatorname{PR}\{S, t\} \rightarrow[' t$ ' or 'not $t$ '], as explained above); Thus, instead of Darwin's Natural Selection SROCS paradigm wherein it is assumed that it is the direct physical interaction between an 'organism' and an (exhaustive hypothetical) series of 'Environmental Factors' that materially causes the "existence" or "extinction" (e.g., non-existence) of a given organism - the Duality Principle points at the existence of a conceptually higher-ordered 'D2 a-causal' computational framework which computes the simultaneous "co-occurrences" of any given 'organism' and corresponding 'Environmental Factors' pair/s (e.g., comprising any particular Universal Simultaneous Computational Frame [USCF's] frame); Likewise, instead of the basic 'Genetic Encoding' hypothesis underlying much of modern Genetics and Biology - wherein it is assumed that it is the material-causal (direct or indirect) relationship/s between a given 'Genetic Factors' and particular 'Phenotypic Property' (or properties) which determines whether any such Phenotypic Property shall "exist" or "not exist"; or wherein it is assumed that it is the direct or indirect physical interaction/s between a particular 'Genetic Encoding' process and certain 'Protein Synthesis' process/es that determines whether or not any given protein/s shall be synthesized (e.g., or vice versa) (Bentwich, 2012b) - the Duality Principle asserts that it is not possible in principle to determine the "existence" or "non-existence" of any such (particular) 'y' entity (e.g., 'Phenotypic Property' or 'Protein Synthesis' etc.) based on its direct or indirect physical interaction with any exhaustive hypothetical series of 'x factors'; Instead, 
the Duality Principle (once again) points at the existence of a conceptually higher-ordered 'D2 a-causal' USCF's based computation of the simultaneous "co-occurrences" of any exhaustive hypothetical pairs series of any such 'Genetic Factors' and (particular) 'Phenotypic Property', or of any 'Genetic Encoding' and 'Protein Synthesis' etc. - all computed simultaneously by the singular conceptually higher-ordered 'D2 a-causal' Universal Computational Principle ('“') which are embedded within its series of rapidly computed USCF's... In much the same manner, the Duality Principle challenges the currently 'materialistic-reductionistic' working hypothesis underlying Neuroscience's assumption whereby any of the (four level) Psycho-Physical Problem's SROCS paradigms asserting that any of the four levels of human Consciousness is necessarily caused by a (direct or indirect) material interaction/s between a certain stimulus and corresponding neural activation pattern (e.g., essentially replicating the above- and previous mentioned SROCS computational structure: Bentwich 2012b); Instead, the Duality Principle evinces that any of these (four leveled SROCS) ' $x-y$ ' pairs relating to various aspects of the human Consciousness is computed simultaneously as "co-occurring" pairs by the singular conceptually higher-ordered 'D2 a-causal' Universal Computational Principle as embedded within the rapid series of USCF's frames... Finally, it was suggested (above) that Gödel's Incompleteness Theorem (GIT) may also replicate the SROCS computational structure and therefore may need to give way to the Duality Principle's (generalized) assertion that for all of those deductive paradigms (or statement/s or instances) for which there is a known empirical capability to determine the "existence" or "non-existence" of any given 'y' entity (or value) or of any given 'truth-value' definition ("t": 'true' or 'false'), there must exist a conceptually higher-ordered (singular) 'D2 a-causal' computational framework which is synonymous to the Universal Computational Principle's that is capable of determining the simultaneous "co-occurrences" of any exhaustive hypothetical series of such deductive ' $\mathrm{X}$ ' and ' $y$ ', or ' $\mathrm{S}$ ' and ' $t$ ' pairs which are necessarily embedded within the rapid series of USCF's). Therefore, the acceptance of the Duality Principle as embedded within the CUFT's rapid series of ('Universal Computational Principle' produced) USCF's and as constraining any of the (abovementioned) scientific SROCS paradigms necessarily calls for the reformulation of each and every one of these SROCS paradigms based on the existence of the CUFT's asserted singular conceptually higher-ordered Universal Computational Principle's 'D2 a-causal' computed rapid series of USCF's (e.g., instead of these scientific SROCS' current asserted 'material-causal' determination of any particular ' $y$ ' factor based on its direct or indirect physical interaction/s with another exhaustive hypothetical series of ' $x$ ' factors)...

Second, based on the Duality Principle's conceptual computational proof for the singularity of the 'D2 a-causal' computational framework -i.e., as necessarily computing all apparent SROCS paradigms' ' $x$ ' and 'y' (direct or indirect) relationship/s, and as embedded within the Universal Computational Principle's rapid computation of the series of USCF's, we must accept the notion wherein all of the abovementioned scientific SROCS paradigms must be computed simultaneously as "co-occurring" (particular) ' $x$ ' and 'y' (inductive or deductive) pairs by the Universal Computational Principle ('") through its computation of the rapid series of USCF's... Indeed, if we were to assemble all of the Duality Principle's (earlier proven: Bentwich, 2012: a \& b) SROCS' conceptually higher-ordered 'D2' computational levels which were shown to (alone) be capable of computing the "co-occurrences" of any (particular) 'x' and 'y' factors we 
would obtain a series of SROCS scientific paradigms that are all shown (by the Duality Principle) to be computed by the conceptually higher-ordered (singular) D2 'a-causal' computational framework:

N.S.:D2: $[\{\mathrm{E}\{1 \ldots \mathrm{n}\}, \mathrm{o}\} \mathrm{st} 1 ;\{\mathrm{E}\{1 \ldots \mathrm{n}\}, \mathrm{o}\} \mathrm{st} 2 \ldots\{\mathrm{E}\{1 \ldots \mathrm{n}\}, \mathrm{o}\} \mathrm{stn}]$.

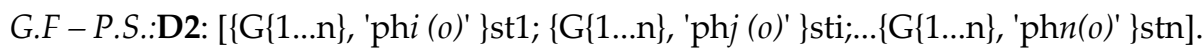

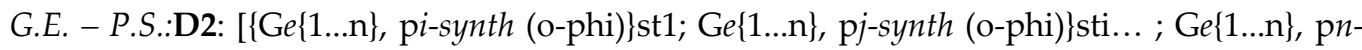
synth (o-phi)\}stn]

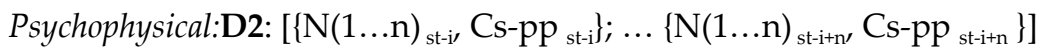

Functional: D2: [\{Cs(pp) $\left.\left.\mathrm{f}_{\mathrm{i}}, \mathrm{Na}(\mathrm{spp}) \mathrm{fi}\right\}_{\mathrm{st}-\mathrm{i}} ; \ldots\left\{\mathrm{Cs}(\mathrm{pp}) \mathrm{f}_{(\mathrm{i}+\mathrm{n})}, \mathrm{Na}(\mathrm{spp}) \mathrm{f}_{(\mathrm{i}+\mathrm{n})}\right\}_{\mathrm{st}(\mathrm{i}+\mathrm{n})}\right]$

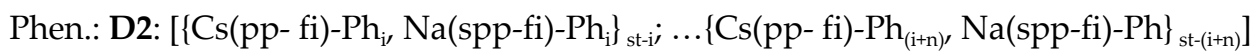

Self: D2: [\{Cs(pp-fi)Ph-Si, Na(pp-fi)Ph-S $\left.\}_{\text {st-i }} ; \ldots\{\mathrm{Cs}(\mathrm{pp}-\mathrm{fi}) \mathrm{Ph}-\mathrm{S}(\mathrm{i}+\mathrm{n}), \mathrm{Na}(\mathrm{pp}-\mathrm{fi}) \mathrm{Ph}-\mathrm{S}(\mathrm{i}+\mathrm{n})\}_{\mathrm{st}-(\mathrm{i}+\mathrm{n})}\right]$

GIT:D2: ([\{S\{1...n $\}, \mathrm{t}\} i \ldots\{\mathrm{S}\{1 \ldots \mathrm{n}\}, \mathrm{t}\} z]$, or $[\{\mathrm{x}\{1 \ldots \mathrm{n}\} i, \mathrm{y} i\} \ldots\{\mathrm{x}\{1 \ldots \mathrm{n}\} z, \mathrm{y} z\}])$

But, since it was already proven by the Duality Principle that this singular conceptually higherordered 'D2 a-causal' computation cannot be reduced to any direct or indirect material interaction/ $s$ between any (particular) exhaustive series of ' $x$ ' factor/s and any ' $y$ ' entity (or between any exhaustive series of logical or mathematical Systems or statement/s and any of their specific 'truth-value' definitions (Bentwich, 2012: a \& b); and since the Computational Unified Field Theory (CUFT) evinced the existence of a rapid series of Universal Simultaneous Computational Frames (USCF's) which was postulated to be computed (e.g., at an extremely rapid rate: ' $\mathrm{c}^{2} / \mathrm{h}$ ') by the singular Universal Computational Principle ('"') - i.e., with no "material" entity existing "in-between" any two subsequent USCF's (frames); then it follows that all of the abovementioned scientific SROCS paradigms must be computed based on the CUFT's singular conceptually higher-ordered 'D2 a-causal' Universal Computational/Consciousness Principle ('") as part of its (rapid) computation of the USCF's series - with no material entity, 'mass', 'energy', 'space' or 'time' object/s or event/s, factor/s or process/es etc. existing "in-between" any USCF's frames...

\section{The universal computational principle's paradigmatic shift: Transcending cartesian dualism}

Based on the above demonstration of the basic constraint imposed by the (singular) Universal Computational/Consciousness Principle ('') upon the computation of any hypothetical (empirically knowable) ' $x-y$ ' relationship (or phenomenon), the next logical question would be: what may be the possible relationship between this conceptually higher-ordered, singular Universal Consciousness Principle and out individual human Consciousness? (Interestingly enough, as we be shown below, posing such a question may have significant theoretical ramifications with regards to some of the most basic tenets underlying modern Cartesian Science, i.e., including the basic tacit assumption wherein the "objective" physical reality may 
be separated from the "subjective" Consciousness observing or measuring such objective phenomenon...);

A natural starting point for exploring this important question may be related to the Duality Principle's (previous) analysis of Neuroscience's current SROCS computational Psychophysical Problem (PPP) of human Consciousness (Bentwich, 2012b): This is because the above analysis of Neuroscience's PPP indicated that all four computational levels of Neuroscience's SROCS PPP computational structure are constrained by the same (basic) Duality Principle, thereby pointing at the (singular) Universal Computational Principle as computing the "simultaneous co-occurrences" of all of these multifarious (e.g., Psychophysical, Functional, Phenomenal, Self) ' $x$-y' pairs. It was moreover shown (above and previously) that the Universal Computational/Consciousness Principle is responsible for the simultaneous computation of all "co-occurring" PPP, (quantum and relativistic) physical relationships, as well as all (empirically knowable) inductive and deductive 'x-y' pairs; We thus arrive at the inevitable conclusion that all (quantum and relativistic) 'x-y' physical relationships, all exhaustive hypothetical inductive or deductive ' $x-y$ ' relationships, as well as all human Consciousness Psychophysical ' $x-y$ ' relationships must be solely produced- sustained- and evolved- by this (singular) Universal Computation/Consciousness Principle, and moreover that this Universal Consciousness Principle comprises the sole "reality" underlying all such (secondary computational) phenomenal relationships...

But, if indeed the sole "reality" underlying all physical, inductive, deductive and individual Consciousness (Psychophysical) ' $x$-y' phenomenal relationships is the Universal Consciousness Principle, then this means that in reality there is only a singular Universal Consciousness Principle which produces all apparent physical, inductive, deductive or individual human Consciousness (secondary computational) phenomena (e.g., comprising of all exhaustive hypothetical empirically knowable ' $x-y$ ' pairs); In this respect, the singular Universal Consciousness Principle becomes the sole "reality" which supersedes- (entirely) constrainsall apparent (quantum or relativistic) phenomenal ' $x-y$ ' relationships, or indeed any inductive or deductive or any individual human Consciousness ' $x-y$ ' relationships... Obviously, such a profound realization signifies a major 'paradigmatic shift' in Cartesian Science's (contemporary) theoretical framework which assumes that all natural phenomena are reducible to the analysis of fundamental (SROCS) ' $x-y$ ' relationships, wherein the "existence" or "non-existence" of any given 'y' element, value, phenomenon or process etc. can be determined solely based on its direct or indirect physical interactions with another (exhaustive hypothetical) series of ' $x$ ' factor/s; Instead, the acceptance of the CUFT's assertion regarding the sole existence of a singular Universal Computational/Consciousness Principle ('') that is (solely) responsible for the production- sustenance- and possible evolution- of all quantum and relativistic physical relationships, all inductive or deductive (empirically knowable) relationships and all individual human Consciousness (psychophysical) relationships - represents a basic shift from a purely 'materialistic-reductionistic' Cartesian approach to the realization that the sole "reality" underlying all phenomenal physical, inductive, deductive or individual human consciousness relationships is only this singular Universal Computational/Consciousness Principle... 
However, in order to fully appreciate the potential theoretical significance of this Universal Computation/Consciousness Principle paradigmatic shift, let's focus our attention on the "mechanics" of this Universal Consciousness Principle's production- sustenance- and evolution- of all (abovementioned) quantum and relativistic physical relationships, inductive or deductive relationships or individual human Consciousness relationships; If we were to take a closer look at the operation of this Universal Computational/Consciousness Principle ("') and its production- sustenance- and (possible) evolution- of any of these physical, inductive, deductive or individual human Consciousness 'x-y' relationships we would realize a few important points:

a. Based on the CUFT's delineation of the operation of this (singular) Universal Computational/Consciousness Principle (alongside the Duality Principle's assertion regarding the Universal Computational/Consciousness Principle's computation of the "simultaneous co-occurrences" of all physical, inductive or deductive 'x-y' pairs) we realize that all exhaustive hypothetical physical, inductive, deductive or individual human Consciousness psychophysical ' $x$-y' pairs must be computed simultaneously by the same Universal Computational/Consciousness Principle - e.g., as embedded within single or multiple USCF's frame/s; As noted above (and previously), such a conceptually higher-ordered (singular) Universal Computational/Consciousness Principle's 'A-Causal D2' computation negates the possibility of any real "material-causal" relationship/s existing between any of these ' $x \rightarrow y$ ' pairs series (but instead advocates the Universal Computational/ Consciousness Principle's sole computation of any singular or multiple USCF's "simultaneous co-occurrences" of an exhaustive hypothetical series of 'x-y' pairs...) Again, as indicated previously, this implies that for instance instead of the Darwin's Natural Selection Principle's postulation of the existence of a 'material-causal' relationship existing between a given organism's Environmental Factors and their determination of that organism's "existence" or "extinction" based on their direct (or indirect) physical interactions with that organism, the Universal Computational/Consciousness Principle indicates that in "reality" there cannot be any "material-causal" relationships between the organism and its Environmental Factors but instead only the Universal Computational/Consciousness Principle's computation of the "simultaneous co-occurrences" of a series of such (particular) 'organism' and Environmental Factors (e.g., as embedded within a series of USCF's). Indeed, based on the (generalized) Duality Principle proof for all inductive or deductive ('Gödel-like' SROCS) computational paradigms, it becomes clear that there cannot exist any "real" "material-causal" relationships between any exhaustive hypothetical (physical quantum or relativistic, inductive or deductive) ' $x-y$ ' entities, but only the conceptually higher-ordered Universal Computational/Consciousness Principle's computation of the "simultaneous co-occurrences" of such pairs across a series of USCF's.

The CUFT's 'Computational Invariance Principle' and 'Universal Consciousness Principle' theoretical postulates which prove that only the 'computationally invariant' Universal Computational Principle ('') may be regarded as "real" whereas the 'computationally variant' (secondary computational) physical properties of 'space', 'time', 'energy' and 'mass' must be regarded as "illusory"; and that since only this singular Universal Com- 
putational Principle exists both "in-between" any two (subsequent) USCF's and (solely) produces any of these "illusory" (secondary computational) physical properties - then this Universal Computational Principle must also possess the 'Universal Consciousness Principle' functions of being capable of producing- sustaining-/retaining- and evolvingany of the numerous spatial pixels properties across any series of USCF frame;

b. Therefore, the CUFT's Universal Consciousness Principle (e.g., augmented by the CUFT's 'Computational Invariance' postulate) asserts that the sole existence of any phenomenal (secondary computational) physical property (of any given object or event) is in truth entirely produced- retained- and evolved- solely and singularly based on the "reality" of this singular Universal Consciousness which solely "exists" both "in-between" any (two subsequent) USCF's and solely produces any of these (secondary computational) physical properties; Note that since none of the 'physical' properties (of 'space', 'time', 'energy' or 'mass') exist "in-between" any (two subsequent) USCF's (e.g., but only the Universal Consciousness Principle which both produces- all of these USCF's secondary computational properties as well as exists "in-between" any two subsequent USCF's), then the sole "reality" that exists both as producing the USCF's and "in-between" any two such USCF's is that Universal Consciousness Principle. Likewise, since according to this Universal Consciousness Principle all four 'physical' properties of 'space', 'time', 'energy' and 'mass' "exist" - only as 'phenomenal' secondary computational properties of the "real" Universal Computational Principle's production of the (rapid series of) USCF's (but "vanish" in between any two such subsequent USCF's), then it is also obvious that no "real" 'materialcausal' relationship can exist between any physical property of an object or an event (e.g., found in a particular USCF frame) and any physical property in any subsequent USCF frame/s... Therefore, we reach the inevitable conclusion that it is only the (singular) Universal Consciousness Principle which truly "exists" and is solely responsible for the production- retention- and evolution- of any of the four (secondary computational) 'physical' properties (e.g., of 'space', 'time', 'energy' and 'mass').

c. Hence, the paradigmatic shift portrayed by the CUFT is that instead of current Cartesian 'materialistic-reductionsitic' Science's basic (implicit) assumption wherein any hypothetical (inductive or deductive) element, entity, phenomenon or process \{'y'\} can be determined solely based on its direct or indirect physical interaction/s with another exhaustive hypothetical ' $x$ ' series (e.g., comprising a SROCS computational structure negated by the CUFT's Duality Principle for all empirically knowable 'x-y' relationships); the CUFT proves the existence of a singular (conceptually higher-ordered) Universal Consciousness (and Computational) Principle which constitutes the sole "reality" that both producesretains- and evolves- the (extremely rapid) series of USCF's giving rise to all four (secondary computational) phenomenal 'physical' properties (e.g., of 'space', 'time', 'energy' and 'mass') and also solely exists "in-between" any (two such) USCF's; Thus, the acceptance of the CUFT's Universal Consciousness Principle overturns the current Cartesian 'materialsitic-reductionistic' scientific paradigm which assumes that the reality is "physical" (e.g., represented by a basic SROCS computational structure) - in favor of a "non-material, acausal" (singular) 'Universal Consciousness Principle' which is the sole "reality" underly- 
ing the phenomenal universe (e.g., including all hypothetical quantum and relativistic physical, inductive or deductive ' $x$-y' relationships), as well exists "independently" of any such secondary computational SROCS derived 'physical phenomenal' properties of the universe...

\section{The CUFT's sixth postulate: 'Ontological relativism'}

But, if indeed we accept the CUFT's (fifth) 'Universal Consciousness Principle' theoretical postulate's assertion regarding the sole "reality" of this 'Universal Consciousness Principle' as (solely) producing- retaining- and evolving- all secondary computational (apparent phenomenal) 'physical' properties (of 'space', 'time', 'energy' and 'mass'), then this may lead to the recognition of a sixth (hypothetical) theoretical postulate of "Ontological Relativism' - i.e., the realization that since the only "valid" principle underlying the phenomenal physical universe is that Universal Consciousness Principle, then our scientific ontological knowledge of the singular "reality" must be based solely on the Universal Consciousness Principle: i.e., specifically on our perception of that Universal Consciousness Principle through our own individual human Consciousness' three states of human Consciousness!

Formally presented, this sixth CUFT's 'Ontological Relativism' postulate appears through a modification of the (previously presented) Universal Computational Formula as the 'Universal Consciousness Formula' thus:

$$
=\left\{(i)\left(\mathrm{w}(1 \ldots \infty): \underline{c}^{2}=\underline{\mathrm{s}} \mathbf{x}\right), d, s\right\}
$$

$\mathrm{h} \mathrm{t} \mathrm{m}$

wherein our sole knowledge of the singular "reality" of the 'Universal Consciousness Principle' (') is gained through our individual human Consciousness which comprises three states of individual human Consciousness (i), namely: "waking" $\{$ 'w'\} (e.g., solely in which we experience the four abovementioned secondary computational 'physical' properties of 'space', 'time', 'energy' and 'mass'), "dream" \{'d'\} (in which we experience quite a similar 'dream-physical' universe - also produced solely by the singular Universal Consciousness Principle), and "deep sleep" \{'S'\} (e.g., in which we only experience solely this singular 'Universal Consciousness Principle' - independently of any of its produced secondary computational 'physical' USCF's derivatives of 'space', 'time', 'energy' and 'mass')... Furthermore, it is hypothesized that the ontological knowledge represented by the 'waking' state of (individual) human Consciousness spans between "1" (e.g., representing our normal individual human Consciousness sensory perception and cognitive ideation) and " $\infty$ " (e.g., representing an 'infinitely' expanded individual human Consciousness state which in fact is hypothesized to be identical with the 'pure' Universal Consciousness Principle ('") existing "in-between" any two subsequent USCF's, see further discussion below).

Indeed, the gist of the CUFT's (sixth) 'Ontological Relativism' postulate is the recognition that given that all four 'physical' properties (of 'space', 'time', 'energy' and 'mass') merely represent 
'computationally variant' (secondary computational) properties that are hence deemed as 'phenomenal' (or even 'illusory') relative to the 'computationally invariant' 'Universal Consciousness Principle' ('י') which solely produces- sustains- and evolves- these apparent phenomenal 'physical' properties (as secondary computational derivatives of the USCF's series) and which also solely exists "in-between" any two such subsequent USCF's; and that our sole knowledge of this singular "reality" of the 'Universal Consciousness Principle' (' ') may only be derived through the three states of (individual) human Consciousness (e.g., 'waking', 'dream' and 'deep sleep'); then there does not exist any "objective" means for preferring the "waking" human Consciousness state (i.e., solely in which there appear those secondary computational phenomenal 'physical' properties of 'space', 'time', 'energy' and 'mass') - upon the two other states of human Consciousness, e.g., "dream" and "deep sleep"! Thus, all three states of (individual) human Consciousness are solely produced by the singular "reality" of the 'Universal Consciousness Principle' ('") and therefore their corresponding 'ontological knowledge' possesses the same relative ontological validity - i.e., it represents an apparent ontological phenomenology which is "unreal" relative to the sole reality of their underlying 'Universal Consciousness Principle'...

Hence, the above formal presentation of the (broader) Universal Consciousness Principle's delineation of the Universal Computational Formula indicates that our sole knowledge of the singular "reality" of the Universal Consciousness Principle ('"') may be derived through the 'Ontological Relativism' of the three states of (individual) human Consciousness (e.g., which are all deemed as 'phenomenal' or "unreal" relative to the singularity of the Universal Consciousness Principle)... Therefore, note that a subset of this 'Universal Consciousness Principle's Formula' constitutes the previously presented special case of the Universal Computational Formula - e.g., which delineates the production of the (four secondary computational) 'physical' properties of 'space', 'time', 'energy' and 'mass':

$$
\begin{aligned}
& \text {, }=\left\{(\mathrm{i})\left(\mathrm{w}(100): \underline{\underline{c}^{2}}=\underline{\underline{x}} \mathrm{x}, \underline{\mathrm{e}}\right) \mathrm{d}, \mathrm{s}\right\} \\
& h \mathrm{t} m
\end{aligned}
$$

However, in addition to the (individual) 'waking' state of human Consciousness (which was previously presented in the Universal Computational Principle), the more generalized Universal Consciousness Formula also incorporates two other individual human Consciousness' (corresponding) forms of ontological knowledge of the sole "reality" of the Universal Consciousness Principle- e.g., which possess the same (relative) ontological validity (namely: the ontological knowledge of the Universal Consciousness Principle arising from the 'dream' and 'deep sleep' states).

The potential significance of this generalized formalization of the Universal Consciousness Principle may be threefold:

a. It fully delineates the various (three) states of our individual human Consciousness' ontological knowledge of the sole "reality" of the (singular) 'Universal Consciousness Principle ('"), thereby providing an exhaustive portrayal of this (newly discovered) higher-ordered "reality" underlying the phenomenal universe and beyond it. 
b. It identifies specific empirical instances of our individual human Consciousness which can validate the Universal Consciousness Principle's (abovementioned) "mechanics" - i.e., as producing- sustaining- or evolving- all four secondary computational 'physical properties in the 'waking' state of individual human Consciousness; as solely existing independently of any such 'physical' properties "in-between" any two USCF's frames; and as also exemplified in the deep sleep state of individual human Consciousness; ; and as producing an equivalent 'ontological relativistic' phenomena universe in the dream state.

c. It identifies particular novel empirical predictions stemming from the possibility of manipulating individual human Consciousness states - i.e., such as for instance in the case of successful meditative states that may 'expand' the individual human Consciousness from its 'standard' 'waking' (i=1) through a spectrum of expanded individual human Consciousness and up to an infinitely expanded individual human Consciousness state $(\mathrm{i}=\infty)$ which is hypothesized to be identical with the 'pure' Universal Consciousness Principle ('') which also exists "in-between" any two subsequent USCF's frames... (This potential individual human Consciousness' expanded spectrum state will be further delineated below.)

\section{The seventh postulate: “Universal Consciousness Spectrum (UCS)"}

Finally, a sixth theoretical postulate is hereby added to the CUFT, namely: the 'Universal Consciousness Spectrum' postulate, which hypothesizes that the Universal Consciousness Principle ('") is capable of expressing a whole spectrum of (individual) human Consciousness (degrees), including (but not limited to) the three (abovementioned) states of individual human Consciousness (e.g., 'waking', 'dream' and 'deep sleep') as well as a myriad of different degrees of "expansiveness" of that individual human Consciousness in the 'waking' state, represented (above) in the Universal Consciousness Formula thus:

$$
\begin{gathered}
'=\left\{(\mathrm{i})\left(w(1 \ldots \infty): \mathrm{c}^{2}=\underline{s} \times \underline{e}\right) \mathrm{d}, \mathrm{s}\right\} \\
\text { h } \mathrm{t} \mathrm{m}
\end{gathered}
$$

The potential significance of the empirical verification of this sixth 'Universal Consciousness Spectrum postulate is that it would indeed enable us to demonstrate that our individual human Consciousness ('i') forms a particular subset of the 'Universal Consciousness Principle' ('') i.e., which ordinarily conforms to our 'standard' (' $i$ ' = 1') (sensory and cognitive) perceptions of the 'waking' state of Consciousness, but which nevertheless has the potential of experiencing the two "non-waking" states of individual human Consciousness (e.g., daily: as the 'dream' and 'deep sleep' states) as well as a whole spectrum of (different degrees of) "expansiveness" of the 'waking' state of individual human Consciousness;

The basic assumption postulated by this 'Universal Consciousness Spectrum' is hence that whereas the Universal Consciousness Principle ('") forms the sole "reality" underling all phenomenal (inductive or deductive), physical or (individual) human Consciousness 'x-y' 
relationships, the individual human Consciousness ('i') possesses the potential of experiencing the full range of this exhaustive Universal Consciousness Principle ('"') including: the three 'standard' states of individual human Consciousness (e.g., 'waking', 'dream' and 'deep sleep'), as well as the multifarious degrees of "expansiveness" of this individual human Consciousness in the 'waking' state: Specifically, it is hypothesized that the varying degrees of individual human Consciousness "expansiveness" (e.g., in the 'waking' state of Consciousness) correspond to its 'inclusiveness' of an increasing number of 'spatial pixels' comprising any single or multiple USCF frame/s (wherein the 'inclusiveness' of all exhaustive spatial pixels comprising such USCF's - represents its "infinite expansiveness", which is precisely equivalent to the Universal Consciousness production- sustenance- and evolution- of the phenomenal physical universe through its series of USCF's)...

Once again, it is suggested that our capacity to verify (e.g., empirically) this (sixth) 'Universal Consciousness Spectrum' theoretical postulate may both validate the complete structure of the CUFT (e.g., as it would demonstrate the fact that the production- sustenance- and evolutionof the phenomenal 'physical' universe is entirely produced by the Universal Consciousness Principle, '" - to which we have "access" through varying degrees of our individual human Consciousness "expansiveness"); as well as open new theoretical "vistas" for exploring the potential effects of modulating our individual human Consciousness on the 'physical' properties of the world. Hence, what follows is a delineation of a (partial) list of specific empirical predictions made by the 'Universal Consciousness Spectrum' postulate:

\section{Critical Predictions of the 'Universal Consciousness Spectrum'}

We last come to delineating a (partial) list of some of the critical empirical predictions of the 'Universal Consciousness Spectrum' postulate, which may (specifically) validate this Universal Consciousness Spectrum postulate, as well as (more generally) validate the complete structure of the 'Computational Unified Field Theory':

a. It may be possible to affect certain (secondary computational) 'physical' properties (e.g., of 'space', 'time', 'energy' or mass') of a human being whose individual Consciousness is being modulated in such a manner as to manipulate that human being's body's 'mass', 'time', 'energy', or 'spatial' values: Essentially, this critical empirical prediction asserts that since according to the above Universal Consciousness Formula the individual human Consciousness (i) is capable of experiencing the full spectrum the 'Universal Consciousness Principle's ('") 'waking' state "expansiveness" - i.e., being inclusive of varying degrees of the Universal Consciousness Principle's generated USCF's spatial pixels; and since all four (secondary computational) 'physical' properties (of 'space', 'time', 'energy' and 'mass' are solely produced- retained- or evolved- by the Universal Consciousness Principle ('"); then it should be possible for a human being to modulate his or her individual Consciousness' "expansiveness" is such a manner as to affect that person's physical body's 'mass', 'time', 'energy' or 'space' values; Empirically, this prediction refers to the potential capacity of qualified "meditators" (e.g., who possess the capacity to modulate their 
individual Consciousness "expansiveness" spectrum) to affect their body's various (four) 'physical' properties (through their manipulation of their individual Consciousness spectrum).

b. Based on these (same) two 'Universal Consciousness Formula' and 'Universal Consciousness Spectrum' tenets it is also predicted that such a qualified "meditator" could also affect their individual Consciousness spectrum "expansiveness" - regarding other spatial pixels that are not associated with their own body, e.g., such as the four 'physical' properties of various objects and events 'external' to their body; Thus, it should be possible (at least in principle) for such a qualified "meditator" to alter any given object's 'spatial', 'temporal', 'mass' or 'energy' values based on their alteration of their individual Consciousness "expansiveness" as it relates to the Universal Consciousness Principle's computation of that object's USCF's physical properties.

c. Finally, since the Universal Consciousness Principle is solely responsible for the production- retention- and evolution- of any physical object or event (across the relevant series of USCF's); and since this Universal Consciousness Principle is exhaustively responsible for this production-sustenance- and evolution of all the spatial pixels in the physical universe i.e., in the "past", "present" and "future"; and since according to the 'Universal Consciousness Spectrum' postulate all of these exhaustive spatial pixels comprising all USCF's pixels comprising the entire physical universe are "accessible" to the individual human Consciousness "expansiveness" degree; then it should be possible (at least in principle), for highly qualified "meditators" to manipulate the physical properties of any physical object or event, e.g., throughout the exhaustive pool of USCF's frames comprising the physical universe in the "past" and "present", and perhaps even in the "future"...

\section{The scientific implications of the CUFT's universal consciousness principle}

We finally arrive at considering some of the potential theoretical ramifications of the CUFT's Universal Consciousness Principle, the Universal Consciousness Formula and the Universal Consciousness Spectrum tenets explored in this chapter (as well as their implications for the generality of the Computational Unified Field Theory and Science in general);

Hence, the current manuscript traces the potential theoretical ramifications of:

a. An 'a-causal' computational framework of the (CUFT's) singular Universal Consciousness Principle's ('") responsible for the (higher-ordered) computation of all exhaustive hypothetical (e.g., empirically knowable) inductive or deductive ' $x$-y' pairs series - which leads to the discovery of a-causal 'Universal Consciousness Principle Computational Program'.

b. An exploration of the CUFT's Universal Consciousness Principle's ('") and Duality Principle's (Bentwich, 2003c, 2004, 2006) reformalization of all (apparent inductive or deductive) major SROCS computational paradigms (e.g., including: Darwin's 'Natural Selection Principle' (Darwin, 1859) and associated Genetic Encoding hypothesis, Neuro- 
science's Psychophysical Problem of human Consciousness and all inductive and deductive Gödel-like SROCS paradigms).

c. Theoretical Ramifications of the Universal Consciousness Principle.

\subsection{A singular 'A-causal' universal consciousness principle computation of all inductive and deductive ' $\mathrm{X}-\mathrm{Y}$ ' relationships}

We thus begin with an exploration of three potential theoretical ramifications of the CUFT's description of the operation of the (singular) Universal Consciousness Principle ("') which has been shown to compute an extremely rapid series of Universal Simultaneous Computational Frames (USCF's);

The Universal Computational/Consciousness Principle was (previously) shown to encapsulate a singular higher-ordered 'D2' computation of an 'a-causal' computation of the "simultaneous co-occurrences" of all exhaustive hypothetical inductive or deductive (e.g., empirically knowable) ' $x-y$ ' pairs series; Therefore, the acceptance of the CUFT's description of the Universal Consciousness Principle necessarily implies that throughout the various (inductive or deductive) disciplines of Science we need to shift from the current basic (Cartesian) "material-causal" scientific theoretical towards a singular (higher-ordered 'D2') 'Universal Consciousness Principle's a-causal computation':

This means that the current (Cartesian) 'material-causal' scientific framework assumes that any given 'y' element (or value) can be explained as a result of its (direct or indirect) 'causal' interaction/s with another (exhaustive hypothetical inductive or deductive) series of ' $x$ ' factor/ $\mathrm{s}$ - which determines whether that 'y' element (or value) "exists" or "doesn't exist", thereby comprising a 'Self-Referential Ontological Computational System' (SROCS) (Bentwich, 2012: $a \& b)$.

SROCS: PR $\{x, y\} \rightarrow[$ 'y' or 'not y']/di1...din.

But, since it was previously shown that such SROCS computational structure inevitably leads to both 'logical inconsistency' and 'computational indeterminacy' that were shown to be contradicted by robust empirical findings indicating the capacity of the major scientific SROCS paradigms to be capable of determining the "existence" or "non-existence" of the particular 'y' element, see Bentwich 2012b) - then the CUFT's 'Duality Principle' asserted the existence of the singular 'Universal Consciousness Principle' ('") which is capable of computing the "simultaneous co-occurrences" of any particular (exhaustive hypothetical) 'x-y' pairs series which are embedded within the Universal Computational/Consciousness Principle's rapid series of USCF's.

What this means is that both specifically for each of the (previously identified) key scientific SROCS paradigms as well as more generally for any hypothetical ('empirically knowable') inductive or deductive ('x-y') phenomenon, we must reformulate our scientific understanding in such a way which will allow us to present any such ' $x-y$ ' relationship/s as being computed by the singular Universal Consciousness Principle (e.g., as the computation of an exhaustivehypothetical "co-occurring" 'x-y' pairs' series); In that respect, this (novel) 'Universal Con- 
sciousness Principle's' scientific framework shifts Science from its current basic (Cartesian) assumption wherein all natural phenomena can be described as 'material-causal' (' $\left.x \rightarrow y^{\prime}\right)$ relationships (e.g., comprising the apparent SROCS computational structure contradicted by the computational Duality Principle) - to an 'a-causal' singular Universal Consciousness Principle which computes the simultaneous "co-occurrences" of any inductive or deductive ' $x$ $y^{\prime}$ pairs series comprising the various 'pixels' of the USCF's frames (e.g., produced by this Universal Consciousness Principle).

Finally, it should be noted that a key principle underlying this shift from the current 'materialcausal' (Cartesian) scientific framework towards the CUFT's (proven) higher-ordered singular Universal Consciousness Principle's ('") 'a-causal' theoretical framework is the acceptance of the impossibility of the existence of any such 'material-causal' ('x-y') relationship/s - i.e., due to the impossibility of any 'physical' entity, attribute (or property) being transferred across any (two subsequent) 'USCF's frames: Thus, apart from the (previously shown) conceptual computational proof of the 'Duality Principle' wherein due to the inevitable 'logical inconsistency' and 'computational indeterminacy' arising from the SROCS computational structure (which is contradicted by empirical evidence indicating the capacity of these key scientific SROCS paradigms to compute the "existence" or "non-existence" of any particular 'y' element or value) - pointing at the existence of the higher-ordered (singular) 'Universal Computational/ Consciousness Principle that computes the "simultaneous co-occurrences" of any (exhaustivehypothetical) ' $x-y$ ' pairs' series; it is suggested that the inclusion of this computational Duality Principle as one of the (seven) theoretical postulates of the CUFT (e.g., specifically alongside the CUFT's 'Computational Invariance' and 'Universal Consciousness' postulates) unequivocally asserts that there cannot (in principle) exist any 'material-causal' effect/s (or relationship/ s) being transferred across any (two subsequent) USCF's frames! This is because the CUFT's very definition of all four 'physical' properties of 'space', 'time', 'energy' and 'mass' - as secondary computational by-products of the (singular) Universal Computational Consciousness' computation of (an extremely rapid series of) 'Universal Simultaneous Computational Frames' (USCF's); and moreover the CUFT's 'Computational Invariance' postulate indication that due to the 'computational variance' of these four (secondary computational) 'physical' properties (e.g., as existing only "during" the appearance of the USCF frames but 'nonexistence' "in-between" any two such subsequent frames, see Bentwich, 2012:a \& b) as opposed to the 'computational invariance' of the 'Universal Consciousness Principle' ('"), we need to regard only this singular (computationally invariant) 'Universal Consciousness Principle' as "real" whereas all four (secondary computationally variant) 'physical' properties must be regarded as merely 'phenomenal' (i.e., as being comprised in reality only from the singular Universal Consciousness Principle); Therefore, the CUFT's 'Universal Consciousness Principle' advocated that none of these four (secondary computationally variant) 'physical' properties (e.g., of 'space', 'time', 'energy' or 'mass') "really" exists - but rather that there is only this one singular Universal Consciousness Principle which exists (solely) "in-between" any (two subsequent) USCF's frames and also solely produces each of these USCF's derived four 'phenomenal physical' properties; Hence, it was evinced (by the CUFT's Universal Consciousness Principle) that there cannot be any 'transference' of any hypothetical 'material' or 'physical' entity, effect, or property across any (two subsequent) USCF's frames! We therefore reach the 
inevitable theoretical conclusion that the current scientific (Cartesian) "material-causality' basic assumption underlying all key scientific SROCS paradigms as well as all (empirically knowable) 'Gödel-like' (inductive or deductive) SROCS 'x-y' relationships, wherein there exists a 'material-causal' effect/s (or relationship/s) between any given ' $x$ ' element and any (exhaustive hypothetical) ' $y$ ' series which determines the "existence" or "non-existence" of that (particular) 'y' element (or value) - is untenable! Instead, we must accept the CUFT's assertion that there can only exists one singular 'Universal Consciousness Principle' ('") which both (solely) produces- all (apparent) secondary computational 'physical' properties (of 'space', 'time', 'energy' and 'mass'), as well as computes the "simultaneous co-occurrences" of any (particular) exhaustive-hypothetical inductive or deductive 'x-y' pairs series (e.g., comprising the exhaustive USCF's frames).

\subsection{The "universal consciousness principle's computational program"}

Therefore, it follows that based on the recognition of the singularity of the Universal Consciousness Principle's 'a-casual' computation of the "simultaneous co-occurrences" of all (inductive or deductive) ' $x$-y' pairs' series (as comprising the exhaustive USCF's frames) - we need to be able to reformulate all of the previously mentioned key scientific SROCS paradigms (Bentwich, 2012: a-b), including: Darwin's 'Natural Selection Principle' and associated 'Genetic Encoding' hypothesis, Neuroscience's Psychophysical Problem of human Consciousness, as well as all (exhaustive hypothetical) 'Gödel-like' (apparent) inductive or deductive SROCS computational paradigms based on this singular (higher-ordered) Universal Consciousness Principle's ('") 'a-causal' USCF's computation;

Hence, what follows is a description of the principle theoretical ramifications of reformulating each of these key scientific (apparent) SROCS computational paradigms, as well as a more generalized description of a tentative 'Universal Consciousness Principle Program' (e.g., which may offer a successful alternative for 'Hilbert's Mathematical Program' to base all of our human scientific knowledge upon the foundations of the operation of the singular Universal Consciousness Principle). First, it may be worthwhile to rearticulate the reformalization of each of these key scientific (apparent) SROCS paradigms in terms of the operation of the singular Universal Consciousness Principle (as previously outlined: Bentwich, 2012b):

N.S.:D2: $[\{\mathrm{E}\{1 \ldots \mathrm{n}\}, \mathrm{o}\} \mathrm{st} 1 ;\{\mathrm{E}\{1 \ldots \mathrm{n}\}, \mathrm{o}\} \mathrm{st} 2 \ldots\{\mathrm{E}\{1 \ldots \mathrm{n}\}, \mathrm{o}\} \mathrm{stn}]$.

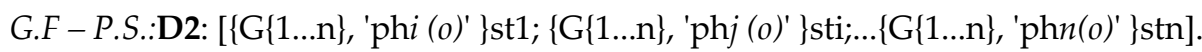

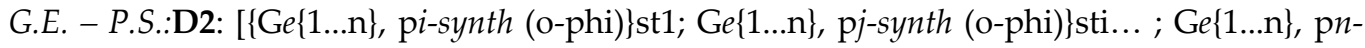
synth (o-phi)\}stn]

Psychophysical:D2: $\left[\left\{\mathrm{N}(1 \ldots \mathrm{n})_{\mathrm{st-i},} \mathrm{Cs}_{\mathrm{p}} \mathrm{pp} \mathrm{st-i}_{\mathrm{i}}\right\} ; \ldots\left\{\mathrm{N}(1 \ldots \mathrm{n})_{\mathrm{st-i+n},} \mathrm{Cs}-\mathrm{pp}_{\mathrm{st}-\mathrm{i}+\mathrm{n}}\right\}\right]$

Functional: D2: [\{Cs(pp) $\left.\left.\mathrm{f}_{\mathrm{i}}, \mathrm{Na}(\mathrm{spp}) \mathrm{fi}\right\}_{\mathrm{st}-\mathrm{i}} ; \ldots\left\{\mathrm{Cs}(\mathrm{pp}) \mathrm{f}_{(\mathrm{i}+\mathrm{n})}, \mathrm{Na}(\mathrm{spp}) \mathrm{f}_{(\mathrm{i}+\mathrm{n})}\right\}_{\mathrm{st}(\mathrm{i}+\mathrm{n})}\right]$

Phen.: D2: [\{Cs(pp- fi)-Ph $\left.\left.\mathrm{i}_{\mathrm{i}} \mathrm{Na}(\mathrm{spp}-\mathrm{fi})-\mathrm{Ph}_{\mathrm{i}}\right\}_{\mathrm{st}-\mathrm{i}}, \ldots\left\{\mathrm{Cs}(\mathrm{pp}-\mathrm{fi})-\mathrm{Ph}_{(\mathrm{i}+\mathrm{n})}, \mathrm{Na}(\mathrm{spp}-\mathrm{fi})-\mathrm{Ph}\right\}_{\mathrm{st}-(\mathrm{i}+\mathrm{n})}\right]$

Self: D2: [\{Cs(pp-fi)Ph-Si, Na(pp-fi)Ph-S $\left.\}_{\text {st-i }} ; \ldots\{\mathrm{Cs}(\mathrm{pp}-\mathrm{fi}) \mathrm{Ph}-\mathrm{S}(\mathrm{i}+\mathrm{n}), \mathrm{Na}(\mathrm{pp}-\mathrm{fi}) \mathrm{Ph}-\mathrm{S}(\mathrm{i}+\mathrm{n})\}_{\mathrm{st}-(\mathrm{i}+\mathrm{n})}\right]$

GIT:D2: $([\{S\{1 \ldots \mathrm{n}\}, \mathrm{t}\} i \ldots\{\mathrm{S}\{1 \ldots \mathrm{n}\}, \mathrm{t}\} z]$, or $[\{\mathrm{x}\{1 \ldots \mathrm{n}\} i, \mathrm{y} i\} \ldots\{\mathrm{x}\{1 \ldots \mathrm{n}\} z, \mathrm{yz}\}])$ 
Indeed, what may be seen from this singular description of all of these key scientific SROCS paradigms, is that it recognize the fact that all of these major (apparent) SROCS paradigms are computed simultaneously as different "co-occurring" ' $x-y$ ' pairs embedded within the same (single or multiple) USCF frame that is produced by the singular Universal Consciousness Principle ('"); What this means is that the recognition of the singularity of this Universal Consciousness Principle as the sole "reality" which computes the "simultaneous co-occurrences" of all of these (particular) exhaustive hypothetical 'x-y' pairs series, and which also exists (solely) "in-between" any two such USCF's - forces us to transcend the 'narrow constraints' of the (current) Cartesian 'material-causal' theoretical framework (e.g., which assumes that any given 'y' entity (or phenomenon) is "caused" by its (direct or indirect) physical interaction/s with (an exhaustive hypothetical ' $x$ ' series); Instead, this singular Universal Consciousness Principle 'a-causal' computation asserts that it is the same singular Universal Consciousness Principle which computes- produces- retains- and evolves- all of these particular scientific (apparent) SROCS 'x-y' pairs series across a series of USCF's...

In other words, instead of the existence of any "real" material-causal relationship between any of these (particular SROCS) ' $x \rightarrow y$ ' entities (e.g., Darwin's Natural Selection Principle's assumed 'material-causal' relationship between an organism's Environmental Factors, ' $x$ ', and own traits or behavior 'y'; or between any exhaustive hypothetical Genetic Factors and any given phenotypic behavior; or between Neuroscience's Psychophysical Problem of Human Consciousness' psychophysical stimulation, ' $x$ ', and Neural Activation, 'y'; or in fact between any hypothetical inductive or deductive Gödel-like SROCS ' $x-y$ ' factors); the CUFT's Universal Consciousness Principle offers an alternative singular (higher-ordered) computational mechanism which computes the "simultaneous co-occurrences" of any of these (exhaustive hypothetical) 'x-y' pairs' series - which are all produced- and embedded- within the Universal Consciousness Principle's computed USCF's frames... Indeed, the shift from the current 'material-causal' (Cartesian) scientific framework towards the Universal Consciousness Principle's singular computation of the "simultaneous co-occurrences" of all exhaustive hypothetical (inductive or deductive) ' $x$-y' pairs' series may lead the way for reformulating all of these key scientific SROCS paradigms (as well as any other hypothetical inductive or deductive ' $x-y$ ' series) within a basic "Universal Consciousness Principle Computational Program";

Essentially, such a 'Universal Consciousness Principle's Computational Program' is based upon the foundations of the CUFT's (abovementioned) three postulates of the 'Duality Principle', the 'Computational Invariance' principle and the 'Universal Consciousness Principle' - all pointing at the fact that all empirically computable (inductive or deductive) ' $x$-y' relationships must necessarily be based upon the singular (conceptually higher-ordered) Universal Consciousness Principle which is solely responsible for the computation of the "simultaneous cooccurrences" of all such (exhaustive hypothetical) inductive or deductive ' $x-y$ ' pairs series comprising the totality of the USCF's (single or multiple) frames.... Moreover, this singular Universal Consciousness Principle ('") was also shown to exist independently of any (secondary computational) 'physical properties' (e.g., of 'space', 'time', 'energy' and 'mass') and therefore constitute the only "reality" that exists invariantly (i.e., both as giving rise to the four 
'phenomenal' physical properties and as existing solely "in-between" any two such subsequent USCF's frames).

In order to appreciate the full (potential) theoretical significance of such a 'Universal Consciousness Principle Computational Program' it may be worthwhile to reexamine Hillbert's famous 'Mathematical Program' to base Mathematics upon the foundations of Logic (e.g., and by extension also all of Science upon the foundations of Mathematics and Logic), and more specifically, to revisit 'Gödel's Incompleteness Theorem' (GIT) which delivered a critical blow to Hilbert's 'Mathematical Program'; It is a well-known that Hilbert's Mathematical Program sought to base Mathematics (e.g., and by extension also the rest of inductive and deductive Science) upon a logical foundation (e.g., of certain axiomatic definitions); It is also well known that Gödel's Incompleteness Theorem (GIT) has failed Hillbert's Mathematical Program due to its proof that there exists certain 'self-referential' logical-mathematical statements that cannot be determined as "true" or "false" (e.g., or logically 'consistent' or 'inconsistent') from within any hypothetical axiomatic logical-mathematical system... Previously (Bentwich, 2012: a \& b) it was suggested that perhaps scientific Gödel -like SROCS computational systems may in fact be constrained by the Duality Principle's (generalized) format, thus:

i.

SROCS: PR $\{x, y\} \rightarrow\left[y^{\prime}\right.$ or 'not y']/di1...din

ii.

SROCS CR $\{S, t\} \rightarrow\left[{ }^{\prime} t\right.$ ' or 'not t']/di1...din

wherein it was shown that both inductive ('i') and deductive (ii) SROCS scientific computational systems are necessarily constrained by the Duality Principle (e.g., as part of the broader CUFT). In other words, the Duality Principle's (generalized format) was shown to constrain all (exhaustive hypothetical) Gödel -like (inductive or deductive) scientific SROCS paradigms, thereby pointing at the existence of a singular (higher-ordered) Universal Consciousness Principle ('") which is solely capable of computing the "simultaneous co-occurrences" of any (exhaustive hypothetical) ' $x$-y' pairs series. It is important to note, however, that the conceptual computational constraint imposed upon all (Gödel -like) inductive or deductive scientific SROCS paradigms was shown to apply for all of those inductive or deductive (apparent) scientific SROCS paradigms - for which there is an empirically known (or 'knowable') ' $x-y$ ' pairs series results!

This latter assertion of the Duality Principle's (generalized proof) may be significant as it both narrows- and emphasized- the scope of the 'scientifically knowable domain'; In other words, instead of the current 'materialistic-reductionistic' scientific framework which is anchored in a basic (inductive or deductive) SROCS computational format (see above) which inevitably leads to both 'logical inconsistency' and 'computational indeterminacy' that are contradicted by robust empirical findings (e.g., pertaining to the key scientific SROCS paradigms); The Duality Principle (e.g., as one of the postulates within the broader CUFT) proves that the only means for computing the "simultaneous co-occurrences" of any (exhaustive hypothetical) ' $x$ $y$ ' pairs series is carried out by the singular (higher-ordered) Universal Consciousness Principle ('"')... Moreover, the (generalized format of the) Duality Principle goes farther to state that for all other (exhaustive hypothetical) inductive or deductive computational SROCS paradigms for which there exists a proven empirical capacity to determine the values of any particular ' $x-y$ ' pairs 
(e.g., empirically "known" or "knowable" ' $x$-y' pairs results)- any of these (hypothetical) scientific SROCS computations must be carried out by the CUFT's identified singular Universal Consciousness Principle ('")!

The (potential) significance of this generalized assertion made by the Computational Unified Field Theory's (CUFT): 'Duality Principle', 'Computational Invariance' principle and Universal Consciousness Principle ('"') is twofold:

a. First, it narrows down the scope of (inductive or deductive) determinable scientific phenomena - to only those (inductive or deductive) ' $x-y$ ' relationships for which there is an empirical capacity to determine their "simultaneously co-occurring" values; essentially the 'Universal Consciousness Principle's Computational Program' anchors itself in the Duality Principle's focus on only those inductive or deductive 'x-y' relationship/s or phenomenon for which there is an empirically 'known' or 'knowable' capacity to determine these 'x-y' pairs values. It is perhaps important to note (in this context) that all of the 'other' inductive or deductive 'x-y' relationship/s which cannot be (empirically) known "naturally" lie outside the scope of our human (scientific) knowledge (and therefore should not be included, anyway within the scope of Science)... Nevertheless, the strict limitation imposed by the 'Universal Consciousness Principle Computational Program' may indeed be significant, as it clearly defines the boundaries of "admissible scientific knowledge" to only that scientific knowledge which is based on empirically known or knowable results pertaining to the "simultaneous co-occurrences" of any ' $x$-y' relationship or phenomenon; (Needless to say that the strict insistence of the Universal Consciousness Computational Program upon dealing only with

b. Second, based on this strict definition of Science as dealing solely with 'empirical knowable' (simultaneously co-occurring) ' $x$-y' relationship/s or phenomenon - the 'Universal Consciousness Computational Program' may in fact offer a broader alternative to GIT (failing of Hilbert's 'Mathematical Program'); This is because once we accept the Universal Consciousness Principle's Computational Program's (above) strict 'empirical constrains', we are led to the Duality Principle's (generalized) conceptual computational proof that any (exhaustive hypothetical) inductive or deductive scientific SROCS' ' $x-y$ ' relationship must be determined by the singular Universal Consciousness Principle ("') computation of the "simultaneous co-occurrences" of any (exhaustive hypothetical) 'x-y' pairs series; then this means that instead of GIT assertion that it is not possible (in principle) to construct a consistent Logical-Mathematical System which will be capable of computing any mathematical (or scientific) claim or theorem, the Universal Consciousness Computational Program asserts that based on a strict definition of Science as dealing solely with empirically knowable 'x-y' relationship/s or phenomenon, we obtain a singular (higherordered) Universal Consciousness Principle which is solely responsible for computing the "simultaneous co-occurrences" of any (exhaustive hypothetical) inductive or deductive ' $x$ y' pairs series (e.g., which were shown by the CUFT to comprise the totality of any single or multiple USCF's frames that are solely produced by this Universal Consciousness Principle). In that sense, it may be said that the Universal Consciousness Principle Computational Program points at the existence of the singular (higher-ordered) Universal 
Consciousness Principle as constraining- and producing- all inductive or deductive scientific relationship/s or phenomena (e.g., which was also shown earlier and previously to constitute the only "reality" which both produces all USCF's derived secondary computational 'physical properties and also solely exists "in-between" any two such USCF's).

\section{Theoretical ramifications of the universal consciousness principle}

The discovery of the singular Universal Consciousness Principle (alongside its 'Universal Consciousness Computational Program') may bear a few significant theoretical ramifications:

a. The Sole "Reality" of the Universal Consciousness Principle: As shown above, all scientific (inductive and deductive) disciplines need to be reformulated based on the recognition that there exists only a singular (higher-ordered) Universal Consciousness Principle ('") which solely produces- sustains- evolves (and constrains) all (apparent) SROCS (inductive or deductive) ' $x-y$ ' relationships; Moreover, this Universal Consciousness Principle is recognized as the sole "reality" that both produces- sustains- and evolves- any of the apparent (four) 'physical' properties of 'space', 'time', 'energy' and 'mass', as well as exists independently of any such 'physical' properties - and is therefore recognized as the only singular "reality", whereas these apparent 'physical' properties are seen as merely 'phenomenal' (secondary computational) manifestations of this singular (higher-ordered) Universal Consciousness Principle "reality".

b. The Transcendence of 'Material-Causality' by the Universal Consciousness Principle 'ACausal' Computation: As shown (above), the acceptance of the Universal Consciousness Principle ('") as the sole "reality" which both produces- (sustains- and evolves-) all USCF's (secondary computational) 'physical' properties, as well as exists independently "inbetween" any (two subsequent) USCF's; (Alongside the Duality Principle's negation of any apparent SROCS' 'causal' relationships and the 'Computational Invariance' principle indication that only the 'computationally invariant' 'Universal Consciousness Principle' "really" exists whereas the secondary 'computationally variant' physical properties are only 'phenomenal') - point at the negation of any "real" material-causal ('x-y') relationships, but instead indicate that there can only exist a singular (higher-ordered) Universal Consciousness Principle 'a-causal' computation of the "simultaneous co-occurrences" of any exhaustive hypothetical inductive or deductive ' $x$-y' pairs' series... (As shown earlier, the strict negation of the existence of any "real" 'material-causal' ' $x \rightarrow y$ ' relationships was evinced by the simple fact that according to the CUFT's model there cannot exist any "real" computationally variant 'physical' or 'material' property that can "pass" across any two subsequent USCF's, but only the computationally invariant "real" Universal Consciousness Principle which exists singularly - as solely producing all apparent secondary computational 'physical' properties as well as existing independently "in-between" any two such subsequent USCF's frames.) Indeed, the need to replace all apparent 'materialcausal' ' $x-y$ ' SROCS relationships by a singular (higher-ordered) Universal Consciousness 
Principle computation of the 'simultaneous co-occurrences' of all possible inductive or deductive ' $x-y$ ' pairs series was shown to apply to all of the key (apparent) scientific SROCS paradigms (including: Darwin's Natural Selection Principle and associated Genetic Encoding hypothesis, Neuroscience's Psychophysical Problem of human Consciousness as well as to all Gödel-like hypothetical inductive or deductive SROCS paradigms; what this implies is that for all of these apparent SROCS scientific paradigms the sole "reality" of the Universal Consciousness Principle forces us to transcend each of the (particular) 'material-causal' $x-y$ relationships in favor of the Universal Consciousness Principle's singular computation of all (exhaustive hypothetical) ' $x-y$ ' pairs series; Thus, for example, instead of Darwin's current 'Natural Selection Principle' SROCS material-causality thesis, which assumes that it is the direct (or indirect) physical interaction between the organism and its Environmental Factors that causes that organism to 'survive' or be 'extinct', the adoption of the Universal Consciousness Principle (and Duality Principle) postulates brigs about a recognition that there is only a singular (Universal Consciousness based) conceptually higher-ordered 'a-causal' computation of the "simultaneous co-occurrences" of an exhaustive hypothetical pairs series of 'organism' and 'Environmental Factors' (e.g., which are computed as part of the Universal Consciousness Principle's production of the series of USCF's frames).

c. Possible Resolution of Physical Conundrums: It is suggested that certain key Physical (and Mathematical) Conundrums including: Physics' "dark energy", "dark matter" and "arrow of time" enigmas may be potentially resolved through the application of this singular 'Universal Consciousness Principle'; this is because according to the CUFT, all (four) 'physical' properties of 'space', 'time', 'energy' and 'mass' are (in reality) solely produced by the Universal Consciousness Principle (e.g., as secondary computational 'phenomenal' properties); Hence, the key enigma of "dark energy" and "dark matter" (e.g., the fact that based on the calculation of the totality of 'mass' and 'energy' in the observable cosmos the expansion of the universe should not be as rapid as is observed - which is currently interpreted as indicating that approximately $70-90 \%$ of the "energy" and "mass" in the universe in "dark", that is not yet observable) - may be explainable based on the CUFT's delineation of the Universal Consciousness Principle's (extremely rapid) computation of the series of USCF's. This is due to the fact that according to the Universal Consciousness Principle's (previously discovered: Bentwich, 2012a) 'Universal Computational Formula' the production of any "mass" or "energy" ("space" or "time") 'physical' properties - are entirely (and solely) produced through the Universal Consciousness Principle's computation of the degree of 'Consistency' (e.g., 'consistent' or 'inconsistent') across two other Computational Dimensions, i.e., 'Framework' ('frame' vs. 'object') and 'Locus' ('global' vs. 'local'): Thus, for instance it was shown that any "mass" measurement of any object in the universe is computed by the Universal Consciousness Principle ('") as the degree of 'consistent-object' measurement (of that particular) object across a series of USCF frames.

Hence, by extension, the totality of the "mass" measured across the entire physical universe should be a measure of the degree of consistent-object/s values across a series of USCF's! Note, 
however, that based on the abovementioned recognition that in "reality" - only the Universal Consciousness Principle ('") "exists" (e.g., both as producing any of the USCF's derived four secondary computational 'physical' properties as well as existing independently "in-between" any two such USCF's frames), and therefore that only this Universal Consciousness Principle "really" produces all of the (apparent) "mass" and "energy" in the 'physical' universe (e.g., rather than the "energy" and "mass" in the 'physical' universe being "caused" by the "material" objects in the cosmos)... Hence, also all of the "energy" in the physical universe is solely produced by this (singular) Universal Consciousness Principle, e.g., as a measure of the degree of 'inconsistent-frame' (changes) of all of the objects (in the universe) across a series of USCF's frames. Therefore, according to the CUFT, the explanation of all of the "mass" and "energy" values observed in the 'physical' universe - should be solely attributed to the operation of the Universal Computational Principle, i.e., through its (extremely rapid) computation of the rapid series of USCF's (respective secondary computational measures of the abovementioned degree of 'consistent-object': "mass", or 'inconsistent-frame': "energy"). We therefore obtain that the (accelerated) rate of expansion of the physical universe - should be explained (according to the CUFT) based on the Universal Consciousness (extremely rapid) computation of the USCF's (e.g., which gives rise to the apparent secondary computational 'physical' measures of 'consistent-object': "mass" or 'inconsistent-frame': "energy"), rather than arise from any 'material-causal' effects of any (strictly hypothetical) "dark mass" or "dark energy"... (Once again, it may be worth pointing at the abovementioned conceptual computational proof that there cannot be any transference of any "physical" property entity or effect etc. across any two subsequent USCF's frames, but only the retention- or evolution- of all of the spatial pixels' "physical" properties by the singular Universal Consciousness Principle across the series of USCF's - which therefore also precludes the possibility of any "real" "material" effects exerted by any "dark" mass or energy on the expansion of the 'physical' universe across a series of USCF frames.)

Similarly, the "arrow of time" conundrum in modern Physics essentially points at the fact that according to the laws of Physics, there should not be any difference between the physical pathways of say the "breaking of a glass cup into a (thousand) small glass' pieces" and the "reintegration of these thousand glass' pieces into a unitary glass cup"! In other words, according to the strict laws of Physics, there should not be any preference for us seeing "glasses" break into a thousand pieces - over our seeing of the thousand pieces become "reintegrated" into whole glass cups (again), which is obviously contradicted by our (everyday) phenomenal experiences (as well as by our empirical scientific observations)... Hence, according to the current state of (quantum and relativistic) models of Physical reality - there is no reasonable explanation for this "arrow of time" apparent empirical "preference" for the "glass breaking into pieces" scenario over the "reintegration of the glass pieces" scenario...

However, it is suggested that according to one of the CUFT critical empirical predictions (previously outlined: Bentwich, 2012b) this "arrow of time" Physical conundrum may be resolved: This is because one (of three) critical empirical predictions of the CUFT assert the possibility of reversing any spatial-temporal sequence associated with any given 'electromagnetic spatial pixel' through the appropriate manipulation of that object's (or event's) electro- 
magnetic spatial pixel values (across a series of USCF's): It was thus indicated that if we were to accurately record the spatial electromagnetic pixels' values of any particular object (e.g., such as an amoeba or any other living organism for instance) across a series of USCF's frames (e.g., or even through a certain sampling from a series of USCF's), and to the extent that we could appropriately manipulate these various electromagnetic spatial pixels' values in such a manner which allows us to reproduce that objects' electromagnetic spatial pixels' values (across the measured series of USCF's) - in the reversed spatial-temporal sequence, then it may be possible to reverse the "flow of time" (e.g., spatial-temporal electromagnetic pixels' sequence). In this way it should be possible (according to one of the critical predictions of the CUFT) to actually "reverse" the "arrow of time" (e.g., at least for particular object/s or event/s: such as for instance, bring about a situation in which a "broken glass cup may in fact be reintegrated"...)

\section{The CUFT's eighth postulate: The 'universal consciousness reality'}

A final (potential) culmination of the CUFT may be given by its seventh (and final) theoretical postulate of the 'Universal Consciousness Reality' - which essentially postulates that there exists only one (and singular) Universal Consciousness Reality which consists of the Universal Consciousness Principle's sole production of the four (secondary computational) 'physical' properties (of 'space', 'time', 'energy' and 'mass'), mass'; that exists invariantly both as producing- maintaining- and evolving- any spatial pixel in the physical universe (through its production of the extremely rapid series of USCF's) as well as exists independently "inbetween" any (two) subsequent USCF's; and that this singular "Universal Consciousness Reality" also pervades- produces- evolves- and alternates- any of the three states of individual human Consciousness (e.g., or even 'four' as will be shown below), thereby constituting the only "real" Universal Consciousness Reality underlying the totality of the physical cosmos, all of our scientific (inductive or deductive) ontological knowledge as well as our own three (or four) individual states of human Consciousness... It is suggested that this final CUFT postulate is a direct continuation of the CUFT's latter theoretical postulates of 'Universal Consciousness', 'Ontological Relativism' and the 'Universal Consciousness Principle Spectrum': This is because the Universal Consciousness Principle asserted that the sole and single "reality" underlying the four 'physical' properties (e.g., of 'space', 'time', 'energy' and 'mass') is only the (singular) Universal Consciousness Principle; that our sole scientific (ontological) knowledge of this singular Universal Consciousness Principle "reality" may only be gained through the three states of individual human Consciousness (e.g., 'waking', 'dream' and 'deep-sleep' - and a fourth potential 'non-dual' state of Consciousness which will be discussed below), and that the ontological validity of these three states of individual human Consciousness is equal (e.g., all being produced- maintained- evolved- and alternated- solely by the Universal Consciousness Principle singular "reality); Next, the CUFT advanced the 'Universal Consciousness Principle Spectrum' theoretical postulate which hypothesized that individual human Consciousness possesses the potential of 'expanding' to encapsulate all of the Universal Consciousness Principle's produced spatial pixels (e.g., comprising the exhaustive series of pixels comprising any single or multiple USCF's - in the waking state of Consciousness). 
Based on these (latter) CUFT theoretical postulates, the Universal Consciousness Reality (final) postulate fully combines these advanced theoretical understandings together with the discovery of the "non-existence" of any real "independent" existence of the individual human Consciousness separately from the sole existence of the Universal Consciousness (Principle) Reality, thereby fully integrating our whole scientific (ontological) knowledge of the physical universe (and all hypothetical inductive or deductive scientific knowledge) into the singular Universal Consciousness Reality (proposed by the CUFT).

In order to arrive at this (potentially far reaching) theoretical conclusion, it is necessary to retrace some of the key theoretical postulates of the CUFT - i.e., specifically, those of the Computational Invariance Principe, the Universal Consciousness Principle, Ontological Relativism and the Universal Consciousness Principle's Spectrum postulate; According to the CUFT's Universal Consciousness Principle ("), there must exist a singular conceptually higherordered Universal Computational Principle which solely exists - both as producing the (extremely rapid) series of USCF's (e.g., and all of their secondary computational 'phenomenal physical' properties of 'space', 'time', 'energy' and 'mass'), as well as existing independently of any such secondary computational 'physical' properties "in-between" any two such subsequent USCF's. Moreover, based on this Universal Consciousness Principle the Universal Computational Principle must (indeed) possess the basic functions of a Universal Consciousness - i.e., retention, production and evolution of any spatial pixel comprising the entirety of all of the USCFs' multifarious spatial pixels (due to the fact that there is no "material" or "physical" property, element or factor that can "pass" across any two subsequent USCF's - based on the CUFT's previous Duality Principle, Universal Computational Principle and Computational Invariance theoretical postulates); Thus, according to the Universal Consciousness Principle, the sole and singular "reality" that comprises- produces- sustains- and evolves- any spatial pixel in the physical universe (and which also importantly exists "in-between" any two subsequent USCF's) is only that "immaterial" Universal Consciousness Principle ('")!

Next, based on the CUFT's realization that there cannot exist any "physical reality" - but only that singular (and sole) reality of the Universal Consciousness Principle ('") it was also recognized that our sole access- (and knowledge-) of this singular Universal Consciousness Principle ('") may only be gained through the three states of individual human Consciousness, e.g., those of 'waking', 'dream' and 'deep sleep'. Moreover, since (based on these abovementioned CUFT latter theoretical postulates) there does not exist any "real" 'physical reality' (e.g., but only its 'phenomenal' appearance as secondary computational 'physical' properties arising from the production of the extremely rapid series of USCF's by this singular higher-ordered Universal Consciousness Principle), then CUFT (final) 'Ontological Relativism' postulate was given which states that there does not exist any "superiority" of the 'waking' state of individual human Consciousness over any of the other (two) states of individual human Consciousness (e.g., 'dream' or 'deep sleep'); Hence, based on the Universal Consciousness Principle (e.g., alongside the other latter CUFT theoretical postulates) there exists only one singular "reality" comprising the entirety of the physical universe (e.g., through its production- maintenanceand evolution- of all USCF's secondary computational 'physical' properties of 'space', 'time', 'energy' and 'mass') and since our sole knowledge of this singular Universal Consciousness 
Principle "reality" is only through our own three states of individual human Consciousness (e.g., consisting of: the waking state which comprises the previously outlined (Bentwich, 2012b) Universal Computational Principle's extremely rapid production of the series of USCF's and their four secondary computational 'physical' properties); and based on the abovementioned (CUFT final) 'Ontological Relativism' theoretical postulate we must reach the inevitable conclusion whereby - not only the ontological contents of these three states of individual human Consciousness are equal (based on the Ontological Relativism postulate), but also these three states of individual human Consciousness must be underlie- comprised- sustained- and evolved- solely based on the singular "Reality" of the Universal Consciousness Principle!

Moreover, based on the (previous mentioned) Universal Consciousness Principle's proof for the sole existence of that sole and singular Universal Consciousness as the only "reality" underlying all secondary computational 'phenomenal physical' properties (e.g., of the USCF's series), as well as of what exists "in-between" any two such subsequent USCF's, then the CUFT's Ontological Relativism theoretical postulate also essentially asserts the fact that each of these three states of individual human Consciousness - is not "different" or "separate" from the singularity of the Universal Consciousness Principle.

Based on these (latter) theoretical postulates - e.g., of 'Computational Invariance', 'Universal Consciousness' and 'Ontological Relativism' - the 'Universal Consciousness Principle Spectrum' (UCPS) postulate was obtained; This UCPS theoretical postulate essentially claims that since (based on the abovementioned CUFT's latter theoretical postulates), there exists only one singular "reality" of the Universal Consciousness Principle ("') which both produces all of the USCF's secondary computational 'physical' properties (e.g., of 'space', 'time', 'energy' and 'mass'), and also exists (solely) "in-between" any two subsequent USCF's; since our only "access" to this singular (higher-ordered) Universal Consciousness Principle - is through the three states of individual human Consciousness (e.g., 'waking', 'dream', and 'deep-sleep'); since (according to the 'Ontological Relativism' postulate), the ontological validity of the 'waking state' (of individual human Consciousness) is equivalent to the ontological validity of the two other states (e.g., 'dream' and 'deep-sleep') - as they are all underlie- constrained- producedmaintained- and evolved- by the singularity of the Universal Consciousness Principle ('') which was shown to constitute the sole "reality" underlying all 'physical' as well as individual human Consciousness phenomena; Therefore, the Universal Consciousness Principle Spectrum (UCPS) theoretical postulate goes further to assert that, in reality, there cannot exist any difference between the phenomenal experiences gained through our individual human Consciousness and that Universal Consciousness Principle ('"') which produces- maintains and evolves- any "spatial pixel" (e.g., as well as the four phenomenal 'physical' properties of 'space', 'time', 'energy' and 'mass') comprising any USCF frame/s; In other words, in contrast to our basic phenomenal experience - i.e., at least in the 'waking' state (and also in the 'dream') of individual human Consciousness: in which we experience our sensory-motor-intellectual (and other individual Consciousness functions, see Bentwich 2012b) as constrained to only a limited body and sensory-physiological functions, the Universal Consciousness Principle Spectrum (UCPS) theoretical postulate actually expands (e.g., 'infinitely') the potential capacity of our individual human Consciousness - to engulf the (unlimited) Universal Consciousness 
Principle ('") which has been shown previously to produce- comprise- sustain- and evolve any and all spatial pixels in the phenomenal universe (as comprising particular single or multiple USCF's)...

It is important to note that despite the fact that this latter assertion made by this 'Universal Consciousness Principle Spectrum' (UCPS) theoretical postulate may seem quite "counterintuitive", it is directly supported also by an application of one of the previous theoretical postulates of the CUFT, namely: through an application of the 'Computational Invariance' principle - i.e., when applied towards the examination of the three states of human Consciousness; Essentially, the 'Computational Invariance' principle asserted that when we contrast between the 'computational invariance' of the Universal Computation/Consciousness Principle (e.g., which both produces- sustains- and evolves- all USCF's secondary computational 'physical' properties, and also exists solely and independently "in-between" any two subsequent USCF's) and the 'computational variance' of the USCF's derived secondary computational 'physical' properties (e.g., of 'space', 'time', 'energy' and 'mass') - based on the basic scientific tenet of 'Ockham's Razor' which states that Science seeks to find the most parsimonious theoretical account for any given phenomenon (or phenomena), the CUFT's Computational Invariance postulate points at the Universal Computational Principle as the only "real" principle that remains invariant -i.e., by both producing- sustaining- and evolvingall USCF's derived secondary computational 'physical' properties (of 'space', 'time', 'energy' and 'mass'), as well as existing independently (of these four basic physical properties) "inbetween" any two subsequent USCF's. It is suggested that in much the same manner, an application of the Computational Invariance Principle towards the Universal Consciousness Principle and the Universal Consciousness Principle Spectrum theoretical postulates may point (unequivocally) at the existence of a singular Universal Consciousness Principle "reality" - which solely underlies- comprises- and produces- all three states of individual human Consciousness (e.g., and therefore proves the complete equivalence of individual human Consciousness with the Universal Consciousness Principle - at least in terms of the potential capacity of individual human Consciousness to "expand" or "experience" the full spectrum of the Universal Consciousness Principle)...

Well, it is hereby hypothesized that a further application of the same Computational Invariance Principle in the case of the three states of (individual) human Consciousness - may indeed prove that underlying the three states of individual human Consciousness there can only exist the singular Universal Consciousness Principle ('"), which is therefore proven to be necessarily equivalent to the three states of individual human Consciousness, e.g., as well as transcend them - thereby comprising the sole and singular "reality" underlying both the entirety of the physical cosmos, as well as constitute the three states of individual human Consciousness...

This is because it is suggested that when we apply the (same) Computational Invariance principle to the 'computationally variant' three states of (individual) human Consciousness i.e., visa vis. the 'computationally invariant' Universal Consciousness Principle ('"); we obtain (once again) that whereas the three states of (individual) human Consciousness are solely produced by the singularity of the Universal Consciousness Principle (e.g., and are constantly alternating), the Universal Consciousness Principle (itself) remains unaltered and exists 
uniformly throughout the three states of individual human Consciousness (and also produces the entirety of the 'physical' cosmos in the waking state of individual human Consciousness). Once again - as in the application of the Computational Invariance Principle to the 'computationally variant' USCF's derived secondary computational 'physical' properties of 'space', 'time', 'energy' and 'mass', e.g., in which it was shown that the when we contrast between the computationally invariance of the Universal Consciousness Principle with the computational variance of the USCF's derived (secondary computational) 'physical' properties (of 'space', 'time', 'energy' and 'mass') we reach the inevitable conclusion whereby the singular computationally invariant Universal Consciousness Principle ('') must be recognized as the sole "reality", whereas the three states of individual human Consciousness are seen as only 'phenomenal' properties of this singular Universal Consciousness Principle... In other words, based on the fact that the three states of individual human Consciousness were already shown to be necessarily produced- sustained- evolved- (and constrained) by the singularity of the Universal Consciousness Principle (e.g., based on the CUFT's previous Universal Consciousness Principle which indicated that the sole and singular "reality" which exists "in-between" any two subsequent USCF's and also produces- and evolves- any USCF derived secondary computational 'physical' property is the singular Universal Consciousness Principle, and based on the 'Ontological Relativism' theoretical postulate which indicated that our sole access to this singular Universal Consciousness Principle can be gained solely through the three states of individual human Consciousness which possess the same ontological validity); a further application of the 'Computational Invariance Principle' (to the three states of individual human Consciousness) points at the fact that whereas there exists a singular (e.g., computationally invariant) Universal Consciousness Principle which produces- sustains- evolves- and alternates- the three states of individual human Consciousness, there are three (computationally variant) individual consciousness states (e.g., of 'waking', 'dream' and 'deep-sleep') which are produced, sustained and evolved etc. by this singular Universal Consciousness Principle; Therefore, an application of the Computational Invariance Principle to the case of the three states of individual human Consciousness points at the only 'phenomenal' stance of each of these three states of (apparent) individual human Consciousness - which are hence seen as "phenomenal" relative to the singular "reality" of the (computationally invariant) 'Universal Consciousness (Principle) Reality' which is recognized as the sole and singular "reality" that produces- sustains- evolves- all (four) waking state's phenomenal secondary computational 'physical' properties (e.g., of 'space', 'time', 'energy' and 'mass'), as well as the three phenomenal states of individual human Consciousness...

Thus, our analysis of the CUFT's (latter) 'Computational Invariance', 'Universal Consciousness Principle', 'Ontological Relativism' and 'Universal Consciousness Principle Spectrum' theoretical postulates has led us to recognize the existence of a singular 'Universal Consciousness Reality' which is solely responsible for the production- maintenance- and evolution- of all USCF's secondary derived computational 'physical' properties (of 'space', 'time', 'energy' and 'mass'), which exists independently of any of these secondary USCF's computational 'physical' properties (e.g., "in-between" any two subsequent frames), and which is also entirely underliessustains- evolves- and alternates- any of the three (or four) individual human Consciousness states (of 'waking', dream', 'deep-sleep' or the "non-dual" state which will be further described 
below); The emphasis of the Universal Consciousness Reality (postulate) is that in "reality" there does not exist any "real" (separate) existence - of either our 'individual' human Consciousness (e.g., comprising the three or four abovementioned states of individual Consciousness), or of the 'phenomenal' physical cosmos (which merely represents the apparent secondary computational properties of 'space', 'time', 'energy' or 'mass' of the Universal Computational Principle's production of the three previously mentioned computationally variant Computational Dimensions). Therefore, it may be said that the culmination of the CUFT may be encapsulated by its seventh 'Universal Consciousness Reality' which highlights the fact that there can only exist one (singular) 'Universal Consciousness Reality' that solely produces- sustains- evolves- (and alternates-) all four (apparent secondary computational) 'physical' properties (of 'space', 'time', 'energy' and 'mass'), as well as all three (or four) individual states of human Consciousness. Needless to say that this latter (potential) equivalence of our individual human Consciousness with the singular Universal Consciousness (Principle) Reality also calls for further scientific exploration of the means for realizing this potential equivalence. Suffice to state (at this point) that some of these potential theoretical ramifications include the (previously stated Universal Consciousness Spectrum postulate's) possibility of modulating human Consciousness in such a manner which enables it to "expand" its scope to encapsulate broader USCF's 'spatial pixels' (than those identified by a particular "person" at a particular 'spatial-temporal' point/s appearing at a single or multiple USCF's frames), thereby potentially affecting any spatial, temporal, mass or energy properties associated with any particular region/s in a given single or multiple USCF's frames...

We've begun this chapter by noting that the discovery of the CUFT's Universal Consciousness Principle ('") may signify a basic "paradigmatic shift" from the current Cartesian "materialisticreductionistic" theoretical framework which assumes that any (hypothetical) 'y' element, phenomenon or process etc. can be determined strictly based on its direct or indirect physical interactions with an exhaustive set of ' $x$ ' factors (e.g., comprising a SROCS computational structure which was negated by the CUFT's Duality Principle for all empirically knowable 'x$y^{\prime}$ relationships) - to a conceptually higher-ordered (singular) Universal Computational/ Consciousness Principle that is solely responsible for the production- sustenance- or evolutionof all 'phenomenal' (secondary computational four 'physical' properties of 'space', 'time', 'energy' or 'mass', or indeed of all (exhaustive hypothetical) inductive or deductive 'x-y' pairs series embedded within any given series of USCF's... We've then emphasized the conceptually higher-ordered ('D2') 'non-material', 'a-causal' computational nature of this singular Universal Computational/Consciousness Principle which computes the "simultaneous co-occurrences" of any exhaustive hypothetical inductive or deductive ' $x-y$ ' pairs' series, thereby negating the possibility of any "real" 'material-causal' relationships existing between any of these exhaustive hypothetical (quantum or relativistic physical, inductive or deductive) ' $x-y$ ' pairs;

Indeed, the application of a generalized format of the Duality Principle has proven that all hypothetical inductive or deductive ' $x$-y' pairs comprising a basic ('Gödel-like') SROCS computational structure must be constrained by the CUFT's Duality Principle which therefore precludes the existence of any "real" 'causal-material' relationship between the ' $x$ ' and ' $y$ ' elements, instead pointing at their sole contingency upon the singular (conceptually higher- 
ordered) Universal Computational/Consciousness Principle (') which computes the "simultaneous co-occurrences" of any of these exhaustive hypothetical inductive or deductive ' $x$ - $y$ ' pairs series comprising a series of USCF's; Moreover, based on the (previous) discovery of the Computational Invariance Principle and Universal Consciousness Principle theoretical postulates and the current chapters delineation of the Universal Consciousness Principle's sole and singular production- sustenance- and (potential) evolution of all the spatial pixels comprising the USCF's portrayal of the physical universe, it was realized that only this Universal Consciousness Principle may be regarded as "real" whereas all of the secondary computational 'physical' properties (e.g., of 'space', 'time', 'energy' or 'mass') as well as all other hypothetical inductive or deductive or any human Consciousness (psychophysical) ' $\mathrm{x}-\mathrm{y}$ ' relationships (or phenomena) must be regarded as (at best) as representing 'phenomenal' (or even "illusory") properties... Likewise, based on the 'Computational Invariance Principle' and the 'Universal Consciousness Principle' which (jointly) indicated that only the Universal Consciousness Principle ('") exists permanently and invariantly both as producing- sustainingand evolving- any of the (secondary computational) USCF's is "real", whereas all (secondary computational) 'physical' properties (of 'space', 'time', 'energy' and 'mass') are 'phenomenal' or "illusory", it was proven that there cannot be any "real" 'material-causal' effects between any (exhaustive hypothetical) ' $x$ ' and 'y' (physical, inductive or deductive) factors that can "pass" across two (or more) USCF's frames, thereby nulling the possibility of any real 'material-causal' ' $x$-y' relationship (e.g., but instead pointing at the abovementioned higher-ordered Universal Consciousness Principle computed 'a-causal' "simultaneous co-occurrences" of any exhaustive hypothetical 'x-y' pairs series).

Indeed, the recognition that only the (singular) Universal Consciousness Principle may be regarded as "real" whereas all of the (secondary computational) 'physical' properties must be seen as 'phenomenal' (e.g., "unreal" relative to their sole production- sustenance- and evolution- by the singular conceptually higher-ordered Universal Consciousness Principle) - has led to the identification of the sixth theoretical postulate of 'Ontological Relativism': i.e., the realization that accepting the Universal Consciousness Principle ('') as the sole and singular "reality" which produces- retains- and evolves- all (phenomenal) 'physical' properties (of 'space', 'time', 'energy' and 'mass') implies that our ontological knowledge of that Universal Consciousness Principle is constrained by three different states of individual human Consciousness (e.g., 'waking', 'dream' and 'deep sleep') whose ontological validity is equivalent... In other words, the 'Ontological Relativism' postulate indicates that there is no longer any "advantage" (or "superiority") for the 'waking' state of individual human Consciousness upon the two other (e.g., 'dream' or 'deep sleep') states - as they are all equivalent in terms of their portrayal of the same singular "reality" of the Universal Consciousness Principle.

Thus, based on this (sixth) 'Ontological Relativism' postulate we arrived at a more comprehensive 'Universal Consciousness Principle Formula' which incorporated the CUFT's (original) 'Universal Computational Formula' within the broader conceptual understanding of (the CUFT's sixth) 'Ontological Relativism' postulate as well as its associated (CUFT's seventh) 'Universal Consciousness Spectrum' postulate; Hence, the broader 'Universal Consciousness Formula' delineated the Universal Consciousness Principle's inclusiveness of the three states 
of (individual) human Consciousness as well as the (new hypothetical) seventh theoretical postulate of the 'Universal Consciousness Spectrum'; Jointly, these two new tenets of the CUFT indicated that over and beyond the individual human Consciousness comprising of three separate states (whose ontological validity is equivalent relative to the "reality" of the singular Universal Consciousness Principle), the individual human Consciousness possesses a full spectrum of 'waking' state "expansiveness" (e.g., spanning from "1 to infinity") which differ in the degree of their "expansiveness" of the number of spatial pixels being included in any given individual human Consciousness portrayal of their perception of the "reality"...

In terms of some of the potential (broader) Scientific implications that may stem from this broader formalization of the CUFT's Universal Consciousness Principle, Universal Consciousness Formula, and 'Universal Consciousness Spectrum' postulates (e.g., as well as from the entirety of the Universal Consciousness Principle based more comprehensive formalization of the CUFT; it is suggested that (first), based on the CUFT (generalized) Duality Principle and Universal Consciousness Principle postulate - e.g., pointing at the computational "invalidity" of any inductive or deductive or indeed any quantum or relativistic physical SROCS' ' $x-y$ ' (materialistic-reductionistic) relationships, Science must accept the need to formalize any such physical - quantum or relativistic, inductive or deductive 'x-y' relationships based on the conceptually higher-ordered (singular) Universal Consciousness 'a-causal' computation of the "simultaneous co-occurrence" of an (exhaustive hypothetical) series of 'x-y' pairs (comprising a segment of a certain USCF/s frame/s); This would also include the reformalization of the (previously and abovementioned) key scientific SROCS paradigms, including: Darwin's Natural Selection Principle and associated genetic encoding hypothesis, Neuroscience's Psychophysical Problem of human Consciousness (and others) based on the sole operation of the singular "reality" of the Universal Consciousness Principle...

Second, the acceptance of the sole "reality" of the singular Universal Consciousness Principle, e.g., visa vis. the realization that all (secondary computational) 'physical' properties (of 'space', 'time', 'energy' and 'mass') are merely 'phenomenal' (or "unreal" - relative to this singular Universal Consciousness Principle which produces- retains- and evolves- all such secondary computational 'physical' properties); And moreover based on the recognition of the (inevitably ensuing) 'Ontological Relativism' which highlights the lack of any "objective-physical" criteria by which to evaluate the ontological validity of any of the three (abovementioned) states of individual human Consciousness (e.g., instead asserting that each of the three states of our individual human Consciousness' is equivalent in terms of its ontological validity relative to the singular "reality" of the Universal Consciousness Principle) - necessitates a basic paradigmatic shift from the (current) Cartesian 'materialistic-reductionsitic' (SROCS) computational paradigms towards the realization that there exist only one singular "reality" of the Universal Consciousness Principle which produces- sustains- and evolves- any of the apparent 'phenomenal' (secondary computational) 'physical' properties of any spatial pixel comprising the (rapid series of) USCF's.

Finally, even above and beyond the (abovementioned) potentially far reaching theoretical ramifications of accepting the sole "reality" of this (singular higher-ordered) Universal Consciousness Principle (e.g., as opposed to the currently accepted Cartesian 'materialistic- 
reductionistic' scientific framework), the discovery of the (broader) 'Universal Consciousness Formula' and 'Universal Consciousness Spectrum' tenets brings about a potentially profound shift in our basic conception of the role of (individual) human Consciousness in modulating the 'physical' properties of 'space', 'time', 'energy' or 'mass', and opens the door for further (important) scientific research regarding the true nature of our individual human Consciousness and its precise relationship to the singular "reality" of the 'Universal Consciousness Principle' (and the phenomenal 'physical' properties).

\section{Acknowledgements}

I would like to thank (wholeheartedly) Dr. Tirza Bentwich, Mr. Brian Fisher, Dr. Talyah UngerBentwich and Mr. Menachem Davorskin whose support and encouragement have allowed me to develop (and pursuit) some of the progressive concepts embedded within the CUFT.

\section{Author details}

Jonathan Bentwich* $^{*}$

Address all correspondence to: drbentwich@gmail.com

Brain-Tech, Israel

\section{References}

[1] Bagger, J, \& Lambert, N. (2007). Modeling multiple M2's. Phys. Rev. D, , 75(4)

[2] Bentwich, J. (2003a). From Cartesian Logical-Empiricism to the'Cognitive Reality': A Paradigmatic Shift, Proceedings of Inscriptions in the Sand, Sixth International Literature and Humanities Conference, Cyprus

[3] Bentwich, J. (2003b). The Duality Principle's resolution of the Evolutionary Natural Selection Principle; The Cognitive 'Duality Principle': A resolution of the 'Liar Paradox' and 'Gödel's Incompleteness Theorem' conundrums; From Cartesian LogicalEmpiricism to the 'Cognitive Reality: A paradigmatic shift, Proceedings of $12^{\text {th }}$ International Congress of Logic, Methodology and Philosophy of Science, August Oviedo, Spain

[4] Bentwich, J. (2003c). The cognitive'Duality Principle': a resolution of the'Liar Paradox' and'Gödel's Incompleteness Theorem' conundrums, Proceedings of Logic Colloquium, Helsinki, Finland, August 2003 
[5] Bentwich, J. (2004). The Cognitive Duality Principle: A resolution of major scientific conundrums, Proceedings of The international Interdisciplinary Conference, Calcutta, January

[6] Bentwich, J. (2006). The 'Duality Principle': Irreducibility of sub-threshold psychophysical computation to neuronal brain activation. Synthese, , 153(3), 451-455.

[7] Bentwich, J. (2012a). Quantum Mechanics / Book 1 (979-9-53307-377-3Chapter title: The'Computational Unified Field Theory' (CUFT): Harmonizing Quantum Mechanics and Relativity Theory.

[8] Bentwich, J. (2012b). Quantum Mechanics / Book 1 (979-9-53307-377-3Chapter 23, Theoretical Validation of the Computational Unified Field Theory., 551-598.

[9] Born, M. (1954). The statistical interpretation of quantum mechanics, Nobel Lecture, December 11, 1954

[10] Brumfiel, G. (2006). Our Universe: Outrageous fortune. Nature, , 439, 10-12.

[11] Ellis, J. (1986). The Superstring: Theory of Everything, or of Nothing? Nature, , 323(6089), 595-598.

[12] Greene, B. (2003). The Elegant Universe, Vintage Books, New York

[13] Heisenberg, W. (1927). Über den anschaulichen Inhalt der quantentheoretischen Kinematik und Mechanik. Zeitschrift für Physik, , 43(3-4), 172-198. 


\title{
Emergent un-Quantum Mechanics
}

\author{
John P. Ralston \\ Additional information is available at the end of the chapter
}

http://dx.doi.org/10.5772/55954

\section{Introduction}

There is great interest in "emergent" dynamical systems and the possibility of quantum mechanics as emergent phenomena. We engage the topic by making a sharp distinction between models of microphysics, and the so-called quantum framework. We find the models have all the information. Given that the framework of quantum theory is mathematically self-consistent we propose it should be viewed as an information management tool not derived from physics nor depending on physics. That encourages practical applications of quantum-style information management to near arbitrary data systems. As part of developing the physics, we show there is no intrinsic distinction between quantum dynamics and classical dynamics in its general form, and there is no observable function for the unit converter known as Planck's constant. The main accomplishment of quantum-style theory is a expanding the notion of probability. A map exists going from macroscopic information as "data" to quantum probability. The map allows a hidden variable description for quantum states, and broadens the scope of quantum information theory. Probabilities defined for mutually exclusive objects equal the classical ones, while probabilities of objects in more general equivalence classes yield the quantum values. Quantum probability is a remarkably efficient data processing device; the Principle of Minimum Entropy explains how it serves to construct order out of chaos.

\section{Complexity and symmetry induce dynamics}

The framework of quantum mechanics is intricately structured and thought the perfection of fundamental theory. It predicts an absolute and unvarying law of time evolution. There is a tightly defined space of possible states, upon which strictly prescribed operators act to produce crisp possibilities for observables. There is an unprecedented universal rule for predicting probabilities of observations. The general predictions of the framework are incompatible with hidden variables defined by distributions, and have been confirmed by every experiment so far conducted. 
Meanwhile, the particular realizations of physical theories are widely believed to be emergent. That means they probably do not really represent fundamental physical law, but instead represent generic outputs of complicated systems not driven by the same laws. The Standard Model of particle physics is the most sophisticated prototype. It explains all data from all experiments done so far, except for a few outliers. Yet it is hard to find anyone involved that will argue the Standard Model is more than a generic derivative expansion embodying certain symmetries of some more complicated theory, of which numberless possibilities exist. Like Hooke's Law, Standard Model Laws are no longer imagined to be serious candidates for Laws, because they are so contrived and of the type that had to occur one way or the other. Nor does one really need the machinery of the Standard Model to understand most of the Universe. For most of what matters, the non-relativistic Schroedinger Equation is a "theory of everything"[1].

Then it is very reasonable to expect that quantum mechanics itself should be a generic, self-defining "emergent" feature of the Universe. In simple terms, an output, not an input.

\subsubsection{Practical goals}

What does that mean? Discussions of emergent quantum mechanics tend to become confused by discord over what we mean by quantum mechanics. Different writers will disagree about what is fundamental and even about what experimental data says. Not far behind is a superstition that quantum objects cannot possibly be understood, so that making them even more obscure and more difficult might be the intellectual high ground. We reject that holdover from the 1920's, but it's not clear whether it has died out, or might be coming back

To skirt the morass we have a new point of view. We want physics to be practical and simple. In this century not many find the mathematics of quantum theory so intimidating. Perhaps physics "quantum-style" is not so profound after all. What is the evidence that the quantum framework itself is so meaningful and fundamental?

Most accomplishments of "quantum mechanics" come from the model details. For example, understanding the Hydrogen atom and calculating $g-2$ of the electron are astounding achievements. However those accomplishments come directly from the model details, and systematic laborious tuning of theory to experimental facts, rather than from the framework of quantum mechanics itself. That is not always noticed while the framework lays claim for every accomplishment. The framework passes every test, especially when tested by thought-experiments set up for validation by pencil and typewriter challenges that recycle the framework. But the framework is rather hard to falsify experimentally. When it might have failed, a little ingenuity never fails to bring it back. That is quite unlike models challenging conventional theory, such as non-linear Schroedinger equations and so on, which have the decency to be able to fail.

Following this and other clues, we propose the quantum framework amounts to descriptive tools and classification tools that categorize data beautifully, but predict very little at all. When the "Laws" of quantum mechanics are considered as procedural and classification structures, it's much easier to guess how they would emerge as human-made bookkeeping.

Not everything in quantum mechanics is procedural, and some of its general workings contain clues to Nature. It is rarely noticed that quantum theory has infinities of hidden variables. They are not classical hidden variables of the usual kind. The "electron" and 
"photon" of the 1920's thought to be so fundamental and ultimate are not fundamental objects. They are approximations that never stop interacting with an infinite ocean of quantum fields, if not something more unknown and more interesting. The self-consistency of the framework suggests that living in peaceful co-existence with what it must ignore may be its main accomplishments.

\subsection{What would emerge?}

Many workers seek to derive quantum mechanics. An active movement suspects or maintains that it is emergent, not fundamental[2-4]. We think it would be a waste to obtain exactly the quantum framework already known. Progress requires new features and new viewpoints. Progress usually involves dropping obsolete views and clearing out deadwood: call it un-Quantization.

We start by considering what can and cannot be given up. Quantum mechanics wiped out the previous vision of a Newtonian universe made of point particles. It's gone forever. There remains some confusion and disagreement about whether the theory is about point particles of some subtle magic kind. While point particles are loosely cited in press releases, and inaccurately associated with theories based on local space-time interactions, we cannot find any evidence for them, and give that up. We believe all the rest of early quantum lore also can be given up, especially those parts leading to pedagogical confusion. We can't explain why it is unconventional or even scandalous to admit that the pre-history of quantum mechanics -meaning that period between 1900 and 1926 - was characterized by great theoretical advances that were all wrong or limiting in some way or other. Being wrong is normal in physics but covering it for generations is very strange.

Many professional physicists are still influenced by the cult of the "quantum of action," forgetting it went away when quantum mechanics found action is not quantized. Physicists have been programmed from birth to hold Einstein's relations $E=\hbar \omega, \vec{p}=\hbar \vec{k}$ as highly fundamental. They actually know these relations are not universal, but derived facts coming from special cases, yet downgrading them is taboo. Physicists often believe that classical Newtonian mechanics shall be a starting point to be "quantized" to predict quantum systems, forgetting this recipe only predicts textbook problems for training purposes. It would forbid the Standard Model of physics to exist at all. Physicists are also trained that Feynman's path integral creates a quantum theory from a classical one, forgetting that what's integrated over nowadays has no relation to the starting point young Mr. Feynman used. When we un-Quantize this, an integral representation of correlation functions is a math tool, not an independent principle, nor does it "come from physics."

We must honor our forebears and we do. Yet why are historical misdirections kept around with special emphasis in quantum physics? The culture of quantum mechanics almost seems to maintain mistakes of pre-quantum history on purpose. Instead of giving them up, misconceptions are kept around as philosophical quandaries and paradoxes because there is no other way to perpetuate mistakes except as paradoxes. All that can be given up.

\subsubsection{Quantum-Style Things Not to Give Up}

Quantum mechanics is a misnomer held-over from the pre-quantum era. The Schroedinger equation explained how and when quantization of physical quantities occurs as an outcome 
of dynamics, and quantization is not the primary new feature of the theory. We have coined the term "quantum-style" to describe things done in the organizational style of quantum mechanics that we don't want to give up.

Quantum-style mechanics describes certain data of the Universe, and the ability to describe experimental data absolutely cannot be given up. But that does not mean in some future time we would interpret success the same way. A certain vagueness of description is probably tied to success. For example, it is rarely noticed that the dramatic demonstrations of beginning quantum probability, such as the Bell inequalities and EPR "paradoxes," are realized experimentally only by virtuoso fiddling and selection of systems nice enough to make them work out. When an experiment produces nothing but the mundane predictions of ordinary classical probability, nobody notices that was also a retrospective quantum prediction of certain dirty density matrices, protecting the framework from falsification. But we have noticed, and it suggests that quantum mechanics must be a framework that is so broad and flexible it does not restrict much. For example, if a future civilization discovered a true and ultimate Newtonian particle, contradicting everything now believed, any competent theorist would find no great difficulty describing it with an appropriately constructed density matrix.

All of this suggests what a new vision of "quantum theory" should include. The restriction of the subject to describing micro-physical objects of fundamental physical character is obsolete. It seems the same mistake as thinking complex numbers have no practical use because they have an "imaginary" part. We believe the framework of quantum theory is mathematically self-consistent. And when there are self-consistent structures, they ought to have more uses than microphysics, and not depend on microphysics for their uses. Even more directly, asking a self-consistent framework to "emerge" means mainly to start using it without fear it could go wrong.

This idea appears radical because it contradicts a few existing ideas. Physicists are educated in the magical antics of quantum objects and convinced they are impossible to understand before they begin serious coursework. As a result, misidentifying mathematical relations as inexplicable Facts of Nature is very common. That is fatal to physics, because Facts and Behaviors of a special Universe are not supposed to work on other Universes. That is why it is generally considered stupid to apply quantum-style methods to non-quantumy objects.

The degree of stupidity depends strongly one's Bayesian priors. 100,000 years ago humans would know the concepts of integers but probably be unable to separate them from empirical facts of Nature. It would be considered absurd and dangerous to imagine integers disassociated from their experimental realization in the number of rocks or rabbits. Yet those humans were not stupid. They lacked the background about the Universe to decide whether integers came from physics or came from human thinking, with physics probably having the edge because it was real. In much the same way the occult mysteries of $\hbar, i=\sqrt{-1}$, and electrons "in all states at once" conditioned the physics community to think "quantum methods are for quantum objects. "

Anyone thoughtful ought to notice that is circular, and ought to be open to using mathematical structures more liberally. But thoughtful people are given false information by the physicists about what's established, and what can be contradicted, and even about what experiments find. We feel it is significant that the early 20th century was the first time physics needed to seriously deal with the details of experiments involving a large number of degrees 
of freedom, equilibrium thermodynamics notwithstanding. Whether or not microphysics had any new and spooky elements, we find that quantum-style methods would need to be invented to handle the complexity. It is very efficient: And then the classification system and information processing power of quantum data organization should be used wherever a useful result might come out.

\subsubsection{How to Proceed}

Towards that end, we have developed an approach which largely avoids the historical path. That path and its traditional presentation interweaves a little dynamics, measurement theory, microphysical facts, experimental claims and the prehistory of failed theories in an alternating web. It is designed not to be challengeable, which is a cheat. To make progress we must change something. We first separate the dynamics, meaning rules of time evolution, from the rest, and identify it as being trivial. This is developed in Section 3. For us the ordinary form of quantum dynamics is a "toy model", which at first seems too simple to be realistic. This is not to say that the models describing microphysics are trivial or easy to solve. Actually the models are the real discovery, while claims that the dynamical framework is the real discovery have things reversed.

The great roadblock to using the organizational scheme of quantum theory more generally is Planck's constant. Once the claim is made that Nature partitions itself into little cells determined by $\hbar$, one cannot do without $\hbar$, nor use quantum theory for anything else but issues of $\hbar$. But the claim is wrong. In Section 4 we discuss a quantum-style Universe without $\hbar$. We claim it is the ordinary Universe, but if that is too provocative, the step of never introducing $\hbar$ is a part of our approach where it never appears.

We also dispense with needlessly obscure definitions of "observables." We define observables (Section 5) as maps from the system coordinates to numbers. This is plain and unpretentious. Satisfying things, including relations of Poisson Brackets usually postulated as independent, can actually be derived using symmetry. In principle the map between system and observables is invertible: the system coordinates (wave function, density matrix) are observable. It is a non-trivial fact that real physical systems seem to have infinite complexity, making exhaustive measurements a bit out of reach, but this "bug" is a "feature."

In the end the new thing that came out of quantum mechanics are new definitions of "probability", Section 6. The working of quantum probability has always been explained by physicists using a self-validating logic that "it works" because "features of microphysical object make it work". (And this is very mysterious and too profound for humans to grasp, etc.) Every time that thinking style is used we find it unprogressive and circular.

Instead of buying it, we seek a rational explanation why certain mathematical tools work sometimes and other rules work other times without depending on circular postulates. In our approach the information management of quantum theory is a topic of mathematical classification, and for that reason mostly devoid of physical content. Since it is mathematics, we can derive the quantum rules of probability as a bookkeeping system that does not need any special features from the objects they describe. And we do this to increase the scope and utility of the rules so we can use them in new applications. 


\subsubsection{Question From the Bottom Up}

It is not always helpful to put the framework of quantum mechanics on a high pedestal. It is sometimes assumed that quantum mechanics might only be "explained" by progress at the far edge of the research frontier involving quantum gravity, foamy space-time, strings, and so on[5]. But if true that would put our topic among those not seeking to deal with what is observable and testable. We are only interested in what is observable and testable.

Progress needs to come from revising the bottom. Successful work at the bottom revises basic notions that are actually harder to challenge than advanced work, because the whole system rests on the base The mathematics of our discussion is not advanced. It is little more than linear algebra, and deliberate choices not to use mathematics that is more advanced than needs to be used.

There is a very elementary point often overlooked. Mathematical subjects can be reduced to a definite minimal number of axioms, which might be swapped around, but not decreased. Early on quantum theory looked ripe for axiomization, and it tends to be accepted today. Yet every effort to make physics into axioms fails because we don't know what the Universe is. However physics can often reduce the number of postulates, axioms or guesses by swapping around the order, which actually changes their meaning and power. The everyday assumption that this was optimized long ago is not true. Thus, it is a form of progress to explore how post-quantum physics-axioms can be eliminated by re-ordering and re-interpreting the logic. The process will help quantum mechanics "emerge" more clearly from its own tangles.

\section{Dynamics}

\subsubsection{Where to Start?}

By very curious structuring, the usual approach to quantum mechanics starts with the doctrine of measurement postulates. What is out of order in those approaches is failing to first define the system and its dynamics. For example the Stern-Gerlach experiment is traditionally developed as a raw mystery of 1922 involving point-like electrons and two spots, then requiring a new principle[6]. If one were given from the start that a two-component wave function was involved the separation into two spots does not really need any new principles. It was known to Fresnel from calcite crystals and explained without requiring a new principle. And if one knew the particular two-component wave functions of electrons were expected from representations of the rotation group none of it would be a terrible surprise. While ordering things to make physics more mysterious and inexplicable was an early promotional tool, we lack any interest in it. That is why we will start with the dynamics, because it can be explained. We will discuss how there is nothing new contributed by quantum mechanics to its own framework of dynamics: at least in our approach.

\subsection{Hamilton's equations in Schroedinger's notation}

Physics predicts little more than evolution of systems with time, symbol $t$. By the end of this work we will argue the predictions (above and beyond the empirically-found model details!) originate in symmetry. 
We assume the reader knows how to get equations by varying an action $S$, expressed using a Lagrangian $L\left(q_{i} \dot{q}_{i}\right)$ :

$$
\delta S=\delta \int d t L\left(q_{i} \dot{q}_{i}\right)
$$

Symbols $q_{i}$ are generalized coordinates, namely numbers describing a system, labeled by $i=1 \ldots N$, and the dot indicates a time derivative. We are not going to suggest that the "action principle" will be our foundation postulate. When and if an emergent quantum system has sufficiently nice dynamics that it gets noticed as an experimental regularity, the action is a fine invariant notation to express it.

By familiar steps, finding the extrema of the action produces Lagrange's equations. Define the Hamiltonian $H$ by

$$
H\left(q_{i}, p_{i}\right)=p_{i} \dot{q}_{i}-L\left(q_{i} \dot{q}_{i}\right)
$$

Repeated indices are summed. When these transformations can be done, then Lagrange's equations are equivalent to Hamilton's:

$$
\dot{q}_{i}=\frac{\partial H}{\partial p_{i}} ; \quad \dot{p}_{i}=-\frac{\partial H}{\partial q_{i}}
$$

We will pause at this point to repeat that $\left(q_{i}, p_{i}\right)$ are real-valued numbers, that everything above was known by (say) 1850, and that our subject is nevertheless quantum dynamics. We will never confuse a classical coordinate with an operator, we will never use the abusive term "quantum particle" except to reject it, and when an operator is intended it will be indicated by a hât.

The thing for our discussion not known in 1850 lies in the number of dynamical degrees of freedom $(d o f)$ we intend to use. A Newtonian particle has three dof usually taken to be its Cartesian coordinates. The Newtonian particle is not a valid prototype and (unlike the early history) we base nothing on making contact with it. In our approach we have no advance information on the number of dof describing a system, because that is an arbitrary defining feature of a system. For $N$ dof the phase-space of $\left(q_{i}, p_{i}\right)$ is $2 N$ dimensional. We also pretend to no advance information on the Hamiltonian, although some properties will be specified to make contact with existing models. We claim this freedom not to commit is a defining fact of basic quantum mechanics: but if that is not agreed, it is a fact of our theory.

Now proceed: Hamilton's equations are invariant under symplectic $(S p)$ transformations. It is usually developed by combining $\left(q_{i}, p_{i}\right)$ into a $2 N$ dimensional multiplet $\Phi=$ $\left(q_{1} \ldots q_{N}, p_{1} \ldots p_{N}\right)$. Hamilton's equations become

$$
\dot{\Phi}=J \frac{\partial \mathcal{H}}{\partial \Phi}
$$


Here $J$ is a matrix with block representation

$$
J=\left(\begin{array}{cc}
0 & 1_{N \times N} \\
-1_{N \times N} & 0
\end{array}\right)
$$

Under a real-valued $2 N \times 2 N$ transformation $S$, the equation transforms

$$
\begin{gathered}
\Phi \rightarrow \Phi_{S}=S \Phi ; \\
\dot{\Phi}_{S}=S \cdot J \cdot S^{T} \frac{\partial \mathcal{H}}{\partial \Phi} .
\end{gathered}
$$

Super- $T$ denotes the transpose. The symplectic group of $2 N$ dimensions is the set of transformations such that

$$
S \cdot J \cdot S^{T}=J
$$

It can be shown the determinant $\operatorname{det}(S)=1$. Transformations satisfying Eq. 5 with determinant -1 will be called $S p$ - parity changing.

\subsubsection{Simplistic Linear Theories Are Not Our Burden To Defend}

By a linear theory we mean that the Hamiltonian $H$ is bilinear in $q_{i}, p_{i}$ :

$$
\begin{gathered}
\mathcal{H}(q, p)=\frac{1}{2} \Phi^{T} H_{\Phi} \Phi \\
H_{\Phi}=\frac{1}{2}\left(\begin{array}{ll}
h_{q q} & h_{q p} \\
h_{q p}^{T} & h_{p p}
\end{array}\right)
\end{gathered}
$$

Matrix multiplication is implied, and $h_{q q}, h_{q p} \ldots$ etc are $N \times N$ arrays of constant parameters. We are not writing linear terms like $\alpha q+\beta p$, which can be removed by translating coordinates. We have no commitment here to the bilinear form, which is presented to make contact with ordinary quantum mechanics.

The most general such theory has a familiar form, seen by writing ${ }^{1}$

$$
h_{q q}=K ; \quad h_{p p}=M^{-1} \quad h_{q p}=-\Gamma^{T} M
$$

Complete the square:

$$
\begin{array}{r}
\mathcal{H}(q, p)=\frac{1}{2} p M^{-1} p+\frac{1}{2} q K q+q M \Gamma p+p \Gamma^{T} M q, \\
=\frac{1}{2}(p-\mathcal{A}(q)) M^{-1}(p-\mathcal{A}(q))+\mathcal{V} ; \\
\text { where } \mathcal{A}(q)=\Gamma q ; \quad \mathcal{V}=\frac{1}{2} q\left(K-\Gamma^{T} M^{-1} \Gamma\right) q .
\end{array}
$$

\footnotetext{
${ }^{1}$ There's no loss of generality using these symbols, as $M_{-1}$ is meant to be the inverse on the space $M$ does not send to zero, i.e. the pseudoinverse.
} 
The Hamiltonian of a classical, 3-dimensional, non-relativistic particle in an external electromagnetic field is $H_{e m}=(\vec{p}-e \vec{A}(\vec{q}))^{2} / 2 m+V(\vec{q})$, where $\vec{A}$ is the vector potential. Except for allowing a tensor mass, a quantum system with three dof (spin-1, say) is dynamically indistinguishable. For more dof the symbol A(q) continues to serves as a vector potential with an associated curvature

$$
\mathcal{F}_{i j}=\frac{\partial \mathcal{A}_{i}}{\partial q_{j}}-\frac{\partial \mathcal{A}_{j}}{\partial q_{i}}=\Gamma_{i j}-\Gamma_{j i}
$$

These theories have gauge symmetries. From Eq. 10 any symmetric part of $\Gamma$ drops out of $\mathcal{F}_{i j}$ and the equations of motion. That is equivalent to gauge transformation $\mathcal{A}(q) \rightarrow$ $\mathcal{A}(q)+\Sigma q$, where $\Sigma=\Sigma^{T}$. As a rule gauge symmetries indicate a system that is being described with more coordinates than are truly dynamical: the redundant coordinates may be hard to eradicate, and easier to treat as "symmetries." That will be a clue.

\subsubsection{Diagonal Frame}

Go to coordinates where the symplectic metric $J$ is diagonal. Since $J$ is antisymmetric, the transformation goes from real to complex numbers:

$$
\Phi=\left(\begin{array}{c}
q_{1} \\
q_{2} \ldots \\
p_{1} \\
p_{2} \\
\ldots
\end{array}\right) \rightarrow \Psi=\left(\begin{array}{c}
\psi_{1} \\
\psi_{2} \\
\ldots \\
\psi_{1}^{*} \\
\psi_{2}^{*} \\
\ldots
\end{array}\right)
$$

An appropriate map is

$$
\begin{aligned}
& \Psi=\mathcal{U} \Phi ; \\
& \mathcal{U}=\frac{1}{\sqrt{2}}\left(\begin{array}{cc}
1_{N \times N} & i 1_{N \times N} \\
1_{N \times N} & -i 1_{N \times N}
\end{array}\right),
\end{aligned}
$$

with

$$
\mathcal{U U}^{\dagger}=1_{2 N \times 2 N}
$$

and then

$$
\mathcal{U} \mathcal{U}^{+}=-\left(\begin{array}{cc}
i 1_{N \times N} & 0 \\
0 & -i 1_{N \times N}
\end{array}\right) .
$$


The transformation produces a remarkable simplification of linear dynamical systems. Hamilton's first equation (Eq. 2) become

$$
i \frac{\partial \Psi}{\partial t}=\frac{\partial H\left(\Psi, \Psi^{*}\right)}{\partial \Psi^{*}} .
$$

Since it is an important point we show the algebra for one dof. We are given

$$
\dot{q}=\frac{\partial H}{\partial p} ; \quad \dot{p}=-\frac{\partial H}{\partial q}
$$

Combine two real numbers into one complex one:

$$
\psi(q+i p) / \sqrt{2}
$$

We call this the "quantum map". It explains how complex numbers came to be essential in quantum theory. Compute

$$
\dot{\psi}=(\dot{q}+i \dot{p}) / \sqrt{2}=\left(\frac{\partial H}{\partial p}-i \frac{\partial H}{\partial q}\right) / \sqrt{2}
$$

The chain rule gives

$$
\frac{\partial}{\partial p}-i \frac{\partial}{\partial q}=\sqrt{2} \frac{\partial}{\partial \psi^{*}}
$$

and then Hamilton's equations are

$$
i \dot{\psi}=\frac{\partial H}{\partial \psi^{*}}
$$

Continuing: When $H(q, p)$ is bilinear then $H\left(\Psi, \Psi^{*}\right)$ is bilinear. In quantum mechanics one always chooses parameters so that

$$
H\left(\Psi, \Psi^{*}\right)=\Psi^{*} \Omega \Psi
$$

where $\Omega$ now contains the parameters. Eq. 14 and 18 give

$$
i \frac{\partial \Psi}{\partial t}=\hat{\Omega} \cdot \Psi
$$

This is Schroedinger's equation, which is nothing more than Hamilton's equation in complex notation. We prefer symbol $\hat{\Omega}$ to $\hat{H}$ for reasons to be explained soon. 


\subsubsection{Conventionally Assumed Properties}

We do not have hermiticity in the form $\hat{\Omega}=\hat{\Omega}^{\dagger}$ automatically. First as Bender and collaborators has emphasized[8], the self-adjoint test does not have a magnificent degree of invariance. If an operator is self-adjoint it will be Hermitian, as defined by having real eigenvalues. It will also remain self-adjoint under unitary transformations. But if a Hermitian operator is subject to arbitrary similarity transformations it may cease to be self-adjoint, while its eigenvalues will not change.

Second, the class of eigenvalues of $\hat{\Omega}$ are a physics decision. The most general solution to Eq. 19 is an expansion in normal modes,

$$
\Psi(t)=\sum_{n} \psi_{n}(0) e^{-i \omega_{n} t}
$$

where $\psi_{n}$ are solutions to the eigenvalue problem

$$
\hat{\Omega} \psi_{n}=\omega_{n} \psi_{n} .
$$

This happens to eliminate a postulate, as we'll explain, and just to re-iterate, we're discussing a system of generalized classical coordinate where the assumption of units $\hbar=1$ has definitely not been imposed. We have no need for $\hbar$ as explained in Section 4. Eq. 21 is self explanatory. Frequencies are the eigenvalues of the frequency operator $\hat{\Omega}$. As Feynman must have said, the textbook business of multiplying $\omega_{n} \rightarrow \hbar \omega_{n} \equiv E_{n}$ so the time evolution appears as $e^{-i E_{n} t / \hbar} \equiv e^{-i \omega_{n} t}$ is a complete waste of time.

If $\omega_{n}$ has a complex part the time evolution contains exponentially growing or damped solutions, which were frowned upon by the authorities in charge of setting up quantum mechanics. That eliminates another postulate (the postulate of Hermiticity), replacing it by the Decision of Hermiticity. To conform with this reasonable decision we specify real $\omega_{n}$, Hermitian $\hat{\Omega}$, which a short exercise shows is equivalent to Eq. 18 .

To reiterate, in our approach we have available every freedom to consider non-linear or non-Hermitian systems, at least up to here. We have taken a more general framework and reduced it to the less general dynamical rules of quantum theory by identifying the restrictions assumed in standard lore. Classical mechanics is a vast general framework not at all the same as Newtonian physics. Understanding that, there is nothing but classical physics in the Schroedinger equation.

\subsubsection{Discussion}

Eq. 19 comes from mere algebraic manipulations. While developed a bit in Ref.[7] it is surprisingly unknown to most physicists. Discussions with many physicists find several frequently asked questions:

- Every interesting quantum theory is non-linear. Right? Why is the linearization $H \rightarrow\langle\psi|\Omega| \psi\rangle$ relevant? No, quantum dynamics is always linear. The mixup about what is linear comes from habitual sloppiness in physics discussions to mix operators with numbers, and then constructing Hamiltonians as non-linear combinations of operators. An operator appears in matrix elements $\hat{\Omega}_{i j}=<i|\hat{\Omega}| j>$, which remains unspecified. 
- Does this method assume a finite-dimensional system, and why would that be relevant? No. The whole point of applying linear algebra and Hilbert space methods to quantum mechanics is one unified notation. The usual infinite dimensional expression for the Hamiltonian is

$$
H\left(\psi, \psi^{*}\right)=\int d x \psi^{*}(x) \hat{H} \psi(x)
$$

Apply Hamilton's equations (Eq. 14) using a functional derivative and you are done.

- Current physics of quantum field theory uses highly non-linear Hamiltonians, for which Eq. 22 fails. Why bother with beginning quantum mechanics? Once again the question is about the matrix elements and dimension of the Hamiltonian operator, which we've left unspecified, and which is generally a non-linear function of the fields. That does not change (repeat) the linearity of the dynamics. It is most directly seen in the functional Schroedinger equation

$$
i \dot{\Psi}(\Phi)=\hat{H}(\Phi,-i \delta / \delta \Phi) \Psi(\Phi) .
$$

This differential equation is equation is linear in the dynamical dof $\Psi$, while exactly equivalent to the non-linear operator relations of the usual approach. Feynman himself was very fond of the Schroedinger picture for the practical reason that wave function equations are easier to solve and approximate than operator equations. For us (up to here) quantum field theory is a very large classical dynamical system.

- How can this be the same as the path integral formulation? Moreover, the field-theoretic path-integral is different from the one of beginning quantum mechanics. We say that basic quantum mechanics is more fundamental than the path integral. Given the Schroedinger equation, the path integral comes to be derived as an integral representation of certain correlations. So we also have path integrals as (up to here) a representation of certain quantities evolving by generalized classical mechanics.

- Where are the operator equations of motion? What role exists for operators? It is interesting that the dis-ordering of material in the education of physicists is such that questions like these come up, while everyone knows the answer. Given the Schroedinger time evolution, and any arbitrary operator, the Heisenberg picture is developed as a definition of time-dependent operators. We must use the Schroedinger picture because it's not really true that Heisenberg operator equations of motion makes an "equivalent theory". The operators lack a wave function to encode a system's initial conditions and state, and which develops proper observables.

- Where is Planck's constant? With classical mechanics and without Planck's constant how are you going to quantize the Hydrogen atom? One of the advantages of our approach is the ability to discard deadwood. "Deriving the Hamiltonian" of the Hydrogen atom is schoolbook bunk: at least in our approach! Planck's constant deserves a separate discussion: the next topic. 


\section{A World without Planck's constant}

Hamilton's equations in its three equivalent forms (Eq. 1, 2, 19) lack Planck's constant. Most physicists believe that Planck's constant is a fundamental feature of our Universe, cannot imagine a world without it, and also have no idea how $\hbar$ could possible "emerge" from the (possible noise and chaos) of theory more fundamental. But a Universe without Planck's constant is not hard to imagine[12]. It is a Universe where human history would have gone differently.

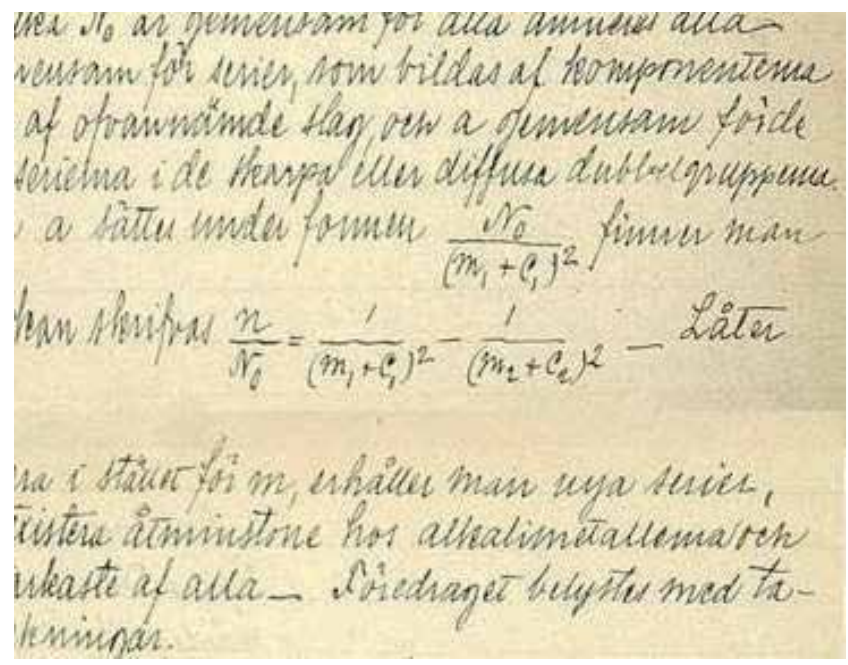

Figure 1. A record of physics from a Universe without Planck's constant. Rydberg's original fits to frequency (wavenumber) data for the Hydrogen spectrum did not need to be converted into Newtonian units and back again to frequency fix the parameters of quantum theory. As Feynman must have said, converting units and the associated conversion constant is a total waste of time.

\subsection{The culprit is mass}

Human history defined a notion of mass as a quantity of matter such as silver or butter long before physics defined mass. Imagine a history where Hamiltonian methods were developed first. Then mass would be more neutral, a particular "coupling constant" appearing in the Hamiltonian. We might find ourselves lacking the Newtonian intuitive picture of "mass," which might be a good thing. We would need to teach ourselves how to get the meaning of parameters from the theory where they appear.

Transformation properties are generally a key. Just as $q$ 's and $p^{\prime}$ s transform under a change of variables, the parameters of a theory transform. However parameters do not transform automatically. Consider the constant $c$ in an ordinary wave equation:

$$
\frac{\partial^{2} \phi}{\partial t^{2}}-c^{2} \vec{\nabla}^{2} \phi=0
$$

Under changes of scale $x \rightarrow x^{\prime}=\lambda_{x} x, t \rightarrow t^{\prime}=\lambda_{t} t$ the equation changes, and becomes false, unless $c$ is transformed by hand. The equation is form-unchanged (has a symmetry) under 
$c \rightarrow c^{\prime}=\lambda_{x} c / \lambda_{t}$. That is read "c has units of length over time." Transforming constants is so familiar the need for derivation escapes notice.

Notice that transforming parameters is not treated as an ordinary symmetry. Under an ordinary symmetry, including space-time symmetries such as Lorentz transformations, the Hamiltonian is unchanged, including the parameters. The reason $c$ must be changed by hand is that the value $c=3 \times 10^{8} \mathrm{~m} / \mathrm{s}$ refers to a particular Universe where units of length and time measured it. Once $c$ is measured and fixed in our particular universe, then changing its value (with fixed scales of space and time) is definitely not a physical symmetry. Educating the math about a passive scale change of coordinates requires we transform parameters measured in old units into new numbers so the Universe described remains the same.

Review such scale changes more generally. We noted that the group of canonical transformations, which preserve the action of our theory, is the symplectic group $S p(2 N)$. Every element $S \in S p(2 N)$ can be written locally as a product of three factors $S=K_{1} \cdot \Lambda \cdot K_{2}$, where $K_{1}, K_{2}$ are "rotations" from the maximal compact subgroup of $S$. The elements $\Lambda$ are scaling transformations of the form

$$
\begin{array}{r}
q_{i} \rightarrow q_{i}^{\prime}=\lambda_{i} q_{i} ; \\
p_{i} \rightarrow p_{i}^{\prime}=p_{i}^{\prime} / \lambda_{i} ; \\
d q_{i} d p_{i}=d q_{i}^{\prime} d p_{i}^{\prime} .
\end{array}
$$

The phase-space volume on every pair (not just the entire space) is preserved by re-scaling and rotations. There are $N$ parameters in $\Lambda$, and $2 N^{2}$ in $K_{1}, K_{2}$, accounting for all $N(2 N+1)$ parameters of $S p(2 N)$. The decomposition is unique up to discrete row-swapping transformations maintaining $\operatorname{det}(S)=1$.

The action-preserving transformations predict momenta $p_{i}$ scale like $1 / q_{i}$. Unless a different definition is made, that requires $p_{i}$ to have units of $1 / q_{i}$. This also follows from the momentum being the generator of translations. Similarly the energy $E$ as the value of the Hamiltonian, and the time $t$ are conjugate. Under scaling transformations preserving the action, they transform with $d t d E=d t^{\prime} d E^{\prime}$, as seen from $L d t=p_{i} d q_{i}-H d t$. The intrinsic units of energy are inverse time, as also seen using the action of a solved system directly: $H=-\partial S / \partial t$.

There is more than one way to apply this. It applies to our complexified dynamical wave functions, $\psi_{i}=\left(q_{i}+i p_{i}\right) / \sqrt{2}$. But it also applies at the most beginning level known as "high school physics." It is astonishing that mixups at the level of high school physics might affect deep questions of quantum mechanics. But this is not as unlikely as it seems. High school students and their teachers are seldom given freedom to challenge what they are taught. Later on it is difficult to give up what we were taught as children.

\subsubsection{Mass Paradox}

Knowing the scaling information of Eq. 25, consider changing the units of a translational coordinate $q$, for example changing the units of length from meters to centimeters. There are $100 \mathrm{~cm} / \mathrm{m}$, hence $q_{c m}=\lambda_{c m / m} q_{m}$ with $\lambda_{c m / m}=100$. Ordinary usage of Newtonian momentum predicts 


$$
\begin{aligned}
p & =m \dot{q} ; \\
q_{i} & \rightarrow q_{i}^{\prime}=\lambda_{i} q_{i} ; \\
p & \rightarrow p^{\prime}=m \lambda \dot{q}=\lambda p .
\end{aligned}
$$

The transformation is precisely the inverse of Eq. 24. That is a good paradox.

The paradox comes because Eq. 26 has re-scaled coordinates without re-scaling parameters. Try it with the speed of light, $x=c t$. Under $t \rightarrow t^{\prime}=\lambda_{t} t$, then $x \rightarrow x^{\prime}=c \lambda_{t} t=\lambda_{t} x$. Changing units of one second to one hour changes the unit of a kilometer by a factor of 3600 .

The correct parameter transformation properties can be found from the Hamiltonian. The Newtonian mass symbol $m$ is defined by $H_{N}=p^{2} / 2 m$. The scaling transformation properties of time $t$, a spatial coordinate $q$, its conjugate momentum $p$, and mass $m$ are:

$$
\begin{aligned}
t \rightarrow t^{\prime} & =\lambda_{t} t ; \\
q \rightarrow q^{\prime} & =\lambda_{q} q ; \\
p=m \frac{d q}{d t} & \rightarrow p^{\prime}=m^{\prime} \frac{\lambda_{q}}{\lambda_{t}} \frac{p}{m}=\frac{p}{\lambda_{q}} \\
m & \rightarrow m^{\prime}=\frac{\lambda_{t}}{\lambda_{q}^{2}} m .
\end{aligned}
$$

The last relation tells us that Newtonian mass has the scaling properties of time over length ${ }^{2}$, or seconds/meter ${ }^{2}$.

Now just as we are accustomed to saying that the number for the speed of light is meaningless until it is expressed as a number of meters per second, or miles per hour, we need to get accustomed to mass as a number of seconds per square meter.

Example Consider a Newtonian object with mass $m=3\left({\left.\text { seconds } / \text { meter }^{2}\right)}^{2}\right.$ moving at 2 meter/second, carrying momentum $p=m v=6 /$ meter at position $q=5$ meter. Transform to $q^{\prime}=5$ meter $(100$ centimeter $/$ meter $)=500$ centimeter and $p^{\prime}=6 /$ meter $(1$ meter $/ 100$ centimeter $)=0.06 /$ centimeter. The area of the initial phase space between the origin and the canonical coordinates is $\Delta A=\Delta p \Delta q=5 \times 6=30$ and equals the area of the final phase space $\Delta p^{\prime} \Delta q^{\prime}=500 \times 0.06=30$.

Example Under the force of gravity on Earth, an object falls with acceleration $g=$ 9.8 meter $/$ second $^{2}$. The gravitational force $F$ on a given mass $m_{1}=1$ second $/$ meter $^{2}$ is

$$
F=m_{1} g=9.8 \frac{\text { seconds }}{\text { meter }^{2}} \frac{\text { meter }}{\text { seconds }^{2}}=\frac{9.8}{\text { meter } \cdot \text { second }} .
$$


The work lifting the mass one meter is

$$
\text { work }=m_{1} g h=9.8 \text { meter } \frac{1}{\text { meter } \cdot \text { second }}=9.8 \frac{1}{\text { second }} .
$$

The power delivered by the force is

$$
\begin{array}{r}
\text { power }=\vec{F} \cdot \vec{v}=\frac{9.8}{\text { meter } \cdot \text { second } \frac{\text { meter }}{\text { second }}}\left(\frac{v}{\text { meter } / \text { second }}\right) \\
=\frac{9.8}{\text { second }^{2}} v_{M S},
\end{array}
$$

where $v_{M S}$ is the dimensionless velocity measured in meter/second. Using a pulley or spring balance to apply the same force to a second object with mass $m_{2}=2$ second $/$ meter $^{2}$ produces an acceleration

$$
a_{2}=F / m_{2}=\frac{9.8}{\text { meter } \cdot \text { second }} \frac{1}{2} \frac{\text { meter }^{2}}{\text { second }^{2}}=4.9 \frac{\text { meter }}{\text { second }^{2}} .
$$

\subsubsection{How This is Related to Planck's Constant}

To see how the discussion is related to quantum theory, use a theory that is relevant. Consider a standard wave equation:

$$
\frac{\partial^{2} \phi}{\partial t^{2}}-c^{2} \vec{\nabla}^{2} \phi+m^{2} c^{4} \phi=0
$$

This equation comes from a ubiquitous linear Hamiltonian model. It is a trap to "derive" this equation using substitution rules of beginning quantum theory: they are circular. It is better to find the equation generic, as indeed it appears in the vibrations of any collection of oscillators that has an "optical" branch. By inspection the dimensions of the combination $m^{2} c^{4}$ are seconds ${ }^{-2}$. Then $m$ scales like a Newtonian mass and we are entitled to call $m$ a "mass parameter."

Make the definition

$$
\begin{aligned}
\phi & =e^{-i m c^{2} t} \psi ; \\
\frac{\partial \phi}{\partial t} & =e^{-i m c^{2} t}\left(-i m c^{2} \psi+\frac{\partial \psi}{\partial t}\right) .
\end{aligned}
$$

This transformation removes the $m^{2} c^{4}$ term. Continue to obtain the time evolution equation for $\psi$. Impose a low frequency approximation that drops the term proportional to $\ddot{\psi}$. The result is

$$
i \dot{\psi}=-\frac{\vec{\nabla}^{2}}{2 m c} \psi
$$


Here we have a model frequency operator $\hat{\Omega}=-\vec{\nabla}^{2} / 2 m c$ familiar from Schroedinger theory. We are still lacking $\hbar$, and in our approach, we will never find it in quantum theory.

Essentially the same analysis is done in Ref[12]. A more complicated frequency operator $\hat{\Omega}=$ $-\vec{\nabla}^{2} / 2 m+U(x)$ represents an ansatz for interacting waves. We would not pretend to know the interaction function $U(x)$ from first principles. (The old predictive recipes, we noted, are just mnemonics and pedagogy.) We find $U$ from data for electrons. Basic scattering theory allows one to invert the Born-level differential cross section of electron-atom scattering into $U(x)$. The same $U(x)$ predicts the observed Hydrogen frequency spectrum, which is quite non-trivial. Figure 1 shows an example of the frequency data of Rydberg[10]. Taken before 1900, the data was of surprisingly high quality. Several other data comparisons are consistent. The entire theory has only two parameters $m$ and $\kappa$. The constant $\kappa$ is dimensionless, as consistent with the results of data-fitting

$$
U(x)=\frac{\kappa c}{|\vec{x}|}
$$

and $U$ correctly has dimensions of frequency.

We said we are only concerned with what is observable and testable. When using experimental data to fit the parameters of quantum theory $\hbar$ is unobservable, and given up, in our theory. Figure 2 shows that we have done the work to fit parameters [12]. With basic information on the frequencies observed in the Rydberg spectrum, and the scattering lengths observed by Geiger and Marsden, etc. one derives $\kappa$ and $\lambda_{e}=c / m_{e}$ directly. The numerical value of $\kappa$ is about $1 / 137$. By a natural coincidence Sommerfeld discovered the dimensionless constant $\kappa$ and called it $\alpha$, the fine structure constant. Dimensionless constants do not depend on the units used to compute them, so that the unobservable unit converter $\hbar$ cancelled out for Sommerfeld. In none of this is the introduction of a conversion to archaic $M K S$ units necessary, nor is it helpful. As Feynman must have said, "bothering to convert units with the meaningless constant known as $\hbar$ is a total waste of time".
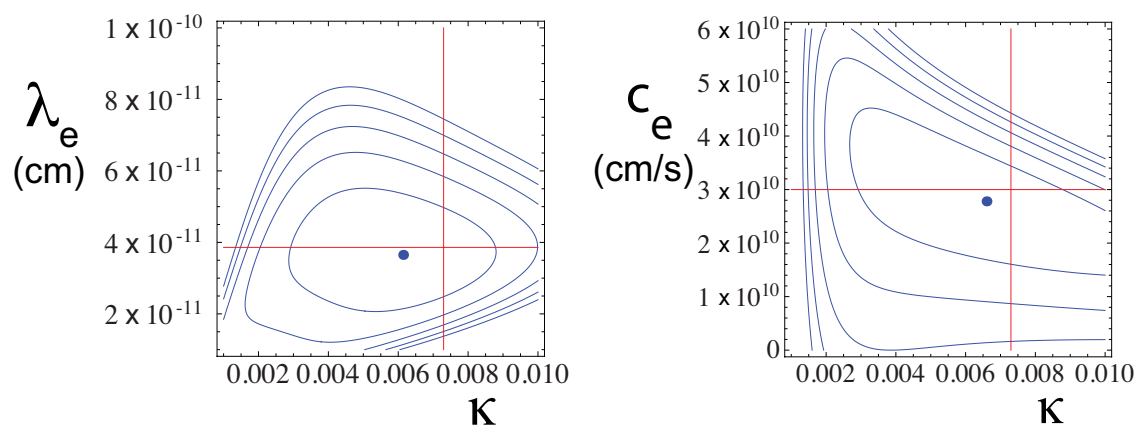

Figure 2. Contours of $\chi^{2}$, the summed-squared differences of data versus fit obtained from the data analysis of Ref. [12]. Left panel: As a function of parameters $\kappa$ and $\lambda_{e}$ with $c_{e} \rightarrow c$. Right panel: As a function of parameters $\kappa$ and $c_{e}$ with $\lambda_{e}$ given by Compton's 1922 experiment. Dots shows the points of minimum $\chi^{2} \sim 0.24$ in both cases. Contours are $\chi^{2}=1,2,3 \ldots$ Lines show modern values $c=3 \times 10^{10} \mathrm{~cm} / \mathrm{s}, \lambda_{e}=3.87 \times 10^{-11}, \kappa=1 / 137$ all lie inside the range of $\chi^{2} \lesssim 1$.

When one redundant unit is dropped, something is gained. The errors in the definition and inconsistent uses of the kilogram drop out. Continuing up the ladder, the system where mass 
is measured in seconds/meter ${ }^{2}$ is such that the accuracy of the best determinations of the electric charge and electron mass were improved[12] by a factor of order 100 compared to the official CODATA determinations[13].

\subsection{A redundant convention}

We come to see where Planck's constant entered human history. The space-time scaling properties of mass were overlooked early, which continues today, due to a Newtonian prejudice that mass is intuitively self-defined. For a long time mass was even thought to be a "constant quantity of matter, unchanging by the principle of conservation of mass." That led to a unit of mass unrelated to meters and seconds, and declared independent by defining a totally arbitrary unit known as the kilogram. Introducing an artificial reference standard was found acceptable for Newtonian physics: using artificial standards to weigh silver and butter was quite ancient and obvious.

Notice that introducing artificial unit conventions cannot be detected as faulty by math or logic. Intelligent technicians and business people use a huge array of unnecessary units daily, in many cases imagining that relations between them (such as 1 pascal $=0.000145$ pounds-per square-inch) must be "laws of physics." Once a redundant unit and its arithmetic enters the scaling laws it can stay around forever.

But as a price for these mistakes, a unit-conversion constant was needed in history to change black-body frequency in terms of temperature, which is energy, which is the frequency of the action, to black body frequency observed in the spectrum, which is frequency. If and only if one insists on measuring mass in kilograms, one needs a new symbol $m_{\mathrm{kg}}=\eta \mathrm{m}$, where eta has units of $\mathrm{kgm}^{2} / \mathrm{s}$. The value of the conversion constant $\eta$ is arbitrary, just as the kilogram is arbitrary, and as Ref. [12] shows, fixing one predicts the other. (And that explains[7] the peculiar phenomenon of international unit standardization from global fits finding 100\% correlation of the "experimental errors in the kilogram" with the "experimental errors in Planck's constant.")

There is a fast way to reach the same conclusions. The action principle is $\delta S=0$. The right hand side " 0 " has no scale, and no units of $S$ can be physically observable. By $S$ we don't mean the action of some subsystem which can be compared to another to define an arbitrary fiducial unit, just like the kilogram. We mean the action of the Universe. That causes one overall constant that was defined in quantum pre-history "with the dimensions of action" to drop out.

At least in our approach, every quantum mechanical relation that involves $\hbar$ is an ordinary relation not involving $\hbar$ that has been multiplied by some power of $\hbar$ on both sides: so that $\hbar$ cancels out of everything observable.

Example: Although Planck is reported to have gotten his constant from black-body data, his original work shows otherwise. From his derivation and data fits [11] the most Planck could get was the ratio of $\hbar / k_{B}$, where $k_{B}$ is Boltzman's constant. Planck thought $k_{B}$ was extremely fundamental, although we now know it is nothing from Nature. It is a conversion constant of energy in temperature units to energy in energy units. The Newtonian convention for mass entered in $k_{B}$ and $\hbar$ both. The kilogram cancels out. When measuring quantum data with quantum data $\hbar$ cannot be obtained [12], and it is nothing but convention to insert the kilogram so as to force a relation. The information was available in 1900. Indeed Wien's Law 
used the classical adiabatic invariance of the action to relate the electromagnetic energy to its frequency. It was already known that the value of the Hamiltonian $H=-\partial S / \partial t$. Hence the clue of a redundant unit existed, and if the connection had been made, it might have led to getting rid of the kilogram before 1900.

Example: Due to pre-quantum nonsense everyone is obliged to learn, the quantization of angular momentum is blamed on the value of Planck's constant. One cannot possibly do without $\hbar$, it is claimed, due to the fundamental commutation relations of angular momentum

$$
\left[J_{i}, J_{i}\right]=i \hbar \epsilon_{i j k} J_{k}
$$

Strictly deductive algebraic relations developed from those commutators produce the possible representations and the spectrum of observables $J_{z}=n \hbar$, where $n$ must be an integer or half-integer. And this reproduces data. And in the limit of $\hbar \rightarrow 0$, the commutators go to zero, which is the classical limit of operators becoming $c$-numbers, etc.

We say: that kind of argumentation fails the quality-control standards of the current millennium. Groups and representations are fine organizing devices that don't originate in claims about physical existence. The representations of $S U(2)$ had been worked out before quantum mechanics wanted them. To get Eq. 29 one takes a set of dimensionless $S U(2)$ generators $\tilde{J}_{i}$ and arbitrarily multiplies them by $\hbar$ :

$$
\left[\hbar \tilde{J}_{i}, \hbar \tilde{J}_{i}\right]=i \hbar \epsilon_{i j k}\left(\hbar \tilde{J}_{k}\right) .
$$

Then $J_{i}=\hbar \tilde{J}_{i}$ come to obey Eq.29. But by remarkable rules of algebra, multiplying both sides of any equation by the same constant does not change the equation. In Eq. 30 one sees that $\hbar^{2}$ cancels out: including that magic limit that $\hbar \rightarrow 0$ or $\hbar \rightarrow-17.3$ or anything else, so it also cancels out in Eq. 29.

The quantization of angular momentum eigenvalues is a fact that has nothing to do with $\hbar$. The "classical limit" has nothing to do with $\hbar$, assuming one can count and distinguish low quantum numbers from huge ones. The reason that $\hbar$ is artificially spliced into Eq. 29, and other algebras, is so that 21st century measurements of angular momentum will be cast into an MKS unit system designed for 17 th century Newtonian physics. Which continues to assess and obstruct quantum mechanical data by interposing the universal kilogram which is not a feature of Nature.

\section{Observables, quantization and bracket relations}

We seek to get as much from the theory as possible without making unnecessary postulates:

\subsubsection{Operators as Physical Observables}

The postulate that operators are "physical observables" is redundant: in our approach. We find using it slyly abuses language that first defined observables as numbers, in order to slip in the operator as a philosophically transcendent realization of physics. That's too cheap. Very simply, the wave function is observable, and using operators to probe a wave function may be convenient, but that is not independent. 


\subsection{Observables as numbers}

An observable is a number extracted from the wave function as a projective representation, meaning that $\psi \equiv z \psi$ for any complex $z$. This symmetry exists in the equation of motion. We wish to call attention to it as a feature of "quantum homogeneity." It makes the information in $\mid \psi>$ equivalent to the information in the density matrix $\rho_{\psi}=|\psi\rangle\langle\psi| /\langle\psi \mid \psi\rangle$. (The bracket notation, suppressed before to reduce clutter, is useful here.) Equivalence exists because if $\rho_{\psi}$ is given then its unit eigenvector predicts $|\psi\rangle$ up to a constant $z$. (Later we discover that density matrices of rank- 1 are too special to base the full theory upon, but that comes with development of quantum probability, which is not yet under discussion.)

It is convenient to extract a number using an "operator sandwich". We define an observable $<\hat{A}>$ as the number from the map

$$
\mid \psi>\rightarrow<\hat{A}>=\frac{<\psi|\hat{A}| \psi>}{<\psi \mid \psi>}=\frac{\operatorname{tr}\left(\hat{A} \rho_{\psi}\right)}{\operatorname{tr}\left(\rho_{\psi}\right)} .
$$

The last relation is general for cases where $\rho$ is not so simple as rank -1 . Note we need nothing from quantum probability to make the map. Instead of prescribing $\langle\psi \mid \psi\rangle$ with a normalization postulate, we maintain it is simply unobservable, and drops out. We may then set $\langle\psi| \psi>\rightarrow 1$ to simplify expressions.

The trace (symbol $t r$ ) acts as an inner product between operators. Let $\hat{A}_{i}$ be a normalized complete set of operators, which is defined by $\operatorname{tr}\left(\hat{A}_{i}^{+} \hat{A}_{j}\right)=\delta_{i j}$. Since the set is complete,

$$
\rho=\sum_{j} A_{j} \operatorname{tr}\left(\hat{A}_{i}^{\dagger} \rho\right)=\sum_{j}<\hat{A}_{j}>\hat{A}_{j}
$$

Thus $\rho$ is equivalent to a number of observables, and $|\psi\rangle$ is observable to the exact extent it is defined. At some point this simple relation seems to have been re-packaged as "quantum holography." The very late date of realizing the wave function is observable (to the extent it is defined) supports our case that quantum mechanics is still "emerging" from its history.

\subsubsection{Eliminating More Postulates}

There is no particular reason for us to postulate that $\hat{A}$ must be Hermitian. If it is not Hermitian the operator sandwich gives a complex number, equivalent to two real numbers and two observables, because any operator is the sum of a Hermitian operator and $i$ times a Hermitian operator. As for complex numbers being observable in the lab, mathematics tells us that complex numbers are real pairs with 2-vector addition and multiplication rules. It is not unusual to observe such number pairs that have phase relations like $q_{i}, p_{i}$ which map directly into $\psi_{i}$, a complex number. So there is no reason for the 18th century trick of scaring people with complex numbers. And yet: given that $\rho$ is Hermitian no harm is done by restricting the operators for observables to being Hermitian. One more grand postulate turns to clay.

At least in our approach, those matters of definition need no foundation postulates. Neither is there a good reason to insist that an observable be an eigenvalue of some especially known 
operator. The interpolation of that rule seems designed to create conflict. Checking a bit of physical data, measuring the eigenvalue of an operator intended is rare. For example experimentalists have been measuring neutrinos for decades while the theorists argued about their operators. We think that measuring a number and calling it an eigenvalue of "some operator " is meaningless. If and when an eigenvalues of a known operator appear in a data set, one obtains that fact that $|\psi\rangle$ will then be the corresponding eigenvector of $\hat{A}$ automatically. We also don't need to discuss "compatible and incompatible" observers in terms of operators that commute. In this century everyone knows how that math works.

\subsubsection{Bracket Relations Are Kinematic}

The quantum map shows that complex $\psi_{i}$ are canonical coordinates, up to $i$ :

$$
\psi_{i}=\left(q_{i}+i p_{i}\right) / \sqrt{2}
$$

A factor of $i$ is tolerable, in that Hamilton's equations are recognizable including it:

$$
i \dot{\psi}=\frac{\partial H}{\partial \psi_{i}^{*}}, \quad i \dot{\psi}^{*}=-\frac{\partial H}{\partial \psi_{i}} .
$$

Thus $i \psi_{i}^{*}$ is the canonical momentum conjugate to $\psi_{i}$. Poisson brackets $(P B)$ are canonically invariant, so that transcribing them to $\psi, \psi^{*}$ involves only a factor of $i$.

In fact the $P B$ relations among our observable are rather simple due to the decision to make observables bilinear in $\psi, \psi^{*}$. The most famous application was the early desire to project the wave function into a few particle-like observables $\vec{Q}, \vec{P}$, mistakenly thought to be important from Newtonian bias. For $\vec{Q}$ to represent a translational coordinate it must transform properly:

$$
\begin{array}{r}
\text { given } \psi(x) \rightarrow \psi_{\vec{a}}(\vec{x})=\psi(\vec{x}-\vec{a}), \\
\text { then } \vec{Q}=d^{3} x \psi^{*} \vec{Q} \psi \rightarrow \vec{Q} \rightarrow \vec{Q}+\vec{a} .
\end{array}
$$

There are few choices but $\vec{Q}=\vec{x}$. The test that a candidate variable $P_{i}$ is conjugate to these $Q_{i}$ needs the bracket $\left\{Q_{i}, P_{j}\right\}_{P B}=\delta_{i j}$.

Write this out, assuming operator sandwiches:

$$
\left\{Q_{i}, P_{j}\right\}_{P B}=-i \sum_{x}\left(\frac{\delta Q_{i}}{\delta \psi_{x}} \frac{\delta P_{j}}{\delta \psi_{x}^{*}}-\frac{\delta P_{j}}{\delta \psi_{x}} \frac{\delta Q_{i}}{\delta \psi_{x}^{*}}\right) .
$$

Computing the derivatives gives

$$
\left\{Q_{i}, P_{j}\right\}_{P B}=-i \int d x \psi_{x}^{*}\left[\hat{Q}_{i}, \hat{P}_{j}\right] \psi_{x}
$$


This must be true for all $\psi$. Quantum homogeneity (the irrelevance of $\langle\psi \mid \psi\rangle$ ) then obtains the map between the operator algebra and Poisson bracket

$$
\left\{Q_{i}, P_{j}\right\}_{P B}=\delta_{i j} \rightarrow\left[\hat{Q}_{i}, \hat{P}_{j}\right]=i \delta_{i j}
$$

The other consistency relations of "quantization" are similar. A $P B$ algebra predicts a commutator algebra, as follows: If $\{A, B\}_{P B}=C$ is true for general $|\psi\rangle$, and all quantities are operator sandwiches, then $[\hat{A}, \hat{B}]=i \hat{C}$ follows by identity. An interesting application comes from the lack of any non-zero commutator with the unit operator "1." It tells us that $\langle\psi \mid \psi\rangle$ does not transform, time evolve, nor give a non-trivial result, so it is a conserved "momentum" of the theory that drops out as unobservable. ${ }^{2}$

The early history of quantum theory found the map between Poisson brackets and commutators profound, and it tends to still be viewed that way. In our approach it emerges on its own as useful, but automatic. If one chooses operators that satisfy the bracket-commutator rules, then their observables transform as they are intended, and vice-versa. It is not really necessary to cast around and discover operators by trial and error. Noether's Theorem will manufacture any number of generalized conjugate $P_{i}$ from point transformations on $Q_{i}$ as the "charges" of conserved (or un-conserved) currents[7].

It is interesting that in retrospect the original Heisenberg program, based on Poisson brackets, guaranteed such an outcome. The virtue of bracket relations lies in generating Lie algebra and related relations that are inherently coordinate-free. Once a given algebra is transcribed to a different notation, it is not going to produce new results. Thus when Heisenberg transcribed the Poisson bracket algebra of Hamiltonian time evolution to a different notation he was building up a classical Hamiltonian dynamics of ordinary kind, if it was not recognized at the time.

The big advance, as mentioned before, comes with the physical model of electrons having an infinite number of dof, as Eq. 31 requires, and as found in a wave theory. That fact was supposed to be evident in the spectrum of atoms showing a (practically) infinite number of normal mode frequencies. That in turn could have been done by 19th century classical physicists, who knew about spectra and normal modes. And indeed Stokes, Kelvin and Lorentz[9] all deduced the facts that atoms are vibrating jello from such clues before 1900. Lacking any technology to test the speculation, they made little of it which is a pity. Immediately after 1900, the cult of the quantum of action went the way of postulating mistakes that could not be expressed without Planck's constant. All of that can be dropped.

\subsubsection{Quantization}

The $P B$-commutator relation of Eq. 31 is commonly called the "quantization" principle according to the recipe of Heisenberg or Dirac, which (being a postulate) cannot be explained. While that is what those gentlemen believed, it is not our approach.

Once the physicists have committed to a linear, Hamiltonian theory, there is very little left to determine except its dimension. As already mentioned Eq. 31 realized with $x_{i}, \partial_{j}=-i \partial / \partial x_{i}$ requires a space of a continuously infinite number of degrees of freedom: waves. Less

\footnotetext{
${ }^{2}$ Similarly, the center of mass momentum of the Universe in Newtonian physics is unobservable.
} 
information exists in the algebra than in the direct and simple model of waves. For one thing, the algebra is kinematic, and will work for any Hamiltonian, including non-local ones that do not seem to be observed. In comparison the wave model predicts the algebra, because it contains everything, so it is superior.

It is sometimes thought that "field quantization" proves that quantization principles are a golden road. But what's kinematic on one space is kinematic on a larger space. If one believes there should be wave functions for classical fields, one defines quantum field theory straightforwardly. It also happens to be equivalent to the space made from products of an arbitrary number of beginning quantum systems, which is neat, but which again shows that invoking the quantization principle was redundant.

Finally, we find there is a perception that abstract operator methods are superior just because they are difficult. It is seldom noticed that an unlimited amount of tortuous and clever operator manipulation can never have more information than just solving the differential equation, which predicts everything, and (in fact) all the differential equations of quantum mechanics are already "solved" by Eq. 20.

These are reason we wrote in the Introduction that the viable models are a higher accomplishment than framework.

In our approach the state space is not going to be predicted by a simplistic algebraic transcription. Finite dimensional quantum models are known and hardly useless: they are models of spin, and molecular rotational dynamics. Finite dimensional models of quantum field theory are known. They are called "lattice theories." The dimensionality of quantum models has no restriction. Leaving the dimension free to grow without limit is what transpired, and one of the reasons the subject is so flexible it cannot fail.

\section{Quantum probability}

In this Section we explore the origins of probability in our approach. Quantum probability is an old subject with many contributions we cannot possibly review. There is some agreement that the Born rule should be "a Theorem, not a Principle"[14], although our approach is not quite the same. In the first place we must dismiss a common misconception that quantum probability contradicts classical probability, or is inconsistent with it, by defining each.

\subsection{Define terms}

Probability itself is a subtle topic. It is not well-defined until "objects" are categorized for the purposes of probability. Classical probability $(C P)$ of frequentist kind is about classifying objects into mutually exclusive $(m e)$ equivalence classes ${ }^{3}$, and assigning numbers to the information by counting. Distributions are a useful tool of $C P$. Quantum probability $(Q P)$ allows such classifications but does not insist on them. Instead $Q P$ is a projective map from a system's state, represented by density matrix, into a number. Distributions sometimes exist in $Q P$, but are not always compatible.

\footnotetext{
${ }^{3}$ Although "equivalence classes" are often mutually exclusive sets by definition, we use the term more broadly, and add $m e$ when the term is intended.
} 
The breakthrough of quantum probability, we believe, lies in generalizing the notion of probability so as not to insist on pre-ordained equivalence classes. Vectors are categorized so they have a great chance of being nearly equivalent. Physicists seem to believe that "quantum objects" from Nature are needed to make sense of quantum probability, and vice-versa. But nothing from physics is involved in developing an accounting system where different vectors are not automatically treated as mutually exclusive. While we will extensively use physics and its examples, the ultimate goal of this Section is not to depend on physics.

\subsection{Discovering quantum probability with a hidden-variable map}

Begin with a remarkably simple map from classical to quantum probability which illustrates the necessary class ideas. Let $\mid D>$ be a big vector we call "data". By means which are quite arbitrary it is partitioned in a collection of smaller vectors $D_{i}^{J}=\langle i J| D>$ with names $J=1 \ldots J_{\max }$ and components $i=1 \ldots i_{\max }$. We tentatively interpret $J$ as labeling the sample number taken from some (deterministic or random) process. The other index is interpreted as describing "objects". The sample space and object space are tentative because certain operations will mix them, as we will see. Formally $D_{i}^{J}$ exists on the direct product of spaces of dimension $i_{\max } \otimes J_{\max }$, upon which there are certain transformation groups and invariants. For convenience the record is normalized $\langle D \mid D\rangle=1$ in the usual way, removing one number set aside.

Now we seek a notion of orderliness or physical regularity. We will expand the vectors in an orthonormal basis set $\left\{\mid e^{\alpha}>\right\}$, where $e_{i}^{\alpha}=\langle i| \alpha>$, and seek some form of statistical repetition. The basis matters, so which basis is used? Every data record actually has two preferred bases, in which the expansion is diagonal:

$$
|D\rangle=\left|e^{\alpha}\right\rangle \Lambda^{\alpha}\left|s^{\alpha}\right\rangle
$$

This is the singular value decomposition (svd), which is unique. It is proven by diagonalizing two correlations (matrices) that automatically have positive real eigenvalues:

$$
\begin{aligned}
& D D^{\dagger}=\sum_{\alpha}\left|e^{\alpha}>\left(\Lambda^{\alpha}\right)^{2}<e^{\alpha}\right| ; \\
& D^{\dagger} D=\sum_{\alpha}\left|s^{\alpha}>\left(\Lambda^{\alpha}\right)^{2}<s^{\alpha}\right| .
\end{aligned}
$$

Notice that the decomposition yields vectors which are orthonormal on their respective spaces. Notice that the vectors are defined up to a symmetry:

$$
\begin{gathered}
\left|e_{\alpha}>\rightarrow z_{e}\right| e_{\alpha}>; \\
\left|s^{\alpha}>\rightarrow z_{s}\right| s^{\alpha}>; \\
|D>\rightarrow| D>.
\end{gathered}
$$

Here $z_{e}, z_{s}$ are arbitrary complex numbers. The factor vectors are eigenvectors which have no normalization except the normalization given by convention. By phase convention the 
singular values are positive real numbers, $\Lambda_{\alpha}>0$. The singular values are invariant with the data is transformed by arbitrary and different unitary transformations on the object and sample spaces.

The interpretation of each term $\left|s^{\alpha}>\right| e_{\alpha}>$ summed in Eq. 32 is a strict correlation of a unique object vector labeled $\alpha$ with a unique sample vector labeled $\alpha$.

Example Suppose the data consists of integer numbers of objects that are |apple $>$ or $\mid$ orange $>$. This is classical me data: by existing in different spaces, $<$ apple|orange $>=0$. Sufficiently fine sampling will produce samples which are either 1 or 0 . Typical data is then

$$
\begin{gathered}
\mid D>=(\mid \text { apple }>, 0, \mid \text { orange }>, \mid \text { apple }>, \mid \text { orange }>, \\
\ldots \mid \text { apple }>) .
\end{gathered}
$$

The expression only makes sense if these me objects are normalized, $<$ apple|apple $>=1$, and so on, else the normalization would conflict with the number of apples. Expand in the natural basis we started with,

$$
\begin{aligned}
\mid D>= & \mid \text { apple }>(1,0,0,1,0, \ldots 1) \\
& + \text { |orange }>(0,0,1,0,1, \ldots 0) .
\end{aligned}
$$

The diagonal form of $s v d$ has appeared, up to a normalization. Whenever data consists of disjoint me objects, one can show those same objects are automatically the svd factors. The fact of strict correlation comes with projecting onto one object such as $\mid$ apple $>$ and producing its sample vector, which is automatically orthogonal to all the other sample vectors:

$$
<\text { apple } \mid D>=(1,0,0,1,0, \ldots 1) .
$$

Conversely, selecting one of the me sampling histories automatically selects a unique object. These are features of classical "events."

To reach the svd form implies samples that are normalized: $\left\langle s^{\alpha} \mid s^{\beta}\right\rangle=\delta^{\alpha \beta}$. Let $N_{\text {apple }}$ be the total number of apples observed. Let $N_{\text {tot }}$ be the total of apples and oranges. Remember that we normalized our data. Then

$$
\begin{aligned}
& (1,0,0,1,0, \ldots 1) \rightarrow \\
& \quad \sqrt{N_{\text {apple }} / N_{\text {tot }}}(1,0,0,1,0, \ldots 1) / \sqrt{N_{\text {tot }} / N_{\text {apple }}} \\
& \quad=\sqrt{N_{\text {apple }} / N_{\text {tot }}} \mid s^{\text {apple }}>.
\end{aligned}
$$

Once normalized we can read off the singular values:

$$
\begin{aligned}
\mid D>= & \sqrt{N_{\text {apple }} / N_{\text {tot }} \mid}\left|s^{\text {apple }}>\right| \text { apple }> \\
& +\sqrt{N_{\text {orange }} / N_{\text {tot }}}\left|s^{\text {orange }}>\right| \text { orange }>.
\end{aligned}
$$


Now suppose there is a unitary transformation of our data on either object of sample or both spaces - but not mixing them. The result will involve linear combinations of the form $\alpha \mid$ apple $>+\beta \mid$ orange $>$, which is classically "taboo." For better or worse, we cannot stop linear transformations from being used or being useful. Whatever the coordinate system, we can construct the svd factors and singular values as invariants.

In many cases (and always in physics) we are actually forced to suppress some detail by summing over unwanted or unrecorded details of the sampling history. That was already done in Eq. 33. We use that observation as the first example in constructing the density matrix $\rho_{\text {object }}$ of the object system:

$$
\rho_{\text {object }}=t r_{S}(|D><D|)=\sum_{\alpha}\left|e^{\alpha}>\left(\Lambda^{\alpha}\right)^{2}<e^{\alpha}\right| .
$$

The form $\rho_{\text {object }}$ we traced-out the sample history. This identity now defines the me $\mid$ object $\left._{\alpha}\right\rangle=\left|e_{\alpha}\right\rangle$. By construction, whenever me data is used, we have a convenient invariant formula for the probability of finding such an object:

$$
P\left(\left|e_{\alpha}>\right| \rho_{\text {object }}\right)=<\mathrm{e}_{\alpha}\left|\rho_{\text {object }}\right| e_{\alpha}>=\operatorname{tr}\left(\rho_{\text {object }}\left|e_{\alpha}><e_{\alpha}\right|\right) .
$$

The symbol $P\left(\left|e_{\alpha}>\right| \rho_{\text {object }}\right)$ is read as the probability of $\mid e_{\alpha}>$ given $\rho_{\text {object }}$, and exactly coincides with counting numbers: thus

$$
P(\mid \text { apple }>\mid \rho)=\left(\sqrt{N_{\text {apple }} / N_{\text {tot }}}\right)^{2}=N_{\text {apple }} / N_{\text {tot }} .
$$

In general form the probability $P$ to get an observable $<\hat{A}>$ is

$$
P=\operatorname{tr}(\rho \hat{A}) / \operatorname{tr}(\rho) .
$$

\subsection{The quantum-style agreement}

We propose an agreement on how data will be managed: we agree to describe a system using its density matrix. We give up the possibility of keeping more information, because it is efficient not to have it.

Review the apples - oranges discussion with a physical example where transformations are natural. Suppose observations consist of events with 3-vector polarizations $\mid \mathcal{E}>$. Moreover, only two orthogonal components $\left|e_{1}>,\right| e_{2}>$ are measured. A generic data set including the sampling history will not generally consist of $m e$ events, but combinations of the form

$$
\mid D>=\left(\left|\mathcal{E}_{1}>, 0\right| \mathcal{E}_{2}>, \ldots \mid \mathcal{E}_{n}>\right)
$$

which is expanded in the basis

$$
\begin{aligned}
\mid D>= & \mid e_{1}>\left(<e_{1}\left|\mathcal{E}_{1}>,<e_{1}\right| \mathcal{E}_{12}>\ldots \ldots<e_{1} \mid \mathcal{E}_{n}>\right) \\
& +\mid e_{2}>\left(<e_{2}\left|\mathcal{E}_{1}>,<e_{2}\right| \mathcal{E}_{2}>, \ldots<e_{2} \mid \mathcal{E}_{n}>\right) .
\end{aligned}
$$


In the form above we have both the events and the sample history. The samples are not mutually exclusive, but naturally fall into the corresponding projections. That can be called the "underlying reality." Meanwhile there exists a unitary transformation on the objects and samples where this arbitrary data will be a sum of strictly correlated, me elements which are indistinguishable from classical events. The difference between that interpretation and the quantum-style one is a coordinate transformation not available from the density matrix, so it becomes meaningless.

Normalize the sample history and take the trace over the sample space to form the density matrix. Upon reaching that level, one cannot distinguish the system from one where every event actually was a product of mutually exclusive object and sample vectors. This information is deliberately lost in forming the data categories. That makes it consistent and unique to define probability using the Born rule. No subsequent experiment can make it false. Probabilities defined by naive counting of integer-valued data will agree exactly.

\subsubsection{Outcome Dependence}

"Outcome dependence" is the name given to statistics that depend on the order of measurement. Our procedure has outcome dependence. Suppose one is selecting channels by simple filters. A "measurement" $\beta$ uses a state $\mid \beta>$ and an associated projector $\pi_{\beta}=|\beta><\beta|$. A series of measurements $\beta, \gamma, \eta \ldots$ in that order, yields

$$
P(\beta, \gamma, \ldots \eta \mid \rho)=\operatorname{tr}\left(\pi_{\eta} \ldots \pi_{\gamma} \pi_{\beta} \rho \pi_{\beta} \pi_{\gamma} \ldots \pi_{\eta}\right)
$$

The projective and non-commutative nature of this kind of probability is self-evident. It follows immediately that no classical distributions can reproduce this kind of probability in general.

The famous Bell inequalities[15] dramatize this fact, yet there was nothing new in finding that distributions fail in general. It would have been extraordinary for probability based on density matrix projections to be equivalent to distributions in the first place. Years after Bell, Werner[16] formulated the criteria called "separability" of density matrices, which when true allows $Q P$ to coincide with $C P$ as formulated with distributions. Thus the classical probability rules exist inside of $Q P$, when and if a special case happens to occur. Conversely $Q P$ in our approach is a more general extension of the concepts and rules of $C P$ that does not contradict any of it. We will argue that $Q P$ is so general there are no restrictions on how it might be used.

About Disturbing Measurements: It is a geometrical fact that any vector can be considered to be any other vector, plus the difference. It takes one step to make the difference orthogonal, by writing

$$
|a>=| b><b \mid a>+(|a>-| b><b \mid a>) .
$$

The term in braces is orthogonal to $\mid b>$, given normalized vectors. Up to an overall scale, the vector $|b\rangle$ that is literally pre-existing in vector $|a\rangle$ is $|b\rangle\langle b \mid a\rangle$. This decomposition, of course, does not come from physics. We see that agreeing vectors are" equivalent" up to a scale is a prelude to counting them as equivalent for probability purposes. 
It is quite important (we repeat) that the coefficient $\langle b \mid a\rangle$ happens to measure the amount of vector $\mid a>$ that is pre-existing and pre-aligned with $\mid b>$. It is very reasonable that a quiet, non-disturbing "physical measurement" of a system will filter out a pre-existing component of a vector variable, and pass it through undisturbed. Undisturbed filtering is precisely what happens in textbook discussions of polarizers, Stern-Gerlach, and diffraction gratings. We wish it would be mentioned it has nothing to do with $\hbar$ (to repeat). Using the undisturbed, pre-existing projection also totally contradicts the old line of thinking that 'measurement" involves an uncontrollable disturbance of the system due to the finite quantum of action. Indeed if one believed that, the overlaps would change by the process of measurement, and the Born rule would fail.

\subsubsection{Division and Reduction}

The conventional approach to "quantum interacting systems" holds that system $A$ "exists on" space $A$, system $B$ "exists on" space $B$, and when they interact the joint system exists on the direct product of spaces $C=A \otimes B$. This is physically puzzling, and we think backwards. Instead we use our idea of equivalence classes developed by partitioning information, or "division".

Given any vector on a space $C$, we may partition it into factor vectors on procedurally-defined spaces $A$ and $B$, as used above. For example a vector of 40 dimensions can be written as the product of vectors on 4 dimensions and vectors on 10 dimensions, or products of $5 \times 8$, etc.

Division is particularly well-developed with Clebsch-series done in the inverse direction: discovering what smaller group representations can be composed to make a given bigger one. A more straightforward division groups a data vector's components into adjacent bins of sub-dimension $i_{\text {max }}$ and names $J$ :

$$
D_{a} \rightarrow D_{i J} \quad J=\operatorname{int}\left(a / i_{\text {max }}\right) ; \quad i=\bmod (a, J),
$$

where int takes the integer part, and $\bmod (a, J)$ returns the remainder of $a / J$. Arnold's famous "cat map" is an example. The freedom to choose the bins and dimensions is very important.

Division is quite coordinate-dependent. Division can be repeated to divide the factors, and make subdivisions. The process of vector "division" is not profound mathematics, but the arbitrariness is important for physics and data manipulation. Given a particular division, the physicist (knowingly or not) inspects the decomposition searching for simplicity and regularity to "emerge". The factor-states that turn out to make physics easy become well known under many terms...electrons, photons, quarks, etc. The interesting question of the ultimate meaning of such entities is discussed in Section 7.2.

Given a density matrix $\rho_{A B}$ defined on $C$, the decision not to study an observable with a non-trivial operator on space $B$ allows us to prepare the density matrix

$$
\rho_{A}=\operatorname{tr}_{B}\left(\rho_{A B}\right)
$$

Here $\operatorname{tr}_{B}$ sums the diagonal elements of the labels on space $B$. This defines reduction in the conventional way. Reduction is inevitable in physics because physics measures very little. 
The rank of a density matrix obtained from reduction depends on how the reduction was done. Obtaining a rank -1 reduced matrix is exceptional. Finding such systems in the laboratory requires great ingenuity. That is why we treat systems that can be described with wave functions as special cases. That may seem to put our dynamical framework discussing wave functions somewhat askew relative to the probabilistic one. Section 6.3.4 explains why and how Hamiltonian time evolution remains relevant.

\subsubsection{What Are Those Hidden Variables?}

It is remarkable that Bell's artful introduction of distribution theory[15], which is inappropriate for quantum-style systems, led to a false perception that "hidden variables" had been excluded. The hidden variables of ordinary quantum mechanics are the physical degrees of freedom (wave function or density matrix projections) on those spaces the physicist ignored in setting up his oversimplified model. There are always such spaces in Nature.

There is no limit to the number of products or their dimensionality that can be used as "data" or "sample vectors." Partitioning vectors on any given space into a number of factors is a highly coordinate-dependent business. It is essentially a map from one sort of index to a number of composite indices, which can always be done linearly: $D_{k} \rightarrow D^{a b c \ldots}=\Gamma_{k}^{a b c \ldots .} D_{k}$, where $\Gamma$ is an array of constants. In a sufficiently large data vector the "sample" and "object" spaces can be re-configured in practically infinite variations. There is very little that is invariant about entanglement when we allow such freedom. Nature cannot possibly care about these coordinate conventions: Pause to consider how it affects physics.

In early days the Hilbert spaces of single electrons or single photons were considered utterly fundamental. They were building blocks for lofty postulates that could not be explained. Yet quantum mechanics was long ago enlarged to develop quantum field theory $(Q F T)$. For some purposes QFT is considered to have no new information on quantum mechanics itself, while defining very complicated quantum models. Yet Nature has subtleties. Basic non-relativistic quantum mechanics is incapable of dealing with the very questions of causality and non-locality that cloud interpretation of measurements. Relativistic QFT deals with issues of causality directly in terms of correlation functions with very well-defined properties. On that basis QFT is the more fundamental topic: it is big enough to support realistic models.

The relation of ordinary quantum mechanics to field theory is then developed by reduction, where unobserved dof are integrated out. As a result all of the phenomenology of ordinary quantum mechanics is subject to the hidden variables known to have been integrated over in developing density matrices that actually occur. This is ignored in ordinary quantum mechanics seeking by itself to be "fundamental." It is not logically consistent to ignore what is known. Now we have shown how naive probabilities of counting emerge from density matrix constructions integrating over quite arbitrary sample spaces. It is hard to escape the inference that the probability interpretation - which beginning quantum mechanics could not explain about its own framework - must certainly originate in reduction of interacting systems of QFT down to the experimentally crude probes developed in beginning quantum mechanics.

Infrared Example: There exists certain pure states of the QFT called bare electrons: the quanta of a free field theory. If such a state actually participated in an experiment we 
doubt we'd have a statistical explanation for its behavior in the same free field theory. But that dynamics is too trivial to describe anything, or even permit participation, because free electrons are free. The so-called bare electron of free field theory has never been observed and cannot in principle be observed. All electrons and all observable states are "dressed." Calculations addressing infrared divergences find that electrons with zero photons are unobservable, or have zero probability to participate in reactions, as known from the ancient time of the Bloch-Nordseik analysis[21]. When the experimenter finally specifies his experimental resolution adequately, the probabilities of events emerge from density matrix steps integrating over unobserved quanta, exactly as we have discussed, yet in such a technical fashion that its relation to beginning questions of quantum probability is never recognized. The same facts also occur for all degrees of freedom not directly pinned down by experimental probes, which is most of them, that are much more difficult to categorize. Every single physical experiment involves so many uncontrolled variables that a statistical description via density matrices cannot be avoided. It is a rare experiment that even finds a single wave function will model the data: and a rare experimenter who can tune his instruments to make that expectation come out.

\subsubsection{Invariants Under Time Evolution}

At the level of QFT one can assert certain wave functions exist, and model them with the Hamiltonian time evolution we cited earlier. That is the state of the art, and returns to how the Hamiltonian dynamics is relevant once again.

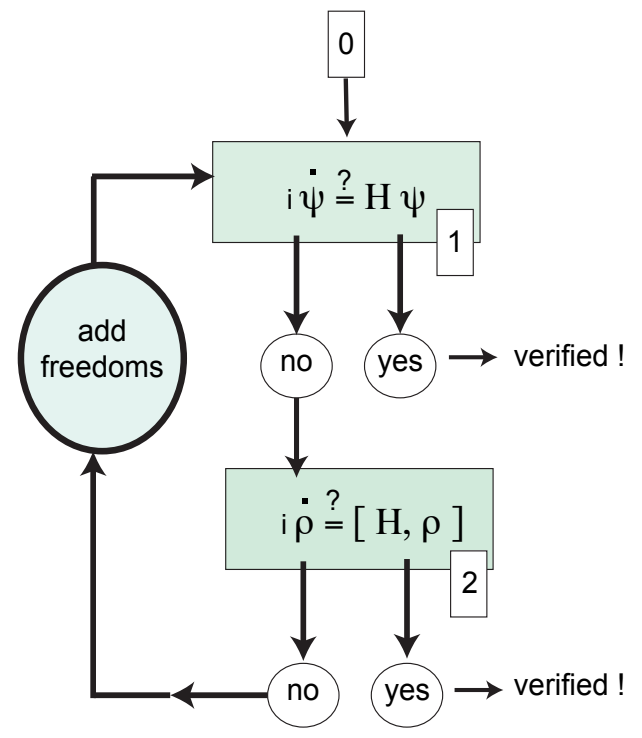

Figure 3. How to test Quantum Dynamics: Tests begin at point 0 with maximal optimism, and assuming the Schroedinger equation applies. At stages 1, 2 tests of dynamics and statistics against observations are made. Dynamical freedoms will be added by the user when a test fails: The system dimension is increased. The procedure flows around a closed loop. The framework cannot be falsified; All outputs verify quantum dynamics.

When probabilities refer to physical measurements, it is important that they be invariants of the system being measured. Invariants refer to some definite transformation group. The 
symmetries of (our approach) to quantum dynamics are $S p(2 N)$. There is no precedent to define quantum probability with that symmetry. By adopting a linear dynamical model with Hermitian $\hat{\Omega}$ the dynamical symmetry group is no worse than $U(N)$. This is easier to work with.

The relation of the groups in our approach is very intimate. Consider the largest set of transformations preserving the unobservable $\langle\Psi \mid \Psi\rangle=\sum_{j}^{N}\left(p_{j}^{2}+q_{j}^{2}\right) / 2$. That is $O(2 N)$, a relatively large group overlooked in ordinary quantum discussions. The intersection with the actual symmetries $O(2 N) \cap S P(2 N) \sim U(N)$, where $\sim$ means isomorphic after complexification. Thus invariance of a probability notion under $U(N)$ is enough for consistency.

Once more our motivation differs from the traditional one. Tradition asserts - blindly and falsely - that since $U(N)$ is the symmetry group of Schroedinger's equation, the notion of total probability must be preserved under $U(N)$. We can't buy that. We don't have a reason to preserve the precious notation of the Schroedinger equation. (It is less general than Hamilton's equations.) What we buy is the fact that $U(N)$ time evolution will not destroy a $U(N)$ invariant probability, if and when the time evolution is that simple. It seems unwise to expect more than that from physics.

Supposing the system is so orderly on a chosen space, one cannot be sure of its effective dynamics for a density matrix on a reduced space. This contradicts lore of the Von Neumann $(v N)$ equation, which is "derived" by methods hoping it might be correct[18]. The equation is equivalent to predicting the time evolution $\rho(t)=U(t) \rho(0) U^{\dagger}(t)$, where $U(t)=\exp (-i \hat{\Omega} t)$. Notice the traditional context assumes that symbol " $\rho$ " refers to a unique object space, while we recognize that concept is procedural. Once a particular division and reduction has been done, it is enough for a single eigenvalue of $\rho$ to be time dependent for the $v N$ equation to fail. While the Von Neumann equation is true by definition in textbooks, it is seldom true in experimental practice. That is because almost all physical systems which are "dirty" enough to need a density matrix are also dirty enough to interact with the environment and spoil the assumptions. There are schemes ("Lindblad theory")[19] to cover the gap. If sometimes a good phenomenology, the cannot be considered general. When energy and interactions leave a subsystem they go into the larger system to return on any number of different time scales. It is not possible in principle for a first order dynamical system to contain enough initial conditions to parameterize all possible cases.

Once every system is a subsystem of a larger system, we should never expect to always predict dynamics.

How the Framework Never Fails: Nevertheless physicists put great faith in the fundamental existence of a wave function on the largest space they are thinking about. That makes a puzzle of why their faith persists. There is a question of whether that framework can be falsified. We do not believe it is possible to falsify the framework. Any system that fails the test of Hamiltonian evolution can be embedded in a larger system. On the larger system it's always possible to "unitarize" any transformation. One method is the "unitary dilation" found by Sz.-Nagy. Figure 3 illustrates the more painful process that physicists follow. By now particle physicists have added numerous quantum fields to the early quantum theory of electrons and photons following the process the figure illustrates. The infinite capacity of theory to expand practically terminates questions of whether such a theory could fail. 


\section{Applications}

As mentioned in the Introduction, our main goal is to take advantage of the efficiency and flexibility of quantum-style data descriptions. We have remarked that description by quantum-style methods is deliberately incomplete. At the same time a great deal of practical experimental information from physics is encoded in wave functions and density matrices by default usage. That is a clue on how to proceed.

\subsection{The optimal patterns defined by data}

Given any "data" as a set of numbers, real or complex, and choosing a method of division, one can start making density matrices and classifying data. But in what sense is this intrinsic and why should it be powerful?

As before let $D_{x}^{J}$ stand for the $J$ th instance of data $D_{x}$. The index $x$ now may stand for multiple labels $x_{1}, x_{2} \ldots x_{n}$ of any dimension. We are interested in the patterns of fluctuations which tend to occur in the entire data set. We define a "pattern " $\mid e>$ as a normalized vector with projections $e(x)=<x \mid e>$. To quantify the importance of a particular pattern, we calculate its overlap-squared summed over the entire data set:

$$
E=\sum_{J}^{J t o t}\left|\left\langle D^{J} \mid e\right\rangle\right|^{2} .
$$

Define the optimal pattern as having the largest possible overlap, subject to the normalization constraint. Algebra gives

$$
\begin{aligned}
E=<e|\rho| e>; \quad & \rho=\sum_{J}^{J t o t}\left|D^{J}><D^{J}\right| \\
\frac{\delta}{\delta \mid e>}\left(\frac{<e|\rho| e>}{<e \mid e>}\right) & =0 ; \\
\rho \mid e_{\alpha}> & =\Lambda_{\alpha}^{2} \mid e_{\alpha}>
\end{aligned}
$$

The eigenvalue problem tells us that a complete set of solutions $\mid e_{\alpha}>$ generally exists; since $\rho=\rho^{\dagger}$, the patterns are automatically orthogonal, and eigenvalues $\Lambda_{\alpha}^{2}$ are real and positive. To interpret the eigenvalues note the overlap $\Omega_{\beta}=<e_{\beta}|\rho| e_{\beta}>=\Lambda_{\beta}^{2}$. Sorting the eigenvalues $\Lambda_{1}^{2}>\Lambda_{2}^{2}>\ldots \Lambda_{N}^{2}$ to makes an optimally convergent expansion of the data expressed in its own patterns.

This result attributed to Karhunen-Loeve[17] is a foundation point of modern signal and image-processing schemes of great effectiveness. Optimally compressing data while retaining a given overlap is done by truncating the expansion of Eq. 37. We think it is no accident that the density matrix appears in Eq. 37. 


\subsection{The principle of minimum entropy}

How does one evaluate a system, and in particular the division of data we mentioned earlier has tremendous flexibility? For that we consider the system's entropy $\mathcal{S}$. The definition is

$$
\mathcal{S}=-\operatorname{tr}(\rho \log (\rho))
$$

For a normalized $N \times N$ density matrix the absolute minimum entropy is $\mathcal{S}=0$, if and only if $\rho$ has rank -1 . The minimum entropy state is the most orderly, least complicated, and is hardly typical. Developing $\mathcal{S}=0$ from samples requires every single sample pattern to be a multiple of every other. The maximum value of $\mathcal{S}=\log (N)$ comes when $\rho \rightarrow 1_{N \times N} / N$ is completely isotropic, and has no preferred basis or pattern associated with it.

In thermodynamics the entropy is recognized as a logarithmic measure of the phase space-volume occupied by the system. The equilibrium distribution is defined by maximizing that volume, the entropy, subject to all constraints such as a fixed total energy or particle number. Experimental science seeks order where it can be found, under which we express the principle of minimum entropy. The principle predicts we should actively use our freedom to partition data to discover low-entropy divisions. They are simple.

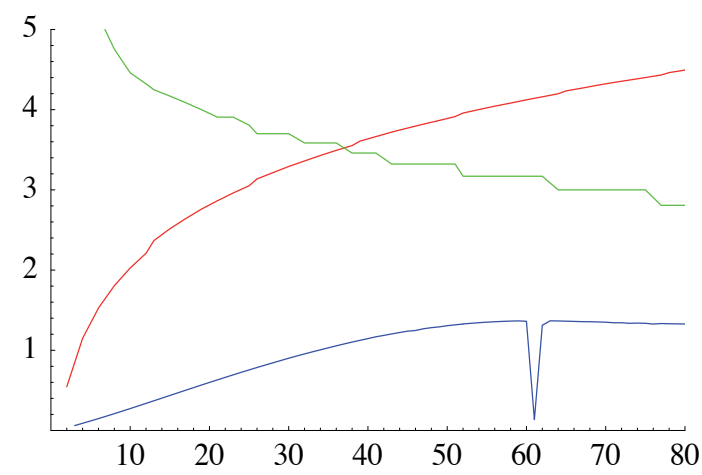

Figure 4. Entropy (in bits) of a coherent data record partitioned on dimension $D$. Bottom curve (blue online): the quantum entropy $-\operatorname{tr}\left(\log _{2}(\rho)\right.$ is comparatively small, and detects the optimal division with a sharp dip. Middle curve (red online): the classical entropy on the same data resolved to $2^{D}$ bit accuracy. Curve highest on left (green online): the entropy of Huffman compression can be less than the classical unprocessed entropy, but it is larger than the quantum entropy.

An Experiment: Figure 4 shows an experiment with Monte Carlo simulation. Two dimensional distributions were defined using sums of Gaussians with randomly generated parameters. A sample from the distribution was extracted from 0.1 unit bins over the interval $-10<x<10,-3<y<3$, making a 12261-point data record $D_{i J}$, which was normalized. Re-partitioning the record on intervals of length $L$ created arrays ${ }^{4}$ Now compare classical and quantum methods. The empirical marginal distribution on index $i$ is $d_{i}=\sum_{j} d_{i j}$. That leads to the classical entropy $\mathcal{S}_{c l}=-\sum_{i} d_{i} \log \left(d_{i}\right)$. Compare the density matrix on the same space, $\rho_{i i^{\prime}}=d_{i j} d_{i j^{\prime}}$, with quantum entropy $\mathcal{S}_{q}=-\operatorname{tr}(\rho \log (\rho))$. The figure shows that $\mathcal{S}_{q}<<\mathcal{S}_{c l}$ unless the data is very noisy. A dip in $\mathcal{S}_{q}$ occurs at favored divisions (Fig. 4). Indeed if one

\footnotetext{
${ }^{4}$ Data were rescaled by a constant to be resolved on exactly $L$ dimensions.
} 
makes data with random linear combinations of specified patterns, the entropy will dip at the division of the pattern's periodicity, or approximate periodicity. Minimum entropy finds simplicity.

Information theory is like experimental physics in manipulating the encoding of repeated patterns to lower the effective entropy. As $L$ increases longer patterns or "words" can be compressed into symbols. Huffman compression is a method to optimize classical information content towards minimum entropy. Huffman coding is a procedure based on me class definitions and therefore classical characterization. Figure 4 shows that using Huffman coding produces $\mathcal{S}_{\text {huffman }}<\mathcal{S}_{c l}$. Yet across the board both classical entropies exceed the quantum value. Both miss the optimal division, because no notion of dividing a product space exists. Similar features have been seen in dozens of different types of data.

An Experiment: A symmetry of a correlation means it is unchanged under a transformation. A symmetry of the density matrix implies it commutes with the generator of the transformation, and then shares eigenstates. Figure 5 shows an experiment in self-organizing or "auto-quantization" of the eigenvectors of the density matrix. To make the figure the first 60 decimal digits of $\pi$ were collected as an array $D_{1 i}$. Random cyclic permutations of the same list produced the samples $D_{2 i}, D_{3 i} \ldots D_{I i}$ for $J=1 \ldots 1000$. The eigenvectors of $\rho_{i i^{\prime}}$ are found to be nearly pure momentum eigenstates: Each shows a peak in Fourier power at a single wave number. The probability to find $\cos (\pi x / 2)$ sampled on the first 60 integers is about $2.3 \%$.

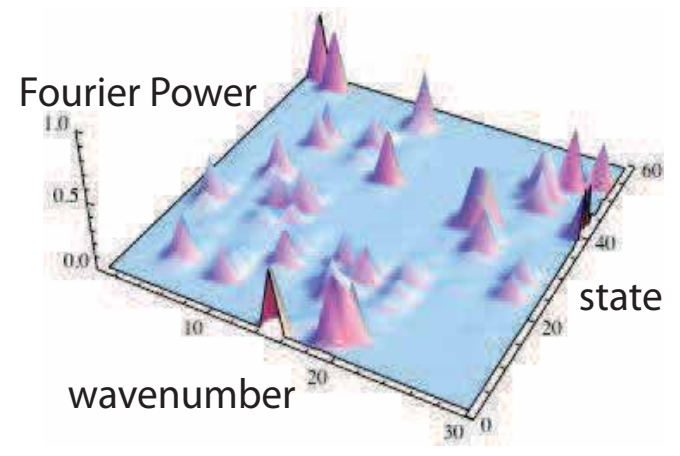

Figure 5. "Auto-quantization" by symmetry. The Fourier power of density matrix eigenvectors made from the first 60 digits of $\pi$ sampled over 1000 random circular permutations is quantized.

Similar results are found with more structured group operations, such as rotations, unitary transformations, or more complicated group operations with elements $R(\omega)$, where $\omega=$ $\alpha_{J}, \beta_{J}, \gamma_{J} \ldots$ are group parameters of sample $J$. Let $|l, m \ldots\rangle$ be an irreducible basis of group representations. Let the objects in the sample be copies of a single object transformed under the group, and let $\sum_{J} \rightarrow d \omega$, the invariant group volume. The density matrix approaches a limit:

$$
\begin{array}{r}
\rho=\int d \omega \sum_{j, j^{\prime}, l, l^{\prime}, m, m^{\prime} \ldots} R(\omega)|l, m \ldots\rangle\langle l, m \ldots| \\
\quad \times|a\rangle\left\langle a|| l^{\prime}, m^{\prime} \ldots\right\rangle\left\langle l^{\prime}, m^{\prime} \ldots\right| R^{\dagger}(\omega) .
\end{array}
$$


Use the completeness property of matrix representations $D_{m^{\prime \prime} m^{\prime \prime \prime}}^{j^{\prime} l^{\prime} \ldots}$ :

$$
\int d \omega D_{m m^{\prime}}^{j, l \ldots}(\omega) \bar{D}_{m^{\prime \prime} m^{\prime \prime \prime}}^{j^{\prime}, l^{\prime} \ldots}(\omega)=\delta^{j j^{\prime}} \delta^{l l^{\prime}} \delta^{m m^{\prime \prime}} \ldots,
$$

Only the diagonal elements survive the sums, with the weights just as dictated by quantum rules:

$$
\rho \rightarrow|j, l, m \ldots\rangle|\langle j, l, m \ldots \mid a\rangle|^{2}\langle j, l, m \ldots|
$$

Data which is many copies of some complicated scalar function of angles, such as the shape of a paramecium or the map of the Earth permuted over all orientations will give a density matrix a diagonal sum of spherical harmonics, with probabilities given by the standard formula. It is kinematic because the machinery defining probability was constructed to expedite linear transformations.

Time Evolution: Turn to time evolution. Earlier we commented that physics predicts little else, and that our Hamiltonian model was a toy. Bounded linear Hamiltonian time evolution is unitary time evolution, also a toy. We offer the idea these features emerge from the Agreement to categorize things with density matrices. A typical correlation in QFT is written

$$
\begin{aligned}
C\left(x_{1}, t_{1}, x_{2}, t_{2}, \ldots x_{n}, t_{n}\right)= & <0\left|\phi\left(x_{1}, t_{1}\right) . . \phi\left(x_{n}, t_{n}\right)\right| 0>, \\
& =\operatorname{tr}\left(\rho_{0} \phi\left(x_{1}, t_{1}\right) . . \phi\left(x_{n}, t_{n}\right)\right) .
\end{aligned}
$$

The state of the art of physics consists of reverting all known correlations into a model for the density matrix $\rho_{0}$. It is very beautiful that the model is relatively simple, but whatever was obtained in the lab, we cannot see how the representation itself could fail.

From space - and time-translational symmetry, which physicists desire to arrange, the correlation is a function of differences $C=C\left(x_{2}-x_{1}, t_{2}-t_{1}, \ldots t_{n}-t_{n-1}\right)$. Any function of $x_{2}-x_{1}$ has a Fourier representation in terms of $\exp ^{i \vec{k}_{12}\left(\vec{x}_{2}-\vec{x}_{1}\right)} \exp ^{-i \omega\left(t_{2}-t_{1}\right)}$. The ultimate time evolution is unitary. By standard steps of the convolution theorem the law of Conservation of Frequency (energy) is kinematic, and inventing a Hamiltonian with enough degrees of freedom cannot fail to describe it ${ }^{5}$, so long as the eigenvalues of $\rho_{0}$ are conserved: otherwise, add freedoms. Thus we don't need to be too embarrassed about the toy dynamics, whose success ought to be judged on the effectiveness and simplicity of the model, not needing higher principles: in our approach.

Many Experiments: In the Introduction we mentioned that the restriction of the quantum methods to describing micro-physical objects of fundamental physical character is obsolete. We've done many experiments to extend the scope. • In Ref. [20] the radio frequency emissions of relativistic protons were used to construct density matrices from signal data and noise data. A combination of techniques improved the signal to noise ratio by about a factor of more than 100, producing the first detection of virtual Cherenkov radiation from protons.

\footnotetext{
${ }^{5}$ One might discover a time-dependent Hamiltonian, which does occur in physics, yet which is invariably embedded in a time-independent larger system.
} 
- In Refs. [22] data from the cosmic microwave background (CMB) was analyzed to test the "cosmological principle" requiring isotropy. The alignment of spherical harmonic multipoles and the entropy of their power distribution contradicts isotropy at a high degree of statistical significance. The origin is unknown, while it cannot be explained by galactic foreground subtractions [23]. • In Ref.[24] the density matrix was constructed from high-dimensional spectroscopic data of a pharmaceutical protein. The principal values were sorted to make projections onto certain subspaces from which the phases of the protein could be determined by inspection. Ref. [25] reviews subsequent progress. By now the method has been used to make empirical phase diagrams towards characterizing the active states, phase transitions and shelf-life of about 100 pharmaceuticals.

\section{Concluding remarks}

Our discussion began with conventional observations that "the framework of quantum mechanics is thought the perfection of fundamental theory" that "predicts an absolute and unvarying law of time evolution." Those observations have been found false in general.

Our Universe seems to need many degrees of freedom for its description. Physics has greatly progressed in the details of models, while giving away credit to a quantum-style framework we claim is independent. The important part of the framework is its extension and enlargement of the definition of probability. In Section 6.1 we stated that the breakthrough of quantum probability lies in generalizing the notion of probability so as not to insist on pre-ordained equivalence classes.

We showed that a map exists going from macroscopic information to quantum probability. The map maintains a hidden variable description for quantum systems, and extends the scope of subjects of quantum information theory. We showed that the probabilistic features of quantum mechanics itself come from the process of reducing hidden freedoms. It is no more profound than a certain method of counting. We believe that quantum physics operates by the same procedures, but so long as parts of physics are unknown, that cannot be "derived." Rather than argue with opinions about what the Universe is, we call it "our approach."

No relation to microphysics is needed. Quantum probability can be viewed as an efficient data-management device, a branch of information theory, reversing a perception that quantum information theory should be a science of microphysical objects.

\subsubsection{What do we mean by the probability of a vector?}

Both classical probability and quantum probability have a feature that certain independent probabilities multiply, using direct product spaces to organize the mathematics. The difference is illustrated by the different way to discriminate between "different" vectors, which finishes our discussion.

Both approaches will decompose a state space into coordinates $v_{i}=\langle i \mid v\rangle$, for $i=1 \ldots . N$ The typical approach to classical probability defines a distribution $f(v)=d N / d v_{1} d v_{2} \ldots d v_{N}$. Break each dimension into $K$ equal bins of resolution $\Delta v_{i}$. The distribution for any situation is a list among $K^{N}$ mutually exclusive possibilities. Completely sampling the distribution needs $K^{N}$ pieces of information, which may well be impossible. There are practical consequences. The classical device made with 102 -state $q$ - bits has $2^{10}$ possible states, all declared distinct. 
One such state is written $v_{i}=(0,0,1,1,0,0,1,0,0,1)$. Another vector is $u_{i}=(0,1,0,0,1,1,0$, $0,1,1)$. These are not the same so their probability of being the same is zero. The probability for any random 2-state vector is of order $2^{-10} \sim 9 \times 10^{-4}$, which is very small.

Compare the quantum probability that one vector can serve for another. It is not based on a distribution. The normalized inner product $\langle u \mid v\rangle=1 /(2 \sqrt{5})$. The probability the vector $\mid u>$ defined above can serve for $|v\rangle$ is $|\langle u \mid v\rangle|^{2}=1 / 20$, which is a much larger probability of coincidence than zero. A numerical calculation finds the average overlap-squared of such random vectors with another one is about 0.3. If one generates 1000 normalized vectors, there will be around 180 with a Born probability exceeding 0.5 that look like a given vector.

Two facts are so basic they tend to escapes notice. First, it is not actually possible in principle to sample and then categorize the spaces of most distributions cited for classical physics. If the data uses 16-bit accuracy and 10-element vectors there are $\left(2^{16}\right)^{10}=1.46 \times 10^{48}$ mutually exclusive class labels set up in the first step. The entire 19th century conception of multidimensional phase spaces for many particles ("moles" of atoms) does not exist in any physically realizable form. While ignoring all dynamics was a success of thermodynamics, the ambition to keep track of some fragment of the vast experimental complexity of many degrees of freedom cannot really be maintained within the framework of distributions: a great deal of classical theory notwithstanding.

Second, the quantum description of any given data is not more complicated, it is vastly less. The language abuse of "quantum particles" greatly confuses this. The 10-dimensional space cited above has no more than $10-1=9$ vectors that are mutually-exclusive of any given vector. The simplification of quantum-style data characterization also occurs with infinitely fine resolution, and with no great sensitivity to the resolution. It is grossly misleading to compare a quantum space of $2^{10}$ dimensions, from spin products $1 / 2 \otimes 1 / 2 \otimes \ldots 1 / 2$ with a classical space of $K^{10}$ mutually-exclusive categories.

That is why we end reiterating the principle of minimum entropy from Section 7.2. Whether or not physics must do it by principle, there is a practical fact that large data sets should be reduced to correlations, and that correlations should be reduced to sub-correlations. When the entropy of dividing data is as low as possible, the experimenter has found order, and statistical regularity, which is the purpose of science. The applications of a new science seeking minimum entropy as defined on flexible quantum-style categories are unlimited.

\section{Acknowledgements}

Research supported in part under DOE Grant Number DE-FG02-04ER14308. We thank Carl Bender, Don Colloday, Danny Marfatia, Phil Mannheim, Doug McKay, Dan Neusenschwander, and Peter Rolnick for comments.

\section{Author details}

John P. Ralston

Department of Physics \& Astronomy, The University of Kansas, Lawrence KS, USA 


\section{References}

[1] R. B. Laughlin and D. Pines, PNAS 97, 28 (1999).

[2] There are too many references to cite. A good introduction is the book by S. L. Adler, Quantum theory as an emergent phenomenon: The statistical mechanics of matrix models as the precursor of quantum field theory, Cambridge, UK: Univ. Pr. (2004) 225 p. See also G. 't Hooft, AIP Conf. Proc. 957, 154 (2007) [arXiv:0707.4568 [hep-th]]

[3] An extensive blbliography with wide discussion is given by the Archiv preprint of B. Hu, Emergence: Key Physical Issues for Deeper Philosphical Enquiries, J. Phys. Conf. Ser. 361 (2012) 012003, arXiv:1204.1077 [physics.hist-ph].

[4] H. -T. Elze, J. Phys. Conf. Ser. 174, 012009 (2009) [arXiv:0906.1101 [quant-ph]].; J. Phys. Conf. Ser. 33, 399 (2006) [gr-qc/0512016]; J. Phys. Conf. Ser. 171, 012034 (2009); M. Blasone, P. Jizba and F. Scardigli, J. Phys. Conf. Ser. 174, 012034 (2009) [arXiv:0901.3907 [quant-ph]]; G. 't Hooft, Int. J. Mod. Phys. A 25, 4385 (2010)

[5] See, e.g. R. Hedrich, Phys. Phil. 2010, 016 (2010) [arXiv:0908.0355 [gr-qc]]; B. Koch, AIP Conf. Proc. 1232, 313 (2010) [arXiv:1004.2879 [hep-th]]; D. Acosta, P. F. de Cordoba, J. M. Isidro and J. L. G. Santander, arXiv:1206.4941 [math-ph].

[6] Modern Quantum Mechanics, by J. J. Sakurai, San Fu Tuan, editor (Addison Wesley, 1998).

[7] J. P. Ralston, J. Phys. A: Math. Theor. 40, 9883 (2007).

[8] C. M. Bender and S. Boettcher, Phys. Rev. Lett. 80, 5243 (1998) [arXiv:physics/9712001]; C. M. Bender, S. Boettcher and P. Meisinger, J. Math. Phys. 40, 2201 (1999) [arXiv:quant-ph/9809072].

[9] Sir George Gabriel Stokes : Memoirs and Scientific Correspondence, edited by Joseph Larmor (Cambridge University Press, 1907); The Theory of Electrons, by H. A. Lorentz (Cosimo Classics, 2007).

[10] For a review of Rydberg's physics, see "Janne Rydberg his life and work", by I. Martinson and L.J. Curtis, NIM B 235, 17 (2005). Graphics is from Lund University Physics, http://www.lth.se/?id=17657.

[11] Max Planck, Annalen der Physik 4, 553 (1901); Translated in http://axion.physics. ubc.ca/200-06/Planck-1901.html. See also Max Planck, The theory of Heat Radiation, translated by Morton Mosius, P. Blackiston's Sons, (1914).

[12] J. P. Ralston, arXiv:1203.5557 [hep-ph].

[13] P. J. Mohr, B. N. Taylor and D. B. Newell, Rev. Mod. Phys. 80, 633 (2008) [arXiv:0801.0028 [physics.atom-ph]].

[14] A. Caticha, Phys. Rev. A 57, 1572 (1998)

[15] J. S. Bell, Physics 1, 195, (1964). 
[16] R. F. Werner, Phys. Rev. A 42, 4777, (1989). A. Peres, Phys. Rev. Lett. 77, 1413 (1996); Phys. Scripta T76, 52 (1998) [arXiv:quant-ph/9707026].

[17] H. Karhunen, Ann. Acad. Science. Fenn, Ser A. I. 37, 1947; M. Loeve, supplement to P. Levy, Processes Stochastic et Mouvement Brownien, Paris, Gauthier Villars, 1948; H. Hotelling, J. Educ. Psychology 24, 417, 1933; ibid 24, 448, 1933; L. Scharfe, Statistical Signal Processing, (Wiley 1990).

[18] J. Von Neumann, Mathematische Grundlagen der Quantenmechanik Springer, Berlin; English translation in The Mathematical Foundations of Quantum Mechanics, Princeton University Press, Princeton (1971).

[19] G. Lindblad, Commun. Math. Phys. 48119 (1976) V. Gorini, A. Kossakowski and E C G Sudarshan, J. Math. Phys. 17821 (1976).

[20] A. Bean, J. P. Ralston and J. Snow, Nucl. Instrum. Meth. A 596, 172 (2008) [arXiv:1008.0029 [physics.ins-det]].

[21] The Bloch-Nordseik treatment of infrared divergences in $Q E D$ is reviewed in many textbooks, including Ralstivistic Quantum Field Theory, by J. D. Bjorken and S. D. Drell,(Wiley, 1965).

[22] P. K. Samal, R. Saha, P. Jain and J. P. Ralston, Mon. Not. Roy. Astron. Soc. 385, 1718 (2008) [arXiv:0708.2816 [astro-ph]]; Mon. Not. Roy. Astron. Soc. 396, 511 (2009) [arXiv:0811.1639 [astro-ph]].

[23] P. K. Aluri, P. K. Samal, P. Jain and J. .P. Ralston, Mon. Not. Roy. Astron. Soc. 414, 1032 (2011) [arXiv:1007.1827 [astro-ph.CO]].

[24] L Kueltzo, J. Fan, J. P. Ralston, M. DiBiase, E. Faulkner, and C. R. Middaugh, J. Pharm. Sci. 94(9), 1893 (2005).

[25] N. Maddux, R Joshi, C. R. Middaugh, J. P. Ralston and R. Volkin, J. Pharm. Sci. 1004171 (2011). 

Chapter 20

\title{
The Wigner-Heisenberg Algebra in Quantum Mechanics
}

\author{
Rafael de Lima Rodrigues \\ Additional information is available at the end of the chapter
}

http://dx.doi.org/10.5772/55994

\section{Introduction}

While the supersymmetry in quantum mechanics (SUSY QM) algebra has thus received much operator applications for potential problems [1-4], another algebra, the general Wigner-Heisenberg(WH) oscillator algebra [5-8], which already possesses an in built structure which generalises the usual oscillators ladder operators, has not, however, in our opinion, received its due attention in the literature as regards its potential for being developed as an effective operator technique for the spectral resolution of oscillator-related potentials. The purpose of the chapter is to bridge this gulf.

The WH algebraic technique which was super-realized for quantum oscillators [9-11], is related to the paraboson relations and a graded Lie algebra structure analogous to Witten's SUSY QM algebra was realized in which only annihilation operators participate, all expressed in terms of the Wigner annihilation operator of a related super Wigner oscillator system [12]. In this reference, the coherent states are investigate via $\mathrm{WH}$ algebra for bound states, which are defined as the eigenstates of the lowering operator, according to the Barut-Girardello approach [13]. Recently, the problem of the construction of coherent states for systems with continuous spectra has been investigated from two viewpoints by Bragov et al. [17]. They adopt the approach of Malkin-Manko [18] to systems with continuous spectra that are not oscillator-like systems. On the other hand, they generalize, modify and apply the approach followed in [19] to the same kind of systems.

To illustrate the formalism we consider here simpler types of such potentials only, of the full 3D isotropic harmonic oscillator problem (for a particle of spin $\frac{1}{2}$ ) [9] and non-relativistic Coulomb problem for the electron [16].

The WH algebra has been considered for the three-dimensional non-canonical oscillator to generate a representation of the orthosympletic Lie superalgebra $\operatorname{csp}(3 / 2)$, and recently 
Palev have investigated the $3 D$ Wigner oscillator under a discrete non-commutative context [20]. Let us now point out the following (anti-)commutation relations $\left([A, B]_{+} \equiv A B+B A\right.$ and $\left.[A, B]_{-} \equiv A B-B A\right)$.

Also, the relevance of $\mathrm{WH}$ algebra to quantization in fractional dimension has been also discussed [21] and the properties of Weyl-ordered polynomials in operators $P$ and $Q$, in fractional-dimensional quantum mechanics have been developed [22].

The Kustaanheimo-Stiefel mapping [25] yields the Schrödinger equation for the hydrogen atom that has been exactly solved and well-studied in the literature. (See for example, Chen [26], Cornish [27], Chen and Kibler [28], D'Hoker and Vinet [29].)

Kostelecky, Nieto and Truax [30] have studied in a detailed manner the relation of the supersymmetric (SUSY) Coulombian problem [31-33] in D-dimensions with that of SUSY isotropic oscillators in D-dimensions in the radial version.

The vastly simplified algebraic treatment within the framework of the WH algebra of some other oscillator-related potentials like those of certain generalised SUSY oscillator Hamiltonian models of the type of Celka and Hussin which generalise the earlier potentials of Ui and Balantekin have been applied by Jayaraman and Rodrigues [10]. Also, the connection of the WH algebra with the Lie superalgebra $s \ell(1 \mid n)$ has been studied in a detailed manner [34].

Also, some super-conformal models are sigma models that describe the propagation of a non-relativistic spinning particle in a curved background [35]. It was conjectured by Gibbons and Townsend that large $n$ limit of an $N=4$ superconformal extension of the $n$ particle Calogero model [36] might provide a description of the extreme Reissner-Nordström black hole near the horizon [37]. The superconformal mechanics, black holes and non-linear realizations have also been investigated by Azcárraga et al. [38].

\section{The abstract WH algebra and its super-realisation}

Six decades ago Wigner [5] posed an interesting question as if from the equations of motion determine the quantum mechanical commutation relations and found as an answer a generalised quantum commutation rule for the one-dimensional harmonic oscillator. Starting with the Schrödinger equation $H\left|\psi_{n}\right\rangle=E_{n} \mid \psi_{n}>$, where the Hamiltonian operator becomes

$$
\hat{H}=\frac{1}{2}\left(\hat{p}^{2}+\hat{x}^{2}\right)=\frac{1}{2}\left[\hat{a}^{-}, \hat{a}^{+}\right]_{+}=\frac{1}{2}\left(\hat{a}^{-} \hat{a}^{+}+\hat{a}^{+} \hat{a}^{-}\right)
$$

(we employ the convention of units such that $\hbar=m=\omega=1$ ) where the abstract Wigner Hamiltonian $\hat{H}$ is expressed in the symmetrised bilinear form in the mutually adjoint abstract operators $a^{ \pm}$defined by

$$
\hat{a}^{ \pm}=\frac{1}{\sqrt{2}}( \pm i \hat{p}-\hat{x}) \quad\left(\hat{a}^{+}\right)^{\dagger}=\hat{a}^{-} .
$$

Wigner showed that the Heisenberg equations of motion 


$$
\left[\hat{H}, \hat{a}^{ \pm}\right]_{-}= \pm \hat{a}^{ \pm}
$$

obtained by also combining the requirement that $x$ satisfies the equation of motion of classical form. The form of this general quantum rule can be given by

$$
\left[\hat{a}^{-}, \hat{a}^{+}\right]_{-}=1+c \hat{R} \rightarrow[\hat{x}, \hat{p}]_{-}=i(1+c \hat{R})
$$

where $c$ is a real constant that is related to the ground-state energy $E^{(0)}$ of $\hat{H}$ and $\hat{R}$ is an abstract operator, Hermitian and unitary, also possessing the properties

$$
\hat{R}=\hat{R}^{\dagger}=\hat{R}^{-1} \rightarrow \hat{R}^{2}=1, \quad\left[\hat{R}, \hat{a}^{\dagger}\right]_{+}=0 \rightarrow[\hat{R}, \hat{H}]_{-}=0 .
$$

It follows from equations (1) and (4) that

$$
\hat{H}=\left\{\begin{array}{l}
\hat{a}^{+} \hat{a}^{-}+\frac{1}{2}(1+c \hat{R}) \\
\hat{a}^{-} \hat{a}^{+}-\frac{1}{2}(1+c \hat{R})
\end{array}\right.
$$

Abstractly $\hat{R}$ is the Klein operator $\pm \exp \left[i \pi\left(\hat{H}-E_{0}\right)\right]$ while in Schrödinger coordinate representation, first investigated by Yang, $\mathcal{R}$ is realised by $\pm P$ where $P$ is the parity operator:

$$
P|x>= \pm| x>, \quad P^{-1}=P, \quad P^{2}=1, P x P^{-1}=-x .
$$

The basic (anti-)commutation relation (1) and (3) together with their derived relation (4) will be referred to here as constituting the WH algebra which is in fact a parabose algebra for one degree of freedom. We shall assume here in after, without loss of generality, that $c$ is positive, i.e. $c=|c|>0$. Thus, in coordinate representation the generalized quantization à la Wigner requires that

$$
\hat{x}=x, \quad \infty-<x<\infty, \quad \hat{p}=-i \frac{d}{d x}+\frac{i c}{2 x} P, \quad R=P .
$$

Indeed, following Yang representation [6] we obtain the coordinate representation for the ladder operators as given by

$$
\hat{a}^{ \pm} \longrightarrow a_{\frac{c}{2}}^{ \pm}=\frac{1}{\sqrt{2}}\left( \pm \frac{d}{d x} \mp \frac{c}{2 x} P-x\right) .
$$

Yang's wave mechanical description was further investigated in $[7,8]$. 
The present author have applied a super-realization so that $R=\Sigma_{3}$ to illustrate the first application of our operator method to the cases of the Hamiltonian of an isotonic oscillator (harmonic plus a centripetal barrier) system. To obtain a super-realisation of the WH algebra, we introduce, in addition to the usual bosonic coordinates $\left(x,-i \frac{d}{d x}\right)$, the fermionic ones $b^{\mp}\left(=\left(b^{ \pm}\right)^{\dagger}\right)$ that commute with the bosonic set and are represented in terms of the usual Pauli matrices $\Sigma_{i},(i=1,2,3)$. Indeed, expressing $a^{ \pm}\left(\frac{c}{2}\right)$ in the following respective factorised forms:

$$
\begin{aligned}
& a^{+}\left(\frac{c}{2}\right)=\frac{1}{\sqrt{2}} \Sigma_{1} x^{(1 / 2) c \Sigma_{3}} \exp \left(\frac{1}{2} x^{2}\right)\left(\frac{d}{d x}\right) \exp \left(\frac{-1}{2} x^{2}\right) x^{-(1 / 2) c \Sigma_{3}} \\
& a^{-}\left(\frac{c}{2}\right)=\frac{1}{\sqrt{2}} \Sigma_{1} x^{(1 / 2) c \Sigma_{3}} \exp \left(\frac{-1}{2} x^{2}\right)\left(\frac{-d}{d x}\right) \exp \left(\frac{1}{2} x^{2}\right) x^{-(1 / 2) c \Sigma_{3}},
\end{aligned}
$$

where these lader operators satisfy the algebra of Wigner-Heisenberg. From (1), (11) and (10) the Wigner Hamiltonian becomes

$$
\begin{aligned}
H\left(\frac{c}{2}\right) & =\frac{1}{2}\left[a^{+}\left(\frac{c}{2}\right), a^{-}\left(\frac{c}{2}\right)\right]_{+} \\
& =\left(\begin{array}{cc}
H_{-}\left(\frac{c}{2}-1\right) & 0 \\
0 & H_{+}\left(\frac{c}{2}-1\right)=H_{-}\left(\frac{c}{2}\right)
\end{array}\right),
\end{aligned}
$$

where the even and odd sector Hamiltonians are respectively given by

$$
H_{-}\left(\frac{c}{2}-1\right)=\frac{1}{2}\left\{-\frac{d^{2}}{d x^{2}}+x^{2}+\frac{1}{x^{2}}\left(\frac{c}{2}\right)\left(\frac{c}{2}-1\right)\right\}
$$

and

$$
H_{+}\left(\frac{c}{2}-1\right)=\frac{1}{2}\left\{-\frac{d^{2}}{d x^{2}}+x^{2}+\frac{1}{x^{2}}\left(\frac{c}{2}\right)\left(\frac{c}{2}+1\right)\right\}=H_{-}\left(\frac{c}{2}\right) .
$$

The time-independent Schrödinger equation for these Hamiltonians of an isotonic oscillator (harmonic plus a centripetal barrier) system becomes the following eigenvalue equation:

$$
H_{ \pm}\left(\frac{c}{2}-1\right)\left|m, \frac{c}{2}-1>=E_{ \pm}\left(\frac{c}{2}-1\right)\right| m, \frac{c}{2}-1>
$$

Thus, from the annihilation condition $a^{-} \mid 0>=0$, the ground-state energy is given by 


$$
E^{(0)}\left(\frac{c}{2}\right)=\frac{1}{2}(1+c)>\frac{1}{2}, \quad c>0
$$

At this stage an independent verification of the existence or not of a zero ground-state energy for $H\left(\frac{c}{2}\right)$ suggested by its positive semi-definite form may be in order.

The question we formulate now is the following: What is the behaviour of the ladder operators on the autokets of the Wigner oscillator quantum states? To answer this question is obtained via WH algebra, note that the Wigner oscillator ladder operators on autokets of these quantum states are given by

$$
\begin{aligned}
a_{\frac{c}{2}}^{-} \mid 2 m, \frac{c}{2}> & =\sqrt{2 m} \mid 2 m-1, \frac{c}{2}> \\
a_{\frac{c}{2}}^{-} \mid 2 m+1, \frac{c}{2}> & =\sqrt{2\left(m+E^{(0)}\right)} \mid 2 m, \frac{c}{2}> \\
a_{\frac{c}{2}}^{+} \mid 2 m, \frac{c}{2}> & =\sqrt{2\left(m+E^{(0)}\right)} \mid 2 m+1, \frac{c}{2}> \\
a_{\frac{c}{2}}^{+} \mid 2 m+1, \frac{c}{2}> & =\sqrt{2(m+1)} \mid 2 m+2, \frac{c}{2}>.
\end{aligned}
$$

Now, from the role of $a^{+}\left(\frac{c}{2}\right)$ as the energy step-up operator (the upper sign choice) the excited-state energy eigenfunctions and the complete energy spectrum of $H\left(\frac{c}{2}\right)$ are respectively given by $\psi^{(n)}\left(\frac{c}{2}\right) \propto\left[a^{+}\left(\frac{c}{2}\right)\right]^{n} \psi^{(0)}\left(\frac{c}{2}\right)$ and $E^{(n)}\left(\frac{c}{2}\right)=E^{(0)}+n, \quad n=0,1,2, \cdots$.

It is known that the operators $\pm \frac{i}{2}\left(a^{ \pm}\left(\frac{c}{2}\right)\right)^{2}$ and $\frac{1}{2} H\left(\frac{c}{2}\right)$ can be chosen as a basis for a realization of the $s o(2,1) \sim s u(1,1) \sim s \ell(2, \mathbf{R})$ Lie algebra. When projected the $-\frac{1}{2}\left(a^{ \pm}\right)^{2}$ operators in the even sector with $\frac{1}{2}\left(\mathbf{1}+\Sigma_{3}\right)$, viz.,

$$
\frac{1}{2}\left(\mathbf{1}+\Sigma_{3}\right) B^{-}=\frac{1}{2}\left(\mathbf{1}+\Sigma_{3}\right)\left(a^{-}\right)^{2}=\left(\begin{array}{rr}
B^{-} & 0 \\
0 & 0
\end{array}\right)
$$

and

$$
\frac{1}{2}\left(\mathbf{1}+\Sigma_{3}\right) B^{+}=\frac{1}{2}\left(\mathbf{1}+\Sigma_{3}\right)\left(a^{+}\right)^{2}=\left(\begin{array}{rr}
B^{+} & 0 \\
0 & 0
\end{array}\right)
$$

we obtain

$$
B^{-}\left(\frac{c}{2}-1\right)=\frac{1}{2}\left\{\frac{d^{2}}{d x^{2}}+2 x \frac{d}{d x}+x^{2}-\frac{\left(\frac{c}{2}-1\right) \frac{c}{2}}{x^{2}}+1\right\}
$$

and 


$$
B^{+}\left(\frac{c}{2}-1\right)=\left(B^{-}\right)^{\dagger}=\frac{1}{2}\left\{\frac{d^{2}}{d x^{2}}-2 x \frac{d}{d x}+x^{2}-\frac{\left(\frac{c}{2}-1\right) \frac{c}{2}}{x^{2}}-1\right\} .
$$

Thus, the Lie algebra becomes

$$
\left[K_{0}, K_{-}\right]_{-}=-K_{-}, \quad\left[K_{0}, K_{+}\right]_{-}=+K_{+}, \quad\left[K_{-}, K_{+}\right]_{-}=2 K_{0},
$$

where $K_{0}=\frac{1}{2} H_{-}, \quad K_{-}=-\frac{1}{2} B^{-}$and $K_{+}=\frac{1}{2} B^{+}$generate once again the $s u(1,1)$ Lie algebra. Therefore, these ladder operators obey the following commutation relations:

$$
\begin{aligned}
{\left[B^{-}\left(\frac{c}{2}-1\right), B^{+}\left(\frac{c}{2}-1\right)\right]_{-} } & =4 H_{-}\left(\frac{c}{2}-1\right) \\
{\left[H_{-}, B^{ \pm}\left(\frac{c}{2}-1\right)\right]_{-} } & = \pm 2 B^{ \pm}\left(\frac{c}{2}-1\right) .
\end{aligned}
$$

Hence, the quadratic operators $B^{ \pm}\left(\frac{c}{2}-1\right)$ acting on the orthonormal basis of eigenstates of $H_{-}\left(\frac{c}{2}-1\right),\left\{\mid m, \frac{c}{2}-1>\right\}$ where $m=0,1,2, \cdots$ have the effect of raising or lowering the quanta by two units so that we can write

$$
B^{-}\left(\frac{c}{2}-1\right)\left|m, \frac{c}{2}-1>=\sqrt{2 m(2 m+c+1)}\right| m-1, \frac{c}{2}-1>
$$

and

$$
B^{+}\left(\frac{c}{2}-1\right)\left|m, \frac{c}{2}-1>=\sqrt{2(m+1)(2 m+c+1)}\right| m+1, \frac{c}{2}-1>
$$

giving

$$
\left|m, \frac{c}{2}-1>=2^{-m}\left\{\frac{\Gamma\left(\frac{c+1}{2}\right)}{m ! \Gamma\left(\frac{c+1}{2}+m\right)}\right\}^{1 / 2}\left\{B^{+}\left(\frac{c}{2}-1\right)\right\}^{m}\right| 0, \frac{c}{2}-1>,
$$

where $\Gamma(x)$ is the ordinary Gamma Function. Note that $B^{ \pm}\left(\frac{c}{2}-1\right) \mid m, \frac{c}{2}-1>$ are associated with the energy eigenvalues $E_{-}^{(m \pm 1)}=\frac{c+1}{2}+2(m \pm 1), \quad m=0,1,2, \ldots$

Let us to conclude this section presenting the following comments: one can generate the called canonical coherent states, which are defined as the eigenstates of the lowering operator $B^{-}\left(\frac{c}{2}-1\right)$ of the bosonic sector, according to the Barut-Girardello approach $[12,13]$ and generalized coherent states according to Perelomov [14,15]. Results of our investigations on these coherent states will be reported separately. 


\section{The 3D Wigner and SUSY systems}

As is well-known, the quantum mechanical (QM) $N=2$ supersymmetry (SUSY) algebra of Witten [1-4]

$$
\begin{aligned}
H_{S S} & =\left[Q_{-}, Q_{+}\right]_{+} \\
\left(Q_{-}\right)^{2} & =\left(Q_{+}\right)^{2}=0 \rightarrow\left[H_{S S}, Q_{\mp}\right]=0,
\end{aligned}
$$

involves bosonic and fermionic sector Hamiltonians of the SUSY Hamiltonian $H_{S S}$ (the even element), which get intertwined through the nilpotent charge operators $Q_{-}=\left(Q_{+}\right)^{\dagger}$ (the odd elements).

The connection between the 3D Wigner Hamiltonian $H(\vec{\sigma} \cdot \vec{L}+\mathbf{1})$ and a 3D SUSY isotropic harmonic oscillator for spin $\frac{1}{2}$ is given by anti-commutation relation (25) and the mutually adjoint charge operators $Q_{\mp}$ in terms of the Wigner system ladder operators:

$$
\begin{aligned}
& Q_{-}=\frac{1}{2}\left(1-\Sigma_{3}\right) a^{-}(\vec{\sigma} \cdot \vec{L}+\mathbf{1}) \\
& Q_{+}=Q_{-}^{+}=\frac{1}{2}\left(1+\Sigma_{3}\right) a^{+}(\vec{\sigma} \cdot \vec{L}+\mathbf{1}), \quad\left(Q_{-}\right)^{2}=\left(Q_{+}\right)^{2}=0 .
\end{aligned}
$$

In this Section, we consider the 3D isotropic spin- $\frac{1}{2}$ oscillator Hamiltonian in the bosonic sector of a Wigner system

$$
H_{-}(\overrightarrow{\boldsymbol{\sigma}} \cdot \vec{L})=\frac{1}{2}\left(p^{2}+r^{2}\right)=\frac{1}{2}\left(-\frac{\partial^{2}}{\partial r^{2}}-\frac{2}{r} \frac{\partial}{\partial r}+\frac{1}{r^{2}}(\vec{\sigma} \cdot \vec{L})(\vec{\sigma} \cdot \vec{L}+\mathbf{1})+r^{2}\right) \quad(0<r<\infty)
$$

for a non-relativistic 3D isotropic oscillator with spin- $\frac{1}{2}$ represented here by $\frac{1}{2} \sigma$. With the use of the following familiar spin- $\frac{1}{2}$ equalities:

$$
\begin{aligned}
& \vec{\sigma} \cdot \vec{p}=\sigma_{r} p_{r}+\frac{i}{r} \sigma_{r}(\vec{\sigma} \cdot \vec{L}+\mathbf{1}), \quad p_{r}=-i\left(\frac{1}{r}+\frac{\partial}{\partial r}\right)=\frac{1}{r}\left(-i \frac{\partial}{\partial r}\right) r=p_{r}^{\dagger} \\
& \sigma_{r}=\frac{1}{r} \vec{\sigma} \cdot \vec{r}, \quad \sigma_{r}^{2}=1, \quad\left[\sigma_{r}, \vec{\sigma} \cdot \vec{L}+\mathbf{1}\right]_{+}=0, \quad L^{2}=\vec{\sigma} \cdot \vec{L}(\vec{\sigma} \cdot \vec{L}+\mathbf{1}) .
\end{aligned}
$$

The 3D fermionic sector Hamiltonian becomes 


$$
H_{+}(\vec{\sigma} \cdot \vec{L})=H_{-}(\vec{\sigma} \cdot \vec{L}+\mathbf{1})=\frac{1}{2}\left(-\frac{\partial^{2}}{\partial r^{2}}-\frac{2}{r} \frac{\partial}{\partial r}+\frac{1}{r^{2}}(\vec{\sigma} \cdot \vec{L}+\mathbf{1})(\vec{\sigma} \cdot \vec{L}+\mathbf{2})+r^{2}\right) .
$$

In this case the connection between $H_{S S}$ and $H_{\mathrm{W}}$ is given by

$$
H_{S S}=H_{\mathrm{W}}-\frac{1}{2} \Sigma_{3}\left\{1+2(\vec{\sigma} \cdot \vec{L}+\mathbf{1}) \Sigma_{3}\right\}
$$

where $H_{\mathrm{W}}=\operatorname{diag}\left(\tilde{H}_{-}(\vec{\sigma} \cdot \vec{L}), \tilde{H}_{+}(\vec{\sigma} \cdot \vec{L})\right)$ is the diagonal 3D Wigner Hamiltonian given by

$$
H_{\mathrm{W}}=H_{\mathrm{W}}(\vec{\sigma} \cdot \vec{L}+\mathbf{1})=\left(\begin{array}{cc}
H_{-}(\vec{\sigma} \cdot \vec{L}) & 0 \\
0 & H_{-}(\vec{\sigma} \cdot \vec{L}+\mathbf{1})
\end{array}\right)
$$

Indeed from the role of $a^{+}$as the energy step-up operator, the complete excited state wave functions $\Psi_{w}^{n}$ are readily given by the step up operation with $a^{+}$:

$$
\psi_{\mathrm{W}}^{(n)} \propto\left(a^{+}\right)^{n} \psi_{\mathrm{W},+}^{(0)}(r, \theta, \varphi)=\left(a^{+}\right)^{n} \widetilde{R}_{1,+}^{(0)}(r)\left(\begin{array}{l}
1 \\
0
\end{array}\right) y_{+}(\theta, \varphi)
$$

On the eigenspaces of the operator $(\vec{\sigma} \cdot \vec{L}+1)$, the 3D Wigner algebra gets reduced to a 1D from with $(\vec{\sigma} \cdot \vec{L}+1)$ replaced by its eigenvalue $\mp(\ell+1), \ell=0,1,2, \ldots$, where $\ell$ is the orbital angular momentum quantum number. The eigenfunctions of $(\vec{\sigma} \cdot \vec{L}+\mathbf{1})$ for the eigenvalues $(\ell+1)$ and $-(\ell+1)$ are respectively given by the well known spin-spherical harmonic $y_{\mp}$. Thus, from the super-realized first order ladder operators given by

$$
a^{ \pm}(\ell+1)=\frac{1}{\sqrt{2}}\left\{ \pm \frac{d}{d r} \pm \frac{(\ell+1)}{r} \Sigma_{3}-r\right\} \Sigma_{1}
$$

where $\frac{c}{2}=\ell+1$, the Wigner Hamiltonian becomes

$$
\begin{aligned}
H(\ell+1) & =\frac{1}{2}\left[a^{+}(\ell+1), a^{-}(\ell+1)\right]_{+} \\
& =\left(\begin{array}{cc}
H_{-}(\ell) & 0 \\
0 & H_{+}(\ell)=H_{-}(\ell+1)
\end{array}\right),
\end{aligned}
$$

where in the representation of the radial part wave functions, $\chi(r)=r R(r)$, the even and odd sector Hamiltonians are respectively given by 


$$
H_{-}(\ell)=\frac{1}{2}\left\{-\frac{d^{2}}{d r^{2}}+r^{2}+\frac{1}{r^{2}} \ell(\ell+1)\right\}
$$

and

$$
H_{+}(\ell)=\frac{1}{2}\left\{-\frac{d^{2}}{d r^{2}}+r^{2}+\frac{1}{r^{2}}(\ell+1)(\ell+2)\right\}=H_{-}(\ell+1) .
$$

In this representation the eigenvalue equation becomes

$$
H_{ \pm}(\ell) \chi(r)=E_{ \pm}(\ell) \chi(r), \quad \chi(r)=r R(r)
$$

The WH algebra ladder relations are readily obtained as

$$
\left[H(\ell+1), a^{ \pm}(\ell+1)\right]_{-}= \pm a^{ \pm}(\ell+1) .
$$

Equations (35) and (39) together with the commutation relation

$$
\left[a^{-}(\ell+1), a^{+}(\ell+1)\right]_{-}=1+2(\ell+1) \Sigma_{3}
$$

constitute the $\mathrm{WH}$ algebra.

The Wigner eigenfunctions that generate the eigenspace associated with even(odd) $\sigma_{3}$-parity for even(odd) quanta $n=2 m(n=2 m+1)$ are given by

$$
\left|n=2 m, \ell+1>=\left(\begin{array}{c}
\mid m, \ell> \\
0
\end{array}\right), \quad\right| n=2 m+1, \ell>=\left(\begin{array}{c}
0 \\
\mid m, \ell>
\end{array}\right)
$$

and satisfy the following eigenvalue equation

$$
H(\ell+1)\left|n, \ell+1>=E^{(n)}\right| n, \ell+1>,
$$

the non-degenerate energy eigenvalues are obtained by the application of the raising operator on the ground eigenstate and are given by

$$
\psi_{W}^{(n)}(r) \propto\left(a^{+}\right)^{n} \psi_{W,+}^{(0)}(r)=\left(a^{+}\right)^{n} \chi_{1,+}^{(0)}(r)\left(\begin{array}{l}
1 \\
0
\end{array}\right)
$$

and 


$$
E^{(n)}=\ell+\frac{3}{2}+n, \quad n=0,1,2, \ldots
$$

The ground state energy eigenvalue is determined by the annihilation condition which reads as:

$$
a^{-} \psi_{\mathrm{w},+}^{(0)}=0, \quad(\vec{\sigma} \cdot \vec{L}+\mathbf{1}) \rightarrow \ell+1 ;
$$

which, after operation on the fermion spinors and the spin-angular part, turns into

$$
\left(\begin{array}{l}
\exp \left(\frac{r^{2}}{2}\right) r^{-\ell-1} \chi_{1,+}^{(0)}(r) \\
\exp \left(\frac{r^{2}}{2}\right) r^{-\ell+1} \chi_{2,+}^{(0)}(r)
\end{array}\right)=\left(\begin{array}{l}
c_{1} \\
c_{2}
\end{array}\right)
$$

Retaining only the non-singular and normalizable $R_{1,+}^{(0)}(r)$, we simply take the singular solution $R_{2,+}^{(0)}(r)$, which is physically non-existing, as identically zero. Hence the Wigner's eigenfunction of the ground state becomes

$$
\psi_{\mathrm{W},+}^{(0)}=\left(\begin{array}{c}
\chi_{1,+}^{(0)}(r) y_{+} \\
0
\end{array}\right), \quad \chi_{1,+}^{(0)}(r) \propto r^{\ell+1} \exp \left(-\frac{r^{2}}{2}\right)
$$

where $0<r<\infty$. For the radial oscillator the energy eigenvectors satisfy the following eigenvalue equations

$$
H_{-}(\ell)\left|m, \ell>=E_{-}^{(m)}\right| m, \ell>,
$$

where the eigenvalues are exactly constructed via WH algebra ladder relations and are given by

$$
E_{-}^{(m)}=\ell+\frac{3}{2}+2 m, \quad m=0,1,2, \ldots
$$

We stress that similar results can be adequately extended for any physical D-dimensional radial oscillator system by the Hermitian replacement of $-i\left(\frac{d}{d r}+\frac{1}{r}\right) \rightarrow-i\left(\frac{d}{d r}+\frac{D-1}{2 r}\right)$ and the Wigner deformation parameter $\ell+1 \rightarrow \ell_{D}+\frac{1}{2}(D-1)$ where $\ell_{D}\left(\ell_{D}=0,1,2, \cdots\right)$ is the D-dimensional oscillator angular momentum.

\section{The constrained Super Wigner Oscillator in $4 D$ and the hydrogen atom}

In this section, the complete spectrum for the hydrogen atom is found with considerable simplicity. Indeed, the solutions of the time-independent Schrödinger equation for the hydrogen atom were mapped onto the super Wigner harmonic oscillator in $4 D$ by using the Kustaanheimo-Stiefel transformation. The Kustaanheimo-Stiefel mapping yields the Schrödinger equation for the hydrogen atom that has been exactly solved and well-studied in the literature. (See for example, [16].) 
Kostelecky, Nieto and Truax have studied in a detailed manner the relation of the SUSY Coulombian problem in D-dimensions with that of SUSY isotropic oscillators in D-dimensions in the radial version. (See also Lahiri et. al. [2].)

The bosonic sector of the above eigenvalue equation can immediately be identified with the eigenvalue equation for the Hamiltonian of the 3D Hydrogen-like atom expressed in the equivalent form given by [16]

The usual isotropic oscillator in $4 D$ has the following eigenvalue equation for it's Hamiltonian $H_{\mathrm{OSC}}^{B}$, described by (employing natural system of units $\hbar=m=1$ ) time-independent Schrödinger equation

$$
H_{o s c}^{\mathrm{B}} \Psi_{o s c}^{\mathrm{B}}(y)=E_{o s c}^{\mathrm{B}} \Psi_{o s c}^{\mathrm{B}}(y)
$$

with

$$
\begin{gathered}
H_{\text {osc }}^{\mathrm{B}}=-\frac{1}{2} \nabla_{4}^{2}+\frac{1}{2} s^{2}, \quad s^{2}=\Sigma_{i=1}^{4} y_{i}^{2}, \\
\nabla_{4}^{2}=\frac{\partial^{2}}{\partial y_{1}^{2}}+\frac{\partial^{2}}{\partial y_{2}^{2}}+\frac{\partial^{2}}{\partial y_{3}^{2}}+\frac{\partial^{2}}{\partial y_{4}^{2}}=\sum_{i=1}^{4} \frac{\partial^{2}}{\partial y_{i}^{2}},
\end{gathered}
$$

where the superscript $B$ in $H_{\mathrm{OSC}}^{B}$ is in anticipation of the Hamiltonian, with constraint to be defined, being implemented in the bosonic sector of the super $4 D$ Wigner system with unitary frequency. Changing to spherical coordinates in 4-space dimensions, allowing a factorization of the energy eigenfunctions as a product of a radial eigenfunction and spin-spherical harmonic.

In (52), the coordinates $y_{i}(i=1,2,3,4)$ in spherical coordinates in $4 \mathrm{D}$ are defined by $[26,29]$

$$
\begin{aligned}
& y_{1}=s \cos \left(\frac{\theta}{2}\right) \cos \left(\frac{\varphi-\omega}{2}\right) \\
& y_{2}=s \cos \left(\frac{\theta}{2}\right) \sin \left(\frac{\varphi-\omega}{2}\right) \\
& y_{3}=s \sin \left(\frac{\theta}{2}\right) \cos \left(\frac{\varphi+\omega}{2}\right) \\
& y_{4}=s \sin \left(\frac{\theta}{2}\right) \sin \left(\frac{\varphi+\omega}{2}\right),
\end{aligned}
$$

where $0 \leq \theta \leq \pi, \quad 0 \leq \varphi \leq 2 \pi$ and $0 \leq \omega \leq 4 \pi$.

The mapping of the coordinates $y_{i}(i=1,2,3,4)$ in $4 D$ with the Cartesian coordinates $\rho_{i}(i=$ $1,2,3)$ in $3 D$ is given by the Kustaanheimo-Stiefel transformation 


$$
\begin{aligned}
& \rho_{i}=\sum_{a, b=1}^{2} z_{a}^{*} \Gamma_{a b}^{i} z_{b}, \quad(i=1,2,3) \\
& z_{1}=y_{1}+i y_{2}, \quad z_{2}=y_{3}+i y_{4},
\end{aligned}
$$

where the $\Gamma_{a b}^{i}$ are the elements of the usual Pauli matrices. If one defines $z_{1}$ and $z_{2}$ as in Eq. (55), $Z=\left(\begin{array}{l}z_{1} \\ z_{2}\end{array}\right)$ is a two dimensional spinor of $S U(2)$ transforming as $Z \rightarrow Z^{\prime}=U Z$ with $U$ a two-by-two matrix of $S U(2)$ and of course $Z^{\dagger} Z$ is invariant. So the transformation (54) is very spinorial. Also, using the standard Euler angles parametrizing $S U(2)$ as in transformations (53) and (55) one obtains

$$
\begin{aligned}
& z_{1}=s \cos \left(\frac{\theta}{2}\right) e^{\frac{i}{2}(\varphi-\omega)} \\
& z_{2}=s \sin \left(\frac{\theta}{2}\right) e^{\frac{i}{2}(\varphi+\omega)} .
\end{aligned}
$$

Note that the angles in these equations are divided by two. However, in $3 \mathrm{D}$, the angles are not divided by two, viz., $\rho_{3}=\rho \cos ^{2}\left(\frac{\theta}{2}\right)-\rho \sin ^{2}\left(\frac{\theta}{2}\right)=\rho \cos \theta$. Indeed, from (54) and (56), we obtain

$$
\rho_{1}=\rho \sin \theta \cos \varphi, \quad \rho_{2}=\rho \sin \theta \sin \varphi, \quad \rho_{3}=\rho \cos \theta
$$

and also that

$$
\begin{aligned}
\rho & =\left\{\rho_{1}^{2}+\rho_{2}^{2}+\rho_{3}^{2}\right\}^{\frac{1}{2}}=\left\{\left(\rho_{1}+i \rho_{2}\right)\left(\rho_{1}-i \rho_{2}\right)+\rho_{3}^{2}\right\}^{\frac{1}{2}} \\
& =\left\{\left(2 z_{1}^{*} z_{2}\right)\left(2 z_{1} z_{2}^{*}\right)+\left(z_{1}^{*} z_{1}-z_{2}^{*} z_{2}\right)^{2}\right\}^{\frac{1}{2}} \\
& =\left(z_{1} z_{1}^{*}+z_{2} z_{2}^{*}\right)=\sum_{i=1}^{4} y_{i}^{2}=s^{2} .
\end{aligned}
$$

The complex form of the Kustaanheimo-Stiefel transformation was given by Cornish [27]. Thus, the expression for $H_{o s c}^{\mathrm{B}}$ in (51) can be written in the form 


$$
\begin{aligned}
H_{o s c}^{\mathrm{B}} & =-\frac{1}{2}\left(\frac{\partial^{2}}{\partial s^{2}}+\frac{3}{s} \frac{\partial}{\partial s}\right) \\
& -\frac{2}{s^{2}}\left[\frac{1}{\sin \theta} \frac{\partial}{\partial \theta} \sin \theta \frac{\partial}{\partial \theta}+\frac{1}{\sin ^{2} \theta} \frac{\partial^{2}}{\partial \varphi^{2}}+\frac{1}{\sin ^{2} \theta}\left(2 \cos \theta \frac{\partial}{\partial \varphi}+\frac{\partial}{\partial \omega}\right) \frac{\partial}{\partial \omega}\right]+\frac{1}{2} s^{2} .
\end{aligned}
$$

We obtain a constraint by projection (or "dimensional reduction") from four to three dimensional. Note that $\psi_{o s c}^{\mathrm{B}}$ is independent of $\omega$ provides the constraint condition

$$
\frac{\partial}{\partial \omega} \Psi_{o s c}^{\mathrm{B}}(s, \theta, \varphi)=0
$$

imposed on $H_{o s c}^{\mathrm{B}}$, the expression for this restricted Hamiltonian, which we continue to call as $H_{o s c}^{\mathrm{B}}$, becomes

$$
H_{o s c}^{\mathrm{B}}=-\frac{1}{2}\left(\frac{\partial^{2}}{\partial s^{2}}+\frac{3}{s} \frac{\partial}{\partial s}\right)-\frac{2}{s^{2}}\left[\frac{1}{\sin \theta} \frac{\partial}{\partial \theta} \sin \theta \frac{\partial}{\partial \theta}+\frac{1}{\sin ^{2} \theta} \frac{\partial^{2}}{\partial \varphi^{2}}\right]+\frac{1}{2} s^{2} .
$$

Identifying the expression in bracket in (61) with $L^{2}$, the square of the orbital angular momentum operator in $3 D$, since we always have

$$
L^{2}=(\vec{\sigma} \cdot \vec{L})(\vec{\sigma} \cdot \vec{L}+1),
$$

which is valid for any system, where $\sigma_{i}(i=1,2,3)$ are the Pauli matrices representing the spin $\frac{1}{2}$ degrees of freedom, we obtain for $H_{o s c}^{\mathrm{B}}$ the final expression

$$
H_{o s c}^{\mathrm{B}}=\frac{1}{2}\left[-\left(\frac{\partial^{2}}{\partial s^{2}}+\frac{3}{s} \frac{\partial}{\partial s}\right)+\frac{4}{s^{2}}(\vec{\sigma} \cdot \vec{L})(\vec{\sigma} \cdot \vec{L}+1)+s^{2}\right]
$$

Now, associating $H_{o s c}^{\mathrm{B}}$ with the bosonic sector of the super Wigner system, $H_{\mathrm{W}}$, subject to the same constraint as in (60), and following the analogy with the Section II of construction of super Wigner systems, we first must solve the Schrödinger equation

$$
H_{\mathrm{W}} \Psi_{\mathrm{W}}(s, \theta, \varphi)=E_{\mathrm{W}} \Psi_{\mathrm{W}}(s, \theta, \varphi),
$$

where the explicit form of $H_{\mathrm{W}}$ is given by 


$$
\begin{aligned}
& H_{\mathrm{W}}\left(2 \vec{\sigma} \cdot \vec{L}+\frac{3}{2}\right)= \\
& \left(\begin{array}{cc}
-\frac{1}{2}\left(\frac{\partial}{\partial s}+\frac{3}{2 s}\right)^{2}+\frac{1}{2} s^{2}+\frac{\left(2 \vec{\sigma} \cdot \vec{L}+\frac{1}{2}\right)\left(2 \vec{\sigma} \cdot \vec{L}+\frac{3}{2}\right)}{2 s^{2}} & 0 \\
0 & -\frac{1}{2}\left(\frac{\partial}{\partial s}+\frac{3}{2 s}\right)^{2}+\frac{1}{2} s^{2}+\frac{\left(2 \vec{\sigma} \cdot \vec{L}+\frac{3}{2}\right)\left(2 \vec{\sigma} \cdot \vec{L}+\frac{5}{2}\right)}{2 s^{2}}
\end{array}\right) \cdot(65
\end{aligned}
$$

Using the operator technique in references $[9,10]$, we begin with the following super-realized mutually adjoint operators

$$
a_{\mathrm{W}}^{ \pm} \equiv a^{ \pm}\left(2 \vec{\sigma} \cdot \vec{L}+\frac{3}{2}\right)=\frac{1}{\sqrt{2}}\left[ \pm\left(\frac{\partial}{\partial s}+\frac{3}{2 s}\right) \Sigma_{1} \mp \frac{1}{s}\left(2 \vec{\sigma} \cdot \vec{L}+\frac{3}{2}\right) \Sigma_{1} \Sigma_{3}-\Sigma_{1} s\right]
$$

where $\Sigma_{i}(i=1,2,3)$ constitute a set of Pauli matrices that provide the fermionic coordinates commuting with the similar Pauli set $\sigma_{i}(i=1,2,3)$ already introduced representing the spin $\frac{1}{2}$ degrees of freedom.

It is checked, after some algebra, that $a^{+}$and $a^{-}$of (66) are indeed the raising and lowering operators for the spectra of the super Wigner Hamiltonian $H_{\mathrm{W}}$ and they satisfy the following (anti-)commutation relations of the WH algebra:

$$
\begin{aligned}
& H_{\mathrm{W}}=\frac{1}{2}\left[a_{\mathrm{W}}^{-}, a_{\mathrm{W}}^{+}\right]_{+} \\
& =a_{\mathrm{W}}^{+} a_{\mathrm{W}}^{-}+\frac{1}{2}\left[1+2\left(2 \vec{\sigma} \cdot \vec{L}+\frac{3}{2}\right) \Sigma_{3}\right] \\
& =a_{\mathrm{W}}^{-} a_{\mathrm{W}}^{+}-\frac{1}{2}\left[1+2\left(2 \vec{\sigma} \cdot \vec{L}+\frac{3}{2}\right) \Sigma_{3}\right] \\
& {\left[H_{\mathrm{W}}, a_{\mathrm{W}}^{ \pm}\right]_{-}= \pm a_{\mathrm{W}}^{ \pm}} \\
& {\left[a_{\mathrm{W}}^{-}, a_{\mathrm{W}}^{+}\right]_{-}=1+2\left(2 \vec{\sigma} \cdot \vec{L}+\frac{3}{2}\right) \Sigma_{3}} \\
& {\left[\Sigma_{3}, a_{\mathrm{W}}^{ \pm}\right]_{+}=0 \Rightarrow\left[\Sigma_{3}, H_{\mathrm{W}}\right]_{-}=0 .}
\end{aligned}
$$


Since the operator $\left(2 \vec{\sigma} \cdot \vec{L}+\frac{3}{2}\right)$ commutes with the basic elements $a^{ \pm}, \Sigma_{3}$ and $H_{\mathrm{W}}$ of the WH algebra (67), (68) and (69) it can be replaced by its eigenvalues $\left(2 \ell+\frac{3}{2}\right)$ and $-\left(2 \ell+\frac{5}{2}\right)$ while acting on the respective eigenspace in the from

$$
\Psi_{\mathrm{Osc}}(s, \theta, \varphi)=\left(\begin{array}{c}
\Psi_{\mathrm{OSC}}^{\mathrm{B}}(s, \theta, \varphi) \\
\Psi_{\mathrm{OSC}}^{\mathrm{F}}(s, \theta, \varphi)
\end{array}\right)=\left(\begin{array}{l}
R_{\mathrm{OSC}}^{\mathrm{B}}(s) \\
R_{\mathrm{OSC}}^{\mathrm{F}}(s)
\end{array}\right) y_{ \pm}(\theta, \varphi)
$$

in the notation where $y_{ \pm}(\theta, \varphi)$ are the spin-spherical harmonics [43],

$$
\begin{aligned}
& y_{+}(\theta, \varphi)=y_{\ell \frac{1}{2} ; j=\ell+\frac{1}{2}, m_{j}}(\theta, \varphi) \\
& y_{-}(\theta, \varphi)=y_{\ell+1 \frac{1}{2} ; j=(\ell+1)-\frac{1}{2}, m_{j}}(\theta, \varphi)
\end{aligned}
$$

so that, we obtain: $(\vec{\sigma} \cdot \vec{L}+1) y_{ \pm}= \pm(\ell+1) y_{ \pm}, \quad\left(2 \vec{\sigma} \cdot \vec{L}+\frac{3}{2}\right) y_{+}=\left(2 \ell+\frac{3}{2}\right) y_{+}$and $(2 \vec{\sigma} \cdot \vec{L}+$ $\left.\frac{3}{2}\right) y_{-}=-\left[2(\ell+1)+\frac{1}{2}\right] y_{-}$. Note that on these subspaces the $3 \mathrm{D} \mathrm{WH}$ algebra is reduced to a formal $1 \mathrm{D}$ radial form with $H_{\mathrm{W}}\left(2 \vec{\sigma} \cdot \vec{L}+\frac{3}{2}\right)$ acquiring respectively the forms $H_{\mathrm{W}}\left(2 \ell+\frac{3}{2}\right)$ and

$$
H_{\mathrm{W}}\left(-2 \ell-\frac{5}{2}\right)=\Sigma_{1} H_{\mathrm{W}}\left(2 \ell+\frac{3}{2}\right) \Sigma_{1}
$$

Thus, the positive finite form of $H_{\mathrm{W}}$ in (67) together with the ladder relations (68) and the form (69) leads to the direct determination of the state energies and the corresponding Wigner ground state wave functions by the simple application of the annihilation conditions

$$
a^{-}\left(2 \ell+\frac{3}{2}\right)\left(\begin{array}{l}
R_{\mathrm{OSC}}^{B^{(0)}}(s) \\
R_{\mathrm{OSC}}^{F^{(0)}}(s)
\end{array}\right)=0 .
$$

Then, the complete energy spectrum for $H_{\mathrm{W}}$ and the whole set of energy eigenfunctions $\Psi_{\mathrm{OSC}}^{(n)}(s, \theta, \varphi)(n=2 m, 2 m+1, m=0,1,2, \cdots)$ follows from the step up operation provided by $a^{+}\left(2 \ell+\frac{3}{2}\right)$ acting on the ground state, which are also simultaneous eigenfunctions of the fermion number operator $N=\frac{1}{2}\left(\mathbf{1}-\Sigma_{3}\right)$. We obtain for the bosonic sector Hamiltonian $H_{\mathrm{OSC}}^{B}$ with fermion number $n_{f}=0$ and even orbital angular momentum $\ell_{4}=2 \ell,(\ell=0,1,2, \ldots)$, the complete energy spectrum and eigenfunctions given by

$$
\left[E_{\text {osc }}^{B}\right]_{\ell_{4}=2 \ell}^{(m)}=2 \ell+2+2 m, \quad(m=0,1,2, \ldots),
$$




$$
\left[\Psi_{o s c}^{B}(s, \theta, \varphi)\right]_{\ell_{4}=2 \ell}^{(m)} \propto s^{2 \ell} \exp \left(-\frac{1}{2} s^{2}\right) L_{m}^{(2 \ell+1)}\left(s^{2}\right)\left\{\begin{array}{l}
y_{+}(\theta, \varphi) \\
y_{-}(\theta, \varphi)
\end{array}\right.
$$

where $L_{m}^{\alpha}\left(s^{2}\right)$ are generalized Laguerre polynomials [9]. Now, to relate the mapping of the 4D super Wigner system with the corresponding system in 3D, we make use of the substitution of $s^{2}=\rho$, Eq. (60) and the following substitutions

$$
\frac{\partial}{\partial s}=2 \sqrt{\rho} \frac{\partial}{\partial \rho}, \quad \frac{\partial^{2}}{\partial s^{2}}=4 \rho \frac{\partial^{2}}{\partial \rho^{2}}+2 \frac{\partial}{\partial \rho},
$$

in (65) and divide the eigenvalue equation for $H_{\mathrm{W}}$ in (64) by $4 s^{2}=4 \rho$, obtaining

$$
\begin{aligned}
& \left(\begin{array}{cc}
-\frac{1}{2}\left(\frac{\partial^{2}}{\partial \rho^{2}}+\frac{2}{\rho} \frac{\partial}{\partial \rho}\right)-\frac{1}{2}\left[-\frac{1}{4}-\frac{\vec{\sigma} \cdot \vec{L}(\vec{\sigma} \cdot \vec{L}+1)}{\rho^{2}}\right] & 0 \\
0 & \left.-\frac{1}{2}\left(\frac{\partial^{2}}{\partial \rho^{2}}+\frac{2}{\rho} \frac{\partial}{\partial \rho}\right)-\frac{1}{2}\left[-\frac{1}{4}-\frac{\left(\vec{\sigma} \cdot \vec{L}+\frac{1}{2}\right)\left(\vec{\sigma} \cdot \vec{L}+\frac{3}{2}\right)}{\rho^{2}}\right]\right)
\end{array}\right)\left(\begin{array}{l}
\Psi B \\
\Psi^{F}
\end{array}\right) \\
& =\frac{1}{4 \rho} E_{\mathrm{W}}\left(\begin{array}{l}
\Psi^{B} \\
\Psi^{F}
\end{array}\right) .
\end{aligned}
$$

The bosonic sector of the above eigenvalue equation can immediately be identified with the eigenvalue equation for the Hamiltonian of the 3D Hydrogen-like atom expressed in the equivalent form given by

$$
\left\{-\frac{1}{2}\left(\frac{\partial^{2}}{\partial \rho^{2}}+\frac{2}{\rho} \frac{\partial}{\partial \rho}\right)-\frac{1}{2}\left[-\frac{1}{4}-\frac{\vec{\sigma} \cdot \vec{L}(\vec{\sigma} \cdot \vec{L}+1)}{\rho^{2}}\right]\right\} \psi(\rho, \theta, \varphi)=\frac{\ell}{2 \rho} \psi(\rho, \theta, \varphi),
$$

where $\Psi^{B}=\psi(\rho, \theta, \varphi)$ and the connection between the dimensionless and dimensionfull eigenvalues, respectively, $\ell$ and $E_{a}$ with $e=1=m=\hbar$ is given by [43]

$$
\ell=\frac{Z}{\sqrt{-2 E_{a}}}, \quad \rho=\alpha r, \quad \alpha=\sqrt{-8 E_{a}},
$$

where $E_{a}$ is the energy of the electron Hydrogen-like atom, $(r, \theta, \varphi)$ stand for the spherical polar coordinates of the position vector $\vec{r}=\left(x_{1}, x_{2}, x_{3}\right)$ of the electron in relative to the nucleons of charge $Z$ together with $s^{2}=\rho$. We see then from equations (75), (76), (79) and (80) that the complete energy spectrum and eigenfunctions for the Hydrogen-like atom given by

$$
\frac{\lambda}{2}=\frac{E_{o s C}^{B}}{4} \Rightarrow\left[E_{a}\right]_{\ell}^{(m)}=\left[E_{a}\right]^{(N)}=-\frac{Z^{2}}{2 N^{2}}, \quad(N=1,2, \ldots)
$$


and

$$
[\psi(\rho, \theta, \varphi)]_{\ell ; æ, m_{j}}^{(m)} \propto \rho^{\ell} \exp \left(-\frac{\rho}{2}\right) L_{m}^{(2 \ell+1)}(\rho)\left\{\begin{array}{l}
y_{+}(\theta, \varphi) \\
y_{-}(\theta, \varphi)
\end{array}\right.
$$

where $E_{\text {osc }}^{B}$ is given by Eq. (75).

Here, $N=\ell+m+1(\ell=0,1,2, \cdots, N-1 ; m=0,1,2, \cdots)$ is the principal quantum number. Kostelecky and Nieto shown that the supersymmetry in non-relativistic quantum mechanics may be realized in atomic systems [44].

\section{The superconformal quantum mechanics from WH algebra}

The superconformal quantum mechanics has been examined in [35]. Another application for these models is in the study of the radial motion of test particle near the horizon of extremal Reissner-Nordström black holes [35, 37]. Also, another interesting application of the superconformal symmetry is the treatment of the Dirac oscillator, in the context of the superconformal quantum mechanics [39-42, 46].

In this section we introduce the explicit supersymmetry for the conformal Hamiltonian in the WH-algebra picture. Let us consider the supersymmetric generalization of $H$, given by

$$
\mathcal{H}=\frac{1}{2}\left\{Q_{c}, Q_{c}^{\dagger}\right\},
$$

where the new supercharge operators are given in terms of the momentum Yang representation

$$
\begin{aligned}
& Q_{c}=\left(-i p_{x}+\frac{\sqrt{g}}{x}\right) \Psi^{\dagger}, \\
& Q_{c}^{\dagger}=\Psi\left(i p_{x}+\frac{\sqrt{g}}{x}\right),
\end{aligned}
$$

with $\Psi$ and $\Psi^{\dagger}$ being Grassmannian operators so that its anticommutator is $\left\{\Psi, \Psi^{\dagger}\right\}=\Psi \Psi^{\dagger}+$ $\Psi^{\dagger} \Psi=1$.

Explicitly the superconformal Hamiltonian becomes

$$
\mathcal{H}=\frac{1}{2}\left(\mathbf{1} p_{x}^{2}+\frac{\mathbf{1} g+\sqrt{g} B(1-c \mathbf{P})}{x^{2}}\right)
$$

where $B=\left[\Psi^{\dagger}, \Psi\right]_{-}$, so that the parity operator is conserved, i.e., $[\mathcal{H}, \mathbf{P}]_{-}=0$.

When one introduces the following operators 


$$
\begin{aligned}
S & =x \Psi^{\dagger}, \\
S^{\dagger} & =\Psi x,
\end{aligned}
$$

it can be shown that these operators together with the conformal quantum mechanics operators $D$ and $K$

$$
\begin{aligned}
D & =\frac{1}{2}\left(x p_{x}+p_{x} x\right), \\
K & =\frac{1}{2} x^{2},
\end{aligned}
$$

satisfy the deformed superalgebra $\operatorname{csp}(2 \mid 2)$ (Actually, this superalgebra is $\operatorname{ssp}(2 \mid 2)$ when we fix $\mathbf{P}=1$ or $\mathbf{P}=-1$.), viz.,

$$
\begin{aligned}
& {[\mathcal{H}, D]_{-}=-2 \imath \mathcal{H},} \\
& {[\mathcal{H}, K]_{-}=-\imath D \text {, }} \\
& {[K, D]_{-}=2 \imath K,} \\
& {\left[Q_{c}, Q_{c}^{\dagger}\right]_{+}=2 \mathcal{H} \text {, }} \\
& {\left[Q_{c}, S^{\dagger}\right]_{+}=-\imath D-\frac{1}{2} B(1+c \mathbf{P})+\sqrt{g},} \\
& {\left[Q_{c}^{+}, S\right]_{+}=\imath D-\frac{1}{2} B(1+c \mathbf{P})+\sqrt{g},} \\
& {\left[Q_{c}^{\dagger}, D\right]_{-}=-\imath Q_{c}^{+} \text {, }} \\
& {\left[Q_{c}^{\dagger}, K\right]_{-}=S^{\dagger} \text {, }} \\
& {\left[Q_{c}^{\dagger}, B\right]_{-}=2 Q_{c}^{\dagger} \text {, }} \\
& {\left[Q_{c}, K\right]_{-}=-S \text {, }} \\
& {\left[Q_{c}, B\right]_{-}=-2 Q_{c} \text {, }} \\
& {\left[Q_{c}, D\right]_{-}=-\imath Q_{c},} \\
& {[\mathcal{H}, S]_{-}=Q_{c}, \quad\left[\mathcal{H}, S^{\dagger}\right]_{-}=-Q_{c}^{\dagger} \text {, }} \\
& {\left[B, S^{\dagger}\right]_{-}=-2 S^{\dagger}, \quad[B, S]_{-}=2 S \text {, }} \\
& {[D, S]_{-}=-\imath S, \quad\left[D, S^{\dagger}\right]_{-}=-\imath S^{\dagger},} \\
& {\left[S^{\dagger}, S\right]_{+}=2 K \text {, }}
\end{aligned}
$$


where, $\mathcal{H}, D, K, B$ are bosonic operators and $Q_{c}, Q_{c}^{+}, S, S^{\dagger}$ are fermionic ones. The supersymmetric extension of the Hamiltonian $L_{0}$ (presented in the previous section) is

$$
\mathcal{H}_{0}=\frac{1}{2}(\mathcal{H}+K), \quad\left[\mathcal{H}_{0}, \mathbf{P}\right]_{-}=0 .
$$

In general, superconformal quantum mechanics has interesting applications in supersymmetric black holes, for example in the problem of a quantum test particle moving in the black hole geometry.

\section{Summary and conclusion}

In this chapter, firstly we start by summarizing the R-deformed Heisenberg algebra or Wigner-Heisenberg algebraic technique for the Wigner quantum oscillator, based on the super-realization of the ladder operators effective spectral resolutions of general oscillator-related potentials.

We illustrate the applications of our operator method to the cases of the Hamiltonians of an isotonic oscillator (harmonic plus a centripetal barrier) system and a 3D isotropic harmonic oscillator for spin $\frac{1}{2}$ embedded in the bosonic sector of a corresponding Wigner system.

Also, the energy eigenvalues and eigenfunctions of the hydrogen atom via Wigner-Heisenberg (WH) algebra in non-relativistic quantum mechanics, from the ladder operators for the 4-dimensional (4D) super Wigner system, ladder operators for the mapped super $3 D$ system, and hence for hydrogen-like atom in bosonic sector, are deduced. The complete spectrum for the hydrogen atom is found with considerable simplicity by using the Kustaanheimo-Stiefel transformation. From the ladder operators for the four-dimensional (4D) super-Wigner system, ladder operators for the mapped super 3D system, and hence for the hydrogen-like atom in bosonic sector, can be deduced. Results of present investigations on these ladder operators will be reported separately.

For future directions, such a direct algebraic method considered in this chapter proves highly profitable for simpler algebraic treatment, as we shall show in subsequent publications, of other quantum mechanical systems with underlying oscillator connections like for example those of a relativistic electron in a Coulomb potential or of certain 3D SUSY oscillator models of the type of Celka and Hussin. This SUSY model has been reported in nonrelativistic context by Jayaraman and Rodrigues [10]. We will also demonstrate elsewhere the application of our method for a spectral resolution complete of the Pöschl-Teller I and II potentials by virtue of their hidden oscillator connections using the $\mathrm{WH}$ algebra operator technique developed in this chapter.

In the work of the Ref. [46], we analyze the Wigner-Heisenberg algebra to bosonic systems in connection with oscillators and, thus, we find a new representation for the Virasoro algebra. Acting the annihilation operator(creation operator) in the Fock basis $\mid 2 m+1, \frac{c}{2}>\left(\left|2 m, \frac{c}{2}\right\rangle\right)$ the eigenvalue of the ground state of the Wigner oscillator appears only in the excited states associated with the even(odd) quanta. We show that only in the case associated with one even index and one odd index in the operator $L_{n}$ the Virasoro algebra is changed. 
Recently, we have analyzed the connection between the conformal quantum mechanics and the Wigner-Heisenberg (WH) algebra [46]. With an appropriate relationship between the coupling constant $g$ and Wigner parameter $c$ one can identify the Wigner Hamiltonian with the simple Calogero Hamiltonian.

The important result is that the introduction of the WH algebra in the conformal quantum mechanics is still consistent with the conformal symmetry, and a realization of superconformal quantum mechanics in terms of deformed $\mathrm{WH}$ algebra is discussed. The spectra for the Casimir operator and the Hamiltonian $L_{0}$ depend on the parity operator. The ladder operators depend on the parity operator, too. It is shown, for example, that the eigenvalues of Calogero-type Hamiltonian is dependent of the Wigner parameter $c$ and the eigenvalues of the parity operator $P$. When $c=0$ we obtain the usual conformal Hamiltonian structure.

We also investigated the supersymmetrization of this model, in that case we obtain a new spectrum for the supersymmetric Hamiltonian of the Calogero interaction's type.

In this case the spectra for the super-Casimir operator and the superhamiltonian depend also on the parity operator. Therefore, we have found a new realization of supersymmetric Calogero-type model on the quantum mechanics context in terms of deformed WH algebra.

\section{Author details}

Rafael de Lima Rodrigues

UFCG-Campus Cuité-PB, Brazil

\section{References}

[1] R. de Lima Rodrigues, "The Quantum Mechanics SUSY Algebra: an Introductory Review," Monograph CBPF-MO-03-01, hep-th/0205017; Witten E 1981 Nucl. Phys. B 185 513; Gendenshteĭn L É and Krive I V 1985 Sov. Phys. Usp. 28645.

[2] A. Lahiri, P. K. Roy and B. Bagchi, J. Phys. A: Math. Gen. 20, 5403 (1987).

[3] E. Drigo Filho e R. M. Ricota, Mod. Phys. Lett. A6, 2137, (1991).

[4] F. Cooper, A. Khare and U. Sukhatme, "Supersymmetry in quantum mechanics," World Scientific, Singapure, 2001; B. Bagchi, "Supersymmetry in quantum and classical mechanics," published by Chapman and Hall, Florida (USA), (2000); G. Junker, "Supersymmetric methods in quantum mechanics and statistical physics," Springer, Berlin (1996).

[5] E. P. Wigner, Phys. Rev. 77, 711 (1950).

[6] L. M. Yang, Phys. Rev. 84, 788 (1951).

[7] O'Raifeartaigh and C. Ryan, Proc. R. Irish Acad. A62, 93 (1963); Y. Ohnuki and S. Kamefuchi, J. Math. Phys. 19, 67 (1978); Y. Ohnuki and S. Watanabe, J. Math. Phys. 33, 3653, (1992). 
[8] Sharma J K, Mehta C L, Mukunda N and Sudarshan E C G 1981 J. Math. Phys. 22 78; Mukunda N, Sudarshan E C G, Sharma J K and Mehta C L 1980 J. Math. Phys. 21 2386; Sharma J K, Mehta C L and Sudarshan E C G 1978 J. Math. Phys. 192089

[9] J. Jayaraman and R. de Lima Rodrigues, J. Phys. A: Math. Gen. 23, 3123 (1990).

[10] J. Jayaraman and R. de Lima Rodrigues, Mod. Phys. Lett. A9, 1047 (1994).

[11] S. M. Plyushchay, Int. J. Mod. Phys. A15, 3679 (2000).

[12] J. Jayaraman, R. de Lima Rodrigues and A. N. Vaidya, J. Phys. A: Math. Gen. 32, 6643 (1999).

[13] A. O. Barut and L. Girardello, Commun. Math. Phys. 21, 41 (1971).

[14] A. M. Perelomov, Commun. Math. Phys. 26, 222 (1972); A. M. Perelomov Generalized Coherent States and Their Applications (Berlin Springer-Verlag, 1986).

[15] K. Fujii, Mod. Phys. Lett. 16A, 1277 (2001).

[16] R. de Lima Rodrigues, J. Phys. A: Math. Theor. 42, 355213 (2009), and references therein.

[17] V. G. Bagrov, J.-P. Gazeau, D M Gitman and A. D. Levin, J. Phys. A: Math. Theor. 45, 125306 (2012).

[18] I. A. Malkin and V. I. ManŠko, Dynamical Symmetries and Coherent States of Quantum Systems (Moscow: Nauka) p 320 (1979).

[19] J. P. Gazeau and J. Klauder, Coherent states for systems with discrete and continuous spectrum J. Phys. A: Math. Gen. 32123 (1999).

[20] R. C. King, T. D. Palev, N. I. Stoilova and J. Van der Jeugt, J. Phys. A: Math. Gen. 36, 4337 (2003), hep-th/0304136.

[21] A. Matos-Albiague, J. Phys. A: Math. Gen. 34, 11059 (2001).

[22] M. A. Lohe and A. Thilagam, J. Phys. A: Math. Gen. 38, 461 (2005).

[23] D. Bergmann and Y. Frishman, Y J. Math. Phys. 6, 1855 (1965).

[24] E. Cahill, J. Phys. A: Math. Gen. 23, 1519 (1990).

[25] P. Kustaanheimo and E. Stiefel, J. Reine Angew. Math. 218, 204 (1965).

[26] A. C. Chen, Phys. Rev. A22, 333 (1980); Erratum Ibid. A22, 2901 (1980).

[27] F. H. J. Cornish, J. Phys. A: Math. Gen. 17, 323 (1984).

[28] A. C. Chen and M. Kibler, Phys. Rev. A31, 3960 (1985).

[29] E. D'Hoker and L. Vinet, Nucl. Phys. B260, 79 (1985). 
[30] V. A. Kostelecky, M. M. Nieto and D. R. Truax, Phys. Rev. D32, 2627 (1985).

[31] R. D. Amado, Phys. Rev. A37, 2277 (1988).

[32] O. L. Lange and R. E. Raab, Operator Methods in Quantum Mechanics, Clarendon Press, Oxford University Press, New York (1991).

[33] R. D. Tangerman and J. A. Tjon, Phys. Rev. A48, 1089 (1993).

[34] R. C. King, N. I. Stoilova and J. Van der Jeugt, J. Phys. A: Math. Gen. 39, 5763 (2006).

[35] P. Claus, M. Derix, R. Kallosh, J. Kumar, P. Townsend and A. Van Proeyen, Phys. Rev. Lett. 81, 4553 (1998), hep-th/9804177.

[36] F. Calogero J. Math. Phys. 10, 2197 (1969).

[37] G. W. Gibbons and P. K. Townsend, Phys. Lett. B454, 187 (1999).

[38] J. A. de Azcarraga, J. M. Izquierdo, J. C. Perez Bueno, Phys. Rev. D59, 084015 (1999).

[39] M. Moshinsky and A. Szczepaniac, J. Phys. A: Math. Gen. 22, L817 (1989).

[40] R. P. Martínez y Romero, Matías Moreno and A. Zentella, Phys. Rev. D43, (1991) 2036.

[41] R. P. Martinez y Romero and A. L. Salas Brito, J. Math. Phys. 33, 1831 (1992).

[42] R. de Lima Rodrigues and A. N. Vaidya, Dirac oscillator via R-deformed Heisenberg algebra, Proceedings of the XXIII Brazilian National Meeting on Particles and Fields (October/2002), site www.sbfisica.org.br/eventos/enfpc/xxiii/procs/trb0013.pdf.

[43] P. M. Mathews and K. Venkatesan, A text book of quantum mechanics, Tata McGraw-Hill publishing company limited (1978).

[44] V. A. Kostelecky and M. M. Nieto, Phys. Rev. D32, 1293 (1985); Phys. Rev. Lett. 35, 2285 (1984).

[45] E. L. da Graça, H. L. Carrion and R. de Lima Rodrigues, Braz. J. Phys. 33, 333 (2003).

[46] H. L. Carrion and R. de Lima Rodrigues, Mod. Phys. Lett. 25A, 2507 (2010). 


\title{
New System-Specific Coherent States by Supersymmetric Quantum Mechanics for Bound State Calculations
}

\author{
Chia-Chun Chou, Mason T. Biamonte, \\ Bernhard G. Bodmann and Donald J. Kouri \\ Additional information is available at the end of the chapter \\ http://dx.doi.org/10.5772/54010
}

\section{Introduction}

Supersymmetric quantum mechanics (SUSY-QM) has been developed as an elegant analytical approach to one-dimensional problems. The SUSY-QM formalism generalizes the ladder operator approach used in the treatment of the harmonic oscillator. In analogy with the harmonic oscillator Hamiltonian, the factorization of a one-dimensional Hamiltonian can be achieved by introducing "charge operators". For the one-dimensional harmonic oscillator, the charge operators are the usual raising and lowering operators. The SUSY charge operators not only allow the factorization of a one-dimensional Hamiltonian but also form a Lie algebra structure. This structure leads to the generation of isospectral SUSY partner Hamiltonians. The eigenstates of the various partner Hamiltonians are connected by application of the charge operators. As an analytical approach, the SUSY-QM approach has been utilized to study a number of quantum mechanics problems including the Morse oscillator ([16]) and the radial hydrogen atom equation ([24]). In addition, SUSY-QM has been applied to the discovery of new exactly solvable potentials, the development of a more accurate WKB approximation, and the improvement of large $N$ expansions and variational methods ([7, 11]). Developments and applications of one-dimensional SUSY-QM can be found in relevant reviews and books ([7, 9, 11, 15, 26, 32, 33]). Recently, SUSY-QM has been developed as a computational tool to provide much more accurate excitation energies using the standard Rayleigh-Ritz variational method $([5,19,20])$.

The harmonic oscillator is fundamental to a wide range of physics, including the electromagnetic field, spectroscopy, solid state physics, coherent state theory, and SUSY-QM. 
The broad application of the harmonic oscillator stems from the raising and lowering ladder operators which are used to factor the system Hamiltonian. For example, canonical coherent states are defined as the eigenstates of the lowering operator of the harmonic oscillator, and they are also minimum uncertainty states which minimize the Heisenberg uncertainty product for position and momentum. In addition, several different approaches have been employed to study generalized and approximate coherent states for systems other than the harmonic oscillator $([3,12,17,18,27-31,34,37])$. Furthermore, algebraic treatments have been applied to the extension of coherent states for shape-invariant systems $([1,4,8,10])$.

The lowering operator of the harmonic oscillator annihilates the ground state, and the ground state minimizes the Heisenberg uncertainty product. Conventional harmonic oscillator coherent states correspond to those states which minimize the position-momentum uncertainty relation. However, these harmonic oscillator coherent states are also constructed by applying shift operators labeled with points of the discrete phase space to the ground state of the harmonic oscillator, termed the "fiducial state" ([18]). Indeed, Klauder and Skagerstam choose to define coherent states in the broadest sense in precisely this manner ([21]). Analogously, the charge operator in SUSY-QM annihilates the ground state of the corresponding system. We therefore expect that the ground state wave function should provide the ideal fiducial function for constructing efficient, overcomplete coherent states for computations of excited states of the system.

In our recent study ([6]), we construct system-specific coherent states for any bound quantum system by making use of the similarity between the treatment of the harmonic oscillator and SUSY-QM. First, since the charge operator annihilates the ground state, the superpotential that arises in SUSY-QM can be regarded as a SUSY-displacement operator or a generalized displacement variable. We show that the ground state for any bound quantum system minimizes the SUSY-displacement-standard momentum uncertainty product. Then, we use the ground state of the system as a fiducial function to generate new system-specific dynamically-adapted coherent states. Moreover, the discretized system-specific coherent states can be utilized as a dynamically-adapted basis for calculations of excited state energies and wave functions for bound quantum systems. Computational results demonstrate that these discretized system-specific coherent states provide more rapidly-converging expansions for excited state energies and wave functions than the conventional coherent states and the standard harmonic oscillator basis.

The organization of the remainder of this chapter is as follows. In Sec. 2, we briefly review the harmonic oscillator, conventional coherent states, and SUSY-QM. We also show that the ground state of a quantum system minimizes the SUSY-displacement-standard momentum uncertainty product. We then construct system-specific coherent states by applying shift operators to the ground state of the system. In Sec. 3, the discretized system-specific coherent state basis is developed for and applied to the Morse oscillator, the double well potential, and the two-dimensional anharmonic oscillator system for calculations of the excited state energies and wave functions. In Sec. 4, we summarize our results and conclude with some comments. 


\section{Theoretical formulation}

\subsection{Harmonic oscillator and conventional coherent states}

The Hamiltonian of the harmonic oscillator is expressed by

$$
H=-\frac{\hbar^{2}}{2 m} \frac{d^{2}}{d x^{2}}+\frac{1}{2} m \omega^{2} x^{2}
$$

where $m$ is the particle's mass, $\omega$ is the angular frequency of the oscillator. The Hamiltonian can be written in terms of the raising and lowering operators as

$$
H=\hbar \omega\left(\hat{a}^{\dagger} \hat{a}+\frac{1}{2}\right)
$$

where $\hat{a}^{\dagger}$ is the raising operator and $\hat{a}$ is the lowering operator. These two operators can be expressed in terms of the position operator $\hat{x}$ and its canonically conjugate momentum operator $\hat{p}_{x}$ by

$$
\begin{aligned}
& \hat{a}=\sqrt{\frac{m \omega}{2 \hbar}} \hat{x}+\frac{i \hat{p}_{x}}{\sqrt{2 m \hbar \omega}}, \\
& \hat{a}^{\dagger}=\sqrt{\frac{m \omega}{2 \hbar}} \hat{x}-\frac{i \hat{p}_{x}}{\sqrt{2 m \hbar \omega}} .
\end{aligned}
$$

Without loss of generality, we set $\hbar=2 m=1$ throughout this study and $\omega=2$ for this case. In particular, the ground state of the harmonic oscillator is annihilated by the lowering operator

$$
\hat{a} \psi_{0}=\frac{1}{\sqrt{2}}\left(\hat{x}+i \hat{p}_{x}\right) \psi_{0}=0 .
$$

By solving this differential equation in the position representation, we obtain the ground state wave function

$$
\psi_{0}(x)=\langle x \mid 0\rangle=N e^{-x^{2} / 2},
$$

where $N$ is the normalization constant.

One of the important properties for the ground state of the harmonic oscillator is that the ground state is a minimum uncertainty state, which minimizes the Heisenberg uncertainty product $\Delta \hat{x} \Delta \hat{p}_{x}$. The usual derivation of the Heisenberg uncertainty principle makes use of Schwarz's inequality ([25])

$$
\left\langle\psi\left|\hat{x}^{2}\right| \psi\right\rangle\left\langle\psi\left|\hat{p}_{x}^{2}\right| \psi\right\rangle \geq\left|\left\langle\psi\left|\hat{x} \hat{p}_{x}\right| \psi\right\rangle\right|^{2}
$$


where zero expectation values of the position and momentum operators are assumed for convenience. The equality holds for the state $|\psi\rangle$, which satisfies the condition

$$
\hat{x}|\psi\rangle=-i \sigma^{2} \hat{p}_{x}|\psi\rangle,
$$

where $\sigma^{2}$ is real and greater than zero. As noted in Eq. (5), the ground state of the harmonic oscillator satisfies the relation with $\sigma^{2}=1$, and hence it is a minimum uncertainty state. In fact, the ground state corresponds to a state centered in the phase space at $x=0$ and $k=0$. Harmonic oscillator coherent states can be constructed by applying shift operators labeled with points of the discrete phase space to a fiducial state, which is taken as the ground state of the harmonic oscillator $([18,21])$. In this sense, harmonic oscillator coherent states are generated by $|\alpha\rangle=\hat{D}(\alpha)|0\rangle$. The shift operator is given by

$$
\hat{D}(\alpha)=e^{\alpha \hat{a}^{+}-\alpha^{*} \hat{a}}
$$

where

$$
\alpha=\frac{1}{\sqrt{2}}\left[\frac{x}{\sigma}+i k \sigma\right]
$$

Here $\alpha$ is a complex-number representation of the phase point $x$ and $k$, and the quantity $\sigma$ is a scaling parameter with the dimensions of length. Thus, the harmonic oscillator coherent states can be constructed by applying the shift operator to the ground state of the harmonic oscillator.

\subsection{Supersymmetric quantum mechanics}

For one-dimensional SUSY-QM, the superpotential $W$ is defined in terms of the ground state wave function by the Riccati substitution

$$
\psi_{0}^{(1)}(x)=N \exp \left[-\int_{0}^{x} W_{1}\left(x^{\prime}\right) d x^{\prime}\right]
$$

where $N$ is the normalization constant. The index "(1)" indicates that the ground state wave function and the superpotential are associated with the sector one Hamiltonian. It is assumed that Eq. (11) solves the Schrödinger equation with energy equal to zero

$$
-\frac{d^{2} \psi_{0}^{(1)}}{d x^{2}}+V_{1} \psi_{0}^{(1)}=0 .
$$

This does not impose any restriction since the energy can be changed by adding any constant to the Hamiltonian. From Eq. (11), the superpotential can be expressed in terms of the ground state wave function by 


$$
W_{1}(x)=-\frac{d}{d x} \ln \psi_{0}^{(1)}(x) .
$$

Substituting Eq. (11) into the Schrödinger equation in Eq. (12), we obtain the Riccati equation for the superpotential

$$
\frac{d W_{1}(x)}{d x}-W_{1}^{2}(x)+V_{1}(x)=0 .
$$

On the other hand, if $W_{1}(x)$ is known, then $V_{1}(x)$ is given by

$$
V_{1}(x)=\left(W_{1}(x)^{2}-\frac{d W_{1}(x)}{d x}\right) .
$$

Obviously, the Schrödinger equation in Eq. (12) is equivalent to

$$
-\frac{d^{2} \psi_{0}^{(1)}}{d x^{2}}+\left(W_{1}^{2}-\frac{d W_{1}}{d x}\right) \psi_{0}^{(1)}=0
$$

Analogous to the harmonic oscillator, the Hamiltonian operator can be factorized by introducing the "charge" operator and its adjoint

$$
\begin{aligned}
& Q_{1}=\frac{d}{d x}+W_{1}=W_{1}+i \hat{p}_{x} \\
& Q_{1}^{\dagger}=-\frac{d}{d x}+W_{1}=W_{1}-i \hat{p}_{x}
\end{aligned}
$$

where $\hat{p_{x}}=-i(d / d x)$ is the coordinate representation of the momentum operator. Throughout this study, the ground state wave function $\psi_{0}(x)$ is assumed to be purely real; hence, the superpotential $W(x)$ is self-adjoint. Then, the sector one Hamiltonian is defined as $H_{1}=Q_{1}^{\dagger} Q_{1}$. Since $E_{0}^{(1)}=0$ for $n=0$, it follows from the Schrödinger equation that for $n>0$

$$
Q_{1}^{\dagger} Q_{1} \psi_{n}^{(1)}=E_{n}^{(1)} \psi_{n}^{(1)},
$$

where $\psi_{n}^{(1)}$ is an eigenstate of $H_{1}$ with $E_{n}^{(1)} \neq 0$. Applying $Q_{1}$ to this equation, we obtain

$$
H_{2}\left(Q_{1} \psi_{n}^{(1)}\right)=Q_{1} Q_{1}^{\dagger}\left(Q_{1} \psi_{n}^{(1)}\right)=E_{n}^{(1)}\left(Q_{1} \psi_{n}^{(1)}\right)
$$

where the sector two Hamiltonian is defined as $H_{2}=Q_{1} Q_{1}^{+}$. Thus, $Q_{1} \psi_{n}^{(1)}$ is an eigenstate of $H_{2}$ with the same energy $E_{n}^{(1)}$ as the state $\psi_{n}^{(1)}$. Analogously, we consider the eigenstates of $\mathrm{H}_{2}$ 


$$
H_{2} \psi_{n}^{(2)}=Q_{1} Q_{1}^{\dagger} \psi_{n}^{(2)}=E_{n}^{(2)} \psi_{n}^{(2)}
$$

Applying $Q_{1}^{\dagger}$ to this equation, we notice that $Q_{1}^{\dagger} \psi_{n}^{(2)}$ is an eigenstate of $H_{1}$

$$
H_{1}\left(Q_{1}^{\dagger} \psi_{n}^{(2)}\right)=\left(Q_{1}^{\dagger} Q_{1}\right)\left(Q_{1}^{\dagger} \psi_{n}^{(2)}\right)=E_{n}^{(2)}\left(Q_{1}^{\dagger} \psi_{n}^{(2)}\right)
$$

It follows that the Hamiltonians $H_{1}$ and $H_{2}$ have identical spectra with the exception of the ground state with $E_{0}^{(1)}=0$. For the ground state, $Q_{1} \psi_{0}^{(1)}=0$, and this shows that the quantity $Q_{1} \psi_{0}^{(1)}$ cannot be used to generate the ground state of the sector two Hamiltonian. Because of the uniqueness of the ground state with $E_{0}^{(1)}=0$, the indexing of the first and second sector levels must be modified. It is clear that the eigenvalues and eigenfunctions of the two Hamiltonians $H_{1}$ and $H_{2}$ are related by

$$
\begin{aligned}
& E_{n}^{(2)}=E_{n+1}^{(1)}, \quad E_{0}^{(1)}=0, \\
& \psi_{n}^{(2)}=\frac{Q_{1} \psi_{n+1}^{(1)}}{\sqrt{E_{n+1}^{(1)}}}, \quad \psi_{n+1}^{(1)}=\frac{Q_{1}^{\dagger} \psi_{n}^{(2)}}{\sqrt{E_{n}^{(2)}}} .
\end{aligned}
$$

Analogously, starting from $\mathrm{H}_{2}$ whose ground state energy is $E_{0}^{(2)}=E_{1}^{(1)}$, we can generate the sector three Hamiltonian $\mathrm{H}_{3}$ as a SUSY partner of $\mathrm{H}_{2}$. This procedure can be continued until the number of bound excited states supported by $H_{1}$ is exhausted.

\subsection{SUSY Heisenberg uncertainty products}

It follows from Eq. (13) that the charge operator annihilates the corresponding ground state

$$
Q \psi_{0}=\left(\hat{W}+i \hat{p}_{x}\right) \psi_{0}=0
$$

Because we concentrate only on the sector one Hamiltonian in the present study, we suppress the sector index. For the harmonic oscillator, the charge operators correspond to the raising and lowering operators for the harmonic oscillator with $W(x)=x$. From the similarity, the superpotential $\hat{W}$ can be regarded as a "SUSY-displacement" operator although such a displacement would, in general, not be generated by the standard momentum operator $\hat{p}_{x}$. In fact, $\hat{W}$ and $\hat{p}_{x}$ are not canonically conjugate variables.

The ground state of the harmonic oscillator is a minimum uncertainty state, which minimizes the Heisenberg uncertainty product $\Delta \hat{x} \Delta \hat{p}_{x}$. Analogously, it is expected that the ground state for a bound quantum system minimizes the SUSY Heisenberg uncertainty product 
$\Delta \hat{W} \Delta \hat{p}_{x}$. For an arbitrary normalized wave function, we consider the square of the SUSY-displacement-standard momentum uncertainty product

$$
\left(\Delta \hat{W} \Delta \hat{p_{x}}\right)^{2}=\left\langle\psi\left|\tilde{W}^{2}\right| \psi\right\rangle\left\langle\psi\left|\tilde{p}_{x}^{2}\right| \psi\right\rangle
$$

where $\tilde{W}=\hat{W}-W_{0}$ and $\tilde{p_{x}}=\hat{p}_{x}-p_{0}$. The quantities $W_{0}=\langle W\rangle$ and $p_{0}=\left\langle\hat{p}_{x}\right\rangle$ correspond to the averaged SUSY-displacement and momentum values, respectively. In order to obtain a lower bound on the uncertainty product in Eq. (24), we employ the Cauchy-Schwarz inequality

$$
\left\langle\psi\left|\tilde{W}^{2}\right| \psi\right\rangle\left\langle\psi\left|\tilde{p}_{x}^{2}\right| \psi\right\rangle \geq\left|\left\langle\psi\left|\tilde{W} \tilde{p}_{x}\right| \psi\right\rangle\right|^{2} .
$$

The equality is satisfied when the two vectors $\tilde{W}|\psi\rangle$ and $\tilde{p}_{x}|\psi\rangle$ are collinear. From this condition, we obtain $\tilde{W}|\psi\rangle=\lambda \tilde{p}_{x}|\psi\rangle$. Rearranging this equation yields

$$
\left(\hat{W}-\lambda \hat{p}_{x}\right)|\psi\rangle=\left(W_{0}-\lambda p_{0}\right)|\psi\rangle
$$

As a special case for $\lambda=-i$, this equation becomes

$$
\left(\hat{W}+i \hat{p}_{x}\right)|\psi\rangle=\left(W_{0}+i p_{0}\right)|\psi\rangle
$$

It follows from Eq. (23) that $\left(W_{0}+i p_{0}\right)=\left\langle\psi_{0}\left|\hat{W}+i \hat{p}_{x}\right| \psi_{0}\right\rangle=0$ for the ground state of the system. Thus, Eq. (23) implies that the ground state satisfies the condition in Eq. (27). Therefore, the ground state of a bound quantum system minimizes the SUSY-displacement-standard momentum uncertainty product $\Delta \hat{W} \Delta \hat{p}_{x}$.

We present some properties of the averaged SUSY-displacement and standard momentum values for the ground state. The averaged SUSY-displacement for the ground state is evaluated by

$$
W_{0}=\left\langle\psi_{0}|W| \psi_{0}\right\rangle=\int_{-\infty}^{\infty} \psi_{0}^{*}(x) W(x) \psi_{0}(x) d x=-\int_{-\infty}^{\infty} \psi_{0}^{*}(x) \frac{d \psi_{0}(x)}{d x} d x
$$

where Eq. (13) has been used. The averaged momentum for the ground state is given by

$$
p_{0}=\left\langle\psi_{0}\left|\hat{p}_{x}\right| \psi_{0}\right\rangle=-i \int_{-\infty}^{\infty} \psi_{0}^{*}(x) \frac{d \psi_{0}(x)}{d x} d x
$$

Again, from Eqs. (28) and (29), $W_{0}+i p_{0}=0$ for the ground state of the system, as indicated in Eq. (23). Furthermore, when the ground state wave function is purely real, it follows from integration by parts that the integral in Eqs. (28) and (29) is equal to zero. Thus, the averaged SUSY-displacement and momentum values for the real-valued ground state wave function are equal to zero, $W_{0}=p_{0}=0$.

The ground state of a quantum system is the minimizer of the SUSY Heisenberg uncertainty product. We can derive the minimum value for the SUSY Heisenberg uncertainty product 
in Eq. (25). For the real-valued ground state wave function, $\tilde{W}=\hat{W}-W_{0}=\hat{W}$ and $\tilde{p_{x}}=$ $\hat{p}_{x}-p_{0}=\hat{p}_{x}$. The right side of the uncertainty product in Eq. (25) becomes

$$
\left\langle\psi_{0}\left|\hat{W} \hat{p}_{x}\right| \psi_{0}\right\rangle=i\left\langle\psi_{0}\left|\hat{W}^{2}\right| \psi_{0}\right\rangle
$$

where $\hat{p}_{x}\left|\psi_{0}\right\rangle=i \hat{W}\left|\psi_{0}\right\rangle$ from Eq. (23) has been used. Thus, the right side of the uncertainty product in Eq. (25) is given by

$$
\left|\left\langle\psi_{0}\left|\hat{W} \hat{p}_{x}\right| \psi_{0}\right\rangle\right|^{2}=\left\langle\hat{W}^{2}\right\rangle^{2}
$$

Similarly, the left side of the uncertainty product in Eq. (25) is given by

$$
\left\langle\psi_{0}\left|\hat{W}^{2}\right| \psi_{0}\right\rangle\left\langle\psi_{0}\left|\hat{p}_{x}^{2}\right| \psi_{0}\right\rangle=\left\langle\hat{W}^{2}\right\rangle\left\langle\hat{W}^{2}\right\rangle
$$

Therefore, the equality in Eq. (25) holds for the ground state, and the SUSY Heisenberg uncertainty product is equal to $\Delta \hat{W} \Delta \hat{p_{x}}=\left\langle\hat{W}^{2}\right\rangle$.

The expectation value of $\hat{W}^{2}$ for the ground state is evaluated by

$$
\left\langle\hat{W}^{2}\right\rangle=\int_{-\infty}^{\infty} \psi_{0}(x) W(x)^{2} \psi_{0}(x) d x=-\int_{-\infty}^{\infty} \psi_{0}(x) W(x) \frac{d \psi_{0}(x)}{d x} d x
$$

where Eq. (13) has been used. From integration by parts, the integral can be expressed by

$$
\int_{-\infty}^{\infty} \psi_{0}(x) W(x) \frac{d \psi_{0}(x)}{d x} d x=-\frac{1}{2} \int_{-\infty}^{\infty} \psi_{0}(x) \frac{d W(x)}{d x} \psi_{0}(x) d x
$$

Thus, the expectation value of $\hat{W}^{2}$ for the ground state is equal to one half of the expectation value for the derivative of the superpotential

$$
\left\langle\hat{W}^{2}\right\rangle=\frac{1}{2}\left\langle\frac{d \hat{W}}{d x}\right\rangle
$$

Moreover, the commutation relation of the SUSY-displacement and the momentum operator is given by

$$
\left[\hat{W}, \hat{p}_{x}\right]=i \frac{d \hat{W}}{d x}
$$

Therefore, the SUSY Heisenberg uncertainty product for the ground state becomes

$$
\Delta \hat{W} \Delta \hat{p_{x}}=\left\langle\hat{W}^{2}\right\rangle=\frac{1}{2}\left\langle\frac{d \hat{W}}{d x}\right\rangle=\frac{1}{2 i}\left\langle\left[\hat{W}, \hat{p}_{x}\right]\right\rangle
$$


For the harmonic oscillator, $W(x)=x$ and $d W / d x=1$. We recover the conventional Heisenberg uncertainty product for the ground state $\Delta \hat{x} \Delta \hat{p_{x}}=1 / 2$. As a special case, a similar derivation has been employed to determine exact minimum uncertainty coherent states for the Morse oscillator ([8]).

\subsection{System-specific coherent states}

Analogous to the harmonic oscillator coherent state, the analysis of a bound quantum system in terms of the SUSY Heisenberg uncertainty principle suggests the construction of system-specific coherent states based on the SUSY-QM ground state. Similarly, the procedure for creating an overcomplete set of such coherent states is to apply the shift operator to the ground state as a fiducial function $([18,21])$

$$
\begin{aligned}
\psi_{\alpha}(x)=\langle x \mid \alpha\rangle=\left\langle x|\hat{D}(\alpha)| \psi_{0}\right\rangle & =N e^{i k_{0}\left(x-x_{0}\right)} e^{-x_{0}(d / d x)} \psi_{0}(x) \\
& =N e^{i k_{0}\left(x-x_{0}\right)} \psi_{0}\left(x-x_{0}\right)
\end{aligned}
$$

where $N$ is the normalization constant. The raising and lowering operators for the shift operator are given by $\hat{a}^{\dagger}=\left(\hat{x}-i \hat{p}_{x}\right) / \sqrt{2}$ and $\hat{a}=\left(\hat{x}+i \hat{p}_{x}\right) / \sqrt{2}$, respectively. The quantity $\alpha=\left(x_{0}+i k_{0}\right) / \sqrt{2}$ is a point in the phase space which completely describes the coherent state. Thus, the functions $\psi_{\alpha}$ form an overcomplete set of the coherent states in the standard phase space which are specifically associated with the quantum-mechanical system described by the SUSY-displacement $W(x)$.

We now consider a coordinate transformation given by $x^{\prime}=x-x_{0}$ for the system-specific coherent states in Eq. (38). The system-specific coherent state becomes

$$
\psi_{\alpha}\left(x^{\prime}\right)=e^{i k_{0} x^{\prime}} \psi_{0}\left(x^{\prime}\right)
$$

where $\psi_{0}\left(x^{\prime}\right)$ is the normalized real-valued ground state wave function, and thus $\psi_{\alpha}\left(x^{\prime}\right)$ is also normalized. The momentum operator is invariant under the coordinate transformation (i.e., $\hat{p}_{x^{\prime}}=\hat{p}_{x}$ ). It is straightforward to show that

$$
\left(\hat{W}\left(x^{\prime}\right)+i \hat{p}_{x^{\prime}}\right)\left|\psi_{\alpha}\right\rangle=i k_{0}\left|\psi_{\alpha}\right\rangle
$$

The averaged SUSY-displacement for the system-specific coherent state is given by

$$
\begin{aligned}
W_{0, \alpha}=\left\langle\psi_{\alpha}|W| \psi_{\alpha}\right\rangle & =\int_{-\infty}^{\infty} \psi_{\alpha}^{*}\left(x^{\prime}\right) W\left(x^{\prime}\right) \psi_{\alpha}\left(x^{\prime}\right) d x^{\prime} \\
& =-\int_{-\infty}^{\infty} \psi_{0}\left(x^{\prime}\right) \frac{d \psi_{0}\left(x^{\prime}\right)}{d x^{\prime}} d x^{\prime}
\end{aligned}
$$


Again, it follows from integration by parts that $W_{0, \alpha}=0$ for all system-specific coherent states. Analogously, the averaged momentum for the system-specific coherent state is given by

$$
p_{0, \alpha}=\left\langle\psi_{\alpha}\left|\hat{p}_{x^{\prime}}\right| \psi_{\alpha}\right\rangle=k_{0}-i \int_{-\infty}^{\infty} \psi_{0}\left(x^{\prime}\right) \frac{d \psi_{0}\left(x^{\prime}\right)}{d x^{\prime}} d x^{\prime} .
$$

Because the integral is equal to zero, $p_{0, \alpha}=k_{0}$. Thus, Eq. (40) can be written as

$$
\left(\hat{W}\left(x^{\prime}\right)+i \hat{p}_{x^{\prime}}\right)\left|\psi_{\alpha}\right\rangle=\left(W_{0, \alpha}+i p_{0, \alpha}\right)\left|\psi_{\alpha}\right\rangle
$$

Analogous to the uncertainty condition for the ground state in Eq. (27), this equation implies that the system-specific coherent state $\left|\psi_{\alpha}\right\rangle$ minimizes the SUSY-displacement-momentum uncertainty product $\Delta \hat{W} \Delta \hat{p}_{x^{\prime}}$ for the displaced coordinate $x^{\prime}=x-x_{0}$.

\subsection{Discretized system-specific coherent states}

A discretized SUSY-QM coherent state basis can be constructed by discretizing the continuous label $\alpha=(q+i k) / \sqrt{2}$ and setting up a von Neumann lattice in phase space with an appropriate density $D$. The discretized system-specific coherent state basis is given by

$$
\psi_{\alpha_{i}}(x)=\left\langle x \mid \alpha_{i}\right\rangle=N e^{i k_{i}\left(x-q_{i}\right)} \exp \left[-\int_{0}^{x-q_{i}} W\left(x^{\prime}\right) d x^{\prime}\right],
$$

where $i=1, \ldots, M$ and $M$ is the number of basis functions. The phase space grid points are defined as ([2])

$$
\left\{\left(q_{i}, k_{i}\right)\right\}=\left\{\left(m \Delta x \sqrt{\frac{2 \pi}{D}}, \frac{n}{\Delta x} \sqrt{\frac{2 \pi}{D}}\right)\right\} \quad m, n \in \mathbb{Z}
$$

where $m$ and $n$ run over all integers, hence $i$ can be thought of as a joint index consisting of $m$ and $n$. The quantity $D$ is the density of grid points in units of $2 \pi \hbar$. As discussed in Klauder and Skagerstam's book ([18]), generalized coherent states constructed by applying displacement operators to a fiducial state are overcomplete; however, completeness of the discretized system-specific coherent states in Eq. (44) has not been established here.

Since the ground state solves the time-independent Schrödinger equation for the corresponding Hamiltonian, the system-specific coherent states build in the dynamics of the system under investigation. This property leads to the expectation that these dynamically-adapted and system-specific coherent states will prove more rapidly convergent in calculations of the excited state energies and wave functions for quantum systems using variational methods. 


\begin{tabular}{lllll}
\hline & $E_{0}$ & $E_{1}$ & $E_{2}$ & $E_{3}$ \\
\hline Exact & $\mathbf{- 5 6 . 2 5}$ & $\mathbf{- 4 2 . 2 5}$ & $\mathbf{- 3 0 . 2 5}$ & $\mathbf{- 2 0 . 2 5}$ \\
\hline SSCS $(M=9)$ & -56.25 & -42.2499824 & -30.2270611 & -19.52261 \\
SSCS $(M=15)$ & -56.25 & -42.2499999 & -30.2499343 & -20.23502 \\
\hline HOCS $(M=9)$ & -54.95 & -37.00 & -21.08 & -10.22 \\
HOCS $(M=15)$ & -56.13 & -41.62 & -28.61 & -17.62 \\
\hline HO $(M=9)$ & -53.79 & -33.34 & -16.45 & -6.40 \\
HO $(M=15)$ & -55.54 & -39.03 & -23.84 & -12.30 \\
\hline
\end{tabular}

Table 1. Comparison of the energy eigenvalues for the Morse oscillator obtained by the system-specific coherent states (SSCS), the harmonic oscillator coherent states (HOCS), and the harmonic oscillator basis functions (HO) with the exact results.

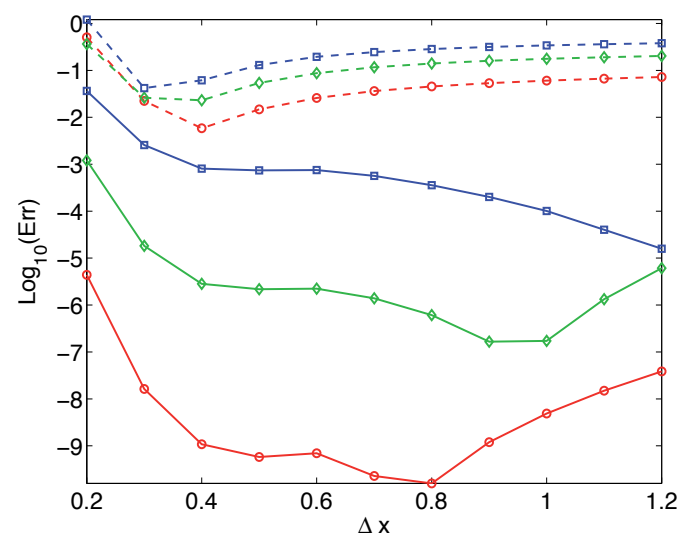

Figure 1. Logarithm of the relative error, versus grid spacing $\Delta x$, of the first (०), second $(\diamond)$, and third $(\square)$ excited state energies for the Morse oscillator using the system-specific coherent states $(-)$ and the harmonic oscillator coherent states (- - -) with 15 basis functions.

To use the Rayleigh-Ritz variational principle, we construct a trial wave function in terms of a linear combination of the system-specific coherent states

$$
|\psi\rangle=\sum_{i=1}^{M} c_{i}\left|\alpha_{i}\right\rangle,
$$

where $c_{i}$ are the coefficients. Because of the non-orthogonality of the system-specific coherent states, the energy eigenvalues and wave functions are determined by solving the generalized eigenvalue problem ([36])

$$
H C=E S C,
$$

where $H_{i j}=\left\langle\alpha_{i}|H| \alpha_{j}\right\rangle$ is the matrix element of the Hamiltonian, $S_{i j}=\left\langle\alpha_{i} \mid \alpha_{j}\right\rangle$ is the overlap matrix, and $C$ is a vector of linear combination coefficients for the eigenvector. Therefore, solving Eq. (47) yields the variational approximation to the eigenvalues and eigenvectors of the Hamiltonian operator. 


\section{Computational results}

\subsection{Morse oscillator}

In order to demonstrate features of system-specific coherent states, computational results will be presented for three quantum systems. The first of these concerns the Morse oscillator. The Hamiltonian of the Morse oscillator is given by

$$
H=-\frac{d^{2}}{d x^{2}}+V(x)=-\frac{d^{2}}{d x^{2}}+64\left(e^{-2 x}-2 e^{-x}\right)
$$

The exact energy eigenvalues are $E_{n}=-(n-15 / 2)^{2}$ where $n=0, \ldots, 7$, and the analytical expression of the ground state wave function is given by

$$
\psi_{0}(x)=N \exp \left[-8 e^{-x}-\frac{15}{2} x\right]
$$

where $N$ is the normalization constant. In this case, the superpotential and its derivative are given by $W(x)=15 / 2-8 \exp (-x)$ and $d W / d x=8 \exp (-x)$, respectively. The minimum SUSY Heisenberg uncertainty product in Eq. (37) is equal to $\Delta \hat{W} \Delta \hat{p_{x}}=15 / 4$. In addition, the discretized system-specific coherent state basis functions in Eq. (44) are expressed by

$$
\psi_{\alpha_{i}}(x)=N e^{i k_{i}\left(x-q_{i}\right)} \exp \left[-8 e^{-\left(x-q_{i}\right)}-\frac{15}{2}\left(x-q_{i}\right)\right]
$$

The phase space grid in Eq. (45) used for the coherent states was $m=-1,0,1$ and $n=-1,0,1$ for $M=9$ basis functions and $m=-1,0,1$ and $n=-2, \ldots, 2$ for $M=15$ basis functions. The phase space density was set to be $D=1$. In contrast with the present system-specific coherent states in Eq. (50), different coherent states for the Morse oscillator defined as eigenstates of the charge operator and minimum uncertainty states have been constructed ([8]).

Table 1 presents the computational results for the energy eigenvalues obtained by solving the generalized eigen-equation in Eq. (47) using the discretized system-specific coherent state basis functions with $\Delta x=0.5$. Since the basis includes the exact ground state wave function, the computational result yields the exact ground state energy. As shown in this table, higher accuracy can be achieved when we increase the number of the basis functions from $M=9$ to $M=15$. In addition, Table 1 presents the computational results obtained using the harmonic oscillator coherent state basis and the standard harmonic oscillator basis. The discretized harmonic oscillator coherent state basis functions are readily determined by substituting $W(x)=x$ into Eq. (44)

$$
\psi_{\alpha_{i}}(x)=N e^{i k_{i}\left(x-q_{i}\right)} e^{-\left(x-q_{i}\right)^{2} / 2} .
$$

The standard harmonic oscillator basis is given by 


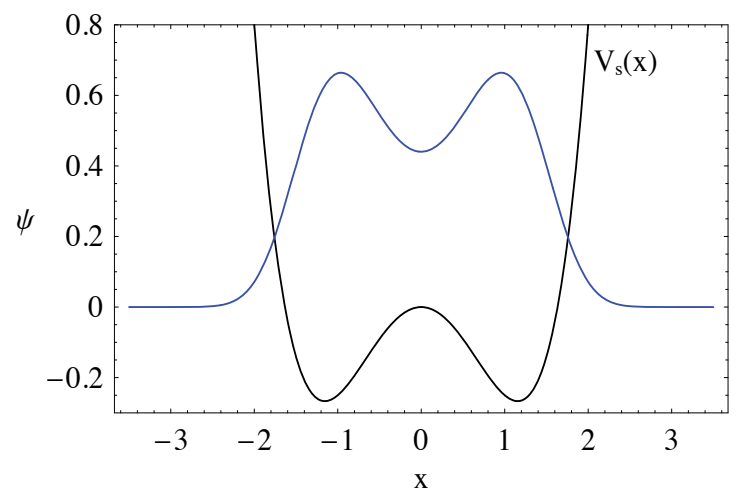

Figure 2. The ground state wave function of the double well potential obtained by the imaginary time propagation method is shown with the scaled potential $V_{s}(x)=V(x) / 20$.

$$
\phi_{n}(x)=\frac{1}{\sqrt{2^{n} n ! \sqrt{\pi}}} H_{n}(x) e^{-x^{2} / 2},
$$

where $H_{n}(x)$ is the Hermite polynomial. Compared with the results obtained from these two basis sets, the computational results from the system-specific coherent states achieve significantly higher accuracy using a small number of basis functions. Thus, the system-specific coherent states provide more accurate approximations of the excited state energies for the Morse oscillator.

Figure 1 displays the logarithm of the relative error of the excited state energies for the system-specific coherent states and the harmonic oscillator coherent states with different values for $\Delta x$ with 15 basis functions. The relative error is defined by

$$
E r r=\frac{E_{\text {numerical }}-E_{\text {exact }}}{\left|E_{\text {exact }}\right|}
$$

As shown in this figure, the system-specific coherent states yield excellent results for the excited state energies. The relative error of the first-excited state energy can even reach $10^{-9}$ for a wide range of $\Delta x$. Additionally, compared with the harmonic oscillator coherent states, the system-specific coherent states give much more accurate results for the first three excited state energies. Also, the system-specific coherent states yield stable computational results for a wide range of $\Delta x$.

\subsection{Double well potential}

As an example of quantum systems without exact analytical solutions, we consider a symmetric double well potential given by

$$
V(x)=3 x^{4}-8 x^{2}
$$




\begin{tabular}{lllll}
\hline & $E_{0}$ & $E_{1}$ & $E_{2}$ & $E_{3}$ \\
\hline DVR & $\mathbf{- 2 . 1 6 9 6 9 3}$ & $\mathbf{- 1 . 4 0 6 4 7 2}$ & $\mathbf{3 . 1 0 2 4 0 6}$ & $\mathbf{7 . 0 8 7 9 3 0}$ \\
\hline SSCS $(M=9)$ & -2.169697 & -1.375254 & 3.106359 & 7.807534 \\
SSCS $(M=15)$ & -2.169697 & -1.406417 & 3.102440 & 7.088186 \\
\hline HOCS $(M=9)$ & -2.1223 & -1.3214 & 3.3931 & 7.5166 \\
HOCS $(M=15)$ & -2.1688 & -1.4048 & 3.1088 & 7.0992 \\
\hline HO $(M=9)$ & -2.1246 & -1.0650 & 3.5063 & 8.6640 \\
HO $(M=15)$ & -2.1543 & -1.3930 & 3.1555 & 7.4491 \\
\hline
\end{tabular}

Table 2. Comparison of the energy eigenvalues for the double well potential obtained by the system-specific coherent states (SSCS), the harmonic oscillator coherent states (HOCS), and the harmonic oscillator basis functions (HO) with the discrete variable representation (DVR) results.

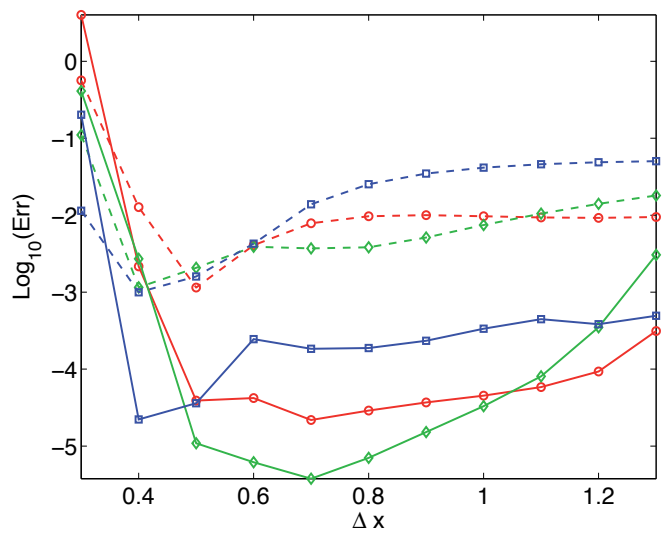

Figure 3. Logarithm of the relative error, versus grid spacing $\Delta x$, of the first (०), second $(\diamond)$, and third $(\square)$ excited state energies for the double well potential using the system-specific coherent states $(-)$ and the harmonic oscillator coherent states $(--)$ with 15 basis functions.

In order to construct the discretized system-specific coherent state basis functions in Eq. (44), we numerically obtain the ground state wave function. We employed the split-operator method ([35]) to integrate the imaginary time Schrödinger equation from $t=0$ to $t=2$ ([36]). The computational grid extends from $x=-8$ to $x=8$ with $2^{13}$ grid points, and the integration time step was $\Delta t=0.01$. The initial state is a Gaussian wave packet given by

$$
\psi(x)=\left(\frac{2}{\pi}\right)^{1 / 4} e^{-x^{2}}
$$

where the wave packet is centered at the origin. Figure 2 presents the resulting ground state wave function of the double well potential with the ground state energy $E_{0}=-2.169694$.

From the computational result for the ground state, we can construct the approximate discretized system-specific coherent states in Eq. (44) used to determine the excited state energies of the double well potential by solving the generalized eigen-equation in Eq. (47). In order to assess the accuracy of the computational results, accurate results were obtained with a Chebyshev polynomial discrete variable representation (DVR) variational calculation 


\begin{tabular}{lllll}
\hline & $E_{0}$ & $E_{1}$ & $E_{2}$ & $E_{3}$ \\
\hline DVR & $\mathbf{0 . 0 0 0 0 0 0}$ & $\mathbf{4 . 7 5 1 8 0 7}$ & $\mathbf{6 . 6 4 6 3 4 9}$ & $\mathbf{8 . 6 7 9 5 7 5}$ \\
\hline SSCS $(M=81)$ & 0 & 4.754974 & 6.647358 & 8.684308 \\
SSCS $(M=225)$ & 0 & 4.751812 & 6.646353 & 8.679596 \\
\hline HOCS $(M=81)$ & 0.0762 & 5.3029 & 6.9378 & 10.4334 \\
HOCS $(M=225)$ & 0.0029 & 4.7915 & 6.6554 & 8.8479 \\
\hline HO $(M=81)$ & 0.0870 & 5.3587 & 7.0307 & 10.5626 \\
HO $(M=225)$ & 0.0144 & 4.8953 & 6.6967 & 9.2190 \\
\hline
\end{tabular}

Table 3. Comparison of the energy eigenvalues for the two-dimensional anharmonic oscillator system obtained by the system-specific coherent states (SSCS), the harmonic oscillator coherent states (HOCS), and the harmonic oscillator basis functions $(\mathrm{HO})$ with the discrete variable representation (DVR) results.

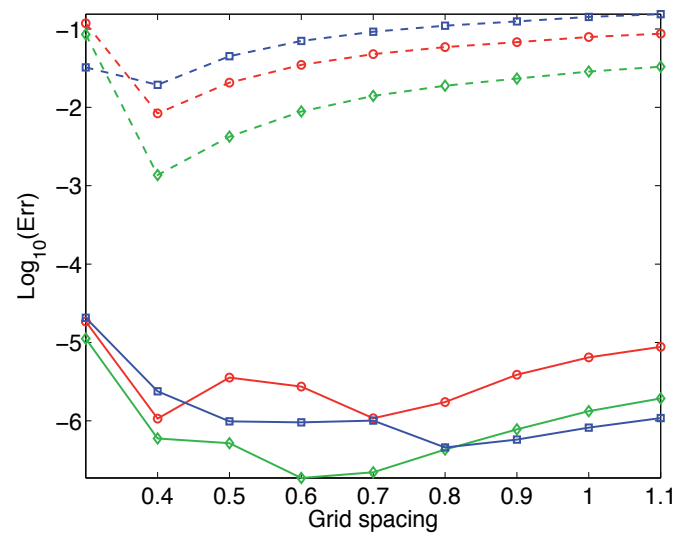

Figure 4. Logarithm of the relative error, versus grid spacing $\Delta x=\Delta y$, of the first (o), second $(\diamond)$, and third ( $\square$ ) excited state energies for the two-dimensional anharmonic oscillator system using the system-specific coherent states ( - ) and the harmonic oscillator coherent states (- - -) with 225 basis functions.

using 1000 grid points on the computational domain extending from $x=-4$ to $x=4$ ([23]). Table 2 presents the computational results for the energy eigenvalues with $\Delta x=0.5$. Again, computational results for the first three excited state energies with significantly higher accuracy were achieved using a small number of the basis functions with $M=15$. In addition, compared with the harmonic oscillator coherent state basis and the standard harmonic oscillator basis, the system-specific coherent states yields more accurate excited state energies for the double well potential. Moreover, Fig. 2 displays the logarithm of the relative error of the excited state energies for the system-specific coherent states and the harmonic oscillator coherent states with different values for $\Delta x$ with 15 basis functions. As shown in this figure, the system-specific coherent states generally yield much more accurate results for the excited state energies than the harmonic oscillator coherent states except for small $\Delta x$. 


\subsection{Two-dimensional anharmonic oscillator system}

As an example of multidimensional systems, we consider a nonseparable nondegenerate two-dimensional anharmonic oscillator system ([19]). The Hamiltonian is given by

$$
\begin{aligned}
H_{1}= & -\nabla^{2}+V(x, y) \\
= & -\frac{\partial^{2}}{\partial x^{2}}-\frac{\partial^{2}}{\partial y^{2}}+\left(4 x y^{2}+2 x\right)^{2} \\
& +\left(4 x^{2} y+2 \sqrt{2} y\right)^{2}-4\left(x^{2}+y^{2}\right)-(2+2 \sqrt{2}) .
\end{aligned}
$$

The exact ground state energy of the system is zero and the analytical expression of the ground state wave function is given by

$$
\psi_{0}^{(1)}(x, y)=N \exp \left(-2 x^{2} y^{2}-x^{2}-\sqrt{2} y^{2}\right)
$$

where $N$ is a normalization constant. Analogous to the one-dimensional case, the discretized system-specific coherent state basis functions are expressed by

$$
\psi_{\alpha_{i}}(x, y)=N e^{i k_{x i}\left(x-q_{x i}\right)} e^{i k_{y i}\left(y-q_{y i}\right)} \psi_{0}\left(x-q_{x i}, y-q_{y i}\right)
$$

In addition, the two-dimensional separable discretized harmonic oscillator coherent state basis functions are given by

$$
\psi_{\alpha_{i}}(x, y)=N e^{i k_{x i}\left(x-q_{x i}\right)} e^{i k_{y i}\left(y-q_{y i}\right)} e^{-\left(x-q_{x i}\right)^{2} / 2} e^{-\left(y-q_{y i}\right)^{2} / 2}
$$

The phase space grid points for these two basis sets are defined by

$$
\left\{\left(q_{x i}, q_{y i}, k_{x i}, k_{y i}\right)\right\}=\left\{\left(m \Delta x \sqrt{\frac{2 \pi}{D}}, m \Delta y \sqrt{\frac{2 \pi}{D}}, \frac{n}{\Delta x} \sqrt{\frac{2 \pi}{D}}, \frac{n}{\Delta y} \sqrt{\frac{2 \pi}{D}}\right)\right\} \quad m, n \in \mathbb{Z}
$$

where $m$ and $n$ are integers. For computational results, we chose $m=-1,0,1$ and $n=-1,0,1$ for $M=81$ basis functions and $m=-1,0,1$ and $n=-2, \ldots, 2$ for $M=225$ basis functions. The phase space density was set to be $D=1$.

Table 3 presents the computational results for the energy eigenvalues obtained using the discretized system-specific coherent states and the harmonic oscillator coherent states with $\Delta x=\Delta y=0.4$. Compared with the DVR results using 50 grid points in $x$ and in $y$ (for a total of 2500 basis functions), the computational results obtained by the system-specific coherent states achieve higher accuracy than the harmonic oscillator coherent states. In addition, Table 3 presents the computational results obtained from the standard harmonic oscillator 
basis of the direct product of the eigenstates of a harmonic oscillator in each dimension with frequency $\omega=2 \sqrt{2}$. These results were obtained by a $\left(N_{x}, N_{y}\right)=(9,9)$ basis set calculation with 81 basis functions and a $\left(N_{x}, N_{y}\right)=(15,15)$ basis set calculation with 225 basis functions. Again, compared with the results obtained from the other two basis sets, the computational results from the system-specific coherent states achieve significantly higher accuracy using a small number of basis functions. Furthermore, Figure 4 displays the logarithm of the relative error of the excited state energies for the system-specific coherent states and the harmonic oscillator coherent states with different values for the grid spacing with 225 basis functions. As shown in this figure, the system-specific coherent states yield much more accurate results for the excited state energies than the harmonic oscillator coherent states for different grid spacings, and the relative errors reach around $10^{-6}$ for a wide range of the grid spacings.

\section{Discussion and perspectives}

The application of SUSY-QM to non-relativistic quantum systems generalizes the powerful ladder operator approach used in the treatment of the harmonic oscillator. The lowering operator of the harmonic oscillator annihilates the ground state, while the charge operator annihilates the ground state of the corresponding ground state for other quantum systems. The similarity between the lowering operator of the harmonic oscillator and the SUSY charge operator implies that the superpotential can be regarded as a system-specific generalized displacement variable. Analogous to the ground state of the harmonic oscillator which minimizes the Heisenberg uncertainty product, the ground state of any bound quantum system was identified as the minimizer of the SUSY Heisenberg uncertainty product. Then, system-specific coherent states were constructed by applying shift operators to the ground state of the system, which serves as a fiducial function. In addition, we employed the discretized system-specific coherent states as a dynamically-adapted basis set to determine the excited state energies and wave functions for the Morse oscillator, the double well potential, and the two-dimensional anharmonic oscillator system. Variational calculations in terms of the discretized system-specific coherent states demonstrated that these dynamically-adapted coherent states yield significantly more accurate excited state energies and wave functions than were obtained with the same number of the conventional coherent states and the standard harmonic oscillator basis.

As presented in the current study, the ladder operator approach of the harmonic oscillator and the SUSY-QM formulation share strong similarity. This observation suggests that the connection of the SUSY-QM with the Heisenberg minimum uncertainty $(\mu-)$ wavelets should be explored ([13, 14, 21, 22]). The SUSY-displacement with the SUSY Heisenberg uncertainty product can lead to the construction of the SUSY minimum uncertainty wavelets and the SUSY distributed approximating functionals. These new functions and their potential applications in mathematics and physics are currently under investigation. In addition, this study presents a practical computational approach for discretized system-specific coherent states in calculations of excited states. The issue of completeness of discretized system-specific coherent states should be examined. These relevant studies will be reported elsewhere in the future. 


\section{Author details}

Chia-Chun Chou, Mason T. Biamonte,

Bernhard G. Bodmann and Donald J. Kouri

University of Houston, USA

\section{References}

[1] Aleixo, A. N. F. \& Balantekin, A. B. [2004]. An algebraic construction of generalized coherent states for shape-invariant potentials, J. Phys. A: Math. Gen. 37: 8513.

[2] Andersson, L. M. [2001]. Quantum dynamics using a discretized coherent state representation: An adaptive phase space method, J. Chem. Phys. 115(3): 1158-1165.

[3] Angelova, M. \& Hussin, V. [2008]. Generalized and gaussian coherent states for the morse potential, J. Phys. A: Math. Theor. 41(30): 304016.

[4] Benedict, M. G. \& Molnár, B. [1999]. Algebraic construction of the coherent states of the morse potential based on supersymmetric quantum mechanics, Phys. Rev. A 60: R1737-R1740.

[5] Bittner, E. R., Maddox, J. B. \& Kouri, D. J. [2009]. Supersymmetric approach to excited states, J. Phys. Chem. A 113: 15276-15280.

[6] Chou, C.-C., Biamonte, M. T., Bodmann, B. G. \& Kouri, D. J. [2012]. New system-specific coherent states for bound state calculations. (submitted).

[7] Cooper, F., Khare, A. \& Sukhatme, U. [2002]. Supersymmetry in Quantum Mechanics, World Scientific, Danvers.

[8] Cooper, I. L. [1992]. A simple algebraic approach to coherent states for the morse oscillator, J. Phys. A: Math. Gen. 25: 1671.

[9] Fernández C, D. J., Hussin, V. \& Nieto, L. M. [1994]. Coherent states for isospectral oscillator hamiltonians, J. Phys. A: Math. Gen. 27: 3547.

[10] Fukui, T. \& Aizawa, N. [1993]. Shape-invariant potentials and an associated coherent state, Phys. Lett. A 180: 308 - 313.

[11] Gangopadhyaya, A., Mallow, J. V. \& Rasinariu, C. [2010]. Supersymmetric Quantum Mechanics: An Introduction, World Scientific, Danvers.

[12] Gerry, C. C. [1986]. Coherent states and a path integral for the morse oscillator, Phys. Rev. A 33: 2207-2211.

[13] Hoffman, D. K. \& Kouri, D. J. [2000]. Hierarchy of local minimum solutions of heisenberg's uncertainty principle, Phys. Rev. Lett. 85: 5263-5267.

[14] Hoffman, D. K. \& Kouri, D. J. [2002]. Hierarchy of local minimum solutions of heisenberg's uncertainty principle, Phys. Rev. A 65: 052106. 
[15] Infeld, L. \& Hull, T. E. [1951]. The factorization method, Rev. Mod. Phys. 23: 21-68.

[16] Jafarpour, M. \& Afshar, D. [2002]. Calculation of energy eigenvalues for the quantum anharmonic oscillator with a polynomial potential, J. Phys. A: Math. Gen. 35: 87.

[17] Kais, S. \& Levine, R. D. [1990]. Coherent states for the morse oscillator, Phys. Rev. A 41: 2301-2305.

[18] Klauder, J. R. \& Skagerstam, B. S. [1985]. Coherent States: Applications in Physics and Mathematical Physics, World Scientific, Singapore.

[19] Kouri, D. J., Maji, K., Markovich, T. \& Bittner, E. R. [2010]. New generalization of supersymmetric quantum mechanics to arbitrary dimensionality or number of distinguishable particles, J. Phys. Chem. A 114: 8202-8216.

[20] Kouri, D. J., Markovich, T., Maxwell, N. \& Bittner, E. R. [2009]. Supersymmetric quantum mechanics, excited state energies and wave functions, and the rayleigh-ritz variational principle: A proof of principle study, J. Phys. Chem. A 113: 15257-15264.

[21] Kouri, D. J., Papadakis, M., Kakadiaris, I. \& Hoffman, D. K. [2003]. Properties of minimum uncertainty wavelets and their relations to the harmonic oscillator and the coherent states, J. Phys. Chem. A 107(37): 7318-7327.

[22] Lee, Y., Kouri, D. \& Hoffman, D. [2011]. Minimum uncertainty wavelets in non-relativistic super-symmetric quantum mechanics, J. Math. Chem. 49: 12-34.

[23] Light, J. C., Hamilton, I. P. \& Lill, J. V. [1985]. Generalized discrete variable approximation in quantum mechanics, J. Chem. Phys 82: 1400-1409.

[24] Liu, Y., Lei, Y. \& Zeng, J. [1997]. Factorization of the radial schrödinger equation and four kinds of raising and lowering operators of hydrogen atoms and isotropic harmonic oscillators, Phys. Lett. A 231: 9-22.

[25] Merzbacher, E. [1997]. Quantum Mechanics, Wiley, New York.

[26] Mielnik, B. [1984]. Factorization method and new potentials with the oscillator spectrum, J. Math. Phys. 25: 3387-3389.

[27] Nieto, M. M. \& Simmons, L. M. [1978]. Coherent states for general potentials, Phys. Rev. Lett. 41: 207-210.

[28] Nieto, M. M. \& Simmons, L. M. [1979a]. Coherent states for general potentials. i. formalism, Phys. Rev. D 20: 1321-1331.

[29] Nieto, M. M. \& Simmons, L. M. [1979b]. Coherent states for general potentials. ii. confining one-dimensional examples, Phys. Rev. D 20: 1332-1341.

[30] Nieto, M. M. \& Simmons, L. M. [1979c]. Coherent states for general potentials. iii. nonconfining one-dimensional examples, Phys. Rev. D 20: 1342-1350. 
[31] Perelomov, A. [1986]. Generalized Coherent States and Their Applications, Springer, New York.

[32] Schrödinger, E. [1941a]. Factorization of the hypergeometric equation, Prod.Roy. Irish Acad. 47 A: 53-54.

[33] Schrödinger, E. [1941b]. Further studies on solving eigenvalue problems by factorization, Prod. Roy. Irish Acad. 46 A: 183-206.

[34] Shreecharan, T., Panigrahi, P. K. \& Banerji, J. [2004]. Coherent states for exactly solvable potentials, Phys. Rev. A 69: 012102.

[35] Tannor, D. J. [2007]. Introduction to Quantum Mechanics: A Time-Dependent Perspective, University Science Books, Sausalito.

[36] Varga, K. \& Driscoll, J. A. [2011]. Computational Nanoscience: Applications for Molecules, Clusters, and Solids, Cambridge University Press, New York.

[37] Zhang, W.-M., Feng, D. H. \& Gilmore, R. [1990]. Coherent states: Theory and some applications, Rev. Mod. Phys. 62: 867-927. 
Quantization and Entanglement 



\title{
Quantum Dating Market
}

\author{
C. M. Arizmendi and O. G. Zabaleta
}

Additional information is available at the end of the chapter

http://dx.doi.org/10.5772/53842

\section{Introduction}

Quantum algorithms have proven to be faster than the fastest known classical algorithms. Clearly, such a superiority means counting on a real quantum computer. Although this essential constraint elimination is in development process, many people is working on that and interesting advances are being made [1-3]. Meanwhile, new algorithms and applications of the existing ones are current research topics $[4,5]$. One of the main goals of quantum computing is the application of quantum techniques to classical troubleshooting: the Shor algorithm [6], for example, is a purely quantum-mechanical algorithm which comes to solve the classical factoring problem, also the contribution of Lov Grover [7,8] to speed up the search for items in an $\mathrm{N}$-item database is very important. Both mathematical finds are the cornerstones of quantum computation, so, considerable amount of work on diverse subjects make use of them. Other algorithms which has been very important for quantum computing progress are Simon's and Deutsch-Jozsa's. Through the quantum games, Meyer in [9] and Eisert in [10], among other, showed that quantum techniques are generalizations of classical probability theory, allowing effects which are impossible in a classical setting. These and many other examples, show that there is no contradiction in using quantum techniques to describe non-quantum mechanical problems and solve hard to solve problems with classical tools. Adding, decision theory and game theory, two examples where probabilities theory is applied, deal with decisions made under uncertain conditions by real humans. Basically, the former considers only one agent and her decisions meanwhile the other considers also the conflicts that two or more players cause to each other through the decisions they take. Due to their inherent complexity this kind of problems results convenient to be analyzed by mean of quantum games models.

Widely observed phenomena of non-commutativity in patterns of behavior exhibited in experiments on human decisions and choices cannot be obtained with classical decision theory [11] but can be adequately described by putting quantum mechanics and decision theory together. Quantum mechanics and decision theory have been recently combined 
[11-13] to take into account the indeterminacy of preferences that are determined only when the action takes place. An agent is described by a state that is a superposition of potential preferences to be projected onto one of the possible behaviors at the time of the interaction. In addition to the main goal of modeling uncertainty of preferences that is not due to lack of information, this formalism seems to be adequate to describe widely observed phenomena of non-commutativity in patterns of behavior.

Within this framework, we study the dating market decision problem that takes into account progressive mutual learning [14,15]. This problem is a variation on the Stable Marriage Problem introduced by Gale and Shapley almost four decades ago [16], that has been recently reformulated in a partial information approach [17, 18]. Specifically, perfect information supposition is very far from being a good approximation for the dating market, in which men and women repeatedly go out on dates and learn about each other.

The dating market problem may be included in a more general category of matching problems where the elements of two sets have to be matched by pairs. Matching problems have broad implications in economic and social contexts [19,20]. As possible applications one could think of job seekers and employers, lodgers and landlords, men and women who want to date, or solitary ciliates courtship rituals [21]. In our model players earn an uncertain payoff from being matched with a particular person on the other side of the market in each time period. Players have a list of preferred partners on the other set. Quantum exploration of partners is compared with classical exploration at the dating set. Nevertheless dating is not just finding, but also being accepted by the partner. The preferences of the chosen partner are important in quantum and classic performances.

Recently [22], we introduced a quantum formulation for decision matching problems, specifically for the dating game that takes into account mutual progressive learning. This learning is accomplished by representing women with quantum states whose associated amplitudes must be modified by men's selection strategies, in order to increase a particular state amplitude and to decrease the others, with the final purpose to achieve the best possible choice when the game finishes. Grover quantum search algorithm is used as a playing strategy. Within the same quantum formulation already used in [22], we will concentrate first on the information associated to the dating market problem. Since we deal with mixed strategies, the density matrix formalism is used to describe the system. There exists a strong relationship between game theories, statistical mechanics and information theories. The bonds between these theories are the density operator and entropy. From the density operator we can construct and understand the statistical behavior about our system by using statistical mechanics. The dating problem is analyzed through information theory under a criterion of maximum or minimum entropy. Even though the decisions players make are based on their payoffs, past experiences, believes, etc., we are not interested in that causes but in the consequences of the decision they take, that is, the influence of the strategies they apply on the quantum system stability. In order to identify the conditions of stability we will use the equivalence between maximum entropy states and those states that obey the Collective Welfare Principle that says that a system is stable only if it maximizes the welfare of the collective above the welfare of the individual [23].

Interesting properties merge when entanglement is considered in quantum models of social decision problems [24]. People decisions are usually influenced by other people actions, opinions, or beliefs, to the extent that they may proceed in ways that they would rarely or never do if moved by their own benefit. Love, hate, envy, or a close friendship, which encase a bit of everything, are examples of relationships between people that may correlate their 
decisions. So, as driven by a no local force, people may make an inconvenient choice in the heat of a competence. In order to formulate in a mathematical way this sort of problem we remodel the quantum dating between men and women with the inclusion of quantum entanglement between men decision states.

The chapter organization is as follows: First of all, to ease game theory unfamiliar readers comprehension a brief introduction to game basics is presented. In the course of the next sections the quantum dating game is particularly studied. In section 3 the Grover quantum search algorithm as a playing strategy is analyzed. In section 5, the system stability is under study. Finally, section 6 explores entangled strategies performance. At the end of each section the results and the consequent section discussions are set. The chapter ends with a final conclusion.

\section{Quantum games}

Game theory [25] is a collection of models (games) designed to study competing agents (players) decisions in some conflict situation. It tries to understand the birth and development of conflicting or cooperative behaviors among a group of individuals who behave rationally and strategically according to their personal interests. Although the theory was conceived in order to analyze and solve social and economy problems, existing applications go beyond [26]. Furthermore, the models reach not only individuals but also governments conflicts, institutions trades or smart machines (phones, computers) access management.

Before starting to explain quantum games basics, the classic games notation is presented. The game can be set in strategic (or normal) form or in extensive form, in any of them it has three elements: a set of players $i \in \mathcal{J}$ which is taken to be a finite set $0, \ldots, N-1$, the set of pure strategies $S_{i}=\left\{s_{0}, s_{1}, \ldots, s_{N-1}\right\}, \quad i=0, \ldots, N-1$ which is the set of all strategies available to the player, and the payoffs function $u_{i}\left(s_{0}, s_{1}, \ldots, s_{N-1}\right), \quad i=0, \ldots, N-1$, where $s_{i} \in S_{i}$. In the strategic form, the game can be denoted by $G(N, S, u)$, where $S=S_{0} \times S_{1} \times \ldots \times S_{N-1}$ and $u=u_{0} \times u_{1} \times \ldots \times u_{N-1}$. Extensive form representation is useful when it is wanted to include not only who makes the move but also when the move is made. Players apply pure strategies when they are certain of what they want, but such condition is not always possible, so mixed strategies must be considered. A mixed strategy is a probability distribution over $S$ which corresponds to how frequently each move is chosen.

As an example, we can mention the well-known Prisoners Dilemma (PD) : Two suspected of committing a crime are caught by the police. As there is insufficient evidence to condemn them, the police place the suspects into separate rooms to convince them to confess. If one of the prisoners confesses, and help the police to condene his partner, he gains his freedom and the other prisoner must serve of 10 years. But if both confess, they must serve a sentence of 3 years. In other case, if both refuse to confess, they both will be convicted of a lesser charge and will have to serve a sentence of only one year in prison. In summary, they can choose between two possible strategies "Confess" (C) or "Not Confess" (N). However, observe that the luck of each player depends both on his election as that of the other. As consequence, confessing is a dominant strategy because regardless the other player decision the one who chooses it avoid the worst conviction. The prisoners know that if neither confesses they must serve a minimum sentence. However, as no one knows the other strategy to do not confess is very risky, specially because camaraderie is not a common quality between criminals. It 
is very common to represent in a bimatrix the possible strategies combinations with their respective reward. The corresponding bimatrix for the prisoners game is 1 .

\begin{tabular}{ccc}
\hline $\mathbf{S}_{\mathbf{1}} \backslash \mathbf{S}_{\mathbf{2}}$ & $\mathbf{C}$ & $\mathbf{N}$ \\
\hline$C$ & 3,3 & 0,10 \\
\hline$N$ & 10,0 & 1,1 \\
\hline
\end{tabular}

Table 1. Prisoners Dilemma: $C \equiv$ confess; $N \equiv$ do not confess. The number on the left is for the years the prisoner $S_{1}$ prisoner must serve.

Quantum game theory is a classic game theory generalization. That is, quantum game strategies and outcomes include the classical as particularities, but also quantum features let the application of new strategies which leads to solutions classically imposible. The $N$ players quantum game si denoted by $G(N, \mathcal{H}, \rho, S(\mathcal{H}), u)$, where $\mathcal{H}$ is the Hilbert space of the physical system and $\rho \in S(\mathcal{H})$ is the system initial condition, being $S(\mathcal{H})$ the associated space state. In quantum games, players strategies are represented by unitary operators, which in quantum mechanics are also known as evolution operators related to the system's Hamiltonian [27]. If we call $U_{i}$ the operator corresponding to player $i$ strategy, the N-players strategies operator results $U=U_{0} \otimes U_{1} \ldots U_{i} \otimes \ldots \otimes U_{N-1}$. Starting from the initial pure state $\left|\Psi_{0}\right\rangle$ of the system, players apply their strategies $U$ in order to modify it according to their preferences, that is modifying the probability amplitudes associated with each base state. As a consequence, evolution from the initial system state to some state $\left|\Psi_{1}\right\rangle$ is given by $\left|\Psi_{1}\right\rangle=U\left|\Psi_{0}\right\rangle$. Quantum games provide new ways to cooperate, to eliminate dilemmas, and as a consequence new equilibriums arise. As can be seen in [10], for example, the dilemma is avoided in the quantum Prisoner's game. That is, the system equilibrium is not longer $(\mathrm{C}, \mathrm{C})$ to be $(\mathrm{N}, \mathrm{N})$.

\section{Quantum search strategy}

In the classic dating market game [28, 29], men choose women simultaneously from $N$ options, looking for those women who would have some "property" they want. Unlike the traditional game, in the quantum version of the dating game, players get the chance to use quantum techniques, for example they can explore their possibilities using a quantum search algorithm. Grover algorithm capitalizes quantum states superposition characteristic to find some "marked" state from a group of possible solutions in considerably less time than a classical algorithm can do [8]. That state space must be capable of being translatable, say to a graph $G$ where to find some particular state which has a searched feature or distinctive mark, throughout the execution of the algorithm. By "distinctive mark" we mean problems whose algorithmic solution are inspired by physical processes. Furthermore it is possible to guarantee that the searched node is marked by a minimum (maximum) value of a physical property included in the algorithm.

Let agents be coded as Hilbert space base states. As a result, men are able to choose from $N_{w}$ women set $W=\left\{|0\rangle,|1\rangle, \ldots,\left|N_{w}-1\right\rangle\right\}$. Table 1 displays four women states in the first column and some feature that makes them unique in the second column which we will code with a letter for simplicity.

If a player is looking for a woman with a feature " $\mathrm{d}$ ", the table must be searched on its second column and when the desired " $\mathrm{d}$ " is found, look at the first column where the corresponding 


\begin{tabular}{cc}
\hline woman & feature \\
\hline$|0\rangle$ & $\mathrm{a}$ \\
$|1\rangle$ & $\mathrm{b}$ \\
$|2\rangle$ & $\mathrm{c}$ \\
$|3\rangle$ & $\mathrm{d}$ \\
\hline
\end{tabular}

Table 2. Sample woman database. Left column contains women states and right column displays a letter representing some feature or a feature set that characterizes each woman on the left.

chosen woman state is: $|3\rangle$ in this example. The procedure is very simple if the table has just a few rows, but when the database gets bigger, the table in the best case would have to be entered $N_{w} / 2$ times [30]. Under this framework we propose to use Grover algorithm in order to achieve man's decision in less time. Without losing generality let $N_{w}=2^{n}$ being $n$ the qubits needed to code $N_{w}$ women. Quantum states transformation are made by applying Hilbert space operators $U$ to them, following $\Psi_{1}=U_{1} \Psi_{0}$ is a new system state starting from $\Psi_{0}$. As a consequence any quantum algorithm can be thought as a set of suitable linear transformations. Grover algorithm starts with $n$ qubits in $|0\rangle$, resulting $\psi_{i n i}=|00 . .00\rangle \equiv|0\rangle^{\otimes n}$ the system initial state, where $\otimes$ symbol denotes Kronecker tensor product. Initially, the woman identified by state $|0\rangle$ is chosen with probability one. The next step is to create superposition states and like many other quantum algorithms Grover uses Hadamard transform to do this task since it maps $n$ qubits initialized with $|0\rangle$ to a superposition of all $n$ orthogonal states in the $|0\rangle,|1\rangle, . .|n-1\rangle$ basis with equal weight, $\psi_{1}=H \psi_{i n i}=\frac{1}{\sqrt{N_{w}}} \sum_{i=0}^{N_{w}-1}|i\rangle$. As an example, when $N_{w}=4$, the state results $\psi_{1}=H|00\rangle=$ $\frac{1}{2} \sum_{i=0}^{3}|i\rangle=\frac{|00\rangle+|01\rangle+|10\rangle+|11\rangle}{2}$. One-qubit Hadamard transform matrix representation is (1), and n-qubits extension is $H^{\bigotimes n}$, see [27],

$$
H=\frac{1}{\sqrt{2}}\left(\begin{array}{cc}
1 & 1 \\
1 & -1
\end{array}\right)
$$

Another quantum search algorithms characteristic, is the "Oracle", which is basically a black box capable of marking the problem solution. We call $U_{f}$ the operator which implement the oracle

$$
U_{f}(|w\rangle|q\rangle)=|w\rangle|q \oplus f(w)\rangle,
$$

where $f(w)$ is the oracle function which takes the value 1 if $w$ correspond to the searched woman, $f(w)=1$, and if it is not the case it takes the value $0, f(w)=0$. The value of $f(w)$ on a superposition of every possible input $w$ may be obtained [27]. The algorithm sets the target qubit $|q\rangle$ to $\frac{1}{\sqrt{2}}(|0\rangle-|1\rangle)$. As a result, the corresponding mathematical expression is:

$$
|w\rangle\left(\frac{|0\rangle-|1\rangle}{\sqrt{2}}\right) \longmapsto U_{f}(-1)^{f(w)}|w\rangle\left(\frac{|0\rangle-|1\rangle}{\sqrt{2}}\right)
$$

Observe that the second register is in an eigenstate, so we can ignore it, considering only the effect on the first register. 


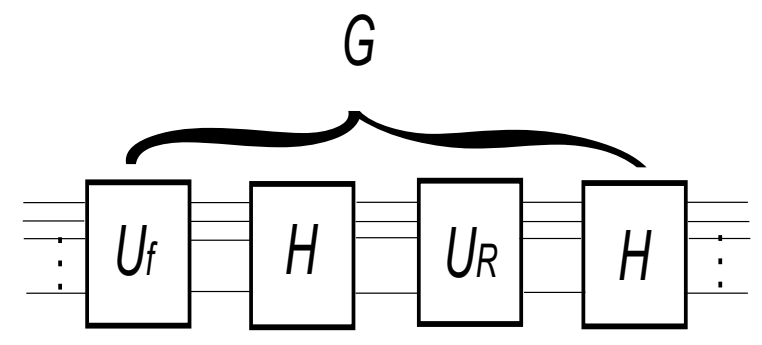

Figure 1. Grover Iteration

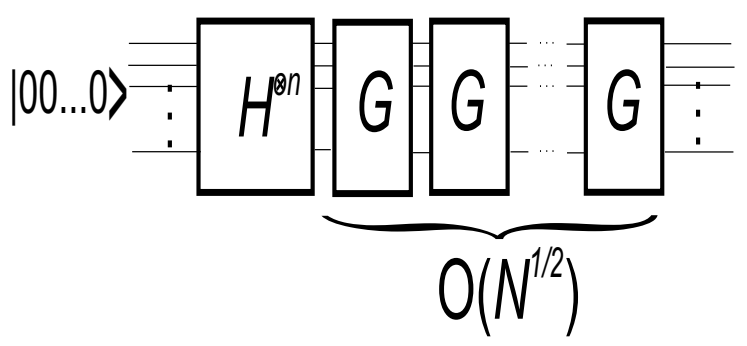

Figure 2. Grover Quantum searching algorithm

$$
|w\rangle \longmapsto U_{f}(-1)^{f(w)}|w\rangle
$$

Consequently, if $f(w)=1$ a phase shift is produced, otherwise nothing happens. As we already stated our algorithm is based on the classical Gale-Shapley (GS) algorithm which assigns the role of proposers to the elements of one set, the men say, and of judges to the elements of the other.

Actually, for a more symmetric formulation of the algorithm where both sets are, at the same time, proposers and judges, it would be necessary another oracle which evaluates women features matching by means of another function $g(x)$ [31], but we will not go into that. As far as we are concerned up to now the Oracle is a device capable of recognizing and "mark" a woman who has some special feature, said hair color, money, good manners, etc. Oracle operator $U_{f}$ makes one of two central operations comprising of a whole operation named Grover iterate $G$ (Fig.1), and a rotation operator $U_{R}$, or conditional phase shift operator represented by equation (5).

$U_{R}$ and $U_{f}$, together with Hadamard transformations represented by $H$ blocks (1), in the order depicted by (Fig. 1), make the initial state vector asymptotically going to reach the solution state vector amplitudes. The symbol $I$ in $U_{R}$ equation is the identity operator.

$$
U_{R}=2|0\rangle\langle 0|-I
$$

Furthermore, after applying Grover iterate, $G, O\left(\sqrt{N_{w}}\right)$ times, the man finds the woman he is looking for. In Figure 1 Grover iterate is shown and Grover quantum algorithm scheme is depicted in 2. 


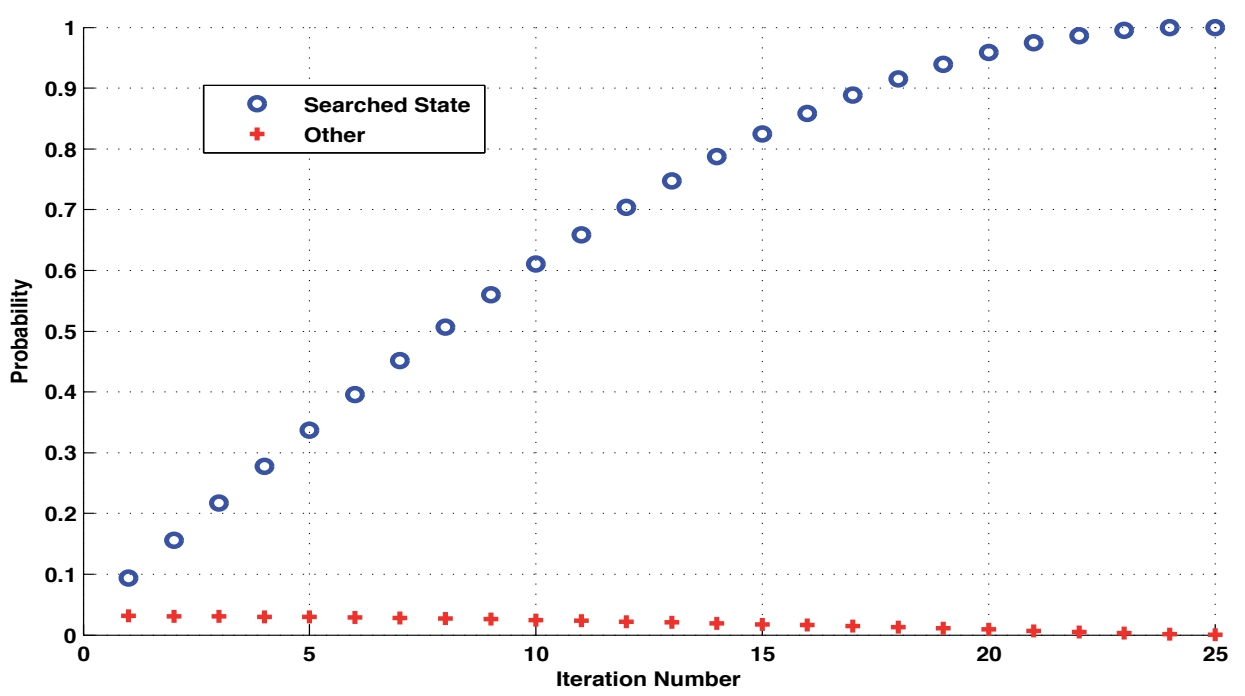

Figure 3. Evolution of the probability to find the chosen woman and the probability to find other woman as a function of the iteration number with Grover's algorithm.

As the number of iterations the algorithm makes depends on the size of the options set, this must be known at the beginning of simulations. Every operator has its matrix representation to be used in simulations. We suppose the player chooses a woman who has some specific particularity that would distinguish her from any other of the group, so we construct matrix $U_{f}$ and other matrixes for that purpose. The evolution of the squared amplitude with the iteration number is shown in Figure 3. The searched state amplitude is initially the same for all possible states $\mid i>$ in the $\Psi_{1}$ expression. The fast increasing of the probability to find the preferred state on each iteration contrasts with the decreasing of the probability to find every other state. The example displayed is for $N_{w}=1024$ women and as the can be seen in Figure 3, the number of iterations needed to get certainty to find the preferred woman are 25. Classically, a statistical algorithm would need approximately $N_{w}=1024$ iterations.

Thus when a given man who wants to date a $N_{w}$ size set selected woman, he must set his own $U_{f}$ operator out, according to his preferences, and then let the algorithm do the job. The case of $N_{m}$ men may be obtained generalizing the single man case: every one of them must follow the same steps. Nevertheless, achieving top choice is hard because of competition from other players and your dream partner may not share your feelings. If all players play quantum, the time to find woman is not an issue and the $N$ stable solutions will be the same as for the classic formulation [32].

\section{Quantum vs classic}

To compare the quantum approach efficiency with the classical one we will consider some players playing quantum and others playing classic. Let us follow the evolution of agents representative from each group, $Q$ and $C$ respectively. 
$Q$, that plays quantum can keep his state as a linear combination of all the prospective results when unitary transforms such as the described above for Grover's algorithm are applied, provided no measurement producing collapse to any of them is done. On the other hand, the only way $C$ has to search such a database is to test the elements sequentially against the condition until the target is found. For a database of size $N_{w}$, this brute force search requires an average of $O\left(N_{w} / 2\right)$ comparisons [7].

Two different games where both men want to date with the same woman are presented: In the first one player $Q$ gives player $C$ the chance to play first and both have only one attempt per turn, which means only one question to the oracle. The second game, in order that $Q$ plays handicapped, is set out in the way that $C$ can play $N_{w} / 2$ times while $Q$ only once, and player $C$ plays first again. For the last case we analyzed two alternatives for the classic player: in the first one he plays without memory of his previous result and therefore, in every try he has $1 / N_{w}$ probability to find the chosen woman to date, the other alternative permits the classic player to discard previous unfavorable outcomes at any try in order to avoid choosing them again and diminish the selection universe.

The player who invites the chosen woman first has more chances to succeed, as well as that who asks the same woman more times. Nevertheless the woman has the last word, and therefore the dating success for each player depends on that woman preferences. So, let us define $P_{c}^{i}$ as the probability that woman $i$ accepts dating the classic player $C$ and $P_{q}^{i}$ as the probability that she accepts the quantum player $Q$ proposal. In order to compare performances, we consider $T=1000$ playing times on turns and count the dating success times, then calculate the mean relative difference between $Q$ and $C$ success total number as $D / T=\frac{\text { Qsuccess-Csuccess }}{T}$, for different woman acceptation probabilities.

Initially, both players begin with the system in the initial state $\psi_{1}=\frac{1}{\sqrt{N_{w}}} \sum_{i=0}^{N_{w}-1}|i\rangle$, therefore the probability to select any woman is the same for both, $p\left(w_{i}\right)=1 / N$. In the next step the Oracle marks one of the prospective women state according men preferences.

The results are highly dependent on the women set size $N_{w}$ because, as mentioned above, Grover algorithm needs $\left.O\left(\sqrt{(} N_{w}\right)\right)$ steps to find the quantum player's chosen partner while the classic player must use $O\left(N_{w}\right)$ for the same task. In the case of only one woman and one man, for example, classic and quantum will not have any advantage on searching and the dating success difference for the first game will depend only on that woman preferences, that is, if $P_{c}>P_{q}$ then $D / T<0$ and the quantum player will do better when $P_{q}>P_{c}$. Similar chances for both players is not usual in most quantum games, such as, for example the coin flip game introduced by Meyer [9] where the quantum player always beats the classic player in a "mano a mano" game. For a two women set $Q$ uses only one step, but $C$ needs two steps to find the right partner. In this case $Q$ does better when $P_{q}>P_{c} / 4$. Winning conditions improve for the quantum player for increasing $N_{w}$, but not in a monotonous way, because the number of steps used by Grover algorithm in $Q$ search is an integer that increases in discrete steps.

In order to facilitate comprehension the set size in the simulations results shown is $N_{w}=8$, that is the biggest $N_{w}$ (taken as $2^{n}$ ) in which $Q$ uses only one step in Grover algorithm.

Under the first game conditions both players have only one attempt by turn. Since $C$ cannot modify state $\psi_{1}$ amplitudes, he has $1 / 8$ chance to be right. On the other hand player $Q$, using Grover algorithm as his strategy, can modify states amplitudes in order to increase 


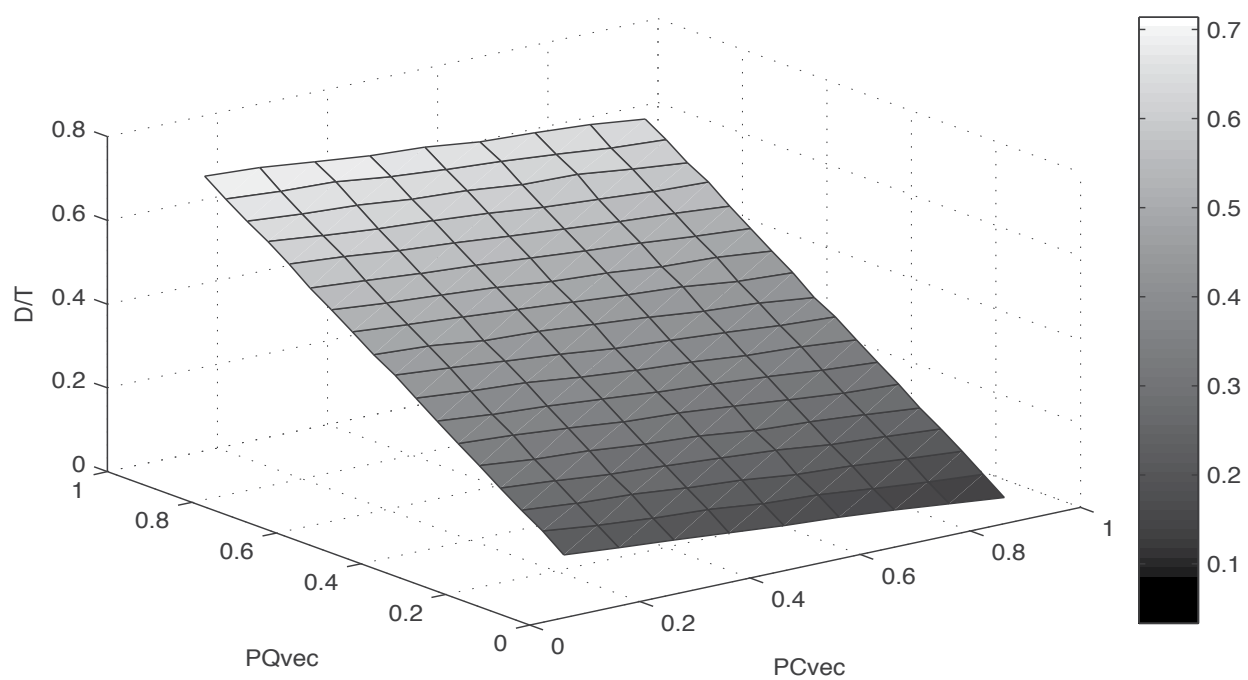

Figure 4. First game: One attempt for both players. Mean relative difference between $Q$ and $C$ success total number as $D / T=\frac{\text { Qsuccess-Csuccess }}{T}$, for different woman acceptation probabilities $P_{c}^{i}$ and $P_{q}^{i}$. Q outperforms $C$ in all shown cases. The small region where $C$ prevails is not shown.

his chances to win, reaching 0.78 as the probability to find his preferred woman in only one iteration. Figure 4 shows that situation outcomes for different $P_{c}^{i}$ and $P_{q}^{i}$ combinations. The vertical axis depicts $D / T$ values as a function of $P_{c}^{i}$ and $P_{q}^{i}$ respectively. $D / T$ is positive for all $P_{c}^{i}$ and $P_{q}^{i}$ values used in the simulation, which means that even at extremes where $P_{c}^{i}>>P_{q}^{i}$ the quantum player performs better. However there is a very small region where $P_{c}^{i} \approx 1$ and $P_{q}^{i} \approx 0$ not shown in the figure that corresponds to a prevailing $C$.

Under the second game conditions player $C$ have $\frac{N_{w}}{2}=4$ attempts before $Q$ plays. After each $C$ attempt the system is forced to collapse to one base state, so a third party, that could be the oracle, arrange the states again and mark the solution. As we explained above, to mark a state means to change its phase but nothing happens to the state amplitude, consequently, for the classic player $C$, the probability that state results the one the Oracle have signaled is, marked or not, $1 / N_{w}=1 / 8$, even though, due to his "insistence", he tries $\frac{N_{w}}{2}=4$ times, his dating success chances increase considerably with respect to the first case. Figure 5 shows the corresponding results, where it is possible to see that classic player $C$ begins to outperform $Q$ when $P_{c}^{i}>>P_{q}^{i}$, that is, when woman has a marked preference for player $C$.

Player $C$ probability to find the chosen woman can increase to $\frac{1}{2}$ when using a classical algorithm like "Brute-Force algorithm". As shown in figure 5, when $C$ has $\frac{N_{w}}{2}=4$ tries while $Q$ has only one, C's odds of success in dating increases, and there are zones on the graph where $D / T<0$. This implies that player $C$ outperforms player $Q$. Nevertheless, to achieve that, the chosen woman preferences must be considerably greater for the classic player, that is $P_{c}^{i}>2 P_{q}^{i}$. 


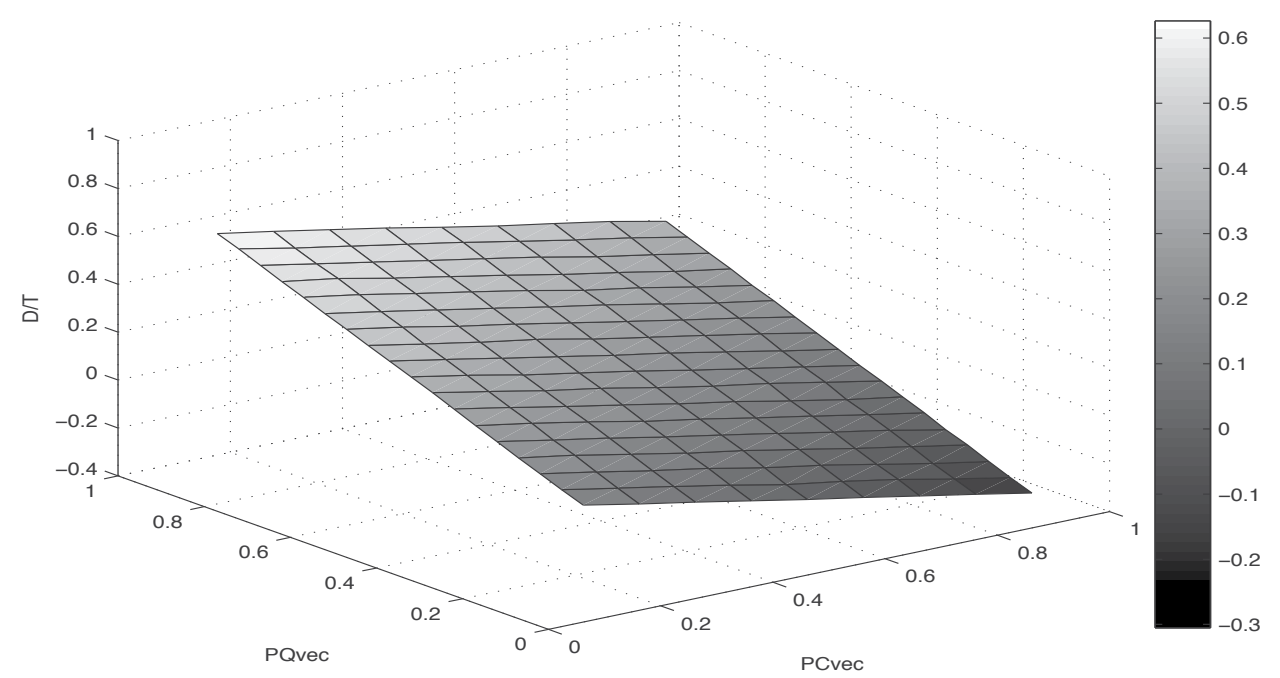

Figure 5. Second game: Classic player $C$ has four tries while $Q$ has only one. Mean relative difference between $Q$ and $C$ success total number as $D / T=\frac{\text { Qsuccess-Csuccess }}{T}$, for different woman acceptation probabilities $P_{c}^{i}$ and $P_{q}^{i}$. C outperforms $Q$ when $P_{c}^{i}>>P_{q}^{i}$

\subsection{Section discussion}

In this section we have introduced a quantum formulation for decision matching problems, specifically for the dating game. In that framework women are represented with quantum states whose associated amplitudes must be modified by men's selection strategies, in order to increase a particular state amplitude and to decrease the others, with the final purpose to achieve the best possible choice when the game finishes. This is a highly time consuming task that takes a $O(N)$ runtime for a classical probabilistic algorithm, being $N$ the women database size. Grover quantum search algorithm is used as a playing strategy that takes the man $O(\sqrt{N})$ runtime to find his chosen partner. As a consequence, if every man uses quantum strategy, no one does better than the others, and stability is quickly obtained.

The performances of quantum vs. classic players depend on the number of players $N$. In a "one on one" game there is no advantage from any of them and the woman preferences rule. Similar chances for quantum and classic players in "one on one" situation is not usual in most quantum games. Winning conditions improve for the quantum player for increasing $N$ and the same number of attempts, but not in a monotonous way. The comparison between quantum and classic performances shows that for the same numbers of attempts, the quantum approach outperforms the classical approach. If the game is set in order that the classic player has $\frac{N}{2}$ opportunities and the quantum player only one, the former player begins to have an advantage over the quantum one when his probability to be accepted by the chosen woman is much higher than the probability for the quantum player. 


\section{Stability of couples}

There is a group of $N_{m}$ men and $N_{w}$ women playing the game. Be $S_{i}=\left\{|0\rangle,|1\rangle, \ldots,\left|N_{w}-1\right\rangle\right\}$ the states in a Hilbert space of man $i$ decisions, where $\left\{0,1, \ldots, N_{w}-1\right\}$ are indexes in decimal notation identifying all the women he may choose. As a result each man has been assigned $\log _{2}\left(N_{w}\right)$ qubits in order to identify each woman. Generally, the state vector of one man decisions will be in quantum superposition of the base states, $\Psi_{i}=\sum_{j=0}^{N_{w}-1} \alpha_{j}|j\rangle$, where $\left|\alpha_{j}\right|^{2}$ is the probability that man $i$ selects woman $j$ when system state is $\Psi_{i}$ so must satisfied the normalization condition $\sum_{j=0}^{N_{w}-1}\left|\alpha_{j}\right|^{2}=1$. If there is no correlation between players, the state space of all men decision system is represented through $S_{M}=S_{0} \otimes S_{1} \otimes \ldots \otimes S_{M-2} \otimes S_{M-1}$, where $\otimes$ is the Kronecker product. Note that the $S_{M}$ extends to any possible combination of men elections. On the other side there are the women who receive men proposals and must decide whether to accept or not one of them. With greater or lesser probability they will receive the all men's proposals, so following the same argument used with the men, be $\Psi_{j}=\sum_{i=0}^{M-1} \alpha_{i}|i\rangle$ the woman $j$ acceptation state and be $S_{W}=S_{0} \otimes S_{1} \otimes \ldots \otimes S_{N_{w}-2} \otimes S_{N_{w}-1}$ the women acceptances space state. Finally, to close the circle, we define the couples possible states which must include so all possible men's elections as all possible women's acceptances. Accordingly, state space of the couples emerge from the Kronecker product of the men and women spaces,i.e. $S_{C}=S_{M} \otimes S_{W}$.

\subsection{Strategies}

In quantum games, players strategies are represented by unitary operators, which in quantum mechanics are also known as evolution operators related to the system's Hamiltonian [27]. If we call $U_{i}$ the operator corresponding to player $i$ strategy, the N-players strategies operator results $U=U_{0} \otimes U_{1} \ldots U_{i} \otimes \ldots \otimes U_{N-1}$. Starting from the initial pure state $\left|\Psi_{0}\right\rangle$ of the system, players apply their strategies $U$ in order to modify it according to their preferences, that is modifying the probability amplitudes associated with each base state. As a consequence, evolution from the initial system state to some state $\left|\Psi_{1}\right\rangle$ is given by $\left|\Psi_{1}\right\rangle=U\left|\Psi_{0}\right\rangle$. Note that, following the reasoning of the preceding paragraph, when $\Psi_{0}$ is the initial state and $\Psi_{1}$ is the final state of the couples system, $U$ arises from men and women strategies $U_{M}$ and $U_{W}$ respectively through $U=U_{M} \otimes U_{W}$. That is, $U_{M}$ is applied by men to the qubits that identify the women states, meanwhile the women action on the qubits that identify men states is given by $U_{W}$.

\subsection{Density matrix and system entropy}

Often, as in life, players are not completely sure about which strategy to apply, that is, by the way of example, the case where someone chooses between the strategy $U_{a}$ with probability $p_{a}$ and $U_{b}$ with probability $p_{b}=1-p_{a}$, that situation is referred in a mixed strategies game. Despite the complete system can be represented by its state vector, when it comes to mixed states the density matrix is more suitable. It was introduced by von Neumann to describe a mixed ensemble in which each member has assigned a probability of being in a determined state. The density operator, as it is also commonly called, represents the statistical mixture of all pure states and is defined by the equation 


$$
\rho=\sum_{i} p_{i}\left|\Psi_{i}\right\rangle\left\langle\Psi_{i}\right|
$$

where the coefficients $p_{i}$ are non-negative and add up to one. From the density operator we can construct and understand the statistical behavior about our system by using statistical mechanics and a criterion of maximum or minimum entropy. Continuing the example, if it is supposed that the system starts in the pure state $\rho_{0}=\left|\Psi_{0}\right\rangle\left\langle\Psi_{0}\right|$, after players mixed actions density matrix evolution is

$$
\rho_{1}=p_{a} U_{a} \rho_{0} U_{a}^{\dagger}+p_{b} U_{b} \rho_{0} U_{b}^{\dagger}
$$

Entropy is the central concept of information theories, [33]. The quantum analogue of entropy was introduced in quantum mechanics by von Neumann,[34] and it is defined by the formula

$$
S(\rho)=-\operatorname{Tr}\left\{\rho \log _{2} \rho\right\}
$$

\section{3. $N=2$ Model}

In order to set up the notation let us look at the following example of two men and two women that interact for $T$ times periods. Let define $\Psi_{0}^{i}=\alpha|0\rangle+\beta|1\rangle$ as the initial decision state of men $i$ which is a linear superposition of the two possible options he has, they are woman 0 or 1 . Without losing generalization consider $\alpha=1$ and $\beta=0$ which is consistent with thinking that they both have preference for the most popular, the most beautiful, the richest, or any superficial feature that most of the time makes men desire a woman at first glance. Consequently, the men's initial state vector is $\Psi_{0}^{M}=\Psi_{0}^{0} \otimes \Psi_{0}^{1}=|00\rangle$, where the first qubit represent man's 0 choice and the second is man's 1 choice. As we explain above, the initial quantum pure state is not stable, so during the game the state will change to the general form $\Psi_{a}^{M}=\sum_{i=0, j=0}^{1} \alpha_{i j}|i j\rangle$ with probability $p_{a}$ and $\Psi_{b}^{M}=\sum_{i=0, j=0}^{1} \beta_{i j}|i j\rangle$ with probability $p_{b}$. As women have the last decision, they must evaluate men proposals and

decide to accept one of them or reject all. We consider, just for the example that woman 0 chooses man 0 with $p_{0 m}$ and man 1 with probability $1-p_{0 m}$, similar condition for woman 1 but in this case being $p_{1 m}$ the probability to choose man 0 . That condition doesn't affect system stability but depending on the probabilities values does affect the maximum and minimum of the couple system's entropy. Equation 9 shows the women density matrix which has no off diagonal elements.

$$
\rho_{w 0}=p_{0 m} p_{1 m}|00\rangle\left\langle 00\left|+p_{0 m}\left(1-p_{1 m}\right)\right| 01\right\rangle\left\langle 01\left|+\left(1-p_{0 m}\right) p_{1 m}\right| 10\right\rangle\left\langle 10\left|+\left(1-p_{0 m}\right)\left(1-p_{1 m}\right)\right| 11\right\rangle\langle 11| .
$$

The direct product of all possible men proposals with all possible women decisions generates a possible partners state vector which in decimal notation is $\Psi_{0}^{P}=\sum_{i=0}^{15}|i\rangle$. Index $i$ is a four qubits number, the first two qubits represent men 0 and 1 choices respectively and the 


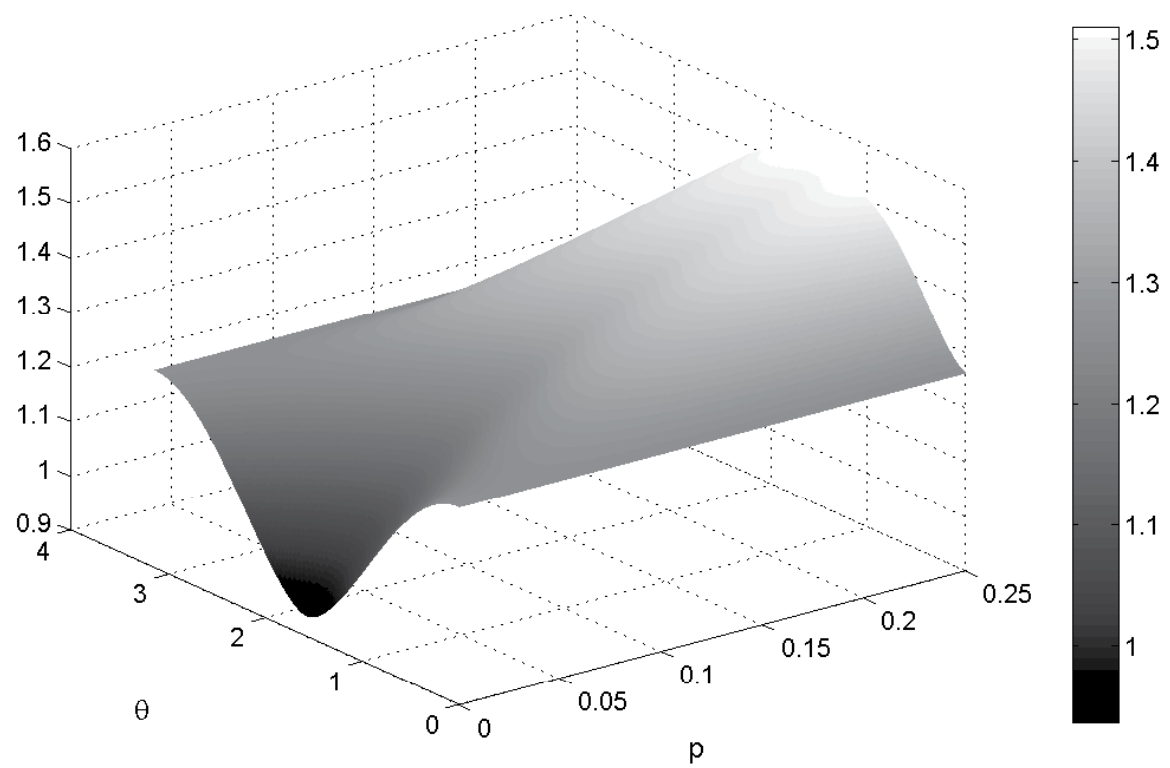

Figure 6. Quantum entropy corresponding to the situation where player 0 varies the probability $p$ to apply strategy $U_{0}^{0}$

other two are the two women possible selections, then 16 are the possible couples states. For example, the state $|0101\rangle$ corresponds to the case that man 0 chooses woman 0 and she accepts him and the same occurs with man 1 and woman 1 . Note that not all states corresponds to possible dates, some of them are considering the cases where there are no date, or the ones where only one couple is formed, the state $|0001\rangle$ is an example of the last case where the man man 0 chooses woman 0 and she accepts but on the other hand man 1 also chooses woman but she doesn't and woman 1 does not receive any proposition. As the game progress, probability amplitudes associated with mismatches must decrease, that because it is considered that people prefer to be coupled.

Single players moves or strategies are associated with unitary operators $U_{i}(\theta)$, with $0 \leq \theta \leq$ $\pi$, applied on each one of their qubits, that in the general case where players have $2^{n}$ options, each pure strategy $U$ is composed by $n$ different $U_{i}\left(\theta_{k}\right)$, being $k$ each state qubit. The general formula of $U_{i}$ is 10, that are rotation operators, as explained in [27] any qubit operation can be decomposed as a product of rotations. In this work we consider $\gamma=0$, therefore in what follows $U(\theta, 0)$ is always replaced by the simplest notation $U(\theta)$.

$$
U(\theta, \gamma)=\left(\begin{array}{cc}
e^{i \gamma} \cdot \cos (\theta / 2) & \sin (\theta / 2) \\
-\sin (\theta / 2) & e^{-i \gamma} \cdot \cos (\theta / 2)
\end{array}\right)
$$

Let $p_{0}$ be the probability of player 0 to apply strategy $U_{0}^{0}$ and $1-p_{0}$ the probability to apply strategy $U_{1}^{0}$ to the initial state $\Psi_{0}^{i}$, while $U_{0}^{1}$ and $U_{1}^{1}$ are the strategies the man 1 applies with probability $p_{1}$ and $1-p_{1}$ respectively. The strategies operators used in the examples are defined below, equations 11 and 12 are applied by man 0 . Both of them transform the initial 


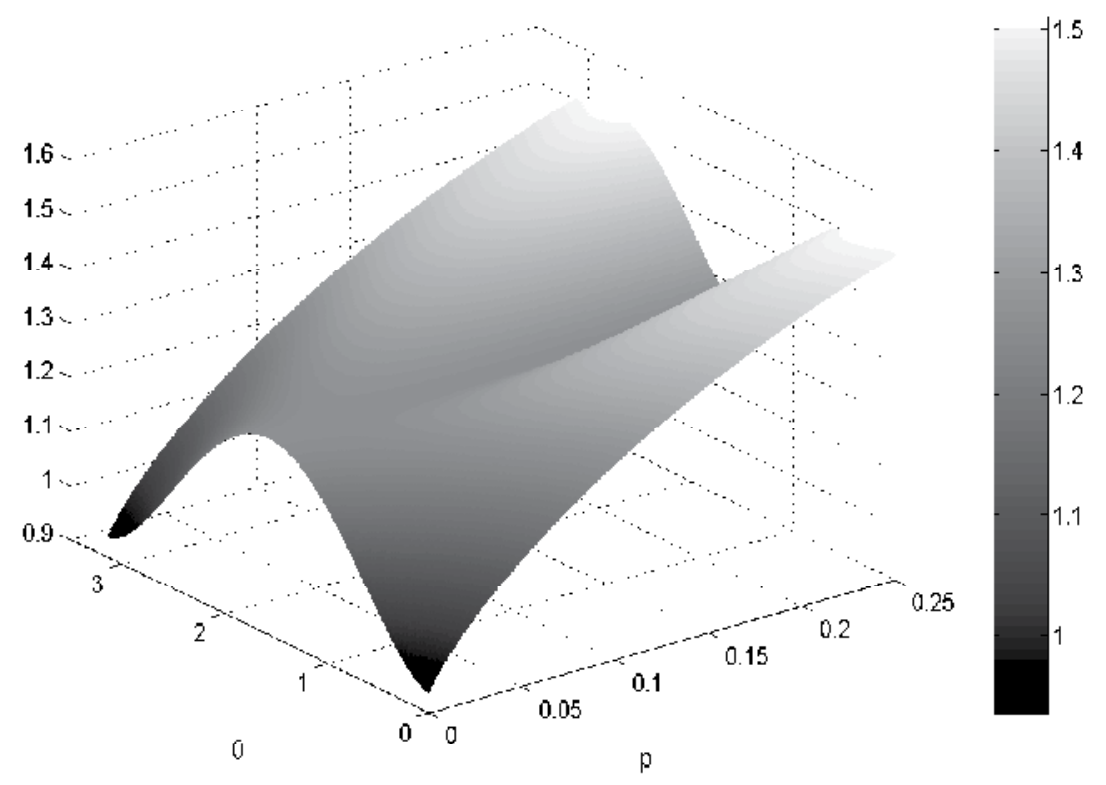

Figure 7. Quantum entropy corresponding to the situation where player 1 varies the probability $p$ to apply strategy $U_{0}^{1}$

state $|0\rangle$ into states that are linear superpositions of 0 and 1 , representing states with different probabilities of choosing one woman or the other. On the other hand, strategies applied by man 1 are presented in 13 and 14 .

Figures 6 and 7 show two situations where the system entropy varies considerably as a function of the strategies the players use. Figure 2, for example, shows the case where the man 1 applies his strategies with fixed probability, just varying the angle $\theta$ while the other man $(0)$ varies both strategies angle and the probability $p$. In all the cases we present here, in order to simplify the outcomes display, women density matrix doesn't change as explained above.

$$
\begin{aligned}
& U_{0}^{0}=U(\theta) \\
& U_{1}^{0}=U(\theta) U(\pi) \\
& U_{0}^{1}=U(\theta) \\
& U_{1}^{1}=U(-\theta)
\end{aligned}
$$

For example, if both men choose the same woman with probability one, this is represented in Fig. 1 with $p=0$. This situation is completely unstable because it is impossible for the 
woman to choose both of them at the same time (we assume). This correspond to minimum entropy as can be easily seen in Fig. 1. Depending on the strategies applied by men, the whole system entropy, that is the couple system entropy changes reaching maximum and minimum limits. As $p$ increases, the mixing of the strategies also increases producing an increase in entropy that indicates a tendency to stability. The mixing of the strategies means that the men proposals are less focused on one woman. Fig. 2 shows the case where men's role change, that is man 0 fixes his strategies probabilities while 1 varies his own. Although for fixed $\theta$ angle, as expected, the minimum entropy points are located where player is applying a pure strategy $(p=0)$, for $\theta=\pi / 2$ the entropy value does not change regardless of the value of $p$, because $U_{0}^{0}$ and $U_{1}^{0}$ are equivalent and therefore player 1 is applying a pure strategy. A result not shown in the figures is that entropy maxima increase when women preferences are the same for every men.

In this way, maxima and minima entropy points may be used to find stable states. Nevertheless, these stable states may not correspond to equilibria states of the game, because the players utilities has not been considered. In order to find Nash equilibria states, these utilities must be considered. This is beyond this chapter goals.

\subsection{Section discussion}

As a continuation of the analysis of a quantum formulation for the dating game that takes into account mutual progressive learning by representing women with quantum states whose associated amplitudes must be modified by men's selection strategies. we concentrate on the information associated to the problem. Since we deal with mixed strategies, the density matrix formalism is used to describe the system. Even though the decisions players make are based on their payoffs, past experiences, believes, etc., we are not interested in that causes but in the consequences of the decision they take, that is, the influence of the strategies they apply on the quantum system stability by means of the equivalence between maximum entropy states and those states that obey the Collective Welfare Principle that says that a system is stable only if it maximizes the welfare of the collective above the welfare of the individual. Maxima and minima entropy points are used to find characteristic strategies that lead to stable and unstable states. Nevertheless, in order to find Nash equilibria states, the players utilities must be considered.

Maxima and minima entropy do not depend only on the strategies of men but also on women preferences, reaching the highest value when they have no preferences, that is when they choose every man with equal probability. On the other hand, minimum entropy correspond to men betting all chips to a single woman, without giving a chance to other woman.

\section{Entangled strategies}

The quantum dating market problem has been formulated as a two-sided bandit model [28], where in one side there are the men who must choose one "item" from the other side, which unlike the one side bandit, is composed by women able to reject the invitations.

The quantum formulation, which was presented in previous section, proceeds by assigning one basis of a Hilbert state space to each woman. As a consequence, if $N_{w}$ is number of women playing, $S_{i}=\left\{|0\rangle,|1\rangle, \ldots,\left|N_{w}-1\right\rangle\right\}$ are the states in the Hilbert space representing a man $i$ decisions, therefore every man needs at least $\log _{2}\left(N_{w}\right)$ qubits to identify each woman. 
The state of man $i$ decisions is a quantum superposition of the base states, $\Psi_{i}=\sum_{j=0}^{N_{w}-1} \alpha_{j}|j\rangle$, where $\left|\alpha_{j}\right|^{2}$ is the probability that man $i$ selects woman $j$ when system state is $\Psi_{i}$ and $|\cdot\rangle$ is known as Dirac's notation. The normalization condition is $\sum_{j=0}^{N_{w}-1}\left|\alpha_{j}\right|^{2}=1$. On the other side of the market, women receive men proposals and must decide which is the best, accept it and reject the others. Thus, $\Psi_{j}=\sum_{i=0}^{N_{m}-1} \alpha_{i}|i\rangle$ is woman $j$ acceptation state. Finally, combining proposals and acceptances the couples space which is the Kronecker product of all men's and all women's spaces is defined, i.e. $S_{C}=S_{M} \otimes S_{W}$.

Men decision states are separable when there is no connection among players, that is, for instance, no man has any emotional bond with some other that could condition their actions, thus all men decision state, $\psi_{M}$, is defined as $\psi_{M}=\bigotimes_{i=1}^{M-1} \psi_{i}$. The same reasoning corresponds to women states. On the other hand, if there is some relationship between two or more men, their actions are non-locally correlated, that is, their decisions are far from being independent. John Stuart Bell shown in 1966 that systems in entangled states exhibit correlations beyond those explainable by local "hidden" properties, or in other words, a non-local connection appears when two quantum particles are entangled, [35]. Therefore, we will study the case with correlation between agents by means of quantum entanglement, in other words, how harmful or beneficial can be for players knowing each other in advance.

As we mention in the previous section, players strategies are represented by unitary operators in quantum games. Starting the system in some state $\left|\Psi_{0}\right\rangle$ at time $t_{0}$, players apply their strategies $U$ in order to modify it according to their preferences, that is modifying the probability amplitudes associated with each base state. Thus, evolution from the initial system state to some state $\left|\Psi_{1}\right\rangle$ in time $t_{1}$ is given by $\left|\Psi_{1}\right\rangle=U\left|\Psi_{0}\right\rangle$. The strategy operator $U$ arises from men and women preferences operators $U_{M}$ and $U_{W}$ respectively through $U=U_{M} \otimes U_{W}$, where $U_{M}$ is applied by men to the qubits that identify the women states, meanwhile the women action on the qubits that identify men states is given by $U_{W}$.

In order to understand the problem we analyze here a simple example of two men and two women. Single players moves or strategies are associated with $2 \times 2$ unitary rotation operators $U_{i}(\theta, \gamma)$ applied on each one of their qubits (15), where $0 \leq \theta \leq \pi$ and $0 \leq$ $\gamma \leq \pi / 2$. Men choices are coded by states $\left|w_{0}\right\rangle=|0\rangle$ and $\left|w_{1}\right\rangle=|1\rangle$, meanwhile women must decide between men $\left|m_{0}\right\rangle=|0\rangle$ and $\left|m_{1}\right\rangle=|1\rangle$. Since any qubit operation can be decomposed as a product of rotations, strategies combinations and possible outcomes are infinite. As a consequence, focusing on men relationship, we study three relevant cases. We suppose, as a measure of satisfaction, that men receive some payoff $p_{w_{i}}$ if accepted by woman $w_{i}$, so for the example we have considered that $p_{w 0}=2$ and $p_{w 1}=5$.

$$
U(\theta, \gamma)=\left(\begin{array}{cc}
e^{i \gamma} \cos (\theta / 2) & \sin (\theta / 2) \\
-\sin (\theta / 2) & e^{-i \gamma} \cos (\theta / 2)
\end{array}\right)
$$

\subsection{Results}

For the first case, let us consider $\psi_{0}=\frac{\sqrt{2}}{2}(|01\rangle+|10\rangle)$ as the initial state of men decisions system, where the left qubit of $\psi_{0}$ is representing man 0 election while the right one represents man 1 choice. As men states are entangled, it is not possible to uncouple their 
single actions. Therefore judging on the probability amplitudes, there is $50 \%$ probability that man 0 chooses woman 0 while man 1 chooses woman 1 and the other $50 \%$ for the other case. Since there is no way that men choose the same woman it is a state of mutual cooperation. Women acceptation state is initialized to $\psi_{w}=0.5(|00\rangle+|01\rangle+|10\rangle+|11\rangle)$, implying that there is initially $25 \%$ chance that they choose the same man. In order to analyze the effect of woman behavior on men payoffs, for this and the following two cases, we consider that men decision state $\psi_{0}$ is invariant, while women strategies and their acceptation state $\psi_{w}$ change. Figure 8 shows the payoff for man 0 as a function of women strategies which are set as $U_{i}=(t \cdot \pi, t \cdot \pi / 2)$ for $t \in[-1,1]$ and $i=0,1$. Different strategies imply changes on women preferences, so some change in $U_{i}(\theta, \gamma)$ implies that woman $i$ acceptation probability distribution is modified. Following [9], equation 16 represents man 0 payoff, where $P_{00}$ and $P_{01}$ are his chances to be accepted for a date with woman 0 and 1 respectively.

$$
\$_{m 0}=2 \cdot P_{00}+5 \cdot P_{01}
$$

In the second example we introduce competition between men. The initial men state is given by $\psi_{0}=\frac{\sqrt{2}}{2}(|00\rangle+|11\rangle)$. Figure 9 depicts again the resulting payoffs for man 0 as a function of women strategies. Finally a third case is considered where men decision state is $\psi_{m}=0.5(|00\rangle+|01\rangle+|10\rangle+|11\rangle)$. In this case men make independent choices choosing one of four possible options with equal probability. Figure 10 show the resulting payoffs as a function of woman strategies.

As the figures show, the different scenarios present significant differences on payoff topology and maximum payoff values.

The cooperative situation presents the highest payoff compared with the competitive and the independent ones as shown in figure 8. Figures 9 and 10 show that a better payoff may be obtained in the competitive setup compared to the independent one, but on the other hand, also a much lower payoff for other women strategies may be available. The independent decision scenario is thus characterized by lowest maxima and less variation on payoffs.

\subsection{Section discussion}

We have considered the dating market decision problem under the quantum mechanics point of view with the addition of entanglement between players states. Women and men are represented with quantum states and strategies are represented by means of unitary operator on a complex Hilbert space. Men final payoff, considering payoff as a measure of satisfaction, depends on the woman he is paired with. If men decision states are entangled, their actions are non-locally correlated modeling competition or cooperation scenarios. Three examples are shown in order to illustrate the more usual scenarios. In two of them the men strategies are correlated in a cooperative and a competitive way respectively. In the other example men strategies are independent. Although cooperative and competitive strategies can drive to higher payoffs, changing of women preferences on those scenarios can lead to very low payoffs. The independent decision scenario is characterized by less variation on payoffs. 


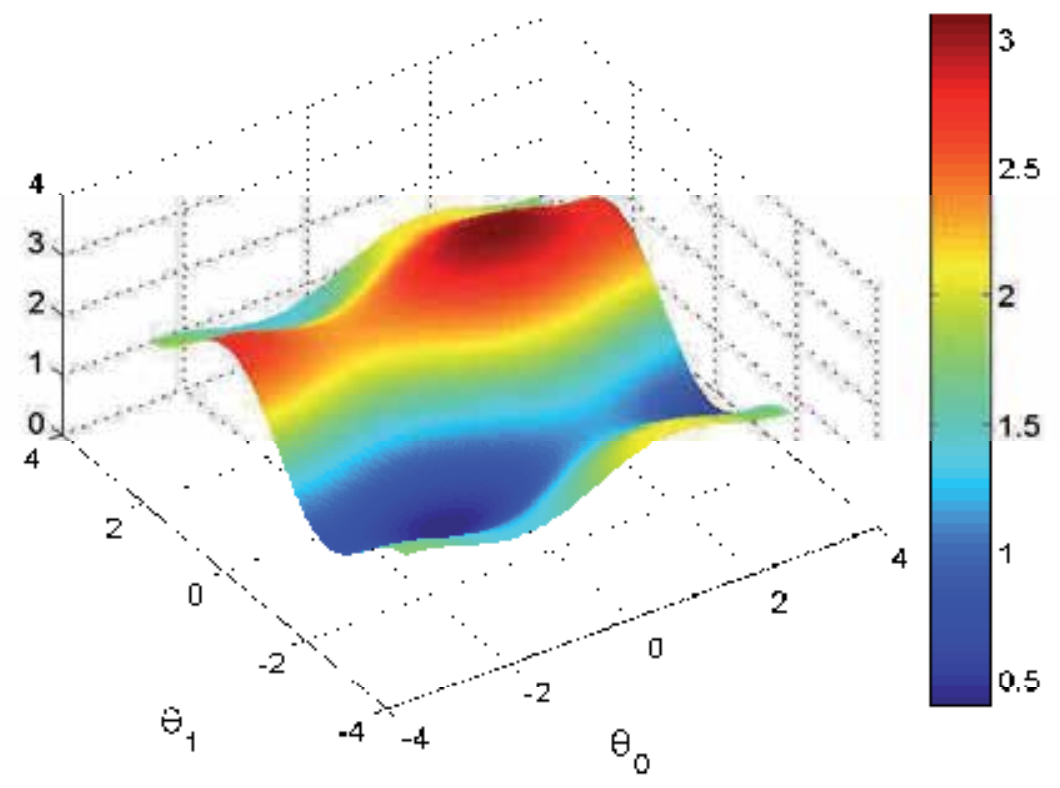

Figure 8. Payoff for man 0 if men never choose the same woman, as function of women acceptation strategies. For the example $\gamma$ varies as $\theta / 2$.

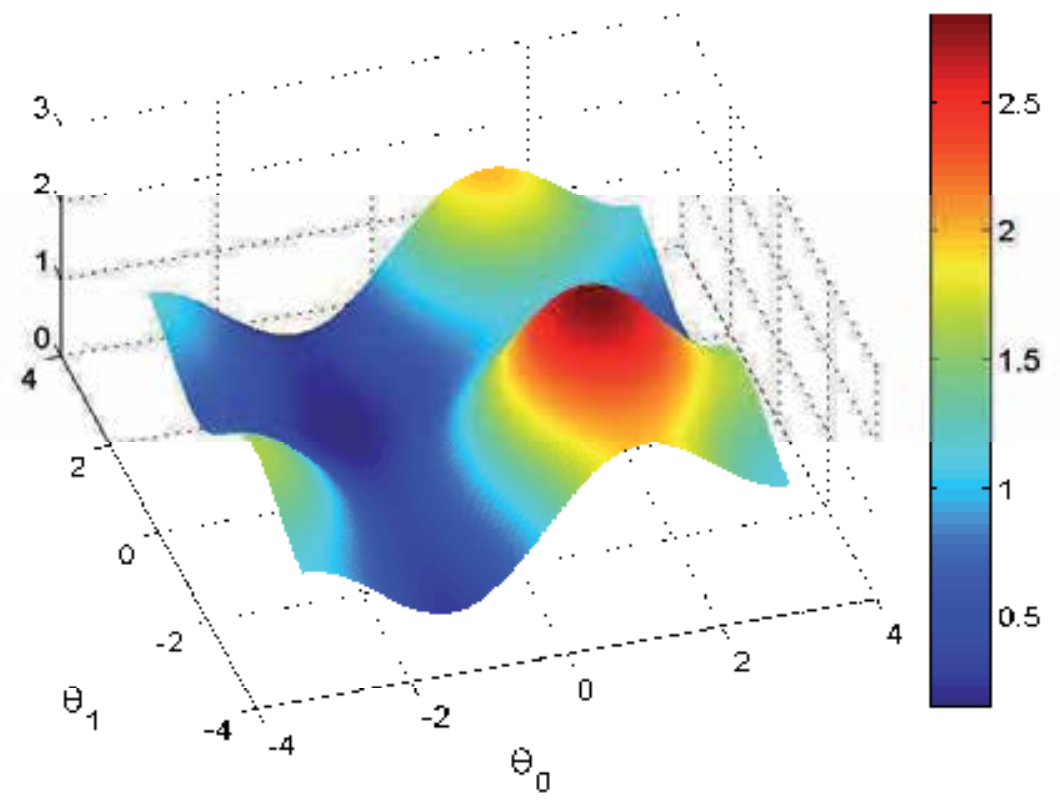

Figure 9. Payoff for man 0 if men always choose the same woman, as function of women acceptation strategies. For the example $\gamma$ varies as $\theta / 2$. 


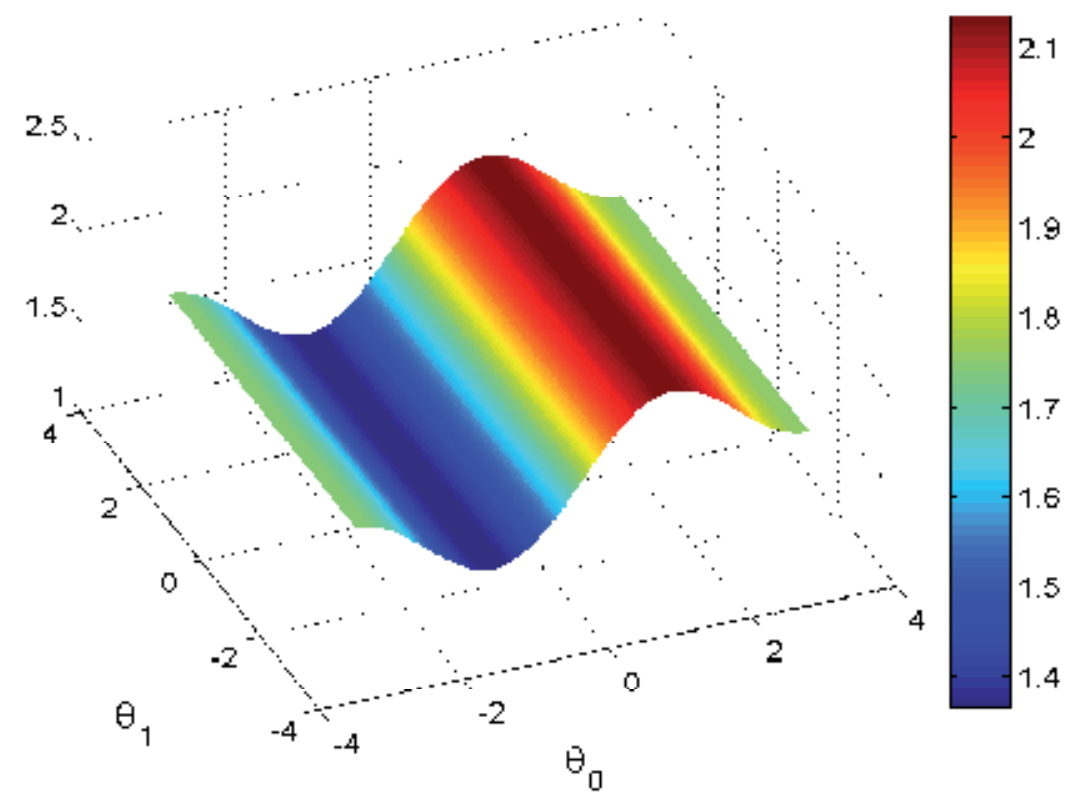

Figure 10. Payoff for man 0 if men choose without restrictions, states are not entangled, as function of women acceptation strategies. For the example $\gamma$ varies as $\theta / 2$.

\section{Final remarks}

The dating market problem may be included in a more general category of matching problems where the elements of two sets have to be matched by pairs. Matching problems have broad implications not only in economic and social contexts but in other very different research fields such as communications engineering or molecular biology, for example. The main goal of this chapter is to introduce and analyze a quantum formulation for the dating market game, whose nearest classical antecedent is the Stable Marriage Problem. Players strategies are represented by unitary operators, which in quantum mechanics are also known as evolution operators related to the Hamiltonian of the system. Significant outcomes arise when classic players play against quantum ones. For instance, when a quantum player uses Grover search algorithm as her strategy, her winning probabilities grow with increasing number of players, but none leads in a "one on one" game. Besides, from stability point of view, maxima and minima entropy points are used to find characteristic strategies that lead to unstable and stable states, resulting the highest entropy values when women have no preferences, that is, when they choose every man with equal probability. On the other hand, minimum entropy correspond to men betting all chips to a single woman, without giving a chance to other woman. Finally, to model relationships between people that may correlate their decisions, our model consider the situation when men decision states are entangled and their actions are non-locally correlated modeling competition or cooperation scenarios. One of the main outcomes is for example that, although cooperative and competitive strategies can drive to higher payoffs, changing of women preferences on those scenarios can lead to very low payoffs. 


\section{Author details}

C. M. Arizmendi and O. G. Zabaleta

Facultad de Ingeniería, Universidad Nacional de Mar del Plata, Argentina

\section{References}

[1] P.I. Hagouel and I.G. Karafyllidis. Quantum computers: Registers, gates and algorithms. In Microelectronics (MIEL), 2012 28th International Conference on, pages 15 -21, may 2012.

[2] D.C. Marinescu. The promise of quantum computing and quantum information theory -quantum parallelism. In Parallel and Distributed Processing Symposium, 2005. Proceedings. 19th IEEE International, page 112, april 2005.

[3] H. Haffner, C.F. Roos, and R. Blatt. Quantum computing with trapped ions. Physics Reports, 469(4):155-203, 2008.

[4] Chao-Yang Pang, Ri-Gui Zhou, Cong-Bao Ding, and Ben-Qiong Hu. Quantum search algorithm for set operation. Quantum Information Processing, pages 1-12, 2012.

[5] B. Criger, O. Moussa, and R. Laflamme. Quantum error correction with mixed ancilla qubits. Phys Rev A, 85(4):5 pp, 2012.

[6] P. W. Shor. Polynomial-time algorithms for prime factorization and discrete logarithms on a quantum computer. arXiv:quant-ph/9508027v2, 1996.

[7] M. Boyer, G. Brassard, P. Hoeyer, and Tapp A. Tight bounds on quantum searching. Fortsch.Phys, 46:493-506, 1998.

[8] L.K. Grover. A fast quantum mechanical algorithm for database search. In Proceedings, 28th Annual ACM Symposium on the Theory of Computing (STOC), pages 212-219, May 1996.

[9] D.A. Meyer. Quantum strategies. Phys. Rev. Lett., 82:1052-1055, 1999.

[10] J. Eisert, M. Wilkens, and M. Lewenstein. Quantum games and quantum strategies. Phys. Rev. Lett., 83:3077-3080, 1999.

[11] A. Lambert-Mogiliansky, S. Zamir, and H. Zwirn. Type indeterminancy: a model of kt-man. J. Math. Psych., 53:349-361, 2009.

[12] V.I. Yukalov and D. Sornette. Quantum decision theory as quantum theory of measurement. Physics Letters A, 372(46):6867-6871, 2008.

[13] T. Temzelides. An uncertainty principle for social science experiments. Available at http://www.owlnet.rice.edu/ tl5, 2005.

[14] C. M. Arizmendi and O. G. Zabaleta. Stability of couples in a quantum dating market. special IJAMAS issue "Statistical Chaos and Complexity, 26:143-149, 2012. 
[15] O.G. Zabaleta and C.M Arizmendi. Quantum decision theory on a dating market. Advances and Applications in Statistical Sciences (AASS), 6:489, 2011.

[16] D. Gale and L.S. Shapley. College admissions and the stability of marriage. Am. Math. Monthly, 69:9-15, 1962.

[17] Y. Zhang and J Leezer. Simulating human-like decisions in a memory-based agent model. Computational and Mathematical Organization Theory, 16(4):373-399, 2009.

[18] P. Laureti and Y.C. Zhang. Matching games with partial information. Physica A, 324:49-65, 2003.

[19] A.E. Roth and M. Sotomayor. Two-sided matching: A study in game-theoretic modeling and analysis. Econometric Society Monograph Series, 324, 1990.

[20] R. V. Mendes. The quantum ultimatum game. Quantum Information Processing, 4(1):1-12, 2005.

[21] K.B. Clark. Origins of learned reciprocity in solitary ciliates searching grouped 'courting' assurances at quantum efficiencies. Biosystems, 99:27-41, 2010.

[22] O.G. Zabaleta and C.M Arizmendi. Quantum dating market. Physica A, 389:2858-2863, 2010.

[23] Esteban Guevara Hidalgo. Quantum games entropy. Physica A, 383:797-804, 2007.

[24] B Arfi. Quantum social game theory. Physica A, 374:794-820, 2007.

[25] M. J. Osborne and A. Rubinstein. A Course in Game Theory. The MIT Press Cambridge, Massachusetts, England, 1998.

[26] Staphane Vialette. On the computational complexity of 2-interval pattern matching problems. Theoretical Computer Science, 312(23):223 - 249, 2004.

[27] M.A. Nielsen and I.L. Chuang. Quantum Computation and Quantum Information. Cambridge University Press, England, 2000.

[28] S. Das and E. Kamenica. Two-sided bandits and the dating market. In Alessandro Saffiotti Leslie Pack Kaelbling, editor, Proceedings of the Nineteenth International Joint Conference on Artificial Intelligence,IJCAI-2005, pages 947-952, Edinburgh, Scotland, UK, August 2005.

[29] C.M Arizmendi. Paradoxical way for losers in a dating game. In Proc. AIP Nonequilibrium Statistical Mechanics and Nonlinear Physics, pages 20-25, Mar del Plata, Argentina, December 2006.

[30] A. Romanelli. Quantum games via search algorithms. Physica A, 379:545-551, 2007.

[31] Chao-Yang Pang, Cong-Bao Ding, and Ben-Qiong Hu. Quantum pattern recognition of classical signal. quant-ph-arXiv:0707.0936v2, 2007. 
[32] M.-J. Omero, M. Dzierzawa, M. Marsili, and Y.-C. Zhang. J. Physique, 7:1723, 1997.

[33] C Shannon. A mathematical theory of communication. Bell System Tech. Jour., 27:379-423, 1948.

[34] J. von Neumann. Mathematische Grundlagen der Quantenmechanik. Springer-Verlag [translated by R. T. Beyer as Mathematical Foundations of Quantum Mechanics (Princeton University Press, Princeton, 1955)], Berlin, 1932.

[35] James D. F. V. and P. G. Kwiat. Quantum state entanglement. Los alamos Science, 27:52-57, 2002. 
Chapter 23

\title{
Quantization as Selection \\ Rather than Eigenvalue Problem
}

\author{
Peter Enders \\ Additional information is available at the end of the chapter \\ http://dx.doi.org/10.5772/53847
}

\section{Introduction}

The experimental, in particular, spectroscopic results about atoms brought Bohr [3] to the following "principal assumptions" for a theory of atoms.

1. That the dynamical equilibrium of the systems in the stationary states can be discussed by help of the ordinary mechanics, while the passing of the systems between different stationary states cannot be treated on that basis.

2. That the latter process is followed by the emission of a homogeneous radiation, for which the relation between the frequency and the amount of energy emitted is the one given by Planck's theory." (p. 7)

Assumption (1) states a strange contraposition of the conservation and of the changes of stationary states. Indeed, the conservation of energy - a generalization of Newton's Law 1 holds true quite general, while the change of stationary states along Newton's Law 2 is restricted to classical mechanics (CM). A smooth transition from representations of $\mathrm{CM}$, which axiomatically fix not only the conditions of conservation, but also the manner of change of stationary states and the equation(s) of motion, respectively (Newton, Lagrange, Hamilton), is impossible, as observed also by Heisenberg [30] and Schrödinger [43]. However, in Leonhard Euler's representation of CM [24][25], only the conditions of conservation of stationary states are fixed, while their change has to be described according to the situation under consideration. This makes it suitable to serve as starting point for a smooth transition from classical to quantum mechanics (QM). Euler's principles of the change of stationary states of bodies will be generalized to classical conservative systems as well as to quantum systems. The latter will be used for deriving the time-dependent from the time-independent Schrödinger equation. 
In his pioneering papers 'Quantization as Eigenvalue Problem' [43], Schrödinger has posed four requirements.

1. The "quantum equation" should "carry the quantum conditions in itself" (Second Commun., p. 511);

2. There should be a special mathematical method for solving the stationary Schrödinger equation, which accounts for the non-classical character of the quantization problem, ie, which is different from the classical eigenvalue methods for calculating the (eigen)modes of strings, resonators and so on (ibid., p. 513);

3. The derivation should uniquely decide, that the energy rather than the frequency values are discretized (ibid., pp. 511, 519), since only the former means true quantization, while the latter corresponds to the classical discretization mentioned in requirement 2;

4. The use of the classical expressions for the kinetic and potential energies should be justified (Fourth Commun., p. 113).

Schrödinger's requirements 1 and 2 mean, that - contrary to the very title of the papers quantization is actually not an eigenvalue problem. For in the latter the discretization is imposed not by the differential equation itself, but by the boundary conditions, and this is the classical discretization for standing waves in organ pipes etc. I will fulfill all four requirements by treating quantization as a selection problem. The number of stationary states of a quantum system is smaller than that of a classical system [6]. I will describe, (i), the selection of quantum systems out of the set of all mechanical systems and, (ii), the selection of their stationary states out of the continuum of classical stationary states. Earlier arguing [19][10] is improved and extended in several essential points.

\section{Elements of an Eulerian representation of classical mechanics}

\subsection{Euler's axioms}

Leonhard Euler [20-25] was the first to apply the calculus to all areas of mathematics and mechanics of his time, and he developed novel methods, such as the calculus of variations and topology. Moreover, he worked out an axiomatic of mechanics, where only Newton's Law (axiom) 1 concerning the conservation of stationary states is retained as an axiom, while Newton's Laws (axioms) 2 and 3 concerning the change of stationary states are treated as problems to be solved (for a detailed account, see [19][10][45]). This allows for introducing alternative equations of motion without loosing the contact to CM.

The existence of stationary states is postulated in the following axioms (as in Newton's axioms, rotatory motion is not considered).

Axiom E0: Every body is either resting or moving.

This means, that the subsequent axioms E1 and E2 are not independent of each other; they exclude each other and, at once, they are in harmony with each other [22]. 
Axiom E1: A body preserves its stationary state at rest, unless an external cause sets it in motion.

Axiom E2: A body preserves its stationary state of straight uniform motion, unless an external cause changes this state.

The stationary-state variable is the velocity vector, $\mathbf{v}$ (the mass of a body is always constant). The equation of stationary state reads $\mathbf{v}=\mathbf{0}$ for the state at rest and $\mathbf{v}=$ const for the state of straight uniform motion. The position, $\mathbf{r}$, is variable of the state of motion, but not of stationary states, because it changes during straight uniform motion, ie, in the absence of (external) causes for changing the stationary state [50].

\subsection{Eulerian principles of change of state for single bodies}

Following [21], the changes of position, $\mathbf{r}$, and velocity, $\mathbf{v}$, of a body of mass $m$ subject to the (external) force, $\mathbf{F}$, during the time interval $d t$ are

$$
d\left(\begin{array}{l}
\mathbf{r} \\
\mathbf{v}
\end{array}\right)=\left(\begin{array}{c}
\mathbf{v} d t \\
\frac{1}{m} \mathbf{F} d t
\end{array}\right)=\left(\begin{array}{ll}
\mathbf{0} & \mathbf{1} \\
\mathbf{0} & \mathbf{0}
\end{array}\right)\left(\begin{array}{l}
\mathbf{r} \\
\mathbf{v}
\end{array}\right) d t+\left(\begin{array}{cc}
\mathbf{0} & \mathbf{0} \\
\mathbf{0} & \frac{1}{m} \mathbf{1}
\end{array}\right)\left(\begin{array}{l}
\mathbf{0} \\
\mathbf{F}
\end{array}\right) d t \equiv \mathbf{U}_{i n t}\left(\begin{array}{l}
\mathbf{r} \\
\mathbf{v}
\end{array}\right)+\mathbf{U}_{e x t}\left(\begin{array}{l}
\mathbf{0} \\
\mathbf{F}
\end{array}\right)
$$

The internal transformation, $\mathbf{U}_{\text {int }}$, describes the internal change, $d \mathbf{r}=\mathbf{v} d t$, that is independent of the external force. The external transformation, $\mathbf{U}_{e x t}$ describes the external change, $d \mathbf{v}=(\mathbf{F} / m) d t$, that depends on the external force. These matrices do not commute: $\mathbf{U}_{\text {int }} \mathbf{U}_{\text {ext }} \neq \mathbf{U}_{\text {ext }} \mathbf{U}_{\text {int }}$. This means, that the internal and external transformations are not reducible onto another; the internal and external changes are independent each of another.

Thus, up to order $d t$,

CB1) The changes of stationary-state quantities $(d \mathbf{v})$ are external changes; they explicitly depend solely on external causes (F), but not on state-of-motion quantities (r);

CB2) The change of the stationary-state quantities $(d \mathbf{v})$ do not explicitly depend on the stationary-state quantities (v) themselves;

CB3) The change of state-of-motion quantities $(d \mathbf{r})$ are internal changes; they explicitly depend solely on stationary-state quantities $(\mathbf{v})$;

CB4) The change of stationary-state $(d \mathbf{v})$ and of state-of-motion quantities $(d \mathbf{r})$ are independent each of another;

CB5) As soon as the external causes (F) vanish, the body remains in the stationary state assumed at this moment: $Z(t)=$ const $=Z\left(t_{1}\right)=\mathbf{v}\left(t_{1}\right)$ for $t \geq t_{1}$, if $\mathbf{F}(t)=\mathbf{0}$ for $t \geq t_{1}$.

Accounting for $d d t=0$ and $d \mathbf{F}=\mathbf{0}$, one obtains from eq. (1) 


$$
d d\left(\begin{array}{l}
\mathbf{r} \\
\mathbf{v}
\end{array}\right)=\left(\begin{array}{ll}
\mathbf{0} & \mathbf{1} \\
\mathbf{0} & \mathbf{0}
\end{array}\right) d\left(\begin{array}{l}
\mathbf{r} \\
\mathbf{v}
\end{array}\right) d t=\left(\begin{array}{cc}
\mathbf{0} & \mathbf{1} \\
\mathbf{0} & \mathbf{0}
\end{array}\right)\left(\begin{array}{cc}
\mathbf{0} & \mathbf{0} \\
\mathbf{0} & \frac{1}{m} \mathbf{1}
\end{array}\right)\left(\begin{array}{l}
\mathbf{0} \\
\mathbf{F}
\end{array}\right) d t^{2}=\left(\begin{array}{c}
\frac{1}{m} \mathbf{F} \\
\mathbf{0}
\end{array}\right) d t^{2}
$$

Thus, the principles CB1...CB5 are compatible with Newton's equation of motion (published first in [20]). For their relationship to Descartes' and Huygens' principles of motion, see [10][45].

\subsection{Eulerian principles of change of state for Hamiltonian systems}

According to Definition 2 and the axioms, or laws of motion (Laws 1 and 2, Corollary 3), the momentum is the stationary-state variable of a body in Newton's Principia. The total momentum "is not changed by the action of bodies on one another" (Corollary 3). The principles CB1...CB5 remain true, if the velocity, $\mathbf{v}$, is replaced with the momentum, $\mathbf{p}$. For this, I will use $\mathbf{p}$ rather than $\mathbf{v}$ in what follows.

For a free body, any function of the momentum, $Z(\mathbf{p})$, is a conserved quantity. If a body is subject to an external force, its momentum is no longer conserved, but becomes a state-ofmotion variable like its position. Correspondingly, $Z(\mathbf{p}) \neq c o n s t$. Suppose, that there is nevertheless a function, $Z_{0}(\mathbf{p}, \mathbf{r})$, that is constant during the motion of the body. It describes the stationary states of the system body \& force. External influences (additional forces) be described through a function $Z_{e x t}(\mathbf{p}, \mathbf{r}, t)$ such, that $Z(\mathbf{p}, \mathbf{r})=Z_{0}(\mathbf{p}, \mathbf{r})+Z_{e x t}(\mathbf{p}, \mathbf{r}, t)$ takes over the role of the stationary-state function.

The following principles - a generalization of CB1...CB5 - will shown to be compatible with Hamilton's equations of motion. Up to order $d t$,

CS1) The change of stationary-state quantities $(d Z)$ depends solely on the external influences $\left(Z_{\text {ext }}\right)$, but not on state-of-motion quantities $(\mathbf{p}, \mathbf{r})$;

CS2) The change of the stationary-state quantities $(d Z)$ is independent of the stationary-state quantities (Z) themselves;

CS3) The changes of state-of-motion quantities $(d \mathbf{p}, d \mathbf{r})$ directly depend solely on stationarystate quantities $(Z)$; the external influences $\left(Z_{e x t}\right)$ affect the state-of-motion quantities $(\mathbf{p}, \mathbf{r})$ solely indirectly (via stationary-state quantities, $Z$ );

CS4) The changes of stationary-state $(d Z)$ and of state-of-motion quantities $(d \mathbf{p}, d \mathbf{r})$ are independent each of another;

CS5) As soon as the external influences $\left(Z_{e x t}\right)$ vanish, the system remains in the stationary state assumed at this moment: $Z(t)=$ const $=Z\left(t_{1}\right)$ for $t \geq t_{1}$, if $Z_{\text {ext }}=0$ for $t>t_{1}$.

These principles imply the equation of change of stationary state to read

$$
d Z=\frac{\partial Z}{\partial \mathbf{p}} \cdot d \mathbf{p}+\frac{\partial Z}{\partial \mathbf{r}} \cdot d \mathbf{r}+\frac{\partial Z}{\partial t} d t=\frac{\partial Z_{e x t}}{\partial t} d t
$$


This equation is fulfilled, if

$$
\frac{\partial Z}{\partial \mathbf{p}}=a \frac{d \mathbf{r}}{d t} ; \quad \frac{\partial Z}{\partial \mathbf{r}}=-a \frac{d \mathbf{p}}{d t}
$$

Compatibility with Newton's equation of motion yields $a=1$ and $Z(\mathbf{p}, \mathbf{r})=H(\mathbf{p}, \mathbf{r})$, the Hamiltonian of the system; (4) becoming Hamilton's equations of motion.

$$
\frac{d \mathbf{p}}{d t}=-\frac{\partial H}{\partial \mathbf{r}} ; \quad \frac{d \mathbf{r}}{d t}=\frac{\partial H}{\partial \mathbf{p}}
$$

It may thus be not too surprising that these principles can cum grano salis be applied also to quantum systems. Of course, the variables, which represent of stationary states and motion, will be other ones, again.

\section{Quantization as selection problem - I. Derivation of the stationary Schrödinger equation}

The usual foundations of QM consider CM to be not sufficient and, consequently, need additional or novel assumptions, for instance,

- to restrict the energy spectrum to the values $n h v$ [41][6] or to $(n / 2) h v$ [3];

- to "distinguish" [31] or to "select" [39][36] the values $n \hbar$ of the action integral, $\oint p d q$ ( $n$ integer; in contrast to $\mathrm{CM}$, the action integral is not subject to a variational principle);

- to suppose the existence of $h$ and to abandon the classical paths [30];

- to suppose the existence of $h$ and of a wave function being the solution of an eigenvalue problem [43];

- to suppose the existence of a quantum logic [2][28] and of a Hilbert space for its representation;

- to suppose the existence of transition probabilities obeying the Chapman-Kolmogorov equation (as in wave mechanics) [27].

All these approaches have eventually resorted to CM in using the classical expressions and the interpretations of position, momentum, potential and kinetic energies, because 'it works'. In contrast, I will present a concrete realization of Schrödinger's $4^{\text {th }}$ requirement. 


\subsection{The relationship between $\mathrm{CM}$ and non-CM as selection problem}

In his Nobel Award speech, Schrödinger ([44] p. 315) pointed to the logical aspect, which is central to the approach exposed here.

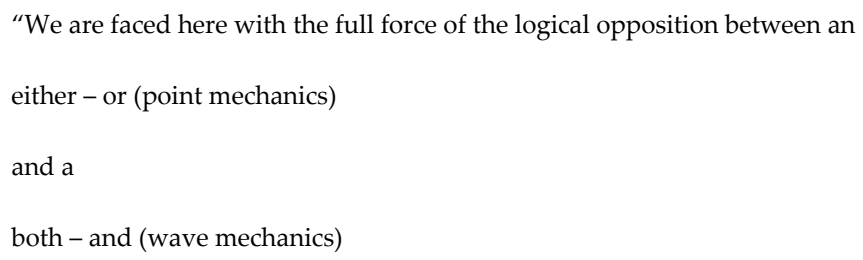

This "logical opposition" consists in a hierarchy of selection problems. ${ }^{1}$

\subsubsection{Selection problem between Newtonian and non-Newtionian CM}

Consider a linear undamped oscillator. For each stationary state of total energy $E$, Newton's equation of motion confines its position, $x$, to the interval between and including the classical turning points: $x_{\min } \leq x \leq x_{\max }$. Its momentum is confined as $p_{\min } \leq p \leq p_{\max }$. More generally speaking, a system obeying Newton's equation of motion moves within the sets $C^{\text {Newton }}=\{\mathbf{r} \mid V(\mathbf{r}) \leq E\}$ and $P^{\text {Newton }}=\{\mathbf{p} \mid T(\mathbf{p}) \leq E\}^{2}$

Alternatively, a classical, though non-Newtonian mechanics is conceivable, where $d p / d t=-\mathbf{F}$ and $E=V-T$. A linear oscillator would move beyond the turning points: $x \leq x_{\min }$ or/and $x \geq x_{\max }$. In general, the set of possible configurations equals $C^{\text {non-Newton }}=\{\mathbf{r} \mid V(\mathbf{r}) \geq E\}$. The momentum configuration is no longer limited: $P^{\text {non }- \text { Newton }}=P^{a l l}=\{\mathbf{p}\}$.

Thus, a conservative CM system obeys either the laws of Newtonian CM, where $d \mathbf{p} / d t=+\mathbf{F}$ and $V(\mathbf{r}(t)) \leq E$, or the laws of non-Newtonian CM, where $d \mathbf{p} / d t=-\mathbf{F}$ and $V(\mathbf{r}(t)) \geq E$. In both cases, the system moves along paths, $\mathbf{r}(t)$.

\subsubsection{Einsteinian selection problem between $C M$ and non-CM}

For both Newtonian and non-Newtonian classical systems, the set of possible energies (the energy spectrum) is continuous. Einstein [6] has observed that this leads to a temperatureindependent specific heat (Dulong-Petit's law) for an ensemble of classical oscillators. In contrast, the discrete set of possible energies of a Planck oscillator yields a specific heat that decreases with decreasing temperature, in agreement with then recent experiments. He

1 I will deviate from the exposition in [19][10] to make it shorter, though clearer and to correct few statements about the momentum configurations.

$2 \mathrm{~V}(\mathrm{r}) \geq 0$, since it equals the "disposable work storage of a system" [33]. ' $r$ ' stays for all configuration variables, ' $p$ ' stays for all momentum configuration variables of a system. 
concluded, "that the set of possible energies of microscopic systems is smaller than that for systems of our everyday experience."

Thus, the set of possible energies of a mechanical system is either continuous, or discrete. ${ }^{3}$

\subsubsection{Selection problem between CM and non-CM in terms of allowed configurations}

Einstein's alternative does not follow from purely mechanical arguing. For this, I continue the reasoning of subsection 3.1.1. The harmony between Newtonian and non-Newtonian CMs consists in that they both build an alternative to a non-CM mechanics, in which the set of allowed configurations comprises the whole configuration space, $C^{\text {all }}=C^{\text {Newton }} \cup C^{\text {non-Newton }}=\{\mathbf{r}\}$. Since the motions along paths in $C^{\text {Newton }}$ and $C^{\text {non-Newton }}$ are incompatible each to another, the motion of non-CM systems does not proceed along paths.

Thus, a mechanical system either moves along paths (CM), or it moves not along paths (non$\mathrm{CM}$ ). The configuration of a non-CM system can assume any element of $C^{\text {all }}$ even in the stationary states.

\subsubsection{Selection problem between mechanics and non-mechanics}

For completeness I note that a system is either a mechanical, or a non-mechanical one.

Like Euler's axioms E1 and E2, these alternatives exclude each other and, at once, are "in harmony each with another". They dialectically determine each another in the sense of Hegel [29].

The question thus is how a linear oscillator without turning points is to be described?

\subsection{Non-classical representation of the potential and kinetic energies}

$V(\mathbf{r})[T(\mathbf{p})]$ is no longer the contribution of the (momentum) configuration $\mathbf{r}(\mathbf{p})$ to the total energy, $E$, since it is unbounded in the domain $C^{\text {all }}\left(P^{\text {all }}\right)$. For this, I define 'limiting factors', $F_{E}(\mathbf{r})$ and $G_{E}(\mathbf{p})$, such, that

$$
\begin{aligned}
& V_{E}^{n c l}(\mathbf{r})=F_{E}(\mathbf{r}) V(\mathbf{r}) \leq E ; \quad \mathbf{r} \in C^{\text {all }} \\
& T_{E}^{n c l}(\mathbf{p})=G_{E}(\mathbf{p}) T(\mathbf{p}) \leq E ; \quad \mathbf{p} \in P^{\text {all }}
\end{aligned}
$$

The contribution of the (momentum) configuration $\mathbf{r}(\mathbf{p})$ of a non-classical system to its total energy, $V_{E}{ }^{n c l}(\mathbf{r})\left[T_{E}{ }^{n c l}(\mathbf{p})\right]$, depends on the energy, because the inequality is no longer realized through the restriction of the (momentum) configuration space.

$F_{E}(\mathbf{r})$ and $G_{E}(\mathbf{p})$ are non-negative. $F_{E}(\mathbf{r})<0$ would mean, that $V_{E}{ }^{n c l}(\mathbf{r})$ is attractive (repulsive), while $V(\mathbf{r})$ is repulsive (attractive). $G_{E}(\mathbf{p})<0$ would mean, that $T_{E}{ }^{n c l}(\mathbf{p})$ becomes negative. For simplicity, I chose the one-dimensional representation of unity and set

3 Because of the finite resolution of measurement apparatus, the set of rational numbers is physically equivalent to the set of real numbers. 


$$
F_{E}(\mathbf{r})=\left|f_{E}(\mathbf{r})\right|^{2} ; \quad G_{E}(\mathbf{p})=\left|g_{E}(\mathbf{p})\right|^{2}
$$

" $\psi \Psi\left[\equiv|\psi|^{2}\right]$ is a kind of weight function in the configuration space of the system. The wavemechanical configuration of the system is a superposition of many, strictly speaking, of all kinematically possible point-mechanical configurations. Thereby, each point-mechanical configuration contributes with a certain weight to the true wave-mechanical configuration, the weight of which is just given through $\psi \Psi$. If one likes paradoxes, one can say, the system resides quasi in all kinematically thinkable positions at the same time, though not 'equally strongly'. " ([43] $4^{\text {th }}$ Commun., p. 135)

Correspondingly, I call $F_{E}$ and $G_{E}$ weight functions, $f_{E}$ and $g_{E}$ - weight amplitudes. Since $F_{E}(\mathbf{r})$ and $G_{E}(\mathbf{p})$ are dimensionless, there are reference values, $r_{\text {ref }}$ and $p_{\text {ref, }}$ such, that actually, $F_{E}(\mathbf{r})=F_{E}\left(\mathbf{r} / r_{r e f}\right)$ and $G_{E}(\mathbf{p})=G_{E}\left(\mathbf{p} / p_{r e f}\right)$. In other words, each such system has got a characteristic length in configuration and in momentum configuration space.

$$
\iiint_{C^{a l l}} F_{E}\left(\frac{\mathbf{r}}{r_{\text {ref }}}\right) \frac{d^{3} r}{r_{\text {ref }}^{3}}=\iiint_{P^{a l l}} G_{E}\left(\frac{\mathbf{p}}{p_{\text {ref }}}\right) \frac{d^{3} p}{p_{\text {ref }}^{3}}=1
$$

In order to simplify the notation, I will omit $r_{\text {ref }}$ and $p_{\text {ref }}$ wherever possible.

\subsection{The stationary Schrödinger equation}

Within $\mathrm{CM}$, the balance between potential, $V(\mathbf{r})$, and kinetic energies, $T(\mathbf{p})$, to yield $V(\mathbf{r})$ $+T(\mathbf{p})=E=$ const is realized through the common path parameter time, $t: \mathbf{r}=\mathbf{r}(t), \mathbf{p}=\mathbf{p}(t) ; E=V(\mathbf{r}(t))$ $+T(\mathbf{p}(t))$. This common parameterization through $t$ is absent for non-classical systems not moving along paths, $\mathbf{r}(t)$. Consequently, the balance between the potential, $V_{E}{ }^{n c l}(\mathbf{r})$, and kinetic energies, $T_{E}{ }^{n c l}(\mathbf{p})$, is not point-wise: $\mathbf{p}(t) \hookrightarrow \mathbf{r}(t)$, but set-wise: $\{\mathbf{p}\} \leftrightarrow\{\mathbf{r}\}$. Set-wise relations are mediated through integral relations. ${ }^{4}$

$$
g_{E}(\mathbf{p})=\frac{1}{\left(2 \pi p_{r e f} r_{r e f}\right)^{\frac{3}{2}}} \iiint_{C^{a l l}} e^{i \frac{\mathbf{p} \cdot \mathbf{r}}{p_{r e f} r_{r e f}}} f_{E}(\mathbf{r}) d^{3} r ; \quad f_{E}(\mathbf{r})=\frac{1}{\left(2 \pi p_{r e f} r_{r e f}\right)^{\frac{3}{2}}} \iiint_{P^{a l l}} e^{-i \frac{\mathbf{r} \cdot \mathbf{p}}{p_{r e f} r_{r e f}}} g_{E}(\mathbf{p}) d^{3} p
$$

In view of the symmetric normalization (8) I have chosen symmetric normalization factors.

Alternatively, it is possible to avoid complex-valued weight amplitudes (wave functions) through using 2-component vectors for them and the Hartley transform in place of the Fourier

4 The most general symmetric Fourier transform contains a free complex-valued parameter [49]. It appears to be merely a rescaling of $\mathrm{r}_{\text {ref }}$ and $\mathrm{p}_{\text {ref }}$ respectively. 
transform. The operators become $2 \times 2$ matrices. It remains to explore whether their free components can be exploited for the description of new effects.

Lacking orbits, such a system does not assume a definite configuration, say, $\mathbf{r}_{1}$, and momentum configuration, $\mathbf{p}_{1}$, at a given time, $t_{1}$, with $E=V_{E}{ }^{n c l}\left(\mathbf{r}_{1}\right)+T_{E}{ }^{n c l}\left(\mathbf{p}_{1}\right)$. Instead, they all contribute to the stationary state, $E$. The partial contribution of the single (momentum) configuration, $\mathbf{r}(\mathbf{p})$, is determined by the weight function according to eq. (6). The total energy thus becomes

$$
E=\frac{\iiint_{C^{a l l}} F_{E}(\mathbf{r}) V(\mathbf{r}) d^{3} r}{\iiint_{C^{a l l}} F_{E}(\mathbf{r}) d^{3} r}+\frac{\iiint_{P^{a l l}} G_{E}(\mathbf{p}) T(\mathbf{p}) d^{3} p}{\iiint_{P^{a l l}} G_{E}(\mathbf{p}) d^{3} p}
$$

The denominators have been added for dimensional reasons. The classical representation is obtained through setting

$$
F_{E}(\mathbf{r})=r_{r e f}^{3} \delta(\mathbf{r}-\mathbf{r}(t)) ; \quad G_{E}(\mathbf{p})=p_{r e f}^{3} \delta(\mathbf{p}-\mathbf{p}(t))
$$

The occurrence of $E$ on the r.h.s. makes eq. (10) to be an implicit equation for $E$. This suggests $E$ to be an internal system parameter being determined solely by system properties like the oscillation frequency of an undamped harmonic oscillator [19]. However, as in CM, the value of $E$ is given by the initial preparation of the system.

The Fourier transform (9) enables me to eliminate one of the weight amplitudes from eq. (10).

$$
\begin{aligned}
E= & \frac{\iiint_{C^{a l l}} \bar{f}_{E}(\mathbf{r}) \hat{H}(\mathbf{r}) f_{E}(\mathbf{r}) d^{3} r}{\iiint_{C^{a l l}} \bar{f}_{E}(\mathbf{r}) f_{E}(\mathbf{r}) d^{3} r} ; \quad \hat{H}(\mathbf{r}) \equiv V(\mathbf{r})+T\left(-i p_{r e f} r_{r e f} \frac{\partial}{\partial \mathbf{r}}\right) \\
= & \frac{\iint_{P^{a l l}} \bar{g}_{E}(\mathbf{p}) \hat{H}(\mathbf{p}) g_{E}(\mathbf{p}) d^{3} p}{\iiint_{P^{a l l}}(\mathbf{p}) g_{E}(\mathbf{p}) d^{3} p} ; \quad \hat{H}(\mathbf{p}) \equiv V\left(i r_{r e f} p_{r e f} \frac{\partial}{\partial \mathbf{p}}\right)+T(\mathbf{p})
\end{aligned}
$$

(Other positions of the weight amplitudes lead to the same results.) Since, in general, $\bar{f}_{E}$ and $\bar{g}_{E}$ are linearly independent of $f_{E}$ and $g_{E}$, respectively, necessary conditions for fulfilling these equations are

$$
E f_{E}(\mathbf{r})=\hat{H}(\mathbf{r}) f_{E}(\mathbf{r}) ; \quad E g_{E}(\mathbf{p})=\hat{H}(\mathbf{p}) g_{E}(\mathbf{p})
$$


Moreover, these equations hold true for the minimum of the r.h.s. of eq. (12), ie, for the ground state. There is no indication for a difference between the stationary-state equation for the ground state and for the states of higher energy.

A comparison with experiments reveals, that $r_{\text {ref }} p_{r e f}=h$, which I will use in what follows. Thus, with $f_{E}(r)=r_{r e f}^{3 / 2} \psi_{E}(r)$ and $g_{E}(p)=p_{r e f}^{3 / 2} \phi_{E}(p)$, eqs. (13) are the stationary Schrödinger equations in configuration and momentum configuration spaces.

\section{Quantization as selection problem - II. Non-classical solution to the stationary Schrödinger equation}

As observed by Schrödinger himself (!), the eigenvalue method used by himself does not properly account for the quantum nature of quantum systems, because it applies to (and had been developed for) classical systems like strings and pipes. In what follows, I will describe a solution method being free of that deficiency.

\subsection{The linear oscillator}

The stationary Schrödinger equation for a linear undamped harmonic oscillator reads

$$
\hat{H}(x) f_{E}\left(\frac{x}{r_{r e f}}\right)=E f_{E}\left(\frac{x}{r_{r e f}}\right) ; \quad \hat{H}(x)=\frac{m}{2} \omega^{2} x^{2}-\frac{\hbar^{2}}{2 m} \frac{\partial^{2}}{\partial x^{2}}
$$

To see its essentials I introduce dimensionless variables as ${ }^{5}$

$$
\xi=\frac{x}{r_{r e f}} ; \quad r_{r e f}=\sqrt{\frac{r_{r e f} p_{r e f}}{2 m \omega}} ; \quad y_{a}(\xi)=f_{E}(\xi) ; \quad a=-\frac{E}{r_{r e f} p_{r e f} \omega}
$$

to obtain

$$
\frac{d^{2} y_{a}(\xi)}{d \xi^{2}}-\left(\frac{1}{4} \xi^{2}+a\right) y_{a}(\xi)=0
$$

This is Weber's equation [48] being one of the equations of the parabolic cylinder [1]. Despite of the reference length, $r_{\text {ref }}$ the stationary states are determined solely through the energy parameter, $-a$. In contrast to the classical oscillator, where $E \sim \omega^{2}$, the quantum oscillator exhibits

5 This yields $\mathrm{p}_{\mathrm{ref}}=2 \mathrm{~m} \omega \mathrm{r}_{\mathrm{ref}} ;$ the classical maximum values are interrelated as $\mathrm{p}_{\max }=\mathrm{m} \omega \mathrm{r}_{\max }$. I deviate from the exposition in [19][10] to make the following clearer. 
$E \sim \omega$. Since $\omega$ does not occur as a self-standing parameter, the quantization is not affecting it; Schrödinger's $3^{\text {rd }}$ requirement is fulfilled.

\subsection{The mathematically distinguished solutions}

For and only for the values $a= \pm 1 / 2$ the 1.h.s. of eq. (16) factorizes. ${ }^{6}$

$$
\left(\frac{d}{d \xi}+\frac{1}{2} \xi\right)\left(\frac{d}{d \xi}-\frac{1}{2} \xi\right) y_{+1 / 2}(\xi)=0 ; \quad\left(\frac{d}{d \xi}-\frac{1}{2} \xi\right)\left(\frac{d}{d \xi}+\frac{1}{2} \xi\right) y_{-1 / 2}(\xi)=0
$$

Therefore, the values $a= \pm 1 / 2$ are mathematically distinguished against all other $a$-values. The corresponding solutions, $y_{ \pm 1 / 2}(\xi)$, are mathematically equivalent, but physically different. $y_{-1 / 2}$ $(\xi)=y_{-1 / 2}(0) \times \exp \left(-\xi^{2} / 4\right)$ is a limiting amplitude, while $y_{+1 / 2}(\xi)=y_{+1 / 2}(0) \times \exp \left(+\xi^{2} / 4\right)$ is not. This distinguishes physically the value $a=-1 / 2$ over the value $a=+1 / 2$.

If there would be no other distinguished $a$-values, there would be only one state $(a=-1 / 2)$. However, a system having got just one state is not able to exchange energy with its environment. In order to find further distinguished $a$-values, I examine two recurrence relations for the standard solutions of eq. (16) ([1] 19.6.1, 19.6.5).

$$
\left(\frac{d}{d \xi}+\frac{1}{2} \xi\right) U(a, \xi)+\left(a+\frac{1}{2}\right) U(a+1, \xi)=0 ; \quad\left(\frac{d}{d \xi}-\frac{1}{2} \xi\right) V(a, \xi)-\left(a-\frac{1}{2}\right) V(a-1, \xi)=0
$$

Such recurrence relations can be obtained without solving the differential equation, viz, from Whittaker's representation of the solutions as contour integrals ([52] 16.61; [1] 19.5). This representation has been developed well before the advance of QM; it is thus independent of the needs of QM.

The recurrence relations

- are not related to the usual, classical solution methods;

- interrelate solution functions with a finite difference between their $a$-values, viz, $\Delta a= \pm 1$ (this becomes $\Delta E= \pm \hbar \omega$ later on);

- reflect the genuine discrete structure wanted; in particular, this structure has nothing to do with boundary conditions, since all solutions exhibit this discrete structure, not only Schrödinger's eigensolutions;

- realize the "conviction", that "the true laws of quantum mechanics would consist not of specific prescriptions for the single orbit; but, in these true laws, the elements of the whole manifold of orbits of a system are connected by equations, so that there is apparently a certain interaction between the various orbits." ([43] Second Commun., p. 508)

6 These factors are closely related to the variables that factorize the classical Hamiltonian. 
Moreover, the recurrence relations divide the set of $a$-values as follows.

Set (1) $a=\ldots,-5 / 2,-3 / 2,-1 / 2$; the $2^{\text {nd }}$ relation (18) breaks at $a=-1 / 2$ being one of the two mathematically distinguished values found above;

Set (2) $a=\ldots, 5 / 2,3 / 2,1 / 2$; the $1^{\text {st }}$ relation (18) breaks at $a=+1 / 2$ being the other mathematically distinguished value found above;

Set (3) $a=\{\ldots,-2+\zeta,-1+\zeta, \zeta, \zeta+1, \zeta+2 \ldots \mid-1 / 2<\varsigma<+1 / 2\}$; there is no break in the recurrence relations (18) for this set.

The smallest interval representing all solutions is the closed interval $a=[-1 / 2,+1 / 2]$, all other solutions being related to it through the recursion formulae. The values $a=-1 / 2$ (set (1)) and $a=$ $+1 / 2$ (set (2)) are mathematically distinguished, again; this time as the boundary points of that interval. All inner interval points, $-1 / 2<a<+1 / 2(\operatorname{set}(3))$, are mathematically equivalent among each another and, consequently, not distinguished. The physically relevant set of $a$-values is a mathematically distinguished set.

\subsection{The physically distinguished solutions}

The mathematically distinguished set (1) contains the physically relevant value $a=-1 / 2$, while the mathematically distinguished set (2) contains the unphysical value $a=+1 / 2$. The recurrence relations (18) show, that all functions $U(a, \xi)$ with $a$-values from set $(1)$ are limiting amplitudes, while all functions $V(a, \xi)$ with $a$-values from set (2) are not. For the $a$-values of set (3), neither $U(a, \xi)$, nor $V(a, \xi)$ is a limiting amplitude.

Moreover, set (1) exhibits a finite minimum of total energy, $E=1 / 2 r_{\text {ref }} p_{\text {ref }} \omega$, while sets (2) and (3) do not. A system of sets (2) or (3) can deliver an unlimited amount of energy to its environment, it is a perpetuum mobile of $1^{\text {st }}$ kind. This makes set (1) to be physically distinguished against sets (2) and (3).

Hence, starting from the ground state, $y_{-1 / 2}(\xi)=y_{-1 / 2}(0) \times \exp \left(-\xi^{2} / 4\right)$, and using the recursion formula (18) for $U(a, \xi)$, the physically relevant solutions are obtained as

$$
y_{-n-\frac{1}{2}}(\xi)=e^{-\frac{1}{4} \xi^{2}} H e_{n}(\xi) ; \quad n=0,1,2, \ldots
$$

where

$$
H e_{n}(\xi)=(-1)^{n} e^{\frac{1}{2} \xi^{2}} \frac{d^{n}}{d \xi^{n}} e^{-\frac{1}{2} \xi^{2}}
$$

is the $n^{\text {th }}$ Hermite polynomial ([1] 19.13.1). Schrödinger's boundary condition (the wave function should vanish at infinity) is fulfilled automatically.

Since ([1] 22.2) 


$$
\int_{-\infty}^{+\infty} e^{-\frac{1}{2} \xi^{2}} H e_{n}(\xi)^{2} d \xi=\sqrt{2 \pi} n !
$$

the normalized solutions [see eq. (8)] read

$$
y_{-n-\frac{1}{2}}(\xi)=\frac{1}{\sqrt{\sqrt{2 \pi} n !}} e^{-\frac{1}{4} \xi^{2}} H e_{n}(\xi) ; \quad n=0,1,2, \ldots
$$

\subsection{The non-classical potential energy and the tunnel effect}

The observation of quantum particles crossing spatial domains, where $V>E$, has led to the notion 'tunnel effect' [34][38]. Being a nice illustration, this wording masks the fact, that the actual contribution of a configuration, $\mathbf{r}$, to the total energy is not $V(\mathbf{r})$, but $V_{E}{ }^{n c l}(\mathbf{r})<E$, see eq. (6).

In terms of the dimensionless variables (15) the dimensionless non-classical potential energy of the oscillator above equals

$$
v_{n}^{n c l}(\xi)=y_{-n-\frac{1}{2}}^{2}(\xi) \frac{1}{2} \xi^{2}=\frac{1}{2 \sqrt{2 \pi} n} \xi^{2} e^{-\frac{1}{2} \xi^{2}} H e_{n}(\xi)^{2}
$$

Using the recurrence formula $\xi H e_{n}(\xi)=H e_{n+1}(\xi)+n H e_{n-1}(\xi)$ ([1] 22.7.14) and the inequality I $H e_{n}(\xi) \mid<\exp \left(\xi^{2} / 4\right) \sqrt{ }(n !) k, k \approx 1.086435$ (ibid., 22.14.17), one can prove, that

$$
v_{n}^{n c l}(\xi)=y_{-n-\frac{1}{2}}(\xi)^{2} \frac{1}{2} \xi^{2}<n+\frac{1}{2} ; \quad-\infty<\xi<+\infty ; \quad n=0,1,2, \ldots
$$

Hence, the inequalities (6) are fulfilled.

The occurrence of the 'smaller than' sign means, that - in contrast to the classical oscillator there are no stationary states with, (i), vanishing potential energy (in particular, the ground state is not a state at rest) and, (ii) vanishing kinetic energy (there are no turning points).

The picture of the tunnel is partially correct, in that the classical turning points are points of inflection such, that beyond them, in the forbidden domains of Newtonian CM, the wave function decreases exponentially.

Notice that these results follow solely from the most general principles of state description according to Leibniz [35], Euler, Helmholtz and Schrödinger, without solving any stationarystate equation or equation of motion and without assuming particular boundary conditions. 


\section{The time dependent case}

While Heisenberg [30] and Schrödinger [43] started from a time-dependent equation ${ }^{7}$, I have worked so far with the set of all possible (momentum) configurations of systems in their stationary states, where time plays no role. In order to incorporate time, I will proceed as Newton and Euler did in the classical case and will consider first the time-dependence of the stationary states, then, the change of these states, and, finally, I will arrive at the timedependent Schrödinger equation as the equation of motion.

\subsection{The time dependence of the stationary states}

According to their definitions (6), the stationary weight functions, $F_{E}(\mathbf{r})$ and $G_{E}(\mathbf{p})$, are time independent. Hence, if there is a time dependence of the stationary weight amplitudes, and correspondingly of the wave functions, it is of the form

$$
\psi_{E}(\mathbf{r}, t)=e^{i \phi_{E}(t)} \psi_{E}(\mathbf{r}) ; \quad \varphi_{E}(\mathbf{p}, t)=e^{i \phi_{E}(t)} \varphi_{E}(\mathbf{p})
$$

The phase, $\varphi_{E}(t)$, is the same for both functions, since the Fourier transform (9) is timeindependent.

For a free particle,

$$
\psi_{E}(\mathbf{r}, t) \sim \exp \left\{i \mathbf{k}_{E} \cdot \mathbf{r}-i \omega_{E} t\right\} ; \quad E=\frac{\hbar^{2} k_{E}^{2}}{2 m}
$$

The group velocity equals the time-independent particle velocity.

$$
\mathbf{v}_{g}=\frac{d \omega_{E}}{d \mathbf{k}_{E}}=\frac{\hbar \mathbf{k}_{E}}{m} \Rightarrow \omega_{E}=\frac{\hbar k_{E}^{2}}{2 m}=\frac{E}{\hbar}
$$

Therefore,

$$
\psi_{E}(\mathbf{r}, t)=e^{-i \frac{E}{\hbar} t} \psi_{E}(\mathbf{r}) ; \quad \phi_{E}(\mathbf{p}, t)=e^{-i \frac{E}{\hbar} t} \phi_{E}(\mathbf{p})
$$

For later use I remark that this can be written as

7 In fn. 2, p. 489, of the $2^{\text {nd }}$ Commun., Schrödinger has distanced himself from the time-independent approach of the $1^{\text {st }}$ Commun. 


$$
\psi_{E}(\mathbf{r}, t)=e^{-\frac{i}{\hbar} \hat{H}(\mathbf{r}) t} \psi_{E}(\mathbf{r}) ; \quad \phi_{E}(\mathbf{p}, t)=e^{-\frac{i}{\hbar} \hat{H}(\mathbf{p}) t} \phi_{E}(\mathbf{p})
$$

\subsection{The equation-of-state-change}

The analog to the Hamiltonian as classical stationary-state function is the function

$$
Z^{n c l}=\frac{\iiint_{C^{a l l}} \bar{f}(\mathbf{r}, t) \hat{H}(\mathbf{r}, t) f(\mathbf{r}, t) d^{3} r}{\iiint_{C^{a l l}} \bar{f}(\mathbf{r}, t) f(\mathbf{r}, t) d^{3} r}=\frac{\iint_{P^{a l l}} \bar{g}(\mathbf{p}, t) \hat{H}(\mathbf{p}, t) g(\mathbf{p}, t) d^{3} p}{\iiint_{P^{a l l}} \bar{g}(\mathbf{p}, t) g(\mathbf{p}, t) d^{3} p}
$$

The analog to the classical principles of change of state, CS1...CS5, reads as follows. Up to first order in $d t$,

QS1) the change of stationary-state quantities $(d Z)$ depends solely on the external causes $\left(Z_{\text {ext }}\right)$, but not on state-of-motion quantities $(f, g)$;

QS2) the changes of the stationary-state quantities $(d Z)$ are independent of the stationary-state quantities (Z) themselves;

QS3) the changes of state-of-motion quantities $(d f, d g)$ depend directly solely on stationarystate quantities (Z);

QS4) the changes of stationary-state $(d Z)$ and of state-of-motion quantities $(d f, d g)$ are independent each of another;

QS5) as soon as the external causes $\left(Z_{e x t}\right)$ vanish, the system remains in the (not necessarily stationary $\left.^{8}\right)$ state assumed in this moment.

Hence, writing

$$
\iiint \bar{f}(\mathbf{r}, t) \hat{H}(\mathbf{r}, t) f(\mathbf{r}, t) d^{3} r=\langle f(\mathbf{r}, t)|\hat{H}(\mathbf{r}, t)| f(\mathbf{r}, t)\rangle
$$

the equation of state change becomes

$$
\begin{aligned}
d Z^{n c l} & =\frac{\langle d f(\mathbf{r}, t)|\hat{H}(\mathbf{r}, t)| f(\mathbf{r}, t)\rangle+\langle f(\mathbf{r}, t)|d \hat{H}(\mathbf{r}, t)| f(\mathbf{r}, t)\rangle+\langle f(\mathbf{r}, t)|\hat{H}(\mathbf{r}, t)| d f(\mathbf{r}, t)\rangle}{\langle f(\mathbf{r}, t) \mid f(\mathbf{r}, t)\rangle} \\
& -\frac{\langle d f(\mathbf{r}, t) \mid f(\mathbf{r}, t)\rangle+\langle f(\mathbf{r}, t) \mid d f(\mathbf{r}, t)\rangle}{\langle f(\mathbf{r}, t) \mid f(\mathbf{r}, t)\rangle} \frac{\langle f(\mathbf{r}, t)|\hat{H}(\mathbf{r}, t)| f(\mathbf{r}, t)\rangle}{\langle f(\mathbf{r}, t) \mid f(\mathbf{r}, t)\rangle} \\
& !\langle f(\mathbf{r}, t)|d \hat{H}(\mathbf{r}, t)| f(\mathbf{r}, t)\rangle \\
& =\frac{f f(\mathbf{r}, t)|f(\mathbf{r}, t)\rangle}{}
\end{aligned}
$$

8 The modification against CS5 is a consequence of the discreteness of the energetic spectrum. 


\subsection{Derivation of the time-dependent Schrödinger equation}

The requirement in eq. (32) implies two conditions.

$$
\begin{aligned}
& \langle d f(\mathbf{r}, t)|\hat{H}(\mathbf{r}, t)| f(\mathbf{r}, t)\rangle+\langle f(\mathbf{r}, t)|\hat{H}(\mathbf{r}, t)| d f(\mathbf{r}, t)\rangle=0 \\
& \langle d f(\mathbf{r}, t) \mid f(\mathbf{r}, t)\rangle+\langle f(\mathbf{r}, t) \mid d f(\mathbf{r}, t)\rangle=d\langle f(\mathbf{r}, t) \mid f(\mathbf{r}, t)\rangle=0
\end{aligned}
$$

The second condition means that there is a unitary time development operator,

$$
\hat{U}\left(\mathbf{r}, t_{2}, t_{1}\right) f\left(\mathbf{r}, t_{1}\right)=f\left(\mathbf{r}, t_{2}\right) ; \quad \hat{U}\left(\mathbf{r}, t_{2}, t_{1}\right)^{\dagger}=\hat{U}\left(\mathbf{r}, t_{2}, t_{1}\right)^{-1}
$$

such, that

$$
\left\langle f\left(\mathbf{r}, t_{2}\right) \mid f\left(\mathbf{r}, t_{2}\right)\right\rangle=\left\langle\hat{U}\left(\mathbf{r}, t_{2}, t_{1}\right) f\left(\mathbf{r}, t_{1}\right) \mid \hat{U}\left(\mathbf{r}, t_{2}, t_{1}\right) f\left(\mathbf{r}, t_{1}\right)\right\rangle=\left\langle f\left(\mathbf{r}, t_{1}\right) \mid f\left(\mathbf{r}, t_{1}\right)\right\rangle
$$

Now I insert eq. (34) into the first requirement (33).

$$
\langle d \hat{U}(\mathbf{r}, t, 0) f(\mathbf{r}, 0)|\hat{H}(\mathbf{r}, t)| \hat{U}(\mathbf{r}, t, 0) f(\mathbf{r}, 0)\rangle+\langle\hat{U}(\mathbf{r}, t, 0) f(\mathbf{r}, 0)|\hat{H}(\mathbf{r}, t)| d \hat{U}(\mathbf{r}, t, 0) f(\mathbf{r}, 0)\rangle=0
$$

The unitary solution to this equation reads $d \hat{U}(r, t, 0)=j u(\hat{H}(r, t)) d t$, where $u(\hat{H})$ is a realvalued rational function of the self-adjoint Hamiltonian, $H$. Compatibility with the stationary case (29) yields $u(\hat{H})=-\frac{1}{\hbar} \hat{H}$. Hence,

$$
\hat{U}(\mathbf{r}, t, 0)=\hat{P}\left(\exp \left\{\frac{-i}{\hbar} \int_{0}^{t} \hat{H}\left(\mathbf{r}, t^{\prime}\right) d t^{\prime}\right\}\right)
$$

where $\hat{P}$ denotes Dyson's time-ordering operator [4]. The time-dependent Schrödinger equation for $f(r, t)$ follows immediately.

The momentum representation can be derived quite analogously.

Both representations of the time-dependent Schrödinger form two equivalent equations of motion. As in the classical case, the equation of motion is a dynamic equation for nonstationary-state entities.

\section{Summary and conclusions}

I have presented a relatively novel approach to quantization, viz, quantization as selection rather than eigenvalue problem. It starts from Euler's rather than Newton's axiomatic and exploits Helmholtz's [32][33] treatment of the energy conservation law. It fulfills all four of Schrödinger's methodical requirements quoted in the Introduction. 
It is often assumed, that the difference between classical and quantum systems is caused by the existence of the quantum of action. I have shown that this assumption is not necessary. It is sufficient to make different assumptions about the set of (momentum) configurations a mechanical system can assume in its stationary states.

The "logical opposition" between CM and QM observed by Schrödinger [44] is actually a dialectic relationship, which resembles that between the Finite and the Infinite. Each determination draws a Limit, where each Limit involves the existence of something beyond it ( $f f$ [29] Logik I p. 145). The notion of the Finite does not exist without the notion of the Infinite (ibid. pp. 139ff.). The Infinite is the Other of the Finite - in turn, the Finite is the Other of the Infinite. Now, the Finite and the Infinite are not simply opposites; a border between them would contradict the very meaning of infinity. The True Infinite includes the Finite, it is the unity of the Finite and the Infinite ( $c f$ ibid. p. 158).

The solution of the stationary Schrödinger equation without using boundary conditions shows, that it actually does "carry the quantum conditions in itself" (Schrödinger's ${ }^{\text {st }}$ requirement, see Introduction). Hence, it has got "maximum strength" in the sense of Einstein [8].

$\mathrm{CM}$ contains the necessary means for going beyond its own frame. This way, the relationship between $\mathrm{CM}$ and non-CM becomes well defined, and the physical content of non-CM is formulated on equal footing with the mathematical method (and vice versa). An example for this is the reformulation of Einstein's criterion (the number of stationary states) in terms of recurrence relations.

Ad-hoc assumptions, which may be suggested by experimental results, but are not supported by the axiomatic of $\mathrm{CM}$, can be avoided. The wave and particle aspects can be obtained from the time-dependent Schrödinger equation and its solutions [10]. The classical path in phase space is replaced with the wave functions in space and momentum representations. The wave functions take also the role of the initial conditions, which "are not free, but also have to obey certain laws" [7].

The dynamics in space and in momentum space are treated in parallel. As a consequence, the Schrödinger equation in momentum representation is obtained at once with the one in position representation. This, too, enables one to keep maximum contact to $\mathrm{CM}$ and to explain, why $\mathrm{QM}$ is a non-classical mechanics of conservative systems, where the classical potential and kinetic energy functions and, consequently, the classical Lagrange and Hamilton functions still apply. This includes a natural explanation of "the peculiar significance of the energy in quantum mechanics" [51].

Modern representations of $\mathrm{CM}$ favor equations of motion as the foundation (the variational principles belong to this class). The state variables are position and velocity (Lagrange, Laplace), or position and momentum (Hamilton). Hence, there are 6 state variables for a single body. In contrast, there are only 3 quantum numbers for a spinless particle. And there are only 3 stationary-state variables for a single body within Newton's (the 3 components of the momentum vector) and Euler's (the 3 components of the velocity vector) representations of $\mathrm{CM}$, respectively. ${ }^{9}$ This is another indication for the fact, that the latter are more suitable for the transition from CM to QM than Lagrange's and Hamilton's representations. 
For the quantization of fields, finally, this approach yields an explanation for the fact, that, within the method of normal-mode expansion, only the temporal, but not the spatial part of the field variables is concerned ( $c f[42])$. Indeed, only those variables are subject to the quantization procedure, the possible values of which are restricted by the energy law. The spatial extension of the normal modes is fixed by the boundary conditions and thus not subject to quantization. The classical field energy (density) is determined by the normal-mode amplitudes and thus limits these. As a consequence, the time-dependent coefficients in the normal-mode expansion are quantized. When formulating this expansion such, that these expansion coefficients get the dimension of length, their quantization can be performed in complete analogy to that of the harmonic oscillator, without invoking additional assumptions or new constants [10]. Moreover, one could try to quantize a field in the space spanned by independent dynamical field variables. This could separate the quantization problem from the spatial and temporal field distributions and, thus, simplify the realization of Einstein's imagination of a "spatially granular" [5] structure of the electromagnetic field.

\title{
Acknowledgements
}

I feel highly indebted to Dr. D. Suisky with whom the basic ideas of 'quantization as selection problem' have been elaborated [46][47][18][19]. Over the years I have benefited from numerous discussions with Dr. M. Altaisky, Prof. Y. Dabaghian, Dr. M. Daumer, Dr. K. Ellmer, Dr. D. B. Fairlie, Dr. A. Förster, Prof. L. Fritsche, Prof. W. Greiner, Dr. H. Hecht, H. Hille, Prof. J. Keller, Prof. J. R. Klauder, Prof. H. Kröger, Dr. Th. Krüger, Prof. H. Lübbig, Prof. G. Mann, Prof. Matone, Prof. S. N. Mayburow, Prof. P. Mittelstaedt, Dr. R. Müller, Prof. J. G. Muroz, Prof. G. Nimtz, Prof. H. Paul, Prof. Th. Pöschel, Prof. J. Rosen, A. Rothenberg, Prof. W. P. Schleich, Prof. J. Schröter, Prof. J. Schnakenberg, Prof. J. Schröter, Dr. W. Smilga, Dr. E. V. Stefanovich, Dr. L. Teufel, Dr. R. Tomulka, Prof. H. Tributsch, Dr. M. Vogt, Prof. R. F. Werner and many more. I am indebted to Profs. M. Müller-Preußker and W. Nolting for their continuous interest and support. Early stages of this work were supported by the Deutsche Akademie der Naturforscher Leopoldina [18], Prof. Th. Elsässer and Prof. E. Siegmund.

\section{Author details}

\author{
Peter Enders*
}

Address all correspondence to: enders@dekasges.de

University of Applied Sciences, Wildau, Königs Wusterhausen, Germany

\footnotetext{
9 Some implications of this similarity between Newton's and Euler's notions of state on the classical and Schrödinger's and Pauli's [39][40] on the quantum sides have been investigated in [9][11][12][15][16].
} 


\section{References}

[1] Abramowitz, M, \& Stegun, I. A. Eds.), Handbook of Mathematical Functions, Washington: NBS 1964; abbreviated reprint (selection by M. Danos \& J. Rafelski): Pocketbook of Mathematical Functions, Thun Frankfurt/Main: Deutsch (1984).

[2] Birkhoff, G, \& Von Neumann, J. The logic of quantum mechanics, Ann. of Math. [2] (1936). , 37(1936), 823-843.

[3] Bohr, N. (1913). On the Constitution of Atoms and Molecules", Phil. Mag. , 26, 1-13.

[4] Dyson, F. J. (1949). The radiation theories of Tomonaga, Schwinger, and Feynman", Phys. Rev. , 75, 486-502.

[5] Einstein, A. (1905). Über einen die Erzeugung und Verwandlung des Lichtes betreffenden heuristischen Gesichtspunkt", Ann. Phys. , 17, 132-148.

[6] Einstein, A. (1907). Die Plancksche Theorie der Strahlung und die Theorie der spezifischen Wärme", Ann. Phys. 22, , $180 \mathrm{ff}$.

[7] Einstein, A. (1923). Bietet die Feldtheorie Möglichkeiten für die Lösung des Quantenproblems?", Sitzungsber. Preuss. Ak. Wiss. phys.-math. K1., 13. Dez., XXXIII, , $359 \mathrm{ff}$.

[8] Einstein, A. (1977). Grundzüge der Relativitätstheorie (Akademie-Verlag, Berlin), , 132.

[9] Enders, P. (2004). Equality and Identity and (In)distinguishability in Classical and Quantum Mechanics from the Point of View of Newton's Notion of State, $6^{\text {th }}$ Int. Symp. Frontiers of Fundamental and Computational Physics, Udine; in: Sidharth, Honsell \& De Angelis (Hrsg.), Frontiers of Fundamental Physics, 2006, , 239-245.

[10] Enders, P. (2006). Von der klassischen Physik zur Quantenphysik. Eine historisch-kritische deduktive Ableitung mit Anwendungsbeispielen aus der Festkörperphysik, Berlin Heidelberg: Springer

[11] Enders, P. Is Classical Statistical Mechanics Self-Consistent? (A paper of honour of C. F. von Weizsäcker, 1912-2007), Progr. Phys. (2007). http://www.allbusiness.com/ science-technology/physics/5518225-1.html, 3(2007), 85-87.

[12] Enders, P. Equality and Identity and (In)distinguishability in Classical and Quantum Mechanics from the Point of View of Newton's Notion of State, Icfai Univ. J. Phys. I ((2008). http://www.iupindia.org/108/IJP_Classical_and_Quantum_Mechanics_71.html

[13] Enders, P. Towards the Unity of Classical Physics, Apeiron 16 ((2009). http:// redshift.vif.com/JournalFiles/V16N1END.pdf

[14] Enders, P. Huygens principle as universal model of propagation, Latin Am. J. Phys. Educ. (2009). http://dialnet.unirioja.es/servlet/articulo?codigo=3688899, 3(2009), 19-32.

[15] Enders, P. Gibbs' Paradox in the Light of Newton's Notion of State, Entropy (2009). http:// www.mdpi.com/1099-4300/11/3/454, 11(2009), 454-456. 
[16] Enders, P. State, Statistics and Quantization in Einstein's 1907 Paper, 'Planck's Theory of Radiation and the Theory of Specific Heat of Solids', Icfai Univ. J. Phys. II ((2009). http:// www.iupindia.org/709/IJP_Einsteins_1907_Paper_176.html

[17] Enders, P. (2010). Precursors of force fields in Newton's 'Principia'", Apeiron 17, 22-27; http://redshift.vif.com/JournalFiles/V17N1END.PDF

[18] Enders, P, \& Suisky, D. (2004). Über das Auswahlproblem in der klassischen Mechanik und in der Quantenmechanik", Nova Acta Leopoldina, Suppl. , 18, 13-17.

[19] Enders, P, \& Suisky, D. (2005). Quantization as selection problem", Int. J. Theor. Phys. , 44, 161-194.

[20] Euler, L. II-1, "Mechanica sive motus scientia analytice exposita", in: Leonardi Euleri Opera Omnia sub auspiciis Societatis Scientarium Naturalium Helveticae (Zürich Basel, 1911 - 1986), ser. II, vol. 1

[21] Euler, L, \& Découverte, I. I-5a. d'une nouveau principe de mécanique", in: Opera Omnia, ser. II, , 5

[22] Euler, L. II-5b, "Harmonie entre les principes généraux de repos et de mouvement de M. de Maupertuis", in: Opera Omnia, ser. II, , 5

[23] Euler, L. II-5c, "Recherches sur l'origine des forces", Mém. ac. sci. Berlin 6 (1750) 1752 , 419-447; in: Opera Omnia, ser. II, , 5

[24] Euler, L. III-1, "Anleitung zur Naturlehre", in: Opera Omnia, ser. III, , 1

[25] Euler, L. III-11, "Lettres à une princesse d'Allemagne sur divers sujets de Physique et de Philosophie", in: Opera Omnia, ser. III, vols. 11 and 12

[26] Faraggi, A. E, \& Matone, M. (1998). The Equivalence Postulate of Quantum Mechanics", arXiv:hep-th/9809127

[27] Feynman, R. P. (1949). Space-Time Approach to Quantum Electrodynamics", Phys. Rev., 76, , 769ff.

[28] FrancisCh. (2010). Quantum Logic, http://rqgravity.net/FoundationsOfQuantumTheory\#QuantumLogic

[29] (G. F. W. Hegel, Werke in 20 Bänden, Frankfurt/M.: Suhrkamp 1969-1971 (stw 601-620).

[30] Heisenberg, W. (1925). Über quantenmechanische Umdeutung kinematischer und mechanischer Beziehungen", Z. Phys. XXXIII, , 879ff.

[31] Heisenberg, W. (1977). Die Geschichte der Quantentheorie", in: Physik und Philosophie (Ullstein, Frankfurt etc), , 15-27.

[32] Helmholtz, H. (1847). Über die Erhaltung der Kraft (Reimer, Berlin) 
[33] Helmholtz, H. von (1911). Vorlesungen über die Dynamik discreter Massenpunkte (Barth, Leipzig, $2^{\text {nd }}$ ed.)

[34] Hund, F. Zur Deutung der Molekelspektren. I, Z. Phys. (1927). III. Bemerkungen über das Schwingungs- und Rotationsspektrum bei Molekeln mit mehr als zwei Kernen, 43 (1927) 805-826, 40(1927), 742-764.

[35] Leibniz, G. W. Specimen dynamicum pro admirandis naturae legibus circa corporum vires et mutuas actiones detegendis et ad suas causas revocandis, Acta erudit. Lipsiens. April ; Engl.: Essay in Dynamics showing the wonderful laws of nature concerning bodily forces and their interactions, and tracing them to their causes, http://www.earlymoderntexts.com/pdf/leibessa.pdftranslated, edited and commented by J. Bennett, June (2006).

[36] Messiah, A. (1999). Quantum Mechanics (Dover, New York), § I.15

[37] Newton, I. (1999). The Principia. Mathematical Principles of Natural Philosophy (A New Translation by I. Bernhard Cohen and Anne Whitman assisted by Julia Buden, Preceded by A Guide to Newton's Principia by I. Bernhard Cohen), Berkeley etc.: Univ. Calif. Press

[38] Nordheim, L. W. Zur Theorie der thermischen Emission und der Reflexion von Elektronen an Metallen, Z. Phys. (1928). , 46(1928), 833-855.

[39] Pauli, W. (1926). Quantentheorie", in: H. Geiger and K. Scheel (Eds.): Handbuch der Physik (Springer, Berlin), , 23, 1-278.

[40] Pauli, W. (1973). Wave Mechanics (Pauli Lectures of Physics, Ed. Ch. P. Enz (MIT Press, Cambridge, Mass., 1973), § 35, 5

[41] Planck, M. (1900). Zur Theorie des Gesetzes der Energieverteilung im Normalspektrum", Verh. Dtsch. Phys. Ges., 2, , $237 \mathrm{ff}$.

[42] Schleich, W. P. (2001). Quantum Optics in Phase Space (Berlin etc., Wiley-VCH), , 282.

[43] Schrödinger, E. (1926). Quantisierung als Eigenwertproblem. Erste Mitteilung, Ann. Phys. [4] Zweite Mitteilung, 489-527; Dritte Mitteilung: Störungstheorie, mit Anwendung auf den Starkeffekt der Balmerlinien, 80 (1926) 437-490; Vierte Mitteilung, 81 (1926) 109-139; reprints in: Abhandlungen zur Wellenmechanik (Barth, Leipzig), 79(1926), 361-376.

[44] Schrödinger, E. (1933). The fundamental idea of wave mechanics" (Nobel award lecture, 1933)

[45] Suisky, D. (2009). Euler as Physicist, Berlin Heidelberg: Springer

[46] Suisky, D, \& Enders, P. (2001). Leibniz's Foundation of Mechanics and the Development of 18th Century Mechanics initiated by Euler", in: H. Poser (Ed.), Nihil sine ratione, Proc. VII Intern. Leibniz Congress, Berlin, http://www.leibniz-kongress.tu- 
berlin.de/webprogramm.html;http://www.information-philosophie.de/philosophie/ leibniz2001.html, 1247.

[47] Suisky, D, \& Enders, P. (2003). On the derivation and solution of the Schrödinger equation. Quantization as selection problem", Proc. $5^{\text {th }}$ Int. Symp. Frontiers of Fundamental Physics, Hyderabad (India), Jan. , 8-11.

[48] Weber, H. Über die Integration der partiellen Differentialgleichung..., Math. Ann. I ((1869).

[49] Weisstein, E. W. (2012). Fourier Transform", From MathWorld-A Wolfram Web Resource; http://mathworld.wolfram.com/FourierTransform.htmlMarch 13, 2012)

[50] Weizsäcker, C. F. v. (2002). Aufbau der Physik (dtv, München, $4^{\text {th }}$ ed.), 235.

[51] Weyl, H. (1950). The Theory of Groups in Quantum Mechanics (Dover, New York), § II.8

[52] Whittaker, E. T, \& Watson, G. N. A Course of Modern Analysis, Cambridge: Cambridge Univ. Press ${ }^{4} 1927$, new ed. (1996). (Cambr. Math. Libr. Ser.) 
Chapter 24

\title{
Entanglement, Nonlocality, Superluminal Signaling and Cloning
}

\author{
GianCarlo Ghirardi
}

Additional information is available at the end of the chapter

http://dx.doi.org/10.5772/56429

\section{Introduction}

Entanglement has been considered by E. Schrödinger [1] as: The most characteristic trait of Quantum Mechanics, the one which enforces its entire departure from classical lines of thought. Actually, the just mentioned unavoidable departure from the classical worldview raises some serious problems when entanglement of far away quantum systems is considered in conjunction with the measurement process on one of the constituents. These worries have been, once more, expressed with great lucidity by Schrödinger himself [1]: It is rather discomforting that the theory should allow a system to be steered or piloted into one or the other type of state at the experimenter's mercy, in spite of his having no access to it.

All those who are familiar with quantum theory will have perfectly clear the formal and physical aspects to which the above sentences make clear reference: they consist in the fact that, when dealing with a composite quantum system whose constituents are entangled and far apart, the free will choice of an observer to perform a measurement at one wing of the apparatus and the quantum reduction postulate imply that the far away state "jumps" in a state which depends crucially from the free will choice of the observer performing the measurement and from the random outcome he has got. Just to present a quite elementary case, let us consider a quantum composite system $S=S^{(1)}+S^{(2)}$, in an entangled state $|\psi(1,2)\rangle$ :

$$
|\psi(1,2)\rangle=\sum_{i} p_{i}\left|\phi_{i}^{(1)}\right\rangle \otimes\left|\gamma_{i}^{(2)}\right\rangle, \quad p_{i} \geq 0, \quad \sum_{i} p_{i}=1
$$

In this equation (the Schmidt biorthonormal decomposition) the sets $\left\{\left|\phi_{i}^{(1)}\right\rangle\right\}$ and $\left\{\left|\gamma_{i}^{(2)}\right\rangle\right\}$ are two orthonormal sets of the Hilbert spaces of system $S^{(1)}$ and $S^{(2)}$, respectively, and, 
as such, they are eigenstates of appropriate observables $\Phi^{(1)}$ and $\Gamma^{(2)}$ of such subsystems. Suppose now that subsystem $S^{(2)}$ is subjected to a measurement of the observable $\Gamma^{(2)}$ and suppose that in the measurement the outcome (one of its eigenvalues) $\Gamma^{(2)}=g_{r}$ is obtained. Then, reduction of the wave packet leads instantly to the state $\left|\phi_{r}^{(1)}\right\rangle \otimes\left|\gamma_{r}^{(2)}\right\rangle$ for which one can claim that if system $S^{(1)}$ is subjected to a measurement of the observable $\Phi^{(1)}$ the outcome $\Phi^{(1)}=f_{r}$ will occur with certainty. Since this outcome, before the measurement on system $S^{(2)}$, has a nonepistemic probability $p_{r}^{2}$ of occurrence, one can state that the observation of $\Gamma^{(2)}$ has caused the instantaneous emergence at-a-distance of a definite property (which, according to quantum mechanics, one cannot consider as possessed in advance) of subsystem $S^{(1)}$, i.e. the one associated to the eigenvalue $f_{r}$ of the observable $\Phi^{(1)}$. An analogous argument can obviously be developed without making reference to the Schmidt decomposition but to an arbitrary measurement on subsystem $S^{(2)}$, and will lead, in general, to the emergence of a different property of subsystem $S^{(1)}$ (typically the outcome of another appropriate measurement on this system becomes certain).

The just described process makes perfectly clear the nonlocal character of quantum mechanics, a fact that subsequently has been precisely identified by the illuminating work of J.S. Bell [2].

The situation we have just described will allow the reader to understand how it has given rise to the so called problem of faster-than-light signaling. If my action on system $B$, which takes place and is completed at a space like separation from system $A$, affects this system making instantaneously actual one of its potentialities, I can hope to be able to take advantage of this quantum peculiarity to make the observer $A$ aware of the fact that I am performing some precise action on subsystem $B$ at a space-like separation from him.

And, actually, this is what happened. From the seventies up to now, an innumerable set of proposals of taking advantage of entanglement and the reduction of the wave packet to achieve superluminal communication between distant observers appeared in the literature, proposals aiming to exploit this exciting possibility and to put into evidence the incompatibility of quantum mechanics with special relativity. Fortunately, as I will show in this paper, all proposal advanced so far, and, in view of some general theorems I will discuss below, all conceivable proposals of this kind, can been proven to be basically flawed in a way or another.

This chapter is devoted to discuss this important and historically crucial aspect of modern physics. As such, it has more an historical than a research interest. However, I believe that the reconsideration of the debate about this issue will be useful for the reader since many not so well known and subtle aspects of quantum mechanics will enter into play.

Before coming to a sketchy outline of the organization of the whole paper I would like to call the attention of the reader to a quite peculiar fact. When the so-called quantum measurement problem arose and was formalized by J. von Neumann [3], the attention of the scientific community was not concentrated on the possible conflicts between quantum mechanics and relativity; quantum mechanics was considered as a fundamentally nonrelativistic description of natural processes. Obviously, everybody had clear that the problem of its relativistic generalization had to be faced, but the debate concerned the nonrelativistic aspects of the theory and nobody had raised the question of possible conflicts between the two pillars of 
modern science ${ }^{1}$. In spite of that, the cleverly devised prescription of wave packet reduction, which was elaborated without having in mind relativistic potential oddities, turned out to be such that, in spite of its nonlocal nature and instantaneity, it did not allow to violate the basic relativistic request of no-faster-than-light signaling.

A brief outline of the organization of the chapter follows. After recalling the relevant aspects of the way in which quantum mechanics accounts for natural processes, we will describe various proposals for achieving faster-than-light signaling which have been put forward, and point out the reasons for which they are basically incorrect. To conclude this part we will present a general theorem showing that quantum mechanics, in its standard version, cannot in principle lead to superluminal communication.

However, the most interesting part of the debate is not the one we have just mentioned. In 1982 an analogous but quite different proposal of faster-than-light signaling has been put forward by N. Herbert [4]. The idea consisted in taking, as usual, advantage of the entanglement of far away subsystems and of wave packet reduction, but a new device was called into play: an hypothetical machine which could perform the task of creating many copies of an arbitrary state of a quantum system (a sort of "quantum xeroxing machine"). The interesting point is that at that time no general argument had been developed proving this task impossible. So, the mistaken suggestion of Herbert triggered the derivation of a theorem, the so-called no-cloning theorem, which was not known and which represents a quite relevant achievement which stays at the very basis of many important recent developments and which, besides proving that Herbert's proposal was unviable, plays a fundamental role for quantum cryptography and quantum computation.

As it is obvious, an hypothetical quantum device allowing faster-than-light communication would give rise to a direct and serious conflict with the special theory of relativity. As already stated, such a device is excluded by quantum mechanics. This, however, does not eliminate completely the potential tension of the nonlocal nature of quantum theory with the basic principles of relativity theory. The central issue is that the instantaneous collapse of the statevector of the far away system, even if it cannot be used to transfer energy or information at a superluminal speed, indicates that, in a way or another, an action performed in a given space-time region has some "effect" on systems at a space-like separation. Einstein has qualified this aspect of the theory as "a spooky action at-a-distance" which he could not accept. A. Shimony, by stressing the fact that the theory cannot be used to actually communicate superluminally has expressed his opinion that there is some sort of "peaceful coexistence of quantum mechanics and relativity" and has suggested to speak, in place of an "action" of a "passion" at-a-distance, to stress the peculiar nature of the perfect correlations of the outcomes, which, before any measurement process, individually have a fundamentally random nature, i.e., only a certain probability of occurrence.

Recently, the just mentioned problem has seen a revival due to the elaboration of the so-called "collapse models", i.e., modifications of quantum mechanics which, on the basis of a unique, universal dynamical principle account both for the quantum evolution of microscopic

\footnotetext{
${ }^{1}$ For this one should wait the celebrated EPR paper, which appeared, just as Schödinger's paper [1] in which the instantaneity of the reduction is seen as problematic, 2 years after von Neumann's precise formalization of the effect of a measurement.
} 
systems as well as for the reduction process when macroscopic systems enter into play. Such theories, the best known of which is the one presented in ref.[ 5] usually quoted as "The GRW Theory", have been worked out with the aim of solving the macro-objectification or measurement problem at the nonrelativistic level, and the fact that they get the desired result in a clean, mathematically rigorous and conceptually precise way has raised the interest of various scientists, among them the one of Bell [6-8]. After the complete formalization of such approaches, it has been natural to start investigating whether they admit relativistic generalizations. Since they, agreeing with the quantum predictions concerning microsystems, exhibit (essentially) the same nonlocal aspects as standard quantum mechanics, the question of wether they actually can be made compatible with relativity has attracted a lot of attention. The serious work of various physicists in recent years has made clear that the program can be pursued, which means that one can have a theory inducing instantaneous collapses at-a-distance which does not violate any relativistic request. We consider it interesting to devote the conclusive part of this chapter to outline the investigations along these lines and to discuss their compatibility with the principles of special relativity.

\section{The relevant formal aspects of the theory}

\subsection{The general rules}

As is well known, quantum mechanics asserts that the most accurate specification of the state of a physical system is given by the statevector $|\Psi\rangle$, an element of the Hilbert space $\mathcal{H}$ associated to the system itself. When one deals with a statistical ensemble of identical systems, an equivalent and practical mathematical object is the statistical operator $\rho$ which is the weighted sum of the statistical operators $\left|\psi_{i}\right\rangle\left\langle\psi_{i}\right|$ corresponding to the pure states $\left|\psi_{i}\right\rangle$ of the members of the ensemble: $\rho=\sum_{i} p_{i}\left|\psi_{i}\right\rangle\left\langle\psi_{i}\right|$, with $p_{i} \geq 0, \sum_{i} p_{i}=1$. For an homogeneous ensemble or an individual system in a pure state $|\Psi\rangle$, the statistical operator is a projection operator: $\rho=|\Psi\rangle\langle\Psi|$.

The observables of the theory are represented by self-adjoint operators of the Hilbert space $\mathcal{H}$, which are characterized by their eigenvalues and eigenvectors. For the observable $\Omega$ one writes its eigenvalue equation as ${ }^{2}$ :

$$
\Omega\left|\omega_{k, \alpha}\right\rangle=\omega_{k}\left|\omega_{k, \alpha}\right\rangle
$$

where the index $\alpha$ is associated to the possible degeneracy of the eigenvalue $\omega_{k}$. A crucial feature implied by the assumption of self-adjointness of the operators representing physical observables is that their spectral family, i.e. the projection operators $P_{r}=\sum_{\alpha}\left|\omega_{r, \alpha}\right\rangle\left\langle\omega_{r, \alpha}\right|$ on their eigenmanifolds, correspond to a resolution of the identity: $\sum_{r} P_{r}=I$, I being the identity operator on $\mathcal{H}$.

For what concerns the physical predictions, it is stipulated that in the case of a system in a pure state $|\Psi\rangle$ one has to express it as a linear combination of the eigensates of the observable (let us call it $\Omega$ ) corresponding to the microscopic physical quantity which one intends to

\footnotetext{
${ }^{2}$ For simplicity we will deal with observables with a purely discrete spectrum, the changes for the continuous case been obvious.
} 
measure: $|\Psi\rangle=\sum_{k, \alpha} c_{k, \alpha}\left|\omega_{k, \alpha}\right\rangle$. Then the theory asserts that the probability $P\left(\Omega=\omega_{r} \mid \Psi\right)$ of getting the outcome $\omega_{r}$ in the measurement of $\Omega$ when the system is in the pure state $|\Psi\rangle$, is given by $\sum_{\alpha}\left|c_{r, \alpha}\right|^{2}$, a quantity which coincides with the square of the norm $\|\left. P_{r}|\Psi\rangle\right|^{2}$ of the projection of the state onto the relevant eigenmanifold. This rule becomes, in the statistical operator language, $P\left(\Omega=\omega_{r} \mid \rho\right)=\operatorname{Tr}\left[P_{r} \rho\right]$, where the symbol $\operatorname{Tr}$ means that the sum of the diagonal elements of the quantity in square brackets in an arbitrary orthonormal complete basis of $\mathcal{H}$ must be taken (this sum is easily proved not to depend on the chosen basis). Note that, using the complete set of the eigenstates of an operator $\Omega$ to evaluate the Trace, one immediately sees that its quantum average can be simply expressed as $\langle\Omega\rangle=\operatorname{Tr}[\Omega \rho]$. It is an important mathematical fact that the Trace operation is linear and enjoys of the following formal feature: given two arbitrary (bounded) operators $\Lambda$ and $\Gamma$ of $\mathcal{H}, \operatorname{Tr}[\Lambda \Gamma]=\operatorname{Tr}[\Gamma \Lambda]$.

Before concluding this subsection we must also mention the effect on the statevector of performing a measurement process. Actually, two kinds of measurements can be carried out: the nonselective and the selective ones, i.e. those in which one measures an observable without isolating the cases in which a precise eigenvalue is obtained or, alternatively, those in which one is interested only in a definite outcome. They are represented, in the statistical operator language, by the two following formal expressions:

$$
\begin{gathered}
\rho_{\text {before }} \rightarrow \rho_{\text {after }}=\sum_{k} P_{k} \rho_{\text {before }} P_{k}, \\
\rho_{\text {before }} \rightarrow \rho_{\text {after }}=P_{k} \rho_{\text {before }} P_{k} / \operatorname{Tr}\left[P_{k} \rho_{\text {before }}\right],
\end{gathered}
$$

with obvious meaning of the symbols.

\subsection{Composite systems}

From now on we will be mainly interested in dealing with quantum systems $S$ composed of two constituents, $S^{(1)}$ and $S^{(2)}$. Accordingly, their statevector $|\Psi(1,2)\rangle$ is an element of the tensor product $\mathcal{H}^{(1)} \otimes \mathcal{H}^{(2)}$ of the Hibert spaces of the constituents. As is well known, in the considered case two radically different situations may occur: in the first one the statevector is simply the direct product of precise statevectors for the constituents $|\Psi(1,2)\rangle=|\phi(1)\rangle \otimes|\gamma(2)\rangle$, and in such a case both constituents possess precise physical properties; alternatively, the statevector is entangled, i.e., it cannot be written in this form but it involves the superposition of factorized states, typically $|\Psi(1,2)\rangle=\sum_{i} c_{i}\left|\phi_{i}(1)\right\rangle \otimes\left|\gamma_{i}(2)\right\rangle$.

An extremely important point concerning composite systems is the following. Suppose one has a composite system and he is interested only in the outcomes of perspective measurement processes on one of the constituents. Then, one can easily convince himself that the simplest way of dealing with this problem is to consider the reduced statistical operator $\tilde{\rho}(1)$, obtained by taking the partial trace of the full statistical operator on the Hilbert space of the subsystem $S^{(2)}$ one is not interested in. At this point, to evaluate the probability of the outcomes of measurements of observables of the system of interest $S^{(1)}$, one can use the reduced statistical 
operator and the same prescriptions we have used for the general case ${ }^{3}$ :

$$
\begin{aligned}
\tilde{\rho}(1) & =\operatorname{Tr}^{(2)}[\rho(1,2)] ; \quad P\left(\Phi^{(1)}=f_{r} \mid \rho(1,2)\right)=\operatorname{Tr}^{(1+2)}\left[P_{r}^{(1)} \rho(1,2)\right] \equiv \operatorname{Tr}^{(1)}\left[P_{r}^{(1)} \tilde{\rho}(1)\right] ; \\
\left\langle\Phi^{(1)}\right\rangle & =\operatorname{Tr}^{(1)}\left[\Phi^{(1)} \tilde{\rho}(1)\right] .
\end{aligned}
$$

It goes without saying that the operator $P_{r}^{(1)}$ in the previous equation is the projection operator onto the linear eigenmanifold associated to the eigenvalue $f_{r}$ of $\Phi^{(1)}$.

\section{3. von Neumann's ideal measurement scheme and its limitations}

For the subsequent analysis it is important to briefly recall the so-called Ideal Measurement Scheme introduced by von Neumann in his celebrated book, ref.[3], and its limitations. The idea is quite simple: we are interested in "measuring" a microscopic observable, which is not directly accessible to our senses. Suppose then we have a microsystem $s$ in a state $\left|\varphi_{i}^{(s)}\right\rangle$ which is in an eigenstate of a micro-observable $\Sigma^{(s)}$ pertaining to the eigenvalue $s_{i}$. How can one ascertain such a value, which, if a measurement is performed, according to the quantum rules will be obtained with certainity? Von Neumann assumed that there exists a macroscopic object $M$ which can be prepared in a ready state $\left|m_{0}\right\rangle$ and can be put into interaction with the microsystem. The interaction leaves unaltered the microstate while it induces, in a quite short time interval, the following evolution of the microsystem+apparatus:

$$
\left|\varphi_{i}\right\rangle \otimes\left|m_{0}\right\rangle \rightarrow\left|\varphi_{i}\right\rangle \otimes\left|m_{i}\right\rangle
$$

where the states $\left|m_{i}\right\rangle$ are assumed to be orthogonal $\left(\left\langle m_{i} \mid m_{j}\right\rangle=\delta_{i, j}\right)$, macroscopically and perceptively different (typically they are associated to different locations of the pointer of the macro-apparatus). Then, an observer, by looking at the measuring apparatus gets immediately the desired information concerning the value $\left(s_{i}\right)$ of the microvariable.

The scheme is usually qualified as ideal because, in practice, the final apparatus states are not perfectly orthogonal and because very often the state of the microsystem is disturbed (or even the system is absorbed) in the measurement. The just mentioned scheme has an immediate important implication; the validity of Eq.(6) and the linear nature of Schrödinger's evolution equation imply that if one triggers the macroapparatus in its ready state with a superposition of the eigenstates of $\Sigma^{(s)}$, one has:

$$
\sum_{i} c_{i}\left|\varphi_{i}\right\rangle \otimes\left|m_{0}\right\rangle \rightarrow \sum_{i} c_{i}\left|\varphi_{i}\right\rangle \otimes\left|m_{i}\right\rangle
$$

which is an entangled state of the microsystem and the macroapparatus.

\footnotetext{
${ }^{3}$ An elementary way to see this is to evaluate the probabilities of the joint outcomes of the measurement of a pair of observables, one for system $S^{(1)}$ and one for $S^{(2)}$, and then to sum on all possible outcomes of the measurement on the system we are not interested in.
} 
Eq.(7) has given rise to one of the most debated problems of quantum mechanics, the so-called measurement or macro-objectification problem. In fact its r.h.s. corresponds to an entangled state of the system and the apparatus and in no way whatsoever to a state corresponding to a precise outcome ${ }^{4}$. The orthodox way out from this puzzle consists in resorting to the postulate of wave packet reduction: when a superposition of different macrostates emerges, a sudden change of the statevector occurs, so that one has to replace the r.h.s. of the previous equation with one of its terms, let us say $\left|\varphi_{j}\right\rangle \otimes\left|m_{j}\right\rangle$. This specific reduction occurs with probability $\left|c_{j}\right|^{2}$. We will not enter, here, in this deep debate, we simply mention that it amounts to accept (as many scientists did) that the linear character of the theory is violated (the reduction process is nonlinear and stochastic while the quantum evolution is linear and deterministic) at an appropriate (but not precisely specified) macroscopic level. Incidentally, von Neumann himself has proposed that the transition from the superposition to one of its terms occurs when a conscious observer becomes aware of the outcome (reduction by consciousness). Recently, various proposals of theories which, on the basis of a unique dynamical principle, account both for the linear nature of the evolution at the microscopic level as well as for the discontinuous changes (collapses) occurring when macrosystems are involved, have been put forward. We refer the reader to ref.[10] for an exhaustive analysis of such model theories.

\subsubsection{Limits to the ideal scheme due to additive conservation laws}

There are limitations to the von Neumann ideal scheme that we must mention because they have played a role in the refutation of some proposals of faster-than-light communication. Such limitations have been identified by Wigner [11], Araki and Yanase [12,13] in a series of interesting papers and subsequently they have been generalized in refs.[14,15]. The analysis by these authors takes into account the existence of additive conserved quantities for the system+apparatus system to derive precise conditions on a process like the one of Eq.(6) which stays at the basis of the von Neumann treatment. Let us summarize the procedure in a sketchy way. The process described by Eq.(6) represents the unitary evolution of the system+apparatus during the measurement process of the observable $\Sigma^{(s)}$ with eigenstates $\left|\varphi_{i}\right\rangle$. Let us therefore write it as: $U\left|\varphi_{i}, m_{0}\right\rangle=\left|\varphi_{i}, m_{i}\right\rangle$. Let us suppose that there exists an additive conserved quantity $\Gamma=\gamma^{(s)} \otimes I^{(A)}+I^{(s)} \otimes \gamma^{(A)}$ of the whole system and let us evaluate the matrix element of $\Gamma,\left\langle\varphi_{i}, m_{0}|\Gamma| \varphi_{j}, m_{0}\right\rangle$ by taking into account that $\Gamma$ commutes with $U$, which implies :

$$
\left\langle\varphi_{i}, m_{0}\left|\left(\gamma^{(s)} \otimes I^{(A)}+I^{(s)} \otimes \gamma^{(A)}\right)\right| \varphi_{j} m_{0}\right\rangle=\left\langle\varphi_{i}, m_{0}\left|U^{\dagger}\left(\gamma^{(s)} \otimes I^{(A)}+I^{(s)} \otimes \gamma^{(A)}\right) U\right| \varphi_{j} m_{0}\right\rangle .
$$

\footnotetext{
${ }^{4}$ Note that in ref.[9] it has been proven that the occurrence of the embarrassing superpositions of macroscopically different states does not require that the measurement proceeds according to the ideal scheme of von Neumann. The same conclusion can be derived as a consequence of the necessary request that quantum mechanics governes the whole process and that one can perform a reasonably reliable measurement ascertaining the microproperty of the measured system.
} 
We then have:

$$
\begin{aligned}
& \left\langle\varphi_{i}, m_{0}\left|\left(\gamma^{(s)} \otimes I^{(A)}+I^{(s)} \otimes \gamma^{(A)}\right)\right| \varphi_{j} m_{0}\right\rangle=\left\langle\varphi_{i}\left|\gamma^{(s)}\right| \varphi_{j}\right\rangle+\delta_{i j}\left\langle m_{0}\left|\gamma^{(A)}\right| m_{0}\right\rangle= \\
& \left\langle\varphi_{i}, m_{0}\left|U^{\dagger}\left(\gamma^{(s)} \otimes I^{(A)}+I^{(s)} \otimes \gamma^{(A)}\right) U\right| \varphi_{j} m_{0}\right\rangle=\left\langle\varphi_{i}, m_{i}\left|\left(\gamma^{(s)} \otimes I^{(A)}+I^{(s)} \otimes \gamma^{(A)}\right)\right| \varphi_{j}, m_{j}\right\rangle= \\
& \delta_{i j}\left\langle\varphi_{i}\left|\gamma^{(s)}\right| \varphi_{j}\right\rangle+\delta_{i j}\left\langle m_{i}\left|\gamma^{(A)}\right| m_{j}\right\rangle .
\end{aligned}
$$

Comparison of the final expression with the one after the equality sign in the first line shows that, in the considered case, one must have, for $i \neq j,\left\langle\varphi_{i}\left|\gamma^{(s)}\right| \varphi_{j}\right\rangle=0$ which amounts to the condition that the observable $\Sigma^{(s)}$ which we want to measure on the microsystem must commute with the microsystem part $\gamma^{(s)}$ of the conserved additive quantity. If this is not the case (as it happens when $\Sigma^{(s)}$ is a component of the angular momentum of the system which does not commute with the other components), a process like the one of Eq.(6) turns out to be impossible; terms must be added to the r.h.s. involving other states of the microsystem besides $\left|\varphi_{i}\right\rangle$ and also other states of the apparatus. In refs.[13-15] it has been shown that in order to go as near as possible to the ideal case one must make more and more large the square of the norm of the state $\gamma^{(A)}\left|m_{0}\right\rangle$. In the case of an angular momentum measurement this means to make the mean value of the square of the angular momentum component extremely large. Actually, in the case of the measurement of the spin component of a spin $1 / 2$ particle, the "distorsion" of the state by the measurement, a quantity which can be estimated by the squared norm $\epsilon^{2}$ of the state which has to be added to the r.h.s. of Eq.(6), must satisfy: $\epsilon^{2} \geq h^{2} / 32 \pi^{2}\left\langle m_{0}\left|L^{2}\right| m_{0}\right\rangle$, where $L^{2}$ is the square of the angular momentum operator of the apparatus: to make the error extremely small one has to make extremely large $\left\langle m_{0}\left|L^{2}\right| m_{0}\right\rangle$.

\subsection{More realistic formalizations of the measurement process}

Up to this point, when accounting for the occurrence of measurement processes, we have always made reference to the projection operators on the eigenmanifolds of the operators associated to the measurement. However, in practice, it is quite difficult to have apparatuses whose effect on the statevector can be accounted precisely by a projection operator. A simple example is the one of a detector of the position of a particle in a given interval $\Delta$ which has different efficiency in different portions of the interval $\Delta$ so that it detects for sure a particle impinging on its central region but only with a certain probability a particle which is detected near its extreme points. Another example is given by a measurement process which corresponds to two different successive measurements of two noncommuting observables, the outcome being represented by the pair of results which have been obtained. Also in this case the probability of "an outcome" cannot be expressed in terms of a single projection operator. The appropriate consideration of situations like those just mentioned has led to the consideration of more general processes affecting the statistical operator than the one of Eq.(3). One can then take advantage of a fundamental theorem by Kraus [16] asserting that the most general map of trace class and trace one semipositive definite operators onto themselves which respects also the condition of complete positivity (which has strong physical reasons to be imposed ${ }^{5}$ ) has the form:

\footnotetext{
${ }^{5}$ For a definition and a discussion of completely positive maps we refer the reader to ref.[16]
} 


$$
\rho \rightarrow \sum_{i} A_{i}^{\dagger} \rho A_{i}, \sum_{i} A_{i} A_{i}^{\dagger}=I
$$

When considering measurement processes we will make reference to Eq.(3) or to the just written equation as expressing the effect of the measurement on the statistical operator.

\section{Proposals of faster-than-light communication and their rebuttal}

As already anticipated, after the clear cut proof by J.S. Bell of the fundamentally nonlocal nature of physical processes involving far away constituents in an entangled state, many proposals have been put forward, either in private correspondence or in scientific papers, suggesting how to put into evidence superluminal effects. We will begin by reviewing a series of proposal whose rebuttal did require only to resort to the standard formalism or to well established facts, such as those put into evidence by the Wigner-Araki-Yanase theorems.

\subsection{Proposals taking advantage of the conservation of angular momentum}

In the year 1979 various papers appeared asserting the possibility of superluminal communication by taking advantage of the change in the angular momentum of a far away constituent due to a measurement performed on its partner. The scientific and social context of these first investigations aiming to take advantage of quantum nonlocality have been described in the interesting and funny book [17] by D. Kaiser How the hippies saved physics, which intends to point out how the actions of a peculiar community of scientists and non scientists trying to justify various sort of paranormal effects on the basis of quantum nonlocality have drawn, in the US, the attention of the scientific community to Bell's fundamental theorem and its implications. The three papers [18-20] that I intend to consider in this section have some strict links with the just mentioned context.

Let us start with refs.[18,19]. Their argument is quite straightforward: one considers two far away spin $1 / 2$ particles in the singlet state which interact with 2 apparatuses aimed to measure the spin $z$-component and are in their "ready" states $\left|A_{0}\right\rangle$ and $\left|B_{0}\right\rangle$, so that the initial state is:

$$
|\Psi\rangle=\frac{1}{\sqrt{2}}\left[\left|1_{+}, 2_{-}\right\rangle-\left|1_{-}, 2_{+}\right\rangle\right] \otimes\left|A_{0}, B_{0}\right\rangle .
$$

Here the indices + and - denote the values (in the usual units) of the $z$-component of the spin of the particles. Suppose now that the interaction of particle 2 with the apparatus $B$ takes place before the other particle reaches $A$ ( $A$ and $B$ being at rest in a given inertial frame). Wave packet reduction occurs, and we are left, with the same probability, with one of the two states $\left|1_{+}, 2_{-}, A_{0}, B_{-}\right\rangle$and $\left|1_{-}, 2_{+}, A_{0}, B_{+}\right\rangle$, where $\left|B_{ \pm}\right\rangle$are the states of the apparatus $B$ after the measurement. We can now evaluate the mean value of the square of the spin angular momentum when the state is the one of Eq.(11) and when it is one of the states of the mixture. In the first case we get: $\left\langle S^{2}\right\rangle_{\text {singlet }}=0$, while in the second case we get the value $\hbar^{2}$. Now one takes advantage of the conservation of the angular momentum $\mathbf{L}=\mathbf{M}+\mathbf{S}$ 
where $\mathbf{M}$ is the angular momentum of the apparatus ${ }^{6}$. Since $\langle\mathbf{M} \cdot \mathbf{S}\rangle=0$ in all above states, one concludes that the measurement induces a change of $\hbar^{2}$ in the apparatus which performs the measurement. This is not the whole story. If one, subsequently, leaves the second particle to interact with the apparatus measuring the spin state of the particle, the expectation value of $\mathbf{L}^{2}$ does not change any more. So, actually, the angular momentum of the apparatus which is the first to perform the measurement changes of the indicated amount, while the one of the other remains unchanged. Now if Alice and Bob, sitting near $A$ and $B$, have at their disposal a source of entangled particles in the singlet state, Bob, who interacts first with his particle, can choose to perform or not the measurement; correspondingly he can choose whether to leave unchanged or to change the angular momentum of the apparatus at $A$. If Alice can detect this change she can get information about the choice made, in each single instance, by $\mathrm{Bob}^{7}$. Superluminal communication becomes possible.

According to the above analysis and the remark in the footnote, the key ingredient which allows to draw the conclusion is the occurrence of an ideal nondistorting measurement of the spin component. This implies that the argument of refs.[18,19] is based on contradictory assumptions, since, as discussed in the previous section, the Wigner-Araki-Yanase theorem asserts that the occurrence of an ideal nondistorting measurement of a spin component of a subsystem contradicts the conservation of total angular momentum. Actually, to have an ideal measurement process one needs apparatuses with a divergent mean value of the square of the angular momentum, but then no change of this quantity can be detected. Alternatively, one should consider nonideal measurements which are compatible with angular momentum conservation, but then the previous argument does not work, just because Eq.(6) has to be modified.

Precisely in the same year in which the above described arguments were presented, N. Herbert circulated a paper [20] which made resort to the functioning of a half wave plate to get the same result. His proposal was stimulated by his reading of a paper [21] written in 1936 by R. Beth and included by the American Association of Physics Teachers in a collection of papers published as Quantum and Statistical Aspects of Light. Beth managed to measure the angular momentum of circularly polarized light due to the fact that when right-circularly polarized light is shone on the half-wave plate it sets the plate spinning in one direction, while left-circularly polarized light spun the half-wave plate in the opposite direction. Moreover, the plate flips the light polarization from left to right and viceversa. Beth had measured the effect for circularly polarized light waves, i.e., by using a huge collection of photons all acting together. Herbert, inspired by this work, suggested, in the paper he called QUICK, to play a similar game with the angular momentum of individual photons to get superluminal effects.

Once more the idea is quite simple: one imagines a source emitting pairs of entangled photons in two opposite directions, their state being the analogous of the singlet state, i.e. the rotationally invariant state: $|\Psi(1,2)\rangle=[1 / \sqrt{2}][|H 1, H 2\rangle+|V 1, V 2\rangle] \equiv[1 / \sqrt{2}][|R 1, L 2\rangle+$ $|L 1, R 2\rangle]$. Here, the symbols $H, V, R$ and $L$ make reference to the states of horizontal, vertical, right and left circular polarizations, respectively. Bob can freely choose whether to perform

\footnotetext{
${ }^{6}$ Here, we disregard the orbital angular momentum of the particles, but the argument holds true also without this limitation.

${ }^{7}$ It is interesting to remark that the same argument can be developed if one does not take into account the reduction process, i.e., if one assumes that the interactions simply take place in accordance with the von Neumann scheme.
} 
a measurement of either plane $(\mathrm{H}, \mathrm{V})$ or circular $(\mathrm{L}, \mathrm{R})$ polarization. As a consequence of his measurement the far away photon is projected either onto a state of plane or of circular polarization. Subsequently, this photon impinges on a half-wave plate (near Alice). Since the photon when plane polarized crosses the plate without transmitting any angular momentum to it, while, when circularly polarized, it imparts a change of $\pm 2 \hbar$ to the angular momentum of the plate, if Alice is able to check whether his plate has not changed or has changed its angular momentum she can know what kind of measurement $(\mathrm{H}, \mathrm{V})$ or $(\mathrm{L}, \mathrm{R})$ Bob has chosen to perform in any single case. Once more, entanglement and reduction of the wave packet allow superluminal transmission of information.

To prove why also this suggestion is inviable one has to analyze the functioning of the half-wave plate. The nice fact is that, as proved in ref.[22], one can develope precisely an argument analogous to the one of Wigner-Araki-Yanase, to prove that a half-wave plate can work as indicated only if a violation of the angular momentum conservation occurs. But such a conservation is necessary for the argument, so, once more, the proposal is contradictory. No superluminal communication is possible by resorting to the QUICK device.

Herbert and the Fundamental Fysiks Group made all they could do to spread out Herbert's conclusions. The debate involved scientists like H. Stapp and P. Eberhard. In june 1979, Stapp challenged the idea, building on Eberhard's argument that statistical averages would wash out any non local effect. But Herbert had worked out his reasoning for individual photons, and the above objection turned out to be not relevant for setting the issue. In the same year we (T. Weber and myself), became acquainted with Herbert's, as well as with Selleri's and others proposals. Accordingly, we wrote the paper [22] which presents the conclusions I have just described concerning refs.[18-20]. Beth's important experiment worked just because the experimenter sent an enormous number of photons at the half-wave plate. But, at the single-photon level, to get the same result, the half-wave plate would have to be infinitely massive, and, as such, it could not be put into rotation by the passage of an individual photon. This conclusion can be made rigorous with a little of mathematics, as we did in ref.[22].

\subsection{Popper enters the game}

Mention should also be made of the position of K. Popper concerning the problem of faster-than-light communication. In some previous writings, but specifically in his famous book [23] Quantum Theory and the Schism in Physics he raised the question of the conflict between quantum theory and special relativity theory, due to his alleged claim that "if quantum mechanical predictions are correct", then one would be able to send superluminal signals putting into evidence a conflict between the two pillars of our conception of the world. Unfortunately he was (mistankingly) convinced that the quantum rules would imply an effect that they actually exclude (a fact which he missed completely to understand), and, consequently, in his opinion they would allow superluminal signaling in an appropriate experimental situation.

The idea is quite simple (see Fig1, a,b): we have two perfectly correlated (in position) particles propagating towards two arrays of detectors placed at left $(L)$ and right $(R)$ of the emitting source at almost equal distances from it. Two slits, orthogonal to the direction $x$ in the figure, are placed at both sides, along the $y$-axis, before the array of the counters, and, initially, only the counters lying behind the opening of the slits get activated. Subsequently, the slit 
at $R$ is narrowed so as to produce an uncertainty principle scatter of the momentum $p_{y}$, which appreciably increases the set of counters which are activated with a non-negligible probability (see Fig.1b). Popper then argues: If quantum mechanics is correct, any increase in the knowledge of the position $y$ at $R$ like the one we get by making more precise the location along the $y$-axis of the particle which is there, implies an analogous increase of the knowledge of the position of the particle at $L$. As a consequence also the scatter at $L$ should increase even though the width of the slit at this side has not been narrowed. This prediction is testable, since new counters would be activated with an appreciable probability, giving rise to a superluminal influence: Alice can know (with an appreciable probability) whether Bob has chosen to narrow or leave unchanged his slit. The conclusion of Popper is quite emblematic: in his opinion the increase of the spread at $L$ would not occur and this would show that quantum theory is wrong. He also contemplates the other alternative: if the scatter at left would increase, then superluminal communication would be possible and relativity theory would be proven false; in both cases, a quite astonishing conclusion.

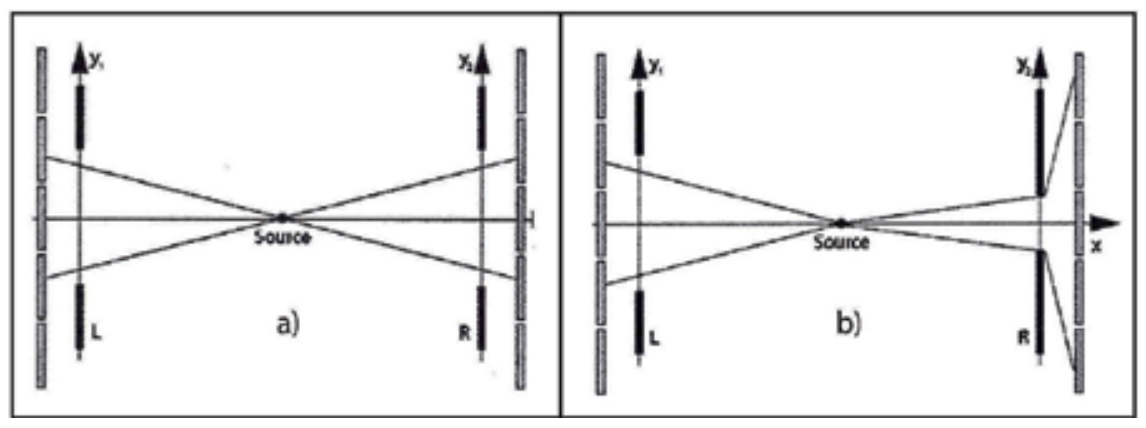

Figure 1. The set up and functioning of Popper's ideal experiment.

Unfortunately, in this passage of his important work, Popper shows his lack of understanding the quantum principles governing the unfolding of the considered experiment. In fact, it can be easily proved that quantum mechanics predicts precisely that no scatter at left will be induced by the narrowing of the slit at right. We do not consider it useful to enter in all technical details of the argument. The reader can look at ref.[24] or to Chapter 11 of ref.[25] for a detailed and punctual discussion. Here, we simply outline the argument: if the positions of the particles are really $100 \%$ correlated (and therefore associated to a Dirac delta like unnormalized state), then they are in a state which implies that, even when the two slits are fully opened, all counters are activated with large probabilities, while, if their correlations are only approximate (even with an extremely high degree of accuracy) the action at $R$ by Bob does not change in any way whatsoever the outcomes at $L$. So, the argument is basically wrong.

\section{The general proof of the impossibility of faster-than-light communication}

To present a completely general proof $[26,27]$ of the fact that instantaneous wave-packet reduction does not allow superluminal signaling we must start by reconsidering all possible actions $[3,16]$ which are permitted, by standard quantum mechanics, on a constituent of a composite system. Quite in general, quantum mechanics allows the possibility of: 
- A unitary transformation describing the free evolution of the system at $R$ under consideration and/or possibly of its interactions with other systems lying in a space-time region which is space like with respect to the one at $L$.

- A transformation corresponding to a non selective projective measurement (with wave packet reduction as described by Eq. (3)) of the considered subsystem.

- A transformation like the one summarized in Eq.(10) corresponding, essentially, to the occurrence of a non ideal measurement.

- A transformation like the one of Eq.(4), corresponding to a selective measurement. To be strict, one should also consider the analogous of this transformation in the case of a non-ideal measurement, but, for the reasons which we will make clear below, this case does not have a physical relevance for faster-than-light signaling.

Now we can proceed to outline our proof, which represents a generalization and a more accurate formulation of some ideas put forward by P. Eberhard [28] about one year before we wrote our paper [26] (see also A. Shimony [29] for an enlightening discussion).

To start with we recall that all probabilistic predictions concerning a subsystem of a composite system can be obtained by considering the reduced statistical operator of the subsystem of interest. We suppose now to have a composite quantum system $S=S^{(1)}+S^{(2)}$ associated to the statistical operator $\rho(1,2)$ and to be interested in predictions concerning prospective measurements on subsystem $S^{(1)}$. As already remarked, the physics of this subsystem is fully described by the reduced statistical operator $\tilde{\rho}^{(1)}=\operatorname{Tr}^{(2)} \rho(1,2)$. We can now consider the following set of equations:

$$
\begin{aligned}
\tilde{\rho}^{(1)} & =\operatorname{Tr}^{(2)} \rho(1,2), \\
\operatorname{Tr}^{(2)}\left[U^{(2) \dagger} \rho(1,2) U^{(2)}\right] & =\operatorname{Tr}^{(2)}\left[U^{(2)} U^{(2) \dagger} \rho(1,2)\right]=\operatorname{Tr}^{(2)} \rho(1,2)=\tilde{\rho}^{(1)}, \\
\operatorname{Tr}^{(2))}\left[\sum_{i} P_{i}^{(2)} \rho(1,2) P_{i}^{(2)}\right] & =\operatorname{Tr}^{(2)}\left[\sum_{i}\left[P_{i}^{(2)}\right]^{2} \rho(1,2)\right]=\operatorname{Tr}^{(2)} \rho(1,2)=\tilde{\rho}^{(1)} \\
\operatorname{Tr}^{(2))}\left[\sum_{i} A_{i}^{(2) \dagger} \rho(1,2) A_{i}^{(2)}\right] & =\operatorname{Tr}^{(2)}\left[\sum_{i} A_{i}^{(2)} A_{i}^{(2) \dagger} \rho(1,2)\right]=\operatorname{Tr}^{(2)} \rho(1,2)=\tilde{\rho}^{(1)} .
\end{aligned}
$$

In these equations we have made use of the cyclic property of the trace over the Hilbert space $\mathcal{H}^{(2)}$ when operators of the same Hilbert space are involved, of the unitarity relation $U^{(2)} U^{(2) \dagger}=I^{(2)}$, of the propery $\left[P_{i}^{(2)}\right]^{2}=P_{i}^{(2)}$ and of the fact that the sum of the operators $P_{i}^{(2)}$ in the case of a projective measurement, as well as of the operators $A_{i}^{(2)} A_{i}^{(2) \dagger}$ of the Kraus theorem [16], must equal the identity operator of the same space. We call the attention of the reader to the fact that in all considered cases, i.e., i). no action on $S^{(2)}$, ii). a unitary evolution of $S^{(2)}$, or iii). the fact that it is subjected to an operation corresponding to a (ideal or non-ideal) nonselective measurement process, the reduced statistical operator $\tilde{\rho}^{(1)}$ of subsystem $S^{(1)}$ does not change in any way whatsoever, and, accordingly, Alice, performing measurements on such a subsystem cannot get any information about the fact that Bob is making some specific action on subsystem $S^{(2)}$. 
Up to now, we have not considered explicitly the case of selective ideal or non-ideal measurement processes, accounted for by Eq. (4) or by its analogue referring to processes like those governed by Eq.(10). If one considers the modifications to the general statistical operator in these cases and one uses the reduced statistical operator to evaluate the probabilities of the measurement outcomes on subsystem $S^{(1)}$, one would easily discover that the physics of such a system is actually changed by the action on its far-away partner. But the probabilistic changes depend crucially on the outcome that Bob has obtained in his measurement, so that Alice might take advantage of this fact only if she would be informed of the outcome obtained by Bob. This implies that Bob must inform Alice concerning his outcome and this can be done only by resorting to standard communication procedures which require a subluminal communication between the two. Accordingly, these cases can safely be disregarded within our context.

It should be clear that the general validity of our theorem implies that all previously discussed attempts to get faster-than-light signaling taking advantage of the instantaneous reduction at-a-distance of the statevector in the case of entangled states of far-away systems, were doomed to fail. We have discussed them in some detail to present an historically complete perspective of the debate on this fundamental issue, i.e. the one of the compatibility of quantum mechanics with relativistic requirements concerning the communication between far-away observers.

\section{A radical change of perspective}

\subsection{Herbert's new proposal}

In 1981 N. Herbert submitted for publication to Foundations of Physics a paper [4] by the title: FLASH-A superluminal communicator based on a new kind of quantum measurement in which he added a new specific device to his previous proposal we have discussed in sect.3.1. The stimulus to do so came probably from our paper, ref.[26], as remarked by D. Kaiser in his book [17]: From Ghirardi's intervention, Herbert came to appreciate the importance of amplifying the tiny distinction between various quantum states, to evade fundamental limits on signaling. The crucial device which, in his opinion, could do the game, was a Laser gain tube exhibiting the following characteristics: if the laser was stimulated by a single photon in any state of polarization (the states which mattered were actually those of plane (V and $\mathrm{H}$ ) and of circular ( $\mathrm{R}$ and $\mathrm{L}$ ) polarization) it would emit a relevant number, let us say $4 \mathrm{~N}$ with $\mathrm{N}$ large, of identical copies (in particular with the same polarization) of the impinging photon. If we summarize the process by means of an arrow leading from the initial photon state to the bunch of final photons, Hebert's Laser gain tube has to work in the following way:

$$
|V, 1\rangle \rightarrow|V, 4 N\rangle,|H, 1\rangle \rightarrow|H, 4 N\rangle,|R, 1\rangle \rightarrow|R, 4 N\rangle,|L, 1\rangle \rightarrow|L, 4 N\rangle,
$$

with obvious meaning of the symbols. Here 1 denotes the photon propagating towards Alice.

By resorting to this machine Herbert's game becomes quite simple. One starts, as in his first proposal, with a source emitting pairs of entangled photons in two opposite directions, their state being the rotationally invariant state: $|\Psi(1,2)\rangle=[1 / \sqrt{2}][|H 1, H 2\rangle+|V 1, V 2\rangle] \equiv$ $[1 / \sqrt{2}][|R 1, L 2\rangle+|L 1, R 2\rangle]$. Obviously, Bob can freely choose whether to perform a 
measurement of either plane $(\mathrm{H}, \mathrm{V})$ or circular $(\mathrm{L}, \mathrm{R})$ polarization. As a consequence of his measurement the far away photon is projected either onto a state of plane or of circular polarization. At this point the far away photon is injected in the Laser gain tube which emits $4 N$ photons with the same polarization, which, in turn, depends on the measurement performed by Bob and the outcome he has got. The $4 \mathrm{~N}$ photons are then separated into 4 beams of $\mathrm{N}$ photons each, directed towards 4 detectors of $V, H, L$ and $R$ (mind the order) polarization, respectively. To see the game coming at an end we have now simply to recall that a detector registers for sure a photon with the polarization it is devised to measure, it does not detect a photon into an orthogonal state and detects with probability $1 / 2$ a photon in a state of polarization which is the equal superposition of the state that it is devised to detect and of the state orthogonal to it.

We analyze the situation in detail specifying the measurements which Bob chooses to perform, the outcomes he gets and the records by the counters near Alice.

- Suppose Bob chooses to perform a polarization measurement aimed to ascertain whether the photon (2) reaching him has vertical or horizontal polarization and that he finds the photon vertically polarized. In this case, the process goes as follows:

a). Initial state: $|\Psi(1,2)\rangle=[1 / \sqrt{2}][|H 1, H 2\rangle+|V 1, V 2\rangle] ;$ b). Measurement with outcome Vertical; c). Reduction of the state: $|V 1, V 2\rangle$; d). Amplification: $|V, 4 N ; V 2\rangle$; e). Number of photons detected by the far away detectors (near Alice) for the 4 beams: N,0,N/2,N/2.

- a). Initial state: $|\Psi(1,2)\rangle=[1 / \sqrt{2}][|H 1, H 2\rangle+|V 1, V 2\rangle]$; b). Measurement with outcome Horizontal; c). Reduction of the state: $|H 1, H 2\rangle$; d). Amplification: $|H, 4 N ; H 2\rangle$; e). Number of photons detected by the far away detectors on the 4 beams: 0,N,N/2,N/2.

- a). Initial state: $[1 / \sqrt{2}][|R 1, L 2\rangle+|L 1, R 2\rangle]$; b). Measurement with outcome Right; $\mathrm{c})$. Reduction of the state: $|L 1, R 2\rangle$; d). Amplification: $|L, 4 N ; R 2\rangle$; e). Number of photons detected by the far away detectors on the 4 beams: N/2,N/2,N, 0 .

- a). Initial state: $[1 / \sqrt{2}][|R 1, L 2\rangle+|L 1, R 2\rangle]$; b). Measurement with outcome Left; c). Reduction of the state: $|R 1 ; L 2\rangle$; d). Amplification: $|R, 4 N ; L 2\rangle$; e). Number of photons detected by the far away detectors on the 4 beams: $\mathrm{N} / 2, \mathrm{~N} / 2,0, \mathrm{~N}$.

Now, one has simply to remark that in the cases listed under the two first items (i.e. when Bob chooses to measure linear polarization) the detector which does not register any photon is either the first or the second, while, in the alternative case in which Bob chooses to measure circular polarization, it is either the third or the fourth detector which does not register any photon. Accordingly, Alice can become aware, instantaneously, of the choice made by Bob: they can communicate superluminally.

\subsection{The no-cloning theorem}

The FLASH paper was sent for refereeing to A. Peres and to me. Peres' answer [30] was rather peculiar: I recommended to the editor that this paper should be published. I wrote that it was obviously wrong, but I expected that it would elicit considerable interest and that finding the error would lead to significant progress in our understanding of physics. I also was rather worried for various reasons. I was not an expert on Lasers and I was informed that A. Gozzini and R. Peierls were trying to disprove Herbert's conclusion by invoking the unavoidable 
noise affecting the Laser which would inhibit its desired functioning. On the other hand, I was convinced that quantum theory in its general formulation and not due to limitations of practical nature would make unviable Herbert's proposal. After worrying for some days about this problem I got the general answer: while it is possible to devise an ideal apparatus which clones two orthogonal states with $100 \%$ efficiency, the same apparatus, if the linear quantum theory governs its functioning, cannot clone states which are linear combinations of the previous ones. Here is my argument, on the basis of which I recommended rejection of Herbert's paper. The assumption that the cloning machine acts as follows:

$$
|V\rangle \rightarrow|V, 4 N\rangle \text { and }|H\rangle \rightarrow|H, 4 N\rangle
$$

when the linear nature of the theory is taken into account, implies:

$$
R \equiv \frac{1}{\sqrt{2}}[i|V\rangle+|H\rangle] \rightarrow \frac{1}{\sqrt{2}}[i|V, 4 N\rangle+|H, 4 N\rangle]
$$

and analogously for the left polarization. Now, the state at the r.h.s. of the last equation is by no means the state $|R, 4 N\rangle$ which Herbert had assumed to occur in the case in which the Laser gain tube is triggered by a right polarized photon. But this is not the whole story: how it has been shown in ref.[31] the very linear nature of the theory implies that no difference in the detections of Alice occurs in dependence of the free will choice of Bob.

This is an account of how Herbert's ingenious, but mistaken, proposal has led me to be the first to derive the no-cloning theorem ${ }^{8}$. About one and half year later Wootters and Zurek [32] and Dieks [33] derived independently the same result and published it $^{9}$. The theorem is of remarkable importance in quantum theory, it has become known as "The no cloning theorem" and it has been quoted an innumerable number of times. Only subsequently I realized that it had been a mistake on my part not to publish my result. I discussed my precise argument with Gozzini and Peierls, by sending them a draft which was a sort of repetition of my referee report and I subsequently published it [31] in collaboration with my collaborator, T. Weber.

\subsubsection{More on quantum cloning}

In a paper like the present one, we believe it useful to mention that E.P. Wigner [34], in an essay of 1961 had already argued that the phenomena of self-replication of biological systems contradict the principles of quantum mechanics. His argument is quite straightforward. Following his notation let us suppose we have a living system in a state $v$ and an environment (assimilated to "food") in a state $w$, so that, the initial statevector of the system, organism +

\footnotetext{
${ }^{8}$ I have chosen to attach at the end of the paper, a document - a letter by A. van der Merwe - which officially attests this fact, since it is known only to a restricted community of physicists.

${ }^{9}$ I must confess that I have never understood why A. Peres, in mentioning my derivation, has stated that it was a special case of the theorems in refs. [32] and [33]. Comparison even only of the short page by A. van der Merwe with the just mentioned papers makes clear that the argument is precisely the same and has the same generality.
} 
nutrient, is: $\Phi=v \times w$. When replication takes place the statevector will have the form: $\Psi=v \times v \times r$, i.e. two organisms each in the statevector $v$ will be present, while the vector $r$ describes both the rest of the system, the rejected part of the nutrient and also the other coordinates (positions, etc.) of the two organisms. One assumes that the system lives in an $N$-dimensional Hilbert space $\mathcal{H}^{(\mathcal{N})}$, the part $r$ in an $R$-dimensional Hilbert space $\mathcal{H}^{(\mathcal{R})}$, while the "food" state $w$ belongs to a $N \cdot R$ dimensional Hilbert space $\mathcal{H}^{(\mathcal{N} R)}$, so that $\Phi$ and $\Psi$ live, as they must, in the same space. Suppose we do not know the state of the living system; however, since it belongs to $\mathcal{H}^{(\mathcal{N})}$ his knowledge requires to know $N$ complex numbers. Analogously we do not know the state $r$, and the state $w$, which require the specification of $R$ and NR complex numbers. We now assume, with Wigner, that the collision matrix which gives the final state resulting from the interaction, which will be denoted as $S$, of the organism and the nutrient is a random matrix, which, however, even though unknown to us explicitly, is completely determined by the laws of quantum mechanics. Obviously $S$ must satisfy:

$$
\Psi=S \Phi .
$$

Choosing a basis for the whole Hilbert space and projecting Eq. (16) on such a basis one gets $N^{2} R$ equations. And now the conclusion follows: our unknown quantities are the components of the states $v, r$ and $w$ on their respective bases and are therefore $N+R+N R$ in number. Thus, according to Wigner, the question is: given the matrix corresponding to $S$, it is possible to find vectors $v, r$ and $w$ such that their components satisfy the above mentioned $N^{2} R$ equations? Since $N^{2} R \gg N+R+N R$, for extremely large $N$ and $R$, according to him: it would be a miracle if such equations could be satisfied. In other words, a self-replicating quantum unit does not exist. One might state that Wigner has "derived" (with the proviso he is making - see below) the no-cloning theorem for a quantum system whose Hilbert space has an extremely high dimensionality $\mathrm{N}$, while we have shown that it holds also for $\mathrm{N}=2$.

Wigner was perfectly aware that the argument is not fully rigorous and cannot be taken too seriously because of the many assumptions on which it is based. However, he seems inclined to attach a certain value to it. This is not surprising because at the time in which he wrote his paper he was adhering to von Neumann's idea that consciousness is responsible for the reduction of the wave packet, so that, in a certain sense, the fact that quantum mechanics is not able to account for the basic property of living organism (the self-reproduction) supported his view that such a theory cannot be used to describe the conscious perceptions of such organisms. In 1971 Eigen [36] responded to Wigner claiming that his choice of resorting to a typical unitary map to account for the process did not take into account the instructive functions of informational macromolecules.

Strictly connected with Wigner argument, even though derived through a much more rigorous and general procedure is the proof of the no-cloning theorem presented in a beautiful paper by R. Alicki [35]. He considers a dynamical transformation $\mathrm{T}$ from an initial state $\varphi \otimes \omega$, where $\varphi$ is the state of the organism and $\omega$ the fixed initial state of the environment designed as "food":

$$
\varphi \otimes \omega \rightarrow T(\varphi \otimes \omega)=\varphi \otimes \varphi \otimes \sigma .
$$

As before, $\sigma$ represents the state of the "food" after the replication. Alicki assumes that any dynamical process of a closed system (typically the one given by $T$ ) cannot reduce the 
indistinguishability of two states $\varphi$ and $\psi$, which can be quantified by the "overlap" $(\varphi \mid \psi)$ of the two states ${ }^{10}$, i.e., $(T(\varphi) \mid T(\psi)) \geq(\varphi \mid \psi)$ (which in Alicki's spirit can be considered as a form of the second law of thermodynamics: indistinguishability cannot decrease with the evolution). Then one has:

$$
\begin{aligned}
\left(\varphi \mid \varphi^{\prime}\right) & =\left(\varphi \otimes \omega \mid \varphi^{\prime} \otimes \omega\right) \leq\left(T(\varphi \otimes \omega) \mid T\left(\varphi^{\prime} \otimes \omega\right)\right) \\
& =\left(\varphi \otimes \varphi \otimes \sigma \mid \varphi^{\prime} \otimes \varphi^{\prime} \otimes \sigma^{\prime}\right)=\left(\varphi \mid \varphi^{\prime}\right)^{2}\left(\sigma \mid \sigma^{\prime}\right) \leq\left(\varphi \mid \varphi^{\prime}\right)^{2},
\end{aligned}
$$

implying $\left(\varphi \mid \varphi^{\prime}\right)=1$ or $\left(\varphi \mid \varphi^{\prime}\right)=0$.

It is interesting to note that if, taking a strictly quantum perspective (which means to replace the round brackets in the above equation by Dirac's bras and kets), one identifies (as it is quite reasonable) the general concept of overlap $\equiv$ indistinguishability with the scalar product of the Hilbert space and one assumes that the unfolding of the process is governed by a unitary transformation (which as such does not change the overlap), the above proof (slightly modified to take into account the complex nature of the scalar product) corresponds to a modern version of the no-cloning theorem which, in place of using the linearity of the evolution as we and the authors of refs.[32,33] did in deriving the theorem, makes resort to unitarity.

\section{Further recent proposals which require new impossibility proofs}

\subsection{A proposal by D. Greenberger}

In spite of the lively debate and the many precise results which should have made fully clear why quantum mechanics does not allow superluminal communication, new papers claiming to have found a new way to achieve this result continue to appear. The first we want to mention is a proposal [37] of D. Greenberger which has been considered as inspiring even quite recently. Actually, in ref.[38] it is claimed that the proposal of Greenberger has not yet been refused and calls into question the universality of the no-signaling theorem, and, accordingly, it represents a stimulus to pursue the investigations on this line.

Greenberger proposal involves the simoultaneous emission of two photons by a source along two different opposite directions $\left(a, a^{\prime}\right)$ and $\left(b, b^{\prime}\right)$, so that the initial state is the entangled state:

$$
|\psi\rangle_{1,2}=\frac{1}{\sqrt{2}}\left[|a\rangle_{1} \otimes\left|a^{\prime}\right\rangle_{2}+|b\rangle_{1} \otimes\left|b^{\prime}\right\rangle_{2}\right]
$$

Subsequently, the two photons impinge on a series of beam splitters, as shown in figure 2. The horizontal gray boxes represent the beam splitters which are assumed to both reflect and transmit half the incident light, and produce a phase shift of $\pi / 2$ upon reflection and none

\footnotetext{
${ }^{10}$ Here , the expressions $(\alpha \mid \beta)$ must not be identified with the Hilbert scalar product, since Alicki is taking a much more general perspective, which, however, requires to quantify the idea of distinguishability and its fundamental properties. He does this by introducing his symbol for the overlap which he takes, for simplicity, to be a real number between 0 and 1 .
} 
upon transmission. On the path of the photon emitted along $b$, after it goes through the first beam splitter, there is a phase-shifter $A$ that shifts the phase of any photon passing through it by $\pi$, and that can be inserted or removed from the beam at will.

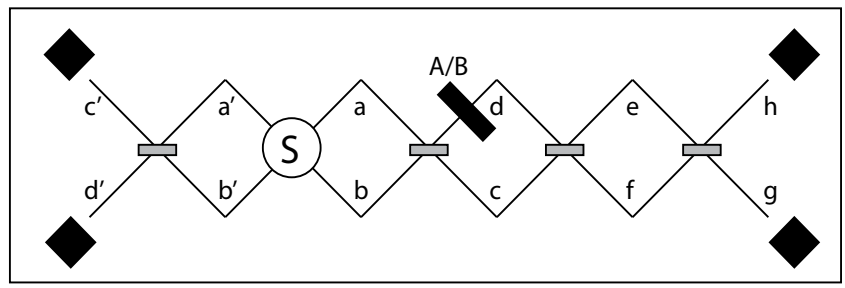

Figure 2. Illustration of Greenberger's proposal as depicted in his paper.

At this point the first crucial assumption of the paper enters into play:

i). The phase shifter can be prepared not only in the states $|A\rangle$ and $|B\rangle$, corresponding to its being inserted or removed from the path of the photon, but also in their orthogonal linear combinations:

$$
|u\rangle_{3}=\frac{1}{\sqrt{2}}[|A\rangle+|B\rangle], \quad|v\rangle_{3}=\frac{1}{\sqrt{2}}[|A\rangle-|B\rangle] .
$$

According to the author of [37], one can also switch on the Hamiltonian $H$ for this macroscopic object, whose eigenstates are $|u\rangle_{3}$ and $|v\rangle_{3}$, corresponding to slightly different energies, implying the development in time of relative phases with respect to each other.

We will not go through the subsequent elementary calculations of the paper; we limit ourselves to mention that the above assumptions lead to the conclusion that, as the photons are nearing their final detectors represented in the figure by the 4 black squares, they will be in the following entangled photon-phase shifter state:

$$
|\psi\rangle_{1,2,3}=\frac{1}{2}\left[\left(-e^{i \alpha}|h\rangle_{1}\left|d^{\prime}\right\rangle_{2}+e^{-i \alpha}|g\rangle_{1}\left|c^{\prime}\right\rangle_{2}\right) e^{i \beta}|u\rangle_{3}+\left(e^{i \alpha}|g\rangle_{1}\left|c^{\prime}\right\rangle_{2}-e^{-i \alpha}|h\rangle_{1}\left|d^{\prime}\right\rangle_{2}\right) e^{-i \beta}|v\rangle_{3}\right]
$$

the phase factors $e^{ \pm i \alpha}$ and $e^{ \pm i \beta}$ being due to the evolution of the states $|u\rangle_{3}$ and $|v\rangle_{3}$.

At this point Greeneberger puts forward his really crucial assumption. In his words:

ii). In accordance with our assumption that one can manipulate these Cat states, one can turn off $H$ for the state $|v\rangle$, while leaving it in place for the state $|u\rangle$. This will rotate the state $|v\rangle$ into the state $e^{i \gamma}|u\rangle$, where $\gamma$ is the accumulated phase difference during this process.

As it is obvious this amounts to accept that a nonunitary transformation $T$ can be performed:

$$
T|u\rangle_{3}=|u\rangle_{3}, \quad T|v\rangle_{3}=e^{i \gamma}|u\rangle_{3}
$$


The conclusion follows. After this transformation the state becomes:

$$
\left|\psi_{\text {final }}\right\rangle_{1,2,3}=e^{i \gamma / 2}\left[-\cos (\alpha+\beta-\gamma / 2)|h\rangle_{1}\left|d^{\prime}\right\rangle_{2}+\cos (\beta-\alpha-\gamma / 2)|g\rangle_{1}\left|c^{\prime}\right\rangle_{2}\right]|u\rangle_{3} .
$$

And now the game is over: by appropriately choosing the angles $\alpha, \beta$ and $\gamma$, one can, at his free will, suppress one of the two terms of the superposition of the photon states, i.e. one can make certain either the firing of the detector in $d^{\prime}$ or the one in $c^{\prime}$ (and correspondingly the one in $h$ or the one in $g$ ) allowing in this way a superluminal transfer of information from the phase shifter, which acts as the signaler, to the photon detectors.

The paper, since nobody had discussed it in spite of its revolutionary character, deserved some attention; we have reconsidered it in ref.[39]. Its weak points are:

- The assumption that one can prepare the linear superposition of two macroscopically different states, corresponding to different locations of the macroscopic phase-shifter. This is impossible to get in practice.

- However, even ignoring the above critical feature of the hypothetical experiment, the really crucial and unacceptable fact is the one embodied in its second assumption, i.e., the possibility of implementing a nonunitary transformation.

We will not go on analyzing all the details of ref.[37] and of the punctual criticisms of ref.[39]. We believe that to show where it fails the simplest way is to resort to an example that we have devised in our paper. We consider an elementary EPR-Bohm like setup for two far away spin $1 / 2$ particles in the singlet state:

$$
\left|\psi_{-}\right\rangle=\frac{1}{\sqrt{2}}\left[\left|\uparrow_{1}\right\rangle\left|\downarrow_{2}\right\rangle-\left|\downarrow_{1}\right\rangle\left|\uparrow_{2}\right\rangle\right]
$$

In strict analogy with what has been assumed by Greenberger, suppose now we can rotate only one of the two spin states of particle 2 making it to coincide, apart from a controllable phase, with the other one:

$$
T\left|\downarrow_{2}\right\rangle=\left|\downarrow_{2}\right\rangle, \quad T\left|\uparrow_{2}\right\rangle=e^{i \gamma}\left|\downarrow_{2}\right\rangle .
$$

Under this transformation the state [25] becomes a factorized state of the two particles:

$$
\left|\psi_{T}\right\rangle=\frac{1}{\sqrt{2}}\left[\left|\uparrow_{1}\right\rangle-e^{i \gamma}\left|\downarrow_{1}\right\rangle\right]\left|\downarrow_{2}\right\rangle
$$

In (26), the state referring to particle 1 is an eigenstate of $\sigma \cdot \mathbf{d}$ for the direction $\mathbf{d}=$ $(\cos \gamma, \sin \gamma, 0)$ pertaining to the eigenvalue -1 . This means that a measurement of this observable by Alice (where particle 1 is) will give with certainty the outcome -1 if Bob has performed the transformation $T$ on his particle, while, if Bob does nothing, the probability of getting such an outcome equals $1 / 2$. Having such a device, one can easily implement superluminal transfer of information. Concluding: if assumption ii) were correct, one would not need all the complex apparatus involved in Greenberger's proposal which, at any rate, cannot work as indicated due to the nonlinear nature of $T$. 


\subsection{A proposal involving a single system}

Another proposal that has to be mentioned is the one [40] by Shiekh. His suggestion is different from all those which have appeared in the literature since the author does not make resort to an entangled state of two systems but he works with a single particle in a superposition of two states corresponding to its being in two far-away regions, and the measurement process involves only one of the two far-away parts of the wave function. So, in a sense, the argument of ref.[40] does not fall under the no-go theorems considered here and requires a separate comment. The author is inspired by the fact that when a single particle is associated to a wavefunction as the one just mentioned, any attempt to test whether it is "here" (at right), or "there" (at left) changes instantaneously the wavefunction on the whole real axis by making it equal to zero or enhancing it "there" according to whether we detect or we do not find the particle "here". The process seems to exhibit some nonlocal aspects due to the instantaneous change at-a-distance. Obviously, that this might lead to superluminal signaling is something that nobody can believe, but it is instructive to show that also in this case, to achieve the desired result, one has to resort to a nonunitary evolution. The elementary analysis which follows will lead once more to the conclusion that the process cannot be used to send superluminal signals.

We briefly review the argument by Shiekh. He considers a particle which is prepared, at time $t=0$, in an equal weights superposition of two normalized states, $|h+\rangle$ and $|h-\rangle$, propagating in two opposite directions, respectively, starting from the common origin of the $x$-axis:

$$
|\psi, 0\rangle=\frac{1}{\sqrt{2}}[|h+\rangle+|h-\rangle] .
$$

Subsequently the state $|h+\rangle$ is injected in an appropriate device behaving in a way rather similar, apart from the final stage, to a Mach-Zender interferometer. One also assumes that an observer, located near to it, can choose, at his free will, to insert or not a phase-shifter along one of the two paths of the interferometer. The two wave functions are then recombined by appropriate deflectors so that, by deciding whether or not to insert the phase-shifter, one can produce a constructive (no phase-shifter in place) or a destructive (the phase-shifter is present) interference of the two terms in which the impinging state $|h+\rangle$ has been split. Finally, a detector is placed along the direction of propagation of the final state and it induces wave packet reduction, since it either detects or fails to detect the particle. We have summarized the situation for the two considered cases in Figs. 3a,b.

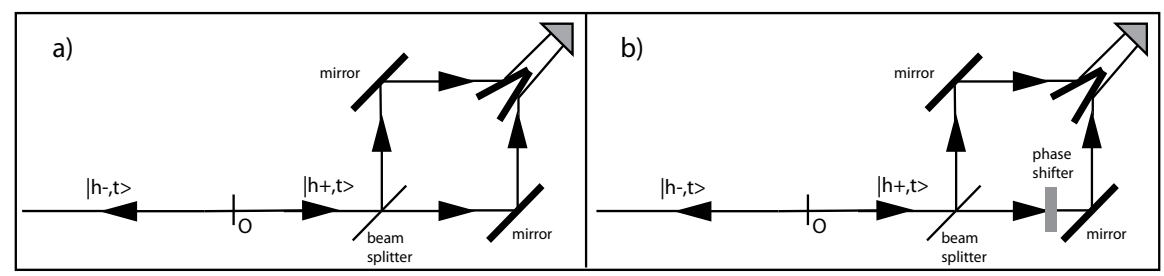

Figure 3. The experimental arrangement of ref.[40]. The two cases refer to no Phase-shifter inserted or phase shifter inserted, respectively.

The author then concludes: If the sender (the guy who can choose to insert or not the phase shifter), arranges for constructive interference, then some of the particles will be "taken up" by the 
sender, but none if destructive interference is arranged; in this way the sender can control the intensity of the beam detected by the receiver (the observer located far away where the evolved of $|h-\rangle$ is concentrated). So, a faster than light transmitter of information (but not energy or matter) might be possible.

We believe that all readers will have clear the trivial mistake of the paper. In fact, what one can govern by deciding whether to insert or not the phase-shifter, is the interference at the central region of the final detector. Let us concentrate our considerations only on the normalized state $|h+\rangle$. If triggered by such a state when it exhibits constructive interference, the counter will register (practically) for sure the particle described by such a state (the wavefunction has a peak just there), while, if there is destructive interference, the counter will not register the particle. But this does not mean that the wavefunction associated to $|h+\rangle$ disappears, as claimed by the author, it simply means that its support lies outside the interval covered by the counter. Actually, no one will doubt that if one places an array of counters covering all the line orthogonal to the final direction of propagation, one of them, different from the one of the experiment, will fire for sure. If one combines these considerations with the fact that actually the whole state of the particle is the superposition of $|h-\rangle$ and $|h+\rangle$ one realizes that the statements we have just made concerning what is going on at right have only probability $1 / 2$ of occurrence, since the particle can be not detected in the right region. Accordingly, the probability that the particle is found at left remains equal to $1 / 2$, as if no specific action would be made at right.

It seems rather peculiar that the author introduces an hypothetical process which can make zero a wavefunction (i.e. the normalized state $|h+\rangle)$, and as such it does not preserve unitarity, and, at the same time, he resorts to the overall conservation of probability (i.e. to unitarity) to claim that the action at right can change the norm of the state at left.

This concludes our analysis of the many proposals which have been presented to send superluminal signals.

\section{Nonlocality and relativistic requirements}

As already stated, quantum mechanics suffers of an internal inconsistency, the one between the linear and deterministic evolution induced by Schrödinger's dynamics and the nonlinear and stochastic collapse of the state in a measurement. Many scientists, among them Einstein, Schrödinger, Bell and many others have been disturbed not only by the formal inconsistency between the two dynamical principles, but especially by the fact that the borderline between what is classical and what is quantum, what is reversible and what is irreversible, what is micro and what is macro is to a large extent ambiguous. Accordingly, many serious attempts to overcome this difficulty have been presented, inspired by the conviction that Bell has expressed [6] so lucidly:

Either the wavefunction, as given by the Schrödinger equation is not everything or it is not right.

\subsection{Bohmian mechanics}

The first alternative corresponds to the idea that the specification of the state of a physical system given by the statevector has to be enriched or replaced by new variables (the 
so-called hidden variables). The best known and rigorous example of this line is represented by Bohmian mechanics [42], a deterministic theory such that the assignement of the wavefunction and of the hidden variables (i.e. the initial positions of all particles which are chosen to be distributed according to the quantum probability $\left.\left|\psi\left(\mathbf{r}_{1}, \mathbf{r}_{2}, \ldots, \mathbf{r}_{N}, t_{0}\right)\right|^{2}\right)$ determines uniquely their positions at any subsequent time. The predictions of the theory concerning the probability distribution of the particles coincide with those of standard quantum mechanics and the theory overcomes the measurement problem in a clean and logically consistent way.

I will spend only few words on the locality issue within Bohmian mechanics. Since this theory agrees with quantum mechanics in general and typically in an EPR-like situation, it must exhibit a specific sort of nonlocality. It has been proved [43] that any deterministic hidden variable theory equivalent to quantum mechanics admits only relativistic generalizations which must resort to a specific foliation of space-time. In other words, such theories are characterized by a preferred reference frame which, however, remains unaccessible. Accordingly, as stressed by Bell [8], they require a change of attitude concerning Lorentz invariance: the situation resembles the one of the theory of relativity in the Fitzgerald, Larmor, Lorentz and Poincare formulation in which there is an absolute aether, but the contraction of space and the dilation of time fooled the moving observers by allowing them to consider themselves at rest. In spite of this remark, explicit and interesting relativistic generalizations of Bohmian mechanics have been presented. In particular bohmian-like relativistic models have been worked out both in first quantized versions [44] as well as in the framework of quantum field theories [45].

\subsection{Collapse theories}

The second alternative corresponds to assuming that Schrödinger's equation has to be changed in such a way not to alter the well established predictions of quantum mechanics for all microscopic systems while leading to the collapse of the statevector with the desired features and probabilities when macroscopic systems enter into play. The first explicit example of this kind is the so called GRW theory [5] which we summarize in a very sketchy way.

The central idea is to modify the linear and deterministic evolution equation of standard quantum mechanics by adding nonlinear and stochastic (i.e. sharing the features of the reduction process) terms to it. As it is obvious, and as it has been stressed by many scientists, since the situations characterizing macro-objects correspond to perceptually different locations of their macroscopic parts (e.g. the pointer) the change in the dynamics must strive to make definite the positions of macroscopic bodies. The model is based on the following assumptions:

- A Hilbert space $\mathcal{H}$ is associated to any physical system and the state of the system is represented by a normalized vector $\left|\psi_{t}\right\rangle$ of $\mathcal{H}$,

- The evolution of the system is governed by Schrödinger's equation. Moreover, at random times, with mean frequency ${ }^{11} \lambda$, each particle of any system is subjected to random

11 Actually this frequency must be made proportional to the mass of the particles entering into play. The value we will choose below refers to nucleons. 
spontaneous localization processes as follows. If particle $i$ suffers a localization then the statevector changes according to:

$$
\left|\psi_{t}\right\rangle \rightarrow \frac{L_{i}(\mathbf{x})\left|\psi_{t}\right\rangle}{\| L_{i}(\mathbf{x})\left|\psi_{t}\right\rangle \|} ; \quad L_{i}(\mathbf{x})=\left(\frac{\alpha}{\pi}\right)^{3 / 4} e^{\left[-\frac{\alpha}{2}\left(\hat{\mathbf{x}}_{i}-\mathbf{x}\right)^{2}\right]},
$$

where $\hat{\mathbf{x}}_{i}$ is the position operator of particle $i$,

- The probability density for a collapse at $\mathbf{x}$ is $p(\mathbf{x})=\| L_{i}(\mathbf{x})\left|\psi_{t}\right\rangle \|^{2}$, so that localizations occur more frequently where the particle has a larger probability of being found in a standard position measurement.

The most relevant fact of the process is its "trigger mechanism", i.e. the fact that, as one can show by passing to the centre-of-mass and relative coordinates, the localization frequency of the c.o.m. of a composite system is amplified with the number of particles, while the internal motion, with the choice for $\alpha$ we will make, remains practically unaffected. We have summarized the situation for a micro (at left) and macroscopic (at right - a pointer) system in Fig.4.
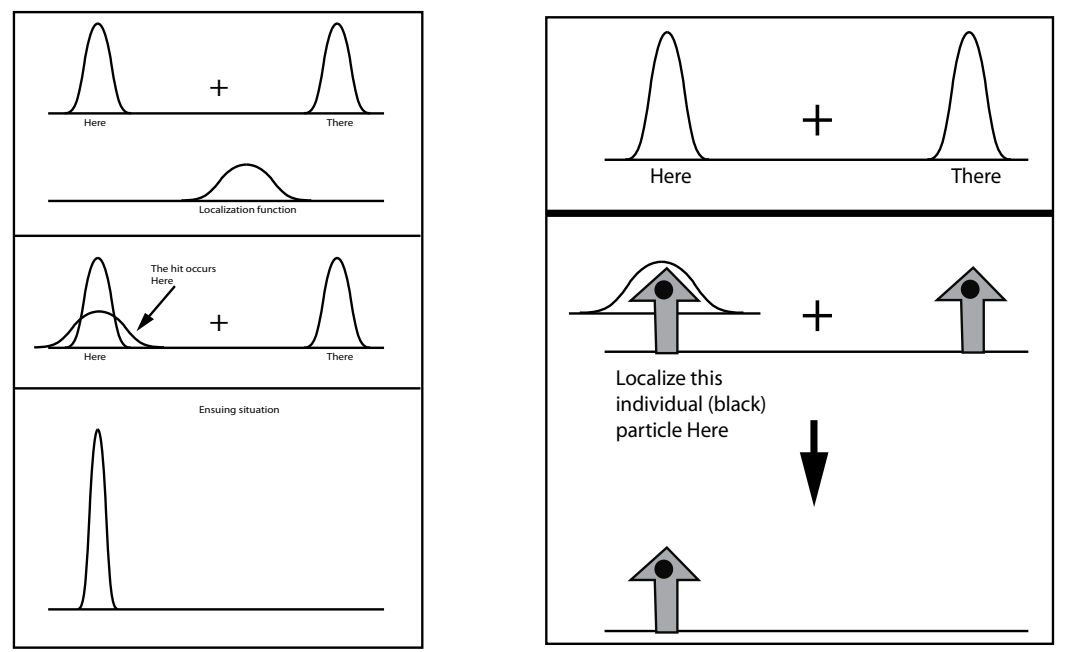

Figure 4. The localization of a single particle and of a macro-object according to GRW.

With these premises we can now make the choice for the parameters $\alpha$ (note that $\frac{1}{\sqrt{\alpha}}$ gives the localization accuracy) and $\lambda$ of the theory. In ref.[5] we have chosen:

$$
\alpha \simeq 10^{10} \mathrm{~cm}^{-2}, \lambda \simeq 10^{-16} \mathrm{sec}^{-1} .
$$

Note that with these choices a microscopic system suffers a localization about every $10^{7}$ years, and this is why the theory agrees with quantum mechanics for such systems, a macroscopic body every $10^{-8} \mathrm{sec}$ (due to the trigger mechanism in the case of an Avogadro number of particles the frequency becomes $10^{24} \cdot 10^{-16}=10^{8} \mathrm{sec}^{-1}$ ). As commented [6] by J. Bell: The cat is not both dead and alive for more than a split second. 
The conclusion should be obvious. A universal dynamics has been worked out which leaves (practically) unaltered all quantum predictions for microsystems but it accounts for wave packet reduction with probabilities in agreement with the quantum ones and for the classical behaviour of macroscopic systems, as well as for our definite perceptions concerning them.

Few remarks: i). The model has been generalized and formulated in a mathematically much more satisfactory but physically equivalent way $[46,47]$ by resorting to stochastic dynamical equations of the Ito or Stratonowich type, ii) The model is manifestly phenomenological but it gives some clear indications concerning the fact that the macro-objectification or measurement problem admits a consistent solution, iii). The model, even though it almost completely agrees with quantum mechanics at the microscopic level qualifies itself as a rival theory of quantum mechanics, one which can be tested against it. Accordingly, it suggests where to look for putting into evidence possible violations of linearity. In recent years a lot of work in this direction is going on.

Obviously, also Collapse theories exhibit nonlocal features. However for them there is no theorem forbidding to get a generalization which does not resort to a preferred reference frame. Lot of work has been done along these lines; I will limit myself to mention some relevant steps. Before doing this, I consider it interesting to stress that the problem of having a theory inducing instantaneous reduction at-a-distance is a quite old one which has seen a lively debate and important contributions by Landau and Peierls [48], Bohr and Rosenfeld [49], Hellwig and Kraus [50] and Aharonov and Albert [51,52].

Soon after the GRW theory has been formulated, P. Pearle [53] has presented a field theoretic relativistic generalization of it which has subsequently [54] been shown to be fully Lorentz invariant. Unfortunately, the model had some limitations arising from the occurrence of divergences which were not easily amendable. In 2000 the author of the present chapter has presented [55] a genuinely relativistic toy model of a theory inducing reductions. The model satisfies all the strict conditions identified in refs.[51,52]. F. Dowker and collaborators [56] have presented, in 2004, a relativistic collapse model on a discrete space that does not require a preferred slicing of space-time.

The really important steps, however, occurred starting from 2007. R. Tumulka has presented [57] a fully satisfactory and genuinely relativistic invariant dynamical reduction model for a system of noninteracting fermions. Another important contribution [58] came from D. Bedingham. Finally, few month ago, a convincing proof of the viability of the Collapse theories in the relativistic domain has been presented [59]. The nice fact is that the conceptual attitude which underlies this attempt is that what the theory assumes to be true of the world around us is the mass density of the whole universe. In this way one recovers a unified, general picture of a quantum universe both at the micro and macro levels.

I believe that the best way to conclude this Chapter, which has dealt in detail with the compatibility of quantum effects and relativistic requirements, is to quote a clarifying sentence from R. Tumulka [57], who has studied in great detail both the Bohmian as well as the Collapse approaches to this fundamental problem:

A somewhat surprising feature of the present situation is that we seem to arrive at the following alternative: Bohmian mechanics shows that one can explain quantum mechanics, exactly and completely, if one is willing to pay with using a preferred reference slicing of space-time; our model suggests that one should be able to avoid a preferred slicing if one is willing to pay with a certain deviation from quantum mechanics. 


\section{Acknowledgements}

We thank Dr. R. Romano for an accurate critical reading of the manuscript.

\section{Author details}

GianCarlo Ghirardi

Professor Emeritus, University of Triest, Italy

\section{References}

[1] Schrödinger E. Discussion of Probability Relations between Separated Systems. Proceedings of the Cambridge Philosophical Society 1935; 31: 555-563.

[2] Bell J.S. On the Einstein-Podolski-Rosen Paradox. Physics 1964; 1: 195-200 .

[3] von Neumann J. Matematische Grundlagen der Quantenmechanik. Berlin: Springer Verlag; 1932. (Engl. trans., Princeton University Press, 1955).

[4] Herbert N. FLASH- A Superluminal Communicator Based Upon a New Kind of Quantum Measurement. Foundations of Physics 1982; 12: 1171-79.

[5] Ghirardi GC., Rimini A. and Weber T. Unified Dynamics for Microscopic and Macroscopic Systems. Physical Review D 1986; 34: 470-491.

[6] Bell J.S. Are there Quantum Jumps? in: Schrödinger: Centenary Celebration of a Polymath. Cambridge: Cambridge University Press; 1987.

[7] Bell J.S. Against Measurement in: Sixty-two Years of Uncertainty. New York: Plenum; 1990.

[8] Bell JS. First and Second Class Difficulties in Quantum Mechanics. Journal of Physics A 2007; 40: 2921-2933.

[9] Bassi A. and Ghirardi GC. A General Argument against the Universal Validity of the Superposition Principle. Physics Letters A 2000; 275: 373-381.

[10] Bassi A. and Ghirardi GC. Dynamical Reduction Models. Physics Reports 2003; 379: 257-426.

[11] Wigner EP. Die Messung Quantenmechanischer Operatoren. Zeitschrift für Physik 1952; 131: 101-108.

[12] Araki H. and Yanase MM. Measurement of Quantum Mechanical Operators. Physical Review 1961; 120: 622-626.

[13] Yanase MM. Optimal Measuring Apparatus. Physical Review 1961; 123: 666-668.

[14] Ghirardi GC., Miglietta F., Rimini A. and Weber T. Limitations on quantum measurements. I. Determination of the Minimal Amount of Nonideality and 
Identification of the Optimal Measuring Apparatuses. Physical Review D 1981; 24: 347-352.

[15] Ghirardi GC., Rimini A. and Weber T. Quantum Evolution in the Presence of Additive Conservation Laws and the Quantum Theory of Measurement. Journal of Mathematical Physics 1982; 23: 1792-1796.

[16] Kraus K. States, Effects and Operations, Berlin: Springer; 1983.

[17] Kaiser D. How the Hippies Saved Physics, New York: W.W. Norton \& Co.; 2011.

[18] Cufaro Petroni N., Garuccio A., Selleri F. and Vigier JP. On a Contradiction between the Classical (idealized) Quantum Theory of Measurement and the Conservation of the Square of the Total Angular Momentum. C.R. Acad. Sci. Ser. B (Sciences Physiques)1980; 290: 111-114.

[19] Selleri F. in: International Seminar on Mathematical Theory of Dynamical Systems and Microphysics. Udine; 1979.

[20] Herbert N. QUICK-a New Superluminal Transmission Concept. Boulder Creek, Cal.: C-Life Institute; 1979.

[21] Beth R. Mechanical Detection and Measurement of the Angular Momentum of Light. Physical Review 1936; 50: 115-25.

[22] Ghirardi GC. and Weber T. On Some Recent Suggestions of Superluminal Communication through the Collapse of the Wave Function. Lettere al Nuovo Cimento 1979; 26: 599-603.

[23] Popper K. Quantum Theory and the Schism in Physics, London: Hutchinson; 1982.

[24] Ghirardi GC., Marinatto L. and de Stefano F. Critical Analysis of Popper's Experiment. Physical Review A 2007; 75: 042107-1-5.

[25] Ghirardi GC. Sneaking a Look at God's Cards, Princeton: Princeton University Press; 2005.

[26] Ghirardi GC., Rimini A. and Weber T. A General Argument against Superluminal Transmission through the Quantum Mechanical Measurement Process. Lett. Nuovo Cimento 1980; 27: 293-298.

[27] Ghirardi GC., Grassi R., Rimini A. and Weber T. Experiments of the EPR Type Involving CP-Violation do not Allow Faster-than-light Communication between Distant Observers. Europhysics Letters 1988; 6: 95-100.

[28] Eberhard PH. Bell's Theorem and the Different Concepts of Locality. Nuovo Cimento B 1978; 46: 392-419.

[29] Shimony A. Controllable and Uncontrollable Nonlocality. in: Foundations of Quantum Mechanics in the Light of New Technology. Tokyo: The physical Society of Japan; 1984. 
[30] Peres A. How the No-Cloning Theorem got its Name. Fortschritte der Physik 2003; 51: 458-61.

[31] Ghirardi GC. and Weber T. Quantum Mechanics and Faster-than-Light Communication: Methodological Considerations. Il Nuovo Cimento B 1983; 78: 9-20.

[32] Wotters WK. and Zurek WH. A Single Quantum Cannot be Cloned. Nature 1982; 299: 802-803.

[33] Dieks D. Communication by EPR Devices. Physics Letters A 1982; 92: 271-272.

[34] Wigner EP. The Probability of the Existence of a Self-Reproducing Unit, in: The Logic of Personal Knowledge. London: Routledge \& Kegan Paul, 1961.

[35] Alicki R. Physical Limits on Self-Replication Processes. Open Systems and Information Dynamics 2006; 13: 113-117.

[36] Eigen M. Selforganization of Matter and Evolution of Biological Macromolecules. Naturwissenschaften 1971; 58: 465-523.

[37] Greenberger DM. If one Could Build a Macroscopic Schrödinger Cat State one could Communicate Superluminally, in: Modern Studies of Basic Quantum Concepts and Phenomena. Singapore: World Scientific Publishing Co. 1998.

[38] Kalamidas DA. A Proposal for a feasible quantum-optical experiment to test the validity of the no-signaling theorem, ArXiv:1110, 4269.

[39] Ghirardi GC. and Romano R. On a Proposal of Superluminal Communication. Journal of Physics A 2012; 45: 232001.

[40] Shiekh AY. The Role of Quantum Interference in Quantum Computing. International Journal of Theoretical Physics 2006; 45: 1653-1655.

[41] Bassi A. and Ghirardi. GC. On a Recent Proposal of Faster-than-Light Quantum Communication. International Journal of Theoretical Physics 2008 47: 2500-2506.

[42] Bohm D. A Suggested Interpretation of the Quantum Theory in Terms of Hidden Variables. Physical Review 1952; 85: 166-193.

[43] Ghirardi GC. and Grassi R. Bohm's Theory versus Dynamical Reduction, in: Bohmian Mechanics and Quantum Theory, an Appraisal. The Netherlands: Kluwer Academic Publishers. 1966.

[44] Dürr, D., Goldstein, S., Münch-Berndl, K., Zanghi, N. Hypersurface Bohm-Dirac Models. Physical Review A 1999; 60: 2729-2736.

[45] Bohm, D., Hiley, B. J. The Undivided Universe. London: Routledge (1993).

[46] Pearle P. Combining Stochastic Dynamical State-Vector Reduction with Spontaneous Localization. Physical Review A 1999; 39: 2277-2289 
[47] Ghirardi GC., Pearle P. and Rimini A. Markov Processes in Hilbert Space and Continuous Spontaneous Localization of Systems of Identical Particles, Physical Review A 1990; 42: 78-89.

[48] Landau and Peierls R. Erweiterung des Unbestimmtheitsprinzips für die relativistische Quantentheorie. Zeischrift für Physik, 1931; 69: 56-69.

[49] Bohr N. and Rosenfeld L. Zur Frage der Messbarkeit der Electromagnetischen Feldgrössen, Kopenaghen. 1933.

[50] Hellwig KE. and Kraus K. Formal description of measurements in local quantum field theory. Physical Review D 1970; 1: 566-571.

[51] Aharonov Y. and Albert DZ. States and observables in relativistic quantum field theories. Physical Review D 1980; 21: 3316-3324,

[52] Aharonov Y. and Albert DZ. Can we Make Sense out of the Measurement Process in Relativistic Quantum Mechanics? Physical Review D 1981; 24:359-370

[53] Pearle P. Toward a Relativistic Theory of Statevector Reduction, in: Sixty-Two Years of Uncertainty. Plenum Press, New York; 1990.

[54] Ghirardi GC., Grassi R. and Pearle P. Relativistic Dynamical Reduction Models: General Framework and Examples. Foundations of Physics 1990; 20: 1271-1316.

[55] Ghirardi GC. Local Measurement of Nonlocal Observables and the Relativistic Reduction Process. Foundations of Physics 2000; 38: 1337-1385.

[56] Dowker, F., Henson, J. Spontaneous Collapse Models on a Lattice. Journal of Statistical Physics 2004; 115: 1327-1339.

[57] Tumulka, R. A Relativistic Version of the Ghirardi-Rimini- Weber Model. Journal of Statistical Physics 2006; 125: 821-840.

[58] Bedingham, DJ. Relativistic state reduction dynamics. ArXiv 1003-2774v2, 2010.

[59] Bedingham DJ., Dürr D., Ghirardi GC., Goldstein S., Tumulka R. and Zanghi. N. Matter Density and Relativistic Models of Wave Function Collapse. ArXiv 1111-1425, 2012. 


\section{FOUNDATIONS OF PHYSICS}

An International Joumal Devoted to the Conceptual Beses and Fundamental Theories of Modern Physics, Biophysics, and Cosmology

Published by Plenum Publishing Corporation, 233 Spring Stroet, New York, N.Y. 10013-1578

March 7, 2002

EOtor: ALWYW VAN DEA MEAWI Depertment of Ptysica Univeraly of Demwer. Denver, Colsanes 80ace Uitied Sistea of America

From: Alwyn van der Merwe

\section{TO WHOM IT MAY CONCERN:}

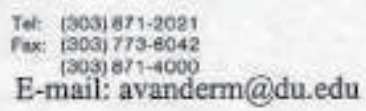

E-mail: avanderm(a)du.edu

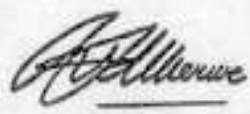

In view of the ever-increasing interest in the so-called "no-cloning theorem" for the crucial role it plays, among others, in the field of quantum computation and quantum information, I consider it my duty at this stage to point out that the above theorem was formulated and proven for the first time in a letter addressed to me by Prof. GianCarlo Ghirardi of the University of Trieste. His letter was dated April 22, 1981, in other words, more than 18 months prior to the public appearance of the paper that is usually credited as the one in which the theorem was first derived. Indeed, this paper, entitled "A single quantum cannot be cloned" and authored by W.K. Wootters and W.H. Zurek, was published in the October 28, 1982 issue of Nature, with the date of receipt given as August 11, 1982.

Accordingly, in order to set the historical record straight as well as to give due credit to GianCarlo Ghirardi for his prior authorship, I am making available for public dissemination a paragraph contained in his April 1981 letter, which responded to my request that he act as a referee for a paper submitted to Foundations of Physics in which the use of an hypothetical apparatus capable of cloning photons was proposed for the purpose of sending superluminal signals. His referee reports contains the following statement, which I literally quote :

"The main idea of the paper consists in the use of a laser gain tube which acts as a device duplicating the triggering states exactly. The problem consists in the fact that such a device must be able to reproduce exactly all 4 types of photons for what concerns their polarizations) which stimulate it. In particular, if a plane polarized ( $H$ or V) photon is incident on the tube, $N$ equally plane polarized photons are emitted by the tube. The same must happen for incident circularly polarized ( $R$ or $L)$ photons.

In my opinion, a gain tube of the type considered by the author cannot exist. In fact, if the gain tube is able, when stimulated by a plane polarized photon, to emit $N$ identically plane polarized photons, the linear nature of quantum mechanics implies that, when it is stimulated by a circularly polarized photon (since this is a linear superposition of plane polarized states $1 / \sqrt{ } 2[i|\mathrm{~V}> \pm| \mathrm{H}>]$ it will not emit $N$ photons with the same circular polarization, but the linear superposition of $N$ photons polarized along $V$ and $N$ photons polarized along $H$. In other words, it is impossible that for all 4 states of polarization (actually for any state which is a superposition of

$|V\rangle$ and $|\mathrm{H}\rangle$ photons) the laser gain tube acts simply as a duplicator producing $N$ photons of the same type.

The basic device of the suggested experiment violates the linear nature of quantum mechanics and therefore all the proposal is incorrect."

Scholars informed about the subject under discussion will have no difficulty in realizing that the above statement is exactly the no-cloning theorem. 
Chapter 25

\title{
The Husimi Distribution: Development and
}

\section{Applications}

\author{
Sergio Curilef and Flavia Pennini
}

Additional information is available at the end of the chapter

http://dx.doi.org/10.5772/53846

\section{Introduction}

The Husimi distribution, introduced by Kôdi Husimi in 1940 [1], is a quasi-probability distribution commonly used to study the correspondence between quantum and classical dynamics [2]. Also, it is employed to describe systems in different areas of physics such as Quantum Mechanics, Quantum Optics, Information Theory [3-8]. Additionally, in nanotechnology it is possible to obtain a clear description of localization -which corresponds to classicality- and is crucial to determine correctly the size of systems when the particle dynamics takes into account mobility boundaries [9]. Among its properties, it is always positive definite and unique, conversely it cannot be considered as a true probability distribution over the quantum-mechanical phase space, reason why it is often considered as a quasi probability distribution. Although it possesses no correct marginal properties, its usefulness is to allow the assessment of the expectation values in quantum mechanics in a way similar to the classical case [10]. The semiclassical Husimi probability distribution refers to a special type of probability, this is for simultaneous but approximate location of position and momentum in phase space.

The Husimi distribution may be obtained in several ways; the strategy that we choose here is to derive it as the expectation value of the density operator in a basis of coherent states [11]. Therefore, the line of working in this chapter is illustrated in the following sequence:

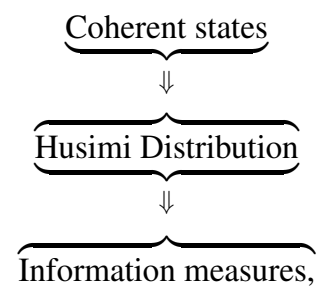


where the transcendence of defining correctly a set of coherent states and the Husimi distribution is evident, being the calculation of measures as Wehrl entropy and/or Fisher information a consequence of this procedure.

Coherent states provide a close connection between classical and quantum formulations of a given system. They were introduced early by Erwin Schrödinger in 1926 [12], but the name coherent state appeared for first time in Glauber's papers [13, 14]- see a detailed study about this in Ref. [15]. It is known that is difficult to construct coherent states for arbitrary quantum mechanical systems. Klauder shows an elegant method for construct it in Ref. [16]. Furthermore, in Ref. [11] Gazeau and Klauder consider essential, among other things, to discuss what an appropriate formulation of coherent states needs [11]. For instance, they suggest a suitable set of requirements. Then, the main interest in this chapter is to discuss, starting from a well defined set of coherent states, some interesting problems related to the Husimi distribution applied to important systems in physics, such as, the harmonics oscillator [5], the Landau diamagnetism model [17, 18] and, the rigid rotator [6, 18]. Also, we will discuss some properties related to systems with continuous spectrum [19]. In each case, the Wehrl entropy is calculated as a possible application.

This chapter is organized as follows. In section 2 we start presenting the background material and methodology that will be employed in the following chapters. In section 3 we revise the Husimi distribution and the Wehrl entropy for the problem of a particle in a magnetic field. In section 4 we discuss phase space delocalization for the rigid rotator within a semiclassical context by recourse to the Husimi distributions of both the linear and the $3 D$-anisotropic instances. In section 5 we propose a procedure to generalize the Husimi distribution to systems with continuous spectrum. We start examining a pioneering work, by Gazeau and Klauder, where the concept of coherent states for systems with discrete spectrum was extended to systems with continuous one. Finally, some concluding remarks and open problems are commented in section 6 .

\section{Background material and methodology}

In this section we center our attention in 3 topics that we consider relevant to understand the problems that will be discussed in the following sections. These are $i$ ) the Husimi distribution and the most direct application, i.e., Wehrl entropy, ii) a special basis to formulate a suitable set of coherent states and iii) a generalization of this concepts to systems with continuous spectrum.

\subsection{Husimi distribution and Wehrl entropy}

The standard statistical mechanics starts conventionally using the Gibbs's canonical distribution, whose thermal density matrix is represented by

$$
\hat{\rho}=Z^{-1} e^{-\beta \hat{H}},
$$

where $Z=\operatorname{Tr}\left(e^{-\beta \hat{H}}\right)$ is the partition function, $\hat{H}$ is the Hamiltonian of the system, $\beta=1 / k_{B} T$ the inverse temperature $T$, and $k_{B}$ the Boltzmann constant [20].

The Husimi distribution is obtained as the expectation value of the density operator in a basis of coherent states as follows [1]

$$
\mu(z)=\langle z|\hat{\rho}| z\rangle,
$$


where $\{|z\rangle\}$ denotes the set of coherent states, which are the eigenstates of the annihilation operator $\hat{a}$, i.e., $\hat{a}|z\rangle=z|z\rangle$ defined for all $z \in \mathbb{C}[11]$. This distribution is normalized to unity according to

$$
\frac{1}{\pi} \int \mathrm{d}^{2} z \mu(z)=1
$$

where the integration is carried out over the complex $z$ plane and the differential is a real element of area proportional to phase space element given by $\mathrm{d}^{2} z=\mathrm{d} x \mathrm{~d} p / 2 \hbar$.

For an arbitrary Hamiltonian $\hat{H}$, with the discrete spectra $\left\{E_{n}\right\}$, being $n$ a positive integer, the Husimi distribution takes the form

$$
\mu(z)=\frac{1}{Z} \sum_{n} e^{-\beta E_{n}}|\langle z \mid n\rangle|^{2},
$$

where $\{|n\rangle \mid\}$ is the set of energy eigenstates with eigenvalues $E_{n}[4,5]$.

The Wehrl entropy is a direct application that we introduce here, which is a useful measure of localization in phase-space [21, 22], whose pertinent definition reads

$$
W=-\frac{1}{\pi} \int \mathrm{d}^{2} z \mu(z) \ln \mu(z)
$$

The uncertainty principle manifests itself through the inequality $W \geq 1$ which was first conjectured by Wehrl [21] and later proved by Lieb (see, for instance Ref. [4]).

In the special case of the Harmonic Oscillator -whose Hamiltonian is $\hat{H}=\hbar \omega\left[\hat{a}^{\dagger} \hat{a}+1 / 2\right]-$ its set of Glauber's coherent states is defined in the form [14]

$$
|z\rangle=e^{-|z|^{2} / 2} \sum_{n=0}^{\infty} \frac{z^{n}}{\sqrt{n !}}|n\rangle,
$$

where $\{|n\rangle\}$ are a complete orthonormal set of phonon-eigenstates, that is,

$$
\left\langle n \mid n^{\prime}\right\rangle=\delta_{n, n^{\prime}}
$$

where $\delta_{n, n^{\prime}}$ is the Kronecker delta function, and the energy-spectrum is given by $E_{n}=\hbar \omega(n+1 / 2)$, with $n=0,1, \ldots$ By definition, Hermitian operator $\hat{H}$ is an observable if this orthonormal system of vectors forms a basis in the state space. This can be expressed by the closure relation

$$
\sum_{n=0}^{\infty}\left|n^{\prime}\right\rangle\langle n|=\hat{1}
$$

where $\hat{1}$ stands for the identity operator in the space formed by eigenvectors. 
In this situation one conveniently resorts to

$$
\begin{gathered}
\mu_{H O}(z)=\left(\hat{1}-e^{-\beta \hbar \omega}\right) e^{-\left(1-e^{-\beta \hbar \omega}\right)|z|^{2}}, \\
W_{H O}=1-\ln \left(1-e^{-\beta \hbar \omega}\right) .
\end{gathered}
$$

which respectively are the useful analytical expressions for Husimi distribution and Wehrl entropy [4].

\subsection{Gazeau and Klauder's coherent states}

Now, we go back to the set of coherent states defined in Eq. (6). Certainly, it is known that coherent states can be constructed in several ways by recourse to different techniques being its formulation of a not unique character. Nevertheless, contrary to this idea and in order to get a unifying perspective, Gazeau and Klauder have suggested that a suitable formalism for coherent states should satisfy at least the following requirements [11]:

1. Continuity of labeling refers to the map from the label space $\mathcal{L}$ into Hilbert space. This condition means that the expression $\|\left|z^{\prime}\right\rangle-|z\rangle \| \rightarrow 0$ whenever $z^{\prime} \rightarrow z$.

2. Resolution of Unity: a positive measure $\tau(z)$ on $\mathcal{L}$ exists such that the unity operator admits the representation

$$
\int_{\mathcal{L}}|z\rangle\langle z| d \tau(z)=1
$$

where $|z\rangle\langle z|$ denotes a projector, which takes a state vector into a multiple of the vector $|z\rangle$.

3. Temporal Stability: the evolution of any coherent state $|z\rangle$ always remains a coherent state, which leads to a relation of the form

$$
|z(t)\rangle=e^{-i \hat{H} t / \hbar}|z\rangle
$$

where $z(0)=z$, for all $z \in \mathcal{L}$ and $t$.

4. Action Identity: this property requires that

$$
\langle z|\hat{H}| z\rangle=\hbar \omega|z|^{2}
$$

At this point, we remark that requirements (3) and (4) are directly satisfied when the spectrum of the Hamiltonian $\hat{H}$ of the system, has the form $E_{n} \sim n \hbar \omega$, where $n$ is the quantum number and $\omega$ is the frequency of the oscillator [11]. In addition, there are some shortcomings about these requirements; for instance, Gazeau and Klauder states cannot be used for degenerate systems. Furthermore, it is questionable that action identity leads to the classical action-angle variable interpretation [23].

\subsection{Continuous spectrum}

Gazeau and Klauder proposed in Ref. [11] a formulation of coherent states for systems with continuous spectrum. They introduced a Hamiltonian $\hat{H}>0$, with a non-degenerate continuous spectrum, thus

$$
\hat{H}|\varepsilon\rangle=\omega \varepsilon|\varepsilon\rangle, \quad 0<\varepsilon<\varepsilon_{M}
$$


where $\{|\varepsilon\rangle\}$ stands for a basis of eigenstates, which we can generalize replacing suitably discrete parameters by continuous ones, sums by integrals and Kronecker by Dirac delta function [46]. In such a case, we can always chose a normalized basis of eigenvectors to rephrase Eqs. (7) and (8) in the following manner [46]

$$
\left\langle\varepsilon \mid \varepsilon^{\prime}\right\rangle=\delta\left(\varepsilon-\varepsilon^{\prime}\right)
$$

and

$$
\int_{0}^{\varepsilon_{M}}\left|\varepsilon^{\prime}\right\rangle\langle\varepsilon|=\hat{1}
$$

where $\varepsilon_{M} \leq \infty$ [11]. In the section 5 and here we use units in which $\hbar=1$.

If we set $M(s)=e^{|z|^{2} / 2}$ and $z=s e^{-i \gamma \varepsilon}$ into coherent states (6), we find

$$
|s, \gamma\rangle=M(s)^{-1} \int_{0}^{\varepsilon_{M}} \mathrm{~d} \varepsilon \frac{s^{\varepsilon} e^{-i \gamma \varepsilon}}{\sqrt{\rho(\varepsilon)}}|\varepsilon\rangle,
$$

where $s>0$. Since $\{|s, \gamma\rangle\}$ are orthonormals, the normalization factor $M(s)$ is given by

$$
M(s)^{2}=\int_{0}^{\varepsilon_{M}} \mathrm{~d} \varepsilon \frac{s^{2 \varepsilon}}{\rho(\varepsilon)},
$$

for $M(s)^{2}<\infty$.

Coherent states (17) must satisfy resolution of identity. In this case, it was introduced in Ref. [11] the following relation

$$
\rho(\varepsilon)=\int_{0}^{s} \mathrm{~d} s^{\prime} s^{2 \varepsilon} \sigma\left(s^{\prime}\right),
$$

where $s^{\prime}$ is a variable of integration with $0 \leq s^{\prime}<s \leq \infty$. In addition, a non-negative weight function $\sigma\left(s^{\prime}\right) \geq 0$ was introduced in order to satisfy the second requirement. Then, the measure of integration takes the form [11]

$$
\mathrm{d} \tau(s, \gamma)=\sigma(s) M(s)^{2} \mathrm{~d} s \frac{\mathrm{d} \gamma}{2 \pi} .
$$

Gazeau and Klauder shown that resolution of unity is satisfied for systems with continuous spectrum in the present formulation of coherent states [11]. In Ref. [19] the authors have proposed a continuous appearance of Eq. (4), replacing the discrete form by the continuous version of variables, functions and operators involved in the formalism. Hence, we are ready to define the Husimi distribution for systems with continuous spectrum in the following manner:

$$
\mu_{Q}(s, \gamma)=\frac{1}{Z} \int_{0}^{\varepsilon_{M}} \mathrm{~d} \varepsilon e^{-\beta \omega \varepsilon}|\langle s, \gamma \mid \varepsilon\rangle|^{2},
$$


where $\varepsilon$ stands for a continuous parameter. The Husimi distribution is normalized according to

$$
\int_{0}^{\infty} \int_{-\infty}^{\infty} \mathrm{d} \tau(s, \gamma) \mu_{Q}(s, \gamma)=1
$$

where the measure $\mathrm{d} \tau(s, \gamma)$ is given by Eq. (20).

We see easily from Eq. (17) that, the projection of eingensatates of the Hamiltonian over coherent states, is given by

$$
\langle s, \gamma \mid \varepsilon\rangle=M(s)^{-1} \frac{s^{\varepsilon} e^{-i \gamma \varepsilon}}{\sqrt{\rho(\varepsilon)}},
$$

where we have considered from Eq. (15) the orthogonality of the continuous states $\{|\varepsilon\rangle\}$. Introducing the above expression into Eq. (21) we finally arrive to [19]

$$
\mu_{Q}(s)=\frac{M(s)^{-2}}{Z} \int_{0}^{\varepsilon_{M}} \mathrm{~d} \varepsilon \frac{e^{-\beta \omega \varepsilon} s^{2 \varepsilon}}{\rho(\varepsilon)}
$$

where we have dropped out the dependence on $\gamma$. The continuous partition function obviously is [20]

$$
Z=\int_{0}^{\varepsilon_{M}} \mathrm{~d} \varepsilon e^{-\beta \omega \varepsilon}
$$

It is important to note that Eq. (24) is consistently normalized in accordance with

$$
\int_{0}^{\infty} \mathrm{d} \tau(s) \mu_{Q}(s)=1
$$

and in this case, the measure is $\mathrm{d} \tau(s)=\sigma(s) M(s)^{2} \mathrm{~d} s$.

\section{Landau diamagnetism: Charged particle in a uniform magnetic field}

Diamagnetism was a problem firstly appointed by Landau who showed the discreteness of energy levels for a charged particle in a magnetic field [24]. By the observation of the diverse scenarios in the framework provided by the Landau diamagnetism we can study some relevant physical properties [2527] as thermodynamic limit, role of boundaries, decoherence induced by the environment. The main motivation for several specialists work even today it is to make an accurate description of its theoretical and practical consequences.

In the past the appropriate partition function for this problem was calculated by Feldman and Kahn appealing to the concept of Glauber's coherent states as a set of basis states [28]. This formulation allows the use of classical concepts to describe electron orbits, even containing all quantum effects [28]. In a previous effort, this approach was used to obtain the Wehrl entropy [21, 22] and Fisher information [29] with the purpose of studying the thermodynamics of the Landau diamagnetism problem, namely, a free spinless charged particle in a uniform magnetic field [7]. In such contribution 
the authors focussed only in the transverse motion of a particle. For this reason, it was necessary to normalize the Husimi distribution in order to arrive to a consistent expression for semiclassical measures $[7,8,32]$.

Certainly, because the relevant effects seem to come only from the transverse motion, several efforts are made to describe this problem in two dimensions [7, 8, 27, 28, 32-34]. Furthermore, since the discovery of interesting phenomena, as the quantum Hall effect, there has been much interest in understanding the dynamics of electrons confined to move in two dimensions in the presence of a magnetic field perpendicular to the motion plane [31]. The confinement is possible at the interface between two materials, typically a semiconductor and an insulator, where a quantum well that traps the particles is formed, forbidding their motion in the direction perpendicular to the interface plane at low energies.

However, we propose here to discuss this problem in the most complete form (three dimensions), some results related to the behavior of the Wehrl entropy. From the present line of reasoning, it is concluded that the two-dimensional formulation is sufficient unto itself to explain the problem whenever the length of the cylindrical geometry of the system is large enough. Nevertheless, as suggested before, electronic devices are based in interfaces. Thus, this fact theoretically imposes a natural lower temperature bound that emerges from the analysis when three dimensions are considered [18].

\subsection{The model of one charged particle in a magnetic field}

We enter the present application by revisiting the complete set of coherent states of a spinless charged particle in a uniform magnetic field. Consider the classical kinetic momentum

$$
\vec{\pi}=\vec{p}+\frac{q}{c} \vec{A}
$$

of a particle of charge $q$, mass $m_{q}$, and linear momentum $\vec{p}$, subject to the action of a vector potential $\vec{A}$. These are the essential ingredients of the well-known Landau model for diamagnetism: a spinless charged particle in a magnetic field $B$ (we follow the presentation of Feldman et al. [28]). The Hamiltonian reads [28]

$$
H=\frac{\vec{\pi} \cdot \vec{\pi}}{2 m_{q}},
$$

and the magnetic field is $\vec{B}=\vec{\nabla} \times \vec{A}$. The vector potential is chosen in the symmetric gauge as $\vec{A}=(-B y / 2, B x / 2,0)$, which corresponds to a uniform magnetic field along the $z$-direction.

By using the quantum formulation of the step-ladder operators [28], one needs to define the step operators as follows [28]

$$
\hat{\pi}_{ \pm}=\hat{p}_{x} \pm i \hat{p}_{y} \pm \frac{i \hbar}{2 \ell_{\mathrm{B}}^{2}}(\hat{x} \pm i \hat{y})
$$

where the length

$$
\ell_{\mathrm{B}}=(\hbar c / q B)^{1 / 2}
$$


is the classical radius of the ground-state Landau orbit [28]. Motion along the $z$-axis is free [28]. For the transverse motion, the Hamiltonian specializes to [28]

$$
\hat{H}_{t}=\frac{\hat{\pi}_{+} \hat{\pi}_{-}}{2 m_{q}}+\frac{1}{2} \hbar \Omega \hat{1},
$$

where an important quantity characterizes the problem, namely,

$$
\Omega=q B / m_{q} c
$$

the cyclotron frequency [33]. The eigenstates $|N, m\rangle$ are determined by two quantum numbers: $N$ (associated to the energy) and $m$ (to the $z$ - projection of the angular momentum). As a consequence, they are simultaneously eigenstates of both $\hat{H}_{t}$ and the angular momentum operator $\hat{L}_{z}$ [28], so that

$$
\hat{H}_{t}|N, m\rangle=\left(N+\frac{1}{2}\right) \hbar \Omega|N, m\rangle=E_{N}|N, m\rangle
$$

and

$$
\hat{L}_{z}|N, m\rangle=m \hbar|N, m\rangle .
$$

We note that the eigenvalues of $\hat{L}_{z}$ are not bounded by below ( $m$ takes the values $\left.-\infty, \ldots,-1,0,1, \ldots, N\right)$ [28]. This agrees with the fact that the energies $(N+1 / 2) \hbar \Omega$ are infinitely degenerate [33]. Such a fact diminishes the physical relevance of phase-space localization for estimation purposes, as we shall see below. Moreover, $L_{z}$ is not an independent constant of the motion [33].

There exists a analogous formulation of an charged particle in a magnetic field by Kowalski that takes into account the geometry of a circle [30] (and for a comparison with the Feldman formulation see Ref.[8]), but at this point, we choose the Feldman formulation to work because the measure is easily defined and the normalization condition and other semiclassical measures are well described.

\subsection{Husimi distribution and Wehrl entropy}

We will start our present endeavor defining the Hamiltonian $\hat{H}=\hat{H}_{t}+\hat{H}_{l}$ for a particle of mass $m_{q}$ and charge $q$ in a magnetic field $B$, where $\hat{H}_{t}=\hbar \Omega(\hat{N}+1 / 2)$ describes the transverse motion, being $\Omega$ the cyclotron frequency as defined by the Eq. (32) and $\hat{N}$ the number operator. In addition, the Hamiltonian $\hat{H}_{l}=\hat{p}_{z}^{2} / 2 m_{q}$ represents a longitudinal one-dimensional free motion. After constructing a coherent state basis, a possible way to define the Husimi function $\eta$, for the complete motion, is given by

$$
\eta\left(x, p_{x} ; y, p_{y} ; p_{z}\right)=\left\langle\alpha, \xi, k_{z}|\hat{\rho}| \alpha, \xi, k_{z}\right\rangle,
$$

where $\hat{\rho}$ is the thermal density operator and the set $\left\{\left|\alpha, \xi, k_{z}\right\rangle\right\}$ represents the coherent states for the motion in three dimensions. Taking the direct product $\left|\alpha, \xi, k_{z}\right\rangle \equiv|\alpha, \xi\rangle \otimes\left|k_{z}\right\rangle$, the set $\{|\alpha, \xi\rangle\}$ corresponds to the coherent states of the transverse motion and $\left\{\left|k_{z}\right\rangle\right\}$ to the longitudinal motion. Therefore, the thermal density operator is given by

$$
\hat{\rho}=\frac{1}{Z} \mathrm{e}^{-\beta\left(\hat{H}_{l}+\hat{H}_{t}\right)},
$$


where $\beta=1 / k_{B} T, k_{B}$ the Boltzmann constant and $T$ the temperature. Besides, $Z$ is the partition function for the particle total motion. If $Z$ is separated in a similar way as other physical properties are separated, it is possible to assure that $Z=Z_{l} Z_{t}$, where $Z_{t}$ is the contribution for the transverse motion and $Z_{l}$ the contribution for the one-dimensional free motion. Thus, the Husimi function [1] is written as

$$
\eta=\frac{\mathrm{e}^{-\beta p_{z}^{2} / 2 m_{q}}}{Z_{l} Z_{t}} \sum_{n, m} \mathrm{e}^{-\beta \hbar \Omega(n+1 / 2)}|\langle n, m \mid \alpha, \xi\rangle|^{2} .
$$

where

$$
\begin{aligned}
& Z_{l}=(\mathcal{L} / h)\left(2 \pi m_{q} k_{B} T\right)^{1 / 2} \quad \text { and } \\
& Z_{t}=\mathcal{A} m_{q} \Omega /(4 \pi \hbar \sinh (\beta \hbar \Omega / 2)),
\end{aligned}
$$

being $\mathcal{L}$ the length of the cylinder, $\mathcal{A}=\pi R^{2}$ the area for cylindrical geometry [28]. In addition, the matrix element $|\langle n, m \mid \alpha, \xi\rangle|^{2}$ represents the probability of finding the charged particle in the coherent state $|\alpha, \xi\rangle$ and we can find its expression as defined previously [34].

It should be noticed that the distribution $\eta$ can be written as follows

$$
\eta=\eta_{l}\left(p_{z}\right) \eta_{t}\left(x, p_{x} ; y, p_{y}\right)
$$

where $\eta$ has been separated as a function of two distributions, namely, $\eta_{l}=\eta_{l}\left(p_{z}\right)$ and $\eta_{t}=$ $\eta_{t}\left(x, p_{x} ; y, p_{y}\right)$. The dependence on the variable $z$ has been missed due to the explicit form of the hamiltonian $\hat{H}_{l}$. Accordingly, after summing in Eq. (37) we find

$$
\begin{aligned}
& \eta_{l}=\frac{\mathrm{e}^{-\beta p_{z}^{2} / 2 m_{q}}}{Z_{l}} \\
& \eta_{t}=\frac{2 \pi \hbar}{\mathcal{A} m_{q} \Omega}\left(1-\mathrm{e}^{-\beta \hbar \Omega}\right) \mathrm{e}^{-\left(1-\mathrm{e}^{-\beta \hbar \Omega}\right)|\alpha|^{2} / 2 \ell_{\mathrm{B}}^{2}},
\end{aligned}
$$

where the length $\ell_{\mathrm{B}}$ is defined by the Eq. (30). From expressions (41) and (42), we emphasize again that $\eta_{l}\left(p_{z}\right)$ describes the free motion of the particle in the magnetic field direction and $\eta_{t}\left(x, p_{x} ; y, p_{y}\right)$ the Landau levels due to the circular motion in a transverse plane to the magnetic field, similar to the harmonic oscillator of Eq. (9) since $|z|^{2} \rightarrow|\alpha|^{2} / 2 \ell_{\mathrm{B}}^{2}$. Consequently Eqs. (40), (41) and (42) together contain the complete description of the system. We noticed both distributions are naturally normalized in a standard form, i.e.,

$$
\int \frac{\mathrm{d} z \mathrm{~d} p_{z}}{h} \eta_{l}\left(p_{z}\right)=1
$$

and

$$
\int \frac{\mathrm{d}^{2} \alpha \mathrm{d}^{2} \xi}{4 \pi^{2} \ell_{\mathrm{B}}^{4}} \eta_{t}\left(x, p_{x} ; y, p_{y}\right)=1
$$


In consequence, both Eqs. (41) and (42), under conditions (43) and (44), bring a promising way to get the exact form of the Wehrl entropy. Furthermore, using the additivity as the most basic property of the entropy, we can state $W_{\text {total }}=W_{l}+W_{t}$. Hence,

$$
\begin{aligned}
& W_{l}=-\int \frac{\mathrm{d} z \mathrm{~d} p_{z}}{h} \eta_{l}\left(p_{z}\right) \ln \eta_{l}\left(p_{z}\right), \\
& W_{t}=-\int \frac{\mathrm{d}^{2} \alpha \mathrm{d}^{2} \xi}{4 \pi^{2} \ell_{\mathrm{B}}^{4}} \eta_{t}\left(x, p_{x} ; y, p_{y}\right) \ln \eta_{t}\left(x, p_{x} ; y, p_{y}\right),
\end{aligned}
$$

where, as before, the subindex $l$ stands for the longitudinal motion and $t$ the transverse.

After evaluating the respective integrals in Eqs. (45) and (46), it is feasible to identify the two particular entropies

$$
\begin{aligned}
& W_{l}=\frac{1}{2}+\ln \left(\frac{\mathcal{L}}{\lambda}\right), \\
& W_{t}=1-\ln \left(1-\mathrm{e}^{-\beta \hbar \Omega}\right)+\ln (g),
\end{aligned}
$$

where $\lambda=h /\left(2 \pi m_{q} k_{\mathrm{B}} T\right)^{1 / 2}$ is the mean thermal wavelength of the particle and $g=\mathcal{A} / 2 \pi \ell_{\mathrm{B}}^{2}$ stands for the degeneracy of a Landau level [35]. Indeed, Eq. (47) coincides with the classical entropy for a free particle in one dimension. Eq. (48) is the Wehrl entropy for the transverse motion and possesses a form for the one close to the harmonic oscillator entropy given by the Eq. (10), with the exception of a term associated with the degeneracy.

\subsection{Semiclassical behavior and consequences}

Although the total Wehrl entropy is expressed simply as follows

$$
W_{\text {total }}=\frac{3}{2}-\ln \left(1-\mathrm{e}^{-\beta \hbar \Omega}\right)+\ln (g)+\ln \left(\frac{\mathcal{L}}{\lambda}\right),
$$

we notice that some of its properties are directly derived from Eqs. (47) and (48). First, as we commented before, $W_{l}$ coincides with the classical entropy for the free motion in one dimension. From this glance, we can add that $W_{l}$ has to be nonnegative, $W_{l} \geq 0$ at all temperatures. This last condition imposes a minimum temperature, given by

$$
T_{0}=\frac{h^{2}}{2 \pi m_{q} e k_{B} \mathcal{L}^{2}},
$$

where $e=2.718281828$. The standard behavior of $W_{l}$ obligates the system to take high values of temperature, wherever the temperature $T$ ought to be greater than $T_{0}$, in such case the conduct of the system is classical. This is equivalent to assert that, if $T / T_{0} \geq 1$, the length of a thermal wave $\lambda$ lower than the average of the spacing among particles and quantum considerations are not relevant [36]. In addition, $T_{0}$ only depends on the size of the system and does not depend on other external or 
internal physical parameters such as transverse area, external magnetic field, charge of the particle, etc. If the system is large then the minimum temperature is low. However, modern electronic systems has junctions where $\mathcal{L}$ is practically zero. In such case the required minimum temperature to make applicable our description is numerically high enough [39].

Nevertheless, the entropy associated with transverse motion satisfies $W_{t} \geq 1+\ln (g)$ for all temperatures in the system of a particle in a magnetic field where the symmetry is polar, which is almost the Lieb condition for systems in one dimension [37] with an additional term associated with the degeneracy $g$. Roughly speaking, the transverse motion is bi-dimensional, but in the Landau approach the quantum motion of the particle in a magnetic field is reduced to a degenerate spectrum in one dimension. This degeneracy essentially recovers the physics of the missing dimension. Resuming the discussion of the behavior of the Wehrl entropy, it is not plausible to adventure any conclusion about the applicability of the present treatment because the Lieb condition is always satisfied. This is the main problem stems from the restricted vision presented in other contributions over this topic which only put its emphasis on the transverse motion $[8,28,30]$ and represent the main difference from the vision obtained in that other contributions that discuss this topic. From the combined reasoning of both motions we conclude that the present description, this is the calculation of $W_{t}$, has sense when the imposition over the temperature is satisfied. Under $T_{0}$ the behavior is intrinsically anomalous and the present proposal is not applicable.

If we consider $k_{B} T \gg \hbar \Omega$, we can apply the first order of approximation as $\ln \left(g /\left(1-e^{-\beta \hbar \Omega}\right)\right) \approx$ $\ln \left(\mathcal{A} T / T_{0} \mathcal{L}^{2}\right)$. Indeed, taking into account that the thermal wave length can be rewritten in terms of the temperature $T_{0}$ this way $\lambda=\mathcal{L}\left(e T_{0} / T\right)^{1 / 2}$, the expression (49) after a bit of algebra reduces to

$$
W_{\text {total }}^{(1)} \approx \frac{3}{2} \ln \left(\frac{T}{T_{0}}\right)+\ln \left(\frac{\mathcal{A}}{\mathcal{L}^{2}}\right) .
$$

Considering that $\mathcal{V}=\mathcal{A} \mathcal{L}$ in Eq. (51), the total Wehrl entropy can be expressed as follows

$$
W_{\text {total }}^{(1)}=\frac{3}{2}+\ln \left(\frac{\mathcal{V}}{\lambda^{3}}\right)
$$

This is a particular expression for the entropy of a free particle in three dimensions related to the motion of a charged particle into a region of the magnetic field making mention of some geometrical properties of the system.

In second order of approximation for high temperatures, considering the special condition $\mathcal{A} \sim \mathcal{L}^{2}$, Wehrl entropy is expressed as follows

$$
W_{\text {total }}^{(2)} \approx \frac{T_{0}}{T} g+\frac{3}{2}+\frac{3}{2} \ln \left(\frac{T}{T_{0}}\right)=\frac{T_{0}}{T} g+W_{\text {total }}^{(1)}
$$

As explained before, the Wehrl entropy takes values that are permitted by the Lieb condition, namely, $W \geq 1$. According to Eq. (53) the slope decreases as temperature increases. This fact illustrates why the disorder slowly increases as the magnetic field increases too. Consequently, at extremely high temperatures as expected, the slope of the present linear dependence tends to zero apparently taking a constant value close to the corresponding classical entropy of the free particle in three dimensions. 
The lower bound of temperature is related to $T / T_{0} \rightarrow 1^{+}$, because this approach does not consider temperature values under $T_{0}$. The total Wehrl entropy is reduced to logarithm behavior of the magnetic field.

To study what occurs close to zero temperature, in accordance with Eq. (50), we need to take systems with $L \rightarrow \infty$ and after this consideration the transverse entropy of Eq. (48) can be seen as follows

$$
W_{t}^{T \rightarrow 0^{+}}=1+\ln (g) .
$$

As we discussed before, this Wehrl entropy is also a kind of harmonic oscillator entropy and the lower bound complies with being greater than a bound limiting value of the temperature, which has been suggested by Wehrl and shown by Lieb, $W \geq 1$ [37]. Starting from this condition it must arrive to the following inequality for the magnetic field

$$
g \geq 1,
$$

where $g=q \mathcal{A} B / h c$ also accounts for the ratio between the flux of the magnetic field $\mathcal{A B}$ and the quantum of the magnetic flux given by $h c / q=4.14 \times 10^{-7}\left[\right.$ gauss $\left./ \mathrm{cm}^{2}\right]$ [17]. Then the inequality (55) adopts the form

$$
B \geq \frac{1}{\mathcal{A}} \frac{h c}{q}=B_{0} .
$$

Therefore, the quantity $B_{0}=h c / \mathcal{A} q$ becomes a bound limiting field that represents the minimum value for the external magnetic field. To study what occurs close to zero magnetic field we need to take systems with $\mathcal{A} \rightarrow \infty$.

For finite values of $\mathcal{A}$ and $B$ lower than $B_{0}$ is manifested the Haas-van Alphen effect, which describes oscillations in the magnetization because at temperatures low enough the particles will tend to occupy the lowest energy states. Whereas if the value of the magnetic field decreases a less number of particles can be in the lowest state due to degeneracy is directly proportional to $B$ [35]. Then, the transverse Wehrl entropy $W_{t}$ is well defined for values of the magnetic field over $B_{0}$, this is $B / B_{0} \geq 1$ and/or $g \rightarrow 1^{+}$.

We can assert that this description of the system is not quantum, we say that it is semiclassical; for instance, it does not contain the Haas-van Alphen effect, the same condition marks the beginning of one description and the ending of the other.

Other relevant effect that emerges from the Landau quantization [38] is the quantum Hall effect [39] which is a quantum-mechanical version of the Hall effect [31], observed in two-dimensional electron systems subjected to low temperatures and strong magnetic fields. The degeneracy is given by [17]

$$
\phi=v \phi_{0},
$$

where $\phi_{0}=h c / q$ is the quantum of the magnetic flux. The factor $v$ is related to the "filling factor" that takes integer values $(v=1,2,3, \ldots)$. The discovery of the fractional quantum Hall effect [32] extend these values to rational fractions $(v=1 / 3,1 / 5,5 / 2,12 / 5, \ldots)$. The integer quantum Hall effect is simply explained in terms of the conductivity quantization $\sigma=v q^{2} / h$. However, the fractional quantum Hall effect relies on other phenomena related to interactions. Consistently, we see that the degeneracy is 
equal to $\mathrm{v}$, which must be greater than 1 due to the inequality (55) obtaining an infinite family of Wehrl entropies

$$
W_{t}=1-\ln \left(1-e^{-\beta \hbar \Omega}\right)+\ln v .
$$

Again, Eq. (55) provides the limiting value of $v$ and, as before, the transverse entropy always satisfies the Lieb bound for all temperatures and large enough systems when the quantum Hall effect is manifested at least for the integer quantum Hall effect. Conversely, fractional values of $v$ less than 1 are left out the present approach.

\section{Description of the molecular rotation: Rigid rotator}

The rigid rotator is a system of a single particle whose quantum spectrum of energy is exactly known. Therefore, the study of typical thermodynamic properties can be analytically derived [40]. Applications lead to the treatment of important aspects of molecular systems [41] and several applications to materials [42].

\subsection{Linear rigid rotator}

We start the present study by exploring a simple model, the linear rigid rotator, based on the excellent discussion concerning the coherent states for angular momenta given in Ref. [43]. The Hamiltonian of the linear rigid rotator is [20]

$$
\hat{H}=\frac{\hat{L}^{2}}{2 I_{x y}}
$$

where $\hat{L}^{2}=\hat{L}_{x}^{2}+\hat{L}_{y}^{2}$ is the angular momentum operator and $I_{x}$ and $I_{y}$ are the associated moments of inertia. We have assumed that $I_{x y} \equiv I_{x}=I_{y}$. Calling $|I K\rangle$ the set of $H$-eigenstates, we recall that they verify the relations

$$
\begin{aligned}
\hat{L}^{2}|I K\rangle & =I(I+1) \hbar^{2}|I K\rangle \\
\hat{L}_{z}|I K\rangle & =K \hbar|I K\rangle,
\end{aligned}
$$

with $I=0,1,2 \ldots$, for $-I \leq K \leq I$, the eigenstates' energy spectrum being given by

$$
\varepsilon_{I}=\frac{I(I+1) \hbar^{2}}{2 I_{x y}} .
$$

Coherent states are constructed in Ref. $[44,45]$ for the lineal rigid rotator, using Schwinger's oscillator model of angular momentum, in the fashion

$$
|I K\rangle=\frac{\left(\hat{a}_{+}^{\dagger}\right)^{I+K}\left(\hat{a}_{-}^{\dagger}\right)^{I-K}}{\sqrt{(I+K) !(I-K) !}}|0\rangle,
$$


with $\hat{a}_{+}, \hat{a}_{-}$the pertinent creation and annihilation operators, respectively, and $|0\rangle \equiv|0,0\rangle$ the vacuum state. The states $|I K\rangle$ are orthogonal and satisfy the closure relation, i.e.,

$$
\begin{aligned}
& \left\langle I^{\prime} K^{\prime} \mid I K\right\rangle=\delta_{I^{\prime}, I} \delta_{K^{\prime}, K}, \\
& \sum_{I=0}^{\infty} \sum_{K=-I}^{I}|I K\rangle\langle I K|=\hat{1} .
\end{aligned}
$$

Since we deal with two degrees of freedom the ensuing coherent states are of the tensor product form (involving $\left|z_{1}\right\rangle$ and $\left|z_{2}\right\rangle$ ) [43, 46]

$$
\left|z_{1} z_{2}\right\rangle=\left|z_{1}\right\rangle \otimes\left|z_{2}\right\rangle
$$

where

$$
\begin{aligned}
& \hat{a}_{+}\left|z_{1} z_{2}\right\rangle=z_{1}\left|z_{1} z_{2}\right\rangle, \\
& \hat{a}_{-}\left|z_{1} z_{2}\right\rangle=z_{2}\left|z_{1} z_{2}\right\rangle .
\end{aligned}
$$

Therefore, the coherent state $\left|z_{1} z_{2}\right\rangle$ writes [43]

$$
\left|z_{1} z_{2}\right\rangle=e^{-\frac{||^{2}}{2}} e^{z_{1} \hat{a}_{+}^{\dagger}} e^{z_{2} \hat{a}_{-}^{\dagger}}|0\rangle
$$

with

$$
\begin{aligned}
& \left|z_{1}\right\rangle=e^{-\frac{\left|z_{1}\right|^{2}}{2}} e^{z_{1} \hat{a}_{+}^{\dagger}}|0\rangle, \\
& \left|z_{2}\right\rangle=e^{-\frac{\left|z_{2}\right|^{2}}{2}} e^{z_{2} \hat{a}_{-}^{\dagger}}|0\rangle .
\end{aligned}
$$

We have introduced the convenient notation

$$
|z|^{2}=\left|z_{1}\right|^{2}+\left|z_{2}\right|^{2}
$$

Using Eqs. (62) and (68) we easily calculate $\left|z_{1} z_{2}\right\rangle$ and, after a bit of algebra, find

$$
\left|z_{1} z_{2}\right\rangle=e^{-\frac{|z|^{2}}{2}} \sum_{n_{+}, n_{-}} \frac{z_{1}^{n_{+}}}{\sqrt{n_{+} !}} \frac{z_{2}^{n_{-}}}{\sqrt{n_{-} !}}|I K\rangle
$$


where $n_{+}=I+K$ and $n_{-}=I-K$. Therefore, the probability of observing the state $|I K\rangle$ in the coherent state $\left|z_{1} z_{2}\right\rangle$ is of the form

$$
\left|\left\langle I K \mid z_{1} z_{2}\right\rangle\right|^{2}=e^{-|z|^{2}} \frac{\left|z_{1}\right|^{2 n_{+}}}{n_{+} !} \frac{\left|z_{2}\right|^{2 n_{-}}}{n_{-} !} .
$$

The present coherent states satisfy resolution of unity

$$
\int \frac{\mathrm{d}^{2} z_{1}}{\pi} \frac{\mathrm{d}^{2} z_{2}}{\pi}\left|z_{1} z_{2}\right\rangle\left\langle z_{1} z_{2}\right|=1
$$

Furthermore, $z_{1}$ and $z_{2}$ are continuous variables.

Following the procedure developed by Anderson et al. [4], we can readily calculate the pertinent Husimi distribution [1]. For our system this is defined, from Eq. (4), as

$$
\mu\left(z_{1}, z_{2}\right)=\left\langle z_{1}, z_{2}|\hat{\rho}| z_{1}, z_{2}\right\rangle
$$

where the density operator is

$$
\hat{\rho}=Z_{2 D}^{-1} \exp (-\beta \hat{H})
$$

The concomitant rotational partition function $Z_{2 D}$ is given in Ref. [20]

$$
Z_{2 D}=\sum_{I=0}^{\infty}(2 I+1) e^{-I(I+1) \frac{\Theta}{T}}
$$

with $\Theta=\hbar^{2} /\left(2 I_{x y} k_{B}\right)$. Remark that in the present context, speaking of the "trace operation" entails performing the sum $\operatorname{Tr} \equiv \sum_{I=0}^{\infty} \sum_{K=-I}^{I}$. Inserting now the closure relation into Eq. (75), and using Eq. (73), we finally get our Husimi distribution in the fashion

$$
\mu\left(z_{1}, z_{2}\right)=e^{-|z|^{2}} \frac{\sum_{I=0}^{\infty} \frac{|z|^{4 I}}{(2 I) !} e^{-I(I+1) \frac{\Theta}{T}}}{\sum_{I=0}^{\infty}(2 I+1) e^{-I(I+1) \frac{\Theta}{T}}} .
$$

It is easy to show that this distribution is normalized to unity

$$
\int \frac{\mathrm{d}^{2} z_{1}}{\pi} \frac{\mathrm{d}^{2} z_{2}}{\pi} \mu\left(z_{1}, z_{2}\right)=1,
$$

where $z_{1}$ and $z_{2}$ are given by Eqs. (66), (67), and (71). Note that we must deal with the binomial expression $\left(\left|z_{1}\right|^{2}+\left|z_{2}\right|^{2}\right)^{4 I}$ firstly and then integrate over the whole complex plane (in two dimensions) in order to verify the normalization condition (79). The differential element of area in the $z_{1}\left(z_{2}\right)$ plane is $\mathrm{d}^{2} z_{1}=\mathrm{d} x \mathrm{~d} p_{x} / 2 \hbar\left(\mathrm{d}^{2} z_{2}=\mathrm{d} y \mathrm{~d} p_{y} / 2 \hbar\right)$ [13]. Moreover, we have the phase-space relationships 


$$
\begin{aligned}
& \left|z_{1}\right|^{2}=\frac{1}{4}\left(\frac{x^{2}}{\sigma_{x}^{2}}+\frac{p_{x}^{2}}{\sigma_{p_{x}}^{2}}\right), \\
& \left|z_{2}\right|^{2}=\frac{1}{4}\left(\frac{y^{2}}{\sigma_{y}^{2}}+\frac{p_{y}^{2}}{\sigma_{p_{y}}^{2}}\right),
\end{aligned}
$$

where $\sigma_{x} \equiv \sigma_{y}=\sqrt{\hbar / 2 m \omega}$ and $\sigma_{p_{x}} \equiv \sigma_{p_{y}}=\sqrt{m \omega \hbar / 2}$.

The profile of the Husimi function is similar to that of a Gaussian distribution.

The Wehrl entropy is a semiclassical measure of localization [21] (so is Fisher's one [5] as well). Indeed, Wehrl's measure is simply a logarithmic Shannon measure built up with Husimi distributions. For the present bi-dimensional model this entropy reads

$$
\mathcal{W}=-\int \frac{\mathrm{d}^{2} z_{1}}{\pi} \frac{\mathrm{d}^{2} z_{2}}{\pi} \mu\left(z_{1}, z_{2}\right) \ln \mu\left(z_{1}, z_{2}\right),
$$

where $\mu\left(z_{1}, z_{2}\right)$ is given by Eq. (78).

\subsection{Rigid rotator in three dimensions}

In the present section we consider a more general problem, the model of the rigid rotator in three dimensions, whose Hamiltonian writes [47]

$$
\hat{H}=\frac{\hat{L}_{x}^{2}}{2 I_{x}}+\frac{\hat{L}_{y}^{2}}{2 I_{y}}+\frac{\hat{L}_{z}^{2}}{2 I_{z}},
$$

where $I_{x}, I_{y}$, and $I_{z}$ are the associated moments of inertia. A complete set of rotator eigenstates is $\{|I M K\rangle\}$. The following relations apply

$$
\begin{aligned}
\hat{L}^{2}|I M K\rangle & =I(I+1) \hbar^{2}|I M K\rangle \\
\hat{L}_{z}|I M K\rangle & =K \hbar|I M K\rangle \\
\hat{J}_{z}|I M K\rangle & =M \hbar|I M K\rangle,
\end{aligned}
$$

where $I=0, \ldots, \infty,-I \leq K \leq I$, and $-I \leq M \leq I$. The states $|I M K\rangle$ satisfy orthogonality and closure relations [47]

$$
\left\langle I^{\prime} M^{\prime} K^{\prime} \mid I M K\right\rangle=\delta_{I^{\prime}, I} \delta_{M^{\prime}, M} \delta_{K^{\prime}, K}
$$




$$
\sum_{I=0}^{\infty} \sum_{M=-I}^{I} \sum_{K=-I}^{I}|I M K\rangle\langle I M K|=\hat{1} .
$$

If we take $\hat{L}^{2}=\hat{L}_{x}^{2}+\hat{L}_{y}^{2}+\hat{L}_{z}^{2}$ and assume axial symmetry, i.e., $I_{x y} \equiv I_{x}=I_{y}$, we can recast the Hamiltonian as

$$
\hat{H}=\frac{1}{2 I_{x y}}\left[\hat{L}^{2}+\left(\frac{I_{x y}}{I_{z}}-1\right) \hat{L}_{z}^{2}\right]
$$

where $\hat{L}^{2}$ is the angular momentum operator and $\hat{L}_{z}$ is its projection on the rotation axis $z$. The concomitant spectrum of energy becomes

$$
\varepsilon_{I, K}=\frac{\hbar^{2}}{2 I_{x y}}\left[I(I+1)+\left(\frac{I_{x y}}{I_{z}}-1\right) K^{2}\right],
$$

where $I=0,1,2, \cdots$ and it represents the eigenvalue of the angular momentum operator $\hat{L}^{2}$, the numbers $m=-I, \cdots,-1,0,1, \cdots, I$ stand for the projections on the intrinsic rotation axis of the rotator. All states exhibit a $(2 I+1)$-degeneracy. The parameters $I_{x}=I_{y} \equiv I_{x y}$ and $I_{z}$ are the inertia momenta. Different "geometrical" instances are characterized through the $I_{x y} / I_{z}-$ ratio. For example, the value $I_{x y} / I_{z}=1$ corresponds to the spherical rotator. Limiting cases can also be considered. This is, $I_{x y} / I_{z}=1 / 2$ and $I_{x y} / I_{z} \rightarrow \infty$, that correspond to the extremely oblate- and prolate cases, respectively.

\subsubsection{Coherent states for the rigid rotator in three dimensions}

In order to obtain the Husimi distribution for this problem we need first of all to have the associated coherent states. Morales et al. have constructed them in Ref. [47] and discussed their mathematical foundations. First, they introduced the auxiliary quantity

$$
X_{I, M, K}=\sqrt{I !(I+M) !(I-M) !(I+K) !(I-K) !},
$$

and then write [47]

$$
\left|z_{1} z_{2} z_{3}\right\rangle=e^{-\frac{|u|^{2}}{2}} \sum_{I M K} \frac{[(2 I) !]^{2} z_{1}^{(I+M)} z_{2}^{I} z_{3}^{(I+K)}}{X_{I, M, K}}|I M K\rangle,
$$

where the following supplementary variable were introduced by Morales et al. in Ref. [47]

$$
|u|^{2}=\left|z_{2}\right|^{2}\left(1+\left|z_{1}\right|^{2}\right)^{2}\left(1+\left|z_{3}\right|^{2}\right)^{2} .
$$

All coherent states share at least two requirements. Continuity of labeling and resolution of unity. In relation to the last property we add

$$
\int d \Gamma\left|z_{1} z_{2} z_{3}\right\rangle\left\langle z_{1} z_{2} z_{3}\right|=1
$$


where $\mathrm{d} \Gamma$ is the measure of integration given by [47]

$$
\mathrm{d} \Gamma=\mathrm{d} \tau\left\{4\left[\left(1+\left|z_{1}\right|^{2}\right)\left(1+\left|z_{3}\right|^{2}\right)\right]^{4}\left|z_{2}\right|^{4}-8\left[\left(1+\left|z_{1}\right|^{2}\right)\left(1+\left|z_{3}\right|^{2}\right)\right]^{2}\left|z_{2}\right|^{2}+1\right\}
$$

with

$$
\mathrm{d} \tau=\frac{\mathrm{d}^{2} z_{1}}{\pi} \frac{\mathrm{d}^{2} z_{2}}{\pi} \frac{\mathrm{d}^{2} z_{3}}{\pi}
$$

and, of course, in this case we have three degrees of freedom. The present formulation satisfy the weaker version of the second requirement, because the measure is defined non positive [47].

\subsubsection{Husimi function, Wehrl entropy}

Using now Eq. (90) we find

$$
\left|\left\langle I M K \mid z_{1} z_{2} z_{3}\right\rangle\right|^{2}=\frac{e^{-|u|^{2}}}{X_{I, M, K}^{2}}[(2 I) !]^{2}\left|z_{1}\right|^{2(I+M)}\left|z_{2}\right|^{2 I}\left|z_{3}\right|^{2(I+K)}
$$

and determine that, in this case, the rotational partition function reads

$$
Z_{3 D}=\sum_{I=0}^{\infty} \sum_{K=-I}^{I} \sum_{M=-I}^{I} e^{-\beta \varepsilon_{I, K}},
$$

i.e.,

$$
Z_{3 D}=\sum_{I=0}^{\infty}(2 I+1) e^{-I(I+1) \frac{\Theta}{T}} \sum_{K=-I}^{I} e^{-\left(\frac{I_{x y}}{I_{z}}-1\right) K^{2} \frac{\Theta}{T}} .
$$

Remark that if we take the "extremely prolate" limiting case $I_{x y} / I_{z} \rightarrow \infty$ just one term that survives in the right sum of the right side in Eq. (97), that for $K=0$, while all terms for $K \neq 0$ vanish. In this special instance case $Z_{2 D}$ is recovered from $Z_{3 D}$. The pertinent Husimi distribution becomes

$$
\mu\left(z_{1}, z_{2}, z_{3}\right)=\frac{e^{-|u|^{2}}}{Z_{3 D}} \sum_{I=0}^{\infty} \frac{(2 I) !}{I !}|v|^{2 I} e^{-I(I+1) \frac{\Theta}{T}} \times g(I),
$$

where

$$
g(I)=\sum_{K=-I}^{I} \frac{\left|z_{3}\right|^{2(I+K)}}{(I+K) !(I-K) !} e^{-\left(\frac{I_{x y}}{I_{z}}-1\right) K^{2} \frac{\Theta}{T}},
$$

with

$$
|v|^{2}=\left(1+\left|z_{1}\right|^{2}\right)^{2}\left|z_{2}\right|^{2},
$$




$$
|u|^{2}=|v|^{2}\left(1+\left|z_{3}\right|^{2}\right)^{2} .
$$

We can easily verify that $\mu\left(z_{1}, z_{2}, z_{3}\right)$ is normalized in the fashion

$$
\int \mathrm{d} \Gamma \mu\left(z_{1}, z_{2}, z_{3}\right)=1
$$

We compute now (i) the Wehrl entropy in the form

$$
\mathcal{W}=\int \mathrm{d} \Gamma \mu\left(z_{1}, z_{2}, z_{3}\right) \ln \mu\left(z_{1}, z_{2}, z_{3}\right)
$$

In the special instance $I_{x y} / I_{z}=1$, that corresponds to the spherical rotator, we explicitly obtain

$$
\mu\left(z_{1}, z_{2}, z_{3}\right)=e^{-|u|^{2}} \frac{\sum_{I=0}^{\infty} \frac{|u|^{2 I}}{I !} e^{-I(I+1) \frac{\Theta}{T}}}{\sum_{I=0}^{\infty}(2 I+1)^{2} e^{-I(I+1) \frac{\Theta}{T}}} .
$$

Having the Husimi functions the Wehrl entropy is straightforwardly computed.

In order to emphasize some special cases associated to possible applications we consider several possibilities.

1. The spherical rotator $I_{x y}=I_{x}=I_{y}=I_{z}$, thus $I_{x y} / I_{z}=1\left(\right.$ e.g. $\left.\mathrm{CH}_{4}\right)$.

2. The oblate rotator $I_{x y}=I_{x}=I_{y}<I_{z}$, specifically $1 / 2 \leq I_{x y} / I_{z}<1\left(\right.$ e.g. $\left.C_{6} H_{6}\right)$.

3. The prolate rotator $I_{x y}=I_{x}=I_{y}>I_{z}$, which corresponds to $I_{x y} / I_{z}>1\left(\right.$ e.g. $\left.P C l_{5}\right)$.

4. The extremely prolate rotator is equivalent to the linear case (all diatomic molecules, $I_{z}=0$, this is $I_{x y} / I_{z} \rightarrow \infty\left(\right.$ e.g. $\left.\mathrm{CO}_{2}, \mathrm{C}_{2} \mathrm{H}_{2}\right)$.

\section{Husimi distribution for systems with continuous spectrum}

In this section we propose a procedure to generalize the Husimi distribution to systems with continuous spectrum. We start extending the concept of coherent states for systems with discrete spectrum to systems with continuous one. In the present section, we see the Husimi distribution as a representation of the density operator in terms of a basis of coherent states. We specially discuss the problem of the continuous harmonic oscillator [20].

\subsection{The exponential weight function: Harmonic oscillator}

From the $\rho(\varepsilon)$ definition expressed in Eq. (19), we can take a non-negative weight function like $\sigma\left(s^{\prime}\right)=$ $\exp \left(-s^{\prime}\right)$. However, this choice is not fully arbitrary, because it relies on , at least, two reasons: 1$)$ it is related to the harmonic oscillator and, 2) it is a useful function that permits exactly to solve the integral (19). The latter reason allows to express such integral in the following way 


$$
\begin{aligned}
\rho(\varepsilon) & =\int_{0}^{s} \mathrm{~d} s^{\prime} s^{\prime 2 \varepsilon} \exp \left(-s^{\prime}\right), \\
& =e^{-s / 2} \frac{s^{\varepsilon}}{2 \varepsilon+1} \mathcal{M}(\varepsilon, \varepsilon+1 / 2, s)
\end{aligned}
$$

where $\mathcal{M}(a, b, x)$ is the Whittaker function [48]. Besides, in relation to the first reason, when we consider $\varepsilon=n$, where $n$ is integer, in the limit $s \rightarrow \infty$; the Eq. (105) drops into the known quantum result for the harmonic oscillator, $\rho(n)=n$ ! [11].

Moreover, the measure in phase space can be explicitly expressed from Eq. (20) as follows

$$
\mathrm{d} \tau(s)=\mathrm{d} s e^{-s / 2} \int_{0}^{\mathcal{\varepsilon}_{F}} \mathrm{~d} \varepsilon \frac{(2 \varepsilon+1) s^{\varepsilon}}{\mathcal{M}(\varepsilon, \varepsilon+1 / 2, s)} .
$$

Although obtaining this explicit form of the measure, a most general expression for the integral of Eq. (106) strongly depends on the particular spectrum of the system. In the present case, a spectrum like $\varepsilon \propto \omega$, the harmonic oscillator in the continuous limit, is considered.

\section{2. $s \rightarrow 0$ approximation for the Husimi distribution}

In order to know the shape of the Husimi distribution in $s=0$, we need to calculate some important quantities. First, we evaluate $\rho(\varepsilon)$ given by Eq. (105) expanding the exponential which appears inside the integral, as follows

$$
\begin{aligned}
\rho(\varepsilon) & \approx \lim _{s \rightarrow 0} \int_{0}^{s} \mathrm{~d} s^{\prime} s^{2 \varepsilon}\left(1-s^{\prime}+\cdots\right) \\
& \approx \frac{s^{2 \varepsilon+1}}{2 \varepsilon+1}\left(1-\frac{2 \varepsilon+1}{2 \varepsilon+2} s+\cdots\right)
\end{aligned}
$$

But, we are interested in evaluating the inverse of $\rho(\varepsilon)$, therefore

$$
\frac{1}{\rho(\varepsilon)} \approx \frac{2 \varepsilon+1}{s^{2 \varepsilon+1}}\left(1+\frac{2 \varepsilon+1}{2 \varepsilon+2} s+\cdots\right) .
$$

Second, we show easily that, in the limit $s \rightarrow 0$, the Husimi distribution is given by

$$
\mu_{Q}(0)=\frac{1}{Z} \frac{\int_{0}^{\varepsilon_{M}} \mathrm{~d} \varepsilon(2 \varepsilon+1) e^{-\beta \omega \varepsilon}}{\int_{0}^{\varepsilon_{M}} \mathrm{~d} \varepsilon(2 \varepsilon+1)} .
$$

Now, after integrating Eq. (25) the partition function is expressed as follows

$$
Z=\frac{1-\exp \left(-\beta \omega \varepsilon_{M}\right)}{\beta \omega}
$$


Then, the substitution of the Eq. (111) into (110) leads us to the appearance

$$
\mu_{Q}(0)=\frac{\left(2 e^{-\beta \omega \varepsilon_{M}} \beta \omega \varepsilon_{M}+2 e^{-\beta \omega \varepsilon_{M}}+e^{-\beta \omega \varepsilon_{M}} \beta \omega-2-\beta \omega\right)}{\beta \omega \varepsilon_{M}\left(e^{-\beta \omega \varepsilon_{M}}-1\right)\left(\varepsilon_{M}+1\right)} .
$$

In the high temperature limit, this becomes

$$
\mu_{Q}(0) \approx \frac{1}{\varepsilon_{M}}-\frac{\beta \omega \varepsilon_{M}}{6\left(\varepsilon_{M}+1\right)}
$$

If we take into account a kind of particles filling a band in the lowest continuous levels of energy (for instance, $\left.\varepsilon_{M} \rightarrow 1\right)$, we find $\mu_{Q}(0)=1-\beta \omega / 12$.

\subsection{Asymptotic behavior of the Husimi function}

In this part of the work, we are considering a particular range for $\varepsilon$; i.e., $0 \leq \varepsilon \leq \varepsilon_{M}=1$ and we study the asymptotic behavior of the Husimi distribution. This trend might be obtained from the limiting case of the Whittaker function [48] defined for $s \rightarrow \infty$, as follows:

$$
\lim _{s \rightarrow \infty} \frac{e^{-s / 2} s^{\varepsilon} \mathfrak{M}(\varepsilon, \varepsilon+1 / 2, s)}{2 \varepsilon+1}=\Gamma(2 \varepsilon+1)
$$

If we replace this result into Eq. (124) we obtain

$$
M(s)^{2}=e^{s / 2} \int_{0}^{\varepsilon_{M}} \mathrm{~d} \varepsilon \frac{s^{2 \varepsilon}}{\Gamma(2 \varepsilon+1)},
$$

and, from Eq. (24) we write

$$
\mu_{Q}(s)=\frac{M(s)^{-2}}{Z} e^{s / 2} \int_{0}^{\varepsilon_{M}} \mathrm{~d} \varepsilon \frac{e^{-\omega \beta \varepsilon} s^{2 \varepsilon}}{\Gamma(2 \varepsilon+1)}
$$

Now, we follow expanding to third order the inverse of the gamma function, $1 / \Gamma(2 \varepsilon+1)$, around its maximum [48]

$$
\frac{1}{\Gamma(2 \varepsilon+1)} \approx \sum_{n=0}^{3} A_{n} \varepsilon^{n}
$$

where $A_{0}=.9963530195, A_{1}=1.221909147, A_{2}=-3.108524622$, and $A_{3}=1.333217620$.

From Eq. (115), we derive a approximate result for $M(s)^{2}$, which is given by

$$
M(s)^{2}=e^{s / 2} \frac{s}{2} \sum_{n=0}^{3} A_{n} \frac{\mathcal{M}\left(\frac{n}{2}, \frac{n}{2}+\frac{1}{2},-2 \ln (s)\right)}{(n+1)(-2 \ln (s))^{1+n / 2}},
$$


and combining all above expressions, we have finally found an expression to third order of approximation for Husimi distribution given by

$$
\mu_{Q}(s)=\frac{M(s)^{-2}}{Z} e^{s / 2-\beta \omega / 2} \frac{s}{2} \sum_{n=0}^{3} A_{n} \frac{\mathcal{M}\left(\frac{n}{2}, \frac{n}{2}+\frac{1}{2}, \beta \omega-2 \ln (s)\right)}{(n+1)(\beta \omega-2 \ln (s))^{1+n / 2}},
$$

where $\mathcal{M}(a, b, c)$ is again the Whittaker function [48].

In the high temperature approximation, Eq. (119) is given by

$$
\mu_{Q}(s) \approx \beta \omega \frac{\exp (-\beta \omega / 2)}{1-\exp (-\beta \omega)} \approx \exp (-\beta \omega / 2)
$$

The present result does not depend on the values of the parameter $s$. Furthermore, this approximation is valid whenever $0 \leq \varepsilon \leq 1$. We notice that the asymptotic trend of the Husimi distribution approaches to the Boltzmann weight in the ground state of the harmonic oscillator.

\subsection{Some applications and consequences}

In Ref. [11], the mean value of energy is obtained from the expectation value of the classical Hamiltonian $\mathcal{H}$ in a coherent state as follows $\mathcal{H}(s)=\langle s, \gamma|\mathcal{H}| s, \gamma\rangle$, therefore they arrive to the relation $\mathcal{H}(s)=s \partial \ln M(s) / \partial s$.

However, it is our interest here to calculate the mean value of energy in a different way, integrating in the variable $s$ with $\mu_{Q}(s)$ as a weigh function. Hence, we have

$$
\langle\mathcal{H}\rangle=\int \mathrm{d} \tau(s) \mu_{Q}(s) \mathcal{H}(s)
$$

where $\mathcal{H}$, expressed in terms of the variable $s$, denotes the classical Hamiltonian of the system. Inserting the Husimi distribution (24) into Eq. (121) and making use the relation (19) we finally get

$$
\langle\mathcal{H}\rangle=\frac{1}{Z} \int_{0}^{\varepsilon_{M}} \mathrm{~d} \varepsilon e^{-\beta \varepsilon} \mathcal{H}(\varepsilon),
$$

that is the classical mean energy [20]. We emphasize that the Husimi distribution, for a system with continuous spectrum, conduces in a natural way to the classical mean value of energy. Obviously, this is not true when the spectrum is discrete.

An extra motivation consists in extending the formulation of coherent states to systems with continuum spectrum considering its explicit form; for instance, we can take a spectrum whose appearance is $E=$ $A \varepsilon^{v}$, where $A$ and $v$ are constant. The values $v= \pm 1$ and $v=2$ might define the continuous limit of three remarkable cases in physics. Certainly, in a general study other values of the parameter $v$ may be conveniently considered as an interesting analytical extension. Thus, for $v=1$ and $A=\omega$ we have the continuous limit of a particle in a harmonic potential; this case is being in detail discussed in the present work. For $v=2$ we have the continuous limit of a particle in a box. For $v=-1$ we have the 
continuous limit of a particle in a Coulomb potential. Therefore, it is necessary to introduce a density of states $g(E)$ in the formulation of continuous coherent states (17) and immediately get the following modification

$$
|s, \gamma\rangle=M(s)^{-1} \int_{0}^{E_{M}} \mathrm{~d} E g(E) \frac{s^{E / A} e^{-i \gamma E / A}}{\sqrt{\rho(E)}}|\varepsilon\rangle,
$$

where the function $M(s)$

$$
M(s)^{2}=\int_{0}^{E_{M}} \mathrm{~d} E g(E)^{2} \frac{s^{2 E / A}}{\rho(E)}
$$

represents the normalization factor.

\section{Final remarks}

We have included in the current work some motivational elements to develop possible future applications to information theory and condensed matter. We have focused attention primarily upon Husimi distribution and its analytical results, beyond the numerical, graphical, or approximate calculations. A semiclassical description undertaking can be tackled, (i) trying to estimate phase-space location via measures as Fisher information and (ii) evaluating the semiclassical Wehrl entropy. A crucial point, in such an estimation, is to define the Husimi distribution in a convenient set of coherent states. Hence, we introduce a formal view of general requirements for formulations of coherent states in the context of the Gazeau and Klauder formalism for the harmonic oscillator - we have included some mathematical details in order to make it easy to follow and instructive in courses of quantum mechanics for graduates- we show some practical elements to apply the present formalisms to specific calculations of semi-classical measures.

By using a suitable formulation of coherent states in every case, we show explicitly the form of the Husimi distribution for i) a spinless charged particle in a uniform magnetic field (Landau diamagnetism), (ii) the linear and the three dimensional rotator (molecular rotation) and (iii) a case of the limiting harmonic oscillator (continuous spectrum).

In addition, we can calculate the probability by projecting the states over the coherent states as a function of a variable related to the coherent states. We see that the localization of probability, in the phase space decreases as temperature increases. Also, as always, the localization of the Husimi distribution in the phase space decreases as temperature increases. The present derivation of Husimi distributions is based on the evaluation of the mean value of the density operator in the basis of a single-particle coherent state. While the Husimi function takes into account collective and environmental effects, the coherent states are independent-particle states. Thus, if the Husimi distribution is delocalized, we need many wave packets (independent-particle states) to represent the state. Furthermore, the thermodynamics of particles in systems, which come from environmentally induced effects, does not depend on the formulation of the coherent states. In this manner, we expect this behavior to become general.

In conclusion, quantal distributions in the phase space, such as the Husimi distribution, have long been recognized as powerful tools for studying the quantum-classical correspondence and semi-classical aspects of quantum mechanics, since they provide a phase-space picture of the density matrix. We aknowledge partial financial support by FONDECYT 1110827. 


\section{Author details}

Sergio Curilef ${ }^{1}$ and Flavia Pennini ${ }^{1,2}$

1 Departamento de Física, Universidad Católica del Norte, Antofagasta, Chile

2 Instituto de Física La Plata-CCT-CONICET, Fac. de Ciencias Exactas, Universidad Nacional de La Plata, La Plata, Argentina

\section{References}

[1] K. Husimi Proceedings of the Physico-Mathematical Society of Japan 22 (1940) 264.

[2] K. Takahashi and N. Saitô, Physical Review Letters 55 (1985) 645.

[3] M.C. Gutzwiller, Chaos in Classical and Quantum Mechanics, (Springer-Verlag, New York, 1990).

[4] A. Anderson and J.J. Halliwell, Physical Review D 48 (1993) 2753.

[5] F. Pennini and A. Plastino, Physical Review E 69 (2004) 057101.

[6] S. Curilef, F. Pennini, A. Plastino and G.L. Ferri, J. Phys. A: Math. Theor. 40 (2007) 5127.

[7] S. Curilef, F. Pennini and A. Plastino, Physical Review B 71 (2005) 024420.

[8] D. Herrera, A.M. Valencia, F. Pennini and S. Curilef, European Journal of Physics 29 (2008) 439.

[9] M. Janssen, Fluctuations and Localization in Mesoscopic Electron Systems, World Scientific Lecture Notes in Physics Vol. 64., 2001

[10] W.P. Scheleich, Quantum Optics in phase space, (Wiley VCH-Verlag, Berlin, 2001).

[11] J.P. Gazeau and J.R. Klauder, Journal of Physics A: Math Gen. 32 (1999) 123.

[12] E. Schrödinger, Naturwissenschaften 14 (1926) 664.

[13] R.J. Glauber, Physical Review Letters 10 (1963) 84.

[14] R.J. Glauber, Physical Review 131 (1963) 2766.

[15] V.V. Dodonov and V.I. Man'ko, Theory of nonclassical states of light, (Taylor \& Francis Group, London and New York, 2003).

[16] J.R. Klauder, J. Phys. A 29 (1996) L293.

[17] F. Olivares, F. Pennini, S. Curilef, Physical Review E 81 (2010) 041134.

[18] S. Curilef, F. Pennini, A. Plastino, G.L. Ferri, Journal of Physics: Conference Series 134 (2008) 012029.

[19] F. Pennini, S. Curilef, Communications in Theoretical physics 53 (2010) 535. 
[20] R.K. Pathria, Statistical Mechanics, (Pergamon Press, Exeter, 1993).

[21] A. Wehrl, Reviews of Modern Physics 50 (1978) 221.

[22] A. Wehrl, Reports on Mathematical Physics 16 (1979) 353.

[23] M. Thaik and A. Inomata, Journal of Physics. A: Math Gen. 38 (2005) 1767.

[24] L.D. Landau, Z. Physik 64 (1930) 629.

[25] S. Dattagupta, A.M. Jayannvar, N. Kumar, Current Science 80 (2001) 861.

[26] J. Kumar, P.A. Sreeram, S. Dattagupta, Physical Review E 79 (2009) 021130.

[27] A. M. Jayannavar, M. Sahoo, Physical Review E 75 (2007) 032102.

[28] A. Feldman, A.H. Kahn, Physical Review B 1 (1970) 4584.

[29] B.R. Frieden, (Cambridge University Press, Crambridge, England, 1998).

[30] K. Kowalski, J. Rembielínski, Journal of Physics A: Math. Gen. 38 (2005) 8247.

[31] K. v. Klitzing, G. Dorda, M. Pepper, Physical Review Letters 45 (1980) 494.

[32] D.C. Tsui, H.L. Stormer, A.C. Gossard, Physical Review Letters 48 (1982) 1559.

[33] M.H. Johnson and B. A. Lippmann, Physical Review 76 (1949) 828.

[34] See Eq. (16) in Ref.[7].

[35] K. Huang, Statistical Mechanics, Wiley, New York, Second edition (1963).

[36] L.E. Reichl, A Modern course in statistical physics, Wiley, New York, Second edition, (1998).

[37] E.H. Lieb, Communications in Mathematical Physics 62 (1978) 35.

[38] F. Lado, Physics Letters A 312 (2003) 101.

[39] T. Chakraborty, P. Pietiläinen, The Quantum Hall Effects, Springer-Verlag, Berlin, (1995).

[40] N. Ullah, Physical Review E 49 (1994) 1743.

[41] F.J. Arranz, F. Borondo, R.M. Benito, Physical Review E 54 (1996) 2458.

[42] J.T. Titantah, M.N. Hounkonnou, Journal of Physics A: Math. Gen. 32 (1999) 897; 30 (1997) 6347; 30 (1997) 6327; 28 (1995) 6345.

[43] M.M. Nieto, Physical Review D 22 (1980) 391.

[44] J.J. Sakurai, Modern Quantum Mechanicsed S.F. Tuan (Reading, MA: Addison-Wesley, 1994) p 217. 
[45] J. Schwinger, In Quantum Theory of Angular Momentumed, L. C. Biedenharn and H van Dam (New York: Academic, 1965) p 229.

[46] C. Cohen-Tannoudji, B. Diu, F. Laloe, Quantum Mechanics vol.1 John Wiley \& Sons, (1977).

[47] J.A. Morales, E. Deumens and Y. Öhrn, Journal of Mathematical Physics 40 (1999) 776.

[48] M. Abramowitz and I.A. Stegun (1964) Handbook of Mathematical Functions (Dover, New York). 
Quantum Information and Related Topics 

Chapter 26

\title{
The Quantum Mechanics Aspect of Structural Transformations in Nanosystems
}

\author{
M. D. Bal'makov \\ Additional information is available at the end of the chapter \\ http://dx.doi.org/10.5772/53704
}

\section{Introduction}

Theoretical and experimental investigations of size effects have made a substantial contribution to the development of nanophysics and nanochemistry. However, a great deal needs to be done in this field. Experimental results are not necessarily consistent with the traditional concepts. In particular, the melting temperature of small nanoparticles unexpectedly turned out to be higher than the melting temperature of a macroscopic sample of the same chemical composition [1].

It is this chemical composition of a macroscopic system that determines its melting temperature $T_{m}$ the specific heat of melting $Q_{m}$, and the entropy of melting $S_{m}$. These quantities do not depend on the number $M$ of atoms (in the limit, $M \rightarrow \infty$ ). This statement ceases to be valid for relatively small systems. In the given case, it is necessary to take into account the dependences of the quantities $T_{m}, Q_{m}$ and $S_{m}$ on the number $M$ of atoms. Moreover, as the size of the system decreases, the interpretation of the physical quantities $T_{m}, Q_{m}$ and $S_{m}$ should be refined. Indeed, one molecule, for example, the hydrogen molecule, cannot melt, because its dissociation occurs with an increase in the temperature. In this respect, it is advisable to analyze the structural transformations in nanosystems within a unified approach of the first principles of quantum mechanics and statistical physics.

\section{Quasiclosed ensembles}

In the framework of classical physics each structural modification is set by the vector

$$
\mathbf{R}=\left(\mathbf{r}_{1}, \mathbf{r}_{2}, \ldots \mathbf{r}_{i}, \ldots \mathbf{r}_{M}\right)
$$


where $\mathbf{r}_{\mathrm{i}}$ are the radius vectors of all atomic nuclei of the polyatomic system. But the atomic nucleus is not a mathematical point whose position is unambiguously determined by the vector $\mathbf{r}_{\mathrm{i}}$. The motion of microparticles is not characterized by the trajectory $\mathbf{r}_{\mathrm{i}}(\mathrm{t})$. One can speak solely about the sites of their localization. In the case of condensed systems the size of the sites of atomic nucleus localization is much smaller than the interatomic distances and is a tenth-hundredth of an angstrom. Therefore, one of the ways to make a brief quantum-mechanical description of the structure $\mathbf{R}$ consists in setting the coordinates $r_{i}(1)$ of the centers of these sites.

As a rule, numerous quantum states forming a quasiclosed ensemble correspond to each memorized macrostate (to each structural modification of $\mathbf{R}_{\mathrm{k}}$ ). This raises the question about the number $G^{(0)}$ of different quasiclosed ensembles.

The magnitude of $G^{(0)}$ cannot be evaluated without application of quantum-mechanical methods. The point is that the components $\mathbf{r}_{i}$ of the vector $\mathbf{R}(1)$ can vary continuously, i.e. there exists a continuum of various structures (different vectors $\mathbf{R}_{k}$ ), which cannot even be numbered with the help of the index $k$, if it has solely integer values. This hampers the determination of the number $G^{(0)}$ of different structural modifications. In a quantum-mechanical description of a structure the superfluous detailing is useless altogether since according to quantum mechanics, a system is usually localized not at one point $\mathbf{R}_{\mathrm{k}}$ but in a certain volume (cell $\Omega_{\mathrm{k}}$ ). The set of all cells $\Omega_{\mathrm{k}}$ is countable. It is this circumstance that allows one to speak about the number $G^{(0)}$ of different structural modifications of the condensed system with a fixed chemical composition.

In order to find the numerical value of $G^{(0)}$ it is necessary to consider primarily the problem of distribution of quantum states over different quasiclosed ensembles. Some of these are formed by the microstates corresponding to one of the free energy minima. The latter holds only for stable and metastable systems. In the overwhelming majority of cases we are dealing however the with nonequilibrium systems, the thermodynamic potentials of which are far from being extreme.

Thus structural modifications of the vitreous state are not characterized by the Gibbs energy minimum. Each of them is described by its intrinsic quasiclosed ensemble. Their macroscopic properties are invariable because a quite definite structural modification corresponds to each ensemble. It is for this reason that glasses are kinetically frozen nonequilibrium systems, the properties of which virtually do not change over the long time interval $t_{\max }$. The same may also be said about the overwhelming majority of noncrystalline substances, many of which are already widely used for recording information.

The class of various quasiclosed ensembles (different macrostates memorized by the system with a fixed chemical composition) is extraordinarily broad. Their number is substantially larger than the number of Gibbs energy minima.

All atomic configurations $\mathbf{R}_{\mathrm{k}}(1)$ of an ideal monatomic gas are equiprobable. Condensed systems are characterized by the totally opposite situation. Therefore, it is not surprising that some of their structural modifications may be frozen (memorized) for a long time interval $t_{\max }$. Let us illustrate what has been said above in the framework of the adiabatic approximation. 


\section{Adiabatic approximation}

Theadiabaticapproximation [2] is based on the considerabledifferencesinthemasses ofelectrons and nuclei, whichmakesit possibletodescribetheirmotionsseparately well.Beinglightparticles, the electrons 'succeed' in adapting themselves to the instantaneous configuration $\mathbf{R}$ (1) of the atomic nuclei, the latter in turn 'notice' only the averaged disposition of electrons.

In the zero approximation the atomic nuclei are regarded to be at rest [ $\mathbf{R}=$ const (1)]. In this case, the wave function $\Phi_{j}(\mathbf{R}, \mathbf{X})$ of the $\mathrm{j}$-th stationary quantum state of the electron subsystem satisfies the equation [3]

$$
\widehat{H} \Phi_{j}(\mathbf{R}, \mathbf{X})=U_{M}^{(j)}(\mathbf{R}) \Phi_{j}(\mathbf{R}, \mathbf{X})
$$

where $\hat{H}$ is the Hamiltonian of electrons at fixed nuclei, which represents the sum of the total Coulomb energy of the interaction of atomic nuclei and electrons, the operator of the spinorbital interaction of electrons and the operator of the kinetic energy of electrons; $\mathbf{X}$ is the sum of spatial and spin variables of all electrons of the system under consideration; $U_{M}\left({ }_{M}\right)(\mathbf{R})$ is the adiabatic electron term (Fig. 1), which in the case of a polyatomic system $(M>3)$ usually has a great number of different physically non-equivalent minima $\mathbf{R}_{\mathrm{k}}$ [4]. The Hamiltonian $H$ does not contain any operator of the kinetic energy of atomic nuclei and, consequently, is the operator of the energy of the system under consideration for the fixed atomic configuration $\mathbf{R}$.

When the motion of atomic nuclei does not induce any transitions between different electronic states, the function $U_{M}^{(j)}(\mathbf{R})(2)$ may be interpreted as the potential energy of the nuclei corresponding to the $j$-th electronic state. In this case their motion takes place in the potential field of $U_{M}^{(j)}(\mathbf{R})$.

Therefore, the nuclear wave function $\chi_{j}(\mathbf{R}, E)$ satisfies the Schrödinger equation [3]

$$
\left(\hat{T}+U_{M}^{(j)}(\mathbf{R})\right) \chi_{j}(\mathbf{R}, E)=E \chi_{j}(\mathbf{R}, E)
$$

in which in contrast to (2), there is no variable $\mathbf{X}$ corresponding to the electron subsystem. Here, $T$ is the operator of the kinetic energy of atomic nuclei; $E$ is the energy of the stationary quantum state. The chemical composition $\mathbf{n}$ determines unequivocally the explicit form of equations (2) and (3). The components of the vector $\mathbf{n}$ are the relative concentrations of atoms of each species which form the system under consideration.

Their different solutions describe various modifications of a substance with a fixed composition. This can serve as the basis for classification of these solutions. Thus in the case of selenium some solutions may be attributed to the fluid state, others - to definite crystalline modifications, to amorphous modifications, to the vitreous state, to films, etc. However, it is most advisable to base the discussed classification of solutions of equations (2) and (3) on the structure $\mathbf{R}(1)$ 
because the information about individual peculiarities of a polyatomic system is eventually stored in the mutual disposition of its atomic nuclei. Any structural modification (e.g., the kth modification) which is preserved at least over the time interval tmax is described by the wave functions $\Phi_{j}(\mathbf{R}, \mathbf{X})$ (2) and $\chi_{j}(\mathbf{R}, E)$ (3) localized near the point $\mathbf{R}_{\mathrm{k}}$. (Fig. 1). The diversity of the latter actually determines all the states belonging to the $\mathrm{k}$-th quasiclosed ensemble.

Usually one or a series of potential $\mathbf{R}_{\mathrm{k}}$ minima correspond to the points $U_{M}^{(j)}(\mathbf{R})$, near which the motion of one or other structural modification takes place. In order to estimate the number of such points (the number $G^{(0)}$ of different quasiclosed ensembles), it is, as a rule, sufficient to consider only the minima of the adiabatic electron term $U_{M}^{(0)}(\mathbf{R})$ corresponding to the ground $(j=0)$ state of the electron subsystem ${ }^{1}$.

The point is that the lifetimes $\tau_{\mathrm{e}}$ of most excited states of the electron subsystem are relatively short $\left(\tau_{\mathrm{e}} \ll \mathrm{t}_{\max }\right)$. Therefore, these states alone cannot form a quasiclosed ensemble, in the framework of which the $k$-th structural modification can be described over a long time interval $t_{\max }$. Its preservation is favored by the potential barriers surrounding the minimum $\mathbf{R}_{k}$ of the adiabatic electron term $U_{M}^{(0)}(\mathbf{R})$ (Fig. 1). If they are sufficiently high, then even the low-energy quasi-steady [5] states localized in the potential well $\mathbf{R}_{\mathrm{k}}$ under consideration have larger [compared to $t_{\max }$ ] lifetimes $\tau_{1}$ which satisfy the inequalities

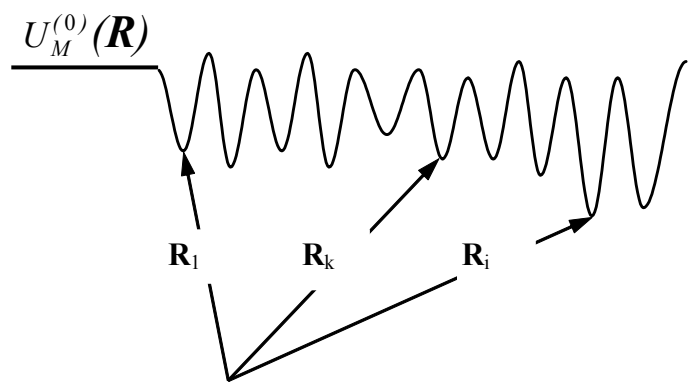

Figure 1. Adiabatic electron term $U_{M}^{(0)}(\mathbf{R})$. This figure is rather conditional because for polyatomic systems $(M>3)$ the function $U_{M}^{(0)}(\mathbf{R})$ is set, in conformity with (1), in the multidimensional space.

$$
\tau_{r}<<t_{\max }<<t_{l}
$$

where $\tau_{\mathrm{r}}$ is the relaxation time of the phonon subsystem, which is usually appreciably shorter than the time $t_{\max }$ required for the preservation of structural modifications.

1 Each minimum of the function $U_{M}^{(0)}(\mathbf{R})$ sets one of the equilibrium configurations $\mathbf{R}_{\mathrm{k}}$. Crystalline nanoparticles correspond to the deepest minima (potential wells). Most minima correspond to different noncrystalline structures. Transition from one potential well to another $\left(\mathbf{R}_{\mathrm{i}} \rightarrow \mathbf{R}_{\mathrm{k}}\right)$ means in the general case the rearrangement of all of the $M$ atomic nuclei of the system. The adiabatic electron term $U_{M}^{(0)}(\mathbf{R})$ does not depend either on temperature or on the thermal prehistory, etc. According to (2), it is unequivocally determined only by the chemical composition. Various scenarios of the system behavior consist in the sequence of passage over potential wells [the minima of the function $\left.\mathrm{U}_{M}^{(0)}(\mathrm{R})\right]$. 
Consequently, quasiclosed ensembles may be formed by the stationary and quasi-steady [5] states with large lifetimes $\tau_{1}(4)$. Usually these are low-energy states, which describe vibrational motion of atomic nuclei near one of the minima of the adiabatic electron term $U_{M}^{(0)}(\mathbf{R})$. Transitions between these states are not accompanied by any substantial changes in the $\mathbf{R}_{k}$ structure [6]. The $k$-th structural modification is preserved when only such transitions take place.

Thus, in order to preserve a polyatomic system, an exact copy and also the recorded information, it is sufficient that all changes occurring in the system do not extend outside the limits of one and the same quasiclosed ensemble. It is this ensemble that characterizes the properties of the system displayed during informational interaction [7].

The magnitude of $G^{(0)}$. can be estimated proceeding from the number $J(\mathbf{n}, M)$ of different minima of the potential $U_{M}^{(0)}(\mathbf{R})$. This approach allows a relatively simple derivation of numerical estimates as the function $J(\mathbf{n}, M)$ depends on only two arguments and, in addition, its determination is actually based on equation (2) when $j=0$. This unambiguous mathematical definition is useful not only for the problem of information copying and recording but also for considering a wide range of other issues [4].

\section{Estimation of the number $G^{(0)}$ of different quasiclosed ensembles}

For the number $J(\mathbf{n}, M)$ of different physically non-equivalent local minima of the adiabatic electron term $U_{M}^{(0)}(\mathbf{R})$, which corresponds to the ground electronic state of the electroneutral system consisting of $M$ atoms, the following asymptotic formula [4] is valid as $M \rightarrow \infty$

$$
\frac{1}{M} \ln J(\mathbf{n}, M) \sim \alpha_{\mathbf{n}}
$$

where $\alpha_{\mathbf{n}}$ is the positive parameter dependent solely on the chemical composition $\mathbf{n}$. The components $n_{i}$ of the vector $\mathbf{n}$ are the relative concentrations of atoms of each type.

It follows from (5) that

$$
J(n, M)=\exp \left(\alpha_{n} M+o(M)\right)
$$

the function $o(M)$ satisfying the condition $\lim _{M \rightarrow \infty} o(M) / M=0$. In other words, the number $J(\mathbf{n}, M)$ of different physically nonequivalent minima of the $U_{M}^{(0)}(\mathbf{R})$ potential exhibits a rapid exponential growth with the increasing number $M$ of atoms forming the system with a fixed ( $\mathbf{n}=$ const) chemical composition. The numerical values of $\alpha_{n}$ in relationship (6) usually differ from $\ln 2 \approx 0.69$ by smaller than one order of magnitude. 
This fact is not surprising because the magnitude $J(\mathbf{n}, M)(6)$ takes into account all potentially possible structural modifications $\mathbf{R}_{\mathrm{k}}(1)$ of a polyatomic system. These are structures of nanoparticles, liquid, glass, perfect crystal, crystals with different concentrations of particular defects, polycrystals, amorphous substances, amorphous and vitreous films, glass-ceramics and many others, including the structures of microheterogeneous materials storing the recorded information. These structures differ from each other not only in the location of particular defects, holes, etc. There exist other differences. For example, there are six equilibrium positions for each oxygen atom in the structure of $\beta$ cristobalite (Fig. 2, positions 1-6) [6].

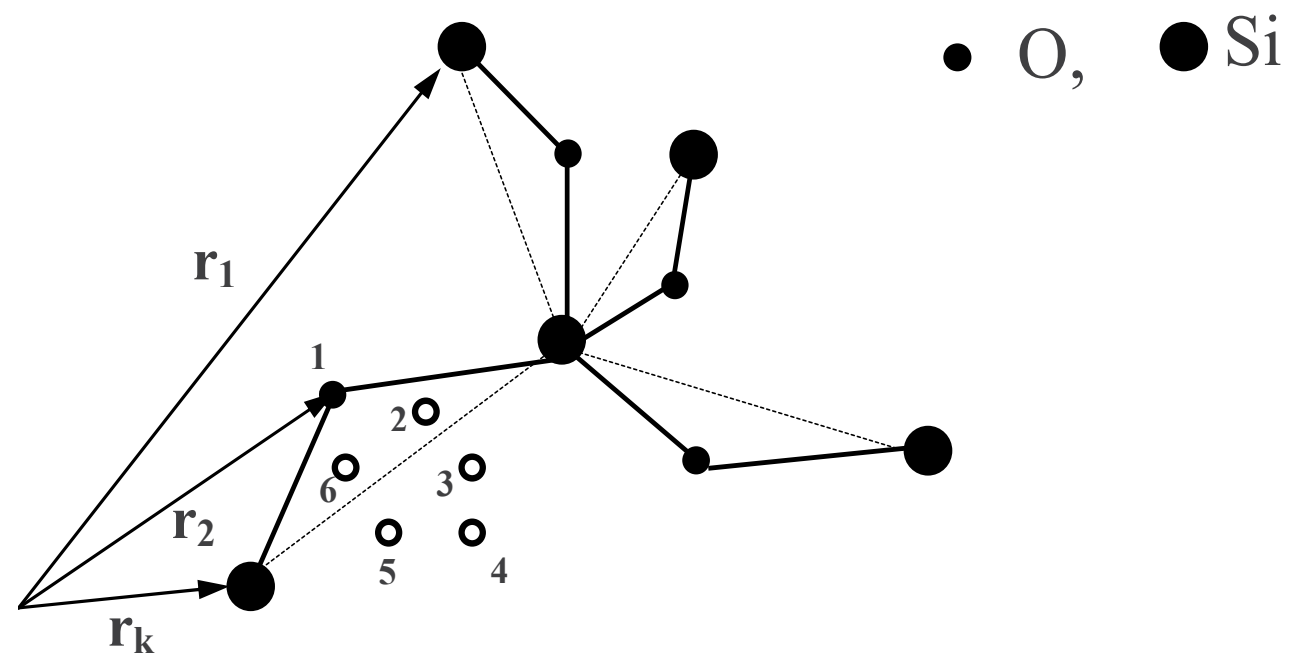

Figure 2. Structural fragment of $\beta$ cristobalite ( $r_{i}$ are the radius vectors of equilibrium atomic positions).

The transitions between them are not accompanied by the formation (disappearance) of defects, holes, dangling chemical bonds, etc. Each structure thus formed is not an exact copy of other structures [7]. These configurations are also taken into account by relationship (6).

The diversity of elementary configurational excitations particularly involves small structural transformations. As a result of these transformations, the transition from one minimum of the adiabatic electronic term $U_{M}^{(0)}(\mathbf{R})$ to another minimum occurs through a correlated rearrangement of many atoms involved in a particular nanofragment. In this case, the distances between any pair of neighboring atoms change insignificantly as compared to the interatomic distances (Fig. 3) and, as a consequence, the short-range order is retained.

Specifically, small structural transformations occur in the glass transition range [4] (upon softening of a glass and melting of a crystal) when the coordination numbers remain virtually unchanged. Uncorrelated small structural transformations that proceed in different nanofragments of the melt upon its rapid cooling lead to generation of internal stresses in the resulting glass. 


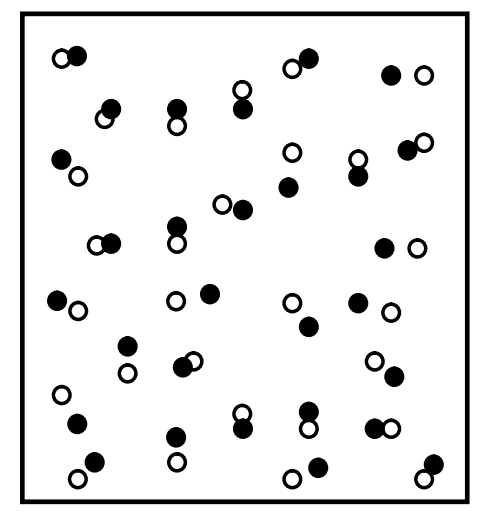

Figure 3. Schematic diagram illustrating a small structural transformation: Oinitial and $\bullet$ final positions of sites of atomic nuclei in a nanofragment.

The diversity of minima of the function $U_{M}^{(0)}(\mathbf{R})$ makes it possible to explain the possibility to vary properties of a material of the same chemical composition through preparation of its various modifications described by different quasiclosed ensembles.

Number $G^{(0)}$ of different quasiclosed ensembles satisfies the following relations :

$$
\ln G^{(0)}\left(t_{\max }, n, M\right) \leq \ln J(n, M)=\left(\alpha_{n} M+o(M)\right) \leq(B \times M+o(M))
$$

where constant $B$ is determined by the identity

$$
B \equiv \sup _{n} \alpha_{\mathbf{n}}
$$

It would not be particularly difficult to find the exact value of constant $B(8)$ if the solutions of equation (2) at $j=0$ were known for the systems of various chemical compositions. Since this is not the case, one has to use model approaches. In their frameworks it is possible to calculate numerical values of the parameter $\alpha_{\mathrm{n}}(5),(8)$ for specific systems. The results of such computations [4] support the following estimate:

$$
B \approx 3
$$

It is difficult to investigate thoroughly all configurations of the polyatomic system, because their number is exponentially large [see relationship (6)]. In this respect, it is necessary to use model approaches. A model based on the Gaussian distribution is convenient for constructing the statistical thermodynamics of melting and softening of nanoparticles. 


\section{Model spectrum for the description of configurational excitations}

In order to describe any equilibrium process, including the melting, in the framework of statistical thermodynamics [8], it is sufficient to know the time dependence of the statistical sum $Z$, which is uniquely determined by the temperature $T$, the energies $E_{j}$ of stationary quantum states, and the multiplicity $g_{j}$ of their degeneracy; that is

$$
Z=\sum_{j} g_{j} \exp \left(-E_{j} / k T\right)
$$

Here, $k$ is the Boltzmann constant and $E_{j}<E_{j+1 . .}$ This holds true for any system with a fixed number of particles from one elementary particle to inhomogeneous (specifically, multiphase) media.

The energy spectrum $\left\{E_{j}, g_{j}\right\}$ depends on the number $M$ of atoms. Since the energy spectrum of a macroscopic system $\left(M \geq 10^{20}\right)$ differs from that of a nanoparticle $\left(10^{8} \geq M \geq 10\right)$, the processes accompanying the melting of a macroscopic sample and a nanoparticle of the same chemical composition cannot not be completely identical. However, the specific features of these processes have much in common: in both cases, upon melting, the structure undergoes transformations, the system becomes microscopically labile, and the entropy and the internal energy increase abruptly.

In expression (10), the summation is performed over all possible configurations corresponding to relationship (6) and different vibrational states. By using the known analytical expression for the statistical sum of an oscillator [8], the sum of all terms associated with the $i$-th configuration $\mathbf{R}_{i}$ can be represented in the form $\tilde{g}_{i} \exp (E / k T)$.

This enables us to change over to the model partition function with due regard only for the configurations in which each configuration is included $\tilde{g}_{i} / \tilde{g}_{1}$ times, where $\tilde{g}_{1}$ corresponds to the configuration with a minimum energy ${ }^{2}$. The ratios $\tilde{g}_{i} / \tilde{g}_{1}$ actually take into account the role of the phonon subsystem in the melting.

The energies $E_{\mathrm{i}}=U_{M}^{(0)}\left(\mathbf{R}_{\mathrm{i}}\right)$ of the equilibrium configurations (2) of the polyatomic system are conveniently calculated per atom; that is,

$$
\varepsilon_{i}=E_{i} / M
$$

The spectrum of numerical values of the energies $\varepsilon_{\mathrm{i}}$ (fig.4) depends on the number of atoms $M$. The level with the minimum energy $E_{1}=0$ is assumed to be nondegenerate: $g_{1}=1$. The distribution of levels located in the energy range between $M \varepsilon_{g}$ and $M \varepsilon_{c}$ is approximated by the Gaussian distribution

\footnotetext{
2 In this case, the number of configurations can be determined from formula (6), in which the numerical value of the parameter $\alpha_{\mathrm{n}}$ changes insignificantly.
} 


$$
N(E) \sim \frac{\sigma\left(E-E_{0}\right)}{\gamma \sqrt{2 \pi M}} \exp \left\{M \alpha_{n}-0.5\left(\frac{E-M \mathrm{~h}}{\gamma \sqrt{M}}\right)^{2}\right\}
$$

Here, $E_{0}$ is the minimum energy necessary for transforming the system from the ground state into the excited state, $\sigma\left(E-E_{0}\right)$ is the step function,

$$
\begin{gathered}
\gamma=0.5\left(\varepsilon_{c}-\varepsilon_{g}\right) /\left(2 \alpha_{n}\right)^{1 / 2} \\
h=0.5\left(\varepsilon_{g}+\varepsilon_{c}\right) .
\end{gathered}
$$

From the distribution density $N(E)(12)$, we derive the following analytical expression for the statistical sum (10) (statistical integral)

$$
\mathrm{Z}=1+\left\{\exp M\left(\alpha_{n}-\frac{\mathrm{h}}{\mathrm{kT}}+\frac{0.5 \gamma^{2}}{(k T)^{2}}\right)\right\} \Phi\left(\frac{M \mathrm{~h}-E_{0}}{\gamma \sqrt{M}}-\frac{\gamma \sqrt{M}}{k T}\right) \mid \Phi\left(\frac{M \mathrm{~h}-E_{0}}{\gamma \sqrt{M}}\right),
$$

where $\Phi(x)=\frac{1}{\sqrt{2 \pi}} \int_{-\infty}^{x} \exp \left(-0.5 x^{2}\right) d x$ is the normal distribution function. The analytical expression (15) permits us to analyze not only the dependence of the statistical sum on the number $M$ of atoms. The corresponding dependences can be obtained for all quantities that are uniquely defined by the statistical sum $Z$. In particular, these are the melting temperature, the heat of melting, and the entropy of melting.

In the limit $M \rightarrow \infty$, the melting temperature $T_{m}$ is given by the expression [4]

$$
T_{m}=\left(\sqrt{\varepsilon_{g}}+\sqrt{\varepsilon_{c}}\right)^{2} / 4 k \alpha_{\mathbf{n}}
$$

In the same limit, the stepwise increments of the energy $\Delta \varepsilon$ (fig.4) and the entropy $\Delta s$ upon melting per atom are represented by the formulas

$$
\begin{gathered}
\Delta \varepsilon=\left(\varepsilon_{g} \varepsilon_{c}\right)^{1 / 2} \\
\Delta s=4 k \alpha_{n}\left(e_{g} e_{c}\right)^{1 / 2}\left(\sqrt{\varepsilon_{g}}+\sqrt{\varepsilon_{c}}\right)^{-2} \leq k \alpha_{\mathbf{n}} .
\end{gathered}
$$

It should be noted that, at the melting temperature $T_{m}$ the following inequalities are satisfied: 


$$
\varepsilon_{c} / k \alpha_{n} \geq T_{m} \geq \max \left\{\left(\varepsilon_{g} \varepsilon_{c}\right)^{1 / 2} / k \alpha_{n}, \varepsilon_{c} / 4 k \alpha_{\mathbf{n}}\right\}
$$

Before melting, the energy is minimum. Without loss of generality, this energy can be taken equal to zero. In the course of melting, there occurs a stepwise transition within the energy band $\left[\varepsilon_{g}, \varepsilon_{c}\right]$, which involves energies of the majority of the equilibrium configurations (fig.4). The width $\left(\varepsilon_{\mathrm{c}}-\varepsilon_{\mathrm{g}}\right)$ of this band is proportional to the root-mean-square deviation of the numerical values of the energies $\varepsilon$ of different configurations.

Upon melting, the structure undergoes transformations. Furthermore, the nanoparticle becomes labile. In particular, the nanoparticle changes in shape, because, after melting, there occur spontaneous transitions between the structural modifications with close energies in the energy band $\left[\varepsilon_{\mathrm{g}}, \varepsilon_{\mathrm{c}}\right]$ (fig.4).

The notion of the "melting of nanoclusters" has already been used [1]. It is obvious that the processes accompanying the melting of a macroscopic sample and a nanoparticle cannot not be completely identical. However, the specific features of these processes have much in common. In both cases, upon melting, the structure undergoes transformations, the system becomes labile, and the entropy and the internal energy increase abruptly.

According to relationships (16-18), the melting temperature $T_{m}$ can be described by one more expression

$$
T_{m}=\Delta \varepsilon / \Delta s,
$$

which coincides with the known expression that relates the heat $\Delta \varepsilon$, the entropy $\Delta s$, and the temperature $T_{m}$ of the transition [9]. Note that, in the case of the macroscopic system, the heat of melting $\Delta \varepsilon$ and the entropy of melting $\Delta s$ per atom in relationship (20) are independent of the number $M$ of atoms forming the macroscopic system, whereas the opposite situation is observed for the nanoparticle. The spectrum of energies $\varepsilon$ of equilibrium configurations can even radically change (fig.4).

For example, the two-atom system $(M=2)$ has only one equilibrium configuration $J(\mathbf{n}, 2)=1$ and the energy band $\left[\varepsilon_{g}, \varepsilon_{\mathrm{c}}\right]$ is absent. Since the structure should change upon melting (there should occur a transition from one equilibrium configuration to another equilibrium configuration), the melting of two-atom systems, in principle, is impossible.

However, the above concept is inapplicable to relatively small nanoclusters consisting of 13 atoms with $J(\mathbf{n}, 13)=1478$ different configurations (different energy levels) [10]. In this case, the energy spectrum can be described by the Gaussian distribution but with parameters different from those used for the macroscopic system.

Relationships (16)-(20) are also valid in the mesoregion where the number of atoms $M$ is larger than two but is not sufficient for the applicability of the asymptotic relationship (5), which allows one to estimate the number of equilibrium configurations of the macroscopic system. 
For nanoclusters, relationships (16) and (18) should contain the parameter $\tilde{\alpha}=\{\ln J(n, M)-1\}$ / $M$ instead of the parameter $\alpha_{n}$ involved in relationship (5). In the case of a two-atom system, we have the parameter $\tilde{\alpha}=\{\ln (J(n, 2)-1)\} / 2=-\infty$. For a nanocluster consisting of 13 atoms, we should use the parameter $\tilde{\alpha}=\{\ln (J(n, 13)-1)\} / 13 \approx 0,56$. The change in sign of the parameter $\tilde{\alpha}$ indicates that, for a relatively small number of atoms $M$, which satisfies the inequality $M>2$, the parameter $\tilde{\alpha}$ can turn out to be close to zero.

Therefore, the numerical value of the parameter in the mesoregion can appear to be considerably smaller than the parameter $\alpha_{n}$ involved in relationship (5) and used for calculating the melting temperature of the macroscopic sample according to relationship (16). This circumstance is responsible for the observed increase in the melting temperature of sufficiently small nanoparticles as compared to the macroscopic sample.

Since the parameter $\alpha_{\mathrm{n}}(\tilde{\alpha})$ is equal to the natural logarithm of the number of energy levels (equilibrium configurations) in the energy band $\left[\varepsilon_{g^{\prime}} \varepsilon_{c}\right]$ (fig.4), the product $k \alpha_{\mathrm{n}}$ in relationship (18) gives an estimate from above for the jump $\Delta s$ in the configurational entropy upon melting. In the case where $\alpha_{\mathrm{n}}(\tilde{\alpha}) \rightarrow 0$, we have $\Delta s \rightarrow 0$. In other words, the decrease in the parameter $\alpha_{\mathrm{n}}(\tilde{\alpha})$, according to relationship (18), leads to a decrease in the entropy of melting $\Delta s$ and, consequently, to an increase in the melting temperature $T_{m}$ in accordance with relationship (20).

Therefore, generally speaking, the melting temperature of macroscopic samples can be lower than the melting temperature of nanoclusters of the same chemical composition. Moreover, there are other specific features of melting of nanoparticles. Particularly, this refers to the melting temperature range $\Delta T_{m}$, which, unlike the corresponding range for macroscopic systems, is not a small quantity.

\section{On the temperature ranges of melting and softening}

A decrease in the number of atoms $M$ results in an increase in the temperature range $\Delta T_{m}$ of the phase transition [10]; that is,

$$
\Delta T_{m} \approx T_{m}(2 n \ln 10) /(M \tilde{\alpha})
$$

Here, $n$ is a natural number which, at the boundaries of this range, determines a low probability $p=10^{-n}$ that the system is in the liquid state before melting and in the solid state after melting, respectively.

At $n=3$ and $\tilde{\alpha}=\ln 2$, expression (21) can be represented in the form

$$
\frac{\Delta T_{m}}{T_{m}} M \approx 20 .
$$


As follows from formula (22), the quantities $\Delta T_{m}$ and $T_{m}$ for nanoparticles containing of the order of ten atoms are comparable in magnitude. By contrast, the temperature range $\Delta T_{m}$ for macroscopic systems $\left(M \sim 10^{23}\right)$ is nearly equal to zero.

According to relationship (22), the temperature range $\Delta T_{m}$ is relatively small

$$
\frac{\Delta T_{m}}{T_{m}}<<10^{-2} .
$$

only for systems involving a considerable number of atoms

$$
M>2000 .
$$

Otherwise, the quantity $\Delta T_{m}$ should not be ignored. Therefore, specific analogy can be drawn between the melting of nanoparticles and the softening of glasses.

Actually, the microscopic mechanism of glass softening is associated with the independent structural excitations in medium-range order nanofragments. Their initial structures, as a rule, are not exact copies of each other [7]. As a consequence, since the glass softening is a thermodynamically nonequilibrium irreversible process, it occurs in a specific temperature range $\Delta T_{g}$ rather than at a fixed temperature $T_{g}$.

Therefore, the glass softening and the transition of the nanoparticle to the microscopically labile state proceed in a particular temperature range rather than at a fixed temperature. Both these phenomena are responsible for the inelastic compliance of the system. This manifests itself as a viscous flow for macroscopic systems and a possibility of changing the shape due to the spontaneous transitions between different structural modifications with close energies within the band $\left[M \varepsilon_{g}, M \varepsilon_{c}\right]$ (Fig. 4) for nanoparticles.

The transition of the nanoparticle to the microscopically decrease in the temperature, the nanoparticle structure does not always revert to the initial state and, as in the case of the glass transition, one of the intermediate structures can turn out to be frozen. The question arises of whether the transition of the nanoparticle to the microscopically labile state in similar situations can be always interpreted as softening.

\section{Admissible states}

The freezing is a thermodynamically nonequilibrium process. The concept of "admissible states" [8] is useful when constructing the statistical thermodynamics of these processes. Not all states can occur for the observation time of a specific system. The states in which $\mathrm{SiO}_{2}$ has a crystalline form are inadmissible at low temperatures if the object was initially in the vitreous form: this compound in experiments at low temperatures does not transform into quartz 


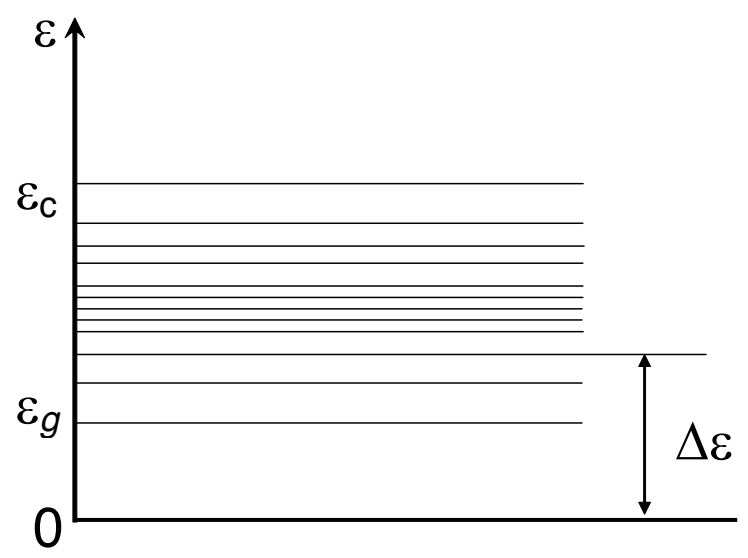

Figure 4. Spectrum of energies $\varepsilon$ per atom for equilibrium configurations of the polyatomic system.

during our life. We assume that all quantum states are admissible if they are not excluded according to the definition of the system or the chosen time scale [8].

Generally speaking, the spectrum of admissible states changes depending on the prehistory of the formation of the polyatomic system. The same holds true for numerical values of the parameters $\gamma, h, \alpha_{n}, \varepsilon_{c}$ and $\varepsilon_{g}(12)-(14)$ used for approximating the spectrum of admissible states. Let us assume that the initial state of the nanoparticle is in the band $\left[M \varepsilon_{g}, M \varepsilon_{c}\right]$ (Fig. 4) and the state with the minimum energy $\varepsilon=0$ is inadmissible ${ }^{3}$. Then, the spectrum of admissible states is approximated only by distribution (12). In this case, we have $\varepsilon_{g}=0$ and the heat of melting $\Delta \varepsilon$ according to relationship (17) is equal to zero. As a consequence, we cannot speak about the melting, even though the thermodynamically nonequilibrium transition to the microscopically labile state is possible upon heating. This transition occurs in the temperature range $\Delta T_{g}$ at the softening temperature $T_{g^{\prime}}$ which can be estimated as follows [4]:

$$
T_{g} \sim \gamma /\left(k\left(2 \alpha_{n}\right)^{1 / 2}\right)
$$

The softening temperature $T_{g}$ and the boundaries of the softening range $\Delta T_{g}$ are kinetic parameters and depend on the prehistory of the compound. By contrast, the melting is a thermodynamically equilibrium process, which proceeds at a fixed temperature. As a result of melting, the structure of the macroscopic system is radically changed from crystalline to disordered. Upon softening, the short-range order in the atomic arrangement is retained. Consequently, the structure undergoes an insignificant transformation. Correspondingly, a change in the internal energy is also small and, therefore, the notion of "the heat of softening" does not exist.

3 The lower level corresponds to the crystal. This level is excluded when constructing the statistical thermodynamics of glasses and glass-forming melts. This level is not used for describing the softening and glass transition. 
In the case of macroscopic systems, the above criteria allow us to distinguish rather simply the melting from softening. By contrast, not all transitions of the nanoparticle from the solid state to the microscopically labile state can be uniquely interpreted as melting or softening, because there are intermediate situations.

Specifically, these situations involve a thermodynamically equilibrium transition that results in an insignificant change in the structure (the short-range order is retained). In this case, the jump $\Delta \varepsilon$ (17) in the internal energy

$$
\Delta \varepsilon<10^{-2} \mathrm{eV}
$$

is small as compared to the heat of melting of the macroscopic system of the same chemical composition per atom. It is unlikely that this transition should be treated as melting. However, since the transition under consideration is thermodynamically equilibrium, it is not advisable to identify this transition with the softening.

Eventually, it is important to know the spectrum of admissible states and the parameters $\gamma$, $h, \alpha_{n}, \varepsilon_{c}$ and $\varepsilon_{g}(12)-(14)$. This makes it possible to reveal the energy characteristics of elementary structural excitations of the nanoparticle. In particular, the transitions between the equilibrium configurations closest in energy in the band $\left[M \varepsilon_{g^{\prime}} M \varepsilon_{c}\right]$ (Fig. 4) are accompanied by the absorption (emission) of longwavelength photons [4]. Their frequencies can be estimated from the relationship

$$
v[H z] \sim 2.10^{14} M\left(\varepsilon_{c}-\varepsilon_{g}\right) \exp \left(-\alpha_{\mathbf{n}} M\right)
$$

According to relationship (27), at $M=100, \alpha_{\mathrm{n}}=0.1$, and $\left(\varepsilon_{c}-\varepsilon_{g}\right)=0.1 \mathrm{eV}$, we obtain a frequency $\sim 10^{11} \mathrm{~Hz}$, which corresponds to the microwave range of electromagnetic radiation.

It follows from relationship (27) that the elementary structural excitations of nanoparticles can be attended by emission (absorption) of photons with frequencies in the microwave, radiofrequency, and low-frequency ranges. It was experimentally demonstrated that the microwave radiation can accelerate chemical reactions by a factor of several tens and even several hundreds [11]. The microscopic mechanism of this phenomenon is not clearly understood. However, it is unquestionable that this mechanism is not reduced only to heating.

\section{Conclusions}

A detailed (on the microscopic level) analysis of the processes that occur upon transition of nanoparticles to the microscopically labile state stimulates consideration of a number of fundamental problems. Their solution provides a deeper insight into the specific features of the nanoworld. Indeed, the melting and softening cannot proceed in the absence of an exponentially large number of various structural modifications (6). However, up to now, most 
attention has been focused on relatively stable structures. The number of these structures for a nanocluster composed of 13 atoms is considerably smaller than 1478 [10].

The other structural modifications have not been adequately investigated, even though their role is important not only for the transition of the nanoparticle to the microscopically labile state. Many chemical transformations represent a sequence of transitions between unstable modifications. They should be taken into account when developing methods for synthesizing nanostructured functional materials with controlled properties.

The majority of nanoparticles of the same chemical composition exhibit similar additive properties. It is sufficient to investigate one of these nanoparticles in order to judge the properties of the other nanoparticles. However, there are "special" nanoparticles. Their properties differ noticeably from the statistical-mean properties and can be unique as compared to those of macromolecules and compact materials. Owing to this uniqueness, it is these nanoparticles that are of most interest for the nanotechnology.

Certainly, special nanoparticles are small in number. Among an exponentially large number (6) of various nanoparticles of the same chemical composition, the choice of a special nanoparticle with required properties is not a simple problem. Moreover, it is not a priori known whether there exists this nanoparticle in principle.

Furthermore, the potential possibility of occurring a large number of similar structural modifications different from the required modification complicates the reproduction of an exact copy [7] of the nanoparticle under consideration. That is why the reproducibility is one of the key problems of the nanotechnology.

In actual practice, the special nanoparticle cannot be synthesized in an accidental way. The traditional methods are not necessarily effective because the vast majority of the currently used chemical reactions belong to "disorganized" reactions in which particles (molecules, ions, atoms, radicals) react as a result of random collisions.

In order to solve many problems of nanotechnologies, it is required to control chemical processes on the microscopic level. It is necessary to design nontraditional methods based on nonequilibrium processes [12].

In particular, it seems likely that the use of electromagnetic radiation holds considerable promise. The methods of microwave chemistry have already been used to produce nanopowders [11].

The problem associated with the synthesis of special nanoparticles would be completely solved if the technique for preparing any controlled equilibrium configuration $\mathbf{R}_{\mathbf{i}}$ of atomic nuclei (Fig. 1) would be developed. In general, for this purpose, it is necessary to know how to operate not with one atom but with many atoms according to a special program [13], which represents algorithmic information. It should be noted that the information aspect of microscopic processes [14] has come under the scrutiny of science only in recent years. 


\title{
Author details
}

\author{
M. D. Bal'makov*
}

Address all correspondence to: Balmak1@yandex.ru

Faculty of Chemistry, St. Petersburg State University, Staryi Peterhof, Universitetskii Pr. 26, St. Petersburg, Russia

\section{References}

[1] Shvartsburg, A. A, \& Jarrold, M. F. Solid Clusters Above the Bulk Melting Point, Phys. Rev. Lett., (2000). , 85(12), 2530-2532.

[2] Born, M, \& Oppenheimer, J. R. Zur Quantentheorie der Molekeln, Ann. Phys. (Leipzig), (1927). , 84(20), 457-483.

[3] Nikitin, E. E. Teoriya elementarnykh atomno-molekulyarnykh protsessov v gazakh (The Theory of Elementary Atomic-Molecular Processes in Gases), Moscow: Khimiya, (1970). , 1-456.

[4] Bal'makov M.D., Stekloobraznoe sostoyanie veshchetsva (The Vitreous State of Matter), St. Petersburg: St. Petersburg State University, (1996). , 1-184.

[5] Landau, L. D, \& Lifshitz, E. M. Kvantovaya mekhanika. Nerelyativistskaya teoriya (Quantum Mechanics: Nonrelativistic Theory), Moscow: Fizmatgiz, (1963). , 1-704.

[6] Wright, A. F, \& Leadbetter, A. J. The Structure of the $\beta$-Cristobalite Phases of $\mathrm{SiO}_{2}$ and $\mathrm{AlPO}_{4}$, Philos. Mag., (1975). , 31(6), 1391-1401.

[7] Bal'makov M.D., Information Capacity of Condensed Systems, Semiconductors and semimetals. Elsevier academic press, (2004). , 79, 1-14.

[8] Kitel Ch., Elementary Statistical Physics, New York: Wiley, 1958. Translated under the title Statisticheskaya termodinamika, Moscow: Nauka, (1977). , 1-336.

[9] Landau, L. D, \& Lifshitz, E. M. Statisticheskaya fizika Moscow: Nauka, (1964). Translated under the title Course of Theoretical Physics, Statistical Physics, Oxford: Butterworth-Heinemann, 1968., 5, 1-568.

[10] Bal'makov M.D., On the Melting Temperature of Nanoparticles, Fiz. Khim. Stekla, (2008). Glass Phys. Chem. (Engl. transl.), 2008, vol. 34, no. 1, pp. 110-112]., 34(1), 140-143.

[11] Berdonosov, S. S. Microwave Chemistry, Soros. Obraz. Zh., (2001). , 7(1), 32-38.

[12] Tver'yanovich A.S., Kim, D., Borisov, E.N., et al., Lazernye i mikrovolnovye metody polucheniya i modifikatsii khal'kogenidnykh poluprovodnikovykh materialov (Laser 
and Microwave Methods for Preparing Chalcogenide Semiconductor Materials), St. Petersburg: BKhV, (2006). , 1-99.

[13] Bal'makov M.D., Algorithmic Approach to the Problem of Control of the Structure of the Nanostate of Matter, Vestn. St. Peterb. Univ., Ser. 4: Fiz., Khim., (2005). (2), 51-59.

[14] Kadomtsev, B. B. Dinamika i informatsiya (Dynamics and Information), Moscow : Usp. Fiz. Nauk Publishing,(1997). in Russian]. 

Chapter 27

\title{
Decoding the Building Blocks of Life from the Perspective of Quantum Information
}

\author{
Rodolfo O. Esquivel, Moyocoyani Molina-Espíritu, \\ Frank Salas, Catalina Soriano, Carolina Barrientos, \\ Jesús S. Dehesa and José A. Dobado
}

Additional information is available at the end of the chapter

http://dx.doi.org/10.5772/55160

\section{Introduction}

Physical theories often start out as theories which only embrace essential features of the macroscopic world, where their predictions depend on certain parameters that have to be either assumed or taken from experiments; as a result these parameters cannot be predicted by such theories. To understand why the parameters have the values they do, we have to go one level deeper - typically to smaller scales where the easiest processes to study are the ones at the lowest level. When the deeper level reduces the number of unknown parameters, we consider the theory to be complete and satisfactory. The level below conventional molecular biology is spanned by atomic and molecular structure and by quantum dynamics. However, it is also true that at the lowest level it becomes very difficult to grasp all the features of the molecular processes that occur in living systems such that the complexity of the numerous parameters that are involved make the endeavour a very intricate one. Information theory provides a powerful framework for extracting essential features of complicated processes of life, and then analyzing them in a systematic manner. In connection to the latter, quantum information biology is a new field of scientific inquiry in which information-theoretical tools and concepts are permitting to get insight into some of the most basic and yet unsolved questions of molecular biology.

Chirality is often glossed over in theoretical or experimental discussions concerning the origin of life, but the ubiquity of homochiral building blocks in known biological systems demands explanation. Information theory can provide a quantitative framework for understanding the role of chirality in biology. So far it has been thought that the genetic code is "unknowable" 
by considering DNA as a string of letters only (... ATTGCAAGC...) and likewise by considering proteins as strings of identifiers (... DYRFQ...), we believe that this particular conclusion might be probably wrong because it entirely fails to consider the information content of the molecular structures themselves and their conformations.

On the other hand, according to molecular biology, living systems consist of building blocks which are encoded in nucleic acids (DNA and RNA) and proteins, which possess complex patterns that control all biological functions. Despite the fact that natural processes select particular building blocks which possess chemical simplicity (for easy availability and quick synthesis) and functional ability (for implementing the desired tasks), the most intriguing question resides in the amino acid selectivity towards a specific codon/anticodon. The universal triplet genetic code has considerable and non-uniform degeneracy, with 64 codons carrying 21 signals (including Stop) as shown in Table 1. Although there is a rough rule of similar codons for similar amino acids, no clear pattern is obvious.

Information theory of quantum many-body systems is at the borderline of the development of physical sciences, in which major areas of research are interconnected, i.e., physics, mathematics, chemistry, and biology. Therefore, there is an inherent interest for applying theoreticinformation ideas and methodologies to chemical, mesoscopic and biological systems along with the processes they exert. On the other hand, in recent years there has been an increasing interest in applying complexity concepts to study physical, chemical and biological phenomena. Complexity measures are understood as general indicators of pattern, structure, and correlation in systems or processes. Several alternative mathematical notions have been proposed for quantifying the concepts of complexity and information, including the Kolmogorov-Chaitin or algorithmic information theory (Kolmogorov, 1965; Chaitin, 1966), the classical information theory of Shannon and Weaver (Shannon \& Weaver, 1948), Fisher information (Fisher, 1925; Frieden, 2004), and the logical (Bennet, 1988) and the thermodynamical (Lloyd \& Pagels, 1988) depths, among others. Some of them share rigorous connections with others as well as with Bayes and information theory (Vitanyi \& Li, 2000). The term complexity has been applied with different meanings: algorithmic, geometrical, computational, stochastic, effective, statistical, and structural among others and it has been employed in many fields: dynamical systems, disordered systems, spatial patterns, language, multielectronic systems, cellular automata, neuronal networks, self-organization, DNA analyses, social sciences, among others (Shalizi et al., 2004; Rosso et al., 2003; Chatzisavvas et al., 2005; Borgoo et al., 2007).

The definition of complexity is not unique, its quantitative characterization has been an important subject of research and it has received considerable attention (Feldman \& Crutchfield, 1998; Lamberti et al., 2004). The usefulness of each definition depends on the type of system or process under study, the level of the description, and the scale of the interactions among either elementary particles, atoms, molecules, biological systems, etc.. Fundamental concepts such as uncertainty or randomness are frequently employed in the definitions of complexity, although some other concepts like clustering, order, localization or organization might be also important for characterizing the complexity of systems or processes. It is not clear how the aforementioned concepts might intervene in the definitions so as to quantita- 
tively assess the complexity of the system. However, recent proposals have formulated this quantity as a product of two factors, taking into account order/disequilibrium and delocalization/uncertainty. This is the case of the definition of López-Mancini-Calbet (LMC) shape complexity [9-12] that, like others, satisfies the boundary conditions by reaching its minimal value in the extreme ordered and disordered limits. The LMC complexity measure has been criticized (Anteonodo \& Plastino, 1996), modified (Catalán et al., 2002; Martin et al., 2003) and generalized (López-Ruiz, 2005) leading to a useful estimator which satisfies several desirable properties of invariance under scaling transfromations, translation, and replication (Yamano, 2004; Yamano, 1995). The utility of this improved complexity has been verified in many fields [8] and allows reliable detection of periodic, quasiperiodic, linear stochastic, and chaotic dynamics (Yamano, 2004; López-Ruiz et al., 1995; Yamano, 1995). The LMC measure is constructed as the product of two important information-theoretic quantities (see below): the so-called disequilibrium D (also known as self-similarity (Carbó-Dorca et al., 1980) or information energy Onicescu, 1996), which quantifies the departure of the probability density from uniformity (Catalán et al., 2002; Martinet al., 2003) (equiprobability) and the Shannon entropy $S$, which is a general measure of randomness/uncertainty of the probability density (Shannon \& Weaver, 1948), and quantifies the departure of the probability density from localizability. Both global quantities are closely related to the measure of spread of a probability distribution.

The Fisher-Shannon product FS has been employed as a measure of atomic correlation (Romera \& Dehesa, 2004) and also defined as a statistical complexity measure (Angulo et al., 2008a; Sen et al., 2007a). The product of the power entropy $J$-explicitly defined in terms of the Shannon entropy (see below)- and the Fisher information measure, I, combine both the global character (depending on the distribution as a whole) and the local one (in terms of the gradient of the distribution), to preserve the general complexity properties. As compared to the $L M C$ complexity, aside of the explicit dependence on the Shannon entropy which serves to measure the uncertainty (localizability) of the distribution, the Fisher-Shannon complexity replaces the disequilibrium global factor $D$ by the Fisher local one to quantify the departure of the probability density from disorder (Fisher, 1925; Frieden, 2004) of a given system through the gradient of the distribution.

The Fisher information $I$ itself plays a fundamental role in different physical problems, such as the derivation of the non-relativistic quantum-mechanical equations by means of the minimum I principle (Fisher, 1925; Frieden, 2004), as well as the time-independent Kohn-Sham equations and the time-dependent Euler equation (Nagy, 2003; Nalewajski, 2003). More recently, the Fisher information has been employed also as an intrinsic accuracy measure for specific atomic models and densities (Nagy \& Sen, 2006; Sen et al., 2007b)), as well as for general quantum-mechanical central potentials (Romera et al. 2006; Dehesa et al., 2007). The concept of phase-space Fisher information has been analyzed for hydrogenlike atoms and the isotropic harmonic oscillator (Hornyak \& Nagy, 2007), where both position and momentum variables are included. Several applications concern atomic distributions in position and momentum spaces have been performed where the $F S$ complexity is shown to provide relevant information on atomic shell structure and ionization processes (Angulo et al., 2008a; Sen et al., 2007a; Angulo \& Antolín, 2008b; Antolín \& Angulo, 2009). 
In line with the aforementioned developments we have undertaken multidisciplinary research projects so as to employ IT at different levels, classical (Shannon, Fisher, complexity, etc) and quantum (von Neumann and other entanglement measures) on a variety of chemical processes, organic and nanostructured molecules. Recently, significant advances in chemistry have been achieved by use of Shannon entropies through the localized/delocalized features of the electron distributions allowing a phenomenological description of the course of elementary chemical reactions by revealing important chemical regions that are not present in the energy profile such as the ones in which bond forming and bond breaking occur (Esquivel et al., 2009). Further, the synchronous reaction mechanism of a $\mathrm{S}_{\mathrm{N}} 2$ type chemical reaction and the nonsynchronous mechanistic behavior of the simplest hydrogenic abstraction reaction were predicted by use of Shannon entropies analysis (Esquivel et al., 2010a). In addition, a recent study on the three-center insertion reaction of silylene has shown that the informationtheoretical measures provide evidence to support the concept of a continuum of transient of Zewail and Polanyi for the transition state rather than a single state, which is also in agreement with other analyses (Esquivel et al., 2010b). While the Shannon entropy has remained the major tool in IT, there have been numerous applications of Fisher information through the "narrowness/disorder" features of electron densities in conjugated spaces. Thus, in chemical reactions the Fisher measure has been employed to analyze its local features (Esquivel et al., 2010c) and also to study the steric effect of the conformational barrier of ethane (Esquivel et al., 2011a). Complexity of the physical, chemical and biological systems is a topic of great contemporary interest. The quantification of complexity of real systems is a formidable task, although various single and composite information-theoretic measures have been proposed. For instance, Shannon entropy $(S)$ and the Fisher information measure $(I)$ of the probability distributions are becoming increasingly important tools of scientific analysis in a variety of disciplines. Overall, these studies suggest that both $S$ and $I$ can be used as complementary tools to describe the information behavior, pattern, or complexity of physical and chemical systems and the electronic processes involving them. Besides, the disequilibrium $(D)$, defined as the expectation value of the probability density is yet another complementary tool to study complexity since it measures its departure from equiprobability. Thus, measuring the complexity of atoms and molecules represents an interesting area of contemporary research which has roots in information theory (Angulo et al., 2010d). In particular, complexity measures defined as products of $S$ and $D$ or $S$ and $I$ have proven useful to analyze complexity features such as order, uncertainty and pattern of molecular systems (Esquivel et al., 2010f) and chemical processes (Esquivel et al., 2011b). On the other hand, the most interesting technological implications of quantum mechanics are based on the notion of entanglement, which is the essential ingredient for the technological implementations that are foreseen in the XXI century. Up to now it remains an open question whether entanglement can be realized with molecules or not and hence it is evident that the new quantum techniques enter the sphere of chemical interest. Generally speaking, entanglement shows up in cases where a former unit dissociates into simpler sub-systems, the corresponding processes are known quite well in chemistry. Although information entropies have been employed in quantum chemistry, applications of entanglement measures in chemical systems are very scarce. Recently, von Neumann measures in Hilbert space have been proposed and applied to small chemical systems (Carrera et al. 
2010, Flores-Gallegos and Esquivel, 2008), showing than entanglement can be realized in molecules. For nanostructures, we have been able to show that IT measures can be successfully employed to analyse the growing behaviour of PAMAM dendrimers supporting the densecore model against the hollow-core one (Esquivel et al., 2009b, 2010g, 2011c).

In the Chapter we will present arguments based on the information content of L- and Daminoacids to explain the biological preference toward homochirality. Besides, we present benchmark results for the information content of codons and aminoacids based on information-theoretical measures and statistical complexity factors which allow to elucidate the coding links between these building blocks and their selectivity.

\section{Information-theoretical measures and complexities}

In the independent-particle approximation, the total density distribution in a molecule is a sum of contribution from the electrons in each of the occupied orbitals. This is the case in both $r$-space and $p$-space, position and momentum respectively. In momentum space, the total electron density, $(p)$, is obtained through the molecular momentals (momentum-space orbitals) $\phi_{i}(p)$, and similarly for the position-space density, $\rho(r)$, through the molecular position-space orbitals $\phi_{i}(r)$. The momentals can be obtained by three-dimensional Fourier transformation of the corresponding orbitals (and conversely)

$$
\varphi_{i}(\mathbf{p})=(2 \pi)^{-3 / 2} \int d \mathbf{r e x p}(-i \mathbf{p} \cdot \mathbf{r}) \phi_{i}(\mathbf{r})
$$

Standard procedures for the Fourier transformation of position space orbitals generated by abinitio methods have been described (Rawlings \& Davidson, 1985). The orbitals employed in ab-initio methods are linear combinations of atomic basis functions and since analytic expressions are known for the Fourier transforms of such basis functions (Kaijser \& Smith, 1997), the transformation of the total molecular electronic wavefunction from position to momentum space is computationally straightforward (Kohout, 2007).

As we mentioned in the introduction, the $L M C$ complexity is defined through the product of two relevant information-theoretic measures. So that, for a given probability density in position space, $\rho(r)$, the $C(L M C)$ complexity is given by (Feldman \& Crutchfield, 1998; Lamberti et al., 2004; Anteonodo \& Plastino, 1996; Catalán et al., 2002; Martin et al., 2003):

$$
C_{r}(L M C)=D_{r} e^{S_{r}}=D_{r} L_{r}
$$

where $D r$ is the disequilibrium (Carbó-Dorca et al., 1980; Onicescu, 1966) 


$$
D_{r}=\int \rho^{2}(\mathbf{r}) d \mathbf{r}
$$

and $S$ is the Shannon entropy (Shannon \& Weaver, 1949)

$$
S_{r}=-\int \rho(\mathbf{r}) \ln \rho(\mathbf{r}) d^{3} \mathbf{r}
$$

from which the exponential entropy $L_{r}=e^{S_{r}}$ is defined. Similar expressions for the LMC complexity measure in the conjugated momentum space might be defined for a distribution $\gamma(p)$

It is important to mention that the $L M C$ complexity of a system must comply with the following lower bound (López-Rosa et al., 2009):

$$
C_{p}(L M C)=D_{p} e^{S p}=D_{p} L_{p}
$$

The FS complexity in position space, $\mathrm{Cr}(F S)$, is defined in terms of the product of the Fisher information (Fisher, 1925; Frieden, 2004)

$$
C(L M C) \geq 1
$$

and the power entropy (Angulo et al. 2008a; Sen et al., 2007a) in position space, $J r$

$$
I_{r}=\int \rho(\mathbf{r})|\vec{\nabla} \ln \rho(\mathbf{r})|^{2} d^{3} \mathbf{r}
$$

which depends on the Shannon entropy defined above. So that, the FS complexity in position space is given by

$$
J_{r}=\frac{1}{2 \pi e} e^{\frac{2}{3} S_{r}}
$$

and similarly

$$
C_{r}(F S)=I_{r} \cdot J_{r}
$$

in momentum space. 
Let us remark that the factors in the power Shannon entropy $J$ are chosen to preserve the invariance under scaling transformations, as well as the rigorous relationship (Dembo et al., 1991).

$$
C_{p}(F S)=I_{p} \cdot J_{p}
$$

with $n$ being the space dimensionality, thus providing a universal lower bound to FS complexity. The definition in Eq. (8) corresponds to the particular case $n=3$, the exponent containing a factor $2 / n$ for arbitrary dimensionality.

It is worthwhile noting that the aforementioned inequalities remain valid for distributions normalized to unity, which is the choice that it is employed throughout this work for the 3dimensional molecular case.

Aside of the analysis of the position and momentum information measures, we have considered it useful to study these magnitudes in the product $r p$-space, characterized by the probability density $f(r, p)=\rho(r) \gamma(p)$, where the complexity measures are defined as

$$
C(F S) \geq n
$$

and

$$
C_{r p}(L M C)=D_{r p} L_{r p}=C_{r}(L M C) C_{p}(L M C),
$$

From the above two equations, it is clear that the features and patterns of both LMC and FS complexity measures in the product space will be determined by those of each conjugated space. However, the numerical analyses carried out in the next section, reveal that the the momentum space contribution plays a more relevant role as compared to the one in position space.

We have also evaluated some reactivity parameters that may be useful to analyze the chemical reactivity of the aminoacids. So that, we have computed several reactivity properties such as the ionization potential (IP), the hardness $(\eta)$ and the electrophilicity index $(\omega)$. These properties were obtained at the Hartree-Fock level of theory (HF) in order to employ the Koopmans' theorem (Koopmans, 1933; Janak, 1978), for relating the first vertical ionization energy and the electron affinity to the HOMO and LUMO energies, which are necessary to calculate the conceptual DFT properties. Parr and Pearson, proposed a quantitative definition of hardness (ๆ) within conceptual DFT (Parr \& Yang, 1989):

$$
C_{r p}(F S)=I_{r p} J_{r p}=C_{r}(F S) C_{p}(F S)
$$


where $\varepsilon$ denotes the frontier molecular orbital energies and $S$ stands for the softness of the system. It is worth mentioning that the factor 1/2 in Eq. (14) was put originally to make the hardness definition symmetrical with respect to the chemical potential (Parr \& Pearson, 1983)

$$
\eta=\frac{1}{2 S} \approx \frac{\varepsilon_{L U M O}-\varepsilon_{H O M O}}{2}
$$

although it has been recently disowned (Ayer et al. 2006: Pearson, 1995). In general terms, the chemical hardness and softness are good descriptors of chemical reactivity. The former has been employed (Ayer et al. 2006: Pearson, 1995; Geerlings et al., 2003) as a measure of the reactivity of a molecule in the sense of the resistance to changes in the electron distribution of the system, i.e., molecules with larger values of $\eta$ are interpreted as being the least reactive ones. In contrast, the $S$ index quantifies the polarizability of the molecule (Ghanty \& Ghosh, 1993; Roy et al., 1994; Hati \& Datta, 1994; Simon-Manso \& Fuentealba, 1998) and hence soft molecules are more polarizable and possess predisposition to acquire additional electronic charge (Chattaraj et al., 2006). The chemical hardness $\eta$ is a central quantity for use in the study of reactivity through the hard and soft acids and bases principle (Pearson, 1963; Pearson, 1973; Pearson, 1997).

The electrophilicity index (Parr et al., 1999), $\omega$, allows a quantitative classification of the global electrophilic nature of a molecule within a relative scale. Electrophilicity index of a system in terms of its chemical potential and hardness is given by the expression

$$
\mu=\left(\frac{\partial E}{\partial N}\right)_{v(r)}=\frac{\varepsilon_{L U M O}+\varepsilon_{H O M O}}{2}
$$

The electrophilicity is also a good descriptor of chemical reactivity, which quantifies the global electrophilic power of the molecules -predisposition to acquire an additional electronic charge(Parr \& Yang, 1989).

\section{Aminoacids}

The exact origin of homochirality is one of the great unanswered questions in evolutionary science; such that, the homochirality in molecules has remained as a mystery for many years ago, since Pasteur. Any biological system is mostly composed of homochiral molecules; therefore, the most well-known examples of homochirality is the fact that natural proteins are composed of L-amino acids, whereas nucleic acids (RNA or DNA) are composed of D-sugars (Root-Bernstein, 2007; Werner, 2009; Viedma et al., 2008). The reason for this behavior continues to be a mystery. Until today not satisfactory explanations have been provided regarding the origin of the homochirality of biological systems; since, the homochirality of the amino acids is critical to their function in the proteins. If proteins (with L-aminoacids) had a 
non-homochiral behavior (with few D-enantiomers in random positions) they would not present biological functionality It is interesting to mention that L-aminoacids can be synthesized by use of specific enzymes, however, in prebiotic life these processes remain unknown. The same problem exists for sugars which have the D configuration. (Hein and Blackmond, 2011; Zehnacker et al., 2008; Nanda and DeGrado, 2004).

On the other hand, the natural amino acids contain one or more asymmetric carbon atoms, except the glycine. Therefore, the molecules are two nonsuperposable mirror images of each other; i.e., representing right-handed ( $\mathrm{D}$ enantiomer) and left-handed (L enantiomer) structures. It is considered that the equal amounts of D- and L- amino acids existed on primal earth before the emergence of life. Although the chemical and physical properties of L-and D amino acids are extremely similar except for their optical character, the reason of the exclusion of Damino acids and why all living organisms are now composed predominantly of L-amino acids are not well-known: however, the homochirality is essential for the development and maintenance of life (Breslow, 2011; Fujii et al., 2010; Tamura, 2008). The essential property of $\alpha$ aminoacids is to form linear polymers capable of folding into 3-dimensional structures, which form catalytic active sites that are essential for life. In the procees, aminoacids behave as hetero bifunctional molecules, forming polymers via head to tail linkage. In contrast, industrial nylons are often prepared from pairs of homo-bifunctional molecules (such as diamines and dicarboxylic acids), the use of a single molecule containing both linkable functionalities is somewhat simpler (Cleaves, 2010; Weber and Miller, 1981; Hicks, 2002).

The concept of chirality in chemistry is of paramount interest because living systems are formed of chiral molecules of biochemistry is chiral (Proteins, DNA, amino acids, sugars and many natural products such as steroids, hormones, and pheromones possess chirality). Indeed, amino acids are largely found to be homochiral (Stryer, 1995) in the L form. On the other hand, most biological receptors and membranes are chiral, many drugs, herbicides, pesticides and other biological agents must themselves possess chirality. Synthetic processes ordinarily produce a 50:50 (racemic) mixture of left-handed and right-handed molecules (so-called enantiomers), and often the two enantiomers behave differently in a biological system.

On the other hand, a major topic of research has been to study the origin of homochirality. In this respect, biomembranes have played an important role for the homochiraility of biopolymers. One of the most intriguing problems in life sciences is the mechanism of symmetry breaking. Many theories have been proposed on these topics and in the attempt to explain the amplification of a first enantiomeric imbalance to the enantiopurity of biomolecules (Bombelli et al., 2004). In all theories on symmetry breaking and on enantiomeric excess amplification little attention has been paid to the possible role of biomembranes, or of simple self-aggregated systems that may have acted as primitive biomembranes. Nevertheless, it is possible that amphiphilic boundary systems, which are considered by many scientists as intimately connected to the emergence and the development of life (Avalos et al. 2000; Bachmann et al., 1992), had played a role in the history of homochirality in virtue of recognition and compartmentalization phenomena (Menger and Angelova, 1998). In general, the major reason for the different recognition of two enantiomers by biological cells is the homochirality of biomolecules such as L-amino acids and D-sugars. The diastereomeric interaction between the 
enantiomers of a bioactive compound and the receptor formed from a chiral protein can cause different physiological responses. The production technology of enantiomerically enriched bioactive compounds one of the most important topics in chemistry. There is great interest in how and when biomolecules achieved high enantioenrichment, including the origin of chirality from the standpoint of chiral chemistry (Zehnacker et al., 2008; Breslow, 2011; Fujii et al., 2010; Tamura, 2008; Arnett and Thompson, 1981)

\subsection{Physical and information-theoretical properties}

Figure 1 illustrates a Venn diagram (Livingstone \& Barton, 1993; Betts \& Russell, 2003) which is contained within a boundary that symbolizes the universal set of 20 common amino acids (in one letter code). The amino acids that possess the dominant properties-hydrophobic, polar and small (<60 ^̊3) - are defined by their set boundaries. Subsets contain amino acids with the properties aliphatic (branched sidechain non-polar), aromatic, charged, positive, negative and tiny $(<35 \AA 3)$. Shaded areas define sets of properties possessed by none of the common amino acids. For instance, cysteine occurs at two different positions in the Venn diagram. When participating in a disulphide bridge $\left(\mathrm{C}_{\mathrm{S}-\mathrm{S}}\right)$, cysteine exhibits the properties 'hydrophobic' and 'small'. In addition to these properties, the reduced form $\left(\mathrm{C}_{\mathrm{S}-\mathrm{H}}\right)$ shows polar character and fits the criteria for membership of the 'tiny' set. Hence, the Venn diagram (Figure l) assigns multiple properties to each amino acid; thus lysine has the property hydrophobic by virtue of its long sidechain as well as the properties polar, positive and charged. Alternative property tables may also be defined. For example, the amino acids might simply be grouped into nonintersecting sets labelled, hydrophobic, charged and neutral.

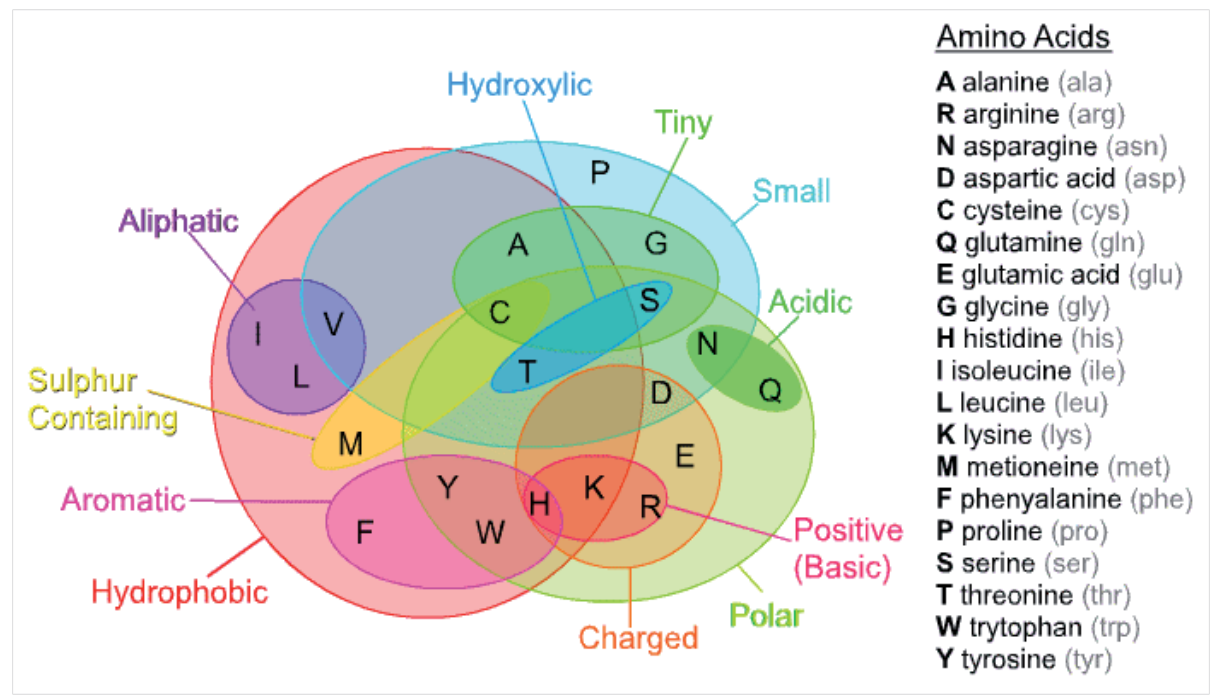

Figure 1. Venn diagram (Livingstone \& Barton, 1993; Betts \& Russell, 2003) of boundaries that symbolizes the universal set of 20 common amino acids (b). The Venn diagram in Fig. (1a) may be simply encoded as the property table or index shown here where the rows define properties and the columns refer to each amino acid. 
In order to perform a theoretical-information analysis of L- and D-aminoacids we have employed the corresponfing L-enantiomers reported in the Protein Data Bank (PDB), which provide a standard representation for macromolecular structure data derived from X-ray diffraction and NMR studies. In a second stage, the D-type enantiomers were obtained from the L-aminoacids by interchanging the corresponding functional groups (carboxyl and amino) of the $\alpha$-carbon so as to represent the D-configuration of the chiral center, provided that steric impediments are taken into account. The latter is achieved by employing the Ramachandran (Ramachandran et al, 1963) map, which represent the phi-psi torsion angles for all residues in the aminoacid structure to avoid the steric hindrance. Hence, the backbone of all of the studied aminoacids represent possible biological structures within the allowed regions of the Ramachandran. In the third stage, an electronic structure optimization of the geometry was performed on all the enantiomers for the twenty essential aminoacids so as to obtain structures of minimum energy which preserve the backbone (see above). In the last stage, all of the information-theoretic measures were calculated by use of a suite of programs which have been discussed elsewhere (Esquivel et al., 2012).

In Figures 2 through 4 we have depicted some selected information-theoretical measures and complexities in position space versus the number of electrons and the energy. For instance, it might be observed from Fig. 2 that the Shannon entropy increases with the number of electrons so that interesting properties can be observed, e.g., the aromatic ones possess more delocalized densities as the rest of the aminoacids (see Figure 1B) which confer specific chemical properties. On the other hand, the disequilibrium diminishes as the number of electron increases (see Fig. 2), which can be related to the chemical stability of the aminoacids, e.g., cysteine and metionine show the larger values (see Fig. 2) which is in agreement with the biological evidence in that both molecules play mutiple functions in proteins, chemical as well as structural, conferring the higher reactivity that is recognized to both molecules. In contrast, aromatic aminoacids (see Fig 1B) are the least reactive, which is in agreement with the lower disequilibrium values that are observed form Fig 2.

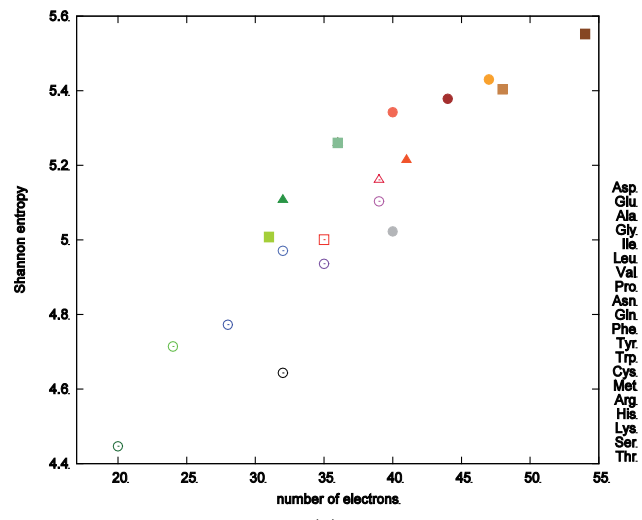

(a)

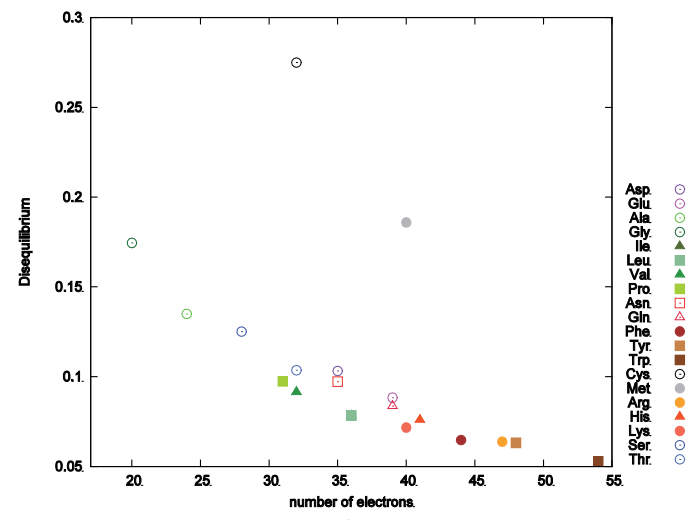

(b)

Figure 2. Shannon entropy (left) and disequilibrum (right), both in position space, versus the number of electrons, for the set of 20 aminoacids. 


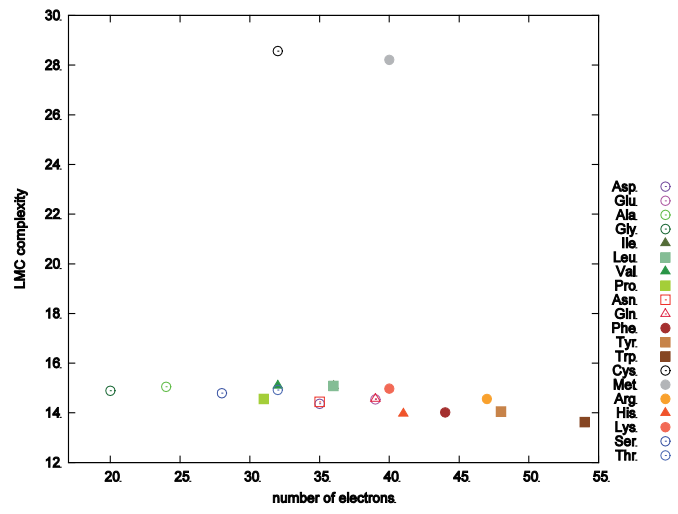

(a)

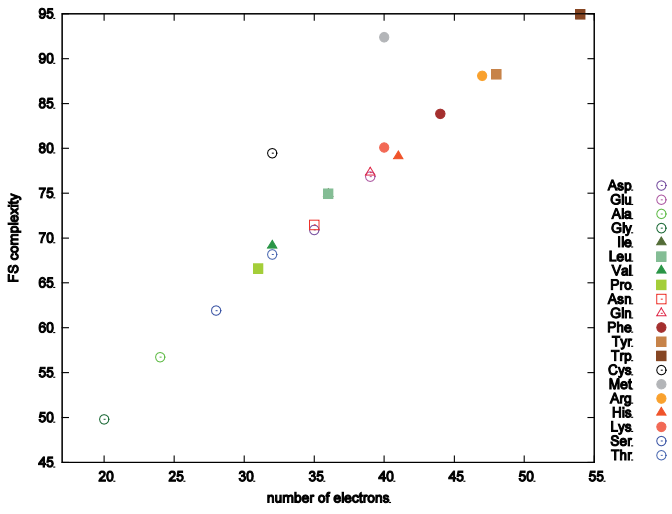

(b)

Figure 3. LMC- (left) and FS-complexities (right), both in position space, versus the number of electrons, for the set of 20 aminoacids.

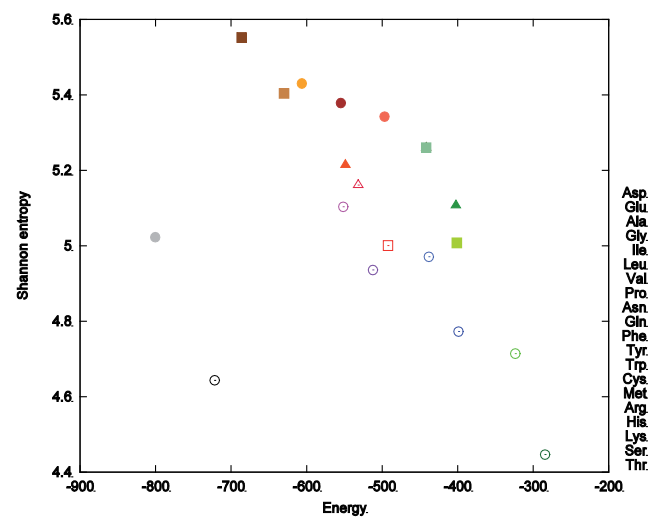

(a)

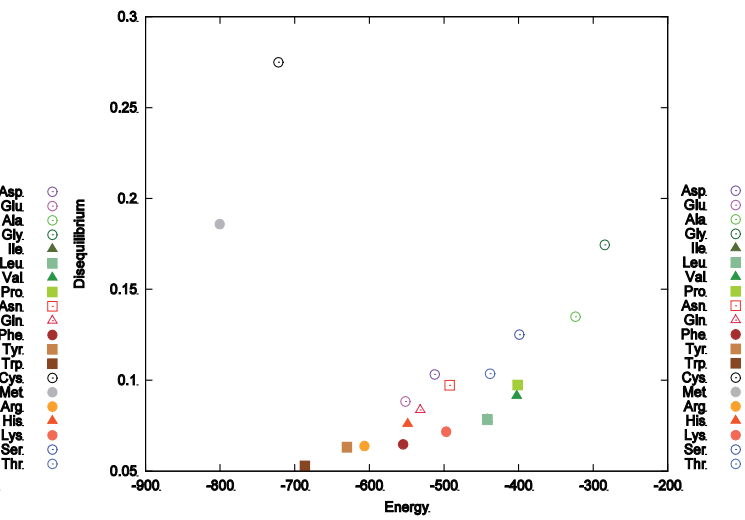

(b)

Figure 4. LMC- (left) and FS-complexities (right), both in position space, versus the total energy, for the set of 20 aminoacids.

In Figures 3 we have plotted the LMC and FS complexities versus the number of electrons for the twenty aminoacids where we can observe that $L M C$ complexity disntinguishes two different groups of aminoacids, where the more reactive (met and cys) hold larger values. In contrast, FS complexity behaves linearly with the number of electrons where the aromatic aminoacids possess the larger values and hence represent the more complex ones. Furthemore, 
the behavior of the LMC and FS complexities with respect to the total energy is analyzed in Figures 4, to note that $L M C$ complexity characterizes two different groups of aminoacids where the most reactive (cys and met) possess the largest values, which incidentally hold the largest energies (negatively). A different behavior is observed for the FS complexity in that the smaller values correspond to the less energetic aminoacids. It is worthy to mention that the FS complexity is related to the Fisher information measure (Eq. 7) which depends on the local behavior of the position space density, i..e., simpler molecules present more ordered chemical structures, and hence these kind of aminoacids are expected to be less complex, e.g., the small and the tiny ones (Ser, Ala, Thr).

\subsection{Homochirality}

In Figures 5 through 8 we have analyzed the homochiral behavior of all aminoacids by plotting the difference between the $L$ and the $D$ values of several physical properties (energy, ionization potential, hardness, electrophilicity) and some relevant information-theoretical measures (Shannon entropy, Fisher, LMC- and FS-complexity). From Figures 5 and 6 one can readily observe that none of the physical properties studied in this work show a uniform enantiomeric behavior, i.e., it is not possible to distinguish the L-aminoacids from the D-ones by using an specific physical property. In contrast, the L-aminoacids can be uniquely characterize $\mathrm{d}$ from the D-ones when informatic-theoretical measures are employed (see Figures 7 and 8) and this is perhaps the most interesting result obtained from our work. To the best of our kowledge no similar observations have been reported elsewhere, showing strong evidence of the utility of Information Theory tools for decoding the essential blocks of life.

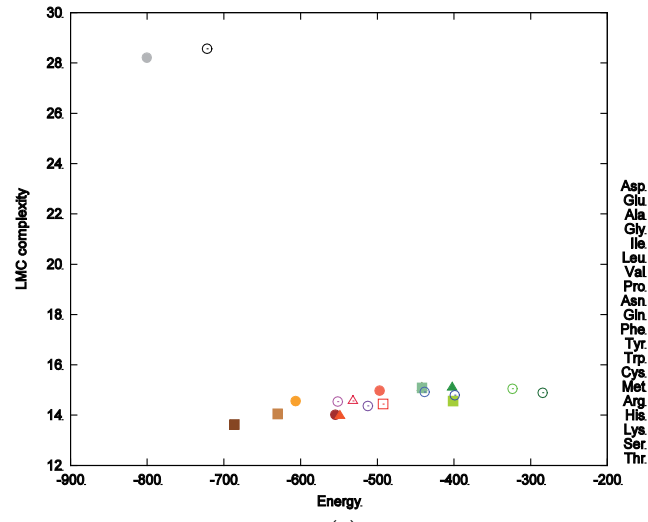

(a)

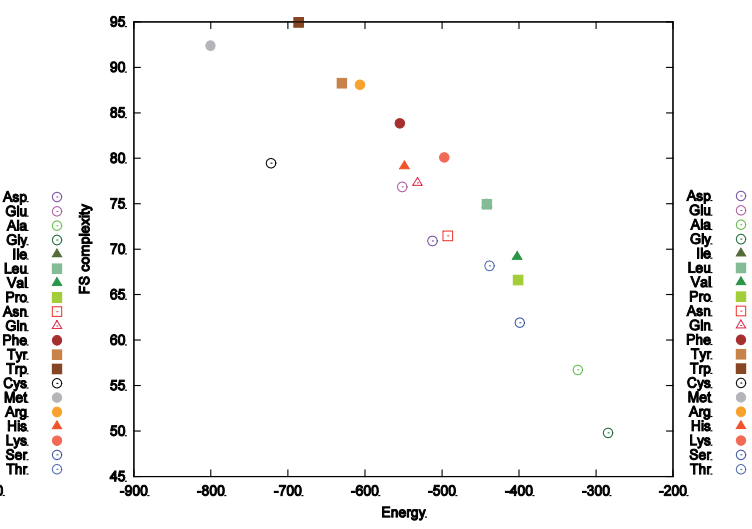

(b)

Figure 5. Differences (L-property - D-property) for the energy (in a.u., left) and the ionization potential (in a.u., right) for the set of 20 aminoacids. 


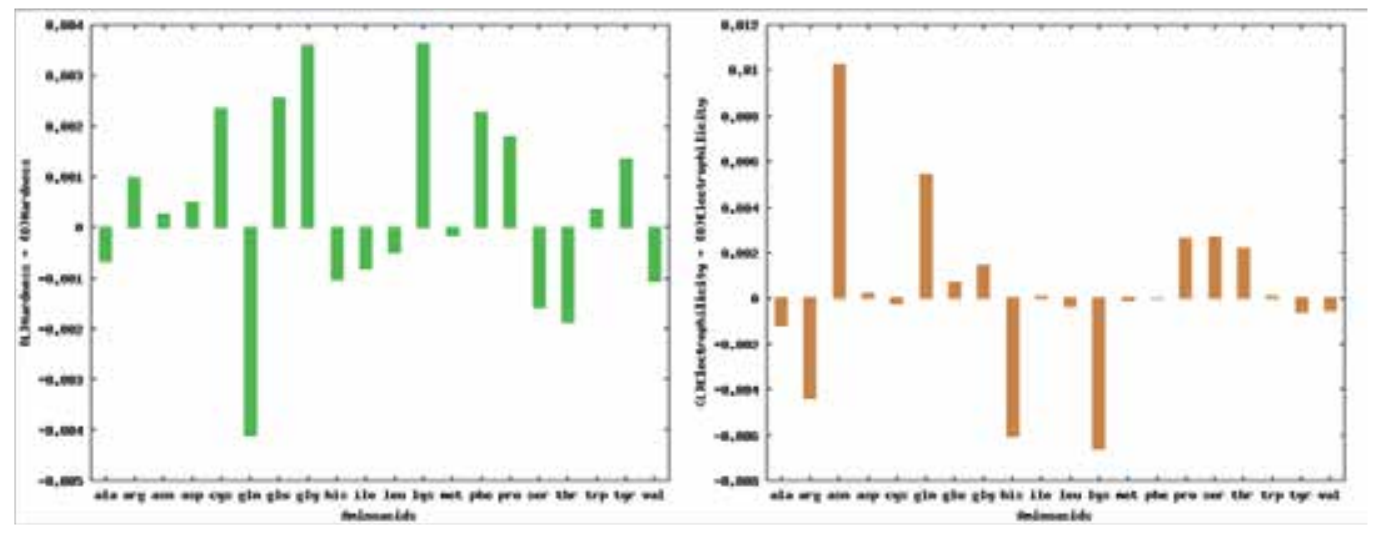

Figure 6. Differences (L-property - D-property) for the hardness (in a.u., left, Eqn. 14) and the electrophilicity (in a.u., right, Eqn. 16) for the set of 20 aminoacids.
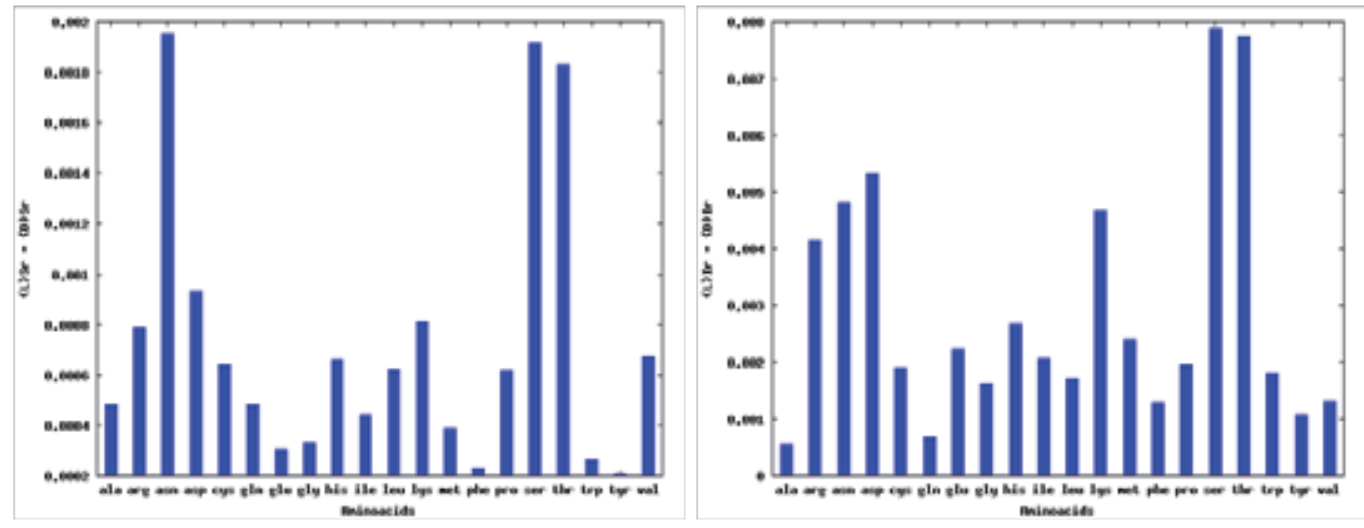

Figure 7. Differences (L-property - D-property) for the Shannon entropy (Eqn. 4, left) and the Fisher information (right, Eqn. 7), both in position space, for the set of 20 aminoacids. 

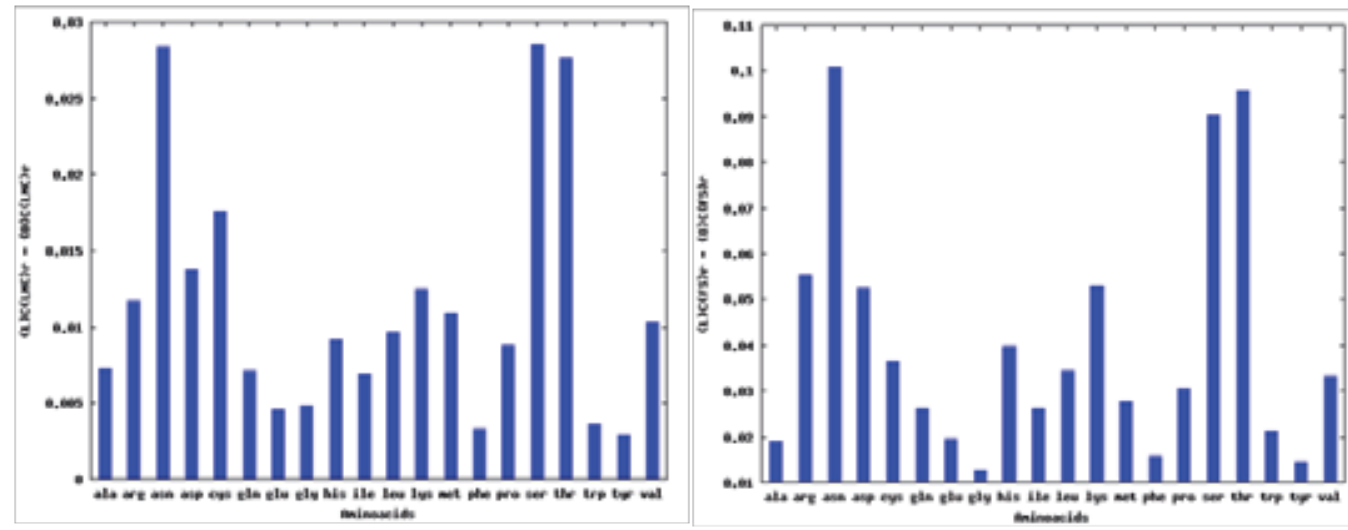

Figure 8. Differences (L-property - D-property) for the LMC (left, Eqn. 2) and the FS-complexities (right, Eqn. 9), both in position space, for the set of 20 aminoacids.

\section{Genetic code}

\subsection{Codons}

The genetic code refers to a nearly universal assignment of codons of nucleotides to amino acids. The codon to amino acid assignment is realized through: (i) the code adaptor molecules of transfer RNAs (tRNAs) with a codon's complementary replica (anticodon) and the corresponding amino acid attached to the $3^{\prime}$ end, and (ii) aminoacyl tRNA synthetases (aaRSs), the enzymes that actually recognize and connect proper amino acid and tRNAs. The origin of the genetic code is an inherently difficult problem (Crick, 1976). Taking into a count that the events determining the genetic code took place long time ago, and due to the relative compactness of the present genetic code. The degeneracy of the genetic code implies that one or more similar tRNA can recognize the same codon on a messenger mRNA. The number of amino acids and codons is fixed to 20 amino acids and 64 codons (4 nucleotides, A.C.U.G per three of each codon) but the number of tRNA genes varies widely 29 to 126 even between closely related organisms. The frequency of synonymous codon use differs between organisms, within genomes, and along genes, a phenomenon known as CUB (codon usage bias) (Thiele et al., 2011).

Sequences of bases in the coding strand of DNA or in messenger RNA possess coded instructions for building protein chains out of amino acids. There are 20 amino acids used in making proteins, but only four different bases to be used to code for them. Obviously one base can't code for one amino acid. That would leave 16 amino acids with no codes. By taking two bases to code for each amino acid, that would still only give you 16 possible codes (TT, TC, TA, TG, CT, CC, CA and so on) - that is, still not enough. However, by taking three bases per amino acid, that gives you 64 codes (TTT, TTC, TTA, TTG, TCT, TCC and so on). That's enough to 
code for everything with lots to spare. You will find a full table of these below. A three base sequence in DNA or RNA is known as a codon.

\begin{tabular}{|c|c|c|c|c|c|c|c|}
\hline \multirow{18}{*}{ 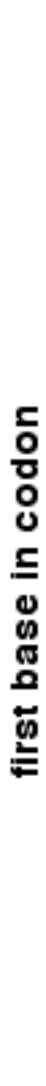 } & & \multicolumn{4}{|c|}{ second base in codon } & \multirow[b]{3}{*}{$\mathbf{U}$} & \multirow{10}{*}{ 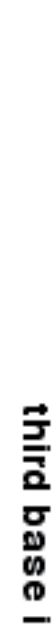 } \\
\hline & & $\mathrm{U}$ & $\mathrm{C}$ & $\mathrm{A}$ & G & & \\
\hline & \multirow{4}{*}{ U } & UUU Phe & UCU Ser & UAU Tyr & UGU Cys & & \\
\hline & & UUC Phe & UCC Ser & UAC Tyr & UGC Cys & C & \\
\hline & & UUA Leu & UCA Ser & UAA stop & UGA stop & A & \\
\hline & & UUG Leu & UCG Ser & UAG stop & UGG Trp & $\mathbf{G}$ & \\
\hline & \multirow{3}{*}{ C } & CUU Leu & CCU Pro & CAU His & CGU Arg & \multirow{4}{*}{$\begin{array}{l}\mathbf{U} \\
\mathbf{C} \\
\mathbf{A} \\
\mathbf{G}\end{array}$} & \\
\hline & & CUC Leu & CCC Pro & CAC His & CGC Arg & & \\
\hline & & CUA Leu & CCA Pro & CAA GIn & CGA Arg & & \\
\hline & & CUG Leu & CCG Pro & CAG GIn & CGG Arg & & \\
\hline & & AUU lle & ACU Thr & AAU Asn & AGU Ser & $\mathbf{U}$ & $\begin{array}{l}\mathbf{3} \\
0\end{array}$ \\
\hline & & AUC lle & ACC Thr & AAC Asn & AGC Ser & C & O요 \\
\hline & A & AUA $\| e$ & ACA Thr & AAA Lys & AGA Arg & A & $\overline{\mathrm{O}}$ \\
\hline & & AUG Met & ACG Thr & AAG Lys & AGG Arg & $\mathbf{G}$ & \\
\hline & & GUU Val & GCU Ala & GAU Asp & GGU Gly & $\mathbf{U}$ & \\
\hline & & GUC Val & GCC Ala & GAC Asp & GGC Gly & C & \\
\hline & $\mathrm{G}$ & GUA Val & GCA Ala & GAA Glu & GGA Gly & A & \\
\hline & & GUG Val & GCG Ala & GAG Glu & GGG Gly & $\mathbf{G}$ & \\
\hline
\end{tabular}

Table 1. Various combinations of three bases in the coding strand of DNA are used to code for individual amino acids - shown by their three letter abbreviation

The codes in the coding strand of DNA and in messenger RNA aren't, of course, identical, because in RNA the base uracil (U) is used instead of thymine (T). Table 1 shows how the various combinations of three bases in the coding strand of DNA are used to code for individual amino acids - shown by their three letter abbreviation. The table is arranged in such a way that it is easy to find any particular combination you want. It is fairly obvious how it works and, in any case, it doesn't take very long just to scan through the table to find what you want. The colours are to stress the fact that most of the amino acids have more than one code. Look, for example, at leucine in the first column. There are six different codons all of which will eventually produce a leucine (Leu) in the protein chain. There are also six for serine (Ser). In fact there are only two amino acids which have only one sequence of bases to code for them methionine (Met) and tryptophan (Trp). Note that three codons don't have an amino acid but 
"stop" instead. For obvious reasons these are known as stop codons. The stop codons in the RNA table (UAA, UAG and UGA) serve as a signal that the end of the chain has been reached during protein synthesis. The codon that marks the start of a protein chain is AUG, that's the amino acid, methionine (Met). That ought to mean that every protein chain must start with methionine.

\subsection{Physical and information-theoretical properties}

An important goal of the present study is to characterize the biological units which codify aminoacids by means of information-theoretical properties. To accomplished the latter we have depicted in Figures 9 through 13 the Shannon entropy, Disequilibrium, Fisher and the LMC and FS complexities in position space as the number of electron increases, for the group of the 64 codons. A general observation is that all codons hold similar values for all these properties as judging for the small interval values of each graph. For instance, the Shannon entropy values for the aminoacids (see Figure 2) lie between 4.4 to 5.6, whereas the corresponding values for the codons (see Figure 9) lie between 6.66 to 6.82, therefore this information measure serves to characterize all these bilogical molecules, providing in this way the first benchmark informational results for the building blocks of life. Further, it is interesting to note from Figures 9 and 10 that entropy increases with the number of electrons (Fig. 9) whereas the opposite behavior is observed for the Disequilibrium measure. Besides, we may note from these Figures an interesting codification pattern within each isolelectronic group of codons where one may note that an exchange of one nucleotide seems to occur, e.g., as the entropy increases in the 440 electron group the following sequence is found: UUU to (UUC, UCU, CUU) to (UCC, CUC, CCU) to CCC. Similar observations can be obtained from Figures 10 and 11 for $\mathrm{D}$ and I, respectively. In particular, Fisher information deserves special analysis, see Figure 11, from which one may observe a more intricated behavior in which all codons seem to be linked across the plot, i.e., note that for each isoelectronic group codonds exchange only one nucleotide, e.g., in the 440 group codons change from UUU to (UUC, UCU, CUU) to (UCC, CUC, CCU) to CCC as the Fisher measure decreaes. Besides, as the Fisher measure and the number of electrons increase linearly a similar exchange is observed, eg., from AAA to (AAG, AGA, GAA) to (AGG, GAG, GGA) to GGG. We believe that the above observations deserve further studies since a codification pattern seems to be apparent.

In Figures 12 and 13 we have depicted the LMC and FS complexities, respectively, where we can note that as the number of electron increases the LMC complexity decreases and the opposite is observed for the FS complexity. It is worth mentioning that similar codification patternsm, as the ones above discussed, are observed for both complexities. Furthermore, we have found interesting to show similar plots in Figures 14 and 15 where the behavior of both complexities is shown with respect to the total energy. It is observed that as the energy increases (negatively) the LMC complexity decreases whereas the FS complexity increases. Note that similar codification patterns are observed in Figure 15 for the FS complexity. 


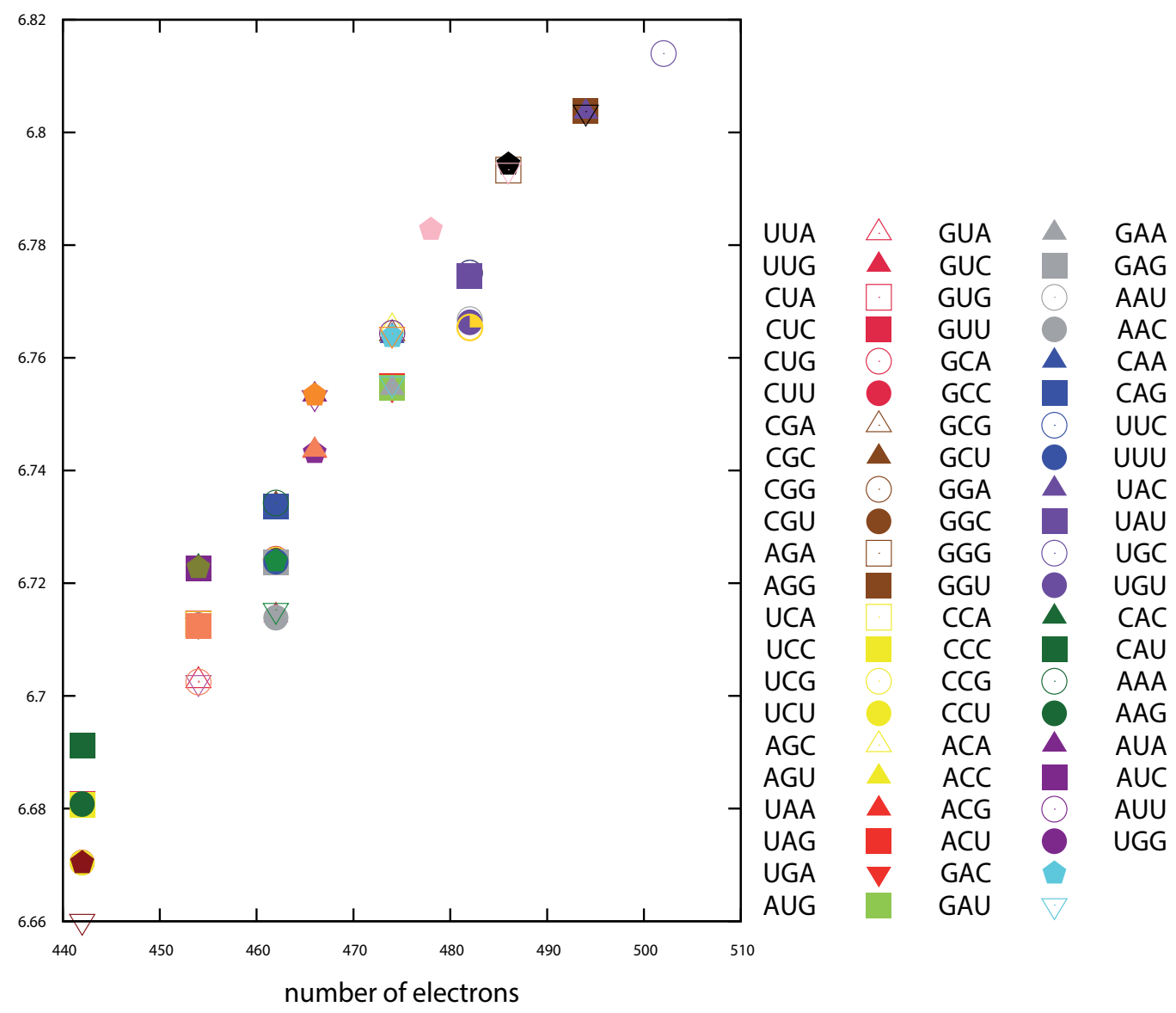

Figure 9. Shannon entropy values in position spaces as a function of the number of electrons for the set of 64 codons. 


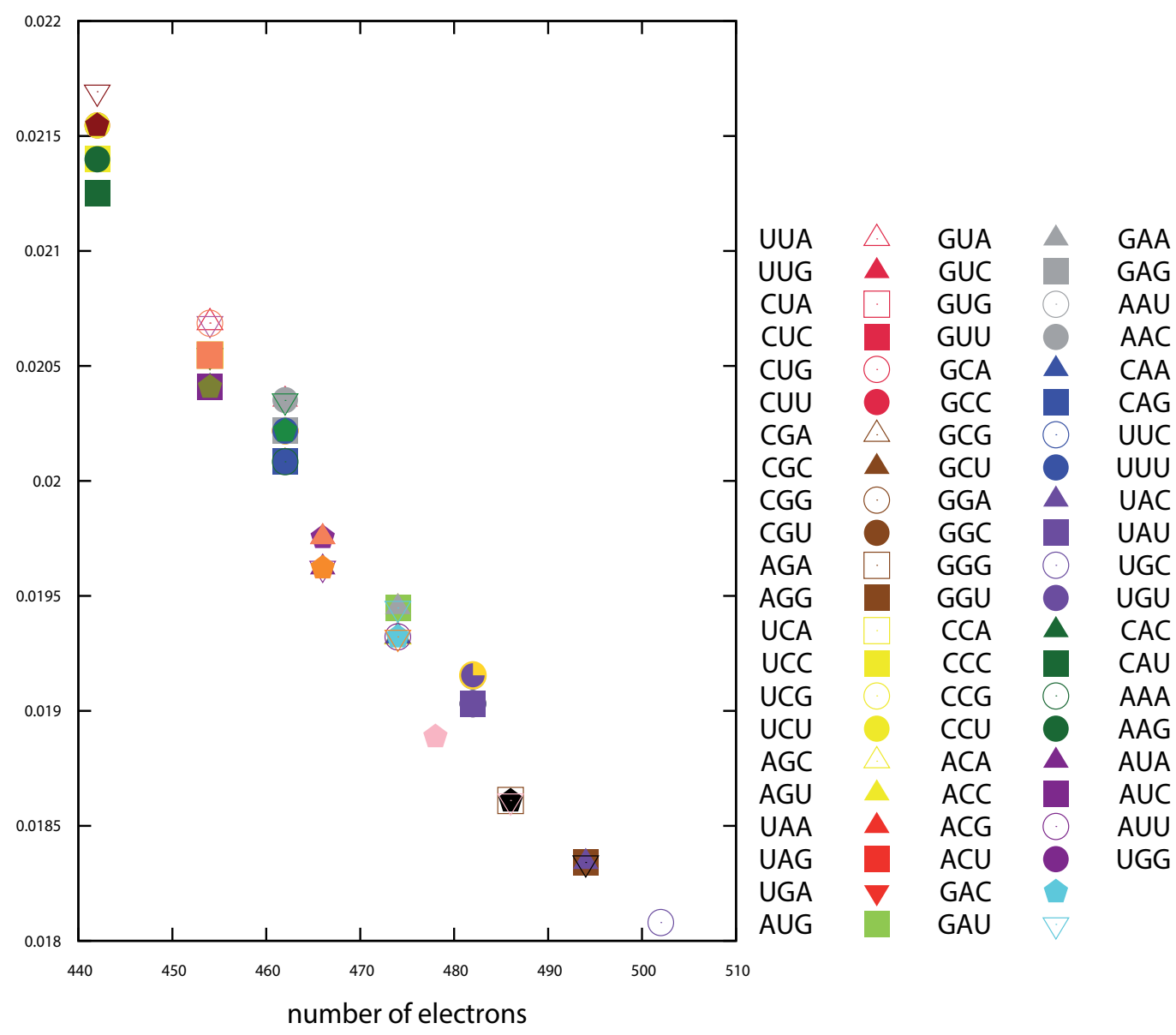

Figure 10. Disequilibrium in position spaces (Eqn. 3)as a function of the number of electrons for the set of 64 codons 


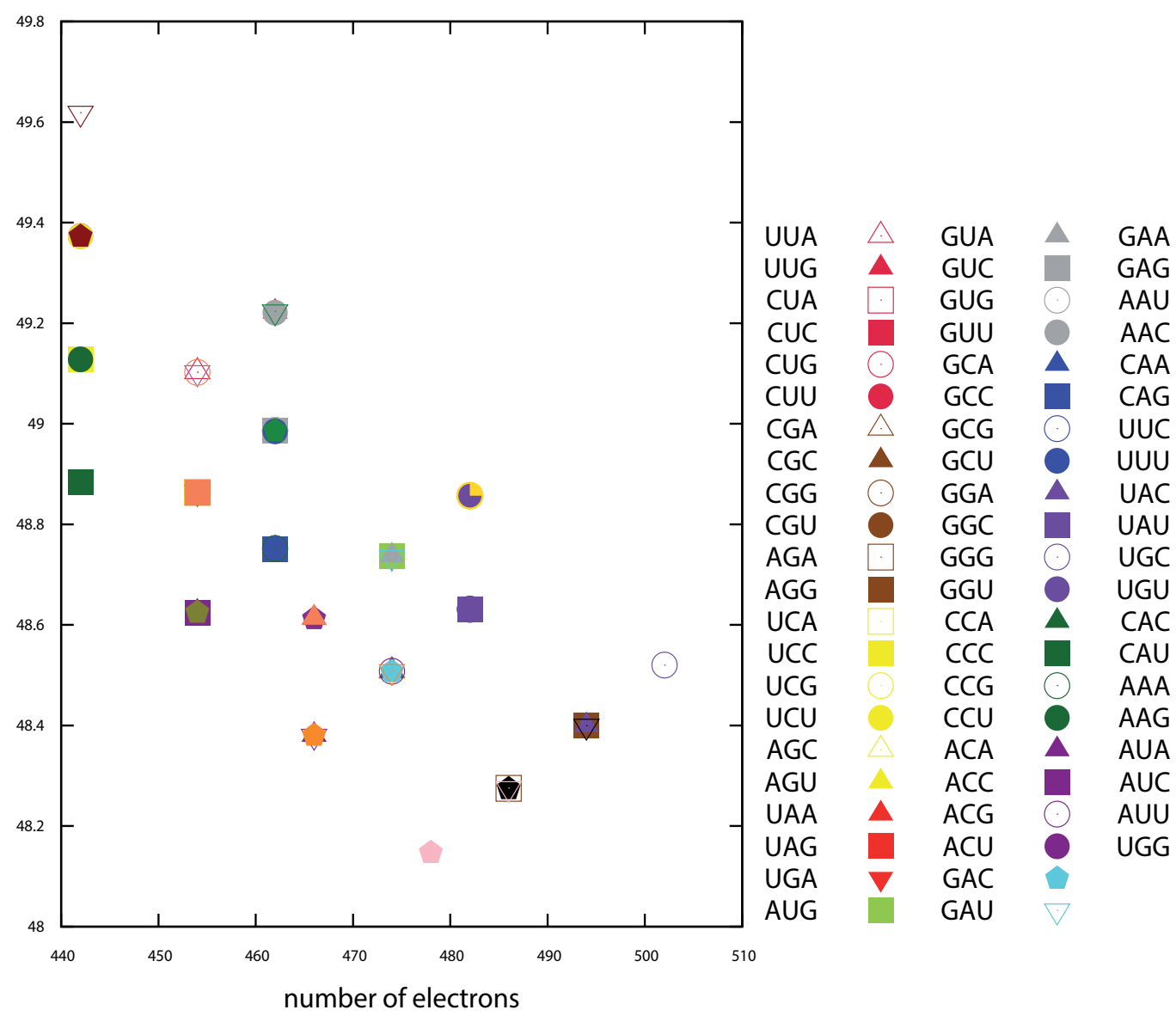

Figure 11. Fisher information in position spaces (Eqn. 7) as a function of the number of electrons for the set of 64 codons 


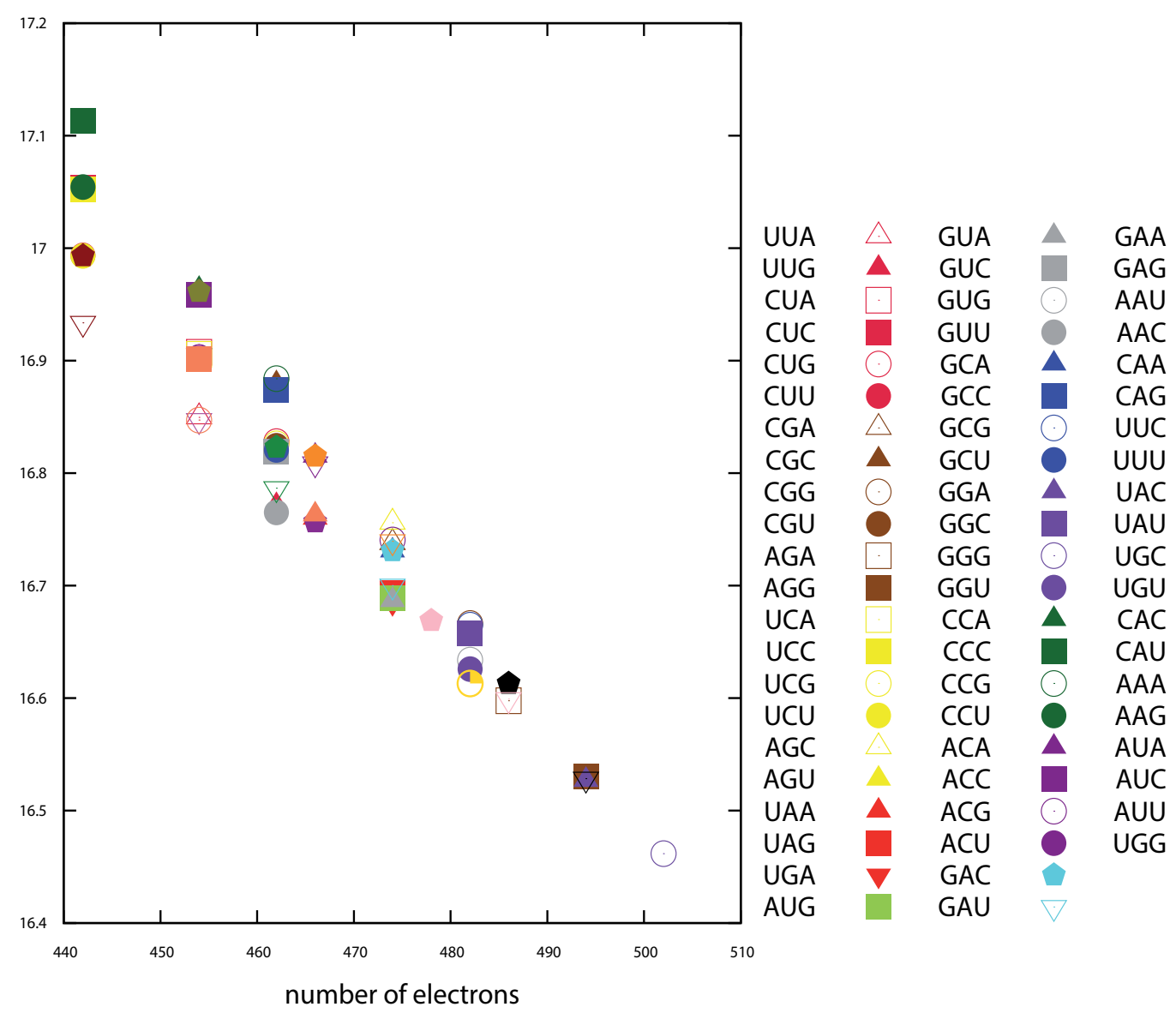

Figure 12. LMC-complexity in position spaces (Eqn.12) as a function of the number of electrons for the set of 64 codons 


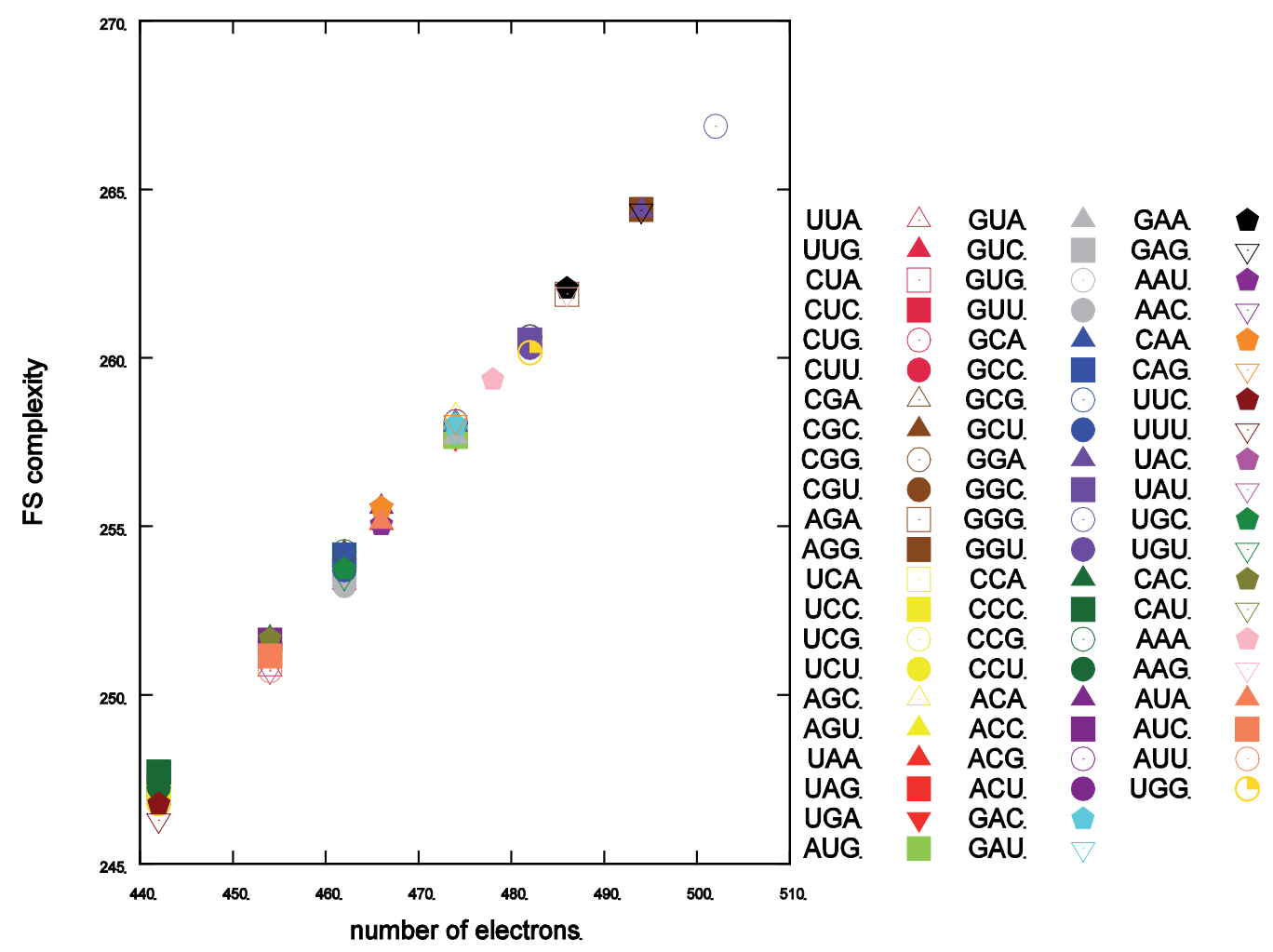

Figure 13. FS-complexity in position spaces (Eqn. 7) as a function of the number of electrons for the set of 64 codons 


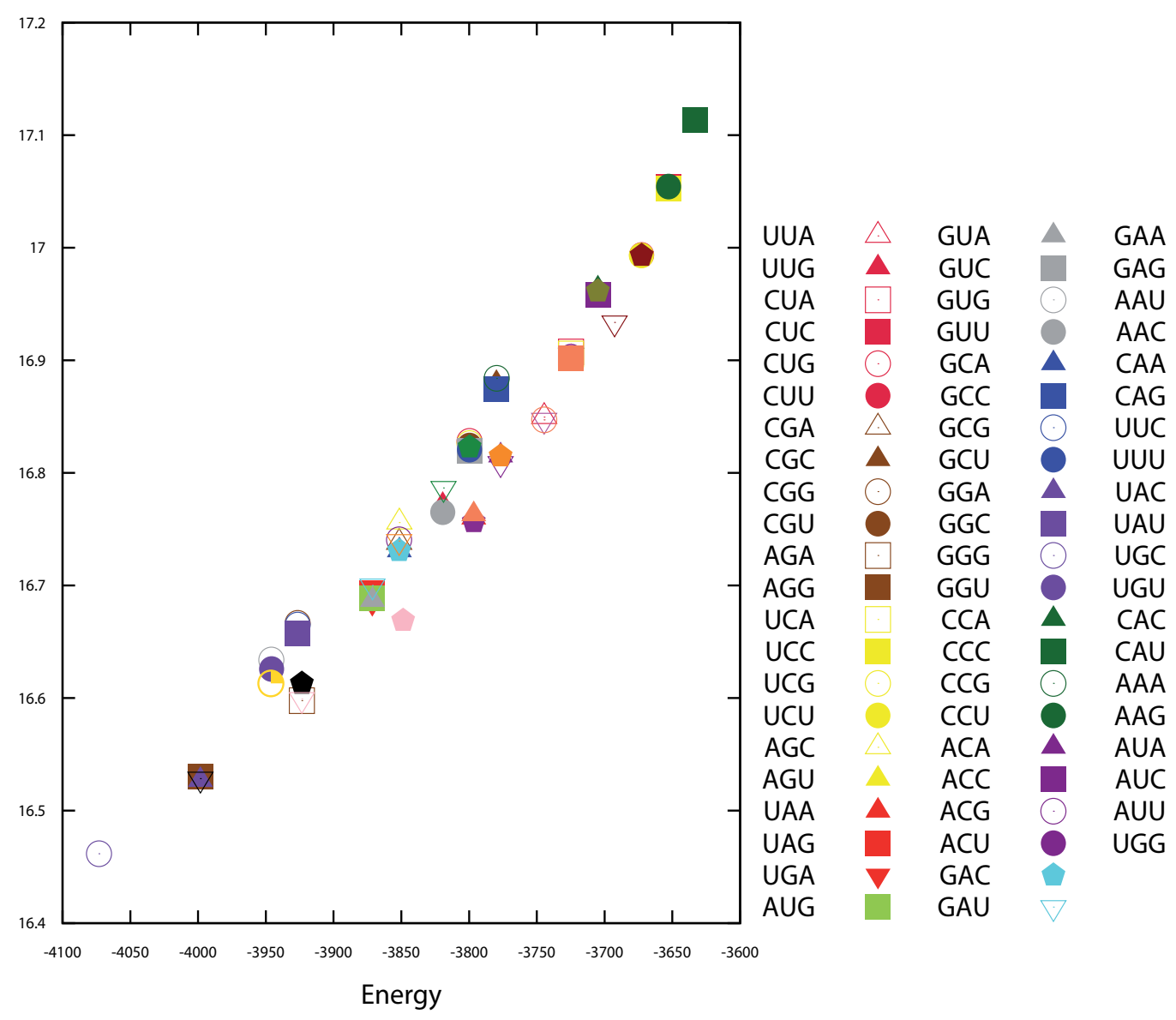

Figure 14. LMC-complexity in position spaces as a function of the total energy for the set of 64 codons. 


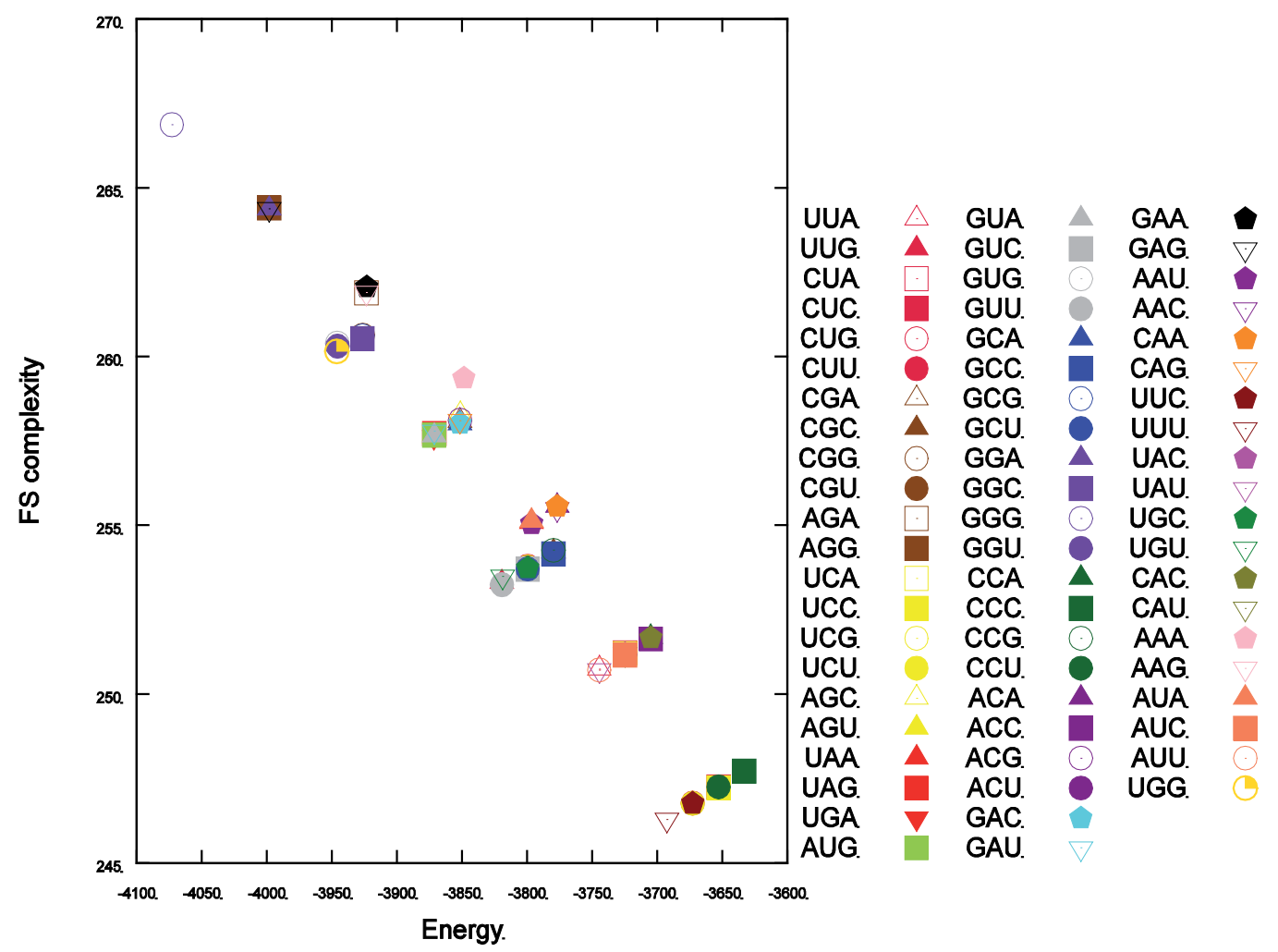

Figure 15. FS-complexity in position space as a function of the total energy for the set of 64 codons.

\section{Concluding remarks}

We have shown throughout this Chapter that information-theoretical description of the fundamental biological pieces of the genetic code: aminoacids and codons, can be analysed in a simple fashion by employing Information Theory concepts such as local and global information measures and statistical complexity concepts. In particular, we have provided for the first time in the literature with benchmark information-theoretical values for the 20 essential aminacids and the 64 codons for the nucleotide triplets. Throughout these studies, we believe that information science may conform a new scientific language to explain essential aspects of biological phenomena. These new aspects are not accessible through any other standard methodology in quantum chemistry, allowing to reveal intrincated mechanisms in which chemical phenomena occur. This envisions a new area of research that looks very promising as a standalone and robust science. The purpose of this research is to provide fertile soil to build this nascent scientific area of chemical and biological inquiry through informationtheoretical concepts towards the science of the so called Quantum Information Biology. 


\section{Acknowledgements}

We wish to thank José María Pérez-Jordá and Miroslav Kohout for kindly providing with their numerical codes. We acknowledge financial support through Mexican grants from CONACyT, PIFI, PROMEP-SEP and Spanish grants MICINN projects FIS2011-24540, FQM-4643 and P06FQM-2445 of Junta de Andalucía. J.A., J.C.A., R.O.E. belong to the Andalusian researchs groups FQM-020 and J.S.D. to FQM-0207. R.O.E. wishes to acknowledge financial support from the CIE-2012. CSC., acknowledges financial support through PAPIIT-DGAPA, UNAM grant IN117311. Allocation of supercomputing time from Laboratorio de Supercómputo y Visualización at UAM, Sección de Supercomputacion at CSIRC Universidad de Granada, and Departamento de Supercómputo at DGSCA-UNAM is gratefully acknowledged.

\section{Author details}

Rodolfo O. Esquivel ${ }^{1,6}$, Moyocoyani Molina-Espíritu ${ }^{1}$, Frank Salas ${ }^{1}$, Catalina Soriano ${ }^{2}$, Carolina Barrientos ${ }^{3}$, Jesús S. Dehesa ${ }^{4,6}$ and José A. Dobado ${ }^{5}$

1 Departamento de Química, Universidad Autónoma Metropolitana-Iztapalapa, México D.F., México

2 Laboratorio de Química Computacional, FES-Zaragoza, Universidad Nacional Autónoma de México, C.P. 09230 Iztapalapa, México, D.F., México

3 Facultad de Bioanálisis-Veracruz, Universidad Veracruzana, Lab. de Química y Biología Experimental, Veracruz, México

4 Departamento de Física Atómica, Molecular y Nuclear, Universidad de Granada, Granada, Spain

5 Grupo de Modelización y Diseño Molecular, Departamento de Química Orgánica, Universidad de Granada, Granada, Spain

6 Instituto "Carlos I" de Física Teórica y Computacional, Universidad de Granada, Granada, Spain

\section{References}

[1] Angulo, J. C, Antolin, J, Sen, K. D, 2008a, Phys, , \& Lett, A.

[2] Angulo, J. C. ; J, Antolín, , \& Chem, J. Phys., 128, 1641092008 
[3] Angulo, J. C, Antolin, J, \& Esquivel, R. O. (2010d). Chapter in the monograph'Statistical Complexity: Applications in Electronic Structure', ed. K.D. Sen (Springer, United Kingdom, 2010) 978-9-04813-889-0

[4] Anteonodo, C, \& Plastino, A. (1996). Phys. Lett. A, 223, 3481996.

[5] Antolín, J, \& Angulo, J. C. Quantum Chem., 109, 5862009.

[6] Arnett, E. M, \& Thompson, O. J. Am. Chem. Soc. , 1981(103), 968-970.

[7] Avalos, M, Babiano, R, Cintas, P, Jiménez, J. L, \& Palacios, J. C. (2000). Tetrahedron: Asymmetry , 2000(11), 2845-874.

[8] Ayers, P. W. ; R. G, Parr, R. G, \& Pearson, J. Chem. Phys. 2006, 124, 194107

[9] Bachmann, P. A, Luisi, P. L, \& Lang, J. Nature , 1992(357), 57-59.

[10] Bennet, C. H. (1988). The Universal Turing Machine a Half Century, edited by R. Herhen, Oxford University Press, Oxford, , 227-257.

[11] Betts, M. J, \& Russell, R. B. (2003). In Bioinformatics for Geneticists, M.R. Barnes, I.C. Gray eds, Wiley, 2003

[12] Bombelli, C, \& Borocci, S. Lupi, Federica.; Mancini, G.; Mannina, L.; Segre, A. L.; Viel, Stéphane ((2004). J. Am. Chem. Soc. , 2004(126), 13354-13362.

[13] Borgoo, A, De Proft, F, Geerlings, P, \& Sen, K. D. (2007). Chem. Phys. Lett. 444, 186 2007.

[14] Breslow, R. Thetrahedron Letters. (2011). , 2011(52), 4228-4232.

[15] Carbó-dorca, R. ; J, \& Arnau, L. Leyda ((1980). Int. J. Quantum Chem., 17, 11851980.

[16] Carrera, E. M, Flores-gallegos, N, \& Esquivel, R. O. Appl. Math. 233, 1483-1490 (2010).

[17] Catalán, R. G. ; J, \& Garay, R. López-Ruiz ((2002). Phys. Rev. E, 66, 0111022002.

[18] Chaitin, G, 1966, \& Acm, J.

[19] Chattaraj, P. K ; U, \& Sarkar, D. R. Roy, Chem. Rev., (2006).

[20] Chatzisavvas, K. Ch.; Ch.C. Moustakidis and C.P. Panos ((2005). J. Chem. Phys., 123, 1741112005

[21] Cleaves, H. J. Biol. , 2010(263), 490-498.

[22] Crick, F, Brenner, S, Klug, A, \& Pieczenik, G. (1976). Orig Life 1976, 7:389-397.

[23] Dembo, A, Cover, T. M, \& Thomas, J. A. (1991). Information theoretic inequalities", IEEE Trans. Inform. Theory, , 37, 1501-1518.

[24] Dehesa, J. S. ; R, González-férez, P, Sánchez-moreno, J, \& Phys, A. 
[25] Esquivel, R O, Flores-gallegos, N, Iuga, C, Carrera, E. M, Angulo, J. C, \& Antolín, J. (2009a). Theoretical Chemistry Accounts 124, 445-460 (2009)

[26] Esquivel, R O, \& Flores-gallegos, N. Iuga, C; Carrera, E.M. ; Angulo, J.C. ; Antolín J. ((2010a). Physics Letters A, 374(2010), 948-951.

[27] Esquivel, R O, Flores-gallegos, N, Sánchez-dehesa, J. S, Angulo, J. C, Antolín, J, Sen, K, 2010b, Phys, ). J, \& Chem, A. 2010(114), 1906-1916.

[28] Esquivel, R. O, Liu, S, Angulo, J. C, Dehesa, J. S, Antolin, J, Molina-espíritu, M, 2011a, Phys, ). J, Chem, A, Phys, J, \& Chem, A.

[29] Esquivel, R. O, Angulo, J. C, Antolin, J, Sánchez-dehesa, J, Flores-gallegos, N, \& Lopez-rosa, S. Chem. Chem. Phys., 12, 7108-7116 (2010)

[30] Esquivel, R. O, Molina-espíritu, M, Angulo, J. C, Antolín, J, Flores-gallegos, N, Dehesa, J. S, \& Mol, b. Phys., (2011). , 109

[31] Esquivel, R. O, Flores-gallegos, N, Carrera, E. M, Dehesa, J. S, Angulo, J. C, Antolín, J, \& Soriano-correa, C. Sim., 35, (2009), 498-511;

[32] Esquivel, R. O, Flores-gallegos, N, Carrera, E. M, \& Soriano-correa, C. (2010g). Journal of Nano Research , 9, 1-15.

[33] Esquivel, R. O, Flores-gallegos, N, Molina-espíritu, M, Plastino, A. R, Dehesa, J. S, Angulo, J. C, Antolín, J, Phys, c). J, \& At, B. Mol. Opt. Phys. 44, 175101 ((2011).

[34] Feldman, D. P. ; J. P. Crutchfield (1998)., Phys. Lett. A, 238, 244 (1998).

[35] Fisher, R. A. (1925). Proc. Cambridge Philos. Soc., 22, 7001925

[36] Flores-gallegos, N, \& Esquivel, R. O. Chem. Soc. (2008), 52(1), 19-30

[37] Frieden, B. R. (2004). Science from Fisher Information (Cambridge University Press, Cambridge, 2004.

[38] Fujii, N, Kaji, Y, Fujii, N, Nakamura, T, \& Motoie, R. Mori, Yuhei.; Kinouchi, T ((2010). Chemistry \& Biodiversity, , 2010(7), 1389-1397.

[39] Geerlings, P. ; F, \& De Proft, W. Langenaeker ((2003). Chem. Rev. 2003, 103, 1793.

[40] Ghanty, T. K, \& Ghosh, S. K. Chem., 1993, 97, 4951

[41] Hati, S, Datta, D, \& Phys, J. Chem., (1994).

[42] He, C. (2010). Nat Chem Biol 2010; , 6, 863-865.

[43] Hein, J. E, \& Blackmond, D. G. (2011). Acc. Chem. Res. 201110.1021/ar200316n

[44] Hicks, J. M. (2002). bk- ch001., 2002-0810.

[45] Hornyak, I, \& Nagy, A. (2007). Chem. Phys. Lett., 437, 1322007.

[46] Janak, J. F. (1978). Phys. Rev. B, 1978, 18, 7165. 
[47] Kaijser, P. ; V. H. Smith, Jr. ((1997). Adv. Quant. Chem. 10, 371997.

[48] Kohout, M. (2007). program DGRID, 2007, version 4.2.

[49] Koopmans, T. A. (1933). Physica, 1933, 1, 104

[50] Kolmogorov, A. N. Probl. Inf. Transm. ((1965).

[51] Lamberti, P. W. ; M. T, Martin, A, \& Plastino, O. A. Rosso ((2004). Physica A, 334, 119 2004.

[52] Livingstone, C D, \& Barton, G J. (1993). CABIOS, 97451993.

[53] López-rosa, S, Angulo, J. C, Antolín, J, 2009, \& Physica, A.

[54] López-rosa, S, \& Esquivel, R. O. Angulo, J.C ; Antolin, J.; Dehesa, J.S. ; Flores-Gallegos, N. ((2010c). J. Chem. Theory Comput. , 6, 145-154.

[55] López-ruiz, R. ; H. L, \& Mancini, X. Calbet ((1995). Phys. Lett. A, 1995, 209, 321.

[56] López-ruiz, R. Chem., 115, 2152005.

[57] Lloyd, S, \& Pagels, H. (1988). Ann. Phys. (N.Y.), 188, 1861988.

[58] Martin, M. T, Plastino, A, \& Rosso, O. A. (2003). Phys. Lett. A, 311, 1262003.

[59] Menger, F. M, \& Angelova, M. I. (1998). Acc. Chem. Res. , 1998(31), 789-797.

[60] Nagy, A. Phys., 2003, 119, 9401.

[61] Nagy, A, \& Sen, K. D. (2006). Phys. Lett. A, 360, 2912006.

[62] Nalewajski, R. (2003). Chem. Phys. Lett., 372, 282003.

[63] Nanda, V, \& Degrado, W. F. Chem. Soc. , 2004(126), 14459-14467.

[64] Onicescu, O. Sci. Paris A 263, 251966.

[65] Parr, R. G, \& Pearson, R. G. Chem. Soc., 1983, 105, 7512.

[66] Parr, R. G. ; W. Yang, ((1989). Density-Functional Theory of Atoms and Molecules (Oxford University Press: New York, 1989).

[67] Parr, R. G. ; L. V, Szentpály, S, \& Liu, J. Am. Chem. Soc., 1999, 121, 1922.

[68] Pearson, R. G. Chem. Soc., 1963, 85, 3533

[69] Pearson, R. G. (1973). Hard and Soft Acids and Bases (Dowen, Hutchinson and Ross: Stroudsberg, 1973)

[70] Pearson, R. G. (1995). Inorg. Chim. Acta 1995, 240, 93.

[71] Pearson, R. G. (1997). Chemical Hardness; (Wiley-VCH; New York, 1997). Ramachandran GN, Ramakrishnan C, Sasisekharan J Mol Biol., 7:95-99., 1963

[72] Rawlings, D. C. ; E. R, \& Davidson, J. Phys. Chem, 89, 9691985. 
[73] Romera, E. ; J. S, \& Dehesa, J. Chem. Phys., 120, 89062004.

[74] Romera, E. ; P, Sánchez-moreno, J. S, \& Dehesa, J. Math. Phys., 47, 1035042006

[75] Rosso, O. A, Martin, M. T, Plastino, A, 2003, \& Physica, A.

[76] Root-bernstein, R. (2007). BioEssays, , 2007(29), 689-698.

[77] Roy, R. ; A. K, Chandra, S, \& Pal, J. Phys. Chem., 1994, 98, 10447

[78] Sen, K. D, Antolín, J, Angulo, J. C, 2007a, Phys, , \& Rev, A.

[79] Sen, K. D. ; C. P, \& Panos, K. Ch. Chatzisavvas and Ch. Moustakidis ((2007b). Phys. Lett. A, 364, 2862007.

[80] Shalizi, C. R. ; K. L, \& Shalizi, R. Haslinger, ((2004). Phys. Rev. Lett., 93, 1187012004.

[81] Simon-manso, Y, Fuenteaelba, P, 1998, Phys, J, \& Chem, A.

[82] Shannon, C. E, \& Weaver, W. (1949). The Mathematical Theory of Communication (University of Illinois Press, Urbana, 1949.

[83] Stryer, L. (1995). Biochemistry, 4th ed., W. H. Freeman and Co.: New York, 1995.

[84] Tamura, K. (2008). BioSystems. , 2008(92), 91-98.

[85] Thiele, I, Fleming, R, Que, R, Bordbar, A, \& Palsson, B. (2011). Nature Precedings 2011;

[86] Viedma, C, Ortiz, J. E, Torrea, T, Izumi, T, \& Blackmond, D. G. Chem. Soc. , 2008(130), 15274-15275.

[87] Vitanyi, P. M. B, \& Li, M. (2000). IEEE Trans. Inf. Theory, 46, 4462000.

[88] Weber, A. L, \& Miller, S. L. Evol. , 1981(17), 273-284.

[89] Werner, F. (2009). Colloids and Surfaces B: Biointerfaces, , 2009(74), 498-503.

[90] Yamano, T, 1995, \& Physica, A.

[91] Yamano, T. Phys., 45, 19742004.

[92] Zehnacker, A, \& Suhm, M. A. (2008). Angew. Chem. Int. Ed. , 2008(47), 6970-6992. 

Chapter 28

\title{
The Theoretical Ramifications of the Computational Unified Field Theory
}

\author{
Jonathan Bentwich \\ Additional information is available at the end of the chapter \\ http://dx.doi.org/10.5772/53804
}

\section{Introduction}

Four previous articles (Bentwich, 2012: a-d) have postulated the existence of a novel 'Computational Unified Field Theory' (CUFT) which is a candidate 'Theory of Everything' (Brumfiel, 2006; Ellis, 1986; Greene, 2003) - i.e., has the potential of unifying between quantum (Born, 1954) and relativistic models of physical reality (and moreover also possesses the potential of opening new 'vistas' for scientific examination connected with its discovery of a new hypothetical 'Universal Computational Principle which carries out an extremely rapid computation, $\mathrm{c}^{2} / \mathrm{h}$ of a series of Universal Simultaneous Computational Frames, 'USCF's, which give rise to all 'apparent' secondary computational 'physical' properties of 'space', 'time', 'energy' and 'mass'); Indeed, the primary focus of the current manuscript is precisely to explore the potential theoretical ramifications of this novel CUFT - based on the recognition that the (singular) Universal Computational Principle ('י') solely produces all (apparent) secondary computational 'physical' properties of 'space, 'time', 'energy' and 'mass', and hence comprises the sole "reality" (which produces all exhaustive hypothetical inductive and deductive phenomenon through a higher-ordered 'a-causal' computational framework; this may subsequently bear significant theoretical ramifications for all (key) 'material-causal' scientific paradigms as well as point at the discovery of a (new) 'Universal Consciousness Principle Computational Program', as well as outline potential resolutions of major Physical 'enigma's;

Hence, the current manuscript traces the potential theoretical ramifications of:

a. An 'a-causal' computational framework of the (CUFT's) singular Universal Consciousness Principle's ('י') responsible for the (higher-ordered) computation of all exhaustive hypothetical (e.g., empirically knowable) inductive or deductive ' $x-y$ ' pairs series - which leads to the discovery of a-causal 'Universal Consciousness Principle Computational Program'. 
b. An exploration of the CUFT's Universal Consciousness Principle's ('י') and Duality Principle's (Bentwich, 2003c, 2004, 2006) reformalization of all (apparent inductive or deductive) major SROCS computational paradigms (e.g., including: Darwin's 'Natural Selection Principle' (Darwin, 1859) and associated Genetic Encoding hypothesis, Neuroscience's Psychophysical Problem of human Consciousness and all inductive and deductive Gödel-like SROCS paradigms).

c. Theoretical Ramifications of the Universal Consciousness Principle.

\section{A singular 'a-causal' universal consciousness principle computation of all inductive and deductive ' $x-y$ ' relationships}

We thus begin with an exploration of three potential theoretical ramifications of the CUFT's description of the operation of the (singular) Universal Consciousness Principle ('י') which has been shown to compute an extremely rapid series of Universal Simultaneous Computational Frames (USCF's);

The Universal Computational/Consciousness Principle was (previously) shown to encapsulate a singular higher-ordered 'D2' computation of an 'a-causal' computation of the "simultaneous co-occurrences" of all exhaustive hypothetical inductive or deductive (e.g., empirically knowable) ' $x-y$ ' pairs series; Therefore, the acceptance of the CUFT's description of the Universal Consciousness Principle necessarily implies that throughout the various (inductive or deductive) disciplines of Science we need to shift from the current basic (Cartesian) "material-causal" scientific theoretical towards a singular (higher-ordered 'D2') 'Universal Consciousness Principle's a-causal computation':

This means that the current (Cartesian) 'material-causal' scientific framework assumes that any given 'y' element(orvalue)canbeexplained as a resultofits(directorindirect)'causal'interaction/ $\mathrm{s}$ with another (exhaustive hypothetical inductive or deductive) series of ' $x$ ' factor/s - which determines whether that 'y' element (or value) "exists" or "doesn't exist", thereby comprising a 'Self-Referential Ontological Computational System' (SROCS) (Bentwich: 2012a-d):

SROCS: PR $\{x, y\} \rightarrow[$ 'y' or 'not y']/di1...din.

But, since it was previously shown that such SROCS computational structure inevitably leads to both 'logical inconsistency' and 'computational indeterminacy' that were shown to be contradicted by robust empirical findings indicating the capacity of the major scientific SROCS paradigms to be capable of determining the "existence" or "non-existence" of the particular 'y' element, see Bentwich 2012b) - then the CUFT's 'Duality Principle' asserted the existence of the singular 'Universal Consciousness Principle' ('י') which is capable of computing the "simultaneous co-occurrences" of any particular (exhaustive hypothetical) ' $x-y$ ' pairs series which are embedded within the Universal Computational/Consciousness Principle's rapid series of USCF's.

What this means is that both specifically for each of the (previously identified) key scientific SROCS paradigms as well as more generally for any hypothetical ('empirically knowable') 
inductive or deductive ('x-y') phenomenon, we must reformulate our scientific understanding in such a way which will allow us to present any such ' $x-y$ ' relationship/s as being computed by the singular Universal Consciousness Principle (e.g., as the computation of an exhaustivehypothetical "co-occurring" 'x-y' pairs' series); In that respect, this (novel) 'Universal Consciousness Principle's' scientific framework shifts Science from its current basic (Cartesian) assumption wherein all natural phenomena can be described as 'material-causal' (' $\left.x \rightarrow y^{\prime}\right)$ relationships (e.g., comprising the apparent SROCS computational structure contradicted by the computational Duality Principle) - to an 'a-causal' singular Universal Consciousness Principle which computes the simultaneous "co-occurrences" of any inductive or deductive ' $x$ $y^{\prime}$ pairs series comprising the various 'pixels' of the USCF's frames (e.g., produced by this Universal Consciousness Principle).

Finally, it should be noted that a key principle underlying this shift from the current 'materialcausal' (Cartesian) scientific framework towards the CUFT's (proven) higher-ordered singular Universal Consciousness Principle's ('י') 'a-causal' theoretical framework is the acceptance of the impossibility of the existence of any such 'material-causal' ('x-y') relationship/s - i.e., due to the impossibility of any 'physical' entity, attribute (or property) being transferred across any (two subsequent) 'USCF's frames: Thus, apart from the (previously shown) conceptual computational proof of the 'Duality Principle' wherein due to the inevitable 'logical inconsistency' and 'computational indeterminacy' arising from the SROCS computational structure (which is contradicted by empirical evidence indicating the capacity of these key scientific SROCS paradigms to compute the "existence" or "non-existence" of any particular 'y' element or value) - which points at the existence of the higher-ordered (singular) 'Universal Computational/Consciousness Principle that computes the "simultaneous co-occurrences" of any (exhaustive-hypothetical) ' $x-y$ ' pairs' series; it is suggested that the inclusion of this computational Duality Principle as one of the (seven) theoretical postulates of the CUFT (e.g., specifically alongside the CUFT's 'Computational Invariance' and 'Universal Consciousness' postulates) unequivocally asserts that there cannot (in principle) exist any 'material-causal' effect/s (or relationship/s) being transferred across any (two subsequent) USCF's frames! This is because the CUFT's very definition of all four 'physical' properties of 'space', 'time', 'energy' and 'mass' - as secondary computational by-products of the (singular) Universal Computational Consciousness' computation of (an extremely rapid series of) 'Universal Simultaneous Computational Frames' (USCF's); and moreover the CUFT's 'Computational Invariance' postulate indication that due to the 'computational variance' of these four (secondary computational) 'physical' properties (e.g., as existing only "during" the appearance of the USCF frames but 'non-existence' "in-between" any two such subsequent frames, see Bentwich, 2012:c-d) as opposed to the 'computational invariance' of the 'Universal Consciousness Principle' ('י'), we need to regard only this singular (computationally invariant) 'Universal Consciousness Principle' as "real" whereas all four (secondary computationally variant) 'physical' properties must be regarded as merely 'phenomenal' (i.e., as being comprised in reality only from the singular Universal Consciousness Principle); Therefore, the CUFT's 'Universal Consciousness Principle' advocated that none of these four (secondary computationally variant) 'physical' properties (e.g., of 'space', 'time', 'energy' or 'mass') "really" exists - but rather that there is only this one singular Universal Consciousness Principle which exists (solely) "in-between" any 
(two subsequent) USCF's frames and also solely produces each of these USCF's derived four 'phenomenal physical' properties; Hence, it was evinced (by the CUFT's Universal Consciousness Principle) that there cannot be any 'transference' of any hypothetical 'material' or 'physical' entity, effect, or property across any (two subsequent) USCF's frames! We therefore reach the inevitable theoretical conclusion that the current scientific (Cartesian) "material-causality' basic assumption underlying all key scientific SROCS paradigms as well as all (empirically knowable) 'Gödel-like' (inductive or deductive) SROCS ' $x-y$ ' relationships, wherein there exists a 'material-causal' effect/s (or relationship/s) between any given ' $x$ ' element and any (exhaustive hypothetical) ' $y$ ' series which determines the "existence" or "non-existence" of that (particular) 'y' element (or value) - is untenable! Instead, we must accept the CUFT's assertion that there can only exists one singular 'Universal Consciousness Principle' ('י') which both (solely) produces- all (apparent) secondary computational 'physical' properties (of 'space', 'time', 'energy' and 'mass'), as well as computes the "simultaneous co-occurrences" of any (particular) exhaustive-hypothetical inductive or deductive 'x-y' pairs series (e.g., comprising the exhaustive USCF's frames).

\section{The "universal consciousness principle's computational program"}

Therefore, it follows that based on the recognition of the singularity of the Universal Consciousness Principle's 'a-casual' computation of the "simultaneous co-occurrences" of all (inductive or deductive) ' $x$-y' pairs' series (as comprising the exhaustive USCF's frames) - we need to be able to reformulate all of the previously mentioned key scientific SROCS paradigms (Bentwich, 2012b-d), including: Darwin's 'Natural Selection Principle' and associated 'Genetic Encoding' hypothesis, Neuroscience's Psychophysical Problem of human Consciousness, as well as all (exhaustive hypothetical) 'Gödel-like' (apparent) inductive or deductive SROCS computational paradigms based on this singular (higher-ordered) Universal Consciousness Principle's ('') 'a-causal' USCF's computation;

Hence, what follows is a description of the principle theoretical ramifications of reformulating each of these key scientific (apparent) SROCS computational paradigms, as well as a more generalized description of a tentative 'Universal Consciousness Principle Program' (e.g., which may offer a successful alternative for 'Hilbert's Mathematical Program' to base all of our human scientific knowledge upon the foundations of the operation of the singular Universal Consciousness Principle). First, it may be worthwhile to rearticulate the reformalization of each of these key scientific (apparent) SROCS paradigms in terms of the operation of the singular Universal Consciousness Principle (as previously outlined: Bentwich, 2012b):

S.:D2: $[\{E\{1 \ldots n\}, o\} s t 1 ;\{E\{1 \ldots n\}, o\} s t 2 \ldots\{E\{1 \ldots n\}, o\} s t n]$.

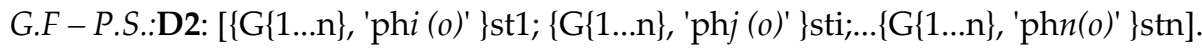

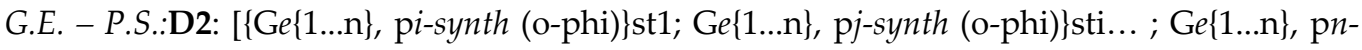
synth (o-phi)\}stn]

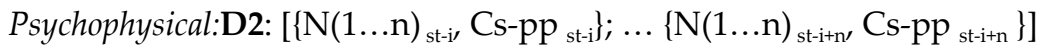


Functional: D2: [\{Cs(pp) $\left.\left.\mathrm{f}_{\mathrm{i}}, \mathrm{Na}(\mathrm{spp}) \mathrm{fi}\right\}_{\mathrm{st}-\mathrm{i}} ; \ldots\left\{\mathrm{Cs}(\mathrm{pp}) \mathrm{f}_{(\mathrm{i}+\mathrm{n})}, \mathrm{Na}(\mathrm{spp}) \mathrm{f}_{(\mathrm{i}+\mathrm{n})}\right\}_{\mathrm{st}(\mathrm{i}+\mathrm{n})}\right]$

Phen.: D2: [\{Cs(pp- fi)-Ph $\left.\left.\mathrm{i}_{\mathrm{i}} \mathrm{Na}(\mathrm{spp}-\mathrm{fi})-\mathrm{Ph}_{\mathrm{i}}\right\}_{\mathrm{st-}-\mathrm{j}}, \ldots\left\{\mathrm{Cs}(\mathrm{pp}-\mathrm{fi})-\mathrm{Ph}_{(\mathrm{i}+\mathrm{n})}, \mathrm{Na}(\mathrm{spp}-\mathrm{fi})-\mathrm{Ph}\right\}_{\mathrm{st}-(\mathrm{i}+\mathrm{n})}\right]$

Self: D2: [ $\left\{\mathrm{Cs}(\mathrm{pp}-\mathrm{fi}) \mathrm{Ph}-\mathrm{Si}, \mathrm{Na}(\mathrm{pp}-\mathrm{fi}) \mathrm{Ph}-\mathrm{S}_{\mathrm{i}}\right\}_{\mathrm{st-i}} ; \ldots$. C s(pp-fi)Ph-S(i+n), Na(pp-fi)Ph-S(i+n) $\left.\}_{\mathrm{st}-(\mathrm{i}+\mathrm{n})}\right]$

GIT:D2: ([\{S\{1...n $\}, \mathrm{t}\} i \ldots\{\mathrm{S}\{1 \ldots \mathrm{n}\}, \mathrm{t}\} z]$, or $[\{\mathrm{x}\{1 \ldots \mathrm{n}\} i, \mathrm{y} i\} \ldots\{\mathrm{x}\{1 \ldots \mathrm{n}\} z, \mathrm{y} z\}])$

Indeed, what may be seen from this singular description of all of these key scientific SROCS paradigms, is that it recognize the fact that all of these major (apparent) SROCS paradigms are computed simultaneously as different "co-occurring" ' $x-y$ ' pairs embedded within the same (single or multiple) USCF frame that is produced by the singular Universal Consciousness Principle (''); What this means is that the recognition of the singularity of this Universal Consciousness Principle as the sole "reality" which computes the "simultaneous co-occurrences" of all of these (particular) exhaustive hypothetical ' $x-y$ ' pairs series, and which also exists (solely) "in-between" any two such USCF's - forces us to transcend the 'narrow constraints' of the (current) Cartesian 'material-causal' theoretical framework (e.g., which assumes that any given 'y' entity (or phenomenon) is "caused" by its (direct or indirect) physical interaction/s with (an exhaustive hypothetical ' $x$ ' series); Instead, this singular Universal Consciousness Principle 'a-causal' computation asserts that it is the same singular Universal Consciousness Principle which computes- produces- retains- and evolves- all of these particular scientific (apparent) SROCS 'x-y' pairs series across a series of USCF's...

In other words, instead of the existence of any "real" material-causal relationship between any of these (particular SROCS) ' $x \rightarrow y$ ' entities (e.g., Darwin's Natural Selection Principle's assumed 'material-causal' relationship between an organism's Environmental Factors, ' $x$ ', and own traits or behavior ' $y$ '; or between any exhaustive hypothetical Genetic Factors and any given phenotypic behavior; or between Neuroscience's Psychophysical Problem of Human Consciousness' psychophysical stimulation, ' $x$ ', and Neural Activation, ' $y$ '; or in fact between any hypothetical inductive or deductive Gödel-like SROCS ' $x$-y' factors); the CUFT's Universal Consciousness Principle offers an alternative singular (higher-ordered) computational mechanism which computes the "simultaneous co-occurrences" of any of these (exhaustive hypothetical) ' $x$-y' pairs' series - which are all produced- and embedded- within the Universal Consciousness Principle's computed USCF's frames... Indeed, the shift from the current 'material-causal' (Cartesian) scientific framework towards the Universal Consciousness Principle's singular computation of the "simultaneous co-occurrences" of all exhaustive hypothetical (inductive or deductive) ' $x$-y' pairs' series may lead the way for reformulating all of these key scientific SROCS paradigms (as well as any other hypothetical inductive or deductive ' $x-y$ ' series) within a basic "Universal Consciousness Principle Computational Program";

Essentially, such a 'Universal Consciousness Principle's Computational Program' is based upon the foundations of the CUFT's (abovementioned) three postulates of the 'Duality Principle', the 'Computational Invariance' principle and the 'Universal Consciousness Principle' - all pointing at the fact that all empirically computable (inductive or deductive) ' $x$-y' relationships must necessarily be based upon the singular (conceptually higher-ordered) Universal Consciousness Principle which is solely responsible for the computation of the "simultaneous co- 
occurrences" of all such (exhaustive hypothetical) inductive or deductive 'x-y' pairs series comprising the totality of the USCF's (single or multiple) frames.... Moreover, this singular Universal Consciousness Principle ('י') was also shown to exist independently of any (secondary computational) 'physical properties' (e.g., of 'space', 'time', 'energy' and 'mass') and therefore constitute the only "reality" that exists invariantly (i.e., both as giving rise to the four 'phenomenal' physical properties and as existing solely "in-between" any two such subsequent USCF's frames).

In order to appreciate the full (potential) theoretical significance of such a 'Universal Consciousness Principle Computational Program' it may be worthwhile to reexamine Hillbert's famous 'Mathematical Program' to base Mathematics upon the foundations of Logic (e.g., and by extension also all of Science upon the foundations of Mathematics and Logic), and more specifically, to revisit 'Gödel's Incompleteness Theorem' (GIT) which delivered a critical blow to Hilbert's 'Mathematical Program'; It is a well-known that Hilbert's Mathematical Program sought to base Mathematics (e.g., and by extension also the rest of inductive and deductive Science) upon a logical foundation (e.g., of certain axiomatic definitions); It is also well known that Gödel's Incompleteness Theorem (GIT) has failed Hillbert's Mathematical Program due to its proof that there exists certain 'self-referential' logical-mathematical statements that cannot be determined as "true" or "false" (e.g., or logically 'consistent' or 'inconsistent') from within any hypothetical axiomatic logical-mathematical system... Previously (Bentwich, 2012c,d) it was suggested that perhaps scientific Gödel -like SROCS computational systems may in fact be constrained by the Duality Principle's (generalized) format, thus:

i.

SROCS: PR $\{x, y\} \rightarrow\left[y^{\prime}\right.$ or 'not y']/di1...din

ii. $\quad$ SROCS CR $\{S, t\} \rightarrow[$ 't' or 'not t']/di1 ...din

wherein it was shown that both inductive ('i') and deductive (ii) SROCS scientific computational systems are necessarily constrained by the Duality Principle (e.g., as part of the broader CUFT). In other words, the Duality Principle's (generalized format) was shown to constrain all (exhaustive hypothetical) Gödel -like (inductive or deductive) scientific SROCS paradigms, thereby pointing at the existence of a singular (higher-ordered) Universal Consciousness Principle ('') which is solely capable of computing the "simultaneous co-occurrences" of any (exhaustive hypothetical) ' $x-y$ ' pairs series. It is important to note, however, that the conceptual computational constraint imposed upon all (Gödel -like) inductive or deductive scientific SROCS paradigms was shown to apply for all of those inductive or deductive (apparent) scientific SROCS paradigms - for which there is an empirically known (or 'knowable') ' $x-y$ ' pairs series results!

This latter assertion of the Duality Principle's (generalized proof) may be significant as it both narrows- and emphasized- the scope of the 'scientifically knowable domain'; In other words, instead of the current 'materialistic-reductionistic' scientific framework which is anchored in a basic (inductive or deductive) SROCS computational format (see above) which inevitably leads to both 'logical inconsistency' and 'computational indeterminacy' that are contradicted by robust empirical findings (e.g., pertaining to the key scientific SROCS paradigms); The Duality Principle (e.g., as one of the postulates within the broader CUFT) proves that the only 
means for computing the "simultaneous co-occurrences" of any (exhaustive hypothetical) ' $x$ $y^{\prime}$ pairs series is carried out by the singular (higher-ordered) Universal Consciousness Principle ('')... Moreover, the (generalized format of the) Duality Principle goes farther to state that for all other (exhaustive hypothetical) inductive or deductive computational SROCS paradigms for which there exists a proven empirical capacity to determine the values of any particular ' $x-y$ ' pairs (e.g., empirically "known" or "knowable" ' $x$-y' pairs results)- any of these (hypothetical) scientific SROCS computations must be carried out by the CUFT's identified singular Universal Consciousness Principle ('')')

The (potential) significance of this generalized assertion made by the Computational Unified Field Theory's (CUFT): 'Duality Principle', 'Computational Invariance' principle and Universal Consciousness Principle ('') is twofold:

a. First, it narrows down the scope of (inductive or deductive) determinable scientific phenomena - to only those (inductive or deductive) ' $x-y$ ' relationships for which there is an empirical capacity to determine their "simultaneously co-occurring" values; essentially the 'Universal Consciousness Principle's Computational Program' anchors itself in the Duality Principle's focus on only those inductive or deductive ' $x-y$ ' relationship/s or phenomenon for which there is an empirically 'known' or 'knowable' capacity to determine these ' $x-y$ ' pairs values. It is perhaps important to note (in this context) that all of the 'other' inductive or deductive 'x-y' relationship/s which cannot be (empirically) known - "naturally" lie outside the scope of our human (scientific) knowledge (and therefore should not be included, anyway within the scope of Science)... Nevertheless, the strict limitation imposed by the 'Universal Consciousness Principle Computational Program' - may indeed be significant, as it clearly defines the boundaries of "admissible scientific knowledge" to only that scientific knowledge which is based on empirically known or knowable results pertaining to the "simultaneous co-occurrences" of any 'x-y' relationship or phenomenon; (Needless to say that the strict insistence of the Universal Consciousness Computational Program upon dealing only with

b. Second, based on this strict definition of Science as dealing solely with 'empirical knowable' (simultaneously co-occurring) ' $x$-y' relationship/s or phenomenon - the 'Universal Consciousness Computational Program' may in fact offer a broader alternative to GIT (failing of Hilbert's 'Mathematical Program'); This is because once we accept the Universal Consciousness Principle's Computational Program's (above) strict 'empirical constrains', we are led to the Duality Principle's (generalized) conceptual computational proof that any (exhaustive hypothetical) inductive or deductive scientific SROCS' 'x-y' relationship must be determined by the singular Universal Consciousness Principle ('') computation of the "simultaneous co-occurrences" of any (exhaustive hypothetical) 'x-y' pairs series. This means that instead of GIT assertion that it is not possible (in principle) to construct a consistent Logical-Mathematical System which will be capable of computing any mathematical (or scientific) claim or theorem, the Universal Consciousness Computational Program asserts that based on a strict definition of Science as dealing solely with empirically knowable 'x-y' relationship/s or phenomenon, we obtain a singular (higherordered) Universal Consciousness Principle which is solely responsible for computing the 
"simultaneous co-occurrences" of any (exhaustive hypothetical) inductive or deductive 'xy' pairs series (e.g., which were shown by the CUFT to comprise the totality of any single or multiple USCF's frames that are solely produced by this Universal Consciousness Principle). In that sense, it may be said that the Universal Consciousness Principle Computational Program points at the existence of the singular (higher-ordered) Universal Consciousness Principle as constraining- and producing- all inductive or deductive scientific relationship/s or phenomena (e.g., which was also shown earlier and previously to constitute the only "reality" which both produces all USCF's derived secondary computational 'physical properties and also solely exists "in-between" any two such USCF's).

\section{Theoretical ramifications of the universal consciousness principle}

The discovery of the singular Universal Consciousness Principle (alongside its 'Universal Consciousness Computational Program') may bear a few significant theoretical ramifications:

1. The Sole "Reality" of the Universal Consciousness Principle: As shown above, all scientific (inductive and deductive) disciplines need to be reformulated based on the recognition that there exists only a singular (higher-ordered) Universal Consciousness Principle ('י') which solely produces- sustains- evolves (and constrains) all (apparent) SROCS (inductive or deductive) ' $x$-y' relationships; Moreover, this Universal Consciousness Principle is recognized as the sole "reality" that both produces- sustains- and evolvesany of the apparent (four) 'physical' properties of 'space', 'time', 'energy' and 'mass', as well as exists independently of any such 'physical' properties - and is therefore recognized as the only singular "reality", whereas these apparent 'physical' properties are seen as merely 'phenomenal' (secondary computational) manifestations of this singular (higherordered) Universal Consciousness Principle "reality".

2. The Transcendence of 'Material-Causality' by the Universal Consciousness Principle 'A-Causal' Computation: As shown (above), the acceptance of the Universal Consciousness Principle ('י') as the sole "reality" which both produces- (sustains- and evolves-) all USCF's (secondary computational) 'physical' properties, as well as exists independently "in-between" any (two subsequent) USCF's; (Alongside the Duality Principle's negation of any apparent SROCS' 'causal' relationships and the 'Computational Invariance' principle indication that only the 'computationally invariant' 'Universal Consciousness Principle' "really" exists whereas the secondary 'computationally variant' physical properties are only 'phenomenal'.) - point at the negation of any "real" material-causal ('x$\left.y^{\prime}\right)$ relationships, but instead indicate that there can only exist a singular (higher-ordered) Universal Consciousness Principle 'a-causal' computation of the "simultaneous cooccurrences" of any exhaustive hypothetical inductive or deductive 'x-y' pairs' series... (As shown earlier, the strict negation of the existence of any "real" 'material-causal' ' $x \rightarrow y$ ' relationships was evinced by the simple fact that according to the CUFT's model there cannot exist any "real" computationally variant 'physical' or 'material' property that can 
"pass" across any two subsequent USCF's, but only the computationally invariant "real" Universal Consciousness Principle which exists singularly - as solely producing all apparent secondary computational 'physical' properties as well as existing independently "in-between" any two such subsequent USCF's frames.) Indeed, the need to replace all apparent 'material-causal' ' $x-y$ ' SROCS relationships by a singular (higher-ordered) Universal Consciousness Principle computation of the 'simultaneous co-occurrences' of all possible inductive or deductive ' $x$-y' pairs series was shown to apply to all of the key (apparent) scientific SROCS paradigms (including: Darwin's Natural Selection Principle and associated Genetic Encoding hypothesis, Neuroscience's Psychophysical Problem of human Consciousness as well as to all Gödel-like hypothetical inductive or deductive SROCS paradigms; what this implies is that for all of these apparent SROCS scientific paradigms the sole "reality" of the Universal Consciousness Principle forces us to transcend each of the (particular) 'material-causal' $x-y$ relationships in favor of the Universal Consciousness Principle's singular computation of all (exhaustive hypothetical) ' $x-y$ ' pairs series; Thus, for example, instead of Darwin's current 'Natural Selection Principle' SROCS material-causality thesis, which assumes that it is the direct (or indirect) physical interaction between the organism and its Environmental Factors that causes that organism to 'survive' or be 'extinct', the adoption of the Universal Consciousness Principle (and Duality Principle) postulates brigs about a recognition that there is only a singular (Universal Consciousness based) conceptually higher-ordered 'a-causal' computation of the "simultaneous co-occurrences" of an exhaustive hypothetical pairs series of 'organism' and 'Environmental Factors' (e.g., which are computed as part of the Universal Consciousness Principle's production of the series of USCF's frames).

3. Possible Resolution of Physical (and Mathematical) Conundrums: It is suggested that certain key Physical (and Mathematical) Conundrums including: Physics "dark energy", "dark matter" and "arrow of time" enigmas may be potentially resolved through the application of this singular 'Universal Consciousness Principle'; this is because according to the CUFT, all (four) 'physical' properties of 'space', 'time', 'energy' and 'mass' are (in reality) solely produced by the Universal Consciousness Principle (e.g., as secondary computational 'phenomenal' properties); Hence, the key enigma of "dark energy" and "dark matter" (e.g., the fact that based on the calculation of the totality of 'mass' and 'energy' in the observable cosmos the expansion of the universe should not be as rapid as is observed - which is currently interpreted as indicating that approximately $70-90 \%$ of the "energy" and "mass" in the universe in "dark", that is not yet observable) - may be explainable based on the CUFT's delineation of the Universal Consciousness Principle's (extremely rapid) computation of the series of USCF's. This is due to the fact that according to the Universal Consciousness Principle's (previously discovered: Bentwich, 2012a) 'Universal Computational Formula' the production of any "mass" or "energy" ("space" or "time") 'physical' properties - are entirely (and solely) produced through the Universal Consciousness Principle's computation of the degree of 'Consistency' (e.g., 'consistent' or 'inconsistent') across two other Computational Dimensions, i.e., 'Framework' ('frame' vs. 'object') and 'Locus' ('global' vs. 'local'): Thus, for instance it was shown that any "mass" measurement of any object in the universe is computed by the Universal Consciousness 
Principle ('י') as the degree of 'consistent-object' measurement (of that particular) object across a series of USCF frames.

Hence, by extension, the totality of the "mass" measured across the entire physical universe should be a measure of the degree of consistent-object/s values across a series of USCF's! Note, however, that based on the abovementioned recognition that in "reality" - only the Universal Consciousness Principle ('י') "exists" (e.g., both as producing any of the USCF's derived four secondary computational 'physical' properties as well as existing independently "in-between" any two such USCF's frames), and therefore that only this Universal Consciousness Principle "really" produces all of the (apparent) "mass" and "energy" in the 'physical' universe (e.g., rather than the "energy" and "mass" in the 'physical' universe being "caused" by the "material" objects in the cosmos)... Hence, also all of the "energy" in the physical universe is solely produced by this (singular) Universal Consciousness Principle, e.g., as a measure of the degree of 'inconsistent-frame' (changes) of all of the objects (I the universe) across a series of USCF's frames. Therefore, according to the CUFT, the explanation of all of the "mass" and "energy" values observed in the 'physical' universe - should be solely attributed to the operation of the Universal Computational Principle, i.e., through its (extremely rapid) computation of the rapid series of USCF's (respective secondary computational measures of the abovementioned degree of 'consistent-object': "mass", or 'inconsistent-frame': "energy"). We therefore obtain that the (accelerated) rate of expansion of the physical universe - should be explained (according to the CUFT) based on the Universal Consciousness (extremely rapid) computation of the USCF's (e.g., which gives rise to the apparent secondary computational 'physical' measures of 'consistent-object': "mass" or 'inconsistent-frame': "energy"), rather than arise from any 'material-causal' effects of any (strictly hypothetical) "dark mass" or "dark energy"... (Once again, it may be worth pointing at the abovementioned conceptual computational proof that there cannot be any transference of any "physical" property (entity or effect etc.) across any (two subsequent) USCF's frames, but only the retention- or evolution- of all of the spatial pixels' "physical" properties by the singular Universal Consciousness Principle across the series of USCF's - which therefore also precludes the possibility of any "real" "material" effects exerted by any "dark" mass or energy on the expansion of the 'physical' universe across a series of USCF frames.)

Similarly, the "arrow of time" conundrum in modern Physics, e.g., which essentially points at the fact that according to the laws of Physics, there should not be any difference between the physical pathways of say the "breaking of a glass cup into a (thousand) small glass' pieces" and the "re-integration of these thousand glass' pieces into a unitary glass cup"! In other words, according to the strict laws of Physics, there should not be any preference for us seeing "glasses" break into a thousand pieces - over our seeing of the thousand pieces become "reintegrated" into whole glass cups (again), which is obviously contradicted by our (everyday) phenomenal experiences (as well as by our empirical scientific observations)... Hence, according to the current state of (quantum and relativistic) models of Physical reality - there is no reasonable explanation for this "arrow of time" apparent empirical "preference" for the "glass breaking into pieces" scenario over the "reintegration of the glass pieces" scenario... 
However, it is suggested that according to one of the CUFT critical empirical predictions (previously outlined: Bentwich, 2012b) this "arrow of time" Physical conundrum may be resolved: This is because one (of three) critical empirical predictions of the CUFT assert the possibility of reversing any spatial-temporal sequence associated with any given 'electromagnetic spatial pixel' through the appropriate manipulation of that object's (or event's) electromagnetic spatial pixel values (across a series of USCF's): It was thus indicated that if we were to accurately record the spatial electromagnetic pixels' values of any particular object (e.g., such as an amoeba or any other living organism for instance) across a series of USCF's frames (e.g., or even through a certain sampling from a series of USCF's), and to the extent that we could appropriately manipulate these various electromagnetic spatial pixels' values in such a manner which allows us to reproduce that objects' electromagnetic spatial pixels' values (across the measured series of USCF's) - in the reversed spatial-temporal sequence, then it may be possible to reverse the "flow of time" (e.g., spatial-temporal electromagnetic pixels' sequence). In this way it should be possible (according to one of the critical predictions of the CUFT) to actually "reverse" the "arrow of time" (e.g., at least for particular object/s or event/s: such as for instance, bring about a situation in which a "broken glass cup may in fact be reintegrated"...)

\section{Author details}

Jonathan Bentwich ${ }^{*}$

Brain Perfection LTD, Israel

\section{References}

[1] Bentwich, J. (2003a). From Cartesian Logical-Empiricism to the'Cognitive Reality': A Paradigmatic Shift, Proceedings of Inscriptions in the Sand, Sixth International Literature and Humanities Conference, Cyprus

[2] Bentwich, J. (2003b). The Duality Principle's resolution of the Evolutionary Natural Selection Principle; The Cognitive 'Duality Principle': A resolution of the 'Liar Paradox' and 'Gödel's Incompleteness Theorem' conundrums; From Cartesian Logical-

[3] Empiricism to the 'Cognitive Reality: A paradigmatic shift Proceedings of $12^{\text {th }}$ International Congress of Logic, Methodology and Philosophy of Science, August Oviedo, Spain

[4] Bentwich, J. (2003c). The cognitive'Duality Principle': a resolution of the'Liar Paradox' and'Gödel's Incompleteness Theorem' conundrums, Proceedings of Logic Colloquium, Helsinki, Finland, August 2003

[5] Bentwich, J. (2004). The Cognitive Duality Principle: A resolution of major scientific conundrums, Proceedings of The international Interdisciplinary Conference, Calcutta, January 
[6] Bentwich, J. (2006). The 'Duality Principle': Irreducibility of sub-threshold psychophysical computation to neuronal brain activation. Synthese, , 153(3), 451-455.

[7] Bentwich, J. (2012a). Quantum Mechanics / Book 1 (979-9-53307-377-3Chapter title: The'Computational Unified Field Theory' (CUFT): Harmonizing Quantum Mechanics and Relativity Theory.

[8] Bentwich, J. (2012b). Quantum Mechanics / Book 1 (979-9-53307-377-3Chapter 23, Theoretical Validation of the Computational Unified Field Theory., 551-598.

[9] Bentwich, J. (2012c). Quantum Mechanics (In Press) Chapter title: The'Computational Unified Field Theory' (CUFT): A Candidate Theory of Everything.

[10] Born, M. (1954). The statistical interpretation of quantum mechanics, Nobel Lecture, December 11, 1954

[11] Brumfiel, G. (2006). Our Universe: Outrageous fortune. Nature, , 439, 10-12.

[12] Darwin, C. (1859). On the Origin of Species by Means of Natural Selection, or the Preservation of Favoured Races in the Struggle for Life John Murray, London; modern reprint Charles Darwin, Julian Huxley (2003). On The Origin of Species. Signet Classics. 0-45152-906-5

[13] Ellis, J. (1986). The Superstring: Theory of Everything, or of Nothing? Nature, , 23 (6089), 595-598.

[14] Greene, B. (2003). The Elegant Universe, Vintage Books, New York 
Chapter 29

\section{Shannon Informational Entropies and Chemical Reactivity}

Nelson Flores-Gallegos

Additional information is available at the end of the chapter

http://dx.doi.org/10.5772/54329

\section{Introduction}

During the last decade, researchers around the world have shown that Information Theory [7] is probably one of the most important models in modern science. This model has given rise to applications and reinterpretations of concepts in Physics, Chemistry, Biology, Mathematics, Telecommunications and many other areas that are not, in principle, related to Information Theory. In the 90's, E. R. Frieden showed that important results such as the Schrödinger equation, the Maxwell-Boltzmann and Boltzmann distributions, the Dirac equation can be derived from principles of Information Theory [1-5].

Indded, information is a general concept that is perfectly applicable to any case. It is possible to ask what is the importance of the concept of information measure in quantum mechanics? What do they have in common the codes used to send messages from a communications' satellite have in common with the bases in a DNA molecule? How does the second law of Thermodynamics relate to Communication, to the extent that it is possible to speak of the entropy of a musical theme? How do the intricate problems of probability relate to the way we express ourselves orally or in writing? The answer to these questions can be found in information, and the fact that this concept can link very different ideas reveals its great generality and power.

In Chemistry, Information Theory has been applied to the characterization of chemical systems and chemical processes [10-13]. It has been shown that it is possible to use informational entropies to characterize processes such as bond breaking and bond formation. Shannon's entropy can be regarded as a general measure of information that can be used to obtain the Fukui function, which is a parameter of chemical reactivity in atomic and simple molecular systems [6]. In practice, the formal development of applications of Shannon's entropies in Density Functional Theory (DFT) is a fertile area of research. In this context the 
maximum entropy method has been applied to DFT [33]. Following this line of research, we have initiated a new investigation to derive formal relationships between Information Theory concepts and Theoretical Chemistry.

In this work, we consider the idea that Shannon's entropy can be directly related to some fundamental DFT concepts. To show this, we present some simple mathematical derivations to prove that the first derivative of the Shannon entropy is directly related to DFT reactivity parameters such as the Fukui function, the hardness, softness, and chemical potential and that it might even be possible to obtain a formal relationship between Shannon's entropy and the electron energy. Finally, chemical applications are presented in which the relationships obtained in this work are used in two case studies involving a simple chemical reaction and a conformational analysis.

\section{The first derivatives of Shannon's entropy and their relationship to chemical reactivity}

For purposes of this chapter we take as a starting point the definition of the unnormalized Shannon's entropy in terms of the electron density in position space, which is defined as:

$$
s(\mathbf{r})=-\int \rho(\mathbf{r}) \ln \rho(\mathbf{r}) d \mathbf{r}
$$

If normalized electron densities are used, $\frac{\rho(\mathbf{r})}{N}$, Shannon's entropy becomes positive: $S(\mathbf{r}) \geq 0$ and the normalized Shannon entropy is

$$
S(\mathbf{r})=-\int \frac{\rho(\mathbf{r})}{N} \ln \frac{\rho(\mathbf{r})}{N} d \mathbf{r},
$$

where $N$ is the number of electrons. This expression can be rewritten as:

$$
\begin{aligned}
S(\mathbf{r}) & =-\frac{1}{N} \int \rho(\mathbf{r}) \ln \left(\frac{\rho(\mathbf{r})}{N}\right) d \mathbf{r} \\
& =-\frac{1}{N} \int \rho(\mathbf{r})[\ln \rho(\mathbf{r})-\ln N] d \mathbf{r} \\
& =-\frac{1}{N} \int \rho(\mathbf{r}) \ln \rho(\mathbf{r}) d \mathbf{r}+\frac{1}{N} \ln N \underbrace{\int \rho(\mathbf{r}) d \mathbf{r}}_{=N} \\
& =-\frac{1}{N} \int \rho(\mathbf{r}) \ln \rho(\mathbf{r}) d \mathbf{r}+\ln N \\
& =-\frac{s(\mathbf{r})}{N}+\ln N .
\end{aligned}
$$

Differentiating the entropy $S(\mathbf{r})$ with respect to $N$ at constant external potential $v(\mathbf{r})$ : 


$$
\left(\frac{\partial S(\mathbf{r})}{\partial N}\right)_{v(\mathbf{r})}=-\frac{s(\mathbf{r})}{N^{2}}-\frac{1}{N} \int\left(\frac{\partial \rho(\mathbf{r})}{\partial N}\right)_{v(\mathbf{r})} \ln \rho(\mathbf{r}) d \mathbf{r}+\frac{1}{N^{\prime}}
$$

The term $\left(\frac{\partial \rho(\mathbf{r})}{\partial N}\right)_{v(\mathbf{r})}$, is the Fukui function, which is a chemical reactivity parameter in DFT [33].

Also, we can set up the follow relationship

$$
\left(\frac{\partial \rho(\mathbf{r})}{\partial \mu}\right)_{v(\mathbf{r})}=\left(\frac{\partial \rho(\mathbf{r})}{\partial N}\right)_{v(\mathbf{r})}\left(\frac{\partial N}{\partial \mu}\right)_{v(\mathbf{r})},
$$

and obtain the variation of Shannon's entropy with respect to the chemical potential:

$$
\begin{aligned}
\left(\frac{\partial S(\mathbf{r})}{\partial N}\right)_{v(\mathbf{r})}\left(\frac{\partial N}{\partial \mu}\right)_{v(\mathbf{r})}= & -\frac{s(\mathbf{r})}{N^{2}}\left(\frac{\partial N}{\partial \mu}\right)_{v(\mathbf{r})}-\frac{1}{N} \int\left(\frac{\partial \rho(\mathbf{r})}{\partial N}\right)_{v(\mathbf{r})}\left(\frac{\partial N}{\partial \mu}\right)_{v(\mathbf{r})} \ln \rho(\mathbf{r}) d \mathbf{r} \\
& +\frac{1}{N}\left(\frac{\partial N}{\partial \mu}\right)_{v(\mathbf{r})}
\end{aligned}
$$

simplify,

$$
\left(\frac{\partial S(\mathbf{r})}{\partial \mu}\right)_{v(\mathbf{r})}=\left[\frac{1}{N}-\frac{S(\mathbf{r})}{N^{2}}\right]\left(\frac{\partial N}{\partial \mu}\right)_{v(\mathbf{r})}-\frac{1}{N} \int\left(\frac{\partial \rho(\mathbf{r})}{\partial \mu}\right)_{v(\mathbf{r})} \ln \rho(\mathbf{r}) d \mathbf{r},
$$

In this expression, we recognize the term $\left(\frac{\partial \rho(\mathbf{r})}{\partial \mu}\right)_{v(\mathbf{r})}$ which is the local softness.

Also, is it interesting to obtain the variation of Shannon's entropy with respect to the electron density,

$$
\left(\frac{\partial S(\mathbf{r})}{\partial \rho(\mathbf{r})}\right)_{v(\mathbf{r})}=-\frac{1}{N} \int 1+\ln \rho(\mathbf{r}) d \mathbf{r}
$$

A similar procedure can be used to obtain the above relations in momentum space as well as for the total Shannon entropy [14], which is defined as

$$
S_{T}=S(\mathbf{r})+S(\mathbf{p})
$$

where $S(\mathbf{r})$ and $S(\mathbf{p})$ are Shannon's entropies in position and momentum spaces, respectively. The variation of the total Shannon entropy with respect to electron number would be: 


$$
\frac{d S_{T}}{d N}=\left(\frac{\partial S(\mathbf{r})}{\partial N}\right)_{v(\mathbf{r})}+\left(\frac{\partial S(\mathbf{p})}{\partial N}\right)_{v(\mathbf{p})}
$$

this permit us, open a door, to the study of this kind of derivatives and chemical descriptors, in momentum space. Results about, the chemical reactivity parameters in momentum space, will be present in other work.

Summarizing, the relations obtained are:

i)

$$
\left(\frac{\partial S(\mathbf{r})}{\partial N}\right)_{v(\mathbf{r})}=-\frac{S(\mathbf{r})}{N^{2}}-\frac{1}{N} \int\left(\frac{\partial \rho(\mathbf{r})}{\partial N}\right)_{v(\mathbf{r})} \ln \rho(\mathbf{r}) d \mathbf{r}+\frac{1}{N}
$$

ii)

$$
\left(\frac{\partial S(\mathbf{r})}{\partial \mu}\right)_{v(\mathbf{r})}=\left[\frac{1}{N}-\frac{s(\mathbf{r})}{N^{2}}\right]\left(\frac{\partial N}{\partial \mu}\right)_{v(\mathbf{r})}-\frac{1}{N} \int\left(\frac{\partial \rho(\mathbf{r})}{\partial \mu}\right)_{v(\mathbf{r})} \ln \rho(\mathbf{r}) d \mathbf{r}
$$

iii)

$$
\left(\frac{\partial S(\mathbf{r})}{\partial \rho(\mathbf{r})}\right)_{v(\mathbf{r})}=-\frac{1}{N} \int 1+\ln \rho(\mathbf{r}) d \mathbf{r} .
$$

In the formalism of DFT, the reactivity parameters are defined as:

$$
\begin{aligned}
\text { Chemical potential } & =\mu=\left(\frac{\partial E}{\partial N}\right)_{v(\mathbf{r})} . \\
\text { Hardness } & =\eta=\left(\frac{\partial \mu}{\partial N}\right)_{v(\mathbf{r})}=\left(\frac{\partial^{2} E}{\partial N^{2}}\right)_{v(\mathbf{r})} . \\
\text { Softness } & =s=\left(\frac{\partial N}{\partial \mu}\right)_{v(\mathbf{r})}=\left(\frac{\partial \rho(\mathbf{r})}{\partial \mu}\right)_{v(\mathbf{r})} . \\
\text { Fukui function } & =f(\mathbf{r})=\left(\frac{\partial \rho(\mathbf{r})}{\partial N}\right)_{v(\mathbf{r})} .
\end{aligned}
$$

where $\mu$ is the Chemical potential, $\eta$ is the Hardness, $s$ is the Softness and $f(\mathbf{r})$ is the Fukui Function. Each one of these equations has a specific interpretation in Chemistry. The Chemical potential, $\mu$, is a measure the escaping tendency of an electron, which is constant, through all space, for the ground state of an atom, molecule, or solid [16]. The Hardness, $\eta$, is related to the polarizability [15] and the Fukui function [23-25], $\left(\frac{\partial \rho(\mathbf{r})}{\partial N}\right)_{v(\mathbf{r})}$, is interpreted as a measure of the sensitivity of the chemical potential with respect to an external perturbation at a particular point. These properties have been included in the chemical vocabulary since the early 1950s. 


\subsection{The second derivatives of Shannon's entropy}

In this section we obtain the second derivatives of the Shannon entropy respect to the electron density, how a first case, we take the Eq. (11),

$$
\begin{aligned}
\left(\frac{\partial^{2} S(\mathbf{r})}{\partial \rho(\mathbf{r}) \partial N}\right)_{v(\mathbf{r})}= & \frac{\partial}{\partial \rho(\mathbf{r})}\left\{-\frac{s(\mathbf{r})}{N^{2}}-\frac{1}{N} \int\left(\frac{\partial \rho(\mathbf{r})}{\partial N}\right)_{v(\mathbf{r})} \ln \rho(\mathbf{r}) d \mathbf{r}+\frac{1}{N}\right\} \\
= & -\frac{1}{N^{2}} \int 1+\ln \rho(\mathbf{r}) d \mathbf{r} \\
& -\frac{1}{N} \int\left(\frac{\partial f(\mathbf{r})}{\partial \rho(\mathbf{r})}\right)_{v(\mathbf{r})} \ln \rho(\mathbf{r})+\frac{f(\mathbf{r})}{\rho(\mathbf{r})} d \mathbf{r} .
\end{aligned}
$$

where $f(\mathbf{r})$ is it the Fukui function.

How a second case, consider the Eq. (12),

$$
\begin{aligned}
\left(\frac{\partial^{2} S(\mathbf{r})}{\partial \rho(\mathbf{r}) \partial \mu}\right)_{v(\mathbf{r})}= & \frac{\partial}{\partial \rho(\mathbf{r})}\left\{\left[\frac{1}{N}-\frac{s(\mathbf{r})}{N^{2}}\right]\left(\frac{\partial N}{\partial \mu}\right)_{v(\mathbf{r})}-\int\left(\frac{\partial \rho(\mathbf{r})}{\partial \mu}\right)_{v(\mathbf{r})} \ln \rho(\mathbf{r}) d \mathbf{r}\right\} \\
= & -\frac{1}{N^{2}}\left(\frac{\partial N}{\partial \mu}\right)_{v(\mathbf{r})} \int 1+\ln \rho(\mathbf{r}) d \mathbf{r} \\
& -\int\left(\frac{\partial s}{\partial \rho(\mathbf{r})}\right)_{v(\mathbf{r})} \ln \rho(\mathbf{r})+\frac{s}{\rho(\mathbf{r})} d \mathbf{r} .
\end{aligned}
$$

where $s$ is the softness.

Finally, for Eq. (13),

$$
\begin{aligned}
\left(\frac{\partial^{2} S(\mathbf{r})}{\partial \rho(\mathbf{r})^{2}}\right)_{v(\mathbf{r})} & =-\frac{1}{N} \int \frac{\partial}{\partial \rho(\mathbf{r})}(1+\ln \rho(\mathbf{r})) d \mathbf{r} \\
& =-\frac{1}{N} \int \frac{d \mathbf{r}}{\rho(\mathbf{r})} .
\end{aligned}
$$

\subsection{The change of Shannon entropy respect to the electron energy using Parr-Gadre-Bartolotti model}

Now, we obtain the variation of the Shannon entropy respect to the energy, in this case, consider the chemical potential $\mu=\left(\frac{\partial E}{\partial N}\right)_{v(\mathbf{r})}$ and the Eq. (4), 


$$
\begin{aligned}
\left(\frac{\partial S(\mathbf{r})}{\partial N}\right)_{v(\mathbf{r})}\left(\frac{\partial N}{\partial E}\right)_{v(\mathbf{r})} & -\frac{1}{N} \int\left(\frac{\partial \rho(\mathbf{r})}{\partial N}\right)_{v(\mathbf{r})}\left(\frac{\partial N}{\partial E}\right)_{v(\mathbf{r})} \ln \rho(\mathbf{r}) d \mathbf{r} \\
& +\left[\frac{1}{N}-\frac{s(\mathbf{r})}{N^{2}}\right]\left(\frac{\partial N}{\partial E}\right)_{v(\mathbf{r})} \\
= & -\frac{1}{N} \int\left(\frac{\partial \rho(\mathbf{r})}{\partial E}\right)_{v(\mathbf{r})} \ln \rho(\mathbf{r}) d \mathbf{r} \\
& +\left[\frac{1}{N}-\frac{s(\mathbf{r})}{N^{2}}\right]\left(\frac{\partial N}{\partial E}\right)_{v(\mathbf{r})},
\end{aligned}
$$

note the importance of this relation, we can establish a formal relation between the Shannon entropy with the electronic energy ${ }^{1}$.

In a similar way, we can obtain the second derivative of the last equation respect to electron density.

$$
\begin{aligned}
\left(\frac{\partial^{2} S(\mathbf{r})}{\partial \rho(\mathbf{r}) \partial E}\right)_{v(\mathbf{r})}= & \frac{\partial}{\partial \rho(\mathbf{r})}\left\{-\frac{1}{N} \int\left(\frac{\partial \rho(\mathbf{r})}{\partial E}\right)_{v(\mathbf{r})} \ln \rho(\mathbf{r}) d \mathbf{r}+\left[\frac{1}{N}-\frac{s(\mathbf{r})}{N^{2}}\right]\left(\frac{\partial N}{\partial E}\right)_{v(\mathbf{r})}\right\} \\
= & -\frac{1}{N} \int\left(\frac{\partial \mu(\mathbf{r})}{\partial \rho(\mathbf{r})}\right)_{v(\mathbf{r})} \ln \rho(\mathbf{r})+\frac{\mu(\mathbf{r})}{\rho(\mathbf{r})} d \mathbf{r} \\
& -\frac{1}{N^{2}}\left(\frac{\partial N}{\partial E}\right)_{v(\mathbf{r})} \int 1+\ln \rho(\mathbf{r}) d \mathbf{r}
\end{aligned}
$$

where $\mu(\mathbf{r})$ it is a local chemical potential.

For obtain a direct application of the last result in the DFT model, we selected the Parr-Gadre-Bartolotti model [26], PGB, this is a local model based in the Thomas-Fermi model $[17,18]$. The expression for the energy in the PGB model is,

$$
d[E]_{P G B}=C \rho(\mathbf{r})^{5 / 3}+\rho(\mathbf{r}) v(\mathbf{r})+B N^{2 / 3} \rho(\mathbf{r})^{4 / 3} d \mathbf{r}
$$

where $B=0.7544$ and $C=3.8738$.

Considering Eq. (21), and Eq. (23), the first step is it obtain $\left(\frac{\partial \rho(\mathbf{r})}{\partial E}\right)_{v(\mathbf{r})}$, for do this, consider

$$
\left(\frac{\partial \rho(\mathbf{r})}{\partial E_{P G B}}\right)_{\nu(\mathbf{r})}^{-1}=\left(\frac{\partial E_{P G B}}{\partial \rho(\mathbf{r})}\right)_{v(\mathbf{r})}
$$

\footnotetext{
${ }^{1}$ A more complete study about of the formal relation between electron energy and Shannon entropy will present in other work.
} 
with this and the Eq. (23),

$$
\begin{aligned}
\left(\frac{\partial E_{P G B}}{\partial \rho(\mathbf{r})}\right)_{\nu(\mathbf{r})} & =\frac{\partial}{\partial \rho(\mathbf{r})}\left\{C \rho(\mathbf{r})^{5 / 3}+\rho(\mathbf{r}) v(\mathbf{r})+B N^{2 / 3} \rho(\mathbf{r})^{4 / 3}\right\} \\
& =\frac{5}{3} C \rho(\mathbf{r})^{2 / 3}+v(\mathbf{r})+\frac{4}{3} B N^{2 / 3} \rho(\mathbf{r})^{1 / 3}
\end{aligned}
$$

and,

$$
\left(\frac{\partial \rho(\mathbf{r})}{\partial E_{P G B}}\right)_{v(\mathbf{r})}=\frac{1}{\frac{5}{3} C \rho(\mathbf{r})^{2 / 3}+v(\mathbf{r})+\frac{4}{3} B N^{2 / 3} \rho(\mathbf{r})^{1 / 3}},
$$

now consider the Eq. (21),

$$
\left(\frac{\partial S(\mathbf{r})}{\partial E_{P G B}}\right)_{v(\mathbf{r})}=-\frac{1}{N} \int \frac{\ln \rho(\mathbf{r})}{\frac{5}{3} C \rho(\mathbf{r})^{2 / 3}+v(\mathbf{r})+\frac{4}{3} B N^{2 / 3} \rho(\mathbf{r})^{1 / 3}} d \mathbf{r}+\left[\frac{1}{N}-\frac{s(\mathbf{r})}{N^{2}}\right]\left(\frac{\partial N}{\partial E}\right)_{v(\mathbf{r})}
$$

The importance of this results, resides in that for the first time we prove that there exist a formal relationship between concepts of the information theory and the chemical reactivity, based on a formal derivation rather than on a phenomenological interpretation.

\subsection{The variation of Shannon's entropy with respect to the electron kinetic energy}

In this case, we consider the famous Thomas-Fermi kinetic energy functional, defined as

$$
d E_{k}^{T F}[\rho(\mathbf{r})]=C_{F} \rho(\mathbf{r})^{5 / 3} d \mathbf{r}, \quad C_{F}=\frac{3}{10}\left(3 \pi^{2}\right)^{2 / 3},
$$

first we use the property (24)

$$
\left(\frac{\partial E_{k}^{T F}}{\partial \rho(\mathbf{r})}\right)_{v(\mathbf{r})}=C_{F} \frac{5}{3} \rho(\mathbf{r})^{2 / 3}
$$

now, we use the Eq. (21)

$$
\begin{aligned}
\left(\frac{\partial S(\mathbf{r})}{\partial E_{k}^{T F}}\right)_{v(\mathbf{r})} & =-\frac{1}{N} \int \frac{\ln \rho(\mathbf{r})}{C_{F} \frac{5}{3} \rho(\mathbf{r})^{2 / 3}} d \mathbf{r}+\left[\frac{1}{N}-\frac{s(\mathbf{r})}{N^{2}}\right] \frac{1}{\mu^{\prime}} \\
& =-\frac{3}{5 N C_{F}} \int \frac{\ln \rho(\mathbf{r})}{\rho(\mathbf{r})^{2 / 3}} d \mathbf{r}-\frac{1}{\mu N^{2}} \int \rho(\mathbf{r}) \ln \rho(\mathbf{r}) d \mathbf{r}+\frac{1}{\mu N}
\end{aligned}
$$




\subsection{The variation of Shannon's entropy with respect to the exchange energy in}

\section{LDA}

For simplicity we take only the exchange energy, in the Local Density Approximation (LDA), in this approximation the total energy of a system can be write as the sum of the correlation and exchange energy: $\varepsilon_{c x}=\varepsilon_{x}+\varepsilon_{c}$. The correlation part, $\varepsilon_{c}$ has been calculated and the results obtained were expressed like complicated expression of $\rho(\mathbf{r})$ [27]. In our case, we only consider the exchange term, defined as

$$
d E_{x}^{L D A}[\rho(\mathbf{r})]=-\frac{3}{4}\left(\frac{3}{\pi}\right)^{1 / 3} \rho(\mathbf{r})^{4 / 3} d \mathbf{r}
$$

again,

$$
\left(\frac{\partial E_{x}^{L D A}}{\partial \rho(\mathbf{r})}\right)_{v(\mathbf{r})}=-\left(\frac{3}{\pi}\right)^{1 / 3} \rho(\mathbf{r})^{1 / 3},
$$

and

$$
\begin{aligned}
\left(\frac{\partial S(\mathbf{r})}{\partial E_{x}^{L D A}}\right)_{v(\mathbf{r})} & =-\frac{1}{N} \int \frac{\ln \rho(\mathbf{r})}{\left(\frac{3}{\pi}\right)^{1 / 3} \rho(\mathbf{r})^{1 / 3}} d \mathbf{r}+\left[\frac{1}{N}-\frac{s(\mathbf{r})}{N^{2}}\right]\left(\frac{\partial N}{\partial E}\right)_{v(\mathbf{r})} \\
& =-\frac{1}{N} \int \frac{\ln \rho(\mathbf{r})}{\left(\frac{3}{\pi}\right)^{1 / 3} \rho(\mathbf{r})^{1 / 3}} d \mathbf{r}-\frac{1}{\mu N^{2}} \int \rho(\mathbf{r}) \ln \rho(\mathbf{r}) d \mathbf{r}+\frac{1}{\mu N}
\end{aligned}
$$

\subsection{Variation of Shannon's entropy with respect to the energy, considering the kinetic and exchange effects.}

Now, we can take the previous results to obtain the variation on Shannon's entropy considering the kinetic and exchange effects using local models. This derivative will be

$$
\begin{aligned}
\left(\frac{\partial S(\mathbf{r})}{\partial E_{k, x}^{T F, L D A}}\right)_{v(\mathbf{r})}= & \left(\frac{\partial S(\mathbf{r})}{\partial E_{k}^{T F}}\right)_{v(\mathbf{r})}+\left(\frac{\partial S(\mathbf{r})}{\partial E_{x}^{L D A}}\right)_{v(\mathbf{r})} \\
= & -\frac{3}{5 N C_{F}} \int \frac{\ln \rho(\mathbf{r})}{\rho(\mathbf{r})^{2 / 3}} d \mathbf{r}-\frac{1}{\mu N^{2}} \int \rho(\mathbf{r}) \ln \rho(\mathbf{r}) d \mathbf{r}+\frac{1}{\mu N} \\
& -\frac{1}{N} \int \frac{\ln \rho(\mathbf{r})}{\left(\frac{3}{\pi}\right)^{1 / 3} \rho(\mathbf{r})^{1 / 3}} d \mathbf{r}-\frac{1}{\mu N^{2}} \int \rho(\mathbf{r}) \ln \rho(\mathbf{r}) d \mathbf{r}+\frac{1}{\mu N} \\
= & {\left[-\frac{3}{5 N C_{F}} \int \frac{\ln \rho(\mathbf{r})}{\rho(\mathbf{r})^{2 / 3}} d \mathbf{r}-\frac{1}{N} \int \frac{\ln \rho(\mathbf{r})}{\left(\frac{3}{\pi}\right)^{1 / 3} \rho(\mathbf{r})^{1 / 3}} d \mathbf{r}\right] }
\end{aligned}
$$




$$
\begin{aligned}
& -\frac{1}{\mu N^{2}} \int \rho(\mathbf{r}) \ln \rho(\mathbf{r}) d \mathbf{r}+\frac{1}{\mu N} \\
= & -\frac{1}{N}\left(\frac{3}{5 C_{F}}+\frac{1}{(3 / \pi)^{1 / 3}}\right) \int\left(\frac{1+\rho(\mathbf{r})^{1 / 3}}{\rho(\mathbf{r})^{2 / 3}}\right) \ln \rho(\mathbf{r}) d \mathbf{r} \\
& -\frac{1}{\mu N^{2}} \int \rho(\mathbf{r}) \ln \rho(\mathbf{r}) d \mathbf{r}+\frac{1}{\mu N} \\
= & -\int\left[A\left(\frac{1+\rho(\mathbf{r})^{1 / 3}}{\rho(\mathbf{r})^{2 / 3}}\right)-B \rho(\mathbf{r})\right] \ln \rho(\mathbf{r}) d \mathbf{r}+B N,
\end{aligned}
$$

where

$$
A=\frac{3}{5 N C_{F}}+\frac{1}{N(3 / \pi)^{2 / 3}}
$$

and

$$
B=\frac{1}{\mu N^{2}}
$$

\subsection{Summary of relationships obtained}

Finally, we present a summary of the different relations obtained in this work. Whit this results, is possible say that the information theory is a model that is subjacent to the Density Functional Theory.

In the follow section we show some results of this relations applied to some chemical process.

$$
\begin{aligned}
\left(\frac{\partial S(\mathbf{r})}{\partial N}\right)_{v(\mathbf{r})} & =-\frac{s(\mathbf{r})}{N^{2}}-\frac{1}{N} \int\left(\frac{\partial \rho(\mathbf{r})}{\partial N}\right)_{v(\mathbf{r})} \ln \rho(\mathbf{r}) d \mathbf{r}+\frac{1}{N} \\
\left(\frac{\partial S(\mathbf{r})}{\partial \mu}\right)_{v(\mathbf{r})} & =\left[\frac{1}{N}-\frac{s(\mathbf{r})}{N^{2}}\right]\left(\frac{\partial N}{\partial \mu}\right)_{v(\mathbf{r})}-\frac{1}{N} \int\left(\frac{\partial \rho(\mathbf{r})}{\partial \mu}\right)_{v(\mathbf{r})} \ln \rho(\mathbf{r}) d \mathbf{r} . \\
\left(\frac{\partial S(\mathbf{r})}{\partial \rho(\mathbf{r})}\right)_{v(\mathbf{r})} & =-\frac{1}{N} \int 1+\ln \rho(\mathbf{r}) d \mathbf{r} . \\
\left(\frac{\partial S(\mathbf{r})}{\partial E}\right)_{v(\mathbf{r})} & =-\frac{1}{N} \int\left(\frac{\partial \rho(\mathbf{r})}{\partial E}\right)_{v(\mathbf{r})} \ln \rho(\mathbf{r}) d \mathbf{r}+\left[\frac{1}{N}-\frac{s(\mathbf{r})}{N^{2}}\right]\left(\frac{\partial N}{\partial E}\right)_{v(\mathbf{r})} . \\
\left(\frac{\partial^{2} S(\mathbf{r})}{\partial \rho(\mathbf{r}) \partial N}\right)_{v(\mathbf{r})} & =-\frac{1}{N} \int\left(\frac{\partial f(\mathbf{r})}{\partial \rho(\mathbf{r})}\right)_{v(\mathbf{r})} \ln \rho(\mathbf{r})+\frac{f(\mathbf{r})}{\rho(\mathbf{r})} d \mathbf{r} . \\
\left(\frac{\partial^{2} S(\mathbf{r})}{\partial \rho(\mathbf{r}) \partial \mu}\right)_{v(\mathbf{r})} & =-\int\left(\frac{\partial s}{\partial \rho(\mathbf{r})}\right)_{v(\mathbf{r})} \ln \rho(\mathbf{r})+\frac{s}{\rho(\mathbf{r})} d \mathbf{r} . \\
\left(\frac{\partial^{2} S(\mathbf{r})}{\partial \rho(\mathbf{r})^{2}}\right)_{v(\mathbf{r})} & =-\frac{1}{N} \int \frac{d \mathbf{r}}{\rho(\mathbf{r})}
\end{aligned}
$$




$$
\begin{aligned}
&\left(\frac{\partial^{2} S(\mathbf{r})}{\partial \rho(\mathbf{r}) \partial E}\right)_{v(\mathbf{r})}=-\frac{1}{N^{2}}\left(\frac{\partial N}{\partial E}\right)_{v(\mathbf{r})} \int 1+\ln \rho(\mathbf{r}) d \mathbf{r} . \\
&\left(\frac{\partial S(\mathbf{r})}{\partial E_{k}^{T F}}\right)_{v(\mathbf{r})}=-\frac{3}{5 N C_{F}} \int \frac{\ln \rho(\mathbf{r})}{\rho(\mathbf{r})^{2 / 3}} d \mathbf{r}-\frac{1}{\mu N^{2}} \int \rho(\mathbf{r}) \ln \rho(\mathbf{r}) d \mathbf{r}+\frac{1}{\mu N} \\
&\left(\frac{\partial S(\mathbf{r})}{\partial E_{x}^{L D A}}\right)_{v(\mathbf{r})}=-\frac{1}{N} \int \frac{\ln \rho(\mathbf{r})}{\left(\frac{3}{\pi}\right)^{1 / 3} \rho(\mathbf{r})^{1 / 3}} d \mathbf{r}-\frac{1}{\mu N^{2}} \int \rho(\mathbf{r}) \ln \rho(\mathbf{r}) d \mathbf{r}+\frac{1}{\mu N} \\
&\left(\frac{S(\mathbf{r})}{E_{k, x}^{T F, L D A}}\right)_{v(\mathbf{r})}=-\int\left[A\left(\frac{1+\rho(\mathbf{r})^{1 / 3}}{\rho(\mathbf{r})^{2 / 3}}\right)-B \rho(\mathbf{r})\right] \ln \rho(\mathbf{r}) d \mathbf{r}+B N . \\
&\left(\frac{\partial S(\mathbf{r})}{\partial E^{P G B}}\right)_{v(\mathbf{r})}=-\frac{1}{N} \int \frac{\ln \rho(\mathbf{r})}{\frac{5}{3} C \rho(\mathbf{r})^{2 / 3}+v(\mathbf{r})+\frac{4}{3} B N^{2 / 3} \rho(\mathbf{r})^{1 / 3}} d \mathbf{r} \\
&+\left[\frac{1}{N}-\frac{s(\mathbf{r})}{N^{2}}\right]\left(\frac{\partial N}{\partial E}\right)_{v(\mathbf{r})} .
\end{aligned}
$$

\section{Description of a simple chemical process}

\subsection{Reaction $\mathrm{CH}_{2} \mathrm{CHF}+\mathrm{CH}_{3} \rightarrow \mathrm{CH}_{3} \mathrm{CHFCH}_{2}$}

To show the application of the relations obtained, we have selected the following radical-molecule chemical reaction: $\mathrm{CH}_{2} \mathrm{CHF}+\mathrm{CH}_{3} \rightarrow \mathrm{CH}_{3} \mathrm{CHFCH}_{2}$.

Structures and energies have been obtained along the reaction path using $\mathrm{m} 062 \mathrm{x} / 6-311++\mathrm{G}(\mathrm{d}, \mathrm{p})$ density functional method with Gaussian 09 [31]. The electron density was calculated with Pérez-Jordá's algorithms [8] and a D-Grid 4.6 [9]. Molecular Electrostatic Potential (MEP) isosurfaces were obtained with Molden 5.0

[22]. The Fukui function condensed was calculated using natural atomics orbitals obtained in a Natural Population Analysis [28].

The condensed Fukui function were calculated according to the following approximations:

$$
\begin{aligned}
f(\mathbf{r})^{+} & =\left|\phi(\mathbf{r})_{\mathrm{LUMO}}\right|^{2}+\sum_{i=1}^{n} \frac{\partial}{\partial N}|\phi(\mathbf{r})|^{2} \\
f(\mathbf{r})^{-} & =\left|\phi(\mathbf{r})_{\mathrm{HOMO}}\right|^{2}+\sum_{i=1}^{n} \frac{\partial}{\partial N}|\phi(\mathbf{r})|^{2} . \\
f(\mathbf{r}) & =\frac{1}{2}\left(f(\mathbf{r})^{+}+f(\mathbf{r})^{-}\right) .
\end{aligned}
$$

where $\phi(\mathbf{r})_{\text {LUMO }}$ correspond to LUMO electron density and $\phi(\mathbf{r})_{\mathrm{HOMO}}$ to HOMO electron density. When a molecule accepts electrons, the electrons tend to go to places where $f(\mathbf{r})^{+}$ is large because it is at these locations that the molecule is most able to stabilize additional electrons. Therefore a molecule is susceptible of a nucleophilic attack at sites where $f(\mathbf{r})^{+}$ 
is large. Similarly, a molecule is susceptible of an electrophilic attack at sites where $f(\mathbf{r})^{-}$ is large, because these are the regions where electron removal destabilizes the molecule the least. In chemical density functional theory, the Fukui functions are the key regioselectivity indicators for electron-transfer controlled reactions. In order to use these expressions we have chosen the Natural Population Analysis, NPA, which involves only matrix diagonalization of small subsets of the density matrix, and also requires a negligible amount of computer time. Although it is more involved than a Mulliken or Löwdin analysis, for a theoretical analysis using von Neumann entropies, NPA is an attractive method [29, 30, 34].

In Figure (1(a)) we present the electron energy profile of the reaction as a function of the reaction coordinate $\mathrm{RX}$ in $\AA$. Trends in the condensed Fukui function are shown in Figure (1(b)). Analogously, trends in Hardness and chemical potential are shown in Figures (1(c)) and $(1(d))$, respectively.

In Figures (2(a)) and (2(b)) we present the trends in the kinetic and exchange energies as a function of the reaction coordinate.

Energy values show that the reaction is exothermic, with the following energy values at stationary points: $\mathrm{E}$ (reactants)=-217.629652 A.U.; $\mathrm{E}$ (transition state)=-217.615808 A.U. and $\mathrm{E}$ (products) $=-217.674564$ A.U. One of the main points of interest for our purposes is the analysis of the structures along the reaction path in terms of descriptors that are related to chemical reactivity. In this specific case, we consider the kinetic and exchange energies (see Figs. (2(a)) and (2(b))). For the kinetic energy we note that there exists a region limited by a maximum at $R X=-0.64230$ and a minimum at $R X=1.44550$, in which important chemical changes occurs. It is associated with bond forming, in the Figures (2(c))-(2(1)) present the Molecular Electrostatic Potential of this process, probably this result permit us establish a kinetically classification criteria of this reaction, that is, the principal parameter that govern this process it is the kinetic. The minima in $R X=1.44550$ is it in relation to important chemical changes that occurs in the frontier orbitals, see the Figures $(2(m)),(2(n))(2(o))$ and (2(p)) where present the isosurfaces of the HOMO and LUMO orbitals.

In the case of the Hardness, we note that not are a correspondence between the maximum or minima of the electron, kinetic or exchange energy with the minimum and maximum of the Hardness. This points, again are related at with the structural changes that occurs in this zone, also, of course, this changes involved important changes in the frontier orbitals. A similar aspect occurs with the condensed Fukui functions, Figure $(1(\mathrm{~b}))$, where we note that in the point $R X=0.32119$ exist a equality $f(\mathbf{r})^{+} \simeq f(\mathbf{r})^{-} \simeq f(\mathbf{r}) \simeq 0.5$ with this numerical result we can note a parallelism with the relation of a hard/soft acids/basis, proposed by Pearson. That in terms of the condensed Fukui functions would be, when a chemical process occurs, exist a point where the active sites of the structures have a equalization of a kind of chemical attack, nucleophilic or electrophilic. See, the Figures (3(a)), (3(b)) and (3(c)).

In this sense, is important to note that, in this case we suspect that the more important changes in the parameters and their equalization occurs in the transition state, this can be suspect by chemical intuition, but how we can see in the different graphics this not occurs, in fact, the hardness, Fig. (1(c)), that exist a minima in $R X=-0.64230$ and a maximum in $R X=3.21128$ this zone permit us define as a zone where the process occurs via nucleophilic attack. 
In Figure (3(e)) and (3(d)) we present, the tendencies of Shannon entropy and the electron energy with the LDA approximation, $E^{L D A}$. In this case, the $E^{L D A}$ increase basically in a linear way, but in the point $R X=2.73035$ have a maximum, this maximum appears to in the chemical potential tendency. Respect to the Shannon entropy we note more structure than $E^{L D A}$, and in this case, we note than the Shannon entropy have a similar tendency than the electron energy, in the same form than the energy the Shannon entropy can detect the transition state, see the Figure (3(f)).

By comparison between the Shannon entropy and electron energy profiles we can note that the Shannon entropy, have a zone delimited in the region $-0.5 \leq R X \leq 0.5$, see Figure (3(f)), that have a correspondence with the transition state zone, this is an important observation, because, the Shannon entropy can detect a transition state zone, where occurs a transfer and redistribution of electron density. Comparing, the tendencies of condensed Fukui function, with the Shannon entropy tendency, we note that this zone, have a relation with a charge transfer between the methyl and the molecule and is in this zone where occurs the process of bond forming. In the same way that the condensed Fukui function, this zone is where the subtle interactions among frontier orbitals occurs, and permit to the molecular system start a complex process of the chemical bond forming, in Figure (4(a)) we present the tendency of a normal mode of vibration, and we note that, the zone predicted by the Shannon entropy exhibit a correspondence with a zone of the negative values of the frequencies, consequently, is possible that we can not speak of a specific point in the reaction path where occurs a bond breaking or bond forming. A similar argumentation, can be applied to the description of the transition state zone. Note the importance of this, is possible that, still with the modern techniques of the experimental chemistry, such the femtochemistry [19-21], we can not detect just a transition state structure, or else, a zone of transition. This zone of transition, is accoted by a zone where the Shannon entropy tendency have a slope approximately to zero, see the Figure (4(b)) accordingly, we can say that the Shannon entropy can detect and predict the zone where the most important chemical changes will be occurs, so, this kind of entropy permit us reveals some chemical aspects that are subjacent in a chemical process. Other important, observation is that the maximum of the electron energy, not correspond to the minimum of the frequencies, it is represents, probability, a conflict between the convectional interpretation, in the sense that the maximum of the energy correspond at one possible transition state structure that, in general, have the most negative value of the frequencies, but how we show in this case, this not occurs, by other hand, the minimum value of the frequencies it is related at one equality with the condensed Fukui functions, see the Figure $(4(\mathrm{c}))$.

In general terms, is important to note, that the frequencies tendencies have a tree zones of negative frequencies, these Hessian values represent the transition vector which show maxima at the vicinity of the transition state. Several features are worth mentioning, the TS corresponds indeed to a saddle point, maxima at the Hessian correspond to high kinetic energy values (largest frequencies for the energy cleavage reservoirs) since they fit with maximal values in the entropy profile, and the Hessian is minimal at the TS, where the kinetic energy is the lowest (minimal molecular frequency) and it corresponds to a saddle point. In this case, the analysis of frequencies can give us, a general idea about that this mechanics occurs in tree steps, in each one, occurs some structural rearrays at expense of a decrease in 
energy exchange and increased kinetic energy. It is relevant note, that the Shannon entropy, see Figure $(4(b))$, can detect three zones where the frequencies are negative, the first of them is between $-3.5 \leq R X \leq-2.2$ the second in $-0.5 \leq R X 0.5$ and the last in $3.8 \leq R X \leq 4.0$. In the first and third zone, the Shannon entropy exhibits a change in their curvature, also, note that this last observation have a correspondence with the other parameters such, hardness, softness, chemical potential and with some Shannon entropy derivatives.

In Figure (5(b)) we compare the trend of the derivative of Snannon's entropy with respect to the number of electrons: $\left(\frac{\partial S(\mathbf{r})}{\partial N}\right)_{v(\mathbf{r})}$ as a function of $R X$, with that of the DFT chemical reactivity descriptor: $\left(\frac{\partial \mu}{\partial N}\right)_{v(\mathbf{r})}$, which is associated to hardness. Even though absolute values are not the same everywhere, there is a perfect coincidence in the region close to the transition state, in terms of the RX position as well as in the absolute values.

A similar situation occurs with the derivative of Shannon's entropy with respect to the chemical potential: $\left(\frac{\partial S(\mathbf{r})}{\partial \mu}\right)_{v(\mathbf{r})}$, and the inverse of the Exchange energy,

$$
\frac{1}{E_{i}^{L D A}}=-\frac{1}{\left(\frac{3}{\pi}\right)^{1 / 3} \int \rho(\mathbf{r})^{4 / 3} d \mathbf{r}}
$$

see Figure (5(d)).

Finally, a plot of the derivative of Shannon's entropy with respect to the electronic energy $\left(\frac{\partial S(\mathbf{r})}{\partial E}\right)_{v(\mathbf{r})}$ as a function of $\mathrm{RX}$, behaves in a manner that is remarkably similar to that of the DFT descriptor for softness: $\left(\frac{\partial N}{\partial \mu}\right)_{v(\mathbf{r})}$ (Figure (5(f)).

The relations that were suggested above based on numerical evidence can be summarized as:

$$
\begin{aligned}
& \left(\frac{\partial S(\mathbf{r})}{\partial N}\right)_{v(\mathbf{r})} \simeq\left(\frac{\partial \mu}{\partial N}\right)_{v(\mathbf{r})} . \\
& \left(\frac{\partial S(\mathbf{r})}{\partial \mu}\right)_{v(\mathbf{r})} \simeq-\frac{3}{4}\left(\frac{3}{\pi}\right)^{1 / 3} \int \rho(\mathbf{r})^{4 / 3} d \mathbf{r} . \\
& \left(\frac{\partial S(\mathbf{r})}{\partial E}\right)_{v(\mathbf{r})} \simeq\left(\frac{\partial N}{\partial \mu}\right)_{v(\mathbf{r})} .
\end{aligned}
$$

From the numerical results obtained from Eqs. (50) and (52), we have been able to establish a linear relationship between the parameters involved, and the following expressions have been obtained: 


$$
\begin{gathered}
\text { Hardness }=\left(\frac{\partial \mu}{\partial N}\right)_{v(\mathbf{r})}=28.3141\left(\frac{\partial S(\mathbf{r})}{\partial N}\right)_{v(\mathbf{r})}+0.0226 \\
\text { Softness }=\left(\frac{\partial N}{\partial \mu}\right)_{v(\mathbf{r})}=24.1088\left(\frac{\partial S(\mathbf{r})}{\partial E}\right)_{v(\mathbf{r})}+0.5876,
\end{gathered}
$$

Thus, the hardness and softness values of this chemical reaction can be obtained with a good level of accuracy from the derivatives of Shannon's entropy.

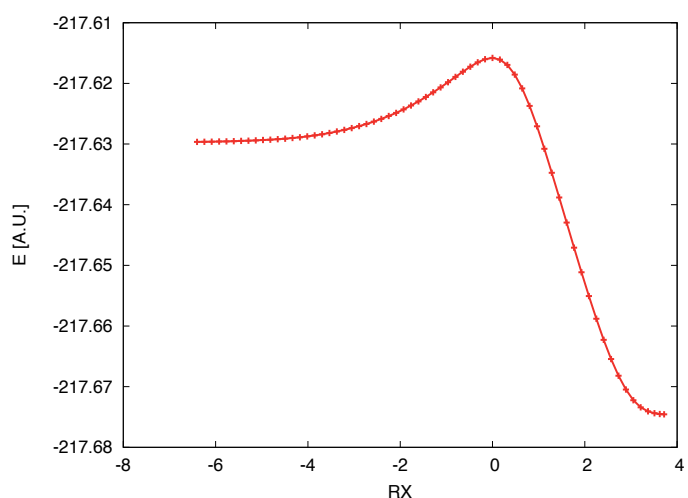

(a) Electron energy profile.

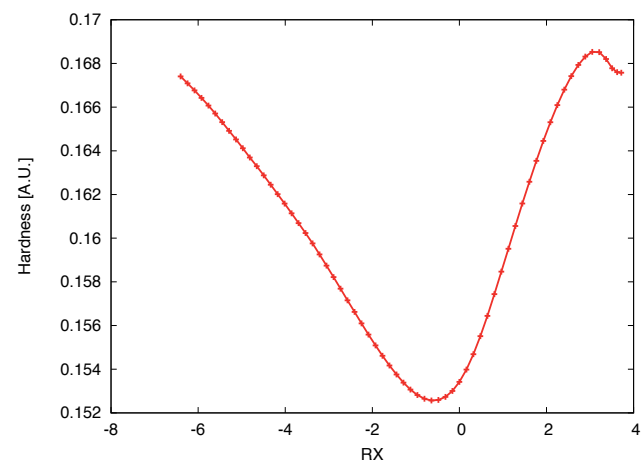

(c) Hardness.

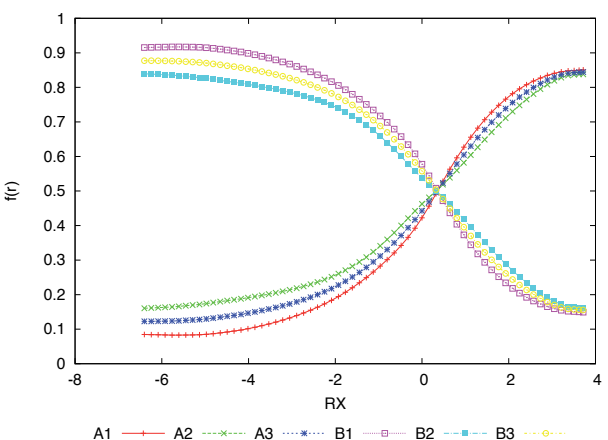

(b) Condensed Fukui function profile $A 1: f(\mathbf{r})^{-}, A 2$ : $f(\mathbf{r})^{+}, A 3: f(\mathbf{r}), B 1: f(\mathbf{r})^{-}, B 2: f(\mathbf{r})^{+}, B 3: f(\mathbf{r})$, where $A$ denote the subsystem $\mathrm{CH}_{2} \mathrm{CHF}$ and $B$ is the subsystem $\mathrm{CH}_{3}$.

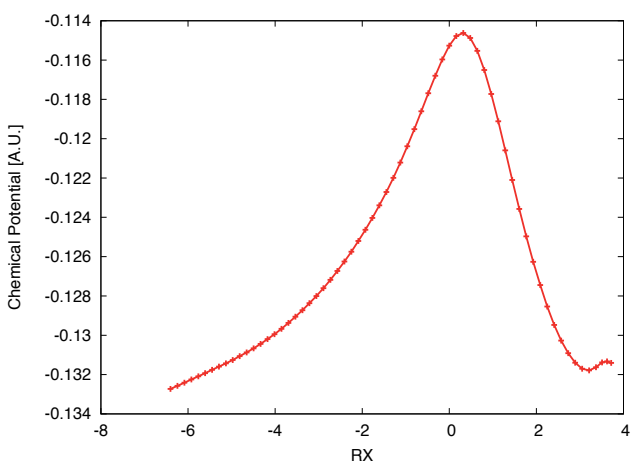

(d) Chemical potential.

Figure 1. Trends of the reaction $\mathrm{CH}_{2} \mathrm{CHF}+\mathrm{CH}_{3} \rightarrow \mathrm{CH}_{3} \mathrm{CHFCH}_{2}$. 


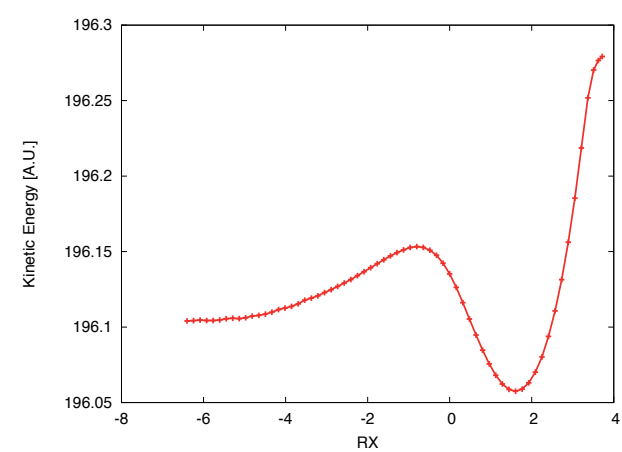

(a) Kinetic Energy.

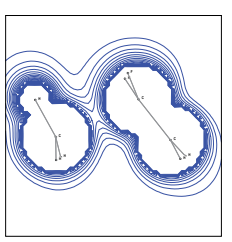

(c) $R X=-0.64230$

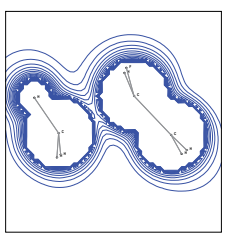

(g) $\mathrm{RX}=0.32119$

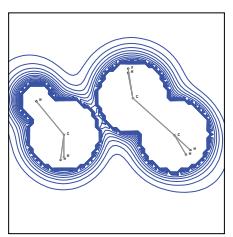

(k) $R X=1.12427$

(l) $\mathrm{RX}=1.44550$

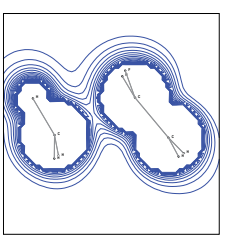

(d) $R X=-0.32112$

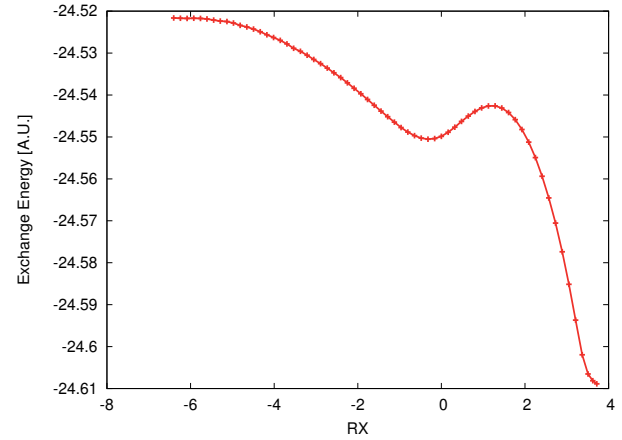

(b) Exchange Energy.

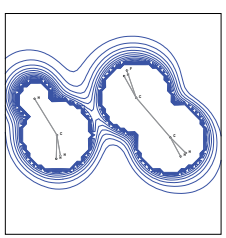

(e) $R X=-0.16058$

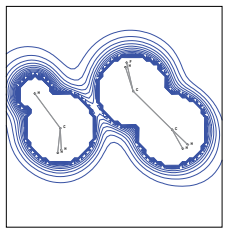

(i) $R X=0.64242$

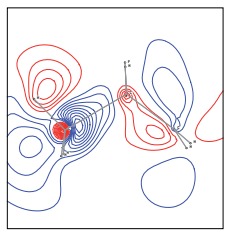

(m) $\mathrm{HOMO}$ in $R X=2.08795$

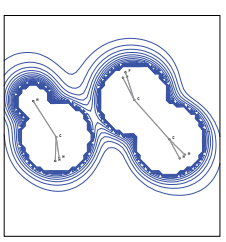

(f) $R X=0.00000$

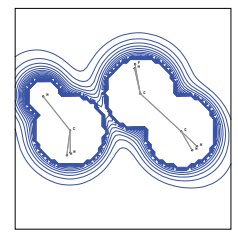

(j) $\mathrm{RX}=0.96366$

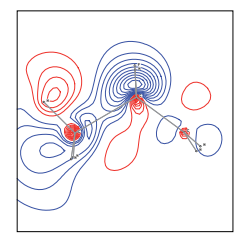

(n) LUMO in

$R X=2.08795$
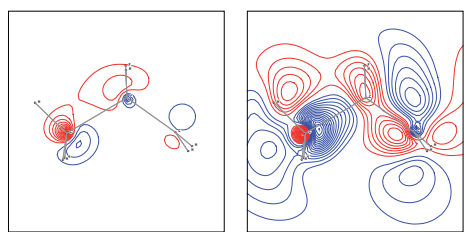

(o) $\mathrm{HOMO}$ in

(p) LUMO in

$R X=2.24856$

$R X=2.24856$

Figure 2. Isosurfaces of the Molecular Electrostatic Potential, in $-0.64230 \leq R X \leq 1.44550$. 


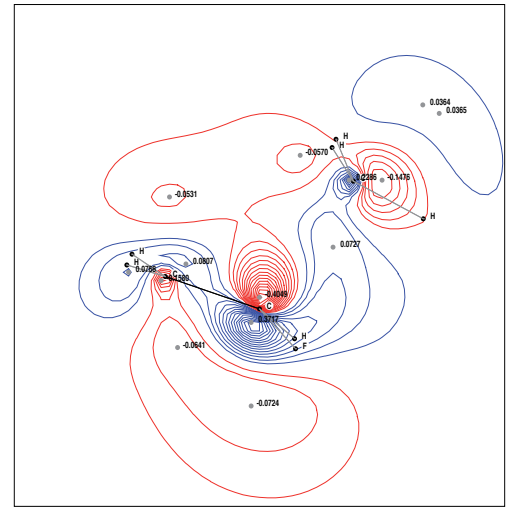

(a) $\mathrm{HOMO}$, in $\mathrm{RX}=0.32119$

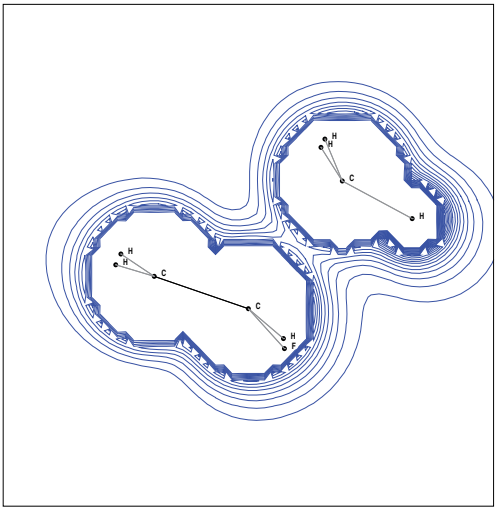

(c) MEP, in $R X=0.32119$

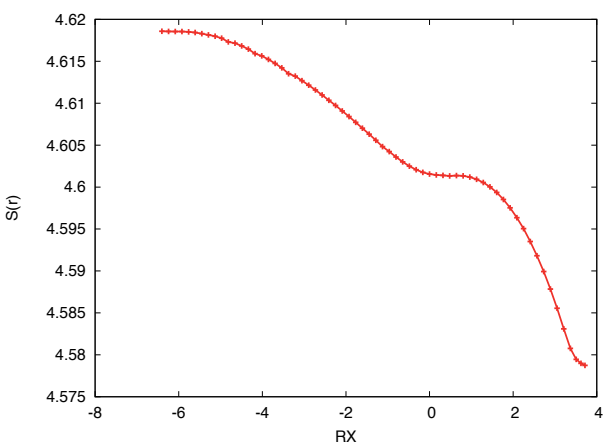

(e) Shannon entropy

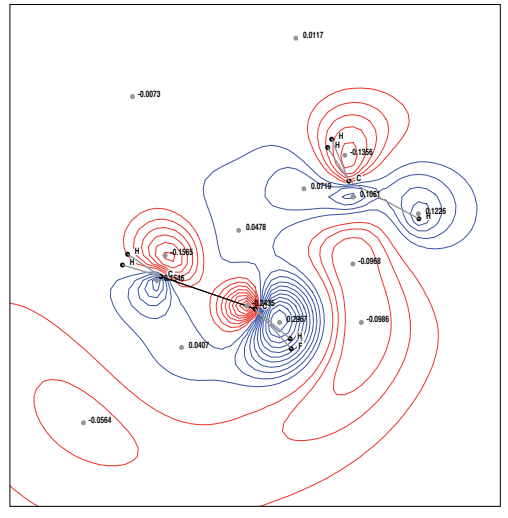

(b) LUMO, in $R X=0.32119$

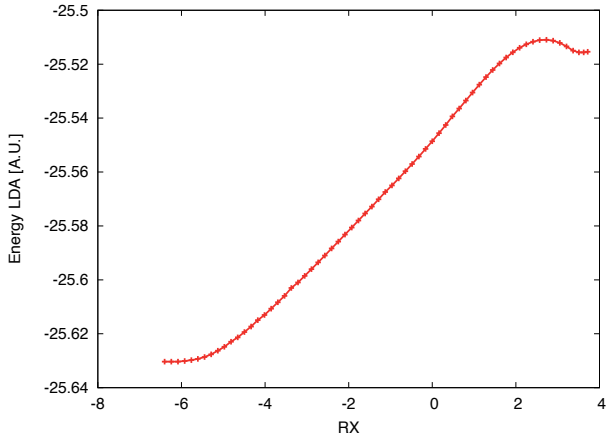

(d) Exchange energy with LDA

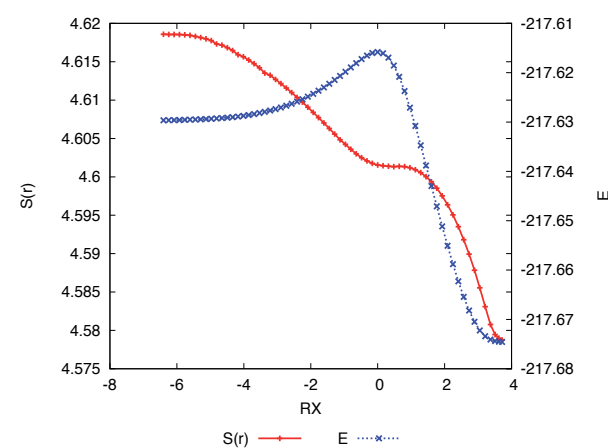

(f) Shannon entropy and the Electron energy

Figure 3. Trends of the reaction $\mathrm{CH}_{2} \mathrm{CHF}+\mathrm{CH}_{3} \rightarrow \mathrm{CH}_{3} \mathrm{CHFCH}_{2}$. 


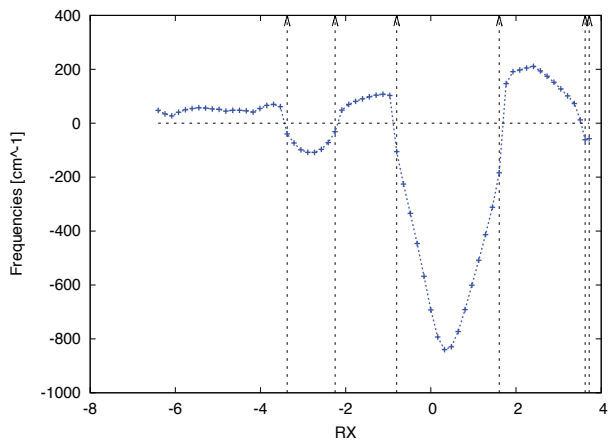

(a) Frequencies.

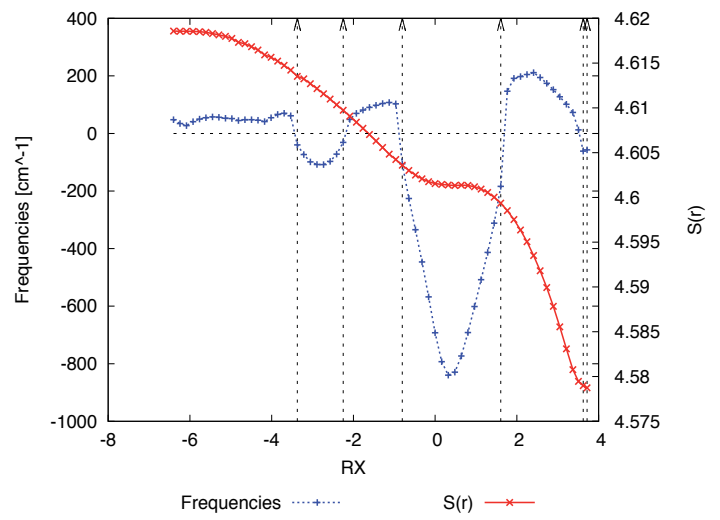

(b) Comparison between the frequencies and the Shannon entropy.

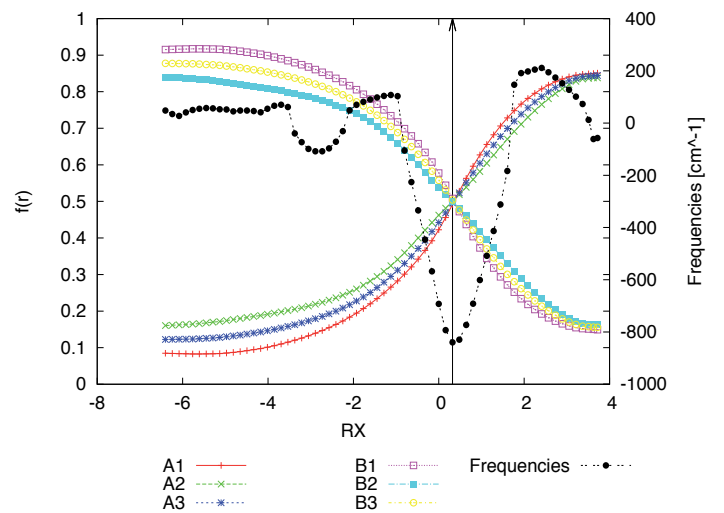

(c) Comparison between the condensed Fukui function and the Frequencies.

Where $A 1: f(\mathbf{r})^{-}, A 2: f(\mathbf{r})^{+}, A 3: f(\mathbf{r}), B 1: f(\mathbf{r})^{-}, B 2: f(\mathbf{r})^{+}$, $B 3: f(\mathbf{r})$, where $A$ denote the subsystem $\mathrm{CH}_{2} \mathrm{CHF}$ and $B$ is the subsystem $\mathrm{CH}_{3}$

Figure 4. Comparison among Frequencies, Shannon entropy and Fukui function. 


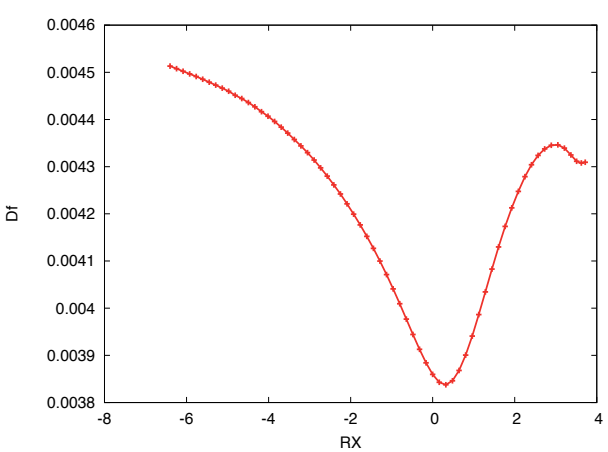

(a) Trend of $\left(\frac{\partial S(\mathbf{r})}{\partial N}\right)_{v(\mathbf{r})}$.

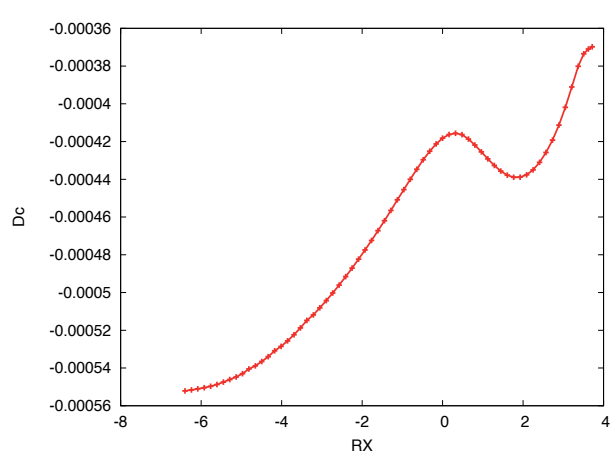

(c) Trend of $\mathrm{Dp}=\left(\frac{\partial S(\mathbf{r})}{\partial \mu}\right)_{v(\mathbf{r})}$.

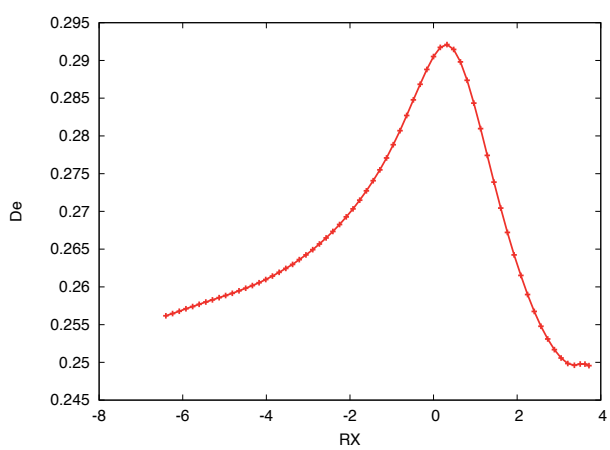

(e) Trend of $\left(\frac{\partial S(\mathbf{r})}{\partial E}\right)_{v(\mathbf{r})}$.

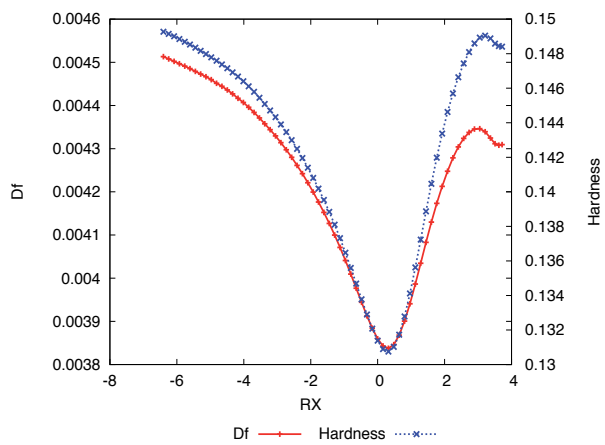

(b) Trend of Df $=\left(\frac{\partial S(\mathbf{r})}{\partial N}\right)_{v(\mathbf{r})}$ and Hardness $=\left(\frac{\partial u}{\partial N}\right)_{v(\mathbf{r})}$.

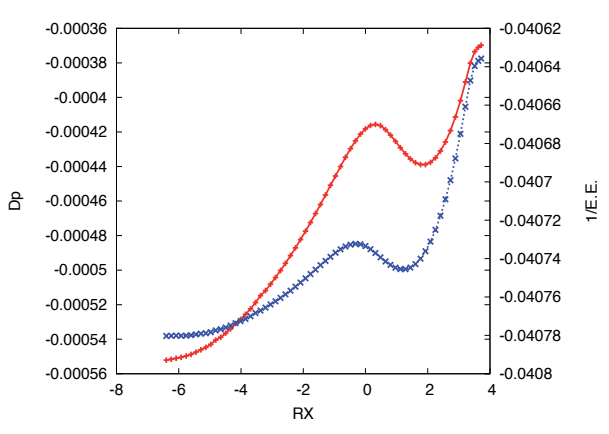

Dp — E.E. ..........

(d) Trend of $\mathrm{Dp}=\left(\frac{\partial S(\mathbf{r})}{\partial \mu}\right)_{v(\mathbf{r})}$ and the inverse of exchange energy.

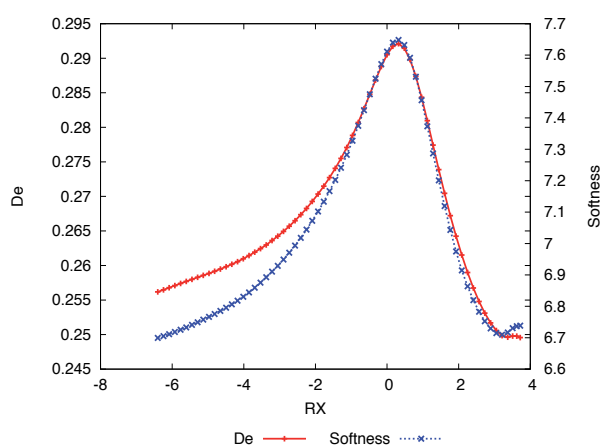

(f) Trend of $\left(\frac{\partial S(\mathbf{r})}{\partial E}\right)_{v(\mathbf{r})}$ and Softness $=\left(\frac{\partial N}{\partial \mu}\right)_{v(\mathbf{r})}$.

Figure 5. Trends of the first derivatives of Shannon entropy.

\subsection{Ethane conformational analysis}

In this section we present a conformational analysis of ethane, as a function of the dihedral rotation angle between the two methyl groups. The initial point corresponds to the eclipsed 
conformer, and an Intrinsic Reaction Coordinate analysis (IRC) has been performed, with ten degrees steps in the dihedral angle. This system was calculated in Gaussian 03 [32], using $\mathrm{B} 3 \mathrm{LYP} / \mathrm{cc}-\mathrm{pVTZ}$.

In Figure (6(a)) we present the electron energy and Shannon entropy profiles. An excellent agreement is observed between the two curves. Furthermore, a clear similarity is also observed between the behavior, along the reaction path, of the hardness and of the first derivative of Shannon's entropy with respect to the number of electrons. This is shown in Figure (6(b)).

In Figure (6(c)) we show the kinetic and exchange energies as a function of RX. Both quantities exhibit inverse behaviors, the position of the minima in one of them coinciding with the maxima of the other.

One of the most important results is shown in Figure (6(d)), where the exchange energy is plotted against Shannon's entropy along the reaction path. A perfect linear correlation is observed between these apparently unconnected quantities, leading to conclude that

i) The Shannon entropy in position space can be used as a measure of the exchange effects in molecular systems.

ii) There exists a direct relationship between the Shannon entropy and the exchange energy.

Based in these conclusions and results, a new research line is being developed, to construct a functional based on an entropic criterion.

The numerical results obtained above cab be summarized in the following manner:

$$
\begin{aligned}
\left(\frac{\partial S(\mathbf{r})}{\partial N}\right)_{v(\mathbf{r})} & \simeq\left(\frac{\partial N}{\partial \mu}\right)_{v(\mathbf{r})} \cdot \\
S(\mathbf{r}) & \simeq-\frac{3}{4}\left(\frac{3}{\pi}\right)^{1 / 3} \int \rho(\mathbf{r})^{4 / 3} d \mathbf{r}
\end{aligned}
$$

The last equation suggests that there may be a fundamental connection between Chemical Density Functional Theory and Information Theory. In Table (1), we present the numerical results of Shannon's entropy and exchange energy. Using these results and a least square regression, is it possible to obtain the following linear relation between Shannon entropy and exchange energy:

$$
E_{e}=A S(\mathbf{r})-B
$$

where $A=2.2127$ and $B=21.0061$. With this equation, we can reproduce the Exchange Energy with a precision of $1 \times 10^{-6}$. 


\begin{tabular}{ccccc}
\hline Angle & $\begin{array}{c}S(\mathbf{r}) \\
\text { [nats] }\end{array}$ & $\begin{array}{c}E . E . \\
\text { [A.U.] }\end{array}$ & $\begin{array}{c}E_{e}=A S(\mathbf{r})-B \\
\text { [A.U.] }\end{array}$ & precision \\
\hline 0 & 4.5902766 & -10.849190 & -10.8492232214 & 0.9999 \\
10 & 4.5901580 & -10.849449 & -10.8494856465 & 0.9999 \\
20 & 4.5898836 & -10.850050 & -10.8500928087 & 0.9999 \\
30 & 4.5895363 & -10.850815 & -10.8508612761 & 0.9999 \\
40 & 4.5892201 & -10.851516 & -10.8515609289 & 0.9999 \\
50 & 4.5890276 & -10.851953 & -10.8519868718 & 0.9999 \\
60 & 4.5889557 & -10.852113 & -10.8521459642 & 0.9999 \\
70 & 4.5890276 & -10.851953 & -10.8519868718 & 0.9999 \\
80 & 4.5892201 & -10.851516 & -10.8515609289 & 0.9999 \\
90 & 4.5895363 & -10.850815 & -10.8508612761 & 0.9999 \\
100 & 4.5898836 & -10.850050 & -10.8500928087 & 0.9999 \\
110 & 4.5901580 & -10.849449 & -10.8494856465 & 0.9999 \\
120 & 4.5902766 & -10.849190 & -10.8492232214 & 0.9999 \\
130 & 4.5901580 & -10.849449 & -10.8494856465 & 0.9999 \\
140 & 4.5898836 & -10.850050 & -10.8500928087 & 0.9999 \\
150 & 4.5895363 & -10.850815 & -10.8508612761 & 0.9999 \\
160 & 4.5892201 & -10.851516 & -10.8515609289 & 0.9999 \\
170 & 4.5890276 & -10.851953 & -10.8519868718 & 0.9999 \\
180 & 4.5889557 & -10.852113 & -10.8521459642 & 0.9999 \\
\hline
\end{tabular}

Table 1. Numerical values of Shannon's entropy and Exchange Energy, in the ethane conformational analysis, where $A=2.2127$ and $B=21.0061$.

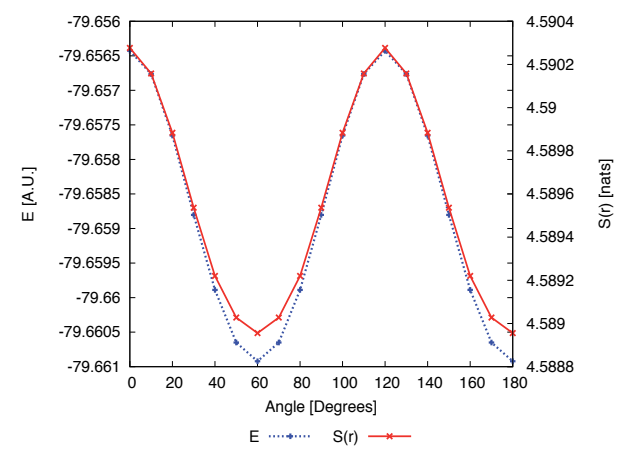

(a) Energy and Shannon entropy profiles

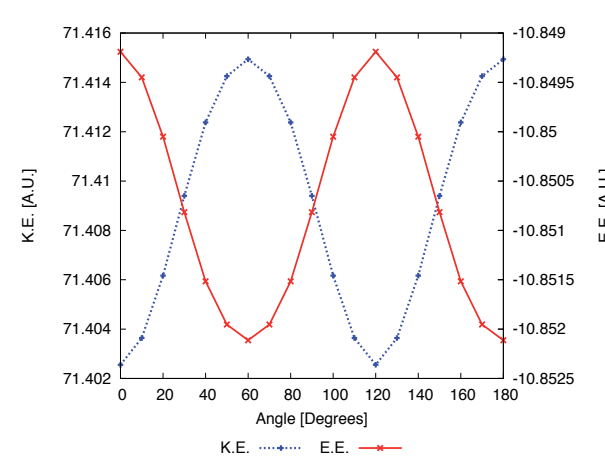

(c) Exchange Energy and Kinetic Energy profiles

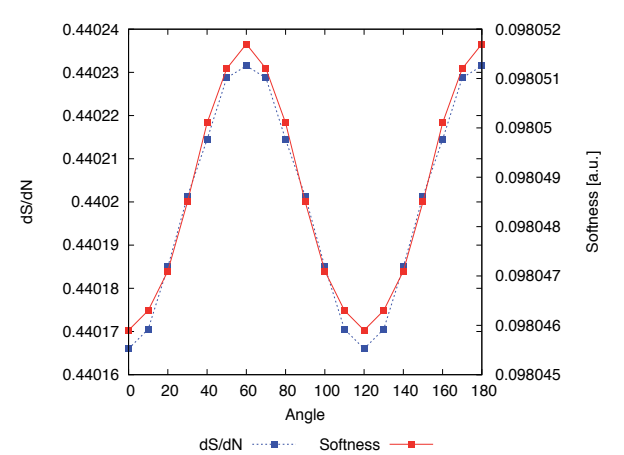

(b) Derivative of Shannon entropy and Softness profile

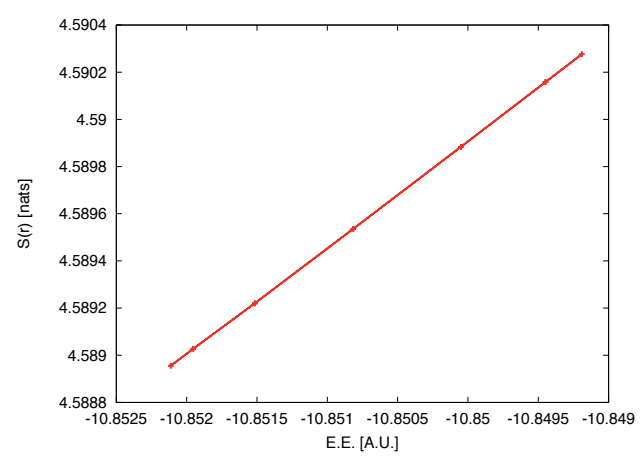

(d) Exchange Energy and Shannon entropy profiles

Figure 6. Tendencies obtained for the Ethane Conformational Analysis. 


\section{Conclusion}

In this work, I have derived relationships that connect Shannon's entropy and its derivatives, with well-known concepts in Density Functional Theory. Numerical applications of these relationships have been performed for two simple problems.

This has been permit us start a new investigation line about it, and with some this results we continue whit the study of the formalism for the construction of a functional based in a principles of physics, and the information theory, also, we pretend continue the develop of the some models that permit us find the direct relation between electron energy with the Shannon entropy.

By other hand, the application of the concepts of the information theory permit do a description more accurate than the description based in only a energetically criteria, and continue with the spirit of some works of Frieden, we speculate that is possible define or found a form that derived the DFT from some fundamental expression, that come form the Information Theory, as Frieden derivations of the fundamental equations of the Thermodynamics, or the derivation of the Scrhödinger equation.

Finally, with this example we have tried to link information from a system that is subjected to a process with the physical and chemical changes. Thus, we have linked the concept of information, which is an epistemological concept completely with ontological concepts and the solution concepts or interpretation of the results allows us feedback on these concepts in ontological terms, according to the author, abstract and more general.

By other part, is probable that today do not exist a ortodoxical definition of what actually is the information, beyond that presented by Shannon and its guidelines, criteria, characterization of it, among other things, the interpretation and the relationship with other concepts such as: energy, electron density, chemical reactivity parameters and many others need be discussed to try of establish a formal relation between concepts.

So, there is no doubt that both knowledge and the praxis and reality as knowledge scientific understanding and also, is it clear that information concept and the model itself is interdisciplinary or transdisciplinary. The concept and moreover, the model itself, promotes a systematic relation with causal analogies and parallelism with scientific knowledge, which transcends the framework of the source domain and extend in various directions, thus making the knowledge acquires an unusual resonance, as this, we believe it is feasible to complement the explanations of natural processes and natural systems.

This model is not intended that the manner of the old school, that using metaphysical substance, the particularities of the processes reveal themselves to us in the end as a progressive manifestation of homogeneous order or a unitary whole and absolute. It is simply to promote and implement a partnership scheme which promises analog route and cover knowledge in a way easier.

\section{Acknowledgments}

I wish to thank José María Pérez Jordá and M. Kohout for kindly providing their numerical codes. I acknowledge financial support from Prof. Annik Vivier-Bunge through project "Red de Química Teórica para el Medio Ambiente y Salud" and for helpful discussions. 


\section{Author details}

Nelson Flores-Gallegos

Universidad Autónoma Metropolitana-Iztapalapa, México

\section{References}

[1] B. Roy Frieden. Fisher information as the basis for the Schrödinger wave equation. Am. J. Phys. 1989, 11, 1004-1008.

[2] B. Roy Frieden. Fisher information, disorder, and the equilibrium distributions of physics. Phys. Rev. A 1990, 41, 4265-4276.

[3] B. Roy Frieden and Roy J. Hughes. Spectral $1 / f$ noise derived from extremized physical information. Phys. Rev. E 1994, 49, 2644-2649.

[4] B. Roy Frieden and Bernard H. Soffer. Lagrangians of physics and the game of Fisher-information transfer. Phys. Rev. E 1995, 52, 2274-2286.

[5] B. Roy Frieden and W. J. Cocke. Foundation for Fisher-information-based derivations of physical laws Phys. Rev. E 1996, 54, 257-260.

[6] Parveen Fazal, S., Sen, K.D., Gutierrez G., and Fuentealba, P. Shannon entropy of 1-normalized electron density. Indian Journal of Chemistry. 2000, 39A, 48-49.

[7] Shannon, C. E. A Mathematical Theory of Communication. The Bell System Thecnical Journal. 1948, 27, 379-423.

[8] Pérez-Jordá, Jose M., Becke, Axel D. and San-Fabian, Emilio. Automatic numerical integration techniques for polyatomic molecules. J. Chem. Phys. 1994, 100, 6520-6534.

[9] M. Kohout. DGrid, version 4.6, Radebeul, 2011.

[10] Nelson Flores-Gallegos and Rodolfo O. Esquivel. von Neumann Entropies Analysis in Hilbert Space for the Dissociation Processes of Homonuclear and Heteronuclear Diatomic Molecules J. Mex. Chem. Soc., 2008, 52, 19-30.

[11] Rodolfo O. Esquivel, Nelson Flores-Gallegos, Cristina Iuga, Edmundo Carrera, Juan Carlos Angulo and Juan Antolín. Phenomenological description of the transition state, and the bond breaking and bond forming processes of selected elementary chemical reactions: An information-theoretic study. Theoretical Chemistry Accounts., 2009, 124, 445-460.

[12] R.O. Esquivel, N. Flores-Gallegos, C. Iuga, E. Carrera, J.C. Angulo and J. Antolín. Phenomenological description of selected elementary chemical reaction mechanisms: An information-theoretic study. Physics Letters A, 2010 , 374, 948-951.

[13] Rodolfo O. Esquivel, Juan Carlos Angulo, Juan Antolín, Jesús S. Dehesa, Sheila López-Rosa and Nelson Flores-Gallegos. Complexity analysis of selected molecules 
in position and momentum spaces. Physical Chemistry Chemical Physics, 2010 , 12, 7108-7116.

[14] Gadre, S.R., Sears, S.B. Some novel characteristics of atomic information entropies. Phys. Rev. A., 1985, 32, 2602-2606.

[15] R. G. Pearson. Hard and Sotf acids and bases. J. Am. Chem. Soc., 1963, 85, 3533-3539.

[16] R. G. Parr and W. Yang. Density functional approach to the frontier electron theory of the chemical reactivity. J. Am. Chem. Soc., 1984, 106, 4049-4050.

[17] L. H. Thomas. The calculation of atomic fields. Proc. Camb. Phil. Soc., 1927, 23, 542-548.

[18] E. Fermi. Un metodo statistce per la determinazione di algune propieta dell'atomo. Rend. Accad., Licei, 1927, 54, 2627-2630.

[19] A. H. Zawail. Laser Femtochemistry. Science, 1988, 242, 1645-1653.

[20] A. H. Zewail. Femtochemistry: Atomic-Scale Dynamics of the Chemical Bond. J Phys. Chem. A, 2000, 104, 5660-5694.

[21] J. S. Baskin and A. H. Zewail. Freezing Time - in a Femtosecond. Sci. Spectra, 1998, 14, 62-71.

[22] Schaftenaar, G. and Noordik, J. H., MOLDEN: a pre- and post-processing program for molecular and electronic structures. J. Comput. Aided Mol. Design., 2000, 123-134.

[23] K. Fukui. The role of frontier orbitals in chemical reactions. Science, 1987, 218, 747-754.

[24] K. Fukui, T. Yonezawa and H. Shingu. A molecular orbital theory of reactivity in aromatic hydrocarbons. J. Chem. Phys., 1952, 20, 722-725.

[25] K. Fukui, T. Yonezawa and C. Nagata. Molecular orbital theory of orientation in aromatic heteroaromatic, and other conjugated molecules. J. Chem. Phys., 1952, 22, 1433-1442.

[26] R. G. Parr, S. R. Gadre and L. J. Bartolotti. Local density functional theory of atoms and molecules. Proc. Natl. Acad. Sci. USA, 1979, 76, 2522-2526.

[27] S. H. Vosko, L. Wilk and M. Nusair. Accurate spin-dependent electron liquid correlation energies for local spin density calculations: a critical analysis. Can. J. Phys., 1980, 58, 1200-1211.

[28] Reed A. E., Weinstock R. B., Weinhold F. Natural Population Analysis. J. Chem. Phys. Vol. 83, No. 2, (1985) 735-747.

[29] Nelson Flores-Gallegos Teoría de información cuántica como lenguaje conceptual en Química. Tesis Doctoral. Universidad Autónoma Metropolitana-Iztapalapa. México D.F. (2010). 
[30] Edmundo M. Carrera, Nelson Flores-Gallegos, Rodolfo O. Esquivel. Natural atomic probabilities in quantum information theory. Journal of Computational and Applied Mathematics., Vol. 233, 1483-1490 (2010).

[31] Gaussian 09, Revision B.01, M. J. Frisch, G. W. Trucks, H. B. Schlegel, G. E. Scuseria, M. A. Robb, J. R. Cheeseman, G. Scalmani, V. Barone, B. Mennucci, G. A. Petersson, H. Nakatsuji, M. Caricato, X. Li, H. P. Hratchian, A. F. Izmaylov, J. Bloino, G. Zheng, J. L. Sonnenberg, M. Hada, M. Ehara, K. Toyota, R. Fukuda, J. Hasegawa, M. Ishida, T. Nakajima, Y. Honda, O. Kitao, H. Nakai, T. Vreven, J. A. Montgomery, Jr., J. E. Peralta, F. Ogliaro, M. Bearpark, J. J. Heyd, E. Brothers, K. N. Kudin, V. N. Staroverov, T. Keith, R. Kobayashi, J. Normand, K. Raghavachari, A. Rendell, J. C. Burant, S. S. Iyengar, J. Tomasi, M. Cossi, N. Rega, J. M. Millam, M. Klene, J. E. Knox, J. B. Cross, V. Bakken, C. Adamo, J. Jaramillo, R. Gomperts, R. E. Stratmann, O. Yazyev, A. J. Austin, R. Cammi, C. Pomelli, J. W. Ochterski, R. L. Martin, K. Morokuma, V. G. Zakrzewski, G. A. Voth, P. Salvador, J. J. Dannenberg, S. Dapprich, A. D. Daniels, O. Farkas, J. B. Foresman, J. V. Ortiz, J. Cioslowski, and D. J. Fox, Gaussian, Inc., Wallingford CT, 2010.

[32] Gaussian 03, Revision C.02, M. J. Frisch, G. W. Trucks, H. B. Schlegel, G. E. Scuseria, M. A. Robb, J. R. Cheeseman, J. A. Montgomery, Jr., T. Vreven, K. N. Kudin, J. C. Burant, J. M. Millam, S. S. Iyengar, J. Tomasi, V. Barone, B. Mennucci, M. Cossi, G. Scalmani, N. Rega, G. A. Petersson, H. Nakatsuji, M. Hada, M. Ehara, K. Toyota, R. Fukuda, J. Hasegawa, M. Ishida, T. Nakajima, Y. Honda, O. Kitao, H. Nakai, M. Klene, X. Li, J. E. Knox, H. P. Hratchian, J. B. Cross, C. Adamo, J. Jaramillo, R. Gomperts, R. E. Stratmann, O. Yazyev, A. J. Austin, R. Cammi, C. Pomelli, J. W. Ochterski, P. Y. Ayala, K. Morokuma, G. A. Voth, P. Salvador, J. J. Dannenberg, V. G. Zakrzewski, S. Dapprich, A. D. Daniels, M. C. Strain, O. Farkas, D. K. Malick, A. D. Rabuck, K. Raghavachari, J. B. Foresman, J. V. Ortiz, Q. Cui, A. G. Baboul, S. Clifford, J. Cioslowski, B. B. Stefanov, G. Liu, A. Liashenko, P. Piskorz, I. Komaromi, R. L. Martin, D. J. Fox, T. Keith, M. A. Al-Laham, C. Y. Peng, A. Nanayakkara, M. Challacombe, P. M. W. Gill, B. Johnson, W. Chen, M. W. Wong, C. Gonzalez, and J. A. Pople, Gaussian, Inc., Wallingford CT, 2004.

[33] Parr, R.G. and Weitao, Y. Density Functional Theory of Atoms and Molecules; Oxford University Press: New York, 1989; pp. 87-104.

[34] Nelson Flores-Gallegos and Carmen Salazar-Hernández. Some Applications of Quantum Mechanics; Chapter 10, Flows of Information and Informational Trajectories in Chemical Processes. InTech, 2012; pp. 233-256. 


\title{
A Novel Isospectral Deformation Chain in Supersymmetric Quantum Mechanics
}

\author{
Bjørn Jensen
}

Additional information is available at the end of the chapter

http://dx.doi.org/10.5772/53845

\section{Introduction}

Supersymmetric quantum mechanics (SUSYQM) has turned out to be surprisingly fertile field which is also able to successfully address challenges in traditional quantum mechanics and beyond. It has its roots in the works of Schrödinger, Infeld and Hull [1] on factorization methods of the Schrödinger equation. The term supersymmetric is due to a work by Witten [2] which brought these methods in contact with contemporary ideas in high energy physics. He showed in particular that the a factorized one-dimensional Schrödinger equation can accompany a super-Lie algebra thus providing a rich toy-model where features and concepts in supersymmetric quantum field theories can be studied in a greatly simplified context. A key ingredient in supersymmetric theories is that every bosonic state has a fermionic superpartner with all properties equal except the spin. In SUSYQM these states emerge as bosonic doublet states. The bosonic and the fermionic states are described in terms of the Schrödinger equation, but they interact with different physical potentials. These potentials are called partner potentials. Not completely surprising, knowing in advance the energy-eigenvalues and functions of the bosonic (fermionic) states the theory provides a map to the fermionic (bosonic) states with exactly the same energy-eigenvalues. Of key interest to us is that the physical partner potentials are expressed in terms of the same superpotential. These expressions are in general not unique. Different superpotentials can give rise to a particular physical potential in the fermionic (bosonic) sector. This does not imply that when the superpotential is changed (deformed) in such a way that the physical potential in the fermionic (bosonic) sector stays unchanged that the physical potential stays invariant in the bosonic (fermionic) sector. Whenever we deform a superpotential in the fermionic (bosonic) sector such that the fermionic (bosonic) potential is invariant the bosonic (fermionic) potential will generally change, but the theory nevertheless assures that the energy-eigenvalues in the bosonic (fermionic) sector stays the same. Such deformations are called isospectral deformations. They are the subject of this chapter. 
Isospectral transformations in the context of SUSYQM has a long history exhibiting methods dating all the way back to Darboux [3]. The dominating approach is to study isospectral Hamilton operators. Different operator methods exist, but the main ones was brought under a single unifying principle by Pursey [4] with the use of isometric operators. A second approach to the study of isospectral transformations is what has been called deformation theory (see [5], e.g.). This is a more direct approach compared with the operator approach in that one studies deformations of the superpotential as described briefly above. It is rather surprising to note that this second approach has not been given much attention in the literature. To the knowledge of this author only one of the simplest deformations possible has been discussed to some extend. In a previous work [6] we initiated a work with the aim to remedy this situation. In [6] we showed that the isospectral deformation which has been considered in previous works is part of a more general deformation scheme. In this work we extend our results in [6]. We explicitly construct an in principle infinite recursively defined isospectral deformation chain where the deformation scheme in [6] emerges as the root of the chain.

This chapter is organized as follows. In the next section we very briefly review some of the basics of SUSYQM, mainly in order to fix notation. We define the notions of partner potentials, superpotential, isospectrality and supersymmetry. In section 2 we briefly remain ourselves about the results in [6]. In section 3 we define the recursive deformation scheme. We also discuss other various deformation schemes but show that a number of other canonical deformation schemes defined along the lines of our recursive scheme either do not allow a recursive structure or either reduces to our scheme. We apply our apparently rather unique recursive deformation scheme to the Coulomb potential and calculate several novel potentials. We summarize our findings and conclude in the last section. No attempt has been made to give an in depth review of the relevant literature due to its immense size. The works which have been acknowledged in the list of references have been so only because of their utility to this author.

\section{SUSYQM - A very brief introduction}

SUSYQM can in its most basic formulation be thought of as the following two factorizations of the Hamiltonian in the stationary Schrödinger equation in appropriate units

$$
\left\{\begin{array}{l}
(-\partial+W(x))(\partial+W(x)) \equiv A^{+} A^{-} \equiv H_{-} \\
(\partial+W(x))(-\partial+W(x)) \equiv A^{-} A^{+} \equiv H_{+}
\end{array}\right.
$$

Here $\partial$ is short hand for differentiation with respect to the single spatial coordinate $x$, and $W(x)$ is the so called superpotential. Both of these factorizations give rise to a Schrödinger equation, but with different potentials $V_{-}(x)$ and $V_{+}(x)$ (the so called superpotentials) given by

$$
V_{ \pm}(x)=W^{2}(x) \pm \partial W(x) .
$$

Let us denote the energy-eigenvalues and eigenstates associated with $H_{ \pm}$by $E_{n}^{ \pm}$and $\psi_{n}^{ \pm}(x)$, respectively. Let $n=0$ denote the ground state. We note that

$$
A^{ \pm} \psi_{0}^{ \pm}(x)=0 \Rightarrow H_{ \pm} \psi_{0}^{ \pm}(x)=0 .
$$


The ground state eigenfunction is thus simply given by

$$
\psi_{0}^{ \pm}(x) \sim e^{ \pm \int^{x} W(x) d x} \sim \frac{1}{\psi_{0}^{\mp}(x)}
$$

The factorization in Eq.(1) carries a symmetry which is not manifestly present in the usual form of the Schrödinger equation. This symmetry is made manifest when Eq.(1) is brought to a matrix form. Defining

$$
Q^{-} \equiv\left(\begin{array}{cc}
0 & 0 \\
A^{-} & 0
\end{array}\right), Q^{+} \equiv\left(\begin{array}{cc}
0 & A^{+} \\
0 & 0
\end{array}\right)
$$

we find that we naturally can construct a matrix-valued Hamiltonian $H$ given by

$$
H \equiv\left(\begin{array}{cc}
H_{-} & 0 \\
0 & H_{+}
\end{array}\right)=\left(\begin{array}{cc}
A^{+} A^{-} & 0 \\
0 & A^{-} A^{+}
\end{array}\right)
$$

It is straightforward to verify that

$$
H=Q^{-} Q^{+}+Q^{+} Q^{-} \equiv\left\{Q^{-}, Q^{+}\right\},\left[Q^{ \pm}, H\right]=0,\left(Q^{ \pm}\right)^{2}=0
$$

This constitutes what is called a super-Lie algebra in contrast to an ordinary Lie algebra which only contains commutators. The commutator in Eq.(7) shows that $Q^{ \pm}$are generators of a symmetry which is left intact under time-translations generated by $H$. We call this symmetry the supersymmetry of the system.

The matrices above naturally act on a two-dimensional vector space with the natural representation

$$
\left(\begin{array}{c}
\psi_{n}^{-}(x) \\
\psi_{n-1}^{+}(x)
\end{array}\right)
$$

It is clear that

$$
\begin{aligned}
& Q^{-}\left(\begin{array}{c}
\psi_{n}^{-}(x) \\
0
\end{array}\right)=\left(\begin{array}{c}
0 \\
\psi_{n-1}^{+}(x)
\end{array}\right) \\
& Q^{+}\left(\begin{array}{c}
0 \\
\psi_{n}^{+}(x)
\end{array}\right)=\left(\begin{array}{c}
\psi_{n+1}^{-}(x) \\
0
\end{array}\right) .
\end{aligned}
$$

Hence, $Q^{ \pm}$relate states with the same eigenvalue of $H$; the energy states are in other words degenerate. An orthogonal basis can naturally be taken to be states on the form

$$
\left(\begin{array}{c}
\alpha(x) \\
0
\end{array}\right),\left(\begin{array}{c}
0 \\
\beta(x)
\end{array}\right) .
$$


It is customary, due to the intimate relation to supersymmetric quantum field theory, to say that the first vector belongs to the bosonic sector and the other to the fermionic sector, even though no fermions appear in this theory. That $Q^{ \pm}$relate states corresponding to the same energy eigenvalue of the $H$-operator can also be seen on the level of the $H_{ \pm}$operators by noting that

$$
H_{+}\left(A^{-} \psi_{n}^{-}\right)=E_{n}^{-}\left(A^{-} \psi_{n}^{-}\right) .
$$

Hence, given an eigenstate $\psi_{n}^{-}$of $H_{-}$with energy eigenvalue $E_{n}^{-}$, the state $A^{-} \psi_{n}^{-}$is an eigenstate of $H_{+}$with energy eigenvalue $E_{n}^{-}$. There is thus a one-to-one correspondence between bosonic and fermionic states with the same energy eigenvalue (Eq.(9-10) above). We call this property the isospectrality of SUSYQM. Much more can be said about SUSYQM, such as the role played by the vacuum in connection with isospectrality. However, for the purpose of this chapter this very brief exposition of some of the basics of SUSYQM is sufficient to fix notation and certain concepts.

\section{A novel isospectral deformation chain}

In [6] we introduced within the framework of SUSYQM an isospectral deformation on the form

$$
W(x) \rightarrow \hat{W}_{0}(x)=F_{0}(x) W(x),
$$

where $W(x)$ is some known superpotential and $F_{0}(x)$ some function to be determined by the isospectrality condition

$$
\hat{V}_{+}(x) \equiv \hat{W}_{0}^{2}(x)+\hat{W}_{0}^{\prime}(x)=W^{2}(x)+W^{\prime}(x) \equiv V_{+}(x) .
$$

It was shown that Eq.(13) includes the only previously explored deformation of this kind, which has the form [5]

$$
W(x) \rightarrow \hat{W}_{0}(x)=W(x)+f(x) .
$$

$f(x)$ is some function which is determined by Eq.(14). In this work we expand the deformation Eq.(13) in various directions and study the implications drawn from the isospectrality condition. We show in particular that the deformation Eq.(13) is the root of an infinitely long and recursively generated chain of deformations. Let us next briefly review some of the findings in [6].

\subsection{Base deformations}

The deformation Eq.(1) implies the following differential equation for $F_{0}(x)[6]^{1}$

$$
\frac{d}{d x} F_{0}(x)+\left(\frac{d}{d x} \ln W(x)\right) F_{0}(x)+W(x) F_{0}^{2}(x)=W(x)+\frac{d}{d x} \ln W(x) .
$$

\footnotetext{
${ }^{1}$ We will often rewrite fractions on the form $W^{\prime}(x) / W(x)$ as the logarithmic derivative of $W(x)$ as a formal tool. Caution must of course be exercised when using the corresponding expressions in actual computations.
} 
This is the generalized Riccati equation [7] . If one particular solution $F_{00}(x)$ of Eq.(16) is known another solution is given by [8]

$$
F_{0}(x)=F_{00}(x)+\frac{1}{X_{0}(x)}
$$

where $X_{0}(x)$ solves the equation

$$
\frac{d}{d x} X_{0}(x)-\left(\frac{d}{d x} \ln W(x)+2 F_{00}(x) W(x)\right) X_{0}(x)=W(x)
$$

Eq.(18) can be solved by elementary means. The resulting superpotential $\hat{W}_{0}(x)$ is given by [6]

$$
\begin{aligned}
\hat{W}_{0}(x) & =\left(F_{00}(x)+\frac{1}{X_{0}(x)}\right) W(x) \equiv \hat{W}_{00}(x)+\frac{1}{X_{0}(x)} W(x)= \\
& =F_{00}(x) W(x)+\frac{e^{-2 \int^{x} F_{00}(t) W(t) d t}}{C_{01}+\int^{x} e^{-2 \int^{u} F_{00}(t) W(t) d t} d u} .
\end{aligned}
$$

$C_{01}$ is an integration constant, which we will assume to be real. We have explicitly introduced upper integration limits in Eq.(19) in order to avoid sign ambiguities. This explains the difference in the sign in the denominator in Eq.(19) compared with Eq.(2.5) in [6] where the reverse order of integration in one of the integrals was implicitly assumed. We do not specify the lower integration limits in Eq.(19). These are not important, of course, since the values of the integrals there can essentially be absorbed into $C_{01}$. We can by simple inspection see that the particular solution $F_{0}(x)=1$, the identity deformation, solves Eq.(16). With $F_{00}(x)=1$ we identically rederive Eq.(15) and the corresponding expression discussed in [5]. The identity deformation corresponds to the limit $C_{01} \rightarrow \infty$ in Eq.(19) with $F_{00}(x)=1$. In the limit $C_{01} \rightarrow \infty$ we generally get $\hat{W}_{0}(x)=\hat{W}_{00}(x)$. This deformation will play a pivotal role in this work; it will represent the base of a recursive scheme for generating novel isospectral deformations. We will therefore refer to a particular $\hat{W}_{00}(x)$ as a base deformation in the following.

In order to expand the space of concrete isospectral deformations further we transform Eq.(16) into an ordinary second order differential equation by the substitution

$$
F_{0}(x)=\frac{1}{W(x)} \frac{d}{d x} \ln U_{0}(x)
$$

This substitution gives rise to the following linear homogeneous second order differential equation

$$
-\frac{d^{2}}{d x^{2}} U_{0}(x)+V_{+}(x) U_{0}(x)=0
$$


This equation coincides of course with the zero-energy eigenfunction equation. However, keep in mind that $U_{0}$ is not in general to be identified with the eigenfunction of the system. This is of particular importance to remember in light of Eq.(16). The special solution $F_{0}(x)=$ 1 is generated by the solution

$$
U_{0}(x) \sim e^{\int^{x} W(t) d t} .
$$

The particular solutions for $F_{0}(x)$ stemming from Eq.(21) can be fed into Eq.(19) (as $F_{00}(x)$ ) and thus expand the space of available concrete deformations. The physical potential $\hat{V}_{-}(x)$ generated by $\hat{W}_{0}(x)$ can in general thus be written $[6]^{2}$

$$
\begin{aligned}
& \hat{V}_{-}(x) \equiv \hat{W}_{0}^{2}(x)-\hat{W}_{0}^{\prime}(x)=\hat{W}_{00}^{2}(x)-\hat{W}_{00}^{\prime}(x)+ \\
& +\frac{4 \hat{W}_{00}(x) e^{-2 \int^{x} \hat{W}_{00}(t) d t}}{C_{01}+\int^{x} e^{-2 \int^{u} \hat{W}_{00}(t) d t} d u}+2\left[\frac{e^{-2 \int^{x} \hat{W}_{00}(t) d t}}{C_{01}+\int^{x} e^{-2 \int^{u} \hat{W}_{00}(t) d t} d u}\right]^{2}
\end{aligned}
$$

with

$$
\hat{W}_{00}(x)=\frac{d}{d x} \ln U_{0}(x)
$$

\subsection{Recursive linear deformations}

Although the Riccati equation can be transformed into an ordinary second order differential equation the non-linearity of the Riccati equation allows for a solution space which is larger than the one associated with linear differential equations of second order, as became evident in the previous section. It is therefore natural to ask whether the non-linearity of the Riccati equation implies even more isospectral deformations than the ones we already have deduced [6]. We will explore this question in this and the next section.

\subsubsection{The sum}

Let us entertain the following idea. Assume that we have derived a particular base deformation $\hat{W}_{00}(x)$ from an explicitly given superpotential $W(x)$. Then assume that we add another term $F_{1}(x) W(x)$ (possibly multiplied with a constant) to that deformation such that we in principle get a novel deformation on the form $\hat{W}(x)=F_{10}(x) W(x)+\hat{W}_{00}(x)$. After determining $F_{10}(x)$ from the isospectrality condition Eq.(14) add yet another term of this kind to the deformation. Let us assume that this process can be repeated indefinitely. Will terms added in this manner give rise to novel deformations? We will in the following show that they do. This represents a recursive deformation scheme.

Following the basic idea, after $m$ iterations we thus have the general recursive linear (in $W(x)$ ) deformation

$$
\hat{W}_{m 0}(x)=\left(\sum_{i=0}^{m} \lambda_{i} F_{i 0}(x)\right) W(x)=\lambda_{m} F_{m 0}(x) W(x)+\hat{W}_{(m-1) 0}(x), \lambda_{0} \equiv 1
$$

\footnotetext{
${ }^{2}$ Note that the corresponding expression in [6] ((2.14)) is misprinted.
} 
The $\lambda_{i}$ 's are assumed to be independent real constants. Starting with a known superpotential $m$ consecutive applications of the isospectrality condition yields the following set of equations

$$
\left\{\begin{array}{l}
F_{00}^{\prime}(x)+[\ln W(x)]^{\prime} F_{00}(x)+W(x) F_{00}^{2}(x)=W(x)+(\ln W(x))^{\prime}, \\
F_{10}^{\prime}(x)+\left[(\ln W(x))^{\prime}+2 F_{00}(x) W(x)\right] F_{10}(x)+\lambda_{1} W(x) F_{10}^{2}(x)=0, \\
F_{20}^{\prime}(x)+\left[(\ln W(x))^{\prime}+2\left(F_{00}(x)+\lambda_{1} F_{10}(x)\right) W(x)\right] F_{20}(x)+\lambda_{2} W(x) F_{20}^{2}(x)=0, \\
\vdots \\
\vdots \\
\left.F_{m 0}^{\prime}(x)+\left[(\ln W(x))^{\prime}+2 \hat{W}_{(m-1) 0}(x)\right)\right] F_{m 0}(x)+\lambda_{m} W(x) F_{m 0}^{2}(x)=0 .
\end{array}\right.
$$

The first equation in Eq.(26) coincides of course per definition with Eq.(16). Note that $F_{j 0}(x)=1$ only solves the first equation in Eq.(26). Let us consider an arbitrary iteration level $n(\neq 0)$ and make the following substitution in Eq.(26)

$$
F_{n 0}(x)=\frac{1}{W(x)}\left(\ln U_{n}(x)\right)^{\prime}
$$

The equation for $F_{n}(x)$ can then be written

$$
U_{n}^{\prime \prime}(x)+\left(\lambda_{n}-1\right) \frac{\left[U_{n}^{\prime}(x)\right]^{2}}{U_{n}(x)}+2 \hat{W}_{(n-1) 0}(x) U_{n}^{\prime}(x)=0
$$

This equation corresponds to Eq.(21) in the case when $n=0$. It reduces in general to an ordinary linear differential equation only when $\lambda_{n}=1, \forall n \neq 0$. We will focus on this special case in this work.

The general solution of Eq.(28) for arbitrary $n \neq 0$, and with $\lambda_{n}$ set to unity, can be found by elementary means, and we deduce that

$$
\begin{aligned}
F_{n 0}(x) W(x) & =\frac{C_{n 2} e^{-2 \int^{x} \hat{W}_{(n-1) 0}(t) d t}}{C_{n 1}+C_{n 2} \int^{x} e^{-2 \int^{u} \hat{W}_{(n-1) 0}(t) d t} d u}= \\
& =\frac{d}{d x} \ln \left(C_{n 1}+C_{n 2} \int^{x} e^{-2 \int^{u} \hat{W}_{(n-1) 0}(t) d t} d u\right) .
\end{aligned}
$$

$C_{n 1}$ and $C_{n 2}$ are integration constants, which we assume to be real. We can reduce the number of integration constants to one at each iteration level, but we will stick to the habit of explicitly writing down the actual number of constants in order to make it easier to compare the various formulas we deduce, which stem from both second and first order differential equations. We also note that the structure of $F_{n 0}(x)$ implies that previous deformations are not regenerated in general. Of course, this does not exclude this possibility to arise, as we will see in Section 5. Hence, $m$ in Eq.(25) has in principle no natural upper bound. From Eq.(25) and Eq.(29) we get the following expression for the superpotential at iteration level $m$ 


$$
\begin{aligned}
\hat{W}_{m 0}(x) & =\hat{W}_{00}(x)+\sum_{j=1}^{m} \frac{d}{d x} \ln \left(C_{j 1}+C_{j 2} \int^{x} e^{-2 \int^{u} \hat{W}_{(j-1) 0}(t) d t} d u\right)= \\
& =\hat{W}_{00}(x)+\frac{d}{d x} \ln \prod_{j=1}^{m}\left(C_{j 1}+C_{j 2} \int^{x} e^{-2 \int^{u} \hat{W}_{(j-1) 0}(t) d t} d u\right) \equiv \\
& \equiv \hat{W}_{00}(x)+\frac{d}{d x} \ln P_{m}(x) .
\end{aligned}
$$

From Eq.(30) we deduce that

$$
e^{-2 \int^{x} \hat{W}_{(j-1) 0}(t) d t}=P_{j-1}^{-2}(x) e^{-2 \int^{x} \hat{W}_{00}(t) d t} ; P_{0}^{2}(x) \equiv 1, j \neq 0
$$

such that

$$
\left\{\begin{array}{l}
P_{1}(x)=C_{11}+C_{12} \int^{x} e^{-2 \int^{u} \hat{W}_{00}(t) d t} d u \\
P_{2}(x)=P_{1}(x)\left(C_{21}+C_{22} \int^{x} P_{1}^{-2}(u) e^{-2 \int^{u} \hat{W}_{00}(t) d t} d u\right) \\
\vdots \\
\vdots \\
P_{m}(x)=P_{m-1}(x)\left(C_{m 1}+C_{m 2} \int^{x} P_{m-1}^{-2}(u) e^{-2 \int^{u} \hat{W}_{00}(t) d t} d u\right)
\end{array}\right.
$$

Hence,

$$
P_{n}(x)=\prod_{j=1}^{n}\left(C_{j 1}+C_{j 2} \int^{x} P_{j-1}^{-2}(u) e^{-2 \int^{u} \hat{W}_{00}(t) d t} d u\right) .
$$

This last form of the $P_{n}(x)$ functions neatly exhibits how the base deformation $\hat{W}_{00}(x)$ generates the higher order deformations. Some of the details we have deduced so far are presented in Figure 1.

Make the following substitution at each iteration level in Eq.(25)

$$
F_{n 0}(x) \rightarrow F_{n 0}(x)+\frac{1}{X_{n}(x)}
$$

This implies (with the $\lambda_{m}$ 's reinstated in Eq.(25)) a generalized form $\hat{W}_{m}(x)$ of the superpotential $\hat{W}_{m 0}(x)$

$$
\hat{W}_{m 0}(x)=\sum_{i=0}^{m} \lambda_{i} F_{i 0} W(x) \Rightarrow \hat{W}_{m}(x)=\hat{W}_{m 0}(x)+\sum_{i=0}^{m} \frac{\lambda_{i}}{X_{i}(x)} W(x) .
$$




$$
\left\{\begin{array}{l}
W \stackrel{\times F_{0}=F_{00}}{\longrightarrow} \quad \hat{W}_{00} \stackrel{+F_{10} W}{\longrightarrow} \hat{W}_{10}=\hat{W}_{00}+\left(\ln P_{1}\right)^{\prime} \stackrel{+F_{20} W}{\longrightarrow} \cdots \stackrel{+F_{m 0} W}{\longrightarrow} \hat{W}_{m 0} \\
W \stackrel{\times F_{0}=1}{\longrightarrow} \hat{W}_{00}=W \stackrel{+F_{10} W}{\longrightarrow} \hat{W}_{10}=W+\left(\ln P_{1}\right)^{\prime} \stackrel{+F_{20} W}{\longrightarrow} \cdots \stackrel{+F_{m 0} W}{\longrightarrow} \hat{W}_{m 0}
\end{array}\right.
$$

Figure 1. The upper line depicts the solvable deformation chain Eq.(25) to iteration level $m$. There is no upper bound on $m$. The $F_{j 0}(x)$ functions are given in Eq.(29). The $P_{j}(x)$ functions are given in Eq.(30) and Eq.(32). They are functions of a base deformation $\hat{W}_{00}(x)$. A base deformation $\hat{W}_{00}(x)$ is generated by the zero-energy Schrödinger equation interacting with the partner potential $V_{+}(x)$, Eq.(21). The second line depicts the important special case when $F_{0}(x)=1$. This particular solution can be derived as a special case of Eq.(19) with $X_{0}^{-1}=0$, which can be achieved by $C_{01} \rightarrow \infty$, and $F_{00}(x)$ determined by Eq.(20) and the solution Eq.(22). $\hat{W}_{10}(x)$ then coincides with Eq.(19) (when $F_{00}(x)=1$ and $C_{01}$ is finite in Eq.(19)); Eq.(19) is thus in this particular case regenerated by the scheme at the next recursion level, i.e.

From Eq.(26) we find that $X_{n}(x)$ satisfies the equation

$$
\frac{d}{d x} X_{n}(x)-\left(\frac{d}{d x} \ln W(x)+2 \hat{W}_{n 0}(x)\right) X_{n}(x)=\lambda_{m} W(x)
$$

This equation is a generalization of Eq.(18). The $n^{\prime}$ th deformation term Eq.(29) thus changes into

$$
F_{n 0}(x) W(x) \rightarrow F_{n 0}(x) W(x)+\frac{d}{d x} \ln \left(C_{n 3}+\lambda_{n} \int^{x} e^{-2 \int^{u} \hat{W}_{n 0}(t) d t} d u\right)
$$

$C_{n 3}$ are integrations constants, which we assume to be real. Eq.(37) implies that the more general expression for the superpotential in Eq.(35) can be written as

$$
\hat{W}_{m}(x)=\hat{W}_{m 0}(x)+\frac{d}{d x} \ln Q_{m}(x)
$$

where

$$
Q_{m}(x) \equiv \prod_{i=0}^{m}\left(C_{i 3}+\lambda_{i} \int^{x} e^{-2 \int^{u} \hat{W}_{i 0}(t) d t} d u\right)
$$

$m=0$ in Eq.(38) $\left(\lambda_{0} \equiv 1\right)$ reproduces Eq.(19). In the special case when $\lambda_{m}=1, \forall m$ in Eq.(25) we get

$$
\hat{W}_{m}(x)=\hat{W}_{00}(x)+\frac{d}{d x} \ln P_{m}(x)+\frac{d}{d x} \ln Q_{m}(x) .
$$

When we compare the expressions for $\left(P_{m}(x)\right)^{\prime}$ and $\left(Q_{m}(x)\right)^{\prime}$ we find that they differ by just the last term in $\left(Q_{m}(x)\right)^{\prime}$. 


\subsubsection{The product}

What happens if we in Eq.(25) assume a product structure instead of a sum structure ? Let us assume that we have determined a base deformation. Let this be the seed superpotential for the deformation

$$
\hat{W}_{00}(x) \rightarrow \hat{W}_{10}(x)=F_{10}(x) \hat{W}_{00}(x)=F_{10}(x) F_{00}(x) W(x),
$$

where $F_{10}(x)$ is some function to be determined by the isospectrality condition. This product scheme can of course in principle be repeated an arbitrary number $m$ times

$$
\hat{W}_{m 0}(x)=\left(\prod_{i=0}^{m} F_{i 0}(x)\right) W(x)=F_{m 0}(x) \hat{W}_{(m-1) 0} .
$$

This structure gives rise to the following set of equations

$$
\left\{\begin{array}{l}
F_{00}^{\prime}(x)+(\ln W(x))^{\prime} F_{00}(x)+W(x) F_{00}^{2}(x)=W(x)+(\ln W(x))^{\prime}, \\
F_{10}^{\prime}(x)+\left(\ln \hat{W}_{00}(x)\right)^{\prime} F_{10}(x)+\hat{W}_{00}(x) F_{10}^{2}(x)=\frac{1}{F_{00}(x)}\left(W+(\ln W(x))^{\prime}\right), \\
\vdots \\
\vdots \\
F_{m 0}^{\prime}(x)+\left(\ln \hat{W}_{(m-1) 0}(x)\right)^{\prime} F_{m 0}(x)+\hat{W}_{(m-1) 0}(x) F_{m 0}^{2}(x)=\frac{W(x)}{\hat{W}_{(m-1) 0}(x)}\left(W(x)+(\ln W(x))^{\prime}\right) .
\end{array}\right.
$$

Clearly, each iteration level depends on all the previous ones, and at each level we are dealing with a non-homogenous non-linear differential equation. Interestingly, by making the following substitution at an arbitrary iteration level $n \neq 0$

$$
F_{n 0}(x)=\frac{1}{\hat{W}_{(n-1) 0}(x)}\left(\ln U_{n}(x)\right)^{\prime}
$$

where $U_{n}(x)$ is some function, the equations Eq.(43) all reduce to Eq.(21). Hence, attempting to generate novel deformations recursively via a product structure, of the kind above, fails. This conclusion was also reached in [6], but at the level of the second order linear differential equation Eq.(21).

\subsection{Recursive non-linear deformations}

We have so far only considered linear (in the superpotential) deformations. In this section we will briefly consider two non-linear deformation schemes. Let us first consider a polynomial kind of deformation. That is, given a superpotential $\hat{W}_{(i-1) 0}(x)$ which we will assume is derived, in some way or another, from some seed superpotential $W(x)$. Consider then the polynomial deformation

$$
\hat{W}_{i 0}(x)=F_{i 0}(x) W^{k}(x)+\hat{W}_{(i-1) 0}(x) ; k \in\{1,2,3, \ldots\}
$$


The isospectrality condition then implies

$$
F_{i 0}^{\prime}(x)+\left[k(\ln W(x))^{\prime}+2 \hat{W}_{(i-1) 0}(x)\right] F_{i 0}(x)+W^{k}(x) F_{i 0}^{2}(x)=0 .
$$

This is a Riccati type equation of the kind we have met earlier in this work. Apparently, different $k$-values give rise to very different equations to solve. However, and rather intriguingly, all the possible $k$-values implies the same deformation. This is seen by making the following substitution

$$
F_{i 0}(x)=\frac{1}{W^{k}(x)} \frac{U_{k}^{\prime}(x)}{U_{k}(x)},
$$

where $U_{k}(x)$ is some function. This expression inserted into Eq.(46) gives

$$
U_{k}^{\prime \prime}(x)+2 \hat{W}_{i-1}(x) U_{k}^{\prime}(x)=0
$$

Hence, $\hat{W}_{i 0}(x)$ is independent of $k$ and we are essentially left with a linear deformation. Clearly, the range of values of $k$ can be expanded to the real numbers.

Another canonical generalization of our work is to consider deformations on the form

$$
\hat{W}(x)=H_{0}(x) \mathcal{F}(W),
$$

where $\mathcal{F}$ is any functional of the seed superpotential $W(x)$. The isospectrality condition then implies

$$
H_{0}^{\prime}(x)+(\ln \mathcal{F}(W))^{\prime} H_{0}(x)+\mathcal{F}(W) H_{0}^{2}(x)=\mathcal{F}(W)^{-1}\left(W^{2}(x)+W^{\prime}(x)\right) .
$$

Note that $H_{0}(x)=1$ does not solve this equation unless $\mathcal{F}(W)=W$, since Eq.(50) with $H_{0}(x)=1$ implies $\mathcal{F}^{\prime}(x)+\mathcal{F}^{2}(x)=V_{+}(x)$. Since $V_{+}(x)$ is uniquely given in Eq.(2) any other choice of functional will fail to satisfy the isospectrality condition. Hence the conclusion. The particular solution $H_{0}(x)=1$ is not forced upon us. We can in principle do without it. It is easily verified that Eq.(50) can be cast into the form Eq.(21) by the substitution

$$
H_{0}(x)=\frac{1}{\mathcal{F}(W)}(\ln U(x))^{\prime}
$$

We can also look for an expanded solution by writing

$$
H_{0}(x)=H_{00}(x)+\frac{1}{Z_{0}(x)},
$$

where $H_{00}(x)$ is a particular solution of Eq.(50). We then get the equation

$$
\frac{d}{d x} Z_{0}(x)-\left(\frac{d}{d x} \ln \mathcal{F}(W)+2 H_{00}(x) \mathcal{F}(W)\right) Z_{0}(x)=\mathcal{F}(W)
$$


which is a generalized form of Eq.(18). The reciprocal solution has the general form

$$
\frac{1}{Z_{0}(x)}=\frac{e^{-2 \int^{x} H_{00}(t) \mathcal{F}(W) d t}}{\mathcal{F}(W)\left(C+\int^{x} e^{-2 \int H_{00}(t) \mathcal{F}(W) d t} d u\right)} .
$$

$C$ is an integration constant, which we assume to be real. Utilizing that $H_{00}(x)=$ $\mathcal{F}^{-1}(x)(\ln U(x))^{\prime}$ the resulting deformation coincides with Eq.(19). We thus therefore conclude that non-linear deformations on the form Eq.(49) does not generate additional deformations to the ones already generated by Eq.(13).

\subsection{Deforming the Coulomb potential}

As a relatively simple application of the linear deformation scheme let us briefly consider deformations of the Coulomb potential. This potential has, within the framework of SUSYQM, been treated in several previous works [5]. The superpotential and the partner potential for the Coulomb potential are given by [5]

$$
\begin{aligned}
W(x) & =\frac{q^{2}}{2(l+1)}-\frac{(l+1)}{x} \\
V_{+}(x) & =\frac{1}{4}\left(\frac{q^{2}}{l+1}\right)^{2}-\frac{q^{2}}{x}+\frac{(l+1)(l+2)}{x^{2}} .
\end{aligned}
$$

$q$ and $l$ in these expressions are the electric charge and the angular momentum quantum numbers, respectively. These potentials result in the following general solution for $U_{0}(x)$ in Eq.(21) [6]

$$
U_{0}(x)=C_{1} M_{l+1, l+\frac{3}{2}}\left(\frac{q^{2} x}{l+1}\right)+C_{2} W_{l+1, l+\frac{3}{2}}\left(\frac{q^{2} x}{l+1}\right) .
$$

The $M(x)$ - and $W(x)$-functions are the Whittaker functions. The solution Eq.(22) is given by [6]

$$
U_{0}(x) \sim e^{\frac{q^{2} x}{2(l+1)}-(l+1) \ln (2 x)} .
$$

We will for simplicity assume this solution in the following. We will let $C_{01} \rightarrow \infty$ in Eq.(19) such that we deal with the identity deformation $\hat{W}_{0}(x)=\hat{W}_{00}(x)=W(x)$. We will also ignore the $Q_{j}(x)$ contributions in the following. Define $A \equiv q^{2} /(2(l+1))$ and $B \equiv l+1$. It then follows that

$$
P_{1}(x)=C_{11}+C_{12} \int^{x} t^{2 B} e^{-2 A t} d t
$$

such that

$$
\hat{W}_{10}(x)=A-\frac{B}{x}+\frac{C_{12} x^{2 B} e^{-2 A x}}{C_{11}+C_{12} \int^{x} t^{2 B} e^{-2 A t} d t}
$$


Let us consider the s-state with $l=0$ in order to get a better grasp on the content buried in Eq.(60). We also set $q \equiv 1$. The expression for $\hat{W}_{10}(x)$ then reduces to

$$
\hat{W}_{10}(x)=\frac{1}{2}-\frac{1}{x}+\frac{C_{12} x^{2} e^{-x}}{C_{11}-C_{12}\left(x^{2}+2 x+2\right) e^{-x}}
$$

after redefining $C_{11}$ such that the lower integration limit of the integral in Eq.(60) does not appear explicitly in the expression for the potential. We will automatically do such redefinitions in the following when it is appropriate. The corresponding physical potential $\hat{V}_{-1}(x)$ can either be derived from the definition $\hat{V}_{-1}(x) \equiv \hat{W}_{10}^{2}(x)-\hat{W}_{10}^{\prime}(x)$ or from Eq.(23) with $\hat{W}_{00}(x)=W(x)$ and $C_{01}$ finite. This is a consequence of a regeneration of Eq.(19) by the recursion scheme which was noted in Figure 1. From the definition it follows that

$$
\hat{V}_{-1}(x)=\frac{1}{4}-\frac{1}{x}+\frac{C_{12} x(2 x-4) e^{-x}}{C_{11}-C_{12}\left(x^{2}+2 x+2\right) e^{-x}}+\frac{2 C_{12}^{2} x^{4} e^{-2 x}}{\left(C_{11}-C_{12}\left(x^{2}+2 x+2\right) e^{-x}\right)^{2}}
$$

In the special case when we set $C_{11}=0$ the last term in Eq.(61) becomes independent of the exponentials (and $C_{12}$ ) and thus reduces to a pure rational function. The physical potential $\hat{V}_{-1}(x)$ generated by $\hat{W}_{10}(x)$ is then given by

$$
\hat{V}_{-1}(x)=\frac{1}{4}-\frac{1}{x}+\frac{4 x(x+2)}{\left(x^{2}+2 x+2\right)^{2}} \equiv V_{-}(x)+\frac{4 x(x+2)}{\left(x^{2}+2 x+2\right)^{2}} .
$$

Let us go to the second iteration level starting from the expression for $\hat{W}_{10}(x)$ in Eq.(61) with $C_{11}=0$, for convenience. It then follows that

$$
\hat{W}_{20}(x)=\hat{W}_{1}(x)+\frac{C_{22} x^{2} e^{x}}{C_{21}\left(x^{2}+2 x+2\right)^{2}+C_{22}\left(x^{2}+2 x+2\right) e^{x}} .
$$

Note that when $C_{21}=0$ we get $\hat{W}_{20}(x)=W(x)$. Hence, the deformation scheme allows in general for the possibility that additional iterations in particular cases may regenerate previous potentials in a nontrivial fashion. The expression for the corresponding physical potential is given by

$$
\begin{aligned}
\hat{V}_{-2}(x)= & \hat{V}_{-1}(x)+\left[\frac{C_{22} x^{2} e^{x}}{C_{21}\left(x^{2}+2 x+2\right)^{2}+C_{22}\left(x^{2}+2 x+2\right) e^{x}}\right] \times \\
& \times\left[-4\left(\frac{1}{x}+\frac{\frac{1}{2} x^{2}}{x^{2}+2 x+2}\right)+\frac{2\left(C_{21}(2 x+2)+C_{22} e^{x}\right)}{C_{21}\left(x^{2}+2 x+2\right)+C_{22} e^{x}}\right] .
\end{aligned}
$$




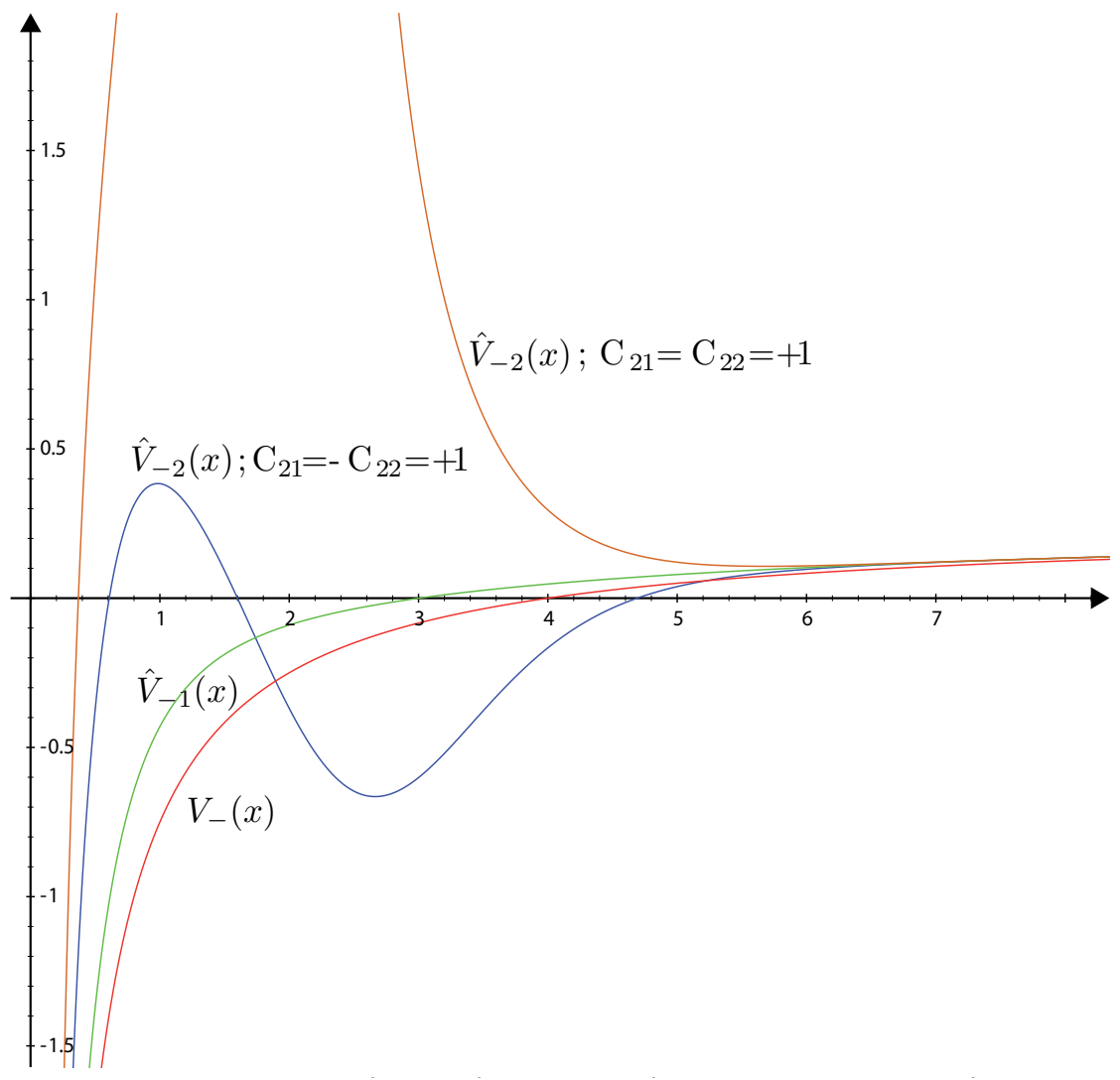

Figure 2. Generic plots depicting $\hat{V}_{-}(x), \hat{V}_{-1}(x)$ and $\hat{V}_{-2}(x)$. The plots for $\hat{V}_{-2}(x)$ show how drastic the nature of a potential might change as the values of the integration constants change.

The superpotential stemming from the third iteration with $\hat{W}_{20}(x)$ in Eq.(64) as the starting point is given by

$$
\hat{W}_{30}(x)=\hat{W}_{20}(x)+\frac{\left(\frac{C_{32} x^{2} e^{x}}{C_{21}\left(x^{2}+2 x+2\right)^{2}+C_{22}\left(x^{2}+2 x+2\right) e^{x}}\right)}{\left(C_{31}+\frac{C_{32}}{C_{22}} \ln \left|\frac{C_{21}\left(x^{2}+2 x+2\right)+C_{22} e^{x}}{x^{2}+2 x+2}\right|\right)} .
$$

This superpotential introduces the possibility for a logarithmic singularity away from the origin when $C_{22} / C_{21}<0$. We note that setting $C_{31}=0$ does not regenerate a previous potential as was possible at the previous iteration level when we correspondingly put $C_{21}=$ 0 . From Eq.(66) we can deduce the physical potential $\hat{V}_{-3}(x)$ at the third iteration level. We do not reproduce it here due to its complexity. Due to the complicated integrals appearing we are not able to provide the analytical expression for $\hat{W}_{40}(x)$. We leave detailed studies of the Coulomb potential for the future. 


\section{Conclusion}

In a previous paper we showed that isospectral deformations on the form Eq.(15) are contained in the space of deformations generated by isospectral deformations on the form Eq.(13). In this work we have shown that Eq.(13) can be considered as the initial, or base, deformation of a novel infinite recursive isospectral deformation chain. This thus answers to some extend the question by which we ended our previous paper [6]; how does the most general isospectral deformation of the kind considered there (Eq.(13) in this paper) look like. The results in this work do obviously only give a partial answer. We deduced in particular that a class of recursive deformations exists which is generated by the solutions of the non-linear differential equations in Eq.(28).

We briefly discussed various ways to construct alternative recursive deformation structures. We considered a linear product structure, polynomial deformations and completely generalized base deformations. They all either failed to provide a recursive structure or they turned out to be identical to the deformation scheme developed in this work.

We applied the linear recursive scheme to the Coulomb potential. We derived novel superpotentials which all per construction satisfy the isospectrality condition. It is straightforward, although very tedious, to check that the corresponding physical potentials $V_{+}$at the various iteration levels all satisfy the isospectrality condition Eq.(26). This application did also demonstrate how easily novel isospectral deformations can be generated in this approach. It did also demonstrate an increased relative complexity of the generated potentials with the number of iterations, as one also naively would expect from the expression Eq.(33).

The results in this work is obviously only a starting point for further research. One issue which needs clarification is the more general implications which can be drawn from Eq.(28). Another obvious issue is the behaviour of the transmission $T$ and reflection $R$ coefficients when the deformation chain is applied to some known initial scattering process. It is known that $T$ and $R$ are invariant under the simple deformation in Eq.(15) [5]. However, it is unclear whether this property is also a property of the general deformation chain. It is also of great interest to study the relation between our chain construction and the conventional operator approaches. One possible strategy one might follow in order to cast some light on this issue is to study the relation between the deformation chain and the concept of intertwining operators. It is known that many exactly solvable potentials are related by intertwining operator transformations including the Darboux transformations which also appear in our context [6]. Clearly, key to our construction in this work is the non-linearity of the Riccati equation. One could contemplate studying the associated JET-space and its deformations. Furthermore, an analysis of the intertwining of the hierarchy of the JET-spaces associated with the system of equations in Eq.(26) might also cast new light on our subject.

\section{Author details}

Bjørn Jensen

* Address all correspondence to: bjorn.jensen@hive.no

Vestfold University College, Norway 


\section{References}

[1] E. Schrödinger, Proc. R. Irish Acad. A46, 9 (1940); A46, 183 (1940); A47, 53 (1941), L.Infeld and T.E. Hull, Rev. Mod. Phys. 23, 21 (1951) (see also the references in this paper).

[2] E. Witten, Nucl. Phys. B188 513 (1981).

[3] G. Darboux, C.R. Acad. Sci. (Paris) 94, 1456 (1882).

[4] D.L. Pursey, Phys. Rev. D33 2267 (1986).

[5] A. Gangopadhyaya, J.V. Mallow and C. Rasinariu, Supersymmetric quantum mechanics World Scientific Publishing Co. (2011).

[6] B. Jensen, JHEP 11, 059 (2011).

[7] E. L. Ince,Ordinary differential equations Dover Publications, Inc. (1956).

[8] W.E. Boyce, R.C. DiPrima, Elementary Differential Equations and Boundary Value Problems New York: Wiley, 4th ed. (1986). 


\title{
Quantum Effects Through a Fractal Theory of Motion
}

\author{
M. Agop, C.Gh. Buzea, S. Bacaita, A. Stroe and M. Popa \\ Additional information is available at the end of the chapter \\ http://dx.doi.org/10.5772/54172
}

\section{Introduction}

Scale Relativity Theory (SRT) affirms that the laws of physics apply in all reference systems, whatever its state of motion and its scale. In consequence, SRT imply [1-3] the followings:

i. Particle movement on continuous and non-differentiable curve (or almost nowhere differentiable), that is explicitly scale dependent and its length tends to infinity, when the scale interval tends to zero.

ii. Physical quantities will be expressed through fractal functions, namely through functions that are dependent both on coordinate field and resolution scale. The invariance of the physical quantities in relation with the resolution scale generates special types of transformations, called resolution scale transformations. In what follows we will explain the above statement.

Let $F(x)$ be a fractal function in the interval $x \in[a, b]$ and let the sequence of values for $x$ be:

$$
x_{a}=x_{0}, x_{1}=x_{0}+\varepsilon, x_{k}=x_{0}+k \varepsilon, x_{n}=x_{0}+n \varepsilon=x_{b}
$$

We can now say that $F(x, \varepsilon)$ is a -scale approximation.

Let us now consider as a $\bar{\varepsilon}$-scale approximation of the same function. Since $F(x)$ is everywhere almost self-similar, if $\varepsilon$ and $\bar{\varepsilon}$ are sufficiently small, both approximations $F(x, \varepsilon)$ and must lead to same results. By comparing the two cases, one notices that scale expansion is related to the increase $d \varepsilon$ of $\varepsilon$, according to an increase $d \bar{\varepsilon}$ of $\bar{\varepsilon}$. But, in this case we have:

$$
\frac{d \varepsilon}{\varepsilon}=\frac{d \bar{\varepsilon}}{\bar{\varepsilon}}=d \rho
$$


situation in which we can consider the infinitesimal scale transformation as being

$$
\varepsilon^{\prime}=\varepsilon+d \varepsilon=\varepsilon+\varepsilon d \rho
$$

Such transformation in the case of function $F(x, \varepsilon)$, leads to:

$$
F\left(x, \varepsilon^{\prime}\right)=F(x, \varepsilon+\varepsilon d \rho)
$$

respectively, if we limit ourselves to a first order approximation:

$$
F\left(x, \varepsilon^{\prime}\right)=F(x, \varepsilon)+\frac{\partial F(x, \varepsilon)}{\partial \varepsilon}\left(\varepsilon^{\prime}-\varepsilon\right)=F(x, \varepsilon)+\frac{\partial F(x, \varepsilon)}{\partial \varepsilon} \varepsilon d \rho
$$

Moreover, let us notice that for an arbitrary but fixed $\varepsilon_{0}$, we obtain:

$$
\frac{\partial \ln \left(\varepsilon / \varepsilon_{0}\right)}{\partial \varepsilon}=\frac{\partial\left(\ln \varepsilon-\ln \varepsilon_{0}\right)}{\partial \varepsilon}=\frac{1}{\varepsilon}
$$

situation in which (5) can be written as:

$$
F\left(x, \varepsilon^{\prime}\right)=F(x, \varepsilon)+\frac{\partial F(x, \varepsilon)}{\partial \ln \left(\varepsilon / \varepsilon_{0}\right)} d \rho=\left[1+\frac{\partial}{\partial \ln \left(\varepsilon / \varepsilon_{0}\right)} d \rho\right] F(x, \varepsilon)
$$

Therefore, we can introduce the dilatation operator:

$$
\hat{D}=\frac{\partial}{\partial \ln \left(\varepsilon / \varepsilon_{0}\right)}
$$

At the same time, relation (8) shows that the intrinsic variable of resolution is not $\varepsilon$, but $\ln \left(\varepsilon / \varepsilon_{0}\right)$.

The fractal function is explicitly dependent on the resolution $\left(\varepsilon / \varepsilon_{0}\right)$, therefore we have to solve the differential equation:

$$
\frac{d F}{d \ln \left(\varepsilon / \varepsilon_{0}\right)}=P(F)
$$


where $P(F)$ is now an unknown function. The simplest explicit suggested form for $P(F)$ is linear dependence [2]

$$
P(F)=A+B F, A, B=\text { const } .
$$

in which case the differential equation (9) takes the form:

$$
\frac{d F}{d \ln \left(\varepsilon / \varepsilon_{0}\right)}=A+B F
$$

Hence by integration and substituting:

$$
\begin{gathered}
B=-\tau, \\
-\frac{A}{B}=F_{0}
\end{gathered}
$$

we obtain:

$$
F\left(\frac{\varepsilon}{\varepsilon_{0}}\right)=F_{0}\left[1+\left(\frac{\varepsilon_{0}}{\varepsilon}\right)^{\tau}\right]
$$

We can now generalize the previous result by considering that $F$ is dependent on parameterization of the fractal curve. If $p$ characterizes the position on the fractal curve then, following the same algorithm as above, the solution will be as a sum of two terms i.e. both classical and differentiable (depending only on position) and fractal, non-differentiable (depending on position and, divergently, on $\left.\varepsilon / \varepsilon_{0}\right)$

$$
F\left(p, \varepsilon / \varepsilon_{0}\right)=F_{0}(p)\left[1+\xi(p)\left(\frac{\varepsilon_{0}}{\varepsilon}\right)^{\tau(p)}\right]
$$

where $\xi(p)$ is a function depending on parameterization of the fractal curve.

The following particular cases are to be considered:

1. in asymptotic small scale regime $\varepsilon\left\langle\left\langle\varepsilon_{0}, \tau\right.\right.$ is constant (with no scale dependence) and power-law dependence on resolution is obtained: 


$$
\begin{array}{ll}
F\left(p, \varepsilon / \varepsilon_{0}\right)=T(p)\left(\frac{\varepsilon_{0}}{\varepsilon}\right)^{\tau} & \text { a } \\
T(p)=F_{0}(p) Q(p) & \text { b }
\end{array}
$$

2. in the asymptotic big scale regime $\varepsilon\rangle\rangle \varepsilon_{0}$, $\tau$ is constant (with no scale dependence) and, in terms of resolution, one obtains an independent law:

$$
F\left(p, \varepsilon / \varepsilon_{0}\right) \rightarrow F_{0}(p)
$$

Particularly, if $F\left(p, \varepsilon / \varepsilon_{0}\right)$ are the coordinates in given space, we can write

$$
X\left(p, \varepsilon / \varepsilon_{0}\right)=x(p)\left[1+\xi(p)\left(\frac{\varepsilon_{0}}{\varepsilon}\right)^{\tau}\right]
$$

In this situation, $\xi(p)$ becomes a highly fluctuating function which can be described by stochastic process while $\tau$ represents (according to previous description) the difference between fractal and topological dimensions. The result is a sum of two terms, a classical, differentiable one (dependent only on the position) and a fractal, non-differentiable one (dependent both on the position and, divergently, on $\varepsilon / \varepsilon_{0}$ ). This represents the importance of the above analysis.

By differentiating these two parts we obtain:

$$
d X=d x+d \xi
$$

where $d x$ is the classical differential element and $d \xi$ is a differential fractal one.

iii. There is infinity of fractal curves (geodesics) relating to any couple of points (or starting from any point) and applied for any scale. The phenomenon can be easily understood at the level of fractal surfaces, which, in their turn, can be described in terms of fractal distribution of conic points of positive and negative infinite curvature. As a consequence, we have replaced velocity on a particular geodesic by fractal velocity field of the whole infinite ensemble of geodesics. This representation is similar to that of fluid mechanics [4] where the motion of the fluid is described in terms of its velocity field $v=(x(t), t)$, density $\rho=(x(t), t)$ and, possibly, its pressure. We shall, indeed, recover the fundamental equations of fluid mechanics (Euler and continuity equations), but we shall write them in terms of a density of probability (as defined by the set of geodesics) instead of a density of matter and adding an additional term of quantum pressure (the expression of fractal geometry). 
iv. The local differential time invariance is broken, so the time-derivative of the fractal field $Q$ can be written two-fold:

$$
\begin{array}{ll}
\frac{d_{+} Q}{d t}=\lim _{\Delta t \rightarrow 0_{+}} \frac{Q(t+\Delta t)-Q(t)}{\Delta t} & \mathrm{a} \\
\frac{d_{-} Q}{d t}=\lim _{\Delta t \rightarrow 0_{-}} \frac{Q(t)-Q(t-\Delta t)}{\Delta t} & \mathrm{~b}
\end{array}
$$

Both definitions are equivalent in the differentiable case $d t \rightarrow-d t$. In the non-differentiable situation, these definitions are no longer valid, since limits are not defined anymore. Fractal theory defines physics in relationship with the function behavior during the "zoom" operation on the time resolution $\delta t$, here identified with the differential element $d t$ (substitution principle), which is considered an independent variable. The standard field $Q(t)$ is therefore replaced by fractal field $Q(t, d t)$, explicitly dependent on time resolution interval, whose derivative is not defined at the unnoticeable limit $d t \rightarrow 0$. As a consequence, this leads to the two derivatives of the fractal field $Q$ as explicit functions of the two variables $t$ and $d t$,

$$
\begin{array}{ll}
\frac{d_{+} Q}{d t}=\lim _{\Delta t \rightarrow 0_{+}} \frac{Q(t+\Delta t, \Delta t)-Q(t, \Delta t)}{\Delta t} & \mathrm{a} \\
\frac{d_{-} Q}{d t}=\lim _{\Delta t \rightarrow 0_{-}} \frac{Q(t, \Delta t)-Q(t-\Delta t, \Delta t)}{\Delta t} & \mathrm{~b}
\end{array}
$$

Notation "+" corresponds to the forward process, while "_" to the backward one.

v. We denote the average of these vectors by $d x_{ \pm}^{i}$, i.e.

$$
\left\langle d X_{ \pm}^{i}\right\rangle=d x_{ \pm}^{i}, i=1,2
$$

Since, according to (19), we can write:

$$
d X_{ \pm}^{i}=d x_{ \pm}^{i}+d \xi_{ \pm}^{i}
$$

and it results:

$$
\left\langle d \xi_{ \pm}^{i}\right\rangle=0
$$

vi. The differential fractal part satisfies the fractal equation: 


$$
d_{ \pm} \xi^{i}=\lambda_{ \pm}^{i}(d t)^{1 / D_{F}}
$$

where $\lambda_{ \pm}^{i}$ are some constant coefficients and $D_{F}$ is a constant fractal dimension. We note that the use of any Kolmogorov or Hausdorff $[1,5,6-8]$ definitions can be accepted for fractal dimension, but once a certain definition is admitted, it should be used until the end of analyzed dynamics.

vii. The local differential time reflection invariance is recovered by combining the two derivatives, $d_{+} / d t$ and $d_{-} / d t$, in the complex operator:

$$
\frac{\hat{d}}{d t}=\frac{1}{2}\left(\frac{d_{+}+d_{-}}{d t}\right)-\frac{i}{2}\left(\frac{d_{+}-d_{-}}{d t}\right)
$$

Applying this operator to the "position vector", a complex velocity yields

$$
\hat{V}=\frac{\hat{d} \boldsymbol{X}}{d t}=\frac{1}{2}\left(\frac{d_{+} \boldsymbol{X}+d_{-} \boldsymbol{X}}{d t}\right)-\frac{i}{2}\left(\frac{d_{+} \boldsymbol{X}-d_{-} \boldsymbol{X}}{d t}\right)=\frac{V_{+}+V_{-}}{2}-i \frac{V_{+}-V_{-}}{2}=V-i \boldsymbol{U}
$$

with:

$$
\begin{array}{ll}
V=\frac{V_{+}+V_{-}}{2} & \text { a } \\
U=\frac{V_{+}-V_{-}}{2} & \text { b }
\end{array}
$$

The real part, $V$, of the complex velocity $\hat{V}$, represents the standard classical velocity, which does not depend on resolution, while the imaginary part, $U$, is a new quantity coming from resolution dependant fractal.

\section{Covariant total derivative}

Let us now assume that curves describing particle movement (continuous but non-differentiable) are immersed in a 3-dimensional space, and that $X$ of components $X^{i}(i=\overline{1,3})$ is the position vector of a point on the curve. Let us also consider a fractal field $Q(X, t)$ and expand its total differential up to the third order:

$$
d_{ \pm} Q=\frac{\partial Q}{\partial t} d t+\nabla Q \cdot d_{ \pm} \mathbf{X}++\frac{1}{2} \frac{\partial^{2} Q}{\partial X^{i} \partial X^{j}} d_{ \pm} X^{i} d_{ \pm} X^{j}+\frac{1}{6} \frac{\partial^{3} Q}{\partial X^{i} \partial X^{j} \partial X^{k}} d_{ \pm} X^{i} d_{ \pm} X^{j} d_{ \pm} X^{k}
$$


where only the first three terms were used in Nottale's theory (i.e. second order terms in the motion equation). Relations (29) are valid in any point both for the spatial manifold and for the points $X$ on the fractal curve (selected in relations 29). Hence, the forward and backward average values of these relations take the form:

$$
\left\langle d_{ \pm} Q\right\rangle=\left\langle\frac{\partial Q}{\partial t} d t\right\rangle+\left\langle\nabla Q \cdot d_{ \pm} X\right\rangle+\frac{1}{2}\left\langle\frac{\partial^{2} Q}{\partial X^{i} \partial X^{j}} d_{ \pm} X^{i} d_{ \pm} X^{j}\right\rangle+\frac{1}{6}\left\langle\frac{\partial^{3} Q}{\partial X^{i} \partial X^{j} \partial X^{k}} d_{ \pm} X^{i} d_{ \pm} X^{j} d_{ \pm} X^{k}\right\rangle
$$

The following aspects should be mentioned: the mean value of function $f$ and its derivatives coincide with themselves and the differentials $d_{ \pm} X^{i}$ and $d t$ are independent; therefore, the average of their products coincides with the product of averages. Consequently, the equations (30) become:

$$
d_{ \pm} Q=\frac{\partial Q}{\partial t} d t+\nabla Q\left\langle d_{ \pm} X\right\rangle+\frac{1}{2} \frac{\partial^{2} Q}{\partial X^{i} \partial X^{j}}\left\langle d_{ \pm} X^{i} d_{ \pm} X^{j}\right\rangle+\frac{1}{6} \frac{\partial^{3} Q}{\partial X^{i} \partial X^{j} \partial X^{k}}\left\langle d_{ \pm} X^{i} d_{ \pm} X^{j} d_{ \pm} X^{k}\right\rangle
$$

or more, using equations (23) with characteristics (24),

$$
\begin{aligned}
& d_{ \pm} Q=\frac{\partial Q}{\partial t} d t+\nabla Q \cdot d_{ \pm} X+\frac{1}{2} \frac{\partial^{2} Q}{\partial X^{i} \partial X^{j}}\left(d_{ \pm} x^{i} d_{ \pm} x^{j}+\left\langle d_{ \pm} \xi^{i} d_{ \pm} \xi^{j}\right\rangle\right)+ \\
& \frac{1}{6} \frac{\partial^{3} Q}{\partial X^{i} \partial X^{j} \partial X^{k}}\left(d_{ \pm} x^{i} d_{ \pm} x^{j} d_{ \pm} x^{k}+\left\langle d_{ \pm} \xi^{i} d_{ \pm} \xi^{j} d_{ \pm} \xi^{k}\right\rangle\right)
\end{aligned}
$$

Even if the average value of the fractal coordinate $d_{ \pm} \xi^{i}$ is null (see 24), for higher order of fractal coordinate average, the situation can still be different. Firstly, let us focus on the averages $\left\langle d_{+} \xi^{i} d_{+} \xi^{j}\right\rangle$ and $\left\langle d_{-} \xi^{i} d_{-} \xi^{j}\right\rangle$. If $i \neq j$, these averages are zero due to the independence of $d_{ \pm} \xi^{i}$ and $d_{ \pm} \xi^{j}$. So, using (25), we can write:

$$
\left\langle d_{ \pm} \xi^{i} d_{ \pm} \xi^{j}\right\rangle=\lambda_{ \pm}^{i} \lambda_{ \pm}^{j}(d t)^{\left(2 / D_{F}\right)-1} d t
$$

Then, let us consider the averages $\left\langle d_{ \pm} \xi^{i} d_{ \pm} \xi^{j} d_{ \pm} \xi^{k}\right\rangle$. If $i \neq j \neq k$, these averages are zero due to independence of $d_{ \pm} \xi^{i}$ on $d_{ \pm} \xi^{j}$ and $d_{ \pm} \xi^{k}$. Now, using equations (25), we can write:

$$
\left\langle d_{ \pm} \xi^{i} d_{ \pm} \xi^{j} d_{ \pm} \xi^{k}\right\rangle=\lambda_{ \pm}^{i} \lambda_{+}^{j} \lambda_{ \pm}^{k}(d t)^{\left(3 / D_{F}\right)-1} d t
$$

Then, equations (32) may be written as follows: 


$$
\begin{aligned}
& d_{ \pm} Q=\frac{\partial Q}{\partial t} d t+d_{ \pm} x \cdot \nabla Q+\frac{1}{2} \frac{\partial^{2} Q}{\partial X^{i} \partial X^{j}} d_{ \pm} x^{i} d_{ \pm} x^{j}+\frac{1}{2} \frac{\partial^{2} Q}{\partial X^{i} \partial X^{j}} \lambda_{ \pm}^{i} \lambda_{ \pm}^{j}(d t)^{\left(2 / D_{F}\right)-1} d t+ \\
& \frac{1}{6} \frac{\partial^{3} Q}{\partial X^{i} \partial X^{j} \partial X^{k}} d_{ \pm} x^{i} d_{ \pm} x^{j} d_{ \pm} x^{k}+\frac{1}{6} \frac{\partial^{3} Q}{\partial X^{i} \partial X^{j} \partial X^{k}} \lambda_{ \pm}^{i} \lambda_{ \pm}^{j} \lambda_{ \pm}^{k}(d t)^{\left(3 / D_{F}\right)-1} d t
\end{aligned}
$$

If we divide by $d t$ and neglect the terms containing differential factors (for details on the method see $[9,10])$, equations $(38 \mathrm{a})$ and $(38 \mathrm{~b})$ are reduced to:

$$
\frac{d_{ \pm} Q}{d t}=\frac{\partial Q}{\partial t}+V_{ \pm} \cdot \nabla Q+\frac{1}{2} \frac{\partial^{2} Q}{\partial X^{i} \partial X^{j}} \lambda_{ \pm}^{i} \lambda_{ \pm}^{j}(d t)^{\left(2 / D_{F}\right)-1}+\frac{1}{6} \frac{\partial^{3} Q}{\partial X^{i} \partial X^{j} \partial X^{k}} \lambda_{ \pm}^{i} \lambda_{ \pm}^{j} \lambda_{ \pm}^{k}(d t)^{\left(3 / D_{F}\right)-1}
$$

These relations also allow us to define the operator:

$$
\frac{d_{ \pm}}{d t}=\frac{\partial}{\partial t}+V_{ \pm} \cdot \nabla+\frac{1}{2} \frac{\partial^{2}}{\partial X^{i} \partial X^{j}} \lambda_{ \pm}^{i} \lambda_{ \pm}^{j}(d t)^{\left(2 / D_{F}\right)-1}+\frac{1}{6} \frac{\partial^{3}}{\partial X^{i} \partial X^{j} \partial X^{k}} \lambda_{ \pm}^{i} \lambda_{ \pm}^{j} \lambda_{ \pm}^{k}(d t)^{\left(3 / D_{F}\right)-1}
$$

Under these circumstances, let us calculate $(\hat{\partial} Q / \partial t)$. Taking into account equations (26), (27) and (37), we shall obtain:

$$
\begin{aligned}
& \frac{\partial Q}{\partial t}=\frac{1}{2}\left[\frac{d_{+} Q}{d t}+\frac{d_{-} Q}{d t}-i\left(\frac{d_{+} Q}{d t}-\frac{d_{-} Q}{d t}\right)\right]= \\
& =\frac{1}{2} \frac{\partial Q}{\partial t}+\frac{1}{2} \boldsymbol{V}_{+} \cdot \nabla Q+\lambda_{+}^{i} \lambda_{+}^{j} \frac{1}{4}(d t)^{\left(2 / D_{F}\right)-1} \frac{\partial^{2} Q}{\partial X^{i} \partial X^{j}}+\lambda_{+}^{i} \lambda_{+}^{j} \lambda_{+}^{k} \frac{1}{12}(d t)^{\left(3 / D_{F}\right)-1} \frac{\partial^{3} Q}{\partial X^{i} \partial X^{j} \partial X^{k}}+ \\
& +\frac{1}{2} \frac{\partial Q}{\partial t}+\frac{1}{2} \boldsymbol{V}_{-} \cdot \nabla Q++\lambda_{-}^{i} \lambda_{-}^{j} \frac{1}{4}(d t)^{\left(2 / D_{F}\right)-1} \frac{\partial^{2} Q}{\partial X^{i} \partial X^{j}}+\lambda_{-}^{i} \lambda_{-}^{j} \lambda_{-}^{k} \frac{1}{12}(d t)^{\left(3 / D_{F}\right)-1} \frac{\partial^{3} Q}{\partial X^{i} \partial X^{j} \partial X^{k}}- \\
& -\frac{i}{2} \frac{\partial Q}{\partial t}-\frac{i}{2} \boldsymbol{V}_{+} \cdot \nabla Q-\lambda_{+}^{i} \lambda_{+}^{j} \frac{i}{2}(d t)^{\left(2 / D_{F}\right)-1} \frac{\partial^{2} Q}{\partial X^{i} \partial X^{j}}-\lambda_{+}^{i} \lambda_{+}^{j} \lambda_{+}^{k} \frac{i}{12}(d t)^{\left(3 / D_{F}\right)-1} \frac{\partial^{3} Q}{\partial X^{i} \partial X^{j} \partial X^{k}}+ \\
& +\frac{i}{2} \frac{\partial Q}{\partial t}+\frac{i}{2} V_{-} \cdot \nabla Q+\lambda_{-}^{i} \lambda_{-}^{j} \frac{i}{2}(d t)^{\left(2 / D_{F}\right)-1} \frac{\partial^{2} Q}{\partial X^{i} \partial X^{j}}+\lambda_{-}^{i} \lambda_{-}^{j} \lambda_{-}^{k} \frac{i}{12}(d t)^{\left(3 / D_{F}\right)-1} \frac{\partial^{3} Q}{\partial X^{i} \partial X^{j} \partial X^{k}}= \\
& =\frac{\partial Q}{\partial t}+\left(\frac{\boldsymbol{V}_{+}+\boldsymbol{V}_{-}}{2}-i \frac{\boldsymbol{V}_{+}-\boldsymbol{V}_{-}}{2}\right) \cdot \nabla Q+\frac{(d t)^{\left(2 / D_{F}\right)-1}}{4}\left[\left(\lambda_{+}^{i} \lambda_{+}^{j}+\lambda_{-}^{i} \lambda_{-}^{j}\right)-i\left(\lambda_{+}^{i} \lambda_{+}^{j}-\lambda_{-}^{i} \lambda_{-}^{j}\right)\right] \frac{\partial^{2} Q}{\partial X^{i} \partial X^{j}}+ \\
& +\frac{(d t)^{\left(3 / D_{F}\right)-1}}{12}\left[\left(\lambda_{+}^{i} \lambda_{+}^{j} \lambda_{+}^{k}+\lambda_{-}^{i} \lambda_{-}^{j} \lambda_{-}^{k}\right)-i\left(\lambda_{+}^{i} \lambda_{+}^{j} \lambda_{+}^{k}-\lambda_{-}^{i} \lambda_{-}^{j} \lambda_{-}^{k}\right)\right] \frac{\partial^{3} Q}{\partial X^{i} \partial X^{j} \partial X^{k}}= \\
& =\frac{\partial Q}{\partial t}+\hat{\boldsymbol{V} \cdot \nabla Q} \cdot \frac{(d t)^{\left(2 / D_{F}\right)-1}}{4}\left[\left(\lambda_{+}^{i} \lambda_{+}^{j}+\lambda_{-}^{i} \lambda_{-}^{j}\right)-i\left(\lambda_{+}^{i} \lambda_{+}^{j}-\lambda_{-}^{i} \lambda_{-}^{j}\right)\right] \frac{\partial^{2} Q}{\partial X^{i} \partial X^{j}}+ \\
& \frac{(d t)^{\left(3 / D_{F}\right)-1}}{12}\left[\left(\lambda_{+}^{i} \lambda_{+}^{j} \lambda_{+}^{k}+\lambda_{-}^{i} \lambda_{-}^{j} \lambda_{-}^{k}\right)-i\left(\lambda_{+}^{i} \lambda_{+}^{j} \lambda_{+}^{k}-\lambda_{-}^{i} \lambda_{-}^{j} \lambda_{-}^{k}\right)\right] \frac{\partial^{3} Q}{\partial X^{i} \partial X^{j} \partial X^{k}}
\end{aligned}
$$


This relation also allows us to define the fractal operator:

$$
\begin{aligned}
& \frac{\hat{\partial}}{\partial t}=\frac{\partial}{\partial t}+\hat{V} \cdot \nabla+\frac{(d t)^{\left(2 / D_{F}\right)-1}}{4}\left[\left(\lambda_{+}^{i} \lambda_{+}^{j}+\lambda_{-}^{i} \lambda_{-}^{j}\right)-i\left(\lambda_{+}^{i} \lambda_{+}^{j}-\lambda_{-}^{i} \lambda_{-}^{j}\right)\right] \frac{\partial^{2}}{\partial X^{i} \partial X^{j}}+ \\
& +\frac{(d t)^{\left(3 / D_{F}\right)-1}}{12}\left[\left(\lambda_{+}^{i} \lambda_{+}^{j} \lambda_{+}^{k}+\lambda_{-}^{i} \lambda_{-}^{j} \lambda_{-}^{k}\right)-i\left(\lambda_{+}^{i} \lambda_{+}^{j} \lambda_{+}^{k}-\lambda_{-}^{i} \lambda_{-}^{j} \lambda_{-}^{k}\right)\right] \frac{\partial^{3}}{\partial X^{i} \partial X^{j} \partial X^{k}}
\end{aligned}
$$

Particularly, by choosing:

$$
\begin{gathered}
\lambda_{+}^{i} \lambda_{+}^{j}=-\lambda_{-}^{i} \lambda_{-}^{j}=2 \mathrm{D} \delta^{\mathrm{j}} \\
\lambda_{+}^{i} \lambda_{+}^{j} \lambda_{+}^{k}=-\lambda_{-}^{i} \lambda_{-}^{j} \lambda_{+}^{k}=2 \sqrt{2} \mathrm{D}^{3 / 2} \delta^{\mathrm{jk}}
\end{gathered}
$$

the fractal operator (39) takes the usual form:

$$
\frac{\hat{\partial}}{\partial t}=\frac{\partial}{\partial t}+\hat{V} \cdot \nabla-i \mathbf{D}(d t)^{\left(2 / D_{F}\right)-1} \Delta+\frac{\sqrt{2}}{3} \mathbf{D}^{3 / 2}(d t)^{\left(3 / D_{F}\right)-1} \nabla^{3}
$$

We now apply the principle of scale covariance and postulate that the passage from classical (differentiable) to "fractal" mechanics can be implemented by replacing the standard time derivative operator, $d / d t$, with the complex operator $\partial / \partial t$ (this results in a generalization of Nottale's [1,2] principle of scale covariance). Consequently, we are now able to write the diffusion equation in its covariant form:

$$
\frac{\partial Q}{\partial t}=\frac{\partial Q}{\partial t}+(\hat{\boldsymbol{V}} \cdot \nabla) Q-i D(d t)^{\left(2 / D_{F}\right)-1} \Delta Q+\frac{\sqrt{2}}{3} D^{3 / 2}(d t)^{\left(3 / D_{F}\right)-1} \nabla^{3} Q=0
$$

This means that at any point on a fractal path, the local temporal $\partial_{t} Q$, the non-linear (convective), $(\hat{V} \cdot \nabla) Q$, the dissipative, $\Delta Q$, and the dispersive, $\nabla^{3} Q$, terms keep their balance.

\section{Fractal space-time and the motion equation of free particles in the dissipative approximation}

Newton's fundamental equation of dynamics in the dissipative approximation is: 


$$
m \frac{\hat{\partial} \boldsymbol{V}}{\partial t}=-\nabla \Phi
$$

where $m$ is the mass, $\hat{V}$ the instantaneous velocity of the particle, $\Phi$ the scalar potential and

$$
\frac{\hat{\partial}}{\partial t}=\frac{\partial}{\partial t}+(\hat{\boldsymbol{V}} \cdot \nabla)-i D(d t)^{\left(2 / D_{F}\right)-1} \Delta
$$

is the fractal operator in the dissipative approximation.

In what follows, we study what happens with equation (44), in the free particle case $(\Phi=0)$, if one considers the space-time where particles move changes from classical to nondifferentiable.

According to Nottale [11], the transition from classical (differentiable) mechanics to the scale relativistic framework is implemented by passing to a fluid-like description (the fractality of space), considering the velocity field a fractal function explicitly depending on a scale variable (the fractal geometry of each geodesic). Separating the real and imaginary parts, (44) becomes:

$$
\begin{aligned}
& \frac{\partial \mathbf{V}}{\partial t}+\mathbf{V} \cdot \nabla \mathbf{V}=0 \\
& \mathbf{U} \cdot \nabla \mathbf{V}=-\mathrm{D} \Delta \mathbf{V}
\end{aligned}
$$

where $\hat{V}=V-i U$ is the complex velocity defined through (27) and $D$ defines the amplitude of the fractal fluctuations $\left(\mathrm{D}=D(d t)^{\left(2 / D_{F}\right)-1}\right)$.

Let us analyze in what follows, the second equation (46) which, one can see, may contain some interesting physics. If we compare it with Navier-Stokes equation, from fluid mechanics [12]

$$
\frac{D v}{D t}=\frac{\partial v}{\partial t}+\boldsymbol{v} \cdot \nabla \boldsymbol{v}=v \nabla^{2} \boldsymbol{v}
$$

we can see the left side of (46) gives the rate at which $V$ is transported through a 'fluid' by means of the motion of 'fluid' particles with the velocity $U$; the right hand side gives the diffusion of $V$, ( $D$ which is the amplitude of the fractal fluctuations, plays here the role of the 'cinematic viscosity' of the 'fluid'). One can notice, in those regions in which the right hand side of (47) is negligible, $D v / D t=0$. This means that in inviscid flows, for instance, $\hat{V}$ is frozen into the 'particles of the fluid'. Physically this is due to the fact that in an inviscid 'fluid' shear stresses are zero, so that there is no mechanism by which $\hat{V}$ can be transferred from one 'fluid' particle to another. This may be the case for the transport of $\boldsymbol{V}$ by $\boldsymbol{U}$ in the second equation (II.3). 
If we consider the flow of $V$ induced by a uniform translational motion of a plane spaced a distance $Y$ above a stationary parallel plane (Fig. 1), and if the 'fluid' velocity increases from zero (at the stationary plane) to $U$ (at the moving plane) like in the case of simple Couette flow, or simple shear flow, then

$$
\text { rate of shear deformation }=\frac{d V}{d y}=\frac{U}{Y}
$$

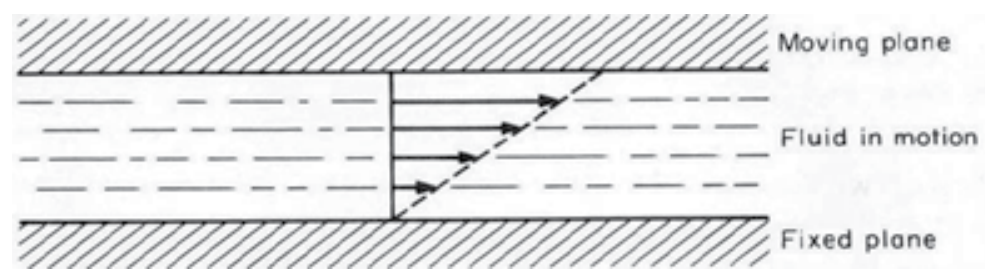

Figure 1. Uniform translational motion of a plane spaced a distance $Y$ above a stationary parallel plane.

For many fluids it is found that the magnitude of the shearing stress is related to the rate of shear proportionally:

$$
T=\eta \frac{d V}{d y}=\eta \frac{U}{Y}
$$

Fluids which obey (49) in the above situation are known as Newtonian fluids, which have a very small coefficient of viscosity. When such 'fluids' flow at reasonable velocities it is found that viscous effects appear only in thin layers on the surface of objects or surfaces over which the 'fluid' flows. That is, if one continues the analogy, and questions how is $V$ transported by the motion of 'fluid' particles with the velocity $U$, in second equation (46), one can assume that the mechanism of transfer of $V$ from one particle of 'fluid' to another is achieved over small distances (in thin layers, as stated above).

We study an important case, of the one-dimensional flow along the Ox axis :

$$
\boldsymbol{V}=\zeta(x) \boldsymbol{k}
$$

To resume, the model considered here consists in analyzing the transport of $V$, along a small elementary distance $\Lambda$, by the 'particles' of a Newtonian fluid moving with velocity $\boldsymbol{U}$, where the stress tensor obeys (49), i.e. 


$$
\frac{d \zeta(x)}{d x}=\frac{\zeta(x)}{\Lambda}
$$

like in the case of simple Couette flow, or simple shear flow.

Consequently, the second eq. (46) reduces to the scalar equation

$$
\zeta^{\prime \prime}(x)+K^{2}(x) \zeta(x)=0
$$

which is the time independent Schrödinger equation, and

$$
K^{2}(x)=\frac{1}{\Lambda D} U(x)
$$

with $\Lambda$ and $D$ having the significance of a small elementary distance and of the 'cinematic viscosity' (or amplitude of the fractal fluctuations), respectively, and $U(x)$ is the velocity of the 'Newtonian fluid', which is nothing but the imaginary part of the complex velocity [13]. In what follows, we solve this equation accurately by means of the WKBJ approximation method with connection formulas.

\subsection{Solving the Schrödinger type equation by means of the WKBJ approximation method}

Let us re-write (53) in the form

$$
K^{2}(x)=\frac{1}{\Lambda D} U(x)=\frac{2 m^{2} c}{\hbar^{2}}(\chi-\gamma(x))
$$

where we take $D=\hbar / 2 m$ and consider the small elementary distance the Compton length $\Lambda=\hbar / m c$ [14]. Therefore, the Schrödinger equation (52) splits into:

$$
\frac{d^{2}}{d x^{2}} \zeta(x)+k^{2}(x) \zeta(x)=0, \quad \chi>\gamma \text { or } \frac{d^{2}}{d x^{2}} \zeta(x)-\rho^{2}(x) \zeta(x)=0, \quad \chi<\gamma
$$

where

$$
k(x)=\sqrt{\frac{2 \mu(\chi-\gamma(x))}{\hbar^{2}}}, \rho(x)=\sqrt{\frac{2 \mu(\gamma(x)-\chi)}{\hbar^{2}}} \text { with } \mu=m^{2} c
$$

$\chi$ is a limit velocity and $\gamma(x)$ a 'velocity potential'. 
Let us try a solution of the form $\zeta(x)=A \exp ((i / \hbar) S(x))$. Substituting this solution into the timeindependent Schrödinger equation (52) we get:

$$
i \hbar \frac{d^{2} S}{d x^{2}}-\left(\frac{d S}{d x}\right)^{2}+\hbar^{2} k^{2}=0 \text { or } i \hbar \frac{d^{2} S}{d x^{2}}-\left(\frac{d S}{d x}\right)^{2}-\hbar^{2} \rho^{2}=0
$$

Assume that $h$ can, in some sense, be regarded as a small quantity and that $S(x)$ can be expanded in powers of $h, S(x)=S_{0}(x)+\hbar S_{1}(x)+\ldots$.

Then,

$$
i \hbar \frac{d}{d x}\left[\frac{d S_{0}}{d x}+\hbar \frac{d S_{1}}{d x}+\ldots\right]-\left(\frac{d S_{0}}{d x}+\hbar \frac{d S_{1}}{d x}+\ldots\right)^{2}+\hbar^{2} k^{2}=0, \quad(\chi>\gamma(x))
$$

We assume that $\left|\frac{d S_{0}}{d x}\right|>>\left|\hbar \frac{d S_{1}}{d x}\right|$ and collect terms with equal powers of $\hbar$.

$$
\begin{aligned}
& -\left[\frac{d S_{0}}{d x}\right]^{2}+\hbar^{2} k^{2}=0 \Rightarrow S_{0}= \pm \int_{x} \hbar k\left(x^{\prime}\right) d x^{\prime} \\
& i \frac{d^{2} S_{0}}{d x^{2}}-2 \frac{d S_{0}}{d x} \frac{d S_{1}}{d x}=0 \Rightarrow S_{1}=\frac{1}{2} i \ln k(x)
\end{aligned}
$$

We have used:

$$
i \frac{d}{d x}\left(\frac{d S_{0}}{d x}\right)=2 \frac{d S_{0}}{d x} \frac{d S_{1}}{d x}, \quad \mathrm{i} \frac{\mathrm{dk}}{\mathrm{dx}}=2 k \frac{d S_{1}}{d x}, \quad d S_{1}=\frac{i}{2} \frac{d k}{k}
$$

Therefore, for $\chi>\gamma(x)$

$$
\zeta(x)=A k^{-\frac{1}{2}} e^{ \pm i \int k\left(x^{\prime}\right) d x^{\prime}}
$$

In the classically allowed region $S_{0}= \pm \int_{x} \hbar k\left(x^{\prime}\right) d x^{\prime}$ counts the oscillations of the velocity wave function. An increase of $2 \pi \hbar$ corresponds to an additional phase of $2 \pi$. 
Similarly, in regions where $\chi<\gamma(x)$ we have:

$$
\zeta(x)=A \rho^{-\frac{1}{2}} e^{ \pm \int \rho\left(x^{\prime}\right) d x^{\prime}}
$$

For our first order expansion to be accurate we need that the magnitude of higher order terms decreases rapidly. We need $\left|\frac{d S_{0}}{d x}\right|>>\left|\hbar \frac{d S_{1}}{d x}\right|$ or $|k|>>\left|\frac{1}{2 k} \frac{d k}{d x}\right|$. The local deBroglie wavelength is $\lambda=2 \pi / k$. Therefore, $\left|\frac{\lambda}{4 \pi} \frac{d \lambda}{d x}\right|<<\lambda$, i.e. the change in $\lambda$ over a distance $\lambda / 4 \pi$ is small compared to $\lambda$. This holds when the velocity potential $\gamma(x)$ varies slowly and the momentum is nearly constant over several wavelengths.

Near the classical turning points the WKBJ solutions become invalid, because $k$ goes to zero here. We have to find a way to connect an oscillating solution to an exponential solution across a turning point if we want to solve barrier penetration problems or find bound states.

\subsection{Velocity potential $\gamma(x)$ and the bound states}

We want to find the velocity wave function in a given velocity potential well $\gamma(x)$. Assuming that the limit velocity of the particle is $\chi$ and that the classical turning points are $x_{1}$ and $x_{2}$, $x_{1}<x_{2}$, i.e. we have a velocity potential well with two sloping sides (Fig. 2).

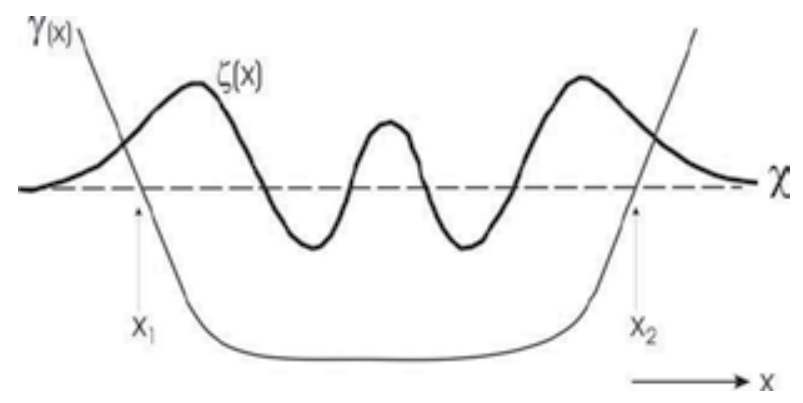

Figure 2. Bound state problem.

For $x<x_{1}$ the velocity wave function is of the form:

$$
\zeta_{1}(x)=A_{1} \rho^{-\frac{1}{2}} e^{\int^{x} \rho\left(x^{\prime}\right) d x^{\prime}}
$$

For $x>x_{2}$ the velocity wave function is of the form:

$$
\zeta_{3}(x)=A_{3} \rho^{-\frac{1}{2}} e^{-\int \rho\left(x^{\prime}\right) d x^{\prime}}
$$


In the region between $x_{1}$ and $x_{2}$ it is of the form:

$$
\zeta_{2}(x)=A_{2} k^{-\frac{1}{2}} e^{+i \int k\left(x^{\prime}\right) d x^{\prime}}+A_{2}^{\prime} k^{-\frac{1}{2}} e^{-i \int k\left(x^{\prime}\right) d x^{\prime}}
$$

At $x=x_{1}$ and $x=x_{2}$ the velocity wave function $\zeta$ and its derivatives have to be continuous. Near $x_{1}$ and $x_{2}$ we expand the velocity potential well $\gamma(x)$ in a Taylor series expansion in $x$ and neglect all terms of order higher than 1 . Near $x_{1}$ we have $\gamma(x)=\chi-K_{1}\left(x-x_{1}\right)$, and near $x_{2}$ we have $\gamma(x)=\chi+K_{2}\left(x-x_{2}\right)$.

In the neighborhood of $x_{1}$ the time-independent Schrödinger equation then becomes:

$$
\frac{d^{2} \zeta}{d x^{2}}+\frac{2 \mu K_{1}}{\hbar^{2}}\left(x-x_{1}\right) \zeta=0
$$

and in the neighborhood of $x_{2}$ the time-independent Schrödinger equation becomes:

$$
\frac{d^{2} \zeta}{d x^{2}}-\frac{2 \mu K_{2}}{\hbar^{2}}\left(x-x_{2}\right) \zeta=0
$$

Let us define $z=-\left(\frac{2 \mu K_{1}}{\hbar^{2}}\right)^{\frac{1}{3}}\left(x-x_{1}\right)$. Then we obtain $\frac{d^{2} \zeta}{d z^{2}}-z \zeta=0$ near $x_{1}$. The solutions of this equation which vanish asymptotically as $z \rightarrow \infty$ or $x \rightarrow-\infty$ are the Airy functions. They are defined through:

$$
\operatorname{Ai}(z)=\frac{1}{\pi} \int_{0}^{\infty} \cos \left(\frac{s^{3}}{3}+s z\right) d s
$$

which for large $|z|$ has the asymptotic form

$$
\operatorname{Ai}(z) \sim \frac{1}{2 \sqrt{\pi} z^{\frac{1}{4}}} \exp \left(-\frac{2}{3} z^{\frac{3}{2}}\right), \quad(z>0)
$$

and

$$
A i(z) \sim \frac{1}{\sqrt{\pi}(-z)^{\frac{1}{4}}} \sin \left(\frac{2}{3}(-z)^{\frac{3}{2}}+\frac{\pi}{4}\right), \quad(z<0)
$$


If the limit velocity $\chi$ is high enough, the linear approximation to the velocity potential well remains valid over many wavelengths. The Airy functions can therefore be the connecting velocity wave functions through the turning point at $x_{1}$.

If we define $z=\left(\frac{2 \mu K_{2}}{\hbar^{2}}\right)^{\frac{1}{3}}\left(x-x_{2}\right)$ then we find $\frac{d^{2} \zeta}{d z^{2}}-z \zeta=0$ near $x=x_{2}$ and the Airy functions can also be the connecting velocity wave functions through the turning point at $x_{2}$. Here $z \rightarrow \infty$ or $x \rightarrow \infty$.

In the neighborhood of $x_{1}$ we have

$$
k^{2}=-\rho^{2}=\left(\frac{2 \mu K_{1}}{\hbar^{2}}\right)^{\frac{1}{3}}\left(x-x_{1}\right)=-\left(\frac{2 \mu K_{1}}{\hbar^{2}}\right)^{\frac{1}{3}} z
$$

Therefore

$$
\int_{x_{1}}^{x} \rho d x^{\prime}=\left(\frac{2 \mu K_{1}}{\hbar^{2}}\right)^{\frac{1}{3}} \int_{x_{1}}^{x} \sqrt{z} d x^{\prime}=-\int_{0}^{x} \sqrt{z^{\prime}} d z^{\prime}=-\frac{2}{3} z^{\frac{3}{2}}
$$

Similarly

$$
\int_{x_{1}}^{x} k d x^{\prime}=\left(\frac{2 \mu K_{1}}{\hbar^{2}}\right)^{\frac{1}{3}} \int_{x_{1}}^{x} \sqrt{-z} d x^{\prime}=-\int_{0}^{x} \sqrt{-z^{\prime}} d z^{\prime}=\frac{2}{3}(-z)^{\frac{3}{2}}
$$

By comparing this with the asymptotic forms of the Airy functions we note that

$$
\zeta_{1}(x)=A_{1} \rho^{-\frac{1}{2}} e^{+\int_{x_{1}}^{x} \rho\left(x^{\prime}\right) d x^{\prime}} \quad\left(x<x_{1}\right)
$$

must continue on the right side as

$$
\zeta_{2}(x)=2 A_{1} k^{-\frac{1}{2}} \sin \left(\int_{x_{1}}^{x} k d x^{\prime}+\frac{\pi}{4}\right)\left(x>x_{1}\right)
$$

In the neighborhood of $x_{2}$ we similarly find that 


$$
\zeta_{3}(x)=A_{3} \rho^{-\frac{1}{2}} e^{-\int_{x_{2}}^{x} \rho\left(x^{\prime}\right) d x^{\prime}}\left(x>x_{2}\right)
$$

must continue in region 2 as

$$
\zeta_{2}(x)=2 A_{3} k^{-\frac{1}{2}} \sin \left(\int_{x}^{x_{2}} k d x^{\prime}+\frac{\pi}{4}\right)\left(x<x_{2}\right)
$$

Both expressions for $\zeta_{2}(x)$ are approximations to the same eigenfunction. We therefore need

$$
2 A_{1} k^{-\frac{1}{2}} \sin \left(\int_{x_{1}}^{x} k d x^{\prime}+\frac{\pi}{4}\right)=2 A_{3} k^{-\frac{1}{2}} \sin \left(\int_{x}^{x_{2}} k d x^{\prime}+\frac{\pi}{4}\right)
$$

For (79) to be satisfied, the amplitudes of each side must have the same magnitude, and the phases must be the same modulo $\pi$ :

$$
\begin{aligned}
& \left|A_{1}\right|=\left|A_{3}\right| \\
& \int_{x_{1}}^{x} k d x^{\prime}+\frac{\pi}{4}=-\int_{x}^{x_{2}} k d x^{\prime}-\frac{\pi}{4}+n \pi
\end{aligned}
$$

Knowing that $\int_{x_{1}}^{x_{2}}=\int_{x_{1}}^{x}+\int_{x}^{x_{2}}$, we have

$$
\int_{x_{1}}^{x_{2}} k d x^{\prime}=\left(n-\frac{1}{2}\right) \pi, \quad n=1,2,3, \ldots
$$

This can be re-written as

$$
\int_{x_{1}}^{x_{2}} \Pi d x=\left(n-\frac{1}{2}\right) \frac{h}{2} \text { or } \oint \Pi d x=\left(n-\frac{1}{2}\right) h
$$

with 


$$
\Pi=[2 \mu(\chi-\gamma(x))]^{1 / 2}=[2 \mu U(x)]^{1 / 2}=m c\left[2 \frac{U(x)}{c}\right]^{1 / 2}
$$

Here $\oint$ denote an integral over one complete cycle of the classical motion. The WKBJ method for $\gamma(x)$ velocity potential well with soft walls, therefore, leads to a Wilson-Sommerfeld type quantization rule except that $n$ is replaced by $n-1 / 2$. It leads to a quantization of the complex velocity $U(x)$.

The factor of $\pi / 2$ arises here due to the two phase changes of $\pi / 4$ at $x_{1}$ and $x_{2}$. In case where only one of the walls is soft and the other is infinitely steep the factor of $1 / 2$ is replaced by $1 / 4$ in (81). If both walls are infinitely steep, the factor of $1 / 2$ in (81) is replaced by 0 .

WKBJ approximation is a semi classical approximation, since it is expected to be most useful in the nearly classical limit of large quantum numbers. The method will not be good for, say, lowest limit velocity states $\chi$, so in order to overcome this shortcomings there is a need for a modified semi classical quantization condition. For oscillations between the two classical turning points $x_{1}$ and $x_{2}$, we obtain the semi classical quantization condition by requiring that the total phase during one period of oscillation to be an integral multiple of $2 \pi$; [15] such that

$$
2 \int_{x_{1}}^{x_{2}} k d x^{\prime}+\phi_{1}+\phi_{2}=2 \pi n
$$

where $\phi_{1}$ is the phase loss due to reflection at the classical turning point $x_{1}$ and $\phi_{2}$ is the phase loss due to reflection at $x_{2}$. Taking $\phi_{1}$ and $\phi_{2}$ to be equal to $\pi / 2$ leads to the modified semiclassical quantization rule, i.e.

$$
\int_{x_{1}}^{x_{2}} k d x^{\prime}=\left(n-\frac{m}{4}\right) \pi
$$

where $m$ is the Maslov index [15], which denotes the total phase loss during one period in units of $\pi / 2$. It contains contributions from the phase losses $\phi_{1}$ and $\phi_{2}$ due to reflections at points $x_{1}$ and $x_{2}$, respectively. It is pertinent to note that taking $\phi_{1}=\phi_{2}=\pi / 2$ and an integer Maslov index $m=2$ in (85), we have the familiar semi classical quantization rule, i.e. (81).

Let us apply the constraint equation (81) to an harmonic oscillator. The condition then is (passing without loss of generality to the limits $-a$ to $+a$ ) 


$$
\int_{-a}^{+a}[2 \mu(\chi-\omega x)]^{1 / 2} d x=\left(n-\frac{1}{2}\right) \pi \hbar
$$

where the energy $W_{c}$ of the oscillator $U(x)$ with the pulsation $\omega$ writes

$$
W_{c}=\frac{1}{2} m \omega^{2} x^{2}=\frac{1}{2} m \gamma^{2}(x)
$$

and we get the expression for the $x$ dependence of the velocity term, $\gamma(x)=\omega x$.

Theleft side term of (86) is an elementary integral and we find:

$$
(\chi+v)^{3 / 2}-(\chi-v)^{3 / 2}=\frac{3 \pi}{2 a(2 \mu)^{1 / 2}}\left(n-\frac{1}{2}\right) \hbar v
$$

where $v=\omega a$ is the liniar velocity (see the graphic in Fig. 3).

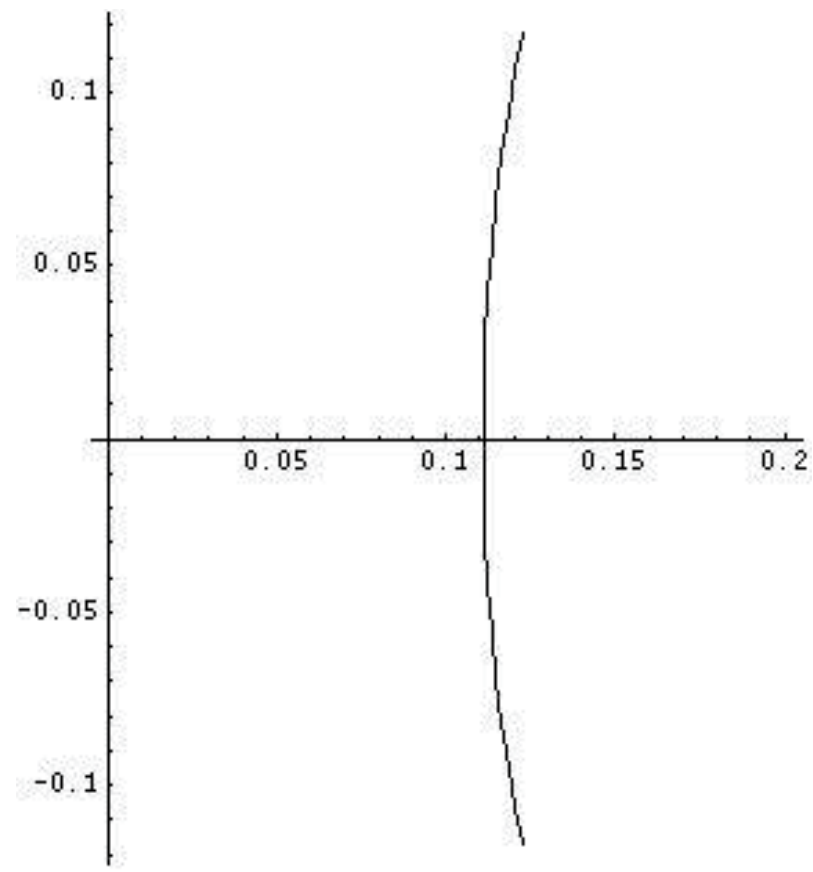

Figure 3. Dependence of the limit velocity $x$ on the linear velocity $u$. 
We try to estimate a value for the limit velocity $\chi$. Let us expand the left side term of (88) in series and keep the first term. If we replace $\mu$ from (56) and take $a=\Lambda$ (the Compton length), we get:

$$
\chi \approx \frac{\pi^{2} \hbar^{2}}{8 \mu a^{2}}\left(n-\frac{1}{2}\right)^{2}=\frac{\pi^{2}}{32}(2 n-1)^{2} c=\left\{\frac{\pi^{2}}{32} c, 9 \frac{\pi^{2}}{32} c, 25 \frac{\pi^{2}}{32} c, \ldots\right\}
$$

It is interesting to note that only the first velocity in (89) is less than the velocity of light, $c$.

Let us analyze now, one more bound state, the velocity wave function in a given velocity double well potential $\gamma(x)$.

We begin by deriving a quantization condition for region 2 analogous to (81). Again, applying the boundary condition for region 1 leaves only the exponentially growing solution. Applying the connection formula at $x_{1}$ then gives an expression for the velocity wave function in region 2:

$$
\zeta_{1}(x)=2 A k^{-1 / 2} \sin \left[\int_{x_{1}}^{x} k d x^{\prime}+\frac{\pi}{4}\right]
$$

However, the solution in region 3 must have both growing and decaying solutions present. Considering the region 3 solutions in terms of $x_{2}$ and letting $B_{L}$ and $C_{L}$ be the amplitudes of the decaying and growing solutions respectively, the connection formulas give another expression for the velocity wave function in region 2 :

$$
\zeta_{2}(x)=2 B_{L} k^{-1 / 2} \cos \theta+C_{L} k^{-1 / 2} \sin \theta
$$

with

$$
\theta=\int_{x}^{x_{2}} k d x^{\prime}-\frac{\pi}{4}
$$

We equate the two expressions (90), (91) for the velocity function in region 2 and cancel common factors giving

$$
2 A \sin \left[\int_{x_{1}}^{x} k d x^{\prime}+\frac{\pi}{4}\right]=2 B_{L} \cos \theta+C_{L} \sin \theta
$$


Using trigonometric identities to simplify the right hand side, gives

$$
2 A \sin \left[\int_{x_{1}}^{x} k d x^{\prime}+\frac{\pi}{4}\right]=\left(4 B_{L}^{2}+C_{L}^{2}\right)^{1 / 2} \sin \left(\theta+\frac{\pi}{2}-\phi_{L}\right)
$$

where

$$
\phi_{L}=\cos ^{-1}\left[\frac{2 B_{L}}{\left(4 B_{L}^{2}+C_{L}^{2}\right)^{1 / 2}}\right]
$$

The magnitude of the sin function must be equal, and the magnitude of the phases must be equal modulo $\pi$ :

$$
\begin{gathered}
4 A^{2}=4 B_{L}^{2}+C_{L}^{2} \\
\int_{x_{1}}^{x} k d x^{\prime}+\frac{\pi}{4}=-\int_{x}^{x_{2}} k d x^{\prime}-\frac{\pi}{4}+\phi_{L}+n \pi
\end{gathered}
$$

Simplifying and combining the integrals gives the quantization condition for region 2:

$$
\theta_{12} \equiv \int_{x_{1}}^{x_{2}} k d x=\pi\left(n-\frac{1}{2}\right)+\phi_{L}
$$

with $n=1,2, \ldots$.

A similar treatment for the turning point $x_{3}$ yields the condition for region 4 :

$$
\theta_{34} \equiv \int_{x_{3}}^{x_{4}} k d x=\pi\left(m-\frac{1}{2}\right)+\phi_{R}
$$

with $m=1,2, \ldots$ and $\phi_{R}$ given by:

$$
\phi_{R}=\cos ^{-1}\left[\frac{2 C_{R}}{\left(4 C_{R}^{2}+B_{R}^{2}\right)^{1 / 2}}\right]
$$


where $B_{R}$ and $C_{R}$ are the amplitudes of the decaying and growing region 3 solutions in terms of $x_{3}$.

We now have the quantization conditions $(98,99)$ for regions 2 and 4 , but they contain the free parameters $\phi_{L}$ and $\phi_{R}$. To eliminate these free parameters, we consider the WKBJ solution in region 3. The coefficients $B_{L}, C_{L}, B_{R}, C_{R}$ define two expressions for solution, which must be equal:

$$
\begin{aligned}
& \zeta_{3}=B_{L} \rho^{-1 / 2} \exp \left[-\int_{x_{2}}^{x} \rho d x^{\prime}\right]+C_{L} \rho^{-1 / 2} \exp \left[\int_{x_{2}}^{x} \rho d x^{\prime}\right] \\
& \zeta_{3}=B_{R} \rho^{-1 / 2} \exp \left[\int_{x}^{x_{3}} \rho d x^{\prime}\right]+C_{R} \rho^{-1 / 2} \exp \left[-\int_{x}^{x_{3}} \rho d x^{\prime}\right]
\end{aligned}
$$

Equations (101) and (102) each contain a term that grows exponentially with $x$ and a term that decays exponentially with $x$. Equating the growing terms from each equation and the decaying term from each equation gives two constraints:

$$
\begin{aligned}
& B_{L} \exp \left[-\int_{x_{2}}^{x} \rho d x^{\prime}\right]=B_{R} \exp \left[\int_{x}^{x_{3}} \rho d x^{\prime}\right] \\
& C_{L} \exp \left[\int_{x_{2}}^{x} \rho d x^{\prime}\right]=C_{R} \exp \left[-\int_{x}^{x_{3}} \rho d x^{\prime}\right]
\end{aligned}
$$

Combining the integrals in these constraints gives

$$
\frac{B_{L}}{B_{R}}=\frac{C_{R}}{C_{L}}=\exp \left(\theta_{23}\right)
$$

with

$$
\theta_{23} \equiv \int_{x_{2}}^{x_{3}} \rho d x^{\prime}
$$


The constraints $(98,99,105)$ may be combined to give a single quantization condition for the allowed WKBJ velocity limits $\chi$ for a double-well velocity potential $\gamma(x)$. Applying trigonometric identities to (95) and (100), and plugging into (105) gives

$$
\tan \phi_{L} \tan \phi_{R}=\left(\frac{C_{L}}{2 B_{L}}\right)\left(\frac{B_{R}}{2 C_{R}}\right)=\frac{1}{4} \exp \left(-2 \theta_{23}\right)
$$

Equation (107) may be combined with (98) and (99) to give the WKBJ quantization condition for a double-well potential in terms of the phase integrals $\theta_{12}$ and $\theta_{34}$ :

$$
\operatorname{ctg} \theta_{12} \operatorname{ctg} \theta_{34}=\frac{1}{4} \exp \left(-2 \theta_{23}\right)
$$

confirming the results given in [16].

Equation (108) is a nonlinear constraint approximately determining the allowed velocity levels $\chi$ of a double-well velocity potential $\gamma(x)$ (see Fig. 4) and can be written (taking $\phi_{R}=\phi_{L}=\pi / 4$ in (98) and (99), i.e. the velocity quarter-wave shift in the connection formulas, which is known to optimize the tunneling effect between two oscillating waves [17] ) as :

$$
\frac{1}{\hbar} \int_{x_{2}}^{x_{3}}[2 \mu(\gamma(x)-\chi)]^{1 / 2} d x=\ln \left\{4 \cdot \operatorname{ctg}\left[\pi\left(n-\frac{1}{2}\right)+\phi_{L}\right] \cdot \operatorname{ctg}\left[\pi\left(m-\frac{1}{2}\right)+\phi_{R}\right]\right\}^{-1 / 2}=-\ln 2 \quad \mathrm{~m}, \mathrm{n}=1,2,3, \ldots
$$

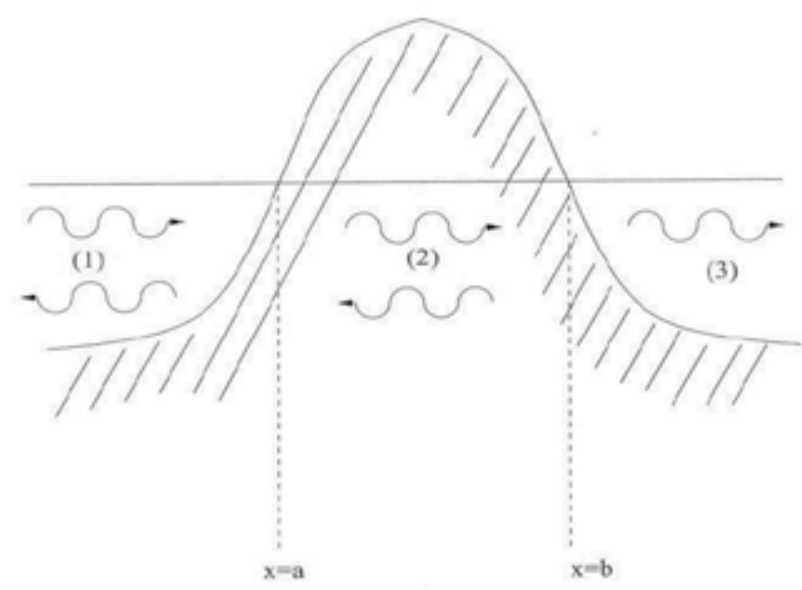

Figure 4. Tunneling potential barrier.

In terms of the momentum $\Pi$ we have : 


$$
\int_{x_{2}}^{x_{3}} \Pi d x=-\hbar \ln 2 \text { or } \oint \Pi d x=-2 \hbar \ln 2
$$

where $\oint$ denotes an integral over one complete cycle of the classical motion, this time

$$
\Pi=[2 \mu(\gamma(x)-\chi)]^{1 / 2}=[2 \mu U(x)]^{1 / 2}=m c\left[2 \frac{U(x)}{c}\right]^{1 / 2}
$$

since $\gamma(x)>\chi$ for the integration limits, i.e. region 3 (see Fig. 5). We get again a quantization of the complex velocity $U(x)$, where the levels are equally spaced at a value of $\hbar \ln 2$.

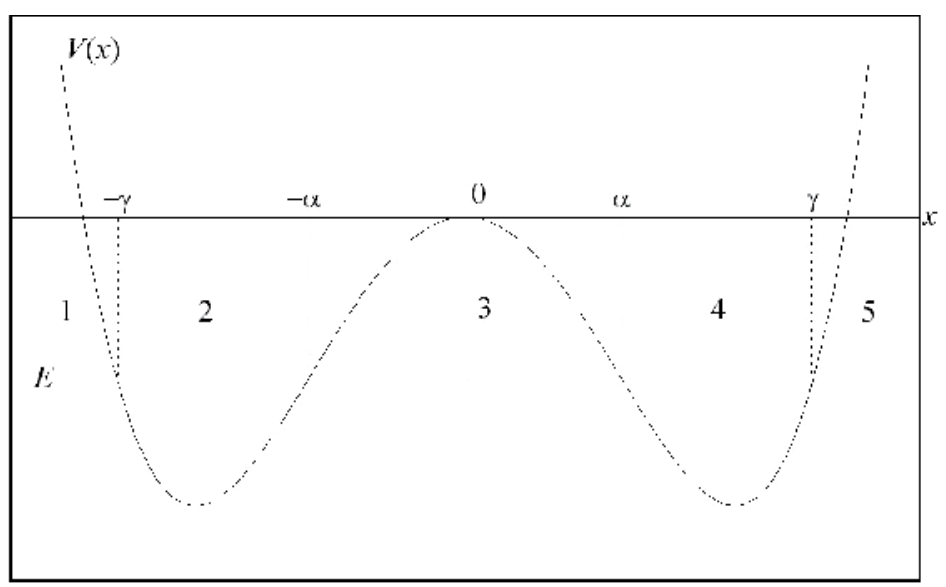

Figure 5. Schematic diagram of a double-well potential with three forbidden regions $(1,3,5)$ and two allowed regions $(2,4)$.

In 1961, Landauer [18] discussed the limitation of the efficiency of a computer imposed by physical laws. In particular he argued that, according to the second law of thermodynamics, the erasure of one bit of information requires a minimal heat generation $k_{B} T \ln 2$, where $k_{B}$ is Boltzmann's constant and $T$ is the temperature at which one erases. Its argument runs as follows. Since erasure is a logical function that does not have a single-valued inverse it must be associated with physical irreversibility and therefore requires heat dissipation. A bit has one degree of freedom and so the heat dissipation should be of order $k_{B} T$. Now, since before erasure a bit can be in any of the two possible states and after erasure it can only be in one state, this implies a change in information entropy of an amount $-k_{B} \ln 2$.

The one-to-one dynamics of Hamiltonian systems [19] implies that when a bit is erased the information which it contains has to go somewhere. If the information goes into observable 
degrees of freedom of the computer, such as another bit, then it has not been erased but merely moved; but if it goes into unobservable degrees of freedom such as the microscopic motion of molecules it results in an increase of entropy of at least $k_{B} \ln 2$.

Inspired by such studies, a considerable amount of work has been made on the thermodynamics of information processing, which include Maxwell's demon problem [20], reversible computation [21], the proposal of the algorithmic entropy [22] and so on.

Here, considering a double-well velocity potential $\gamma(x)$ and the velocity quarter-wave shift in the connection formulas, a quanta of $\hbar \ln 2$ for the complex velocity $U(x)$ of the moving Newtonian 'fluid' occurs. It can be argued that it can be put into a one-to-one correspondence to the quanta of information Landauer and other authors discussed about [23, 24].

Furthermore, one gets an interesting result when taking $\phi_{R}=\phi_{L}=\pi / 2$, i.e. the velocity halfwave shift in the connection formulas, when singularities occur in (II.66). We try to solve this case by making use of the vortices theory. Benard in 1908 was the first to investigate the appearance of vortices behind a body moving in a fluid [12]. The body he used was a cylinder. He observed that at a high enough fluid velocity (or Reynolds number based on the cylinder diameter), which depends on the viscosity and width of the body, vortices start to shed behind the cylinder, alternatively from the top and the bottom of the cylinder.

Consequently, we write (109) in the form

$$
\int_{x}[2 \mu(\gamma(x)-\chi)]^{1 / 2} d x=-\hbar \ln 2-\hbar \ln \left\{\operatorname{tg}\left[\frac{\pi}{l}\left(x-x_{0}\right)\right]\right\}
$$

where we use $\operatorname{ctg}(\alpha+\pi / 2)=-\operatorname{tg}(\alpha)$, take $\mathrm{m}=\mathrm{n}$, make the notations $x=n l, \quad x_{0}=l / 2$ and consider again the one-dimensional case, motion along the Ox axis.

Solving (112) one gets

$$
U(x)=c\left(1+\operatorname{ctg}^{2}\left[\frac{2 \pi}{\Lambda}\left(x-x_{0}\right)\right]\right)
$$

where we assume $l=\Lambda$ (the Compton length), $U(x)=[\gamma(x)-\chi] / 2 \pi^{2}$ and replace $\mu=m^{2} c$, where $c$ is the velocity of light. When plotting (113) (see Fig. 6) we see that indeed, singularities are obtained for $x-x_{0}=\Lambda / 2$ and for $x-x_{0}=\Lambda / 4$ we get for $U(x)$ minima of value the velocity of light, $c$. Usually, at some distance behind a body placed in a fluid, vortices are arranged at a definite distance $l$ apart and with a definite separation $h$ between the two rows. The senses of the rotation in the two rows are opposite (see Fig. 7). 


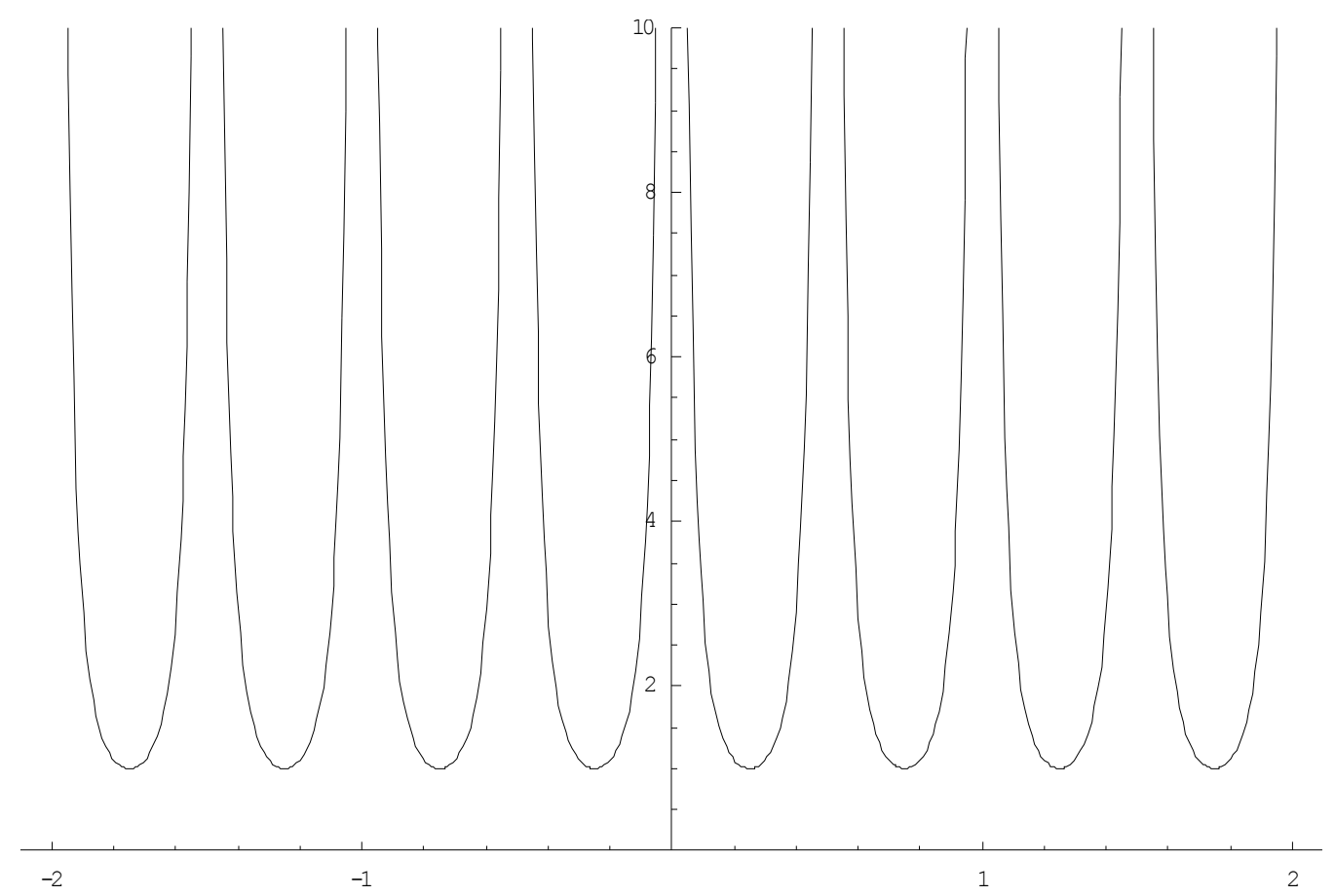

Figure 6. The complex velocity $U(x)$ singularities' distribution along the Ox axis.

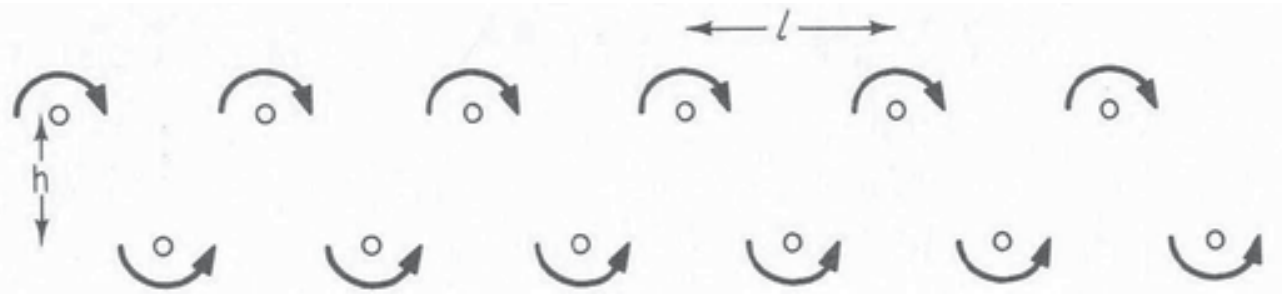

Figure 7. Von Karman vortex streets.

In 1912 von Karman expounded a theory of such vortex streets and the drag which a cylinder would experience due to their formation [12]. Since we considered here the one-dimensional case, we get the solution of a single row of rectilinear vortices, which has already been referred to as characterizing a surface of discontinuity (see Fig. 8).

A typical bound state in a double-well velocity potential has two classically allowed regions, where the velocity potential $\gamma(x)$ is less than the limit velocity $\chi$. These regions are separated by a classically forbidden region, or barrier, where the velocity potential is larger than the limit velocity. As we can see, quantum mechanics predicts that a velocity wave $\zeta(x)$ travelling in such a potential is most likely to be found in the allowed regions. However, unlike classical mechanics, quantum mechanics predicts that this velocity wave can also be found in the 


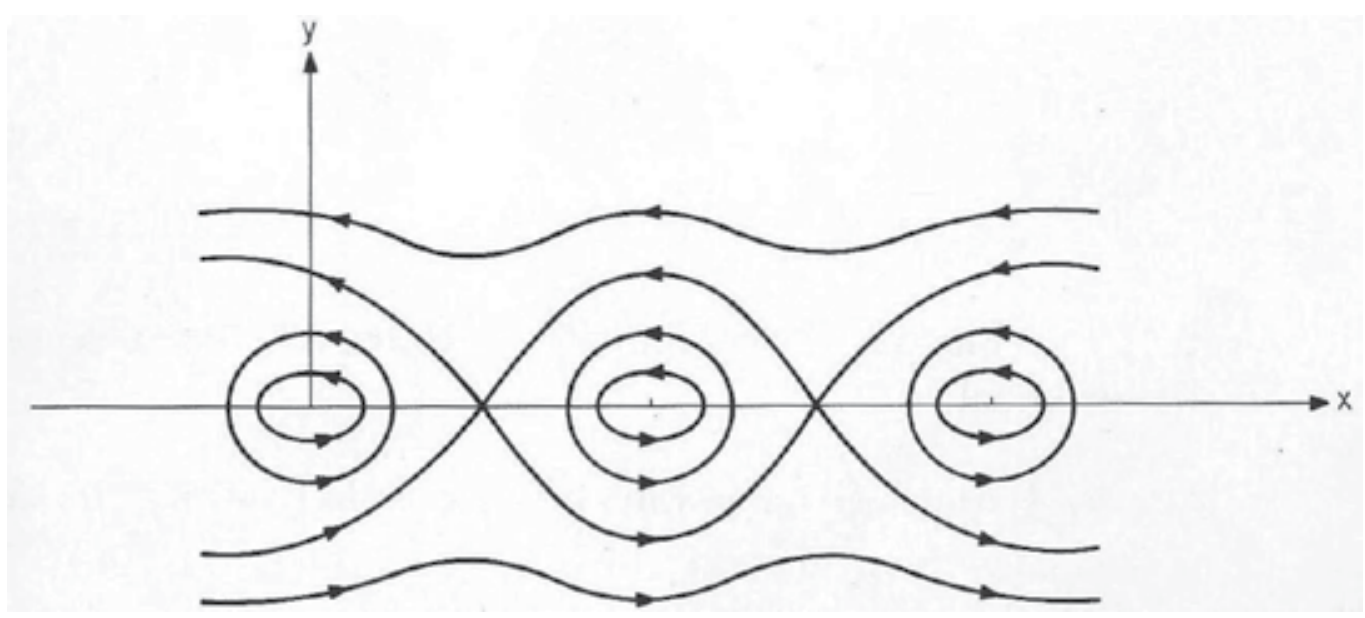

Figure 8. Single row of rectilinear vortices.

forbidden region. This uniquely quantum mechanical behavior allows a velocity wave, initially localized in one potential well, to penetrate through the barrier, into the other well (as we will see in what follows).

\subsection{Velocity potential $\gamma(x)$ and the quantum barrier}

We already know at the points where $\chi-\gamma(x)=0$, special treatment is required because $k$ is singular. The way of handling the solution near the turning point is a little bit more technical, but the basic idea is that we have a solution to the left and to the right of the turning point, and one needs a formula that interpolates between them. In other words, in the vicinity of the turning point one approximates $\sqrt{2 \mu(\chi-\gamma(x)) / \hbar^{2}}$ by a straight line over a small interval and solves TISE (time independent Schrodinger equation) exactly. This leads to the following connection formulas:

Barrier to the right $(\mathrm{x}=\mathrm{b}$ turning point $)$

$$
\begin{aligned}
& \frac{2}{\sqrt{k}} \cos \left[\int_{x}^{b} k(x) d x-\frac{\pi}{4}\right] \Leftrightarrow \frac{1}{\sqrt{\rho}} e^{-\int_{b}^{x} \rho(x) d x} \\
& \frac{1}{\sqrt{k}} \sin \left[\int_{x}^{b} k(x) d x-\frac{\pi}{4}\right] \Leftrightarrow-\frac{1}{\sqrt{\rho}} e^{\int^{b} \rho(x) d x}
\end{aligned}
$$

Barrier to the left ( $x=$ a turning point $)$ 


$$
\begin{aligned}
& \frac{2}{\sqrt{k}} \cos \left[\int_{a}^{x} k(x) d x-\frac{\pi}{4}\right] \Leftrightarrow \frac{1}{\sqrt{\rho}} e^{-\int^{a} \rho(x) d x} \\
& \frac{1}{\sqrt{k}} \sin \left[\int_{a}^{x} k(x) d x-\frac{\pi}{4}\right] \Leftrightarrow-\frac{1}{\sqrt{\rho}} e^{\int^{x} \rho(x) d x}
\end{aligned}
$$

The connection formulas enable us to obtain relationships between the solutions in a region at some distance to the right of the turning point with those in a region at some distance to the left [25-27].

One of the most important problems to which connection formulas apply is that of the penetration of a potential barrier. The barrier is shown in Fig. 4 and the limit velocity $\chi$ is such that the turning points are at $x=a$ and $x=b$.

Suppose that the motion is incident from the left. Some waves will be reflected and some transmitted, so that in region III we will have:

$$
\zeta_{3}(x)=\frac{1}{\sqrt{k}} e^{i \int^{x} k d x-i \frac{\pi}{4}}=\frac{1}{\sqrt{k}} \cos \left[\int_{b}^{x} k d x-\frac{\pi}{4}\right]+\frac{i}{\sqrt{k}} \sin \left[\int_{b}^{x} k d x-\frac{\pi}{4}\right]
$$

The phase factor is included for convenience of applying the connection formulas.

In region II (using (114) and (115) on (118)) we have:

$$
\zeta_{2}(x)=\frac{1}{2} \frac{1}{\sqrt{\rho}} e^{-\int^{b} \rho d x}-i \frac{1}{\sqrt{\rho}} e^{\int^{x} \rho d x}
$$

Now using

$$
\int_{x}^{b} \rho d x=\int_{x}^{a} \rho d x+\int_{a}^{b} \rho d x=-\int_{a}^{x} \rho d x+\alpha
$$

we can write

$$
\zeta_{2}(x)=\frac{1}{2} \frac{1}{\sqrt{\rho}} e^{\int^{x} \rho d x} e^{-\alpha}-i \frac{1}{\sqrt{\rho}} e^{-\int_{a}^{x} \rho d x} e^{\alpha}
$$


Again, using the connection formulas for the case barrier to the right (using (116) and (117) on (121)), we get for region I:

$$
\begin{aligned}
\zeta_{1}(x)= & \frac{1}{2} e^{-\alpha} \frac{1}{\sqrt{k}} \sin \left[\int_{x}^{a} k d x-\frac{\pi}{4}\right]-i \frac{2}{\sqrt{k}} e^{\alpha} \cos \left[\int_{x}^{a} k d x-\frac{\pi}{4}\right]= \\
= & \frac{1}{2} \frac{1}{\sqrt{k}} e^{-\alpha} \sin (u)-i \frac{2}{\sqrt{k}} e^{\alpha} \cos (u)= \\
= & -\frac{i}{\sqrt{k}}\left[e^{i u}\left(e^{\alpha}+\frac{1}{4} e^{-\alpha}\right)+e^{-i u}\left(e^{\alpha}-\frac{1}{4} e^{-\alpha}\right)\right]
\end{aligned}
$$

Hence

$$
\left\{\begin{array}{l}
\zeta_{1}^{i n c}(x)=-\frac{i}{\sqrt{k}}\left(e^{\alpha}+\frac{1}{4} e^{-\alpha}\right) e^{i \int^{a} k d x-i \frac{\pi}{4}} \\
\zeta_{1}^{r e f}(x)=-\frac{i}{\sqrt{k}}\left(e^{\alpha}-\frac{1}{4} e^{-\alpha}\right) e^{-i \int^{a} k d x+i \frac{\pi}{4}}
\end{array}\right.
$$

Having obtained the expression for $\zeta_{1}^{\text {inc }}(x)$ and $\zeta_{1}^{\text {ref }}(x)$ we are now in position to calculate the transmission coefficient using:

$$
T=\left|\frac{\zeta_{3}(x)}{\zeta_{1}^{i n c}(x)}\right|^{2}=\frac{e^{-2 \alpha}}{\left(1+\frac{1}{4} e^{-2 \alpha}\right)^{2}}
$$

To summarize, for a barrier with large attenuation $e^{-2 \alpha} \rightarrow 0$, the tunneling probability equals

$$
T=\frac{e^{-2 \alpha}}{\left(1+\frac{1}{4} e^{-2 \alpha}\right)^{2}} \approx e^{-2 \alpha}=\exp \left(-\frac{2}{\hbar} \int_{a}^{b} \rho d x\right)=\exp \left(-\frac{2}{\hbar} \int_{a}^{b}[2 \mu(\gamma(x)-\chi)]^{1 / 2} d x\right)
$$

The reflection coefficient is:

$$
R=\left|\frac{\zeta_{1}^{r e f}(x)}{\zeta_{1}^{\text {inc }}(x)}\right|^{2} \cong \frac{\left(e^{\alpha}-\frac{1}{4} e^{-\alpha}\right)^{2}}{\left(e^{\alpha}+\frac{1}{4} e^{-\alpha}\right)^{2}}, \quad T+R=1
$$


and also in the same large attenuation limit, we have:

$$
R \approx 1-e^{-2 \alpha}=1-\exp \left(-\frac{2}{\hbar} \int_{a}^{b} \rho d x\right)=1-\exp \left(-\frac{2}{\hbar} \int_{a}^{b}[2 \mu(\gamma(x)-\chi)]^{1 / 2} d x\right)
$$

One can see from (125) and (127) thatthe velocity wave $\zeta(x)$ on small distances, with the same order of magnitude as $\Lambda$, may be influenced by $U(x)$,i.e. it can be transmitted, attenuated or reflected at this scale length. In other words, we get from the calculus, that the velocity field $V$ is indeed transported by the motion of the 'Newtonian fluid' particles with the velocity $U(x)$ (the imaginary part of the complex velocity [13]).

\section{Casimir type effect in scale relativity theory}

In recent years, new and exciting advances in experimental techniques [28] prompted a great revival of interest in the Casimir effect, over fifty years after its theoretical discovery (for a recent review on both theoretical and experimental aspects of the Casimir effect, see Refs. [29-31]). As is well known, this phenomenon is a manifestation of the zero-point fluctuations of the electromagnetic field: it is a purely quantum effect and it constitutes one of the rare instances of quantum phenomena on a macroscopic scale.

In his famous paper, Casimir evaluated the force between two parallel, electrically neutral, perfectly reflecting plane mirrors, placed a distance $L$ apart, and found it to be attractive and of a magnitude equal to:

$$
F_{C}=\frac{\hbar c \pi^{2} A}{240 L^{4}}
$$

Here, $A$ is the area of the mirrors, which is supposed to be much larger than $L^{2}$, so that edge effects become negligible. The associated energy $E_{C}$

$$
E_{C}=-\frac{\hbar c \pi^{2} A}{720 L^{3}}
$$

can be interpreted as representing the shift in the zero-point energy of the electromagnetic field, between the mirrors, when they are adiabatically moved towards each other starting from an infinite distance. The Casimir force is indeed the dominant interaction between neutral bodies at the micrometer or submicrometer scales, and by modern experimental techniques it has now been measured with an accuracy of a few percent (see [28] and references therein). 
Since this effect arises from long-range correlations between the dipole moments of the atoms forming the walls of the cavity, that are induced by coupling with the fluctuating electromagnetic field, the Casimir energy depends in general on the geometric features of the cavity. For example, we see from (129) that, in the simple case of two parallel slabs, the Casimir energy $E_{C}$ is negative and is not proportional to the volume of the cavity, as would be the case for an extensive quantity, but actually depends separately on the area and distance of the slabs. Indeed, the dependence of $E_{C}$ on the geometry of the cavity can reach the point where it turns from negative to positive, leading to repulsive forces on the walls. For example [29], in the case of a cavity with the shape of a parallelepiped, the sign of $E_{C}$ depends on the ratios among the sides, while in the case of a sphere it has long been thought to be positive. It is difficult to give a simple intuitive explanation of these shape effects, as they hinge on a delicate process of renormalization, in which the finite final value of the Casimir energy is typically expressed as a difference among infinite positive quantities. In fact, there exists a debate, in the current literature, whether some of these results are true or false, being artifacts resulting from an oversimplification in the treatment of the walls [33].

There are three well-known technical types of derivation of the Casimir force for different geometries including the simplest geometry of two parallel, uncharged, perfectly conducting plates firstly explored by Casimir. One modern method is the quantum field theoretical approach based on the appropriate Green's function of the geometry of problem [34]. The other technical type is the dimensional regularization method that involves the mathematical complications of the Riemann zeta function and the analytical continuation [34]. The last (the most elementary/the simplest) method is based on modes summation by using the EulerMaclurian integral formula [35-37].

The problem of finding the Casimir force, not only for the simplest geometry of two plates or rectangular prism, that we want to study here, but also for other more complicated geometries, indispensably/automatically involves some infinities/irregularities; thus, one should regularize the calculation for arriving at the desired finite physical result(s). In the Green' function method, one uses the subtraction of two terms (two Green's functions) to do the required regularization. In the dimensional regularization method, although there isn't an explicit subtraction for the regularization of the problem, as is clear from its name, the calculation is regularized dimensionally by going to a complex plane with a mathematically complicated/ ambiguous approach. In the simplest method in which the Euler-Maclurian formula is used, the regularization is performed by the subtraction of the zero-point energy of the free space (no plates) from the energy expression under consideration/calculation (e.g. summation of the interior and exterior zero-point energies of the two parallel plates).

Navier-Stokes equations in scale relativity theory predict that the (vector) velocity field $V$ and/ or the (scalar) density field $\rho$, on small distances (the same magnitude as the Compton length) behave like a wave function and are transported by the motion of the Newtonian fluid with velocity $U$.

Furthermore, when considering vacuum from the Casimir cavity, a non-differentiable, Newtonian, $2 D$ non-coherent quantum fluid whose entities (cvasi-particles) assimilated to vortex-type objects, initially non-coherent, become coherent (the coherence of the quantum 
fluid reduces to its ordering in vortex streets) due to the constraints induced by the presence of slabs. Casimir type forces are derived which are in good agreement with other theoretical results and experimental data, for both cases: two metallic slabs, parallel to each other, placed at a distance $d$ apart, that constitute the plates of the cavity and a rectangle of sides $d_{1}, d$.

In other words, non-differentiability and coherence of the quantum fluid due to constraints generate pressure along the $O x$ and $O y$ axis.

For viscous compressible fluids, Navier-Stokes equations

$$
\rho \frac{D \boldsymbol{v}}{D t}=\rho \boldsymbol{X}-\nabla p+\mu \nabla^{2} \boldsymbol{v}+\frac{\mu}{3} \nabla(\nabla \cdot \boldsymbol{v})
$$

together with the equation of continuity

$$
\frac{D \rho}{D t}+\rho \nabla \cdot v=0
$$

where $\rho$ is the density, $\boldsymbol{v}$ the velocity of the fluid, $X$ the body force, $p$ the pressure, $\mu$ the shear viscosity and $D / D t \equiv d / d t+v \cdot \nabla$ the Eulerian derivative, apply to Newtonian (or near) fluids, that is, to fluids in which the stress is linearly related to the rate of strain (as will be assumed further in this section) [12].

Let us see first, what happens with the set of equations (130) and (131), if one considers that the space-time, where particles move, changes from classical to non-differentiable.

We already know, according to Nottale [11], that a transition from classical (differentiable) mechanics to the scale relativistic framework is implemented by passing to a fluid-like description (the fractality of space), considering the velocity field a fractal function explicitly depending on a scale variable (the fractal geometry of each geodesic) and defining two fractal velocity fields which are fractal functions of the scale variable $d t$ (the non-differentiability of space).

Consequently, replacing $d / d t$ with the fractal operator (42) and solving for both real and imaginary parts, (130) and (131) become, in a stationary isotropic case, taking the body force $X=0$ (constant gravitational field) and $\nabla \boldsymbol{U}=0$ (assuming a constant density of states for the "fluid particles" moving with the velocity $\boldsymbol{U}$ - see further in this section):

$$
\begin{aligned}
& \boldsymbol{V} \cdot \nabla \boldsymbol{V}=-\frac{\nabla p}{\rho}+v \nabla^{2} \boldsymbol{V} \\
& \boldsymbol{U} \cdot \nabla \boldsymbol{V}+D \nabla^{2} \boldsymbol{V}=0
\end{aligned}
$$

and 


$$
\begin{array}{ll}
\boldsymbol{V} \cdot \nabla \rho+\rho \nabla \cdot \boldsymbol{V}=0 & \mathrm{a} \\
\boldsymbol{U} \cdot \nabla \rho+D \nabla^{2} \rho=0 & \mathrm{~b}
\end{array}
$$

where $V$ represents the standard classical velocity, which does not depend on resolution, while the imaginary part, $U$, is a new quantity coming from resolution dependant fractal, $v=$ $\mu / \rho$ the kinematic viscosity and $D=\hbar / 2 m$ defines the amplitude of the fractal fluctuations.

The causes of the Casimir effect are described by quantum field theory, which states that all of the various fundamental fields, such as the electromagnetic field, must be quantized at each and every point in space. In a simplified view, a "field" in physics may be envisioned as if space were filled with interconnected vibrating balls and springs, and the strength of the field can be visualized as the displacement of a ball from its rest position. Vibrations in this field propagate and are governed by the appropriate wave equation for the particular field in question. The second quantization of quantum field theory requires that each such ballspring combination to be quantized, that is, that the strength of the field to be quantized at each point in space. Canonically, the field at each point in space is a simple harmonic oscillator, and its quantization places a quantum harmonic oscillator at each point. Excitations of the field correspond to the elementary particles of particle physics. However, even the vacuum has a vastly complex structure, so all calculations of quantum field theory must be made in relation to this model of vacuum. The vacuum has, implicitly, all of the properties that a particle may have: spin, or polarization in the case of light, energy, and so on. On average, all of these properties cancel out: the vacuum is, after all, "empty" in this sense. One important exception is the vacuum energy or the vacuum expectation value of the energy.

Let us consider here, vacuum, as a non-differentiable, Newtonian, $2 D$ non-coherent quantum fluid whose entities (cvasi-particles) assimilate to vortex-type objects [38] (see Fig.9) and are described by the wave function $\Psi[39,40]$

$$
\Psi=c n(\underline{u} ; k)
$$

with

$$
\begin{array}{ll}
\underline{u}=\frac{K}{a} \underline{z}, & \mathrm{a} \\
\underline{z}=x+i y, & \mathrm{~b} \\
\frac{K}{a}=\frac{K^{\prime}}{b} & \mathrm{c} \\
K=\int_{0}^{\pi / 2}\left(1-k^{2} \sin ^{2} \phi\right)^{-1 / 2} d \phi, & \mathrm{d} \\
K^{\prime}=\int_{0}^{\pi / 2}\left(1-k^{\prime 2} \sin ^{2} \phi\right)^{-1 / 2} d \phi, & \mathrm{e} \\
k^{2}+k^{\prime 2}=1 & \mathrm{f}
\end{array}
$$


and $K, K^{\prime}$ complete elliptic integrals of the first kind of modulus $k$ [41], form a vortex lattice of constants $a, b$.

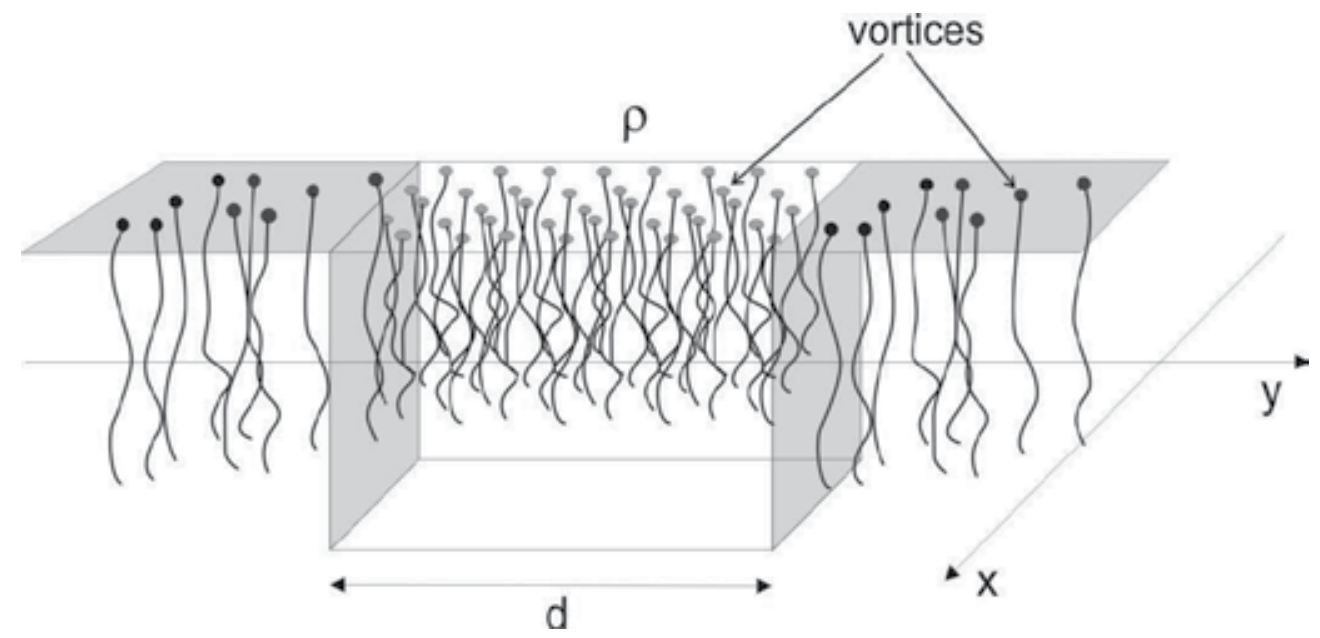

Figure 9. The vacuum from a Casimir cavity whose entities (cvasi-particles) are assimilated to vortex-type objects.

Applying in the complex plane [42], the formalism developed in [13] by means of the relation $\Psi=e^{F(z) / \Gamma}=c n(\underline{u} ; k)$ one introduces the complex potential

$$
F(\underline{z})=G(x, y)+i H(x, y)=\Gamma \ln [c n(\underline{u} ; k)]
$$

with $\Gamma$ the vortex constant. In the general case $\Gamma=c \Lambda=\hbar / m$ [38-40], the interaction scale being specified through $\Gamma$ 's value ( $\Lambda$ being considered as the Compton length).

Based on the complex potential (136), one defines the complex velocity field of the noncoherent quantum fluid, through the relation:

$$
v_{x}-i v_{y}=\frac{d F(\underline{z})}{d \underline{z}}=-\frac{\Gamma K}{a} \frac{s n(\underline{u} ; k) d n(\underline{u} ; k)}{c n(\underline{u} ; k)}
$$

or explicitly, using the notations [41, 42]: 


$$
\begin{aligned}
& s=\operatorname{sn}(\alpha, k), \quad \text { a } \\
& c=c n(\alpha, k), \quad \mathrm{b} \\
& d=d n(\alpha, k), \quad \text { c } \\
& \alpha=\frac{K}{a} x, \quad \mathrm{~d} \\
& s_{1}=\operatorname{sn}\left(\beta, k^{\prime}\right), \quad \text { e } \\
& c_{1}=c n\left(\beta, k^{\prime}\right), \quad \mathrm{f} \\
& d_{1}=d n\left(\beta, k^{\prime}\right), \quad \mathrm{g} \\
& \beta=\frac{K}{a} y \quad \mathrm{~h} \\
& v_{x}-i v_{y}=-\Gamma \frac{K}{a}\left\{\frac{s c d\left[c_{1}^{2}\left(d_{1}^{2}+k^{2} c^{2} s_{1}^{2}\right)-s_{1}^{2} d_{1}^{2}\left(d^{2} c_{1}^{2}-k^{2} s^{2}\right)\right]}{\left(1-d^{2} s_{1}^{2}\right)\left(c^{2} c_{1}^{2}+s^{2} d^{2} s_{1}^{2} d_{1}^{2}\right)}\right. \\
& \left.+i \frac{s_{1} c_{1} d_{1}\left[c^{2}\left(d^{2} c_{1}^{2}-k^{2} s^{2}\right)+s^{2} d^{2}\left(d_{1}^{2}+k^{2} c^{2} s_{1}^{2}\right)\right]}{\left(1-d^{2} s_{1}^{2}\right)\left(c^{2} c_{1}^{2}+s^{2} d^{2} s_{1}^{2} d_{1}^{2}\right)}\right\}
\end{aligned}
$$

Having in view that $c n(\underline{u}+\underline{\Omega})=c n(\underline{u})$, where $\underline{\Omega}=2(2 m+1) K+2 n i K^{\prime}$ and $m, n= \pm 1, \pm 2 \ldots$, for $k \rightarrow$ 0 and $k^{\prime} \rightarrow 1$ limits, respectively, the quantum fluid, initially non-coherent (the amplitudes and phases of quantum fluid entities are independent) becomes coherent (the amplitudes and phases of quantum fluid entities are correlated [43]). In this context, from Fig. 10a,b of the equipotential curves $G\left(x_{r}, y_{r}\right)=$ const., for $k^{2}=0,1$, it results that the coherence of the quantum fluid reduces to its ordering in vortex streets - see Fig. III.2a for vortex streets aligned with the $O x$ axis and Fig. 10b for vortex streets aligned with the $O y$ axis. This process of ordering is achieved by generation of quasi-particles. Indeed, in the usual quantum mechanics the imaginary term $(i \Theta)$ from the energy, i.e. $E=E_{0}+i \Theta$, induces elementary excitations named resonances (for details see the collision theory [44]). Similarly, by extending the collision theory to the fractal space-time $[1,45]$, will imply that the presence of the imaginary term $H\left(x_{r}, y_{r}\right)$ in the potential $F(\underline{z})$ will generate quasi-particles, as well.

Now, writing the Navier-Stokes equation (132a) and the equation of continuity (133a) in scale relativity theory for constant density (incompressible fluids) in two dimensions, one gets

$$
\begin{array}{ll}
\frac{\partial p}{\partial x}=\rho \mathrm{D}\left(\frac{\partial^{2} v_{x}}{\partial x^{2}}+\frac{\partial^{2} v_{x}}{\partial y^{2}}\right)-\rho\left(v_{x} \frac{\partial v_{x}}{\partial x}+v_{y} \frac{\partial v_{x}}{\partial y}\right) & \mathrm{a} \\
\frac{\partial p}{\partial y}=\rho \mathrm{D}\left(\frac{\partial^{2} v_{y}}{\partial x^{2}}+\frac{\partial^{2} v_{y}}{\partial y^{2}}\right)-\rho\left(v_{x} \frac{\partial v_{y}}{\partial x}+v_{y} \frac{\partial v_{y}}{\partial y}\right) & \mathrm{b}
\end{array}
$$




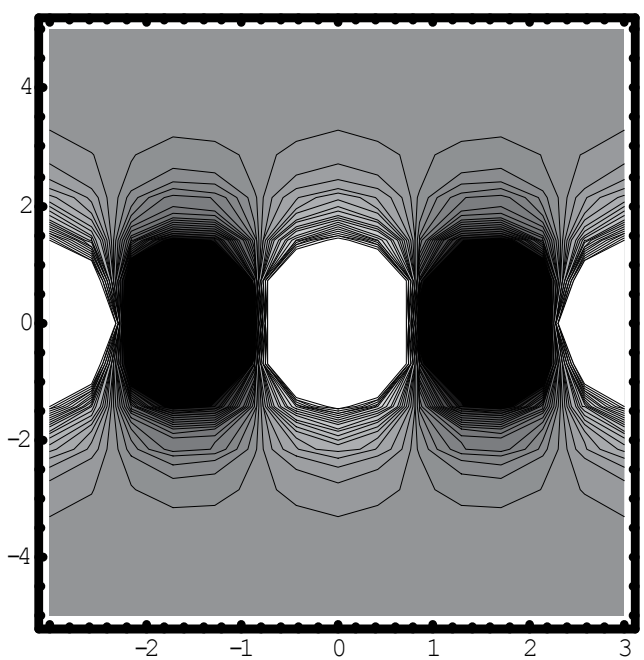

(a)

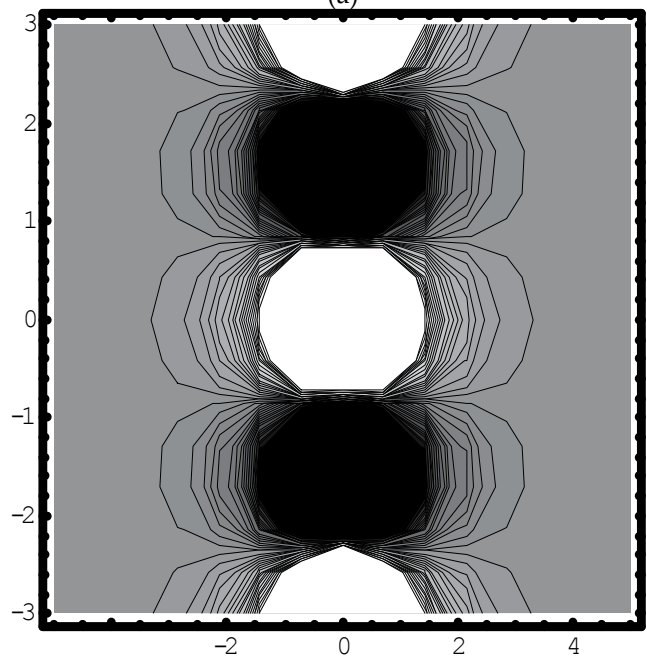

(b)

Figure 10. The equipotential curves $G\left(x_{r} y_{r}\right)=$ const., a) for vortex streets aligned with the $O x$ axis and b) for vortex streets aligned with the Oy axis.

$$
\frac{\partial v_{x}}{\partial x}+\frac{\partial v_{y}}{\partial y}=0
$$

where the shear viscosity $v$ is replaced by $D$ since we are dealing here with a non-differentiable quantum fluid.

Then, after some rather long yet elementary calculus one gets from $(140 a, b)$ through the degenerations : 
i.

$$
\begin{aligned}
& k=0, \quad k^{\prime}=1, \quad K=\frac{\pi}{2}, \quad K^{\prime}=\infty \\
& p_{y}(\alpha)=-p_{0} \sinh ^{2}\left(\frac{\pi d}{2 a}\right) \frac{1-\tan ^{2} \alpha}{\cos (2 \alpha)+\cosh \left(\frac{\pi d}{a}\right)} \\
& p_{x}(\beta)=-p_{0} \sin ^{2}\left(\frac{\pi d_{1}}{2 a}\right) \frac{1+\tanh ^{2} \beta}{\cos \left(\frac{\pi d_{1}}{a}\right)+\cosh (2 \beta)}
\end{aligned}
$$

with

$$
\begin{array}{ll}
p_{0}=\frac{\hbar^{2} \pi^{2} \rho}{4 M^{2} a^{2}} ; & \mathrm{a} \\
\alpha=\frac{\pi x}{2 a} ; & \mathrm{b} \\
\beta=\frac{\pi y}{2 a} & \mathrm{c}
\end{array}
$$

and

ii.

$$
\begin{gathered}
k=1, \quad k^{\prime}=0, \quad K=\infty, \quad K^{\prime}=\frac{\pi}{2} \\
p_{y}\left(\alpha^{\prime}\right)=-p_{0}^{\prime} \sin ^{2}\left(\frac{\pi d}{2 b}\right) \frac{1+\tanh ^{2} \alpha^{\prime}}{\cos \left(\frac{\pi d}{b}\right)+\cosh \left(2 \alpha^{\prime}\right)} \\
p_{x}\left(\beta^{\prime}\right)=-p_{0}^{\prime} \sinh ^{2}\left(\frac{\pi d_{1}}{2 b}\right) \frac{1-\tan ^{2} \beta^{\prime}}{\cos \left(2 \beta^{\prime}\right)+\cosh \left(\frac{\pi d_{1}}{b}\right)}
\end{gathered}
$$

with

$$
\begin{array}{ll}
p_{0}^{\prime}=\frac{\hbar^{2} \pi^{2} \rho}{4 M^{2} b^{2}} ; & \text { a } \\
\alpha^{\prime}=\frac{\pi x}{2 b} ; & \text { b } \\
\beta^{\prime}=\frac{\pi y}{2 b} & \text { c }
\end{array}
$$


Here, $\rho$ is the quantum fluid's density, $M$ the mass of the quantum fluid entities, $d$ and $d_{1}$ are the elementary space intervals considered along the $O y$ and $O x$ axis, respectively.

In other words, non-differentiability and coherence of the quantum fluid due to constraints, generate pressure along the $O x$ and $O y$ axis.

Moreover, one can show that the equation of continuity (141) is identically satisfied for both cases of degeneration.

Let us consider a Casimir cavity consisting of the vacuum with the vortex lattice depicted above and two metallic slabs, that constitute the plates of the cavity, placed at a distance $d$ apart, parallel to each other and to the $x \mathrm{Oz}$ plane (see Fig. III.1). According to the analysis from the previous section, one can see that if the quantum fluid is placed in a potential well with infinite walls (the case of the Casimir cavity analyzed here, where the two plates are the constraints of the quantum fluid), along a direction perpendicular to the walls (the $\mathrm{Oy}$ axis here) a coherent structure, a vortex street forms (see Fig. III.2b). Consequently, by integrating (144a,b) with (145a-c) over $\alpha_{r}$ and $\beta_{r}$, and using the result in the quantization rule:

$$
\int_{d_{1}}^{d_{2}} k d x=n \pi, \quad n=1,2,3, \ldots
$$

where $d_{1} \sim m \pi a, d \sim n \pi b$, with $m, n=1,2, \ldots .$, one gets

$$
\begin{aligned}
& \frac{\pi}{2} \frac{p_{y}}{p_{0}^{\prime}}=2 r \arctan \left[\tan \left(\frac{n \pi^{2}}{2}\right) \tanh \left(\frac{m \pi^{2}}{4 r}\right)\right] \tan ^{-1}\left(n \pi^{2}\right)-r \tanh \left(\frac{m \pi^{2}}{4 r}\right) \\
& \frac{\pi}{2} \frac{p_{x}}{p_{0}^{\prime}}=-2 \arctan \left[\tan \left(\frac{n \pi^{2}}{4}\right) \tanh \left(\frac{m \pi^{2}}{2 r}\right)\right] \tanh ^{-1}\left(\frac{m \pi^{2}}{r}\right)+\tan \left(\frac{n \pi^{2}}{4}\right)
\end{aligned}
$$

where

$$
\begin{array}{ll}
p_{0}^{\prime}=\frac{\hbar^{2} \pi^{2} \rho}{4 M^{2} b^{2}} ; & \mathrm{a} \\
\alpha_{r}=\frac{\pi}{2} \frac{x}{a} ; & \mathrm{b} \\
\beta_{r}=\frac{\pi}{2} \frac{y}{b} ; & \mathrm{c} \\
r=\frac{b}{a} & \mathrm{~d}
\end{array}
$$

Graphically this is presented in Fig. III.3a,b for different values of the parameters $m, n=1,2, \ldots$. and $r$. 
If the plates were in the $y O z$ plane the constraints being along the $O x$ axis, vortex streets would form along this axis and the result in $(142 a, b)$ with $(143 a-c)$ would have been applied, i.e. the cases i) or ii) are identical, yet they depend on the geometry chosen.

Firstly, one can notice that the pressure $p_{y}$ on the plates, given by (147a), stabilizes for great $r$ values, is always negative and an attractive force results (see Fig. $11 \mathrm{a}$ ), as is the case of the Casimir force (128).

Secondly, the theory predicts, that besides the pressure $p_{y}$ acting on the plates, there must be yet another pressure, $p_{x}$ (see Fig. $11 \mathrm{~b}$ ), acting along the $O x$ axis and given by (147b). One can see that this pressure annuls for great $r$ values, and has a minimum for some values of the parameters $m, n$. This result is new and should be checked by experiments.

Moreover, if one tries to compute the order of magnitude of this force, and replaces in (144a) : $\hbar=1.05410^{-34} \mathrm{~J} . \mathrm{s}, m=9.110^{-31} \mathrm{~kg}, \rho \sim 10^{21} \mathrm{~cm}^{-3}, b=1 \AA \AA$ (values specific to a bosonic gas, i.e. found in high-Tc superconductors [46]) and $d \sim 5 b$ (the distance between the plates), gets a value for $p_{y} \cong 6.1810^{10} \mathrm{~N} \mathrm{~m}^{-2}$ the same order of magnitude as the value calculated using (128), $F_{C} \cong 2.08$ $10^{10} \mathrm{~N} \mathrm{~m}^{-2}$.

As a final test, let us study the case of a Casimir cavity, as a rectangle of sides $d_{1}, d$. Now, the plates induce constraints along both $O x$ and $O y$ axis, thus correlations (vortex streets) form along these directions and one should use the degenerations i) and ii), simultaneously. Consequently, from $(142 a, b)$ with $(143 a-c)$ and $(144 a, b)$ with $(145 a-c)$ one gets

$$
p_{y \text { rect }}\left(\alpha, \alpha^{\prime}\right)=-\frac{\hbar^{2} \pi^{2} \rho}{4 m^{2}}\left(\frac{1}{a^{2}} \frac{\sinh ^{2} A}{\cos ^{2} \alpha}\left(1+\frac{\cosh 2 A}{\cos 2 \alpha}\right)^{-1}+\frac{1}{b^{2}} \frac{\sin ^{2} B}{\cosh ^{2} \alpha^{\prime}}\left(1+\frac{\cos 2 B}{\cosh 2 \alpha^{\prime}}\right)^{-1}\right)
$$

with

$$
A=\frac{\pi d}{2 a} ; \quad B=\frac{\pi d}{2 b}
$$

and

$$
p_{\text {x rect }}\left(\beta, \beta^{\prime}\right)=-\frac{\hbar^{2} \pi^{2} \rho}{4 m^{2}}\left(\frac{1}{a^{2}} \frac{\sin ^{2} A^{\prime}}{\cosh ^{2} \beta}\left(1+\frac{\cos 2 A^{\prime}}{\cosh 2 \beta}\right)^{-1}+\frac{1}{b^{2}} \frac{\sinh ^{2} B^{\prime}}{\cos ^{2} \beta^{\prime}}\left(1+\frac{\cosh 2 B^{\prime}}{\cos 2 \beta^{\prime}}\right)^{-1}\right)
$$

with

$$
A^{\prime}=\frac{\pi d_{1}}{2 a} ; \quad B^{\prime}=\frac{\pi d_{1}}{2 b}
$$



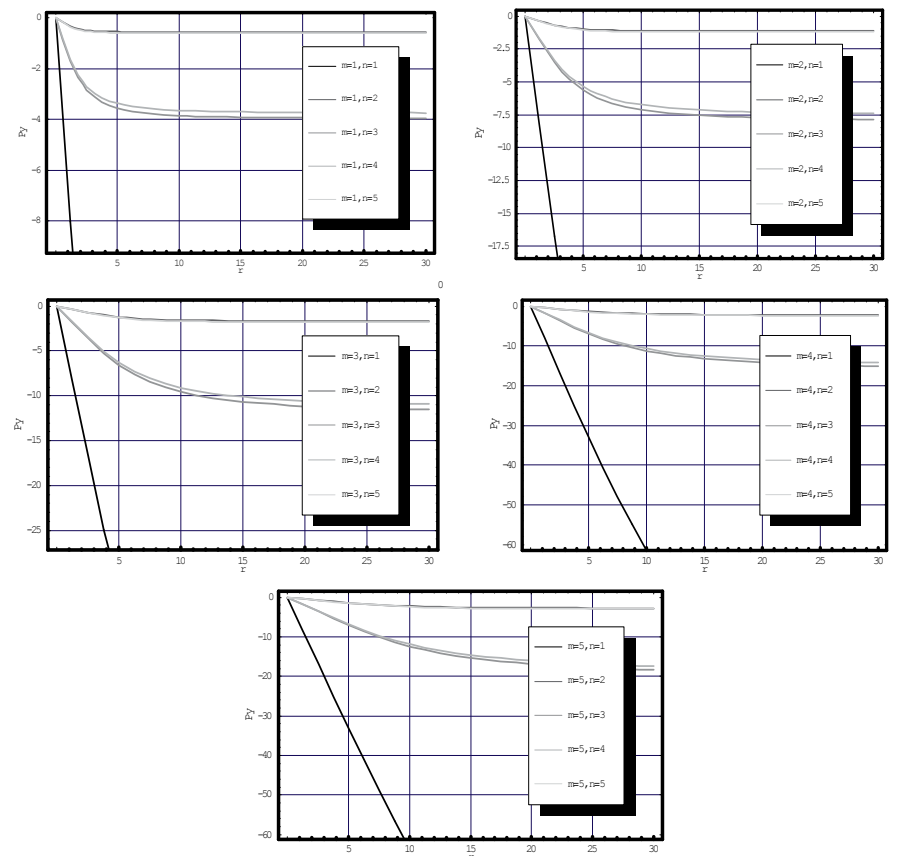

(a)
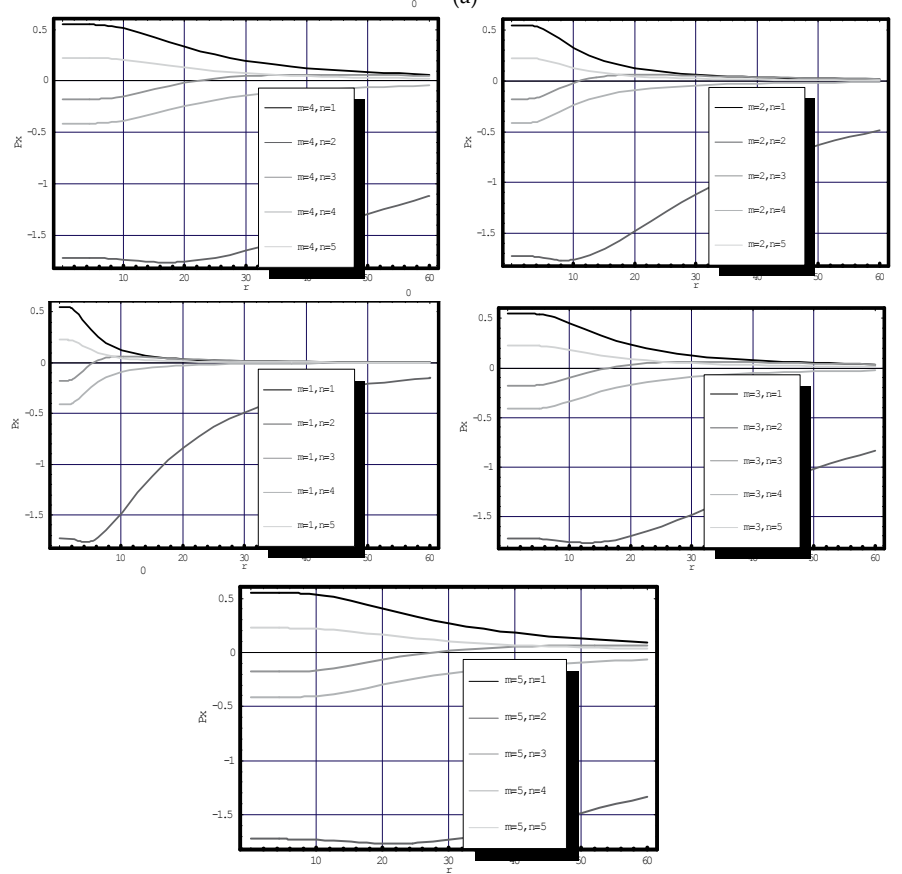

(b)

Figure 11. a) Plot of the pressure $p_{y}$ on the plates, versus the parameter $r$ for different values of parameters $m, n ; b$ ) Plot of the pressure $p_{x}$ versus the parameter $r$ for different values of parameters $m, n$. 
At every point $(x, y)$ there is a pressure formed of the two constraints. Consequently, adding the pressures in (149) and (151) and using again the result in (146) (i.e. $d_{1} \sim m \pi a, d \sim n \pi b$, where $m, n=1,2, \ldots$.$) one gets:$

$$
\begin{aligned}
& -\frac{p_{r e c t}\left(\alpha_{r}, \beta_{r}\right)}{p_{0}^{\prime}}=r^{2} \frac{\sinh ^{2}\left(\frac{n \pi^{2}}{2} r\right)}{\cos ^{2} \alpha_{r}}\left(1+\frac{\cosh \left(n \pi^{2} r\right)}{\cos \left(2 \alpha_{r}\right)}\right)^{-1}+\frac{\sin ^{2}\left(\frac{n \pi^{2}}{2}\right)}{\cosh ^{2}\left(\frac{\alpha_{r}}{r}\right)}\left(1+\frac{\cos \left(n \pi^{2}\right)}{\cosh \left(\frac{2 \alpha_{r}}{r}\right)}\right)^{-1}+ \\
& +r^{2} \frac{\sin ^{2}\left(\frac{m \pi^{2}}{2}\right)}{\cosh ^{2}\left(r \beta_{r}\right)}\left(1+\frac{\cos \left(m \pi^{2}\right)}{\cosh \left(2 r \beta_{r}\right)}\right)^{-1}+\frac{\sinh ^{2}\left(\frac{1}{r} \frac{m \pi^{2}}{2}\right)}{\cos ^{2} \beta_{r}}\left(1+\frac{\cosh \left(\frac{m \pi^{2}}{r}\right)}{\cos \left(2 \beta_{r}\right)}\right)^{-1}
\end{aligned}
$$

where

$$
p_{0}^{\prime}=\frac{\hbar^{2} \pi^{2} \rho}{4 M^{2} b^{2}} ; \quad \alpha_{\mathrm{r}}=\frac{\pi}{2} \frac{x}{a}=\frac{\pi}{2} x_{r} ; \quad \beta_{\mathrm{r}}=\frac{\pi}{2} \frac{y}{b}=\frac{\pi}{2} y_{r} ; \quad r=\frac{b}{a}
$$

Furthermore, we integrate (153) over $x_{r}$ and $y_{r}$, respectively, in order to find a value of the pressure acting on the sides of the rectangular enclosure. After some long, yet elementary calculus, one finds:

$$
\begin{gathered}
\frac{p_{r e c t}}{p_{0}^{\prime}}=-4 n r^{2} \frac{\operatorname{arctg}\left[\operatorname{tg}\left(\frac{m \pi^{2}}{4}\right) \operatorname{th}\left(\frac{n \pi^{2}}{2} r\right)\right]}{\operatorname{th}\left(n \pi^{2} r\right)}+4 n r \frac{\operatorname{arctg}\left[\operatorname{tg}\left(\frac{n \pi^{2}}{2}\right) \operatorname{th}\left(\frac{m \pi^{2}}{4} \frac{1}{r}\right)\right]}{\operatorname{tg}\left(n \pi^{2}\right)}+ \\
+2 n r^{2} \operatorname{tg}\left(\frac{m \pi^{2}}{4}\right)-2 n r \operatorname{th}\left(\frac{m \pi^{2}}{4} \frac{1}{r}\right)- \\
-4 m \frac{\operatorname{arctg}\left[\operatorname{tg}\left(\frac{n \pi^{2}}{4}\right) \operatorname{th}\left(\frac{m \pi^{2}}{2} \frac{1}{r}\right)\right]}{\operatorname{th}\left(m \pi^{2} \frac{1}{r}\right)}+4 m r \frac{\operatorname{arctg}\left[\operatorname{tg}\left(\frac{m \pi^{2}}{2}\right) \operatorname{th}\left(\frac{n \pi^{2}}{4} r\right)\right]}{\operatorname{tg}\left(m \pi^{2}\right)}+ \\
+2 m \operatorname{tg}\left(\frac{n \pi^{2}}{4}\right)-2 m r \operatorname{th}\left(\frac{n \pi^{2}}{4} r\right)
\end{gathered}
$$

Plots of (155) for various values of parameters $m, n=1,2, \ldots$ and $r$ are depicted in Fig. III.4a,b. 

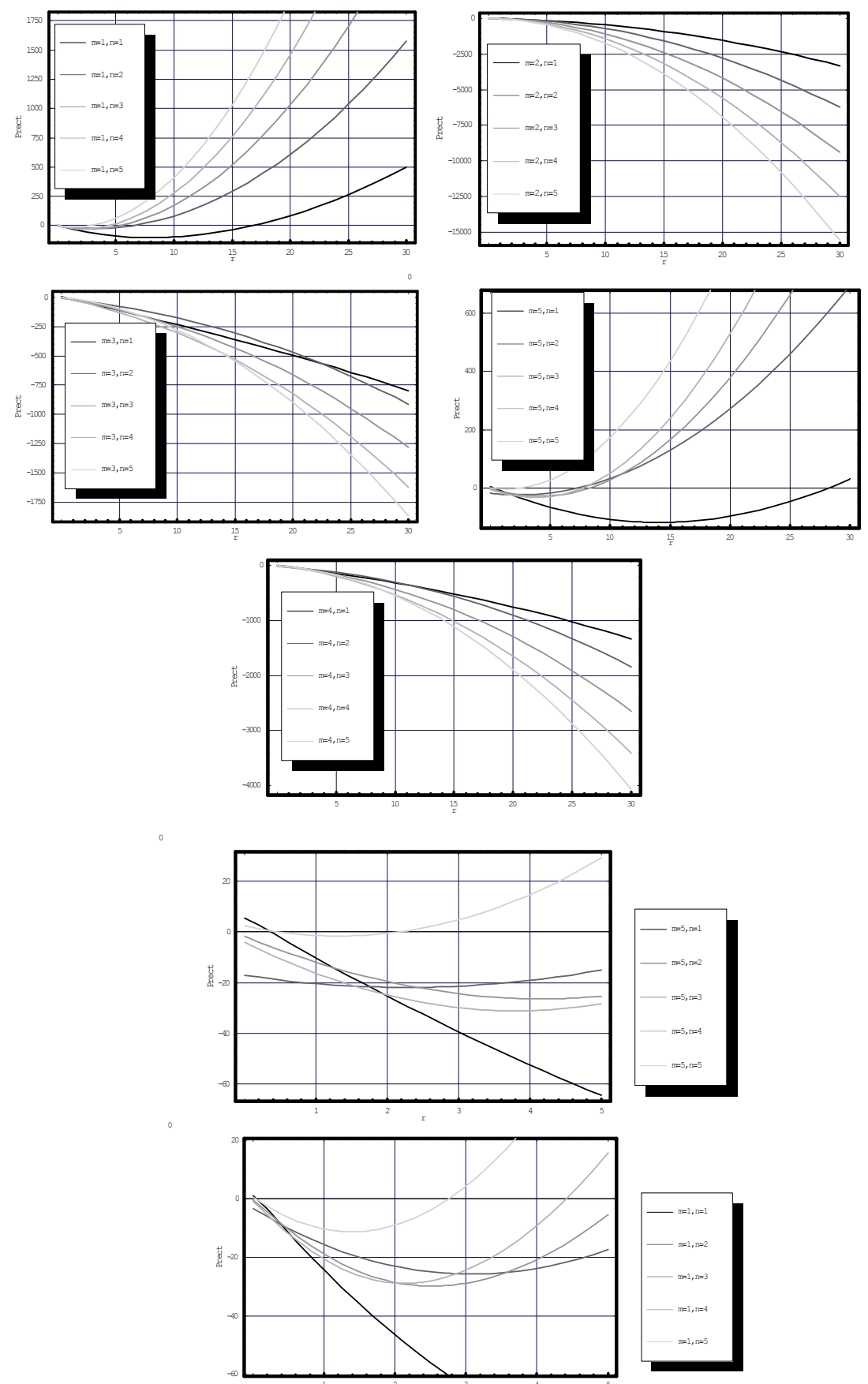

Figure 12. a) Plots of $p_{\text {rect }}$ versus the parameter $r$ for various values of parameters $m, n=1,2, \ldots ; b$ ) the same plot, yet we present here a magnification of the domain of $r$ for highly asymmetric values of $m, n(1,5$ and 5,1$)$. 
One can notice that if the two parameters $m$ and $n$ have close values, the force acting on the Casimir rectangle is always negative and decreases exponentially for increasing $r$. For parameters $m$ and $n$ (1,5 and 5,1, i.e. very asymmetric) the force has negative and positive domains (see Fig. 12 b) and increases exponentially for increasing $r$. Moreover, if one tries to find the positive and negative domains, and solve (155) for $m=5, n=5$ finds $p_{\text {rect }}<0$ for $0.45753 \leq r \leq 2.18565$ and $p_{\text {rect }}>0$ for $r>2.18565$ and $r<0.45753$. This result is in agreement with the calculus of regularization using the Abel-Plana formula where $E<0$ for $0.36537 \leq L / l \leq$ 2.73686 and $E>0$ for $L / l>2.73686$ and $L / l<0.36537$ [47].

\section{Fractal approximation of motion in mass transfer: release of drug from polimeric matrices}

Polymer matrices can be produced in one of the following forms: micro/nano-particles, micro/ nano capsules, hydro gels, films, patches.Our new approach considers the entire system (drug loaded polymer matrix in the release environment) as a type of "fluid" totally lacking interaction or neglecting physical interactions among particles. At the same time, the induced complexity is replaced by fractality. This will lead to particles moving on certain trajectories called geodesics within fractal space. This assumption represents the basis of the fractal approximation of motion in Scale Relativity Theory (SRT) [1, 2], leading to a generalized fractal "diffusion" equation that can be analyzed in terms of two approximations (dissipative and dispersive).

\subsection{The dissipative approximation}

In the dissipative approximation the fractal operator (42) takes the form [48, 49]:

$$
\frac{\hat{\partial}}{\partial t}=\frac{\partial}{\partial t}+\hat{\boldsymbol{V}} \cdot \nabla-i \mathrm{D}(d t)^{\left(2 / D_{F}\right)-1} \Delta
$$

As a consequence, we are now able to write the fractal "diffusion" type equation in its covariant form:

$$
\frac{\partial Q}{d t}=\frac{\partial Q}{\partial t}+(\hat{V} \cdot \nabla) Q-i D(d t)^{\left(2 / D_{F}\right)-1} \Delta Q=0
$$

Separating the real and imaginary parts in (157), i.e.

$\frac{\partial Q}{\partial t}+V \cdot \nabla Q=0$ 


$$
-\boldsymbol{U} \cdot \nabla Q=\mathrm{D}(d t)^{\left(2 / D_{F}\right)-1} \Delta Q
$$

we can add these two equations and obtain a generalized "diffusion" type law in the form:

$$
\frac{\partial Q}{\partial t}+(\boldsymbol{V}-\boldsymbol{U}) \cdot \nabla Q=\mathrm{D}(d t)^{\left(2 / D_{F}\right)-1} \Delta Q
$$

\subsubsection{Standard "diffusion" type equation. Fick type law}

The standard "diffusion" law, i.e.:

$$
\frac{\partial Q}{\partial t}=\mathrm{D} \Delta Q
$$

results from (159) on the following assertions:

i. the diffusion path are the fractal curves of Peano's type. This means that the fractal dimension of the fractal curves is $D_{F}=2$.

ii. the movements at differentiable and non-differentiable scales are synchronous, i.e. $V=U$;

iii. the structure coefficient $\mathrm{D}$, proper to the fractal-nonfractal transition, is identified with the diffusion coefficient, i.e.

$D \equiv D$.

\subsubsection{Anomalous "diffusion" type equation. Weibull relation}

The anomalous diffusion law results from (IV.4) on the following assumptions:

i. the diffusion path are fractal curves with fractal dimension $D_{F} \neq 2$;

ii. the time resolution, $\delta t$, is identified with the differential element $d t$, i.e. the substitution principle can be applied also, in this case;

iii. the movements at differentiable and non-differentiable scales are synchronous, i.e. $V=U$.

Then, the equation (IV.4) can be written:

$$
\frac{\partial Q}{\partial t}=\mathrm{D}(d t)^{\left(2 / D_{F}\right)-1} \Delta Q
$$

In one-dimensional case, applying the variable separation method [50] 


$$
Q(t, x)=T(t) \cdot X(x)
$$

with the standard initial and boundary conditions:

$$
Q(t, 0)=0, Q(t, L)=0, Q(0, x)=F(x), 0 \leq x \leq L
$$

implies:

$$
\frac{1}{\mathrm{D}(d t)^{\left(2 / D_{F}\right)-1}} \frac{1}{T(t)} \frac{d T(t)}{d t}=\frac{1}{X(x)} \frac{d^{2} X(x)}{d x^{2}}=-m^{2}=-\left(\frac{n \pi}{L}\right)^{2}, n=1,2
$$

where $L$ is a system characteristic length, $m$ a separation constant, dependent on diffusion order $n$.

Accepting the viability of the substitution principle, from (164), through integration, results:

$$
\ln T=-m^{2} \mathrm{D} \int(d t)^{2 / D_{F}}
$$

Taking into consideration some results of the fractional integro-differential calculus [51, 52], (165) becomes:

$$
\begin{aligned}
& \ln T=-\frac{m^{2} \mathrm{D}}{\Gamma\left(\frac{2}{D_{F}}+1\right)} t^{\frac{2}{D_{F}}}, \quad \mathrm{a} \\
& \Gamma\left(\frac{2}{D_{F}}\right)=\int_{0}^{\infty} x^{\left(\frac{2}{D_{F}}\right)-1} e^{-x} d x \quad \mathrm{~b}
\end{aligned}
$$

Moreover, $(166 \mathrm{a}, \mathrm{b})$ can be written under the form:

$$
T(t)=\exp \left[-\frac{m^{2} \mathrm{D}}{\Gamma\left(\frac{2}{D_{F}}+1\right)} t^{\frac{2}{D_{F}}}\right]
$$

The relative variation of concentrations, time dependent, is defined as: 


$$
T(t)=\frac{Q_{\infty}-Q_{t}}{Q_{\infty}}
$$

where $Q_{t}$ and $Q_{\infty}$ are cumulative amounts of drug released at time $t$ and infinite time.

From (167) and (168) results:

$$
\frac{Q_{t}}{Q_{\infty}}=1-\exp \left[-\frac{m^{2} \mathrm{D}}{\Gamma\left(\frac{2}{D_{F}}+1\right)} t^{\frac{2}{D_{F}}}\right]
$$

equation similar to Weibull relation $\frac{Q_{t}}{Q_{\infty}}=1-\exp \left(-a t^{b}\right), a$ and $b$ representing constants specific for each system that are defined by:

$$
\begin{array}{ll}
a=\frac{m^{2} D}{\Gamma\left(\frac{2}{D_{F}}+1\right)}=\left(\frac{n \pi}{L}\right)^{2} \frac{D}{\Gamma\left(\frac{2}{D_{F}}+1\right)} & \mathrm{a} \\
b=\frac{2}{D_{F}} & \mathrm{~b}
\end{array}
$$

We observe that both constants, $a$ and $b$, are functions of the fractal dimension of the curves on which drug release mechanism take place, dimension that is a measure of the complexity and nonlinear dynamics of the system. Moreover, constant $a$ depends, also, on the "diffusion" order $n$.

\subsubsection{The correspondence between theoretical model and experimental results}

The experimental and Weibull curves for HS (starch based hydrogels loaded with levofloxacin) and GA (GEL-PVA microparticles loaded with chloramphenicol) samples are plotted in Fig. 13.

The experimental data allowed to determine the values of Weibull parameters ( $a$ and $b$ ), and implicitly, the value of the fractal dimension from the curve on which release takes place [55].

These values confirmed that the complexity of the phenomena determines, also, naturally, a complex trajectory for the drug particles. Most values are between 1 and 3, in agreement with the values usually accepted for fractal process; higher values denotes the fact that, either fractal dimension must be redefined as function of structure "classes", or the drug release process is complex, involving many freedom degrees in the phase space [56]. Another observation that can be made based on this results is that the samples with $D_{F}<2$ manifests a "sub-diffusion" 

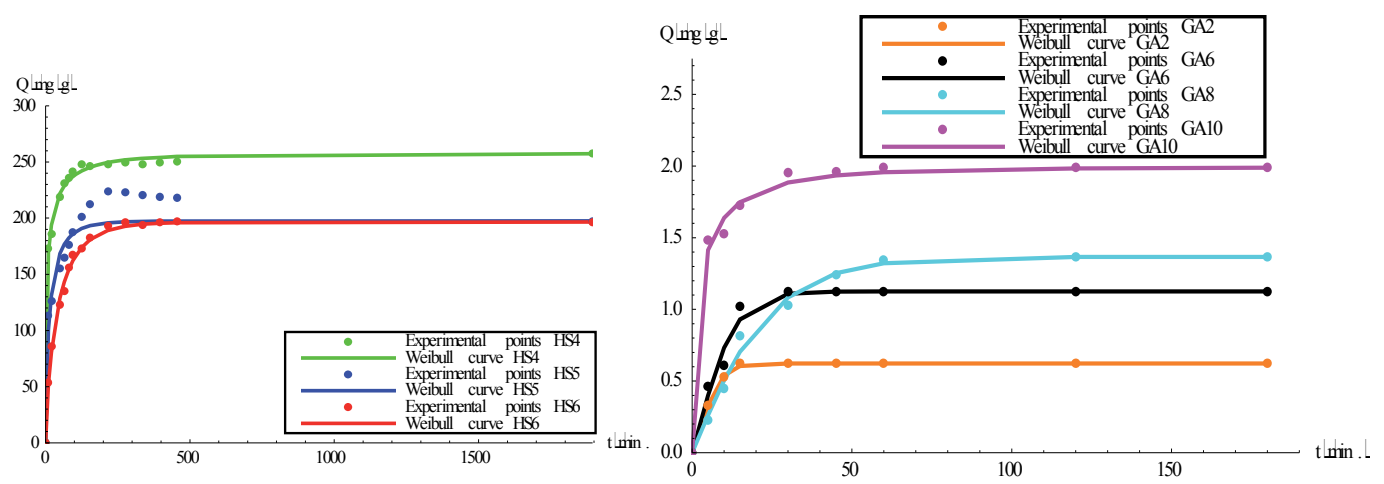

Figure 13. Experimental and Weibull curves for HS (left plot) and GA samples (right plot).

and, in the other, with $D_{F}>2$, the release process is of super-diffusion, classification in concordance with the experimental observation that this samples exhibit a "faster" diffusion, with a higher diffusion rate, in respect with the other samples [55].

\subsection{The dispersive approximation}

Let us now consider that, in comparison with dissipative processes, convective and dispersive processes are dominant ones. In these conditions, the fractal operator (42) takes the form:

$$
\frac{\hat{\partial}}{d t}=\frac{\partial}{\partial t}+(\hat{\boldsymbol{V}} \cdot \nabla)+\frac{\sqrt{2}}{3} \mathbf{D}^{3 / 2}(d t)^{\left(3 / D_{F D}\right)-1} \nabla^{3}
$$

Consequently, we are now able to write the diffusion equation in its covariant form, as a Korteweg de Vries type equation:

$$
\frac{\hat{\partial} Q}{d t}=\frac{\partial Q}{\partial t}+(\hat{V} \cdot \nabla) Q+\frac{\sqrt{2}}{3} D^{3 / 2}(d t)^{\left(3 / D_{F D}\right)-1} \nabla^{3} Q=0
$$

If we separate the real and imaginary parts from Eq. (172), we shall obtain:

$$
\begin{array}{ll}
\frac{\partial Q}{\partial t}+\boldsymbol{V} \cdot \nabla Q+\frac{\sqrt{2}}{3} D^{3 / 2}(d t)^{\left(3 / D_{F}\right)-1} \nabla^{3} Q=0 & \mathrm{a} \\
-\boldsymbol{U} \cdot \nabla Q=0 & \mathrm{~b}
\end{array}
$$

By adding them, the fractal diffusion equation is: 


$$
\frac{\partial Q}{\partial t}+(\boldsymbol{V}-\boldsymbol{U}) \cdot \nabla Q+\frac{\sqrt{2}}{3} \mathbf{D}^{3 / 2}(d t)^{\left(3 / D_{F}\right)-1} \nabla^{3} Q=0
$$

From Eq. (173b) we see that, at fractal scale, there will be no $Q$ field gradient.

Assuming that $|\boldsymbol{V}-\boldsymbol{U}|=\sigma \cdot Q$ with $\sigma=$ constant (in systems with self structuring processes, the speed fluctuations induced by fractal - non fractal are proportional with the concentration field [55]), in the particular one-dimensional case, equation (174) with normalized parameters:

$$
\begin{array}{ll}
\bar{\tau}=\omega t, & \mathrm{a} \\
\bar{\xi}=k x, & \mathrm{~b} \\
\Phi=\frac{Q}{Q_{0}} & \mathrm{c}
\end{array}
$$

and normalizing conditions:

$$
\frac{\sigma Q_{0} k}{6 \omega}=\frac{\sqrt{2}}{3} \frac{D^{3 / 2}(d t)^{\left(3 / D_{F}\right)-1} k^{3}}{\omega}=1
$$

take the form:

$$
\partial_{\tau}^{-} \phi+6 \phi \partial_{\bar{\xi}} \phi+\partial_{\bar{\xi} \bar{\xi} \bar{\xi}} \phi=0
$$

In relations $(175 \mathrm{a}, \mathrm{b}, \mathrm{c})$ and $(176) \omega$ corresponds to a characteristic pulsation, $k$ to the inverse of a characteristic length and $Q_{0}$ to balanced concentration.

Through substitutions:

$$
\begin{array}{ll}
w(\theta)=\phi(\bar{\tau}, \bar{\xi}), & \mathrm{a} \\
\theta=\bar{\xi}-u \bar{\tau} & \mathrm{b}
\end{array}
$$

eq.(177), by double integration, becomes:

$$
\frac{1}{2} w^{\prime 2}=F(w)=-\left(w^{3}-\frac{u}{2} w^{2}-g w-h\right)
$$


with $g, h$ two integration constants and $u$ the normalized phase velocity. If $F(w)$ has real roots, equation (177) has the stationary solution:

$$
\phi(\bar{\xi}, \bar{\tau}, s)=2 a\left(\frac{E(s)}{K(s)}-1\right)+2 a \cdot c n^{2}\left[\frac{\sqrt{a}}{s}\left(\bar{\xi}-\frac{\bar{u}}{2} \bar{\tau}+\overline{\xi_{0}}\right) ; s\right]
$$

where $c n$ is Jacobi's elliptic function of $s$ modulus [41], $a$ is the amplitude, $\xi_{0}$ is a constant of integration and

$$
\begin{aligned}
& K(s)=\int_{0}^{\pi / 2}\left(1-s^{2} \sin ^{2} \phi\right)^{-1 / 2} d \phi \quad \mathrm{a} \\
& E(s)=\int_{0}^{\pi / 2}\left(1-s^{2} \sin ^{2} \phi\right)^{1 / 2} d \phi \quad \mathrm{b}
\end{aligned}
$$

are the complete elliptic integrals [41].

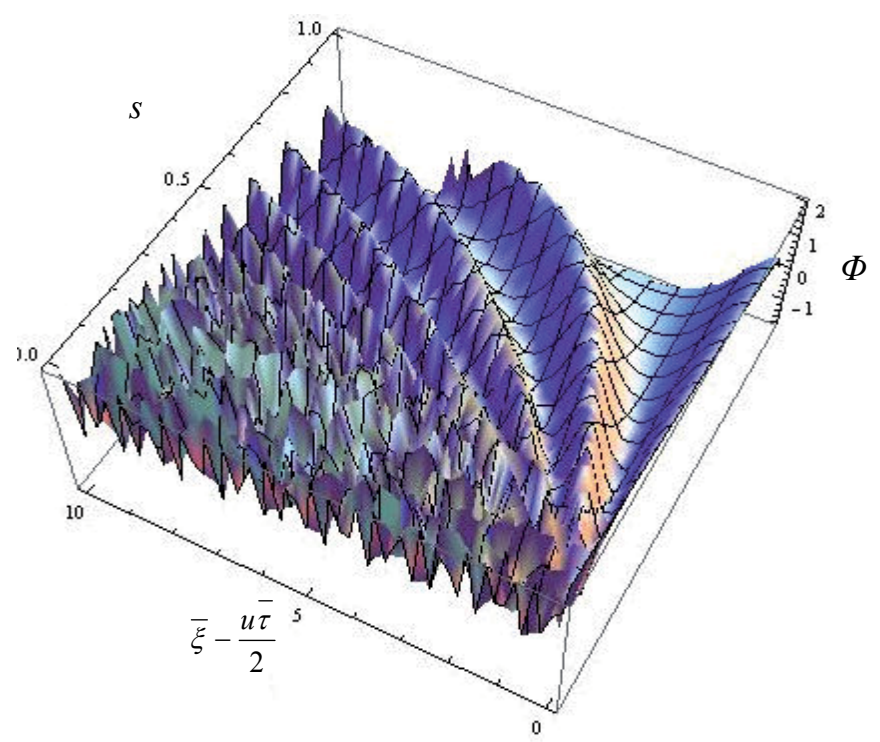

Figure 14. One-dimensional cnoidal oscillation modes of the field $\Phi$ 
Parameter $s$ represents measure characterizing the degree of nonlinearity in the system. Therefore, the solution (180) contains (as subsequences for $s=0$ ) one-dimensional harmonic waves, while for $s \rightarrow 0$ one-dimensional wave packet. These two subsequences define the nonquasi-autonomous regime of the drug release process [48, 49, 55], i.e. the system should receive external energy in order to develop. For $s=1$, the solution (180) becomes one-dimensional soliton, while for $s \rightarrow 1$, one-dimensional soliton packet will be generated. The last two imply a quasi-autonomous regime (self evolving and independent [48]) for drug particle release process $[48,49,55]$.

The three dimensional plot of solution (180) shows one-dimensional cnoidal oscillation modes of the concentration field, generated by similar trajectories of the drug particles (see Fig. 14). We mention that cnoidal oscillations are nonlinear ones, being described by the elliptic function $c n$, hence the name (cnoidal).

It is known that in nonlinear dynamics, cnoidal oscillation modes are associated with nonlinear lattice of oscillators (the Toda lattice [56]). Consequently, large time scale drug particle ensembles can be compared to a lattice of nonlinear oscillators which facilitates drug release process.

\subsubsection{The correspondence between theoretical model and experimental results}

In what follows we identify the field $\Phi$ from relation (180) with normalized concentration field of the released drug from micro particles.

For best correlation between experimental data and the theoretical model (for each sample) we used a planar intersection of the graph in Fig. 14 [57], in order to obtain two-dimensional plots.

The highest value of the correlation coefficient (for two data sets: one obtained from the planar intersection, the other from experimental data) will represent the best approximation of experimental data with the theoretical model.

Our goal was to find the right correlation coefficient which should be higher than $0.6-0.7$, in order to demonstrate the relevance of the model we had in view. Figs. 15 show experimental and theoretical curves that were obtained through this method, where $R^{2}$ represents the correlation coefficient and $\eta$ a normalized variable which is simultaneously dependent on normalized time and on nonlinear degree of the system (s parameter). Geometrically, $\eta$ represents the congruent angle formed by the time axis and the vertical intersection plane. 

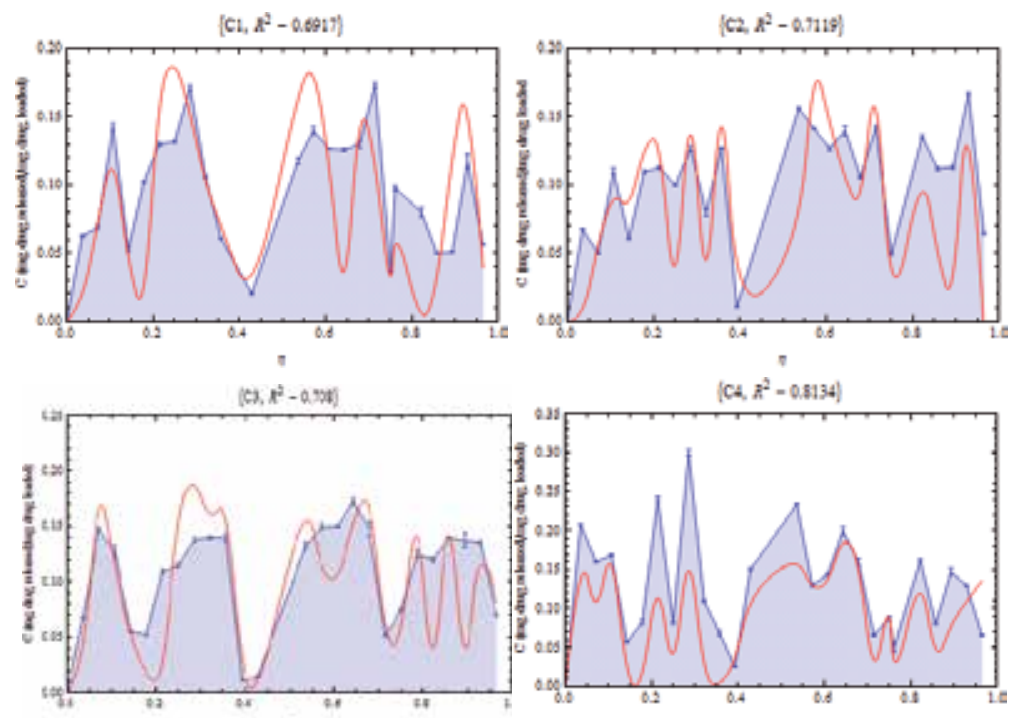

\{Cs, $\left.x^{2}-0.7207\right\}$

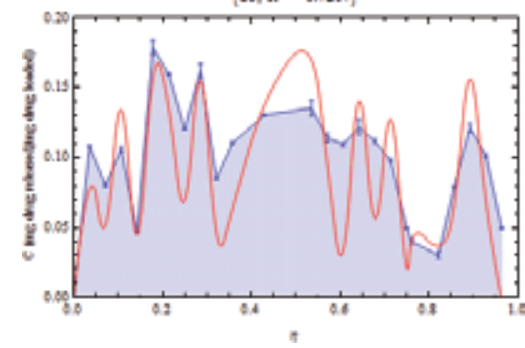

$\left[c 7, x^{2}-0.52491\right\}$
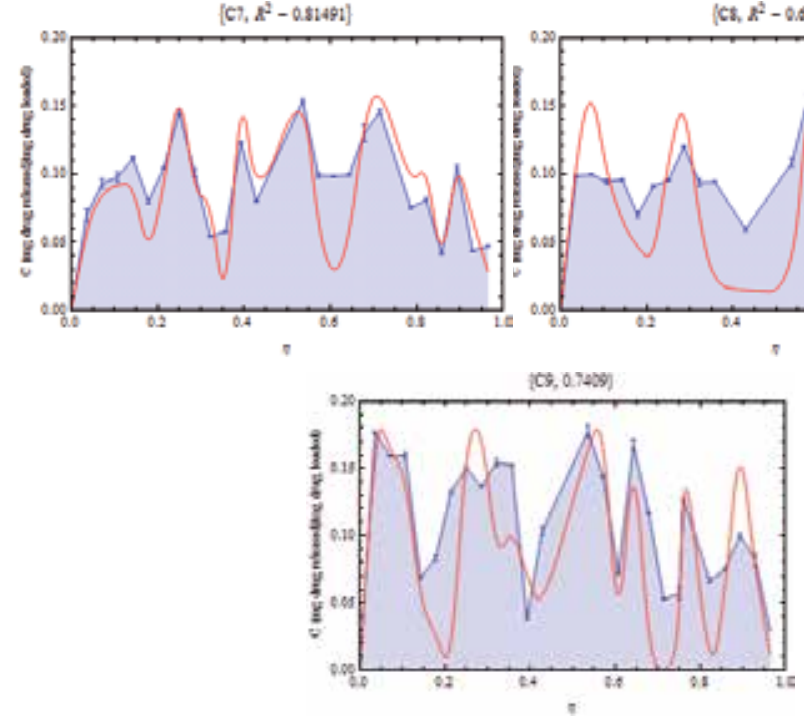

(C4, $z^{2}=0.8434$ )

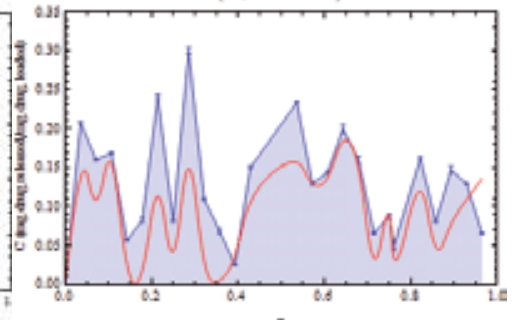

$\left.\left\{C s, t^{2}-0 . \Delta 0^{2}\right)\right\}$

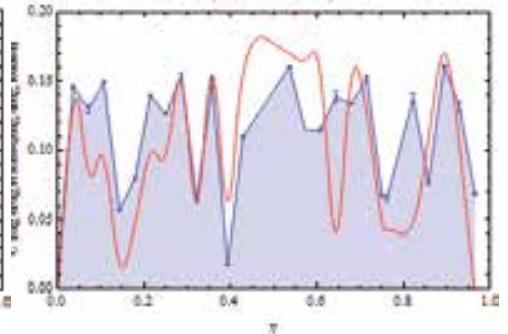

(cs, $x^{2}-0.6755$ )

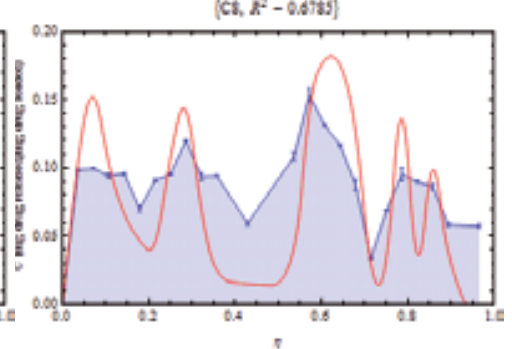




\section{Conclusions}

i. Scale relativistic framework is implemented by passing to a fluid-like description (the fractality of space), considering the velocity field a fractal function explicitly depending on a scale variable (the fractal geometry of each geodesic) and defining two fractal velocity fields which are fractal functions of the scale variable $d t$ (the non-differentiability of space).

An application of these principles to the motion equation of free particles leads to the occurence of a supplementary TISE (time independent, Schrödinger-type equation) and the following interesting results :

- $\zeta(x)$ behaves like a wave function on small distances (the same magnitude as the Compton length);

- for $\gamma(x)$ a velocity potential well, $U(x)$ is quantified;

- for the harmonic oscillator case, the limit velocity $\chi$ has discrete values, and only the first value is less than the velocity of light, $c$;

- in the double-well velocity potential, the complex velocity $U(x)$ is again quantized, this time the levels are equally spaced at a value of $\hbar \ln 2$;

- if one takes $\phi_{R}=\phi_{L}=\pi / 2$, singularities are obtained for $x-x_{0}=\Lambda / 2$ and for $x-x_{0}=$ $\Lambda / 4$ one gets minima for $U(x)=c$ in a double-well velocity potential;

- since we considered here the one-dimensional case we get the solution of a single row of rectilinear vortices, which has already been referred to as characterizing a surface of discontinuity;

- a typical bound state in a double-well has two classically allowed regions, where the velocity potential is less than the limit velocity; these regions are separated by a classically forbidden region, or barrier, where the velocity potential is larger than the limit velocity;

- for tunneling case, there is a nonzero transmission, reflection coefficient, whichleads to the proof of the transport of the $V$ field by the motion of the Newtonian fluid with velocity $U(x)$, on small distances (of the order of magnitude of Compton length).

ii. We analyzed vacuum from the Casimir cavity, considered a non-differentiable, Newtonian, 2D non-coherent quantum fluid, by writing the Navier-Stokes equations inscalerelativity theory's framework. As a result the following results may beextracted:

- the (vector) velocity field $V$ and/or the (scalar) density field $\rho$ behave like a wave function on small distances (the same magnitude as the Compton length);

- the (vector) velocity field $V$ and/or the (scalar) density field $\rho$ are transported by the motion of the Newtonian fluid with velocity $U$, on small distances (the same magnitude as the Compton length);

Also, the entities assimilated to vortex-type objects from the Casimir cavity, initially non-coherent, become coherent due to constraints induced by the presence of walls and generate pressure along the $O x$ and $O y$ axis, thus one can stress out : 
- the pressure $p_{y}$ on the plates, is negative and an attractive force results, as is the case of the Casimir force;

- besides the pressure $p_{y}$ acting on the plates, there must be yet another pressure, $p_{x \prime}$ acting along the $O x$ axis;

- the order of magnitude of this force, $p_{y} \cong 6.1810^{10} \mathrm{~N} \mathrm{~m}^{-2}$ is the same with the value of the classical Casimir force calculation, $F_{C} \cong 2.0810^{10} \mathrm{~N} \mathrm{~m}^{-2}$;

- in the case of the Casimir cavity from inside a rectangular enclosure of sides $d_{1}, d$, the plates induce constraints along both $O x$ and $O y$ axis, and one can notice that if the two parameters $m$ and $n$ have close values, the force acting on the Casimir rectangle is always negative and for parameters $m$ and $n$ very asymmetric the force has negative and positive domains, in agreement with the calculus of regularization using the Abel-Plana formula.

iii. Using fractional calculus, the fractal "diffusion" equation give rise to Weibull relation, a statistical distribution function of wide applicability, inclusively in drug release studies. In this approach, we consider all the simultaneous phenomena involved, equivalent with complexity and fractality, offering, in this way, a physical base to this equation and for its parameters. They are functions of fractal dimension of the curves on which drug release mechanism takes place, dimension that is a measure of the complexity and nonlinear dynamics of the system, dependent on the diffusion order.

This theory offers new alternatives for the theoretical study of drug release process (on large time scale) in the presence of all phenomena and considering a highly complex and implicitly, non linear system. Consequently, the concentration field has cnoidal oscillation modes, generated by similar trajectories of drug particles. This means that the drug particle ensemble (at time large scale) works in a network of non linear oscillators, with oscillations around release boundary. Moreover, the normalized concentration field simultaneously depends on normalized time non linear system (through s parameter).

\section{Author details}

M. Agop ${ }^{1}$, C.Gh. Buzea ${ }^{2}$, S. Bacaita ${ }^{1}$, A. Stroe ${ }^{3}$ and M. Popa ${ }^{4}$

1 Department of Physics, Faculty of Machine Manufacturing and Industrial Management, "Gheorghe Asachi" Technical University of Iasi, Iasi, Romania

2 National Institute of Research and Development for Technical Physics, Romania

3 National College “Nicolae Balcescu”, Al. I. Cuza Bvd., Braila, Romania

4 Department of Natural and Synthetic Polymers, Faculty of Chemical Engineering and Environmental Protection, “Gheorghe Asachi” Technical University of Iasi, Iasi, Romania 


\section{References}

[1] L. Nottale, Fractal Space-Time and Microphysics: Towards a Theory of Scale Relativity, World Scientific Publishing, Singapore, 1993.

[2] L. Nottale, Scale Relativity and Fractal Space-Time - A New Approach to Unifying Relativity and Quantum Mechanics, Imperial College Press, London, 2011.

[3] L. Nottale, Fractals and the quantum theory of space time, Int. J. Mod. Phys. A, vol. 4, no. 19, pp. 5047-5117, 1989.

[4] L. D. Landau, E. M. Lifshitz, Fluid Mechanics, $2^{\text {nd }}$ Edition, Butterworth Heinemann Publishing, Oxford, 1987.

[5] B.B. Mandelbrot, The Fractal Geometry of Nature, Freeman, San Francisco, USA, 1983.

[6] J. F. Gouyet, Physique et Structures Fractals, Masson, Paris, 1992.

[7] M.S. El Naschie, O. E. Rössler, I. Prigogine, Quantum Mechanics, Diffusion and Chaotic Fractals, Elsevier, Oxford, 1995.

[8] P. Weibel, G. Ord, O. E. Rösler, Space Time Physics and Fractality, Springer Dordrecht, 2005.

[9] M. Agop, N. Forna, I. Casian Botez, C. Bejenariu, New theoretical approach of the physical processes in nanostructures, J. Comput. Theor. Nanosci., vol. 5, no. 4, pp. 483-489, 2008.

[10] I. Casian-Botez, M. Agop, P. Nica, V. Paun, G.V. Munceleanu, Conductive and convective types behaviors at nano-time scales, J. Comput. Theor. Nanosci., vol. 7, no. 11, pp. 2271-2280, 2010.

[11] L. Nottale, Scale-relativity and quantization of the universe. I. Theoretical framework, Astron. Astrophys., vol. 327, no. 3, pp. 867-889, 1997.

[12] P.D.McCormack, L. Crane, Physical Fluid Mechanics, Academic Press, LondonNewYork, 1973.

[13] M. Agop, P.D.Ioannou, C.Gh.Buzea, P.Nica, Hydrodynamic formulation of scale relativity theory and unified superconductivity by means of a fractal string, Physica C, vol. 390, no. 1, pp. 37-55, 2003.

[14] M. Agop, P.E. Nica, P.D. Ioannou, A. Antici, V.P. Paun, Fractal model of the atom and some properties of the matter through an extended model of scale relativity, The European Phys. J. D, vol. 49, no. 2, pp. 239-248, 2008.

[15] V. P. Maslov, M. V.Fedoriuk, Semiclassical Approximation in Quantum Mechanics, Reidel, Dordrecht, 1981.

[16] W. Nolting, Quantenmechanik-Methoden und Anwendungen., Grundkurs Theoretische Physik Springer, 2004. 
[17] C. S. Park, M. G. Jeong, S.-K. Yoo, D.K. Park, arXiv:hep-th/9808137v1, 1998.

[18] R. Landauer, Irreversibility and Heat Generation in the Computing Process, IBM J. Res. Dev., vol. 5, no. 3, pp. 183-192, 1961.

[19] S. Lloyd, Use of mutual information to decrease entropy: Implications for the second law of thermodynamics, Phys. Rev. A, vol. 39, no. 10, pp. 5378-5386, 1989.

[20] C.H. Bennett, The thermodynamics of computation - a review, Int. J. Theor. Phys., vol. 21, no. 12, pp. 905-940, 1982.

[21] E. Fredkin, T. Toffoli, Conservative logic, Int. J. Theor. Phys., vol. 21, no. 3-4, pp. 219-253, 1982.

[22] W.H. Zurek, Algorithmic randomness and physical entropy, Phys. Rev. A, vol. 40, no. 8, pp. 4731-4751, 1989.

[23] C.R. Calidonna, A. Naddeo, Towards reversibility in a JJL qubit qualitative model by means of CAN2 paradigm, Phys. Lett. A, vol. 358, no. 5-6, pp. 463-469, 2006.

[24] R. Alicki, M. Horodecki, P. Horodecki, R. Horodecki, Thermodynamics of Quantum Information Systems - Hamiltonian Description, Open Sys. \& Information Dyn., vol. 11, no. 3, pp. 205-217, 2004.

[25] V. S. Popov, B. M. Karnakov, V. D. Mur, On matching conditions in the WKB method, Phys. Lett. A, vol. 210, no. 6, pp. 402-408, 1996.

[26] C. Eltschka, H. Friedrich, M. J. Moritz, J. Trost, Tunneling near the base of a barrier, Phys. Rev. A, vol. 58, no. 2, pp. 856-861, 1998.

[27] M. J. Moritz, Tunneling and reflection of long waves, Phys. Rev. A, vol. 60, no.2, pp. 832-841, 1999.

[28] S.K. Lamoreaux, Demonstration of the Casimir force in the 0.6 to $6 \mu \mathrm{m}$ range, Phys. Rev. Lett., vol. 78, no. 1, pp. 5-8, 1997.

[29] M. Bordag, U. Mohideen, V.M. Mostepanenko, New developments in the Casimir effect, Phys. Rep., vol. 353, no. 1-3, pp. 1-206, 2001.

[30] K. Milton, The Casimir effect: recent controversies and progress, J. Phys. A, vol. 37, no. 38, pp. R209-R277, 2004.

[31] V.V. Nesterenko, G. Lambiase, G. Scarpetta, Calculation of the Casimir energy at zero and finite temperature: Some recent results, Riv. Nuovo Cimento, vol. 027, no. 06, pp. 1-74, 2004.

[32] S.K. Lamoreaux, The Casimir force: background, experiments and applications, Rep. Prog. Phys., vol. 68, no. 1, pp. 201-236, 2005.

[33] G. Barton, Perturbative Casimir energies of dispersive spheres, cubes and cylinders, J. Phys. A: Math. Gen., vol. 34, no., pp. 4083-4114, 2001. 
[34] K. A. Milton, The Casimir Effect: Physical Manifestations of Zero-Point Energy (chapter 2), World Scientific, 2001.

[35] L. E. Ballentine, Quantum Mechanics (chapter 19), Prentice-Hall, 1990.

[36] C. Itzykson, J. B. Zuber, Quantum Field Theory (chapter 3), McGraw-Hill, 1985.

[37] K. Huang, Quantum Field Theory (chapter 5), John Wiley, 1998.

[38] M. Ignat, N. Rezlescu, C.Gh.Buzea, C. Buzea, About the pair breaking-time in superconductors, Phys. Lett. A vol. 195, no. 2, pp. 181-183, 1994.

[39] C. Gh. Buzea, M. Agop, N. Rezlescu, C. Buzea, T. Horgos, V. Bahrin, The Time of Diffusion and Infinite Conductivity of High-Tc Superconductors, Phys. Stat. Sol.(b), vol. 205, no. 2, pp. 595-602, 1998.

[40] M. Agop, C. Gh. Buzea, N. Rezlescu, C. Buzea, C. Marin, Wave guide perturbative solutions for the Ginzburg-Landau equation.: Infinite conductivity and discrete values of the critical temperature in superconductors, Physica C, vol. 313, no. 3-4, pp. 219-224, 1999.

[41] F. Bowman, Introduction to elliptic functions with applications, English University Press London, 1961.

[42] O. Mayer, Special issues in the theory of the functions with one complex variable, vol. II, Academic Press Bucharest, 1990.

[43] M. Agop, V. Griga, C. Buzea, C. Stan, D. Tatomir, The uncertainty relation for an assembly of Planck-type oscillators. A possible GR-quantum mechanics connection, Chaos, Solitons \& Fractals, vol. 8, no. 5, pp. 809-821, 1997.

[44] S. Titeica, Quantum Mechanics, Academic Press Bucharest, 1984.

[45] M. Agop, N. Rezlescu, G. Kalogirou, Nonlinear Phenomena in Materials Science, Graphics Art Publishing House Athens, 1999.

[46] G. Burns, High-Temperature Superconductivity, Academic Press San Diego, 1992.

[47] M. Bordag, G. L. Klimchitskaya, U. Mohideen, V. M. Mostepanenko, Advances in the Casimir Effect., Oxford Univ. Press, 2009.

[48] M. Agop, N. Forna, I. Casian Botez, C. Bejenariu, New theoretical approach of the physical processes in nanostructures, J. Comput. Theor. Nanosci., vol. 5, no. 4, pp. 483-489, 2008.

[49] I. Casian-Botez, M. Agop, P. Nica, V. Paun, G.V. Munceleanu, Conductive and convective types behaviors at nano-time scales, J. Comput. Theor. Nanosci., vol. 7, no. 11, pp. 2271-2280, 2010.

[50] L. Jude, Mathematics physics equations. Theory and applications, Matrix Rom Publishing Bucharest, 2010. 
[51] K. B. Oldham, J. Spanier, The Fractional Calculus: Theory and Applications of Differential and Integration to Arbitrary Order, Dover Publications New York, 2006.

[52] A. A. Kilbas, H. M. Srivastava, J. J. Trujilto, Theory and Applications of Fractional Differential Equations, Elsevier Armsterdam, 2006.

[53] S. Bacaita, C. Uritu, M.Popa, A. Uliniuc, C. Peptu, M. Agop, Drug release kinetics from polymer matrix through the fractal approximation of motion, Smart Materials Research, article ID 264609, doi:10.1155/2012/264609, 2012.

[54] A. J. Lichtenberg, Phase-Space Dynamics of Particle, John Wiley and Sons Inc. New York, 1969.

[55] S. Popescu, Actual issues in the physics of self-structured systems, Tehnopress Publishing, Iasi, Romania, 2003.

[56] M. Toda, Theory of Nonlinear Lattices, Springer, Berlin, 1989.

[57] S. Bacaita et. al., Nonlinearities in Drug Release Process from Polymeric Microparticles: Long-Time-Scale Behaviour, Journal of Applied Mathematics, vol. 2012, article ID 653720, 2012, doi:10.1155/2012/653720. 




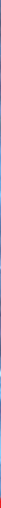

\section{Edited by Paul Bracken}

The development of quantum mechanics has taken physics in a vastly new direction from that of classical physics from the very start. In fact, there continue at present

to be many developments in the subject of a very fundamental nature, such as implications for the foundations of physics, physics of entanglement, geometric phases, gravity and cosmology and elementary particles as well. It is hoped the papers in this volume will provide a much needed resource for researchers with regard to current topics of research in this growing area.

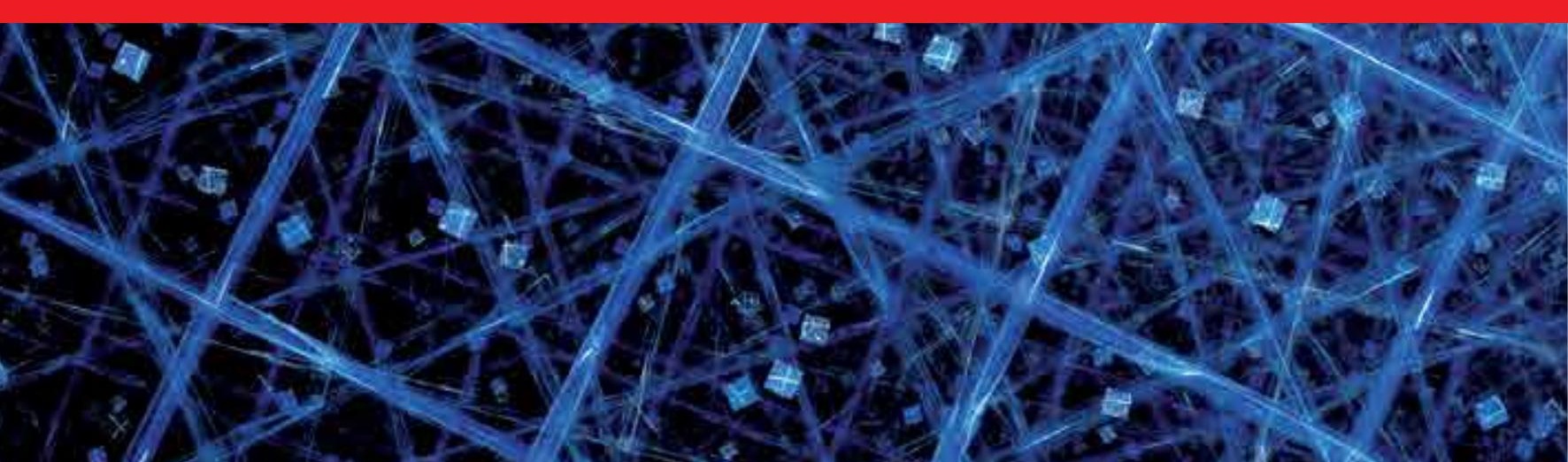

